LBL-90 Revised

October 1999

\title{
A GUIDE TO
}

\section{EXPERIMENTAL PARTICLE PHYSICS LITERATURE}

\section{4-1998}

\author{
V.V. Ezhela, B.B. Filimonov, S.B. Lugovsky, V.S. Lugovsky, \\ B.V. Polishchuk, E.A. Razuvaev, S.R. Slabospitsky, S.I. Striganov, \\ Yu.G. Stroganov, and O.V. Zenin \\ Compilation, Analysis and Systematization Group, ${ }^{*}$ Institute for High Energy Physics \\ Protvino, Moscow Region, Russian Federation \\ G.A. Arutyunyants and S.M. Kiselev \\ Institute of Theoretical and Experimental Physics \\ Moscow, Russian Federation \\ A.P. Jerusalimov \\ Joint Institute for Nuclear Researh \\ Dubna, Moscow Region, Russian Federation \\ B. Armstrong, R.M. Barnett, P.S. Gee, \\ D. E. Groom, T.G. Trippe, and W. Yao \\ Berkeley Particle Data Group,$^{\dagger}$ Lawrence Berkeley National Laboratory \\ Berkeley, California, 94720, USA
}

\begin{abstract}
This is an indexed guide to experimental particle physics literature for the years 1994-1998. About 4100 papers are indexed by Beam/Target/Momentum, Reaction/Momentum/Data-Descriptor, Particle/Decay, Accelerator/Experiment/Detector. All indices are cross-referenced to the paper's title and reference in an ID/Reference/Title index. The information is also available on a regularly updated DATAGUIDE database from the World Wide Web http://wwwppds . ihep.su:8001/ppds.html.
\end{abstract}

*The COMPAS Group is supported in part by the Russian Foundation for Basic Research under contract FRBR1999-07-90356.

${ }^{\dagger}$ The Berkeley Particle Data Group is supported by the Director, Office of Science, Office of High Energy and Nuclear Physics, Division of High Energy Physics of the U.S. Department of Energy under Contract No. DE-AC03-76SF00098, and by the U.S. National Science Foundation under Agreement No. PHY-9703140. Partial funding to cover the cost of this publication is also provided by an implementing arrangement between the governments of Japan (Monbusho) and the United States (DOE) on cooperative research and development. 


\section{DISCLAIMER}

This report was prepared as an account of work sponsored by an agency of the United States Government. Neither the United States Government nor any agency thereof, nor any of their employees, make any warranty, express or implied, or assumes any legal liability or responsibility for the accuracy, completeness, or usefulness of any information, apparatus, product, or process disclosed, or represents that its use would not infringe privately owned rights. Reference herein to any specific commercial product, process, or service by trade name, trademark, manufacturer, or otherwise does not necessarily constitute or imply its endorsement, recommendation, or favoring by the United States Government or any agency thereof. The views and opinions of authors expressed herein do not necessarily state or reflect those of the United States Government or any agency thereof. 


\section{DISCLAIMER}

Portions of this document may be illegible in electronic image products. Images are produced from the best available original document. 


\section{TABLE OF CONTENTS}

\section{Introduction}

1. Overview ............................. 1

2. Scope of this Compilation . . . . . . . . . . . . . . . . . . . . . . . 1

3. Using this Compilation . . . . . . . . . . . . . . . . . . . . . . . . . 1

4. Particle Physics Data System . . . . . . . . . . . . . . . . . . . . . . . . . . 2

5. Accessing the PPDS Databases . . . . . . . . . . . . . . . . . . . . . . . 2

References .............................. 3

\section{Indices}

ID/Reference/Title Index . . . . . . . . . . . . . . . . . . . . . . . . . . . . . . . . 5

Beam/Target/Momentum Index . . . . . . . . . . . . . . . . . . . . . . . 117

Reaction/Momentum/Data-Descriptor Index . . . . . . . . . . . . . . . . . . . 157

Particle/Decay Index . . . . . . . . . . . . . . . . . . . . . . . . . . . . . . . . . . 323

Accelerator/Experiment/Detector Index . . . . . . . . . . . . . . . . . . . . . . 399

\section{Vocabularies}

Particle Vocabulary . . . . . . . . . . . . . . . . . . . . . . . . . . . . . . 425

Accelerator Vocabulary . . . . . . . . . . . . . . . . . . . . . . . . . . . . . . . 443

Detector Vocabulary . . . . . . . . . . . . . . . . . . . . . . . . . . . . 447

Data Descriptor Vocabulary . . . . . . . . . . . . . . . . . . . . . . 453 


\section{Overview}

This report is a guide to experimental particle physics papers containing new experimental data published or preprinted during the years 1994-1998. Earlier papers may be found in our previous editions. ${ }^{1}$ No actual data are presented in this report. The report is based on the DATAGUIDE database, maintained by the COMPAS Group at IHEP Protvino using the BDMS system under OpenVMS. The database is accessible from the World Wide Web:

http://wwwppds. ihep.su:8001/ppds.html.

In this Introduction, Sec. 2 discusses the scope of this compilation and its sources of information. Sec. 3 tells how to use this book. Sec. 4 describes publicly-accessible Particle Physics Data System databases. Sec. 5 tells how to access those databases, including the DATA GUIDE database on which this book is based.

The body of this report contains five Indices followed by four Vocabularies. In the Indices, papers are referenced by an identifier (ID) consisting of the first author's name and the year of first preprinting or publication, e.g. Smith 91 .

- The ID/Reference/Title Index lists IDs, each with the title and preprint number and/or publication reference. From the ID of a paper in any of the other Indices, you can find the full reference in the this Index.

- The Beam/Target/Momentum Index lists papers by beam particle, target particle, and beam momentum (or center-of-mass energy).

- The Reaction/Momentum/Data-Descriptor Index lists papers by the full reaction.

- The Particle/Decay Index lists papers by a specific particle and its decay mode.

- The Accelerator/Experiment/Detector Index lists papers by the facility at which the experiment was done.

The four Vocabularies that follow the Indices are:

- The Particle Vocabulary gives our spelling conventions for particle names. We use the same nomenclature for particles as does the "Review of Particle Physics." 2

- The Accelerator Vocabularies give the names and abbreviations assigned to accelerators.

- The Detector Vocabularies give the names and abbreviations assigned to detectors.

- The Data Descriptor Vocabulary gives abbreviations used in the Reaction/Momentum/Data-Descriptor Index.

Please bring any errors and omissions you may find to our attention by e-mail to ezhela@mx. ihep.su.

\section{Scope of this Compilation}

Compilation of this report begins with extraction of bibliographic data from a scan of the literature made by the COMPAS Group at IHEP Protvino. Additional bibliographic data comes from the SLAC-SPIRES $H E P$ database, a joint project of the SLAC and DESY libraries. From the papers in these databases, we select those with experimental data. When in doubt about the newness or originality of data, we include the paper. All decisions are made by a physicist.
"Data" means not only the obvious experimentally measured quantities, but also some derived quantities, such as partial-wa ve amplitudes. Also, theoretical papers that extract new information from experiments are included. We exclude instrumentation papers and papers mainly of interest only to nuclear physicists, such as nuclear-level or other nuclear-structure measurements. There are, of course, gray areas: many particle physics experiments measure scattering phenomena off nuclei. Heavy-ion experiments are also sometimes of interest to elementary particle physicists. In these areas, we generally include papers that report more than just nuclear-structure parameters and that involve beam energies above about $1 \mathrm{GeV} /$ nucleon, or that report measurements on light nuclear targets, such as the isotopes of hydrogen, helium, or lithium. Other papers are decided on a case-by-case basis by a physicist.

We also publish "Current Experiments in Particle Physics," LBL-91 Revised (1996), ${ }^{3}$ covering current, approved experiments at the major world accelerators and underground/under water detectors. This includes a Beam/Target/Momentum Index, a Spokesperson Index, and summaries of each of the experiments.

\section{Using this Compilation}

Each paper is assigned a unique ID, consisting of the first author's name and the year of the first preprinting or publication. In case of duplicates, we append a letter to distinguish IDs, as in:

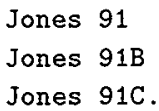

The maximum length of the ID is 16 characters; thus long names are truncated.

All references to a paper use this single ID given in the ID/Reference/Title Index. When a paper has been both preprinted and published, both references are given. In these cases, the year in the ID, which is usually that of the preprint, may not match the year of the published reference. In the very few cases where the first author of the preprint is not the same as that of the publication, the ID usually contains the preprint's first author.

To see a paper's full author list or to search for a set of papers by the name of one or more authors, one may query the $D A T A G U I D E$ (see Sections 4 and 5 below) or HEP databases:

http://ww-spires.slac.stanford.edu/find/hep.

The first page of each Index explains its use. Here are a few of our conventions.

- We follow the particle naming scheme used in the "Review of Particle Physics"2 and in the previous three editions of this report; see the latest edition of the "Review" for a complete description. A full list of particle terms, including terms used to describe collisions and decays, is given in the Particle Vocabulary.

- Some particle names actually represent groups of particles. For example,

* " $\mathrm{X}$ " is used for inclusive measurements or, if used as the only particle in the final state, for total-crosssection measurements; 
* "inelastic" represents a sum over all inelastic final states;

* "jet" represents a jet of particles, treated as a single entity;

* "(vees)" represents zero or more unspecified neutral vees, "vee (vees)" represents one or more unspecified neutral vees, and "2vee (vees)" represents two or more of the same;

* "mult[charged-hadron]" represents a collection of reactions for which the multiplicity distribution of charged hadrons has been measured, or represents a sum over final states with the lower and upper bounds on multiplicity specified;

* " $0 \gamma$ " means final states in which the occurrence of photons in the inclusive part of the final state has been excluded.

- In using the computer database, antiparticles that are commonly written with a bar over the name are spelled with the letters "BAR" appended to the particle name, e.g., PBAR, LAMBDA/CBAR-.

- Particles tend to be encoded in the same language that the experimenters used. Thus, some inevitable ambiguity occurs; for example, "charged" in one paper may be called "charged-hadron" in another.

- Reactions are listed in the shortest form possible. Identical particles are grouped together, so the reaction $\pi^{-} p \rightarrow$ $\pi^{+} \pi^{+} \pi^{-} \pi^{-} \pi^{-} p$ will appear as $\pi^{-} p \rightarrow p 2 \pi^{+} 3 \pi^{-}$.

\section{Particle Physics Data System}

The PPDS databases are maintained by the IHEP Protvino COMPAS Group under the Berkeley Database Management System (BDMS) with input from the worldwide Particle Data Group collaboration. This section describes publicly accessible PPDS databases. See Sec. 5 for information on accessing these databases.

- DATAGUIDE, on which this report is based, contains two groups of keys:

* Bibliographic: ID, references, year of preprinting or publication, authors and affiliations, and experiment number.

* Topical: beam particle, target particle, reactions, particles in the final states of reactions, momenta in initial states, types of data obtained, particles whose property has been measured, accelerator and/or detector, and initial state polarization.

- REACTIONS is a compilation of numerical experimental particle physics reaction data, including data from 2-body (and quasi-2-body) scattering, $e^{+} e^{-}$annihilation, and inclusive hadron, photon, and lepton physics such as total and differential cross sections, fragmentation functions, structure functions, and polarization measurements. It covers 1952 to the present and is updated approximately quarterly. This database is a collaboration of Durham/RAL and the COMPAS group.

- CS, regularly updated from the REACTIONS database, contains data from CERN-HERA, UCRL, and LBL crosssection compilations covering 1950 to the present.
- $P P$ contains information on particle properties derived from the "Review of Particle Physics" Summary Tables.

- VOCABULARY controls usage of particle names, accelerator names, detector names, and data descriptors in the above databases.

\section{Accessing the PPDS Databases}

The DATAGUIDE, REACTIONS, and CS databases are reachable from the World Wide Web:

http://wwwppds . ihep .su: 8001/ppds.html.

Unfortunately, searches via the WWW are less powerful and more limited in scope than searches done by telnetting to our databases directly. The PPDS databases can be accessed interactively via INTERNET:

telnet $\mathrm{m} 10$. ihep.su.

Then login to the captive account PPDS_PUBLIC (no password is required).

In the following description, words in Typewriter Font must be typed as given. Only the letters in UPPER CASE are necessary and these must be entered in upper case. Italic words are variables for which the user substitutes an appropriate value, again in upper case.

- For a short explanation of the database, type: HELP

- For a list of BDMS commands, type: ?

- For an explanation of a particular BDMS command, type: ? command-word (e.g., ?FInd, ?HELpbase, ??)

- To see the record structure and names of keys for searching, type:

FDT

- To browse the index of a key, type: INDex, key-name (e.g., INDex , $\mathrm{AC}$ )

- To search an index, type: FInd key-name $=k e y$-value

Note the use of ' $* *$ ' to terminate each search statement and the use of ' $;$ ' to separate data elements.

The following examples typify the FIND search command: FInd $\mathrm{AC}=\mathrm{BNL} ; * *$

FInd $\mathrm{AC}=\mathrm{BNL} ; \mathrm{OR} \mathrm{AC}=\mathrm{BONN} ; * *$

Each successful search produces a list of all previous searches and labels them with a 'set number.' A previous search result can then be combined with a current search by use of set numbers:

FInd (1) and RE=PI+ P $\rightarrow P I+P$; **

FInd (1) and (2) **

Note that ';' is not used in searches that only use 'sets.'

Enter DIR to get a list of these set numbers and search commands.

- To do a truncated search, use a slash after the key value: FInd $\mathrm{DE}=\mathrm{HBC} / ; * *$

This finds all detectors that begin with $\mathrm{HBC}$.

- To do a string search, use $/ C$ after the key name: FInd $\mathrm{DE} / \mathrm{C}=\mathrm{BC}$; $* *$ 
This finds all detectors that have $\mathrm{BC}$ anywhere in the name.

- The following examples are WRONG:

find ac=bnl; $* *$

FInd $A C$ BNL; $* *$

FInd $\mathrm{AC}=\mathrm{BNL} * *$

(Error: uses lowercase)

(Error: no '=')

(Error: no ';')

FInd $\mathrm{AC}=\mathrm{BNL}$ OR BONN;** (Error: no ';' \& no ' $\mathrm{AC}=$ ')

- To see the results of a search with key names, type: LISt

- Or to restrict data elements shown, append the desired key names. For example:

LISt, AC, RE, SC.

The leading comma and terminal period are required.

- Or for an attractive listing, type:

Document then

Lookfile

- To save the result of a search in a file, type one of the following:

Document

DUmp

PRInt

The results are stored in the files DOC.DOC, DOC. DUM, or DOC.PRN. The first file contains a user-friendly listing, the second contains a highly compressed dump of each record (with data element and value), and the third contains a line-by-line decompressed version of the second file. Another file automatically created, DOC.AUD, contains a history of your commands.

\section{REFERENCES}

1. V.V. Ezhela et al., "A Guide to Experimental Particle Physics Literature," LBL-90 Revised (1996).

S.I. Alekhin et al., "A Guide to Experimental Elementary Particle Physics Literature," LBL-90 Revised (1993).

S.I. Alekhin et al., "A Guide to Experimental Elementary Particle Physics Literature," LBL-90 Revised (1990).

G.P. Yost et al., "A Guide to Data in Elementary Particle Physics," LBL-90 Revised (1986).

C.P. Horne et al., "Indexed Compilation of Experimental High Energy Physics Literature," LBL-90, 1978.

2. C. Casso et al. (Particle Data Group), "Review of Particle Physics," Eur. Phys. C3, 1 (1998).

3. H. Galić et al., "Current Experiments in Particle Physics," LBL-91 Revised (1996). 
This Index provides the full reference and title and a short identifier (ID) for each of the papers referred to in the later Indices. The ID consists of the name of the first author and the year the paper appeared, as in JONES 91. Other papers with the same first author and year are listed as JONES 91B, JONES 91C, etc.

One may use this index to see if a preprint has been published. Note, however, that the year of preprinting and the year of publishing are often different, and our ID is usually that of the year of preprinting.

Due to text processing procedures, titles of papers in this index may differ slightly from the original titles, especially regarding particle names.

\section{Illustrative Key}

Document ID: all other indices in this volume refer to this paper by this ID.

Primary Reference: the journal reference for this paper, if the paper was published.

Additional References: the preprint number (occasionally there is more than one).

Title of Paper
Dougherty 88

Dowell 88

Drechsel 85

Dropesky 86

Druzhinin 88

Dubar 89

Dubinina 88

Duffy 85

Dugan 85B

Dukhovskoj 87
LBL-26303

An Experimental Investigation of Double Beta Decay of ${ }^{100}$ Mo

CERN-EP-88-154

Recent Results from the UA1 Experiment

Phys. Rev. Lett. 54:30,1985

Search for Anomalous Fragments of ${ }^{56} \mathrm{Fe}$ Using Plastic Nuclear Track Detectors

Phys. Rev. C32:1305,1985

Excitation Functions for the Production of ${ }^{18} \mathrm{Fl}$ and ${ }^{24} \mathrm{Na}$ from $\mathrm{Al}$ and $\mathrm{Si}$ with Fast Pions

Z. Phys. C37:1,1988; NOVO-87-52

Search for Rare Radiative Decays of the $\phi$ Meson at VEPP-2M

Yad. Phys. 49:1239,1989

Parametrization of Total Cross Sections at Intermediate Energies

Pisma Zh. Eksp. Teor. Fiz. 48:233,1988

Observation of the Slow Pion Production in the Nucleus Nucleus Interactions

Phys. Rev. Lett. 55:1816,1985

$A$-dependence of Charm Production

Phys. Rev. Lett. 55:170,1985; HUTP-85/A033

New Neutrino Constraints on Majorana Mass Matrices

Yad. Phys. 47:1816.1988: ITEP-87-198

Measurement of the Total Cross Sections of the Proton Interactions with Nuclei ${ }^{6} \mathrm{Li},{ }^{7} \mathrm{Li}$, and ${ }^{9} \mathrm{Be}$ at $2 \mathrm{GeV} / c$ 
Abachi 93B

Abachi 94

Abachi 94B

Abachi $94 \mathrm{C}$

Abachi 94D

Abachi 95B

Abachi 95C

Abachi 95E

Abachi $95 \mathrm{~F}$

Abachi $95 \mathrm{G}$

Abachi 95H

Abachi 95I

Abachi 95J

Abachi 95K

Abachi $95 \mathrm{~L}$

Abachi 95M

Abachi $95 \mathrm{~N}$

Abachi 950

Abachi 95P

Abachi 95Q

Abachi 95R

Abachi 95S

Abachi 95T

Abachi 95U

Abachi 95V

Abachi 95W

Abachi 95X

Abachi 95Y

Abachi $95 \mathrm{Z}$

Abachi 95ZB

Abachi 95ZC

Abachi 952D

Abachi 95ZE

Abachi 95ZF

Abachi $952 \mathrm{Z}$

Abachi 95ZH

Abachi 95ZI

Abachi 95ZK

Abachi 95ZL
Phys. Rev. Lett. 72:965, 1994; FERMILAB-PÜB-93-340-E; D0-NOTE-1974

First Generation Leptoquark Search in $p \bar{p}$ Collisions at $\sqrt{s}=1.8 \mathrm{TeV}$

Phys. Rev. Lett. 72:2332, 1994; FERMILAB-PUB-94-005-E; D0-NOTE-2021

Rapidity Gaps between Jets in $p \bar{p}$ Collisions at $\sqrt{s}=1.8 \mathrm{TeV}$

Phys. Rev. Lett. 74:2422, 1995; FERMILAB-PUB-94-354-E Search for High Mass Top Quark Production in $p \bar{p}$ Collisions at $\sqrt{s}=1.8 \mathrm{TeV}$

Phys. Rev. Lett. 74:3548, 1995; FERMILA B-PUB-94-409-E

Inclusive $\mu^{-}$and $b$-Quark Production Cross Sections in $p \bar{p}$ Collisions at $\sqrt{s}=1.8 \mathrm{TeV}$

Phys. Rev. Lett. 72:2138, 1994; FERMILAB-PUB-94-004-E

Search for the Top Quark in $p \bar{p}$ Collisions at $\sqrt{s}=1.8 \mathrm{TeV}$

Phys. Rev. Lett. 75:1028, 1995; FERMILAB-PUB-95-042-E

Measurement of the $Z^{0} Z^{0} \gamma$ and $Z^{\circ} \gamma \gamma$ Couplings in $\bar{p} p$ Collisions at $\sqrt{s}=1.8 \mathrm{TeV}$

Phys. Rev. Lett. 75:1023, 1995; FERMILAB-PUB-95-044-E

Search for $W^{ \pm}$Boson Pair Production in $p \bar{p}$ Collisions at $\sqrt{s}=1.8 \mathrm{TeV}$

Phys. Rev. Lett. 75:1034, 1995; FERMILAB-PUB-95-101-E; HEPEX-9505007

Measurement of the $W W \gamma$ Gauge Boson Couplings in $p \bar{p}$ Collisions at $\sqrt{s}=1.8 \mathrm{TeV}$

Phys. Rev. Lett. 75:618, 1995; FERMILAB-PUB-95-057-E

Search for Squarks and Gluinos in $p \bar{p}$ Collisions at $\sqrt{s}=1.8 \mathrm{TeV}$

Phys. Rev. Lett. 74:2632, 1995; FERMILAB-PUB-95-028-E

Observation of the Top Quark

FERMILAB-CONF-95-217-E

Measurement of the Inclusive Triple Differential Dijet Cross Section, $d^{3} \sigma / d E_{T} d \eta_{1} d \eta_{2}$ in $p \bar{p}$ Collisions at $\sqrt{s}=1.8 \mathrm{TeV}$

Phys. Rev. Lett. 75:1456, 1995; FERMILAB-PUB-95-130-E

$W^{ \pm}$and $Z^{0}$ Boson Production in $p \bar{p}$ Collisions at $\sqrt{s}=1.8 \mathrm{TeV}$

FERMILAB-CONF-95-206-E

Measurement of the $\Upsilon$ Cross Section at Do using Dimuons

FERMILAB-CONF-95-210-E

Searches for New Gauge Bosons using the Do Detector

FERMILAB-CONF-95-193-E

Search for Squarks and Gluinos in $p \bar{p}$ Collisions at the Do Detector

FERMILAB-CONF-95-207-E

Inclusive Dimuon and $b$ Quark Production Cross Sections in $p \bar{p}$ Collisions at $\sqrt{s}=1.8 \mathrm{TeV}$

FERMILAB-CONF-95-251-E

Diphoton Production in $p \bar{p}$ Collisions at $\sqrt{s}=1.8 \mathrm{TeV}$

FERMILAB-CONF-95-249-E

Limits on the Anomalous $Z^{0} Z^{0} \gamma$ and $Z^{0} \gamma \gamma$ Couplings in $p \bar{p}$ Collisions at $\sqrt{s}=1.8 \mathrm{TeV}$

FERMILAB-CONF-95-208-E

Inclusive Muon and $b$ Quark Production Cross Sections in $p \bar{p}$ Collisions at $\sqrt{s}=1.8 \mathrm{TeV}$

FERMILAB-CONF-95-216-E

Rapidity Gaps between Jets at DO

FERMILAB-CONF-95-209-E

Measurement of $B^{0}-\bar{B}^{0}$ Mixing using Dimuons at Do

Phys. Lett. 357B:500, 1995; FERMILAB-PUB-95-203-E

Transverse Energy Distributions within Jets in $p \bar{p}$ Collisions at $\sqrt{s}=1.8 \mathrm{TeV}$

FERMILAB-CONF-95-215-E

Single Photon, Photon Jet and Diphoton Production at DO

FERMILAB-CONF-95-242-E

Search for $W^{ \pm}$Boson Pair Production in $p \bar{p}$ Collisions at $\sqrt{s}=1.8 \mathrm{TeV}$

Phys. Rev. D52:4877, 1995; FERMILAB-PUB-95-020-E

Top Quark Search with the DO 1902 - 1903 Data Sample

FERMILAB-CONF-95-214-E

Studies of Topological Distributions of the Three and Four Jet Events in $\bar{p} p$ Collisions at $\sqrt{s}=1800 \mathrm{GeV}$ with the Do Detector

Search for Fourth Generation Neutral Heavy Leptons

FERMILAB-CONF-95-212-E

A Study of the Strong Coupling Constant using $W^{ \pm}+$Jets Processes

Phys. Rev. Lett. 75:3618, 1995; FERMILAB-PUB-95-185-E; HEPEX-9507002

Second Generation Leptoquark Search in $p \bar{p}$ Collisions at $\sqrt{s}=1.8 \mathrm{TeV}$

FERMILAB-CONF-95-205-E

$J / \psi(1 S)$ Production in $p \bar{p}$ Collisions at $\sqrt{s}=1.8 \mathrm{TeV}$

Phys. Rev. Lett. 76:734, 1996; FERMILAB-PUB-95-302-E; HEPEX-9509013

Jet Production via Strongly Interacting Color Singlet Exchange in $p \bar{p}$ Collisions

Phys. Lett. 358B:405, 1995; FERMILAB-PUB-95-283-E

Search for Heavy $W^{ \pm}$Boson in $1.8 \mathrm{TeV} p \bar{p}$ Collisions

FERMILAB-CONF-95-259-E; HEPEX-9508002

Tests of QCD in $W^{ \pm}$and $Z^{0}$ Production at Tevatron

FERMILAB-CONF-95-250-E

Search for Anomalous $W W$ and $W Z^{0}$ Production at Do

FERMILAB-CONF-95-184-E

Search for First and Second Generation Leptoquarks at Do

FERMILAB-CONF-95-218-E

Rapidity Correlations between High $p_{T}$ Intermediate Vector Bosons and Jets in $\bar{p} p$ Collisions at $\sqrt{s}=$ $1.8 \mathrm{TeV}$

FERMILAB-CONF-95-204-E

Transverse Energy Distributions within Jets in $p \bar{p}$ Collisions at $\sqrt{s}=1.8 \mathrm{TeV}$

Phys. Rev. D53:6000, 1996; FERMILAB-PUB-95-296-E; HEPEX-9509005

Studies of Topological Distributions of Inclusive Three- and Four-Jet Events in $\bar{p} p$ Collisions at $\sqrt{s}=1800$

$\mathrm{GeV}$ with the Do Detector

FERMILAB-CONF-95-361-E

Rapidity Dependence of the Inclusive $J / \psi(1 S)$ Production in $p \bar{p}$ Collisions at $\sqrt{s}=1.8 \mathrm{TeV}$ 
Abachi 95ZM

Abachi 95ZN

Abachi 9520

Abachi 95ZP

Abachi 95ZQ

Abachi 96

Abachi 96B

Abachi 96C

Abachi 96D

Abachi $96 \mathrm{E}$

Abachi 96F

Abachi 96G

Abachi 96H

Abachi 96I

Abachi 96J

Abachi $96 \mathrm{~K}$

Abachi $96 \mathrm{~L}$

Abachi $96 \mathrm{M}$

Abachi $96 \mathrm{~N}$

Abachi 960

Abachi 96P

Abachi 96Q

Abachi 96R

Abachi $96 \mathrm{~S}$

Abachi $96 \mathrm{~T}$

Abachi $96 \mathrm{U}$

Abachi $96 \mathrm{~V}$

Abachi 96W

Abachi 97

Abachi 97B

Abachi 97C

Abachi 97D

Abaev 95

Abaev 95B

Abatzis 94

Abatzis $94 \mathrm{~B}$

Abatzis $94 \mathrm{C}$

Abatzis 94D
Phys. Rev. Lett. 75:3226, 1995; FERMILAB-PUB-95-085-E; FERMILAB-PUB-95-219-E; FSU-SCRI-95-49 A Study of the Strong Coupling Constant using $W^{ \pm}+$Jets Processes

Phys. Rev. Lett. 76:3271, 1996; FERMILAB-PUB-95-412-E; HEPEX-9512007 Search for Right-Handed $W^{ \pm}$Bosons and Heavy $W^{\prime \pm}$ in $\bar{p} p$ Collisions at $\sqrt{s}=1.8 \mathrm{TeV}$

Phys. Rev. Lett. 76:2228, 1996; FERMILAB-PUB-95-385-E Search for Supersymmetric wino $_{1}$ zino $_{2}$ Production via Trilepton Final States in $p \bar{p}$ Collisions at $\sqrt{s}=$ $1.8 \mathrm{TeV}$

Phys. Rev. Lett. 76:2222, 1996; FERMILAB-PUB-95-380-E

Search for Light Top Squarks in $p \bar{p}$ Collisions at $\sqrt{s}=1.8 \mathrm{TeV}$

FERMILAB-CONF-95-187-E

Search for Light Top Squarks with the Do Detector

Phys. Lett. 370B:239, 1996; FERMILAB-PUB-96-003-E

$J / \psi(1 S)$ Production in $p \bar{p}$ Collisions at $\sqrt{s}=1.8 \mathrm{TeV}$

Phys. Rev. Lett. 77:595, 1996; FERMILAB-PUB-96-038-E

The Azimuthal Decorrelation of Jets Widely Separated in Rapidity

Phys. Rev. Lett. 77:5011, 1996; FERMILAB-PUB-96-072-E

The Isolated Photon Cross Section in the Central and Forward Rapidity Regions in $p \bar{p}$ Collisions at $\sqrt{s}$ $=1.8 \mathrm{TeV}$

Phys. Rev. Lett. 77:3303, 1996; FERMILAB-PUB-96-115-E; HEPEX-9606013

Search for Anomalous $W W$ and $W Z^{\circ}$ Production in $p \bar{p}$ Collisions at $\sqrt{s}=1.8 \mathrm{TeV}$

Phys. Lett. 385B:471, 1996; FERMILAB-PUB-96-187-E

Search for Additional Neutral Gauge Bosons

FERMILAB-CONF-96-163-E

Search for a Fourth Generation Charge -1/3 Quark via Flavor Changing Neutral Currents

FERMILAB-CONF-96-172-E

A Measurement of the Ratio of $W^{ \pm}+1$ Jet to $W^{ \pm}+0$ Jets Cross Sections and Comparisons to QCD

FERMILAB-CONF-96-249-E

Rapidity Dependence of the Inclusive $J / \psi(1 S)$ Production in $p \bar{p}$ Collisions at $\sqrt{s}=1.8 \mathrm{TeV}$

FERMILAB-CONF-96-253-E

Search for $b \rightarrow \mathrm{X}^{+} \mu^{-}$and $B^{0} \rightarrow \mu^{+} \mu^{-}$Decays in $p \bar{p}$ Collisions at $\sqrt{s}=1.8 \mathrm{TeV}$

FERMILAB-CONF-96-278-E

Color Coherence in $p \bar{p}$ Collisions at $\sqrt{s}=1.8 \mathrm{TeV}$

FERMILAB-CONF-96-258-E

Results from a Search for a Neutral Scalar Produced in Association with a $W^{ \pm}$Boson in $p \bar{p}$ Collisions at $\sqrt{s}=1.8 \mathrm{TeV}$

FERMILAB-CONF-96-252-E

Small Angle Muon and $b$ Quark Production in $p \bar{p}$ Collisions at $\sqrt{s}=1.8 \mathrm{TeV}$

FERMILAB-CONF-96-280-E

Inclusive Jet Cross Section in $p \tilde{p}$ Collisions with the Do Detector

FERMILAB-CONF-96-255-E

Search for Chargino-Neutralino Associated Production via Trileptonic Final States with the Do Detector

FERMILAB-CONF-96-254-E

SUGRA-GUT Motivated SUSY Search in the Dielectron Channel at Do

FERMILAB-CONF-96-257-E

Search for Squarks and Gluinos in $p \bar{p}$ Collisions at the Do Detector with the Jets and Missing Energy Signature

Phys. Rev. Lett. 78:3818, 1997; FERMILAB-PUB-96-430-E; HEPEX-9611021

Search for a Fourth Generation Charge $-1 / 3$ Quark via Flavor Changing Neutral Current Decay

FERMILAB-CONF-96-248-E

The $b \bar{b}$ Production Cross Section and Correlations in $p \bar{p}$ Collisions at $\sqrt{s}=1.8 \mathrm{TeV}$

FERMILAB-CONF-96-247-E

Hard Single Diffractive Jet Production at $D^{0}$

Phys. Rev. Lett. 78:2070, 1997; FERMILA B-PUB-96-446-E; HEPEX-9612011

Search for Diphoton Events with Large Missing Transverse Energy in $p \bar{p}$ Collisions at $\sqrt{s}=1.8 \mathrm{TeV}$

Phys. Rev. D57:589, 1998; FERMILA B-PUB-96-449-E; HEPEX-9612009

Search for Top Squark Pair Production in the Dielectron Channel

Phys. Rev. Lett. 78:3634, 1997; FERMILAB-PUB-96-434-E; D0-PUB-96-14; HEPEX-9612002

Limits on Anomalous $W W \gamma$ Couplings from $\bar{p} p \rightarrow W \gamma$ X Events at $\sqrt{s}=1.8 \mathrm{TeV}$

Phys. Rev. Lett. 77:3309, 1996; FERMILAB-PUB-96-177-E

Measurement of the $W^{ \pm}$Boson Mass

Phys. Rev. Lett. 78:3640, 1997; FERMILAB-PUB-97-047-E; HEPEX-9702011

Study of the $Z^{0} Z^{0} \gamma$ and $Z^{0} \gamma \gamma$ Couplings in $Z^{\circ}(\nu \bar{\nu}) \gamma$ Production

Phys. Rev. Lett. 79:1197, 1997; FERMILAB-PUB-97-059-E; HEPEX-9703008 Direct Measurement of the Top Quark Mass

Phys. Rev. Lett. 79:1203, 1997; FERMILA B-PUB-97-109-E; HEPEX-9704015

Measurement of the Top Quark Pair Production Cross Section in $\bar{p} p$ Collisions

Phys. Rev. D56:6742, 1997; FERMILAB-PUB-97-088-E; HEPEX-9704004

Studies of Gauge Boson Pair Production and Trilinear Couplings

Phys. At. Nucl. 58:1542, 1995; Yad. Phys. 58:1635, 1995

Measurement of the Spin Rotation Parameter $A_{+}$in $\pi^{+} p$ Elastic Scattering at $1.43 \mathrm{GeV} / c$

Z. Phys. A352:85, 1995

Phase-shift Analysis of $\pi p$ Scattering from 160 to $600 \mathrm{MeV}$

Nucl. Phys. A566:225C, 1994

New Results from WA85 on Multistrange Hyperon Production in $200 \mathrm{~A} \cdot \mathrm{GeV} / \mathrm{c} \mathrm{S}-\mathrm{Wt}$ Interactions

Nucl. Phys. A566:491C, 1994

$\Omega^{-}$Signal in WA85

Nucl. Phys. A566:499C, 1994

Strange Particle Production in Sulphur-Sulphur Interactions at $200 \mathrm{GeV} / c$ per Nucleon

Phys. Lett. 324B:509, 1994; CERN-PPE-94-28

Observation of a Narrow Scalar Meson at $1450 \mathrm{MeV}$ in the Reaction $p p \rightarrow p_{f}\left(\pi^{+} \pi^{-} \pi^{+} \pi^{-}\right) p_{s}$ at $450 \mathrm{GeV} / \mathrm{c}$ using the CERN Omega Spectrometer

See the legend on page 5. 
Abatzis 95

Abatzis 95B

Abatzis $95 \mathrm{C}$

Abatzis $95 \mathrm{D}$

Abatzis $95 \mathrm{E}$

A batzis 96

Abatzis $96 \mathrm{~B}$

Abatzis 97

Abazov 96

Abbiendi 98

Abbiendi $98 \mathrm{~B}$

Abbiendi $98 \mathrm{C}$

Abbiendi $98 \mathrm{D}$

Abbiendi $98 \mathrm{E}$

Abbiendi $98 \mathrm{~F}$

Abbiendi $98 \mathrm{G}$

Abbiendi $98 \mathrm{H}$

Abbiendi 98I

Abbiendi 98J

Abbiendi $98 \mathrm{~K}$

Abbiendi $98 \mathrm{~L}$

Abbiendi $98 \mathrm{M}$

Abbiendi $98 \mathrm{~N}$

Abbiendi 980

Abbiendi $98 \mathrm{P}$

Abbiendi $98 \mathrm{R}$

Abbiendi 99

Abbiendi $99 \mathrm{~B}$

Abbiendi $99 \mathrm{C}$

Abbiendi 99D

Abbott 94

Abbott $94 \mathrm{~B}$

Abbott 95

Abbott 97

Abbott $97 \mathrm{~B}$

Abbott $97 \mathrm{C}$

Abbott $97 \mathrm{D}$

Abbott $97 \mathrm{E}$
Phys. Lett. 354B:178, 1995; CERN-PPE-95-66

A Study of Cascade and Strange Baryon Production in Sulphur-Sulphur Interactions at 200 GeV/c per Nucleon

Phys. Lett. 355B:401, 1995; CERN-PPE-95-074

Charged Kaon Production in $\mathrm{S}$ Wt Collisions at $200 \mathrm{GeV} / \mathrm{c}$ per Nucleon

Nucl. Phys. A590:317C, 1995

Strange Particle Production in Sulphur-Sulphur Interactions at $200 \mathrm{GeV} / c$

Nucl. Phys. A590:307C, 1995

Results on the Production of Baryons with $S=1,2,3$ and Strange Mesons in S-Wt Collisions at $200 \mathrm{GeV} / c$ per Nucleon

Phys. Lett. 347 B:158, 1995

Measurement of the $\Omega / \Xi$ Production Ratio in Central S-Wt Interactions at $200 \mathrm{~A} \mathrm{GeV} / c$

Phys. Lett. 376B:251, 1996; CERN-PPE-96-36

Study of $K_{S}, \Lambda$, and $\bar{\Lambda}$ Production in $\mathrm{S}-\mathrm{Wt}$ Collisions at $200 \mathrm{Gev} / \mathrm{c}$ per Nucleon

Phys. Lett. 400B:239, 1997; CERN-PPE-96-165

Hyperon Production in Proton-Sulphur Collisions at $200 \mathrm{GeV} / \mathrm{c}$

Phys. Lett. 393B:210, 1997

Hyperon Production in Proton-Tungsten Interactions at $200 \mathrm{GeV} / \mathrm{c}$

JINR-E1-96-104

Observation of a Resonance-Like Structure in the $p p \rightarrow p p \gamma \gamma$ Reaction at Proton Energy 188 MeV: Is the Dibaryon of Mass $1923 \mathrm{MeV}$ Found?

Eur. Phys. J. C6:1, 1999; CERN-EP-98-108; HEPEX-9808023

Test of the Standard Model and Constraints on New Physics from Measurements of Fermion-Pair Production at $183 \mathrm{GeV}$ at LEP

CERN-EP-98-113; HEPEX-9808027

Di-Jet Production in Photon-Photon Collisions at $\sqrt{s_{e e}}=161$ and $172 \mathrm{GeV}$

Phys. Lett. 438B:391, 1998; CERN-EP-98-120

First Measurement of $Z^{0} / \gamma^{*}$ Production in Compton Scattering of Quasi-Real Photons

CERN-EP-98-114; HEPEX-9808031

A Study of Parton Fragmentation in Hadronic $Z^{0}$ Decays using $\Lambda \bar{\Lambda}$ Correlations

Phys. Lett. 444B:539, 1998; CERN-EP-98-119; HEPEX-9808006

Measurement of the Average Polarization of $b$ Baryons in Hadronic $Z^{0}$ Decay

Eur. Phys. J. C8:573, 1999; CERN-EP-98-146; HEPEX-9810016

Measurement of the Semileptonic Branching Ratio of Charm Hadrons Produced in $Z^{\circ} \rightarrow c \bar{c}$ Decays

Eur. Phys. J. C8:191, 1999; CERN-EP-98-167; HEPEX-9811028

$W^{+} W^{-}$Production and Triple Gauge Boson Couplings at LEP Energies up to $183 \mathrm{GeV}$

Eur. Phys. J. C9:1, 1999; CERN-EP-98-186; HEPEX-9812018

A Measurement of the Product Branching Ratio $f\left(b \rightarrow \Lambda_{b}\right) \cdot \operatorname{BR}\left(\Lambda_{b} \rightarrow \Lambda X\right) Z^{0}$ Decays

Phys. Lett. 447B:134, 1999; CERN-EP-98-175; HEPEX-9812017

A Measurement of the $\tau^{-} \rightarrow e^{-\nu} e_{\tau}$ Branching Ratio

Eur. Phys. J. C8:23, 1999; CERN-EP-98-143; HEPEX-9810021

Search for Anomalous Photonic Events with Missing Energy in $e^{+} e^{-}$Collisions at $\sqrt{s}=130$, 136 and $183 \mathrm{GeV}$

183 GeV
CERN-EP-98-195; HEPEX-9901017

Measurement of the $B^{+}$and $B^{\circ}$ Lifetimes and Search for CP(T) Violation using Reconstructed Secondary Vertices

Phys. Lett. 453B:153, 1999; CERN-PPE-98-196; HEPEX-9901019

Color Reconnection Studies in $e^{+} e^{-} \rightarrow W^{+} W^{-}$at $\sqrt{s}=183 \mathrm{GeV}$

Phys. Let t. 453B:138, 1999; CERN-EP-98-197; HEPEX-9901025

Measurement of the $W$ Mass and Width in $e^{+} e^{-}$Collisions at $183 \mathrm{GeV}$

CERN-EP-98-122; HEPEX-9808036

Search for Acoplanar Lepton Pair Events in $e^{+} e^{-}$Annihilation at $\sqrt{s}=161,172$ and $183 \mathrm{GeV}$

Eur. Phys. J. C8:217, 1999; CERN-EP-98-137; HEPEX-9810002

A Measurement of $R_{b}$ using a Double Tagging Method

Eur. Phys. J. C8:255, 1999; CERN-EP-98-136; HEPEX-9809031

Search for Chargino and Neutralino Production at $\sqrt{s}=181-184 \mathrm{GeV}$ at LEP

Eur. Phys. J. C7:407, 1998; CERN-EP-98-173

Search for Higgs Bosons in $e^{+} e^{-}$Collisions at $183 \mathrm{GeV}$

CERN-EP-99-010; HEPEX-9902024

Measurements of the QED Structure of the Photon

CERN-EP-99-009

Intermittency and Correlations in Hadronic $Z^{\circ}$ Decays

CERN-EP-99-028; HEPEX-9903027

Experimental Properties of Gluon and Quark Jets from a Point Source

CERN-EP-99-045

Test of the Flavour Independence of $\alpha_{s}$ using Next-to-leading Order Calculations for Heavy Quarks

Phys. Rev. C50:1024, 1994; BNL-60277

Charged Hadron Distributions in Central and Peripheral Si + A Collisions at 14.6 A GeV/c

Phys. Lett. 337 B:254, 1994; BNL-49897

Intermittency in Central Collisions of ${ }^{16} \mathrm{O}+\mathrm{A}$ at $14.6 \mathrm{~A} \mathrm{GeV} / \mathrm{c}$

Phys. Rev. C52:2663, 1995; BNL-61896

Multiplicity Distributions from Central Collisions of ${ }^{16} \mathrm{O} \mathrm{Cu}$ at $14.6 \mathrm{~A} \mathrm{GeV} / c$ and Intermittency

Phys. Rev. Lett. 80:2063, 1998; FERMILA B-PUB-97-172-E

Measurement of the Top Quark Mass using Dilepton Events

Phys. Lett. 414B:419, 1997; FERMILAB-PUB-97-201-E; HEPEX-9706012

Color Coherent Radiation in Multijet Events from $p \bar{p}$ Collisions at $\sqrt{s}=1.8 \mathrm{TeV}$

Phys. Rev. Lett. 79:1441, 1997; FERMILAB-PUB-97-136-E; HEPEX-9705010

Limits on $W W Z$ and $W W \gamma$ Couplings from $p \bar{p} \rightarrow e^{-} \nu$ jet jet X Events at $\sqrt{s}=1.8$ TeV

Phys. Rev. Lett. 79:4321, 1997; FERMILAB-PUB-97-252-E; D0PUB-97-5; HEPEX-9707033

Search for Scalar Leptoquark Pairs Decaying to Electrons and Jets in $\bar{p} p$ Collisions

Phys. Rev. Lett. 80:1591, 1998; FERMILAB-PUB-97-153-E; HEPEX-9705015

Search for the Trilepton Signature from the Associated Production of SUSY chargino ${ }^{ \pm}$neutralino Gauginos 
Abbott $97 \mathrm{~F}$

Abbott $97 \mathrm{G}$

Abbott $97 \mathrm{H}$

Abbott 97I

Abbott 97J

Abbott $97 \mathrm{~K}$

Abbott $97 \mathrm{~L}$

Abbott 98

Abbott $98 \mathrm{~B}$

Abbott $98 \mathrm{C}$

Abbott 98D

Abbott $98 \mathrm{E}$

Abbott $98 \mathrm{~F}$

Abbott $98 \mathrm{G}$

Abbott $98 \mathrm{H}$

Abbott $98 \mathrm{I}$

Abbott 98J

Abbott $98 \mathrm{~K}$

Abbott $98 \mathrm{~L}$

Abbott $98 \mathrm{M}$

Abbott $98 \mathrm{~N}$

Abbott 980

Abbott 98P

Abbott $98 \mathrm{Q}$

Abbott 98R

Abbott 99

Abbott 99B

Abbott $99 \mathrm{C}$

Abdinov 95

Abdullin 92

Abdurashitov 94

Abdurashitov 96

Abe $93 \mathrm{~N}$

Abe $93 \mathrm{~S}$

Abe 93T

Abe $93 \mathrm{U}$

Abe 93W

Abe 94

Abe $94 \mathrm{~B}$
Phys. Rev. Lett. 80:666, 1998; FERMILAB-PUB-97-237-E; HEPEX-9707016

Measurement of Dijet Angular Distributions and Search for Quark Compositeness

Phys. Rev. Lett. 80:442, 1998; FERMILAB-PUB-97-273-E; HEPEX-9708005

Experimental Search for Chargino and Neutralino Production via Gauge-Mediated Supersymmetry Breaking Models

Phys. Rev. D58:012002, 1998; FERMILAB-PUB-97-328-E; HEPEX-9710007

Determination of the Mass of the $W^{ \pm}$Boson using the Do Detector at the Tevatron

Phys. Rev. D57:3817, 1998; FERMILAB-PUB-97-363-E; HEPEX-9710031

$Z^{0} \gamma$ Production in $p \bar{p}$ Collisions at $\sqrt{s}=1.8 \mathrm{TeV}$ and Limits on Anomalous $Z^{0} Z^{0} \gamma$ and $Z^{\circ} \gamma \gamma$ Couplings Phys. Rev. Lett. 80:2051, 1998; FERMILAB-PUB-97-344-E; HEPEX-9710032

Search for First Generation Scalar Leptoquark Pairs in $p \bar{p}$ Collisions at $\sqrt{s}=1.8 \mathrm{TeV}$

Phys. Rev. Lett. 80:3008, 1998; FERMILAB-PUB-97-423-E; HEPEX-9712028

A Measurement of the $W^{ \pm}$Boson Mass

Phys. Rev. D58:092003, 1998; FERMILAB-PUB-97-422-E; HEPEX-9712029

A Measurement of the $W^{ \pm}$Boson Mass

Phys. Rev. D58:052001, 1998; FERMILAB-PUB-98-031-E; HEPEX-9801025

Direct Measurement of the Top Quark Mass at $D^{\circ}$

Phys. Lett. 423B:419, 1998; FERMILAB-PUB-98-033-E; HEPEX-9801027

Search for the Decay $b \rightarrow X_{s} \mu^{+} \mu^{-}$

Phys. Rev. Lett. 80:5498, 1998; FERMILAB-PUB-98-075-E; HEPEX-9803003 Measurement of the Shape of the Transverse Momentum Distribution of $W$ bosons produced in $p \bar{p}$ collisions at $\sqrt{s}=1.8 \mathrm{TeV}$

Phys. Rev. D58:051101, 1998; FERMILAB-PUB-98-076-E; HEPEX-9803004

Limits on $W W \gamma$ and $W W Z^{\circ}$ Couplings from $W$ Boson Pair Production

Phys. Rev. Lett. 81:524, 1998; FERMILAB-PUB-98-095-E; HEPEX-9803023

A Search for Heavy Pointlike Dirac Monopoles

Phys. Rev. Lett. 81:38, 1998; FERMILAB-PUB-98-081-E; HEPEX-9803009

Search for Charge $1 / 3$ Third Generation Leptoquarks in $p \bar{p}$ Collisions at $\sqrt{s}=1.8 \mathrm{TeV}$

Phys. Rev. D58:031102, 1998; FERMILA B-PUB-98-094-E; HEPEX-9803017

Limits on Anomalous $W W \gamma$ and $W W Z^{\circ}$ Couplings

Phys. Rev. Lett. 82:2457, 1999; FERMILAB-PUB-98-220-E; HEPEX-9807014

The Dijet Mass Spectrum and a Search for Quark Compositeness in $\bar{p} p$ Collisions at $\sqrt{s}=1.8 \mathrm{TeV}$

Phys. Rev. Lett. 82:1379, 1999; DAPNIA-SPHN-98-81-SACLAY; NUCLEX-9810017

A Precise Measurement of the Deuteron Elastic Structure Function $A\left(Q^{2}\right)$

Phys. Rev. Lett. 82:2451, 1999; FERMILA B-PUB-98-207-E; HEPEX-9807018

The Inclusive Jet Cross Section in $\bar{p} p$ Collisions at $\sqrt{s}=1.8 \mathrm{TeV}$

Phys. Rev. Lett. 82:35, 1999; FERMILA B-PUB-98-237-E; HEPEX-9807029

Small Angle $J / \psi(1 S)$ Production in $\bar{p} p$ Collisions at $\sqrt{s}=1.8 \mathrm{TeV}$

Phys. Rev. Lett. 82:2244, 1999; FERMILAB-PUB-98-362-E; HEPEX-9811029

Search for High Mass Photon Pairs in $p \bar{p} \rightarrow \gamma \gamma$ jet jet Events at $\sqrt{s}=1.8 \mathrm{TeV}$

Phys. Rev. Lett. 82:4769, 1999; FERMILAB-PUB-98-391-E; HEPEX-9812010

Measurement of the High-Mass Drell-Yan Cross Section and Limits on Quark-Electron Compositeness Scales

Phys. Rev. D60:052001, 1999; FERMILAB-PUB-98-261-E; HEPEX-9808029

Measurement of the Top Quark Mass in the Dilepton Channel

Phys. Rev. Lett. 80:5072, 1998

Quasifree $\left(e, e^{\prime} p\right)$ Reactions and Proton Propagation in Nuclei

Phys. Lett. 440B:189, 1998; FERMILAB-PUB-98-285-E; HEPEX-9809016

Probing Hard Color Singlet Exchange in $p \bar{p}$ Collisions at $\sqrt{s}=630$ and $1800 \mathrm{GeV}$

FERMILAB-PUB-98-402-E; HEPEX-9902013

Search for Squarks and Gluinos in Events Containing Jets and a Large Imbalance in Transverse Energy

Phys. Rev. D60:012001, 1999; FERMILA B-PUB-98-130-E; HEPEX-9808034

Measurement of the Top Quark Pair Production Cross Section in $p \bar{p}$ Collisions using Multijet Final States

Phys. Rev. Lett. 82:4975, 1999; FERMILA B-PUB-99-029-E; HEPEX-9902028

Search for Charged Higgs Bosons in Decays of Top Quark Pairs

Phys. Rev. D60:031101, 1999; FERMILAB-PUB-99-046-E; HEPEX-9903041

Search for Bottom Squarks in $p \bar{p}$ Collisions at $\sqrt{s}=1.8 \mathrm{TeV}$

HEPEX-9904023

Search for Second Generation Leptoquark Pairs Decaying to $\mu \nu$ jets in $p \bar{p}$ Collisions at $\sqrt{s}=1.8 \mathrm{TeV}$

Turk. J. Phys. 19:936, 1995

Determination of Mean "Temperature" of System of Charged $\pi^{-}$-Mesons, Emitted in $\pi^{-12} \mathrm{C}$ Interactions at $P_{x^{-}}=40 \mathrm{GeV} / \mathrm{c}$ for Different Intervals of their Transverse Momenta

Phys. At. Nucl. 56:536, 1993; Yad. Phys. 56-4:204, 1993; Nucl. Phys. A569:753, 1994; ITEP.92.86 Cross Sections of ${ }^{4} \mathrm{He}$ Interaction with Protons and He $p$ Elastic Scattering at $2.7 \mathrm{GeV} / \mathrm{c}$

Phys. Lett. 328B:234, 1994

Results from SAGE

Phys. Rev. Lett. 77:4708, 1996

The Russian-American Gallium Experiment (SAGE) Cr Neutrino Source Measurement

Phys. Rev. Lett. 71:3421, 1993; FERMILAB-PUB-93-158-E; ANL-HEP-PR-94-22

Measurement of the Average Lifetime of $B$ Hadrons Produced in $p \bar{p}$ Collisions at $\sqrt{s}=1.8 \mathrm{TeV}$

Phys. Rev. D50:5550, 1994; FERMILAB-PUB-93-234-E Measurement of the Antiproton-Proton Total Cross Section at $\sqrt{s}=546$ and $1800 \mathrm{GeV}$

Phys. Rev. D50:5535, 1994; FERMILAB-PUB-93-233-E; CDF-PUB-MIN-BIAS-PUBLIC-2050

Measurement of $\bar{p} p$ Single Diffraction Dissociation at $\sqrt{s}=546$ and $1800 \mathrm{GeV}$

Phys. Rev. D50:5518, 1994; FERMILA B-PUB-93-232-E; CDF-PUB-MIN-BIAS-PUBLIC-2049

Measurement of Small Angle Antiproton-Proton Elastic Scattering at $\sqrt{s}=546$ and $1800 \mathrm{GeV}$

Phys. Rev. D49:1, 1994; FERMILA B-PUB-93-133-E; CDF-PUB-ELECTROWEAK-PUBLIC-1760; ANL-HEP-PR-94-88 Measurement of Drell-Yan Electron and Muon Pair Differential Cross Sections in $p \bar{p}$ Collisions at $\sqrt{s}=$

1.8 TeV
FERMILAB-CONF-94-146-E; CDF-PUB-ELECTROWEAK-PUBLIC-2628

Tests of Structure Functions using Leptons at CDF: $W^{ \pm}$Asymmetry and Drell-Yan Production

Phys. Rev. Lett. 73:225, 1994; FERMILAB-PUB-94-116-E; ANL-HEP-PR-94-61; CDF-PUB-TOP-PUBLIC-2595; 
Abe $94 \mathrm{~B}$ (cont'd)

A be $94 \mathrm{C}$

Abe $94 \mathrm{D}$

A be $94 \mathrm{E}$

A be $94 \mathrm{~F}$

A be $94 \mathrm{G}$

A be $94 \mathrm{H}$

Abe $94 \mathrm{I}$

A be $94 \mathrm{~J}$

A be $94 \mathrm{~K}$

A be $94 \mathrm{~L}$

A be $94 \mathrm{M}$

A be $94 \mathrm{~N}$

A be 940

Abe $94 \mathrm{P}$

A be $94 Q$

Abe $94 \mathrm{R}$

Abe $94 \mathrm{~S}$

Abe $94 \mathrm{~T}$

A be $94 \mathrm{U}$

A be $94 \mathrm{~V}$

A be $94 \mathrm{~W}$

A be $94 \mathrm{X}$

A be $94 \mathrm{Y}$

A be $94 Z$

Abe $94 Z B$

Abe $947 \mathrm{C}$

Abe $942 \mathrm{D}$

A be $94 Z E$

A be $94 \mathrm{ZF}$

Abe $942 \mathrm{G}$

Abe $942 \mathrm{H}$

Abe 9421

Abe 95

Abe $95 \mathrm{~B}$

Abe $95 \mathrm{C}$

Abe $95 \mathrm{D}$
HEPEX-9405005

Evidence for Top Quark Production in $\bar{p} p$ Collisions at $\sqrt{s}=1.8 \mathrm{TeV}$

Phys. Rev. Lett. 73:25, 1994; SLAC-PUB-6456

Precise Measurement of the Left-Right Cross Section Asymmetry in $Z^{0}$ Boson Production by $e^{+} e^{-}$ Collisions

Phys. Rev. Lett. 72:3456, 1994; FERMILAB-PUB-94-034-E; CDF-PUB-BOTTOM-PUBLIC-2381; ANL-HEP-PR-94-50 Measurement of the $B^{+}$and $B^{0}$ Meson Lifetimes

Phys. Rev. Lett. 72:1977, 1994; FERMILAB-PUB-93-338-E; CDF-PUB-EXOTIC-PUBLIC-2283; ANL-HEP-PR-94-35

$A$ Search for the Top Quark Decaying to Charged Higgs in $p \bar{p}$ Collisions at $\sqrt{3}=1.8 \mathrm{TeV}$

Phys. Rev. D50:5562, 1994; FERMILAB-PUB-94-072-E; CDF-ANAL-JET-PUBLIC-2413 Evidence for Color Coherence in $p \vec{p}$ Collisions at $\sqrt{s}=1.8 \mathrm{TeV}$

Phys. Rev. Lett. 73:2296, 1994; FERMILAB-PUB-94-126-E; CDF-PUB-ELECTROWEAK-PUBLIC-2537 $W$ Boson + Jet Angular Distribution in $p \bar{p}$ Collisions at $\sqrt{s}=1.8 \mathrm{TeV}$

Phys. Rev. Lett. 73:2662, 1994; Phys. Rev. Lett. 74:1891, 1995; FERMILAB-PUB-94-208-E; CDF-PUB-JET-PUBLIC2656

A. Precision Measurement of the Prompt Photon Cross Section in $p \bar{p}$ Collisions at $\sqrt{s}=1.8 \mathrm{TeV}$

FERMILAB-PUB-94-202-E; CDF-PUB-EXOTIC-PUBLIC-1491

Search for Radiative Decays of Neutralinos in Proton-Antiproton Collisions at $\sqrt{s}=1.8 \mathrm{TeV}$

Phys. Rev. D51:R949, 1995; FERMILAB-PUB-94-198-E; CDF-PUB-ELECTROWEAK-PUBLIC-2651

Search for New Gauge Bosons Decaying into Dielectrons in $\bar{p} p$ Collisions at $\sqrt{s}=1.8 \mathrm{TeV}$

Phys. Rev. Lett. 74:855, 1995; FERMILAB-PUB-94-194-E; RU-94-52; CDF-PUB-JET-CDFR-2639 Observation of Rapidity Gaps in $\bar{p} p$ Collisions at $1.8 \mathrm{TeV}$

FERMILAB-PUB-94-171-E; CDF-PUB-JET-PUBLIC-2216

Analysis of Jet Charged Particle Momentum Distributions for Quark-Gluon Separation in $\bar{p} p$ Collisions at $\sqrt{s}=1.8 \mathrm{TeV}$

Phys. Rev. Lett. $74: 850,1995$; FERMILAB-PUB-94-313-E

The Charge Asymmetry in $W^{ \pm}$-Boson Decays Produced in $p \bar{p}$ Collisions at $\sqrt{s}=1.8 \mathrm{TeV}$

Phys. Rev. Lett. 74:1936, 1995; FERMILAB-PUB-94-236-E

Measurement of $W^{ \pm}$Photon Couplings with CDF in $p \bar{p}$ Collisions at $\sqrt{s}=1.8 \mathrm{TeV}$

Phys. Rev. Lett. 74:1941, 1995; FERMILAB-PUB-94-304-E

Limits on $Z^{0}$ - Photon Couplings from the $p \bar{p}$ Interactions at $\sqrt{s}=1.8 \mathrm{TeV}$

FERMILAB-PUB-94-244-E

Measurement of $W^{ \pm}+\gamma$ and $Z^{\circ}+\gamma$ Cross Sections in the Electron and Muon Channels in $\sqrt{s}=1.8 \mathrm{TeV}$ $\bar{p} p$ Collisions

FERMILAB-PUB-94-131-E

$b \bar{b}$ Quark Pair Correlations in $p \bar{p}$ Collisions at $\sqrt{s}=1.8 \mathrm{TeV}$

Phys. Rev. Lett. 74:2900, 1995; FERMILAB-PUB-94-268-E

Search for Charged Bosons Heavier than the $W^{ \pm}$in $p \bar{p}$ Collisions at $\sqrt{s}=1800 \mathrm{GeV}$

Phys. Rev. Lett. 74:341, 1995; FERMILAB-PUB-94-301-E

A Direct Measurement of the $W^{ \pm}$Boson Width $\Gamma_{W \pm}$

Phys. Rev. Lett. 73:2667, 1994; ANL-HEP-PR-95-15

A Search for the Top Quark Decaying to Charged Higgs in $p \bar{p}$ Collisions at $\sqrt{3}=1.8 \mathrm{TeV}$

Phys. Rev. Lett. 74:2890, 1995; SLAC-PUB-6644

Measurement of $A_{b}$ from the Left-Right Forward-Backward Asymmetry of $b$ Quark Production in $Z^{0}$

Decays using a Momentum-Weighted Track Change Technique

Phys. Rev. Lett. 74:2895, 1995; SLAC-PUB-6607

Measurement of $A_{b}$ and $A_{c}$ from the Left-Right Forward-Backward Asymmetry of Leptons in Hadronic

Events at the $Z^{0}$ Resonance

Phys. Rev. D51:962, 1995; SLAC-PUB-6641

Measurement of $\alpha_{s}$ from Hadronic Event Observables at the $Z^{0}$ Resonance

Phys. Rev. Lett. 74:4988, 1995; FERMILAB-PUB-94-420-E

Measurement of the $B_{3}$ Meson Lifetime

Phys. Rev. Lett. 74:3538, 1995; FERMILAB-PUB-94-405-E

Search for New Particles Decaying to Dijets in $p \bar{p}$ Collisions at $\sqrt{s}=1.8 \mathrm{TeV}$

Phys. Rev. D51:4623, 1995; FERMILAB-PUB-94-411-E

Kinematic Evidence for Top Quark Pair Production in $W+$ Multijet Events in $p \bar{p}$ Collisions at $\sqrt{s}=$ $1.8 \mathrm{TeV}$

Phys. Rev. D53:R2271, 1996; SLAC-PUB-6687

$A$ Test of the Flavor Independence of Strong Interactions

Phys. Rev. Lett. 72:3145, 1994; SLAC-PUB-6372; LBL-34732

Measurement of the Charged Multiplicity of $Z^{0} \rightarrow b \bar{b}$ Events

Phys. Rev. Lett. 72:3004, 1994; FERMILAB-PUB-93-341-E

Search for Excited Quarks in $p \bar{p}$ Collisions at $\sqrt{s}=1.8 \mathrm{TeV}$

Phys. Rev. D50:2966, 1994; FERMILA B-PUB-94-097-E; CDF-PUB-TOP-PUBLIC-2561

Evidence for Top Quark Production in $\bar{p} p$ Collisions at $\sqrt{s}=1.8 \mathrm{TeV}$

Phys. Rev. D50:4252, 1994; ANL-HEP-PR-94-89

Measurement of the $B$ Meson and $b$-Quark Cross Sections at $\sqrt{3}=1.8 \mathrm{TeV}$ using the Exclusive Decays $B^{0}$ $\rightarrow J / \psi(1 S) K^{*}(892)^{0}$

Phys. Rev. Lett. 73:220, 1994; FERMILAB-PUB-94-051-E; CDF-PUB-ELECTROWEAK-PUBLIC-2182

Measurement of the Ratio $\sigma \cdot B\left(W^{ \pm} \rightarrow e^{ \pm} \nu\right) / \sigma \cdot B\left(Z^{\circ} \rightarrow e^{+} e^{-}\right)$in $\bar{p} p$ Collisions at $\sqrt{s}=1.8 \mathrm{TeV}$

FERMILAB-CONF-94-138-E; CDF-PUB-BOTTOM-PUBLIC-2613

Measurement of the $B$, Meson Lifetime at CDF

Phys. Rev. D50:5580, 1994; SLAC-PUB-6451

Measurement of $\alpha_{s}$ from Energy-Energy Correlations at the $Z^{0}$ Resonance

Phys. Rev. Lett. 74:2626, 1995; FERMILAB-PUB-95-022-E

Observation of Top Quark Production in $\bar{p} p$ Collisions

Phys. Rev. Lett. 75:1012, 1995; FERMILAB-PUB-95-050-E

A Search for Second Generation Leptoquarks in $p \bar{p}$ Collisions at $\sqrt{s}=1.8 \mathrm{TeV}$

Phys. Rev. Lett. 75:11, 1995; FERMILAB-PUB-95-035-E

Measurement of the $W^{ \pm}$Boson Mass

Phys. Rev. Lett. 75:608, 1995; FERMILAB-PUB-95-038-E

Properties of High-Mass Multijet Events at the Fermilab Proton-Antiproton Collider 
Abe $95 \mathrm{E}$

Abe $95 \mathrm{~F}$

Abe $95 \mathrm{G}$

Abe $95 \mathrm{H}$

Abe 951

Abe $95 \mathrm{~J}$

Abe $95 \mathrm{~L}$

Abe $95 \mathrm{M}$

Abe $95 \mathrm{~N}$

Abe 950

Abe $95 \mathrm{P}$

Abe $95 \mathrm{Q}$

Abe $95 \mathrm{R}$

Abe $95 \mathrm{~S}$

Abe $95 \mathrm{~T}$

Abe $95 \mathrm{U}$

A be $95 \mathrm{~V}$

Abe $95 \mathrm{~W}$

Abe $95 \mathrm{X}$

Abe $95 \mathrm{Y}$

Abe $95 \mathrm{Z}$

Abe 95ZB

A be $95 Z C$

Abe 95ZD

Abe 95ZE

Abe $95 \mathrm{ZF}$

Abe $952 \mathrm{G}$

Abe $952 \mathrm{H}$

Abe 95ZI

Abe $95 \mathrm{ZJ}$

Abe $95 \mathrm{ZK}$

Abe $95 \mathrm{ZL}$

Abe $95 \mathrm{ZM}$

Abe $95 \mathrm{ZN}$

Abe 9520

Abe 95ZP
Phys. Rev. Lett. 75:1451, 1995; FERMILAB-PUB-95-048-E

Measurement of the $B$ Meson Differential Cross-Section, $d \sigma / d p_{T}$, in $p \bar{p}$ Collisions at $\sqrt{s}=1800 \mathrm{GeV}$

Phys. Rev. Lett. 74:346, 1995; SLAC-PUB-6508 Precision Measurement of the Proton Spin Structure Function $g_{1}^{p}$

Phys. Rev. D52:2624, 1995; FERMILAB-PUB-95-025-E; CDF-PUB-ELECTROWEAK-PUBLIC-2812 A Measurement of the Ratio $\sigma \cdot B\left(p \bar{p} \rightarrow W^{ \pm} \rightarrow e^{ \pm} \nu\right) / \sigma \cdot B\left(p \bar{p} \rightarrow Z^{0} \rightarrow e^{+} e^{-}\right)$in $p \bar{p}$ Collisions at $\sqrt{s}=$ $1800 \mathrm{GeV}$

Phys. Rev. D52:4784, 1995; FERMILAB-PUB-95-033-E Measurement of the $W^{ \pm}$Boson Mass

Phys. Rev. D52:R2605, 1995; FERMILAB-PUB-95-083-E

Identification of Top Quarks at CDF using Kinematic Variables

Phys. Rev. Lett. 75:3997, 1995; FERMILAB-PUB-95-149-E; CDF-PUB-TOP-CDFR-3110 Study of $t \bar{t}$ Production in $p \bar{p}$ Collisions using Total Transverse Energy

Phys. Rev. Lett. 75:1017, 1995; FERMILAB-PUB-95-036-E; CDF-ANAL-ELECTROWEAK-CDFR-2951 Limits on $W W Z^{0}$ and $W W \gamma$ Couplings from $W W$ and $W Z^{0}$ Production in $p \bar{p}$ Collisions at $\sqrt{s}=$ $1.8 \mathrm{TeV}$

FERMILAB-CONF-95-229-E; CDF-PUB-BOTTOM-PUBLIC-3266

Search for the Decay $B^{0} \rightarrow \mu^{+} \mu^{-}$

FERMILAB-CONF-95-222-E; CDF-PUB-BOTTOM-PUBLIC-3241

Measurement of the $B^{+}$and $B^{\circ}$ Meson Lifetimes using Exclusive $B \rightarrow \psi K$ Decays at CDF

FERMILAB-CONF-95-224-E

Observation of $B^{+} \rightarrow J / \psi(1 S) \pi^{+}$

FERMILAB-CONF-95-228-E; CDF-BOTTOM-PUB-3236

Measurement of the Mass of the $B_{s}$ Meson in $p \bar{p}$ Collisions at $\sqrt{s}=1.8 \mathrm{TeV}$

Phys. Rev. D53:1051, 1996; FERMILAB-PUB-95-289-E; HEPEX-9508017

Measurement of Correlated $\mu-\bar{b}$-Jet Cross Sections in $p \bar{p}$ Collisions at $\sqrt{s}=1.8 \mathrm{TeV}$

FERMILAB-CONF-95-233-E; CDF-DOC-BOTTOM-PUBLIC-3261 Measurement of the Polarization in the Decays $B^{0} \rightarrow J / \psi(1 S) K^{*}(892)^{\circ}$ and $B_{s} \rightarrow J / \psi(1 S) \phi(1020)$

Phys. Rev. Lett. 75:25, 1995; SLAC-PUB-95-6734 Precision Measurement of the Deuteron Spin Structure Function $g_{1}$ (deuteron)

FERMILAB-CONF-95-226-E

Production of $J / \psi(1 S)$ from $\chi_{\mathrm{c}}$ Decays at CDF

FERMILAB-CONF-95-223-E; CDF-PUB-BOTTOM-PUBLIC-3244 $B \bar{B}$ Production Correlations, $B \bar{B}$ Mixing, and $\epsilon(B)$ at $\mathrm{CDF}$

Phys. Rev. Lett. 75:4173, 1995; SLAC-PUB-95-6969; HEPEX-9510005

First Measurement of the T-odd Correlation between the $Z^{\circ}$ Spin and the Three-Jet Plane Orientation in Polarized $Z^{\circ}$ Decays to Three Jets

Phys. Rev. Lett. 75:4358, 1995; FERMILAB-PUB-95-271-E; CDF-PUB-BOTTOM-PUBLIC-3215 $\Upsilon$ Production in $p \bar{p}$ Collisions at $\sqrt{s}=1.8 \mathrm{TeV}$

FERMILAB-CONF-95-202-E

A Limit on $\left.\sigma \cdot B r(B) \rightarrow J / \psi(1 S) \pi^{ \pm}\right) / \sigma \cdot B r\left(B^{ \pm} \rightarrow J / \psi(1 S) K^{ \pm}\right)$in $\sqrt{s}=1.8 \mathrm{TeV}$ Proton Antiproton Collisions FERMILA B-CONF-95-231-E; CDF-PUB-BOTTOM-PUBLIC-3255

Measurement of $B^{0} \bar{B}^{0}$ Mixing via Time Evolution

Phys. Rev. D52:4240, 1995; SLAC-PUB-95-6739 Comparison of a New Calculation of Energy-Energy Correlations with $e^{+} e^{-} \rightarrow$ Hadrons Data at the $Z^{0}$ Resonance

Phys. Rev. Lett. 75:3068, 1995; FERMILAB-PUB-95-270-E; CDF-PUB-BOTTOM-PUBLIC-3231

Measurement of the Polarization in the Decays $B^{\circ} \rightarrow J / \psi(1 S) K^{*}(8 \theta 2)^{\circ}$ and $B_{s} \rightarrow J / \psi(1 S) \phi(1020)$

Phys. Rev. D53:3496, 1996; FERMILAB-PUB-95-317-E

Measurement of the Mass of the $B_{s}$ Meson

SLAC-PÜB-95-6979

Measurement of the Polarized Forward-Backward Asymmetry of $Z^{\circ} \rightarrow B \bar{B}$ using a Lifetime Tag and Momentum Weighted Track Charge

Phys. Rev. Lett. 76:3070, 1996; FERMILAB-PUB-95-301-E; CDF-PUB-ELECTROWEAK-PUBLIC-3312

Measurement of $\sigma \mathrm{B}(\mathrm{W} \rightarrow e \nu)$ and $\sigma \mathrm{B}\left(Z \rightarrow e^{+} e^{-}\right)$in $p \bar{p}$ Collisions at $\sqrt{s}=1.8 \mathrm{TeV}$

Phys. Rev. D52:4828, 1995; SLAC-PUB-95-6767 Measurement of the $\tau^{ \pm}$Lifetime at SLD

Phys. Rev. D53:1023, 1996; SLAC-PUB-95-6569

Measurements of $R(B)$ with Impact Parameters and Displaced Vertices

Phys. Rev. Lett. 75:613, 1995

Search for Squarks and Gluinos via Radiative Decays of Neutralinos in Proton-Antiproton Collisions at $\sqrt{s}=1.8 \mathrm{TeV}$

Phys. Rev. Lett. 76:2015, 1996; FERMILAB-PUB-95-368-E

Reconstruction of $B^{0} \rightarrow J / \psi(1 S) K_{S}$ and Measurement of Ratios of Branching Ratios Involving $B \rightarrow$ $J / \psi(1 S) K^{*}(892)$

Phys. Lett. 361B:199, 1995 A Study of Single Photon Production in $e^{+} e^{-}$Collisions at $\sqrt{s}=58 \mathrm{GeV}$ with the TOPAZ Detector at TRISTAN

Phys. Rev. Lett. 76:2852, 1996; FERMILAB-PUB-95-386-E; CDF-3131

Search for the Rare Decay $W^{ \pm} \rightarrow \pi^{ \pm}+\gamma$

Phys. Rev. Lett. 76:587, 1996; SLAC-PUB-95-6982; HEPEX-9511013

Measurements of the Proton and Deuteron Spin Structure Function $g_{2}$ and Asymmetry $A_{2}$

Phys. Rev. Lett. 74:2880, 1995; SLAC.PUB-6605 Polarized Bhabha Scattering a Precision Measurement of the Electron Neutral Current Couplings

SLAC-PUB-95-6972

Preliminary Measurements of $B^{0}$ and $B^{+}$Lifetimes at SLD

Phys. Lett. 364 B:61, 1995; SLAC-PUB-95-6997; HEPEX-9511015

Measurements of the $Q^{2}$-Dependence of the Proton and Deuteron Spin Structure Functions $g_{p}^{1}$ and $g_{\mathrm{deuteron}}^{1}$ Phys. Rev. Lett. 74:1512, 1995; SLAC-PUB-6643

A Search for Jet Handedness in Hadronic $Z^{0}$ Decays 
A be $95 Z Q$

Abe $95 Z R$

Abe 96

Abe $96 \mathrm{~B}$

A be $96 \mathrm{C}$

Abe $96 \mathrm{D}$

A be $96 \mathrm{E}$

A be $96 \mathrm{~F}$

Abe $96 \mathrm{G}$

Abe $96 \mathrm{H}$

Abe $96 \mathrm{I}$

A be $96 \mathrm{~J}$

A be $96 \mathrm{~K}$

Abe $96 \mathrm{~L}$

Abe $96 \mathrm{MI}$

Abe $96 \mathrm{~N}$

A be 960

A be $96 \mathrm{P}$

Abe 960

Abe $96 R$

Abe $96 \mathrm{~S}$

Abe $96 \mathrm{~T}$

Abe $96 \mathrm{~L}$

A be $96 \mathrm{~V}$

Abe $96 \mathrm{~W}$

Abe $96 \mathrm{X}$

Abe $96 \mathrm{Y}$

A be $96 \mathrm{Z}$

Abe $96 Z B$

Abe $96 \mathrm{ZC}$

A be $962 \mathrm{D}$

Abe $962 \mathrm{E}$

Abe $96 \mathrm{ZF}$

A be $96 \mathrm{ZG}$

Abe 97

Abe $97 \mathrm{~B}$

Abe $97 \mathrm{C}$
Phys. Rev. Lett, 75:3609, 1995; SLAC-PUB-95-6681

Measurement of the Left-Right Forward-Backward Asymmetry for Charm Quarks with $D^{*}(2010)+$ and $D^{+}$Mesons

Phys. Rev. Lett. 75:3624, 1995; SLAC-PUB-95-6976; HEPEX-9511005

Measurement of the Average $B$ Hadron Lifetime in $Z^{0}$ Decays using Reconstructed Vertices

Phys. Rev. D54:735, 1996; FERMILAB-PUB-96-004-E; HEPEX-9601003

Search for Charged Higgs Decays of the Top Quark using Hadronic $\tau$-Decays

Phys. Rev. Lett. 77:438, 1996; FERMILAB-PUB-96-020-E

Inclusive Jet Cross Section in $\bar{p} p$ Collisions at $\sqrt{s}=1.8 \mathrm{TeV}$

Phys. Rev. Lett. 76:4307, 1996; FERMILAB-PUB-96-029-E; CDF-PUB-EXOTIC-CDFR-3440

Search for Chargino-Neutralino Production in $p \bar{p}$ Collisions at $\sqrt{s}=1.8 \mathrm{TeV}$

Phys. Rev. Lett. 76:4675, 1996; FERMILAB-PUB-96-040-E; CDF-PUB-BOTTOM-PUBLIC-3332

Search for Flavor Changing Neutral Current $B$ Meson Decays in $p \bar{p}$ Collisions at $\sqrt{s}=1.8$ TeV

Phys. Rev. Lett. 76:4462, 1996; FERMILAB-PUB-96-041-E; CDF-PUB-BOTTOM-PUB-3492

Measurement of the $B^{-}$and $\bar{B}^{\circ}$ Meson Lifetimes using Semileptonic Decays

Phys. Rev. Lett. 77:448, 1996; FERMILA B-PUB-96-056-E

Properties of Jets in $Z^{\circ}$ Boson Events from $1.8 \mathrm{TeV} \bar{p} p$ Collisions

Phys. Rev. Lett. 78:4307, 1997; Phys. Rev. Lett. 77:5336, 1996; FERMILAB-PUB-96-317-E; HEPEX-9609011

Measurement of Dijet Angular Distributions at CDF

Phys. Rev. Lett. 77:1945, 1996; FERMILAB-PUB-96-101-E

Measurement of the Lifetime of the $B_{s}$ Meson using the Exclusive Decay Mode $B_{s} \rightarrow J / \psi(1 S) \phi(1020)$

Phys. Rev. D54:4221, 1996; FERMILA B-PUB-96-098-E

Further Properties of High Mass Multi Jet Events at the Fermilab Proton Antiproton Collider

Phys. Rev. Lett. 77:1439, 1996; FERMILAB-PUB-96-089-E

Measurement of $\Lambda_{b}$ Lifetime using $\Lambda_{b} \rightarrow \Lambda_{c}^{+} \ell^{-} \bar{\nu}$

Phys. Rev. D54:6596, 1996; FERMILAB-PUB-96-119.E

Ratios of Bottom Meson Branching Fractions Involving $J / \psi(1 S)$ Mesons and Determination of $b$ Quark Fragmentation Fractions

Phys. Rev. Lett. 77:2616, 1996; FERMILAB-PUB-96-071-E; CDF-PUB-ELECTROWEAK-CDFR-3493

Forward Backward Charge Asymmetry of Electron Pairs above the $Z$ Pole

Phys. Rev. Lett. 77:5005, 1996; FERMILAB-PUB-96-152-E

Measurement of the $\gamma D^{*}(2010)^{ \pm}$Cross Section in $\bar{p} p$ Collisions at $\sqrt{s}=1.8 \mathrm{TeV}$

Phys. Rev. Lett. 77:5176, 1996; FERMILAB-PUB-96-300-E; CDF-PUB-BOTTOM-CDFR-3690

Measurement of the Branching Fraction $B r\left(B^{+} \rightarrow J / \psi(1 S) \pi^{+}\right)$and Search for $B_{c}^{+} \rightarrow J / \psi(1 S) \pi^{+}$

Phys. Rev. Lett. 78:17, 1997; SLAC-PUB-7069; COLO-HEP-367; HEPEX-9609019

First Measurement of the Left-Right Charge Asymmetry in Hadronic $Z^{\circ}$ Boson Decays and a New Determination of $\sin ^{2} \theta_{W}$ (eff)

Phys. Rev. D55:1142, 1997; FERMILAB-PUB-96-270-E; CDF-PUB-BOTTOM-PUBLIC-3724

Observation of $\Lambda_{b} \rightarrow J / \psi(1 S) \Lambda$ at the Fermilab Proton Antiproton Collider

Phys. Rev. Lett. 76:2006, 1996; FERMILAB-PUB-95-399-E; CDF-PUB-EXOTIC-3404

Search for Gluino and Squark Cascade Decays at the Fermilab Tevatron Collider

Phys. Rev. Lett. 78:4536, 1997; FERMILAB-PUB-96-311-E

Observation of $W^{+} W^{-}$Production in $\bar{p} p$ Collisions at $\sqrt{s}=1.8 \mathrm{TeV}$

Phys. Rev. D55:2546, 1997; FERMILAB-PUB-96-216-E

Measurement of $b \bar{b}$ Production Correlations, $B^{0} \bar{B}^{0}$ Mixing, and a Limit on $\epsilon_{B}$ in $p \bar{p}$ Collisions at $\sqrt{s}=$ $1.8 \mathrm{TeV}$

SLAC.PUB-7226

Measurement of the $B^{+}$and $B^{0}$ Lifetimes from Semileptonic Decays at SLD

SLAC-PUB-7227

Measurement of the $B^{+}$and $B^{0}$ Lifetimes with Topological Vertexing at SLD

SLAC-PUB-7228

Measurement of Time Dependent $B^{0}-\bar{B}^{0}$ Mixing using Inclusive Semileptonic Decays

SLAC-PUB-7230

Preliminary Measurements of the Time Dependence of $B^{0}-\bar{B}^{0}$ Mixing with Kaon and Charge Dipole Tags

Phys. Rev. Lett. 78:2075, 1997; SLAC-PUB-7291; HEPEX-9611011

An Improved Measurement of the Left-Right $Z^{0}$ Cross Section Asymmetry

Phys. Lett. 386B:475, 1996; SLAC-PUB-7172; HEPEX-9608008

Measurement of the Charged Multiplicities in $b, c$ and Light Quark Events from $Z^{\circ}$ Decays

SLAC-PUB-7213

Updated Measurement of the $\tau \pm$ Lifetime at SLD

Phys. Lett. 371B:149, 1996; SLAC-PUB-95-7027; SLAC-PUB-7027; HEPEX-9601010

Factorial and Cumulant Moments in $e^{+} e^{-} \rightarrow$ Hadrons at the $Z^{0}$ Resonance

Phys. Rev. Lett. 78:815, 1997; SLAC-PUB-7242; DAPNIA-SPHN-96-41; HEPEX-9701004

Measurements of the Proton and Deuteron Spin Structure Function $g_{1}$ in the Resonance Region

Phys. Rev. Lett. 78:2906, 1997; FERMILAB-PUB-96-450-E

Search for Third Generation Leptoquarks in $\bar{p} p$ Collisions at $\sqrt{s}=1.8 \mathrm{TeV}$

SLAC-PUB-7266; HEPEX-9702002

Measurement of the $B^{+}$and $B^{0}$ Lifetimes using Topological Vertexing

Phys. Rev. Lett. 79:590, 1997; SLAC-PUB-7674

Measurement of the $B^{+}$and $B^{\circ}$ Lifetimes using Topological Reconstruction of Inclusive and Semileptonic Decays

Phys. Rev. Lett. 76:4886, 1996

First Study of Rapidity Gaps in $e^{+} e^{-}$Annihilation

Phys. Rev. Lett. 79:572, 1997; FERMLLAB-PUB-97-024-E; CDF-PUB-BOTTOM-PUBLIC-3792

$J / \psi(1 S)$ and $\psi(2 S)$ Production in $p \bar{p}$ Collisions at $\sqrt{s}=1.8 \mathrm{TeV}$

SLAC-PUB-7117; HEPEX-9702001

Measurement of the $B^{+}$and $B^{0}$ Lifetimes from Semileptonic Decays

Phys. Rev. Lett. 78:2698, 1997; FERMILAB-PUB-97-025-E; CDF-ANAL-JET-CDFR-3799; HEPEX-9703010 Observation of Diffractive $W-B o s o n$ Production at the TEVATRON 
Abe 97D

Abe $97 \mathrm{E}$

Abe $97 \mathrm{~F}$

A be $97 \mathrm{G}$

Abe $97 \mathrm{H}$

A be 97I

Abe $97 \mathrm{~J}$

Abe $97 \mathrm{~K}$

Abe $97 \mathrm{~L}$

Abe $97 \mathrm{M}$

Abe $97 \mathrm{~N}$

A be 970

Abe $97 \mathrm{P}$

Abe $97 \mathrm{Q}$

Abe $97 \mathrm{R}$

Abe $97 \mathrm{~S}$

Abe $97 \mathrm{~T}$

Abe $97 \mathrm{U}$

A be $97 \mathrm{~V}$

Abe $97 \mathrm{~W}$

Abe $97 \mathrm{X}$

A be $97 \mathrm{Y}$

Abe $97 \mathrm{Z}$

Abe 97ZB

A be $972 \mathrm{C}$

Abe 97ZD

A be 97ZE

A be $972 \mathrm{~F}$

A be $972 G$

Abe $972 \mathrm{H}$

A be $97 \mathrm{ZI}$

Abe $97 \mathrm{ZJ}$

Abe $972 \mathrm{~K}$

A be $97 \mathrm{ZL}$

Abe 98

A be $98 \mathrm{~B}$

A be $98 \mathrm{C}$

A be $98 \mathrm{D}$
Phys. Rev. D55:R5263, 1997; FERMILA B-PUB-97-023-E; HEPEX-9702004 Search for New Particles Decaying to Dijets at CDF

Phys. Rev. Lett. 78:3442, 1997; Phys. Rev. Lett. 79:959, 1997; SLAC-PUB-7395; HEPEX-9702009 Measurement of Leading Particle Effects in Decays of $Z^{\circ}$ Bosons into Light Flavors

Phys. Rev. Lett. 79:578, 1997; FERMILAB-PUB-97-026-E

Production of $J / \psi(1 S)$ Mesons from $\chi_{c}$ Meson Decays in $p \bar{p}$ Collisions at $\sqrt{s}=1.8 \mathrm{TeV}$

Phys. Rev. Lett. 78:4691, 1997; SLAC-PUB-7333; HEPEX-9701020

Measurement of the $\tau$ Neutrino Helicity and the Michel Parameters in Polarized $e^{+} e^{-}$Collisions

Phys. Rev. Lett. 79:1992, 1997; FERMILAB-PUB-97-075-E; CDF-ANAL-TOP-CDFR-4025

First Observation of the All Hadronic Decay of $t \bar{t}$ Pairs

Phys. Rev. Lett. 79:2636, 1997; FERMILA B-PUB-97-076-E

Measurement of Diffractive Dijet Production at the Tevatron

Phys. Rev. Lett. 80:660, 1998; SLAC-PUB-7481; HEPEX-9708015

Measurements of $R(B)$ using a Vertex Mass Tag

Phys. Rev. Lett. 79:357, 1997; FERMILAB-PUB-97-058-E; HEPEX-9704003

Search For Charged Higgs Decays of the Top Quark using Hadronic Decays of the Tau Lepton

Phys. Rev. Lett. 79:2192, 1997; FERMILAB-PUB-97-122-E

Search for New Gauge Bosons Decaying into Dileptons in $\bar{p} p$ Collisions at $\sqrt{s}=1.8 \mathrm{TeV}$

Phys. Rev. Lett. 79:2198, 1997; FERMILAB-PUB-97-171-E

Limits on Quark-Lepton Compositeness Scales from Dileptons Produced in $1.8 \mathrm{TeV} p \bar{p}$ Collisions

Phys. Rev. D56:2532, 1997; FERMILAB-PUB-97-093-E

Properties of Six-Jet Events with Large Six-Jet Mass at the Fermilab Proton Antiproton Collider

Phys. Rev. Lett. 79:3585, 1997; FERMILAB-PUB-97-096-E; CDF-ANAL-TOP-PUBLIC-4011; HEPEX-9704007

The $\mu \tau$ and $e \tau$ Decays of Top Quark Pairs Produced in $p \bar{p}$ Collisions at $\sqrt{s}=1.8 \mathrm{TeV}$

Phys. Rev. Lett. 79:584, 1997; FERMILAB-PUB-97-083-E

Measurement of Double Parton Scattering in in $\bar{p} p$ Collisions at $\sqrt{s}=1.8 \mathrm{TeV}$

Phys. Rev. D57:67, 1998; FERMILAB-PUB-97-106-E

Properties of Photon Plus Two-Jet Events in $\bar{p} p$ Collisions at $\sqrt{s}=1.8 \mathrm{TeV}$

Phys. Rev. Lett. 79:3819, 1997; FERMILAB-PUB-97-247-E; CDF-ANAL-EXOTIC-CDFR-4157

Search for New Particles Decaying into $b \bar{b}$ and Produced in Association with $W$ Bosons Decaying into $e$ $\nu$ or $\mu \nu$ at the Tevatron

Phys. Rev. D56:3811, 1997; FERMILA B-PUB-97-094-E

Double Parton Scattering in $\bar{p} p$ Collisions at $\sqrt{s}=1.8 \mathrm{TeV}$

Phys. Rev. Lett. 79:804, 1997; SLAC-PUB-7418; HEPEX-9704012

Direct Measurement of Leptonic Coupling Asymmetries with Polarized $Z^{\circ}$

Phys. Rev. D56:5310, 1997; SLAC-PUB-7489; HEPEX-9707011

Measurement of the $B$ Hadron Energy Distribution in $Z^{0}$ Decays

Phys. Rev. D56:R1357, 1997; FERMILAB-PUB-97-031-E

Search for Gluinos and Squarks at the Fermilab Tevatron Collider

Phys. Rev. Lett. 80:1156, 1998; FERMILAB-PUB-97-283-E

Dijet Production by Color-Singlet Exchange at the Fermilab Tevatron

Phys. Rev. Lett. 79:4327, 1997; FERMILAB-PUB-97-280-E; HEPEX-9708017

Search for First Generation Leptoquark Pair Production in $p \bar{p}$ Collisions at $\sqrt{s}=1.8 \mathrm{TeV}$

Phys. Rev. Lett. 80:2525, 1998; FERMILAB-PUB-97-270-E

Search for Flavor-Changing Neutral Current Decays of the Top Quark in $p \bar{p}$ Collisions at $\sqrt{s}=1.8 \mathrm{TeV}$

Phys. Rev. Lett. 79:4760, 1997; FERMILAB-PUB-97-301-E; HEPEX-9709016

Properties of Jets in $W^{ \pm}$Boson Events from $1.8 \mathrm{TeV} \bar{p} p$ Collisions

Phys. Rev. Lett. 80:2057, 1998; FERMILA B-PUB-97-312-E

Measurement of the $B^{0} \bar{B}^{0}$ Oscillation Frequency in $p \bar{p}$ Collisions using $\pi B$ Meson Charge Flavor Correlations at $\sqrt{s}=1.8 \mathrm{TeV} / c^{2}$

Phys. Rev. Lett. 80:2767, 1998; FERMILAB-PUB-97-284-E; HEPEX-9801014

Measurement of the Top Quark Mass

Phys. Rev. Lett. 80:3461, 1998; FERMILAB-PUB-97-290-E

Measurement of the Differential Cross Section for Events with Large Total Transverse Energy in $p \bar{p}$ Collisions at $\sqrt{s}=1.8 \mathrm{TeV}$

Phys. Rev. Lett. 80:2773, 1998; FERMIALB-PUB-97-286-E; HEPEX-9710008

Measurement of the $t \bar{t}$ Production Cross Section in $p \bar{p}$ Collisions at $\sqrt{s}=1.8 \mathrm{TeV}$

Phys. Lett. 404B:377, 1997; SLAC-PUB-7460; HEPEX-9705017

Measurement of the Neutron Spin Structure Function $g_{2}^{n}$ and Asymmetry $A_{2}^{n}$

Phys. Rev. Lett. 79:26, 1997; SLAC-PUB-7459; HEPEX-9705012

Precision Determination of the Neutron Spin Structure Function $g_{1}^{n}$

Phys. Rev. Lett. 80:2779, 1998; FERMILA B-PUB-97-304-E; HEPEX-9802017

Measurement of the Top Quark Mass and $t \bar{t}$ Production Cross Section from Dilepton Events at the Collider Detector at Fermilab

Phys. Rev. D57:5382, 1998; FERMILAB-PUB-97-352-E

Measurement of $B$ Hadron Lifetimes using $J / \psi(1 S)$ Final States at CDF

Phys. Rev. Lett. 80:5720, 1998; FERMILAB-PUB-97-285-E; HEPEX-9711004

Observation of Fadronic $W^{ \pm}$Decays in $t \bar{t}$ Events with the Collider Detector at Fermilab

Phys. Rev. D57:R3811, 1998; FERMILAB-PUB-97-397-E

Search for the Decays $B^{\circ} \rightarrow \mu^{+} \mu^{-}$and $B s \rightarrow \mu^{+} \mu^{-}$in $p \bar{p}$ Collisions at $\sqrt{s}=1.8 \mathrm{TeV}$

Phys. Lett. 405B:180, 1997; SLAC-PUB-7461; HEPPH-9705344

Next-to-Leading Order QCD Analysis of Polarized Deep Inelastic Scattering Data

Phys. Rev. Lett. 81:1791, 1998; FERMILAB-PUB-98-024-E; HEPEX-9801019

Searches for New Physics in Diphoton Events in $p \bar{p}$ Collisions at $\sqrt{s}=1.8 \mathrm{TeV}$

Phys. Rev. D58:112003, 1998; SLAC-PUB-7753; HEPEX-9802357

Measurement of the Proton and Deuteron Spin Structure Functions $g_{1}$ and $g_{2}$

Phys. Rev. D57:1359, 1998; FERMILAB-PUB-98-009-E

The Jet Pseudorapidity Distribution in Direct Photon Events in $p \bar{p}$ Collisions at $\sqrt{s}=1.8 \mathrm{TeV}$

Phys. Rev. D58:031 101, 1998; FERMILAB-PUB-98-092-E

Search for the Rare Decay $W^{ \pm} \rightarrow \pi^{ \pm} \gamma$ in Proton-Antiproton Collisions at $\sqrt{s}=1.8 \mathrm{TeV}$ 
Abe $98 \mathrm{E}$

A be $98 \mathrm{~F}$

A be 98G

Abe $98 \mathrm{H}$

Abe $98 \mathrm{I}$

Abe $98 \mathrm{~J}$

A be $98 \mathrm{~K}$

A be $98 \mathrm{~L}$

A be $98 \mathrm{M}$

Abe $98 \mathrm{~N}$

Abe 980

A be $98 \mathrm{P}$

A be $98 \mathrm{Q}$

Abe $98 \mathrm{R}$

Abe $98 \mathrm{~S}$

Abe $98 \mathrm{~T}$

Abe $98 \mathrm{U}$

A be $98 \mathrm{~V}$

Abe $98 \mathrm{WV}$

A be $98 \mathrm{X}$

A be $98 \mathrm{Y}$

Abe 982

Abe $98 \mathrm{ZB}$

Abe $98 \mathrm{ZC}$

Abe $982 \mathrm{D}$

A be $98 \mathrm{ZE}$

A be $987 \mathrm{~F}$

A be 99

A be $99 \mathrm{~B}$

Abe $99 \mathrm{C}$

A be $99 \mathrm{D}$

Abe $99 \mathrm{E}$

Abegg 94

Abegg 94B

Abegg 95

Abegg 95B

Abela 96
Phys. Rev. D58:072001, 1998; FERMILAB-PUB-98-091-E; CDF-PUB-BOTTOM-PUBLIC-4425; HEPEX-9803013 Observation of $B^{+} \rightarrow \psi(2 S) K^{+}$and $B^{\circ} \rightarrow \psi(2 S) K^{*}(892)^{\circ}$ Decays and Measurements of $B$-Meson Branching Fractions into $J / \psi(1 S)$ and $\psi(2 S)$ Final States

Phys. Rev. D58:112004, 1998; FERMILAB-PUB-98-121-E; HEPEX-9804014

Observation of $B_{c}$ Mesons in $p \bar{p}$ Collisions at $\sqrt{s}=1.8 \mathrm{TeV}$

Phys. Rev. D58:091101, 1998; FERMILAB-PUB-98-110-E

Search for the Rare Decay $W^{ \pm} \rightarrow D_{s}^{ \pm} \gamma$ in $p \bar{p}$ Collisions at $\sqrt{s}=1.8 \mathrm{TeV}$

Phys. Rev. Lett. 81:942, 1998; SLAC-PUB-7794

A Direct Measurement of Parity Violation in the Coupling of $Z^{0}$ Bosons to $b$ Quarks using a Mass Tag and Momentum Weighted Track Charge

Phys. Rev. Lett. 81:1367, 1998; FERMILAB-PUB-98-133-E

Measurement of the $\sigma(W+\geq 1$ jet $) / \sigma(W)$ Cross Section Ratio from $\bar{p} p$ Collisions at $\sqrt{s}=1.8 \mathrm{TeV}$

Phys. Rev. D58:051102, 1998; FERMILAB-PUB-98-145-E

Search for Long-Lived Parents of $Z^{\circ}$ Bosons in $p \bar{p}$ Collisions at $\sqrt{s}=1.8 \mathrm{TeV}$

Phys. Rev. D59:012002, 1999; SLAC-PUB-7660; HEPEX-9805023

An Improved Test of the Flavor Independence of Strong Interactions

Phy's. Rev. D59:052001, 1999; SLAC-PUB-7766; IIEPEX-9805029

Production of $\pi^{+}, K^{+}, K^{0}, K^{*}(892)^{\circ}, \phi(1020), p$ and $\Lambda$ in Hadronic $Z^{0}$ Decays

Phys. Rev. Lett. 80:5275, 1998; FERMILA B-PUB-98-084-E; HEPEX-9803015

Search for Chargino-Neutralino Associated Production at the Fermilab Tevatron Collider

Phys. Rev. Lett. 81:2432, 1998; FERMILA B-PUB-98-157-E; HEPEX-9805034

Observation of $B_{c}$ Mesons in $p \bar{p}$ Collisions at $\sqrt{s}=1.8 \mathrm{TeV}$

Phys. Rev. D58:092002, 1998; FERMILAB-PUB-98-167-E; HEPEX-9806018

Improved Measurement of the $B^{-}$and $\bar{B}^{\circ}$ Meson Lifetimes using Semileptonic Decays

Phys. Rev. Lett. 81:5513, 1998; FERMILAB-PUB-98-189-E; CDF-PUB-BOTTOM-CDFR-4574; HEPEX-9806025

Measurement of the CP Violation Parameter $\sin (2 \beta)$ in $B^{0} / \bar{B}^{0} \rightarrow J / \psi(1 S) K_{S}$ Decays

Phys. Rev. Lett. 81:4806, 1998; FERMILA B-PUB-98-219-E

A Search for Second Generation Leptoquarks in the Dimuon Plus Dijet Channel of $p \bar{p}$ Collisions at $\sqrt{s}=$ $1.8 \mathrm{TeV}$

Phys. Rev. D59:092002, 1999; FERMILAB-PUB-98-206-E; HEPEX-9806034

Searches for New Physics in Diphoton Events in $p \bar{p}$ Collisions at $\sqrt{s}=1.8 \mathrm{TeV}$

Phys. Rev. Lett. 81:5748, 1998; FERMILAB-PUB-98-252-E

Search for Higgs Boson Produced in Association with a Vector Boson in $p \bar{p}$ Collisions at $\sqrt{s}=1.8 \mathrm{TeV}$

FERMILA B-PUB-98-321-E; HEPEX-9810031

Search for a Technicolor techniomega Particle in Events with a Photon and a $b$-quark Jet at CDF

Phys. Rev. Lett. 82:2038, 1999; FERMILAB-PUB-98-290-E; HEPEX-9809022

Search for New Particles Decaying to $b \bar{b}$ in $p \bar{p}$ Collisions at $\sqrt{s}=1.8 \mathrm{TeV}$

Phys. Rev. Lett. 82:3206, 1999; FERMILAB-PUB-98-352-E

Search for Third-Generation Leptoquarks from Technicolor Models in $p \bar{p}$ Collisions at $\sqrt{s}=1.8 \mathrm{TeV}$

Phys. Rev. Lett. 81:5278, 1998; FERMILAB-PUB-98-334-E

Events with a Rapidity Gap between Jets in $\bar{p} p$ Collisions at $\sqrt{s}=630 \mathrm{GeV}$

Phys. Rev. Lett. 81:5754, 1998; FERMILAB-PUB-98-256-E; HEPEX-9809001

Measurement of the Lepton Charge Asymmetry in $W^{ \pm}$-Boson Decays Produced in $p \bar{p}$ Collisions

FERMILAB-PUB-98-392-E

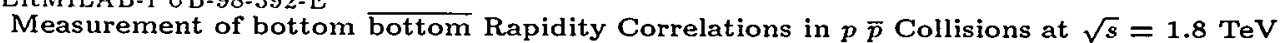

Phys. Rev. D59:052002, 1999; FERMILAB-PUB-98-280-E

Measurement of $Z^{0}$ and Drell-Yan Production Cross Section using Dimuons in $p \bar{p}$ Collisions at $\sqrt{s}=$ $1.8 \mathrm{TeV}$

Phys. Rev. Lett. 82:271, 1999; Phys. Rev. Lett. 82:2808, 1999; FERMILAB-PUB-98-319-E; HEPEX-9810029

Measurement of the Top Quark Mass with the Collider Detector at Fermilab

Phys. Rev. D59:032001, 1999; FERMILAB-PUB-98-188-E; HEPEX-9806026

Measurement of the $B^{0}-\bar{B}^{0}$ Flavor Oscillation Frequency and Study of Same Side Flavor Tagging of $B$ Mesons in $p \bar{p}$ Collisions

Phys. Rev. Lett. 81:5742, 1998; FERMILAB-PUB-98-263-E

Search for the Decays $B_{s}, B^{\circ} \rightarrow e^{ \pm} \mu^{\mp}$ and Pati-Salam Leptoquarks

Phys. Rev. D59:092001, 1999; FERMILAB-PUB-98-327-E

Kinematics of $t \bar{t}$ Events at CDF

Phys. Lett. 452B:194, 1999; SLAC-PUB-7927; HEPEX-9808028

Measurements of $R=\sigma_{L} / \sigma_{T}$ for $0.03 \leq x \leq 0.1$ and Fit to World Data

FERMILAB-PUB-99-021-E; HEPEX-9902001

Measurement of the Associated $\gamma \mu^{ \pm}$Production Cross Section in $p \bar{p}$ Collisions at $\sqrt{s}=1.8 \mathrm{TeV}$

FERMILAB-PUB-99-030-E

Measurement of the $B^{\circ} \bar{B}^{0}$ Oscillation Frequency using Dimuon Data in $p \bar{p}$ Collisions at $\sqrt{s}=1.8 \mathrm{TeV}$

SLAC-PUB-7920; HEPEX-9903004

First Study of the Structure of $e^{+} e^{-} \rightarrow b \bar{b}$ gluon Events and Limits on the Anomalous Chromomagnetic Moment of the $b$-Quark

FERMILAB-PUB-99-043-E

A Measurement of $b$ Quark Fragmentation Fractions in the Production of Strange and Light $B$ Mesons in $p \bar{p}$ Collisions at $\sqrt{s}=1.8 \mathrm{TeV}$

Phys. Rev. D59:032004, 1999; FERMILAB-PUB-98-172-E; HEPEX-9808003

Measurement of the $B_{s}$ Meson Lifetime using Semileptonic Decays

Phys. Rev. D50:92, 1994; TRI-PP-94-10

Measurement of the Branching Ratio for the Decay $\eta \rightarrow \mu^{+} \mu^{-}$

TRI-PP-94-96

Measurement of Charge Symmetry Breaking in $n p$ Elastic Scattering at $350 \mathrm{MeV}$

Phys. Rev. Lett. 75:1711, 1995; TRI-PP-95-43

Precision Measurement of Charge Symmetry Breaking in $n p$ Elastic Scattering at $347 \mathrm{MeV}$ TRI-PP-95-52

Precision Measurement of Charge Symmetry Breaking in $n p$ Elastic Scattering at $347 \mathrm{MeV}$

Phys. Rev. Lett. 77:1950, 1996; NUCLEX-9805005

Improved Upper Limit on Muonium to Antimuonium Conversion 
Abele 96

Abele $96 \mathrm{~B}$

Abele $96 \mathrm{C}$

Abele 97

Abele 97B

Abele $97 \mathrm{C}$

Abele 97D

Abele $97 \mathrm{E}$

A bele $97 \mathrm{~F}$

Abele $97 \mathrm{G}$

Abele $97 \mathrm{H}$

Abele 98

A bele 98B

Abele $98 \mathrm{C}$

Abele 98D

Abele $98 \mathrm{E}$

Abele 99

Abele 99B

Ableev 94

Ableev 94B

Ableev $94 \mathrm{C}$

Ableev 94D

Ableev $94 \mathrm{E}$

Ableev $94 \mathrm{~F}$

Ableev 94G

Ableev $94 \mathrm{H}$

Ableev 94I

Ableev 95

Ableev 95C

Abraamyan 92

Abraamyan 94B

Abraamyan $94 \mathrm{C}$

Abraamyan 96

Abraamyan 98

Abramov 94

Abramov 96

Abramov 96B

Abramov 98
Phys. Lett. 380B:453, 1996

A. Study of $f_{0}(1500)$ Decays into $4 \pi^{\circ}$ in $\bar{p} p \rightarrow 5 \pi^{\circ}$ at Rest

Phys. Lett. 385B:425, 1996

Observation of $f_{0}(1500)$ Decay into $K_{L} K_{L}$

Nucl. Phys. A609:562, 1996; Nucl. Phys. A625:889, 1997

Further Analysis of $\bar{p} p \rightarrow 3 \pi^{0}, \eta \eta \pi^{0}$ and $\eta \pi^{0} \pi^{0}$ at Rest

Phys. Lett. 391B:191, 1997

High-Mass $\rho$-Meson States from $\bar{p}$ deuteron Annihilation at Rest into $\pi^{-} \pi^{0} \pi^{0} p_{\text {spectator }}$

Phys. Lett. 402B:195, 1997

Measurement of the Decay Distribution of $\eta^{\prime} \rightarrow \pi^{+} \pi^{-} \gamma$ and Evidence for the Box Anomaly

Phys. Lett. 404B:179, 1997

Study of the $\pi^{\mathbf{0}} \pi^{\mathbf{0}} \eta^{\prime}$ Final State in $\bar{p} p$ Annihilation at Rest

Phys. Lett. 411B:354, 1997

$\rho-\omega$ Interference in $\bar{p} p$ Annihilation at Rest into $\pi^{+} \pi^{-} \eta$

Phys. Lett. 411B:361, 1997

Measurement of the $\omega \rightarrow \eta \gamma$ Decay Branching Ratio

Phys. Lett. 415B:289, 1997

Antiproton-Proton Annihilations at Rest into $K_{L} K^{ \pm} \pi^{\mp} \pi^{0}$

Phys. Lett. 415B:280, 1997

Antiproton-Proton Annihilations at Rest into $K_{L} K_{S} \pi^{0} \pi^{0}$

Phys. Lett. 407 B:212, 1997

$A$ Measurement of the Beta Asymmetry $A$ in the Decay of Free Neutrons

Phys. Lett. 417B:193, 1998

Decay Dynamics of the Process $\eta \rightarrow 3 \pi^{0}$

Phys. Lett. 417 B:197, 1998

Momentum Dependence of the Decay $\eta \rightarrow \pi^{+} \pi^{-} \pi^{0}$

Phys. Rev. D57:3860, 1998

$\bar{p} p$ Annihilation at Rest into $K_{L} K^{ \pm} \pi^{\mp}$

Nucl. Phys. B514:45, 1998

Study of $\bar{p} p \rightarrow \eta \pi^{0} \pi^{0} \pi^{0}$ at Rest

Phys. Lett. 423B:175, 1998

Exotic $\eta \pi$ State in $\bar{p}$ deuteron Annihilation at Rest into $\pi^{-} \pi^{0} \eta p_{\text {spectator }}$

Phys. Lett. 446B:349, 1999

Evidence for a $\pi \eta$ P-Wave in $\bar{p} p$ Annihilations at Rest into $\pi^{0} \pi^{0} \eta$

Phys. Lett. 450B:275, 1999

$\bar{p}$ deuteron Annihilation at Rest into $\pi^{-} \pi^{+} \pi^{-} p_{\text {spectator }}$

Phys. At. Nucl. 57:1614, 1994; Yad. Phys. 57:1684, 1994

Recent Results on the Analysis of $\bar{p} p \rightarrow \pi^{+} \pi^{-} \pi^{0}$

Phys. At. Nucl. 57:1619, 1994; Yad. Phys. 57:1689, 1994

Two-Body Final State from nucleon nucleon Annihilations in Gaseous Hydrogen and $S / P$ Wave Problem Phys. Lett. 329B:407, 1994

Measurement of the $\bar{p} p \rightarrow \pi^{+} \pi^{-}$and $\bar{p} p \rightarrow K^{+} K^{-}$Annihilation Frequencies in a 5 mb Hydrogen Gas Target

Phys. Lett. 334B:237, 1994

$\phi(1020)$ and $\omega$ Meson Production in $\bar{n} p$ Annihilation and the OZI Rule

Nuovo Cim. 107 A:2837, 1994

A Study of Pontecorvo Reactions in Antiproton Deuterium Annihilations at Rest

Phys. At. Nucl. 57:1745, 1994; Yad. Phys. 57:1816,1994

Recent Results from OBELIX in Atomic Physics

Phys. At. Nucl. 57:1716, 1994; Yad. Phys. 57:1787, 1994

Measurement of the $\phi(1020)$ - and $\omega$-Meson Production in Antiproton Annihilation at Rest on Deuterium Nuovo Cim. 107A:943, 1994

Annihilation Cross Sections of Antineutrons on C, Al, Cu, Sn and Pb at Low Momenta (180 - 280 MeV/c) with the OBELIX Spectrometer

Nuovo Cim. 107 A:1325, 1994

Changes in the Annihilation's Delay Time Distribution of Stopped Antiprotons in Helium Gas due to Contaminants.

Nucl. Phys. A585:577, 1995

Measurements of the $\bar{p}$ deuteron Annihilation at Rest

Nucl. Phys. A594:375, 1995

$\phi(1020) \pi^{\circ}$ and $\phi(1020) \eta$ Production in Antiproton Annihilation at Rest in a Hydrogen Gas Target at NTP

Phys. Lett. 323B:1, 1994; JINR-E1-92-307

Inclusive Neutral Pion Production at Forward Angles at $4.5 \mathrm{GeV} / c$ per Nucleon in $\alpha+\mathrm{C} \rightarrow \pi^{0} \mathrm{X}$ and $\alpha$ $+\mathrm{Cu} \rightarrow \pi^{0}$ X Reactions

Phys. At. Nucl. 59:252, 1996; Yad. Phys. 59:271, 1996; JINR-P1-94-289

Inclusive $\pi^{\circ}$ Production in $p \mathrm{C}$ and $p \mathrm{Cu}$ Interactions at $4.5 \mathrm{GeV} / c$

Kr. Soob. JINR 68:29, 1994

Investigation of Neutral Particle Production by Relativistic Nuclei on the LHE 90-Channel $\gamma$ Spectrometer. Results and Perspectives

Phys. At. Nucl. 60:1843, 1997; Yad. Phys. 60:2014, 1997; JINR-P1-96-493

Inclusive $\pi^{0}$ Production in $\mathrm{C} \mathrm{C}$ and $\mathrm{C}$ Cu Interactions at a Momentum of $4.5 \mathrm{GeV} / \mathrm{c}$

DUBNA-96, v. II, p. 47

Inclusive Neutral Pion Production in Proton-Nucleus and Nucleus-Nucleus Interactions at a Momentum of $4.5 \mathrm{GeV} / \mathrm{c}$ per Nucleon

Phys. At. Nucl. 57:797, 1994; Yad. Phys. 57:850, 1994

Search for Dibaryons in Two-Protons Mass-Spectra from $p n \rightarrow 2 p \pi^{-}$(Backward) at $1.98 \mathrm{GeV} / \mathrm{c}$

Nucl. Phys. B492:3, 1997; IFVE-96-82

Production Asymmetry Measurement of High $x_{T}$ Hadrons in $p \uparrow p$ Collisions at $40 \mathrm{GeV}$

Phys. At. Nucl. 59:376, 1996; Yad. Phys. 59:399, 1996

Inclusive Pion Double Charge Exchange on ${ }^{16} \mathrm{O}$ at 800 and $750 \mathrm{MeV}$

Eur. Phys. J. A1:115, 1998

Search for $d^{\prime}$ in Pion Inclusive Spectra from $p p$ Interactions

See the legend on page 5 . 
Abramov $98 \mathrm{~B}$

Abreu $93 \mathrm{G}$

Abreu 93L

Abreu 94

Abreu $94 \mathrm{~B}$

Abreu $94 \mathrm{C}$

Abreu 94D

Abreu $94 \mathrm{E}$

Abreu $94 \mathrm{~F}$

Abreu $94 \mathrm{G}$

Abreu $94 \mathrm{H}$

Abreu 94I

Abreu 94J

Abreu $94 \mathrm{~K}$

Abreu 94L,

Abreu $94 \mathrm{M}$

Abreu $94 \mathrm{~N}$

Abreu 940

Abreu $94 \mathrm{P}$

Abreu $94 Q$

Abreu $94 \mathrm{R}$

Abreu $94 \mathrm{~S}$

Abreu 95

Abreu 95B

Abreu $95 \mathrm{C}$

Abreu $95 \mathrm{D}$

Abreu $95 \mathrm{E}$

Abreu 95F

Abreu $95 \mathrm{G}$

Abreu $95 \mathrm{H}$

Abreu 951

Abreu 95J

Abreu $95 \mathrm{~K}$

Abreu 95L

Abreu $95 \mathrm{M}$

Abreu $95 \mathrm{~N}$

Abreu 950

Abreu $95 \mathrm{P}$

Abreu $95 \mathrm{Q}$

DUBNA-96, v. II, p. 18

Search for dibaryon in Pion Inclusive Spectra from $p$-Interactions

Z. Phys. C59:533, 1993; Z. Phys. C65:709, 1995; CERN-PPE-93-70

A Measurement of $D$ Meson Production in $Z^{\circ}$ Hadronic Decays

Phys. Lett. 322 B:459, 1994; CERN-PPE-93-220

Measurement of the $B^{0} \bar{B}^{0}$ Mixing using the Average Electric Charge of Hadron Jets in $Z^{\circ}$ Decays

Z. Phys. C61:407, 1994; CERN-PPE-93-176

Production Rate and Decay Lifetime Measurements of $B_{s}$ Mesons at LEP using $D_{B}^{ \pm}$and $\phi(1020)$ Mesons

Z. Phys. C63:17, 1994; CERN-PPE-94-02

Invariant Mass Dependence of Particle Correlations in Hadronic Final States from the Decay of the $Z^{\circ}$

Phys. Lett. 323B:242, 1994; CERN-PPE-94-03

Interference of Neutral Kaons in the Hadronic Decays of the $Z^{\circ}$

2. Phys. C62:357, 1994; CERN-PPE-94-04

Study of Hard Scattering Processes in Multihadron Production from $\gamma \gamma$ Collisions at LEP

Nucl. Phys. B417:3, 1994; Nucl. Phys. B426:244, 1994; CERN-PPE-94-08 Measurements of the Line Shape of the $Z^{\circ}$ and Determination of Electroweak Parameters from Its Hadronic and Leptonic Decays

Z. Phys. C63:3, 1994; CERN-PPE-94-24

A Precision Measurement of the Average Lifetime of $B$ Hadrons

Nucl. Phys. B418:403, 1994; CERN-PPE-94-031

Improved Measurement of Cross Sections and Asymmetries at the $Z^{0}$ Resonance

Phys. Lett. 327B:386, 1994; CERN-PPE-94-036

Measurement of the $e^{+} e^{-} \rightarrow \gamma \gamma$ ( $\gamma^{\prime}$ 's) Cross Section at LEP Energies

Phys. Lett. 324B:500, 1994; CERN-PPE-94-22

A Measurement of the $B_{3}$ Meson Mass

Phys. Lett. 332B:488, 1994; CERN-PPE-94-067 Measurement of the $B^{\circ} \bar{B}^{0}$ Mixing Parameter in DELPHI

Phys. Lett. 338B:409, 1994; CERN-PPE-94-132

Measurement of Time Dependent $B^{0} \bar{B}^{0}$ Mixing

Z. Phys. C64:183, 1994; CERN-PPE-94-83 Search for Pair-Produced Heavy Scalars in $Z^{0}$ Decays

Phys. Lett. 341 B:109, 1994; CERN-PPE-94-99 $J / \psi(1 S)$ Production in the Hadronic Decays of the $Z^{0}$

Z. Phys. C65:603, 1995; CERN-PPE-94-121

A Study of Radiative Muon Pair Events at $Z^{0}$ Energies and Limits on an Additional $Z^{\prime}$ Gauge Boson

Phys. Lett. 334B:435, 1994; CERN-PPE-94-88

Charged Kaon Production in $\tau \pm$ Decays at LEP

Nucl. Phys. B421:3, 1994; CERN-PPE-94-046-REV

Search for the Standard Model Higgs Boson in $Z^{0}$ Decays

2. Phys. C65:587, 1995; CERN-PPE-94-130

Production Characteristics of $K^{0}$ and Light Meson Resonances in Hadronic Decays of the $Z^{0}$

Z. Phys. C65:555, 1995; CERN-PPE-94-131

Measurement of the $\Gamma(B \bar{B}) / \Gamma$ (hadron) Branching Ratio of the $Z^{0}$ by Double Hemisphere Tagging

Z. Phys. C65:569, 1995; CERN-PPE-94-161

Measurement of the Forward-Backward Asymmetry of $e^{+} e^{-} \rightarrow z^{0} \rightarrow b \bar{b}$ using Prompt Leptons and a Lifetime Tag

Phys. Lett. 342B:402, 1995; CERN-PPE-94-163

First Evidence of Hard Scattering Processes in Single Tagged $\gamma \gamma$ Collisions

Z. Phys. C66:323, 1995; CERN-PPE-95-08

Measurement of $\Gamma(B \bar{B}) / \Gamma$ (hadrons) using Impact Parameter Measurements and Lepton Identiflcation

Z. Phys. C67:543, 1995; CERN-PPE-95-39

Strange Baryon Production in $Z^{\circ}$ Hadronic Decays

Nucl. Phys. B444:3, 1995; CERN-PPE-95-028

Inclusive Measurements of the $K^{ \pm}$and $p / \bar{p}$ Production in Hadronic $Z^{0}$ decays

Phys. Lett. 357B:255, 1995; CERN-PPE-95-91

Search for Exclusive Charmless $B$ Meson Decays with the DELPHI Detector at LEP

Phys. Lett. 357B:715, 1995; CERN-PPE-95-114

A Measurement of the $\tau^{ \pm}$Leptonic Branching Fractions

Z. Phys. C69:223, 1995; CERN-PPE-95-087; PHOTON-95 p. 16

A Measurement of the Photon Structure Function $F_{2}^{\gamma}$ at an Average $Q^{2}$ of $12 \mathrm{GeV}^{2} / c^{4}$

Z. Phys. C68:353, 1995; CERN-PPE-95-53

$B^{*}$ Production in $Z^{\circ}$ Decays

Phys. Lett. 355B:415, 1995; CERN-PPE-95-7 7

Observation of Short Range Three Particle Correlations in $e^{+} e^{-}$Annihilations at LEP Energies

Phys. Lett. 361 B:207, 1995; CERN-PPE-95-130

Measurement of $\Delta\left(1232 P_{33}\right)$ Production in Hadronic $Z$ Decays

Phys. Lett. 359B:411, 1995; CERN-PPE-95-115

Upper Limits on the Branching Ratios $\tau^{ \pm} \rightarrow \mu^{ \pm} \gamma$ and $\tau^{ \pm} \rightarrow e^{ \pm} \gamma$

Z. Phys. C68:13, 1995; CERN-PPE-95-060

A Measurement of $B^{+}$and $B^{0}$ Lifetimes using $\bar{D} \ell^{+}$Events

Z. Phys. C68:375, 1995; CERN-PPE-95-054

Lifetime and Production Rate of Beauty Baryons from $Z$ Decays

Z. Phys. C68:541, 1995; CERN-PPE-95-29

Production of Strange $B$ Baryons Decaying into $\Xi \ell$ Pairs at LEP

Z. Phys. C69:575, 1996; CERN-PPE-95-145

Search for Promptly Produced Heavy Quarkonium States in Hadronic $Z^{\circ}$ Decays

Phys. Lett. 365B:448, 1996; CERN-PPE-95-154

A Precise Measurement of the Tau Lepton Lifetime

Phys. Lett. 347 B:447, 1995; CERN-PPE-95-01

Production of Charged Particles, $K_{S}, K^{ \pm}, p$ and $\Lambda$ in $Z^{0} \rightarrow B \bar{B}$ Events and in the Decay of $B$ Hadrons 
Abreu 95R

Abreu 95S

Abreu 95T

Abreu $95 \mathrm{U}$

Abreu 95V

Abreu $95 \mathrm{X}$

Abreu 96

Abreu $96 \mathrm{~B}$

Abreu $96 \mathrm{C}$

Abreu 96D

Abreu $96 \mathrm{E}$

Abreu $96 \mathrm{~F}$

Abreu $96 \mathrm{G}$

Abreu $96 \mathrm{H}$

Abreu 96I

Abreu 96J

Abreu $96 \mathrm{~K}$

Abreu 96L

Abreu $96 \mathrm{M}$

Abreu $96 \mathrm{~N}$

Abreu 960

Abreu 96P

Abreu 96Q

Abreu 96R

Abreu 96S

Abreu 96T

Abreu $96 \mathrm{U}$

Abreu $96 \mathrm{~V}$

Abreu $96 \mathrm{~W}$

Abreu $96 \mathrm{X}$

Abreu 96Y

Abreu 962

Abreu 96ZB

Abreu 96ZC

Abreu 962D

Abreu 96ZE

Abreu 96ZF

Abreu 97

Abreu 97B
Phys. Lett. 345B:598, 1995; CERN-PPE-94-210

Observation of Orbitally Excited $B$ Mesons

Z. Phys. C66:341, 1995; CERN-PPE-94-193 Measurement of the Forward-Backward Asymmetry of Charm and Bottom Quarks at the $Z^{\circ}$ Pole using $D^{*}(\mathbf{2 0 1 0})^{ \pm}$Mesons

Z. Phys. C67:183, 1995; CERN-PPE-95-30

Measurements of the $\tau^{ \pm}$Polarization in $Z^{\circ}$ Decays

Z. Phys. C69:1, 1995; CERN-PPE-95-101

Study of Prompt Photon Production in Hadronic $Z^{0}$ Decays

Z. Phys. C67:1, 1995; CERN-PPE-94-219

First Measurement of the Strange Quark Asymmetry at the $Z^{0}$ Peak

Z. Phys. C67:69, 1995; CERN-PPE-94-218

Search for Heavy Neutral Higgs Bosons in Two-Doublet Models

Z. Phys. C74:577, 1997; CERN-PPE-96-003

Search for New Phenomena using Single Photon Events in the DELPHI Detector at LEP

Z. Phys. C70:179, 1996; CERN-PPE-95-164

Energy Dependence of the Differences between the Quark and Gluon Jet Fragmentation

Z. Phys. C71:539, 1996; CERN-PPE-96-11

Determination of $V_{c b}$ from the Semileptonic Decay $B^{0} \rightarrow D^{*}(2010)^{-} \ell^{+} \nu$

Phys. Lett. 380B:480, 1996; CERN-PPE-96-60

Study of Radiative Leptonic Events with Hard Photons and Search for Excited Charged Leptons at $\sqrt{s}=$ $130-136 \mathrm{GeV}$

Phys. Lett. 379B:309, 1996; CERN-PPE-96-026

First Measurement of $f_{2}^{\prime}(\mathbf{1 5 2 5})$ Production in $Z^{0}$ Hadronic Decays

Phys. Lett. 374B:351, 1996; CERN-PPE-96-16

Search for Exclusive Decays of the $\Lambda_{b}$ Baryon and Measurement of its Mass

Phys. Lett. 382B:323, 1996; CERN-PPE-96-75

Search for the Lightest Chargino at $\sqrt{s}=130$ and $136 \mathrm{GeV}$ in DELPHI

Phys. Lett. 379B:330, 1996; CERN-PPE-96-54

Kaon Interference in the Hadronic Decays of the $Z^{0}$

Z. Pliys. C72:31, 1996; CERN-PPE-96-44

First Study of the Interference between Initial and Final State Radiation at the $Z^{0}$ Resonance

Z. Phys. C73:61, 1996; CERN-PPE-96-77

Measurement of Inclusive $K^{*}(892)^{\circ}, \phi(1020)$ and $K_{2}^{*}(1430)^{\circ}$ Production in Hadronic $Z^{\circ}$ Decays

Z. Phys. C72:179, 1996; CERN-PPE-96-76

Search for High Mass $\gamma \gamma$ Resonances in $e^{+} e^{-} \rightarrow \ell^{+} \ell^{-} \gamma \gamma, \nu \bar{\nu} \gamma \gamma$ and $q \bar{q} \gamma \gamma$ at LEP I

Phys. Lett. 377B:195, 1996; CERN-PPE-96-13

Updated Precision Measurement of the Average Lifetime of $B$ Hadrons

Phys. Lett. 387B:651, 1996; CERN-PPE-96-110

Search for Neutralinos, Scalar Leptons and Scalar Quarks in $e^{+} e^{-}$Interactions at $\sqrt{s}=130$ and 136 GeV

Z. Phys. C71:199, 1996; CERN-PPE-96-21

Determination of the Average Lifetime of $B$ Baryons

Z. Phys. C71:11, 1996; CERN-PPE-96-32

Mean Lifetime of the $B$, Meson

Z. Phys. C70:531, 1996; CERN-PPE-96-015

Measurement of the Partial Decay Width $R_{b}=\Gamma(b \bar{b}) / \Gamma$ (hadrons) of the $Z^{0}$ with the DELPHI Detector at LEP

Phys. Lett. 389B:405, 1996; CERN-PPE-96-131

An Upper Limit for $\operatorname{Br}\left(Z^{0} \rightarrow\right.$ gluon gluon gluon $)$ from Symmetric Three Jet $Z^{\circ}$ Hadronic Decays

Z. Phys. C73:229, 1997; CERN-PPE-96-130

Measurement of Event Shape and Inclusive Distributions at $\sqrt{s}=130$ and $136 \mathrm{GeV}$

Z. Phys. C73:243, 1997; CERN-PPE-96-129

Search for Lepton Flavor Number Violating $Z^{0}$ Decays

Phys. Lett. 372B:172, 1996; CERN-PPE-96-005

Charged Particle Multiplicity in $e^{+} e^{-}$Interactions at $\sqrt{s}=130 \mathrm{GeV}$

Z. Phys. C72:17, 1996; CERN-PPE-96-06

Measurement of the $B^{0}$ Oscillation Frequency using Kaons, Leptons and Jet Charge

Z. Phys. C74:19, 1997; Z. Phys. C75:579, 1997; CERN-PPE-96-139

A Precise Measurement of the $B^{\circ}$ Meson Lifetime using a New Technique

Phys. Lett. 393B:245, 1997; CERN-PPE-96-169

Search for Excited Leptons in $e^{+} e^{-}$Collisions at $\sqrt{s}=161 \mathrm{GeV}$

Phys, Lett. 396B:315, 1997; CERN-PPE-96-188

Search for Stable Heavy Charged Particles in $e^{+} e^{-}$Collisions at $\sqrt{s}=130-136,161$ and $172 \mathrm{GeV}$

Phys. Lett. 398B:194, 1997; CERN-PPE-96-185

A Measurement of $\alpha_{s}$ from the Scaling Violation in $e^{+} e^{-}$Annihilation

Phys. Lett. 398B:207, 1997; CERN-PPE-96-194

Search for the $B_{c}$ Meson

Z. Phys. C74:57, 1997; Z. Phys. C75:580, 1997; CERN-PPE-96-195

Search for Neutral Heavy Leptons Produced in $Z^{\circ}$ Decays

Phys. Lett. 401B:118, 1997; CERN-PPE-96-193

Identifled Particles in Quark and Gluon Jets

Z. Phys. C73:11, 1996; CERN-PPE-96-120

Tuning and Test of Fragmentation Models Based on Identifled Particles and Precision Event Shape Data

Phys. Lett. 368B:230, 1996

Muon Pair and Vector Meson Cross Sections in $p$ Wt and S U Collisions at 200 GeV/Nucleon

Phys. Lett. 368B:239, 1996

Production of $\rho+\omega$ and $\phi(1020)$ in $p$ Wt and $S U$ Collisions at $200 \mathrm{GeV} / \mathrm{Nucleon}$

Phys. Lett. 397B:158, 1997; CERN-PPE-97-009

Measurement and Interpretation of the W-Pair Cross Section in $e^{+} e^{-}$Interactions at $161 \mathrm{GeV}$

Phys. Lett. 416B:247, 1998; CERN-PPE-97-027

Rapidity Correlations in $\Lambda$ Baryon and Proton Production in Hadronic $Z^{\circ}$ Decays

See the legend on page 5. 
Abreu $97 \mathrm{C}$

Abreu 97D

Abreu 97E

Abreu $97 \mathrm{~F}$

Abreu $97 \mathrm{G}$

Abreu $97 \mathrm{H}$

Abreu 97I

Abreu 97J

Abreu $97 \mathrm{~K}$

Abreu $97 \mathrm{~L}$

Abreu $97 \mathrm{M}$

Abreu $97 \mathrm{~N}$

Abreu $97 \mathrm{O}$

Abreu 97P

Abreu 97Q

Abreu $97 R$

Abreu 97S

Abreu 97T

Abreu 98

Abreu $98 \mathrm{~B}$

Abreu 98C

Abreu 98D

Abreu $98 \mathrm{E}$

Abreu $98 \mathrm{~F}$

Abreu 98G

Abreu $98 \mathrm{H}$

Abreu 98I

Abreu 98J

Abreu $98 \mathrm{~K}$

A breu $98 \mathrm{~L}$

Abreu $98 \mathrm{M}$

Abreu $98 \mathrm{~N}$

Abreu 980

Abreu 98P

Abreu $98 \mathrm{Q}$

Abreu $98 \mathrm{R}$

Abreu $98 \mathrm{~S}$

Abreu $98 \mathrm{~T}$

Abreu $98 \mathrm{U}$
Phys. Lett. 401B:181, 1997; CERN-PPE-97-030 Measurement of Correlations between Pions from Different W's in $e^{+} e^{-} \rightarrow W^{+} W^{-}$Events Phys. Lett. 404B:194, 1997; CERN-PPE-97-034 Measurement of the Transverse Spin Correlation in $Z^{0} \rightarrow \tau^{+} \tau^{-}$Decays

2. Phys. C75:581, 1997; CERN-PPE-97-045 A Study of the Reaction $e^{+} e^{-} \rightarrow \mu^{+} \mu^{-} \gamma_{I S R}$ at LEP and Search for New Physics at Annihilation Energies near $80 \mathrm{GeV}$

207 1997. CERN-PPE-97-039

Measurement of the Multiplicity of Gluons Splitting to Bottom Quark Pairs in Hadronic $Z^{0}$ Decays

Phys. Lett. 406B:271, 1997; CERN-PPE-97-055 Measurement of the Spin Density Matrix for the $\rho^{\circ}, K^{*}(892)^{\circ}$ and $\phi(1020)$ Produced in $Z^{\circ}$ Decays

Z. Phys. C76:579, 1997; CERN-PPE-97-051 Measurement of $B^{0}-\bar{B}^{0}$ Oscillations

Phys. Lett. 407B:174, 1997; CERN-PPE-97-062 Observation of Charge Ordering in Particle Production in Hadronic $Z^{\circ}$ Decay

Phys. Lett. 414B:401, 1997; CERN-PPE-97-112 Measurement of the Triple Gluon Vertex from Double Quark Tagged 4-Jet Events Eur. Phys. J. C6:19, 1999; CERN-PPE-97-108 Measurement of the Quark and Gluon Fragmentation Functions in $Z^{0}$ Hadronic Decays

Eur. Phys. J. C2:1, 1998; CERN-PPE-97-085 Search for Neutral and Charged Higgs Bosons in $e^{+} e^{-}$Collisions at $\sqrt{s}=161$ and $172 \mathrm{GeV}$

Eur. Phys. J. C1:1, 1998; CERN-PPE-97-107 Search for Charginos, Neutralinos and Gravitinos at LEP

Phys. Lett. 416B:233, 1998; CERN-PPE-97-113 Charged Particle Multiplicity in $e^{+} e^{-} \rightarrow q \bar{q}$ Events at 161 and $172 \mathrm{GeV}$ and from the Decay of the $W^{ \pm}$ Boson

Phys. Lett. 418B:430, 1998; CERN-PPE-97-141 $m_{b}$ at $M_{Z}$

Phys. Lett. 420B:140, 1998; CERN-PPE-97-145 Search for Charged Higgs Bosons in $e^{+} e^{-}$Collisions at $\sqrt{s}=172 \mathrm{GeV}$

Eur. Phys. J. C2:581, 1998; CERN-PPE-97-160 Measurement of the $W^{ \pm}$Pair Cross-Section and of the $W^{ \pm}$Mass in $e^{+} e^{-}$Interactions at $172 \mathrm{GeV}$

Phys. Lett. 423B:194, 1998; CERN-PPE-97-163 Measurement of Trilinear Gauge Couplings in $e^{t} e^{-}$Collisions at 161 and $172 \mathrm{GeV}$

Phys. Lett. 410B:327, 1997 $J / \psi(1 S)$ and Drell-Yan Cross-Sections in $\mathrm{Pb} \mathrm{Pb}$ Interactions at $158 \mathrm{GeV} / \mathrm{c}$ per Nucleon

Phys. Lett. 410B:337, 1997 Anomalous $J / \psi(1 S)$ Suppression in $\mathrm{Pb} \mathrm{Pb}$ Interactions at $158 \mathrm{GeV} / c$ per Nucleon

Phys. Lett. 426B:193, 1998; CERN-EP-98-007 Measurement of the Inclusive Charmless and Double-Charm $B$ Branching Ratios

Phys. Lett. 426B:411, 1998; CERN-EP-98-014 A Study of the Hadronic Resonance Structure in the Decay $\tau \rightarrow 3 \pi \nu_{\tau}$

Phys. Lett. 425B:399, 1998; CERN-EP-98-034 Measurement of the Charged Particle Multiplicity of Weakly Decaying B Hadrons

Phys. Lett. 426B:231, 1998; CERN-EP-98-030 First Evidence for a Charm Radial Excitation, $D^{*}(2600)$

Eur. Phys. J. C4:1, 1998; CERN-EP-98-024

Investigation of the Splitting of Quark and Gluon Jets

Phys. Lett. 433B:429, 1998; CERN-EP-98-075 Measurement of the $e^{+} e^{-} \rightarrow \gamma \gamma(\gamma$ 's) Cross Section at the LEP Energies

Phys. Lett. 423B:207, 1998; IPN L-98-02; LYCEN-9802 Transverse Momentum of $J / \psi(1 S), \psi(2 S)$ and Mass Continuum Muon Pairs Produced in ${ }^{32} \mathrm{~S} \mathrm{U}$ Collisions at $200 \mathrm{GeV} / c$ per Nucleon

Phys. Lett. 441B:479, 1998; CERN-EP-98-151; HEPEX-9810030

A Search for $\eta_{c}(2 S)$ Production in Photon-Photon Fusion at LEP

Phys. Lett. 444B:516, 1998; CERN-EP-98-161

Charmonia Production in $450 \mathrm{GeV} / \mathrm{c}$ Proton Induced Reactions

Phys. Lett. 446B:62, 1999; CERN-EP-98-177

Search for Leptoquarks and FCNC in $e^{+} e^{-}$Annihilations at $\sqrt{s}=183 \mathrm{GeV}$

Phys. Lett. 439B:209, 1998; CERN-EP-98-115

Measurement of $\left|V_{c s}\right|$ using $W^{ \pm}$Decays at LEP2

CERN-EP-98-180

A. Precise Measurement of the Partial Decay Width Ratio $R_{b}^{0}=\Gamma_{b \bar{b}} / \Gamma_{h a d}$

Eur. Phys. J. C9:367, 1999; CERN-EP-98-189 Measurement of $A_{F B}^{b b}$ in Hadronic $Z^{0}$ Decays using a Jet Charge Technique

Eur. Phys. J. C8:41, 1999; CERN-EP-98-169

Search for Composite and Exotic Fermions at LEP 2

Eur. Phys. J. C5:585, 1998; CERN-EP-98-095

$\pi^{ \pm}, K^{ \pm}, p$ and $\bar{p}$ Production in $Z^{0} \rightarrow q \bar{q}, Z^{0} \rightarrow b \bar{b}, Z^{0} \rightarrow u \bar{u}, d \bar{d}, s \bar{s}$

Phys. Lett. 438B:35, 1998

$J / \psi(1 S), \psi(2 S)$ and Drell-Yan Production in $p p$ and $p$ deuteron Interactions at $450 \mathrm{GeV} / c$

Phy's. Lett. 440B:203, 1998; CERN-EP-98-138

Two-Particle Angular Correlations in $e^{+} e^{-}$Interactions Compared with QCD Predictions

Phys. Lett. 449B:128, 1999; CERN-EP-98-190 $J / \psi(1 S), \psi(2 S)$ and Drell-Yan Production in SU Interactions at $200 \mathrm{GeV}$ per Nucleon

Phys. Lett. 449B:364, 1999; CERN-EP-98-199

Measurement of Inclusive $\rho^{0}, f_{0}(980), f_{2}(1270), K_{2}^{*}(1430)^{\circ}$, and $f_{2}^{\prime}(1525)$ Production in $Z^{0}$ Decays

Eur. Phys. J. C7:595, 1999; CERN-EP-98-170; HEPEX-9811006

Search for Lightest Neutralino and Stau Pair Production in Light Gravitino Scenarios with $\tilde{\tau}$ NLSP

Jour. of Phys. G 25:235, 1999; CERN-EP-98-188

Intermediate Mass Dimuons in NA38/NA50 
Abreu 98V

Abreu $98 \mathrm{~W}$

Abreu 99

Abreu 99B

Abreu 99C

Abreu 99D

Abreu 99E

Abreu 99F

Abreu 99G

Abreu 99H

Abreu 99I

Abreu 99J

Abreu $99 \mathrm{~K}$

Abt 93B

Abt 94

Abt $94 \mathrm{~B}$

Abt $94 \mathrm{C}$

Acciarri 94

Acciarri 94B

Acciarri $94 \mathrm{C}$

Acciarri 94D

Acciarri $94 \mathrm{E}$

Acciarri $94 \mathrm{~F}$

Acciarri 95

Acciarri 95B

Acciarri 95C

Acciarri 95D

Acciarri 95E

Acciarri 95F

Acciarri 95G

Acciarri $95 \mathrm{H}$

Acciarri 95I

Acciarri 95J

Acciarri $95 \mathrm{~K}$

Acciarri 95L

Acciarri $95 \mathrm{M}$

Acciarri $95 \mathrm{~N}$

Acciarri 950
Phys. Lett. 446B:75, 1999; Phys. Lett. 451B:447, 1999; CERN-EP-98-176

Search for Charginos, Neutralinos and Gravitinos in $e^{+} e^{-}$Interactions at $\sqrt{s}=183 \mathrm{GeV}$

Phys. Lett. 444B:491, 1998; CERN-EP-98-171; HEPEX-9811007

A Search for Heavy Stable and Longlived Squarks and Sleptons in $e^{+} e^{-}$Collisions at Energies from 130 to $183 \mathrm{GeV}$

CERN-EP-99-005

Measurement and Interpretation of Fermion-Pair Production at LEP Energies from 130 to $172 \mathrm{GeV}$

Phys. Lett. 448B:311, 1999; CERN-EP-98-198

Study of the Four-Jet Anomaly Observed at LEP Centre-of-Mass Energies of 130 and $136 \mathrm{GeV}$

Phys. Lett. 449B:383, 1999; CERN-EP-99-003; HEPEX-9903073

The Scale Dependence of the Hadron Multiplicity in Quark and Gluon Jets and a Precise Determination of $C_{A} / C_{F}$

CERN-EP-99-007; HEPEX-9903074

Measurement of the Forward Backward Asymmetry of $c$ and $b$ Quarks at the $Z^{0}$ Pole using Reconstructed Mesons

Search for Neutral Higgs Bosons in $e^{+} e^{-}$Collisions at $\sqrt{s}=183 \mathrm{GeV}$

Phys. Lett. 450B:456, 1999; CERN-EP-99-013

Observation of a Threshold Effect in the Anomalous $J / \psi(1 S)$ Suppression

Phys. Rev. C59:876, 1999

Observation of Fission in $\mathrm{Pb} \mathrm{Pb}$ Interactions at $158 \mathrm{~A} \mathrm{GeV}$

Eur. Phys. J. C6:385, 1999; CERN-EP-98-116

Search for Scalar Fermions and Long-Lived Scalar Leptons at Centre-of-Mass Energies of 130 to $172 \mathrm{GeV}$

Eur. Phys. J. C6:371, 1999; CERN-EP-98-142

Search for Pair-Produced Neutralinos in Events with Photons and Missing Energy from $\mathrm{e}^{+} \mathrm{e}^{-}$Collisions at $\sqrt{s}=130-183 \mathrm{GeV}$

Phys. Lett. 456B:310, 1999; CERN-EP-99-047

$W$ Pair Production Cross Section and $W$ Branching Fractions in $e^{+} e^{-}$Interactions at $183 \mathrm{GeV}$

Phys. Lett. 456B:322, 1999; CERN-EP-99-044

Energy Dependence of Event Shapes and $\alpha_{s}$ at LEP2

Phys. Lett. 321B:161, 1994; DESY-93-146

Scaling Violations of the Proton Structure Function $F_{2}$ at Small $x$

Z. Phys. C61:59, 1994; DESY-93-137

A Measurement of Multi-Jet Rates in Deep-Inelastic Scattering at HERA

2. Phys. C63:377, 1994; DESY-94-033

Energy Flow and Charged Particle Spectrum in Deep Inelastic Scattering at HERA

Phys. Lett. 328B:176, 1994; DESY-94-030

Inclusive Charged Particle Cross Sections in Photoproduction at HERA

Z. Phys. C62:551, 1994; CERN-PPE-94-045

Measurement of Cross-Sections and Leptonic Forward-Backward Asymmetries at the $Z^{0}$ Pole and Deter-

mination of Electroweak Parameters
Phys. Lett. 328B:223, 1994; CERN-PPE-94-53

Measurement of Inclusive Production of Neutral Hadrons from $Z^{0}$ Decays

Phys. Lett. 332B:201, 1994; CERN-PPE-94-068

Measurement of the Inclusive $b \rightarrow \tau \nu_{\tau}$ X Branching Ratio

Phys. Lett. 335B:542, 1994; CERN-PPE-94-89

Measurement of the $B^{0}-\bar{B}^{0}$ Mixing Parameter and the $Z^{\circ} \rightarrow b \bar{b}$ Forward-Backward Asymmetry

Phys. Lett. 346B:190, 1995; CERN-PPE-94-216

Measurement of Energetic Single Photon Production at LEP

Phys. Lett. 341B:245, 1994; CERN-PPE-94-145

$A$ Measurement of $\tau \pm$ Polarization at LEP

Phys. Lett. 345B:93, 1995; CERN-PPE-94-178

Measurement of Exclusive Branching Fractions of Hadronic One Prong Tau Decays

Phys. Lett. 345B:609, 1995; CERN-PPE-94-186

Search for Anomalous $Z^{0} \rightarrow \gamma \gamma \gamma$ Events at LEP

Phys. Lett. 351B:375, 1995; CERN-PPE-95-05

Measurement of Weak Charged Current Structure in Semileptonic $b$ Hadron Decays at the $Z^{0}$ Peak

Phys. Lett. 345B:589, 1995; CERN-PPE-94-143

$B^{*}$ Production in $Z^{\circ}$ Decays at LEP

Phys. Lett. 345B:74, 1995; CERN-PPE-94-164

Energy and Particle Flow in Three Jet and Radiative Two Jet Events from Hadronic $Z^{\circ}$ Decays

Phys. Lett. 350B:109, 1995; CERN-PPE-95-14

Search for Neutralinos in $Z^{\circ}$ Decays

Phys. Lett. 353B:136, 1995; CERN-PPE-95-41

Tests of QED at LEP Energies using $e^{+} e^{-} \rightarrow \gamma \gamma(\gamma)$ and $e^{+} e^{-} \rightarrow \ell^{+} \ell^{-} 2 \gamma$

Phys. Lett. 363B:137, 1995; CERN-PPE-95-136

Search for the Decays $B^{\mathrm{O}} \rightarrow \gamma \gamma$ and $B_{s} \rightarrow \gamma \gamma \gamma$

Phys. Lett. 352B:487, 1995; CERN-PPE-95-42

One Prong $\tau^{ \pm}$Decays with Neutral Kaons

Phys. Lett. 353B:145, 1995; CERN-PPE-95-49

Evidence for Gluon Interference in Hadronic $Z^{\circ}$ Decays

Phys. Lett. 370B:195, 1996; CERN-P PE-95-191

Measurement of Hadron and Lepton-Pair Production at $130 \mathrm{GeV} \leq \sqrt{s} \leq 140 \mathrm{GeV}$ at LEP

Phys. Lett. 370B:211, 1996; CERN-PPE-95-190

Search for Excited Leptons in $e^{+} e^{-}$Annihilation at $\sqrt{s}=130 \mathrm{GeV}-140 \mathrm{GeV}$

Phys. Lett. 371B:126, 1996; CERN-PPE-95-182

Measurement of $\eta$ Production in Two and Three Jet Events from Hadronic $Z^{\circ}$ Decays at LEP

Phys. Lett. 371B:137, 1996; CERN-PPE-95-192

Study of the Structure of Hadronic Events and Determination of $\alpha_{s}$ at $\sqrt{s}=130$ and $136 \mathrm{GeV}$

Phys. Lett. 363B:118, 1995; CERN.PPE-95-118

Study of the $K_{S} K_{S}$ Final State in Two-Photon Collisions 
Acciarri 95P

Acciarri 96

Acciarri $96 \mathrm{~B}$

Acciarri $96 \mathrm{C}$

Acciarri 96D

Acciarri $96 \mathrm{E}$

Acciarri 96F

Acciarri $96 \mathrm{G}$

Acciarri $96 \mathrm{H}$

Acciarri 96I

Acciarri 96J

Acciarri $96 \mathrm{~K}$

Acciarri 96L

Acciarri $96 \mathrm{M}$

Acciarri $96 \mathrm{~N}$

Acciarri 960

Acciarri 97

Acciarri 97B

Acciarri $97 \mathrm{C}$

Acciarri 97D

Acciarri 97E

Acciarri 97F

Acciarri 97G

Acciarri $97 \mathrm{H}$

Acciarri 971

Acciarri 97J

Acciarri $97 \mathrm{~K}$

Acciarri 97L

Acciarri $97 \mathrm{M}$

Acciarri $97 \mathrm{~N}$

Acciarri 970

Acciarri $97 \mathrm{P}$

Acciarri $97 \mathrm{Q}$

Acciarri 97R

Acciarri $97 \mathrm{~S}$

Acciarri $97 \mathrm{~T}$

Acciarri $97 \mathrm{U}$

Acciarri $97 \mathrm{~V}$
Phys. Lett. 363B:127, 1995; CERN-PPE-95-124 Search for Neutral Charmless $B$ Decays at LEP

Phys. Lett. 384B:323, 1996; CERN-PPE-96-48 Observation of Multiple Hard Photon Final States at $\sqrt{s}=130-140 \mathrm{GeV}$ at LEP

Phys. Lett. 385B:454, 1996; CERN-PPE-96-095 Search for Neutral Higgs Boson Production through the Process $e^{+} e^{-} \rightarrow Z^{0}$ higgs

Phys. Lett. 377B:313, 1996; CERN-PPE-96-46 Measurement of the Michel Parameters and the Average $r^{ \pm}$Neutrino Helicity from $\tau^{ \pm}$Decays in $e^{+} e^{-}$ $\rightarrow \tau^{+} \tau^{-}$

Phys. Lett. 377B:289, 1996; CERN-PPE-96-29

Search for Supersymmetric Particles at $130 \mathrm{GeV} \leq \sqrt{s} \leq 140 \mathrm{GeV}$ at LEP

Phys. Lett. 374B:331, 1996; CERN-PPE-96-24 Measurement of Muon-Pair Production at $50 \mathrm{GeV} \leq \sqrt{s} \leq 80 \mathrm{GeV}$ at LEP

Phys. Lett. 377B:304, 1996; CERN-PPE-96-38 Search for Unstable Sequential Neutral and Charged Heavy Leptons in $e^{+} e^{-}$Annihilation at $\sqrt{s}=130$ and $136 \mathrm{GeV}$

Phys. Lett. 388B:409, 1996; CERN-PPE-96-50 Search for New Particles in Hadronic Events with Isolated Photons

2. Phys. C71:379, 1996; CERN-PPE-96-49

Measurement of the Branching Ratios $B \rightarrow e \nu \mathrm{X}, \mu \nu \mathrm{X}, \tau \nu \mathrm{X}$ and $\nu \mathrm{X}$

Phys. Lett. 391B:474, 1997; CERN-PPE-96-151 Search for Neutral $B$ Meson Decays to Two Charged Leptons

Phys. Lett. 391B:481, 1997; CERN-PPE-96-152 Search for Exclusive $B$ Decays to $J / \psi(1 S)$ and $\eta$ or $\pi^{0}$ with the L3 Detector

Phys. Lett. 389B:187, 1996; CERN-PPE-96-124 Measurement of the Lifetime of the $\tau^{ \pm}$Lepton

Phys. Lett. 396B:327, 1997; CERN-PPE-96-198 Measurement of $D_{s}^{-} \rightarrow \tau^{-} \bar{\nu}_{\tau}$ and a New Limit for $B^{-} \rightarrow \tau^{-} \bar{\nu}_{\tau}$

Phys. Lett. 393B:465, 1997; CERN-PPE-96-171 Measurement of Inclusive $\omega$ and $\eta^{\prime}$ Production in Hadronic $Z^{0}$ Decays

Phys. Lett. 393B:477, 1997; CERN-PPE-96-170 Study of the Weak Charged Hadronic Current in $b$ Decays

Phys. Lett. 383B:487, 1996; CERN-PPE-96-073 Measurement of the $B^{\circ}$ Meson Oscillation Frequency

Phys. Lett. 398B:223, 1997; CERN-PPE-97-014

Pair-Production of $W^{ \pm}$Bosons in $e^{+} e^{-}$Interactions at $\sqrt{s}=161 \mathrm{GeV}$

Phys. Lett. 401 B:139, 1997; CERN-PPE-97-012 Search for Excited Leptons in $e^{+} e^{-}$Annihilation at $\sqrt{s}=161 \mathrm{GeV}$

Phys. Lett. 403B:168, 1997; CERN-PPE-97-028 Production of Single $W^{ \pm}$Bosons at LEP

Phys. Lett. 407B:341, 1997; CERN-PPE-97-043

Production of $e^{ \pm}, \mu^{ \pm}$and $\tau^{ \pm}$Pairs in Untagged Two-Photon Collisions at LEP

Phys. Lett. 408B:450, 1997; CERN-PPE-97-048 Cross Section of Hadron Production in $\gamma \gamma$ Collisions at LEP

Phys. Lett. 407B:351, 1997; CERN-PPE-97-044 Inclusive $J / \psi(1 S), \psi(2 S)$, and $\chi_{c}$ Production in Hadronic $Z^{\circ}$ Decays

Phys. Lett. 407B:389, 1997 ; CERN-PPE-97-050 $K_{S}$ and $\Lambda$ Production in Quark and Gluon Jets at LEP

Phys. Lett. 411B:330, 1997; CERN-PPE-97-057 Search for Anomalous Four Jet Events in $e^{+} e^{-}$Annihilation at $\sqrt{s}=130-172 \mathrm{GeV}$

Phys. Lett. 413B:167, 1997; CERN-PPE-97-078 Upsilon Production in $Z^{0}$ Decays

Phys. Lett. 413B:159, 1997; CERN-PPE-97-077 Hard-Photon Production at $\sqrt{s}=161$ and $172 \mathrm{GeV}$ at LEP

Phys. Lett. 415B:299, 1997; CERN-PPE-97-076 Single and Multi-Photon Events with Missing Energy in $e^{+} e^{-}$Collisions at $161 \mathrm{GeV} \leq \sqrt{3} \leq 172 \mathrm{GeV}$

Phys. Lett. 412B:189, 1997; CERN-PPE-97-075 Search for Heavy Neutral and Charged Leptons in $e^{+} e^{-}$Annihilation at $\sqrt{s}=161$ and $172 \mathrm{GeV}$

Phys. Lett. 411B:339, 1997; CERN-PPE-97-074 Study of Hadronic Events and Measurements of $\alpha_{s}$ between 30 and $91 \mathrm{GeV}$

Phys. Lett. 407B:419, 1997; CERN-PPE-97-067 Measurement of $W^{ \pm}$Pair Cross Sections in $e^{+} e^{-}$Interactions at $\sqrt{s}=172 \mathrm{GeV}$ and $W^{ \pm}$Decay Branching Fractions

Phys. Lett. 413B:147, 1997; CERN-PPE-97-068

Resonance Formation in the $\pi^{+} \pi^{-} \pi^{0}$ Final State in Two-Photon Collisions

Phys. Lett. 412B:201, 1997; CERN-PPE-97-082

Search for New Physics in Energetic Single Photon Production in $e^{+} e^{-}$Annihilation at the $Z^{\circ}$ Resonance Phys. Lett. 411B:373, 1997; CERN-PPE-97-081

Search for the Standard Model Higgs Boson in $e^{+} e^{-}$Interactions at $161 \leq \sqrt{s} \leq 172 \mathrm{GeV}$

Phys. Lett. 418B:399, 1998; CERN-PPE-97-110

Measurement of $\eta^{\prime}$ Formation in Two-Photon Collisions at LEP 1

Phys. Lett. 413B:191, 1997; CERN-PPE-97-106

Neutral Current Four Fermion Production in $e^{+} e^{-}$Interactions at $130 \mathrm{GeV} \leq \sqrt{s} \leq 172 \mathrm{GeV}$

Phys. Lett. 413B:176, 1997; CERN-PPE-97-098

Measurements of Mass, Width and Gauge Couplings of the $W^{ \pm}$Boson at LEP

Phys. Lett. 418B:389, 1998; CERN-PPE-97-097

Missing Mass Spectra in Hadronic Events from $e^{+} e^{-}$Collisions at $\sqrt{s}=161-172 \mathrm{GeV}$ and Limits on Invisible Higgs Decay

Phys. Lett. 414B:373, 1997; CERN-PPE-97-099

Search for $R$ Parity Breaking Sneutrino Exchange at LEP 
Acciarri $97 \mathrm{~W}$

Acciarri 97X

Acciarri 97Y

Acciarri 972

Acciarri 97ZB

Acciarri 98

Acciarri 98B

Acciarri 98C

Acciarri 98D

Acciarri 98E

Acciarri $98 \mathrm{~F}$

Acciarri 98G

Acciarri $98 \mathrm{H}$

Acciarri 98I

Acciarri 98J

Acciarri 98K

Acciarri 98L

Acciarri $98 \mathrm{M}$

Acciarri $98 \mathrm{~N}$

Acciarri 980

Acciarri 98P

Acciarri 98Q

Acciarri 98R

Acciarri $98 \mathrm{~S}$

Acciarri 98T

Acciarri $98 \mathrm{U}$

Acciarri $98 \mathrm{~V}$

Acciarri $98 \mathrm{~W}$

Acciarri 98X

Acciarri $98 \mathrm{Y}$

Acciarri $98 \mathrm{Z}$

Acciarri 982B

Acciarri $98 \mathrm{ZC}$

Acciarri $982 \mathrm{D}$

Acciarri 98ZE

Acciarri 98ZF

Acciarri 99
Phys. Lett. 416B:220, 1998; CERN-PPE-97-127 Measurement of the Average Lifetime of $b$-Hadrons in $Z^{\circ}$ Decays

Eur. Phys. J. C4:207, 1998; CERN.PPE-97-130 Search for Scalar Leptons, Charginos and Neutralinos in $e^{+} e^{-}$Collisions at $\sqrt{s}=161-172 \mathrm{GeV}$ Phys. Lett. 404B:390, 1997; CERN-PPE-97-042 QCD Studies and Determination of $\alpha_{s}$ in $e^{+} e^{-}$Collisions at $\sqrt{s}=161$ and $172 \mathrm{GeV}$

Phys. Lett. 407B:361, 1997; CERN-PPE-97-052 Measurement of Hadron and Lepton Pair Production at $161 \leq \sqrt{3} \leq 172 \mathrm{GeV}$ at LEP

Phys. Lett. 429B:375, 1998; CERN-PPE-97-165 Local Multiplicity Fluctuations in Hadronic $Z^{0}$ Decay

Phys. Lett. 431B:199, 1998; CERN-EP-98-025 Determination of the Number of Light Neutrino Species from Single Photon Production at LEP

Phys. Lett. 433B:163, 1998; CERN-EP-98-031 Search for New Physics Phenomena in Fermion-Pair Production at LEP

Phys. Lett. 426B:207, 1998; CERN-EP-98-015 Measurement of the Weak Dipole Moments of the $\tau^{ \pm}$Lepton

Phys. Lett. 429B:387, 1998; CERN-EP-98-026 Measurement of $\tau$ Polarization at LEP

Eur. Phys. J. C5:195, 1998; CERN-EP-98-028 Measurement of the $B^{0} \bar{B}^{0}$ Oscillation Frequency

Phys. Lett. 431B:437, 1998; CERN-EP-98-052 Search for the Standard Model Higgs Boson in $e^{+} e^{-}$Interactions at $\sqrt{s}=183 \mathrm{GeV}$

Phys. Lett. 428B:186, 1998; CERN-EP-98-023 Angular Multiplicity Fluctuations in Hadronic $Z^{\circ}$ Decays and Comparison to QCD Models and Analytical Calculations

Phys. Lett. 434B:169, 1998; CERN-EP-98-045

Measurement of the Anomalous Magnetic and Electric Dipole Moments of the $\tau$ Lepton

Phys. Lett. 438B:363, 1998; CERN-EP-98-060 Photon Structure Functions and Azimuthal Correlations of Lepton Pairs in Tagged $\gamma \gamma$ Collisions

Phys. Lett. 436 B:389, 1998; CERN-EP-98-072 Search for Neutral Higgs Bosons of the Minimal Supersymmetric Standard Model in $e^{+} e^{-}$Interactions at $\sqrt{s}=130-183 \mathrm{GeV}$

Phys. Lett. 436B:187, 1998; CERN-EP-98-096

Study of Anomalous $Z^{\circ} Z^{0} \gamma$ and $Z^{0} \gamma \gamma$ Couplings at LEP

Phys. Lett. 436 B:428, 1998; CERN-EP.98-100

Test of CP Invariance in $Z^{\circ} \rightarrow \mu^{+} \mu^{-} \gamma$ Decay

Phys. Let.t. 436 B:417, 1998; CERN-EP-98-099

Production of Single $W$ Bosons in $e^{+} e^{-}$Interactions at $130 \mathrm{GeV} \leq \sqrt{s} \leq 183 \mathrm{GeV}$ and Limits on Anomalous $W W_{\gamma}$ Couplings

Phys. Lett. 436B:403, 1998; CERN-EP-98-098

Study of the Hadronic Photon Structure Function $F_{2}^{\gamma}$ at LEP

Phys. Lett. 439B:183, 1998; CERN-EP-98-109

Measurement of Radiative Bhabha and Quasi-Real Compton Scattering

Phys. Lett. 436B:174, 1998; CERN-EP-98-097 Measurement of the Inclusive Charmless Semileptonic Branching Fraction of Beauty Hadrons and a Determination of $\left|V_{u b}\right|$ at LEP

Phys, Lett. 436B:437, 1998; CERN-EP-98-121

Measurement of $W^{ \pm}$Pair Cross Sections in $e^{+} e^{-}$Interactions at $\sqrt{s}=183 \mathrm{GeV}$ and $W^{ \pm}$Decay Branching

Fractions
Phys. Lett. 447B:147, 1999; CERN-EP-98-168

The $Q^{2}$ Evolution of the Hadronic Photon Structure Function $F_{2}^{\gamma}$ at LEP

Phys. Lett. 453B:73, 1999; CERN-EP-98-184

$\chi_{\mathrm{c} 2}(1 P)$ Formation in Two-Photon Collisions at LEP

Phys. Lett. 453B:83, 1999; CERN-EP-98-185

Inclusive Charm Production in Two-Photon Collisions at LEP

Phys. Lett. 446B:368, 1999; CERN-EP-98-149

Search for Charged Higgs Bosons in $e^{+} e^{-}$Collisions at Centre-of-Mass Energies between 130 and $183 \mathrm{Gev}$

Phys. Lett. 448B:152, 1999; CERN-EP-98-156

Measurement of the $e^{+} e^{-} \rightarrow Z^{0} \rightarrow b \bar{b}$ Forward-Backward Asymmetry and the $B^{0} \bar{B}^{0}$ Mixing Parameter using Prompt Leptons

Phys. Lett. 444B:503, 1998; CERN-EP-98-150

Single and Multi-Photon Events with Missing Energy in $e^{+} e^{-}$Collisions at $\sqrt{s}=183 \mathrm{GeV}$

Phys. Lett. 438B:417, 1998; CERN-EP-98-127

Upper Limit on the Lifetime Difference of Short- and Long-Lived $B$, Mesons

Phys. Lett. 439B:225, 1998; CERN-EP-98-134

Measurement of the Effective Weak Mixing Angle by Jet-Charge Asymmetry in Hadronic Decays of the $Z^{\circ}$ Boson

Phys. Lett. 444B:569, 1998; CERN-EP-98-148

QCD Results from Studies of Hadronic Events Produced in $e^{+} e^{-}$Annihilations at $\sqrt{s}=183 \mathrm{GeV}$

Phys. Lett. 453B:94, 1999; CERN-EP-98-204

Heavy $Q$ uarkonium Production in $Z^{\circ}$ Decays

Phys. Lett. 453B:333, 1999; CERN-EP-98-205

Measurement of the Cross-Section for the Process $\gamma^{*} \gamma^{*} \rightarrow$ Hadrons at LEP

Phys. Lett. 450B:281, 1999; CERN-EP-98-206

Study of Neutral-Current Four-Fermion and $Z^{\circ} Z^{\circ}$ Production in $e^{+} e^{-}$Collisions at $\sqrt{s}=183 \mathrm{GeV}$

Phys. Lett. 438B:405, 1998; CERN-EP-98-123

Measurement of the Michel Parameters and the Average $\nu_{\tau}$ Helicity from $\tau$ Decays at LEP

Phys. Lett. 445B:428, 1999; CERN-EP-98-135

Searches for Scalar Top and Scalar Bottom Quarks in $e^{+} e^{-}$Interactions at $161 \mathrm{GeV} \leq \sqrt{(s)} \leq 183 \mathrm{GeV}$

Phys. Lett. 454B:386, 1999; CERN-EP-99-017

Measurement of Mass and Width of the $W^{ \pm}$Boson

See the legend on page 5. 
Achasov 94

Achasov 96

Achasov 97

Achasov 98

Achasov $98 \mathrm{~B}$

Achasov $98 \mathrm{C}$

Achasov 98D

Achasov 99

Achkar 95

Achkar 96

Ackerbauer 97

Ackerstaff 96

Ackerstaff 96B

Ackerstaff $96 \mathrm{C}$

Ackerstaff $96 \mathrm{D}$

Ackerstaff $96 \mathrm{E}$

Ackerstaff $96 \mathrm{~F}$

Ackerstaff $96 \mathrm{G}$

Ackerstaff $96 \mathrm{H}$

Ackerstaff 96 I

Ackerstaff $96 \mathrm{~J}$

Ackerstaff $96 \mathrm{~K}$

Ackerstaff $96 \mathrm{~L}$

Ackerstaff $96 \mathrm{M}$

Ackerstaff $96 \mathrm{~N}$

Ackerstaff 960

Ackerstaff $96 \mathrm{P}$

Ackerstaff $96 Q$

Ackerstaff 96R

Ackerstaff 97

Ackerstaff $97 \mathrm{C}$

Ackerstaff $97 \mathrm{D}$

Ackerstaff $97 \mathrm{E}$

Ackerstaff $97 \mathrm{~F}$

Ackerstaff $97 \mathrm{G}$

Ackerstaff $97 \mathrm{H}$

Ackerstaff 97 I
Phys. Lett. 333B:259, 1994

Did the VES(IHEP) Group Observe the Reaction $\pi^{-}$nucleon $\rightarrow \pi^{-} \eta$ nucleon and $\pi^{-}$nucleon $\rightarrow \pi^{-} \eta^{\prime}$

nucleon ?

Rho Primes in Analyzing $e^{+} e^{-}$Annihilation, MARK III, LASS and ARGUS Data

BUDKERIN P-97-78; BIN P-97-78; HEPEX-9710017

First Physical Results from SND Detector at VEPP-2M

Phys. Lett. 425B:388, 1998; HEPEX-9803008
Upper Limit on the $\eta \rightarrow \pi^{\circ} \pi^{\circ}$ Decay

Phys. Lett. 438B:441, 1998; HEPEX-9809010 Evidence of the $\phi(1020) \rightarrow \eta \pi^{\circ} \gamma$ Decay

Jetp Lett. 68:573, 1998; Pisma Zh. Eksp. Teor. Fiz. 68:549, 1998; HEPEX-9809002

Experimental Study of the Decay $\phi(1020) \rightarrow \eta \gamma$ in Multiphoton Final State

Jetp Lett. 67:777, 1998; Pisma Zh. Eksp. Teor. Fiz. 67:737, 1998; HEPEX-9804002

Study of $\Delta\left(1232 P_{33}\right)$ Isobar Electroproduction at VEPP-2M $e^{+} e^{-}$Collider

Phys. Lett. 449B:122, 1999; HEPEX-9901020

Observation of the Decay $\phi(1020) \rightarrow \omega \pi^{\circ}$

Nucl. Phys. B434:503, 1995

Search for Neutrino Oscillations at 15, 40 and 95 Meters from a Nuclear Power Plant at Bugey

Phys. Lett. 374B:243, 1996; LAPP-EXP-96-02; CPPM-96-02; ISN-96-10; LPC-96-05

Comparison of Antineutrino Reactor Spectrum Models with the Bugey 3 Measurements

Phys. Lett. 417 B:224, 1998; PSI-PR-97-21; HEPPH-9708487

A Precision Measurement of Nuclear Muon Capture on ${ }^{3} \mathrm{He}$

Z. Phys. C73:433, 1997; CERN-PPE-96-132

Inclusive Jet Production in Photon Photon Collisions at $\sqrt{s}=130$ and $136 \mathrm{GeV}$

Phys. Lett. 389B:197, 1996; CERN-PPE-96-133

Search for Scalar Top and Scalar Bottom Quarks using the OPAL Detector at LEP

Phys. Lett. 391 B:197, 1997; CERN-PPE-96-138

Search for Excited Leptons in $e^{+} e^{-}$Collisions at $\sqrt{s}=181 \mathrm{GeV}$

Z. Phys. C73:397, 1997; CERN-PPE-96-137

A Measurement of the Average Bottom Hadron Lifetime

Phys. Lett. 393B:217, 1997; CERN-PPE-96-140

Search for Unstable Neutral and Charged Heavy Leptons in $e^{+} e^{-}$Collisions at $\sqrt{s}=101 \mathrm{GeV}$

Phys. Lett. 389B:416, 1996; CERN-PPE-96-141

Measurement of the Mass of the $W$ Boson in $e^{+} e^{-}$Collisions at $\sqrt{s}=161 \mathrm{GeV}$

Z. Phys. C74:49, 1997; CERN-PPE-96-153

Measurement of the QED Longitudinal Structure Function of the Photon using Azimuthal Correlations at LEP

Phys. Lett. 391B:210, 1997; CERN-PPE-96-154

Photonic Events with Large Missing Energy in $e^{+} e^{-}$Collisions at $\sqrt{s}=161 \mathrm{GeV}$

Phys. Lett. 391 B:221, 1997; CERN-PPE-96-156 Production of Fermion-Pair Events in $e^{+} e^{-}$Collisions at $161 \mathrm{GeV}$ Centre-of-Mass Energy

Phys. Lett. 395B:128, 1997; CERN-PPE-96-162

A Measurement of $V_{c b}$ using $\bar{B}^{0} \rightarrow D^{*}(2010)^{+} \ell^{-} \bar{\nu}$ Decays

Phys. Lett. 389B:616, 1996; CERN-PPE-96-135

Search for Chargino and Neutralino Production in $e^{+} e^{-}$Collisions at $\sqrt{s}=161 \mathrm{GeV}$

Z. Phys. C74:33, 1997; CERN-PPE-96-155

Analysis of Hadronic Final States and the Photon Structure Function $F_{2}^{\gamma}$ in Deep Inelastic Electron Photon Scattering at LEP

Z. Phys. C74:423, 1997; CERN-PPE-96-166

Measurement of the Semileptonic Branching Fraction of Inclusive $B$ Baryon Decays to $\Lambda$

Phys. Lett. 393B:231, 1997; CERN-PPE-96-161

Search for the Standard Model Higgs Boson in $e^{+} e^{- \text {Collisions at } \sqrt{s}}=161 \mathrm{GeV}$

2. Phys. C74:1, 1997; CERN-PPE-96-167

An Improved Measurement of $R_{b}$ using a Double Tagging Method

Z. Phys. C74:403, 1997; CERN-PPE-96-184

Search for $C P$ Violation in $Z^{\circ} \rightarrow \tau^{+} \tau^{-}$and an Upper Limiton the Weak Dipole Moment of the $\tau$ Lepton

Phys. Lett. 396B:301, 1997; CERN-PPE-96-182

Search for Charged Scalar Leptons using the OPAL Detector at $\sqrt{s}=161 \mathrm{GeV}$

Z. Phys. C74:437, 1997; CERN-PPE-97-005

Study of $\phi(1020), D^{*}(\mathbf{2 0 1 0})^{ \pm}$and $B^{*}$ Spin Alignment in Hadronic $Z^{0}$ Decays

Phys. Lett. 397B:147, 1997; CERN-PPE-97-004

Measurement of the Triple Gauge Boson Coupling $\alpha_{\left(W_{\phi}\right)}$ from $W^{+} W^{-}$Production in $e^{+} e^{-}$Collisions at $\sqrt{3}=161 \mathrm{GeV}$

2. Phys. C75:385, 1997; CERN-PPE-97-006

Measurements of the b-Quark Forward-Backward Asymmetry around the $Z^{\circ}$ Peak using Jet Charge and

Vertex Charge

Z. Phys. C75:593, 1997; CERN-PPE-97-020

A Measurement of the Hadronic Decay Current and the $\nu_{\tau}$ Helicity in $\tau^{-} \rightarrow \pi^{-} \pi^{-} \pi^{+} \nu_{\tau}$.

Phys. Lett. 411B:387, 1997; CERN-PPE-97-087; HEPEX-9708019

Measurement of the $Q^{2}$ Evolution of the Photon Structure Function $F_{2}^{\gamma}$

Eur. Phys. J. C2:39, 1998; CERN-PPE-97-086; HEPEX-9708020

Measurement of the Quark to Photon Fragmentation Function through the Inclusive Production of Prompt

Photons in Hadronic $Z^{\circ}$ Decays

Phys. Lett. 412B:225, 1997; CERN-PPE-97-103; HEPEX-9708028

Measurement of the Photon Structure Function $F_{2}^{\gamma}$ at Low

Eur. Phys. J. C2:49, 1998; CERN-PPE-97-104; HEPEX-9708027

Polarization and Forward-Backward Asymmetry of $\Lambda$ Baryons in Hadronic $Z^{0}$ Decays

Phys. Lett. 404B:213, 1997; CERN-PPE-97-049; HEPEX-9707023

An Upper Limit on the Branching Ratio for $\tau^{ \pm}$Decays into Seven Charged Particles 
Ackerstaff $97 \mathrm{~J}$

Ackerstaff $97 \mathrm{~K}$

Ackerstaff 97L

Ackerstaff 97M

Ackerstaff $97 \mathrm{~N}$

Ackerstaff 970

Ackerstaff 97P

Ackerstaff $97 \mathrm{Q}$

Ackerstaff 97R

Ackerstaff 97S

Ackerstaff $97 \mathrm{~T}$

Ackerstaff $97 \mathrm{U}$

Ackerstaff $97 \mathrm{~V}$

Ackerstaff 97W

Ackerstaff 97X

Ackerstaff $97 Y$

Ackerstaff 972

Ackerstaff $97 \mathrm{ZB}$

Ackerstaff $97 Z C$

Ackerstaff 97ZD

Ackerstaff $97 \mathrm{ZE}$

Ackerstaff $97 Z \mathrm{~F}$

Ackerstaff $97 \mathrm{ZG}$

Ackerstaff $97 \mathrm{ZH}$

Ackerstaff 97ZI

Ackerstaff 98

Ackerstaff 98B

Ackerstaff 98C

Ackerstaff 98D

Ackerstaff $98 \mathrm{E}$

Ackerstaff $98 \mathrm{~F}$

Ackerstaff $98 \mathrm{G}$

Ackerstaff $98 \mathrm{H}$

Ackerstaff 98I

Ackerstaff 98J

Ackerstaff $98 \mathrm{~K}$

Ackerstaff 98L

Ackerstaff $98 \mathrm{M}$
Z. Phys. C75:193, 1997; CERN-PPE-97-015

QCD Studies with $e^{+} e^{-}$Annihilation Data at $161 \mathrm{GeV}$

Z. Phys. C76:387, 1997; CERN-PPE-97-063; HEPEX-9707019

Measurement of the Branching Fractions and Forward-Backward Asymmetries of the $Z^{\circ}$ into Light Quarks

Z. Phys. C76:401, 1997; CERN-PPE-97-036; HEPEX-9707009

A Study of $B$ Meson Oscillations using Hadronic $Z^{0}$ Decays Containing Leptons

Z. Phys. C76:417, 1997; CERN-PPE-97-064; HEPEX-9707010

An Updated Study of $B$ Meson Oscillations using Dilepton Events

Eur. Phys. J. C2:407, 1998; CERN-PPE-97-095; HEPEX-9708023

A Measurement of the $B_{s}$ Lifetime using Reconstructed $D_{s}^{-}$Mesons

Eur. Phys. J. C1:439, 1998; CERN-PPE-97-093; HEPEX-9708021

Measurement of $\mathrm{f}\left(c \rightarrow D^{*}(2010)^{+} \mathrm{X}\right), \mathrm{f}\left(b \rightarrow D^{*}(2010)+\mathrm{X}\right)$ and $\Gamma_{c c} / \Gamma_{h a d}$ using $D^{*}(2010)^{ \pm}$Mesons

Eur. Phys. J. C2:597, 1998; CERN-PPE-97-125; HEPEX-9709023

Measurement of Triple Gauge Boson Couplings from $W^{+} W^{-}$Production at $\sqrt{s}=172 \mathrm{GeV}$

Eur. Phys. J. C1:45, 1998; CERN-PPE-97-123; HEPEX-9709027

Search for Unstable Heavy and Excited Leptons in $e^{+} e^{-}$Collisions at $\sqrt{s}=170-172 \mathrm{GeV}$

Eur. Phys. J. C1:31, 1998; CERN-PPE-97-121; HEPEX-9709022

Search for a Massive Diphoton Resonance at $\sqrt{s}=81-172 \mathrm{GeV}$

Eur. Phys. J. C1:21, 1998; CERN-PPE-97-109; HEPEX-9709002

Multi-Photon Final States in $e^{+} e^{-}$Collisions at $\sqrt{s}=130-172 \mathrm{GeV}$

Eur. Phys. J. C1:395, 1998; CERN-PPE-97-116; HEPEX-9709006

Measurement of the $W$ Boson Mass and Production and Decay Properties in $e^{+} e^{-}$Collisions at $\sqrt{s}=$

172 GeV
Eur. Phys. J. C2:441, 1998; CERN-PPE-97-101; HEPEX-9708024

Tests of the Standard Model and Constraints on New Physics from Measurements of Fermion-pair Production at $130-172 \mathrm{GeV}$ at LEP

Eur. Phys. J. C1:425, 1998; CERN-PPE-97-115; HEPEX-9709003

Search for the Standard Model Higgs Boson in $e^{+} e^{-}$Collisions at $\sqrt{s}=161-172 \mathrm{GeV}$

Eur. Phys. J. C2:213, 1998; CERN-PPE-97-083; HEPEX-9708018

Search for Chargino and Neutralino Production at $\sqrt{s}=170$ and $172 \mathrm{GeV}$

Phys. Lett. 412B:210, 1997; CERN-PPE-97-094; HEPEX-9708022

Spin Alignment of Leading $K^{*}(892)^{\circ}$ Mesons in Hadronic $Z^{\circ}$ Decays

Z. Phys. C76:425, 1997; CERN-PPE-97-035

Production of $P$ Wave Charm and Charm-Strange Mesons in Hadronic $Z^{\circ}$ Decays

Eur. Phys. J. C4:47, 1998; CERN-PPE-97-124

Search for Anomalous Production of Dilepton Events with Missing Transverse Momentum in $e^{+} e^{-}$Collisions at $\sqrt{s}=161$ and $172 \mathrm{GeV}$

Phys. Lett. 420B:157, 1998; CERN-PPE-97-137; HEPEX-9801026

Search for the $B_{c}$ Meson in Hadronic $Z^{\circ}$ Decays

Z. Phys. C75:409, 1997; CERN-P PE-97-046

Search for Scalar Top and Scalar Bottom Quarks at $\sqrt{s}=170-172 \mathrm{GeV}$ in $e^{+} e^{-}$Collisions

Eur. Phys. J. C2:607, 1998; CERN-PPE-97-132

Search for Anomalous Production of Photonic Events with Missing Energy in $e^{+} e^{-}$Collisions at $\sqrt{s}=$ $130-172 \mathrm{GeV}$

Eur. Phys. J. C4:193, 1998; CERN-PPE-97-152; HEPEX-9801029

Measurement of the One-Prong Hadronic Tau Branching Ratios at LEP

Phys. Lett. 426B:161, 1998; CERN-PPE-97-159; HEPEX-9802002

Measurements of the $B_{s}$ and $\Lambda_{b}$ Lifetimes

Phys. Lett. 426B:180, 1998; CERN-PPE-97-168; HEPEX-9802004

Search for Charged Higgs Bosons in $e^{+} e^{-}$Collisions at $\sqrt{s}=130-172 \mathrm{GeV}$

Z. Phys. C74:413, 1997; CERN-PPE-96-192

$B^{*}$ Production in $Z^{\circ}$ Decays

Phys. Lett. 404B:383, 1997; OAP-741; HEPEX-9703005

Measurement of the Neutron Spin Structure Function $g_{1}^{n}$ with a Polarized ${ }^{3}$ He Internal Target

Eur. Phys. J. C5:379, 1998; CERN-EP-98-001; HEPEX-9801022

Investigation of CP Violation in $B^{0} \rightarrow J / \psi(1 S) K_{S}$ Decays at LEP

Eur. Phys. J. C4:19, 1998; CERN-EP-98-010; HEPEX-9802013

Production of $f_{0}(980), f_{2}(1270)$ and $\phi(1020)$ in Hadronic $Z^{0}$ Decay

Phys. Lett. 429B:399, 1998; CERN-EP-98-013; HEPEX-9802015

Search for an Excess in the Production of Four-Jet Events from $e^{+} e^{-}$Collisions at $\sqrt{s}=130-184 \mathrm{GeV}$

Eur. Phys. J. C5:1, 1998; CERN-EP-98-006; HEPEX-9802008

Determination of the Production Rate of $D^{*}$ Mesons and of the Ratio $V /(V+P)$ in $Z^{\circ} \rightarrow c \bar{c}$ Decays

Phys. Lett. 433B:195, 1998; CERN-EP-98-039; HEPEX-9803026 Search for Stable and Long-Lived Massive Charged Particles with the OPAL Detector at LEP in $e^{+} e^{-}$ Collisions at $\sqrt{3}=130-183 \mathrm{GeV}$

Phys. Lett. 431B:188, 1998; CERN-EP-98-033; HEPEX-9803020

An Upper Limit on the Anomalous Magnetic Moment of the $\tau$ Lepton

Eur. Phys. J. C5:19, 1998; CERN-EP-98-029; HEPEX-9803019

A Search for Neutral Higgs Bosons in the MSSM and Models with Two Scalar Field Doublets

Eur. Phys. J. C5:229, 1998; CERN-EP-98-055; HEPEX-9806035

An Upper Limit for the $\nu_{\tau}$ Mass from $\tau^{ \pm} \rightarrow 5 \pi^{ \pm} \nu_{r}$ Decays

Eur. Phys. J. C5:411, 1998; CERN-EP-98-054; HEPEX-9805011

Photon and Light Meson Production in Hadronic $Z^{0}$ Decays

Eur. Phys. J. C8:241, 1999; CERN-EP-98-058

Production of $K_{S}$ and $\Lambda$ in Quark and Gluon Jets from $Z^{\circ}$ Decay

Eur. Phys. J. C5:239, 1998; CERN-EP-98-068

Bose-Einstein Correlations of Three Charged Pions in Hadronic $Z^{\circ}$ Decay

Phys. Rev. Lett. 81:5519, 1998; DESY-98-078; HEPEX-9807013

The Flavor Asymmetry of the Light Quark Sea from Semi-Inclusive Deep-Inelastic Scattering

Phys. Lett. 444B:531, 1998; DESY-98-122; HEPEX-9809015

Determination of the Deep Inelastic Contribution to the Generalized Gerasimov-Drell-Hearn Integral for the Proton and Neutron

See the legend on page 5 . 
Ackerstaff $98 \mathrm{~N}$

Ackerstaff 980

Ackerstaff $98 \mathrm{P}$

Ackerstaff $98 \mathrm{Q}$

Ackerstaff $98 \mathrm{R}$

Ackerstaff $98 \mathrm{~S}$

Ackerstaff $98 \mathrm{~T}$

Ackerstaff $98 \mathrm{U}$

Ackerstaff $98 \mathrm{~V}$

Ackerstaff $98 \mathrm{~W}$

Aclander 98

Acosta 93

Adachi 94

Adachi 99

Adam 93

Adam 95

Adam 95B

Adam 96

Adam 96B

Adam $96 \mathrm{C}$

Adam $96 \mathrm{D}$

Adam 97

Adamo 94

Adamov 94

Adamovich 94

Adamovich $94 \mathrm{~B}$

Adamovich $94 \mathrm{C}$

Adamovich $94 \mathrm{D}$

Adamovich $94 \mathrm{E}$

Adamovich 95

Adamovich $95 \mathrm{~B}$

Adamovich $95 \mathrm{C}$

Adamovich 95D

Adamovich $95 \mathrm{E}$

Adamovich $95 \mathrm{~F}$

Adamovich 95G

Adamovich $95 \mathrm{H}$

Adamovich 95I
Phys. Lett. 438B:379, 1998; CERN-EP-98-092

Multi-photon Production in $e^{+} e^{-}$Collisions at $\sqrt{s}=183 \mathrm{GeV}$

Eur. Phys. J. C6:253, 1999; CERN-EP-98-091

Inclusive Production of Charged Hadrons and $K_{S}$ Mesons in Photon Photon Collisions

Eur. Phys. J. C7:369, 1999; CERN-EP-98-089

Measurements of Flavour Dependent Fragmentation Functions in $Z^{0} \rightarrow q \bar{q}$ Events

Eur. Phys. J. C8:183, 1999; CERN-EP-98-090

Measurement of Tau Branching Ratios to Five Charged Hadrons

Phys. Lett. 437B:218, 1998; CERN-EP-98-093

Search for Higgs Bosons and New Particles Decaying into Two Photons at $\sqrt{s}=183 \mathrm{GeV}$

Eur. Phys. J. C8:3, 1999; CERN-EP-98-104; HEPEX-9808016

Measurement of the Michel Parameters in Leptonic $\tau$ Decays

Eur. Phys. J. C7:571, 1999; CERN-EP-98-102; HEPEX-9808019

Measurement of the Strong Coupling Constant $\alpha_{s}$ and the Vector and Axial-Vector Spectral Functions in Hadronic $\tau$-Decays

Eur. Phys. J. C6:225, 1999; CERN-EP-98-107; HEPEX-9808026

Search for Scalar Top and Scalar Bottom Quarks at $\sqrt{s}=183 \mathrm{GeV}$ at LEP

Phys. Lett. 439B:197, 1998; CERN-EP-98-106; HEPEX-9808025 Production of $\chi_{c 2}(1 P)$ Mesons in Photon-Photon Collisions at LEP

Phys. Rev. Lett. 82:3025, 1999; DESY-98-178; HEPEX-9811011 Observation of a Coherence Length Effect in Exclusive $\rho$ Electroproduction

EVA-98-1; NUCLEX-9801003

A Direct Measurement of Short Range $N N$ Correlations in Nuclei via the Reaction ${ }^{12} \mathrm{C}(p, 2 p m)$

Phys. Rev. D49:5690, 1994; CLNS-93-1238; CLEO-93-14 First Measurement of $\Gamma\left(D_{s}^{+} \rightarrow \mu^{+} \nu\right) / \Gamma\left(D_{s}^{+} \rightarrow \phi(1020) \pi^{+}\right)$

Nucl. Phys. A577:433C, 1994 Test of Parity Violation and Time Reversal Invariance in Slow Neutron Absorption Reaction

Phys. Lett. 451B:256, 1999; KEK-98-208; NWU-HEP-98-02; TUAT-HEP-98-03; DPNU-98-48; TIT-HPE-98-013; OCU. HEP-98-01; PU-98-708; HEPEX-9901036

Measurement of the Jet Width in $\gamma \gamma$ Collisions and in $e^{+} e^{-}$Annihilation Process at TRISTAN

Phys. Lett. 321B:283, 1994; CERN-PPE-93-207; L3-068

A Study of Four Fermion Processes at LEP

Z. Phys. C69:561, 1996; CERN-PPE-95-144

Measurement of Inclusive $\pi^{0}$ Production in Hadronic $Z^{0}$ Decays

Z. Phys. C68:363, 1995; CERN-PPE-95-059

Lifetimes of Charged and Neutral $B$ Hadrons using Event Topology

2. Phys. C70:371, 1996; CERN-PPE-96-12

Production of $\Sigma^{0}$ and $\Omega^{-}$in $Z^{\circ}$ Decays

Phys. Lett. 380B:471, 1996; CERN-PPE-96-61

Search for Anomalous Production of Single Photons at $\sqrt{s}=130$ and $136 \mathrm{GeV}$

Z. Phys. C73:1, 1996; CERN-PPE-96-119

Search for Pair Production of Heavy Objects in 4-Jet Events at $\sqrt{s}=130-136 \mathrm{GeV}$

Z. Phys. C72:207, 1996; CERN-PPE-96-067

Study of Rare $b$ Decays with the DELPHI Detector at LEP

Phys. Lett. 414B:382, 1997; CERN-PPE-97-114 Search for $B_{s}-\bar{B}_{s}$ Oscillations

Nucl. Phys. A569:761, 1994

An Experimental Study of Antiproton ${ }^{4} \mathrm{He}$ Annihilation at Rest

Izv. RAN, Fiz. 58-12:54, 1994; Bull. Russ. Acad. Sci. Phys. Ser. 58:1995, 1994

Simultaneous Analysis of EAS Electron, Photon, Muon, and Hadron Components to Study the Mass Composition of Primary Cosmic Ray

Nucl. Phys. A566:419C, 1994

Rapidity Density Distributions in $\mathrm{Au}+\mathrm{Au}$ and $\mathrm{Au}+\mathrm{Ag}$ Interactions at $11.6 \mathrm{~A} \mathrm{GeV} / \mathrm{c}$

Phys. Lett. 322B:166, 1994

Rapidity Density Distribution and their Fluctuation in Violent Au-Induced Nuclear Interactions at 11.6 A GeV/c

Phys. Lett. 338B:397, 1994

Helium Production in 10.7 A GeV Au Induced Nucleus-Nucleus Collisions

Nuovo Cim. 107 A:1953, 1994

Results on Charmed Meson Decay from the WA82 Experiment at the CERN Omega Spectrometer

KOSICE-94 p. 188

Aspects on Au-Induced Interactions with Emulsion and Heavy Nuclei at $11.6 \mathrm{~A} \cdot \mathrm{GeV} / \mathrm{c}$

Phys. Lett. 348B:256, 1995; CERN-PPE-94-214

Study of Charm Correlations in $\pi^{-}$nucleon Interactions at $\sqrt{s}=26 \mathrm{GeV}$

Phys. Lett. 353B:563, 1995; CERN-PPE-95-71

Search for the Decay $D^{0} \rightarrow \mu^{+} \mu^{-}$

Phys. Lett. 358B:151, 1995; CERN-PPE-95-105

Measurement of $\Omega_{c}$ Lifetime

Phys. Lett. 352B:472, 1995

Charged Particle Density Distributions in Au Induced Interactions with Emulsion Nuclei at $10.7 \mathrm{~A} \mathrm{GeV}$

Nucl. Phys. A590:597C, 1995

Particle Production in Gold and Lead Induced Interactions at AGS and SPS

Z. Phys. C65:421, 1995

On the Production of Slow Particles in High Energy Heavy Ion Collisions

2. Phys. A351:311,1995 ${ }^{28} \mathrm{Si}\left({ }^{32} \mathrm{~S}\right)$ Fragmentation at 3.7 A, 14.6 A and $200 \mathrm{~A} \mathrm{GeV}$

Nucl. Phys. A593:535, 1995

Charged Particle Multiplicity and Pseudorapidity Density Distributions in ${ }^{16} \mathrm{O},{ }^{28} \mathrm{Si}$ and ${ }^{197} \mathrm{Au}-\mathrm{Induced}$ Nuclear Interactions at 14.6 and $11.6 \mathrm{GeV} / \mathrm{c}$

Phys. Lett. 363B:230, 1995

Rescattering Probed by the Emission of Slow Target Associated Particles in High-Energy Heavy-Ion Interactions 
Adamovich 96

Adamovich 96B

Adamovich 97

Adamovich $97 \mathrm{~B}$

Adamovich $97 \mathrm{C}$

Adamovich 97D

Adamovich $97 \mathrm{E}$

Adamovich 98

Adamovich $98 \mathrm{~B}$

Adamovich $98 \mathrm{C}$

Adamovich 99

Adams $93 \mathrm{C}$

Adams 93D

Adams 93F

Adams 94

Adams 94B

Adams $94 \mathrm{C}$

Adams 94D

Adams 94E

Adams 94F

Adams $94 \mathrm{G}$

Adams $94 \mathrm{H}$

Adams 94I

Adams 95

Adams 95B

Adams $95 \mathrm{C}$

Adams 95D

Adams 95E

Adams 95F

Adams 95G

Adams $95 \mathrm{H}$

Adams 96

Adams 96B

Adams 97

Adams 97B

Adams 97C
Phys. Lett. 385B:487, 1996; CERN-PPE-96-108

A Study of Kinematical Correlations between Charmed Particles Produced in $\pi^{-}$Cu Interactions at $\sqrt{s}$ $=28 \mathrm{GeV}$

Nucl. Phys. B495:3, 1997; CERN-PPE-96-180

Measurement of Charmed-Meson Production in Interactions between $350 \mathrm{GeV} / c \pi^{-}$Particles and Nuclei

Z. Phys. C76:35, 1997; Nucl. Phys. (PS) 55A:14, 1997; CERN-PPE-97-023; HEPEX-9703007

$\Xi^{-}$Production by $\Sigma^{-}, \pi^{-}$and Neutrons in the Hyperon Beam Experiment at CERN

Phys. Lett. 408B:469, 1997; CERN-PPE-97-058

Search for the Flavour Changing Neutral Current Decay $D^{0} \rightarrow \mu^{+} \mu^{-}$

Eur. Phys. J. C5:621, 1998; CERN-PPE-97-134; MPI-H-V38-1997; HEPEX-9710024

First Observation of the $\Xi^{-} \pi^{+}$Decay Mode of the $\Xi(1690)^{0}$ Hyperon

Phys. Lett. 390B:445, 1997

He Production in $158 \mathrm{~A} \mathrm{GeV} / \mathrm{c} \mathrm{Pb}$ on $\mathrm{Pb}$ Interactions

Phys. At. Nucl. 60:1435, 1997; Yad. Phys. 60:1580, 1997

Collective Motion in ${ }^{84} \mathrm{Kr}$ Induced Interactions with $\mathrm{Ag}-\mathrm{Br}$ Nuclei at $0.85 \mathrm{GeV}$ per Nucleon

Nucl. Phys. B519:19, 1998; CERN-EP-98-019

Measurement of the Beauty Production Cross-Section in $350 \mathrm{GeV} / \mathrm{c} \pi^{-} \mathrm{Cu}$ Interactions

Eur. Phys. J. C8:593, 1999; CERN-EP-98-041; MPI-K-H-V5-1998; HEPEX-9803021

Charge Asymmetries for $D, D_{s}^{ \pm}$and $\Lambda_{c}$ Production in $\Sigma^{-}$Nucleus Interactions at $340 \mathrm{GeV} / c$

Eur. Phys. J. C6:35, 1999; CERN-EP-98-112

A Measurement of the Form-Factor Ratios in the Decay $D^{+} \rightarrow \bar{K}^{*}(892)^{0} \mu^{+} \nu_{\mu}$

Eur. Phys. J. C8:59, 1999

First Observation of $\Sigma^{-} e^{-}$Elastic Scattering in the Hyperon Beam Experiment WA89 at CERN

Phys. Rev. Lett. 72:466, 1994; FERMILAB-PUB-93-171-E

$Q^{2}$ Dependence of the Average Squared Transverse Energy of Jets in Deep-Inelastic Muon-Nucleon Scattering with Comparison to QCD

Z. Phys. C61:179, 1994; FERMILAB-PUB-93-246

Production of Charged Hadrons by Positive Muons on Deuterium and Xenon at $490 \mathrm{GeV}$

Phys. Lett. 308B:418, 1994; MPI-PHE-93-10; FERMILAB-PUB-93-170-E

An Investigation on Bose-Einstein Correlations in Muon-Nucleon Interactions at $480 \mathrm{GeV}$

Phys. Rev. Lett. 72:2337, 1994; FERMILAB-PUB-94-025-E

Observation of Jet Production by Real Photons

Phys. Lett. 336 B:269, 1994

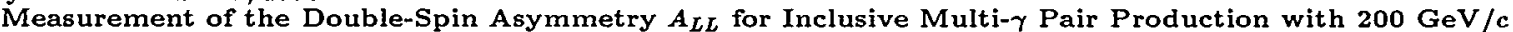
Polarized Proton Beam and Polarized Proton Target

Phys. Rev. D53:4747, 1996; IFVE-94-88

Single-Spin Asymmetries and Invariant Cross Sections of the High-Transverse-Momentum Inclusive $\pi^{0}$ Production in $200 \mathrm{GeV} / \mathrm{c} p$ and $\bar{p} p$ Interactions

Z. Phys. C61:539, 1994; FERMILAB-P UB-93-392-E

Production of Neutral Strange Particles in Muon-Nucleon Scattering at $490 \mathrm{GeV}$

Phys. Lett. 335B:535, 1994; MPI-PHE-94-12; FERMILAB-PUB-94-217-E

Density and Correlation Integrals in Deep-Inelastic Muon-Nucleon Scattering at $490 \mathrm{GeV}$

Z. Phys. C65:225, 1995; FERMILAB-PUB-94-218-E

Nuclear Shadowing, Diffractive Scattering and Low Momentum Protons in $\mu^{-}$Xe Interactions at $490 \mathrm{GeV}$

Phys. Lett. 329B:399, 1994; Phys. Lett. 339B:332, 1994; DAPNIA-94-04; CERN-PPE-94-057; HEPPH-9404270 Measurement of the Spin Dependent Structure Function $g_{1}(x)$ of the Proton

Phys. Rev. D50:1836, 1994; FERMILAB-PUB-93-245-E

Scaled Energy $(z)$ Distributions of Charged Hadrons Observed in Deep-Inelastic Muon Scattering at 490 $\mathrm{GeV}$ from Xenon and Deuterium Targets

Phys. Lett. 336B:125, 1994; CERN-PPE-94-116

Spin Asymmetry in Muon Proton Deep Inelastic Scattering on a Transversely Polarized Target

Phys. Rev. Lett. 75:1466, 1995; FERMILAB-PUB-95-017-E

Extraction of the Ratio $F_{2}(n) / F_{2}(p)$ from Muon-Deuteron and Muon-Proton Scattering at Small $x$ and $Q^{2}$

Phys. Rev. C51:17, 1995

Measurement of $\gamma \uparrow$ deuteron $\rightarrow p n \pi^{0}$ at Large Nucleon Momenta

Z. Phys. C67:403, 1995

Shadowing in Inelastic Scattering of Muons on Carbon, Calcium and Lead at Low $x_{B j}$

Phys. Rev. Lett. 74:5198, 1995; Phys. Rev. Lett. 80:2020, 1998

Nuclear Decay Following Deep Inelastic Scattering of $470 \mathrm{GeV}$ Muons

Phys. Lett. 357B:248, 1995; CERN-PPE-95-97

A New Measurement of the Spin-Dependent Structure Function $g_{1}(x)$ of the Deuteron

Z. Phys. C71:391, 1996; FERMILAB-PUB-95-395-E

Determination of the Gluon Distribution Function of the Nucleon using Energy-Energy Angular Pattern in Deep-Inelastic Muon-Deuteron Scattering

Phys. Rev. Lett. 74:1525, 1995; FERMILA B-PUB-94-233-E

Measurement of Nuclear Transparencies from Exclusive $\rho^{\circ}$ Meson Production in Muon-Nucleus Scattering at $470 \mathrm{GeV}$

Phys. Lett. 345B:569, 1995

Measurement of Single Spin Asymmetry for Direct Photon Production in $p$ p Collisions at $200 \mathrm{GeV}$

Phys. Rev. D54:3006, 1996; FERMILAB-PUB-95-396-E

Proton and Deuteron Structure Functions in Muon Scattering at $470 \mathrm{GeV}$

MANCHESTER-95 p. 491

Resonance Production in the Reaction $\pi^{-} p \rightarrow n \pi^{-} \pi^{+} \pi^{0} \pi^{0}$

Phys. Lett. 396B:338, 1997; CERN.PPE-97-008

The Spin-Dependent Structure Function $g_{1}(x)$ of the Deuteron from Polarized Deep-Inelastic Muon Scattering

Phys. Rev. D56:5330, 1997; CERN-PPE-97-022; HEPEX-9702005

Spin Structure of the Proton from Polarized Inclusive Deep-Inelastic Muon-Proton Scattering

Z. Phys. C74:237, 1997; FERMILAB-PUB-97-103-E; MPI-PHE-97-03

Diffractive Production of $\rho^{\mathrm{o}}$ Mesons in Muon-Proton Interactions at $470 \mathrm{GeV}$ 
Adams 97D

Adams $97 \mathrm{E}$

Adams $97 \mathrm{~F}$

Adams 98

Adams 98B

Adarkar 98

Adeva $93 \mathrm{~B}$

Adeva 95

Adeva 97

Adeva $97 \mathrm{~B}$

Adeva 98

Adeva $98 \mathrm{~B}$

Adeva $98 \mathrm{C}$

Adinolfi 99

Adler 94

Adler $94 \mathrm{~B}$

Adler 95

Adler $95 \mathrm{~B}$

Adler 95C

Adler $95 \mathrm{D}$

Adler $95 \mathrm{E}$

Adler 95F

Adler 95G

Adler 96

Adler 96B

Adler $96 \mathrm{C}$

Adler $96 \mathrm{D}$

Adler $96 \mathrm{E}$

Adler $96 \mathrm{~F}$

Adler 97

Adler $97 \mathrm{~B}$

Adler $97 \mathrm{C}$

Adler $97 \mathrm{D}$

Adler 98

Adloff 96
Phys. Rev. Lett. 79:4083, 1997; FERMILAB-PUB-97-320-E; RUTGERS-97-26; HEPEX-9709028 Search for Light Gluinos via the Spontaneous Appearance of $\pi^{+} \pi^{-}$Pairs with an $800 \mathrm{GeV} / \mathrm{c}$ Proton Beam at FERMILAB

Nucl. Phys. B510:3, 1998; IFVE-97-56

Measurement of Single Spin Asymmetry in $\eta$ Meson Production in $p \uparrow p$ and $p\rceil p$ Interactions in the Beam Fragmentation Region at $200 \mathrm{GeV} / \mathrm{c}$

Z. Phys. C76:441, 1997; MPI-PHE-97-19 Inclusive Single-Particle Distributions and Transverse Momenta of Forward Produced Charged Hadrons in $\mu^{ \pm} p$ Scattering at $470 \mathrm{GeV}$

Phys. Rev. Lett. 80:4123, 1998; FERMILAB-PUB-98-058-E Measurement of the Branching Fraction of the Decay $K_{L} \rightarrow \pi^{+} \pi^{-} e^{+} e^{-}$

Phys. Let.t. 447B:240, 1999; FERMILAB-PUB-98-178-E; EFI-98-21; HEPEX-9806007 Search for the Decay $K_{L} \rightarrow \pi^{0} \nu \bar{\nu}$

Phys. Rev. D57:2653, 1998 Cosmic Ray Composition from Multiple Muon Data with the KGF Underground Detector

Phys. Lett. 320B:400, 1994; CERN-PPE-93-206 Combined Analysis of World Data on Nucleon Spin Structure Functions

Phys. Lett. 369B:93, 1996; CERN-PPE-95-187 Polarization of Valence and Nonstrange Sea Quarks in the Nucleon from Semiinclusive Spin Asymmetries

Phys. Lett. 412B:414, 1997; CERN-PPE-97-118 The Spin Dependent Structure Function $g_{1}(x)$ of the Proton from Polarized Deep-Inelastic Muon Scattering

Phys. Lett. 420B:180, 1998; CERN-PPE-97-147; HEPEX-9711008 Polarised Quark Distributions in the Nucleon from Semi-Inclusive Spin Asymmetries

Phys. Rev. D58:112001, 1998; CERN-EP-98-085 Spin Asymmetries $A_{1}$ and Structure Functions $g_{1}$ of the Proton and the Deuteron from Polarized High Energy Muon Scattering

Phys. Rev. D58:112002, 1998; CERN-EP-98-086 A Next-to-Leading Order QCD Analysis of the Spin Structure Function $g_{1}$

DUBNA-96, v. I, p. 252 The Spin-Dependent Structure Function $g_{1}(x)$ of the Deuteron from Polarized Deep-Inelastic Muon Scattering

Nucl. Phys. B547:3, 1999; CERN-EP-99-020

$D^{*}(\mathbf{2 0 1 0})^{ \pm}$Production in $350 \mathrm{GeV} / c \pi^{-} N$ Interactions

Z. Phys. C63:541, 1994; CERN-PPE-94-064

Bose-Einstein Correlations in $\widetilde{p} p$ Annihilations at Rest

Phys. At. Nucl. 57:1772, 1994; Yad. Phys. 57:1842, 1994

Recent Results of the CPLEAR Experiment

Phys. Lett. 363B:237, 1995; CERN-PPE-95-103

Measurement of $K_{L}-K_{S}$ Mass Difference using Semileptonic Decays of Tagged Neutral Kaons

Phys. Rev. Lett. 76:1421, 1996; BNL-62327; PRINCETON-HEP-95-8; TRI-PP-95-83; HEPEX-9510006 Search for Decay $K^{+} \rightarrow \pi^{+} \nu \bar{\nu}$

Z. Phys. C70:211, 1996; CERN-PPE-95-134

First Observation of a Particle-Antiparticle Asymmetry in the Decay of Neutral Kaons into $\pi^{\circ} \pi^{\circ}$

Phys. Lett. 363B:243, 1995; CERN-PPE-95-107

Measurement of the $C P$ Violation Parameter $\eta_{+-}$using Tagged $K^{0}$ and $\bar{K}^{0}$

Phys. Lett. 364B:239, 1995; CERN-PPE-95-149

Test of CPT Symmetry and Quantum Mechanics with Experimental Data from CPLEAR

Z. Phys. C65:199, 1995; CERN-PPE-94-85

Inclusive Measurement of $\bar{p}$ Annihilation at Rest in Gaseous Hydrogen to Einal States Containing $\rho^{\circ}$ and $f_{2}(1270)$

CERN-PPE-95-112

A New Determination of $K_{L}-K_{S}$ Mass Difference and the Phase of the CP Violation Parameter $\eta_{+}-$from an Evaluation of Experimental Data

Phys. Lett. 370B:167, 1996; CERN-PPE-95-189

Search for $C P$ Violation in the Decay of Neutral Kaons to $\pi^{+} \pi^{-} \pi^{0}$

Phys. Lett. 374B:313, 1996; CERN-PPE-96-08 Observation of the $C P$ Conserving $K_{S} \rightarrow \pi^{+} \pi^{-} \pi^{0}$ Decay Amplitude

Phys. At. Nucl. 59:1363, 1996; Yad. Phys. 59:1422, 1996 Bose-Einstein Pion Correlations

Yad. Phys. 59:1588, 1996; Phys. At. Nucl. 59:1588, 1996 $C P, T$ and $C P T$ Violation in the Neutral Kaon System at the CPLEAR Experiment

Phys. Lett. 369B:367, 1996

Evaluation of the Phase of the CP Violation Parameter $\eta_{+}-$and $K_{L}-K_{S}$ Mass Difference from a Correlation Analysis of Different Experiments

Nuovo Cim. 109A:981, 1996; PISA-95 p. 981

Measurement of $C P$ Violation Parameters and Test of $C P T$ Invariance with $\bar{K}^{0}$ and $K^{\circ}$ at $\mathrm{LEAR}$

Phys. Let.t. 407 B:193, 1997; CERN-PPE-97-054

CPLEAR Results on the CP Parameters of Neutral Kaons Decaying to $\pi^{+} \pi^{-} \pi^{\circ}$

Phys. Rev. Lett. 79:4756, 1997; BNL-64627; PRINCETON-HEP-97-11; TRI-PP-97-27; KEK-97-114; HEPEX-9708012 Observation of the Decay $K^{+} \rightarrow \pi^{+} \mu^{+} \mu^{-}$

Phys. Rev. Lett. 79:2204, 1997; BNL-64631; PRINCETON-HEP-97-12; TRI-PP-97-28; KEK-97-115; HEPEX-9708031 Evidence for the Decay $\mathrm{K}^{+} \rightarrow \pi^{+} \nu \bar{\nu}$

Phys. Lett. 403B:383, 1997

Experimental Measurement of the $K_{S} K_{S} / K_{S} K_{L}$ Ratio in Antiproton Annihilations at Rest in Gaseous Hydrogen at 15 and 27 bar

Phys. Rev. D58:012003, 1998; BNL-65204; PRINCETON-HEP-98-1; TRI-PP-98-1; HEPEX-9802011

Upper Limit on the Decay $K^{+} \rightarrow e^{+} \nu \mu^{+} \mu^{-}$

Nucl. Phys. B485:3, 1997; DESY-96-215; HEPEX-9610006

Measurement of Charged Particle Transverse Momentum Spectra in Deep Inelastic Scattering 
Adloff 96B

Adloff $96 \mathrm{C}$

Adloff 97

Adloff 97B

Adloff $97 \mathrm{C}$

Adloff 97D

Adloff $97 \mathrm{E}$

Adloff $97 \mathrm{~F}$

Adloff $97 \mathrm{G}$

Adloff $97 \mathrm{H}$

Adloff 97I

Adloff 97J

Adloff $97 \mathrm{~K}$

Adloff 97L

Adloff $97 \mathrm{M}$

Adloff 98

Adloff 98B

Adloff 98C

Adloff 98D

Adloff 98E

Adloff $98 \mathrm{~F}$

Adloff $98 \mathrm{G}$

Adloff $98 \mathrm{H}$

Adloff 98I

Adloff 98J

Adloff $98 \mathrm{~K}$

Adloff $98 \mathrm{~L}$

Adomeit 98

Adyasevich 94

Adyasevich 94B

Adyasevich 96

Afanasiev 97

Afanasiev 98

Afanasiev 98B

Afanasiev $98 \mathrm{C}$

Afanasyev 94

Afanasyev 96
Z. Phys. C72:593, 1996; DESY-96-138

Inclusive $D^{0}$ and $D^{*}(2010)^{ \pm}$Production in Deep Inelastic $e^{-} p$ Scattering at HERA

Phys. Lett. 393B:452, 1997; DESY-96-236; HEPEX-9611017

Determination of the Longitudinal Proton Structure Function $F_{L}\left(x, Q^{2}\right)$ at Low $x$

2. Phys. C74:221, 1997; DESY-97-009; HEPEX-9702003

Diffraction Dissociation in Photoproduction at HERA

Z. Phys. C74:191, 1997; DESY-97-024; HEPEX-9702012

Observation of Events at Very High $Q^{2}$ in ep Collisions at HERA

Nucl. Phys. B497:3, 1997; DESY-97-042; HEPEX-9703012

A Measurement of the Proton Structure Function $F_{2}\left(x, Q^{2}\right)$ at Low $x$ and Low $Q^{2}$ at HERA

2. Phys. C75:607, 1997; DESY-97-082; HEPEX-9705014 Proton Dissociative $\rho$ and Elastic $\phi(1020)$ Electroproduction at HERA

Nucl. Phys. B504:3, 1997; DESY-97-108; HEPEX-9707005

Evolution of $e^{+} p$ Fragmentation and Multiplicity Distribution in the Breit Frame

Phys. Lett. 406B:256, 1997; DESY-97-098; HEPEX-9706002

Measurement of Event Shape Variables in Deep Inelastic $e^{-} p$ Scattering

Z. Phys. C76:613, 1997; DESY-97-158; HEPEX-9708016

Inclusive Measurement of Diffractive Deep-Inelastic $e^{-} p$ Scattering

Eur. Phys. J. C1:97, 1998; DESY-97-164; HEPEX-9709004

Measurement of the Inclusive Dijet Cross Section in Photoproduction and Determination of an Effective Parton Distribution in the Photon

Z. Phys. C76:213, 1997; DESY-97-095; HEPEX-9705018

Photoproduction of $K^{\circ}$ and $\Lambda$ at HERA and a Comparison with Deep Inelastic Scattering

Phys. Lett. 415B:418, 1997; DESY-97-179; HEPEX-9709017

Low $q^{2}$ Jet Production at HERA and Virtual Photon Structure

Eur. Phys. J. C1:495, 1998; DESY-97-210; HEPEX-9711006

Thrust Jet Analysis of Deep-Inelastic Large-Rapidity-Gap Events

Phys. Lett. 421B:385, 1998; DESY-97-228; HEPEX-9711012

Photoproduction of $\psi(2 S)$ Mesons at HERA

Z. Phys. C75:437, 1997; DESY-97-075; HEPEX-9705001

Bose-Einstein Correlations in Deep Inelastic $e^{-} p$ Scattering at HERA

Eur. Phys. J. C5:439, 1998; DESY-98-044; HEPEX-9804012 Multiplicity Structure of the Hadronic Final State in Diffractive Deep Inelastic Scattering at HERA

DESY-98-076; HEPEX-9806029

Dijet Event Rates in Deep-Inelastic Scattering at HERA

Eur. Phys. J. C5:575, 1998; DESY-98-063; HEPEX-9806009

Observation of Events with an Isolated High Energy Lepton and Missing Transverse Momenta at HERA

Eur. Phys. J. C5:625, 1998; DESY-98-075; HEPEX-9806028

Differential $(2+1)$ Jet Event Rates and Determination of $\alpha_{s}$ in Deep Inelastic Scattering at HERA

Eur. Phys. J. C6:575, 1999; DESY-98-087; HEPEX-9807019

Multijet Event Rates in Deep Inelastic Scattering and Determination of the Strong Coupling Constant

Eur. Phys. J. C6:587, 1999; DESY-98-169; HEPEX-9811013 Measurement of Leading Proton and Neutron Production in Deep Inelastic Scattering at HERA

Nucl. Phys. B538:3, 1999; DESY-98-143; HEPEX-9809028

Forward Jet and Particle Production at HERA

Nucl. Phys. B545:21, 1999; DESY-98-204; HEPEX-9812023

Measurement of $D^{*}$ Cross Sections at HERA and Determination of the Gluon Density in the Proton using NLO QCD

Eur. Phys. J. C6:421, 1999; DESY-98-092; HEPEX-9808013 Diffractive Dijet Production at HERA

DESY-98-205; HEPEX-9812024

Measurement of Dijet Cross-Sections at Low $Q^{2}$ and the Extraction of an Effective Parton Density for the Virtual Photon

DESY-98-148; HEPEX-9810020

Charged Particle Cross-Sections in Photoproduction and Extraction of the Gluon Density in the Photon

Phys. Lett. 428B:206, 1998; DESY-98-029; HEPEX-9803032

Hadron Production in Diffractive Deep Inelastic Scattering

Z. Phys. C71:227, 1996

Evidence for Two Isospin Zero $J^{P C}=2^{-+}$Mesons at 1645 and $1875 \mathrm{MeV}$

Phys. At. Nucl. 57:253, 1994; Yad. Phys. 57:268, 1994

Two-Particle Correlations for Light Nuclear Fragments in Nucleus-Nucleus Collisions at 3.6 GeV/Nucleon

Phys. At. Nucl. 57:1981, 1994; Yad. Phys. 57:2057, 1994

Two-Particle Correlations with Participation of Pions and Light Nuclear Fragments in Nucleus-Nucleus Collisions at $3.6 \mathrm{GeV} / \mathrm{Nucleon}$

Z. Phys. C71:65, 1996; DAPNIA-SPP-96-03

Measurement of the Total Cross Section Difference $\Delta \sigma_{L}$ in $n p$ Transmission at $1.19,2.49$ and $3.65 \mathrm{GeV}$

Nucl. Phys. A625:817, 1997; DPNU-97-31

Tensor Analyzing Power $T_{20}$ for Cumulative Pion Production from Deuterons in the GeV Energy Region

Phys. Lett. 434B:21, 1998; JINR-E1-98-75

Measurement of the Tensor Analyzing Power $A_{y y}$ in Inclusive Breakup of $\theta \mathrm{GeV} / \mathrm{c}$ Deuterons on Carbon at Large Transverse Momenta of Protons

Kr. Soob. JINR 88:5, 1998

Tensor and Vector Analyzing Powers $A_{y y}$ and $A_{y}$ in the ${ }^{12} \mathrm{C}$ (deuteron, $p$ ) X and ${ }^{12} \mathrm{C}$ (deuteron, deuteron) X Reactions at Initial Deuteron Momentum of $9 \mathrm{GeV} / c$ and Emission Angle of 85 mrad

Phys. Lett. 445B:14, 1998; JINR-E1-98-319

Fragmentation of Tensor Polarized Deuterons into Cumulative Pions

Phys. Lett. 338B:478, 1994; JINR-E1-94-423

Experimental Estimation of the Lifetime of Atoms Formed by $\pi^{+}$and $\pi^{-}$Mesons

Phys. At. Nucl. 60:938, 1997; Yad. Phys. 60:1049, 1997; JINR-E1-96-256

Measurement of the Coulomb Interaction Effect in $\pi^{+} \pi^{-}$Pairs from the Reaction $p$ Ta $\rightarrow \pi^{+} \pi^{-} \mathrm{X}$ at 70 $\mathrm{GeV}$

See the legend on page 5. 
Afanasyev 97

Agababyan 94

Agababyan 94B

Agababyan $94 \mathrm{C}$

Agababyan $94 \mathrm{D}$

Agababyan 95

Agababyan 95B

Agababyan 95C

Agababyan $95 \mathrm{D}$

Agababyan 96

Agababyan $96 \mathrm{~B}$

Agababyan 96C

Agababyan 97

Agababyan 98

Agakichiev 95

Agakichiev 96

Agakichiev 97

Agakichiev 98

Agakichiev 98B

Agakishiev 98

Aggarwal 95

Aggarwal 97

Aggarwal 97B

Aggarwal 97C

Aggarwal 98

Aggarwal 98B

Aggarwal 98C

Aggarwal 98D

Aggarwal $98 \mathrm{E}$

Aggarwal 98F

Aglietta 94

Aglietta $94 \mathrm{~B}$

Aglietta 95

Aglietta 96

Aglietta $96 \mathrm{~B}$

Aglietta $96 \mathrm{C}$

Aglietta $96 \mathrm{D}$

Aglietta 97
JINR-P1-97-306

Ratio of Production Cross Sections for Pairs of Opposite Charged Pions in the Free and Atomic-Bound States

Phys. Lett. 320B:411, 1994; HEN-361 Collective Sea-Gull Effect in $\pi^{+} p$ Interactions at $250 \mathrm{GeV} / \mathrm{c}$

Phys, Lett. 328B:199, 1994; HEN-369 Angular Dependence of Factorial Moments in $\pi^{+} / K^{+} p$ Interactions at $250 \mathrm{GeV} / \mathrm{c}$

Phys. Lett. 332B:458, 1994; HEN-368 Genuine Higher Order Correlations in $\pi^{+} p$ and $K^{+} p$ Collisions at $250 \mathrm{GeV} / c$

Z. Phys. C64:381, 1994; HEN-363

Transverse Momentum Compensation in $\pi^{+} p$ Interactions at $250 \mathrm{GeV} / \mathrm{c}$

Z. Phys. C66:385, 1995; HEN-372

Backward Proton Production in $\pi^{+}$and $K^{+}$Collisions with $\mathrm{Al}$ and Au Nuclei at $250 \mathrm{GeV} / \mathrm{c}$

Z. Phys. C66:409, 1995; HEN-371

Angular Dependence of Bose-Einstein Correlations in Interactions of $\pi^{+}$and $K^{+}$Mesons with Protons and Nuclei at $250 \mathrm{GeV} / \mathrm{c}$

Z. Phys. C68:229, 1995; HEN-375

Higher Order Bose-Einstein Correlations in $\pi^{+} p$ and $K^{+} p$ Collisions at $250 \mathrm{GeV} / c$

Phys. Lett. 353B:397, 1995; HEN-382

Angular Dependence of Particle Correlations in $\pi^{+} / K^{+} p$ Interactions at $250 \mathrm{GeV} / \mathrm{c}$

Phys. Lett. 382 B:305, 1996; HEN-387; HEPEX-9606005 Self-Affine Fractality in $\pi^{+} p$ and $K^{+} p$ Collisions at $250 \mathrm{GeV} / \mathrm{c}$

Phys. Lett. 393B:205, 1997; HEN-395

Factorial Moments and Correlations in Transverse Momentum in $\pi^{+} p$ and $K+p$ Collisions at $250 \mathrm{GeV} / c$

Z. Phys. C71:405, 1996; HEN-390; HEN-96-390

Two- and Three-Dimensional Analysis of Bose-Einstein Correlations in $\pi^{+} / K^{+} p$ Interactions at 250 GeV/c

Phys. Lett. 422B:359, 1998; HEN-405; HEN-97-405; HEPEX-9711009

Estimation of Hydrodynamical Model Parameters from the Invariant Spectrum and the Bose-Einstein Correlations of $\pi^{-}$Mesons Produced in $\left(\pi^{+} / K^{+}\right) p$ Interactions at $250 \mathrm{GeV} / c$

Phys. Lett. 431 B:451, 1998; HEN-406; HEPEX-9805030 Self-Affine Scaling from Non-Integer Phase Space Partition in $\pi^{+} p$ and $\mathrm{K}^{+}{ }_{p} \mathrm{Collisions}$ at $250 \mathrm{GeV} / \mathrm{c}$

Phys. Rev. Lett. 75:1272, 1995; CERN-PPE-95-26 Enhanced Production of Low Mass Electron Pairs in $200 \mathrm{GeV} / \mathrm{u}$ S Au Collisions at the CERN SPS

Nucl. Phys. A610:317C, 1996 First Results from CERES/NA45 on Low-Mass Electron Pair Production in Pb Au Collisions

Phys. Lett. 422B:405, 1998; NUCLEX-9712008

Low-Mass $e^{+} e^{-}$Pair Production in $158 \mathrm{~A} \mathrm{GeV} \mathrm{Pb} \mathrm{Au} \mathrm{Collisions} \mathrm{at} \mathrm{the} \mathrm{CERN} \mathrm{SPS,} \mathrm{its} \mathrm{Dependence} \mathrm{on}$ Multiplicity and Transverse Momentum

Nucl. Phys. A638:159C, 1998; TSUKUBA-97 p. $159 \mathrm{C}$ CERES Results on Low-Mass Electron Pair Production in $\mathrm{Pb}-\mathrm{Au}$ Collisions

Nucl. Phys. A638:467C, 1998; TSUKUBA-97 p. $467 \mathrm{C}$ Hadron Physics with CERES: Spectra and Collective Flow

Eur. Phys. J. C4:231, 1998 Systematic Study of Low-Mass Electron Pair Production in $p \mathrm{Be}$ and $p$ Au Collisions at $450 \mathrm{GeV} / c$

Nucl. Phys. A590:503C, 1995 Results from the WA93 Photon Multiplicity Detector

Phys. Rev. C56:1160, 1997; IKP-MS-96-1001 Soft Photon Production in Central $200 \mathrm{GeV} /$ nucleon $^{32} \mathrm{~S}$ Au Collisions

Phys. Lett. 403B:390, 1997 Azimuthal Anisotropy in S + Au Reactions at $200 \mathrm{~A} \mathrm{GeV}$

Phys. Lett. 404B:207, 1997

Event by Event Measurement of $\left\langle p_{\Upsilon}\right\rangle$ of Photons in S A A Collisions at $200 \mathrm{~A} \cdot \mathrm{GeV}$

Phys. Rev. Let. 81:4087, 1998; IKP-MS-980601; NUCLEX-9806004 Centrality Dependence of Neutral Pion Production in $158 \cdot \mathrm{A} \mathrm{GeV}{ }^{208} \mathrm{~Pb}+{ }^{208} \mathrm{~Pb} \mathrm{Collisions}$

NUCLEX-9807004

Directed Flow in $158 \mathrm{~A} \cdot \mathrm{GeV}{ }^{208} \mathrm{~Pb}+{ }^{208} \mathrm{~Pb}$ Collisions.

Nucl. Phys. A638:147C, 1998 Recent Results on Pb + Pb Collisions at $158 \mathrm{~A}$ GeV from the WA98

Nucl. Phys. A638:415C, 1998 Systematics of Hadron Production - Probing the Final and Initial States

Nucl. Phys. A638:459C, 1998 Collective Flow in $158 \mathrm{~A} \mathrm{GeV} \mathrm{Pb+Pb} \mathrm{Collisions}$

Phys. Rev. C58:1146, 1998 Multiplicity and Pseudorapidity Distribution of Photons in S + Au Reaction at $200 \mathrm{~A} \cdot \mathrm{GeV}$

Phys. Lett. 333B:555, 1994; LNGS-94-96 The Limit to the UHE Extraterrestrial Neutrino Flux from the Observations of Horizontal Air Showers at EAS-TOP

Phys. Lett. 337B:376, 1994; LNGS-94-100

Study of the Primary Cosmic Ray Composition around the Knee of the Energy Spectrum

Astropart. Phys. 3:311, 1995; LNGS-95-06

Neutrino-Induced and Atmospheric Single-Muon FIuxes Measured over Five Decades of Intensity by LVD at Gran Sasso Laboratory

Astropart. Phys. 6:71, 1996; INFN-AE-96-21

A Limit to the Rate of Ultra High Energy $\gamma$-Rays in the Primary Cosmic Radiation

Astroph. Jour. 470:501, 1996; INFN-A E-96-14

A Measurement of the Solar and Sidereal Cosmic Ray Anisotropy at $E_{0} \sim 10^{14} \mathrm{eV}$

Astroph. Jour. 469:305, 1996; IN FN-AE-96-15

Search for Gamma Ray Bursts at Photon Energies $E \geq 10 \mathrm{GeV}$ and $E \geq 10 \mathrm{TeV}$

INFN-AE-96-30

Multicomponent Extensive Air Shower Observations at EAS-TOP

Izv. RAN, Fiz. 61:597, 1997

The Upper Limit of the Solar Antineutrino FIux According to the LSD Array Data 
Aglietta 97B

Aglietta 97C

Aglietta 98

Agnello 94

Aharonov 95

Aharonov 95B

Ahle 94

Ahle 98

Ahle 99

Ahlen 94

Ahlen 95

Ahmad 94

Ahmad 95

Ahmad 95B

Ahmad 90

Ahmad 97

Ahmad 98

Ahmed 94

Ahmed 94B

Ahmed $94 \mathrm{C}$

Ahmed 94D

Ahmed $94 \mathrm{E}$

Ahmed $94 \mathrm{~F}$

Ahmed $94 \mathrm{G}$

Ahmed $94 \mathrm{H}$

Ahmed 95

Ahmed 95B

Ahmed 95C

Ahmed 95D

Ahmidouch 95

Ahmidouch 95B

Ahmidouch 96

Ahmidouch 98

Ahn 97

Ahn 98

Ahn 98B

Ahn $98 \mathrm{C}$

Ahn 98D
Izv. RAN, Fiz. 61:580, 1997

Study of High Energy Muons in the LVD Experiment

Astropart. Phys. 6:143, 1997; INFN-AE-96-20

The Shapes of the Atmospheric Cherenkov Light Images from Extensive Air Showers

Phys. Rev. D58:092005, 1998; HEPEX-9806001

Muon "Depth - Intensity" Relation Measured by LVD Underground Experiment and Cosmic-Ray Muon Spectrum at Sea Level

Phys. Lett. 337B:226, 1994

Measurement of the Frequency of the Annihilation Reaction $\bar{p} p \rightarrow \pi^{0} \pi^{0}$ at Rest in a NTP Hydrogen Target

Phys. Lett. 353B:168, 1995

New Experimental Limits for the Electron Stability

Phys. Rev. D52:3785, 1995

New Laboratory Bounds on the Stability of the Electron

Phys. Lett. 332B:258, 1994; BNL-49567

Global Transverse Energy Distributions in $\mathrm{Si}+\mathrm{Al}, \mathrm{Au}$ at $14.6 \mathrm{~A} \cdot \mathrm{GeV} / \mathrm{c}$ and $\mathrm{Au}+\mathrm{Au}$ at $11.6 \mathrm{~A} \cdot \mathrm{GeV} / c$

Phys. Rev. Lett. 81:2650, 1998

Antiproton Production in $\mathrm{Au}+\mathrm{Au}$ Collisions at $11.7 \mathrm{~A} \mathrm{GeV} / \mathrm{c}$

Phys. Rev. C59:2173, 1999

Simultaneous Multiplicity and Forward Energy Characterization of Particle Spectra in Au + Au Collisions at $11.6 \mathrm{~A} \cdot \mathrm{GeV} / \mathrm{c}$

Phys. Rev. Lett. 72:608, 1994; LNGS-93-84

Search for Slowly Moving Magnetic Monopoles with the MACRO Detector

Phys. Lett. 357B:481, 1995

Atmospheric Neutrino Flux Measurement using Upgoing Muons

Nuovo Cim. 107 A:683, 1994

Peripheral Collisions Caused by $4.5 \mathrm{~A} \mathrm{GeV/c} \mathrm{Carbon} \mathrm{and} \mathrm{Silicon} \mathrm{Nuclei}$

Int. Jour. Mod. Phys. A10:845, 1995

Multiplicity Characteristics of Particles Produced in ${ }^{12} \mathrm{C}$ Emulsion Collisions at $4.5 \mathrm{~A} \mathrm{GeV} / \mathrm{c}$

Phys. Rev. Lett. 75:2658, 1995

Search for Narrow Sum Energy Lines in Electron Positron Pair Emission from Heavy Ion Collisions Near the Coulomb Barrier

Phys. Lett. 382B:35, 1996; Phys. Lett. 386B:496, 1996

$\Lambda$ Production by $11.6 \mathrm{~A} \mathrm{GeV} / c$ Au Beam on Au Target

Phys. Rev. Lett. 78:618, 1997

Search for Monoenergetic Positron Emission from Heavy-Ion Collisions at Coulomb-Barrier Energies

Nucl. Phys. A636:507, 1998; NUCLEX-9803006

Nuclear Matter Expansion Parameters from the Measurement of Differential Multiplicities for Lambda Production in Central Au+Au Collisions at AGS

Z. Phys. C64:545, 1994; DESY-94-154

A Search for Leptoquarks and Squarks at HERA

Phys. Lett. 340B:205, 1994; DESY-94-138

A Search for Heavy Leptons at HERA

Nucl. Phys. B435:3, 1995; DESY-94-198

Observation of Hard Processes in Rapidity Gap Events in $\gamma p$ Interactions at HERA

Phys. Lett. 338B:507, 1994; DESY-94-153

Photoproduction of $J / \psi(1 S)$ Mesons at HERA

Phys. Lett. 346B:415, 1995; DESY-94-220

Determination of the Strong Coupling Constant from Jet Rates in Deep Inelastic scattering

DESY-94-248

Observation of an $e^{+} p \rightarrow \mu^{+}$X Event with High Transverse Momenta at HERA

Phys. Lett. 324B:241, 1994; DESY-94-012

First Measurement of the Charged Current Cross Section at HERA

Nucl. Phys. B429:477, 1994; DESY-94-133

Deep Inelastic Scattering Events with a Large Rapidity Gap at HERA

Z. Phys. C66:529, 1995; DESY-95-024

Experimental Study of Hard Photon Radiation Processes at HERA

Nucl. Phys. B439:471, 1995; DESY-95-006

A Measurement of the Proton Structure Function $F_{2}\left(x, Q^{2}\right)$

Nucl. Phys. B445:195, 1995; DESY-95-062

Inclusive Parton Cross Sections in Photoproduction and Photon Structure

Phys. Lett. 348B:681, 1995; DESY-95-036

First Measurement of the Deep Inelastic Structure of Proton Diffraction

Phys. Lett. 364B:116, 1995

Charge-Exchange $\bar{p} p \rightarrow \bar{n} n$ Differential Cross Sections between 546 and $1287 \mathrm{MeV} / \mathrm{c}$

Nucl. Phys. B444:27, 1995

First Measurement of the $\bar{p} p \rightarrow \bar{n} n$ Depolarization Parameter $D_{0 n o n}$ at $546 \mathrm{MeV} / c$ and $875 \mathrm{MeV} / \mathrm{c}$

Phys. Lett. 380B:235, 1996

First Measurement of the Spin-Transfer Parameter $K_{n o o n}$ in the $\bar{p} p \rightarrow \bar{n} n$ Reaction at $875 \mathrm{MeV} / c$

Eur. Phys. J. C2:627, 1998

Spin Observables in Neutron-Proton Elastic Scattering

Nucl. Phys. A625:231, 1997; KEK-96-158

Scalar and Vector Meson Production and Two-Step Processes in $\left(K^{-}, K^{+}\right)$Reaction on ${ }^{12} \mathrm{C}$

Phys. Lett. 444B:267, 1998; KEK-98-24

Enhanced $\Lambda \Lambda$ Production near Threshold in the ${ }^{12} \mathrm{C}\left(K^{-}, K^{+}\right)$Reaction

Nucl. Phys. A648:263, 1999; KEK-97-224

A Study of $\Sigma t_{p}$ Elastic Scattering in the Region of $300 \leq P_{\Sigma} \leq 600 \mathrm{MeV} / c$ with a Scintillating Fiber Target

Phys. Lett. 440B:179, 1998

An Experimental Study of the Process $e^{+} e^{-} \rightarrow e^{+} e^{-} \mu^{+} \mu^{-}$

Nucl. Phys. A639:379C, 1998; UP'TON-97 p. 379C

Enhanced Production of $\Lambda \Lambda$ Pairs near Threshold in the $\left(K^{-}, K^{+}\right)$Reaction on ${ }^{12} \mathrm{C}$ 
Ahner 97

Aid 95

Aid 95B

Aid $95 \mathrm{C}$

Aid 95D

Aid $95 \mathrm{E}$

Aid 95F

Aid 95G

Aid $95 \mathrm{H}$

Aid 95I

Aid 95J

Aid 96

Aid 96B

Aid $96 \mathrm{C}$

Aid 96D

Aid $96 \mathrm{E}$

Aid $96 \mathrm{~F}$

Aid $96 \mathrm{G}$

Aid $96 \mathrm{H}$

Aid 96I

Aid 96J

Aid $96 \mathrm{~K}$

Aid $96 \mathrm{~L}$

Aid $96 \mathrm{M}$

Aihara 95

Airapetyan 98

Aitala 95

Aitala 96

Aitala $96 \mathrm{~B}$

Aitala $96 \mathrm{C}$

Aitala $96 \mathrm{D}$

Aitala $96 \mathrm{E}$

Aitala $96 \mathrm{~F}$

Aitala 97

Aitala $97 \mathrm{~B}$

Aitala $97 \mathrm{C}$

Aitala $97 \mathrm{D}$

Phys. Lett. 393B:31, 1997

Pion and Kaon Emission from the Fireball Formed in Ne $+\mathrm{NaFl}$ Collisions at $1-2 \mathrm{GeV} / \mathrm{Nucleon}$

Nucl. Phys. B449:3, 1995; DESY-95-086

A Direct Determination of the Gluon Density in the Proton at Low $x$

Z. Phys. C67:565, 1995; DESY-95-102

Measurement of the $e^{+}$and $e^{-}$Induced Charged Current Cross Sections at HERA

Phys. Lett. 354B:494, 1995; DESY-95-081

The Gluon Density of the Proton at Low $x$ from a QCD Analysis of $F_{2}$

Phys. Lett. 356B:118, 1995; DESY-95-108

Transverse Energy and Forward Jet Production in the Low $x$ Regime at HERA

Nucl. Phys. B445:3, 1995; DESY-95-072

A Study of the Fragmentation of Quarks in $e^{-} p$ Collisions at HERA

Z. Phys. C69:27, 1995; DESY-95-162

Measurement of the Total Photon Proton Cross Section and Its Decomposition at $200 \mathrm{GeV}$ Center of Mass Energy

Phys. Lett. 353B:578, 1995; DESY-95-079

Leptoquarks and Compositeness Scales from a Contact Interaction Analysis of Deep Inelastic $e^{ \pm} p$ Scattering at HERA

Phys. Lett. 358B:412, 1995; DESY-95-156

Comparison of Deep Inelastic Scattering with Photoproduction Interactions at HERA

Z. Phys. C70:17, 1996; DESY-95-219

Jets and Energy Flow in Photon-Proton Collisions at HERA

Phys. Lett. 369B:173, 1996; DESY-95-233

A Search for Leptoquarks at HERA

Nucl. Phys. B463:3, 1996; DESY-95-251; HEPEX-9601004

Elastic Photoproduction of $\rho^{0}$ Mesons at HERA

Z. Phys. C70:609, 1996; DESY-96-014

Energy Flow in the Hadronic Final State of Diffractive and Non-Diffractive Deep-Inelastic Scattering at HERA

Nucl. Phys. B468:3, 1996; Nucl. Phys. B548:639, 1999; DESY-96-023; HEPEX-9602007

Elastic Electroproduction of $\rho$ and $J / \psi(1 S)$ Mesons at Large $Q^{2}$ at HERA

Nucl. Phys. B472:32, 1996; DESY-96-055

Photoproduction of $D^{*}(2010)^{\ddagger}$ Mesons in Electron Proton Collisions at HERA

Phys. Lett. 380B:461, 1996; DESY-96-082; HEPEX-9605002

A Search for Selectrons and Squarks at HERA

Z. Phys. C71:211, 1996; DESY-96-056

A Search for Squarks of $R p$-Violating SUSY at HERA

Nucl. Phys. B483:44, 1997; DESY-96-163; HEPEX-96090008

Search for Excited Fermions with the H1 Detector

Nucl. Phys. B480:3, 1996; DESY-96-122

Strangeness Production in Deep Inelastic Positron-Proton Scattering at HERA

Z. Phys. C72:573, 1996; DESY-96-160

Charged Particle Multiplicities in Deep Inelastic Scattering at HERA

Phys. Lett. 392B:234, 1997; DESY-96-224; HEPEX-9611004

Scale Influence on the Energy Dependence of Photon - Proton Cross Sections

Nucl. Phys. B472:3, 1996; DESY-96-037

Elastic and Inelastic Photoproduction of $J / \psi(1 S)$ Mesons at HERA

Phys. Lett. 379B:319, 1996; DESY-96-046; HEPEX-9603009

Measurement of the $Q^{2}$ Dependence of the Charged and Neutral Current Cross Sections in $e^{ \pm} p$ Scattering at HERA

Nucl. Phys. B470:3, 1996; DESY-96-039

A Measurement and $\mathrm{QCD}$ analysis of the proton structure function $\mathrm{F}_{2}\left(x, q^{2}\right)$ at $\mathrm{HERA}$

LBL-37244

$W^{ \pm} \gamma$ and $Z^{0} \gamma$ Production at Tevatron

Phys. Lett. 442B:484, 1998; DESY-98-072; HEPEX-9807015

Measurement of the Proton Spin Structure Function $g_{1}^{p}$ with a Pure Hydrogen Target

Phys. Rev. Lett. 76:364, 1996; FERMILA B-PUB-95-142-E; KSU-HEP-95-01

Search for the Flavor Changing Neutral Current Decays $D^{+} \rightarrow \pi^{+} \mu^{+} \mu^{-}$and $D^{+} \rightarrow \pi^{+} e^{+} e^{-}$

Phys. Lett. 371B:157, 1996; FERMILAB-PUB-96-001-E; HEPEX-9601001

Asymmetries between the Production of $D^{+}$and $D^{-}$Mesons from $500 \mathrm{GeV} / c \pi^{-} \mathrm{Nucleon}$ Interactions as Function of $x_{F}$ and $p_{T}^{2}$

Phys. Lett. 379B:292, 1996; FERMILAB-PUB-96-076-E; HEPEX-9604007

Mass Splitting and Production of $\Sigma_{c}(2455)^{\circ}$ and $\Sigma_{c}(2455)^{++}$Measured in $500 \mathrm{GeV} \pi^{-}$nucleon Interactions

Phys. Lett. 403B:185, 1997; FERMILAB-PUB-96-206-E

Observation of $D-\pi$ Production Correlations in $500 \mathrm{GeV} \pi^{-}$nucleon Interactions

Phys. Lett. 397B:325, 1997; FERMILAB-PUB-96-399-E; HEPEX-9611002

Measurement of the Branching Ratio $B\left(D^{+} \rightarrow \rho^{\circ} \ell^{+} \nu\right) / B\left(D^{+} \rightarrow K^{*}(892)^{\circ} \ell^{+} \nu\right)$

Phys. Rev. Lett. 77:2384, 1996; FERMILAB-PUB-96-109.E; KSU-HEP-96-01; HEPEX-9606016

Search for $D^{0} \bar{D}^{0}$ Mixing in Semileptonic Decay Modes

Phys. Lett. 403B:377, 1997; FERMILAB-PUB-96-442-E; USCHEP-9602; HEPEX-9612005 Search for $C P$ Violation in Charged $D$ Meson Decays

Phys. Lett. 404B:187, 1997; FERMILAB-PUB-97-053-E; CBFR-NF-013-97; HEPEX-9706025

The Doubly Cabibbo-Suppressed Decay $D^{+} \rightarrow K^{+} \pi^{-} \pi^{+}$

Phys. Lett. $411 \mathrm{~B}: 230,1997$; FERMILAB-PUB-97-105-E; HEPEX-9708040

Asymmetries between the Production of $D_{s}^{-}$and $D_{s}^{+}$Mesons from $500 \mathrm{GeV} / \mathrm{c} \pi^{-} \mathrm{Nucleon}$ Interactions as Functions of $x_{F}$ and square of $p_{t}^{2}$

Phys. Rev. Lett. 81:44, 1998; FERMILAB-PUB-97-118-E; HEPEX-9709013

Search for the Pentaquark via the $P_{\bar{c} s}^{0} \rightarrow \phi(1020) \pi p$ Decay

Phys. Rev. Lett. 80:1393, 1998; FERMILAB-PUB-97-267

Measurement of the Form-Factor Ratios for $D^{+} \rightarrow \bar{K}^{*}(892)^{0} e^{+} \nu_{e}$ 
Aitala 97E

Aitala 97F

Aitala 98

Aitala 98B

Aitala $98 \mathrm{C}$

Aitala 98D

Aitala $98 \mathrm{E}$

Ajaka 98

Ajimura 94

Ajimura 98

Ajinenko 94

Ajitanand 98

Akagi 94

Akerlof 97

Akers 93D

Akers 93F

Akers 93G

Akers $93 \mathrm{H}$

Akers 93J

Akers 94

Akers 94B

Akers $94 \mathrm{C}$

Akers 94D

Akers $94 \mathrm{E}$

Akers $94 \mathrm{~F}$

Akers $94 \mathrm{G}$

Akers $94 \mathrm{H}$

Akers 94I

Akers 94J

Akers $94 \mathrm{~K}$

Akers 94L

Akers $94 \mathrm{M}$

A kers $94 \mathrm{~N}$

Akers 940

Akers $94 \mathrm{P}$

Akers $94 \mathrm{Q}$

Akers 94R

A kers 94S
Phys. Lett. 421B:405, 1998; FERMILAB-PUB-97-345-E; HEPEX-9711003

Branching Fractions for $D^{0} \rightarrow K^{+} K^{-}$and $D^{0} \rightarrow \pi^{+} \pi^{-}$, and a Search for $C P$ Violation in $D^{0}$ Decays

Phys. Lett. 423B:185, 1998; FERMILAB-PUB-97-373-E

Study of the Decay $D^{0} \rightarrow K^{-} K^{+} \pi^{-} \pi^{+}$

Phys. Lett. 445B:449, 1999; FERMILAB-PUB-98-320-E; USCHEP-9801; HEPEX-9811016

Measurement of the $D_{s}^{ \pm}$Lifetime

Phys. Lett. 450B:294, 1999; KSU-HEP-98-02; FERMILAB-PUB-98-330-E; HEPEX-9812013

Measurement of the Form-Factor Ratios for $D_{s}^{+} \rightarrow \phi(1020) \ell^{+} \nu_{l}$

Phys. Lett. 440B:435, 1998; KSU-HEP-98-001; FERMILAB-PUB-98-289-E

Measurement of the Form-Factor Ratios for $D^{+} \rightarrow \bar{K}^{*}(882)^{\circ} \ell^{+} \nu$

Phys. Rev. D57:13, 1998; FERMILAB-PUB-96-214-E; HEPEX-9608018

$A$ Search for $D^{0}-\bar{D}^{\circ}$ Mixing and Doubly Cabibbo Suppressed Decays of the $D^{0}$ in Hadronic Final States FERMILAB-PUB-98-297-E; HEPEX-9809029

Correlations between $D$ and $\bar{D}$ Mesons Produced in $500 \mathrm{GeV} / \mathrm{c} \pi^{-}$nucleon Interactions

Phys. Rev. Lett. 81:1797, 1998

New Measurement of $\Sigma$ Beam Asymmetry for $\eta$ Meson Photoproduction on the Proton

Nucl. Phys. A577:271C, 1994

A Hypernuclei by Quasifree $\left(\pi^{+}, K^{+}\right)$Reaction on ${ }^{12} \mathrm{C}$

Phys. Rev. Lett. 80:3471, 1998; KEK-97-226

Polarization of ${ }_{0}^{5} \mathrm{He}$ Produced by the $\left(\pi^{+}, K^{+}\right)$Reaction

Z. Phys. C61:567, 1994

Invariant Mass Dependence of Particle Correlations in $\pi^{+} p$ and $K^{+} p$ Interactions at $250 \mathrm{GeV} / \mathrm{c}$

Nucl. Phys. A638:451C, 1998; TSUKUBA-97 p. 451C

Collective Flow in Au + Au Collisions between $2-8 \mathrm{~A} \cdot \mathrm{GeV}$ at AGS

Phys. Rev. D51:2061, 1995; KEK-94-151

Experimental Study of the Rare Decays $K_{L} \rightarrow \mu^{-} e^{-}, K_{L} \rightarrow e^{-} e^{-}, K_{L} \rightarrow \mu^{-} \mu^{-}$and $K_{L} \rightarrow e^{-} e^{-} e^{-} e^{-}$

ASTROPH-9706123

A Search for Correlations of TeV Gamma Rays with Ultrahigh-Energy Cosmic Rays

2. Phys. C61:19, 1994; CERN-PPE-93-146

Improved Measurements of the Neutral Current from Hadron and Lepton Production at LEP

Z. Phys. C61:357, 1994; CERN-PPE-93-155

Measurement of $\Gamma\left(Z^{\circ} \rightarrow b \vec{b}\right) / \Gamma\left(Z^{\circ} \rightarrow\right.$ hadrons $)$ using Impact Parameters and Leptons

Z. Phys. C61:199, 1994; CERN-PPE-93-156

Measurement of the Photon Structure Function $F_{2}^{\gamma}$ in the Reaction $e^{+} e^{-} \rightarrow e^{+} e^{-}$hadron (hadrons) at

Z. Phys. C61:209, 1994; CERN-PPE-93-174

Studies of Charged Particle Multiplicity in $b$-Quark Events

Phys. Lett. 320B:417, 1994; CERN-PPE-93-183

Multiplicity and Transverse Momentum Correlations in Multihadronic Final States in $e^{+} e^{-}$Interactions at $\sqrt{s}=91.2 \mathrm{GeV}$

Phys. Lett. 328B:207, 1994; CERN-PPE-94-042

Measurement of the $\tau^{-} \rightarrow$ hadron ${ }^{-} \pi^{0} \nu_{\tau}$ and $\tau^{-} \rightarrow$ hadron ${ }^{-} 2 \pi^{0}\left(\pi^{0}, s\right) \nu_{\tau}$ Branching Ratios

Phys. Lett. 327B:411, 1994; CERN-PPE-94-043

Measurement of the Time Dependence of $B^{0} \leftrightarrow \bar{B}^{0}$ Mixing using a Jet Charge Technique

Z. Phys. C63:181, 1994; CERN-PPE-94-049

Measurement of the Production Rates of Charged Hadrons in $e^{+} e^{-}$Annihilation at the $Z^{\circ}$

Z. Phys. C63:197, 1994; CERN-PPE-94-51

QCD Studies using a Cone Based Jet Finding Algorithm for $e^{+} e^{-}$Collisions at LEP

Z. Phys. C63:363, 1994; CERN-PPE-94-52

A Study of Mean Sub-Jet Multiplicities in Two and Three Jet Hadronic $Z^{\circ}$ Decays

Phys. Lett. 327B:397, 1994; CERN-PPE-94-048

Search for the Minimal Standard Model Higgs Boson

Phys. Lett. 336B:585, 1994; CERN-PPE-94-090

Measurement of the Time Dependence of $B_{d}^{0} \leftrightarrow \bar{B}_{d}^{0}$ Mixing using Leptons and $D^{*}(2010)^{ \pm}$Mesons

Phys. Lett. 337B:196, 1994; CERN-PPE-94-91

Observation of Exclusive Decays of $B$ Mesons at LEP

Phys. Lett. 339B:278, 1994; CERN-PPE-94-108

Measurements of the Inclusive Branching Ratios of $\tau^{ \pm}$Leptons to $K_{S}$ and Charged $K^{*}(892)$

Phys. Lett. 337B:207, 1994; CERN-PPE-94-103

Search for a Scalar Top Quark using the OPAL Detector

Phys. Lett. 337B:393, 1994; CERN-PPE-94-98

Search for Rare Hadronic $B$ Decays

Phys. Lett. 338B:497, 1994; CERN-PPE-94-129

Updated Measurement of the $\tau^{ \pm}$Lifetime

Z. Phys. C65:47, 1995; CERN-PPE-94-105

Measurement of Single Photon Production in $e^{+} e^{-}$Collisions Near the $Z^{0}$ Resonance

Z. Phys. C65:17, 1995; CERN-PPE-94-106

Measurement of $\Gamma\left(Z^{0} \rightarrow b \bar{b}\right) / \Gamma\left(Z^{\circ} \rightarrow\right.$ Hadrons $)$ using a Double Tagging Method

Z. Phys. C65:183, 1995; CERN-PPE-94-107

Determination of an Upper Limit for the Mass of the $\nu_{\tau}$ at LEP

Z. Phys. C66:31, 1995; CERN-PPE-94-171

A Test of $C P$ Invariance in $Z^{\circ} \rightarrow \tau^{+} \tau^{-}$using Optimal Observables

Z. Phys. C64:1, 1994; CERN-PPE-94-104

Search for Neutral Higgs Bosons in the Minimal Supersymmetric Extension of the Standard Model

Z. Phys. C66:19, 1995; CERN-PPE-94-206

Observations of $\pi-B$ Charge-Flavor Correlations and Resonant $B \pi$ and $B K$ Production

Z. Phys. C67:27, 1995; CERN-PPE-94-217

A Measurement of the Production of $D^{*}(2010)^{ \pm}$Mesons on the $Z^{\circ}$ Resonance 
A kers 95

Akers 95B

Akers 95C

A kers 95D

Akers $95 \mathrm{E}$

A kers $95 \mathrm{~F}$

Akers $95 \mathrm{G}$

Akers $95 \mathrm{H}$

Akers 951

Akers 95J

Akers $95 \mathrm{~K}$

Akers 95L

Akers $95 \mathrm{M}$

A kers $95 \mathrm{~N}$

Akers 950

Akers $95 \mathrm{P}$

Akers $95 \mathrm{Q}$

Akers $95 \mathrm{R}$

Akers $95 \mathrm{~S}$

Akers $95 \mathrm{~T}$

Akers $95 \mathrm{U}$

A kers $95 \mathrm{~V}$

Akers $95 \mathrm{~W}$

Akers $95 \mathrm{X}$

Akesson 94

Akesson 96

Akhmadaliev 98

Akhmetshin 95

Akhmetshin 95B

Akhmetshin 97

Akhmetshin 97B

Akhmetshin $97 \mathrm{C}$

Akhmetshin 98

Akhobadze 97

Akhobadze 97B
Z. Phys. C65:367, 1995; CERN-PPE-94-135 A Measurement of the QCD Color Factor Ratios $C_{A} / C_{F}$ and $T_{F} / C_{F}$ from Angular Correlations in Four-Jet Events

Z. Phys. C65:1, 1995; CERN-PPE-94-120

Measurement of the Tau Lepton Polarization and Its Forward-Backward Asymmetry from $Z^{\circ}$ Decays

Z. Phys. C65:31, 1995; CERN-PPE-94-123

Determination of Event Shape Distributions and $\alpha_{S}(B)$ from $Z^{0} \rightarrow b \bar{b}$ Events at LEP

Z. Phys. C66:543, 1995; CERN-P PE-95-06

Measurement of the Leptonic Branching Ratios of the Tau Lepton

Z. Phys. C66:555, 1995; CERN-PPE-95-12

A Study of $B$ Meson Oscillations using Dilepton Events

Z. Phys. C67:57, 1995; CERN-PPE-95-02

A Study of Charm Meson Production in Semileptonic $B$ Decays

Z. Phys. C68:1, 1995; CERN-PPE-95-27

Inclusive Strange Vector and Tensor Meson Production in Hadronic $Z^{0}$ Decays

2. Phys. C67:203, 1995; CERN-P PE-95-21

Search for Heavy Charged Particles and for Particles with Anomalous Charge in $e^{+} e^{-}$Collisions at LEP

Phys. Lett. 352B:176, 1995; CERN-PPE-95-038

A Measurement of Charged Particle Multiplicity in $Z^{\circ} \rightarrow c \bar{c}$ and $Z^{\circ} \rightarrow b \bar{b}$ Events

Z. Phys. C67:389, 1995; CERN-PPE-95-024

The Production of Neutral Kaons in $Z^{0}$ Decays and their Bose-Einstein Correlations

Phys. Lett. 353B:595, 1995; CERN-PPE-95-058

Measurement of the Multiplicity of Charmed Quarks Pairs Produced from Gluon Splitting in Hadronic $Z^{\mathrm{O}}$ Decay

Z. Phys. C68:203, 1995; CERN-PPE-95-57

Measurement of the Longitudinal, Transverse and Asymmetry Fragmentation Functions at LEP

Z. Phys. C68:179, 1995; CERN-PPE-95-75

A Model Independent Measurement of Quark and Gluon Jet Properties and Differences

Z. Phys. C68:519, 1995; CERN-PPE-95-069

A Study of QCD Structure Constants and a Measurement of $\alpha_{s}\left(m_{z^{0}}\right)$ at LEP using Event Shape Observables

Z. Phys. C68:555, 1995; CERN-PPE-95-070

Measurement of the $\tau^{-} \rightarrow$ 2hadron hadron $^{+} \nu_{\tau}$ and $\tau^{-} \rightarrow$ 2hadron ${ }^{-}$hadron ${ }^{+} \pi^{0}$ ( $\pi^{0}$ 's) $\nu_{\tau}$ Branching Ratios

Phys. Lett. 350B:273, 1995; CERN-PPE-95-020

An Improved Measurement of the $B_{s}$ Lifetime

Z. Phys. C67:15, 1995; CERN-PPE-95-13

Comparisons of the Properties of Final State Photons in Hadronic Z Decays with Predictions from Matrix

2. Element Calculations

Improved Measurements of the $B^{\circ}$ and $B^{+}$Meson Lifetimes

Z. Phys. C67:45, 1995; CERN-PPE-95-22

Measurement of the Hadronic Decay Current in $\tau^{-} \rightarrow 2 \pi^{-} \pi^{+}{ }_{\nu \tau}$

2. Phys. C67:555, 1995; CERN-PPE-95-43

A Search for Lepton Flavor Violating $Z$ Decays

Phys. Lett. 353B:402, 1995; CERN-PPE-95-51

A Measurement of the $\Lambda_{b}$ Lifetime

Z. Phys. C68:531, 1995; CERN-PPE-95-083

Investigation of the String Effect using Final State Photons

Z. Phys. C69:195, 1995; CERN-PPE-95-90

Measurement of the Average $b$-Baryon Lifetime and the Product Branching Ratio $f\left(b \rightarrow \Lambda_{b}\right) \cdot \operatorname{Br}\left(\Lambda_{b} \rightarrow\right.$ $\left.\Lambda \ell^{-} \nu \mathrm{X}\right)$

Z. Phys. C67:365, 1995; CERN-PPE-95-50

A Measurement of the Forward-Backward Asymmetry of $e^{+} e^{-} \rightarrow b \vec{b}$ by Applying a Jet Charge Algorithm to Lifetime Tagged Events

Z. Phys. C68:47, 1995; CERN-PPE-94-140

Low-Mass Lepton-Pair Production in $p$-Be Collisions at $450 \mathrm{GeV} / c$

Z. Phys. C72:429, 1996; CERN-PPE-96-23

A Study of Electron-Muon Pair Production in $450 \mathrm{GeV} / \mathrm{c} p$ Be Collisions

Phys. Rev. C58:2844, 1998; BUDKERINP-98-51; HEPEX-9806037

Delbrück Scattering at Energies $140-450 \mathrm{MeV}$

Phys. Lett. 364 B:199, 1995; BUDKERIN P-95-35

Measurement of $\phi(1020)$-Meson Parameters with CMD-2 Detector at VEPP-2M Collider

BUDKERINP-95-62

Recent Results of the $\phi$-Meson Study with CMD-2 at VEPP-2M and Relevance to Future $C P, C P T \phi$ Factory Studies

Phys. Lett. 398B:423, 1997; BUDKERINP-96-93; HEPEX-9702006

Study of $K_{S} K_{L}$ Coupled Decays and $K_{L}$ Be Interactions with the CMD-2 Detector at VEPP-2M Collider

Phys. Lett. 415B:452, 1997; HEPEX-9711007

Search for Rare $\phi(1020)$ Decays in $\pi^{+} \pi^{-} \gamma$ Final State

Phys. Lett. 415B:445, 1997

First Observation of the Decay $\phi(1020) \rightarrow \eta^{\prime} \gamma$

Phys. Lett. 434B:426, 1998; BUDKERINP-98-30

Study of Dynamics of $\phi(1020) \rightarrow \pi^{+} \pi^{-} \pi^{0}$ Decay with CMD-2 Detector

Yad. Phys. 60:1271, 1997; Phys. At. Nucl. 60:1145, 1997

The Associative Multiplicity of Negative Particles in Events with Production of Neutral Strange Particles in $\pi^{-}$nucleus Interaction at the Momentum $40 \mathrm{GeV} / c$ with Trigger Particle $\left|p_{t}\right| \geq 1.1 \mathrm{GeV} / c$

Phys. At. Nucl. 60:53, 1997; Yad. Phys. 60:60, 1997

Strange Neutral Particle Production in the $\pi^{-} A$ Interactions at $40 \mathrm{GeV} / c$ in Events with $p_{t} \geq 1.1 \mathrm{GeV} / c$ Trigger Particle 
Akhobadze 98

Akiba 96

Akkus 98

Alam 94B

Alam 94C

Alam 95

Alam 97

Alam 97B

Alam 98

Alanakyan 97

Alanakyan 98

Alaviharati 99

Albaaj 94

Albajar 93B

Albajar 95

Alber 94

Alber 95

Alber 95C

Alber 95D

Alber 96

Alber 97

Alberico 98

Alberico 98B

Albers 97

Albert 95

Albrecht 93D

Albrecht 94B

Albrecht 94C

Albrecht 94D

Albrecht 94E

Albrecht 94F

Albrecht 94G

Albrecht $94 \mathrm{H}$

Albrecht 94I

Albrecht 94J

Albrecht $94 \mathrm{~K}$

Albrecht $94 \mathrm{~L}$

Albrecht 94M
Phys. At. Nucl. 61:196, 1998; Yad. Phys. 61:245, 1998; JINR-E1-96-451 Investigation of the Dynamics of Hadron-Nucleus Interactions at $40 \mathrm{GeV}$ Utilizing the Characteristics of Neutral Strange Particles

Phys. Rev. Lett. 76:2021, 1996

Production of $\phi(1020)$ Mesons in Central ${ }^{28} \mathrm{Si}+{ }^{108} \mathrm{Au}$ Collisions at $14.6 \mathrm{~A} \cdot \mathrm{GeV} / \mathrm{c}$

Phys. Lett. 434B:200, 1998; CERN-EP-98-084 Experimental Search for Muonic Photons

Phys. Rev. Lett. 74:2885, 1995; CLNS-94-1314; CLEO-94-25 First Measurement of the Rate for the Inclusive Radiative Penguin Decay $b \rightarrow s \gamma$

Phys. Rev. D50:43, 1994; CLNS-94-1270; CLEO-94-5 Exclusive Hadronic $B$ Decays to Charm and Charmonium Final States

Phys. Rev. Lett. 76:2637, 1996; CLNS-95-1370; CLEO-95-19

Tau Decays into Three Charged Leptons and Two Neutrinos

Phys. Rev. D56:17, 1997; CLNS-96-1452; CLEO-96-22; HEPEX-9701006

Study of Gluon versus Quark Fragmentation in $\Upsilon(1 S) \rightarrow$ gluon gluon $\gamma$ and $e^{+} e^{-} \rightarrow q \bar{q} \gamma$ Events at $\sqrt{s}=$ $10 \mathrm{GeV}$

Phys. Rev. D57:4467, 1998; CLNS-97-1502; CLEO-97-18; HEPEX-9709012

Measurement of the Branching Fractions of $\Lambda_{c}^{+} \rightarrow p \bar{K}$ pions

Phys. Rev. Lett. 81:3328, 1998; CLNS-98-1560; CLEO-98-8; HEPEX-9805033

Further Search for the Two-Photon Production of the Glueball Candidate $f_{J}(2220)$

Phys. At. Nucl. 60:1069, 1997; Yad. Phys. 60:1194, 1997

Effects of Nuclear Matter on Electron-Nucleon Interaction in the Reaction ${ }^{12} \mathrm{C}\left(e^{-}, e^{-1} p\right)$

Phys. At. Nucl. 61:207, 1998; Yad. Phys. 61:256, 1998 Reaction ${ }^{12} \mathrm{C}\left(e^{-}, e^{-1} p\right)$ with Cumulative Proton

HEPEX-9902029

Measurement of the Decay $K_{L} \rightarrow \pi^{0} \gamma \gamma$

Nuovo Cim. 107A:1611, 1994

Particle Production in He Li Collisions at $4.5 \mathrm{~A} \mathrm{GeV} / \mathrm{c}$

2. Phys. C61:41, 1994; CERN-PPE-93-153

Measurement of $B^{0}-\bar{B}^{0}$ Correlations at the CERN $S p \bar{p} S$ Collider

Phys. Lett. 369B:46, 1996; CERN-PPE-95-158

Measurement of $\alpha_{S}$ from $B \bar{B}$ Production at the CERN $p \bar{p}$ Collider

Z. Phys. C64:195, 1994

Strange Particle Production in Nuclear Collisions at $200 \mathrm{GeV}$ per Nucleon

Nucl. Phys. A590:453C, 1995

Two-Pion Interferometry in Central Nucleus-Nucleus Collisions at the CERN SPS - Results from Experiments NA35 and NA49

Phys. Rev. Lett. 74:1303, 1995; LBL-36062

Transverse Momentum Dependence of Bose-Einstein Correlations in $200 \mathrm{~A} \mathrm{GeV} / \mathrm{c} \mathrm{S}+\mathrm{A}$ collisions

Phys. Rev. Lett. 75:3814, 1995

Transverse Energy Production in ${ }^{208} \mathrm{~Pb} \mathrm{~Pb}$ Collisions at $158 \mathrm{GeV}$ per Nucleon

Phys. Lett. 366 B:56, 1996 Antibaryon Production in Sulphur-Nucleus Collisions at $200 \mathrm{GeV}$ per Nucleon

Eur. Phys. J. C2:643, 1998; IKF-HENPG-6-94; HEPEX-9711001

Charged Particle Production in Proton-, Deuteron-, Oxygen- and Sulphur Nucleus Collisions at $200 \mathrm{GeV}$ per Nucleon

Phys. Lett. 432B:427, 1998; HEPEX-9806017 Measurements of the Reaction $\bar{p} p \rightarrow \phi(1020) \eta$ of Antiproton Annihilation at Rest at Three Hydrogen Target Densities

Phys. Lett. 438B:430, 1998

Study of $\phi(1020)$ and $f_{2}(1565)$ Meson Production in $\bar{p} p$ Annihilation at Rest

Phys. Rev. Lett. 78:1652, 1997

Proton-Proton Elastic Scattering Excitation Functions at Intermediate Energies

Phys. Rev. C51:R1065, 1995; NUCLTH-9410039 Measurement of the Reaction ${ }^{12} \mathrm{C}\left(\nu_{\mu}, \mu^{-}\right) \mathrm{X}$ Near Threshold

Phys. Lett. 324B:249, 1994; DESY-93-149 A Study of $\bar{B}^{\circ} \rightarrow D^{*}(\mathbf{2 0 1 0})^{+} \ell^{-} \bar{\nu}$ and $B^{\circ} \bar{B}^{0}$ Mixing using Partial $D^{*}(2010)^{+}$Reconstruction

Phys. Lett. 332B:451, 1994; DESY-94-029 Observation of $\gamma \gamma \rightarrow \phi(1020) \rho^{0}$ and $\gamma \gamma \rightarrow \phi(1020) \omega$

Nucl. Phys. A566:61C, 1994

Single Photon and Neutral Meson Data from WA80

Nucl. Phys. A566:355C, 1994

Comparison of $\pi^{\circ}$ and $\eta$ Spectra from $\mathrm{S}+\mathrm{Au}$ Collisions at $200 \mathrm{GeV} / \mathrm{c}$

Nucl. Phys. A566:519C, 1994

Effective Source Sizes of Low Rapidity Soft Particle Emission

Phys. Lett. 326B:320, 1994; DESY-94-004

Observation of Polarization Effects in $\Lambda_{c}^{+}$Semileptonic Decay

Z. Phys. C61:1, 1994; DESY-93-084

Inclusive Production of $K^{*}(\mathbf{8 9 2}), \rho^{0}$, and $\omega$ Mesons in the $\Upsilon$ Energy Region

Phys. Rev. C50:1048, 1994; LBL-35214

Intermittency and Correlations in $200 \mathrm{GeV} /$ Nucleon $\mathrm{S}+\mathrm{S}$ and $\mathrm{S}+\mathrm{Au}$ Collisions

Phys. Lett. 335 B:526, 1994; DESY-94-069

Reconstruction of the Decay $B^{-} \rightarrow D_{1}(0)(2414) \pi^{-}$

Z. Phys. C64:375, 1994; DESY-94-052

Study of $D^{\mathbf{0}}$ and $D^{+}$Decays into Final States with Two or Three Kaons

Phys. Lett. 341B:441, 1994; DESY-94-100

The First Measurement of the Michel Parameter $\eta$ in $\tau^{ \pm}$Decays

Phys. Lett. 340B:125, 1994; DESY-94-094

Measurement of the Absolute Branching Fractions for $D^{\circ}$ Decays into $K^{-} \pi^{+}, K^{-} \pi^{+} \pi^{+} \pi^{-}, \bar{K}^{0} \pi^{+} \pi^{-}$

Phys. Lett. 338B:390, 1994; DESY-94-110

Determination of the Radiative Decay Width of the $\eta_{c}(1 S)$ Meson 
Albrecht $94 \mathrm{~N}$

Albrecht $94 \mathrm{O}$

Albrecht 94P

Albrecht 94Q

Albrecht 94R

Albrecht $94 \mathrm{~S}$

Albrecht $94 \mathrm{U}$

Albrecht 95

Albrecht $95 \mathrm{~B}$

Albrecht 95C

Albrecht 95D

Albrecht $95 \mathrm{E}$

Albrecht $95 \mathrm{~F}$

Albrecht 95G

Albrecht $95 \mathrm{H}$

Albrecht 95I

Albrecht 96

Albrecht 96B

Albrecht 97

Albrecht 97B

Albrecht 98

Albrow 96

Albuquerque 94

Albuquerque 96

Alcaraz 95

Alcock 95

Alde 93

Alde 94

Alde $94 \mathrm{~B}$

Alde $94 \mathrm{C}$

Alde 95

Alde 95B

Alde 96

Alde 97

Alde 98

Aleev 93C

Aleev $96 \mathrm{~B}$

Aleev $96 \mathrm{C}$
Z. Phys. C66:63, 1995; DESY-94-111

Measurement of the Decay Fractions of $D^{*}$ Mesons

Z. Phys. C65:619, 1995; DESY-94-121

A Measurement of the Electronic Widths $\Gamma_{e e}$ of the $\Upsilon(1 S), \Upsilon(2 S)$, and $\Upsilon(4 S)$ Resonances, and of the Total Decay Width $\Gamma$ of the $\Upsilon(4 S)$

Phys. Lett. 340B:217, 1994; -DESY-94-139

Measurement of the Polarization in the Decay $B \rightarrow J / \psi(1 S) K^{*}(892)$

Phys. Lett. 337 B:383, 1994; DESY-94-120

Determination of the Structure of $\tau^{ \pm}$Decays in the Reaction $e^{+} e^{-} \rightarrow \tau^{+} \tau^{-} \rightarrow \rho^{+} \bar{\nu}_{\tau} \rho^{-} \nu_{\tau}$ and a Precision Measurement of the $\nu_{\tau}$ Helicity

Z. Phys. C62:371, 1994; DESY-93-052

Kaons in Flavor Tagged $B$ Decays

Phys. Lett. 342B:397, 1995; DESY-94-177

Evidence for $W^{ \pm}$Exchange in Charmed Baryon Decays

Phys. Lett. 353B:554, 1995; DESY-94-246

Search for Rare $B$ Decays

Phys. Lett. 349B:576, 1995; DESY-95-011

Determination of the Michel Parameters $\xi$ and $\delta$ in Leptonic $\tau^{ \pm}$Decays

2. Phys. C68:25, 1995; DESY-95-071

A Search for Lepton Flavor Violating Decays $\tau \rightarrow e \alpha, \tau \rightarrow \mu \alpha$

Z. Phys. C68:215, 1995; DESY-95-087

$\tau^{ \pm}$Decays into $K^{*}(802)$ Mesons

Z. Phys. C69:405, 1996; DESY-95-129

Measurement of the Decay $D_{s, 5}(2573)^{+} \rightarrow D^{0} K^{+}$

Phys. Lett. 374B:249, 1996; DESY-95-187

Measurement of the Semileptonic Branching Fractions of the $D^{\circ}$ Meson

Phys. Lett. 361 B:14, 1995; HEPEX-9507009

Production of $\eta$ Mesons in $200 \mathrm{~A} \mathrm{GeV} / \mathrm{c} \mathrm{S}+\mathrm{S}$ and $\mathrm{S}+\mathrm{Au}$ Reactions

Phys. Lett. 374B:265, 1996; DESY-95-227

Two Photon Production of $\omega$ Pairs

Phys. Lett. 374B:256, 1996; DESY-95-223

Two Measurements of $B^{0}-\bar{B}^{0}$ Mixing using Kaon Tagging

Nucl. Phys. A590:81C, 1995

Search for Direct Photon Production in 200 A GeV S Au Reactions: A Status Report

Phys. Rev. Lett. 76:3506, 1996; CERN-PPE-95-186

Limits on the Production of Direct Photons in $200 \mathrm{~A} \mathrm{GeV}{ }^{32} \mathrm{~S}+\mathrm{Au}$ Collisions

Z. Phys. C74:469, 1997; DESY-96-112

A Partial Wave Analysis of the Reaction $\gamma \gamma \rightarrow \pi^{+} \pi^{-} \pi^{0}$

Phys. Lett. 402B:207, 1997; DESY-97-008

Evidence for $\Lambda_{c}(\mathbf{2 5 9 3})+$ Production

Phys. Lett. 431B:179, 1998; DESY-97-194; HEPEX-9711022

Determination of the Michel Parameters $\rho, \xi$, and $\delta$ in $\tau^{ \pm}$-Lepton Decays with $\tau^{ \pm} \rightarrow \rho \nu$ Tags

Eur. Phys. J. C5:255, 1998; IKP-MS-980501; NUCLEX-9805007

Transverse Momentum Distributions of Neutral Pions from Nuclear Collisions at $200 \mathrm{~A} \mathrm{GeV}$

FERMILAB-CONF-96-138-E

Diffractive Production of Massive States

Phys. Rev. D50:R18, 1994; IFUSP-P-1104

New Upper Limit for the Branching Ratio of the $\Omega^{-} \rightarrow \Xi^{-} \gamma$ Radiative Decay

Phys. Rev. Lett. 78:3252, 1997; FERMILAB-PUB-96-047-E

A Search for Light Supersymmetric Baryons

Nucl. Phys. (PS) B40:237, 1995

Tau Lepton Branching Fractions from L3

Phys. Rev. Lett. 74:2867, 1995; ASTROPH-9501091

Experimental Limits on the Dark Matter Halo of the Galaxy from Gravitational Microlensing

2. Phys. C61:35, 1994; Phys. At. Nucl. 56:1229, 1993; Yad. Phys. 56-9:137, 1993; IFVE-93-29; CERN-PPE-93-143 Model-Independent Measurement of $\omega \rightarrow \eta \gamma$ Decay Branching Ratio

Phys. Lett. 340B:122, 1994; CERN-PPE-94-149 Observation of the $\omega \rightarrow \pi^{0} \pi^{0} \gamma$ Decay

2. Phys. C66:375, 1995; CERN-PPE-94-157

Study of the $f_{0}(995)$ Resonance in the $\pi^{0} \pi^{0}$ Decay Channel

2. Phys. C66:379, 1995; Nuovo Cim. 107A:1867, 1994; CERN-PPE-94-158 Partial-Wave Analysis of the $\omega \pi^{0}$ System at High Energies

Phys. At. Nucl. 59:982, 1996; Yad. Phys. 59:1027, 1996; IFVE-95-93

Observation of $a_{4}(2040)^{b}$-Meson in the $\eta \pi^{0}$ Decay Channel

MANCHESTER-95 p. 448; KEK-95-160

Study of the $\eta \pi^{\circ}$ System in the $p$ p Central Collision Reaction at $450 \mathrm{GeV} / \mathrm{c}$

Phys. At. Nucl. 60:386, 1997; Yad. Phys. 60:459, 1997; IFVE-96-39 Spin-Parity Analysis of $\eta \pi^{0} \pi^{0}$-System in $\pi^{-} p$ Charge Exchange Reaction at $100 \mathrm{GeV} / \mathrm{c}$ by Zemach Method

Phys. Lett. 397B:350, 1997; KEK-96-174

Study of the $\pi^{\circ} \pi^{\circ}$ System in $p$ Central Collisions at $450 \mathrm{GeV} / \mathrm{c}$

Eur. Phys. J. A3:361, 1998; IFVE-98-23

Study of the $\pi^{\circ} \pi^{\circ}$ System with the GAMS-4000 Spectrometer at $100 \mathrm{GeV} / c$

Phys. At. Nucl. 57:1370, 1994; Yad. Phys. 57:1443, 1994; JINR-P1-93-26 Measurements of Relative Branching Rations of Charm Particles $\Lambda_{c}^{+}$and $D^{-}$

JINR-P1-96-437 Investigation of $\phi(1020)$-Meson Inclusive Production by Neutrons on the Serpukhov Accelerator

Kr. Soob. JINR $77: 31,1996$ Observation $\Sigma_{c}(2455)^{\circ}$ Charmed Baryon in the Experiment EXCHARM 
Alekseev 95

Alekseev 97

Alekseev 99

Alemany 94

Alemany 97

Aleshin 94

Aleshin 94B

Aleshin 95B

Aleshin 96

Aleshin 96B

Aleshin 96C

Aleshin 97

Alessandrell 94

Alessandrell 96

Alessandrell 98

Alexa 98

Alexander 94

Alexander 94B

Alexander 95

Alexander 95B

Alexander $95 \mathrm{C}$

Alexander 95D

Alexander $95 \mathrm{E}$

Alexander 95F

Alexander 95G

Alexander $95 \mathrm{H}$

Alexander 95I

Alexander 96

Alexander 96B

Alexander $96 \mathrm{C}$

Alexander 96D

Alexander $96 \mathrm{E}$

Alexander 96F

Alexander 96G

Alexander $96 \mathrm{H}$

Alexander 96I

Alexander $96 \mathrm{~J}$
Phys, Lett. 351B:585, 1995

Measurement of the Spin Rotation Parameter $A_{+}$in the Elastic Scattering of Positive Pions on a Longitudinally Polarized Proton Target in the Second Resonance Region

Yad. Phys. 61:223, 1998; Phys. At. Nucl. 61:174, 1998; ITEP-97-19; HEPPH-9802396

The Pion Production in $\pi^{-} p \rightarrow \pi^{-} \pi^{+} n$ on the Polarized Proton Target at $1.78 \mathrm{GeV} / \mathrm{c}$

ITEP-7.99

Measurement of the Carbon Analyzing Power for the Momenta Range $1.35-2.02 \mathrm{GeV} / \mathrm{c}$

GLASGOW-94, v. 2, p. 1081; LAL-94-68

Tau Decays into Kaons

Nucl. Phys. (PS) B55:341, 1997; ESTES PARK-96 p. 341

$\tau$ Vector Spectral Functions and the Hadronic Contributions to $(g-2)_{\mu}$ and $\alpha\left(M_{Z}^{2}\right)$

ITEP-94-18

The Existence of Exotic States in Decays of Heavy Baryon Produced in the Reaction $\pi^{-} p \rightarrow^{n} 2 \pi^{-} 2 \pi^{+}$ at $4.35 \mathrm{GeV} / \mathrm{c}$ Momentum

Nucl. Phys. A568:809, 1994; DAPNIA-SPHN-93-03

Study of the Deuteron Structure in Quasi-Elastic Breakup Reaction $p$ deuteron $\rightarrow p p n$ at 1 GeV

Zh. Eksp. Teor. Fiz. 108:1899, 1995

Investigation of the Neutral Strange Particle Production in the Neutrino-Nucleus Interactions at SKIF Spectrometer at the Energies of $3-30 \mathrm{GeV}$

ITEP-96-23

The Search for Neutrino Interactions at MAS-1 setup

Zh. Eksp. Teor. Fiz. 110:385, 1996

Investigation of Inclusive Characteristics of Neutrino Nucleus Scattering on Photoemulsion Nulcei at Energy $E_{\nu}=3-30 \mathrm{GeV}$ (Experiment E-128)

Zh. Eksp. Teor. Fiz. 110:391, 1996

Investigation of Average Multiplicities of Hadronic Final State in the Neutrino Nucleus Interactions at $\left\langle E_{\nu}\right\rangle=8.7 \mathrm{GeV}$ (Experiment E-128)

ITEP-97-10

Analysis of Neutrino Events from WA-95 Experiment with Nuclear Photoemulsion, Prepared by ITEP

Phys. Lett. 335B:519, 1994

A New Search for Neutrinoless $\beta \beta$-Decay with Thermal Detector

Phys. Lett. 384B:316, 1996

Preliminary Results on the Performance of a $\mathrm{TeO}_{2}$ Thermal Detector in a Search for Direct Interactions

of WIMPs
Phys. Lett. $433 \mathrm{~B}: 156,1998$

Preliminary Results on Double Beta Decay of ${ }^{130}$ Te with an Array of Twenty Cryogenic Detectors

Phys. Rev. Lett. 82:1374, 1999; NUCLEX-9812002

Large Momentum Transfer Measurements of the Deuteron Elastic Structure Function $A\left(Q^{2}\right)$ at Jefferson Laboratory

Phys. Rev. Lett. 75:4155, 1995; Phys. Rev. Lett. 74:3113, 1995; CLNS-94-1288; CLEO-94-14

First Observation of $\Xi_{c}^{+} \rightarrow \Xi^{0} e^{+} \nu_{e}$ and a Measurement of the $\Xi_{c}^{+} / \Xi_{c}^{0}$ Lifetime Ratio

Phys. Lett. 341B:435, 1994; Phys. Lett. 347B:469, 1995; CLNS-94-1291; CLEO-94-18

Observation of bottom $\rightarrow J / \psi(1 S) \pi$ Decays

Phys. Rev. D53:1013, 1996; CLNS-95-1343; CLEO-95-9; HEPEX-9508005

Observation of the Cabibbo Suppressed Charmed Baryon Decay $\Lambda_{c}^{+} \rightarrow p \phi(1020)$

Phys. Lett. 358B:162, 1995; CERN-PPE-95-99

$\Delta\left(1232 P_{33}\right)^{++}$Production in Hadronic $Z^{0}$ Decays

Phys. Lett. 364B:93, 1995; CERN-PPE-95-122

A Study of $b$ Quark Fragmentation into $B^{\circ}$ and $B^{+}$Mesons at LEP

Z. Phys. C69:543, 1996; CERN-PPE-95-126

A Comparison of $b$ and $u d s$ Quark Jets to Gluon Jets

Z. Phys. C70:197, 1996; CERN-PPE-95-153

$J / \psi(1 S)$ and $\psi(2 S)$ Production in Hadronic $Z^{\circ}$ Decays

Phys. Lett. 368B:244, 1996; CERN-PPE-95-155

Test of the Exponential Decay Law at Short Decay Times using Tau Leptons

Phys. Lett. 369B:163, 1996; CERN-PPE-95-142

Measurement of the $\tau^{-} \rightarrow e^{-} \bar{\nu}_{e} \nu_{\tau}$ Branching Ratio

2. Phys. C70:357, 1996; CERN-PPE-95-179

Measurement of the Heavy Quark Forward-Backward Asymmetries and Average $B$ Mixing using Leptons

in Multi-Hadronic Events

Z. Phys. C71:1, 1996; CERN-PPE-95-193

Search for a Narrow Resonance in $Z^{0}$ Decays into Hadrons and Isolated Photons

Phys. Lett. 376B:315, 1996; CERN-PPE-96-031

A Study of Four Fermion Final States with High Multiplicity at LEP

Phys. Lett. 387B:432, 1996; CERN-PPE-96-098

Test of the Four Fermion Contact Interaction in $e^{+} e^{-}$Collisions at $130-140 \mathrm{GeV}$

2. Phys. C73:569, 1997; CERN-PPE-96-099

Strange Baryon Production in Hadronic $Z^{\circ}$ Decays

Z. Phys. C73:587, 1997; CERN-PPE-96-100

$\Sigma^{+}, \Sigma^{0}$, and $\boldsymbol{\Sigma}^{-}$Hyperon Production in Hadronic $Z^{0}$ Decays

Phys. Lett. 384B:343, 1996; CERN-PPE-96-062

Prompt $J / \psi(1 S)$ Production in Hadronic $Z^{\circ}$ Decays

Phys. Rev. Lett. 77:5000, 1996; CLNS-96-1419; CLEO-96-9

First Measurement of the $B \rightarrow \pi \ell \nu$ and $B \rightarrow \rho(\omega \ell \nu)$ Branching Fractions

Phys. Lett. 374B:341, 1996; CERN-PPE-96-018

Improved Measurement of the Lifetime of the $\tau^{ \pm}$Lepton

Phys. Lett. 377B:273, 1996; CERN-PPE-96-019

Topological Search for the Production of Neutralinos and Scalar Particles

Z. Phys. C72:231, 1996; CERN-PPE-96-042

Upper Limit on the $\nu_{\tau}$ Mass from $\tau^{ \pm} \rightarrow$ 3hadron $\nu_{\tau}$ Decays

Z. Phys. C72:389, 1996; CERN-PPE-96-90

Multiplicity Dependence of Bose-Einstein Correlations in Hadronic $Z$ Decays

See the legend on page 5 . 
Alexander $96 \mathrm{~K}$

Alexander 96L

Alexander $96 \mathrm{M}$

Alexander $96 \mathrm{~N}$

Alexander 960

Alexander $96 \mathrm{P}$

Alexander $96 \mathrm{Q}$

Alexander $96 \mathrm{R}$

Alexander $96 \mathrm{~S}$

Alexander 96T

Alexander $96 \mathrm{U}$

Alexander $96 \mathrm{~V}$

Alexander $96 \mathrm{~W}$

Alexander $96 \mathrm{X}$

Alexander $96 \mathrm{Y}$

Alexander 967

Alexander $96 \mathrm{ZB}$

Alexander 97

Alexander 98

Alexander $98 \mathrm{~B}$

Alexandrov 98

Alexandryan 94

Alexandryan 96

Alexopoulos 94

Alexopoulos $94 \mathrm{~B}$

Alexopoulos $94 \mathrm{C}$

Alexopoulos 95

Alexopoulos 95B

Alexopoulos $95 \mathrm{C}$

Alexopoulos $95 \mathrm{D}$

Alexopoulos 97

Alexopoulos $97 \mathrm{~B}$

Alexopoulos 98

Alfimenkov 96

Alibekov 97

Alkhazov 97

Allet 94

Allet 96
Z. Phys. C72:365, 1996; CERN-PPE-96-078

A Precise Measurement of the Tau Polarization and Its Forward-Backward Asymmetry at LEP

Z. Phys. C72:191, 1996; CERN-PPE-96-47

$Q C D$ Studies with $e^{+} e^{-}$Annihilation Data at 130 and $138 \mathrm{GeV}$

Phys. Lett. 384B:377, 1996; CERN-PPE-96-068

A First Measurement of the $\Lambda \bar{\Lambda}$ and $\Lambda \Lambda(\bar{\Lambda} \bar{\Lambda})$ Spin Compositions in Hadronic $Z^{0}$ Decays

Z. Phys. C72:377, 1996; CERN-PPE-96-074

A Measurement of the $B^{0}$ Oscillation Frequency using Leptons and $D^{*}(2010)^{ \pm}$Mesons

Phys. Lett. 385B:433, 1996; CERN-PPE-96-093

Search for Unstable Neutral and Charged Heavy Leptons in $e^{+} e^{-}$Collisions at $\sqrt{s}=130$ and $138 \mathrm{GeV}$

Phys. Lett. 377B:222, 1996; CERN-PPE-96-039

Measurements with Photonic Events in $e^{+} e^{-}$Collisions at Center-of-Mass Energies of $130-140 \mathrm{GeV}$

Z. Phys. C72:1, 1996; CERN-PPE-96-51

A Study of Charm Hadron Production in $Z^{0} \rightarrow c \bar{c}$ and $Z^{0} \rightarrow b \bar{b}$ Decays at LEP

Phys. Lett. 388B:437, 1996; CERN-PPE-96-121

Measurement of the Branching Fraction of the Radiative Decay $\tau^{-} \rightarrow \mu^{-} \bar{\nu}_{\mu} \nu_{\tau} \gamma$

Z. Phys. C73:379, 1997; CERN-PPE-96-101

A Measurement of the Charm and Bottom Forward-Backward Asymmetries using $D$ Mesons at LEP

Phys. Lett. 386 B:463, 1996; CERN-PPE-96-094

Search for Excited Leptons in $e^{+} e^{-}$Collisions at $\sqrt{s}=130$ and $136 \mathrm{GeV}$

Phys. Lett. 376B:232, 1996; CERN-PPE-96-025

Measurement of Cross Sections and Asymmetries in $e^{+} e^{-}$Collisions at $130-140 \mathrm{GeV}$ Center-of-Mass Energy

Phys. Lett. 377B:181, 1996; CERN-PPE-96-20

Search for Chargino and Neutralino Production using the OPAL Detector at $\sqrt{s}=130-138$ GeV at LEP

Z. Phys. C73:189, 1997; CERN-PPE-96-118

Search for Neutral Higgs Bosons in $Z^{\circ}$ Decays using the OPAL Detector at LEP

Phys. Let.t. 388B:659, 1996; CERN-PPE-96-116

Test of QCD Analytic Predictions for Multiplicity Ratio between Gluon and Quark Jets

Z. Phys. C73:201, 1997; CERN-PPE-96-096

Searches for Supersymmetric Particles and Anomalous Four-Jet Production at $\sqrt{s}=130$ and $136 \mathrm{GeV}$ at LEP

Phys. Lett. 370B:185, 1996; CERN-PPE-95-181

Observation of $\Upsilon$ Production in Hadronic $Z^{\circ}$ Decays

Phys. Lett. 370B:174, 1996; CERN-PPE-95-180

Search for Charged Higgs Bosons using the OPAL Detector at LEP

Phys. Rev. D56:5320, 1997; CLNS-97-1480; CLEO-97-9; HEPEX-9705009

Determination of the Michel Parameters and the $\tau^{ \pm}$Neutrino Helicity in $\tau^{ \pm}$Decay

Phys. Rev. D58:052004, 1998; CLNS-98-1540; CLEO-98-1; HEPEX-9802024

The Hadronic Transitions $\Upsilon(2 S) \rightarrow \Upsilon(1 S)$

Phys. Lett. 440B:393, 1998; CERN-EP-98-124; HEPEX-9808035

Measurement of the Longitudinal Cross-Section using the Direction of the Thrust Axis in Hadronic Events at LEP

Phys. Lett. 433B:217, 1998; CERN-EP-98-083

Azimuthal Correlation between Beauty Particles Produced in $350 \mathrm{GeV} / \mathrm{c} \pi^{-}$Cu Interactions

Phys. At. Nucl. 57:2037, 1994; Yad. Phys. 57:2115, 1994

Study of Photonuclear Reactions on the Sn Nucleus

Phys. At. Nucl. 59:560, 1996; Yad. Phys. 59:592, 1996

Study of Isomer Ratios of Proton-Nuclear Cross Sections on Tin Isotopes

FERMILAB-CONF-94-124-E

Mass Identifled Particle Production and Bose-Einstein Correlations at $1800 \mathrm{GeV}$

FERMILAB-CONF-94-178-E

Production of $J / \psi(1 S)$ in $800 \mathrm{GeV} / c$-Si Interactions

Phys. Lett. 336B:599, 1994

Multiplicity Dependence of the Transverse Momentum Spectra of Centrally Produced Hadrons in $\bar{p} p$ Collisions at $0.3,0.4,0.0$, and $1.8 \mathrm{TeV}$ Center of Mass Energy

Phys. Lett. 353B:155, 1995

Charged Particles Multiplicity Correlations in $\bar{p} p$ Collisions at $\sqrt{s}=0.3-1.8 \mathrm{TeV}$

Phys. Rev. Lett. 77:2380, 1996; FERMILA B-PUB-95-286-E

Search for the Flavor Changing Neutral Current Decay $D^{0} \rightarrow \mu^{+} \mu^{-}$in $800 \mathrm{GeV}$ Proton-Silicon Interactions

Z. Phys. C67:411, 1995

$\phi(1020)$ Meson Production form $\bar{p} p$ Collisions at $\sqrt{s}=1.8 \mathrm{TeV}$

Phys. Lett. 374B:271, 1996; FERMILAB-PUB-95-297-E

Measurement of $J / \psi(1 S), \psi(2 S)$ and $\Upsilon$ Total Cross Sections in $800 \mathrm{GeV} / c p$ Si Interactions

Phys. Rev. D55:3927, 1997; FERMILAB.PUB-97-245-E

Differential Cross Sections of of $J / \psi(1 S)$ and $\psi(2 S)$ in $800 \mathrm{GeV} / c p$ Si Interactions

Phys. Rev. Lett. 82:41, 1999; FERMILAB-PUB-97-246-E

$A$ Measurement of the $B \bar{B}$ Cross Section in $800 \mathrm{GeV} / c$ Proton-Silicon Interactions

Phys. Lett. 435B:453, 1998

The Role of Double Parton Collisions in Soft Hadron Interactions

Phys. At. Nucl. 59:1861, 1996; Yad. Phys. 59:1929, 1996

Study of Parity Violation Effect at Interaction of Neutrons with a Polarized Lanthanum

Izv. RAN, Fiz. 61:474, 1997

Leading Neutral Pions from Hadron Interactions with Iron Nuclei

Phys. Rev. Lett. 78:2313, 1997

Nuclear Matter Distributions in ${ }^{6} \mathrm{He}$ and ${ }^{8} \mathrm{He}$ from Small Angle $p$ He Scattering in Inverse Kinematics at Intermediate Energy

Phys. Rev. C50:602, 1994

Cross Section and Analyzing Power $A_{y}$ in the Break up Reaction ${ }^{2} \mathrm{H}(p \nmid, p p) n$ at $65 \mathrm{MeV}$ : Collinearity Conflgurations

Phys. Lett. 383B:139, 1996

Search for Right-Handed Weak Currents in the $\beta$-Asymmetry-Polarization Correlation from ${ }^{12} \mathrm{Nit}$ Decay 
Allgower 98

Allgower 98B

Allgower $98 \mathrm{C}$

Allison 96B

Allison 98

Allport 96

Altarelli 93

Altarev 96

Altegoer 98

Altegoer $98 \mathrm{~B}$

Altegoer $98 \mathrm{C}$

Alteholz 94

Althoff 94

Altmann 95

Alton 96

Alvarezdelar 95

Alvaro 97

Alverson 93B

Alves 93

Alves 93B

Alves 96

Alves 96B

Alves 97

A maglobeli 96

Amaudruz 95

A mbrogiani 96

Ambrogiani 99

Ambrose 98

Ambrose 98B

Ambrosini 97

Ambrosini 98

Ambrosini 98B

Ambrosini $98 \mathrm{C}$

Ambrosio 95

Ambrosio 97

Ambrosio 97B

Ambrosio $97 \mathrm{C}$
Eur. Phys. J. C1:131, 1998

Angular Dependence of $p$ spin Correlation and Rescattering Observables between 1.80 and $2.10 \mathrm{GeV}$ Eur. Phys. J. C5:453, 1998

$p p$ Elastic Scattering Polarization Transfer $K_{\text {onno }}$ and Depolarization $D_{o n o n}$ between 1.94 and $2.80 \mathrm{GeV}$

Nucl. Phys. A637:231, 1998

The $p$ Elastic Scattering Analyzing Power Measured with the Polarized Beam and the Unpolarized Target between 1.08 and $2.80 \mathrm{GeV}$

Phys. Lett. 391B:491, 1997; RAL-P-96-007; PDK-570; HEPEX-9611007

Measurement of the Atmospheric Neutrino Flavour Composition in Soudan 2

Phys. Lett. 427B:217, 1998; ANL-HEP-PR-98-24; PDK-698; HEPEX-9803030

Search for the Proton Decay Mode $p \rightarrow \nu K^{+}$in Soudan-2

MANCHESTER-95 p. 343

Strange Baryon Production at DELPHI

Phys. Lett. 320B:152, 1994; Phys. Lett. 325B:538, 1994; CERN-TH-7023-93

On the $q^{2}$ Dependence of the Measured Polarized Structure Functions

Phys. At. Nucl. 59:1152, 1996; Yad. Phys. 59:1204, 1996

Search for the Neutron Electric Dipole Moment

Phys. Lett. 428B:197, 1998; CERN-EP-98-047; HEPEX-9804003

Search for a New Gauge Boson in $\pi^{\circ}$ Decays

Phys. Lett. 431B:219, 1998; CERN-EP-98-057

A Search for $\nu_{\mu} \rightarrow \nu_{\tau}$ Oscillations using the NOMAD Detector

Phys. Lett. 445B:439, 1999; CERN-EP-98-179

Precision Measurement of Scaled Momentum, Charge Multiplicity and Thrust in $\nu_{\mu}$ nucleon and $\bar{\nu}_{\mu}$ nucleon Interactions

Phys. Rev. Lett. 73:1336, 1994; PSI-PR-94-11; MIT-LNS-94-56

A Large Solid Angle Study of Pion Absorption on ${ }^{3} \mathrm{He}$

WILLIAMSBURG-94 p. 505

Target Asymmetry Measurements of $\gamma p \rightarrow \pi^{+} n$ with PHOENICS at ELSA

Z. Phys. C68:221, 1995

Search for the Electron Positron Decay of Axions and Axion-Like Particles at a Nuclear Power Reactor at Bugey

Phys. Rev. D56:5301, 1997; FERMILAB-PUB-96-188-E

The Emergence of Jet Dominance in $\gamma p$ Interactions at Fixed-Target Energies

Phys. Lett. 351 B:418, 1995

Study of Very Peripheral S Pb and S S Interactions at $200 \mathrm{GeV} / c$ per Nucleon

Nucl. Phys. (PS) B55:304, 1997; ESTES PARK-96 p. 304

Weak Electric and Magnetic Dipole Moments of the $\tau^{ \pm}$Lepton from Azimuthal Asymmetries

Phys. Rev. D49:3106, 1994; FERMILA B-PUB-93-284-E

Structure of the Recoiling System in Direct-Photon and $\pi^{0}$ Production by $\pi^{-}$and $p$ Beams at $500 \mathrm{GeV} / \mathrm{c}$

Phys. Rev. D49:R4317, 1994; FERMILAB-PUB-93-081-E $D^{*}(2010)^{ \pm}$Production in $250 \mathrm{GeV} \pi^{ \pm} \mathrm{N}$ Interactions

Phys. Rev. Lett. 72:812, 1994; Phys. Rev. Lett. 72:1946, 1994; FERMILAB-PUB-93-310-E Enhanced Leading Production of $D^{ \pm}$and $D^{*}(2010)^{ \pm}$in $250 \mathrm{GeV} \pi^{ \pm}$Nucleon Interactions

Phys. Rev. Lett. 77:2392, 1996; FERMILAB-PUB-96-084 Feynman- $x$ and Transverse Momentum Dependence of $D$ Meson Production in $250 \mathrm{GeV} \pi$, kaon and $p$ nucleon Interactions

Phys. Rev. Lett. 77:2388, 1996; Phys. Rev. Lett. 81:1537, 1998; FERMILAB-PUB-96-083 Forward Cross Sections for Production of $D^{+}, D^{0}, D_{s}^{+}, D^{*}(2010)^{+}$and $\Lambda_{c}^{+}$in $250 \mathrm{GeV} \pi^{ \pm}, K^{ \pm}$, and $p$ Nucleon Interactions

Phys. Rev. D56:6003, 1997; FERMILAB-PUB-97-086-E; TUHEP-97-01; HEPEX-9703015 Atomic Mass Dependence of $\Xi^{-}$and $\Xi^{+}$Production in Central $250 \mathrm{GeV} \pi^{-}-$Nucleon Interactions

Phys. At. Nucl. 59:973, 1996; Yad. Phys. 59:1018, 1996

Inclusive Production of $K$ Mesons in $p p$ Interactions at $24 \mathrm{GeV} / c$

Nucl. Phys. B441:3, 1995

A Re-Evaluation of the Nuclear Structure Function Ratios for deuteron, $\mathrm{He},{ }^{8} \mathrm{Li}, \mathrm{C}$ and $\mathrm{Ca}$

Phys. At. Nucl. 59:1307, 1996; Yad. Phys. 59:1366, 1996 Light Quark Meson Spectroscopy

FERMILAB-PUB-99-027-E

Measurements of the Magnetic Form Factor of the Proton in the Timelike Region at Large Momentum Transfer

Phys. Rev. Lett. 81:4309, 1998; UTEXAS-HEP-98-14; HEPEX-9810007

First Observation of the Rare Decay Mode $K_{L} \rightarrow e^{+} e^{-}$

Phys. Rev. Lett. 81:5734, 1998; HEPEX-9811038

New Limit on Muon and Electron Lepton Number Violation from $K_{L} \rightarrow \mu^{ \pm} e^{\mp}$

Phys. Lett. 417B:202, 1998; BUHE-97-05

Baryon and Antibaryon Production in Lead-Lead Collisions at $158 \mathrm{~A} \mathrm{GeV} / \mathrm{c}$

Phys. Lett. 420B:225, 1998; CERN-EP-98-018

$K / \pi$ Production Ratios from $450 \mathrm{GeV} / c$ Protons on Beryllium

Phys. Lett. 425B:208, 1998; CERN-EP-98-065

Pion Yield from $450 \mathrm{GeV} / \mathrm{c}$ Protons on Beryllium

Nucl. Phys. A638:411C, 1998; TSUKUBA-97 p. 411C

New Results from NA52 on Particle Production in $\mathrm{Pb}+\mathrm{Pb}$ Collisions at $158 \mathrm{GeV}$ per Nucleon

Phys. Rev. D52:3793, 1995

Vertical Muon Intensity Measured with MACRO at the Gran Sasso Laboratory

Phys. Lett. 406B:249, 1997; IN FN-AE-97-16

Magnetic Monopole Search with the MACRO Detector at Gran Sasso

Phys. Rev. D56:1407, 1997

High Energy Cosmic Ray Physics with Underground Muons in MACRO. I. Analysis Methods and Experimental Results

Phys. Rev. D56:1418, 1997

High Energy Cosmic Ray Physics with Underground Muons in MACRO. II. Primary Spectra and Composition

See the legend on page 5. 
Ambrosio 97D

A mbrosio $97 \mathrm{E}$

A mbrosio $97 \mathrm{~F}$

Ambrosio $97 \mathrm{G}$

Ambrosio $97 \mathrm{H}$

Ambrosio 98

Ambrosio 98B

Ambrosio 98C

Ambrosio $98 \mathrm{D}$

Ameeva 94

A melin 94

A melin 95

Amelin 95B

Amelin 95C

Amelin 98

Amelin 98B

Ammar 93B

Ammar 95

Ammar 96

Ammar 97

A mmar 98

Amroun 94

Amsler 94

Amsler 94B

Amsler $94 \mathrm{C}$

Amsler $94 \mathrm{D}$

Amsler 94E

Amsler $94 \mathrm{~F}$

Amsler $94 \mathrm{G}$

Amsler $94 \mathrm{H}$

Amsler 94I

Amsler 94J

Amsler 95

Amsler 95B

Amsler 95C

Amsler 95D

Amsler 95E

Amsler 95F

Amsler 95G
INFN-AE-97-27

A Sky Survey using Muons in the MACRO Detector

INFN-AE-97-20

Search for Nuclearites with the MACRO Detector at Gran Sasso

INFN-AE-97-28

The Search for Sidereal Anisotropy in the Underground Muon Intensity as Seen by MACRO

INFN-AE-97-26

Measurement of Underground Muon Energies using a TRD in MACRO

INFN-AE-97-24

Observation of Upgoing Charged Particles in MACRO Produced by High Energy Interactions of Muons

Phys. Lett. 434B:451, 1998; INFN-AE-98-13; HEPEX-9807005

Measurement of the Atmospheric Neutrino-Induced Upgoing Muon Flux using MACRO

Phys. Rev. D59:012003, 1999; INFN-AE-98-14; HEPEX-9807006

Observation of the Shadowing of Cosmic Rays by the Moon using a Deep Underground Detector

HEPEX-9812020

Limits on Dark Matter WIMPs using Upward-Going Muons in the MACRO Detector

Astropart. Phys. 9:105, 1998; INFN-AE-97-55; HEPEX-9807032

The Observation of Up-Going Charged Particles Produced by High Energy Muons in Underground De-

Kr. Soob. JINR 68:43, 1994

The Relativistic Projectile Nuclei Fragmentation and A-Dependence of Nucleon Fermi Momenta

Z. Phys. C66:71, 1995; IFVE-94-91 Study of the Decay $f_{1}(1285) \rightarrow \rho^{0} \gamma$

Z. Phys. C70:71, 1996; IFVE-95-71

Partial-Wave Analysis of the Reaction $\pi^{-} p \rightarrow \pi^{+} \pi^{-} \pi^{0} n$ at $p_{\pi_{-}}=36 \mathrm{GeV} / c$

Phys. Lett. 356 B:595, 1995; IFVE-95-78

Study of Resonance Production in Diffractive Reaction $\pi^{-}$nucleus $\rightarrow \pi^{+} \pi^{-} \pi^{-}$nucleus

Phys. At. Nucl. 59:976, 1996; Yad. Phys. 59:1021, 1996; IFVE-95-112

Study of Diffractive Reaction $\pi^{-}$nucleus $\rightarrow \eta \eta \pi^{-}$nucleus at the Momentum $P_{\pi-}=37 \mathrm{GeV} / \mathrm{c}$

IFVE-98-62; HEPEX-9810032

Study of $\eta \pi^{-}$Production by Pions in the Coulomb Field

IFVE-97-80; HEPEX-9810013

Study of the Reaction $\pi^{-\mathrm{Be}} \rightarrow \omega \pi^{-} \pi^{0} \mathrm{Be}$

Phys. Rev. D49:5701, 1994; CLNS-93-1258; CLEO-93-21

Search for $B^{\circ}$ Decays to Two Charged Leptons

Phys. Rev. Lett. 74:3534, 1995; CLNS-94-1305; CLEO-94-23

New Decay Modes of the $\Lambda_{c}^{+}$Charmed Baryon

Phys. Rev. D55:13, 1997; CLNS-96-1401; CLEO-96-07

Study of Flavor-Tagged Baryon Production in $B$ Decay

Phys. Rev. D57:1350, 1998; CLNS-97-1493; CLEO-97-14; HEPEX-9707018

A Measurement of the Total Cross Section for $e^{t} e^{-} \rightarrow$ hadrons at $\sqrt{s}=10.52 \mathrm{GeV}$

Phys. Lett. 431B:209, 1998; CLNS-98-1551; CLEO-98-6; HEPEX-9803031

A Limit on the Mass of the $\nu_{\tau}$

Nucl. Phys. A579:369, 1994; DAPNIA-SPHN-94-05; PCFF-RI-9403

${ }^{3} \mathrm{H}$ and ${ }^{3} \mathrm{He}$ Electromagnetic Form Factors

Phys. Lett. 322B:431, 1994

Observation of a Scalar Resonance Decaying to $\pi^{+} \pi^{-} \pi^{0} \pi^{0}$ in $\bar{p} p$ Annihilation at Rest

Yad. Phys. 57:1542, 1994; Phys. At. Nucl. 57:1465, 1994

Physics with Crystal Barrel: New Mesons in $\bar{p} p$ Annihilation

Phys. At. Nucl. 57:1602, 1994; Yad. Phys. 57:1672, 1994

Pontecorvo Reactions in $\bar{p}$ deuteron Annihilation at Rest

Phys. At. Nucl, 57:1711, 1994; Yad. Phys. 57:1782, 1994 $\eta$-Decay Branching Ratio

Phys. At. Nucl. 57:1497, 1994; Yad. Phys. 57:1572, 1994 Search for Hybrids

Phys. Lett. 327 B:425, 1994 Study of $\bar{p} p$ Annihilation at Rest into $\omega \eta \pi^{\circ}$

Phys. Lett. 333B:271, 1994; CERN-PPE-94-078 Search for a New Light Gauge Boson in Decays of $\pi^{0}$ and $\eta$

Phys. Lett. 333B:277, 1994 Observation of a New $I^{G}\left(J^{P C}\right)=1^{-}\left(0^{++}\right)$Resonance at $1450 \mathrm{MeV}$

Phys. Lett. 340B:259, 1994 $\eta \bar{\eta}^{\prime}$ Threshold Enhancement in $\bar{p} p$ Annihilations into $\pi^{0} \dot{\eta} \eta^{\prime}$ at Rest

Nuovo Cim. 107A:1815, 1994 New Results from Crystal Barrel

Phys. Lett. 342B:433, 1995

High-Statistical Study of $f_{0}(1500)$ Decay into $\pi^{0} \pi^{0}$

Phys. Lett. 346B:203, 1995 $\eta$-Decays into Three Pion

Phys. Lett. 346B:363, 1995 Observation of Radiative $\bar{p} p$ Annihilation into a $\phi(1020)$ Meson

Phys. Lett. 352B:187, 1995 First Observation of the Production of Nucleon Resonances in Antiproton Annihilation in Liquid Deuterium

Phys. Lett. 355B:425, 1995

Coupled Channel Analysis of $\bar{p} p$ Annihilation into $\pi^{0} \pi^{0} \pi^{0}, \pi^{0} \eta \eta$ and $\pi^{0} \pi^{0} \eta$

Phys. Lett. 358B:389, 1995

$E$ Decays to $\eta \pi \pi$ in $\bar{p} p$ Annihilation at Rest

Z. Phys. A351:325, 1995

First Observations of Pontecorvo Reactions with a Recoiling Neutron 
Amsler $95 \mathrm{H}$

Amsler 96

Amsler 96B

Amsler $96 \mathrm{C}$

Amsler $96 \mathrm{D}$

Anagnastopou 97

Anagnostopou 96

Anastassov 97

Anastassov 97B

Anastassov 98

Andersen 94

Andersen 94B

Andersen $94 \mathrm{C}$

Andersen 95

Andersen 98

Anderson 96

Anderson 96B

Anderson 97

Anderson 97B

Anderson 98

Anderson 98B

Andivahis 94

Andreazza 95

Andreev 94

Andreeva 95B

Andreeva 97

Andreeva 97B

Andreeva 98

Andreeva 98B

Andrighetto 97

Androic 96

Andronenko 94

Andronic 97

Andronic 97B

Andronic 98

Angelescu 96

Angelique 97

Angelis 96

Angelis 97
Phys. Lett. 353B:571, 1995

High Statistics Study of $f_{0}(1500)$ Decay into $\eta \eta$

Z. Phys. C70:219, 1996

Search for a New Light Gauge Boson in $\pi^{\circ}, \eta$ and $\eta^{\prime}$ Decays

MANCHESTER-95 p. 328

$f_{1}(1420)$ Decay to $\eta \pi \pi$

Phys. At. Nucl. 59:1415, 1996; Yad. Phys. 59:1473, 1996

Antiproton Annihilation at Rest in Liquid Deuterium

Phys. At. Nucl. 59:1822, 1996; Yad. Phys. 59:1886, 1996

Two Body Annihilation in Flight

Nucl. Phys. A626:375C, 1997

A New Determination of the Pion Mass

Yad. Phys. 59:1562, 1996; Phys. At. Nucl. 59:1503, 1996

Protonium $X$-Ray Spectroscopy

Phys. Rev. Lett. 80:4127, 1998; CLNS-97-1501. CLEO-97-17; HEPEX-9708035

Investigation of Semileptonic $B$ Meson Decay to $P$-Wave Charm Mesons

Phys. Rev. D55:2559, 1997; Phys. Rev. D58:119904, 1998; CLNS-96-1431; CLEO-96-16

Experimental Test of Lepton Universality in $\tau$ Decay

Phys. Rev. Lett. 82:286, 1999; CLNS-98-1572; CLEO-98-9; HEPEX-9807031 First Observation of $\Upsilon(1 S) \rightarrow \gamma \pi \pi$

Nucl. Phys. A566:217C, 1994

Results from the NA36 Experiment on the Production of Strangeness $|S|=1$ and $|S|=2$ Particles

Nucl. Phys. A566:487C, 1994

Strangeness Production in $p+\mathrm{Pb}$ Reactions at $200 \mathrm{GeV} / \mathrm{c}$

Phys. Lett. 327B:433, 1994

Production of $\Lambda, \bar{\Lambda}$, and $\Xi^{-}, \bar{\Xi}^{+}$Particles in S+Pb Collisions at $200 \mathrm{GeV} / c$ per Nucleon

Nucl. Phys. A590:291C, 1995

Recent Results from the NA36 Experiment

Phys. Lett. 433B:209, 1998; CERN-EP-98-064

Enhancement of Central $\Lambda, \Xi$ and $\Omega^{-}$Yields in $\mathrm{Pb} \mathrm{Pb}$ Collisions at $158 \mathrm{~A} \mathrm{GeV} / \mathrm{c}$

Phys. Rev. C54:237, 1996 ${ }^{2} \mathrm{C}(p, n){ }^{12} \mathrm{Nit}$ Reaction at $135 \mathrm{MeV}$

Phys. Rev. C54:1531, 1996

Cross Section Measurements for the deuteron $(p, n) 2 p$ Reaction at $135 \mathrm{MeV}$

Phys. Rev. D56:2485, 1997; CLNS-96-1448; CLEO-96-19; HEPEX-9701013

$\bar{\Lambda} \Lambda$ Production in Two-Photon Interactions at CLEO

Phys. Rev. Lett. 79:3814, 1997; CLNS-97-1497; CLEO-97-16; HEPEX-9707027

Study of the Decay $\tau^{-} \rightarrow 2 \pi^{-} \pi^{+} 3 \pi^{0} \nu_{r}$

Phys. Rev. Lett. 81:3823, 1998; CLNS-98-1557; CLEO-98-7; HEPEX-9805027

First Search for CP Violation in $\tau^{ \pm}$Lepton Decay

Nucl. Phys. A631:752C, 1998; GRONINGEN-97 p. 752C

Measurement of a Complete Set of Spin Observables in the deuteron $(p, n) 2 p$ Reaction at $200 \mathrm{MeV}$

Phys. Rev. D50:5491, 1994; SLAC-PUB-6462

Measurements of the Electric and Magnetic Form-Factors of the Proton from $q^{2}=1.75$ to $8.83 \mathrm{GeV} / c^{2}$

Nucl. Phys. (PS) B40:321, 1995

$\tau^{ \pm}$Lifetime with the Impact Parameter Difference Method

Phys. Rev. C50:15, 1994 Experimental Study of the Reaction $p p \rightarrow p p \pi^{0}$ in the Energy Region $600-900 \mathrm{MeV}$

Phys. At. Nucl. 58:951, 1995; Yad. Phys. 58:1024, 1995

Peculiarities of Characteristics of Interactions of Relativistic Nuclei with Nuclei Ag-Br

Z. Phys. A358:337, 1997 Complex Analysis of Gold Interactions with Photoemulsion Nuclei at $10.7 \mathrm{GeV} /$ Nucleon within the Framework of Cascade and FRITIOF Models

Z. Phys. A359:277, 1997

Multifragmentation of Gold Nuclei in the Interactions with Photoemulsion Nuclei at 10.7 GeV/Nucleon

Eur. Phys. J. A1:77, 1998

Critical Behaviour in Au Fragmentation at $10.7 \mathrm{~A} \mathrm{GeV}$

Eur. Phys. J. A2:61, 1998

Bounce-off in ${ }^{19}$ Au Induced Collisions with $\mathrm{Ag}(\mathrm{Br}) \mathrm{Nuclei}$ at $11.6 \mathrm{~A} \cdot \mathrm{GeV} / \mathrm{c}$

Phys. Lett, 412B:148, 1997 Charged Particle Production in SS Collisions at $200 \mathrm{GeV} / \mathrm{c}$ per Nucleon

Phys. Rev. C53:2591, 1996

Evidence of Initial State Interactions in Multinucleon Pion Absorption

2. Phys. A350:1, 1994

Isotopic Ratios of Intermediate Mass Fragments Produced in $p+$ nucleus Reactions at $1 \mathrm{GeV}$

Z. Phys. A359:55, 1997; NUCLEX-9704009

Charged Pions from $\mathrm{Ni}$ on $\mathrm{Ni}$ Collisions between 1 and $2 \mathrm{~A} \mathrm{GeV}$

Z. Phys. A357:215, 1997

Charged Pion Production in Au on Au Collisions at $1 \mathrm{~A} \cdot \mathrm{GeV}$

Eur. Phys. J. A3:335, 1998; NUCLEX-9809005

Identiflcation of Baryon Resonances in Central Heavy-Ion Collisions at Energies between 1 and 2 A.GeV UB-PUB-EPPG-PHYS-35

Influence of Two-Prong Inelastic Events on $\left(\pi^{+},{ }^{3} \mathrm{He}\right)$ Reaction at $120 \mathrm{MeV}$

Nucl. Phys, A614:261, 1997

Directed Collective Flow and Azimuthal Distributions in ${ }^{36} \mathrm{Ar}{ }^{27} \mathrm{Al}$ Collisions from 55 to $95 \mathrm{MeV} / \mathrm{u}$

Nucl. Phys. A610:200C, 1996; IKP-MS-96-0801

Photon and Neutral Meson Production in $158 \mathrm{GeV} / \mathrm{Nucleon}{ }^{208} \mathrm{~Pb} \mathrm{~Pb}$ Collisions

Eur. Phys. J. C5:63, 1998; CERN-PPE-97-117

Study of Vector Mesons in Dimuon Production in a Large Kinematic Region in $p$ Wt and S Wt interactions at $200 \mathrm{GeV} / \mathrm{c} /$ nucleon 
Angelis 98

Angelis 98

Angelopoulos 97

Angelopoulos $97 \mathrm{~B}$

Angelopoulos 97C

Angelopoulos $97 \mathrm{D}$

Angelopoulos $97 \mathrm{E}$

Angelopoulos 98

Angelopoulos $98 \mathrm{~B}$

Angelopoulos 98C

Angelopoulos 98D

Angelopoulos $98 \mathrm{E}$

Angelov 94

Anghinolfi 95

Anghinolfi 96

Anikeyev 95

Anikeyev $95 \mathrm{~B}$

Anikeyev 98

Anikina 95

Anikina $95 \mathrm{~B}$

Anikina 98

Anikina 98B

Aniol 98

Anisimov 95

Anisimov 97

Anisimov 98

Anisovich 94

Anisovich 95

Anklin 94

Anklin 98

Anklin 98B

Anne 94

Annis 98

Anoshina 97

Anraku 96

Anselmann 94

Anselmann 95

Anselmann 9.5B
CERN-EP-98-082

Excess of Continuum Dimuon Production at Masses between Threshold and the $J / \psi(1 S)$ in $S$ Wt Interactions at $200 \mathrm{GeV} / \mathrm{c} /$ nucleon

Phys. Lett. 413B:422, 1997; CERN-PPE-97-131

Measurement of the Neutral Kaon Regeneration Amplitude in Carbon at Momenta below 1 GeV/c

Eur. Phys. J. C1:139, 1998; CERN-PPE-97-135

Direct Determination of Two-Pion Correlations for $\bar{p} p \rightarrow 2 \pi^{+} 2 \pi^{-}$Annihilation at Rest

Phys. Lett. 420B:191, 1998; CERN-PPE-97-148

Measurement of the $C P$-Violation Parameter $\eta_{0 o}$ using Tagged $\bar{K}^{\circ}$ and $K^{\circ}$

Phys. Lett. 413B:232, 1997

An Upper Limit for the Branching Ratio of the Decay $K_{S} \rightarrow e^{+} e^{-}$

Nucl. Phys. A626:157C, 1997

Measurement of $C P, T$ and $C P T^{*}$ Violation Parameters in the Neutral Kaon System at CPLEAR

Phys. Lett. 425B:391, 1998; CERN-EP-98-004

Search for CP violation in the decay of tagged $K^{0}$ and $\bar{K}^{0}$ to $\pi^{0} \pi^{0} \pi^{0}$

Eur. Phys. J. C5:389, 1998; CERN-EP-98-074

The Neutral Kaon Decays to $\pi^{+} \pi^{-} \pi^{0}$ : a Detailed Analysis of the CPLEAR data

Phys. Lett. 444B:38, 1998; CERN-EP-98-152

Measurement of the $K_{L}-K_{S}$ Mass Difference using Semileptonic Decays of Tagged Neutral Kaons

Phys. Lett. $444 \mathrm{~B}: 43,1998$; CERN-EP-98-153 First Direct Observation of Time-Reversal Non-Invariance in the Neutral-Kaon System

Phys. Lett. 444B:52, 1998; CERN-EP-98-154

A Determination of the CPT Violation Parameter Re $(\delta)$ from the Semileptonic Decay of Strangeness

Tagged Neutral Kaons

Kr. Soob. JINR 65:40, 1994

Clusterization in Processes of Multiparticle Production in Nuclei. Two-Cluster Correlations

Jour, of Phys, G 21:L9, 1995

Inclusive Electron Scattering from an Oxygen and Argon Jet Target

Nucl. Phys. A602:405, 1996; INFN-ISS-95-13; NUCLTH-9603001

Quasi-Elastic and Inelastic Inclusive Electron Scattering from an Oxygen Jet Target

LOHUSALU -95 p. 17

Study of Neutrino and Antineutrino Charged-Current Interactions in U70 Wide Band Beams

Z. Phys. C70:39, 1996; IFVE-95-50

Total Cross Section Measurements for $\nu_{\mu}, \bar{\nu}_{\mu}$ Interactions in $3-30$ GeV Energy Range with IHEP-JINR Neutrino Detector

Kí. Soob. JINR 87:13,1998

A New Experimental Study of Charged $K \rightarrow 3 \pi$ Decays

JINR-E1-95-311

A Measurement of the Expansion Velocity of Pion Production Volume

Phys. Lett. 397B:30, 1997; JINR-E1-96-223

HBT Measurement of the Expansion Velocity of Pion Production Volume

HEPI-98-07; HEPEX-9811019

The Light Front Analysis of $\pi^{-}$Mesons Produced in the Relativistic Nucleus-Nucleus Collisions

Nucl. Phys. A640:117, 1998

Light-Front Analysis of $\pi^{-}$Mesons Produced in $\mathrm{Mg}-\mathrm{Mg}$ Collisions at $4.3 \mathrm{~A} \cdot \mathrm{GeV} / \mathrm{c}$

Phys. Rev. Lett. 82:1096, 1999; WM-98-117; NUCLEX-9810012

Measurement of the Neutral Weak Form Factors of the Proton

Kr. Soob. JINR 73:31, 1995

Measurement of the Tensor Analyzing Power for the Reaction of Fragmentation of Tensor Polarized Deuterons with Momenta from 6.2 to 9.0 GeV/c into Cumulative Mesons

Phys. At. Nucl. 60:957, 1997; Yad. Phys. 60:1070, 1997

The Study of Dependence of Relativistic Deuteron Fragmentation Cross Section into Cumulative $\pi-$ Mesons on Target Atomic Mass

Kr. Soob. JINR 91:25, 1998

First Results of Study of Transversal Dimension of Region of Cumulative Particles Production in deuteron $+\mathrm{C}$ and deuteron $+\mathrm{Cu}$ Reactions for Energy $2 \mathrm{GeV} /$ nucleon

Phys. Lett. 323B:233, 1994

Observation of $\mathrm{Two} J^{P C}=0^{++}$Isoscalar Resonances at 1365 and $1520 \mathrm{MeV}$

Phys. Lett. 355B:363, 1995

Two-Resonance Structure of the $I J^{P C}=0^{++} \pi \pi$ Amplitude in Mass Region around $1 \mathrm{GeV}$

Phys. Lett. 336 B:313, 1994

Precision Measurement of the Neutron Magnetic Form Factor

Phys. Lett. 428B:248, 1998

Precise Measurements of the Neutron Magnetic Form Factor

Nucl. Phys. A636:189, 1998

Tensor Analyzing Power $A_{y y}$ of deuteron $\rightarrow p$ Radiative Capture

Nucl. Phys. A575:125, 1994

Exclusive and Restricted-Inclusive Reactions Involving the ${ }^{11} \mathrm{Be}$ One-Neutron Halo

Phys. Lett. 435B:458, 1998; CERN-EP-98-087

Observation of Neutrino Induced Diffractive $D_{s}^{*+}$ Production and Subsequent Decay $D_{s}^{*+} \rightarrow D_{s}^{+} \rightarrow \tau^{+} \rightarrow \mu^{+}$

Phys. At. Nucl. 60:224, 1997; Yad. Phys. 60:283, 1997

Analyzing Power of Proton-Carbon Interaction at the Energies of $0.71-3.61 \mathrm{GeV}$

KEK-96-12

Search for Antihelium of Cosmic Origin

Phys. Lett. 327B:377, 1994; LNGS-94-89

GALLEX Results from the First 30 Solar Neutrino Runs

Phys. Lett. 342B:440, 1995; DAPNIA-SPP-94-40; GX-65-1994; LNGS-94-111; MPI-H-V30-1994; ROM2F-94-47

First Results from the ${ }^{51} \mathbf{C r}$ Neutrino Source Experiment with the GALLEX Detector

Phys. Lett. 361B:235, 1995; Phys. Lett. 357 B:237, 1995

GALLEX Solar Neutrino Observations: Comlpete Results for GALLEX II 
Anthony 95

Anthony 96

Anthony 99

Antinori 95

Antinori 97

Antipov 93B

Antipov 95

Antonelli 94

Antonelli 96

Antonelli 98

Antoniazzi 94

Antoniazzi 94B

Antonov 94

Antonov 95

Antonov 95B

Antos 94

Anwaywiese 94

Anwaywiese 95

Aoki 94

Aoki 95

Aoki 95B

Aoki 98

Aoki 98B

Aono 95

Aoyagi 94

Apanasenko 94

A panasenko 97

A panasevich 97

A panasevich 97B

A pollonio 97

Apostolakis 97

Apostolakis 99

Appel 94

Appelquist 94

Appelquist 95

Appelquist 96
Phys. Rev. Lett. 75:1949, 1995; SLAC-PUB-95-6796; LBL-37178

An Accurate Measurement of the Landau-Pomeranchuk-Migdal Effect

Phys. Rev. D54:6620, 1996; SLAC-PUB-7103; HEPEX-9610007

Deep Inelastic Scattering of Polarized Electrons by Polarized ${ }^{3} \mathrm{He}$ and the Study of the Neutron Spin Structure

SLAC-PUB-7983; HEPEX-9901006

Measurement of the Proton and Deuteron Spin Structure Functions $g_{2}$ and Asymmetry $A_{2}$

Phys. Lett. 353B:589, 1995; CERN-PPE-95-33; MANCHESTER-95 p. 120

A Further Study of the Centrally Produced $\pi^{+} \pi^{-}$and $\pi^{+} \pi^{-} \pi^{+} \pi^{-}$Channels in $p$ Interactions at 300 and $450 \mathrm{GeV} / \mathrm{c}$

Phys. Lett. 412B:407, 1997

Pion and Proton Production in Proton-Tungsten and Sulphur-Tungsten Interactions at $200 \mathrm{GeV} / \mathrm{c}$ per Nucleon

Yad. Phys. 57:106, 1994; Phys. At. Nucl. 57:100, 1993; IFVE-93-52

Cumulative Protons Production Accompanied by Fast $\rho^{0}$-Mesons in $40 \mathrm{GeV} / c \pi^{-} A$ Interactions

Phys. At. Nucl. 58:798, 1995; Yad. Phys. 58:863, 1995

Study of Fast $p, \bar{p}$ Yields in $\pi^{-}$Be Interaction at $40 \mathrm{GeV} / c$ with Cumulative Particle Production

Phys. Lett. 334B:431, 1994; LNF-94-032-P

Measurement of the Electromagnetic Form-Factor of the Proton in the Time-Like Region

Phys. Lett. 365B:427, 1996; LNF-95-047-P

Measurement of the Total $e^{+} e^{-} \rightarrow$ hadrons Cross Section Near the $e^{+} e^{-} \rightarrow$ nucleon $\overline{\text { nucleon }}$ Threshold

Nucl. Phys. B517:3, 1998

First Measurement of the Neutron Electromagnetic Formfactor in the Time-Like Region

Phys. Rev. D49:543, 1994; FERMILAB-PUB-93-083-E

Production of $\chi$ Charmonium via $300 \mathrm{GeV} / c$ Pion and Proton Interactions on a Lithium Target

Phys. Rev. D50:4258, 1994; FERMILAB-PUB-92-265-E

Search for Hidden Charm Resonance States Decaying into $J / \psi(1 S)$ or $\psi(2 S)$ Plus Pions

Izv. RAN, Fiz. 58-12:174, 1994; Bull. Russ. Acad. Sci. Phys. Ser. 58:2101, 1994

Primary Cosmic Ray Energy Spectrum and Spatial-Temporal Characteristics of EAS Cherenkov Light According to TACT Telescope Measurements

Astropart. Phys. 3:231, 1995

The New Tien-Shan Atmospheric Cerenkov Telescope (TACT). Contemporary Status: All-Particle Spectrum Measured

Vestn. of Moscow Univ. Fiz., Astr. 36-2:33, 1995

Energy Spectrum of Primary Cosmic Ray at Energies 100 - 2000 TeV Measured with TACT Array

FERMILAB-CONF-94-374-E

Search for Top Quark at CDF

FERMILAB-CONF-94-145-E; CDF-PUB-BOTTOM-PUBLIC-2522

Limit on the Rare Decay $B \rightarrow \mu^{+} \mu^{-} K^{ \pm}$

FERMILAB-CONF-95-201-E; CDF-PUB-BOTTOM-PUBLIC-3248

Limits on Rare $B$ Decays $B \rightarrow \mu^{+} \mu^{-} K^{ \pm}$and $B \rightarrow \mu^{+} \mu^{-} K^{*}(892)^{\circ}$

Phys. Rev. D50:69, 1994; KEK-93-181

Search for Right-Handed Currents in the Decay Chain of $K^{+} \rightarrow \mu^{+} \nu_{\mu}, \mu^{+} \rightarrow e^{+} \nu_{e} \bar{\nu}_{\mu}$

Nuovo Cim. 108A:1125, 1995; CERN-PPE-95-073

Charged Particle Multiplicity and Transverse Energy Measured in ${ }^{32} \mathrm{~S}$ Central Interactions at $200 \mathrm{GeV}$ per Nucleon

Phys. Lett. 355B:45, 1995

Production of Twin Single Hypernuclei and the $\Xi^{-}$Nuclear Interaction

Phys. Lett. 440B:386, 1998; KEK-98-173

Study of Baryon Production Mechanism in $e^{+} e^{-}$Annihilation into Hadrons

Nucl. Phys. A644:365, 1998

Quasifree $p\left(K^{-}, K^{+}\right) \Xi^{-}$Reaction in Nuclear Emulsion

Phys. Rev. Lett. 74:4997, 1995

Measurement of the Tensor Analyzing Power $T_{20}$ for deuteron $\uparrow+{ }^{12} \mathrm{C} \rightarrow p\left(0^{\circ}\right)+\mathrm{X}$ in the Region of High Internal Momenta in the Deuteron

Nuovo Cim. 107A:1925, 1994

New Results on Spectroscopy from BENKEI

Izv. RAN, Fiz. 58-12:32, 1994; Bull. Russ. Acad. Sci. Phys. Ser. 58:1976, 1994

Interactions of Energetic Primary Cosmic Ray Nuclei with Nuclei of $\mathrm{Pb}$ and $\mathrm{Fe}$ Atoms of Stratospheric XEC Targets

Izv, RAN, Fiz. 61:477, 1997

The Investigation of Central Nucleus-Nucleus Interactions at $1 \mathrm{TeV} / \mathrm{n}$ in Cosmic Rays by the Stratospheric Emulsion Chamber

Phys. Rev. D56:1391, 1997; FERMILAB-PUB-97-030-E; HEPEX-9702014

Production of Charm Mesons at High Transverse Momentum in $515 \mathrm{GeV} / \mathrm{c} \pi^{-}-\mathrm{Nucleon}$ Collisions

Phys. Rev. Lett. 81:2642, 1998; FERMILAB-PUB-97-351-E; HEPEX-9711017 Evidence for Parton $k_{T}$ Effects in High- $p_{T}$ Particle Production

Phys. Lett. 420B:397, 1998; HEPEX-9711002

Initial Results from the CHOOZ Long Baseline Reactor Neutrino Oscillation Experiment

Phys. Lett. 422B:339, 1998; CERN-PPE-97-140; DAPNIA-SPP-97-29

An EPR Experiment Testing the Non-separability of the $K^{0} \bar{K}^{0}$ Wave Function

Eur. Phys. J. C6:437, 1999; PSI-PR-98-17

Pion Correlations and Resonance Effects in $\bar{p} p$ Annihilation to $2 \pi^{+} 2 \pi^{-} \pi^{0}$

FERMILAB-CONF-94-347-E

New Preliminary Results on the Physics of Charm Hadroproduction Subprocesses

Nucl. Phys. A566:507C, 1994

Strangelet Search in S Wt Collisions at $200 \mathrm{~A} \mathrm{GeV} / \mathrm{c}$

Nucl. Phys. A 590:347C, 1995

First Look at NA52 Data on Pb-Pb Interactions at $158 \mathrm{GeV} / c$ per Nucleon

Phys. Lett. 376B:245, 1996; BUHE-96-03

Antinuclei Production in $\mathrm{Pb} \mathrm{Pb}$ Collisions at $158 \mathrm{~A} \mathrm{GeV} / c$ 
Appelquist $96 \mathrm{~B}$

Appelshauser 97

Appelshauser 98

A ppelshauser $98 \mathrm{~B}$

Apsimon 93

A rakelyan 95

Ardashev 97

Arena 95

Arima 96

A risaka 98

A risawa 94

Armbruster 94

Armbruster 98

Armbruster 98B

Armbruster $98 \mathrm{C}$

Armbruster $98 \mathrm{D}$

Armstrong 94

Armstrong $94 \mathrm{~B}$

Armstrong 95

Armstrong 96

Armstrong $96 \mathrm{~B}$

Armstrong 97

Armstrong $97 \mathrm{~B}$

Armstrong 97C

Armstrong $97 \mathrm{D}$

Armstrong 98B

Armstrong 99

Arndt 93

Arndt 94

Arndt 95

Arndt 95B

Arneodo 92

Arneodo 94

Arneodo $94 \mathrm{~B}$

Arneodo $94 \mathrm{C}$

Arneodo 95

Arneodo $95 \mathrm{C}$

Arneodo $95 \mathrm{D}$
Phys. Rev. Lett. 76:3907, 1996; BUHE-96-02

Strangelet Search in $\mathrm{Pb} \mathbf{P b}$ Interactions at $158 \mathrm{GeV} / c$ per Nucleon

Eur. Phys. J. C2:661, 1998; IKF-HENPG-6-97; HEPEX-9711024

Hadronic Expansion Dynamics in Central $\mathrm{Pb}+\mathrm{Pb}$ Collisions at $158 \mathrm{GeV}$ per Nucleon

Phys. Rev. Lett. 82:2471, 1999; NUCLEX-9810014

Baryon Stopping and Charged Particle Distributions in Central Pb+Pb Collisions at $158 \mathrm{GeV}$ per Nucleon

Phys. Lett. 444B:523, 1998; NUCLEX-9810005

$\Xi$ and $\Xi$ Production in $158 \mathrm{GeV} /$ Nucleon $\mathrm{Pb}+\mathrm{Pb}$ Collisions

Z. Phys. C61:383, 1994; CERN-PPE-93-185

Photo- and Hadro-Production of $\phi(1020), K^{*}(892)^{0}$ and $\bar{K}^{*}(892)^{\circ}$ Mesons in the Energy Range 65 to $175 \mathrm{GeV}$

Yad. Phys. 58:263, 1995; Phys. At. Nucl. 58:219, 1995

Multifragmentation of Heavy Nuclei in the Bremsstrahlung Beam with $E_{\max }(\gamma)=1.85 \mathrm{GeV}$

MSU-97-40-491

A Study of the Charmed Particles Production in $p p$ Interactions at $70 \mathrm{GeV} / \mathrm{c}$ with the SVD Set-UP

Nuovo Cim. 108A:417, 1995

Semiinclusive Analysis of Multiparticle Production in hadron-hadron Collisions at $\sqrt{s}=16.7 \mathrm{GeV}$ in Terms of Universal Multifractals

Phys. Rev. D55:19, 1997; KEK-96-80; HUPD-9611; KOBE-HEP-96-03; KUNS-1393; NGTHEP-96-01; OULNS-96-01 Precise Measurement of Bhabha Scattering at a Center-of-Mass Energy of $57.77 \mathrm{GeV}$

Phys. Lett. 432B:230, 1998

Search for the Lepton-Family Number Violating Decays $K_{L} \rightarrow \pi^{0} \mu^{ \pm} e^{\mp}$

Nucl. Phys. B424:241, 1994

Observation of Attenuation Behaviour of Hadrons in Extremely High Energy Cosmic Ray Interactions: New Hadronic State?

Phys. Lett. 348B:19, 1994

Anomaly in the Time Distribution of Neutrinos from a Pulsed Beam Stop Source

Phys. Rev. C57:3414, 1998; HEPEX-9801007

KARMEN Limits on $\nu_{e} \rightarrow \nu_{\tau}$ Oscillations in 2- $\nu$ and 3- $\nu$ Mixing Schemes

Phys. Rev. Lett. 81:520, 1998; HEPEX-9806024

Measurement of the Energy Spectrum of $\nu_{e}$ from Muon Decay and Implications for the Lorentz Structure of the Weak Interaction

Phys. Lett. 423B:15, 1998

Measurement of the Weak Neutral Current Excitation ${ }^{12} \mathrm{C}\left(\nu_{\mu}, \nu_{\mu}^{\prime}\right)^{12} \mathrm{C}^{*}\left(1^{+}, 1,15.1 \mathrm{MeV}\right)$ at $E_{\nu_{\mu}}=$ $29.8 \mathrm{MeV}$

Nucl. Phys. A629:507C, 1998; OSAKA-97 p. 507C

The Advantage of a Pulsed Neutrino Source in the Search for Neutrino Oscillations and Neutrino Nuclear Interactions

Phys. At. Nucl. 57:1513, 1994; Yad. Phys. 57:1587, 1994

Recent Results in Light Quark Meson Spectroscopy from FERMILAB Experiment $E-780$

WILLIAMSBURG-94 p. 628

Pontecorvo Reactions in Antiproton Annihilation on Deuterium

Phys. Rev. D52:4839, 1995; FERMILAB-PUB-95-092-E

Study of the $\eta_{c}(1 S)$ State of Charmonium Formed in $\bar{p} p$ Annihilations and a Search for the $\eta_{c}(2 S)$

Phys. Lett. 385B:479, 1996

Precision Measurements of Antiproton-Proton Forward Elastic Scattering Parameters in the 3.7 to 6.2 GeV/c Region

Phys. Rev. D54:7067, 1996

Observation of the Radiative Decay $J / \psi(1 S) \rightarrow e^{\dagger} e^{-\gamma}$

Phys. Rev. Lett. 79:3351, 1997; NUCLEX-9709005

Antiproton Production in 11.5 A GeV/c Au Pb Nucleus Nucleus Collisions

Phys. Rev. Lett. 79:3612, 1997; NUCLEX-9706004

Search for Charged Strange Quark Matter Produced in $11.5 \mathrm{~A} \mathrm{GeV} / c \mathrm{Au} \mathrm{Pb}$ Collisions

Phys. Rev. D55:1153, 1997

Measurement of the Branching Ratios $\psi(2 S) \rightarrow e^{+} e^{-}, \psi(2 S) \rightarrow J / \psi(1 S) \pi \pi, \psi(2 S) \rightarrow J / \psi(1 S) \eta$

Phys. Rev. D56:2509, 1997

Two-body Neutral Final States Produced in $\bar{p} p$ Annihilations at $2.911 \leq \sqrt{s} \leq 3.686 \mathrm{GeV}$

NUCLEX-9811001

Electroproduction of the $S_{11}(1535)$ Resonance at High Momentum Transfer

Phys. Rev. C59:1829, 1999

Search for Neutral Strange Quark Matter in High Energy Heavy Ion Collisions

Phys. Rev. C48:1926, 1993; Phys. Rev. C49:1229, 1994; VPICAPS-93.1

Analysis of the Reaction $\pi^{+}$deuteron $\rightarrow p p$ to $500 \mathrm{MeV}$

Phys. Rev. C50:2731, 1994; VPI-CAPS-7-2

An Updated Analysis of nucleon nucleon Elastic Scattering Data to $1.6 \mathrm{GeV}$

Phys. Rev. C52:2120, 1995; VPI-CAPS-95-2

Updated Analysis of $\pi$ nucleon Elastic Scattering Data to $2.1 \mathrm{GeV}$ : The Baryon Spectrum

Phys. Rev. C52:2246, 1995

Extraction of the $\pi$ nucleon nucleon Coupling Constant from nucleon nucleon Scattering Data

Phys. Rept. 240:301, 1994; CERN-PPE-92-113

Nuclear Effects in Structure Functions

Phys. Lett. 332B:195, 1994

Quasielastic $J / \psi(1 S)$ Muoproduction from Hydrogen, Deuterium, Carbon and Tin

Nucl. Phys. B429:503, 1994; CERN-PPE-94-146 Exclusive $\rho^{0}$ and $\phi(1020)$ Muoproduction at Large $q^{2}$

Phys. Rev. D50:R1, 1994; CERN-PPE-94-032 A Re-Evaluation of $F_{2}^{n} / F_{2}^{p}$ and $F_{2}^{p}-F_{2}^{n}$

Nucl. Phys. B441:12, 1995; CERN-PPE-95-32

The Structure Function Ratios $F_{2}^{\mathrm{Li}} / F_{2}^{\text {deuteron }}$ and $F_{2}^{\mathrm{C}} / F_{2}^{\text {deuteron }}$ at Small $x$

Phys. Lett. 364B:107, 1995; CERN.PPE-95-138

Measurement of the Proton and the Deuteron Structure Functions, $F_{2}^{p}$ and $F_{2}^{\text {deuteron }}$

Nuovo Cim. 108A:1247, 1995

Diffractive Production of Vector Mesons in Muon Scattering on Nucleons and Nuclei 
Arneodo 96

Arneodo 96B

Arneodo $96 \mathrm{C}$

Arneodo 97

Arnold 95

Arnold 96

Arnold 98

Arpesella 94

Arpesella 96

Arpesella 97

Arrington 96

Arrington 98

Arroyo 94

Artemiev 94

Artemiev 96

Artemov 95

Artemov 96

Artuso 93

Artuso 94

Artuso 95

Artuso 95B

Artuso 96

Artuso 97

Artuso 97B

Artuso 98

Arvieux 97

Arvieux 98

Asai 94

Asai 95

Asakawa 96

Asakimori 94

Asakimori 95

Aseev 97

Ashitkov 98

Ashktorab 96

Ashktorab 98

Ashktorab 98B

Ashktorab $98 \mathrm{C}$
Nucl. Phys. B487:3, 1997; HEPEX-9611022

Accurate Measurement of $F_{2}^{\text {deuteron }} / F_{2}^{p}$ and $R^{\text {deuteron }}-R^{p}$

Nucl. Phys. B481:3, 1996

The A Dependence of the Nuclear Structure Function Ratios

Nucl. Phys. B481:23, 1996

The $Q^{2}$ Dependence of the Structure Function Ratio $F_{S_{n}}^{2} / F_{C}^{2}$ and the Difference $R_{S_{n}}-R_{C}$ in Deep Inelastic Muon Scattering

Nucl. Phys. B483:3, 1997; HEPPH-9610231

Measurement of the Proton and Deuteron Structure Functions, $F_{2}^{p}$ and $F_{2}^{\text {deuteron, }}$, and of the Ratio $\sigma_{L} / \sigma_{T}$

Pisma Zh. Eksp. Teor. Fiz. 61:168, 1995; Jetp Lett. 61:170, 1995

Observation of Two Neutrino Double Beta Decay of ${ }^{118} \mathrm{Cd}$ with the Tracing Detector NEMO-2

Z. Phys. C72:239, 1996

Double- $\beta$ Decay of ${ }^{116} \mathrm{Cd}$

Eur. Phys. J. A2:411, 1998

Kinetic Energy Spectrum and Polarization of Neutrons from the Reaction ${ }^{12} \mathrm{C}(p, n) \mathrm{X}$ at $590 \mathrm{MeV}$

Eur. Lett. 27:29, 1994; LNGS-94-95

Search for $\beta \beta$ Decay of ${ }^{86} \mathrm{Zr}$ and ${ }^{150} \mathrm{Nd}$ to Excited States of ${ }^{98} \mathrm{Mo}$ and ${ }^{150} \mathrm{Sm}$

Phys. Lett. 389B:452, 1996

Measurement of the ${ }^{3} \mathrm{He}\left({ }^{3} \mathrm{He}, 2 p\right){ }^{4} \mathrm{He}$ Cross Section within the Solar Gamow Peak

Phys. Rev. C57:2700, 1998; NUCLEX-9707003

The Cross Section of ${ }^{3} \mathrm{He}\left({ }^{3} \mathrm{He}, 2 p\right){ }^{4} \mathrm{He}$ Measured at Solar Energies

Phys. Rev. C53:2248, 1996; NUCLEX-9504003

Inclusive Electron Scattering from Nuclei at $x=1$

Phys. Rev. Lett. 82:2056, 1999; OAP-747; NUCLEX-9811008

Inclusive Electron - Nucleus Scattering at Large Momentum Transfer

Phys. Rev. Lett. 72:3452, 1994; NEVIS-1498

A Precise Measurement of the Weak Mixing Angle in Neutrino Nucleon Scattering

Phys. Lett. 345B:564, 1995; ITEP-94-66

Observation of ${ }^{150} \mathrm{Nd} 2 \beta 2 \nu$ Decay in the Time Projection Chamber Experiment

Phys. At. Nucl. 59:6, 1996; Yad. Phys. 59:10, 1996

Half-Life Measurement $T_{1 / 2}\left(2 \beta 2 \nu{ }^{150} \mathrm{Nd}\right)$ in the Experiment with Time Projection Chamber in Magnetic

Field
Yad. Phys. 60:277, 1997; Phys. At. Nucl. 60:218, 1997; JINR-P1-95-330

Determination of Formfactors Parameters $\lambda^{+}, \lambda^{\circ}$ in $K_{\mu 3}$-Decay

Yad. Phys. 60:2205, 1997; Phys. At. Nucl. 60:2023, 1997; JINR-P1-96-512

Detemination of Form Factor Parameters $\lambda_{+}$and $\lambda_{0}$ in $K_{\mu 3^{-}}$and $K_{e 3}$-Decays

Phys. Rev. D50:5484, 1994; CLEO-93-17; CLNS-93-1245

Measurement of Cross Section for $\gamma \gamma \rightarrow p \bar{p}$

Phys. Rev. Lett. 72:3762, 1994; CLNS-94-1281; CLEO-94-11

A Measurement of the Branching Fraction $B\left(\tau^{-} \rightarrow\right.$ hadron $\left.^{-} \pi^{0} \nu_{\tau}\right)$

Phys. Rev. Lett. 75:785, 1995; CLNS-95-1331; CLEO-95-5

A Search for $B \rightarrow \ell \bar{\nu}$

Phys. Lett. 378B:364, 1996; CLNS-95-1387; CLEO-95-23

Measurement of the Branching Fraction for $D_{-}^{-} \rightarrow \phi(1020) \pi^{-}$

Nucl. Instr. and Meth. A384:39, 1996; HEPSY-96-2; HEPEX-9610009

$B$ Decay Studies at CLEO

Phys. Lett. 399B:321, 1997; CLNS-97-1461; CLEO-97-2; HEPEX-9702007

Study of the $B^{0}$ Semileptonic Decay Spectrum at the $\Upsilon(4 S)$ Resonance

Phys. Rev. Lett. 80:3193, 1998; CLNS-97-1517; CLEO-97-24; HEPEX-9712023

Measurement of $\operatorname{Br}\left(D^{\circ} \rightarrow K^{-} \pi^{+}\right)$using Partial Reconstruction of $\bar{B} \rightarrow D^{*}(2010)^{+} \times \ell^{-} \bar{\nu}$

Phys. Rev. Lett. 82:3020, 1999; CLNS-98-1589; CLEO-98-17; HEPEX-9811027

First Observation of the Decay $B^{0} \rightarrow D^{*}(2010)^{+} D^{*}(2010)^{-}$

Z. Phys. C76:465, 1997; ANL-HEP-PR-98-16

Proton-Proton Elastic Scattering Analyzing Power in the 2.16 to $2.28 \mathrm{GeV}$ Energy Region

DUBNA.96, v. II, p. 120

Proton-Proton Elastic Scattering Analyzing Power in the 2.16 to $2.28 \mathrm{GeV}$ Energy Region

Phys. Lett. 323B:90, 1994; UT-ICEPP-93-09

Search for Shortlived Neutral Bosons in Orthopositronium Decay

Phys. Lett. 357B:475, 1995

New Measurement of the Orthopositronium Decay Rate

FERMILAB-CONF-96-193-E; CDF-PUB-QCD-PUB-3758

High Mass Multi-Jet Events at the Fermilab Proton-Antiproton Collider

Jour. of Phys. G 20:1257, 1994

Multiple Photon Emission in Heavy Particle Decays

UWSEA-PUB-95-07; ASTROPH-9509091

Energy Spectra and Elemental Composition of Nuclei above $100 \mathrm{TeV}$ from a Series of the JACEE Balloon Flight

Phys. Rev. C56:596R, 1997

Search for a Narrow Resonance Structure in Pion Production from $p$ Cu Near $350 \mathrm{MeV}$

Phys. At. Nucl. 61:910, 1998; Yad. Phys. 61:1002, 1998

Search for Double Beta Decay of ${ }^{100}$ Mo with Liquid Argon Ionization Chamber (First Results)

Nucl. Phys. A610:139C, 1996

Particle Production in Au Au Collisions From BNL E866

Phys. Rev. C57:466, 1998

Particle Production at High Baryon Density in Central Au Au Reactions at $11.6 \mathrm{~A} \mathrm{GeV} / \mathrm{c}$

Phys. Rev. C57:1416, 1998

Proton, Deuteron, and Triton Emission at Target Rapidity in Au Au Collisions at 10.2 A GeV: Spectra and Directed Flow

Nucl. Phys. A638:427C, 1998; TSUKUBA-97 p. 427C

Centrality and Collision System Dependence of Antiproton Production from $p+A$ to Au Au Collisions at AGS Energies 
Ashktorab $98 \mathrm{D}$

Ashktorad 95

Aslanyan 96

Aslanyan 98

Asner 95

Asner 96

Asner 97

Asner 98

Asner 99

A so 95

Aso $95 B$

Asratyan 94

Asratyan 95

Asratyan 97

Assamagan 94

Assamagan 96

Assamagan 98

Aston 94

Astone 96

Astur 95

Athanas 94

Athanas 97

Athanas 98

Athanassopou 95

Athanassopou 96

Athanassopou 96B

Athanassopou 97

Athanassopou $97 \mathrm{~B}$

Athanassopou $97 \mathrm{C}$

Atrashkevich 94

Auce 94

Auce 96

Audit 97

Augier 94

Aulchenko 95

Aulchenko 98

Aulchenko 98B

Aulchenko 99

Aumann 94
Phys. Rev. C58:3523, 1998

Kaon Production in $\mathrm{Au}+\mathrm{Au}$ Collisions at $11.6 \mathrm{~A} \cdot \mathrm{GeV} / \mathrm{c}$

Nucl. Phys. A590:249C, 1995

Recent Results from E866

JINR-P1-96-150

Analysis of Nucleon Clusters in Nucleus-Nucleus Interactions $\left({ }^{4} \mathrm{He},{ }^{12} \mathrm{C}\right)+{ }^{12} \mathrm{C}$ at $4.2 \mathrm{~A} \mathrm{GeV} / \mathrm{c} \mathrm{Momentum}$

Kr. Soob. JINR $87: 26,1998$

Stable dibaryon $(S=-2)$ Found in Dubna

Phys. Rev. D53:1039, 1996; CLNS-95-1338; CLEO-95-8

Search for Exclusive Charmless Hadronic $B$ Decays

Phys. Rev. D54:4211, 1996; CLNS-96-1390; CLEO-96-2

Analysis of $D^{\circ} \rightarrow K \bar{K} \mathrm{X}$ Decays

Phys. Rev. Lett. 79:799, 1997; CLNS-97-1474; CLEO-97-6; HEPEX-9704014

Search for the Decays $B^{\mathbf{0}} \rightarrow D^{(*)}+D^{(*)-}$

Phys. Rev. D58:092001, 1998; CLNS-97-1531; CLEO-97-30; HEPEX-9803022

Radiative Decay Modes of the $D^{0}$ Meson

CLNS-99-1601; CLEO-99-1; HEPEX-9902022

Hadronic Structure in the Decay $\tau^{-} \rightarrow \nu_{\tau} \pi^{-} \pi^{0} \pi^{0}$ and the Sign of the $\tau$ Neutrino Helicity

KEK-95-19

Charm Production in Two Photon Processes

Phys. Lett. 363B:249, 1995; KEK-95-119

Measurement of Charm Production in Two Photon Processes using Inclusive Lepton Events at TRISTAN

Z. Phys. C61:563, 1994; CERN-PPE-93-190

Observation of $D_{31}(2536)+$ Meson Production by Neutrinos in BEBC

Z. Phys. C68:43, 1995; CERN-PPE-95-48

Study of $D^{*}(2010)^{+}$and Search for $D_{2}^{*}(2460)^{\circ}$ Production by Neutrinos in BEBC

Z. Phys. C76:647, 1997; FERMILAB-PUB-97-289-E; CERN-PPE-97-037

Production of $D^{*}(\mathbf{2 0 1 0})^{+}$Mesons by High Energy Neutrinos from the Tevatron

Phys. Lett. 335B:231, 1994; PSI-PR-94-19

Measurement of the Muon Momentum in Pion Decay at Rest using a Surface Muon Beam

Phys. Rev. D53:6065, 1996; PSI-PR-95-28 Upper Limit of the Muon-Neutrino Mass and Charged Pion Mass from Momentum Analysis of a Surface Muon Beam

Phys. Lett. 434B:158, 1998; PSI-PR-98-11

Search for a Heavy Neutrino State in the Decay $\pi^{+} \rightarrow \mu^{+} \nu_{\mu}$

SLAC-PUB-5606

Evidence for $\rho(1300)$ in the Reaction $K^{-} p \rightarrow \pi^{+} \pi^{-} \Lambda$ at $11 \mathrm{GeV} / c$

Phys. Lett. 385B:421, 1996; LNF-96-48-P Upper Limit for a Gravitational-Wave Stochastic Background with the EXPLORER and NAUTILUS Resonant Detectors

Nucl. Phys. (PS) B39:172, 1995

Overview of New QCD Analyses at Do

Phys. Rev. Lett. 73:3503, 1994; Phys. Rev. Lett. 74:3090, 1995; CLNS-94-1286; CLEO-94-16; HEPEX-9406004

Semileptonic Branching Fractions of Charged and Neutral $B$ Mesons

Phys. Rev. Lett. 79:2208, 1997; CLNS-97-1486; CLEO-97-12; HEPEX-9705019

Measurement of the $\bar{B} \rightarrow D \ell \bar{\nu}$ Partial Width and Form Factor Parameters

Phys. Rev. Lett. 80:5493, 1998; CLNS-98-1541; CLEO-98-2; HEPEX-9802023

First Observation of the Cabibbo Suppressed decay $B^{+} \rightarrow \bar{D}^{0} K^{+}$

Phys. Rev. Lett. 75:2650, 1995

Candidate Events in a Search for $\bar{\nu}_{\mu} \rightarrow \bar{\nu}_{e}$ Oscillations

Phys. Rev. Lett. 77:3082, 1996; LA-UR-96-1582; NUCLEX-9605003

Evidence for $\bar{\nu}_{\mu} \rightarrow \bar{\nu}_{e}$ Oscillations from the LSND Experiment at LAMPF

Phys. Rev. C54:2685, 1996; LA-UR-96-1326; NUCLEX-9605001 Evidence for Neutrino Oscillations from Muon Decay at Rest

Phys. Rev. C55:2078, 1997; LA-UR-96-3600; NUCLEX-9705001

Measurements of the Reactions ${ }^{12} \mathrm{C}\left(\nu_{e}, e^{-}\right){ }^{12} \mathrm{Nit}$ and ${ }^{12} \mathrm{C}\left(\nu_{e}, e^{-}\right){ }^{12} \mathrm{Nit}^{*}$

Phys. Rev. C58:2489, 1998; UCRHEP-E191; LA-UR-97-1998; NUCLEX-9706006

Evidence for $\nu_{\mu} \rightarrow \nu_{e}$ Oscillations from Pion Decay in Flight Neutrinos

Phys. Rev. Lett. 81:1774, 1998; UCRHEP-E197; NUCLEX-9709006

Evidence for $\nu_{\mu} \rightarrow \nu_{e}$ Oscillations from the LSND

Izv. RAN, Fiz. 58-12:45, 1994; Bull. Russ. Acad. Sci. Phys. Ser. 58:1988, 1994

The Mass Composition of Primary Cosmic Rays around the Knee of the Energy Spectrum

Phy's. Rev. C50:871, 1994

Reaction Cross Sections for $75-180 \mathrm{MeV} \alpha$ Particles on Targets from ${ }^{12} \mathrm{C}$ to ${ }^{208} \mathrm{~Pb}$

Phys. Rev. C53:2919, 1996

Reaction Cross Section for 38, 65, and $97 \mathrm{MeV}$ Deuterons on Targets from ${ }^{9} \mathrm{Be}$ to ${ }^{208} \mathrm{~Pb}$

Nucl. Phys. A614:461, 1997; DAPNIA-SPHN-96-19

Study of Three-Nucleon Mechanisms in the Photodisintegration of ${ }^{3} \mathrm{He}$

Phys. Let t. 344B:451, 1995; CERN-PPE-94-160

Measurement of the Proton-Antiproton Total Cross Section at the Sp̄pS Collider by a Luminosity Dependent Method

BUDKERINP-95-56

Beginning of the Experiments with SND Detector at $e^{+} e^{-}$Collider VEPP-2M

Phys. Lett. 440B:442, 1998 ; HEPEX-9807016

First Observation of $\phi(1020) \rightarrow \pi^{0} \pi^{0} \gamma$ Decay

Phys. Lett. 436 B:199, 1998

Search for the Decay $\phi(1020) \rightarrow \eta \pi^{0} \pi^{0} \gamma$

Pisma Zh. Eksp. Teor. Fiz. 69:87, 1999

Decay $\phi(1020) \rightarrow \eta^{\prime} \gamma$

Nucl. Phys. A569:157C, 1994

Evidence for Two-Phonon Giant-Dipole Resonance from Inclusive Measurements of ${ }^{187}$ Au Target Dissociation 
Aunola 95

Avdeyev 97

Avdeyev 98

A verbeck 97

A verichev 95

A verichev 95B

A verichev 95C

Aversa 95

A very $93 \mathrm{~B}$

A very 94

A very $94 \mathrm{~B}$

Avery 95

Avery 97

A vetyan 96

A vignone 98

A vignone 98B

Avila 99

Avramenko 94

Avramenko 96

Avrigeanu 98

Awes 95

Awes 95B

A wes 96

Azhgirey 94

Azhgirey 94B

Azhgirey 95

Azhgirey $95 \mathrm{~B}$

Azhgirey 96

Azhgirey 96B

Azhgirey 97

Azhgirey 97B

Azhgirey 98

Azhgirey 98B

Azzi 96

Baarmand 96

Babu 94
Pisma Zh. Eksp. Teor. Fiz. 62:690, 1995; Jetp Lett. 62:706, 1995

Double Beta Processes in ${ }^{82}$ Mo

Kr. Soob. JINR 82:71, 1997

"Thermal" Multifragmentation in $p+\mathrm{Au}$ Collisions at Relativistic Energies

Eur. Phys. J. A3:75, 1998

Thermal Multifragmentation in $p$ Au Interactions at 2.16, 3.6 and $8.1 \mathrm{GeV}$ Incident Energies

Z. Phys. A 359:65, 1997

Production of $\pi^{\mathbf{0}}$ and $\eta$ Mesons in Carbon-Induced Relativistic Heavy-Ion Collisions

Kr. Soob. JINR 69:37, 1995

Cumulative Pion and Proton Production in $p$ deuteron Collisions at 4.45 and $8.9 \mathrm{GeV} / \mathrm{c}$

Kr. Soob. JINR 69:27, 1995

One Spin Pion Asymmetry in the deuteron $\uparrow$ atom $\rightarrow \pi^{ \pm}\left(90^{\circ}\right)$ ] X Process

Phys. At. Nucl. 60:1643, 1997; Yad. Phys. 60:1799, 1997; JINR-E1-95-506

One-Spin Pion Asymmetry in deuteront nucleus $\rightarrow \pi^{ \pm}$X Processes near $\theta_{\pi}=90^{\circ}$

LNF-95-051-PC; ROME-95 p. 11

Observations of Cosmic Ray Positrons during the 1993 Flight of the NMSU/WIZARD TS 3 Balloon-Borne A pparatus

Phys. Lett. 325B:257, 1994; CLNS-93-1260; CLEO-93-22

Observation of $\Lambda_{c}^{+}$Decays to $\Lambda \pi^{+} \pi^{0}, \Sigma^{0} \pi^{+}, \Sigma^{0} \pi^{+} \pi^{0}$, and $\Sigma^{0} \pi^{-} \pi^{+} \pi^{+}$

Phys. Lett. 331 B:236, 1994; Phys. Lett. 342B:453, 1995; CLNS-94-1280; CLEO-94-10

Production and Decay of $D_{1}(2420)^{\circ}$ and $D_{2}^{*}(2460)^{\circ}$

Phys. Lett. 337B:405, 1994; CLNS-94-1290

Measurement of the Ratios of Form-Factors in the Decay $D_{s}^{+} \rightarrow \phi(1020) e^{+} \nu_{e}$

Phys. Rev. Lett. 75:4364, 1995; CLNS-95-1352; CLEO-95-14; HEPEX-9508010

Observation of a Narrow State Decaying into $\Xi_{c}(2460)^{+} \pi^{-}$

Phys. Rev. D55:R1119, 1997; CLNS-96-1434; CLEO-96-17

Search for $\phi(1020)$ Mesons in $\tau^{ \pm}$-Lepton Decay

Yad. Phys. 59:110, 1996; Phys. At. Nucl. 59:102, 1996

Coherent Dissociation ${ }^{16} \mathrm{O} \rightarrow 4 \alpha$ in Emulsion at $4.5 \mathrm{GeV} / \mathrm{c}$ per Nucleon

Phys. At. Nucl. 61:1137, 1998; Yad. Phys. 61:1237, 1998

First Results from SOLAX: A New Technique to Detect Axions from the Sun

Phys. Rev. Lett. 81:5068, 1998

Experimental Search for Solar Axions via Coherent Primakoff Conversion in a Germanium Spectrometer

Phys. Lett. 445B:419, 1999; FERMILAB-PUB-98-313-E

A Measurement of the Proton-Antiproton Total Cross Section at $\sqrt{s}=1.8 \mathrm{TeV}$

JINR-E1-94-311

Pion Momentum Spectra in Nuclear Charge Exchange Reactions $A\left({ }^{3} \mathrm{H},{ }^{3} \mathrm{He}\right)$

Nucl. Phys. A596:355, 1996

Pion Production in Nuclear Charge Exchange Reactions $A\left({ }^{3} \mathrm{H},{ }^{3} \mathrm{He}\right)$

Phys. Rev. C60:017602, 1999; NUCLEX-9812003

Energy Dependence of the Isomeric Cross Section Ratio in the ${ }^{58} \mathrm{Ni}(n, p)^{58} \mathrm{Co}, g$ Reactions

Z. Phys. C65:207, 1995

Two-Proton Correlations in the Target Fragmentation Region of Nuclear Collisions at $200 \mathrm{~A} \mathrm{GeV}$

Z. Phys. C69:67, 1995; IKP-MS-95-0601

Bose-Einstein Correlations of Soft Pions in Ultrarelativistic Nucleus-Nucleus Collisions

Phys. Lett. 381B:29, 1996; HEPEX-9601007

Azimuthal Correlations in the Target Fragmentation Region of High Energy Nuclear Collisions

WILLIAMSBURG-94 p. 415

New Data on $T_{20}$ in Inclusive Deuteron Breakup at $9 \mathrm{GeV} / \mathrm{c}$ on Protons and Correlation between Polarization Observables

WILLIAMSBURG-94 p. 423

Measurements of $T_{20}$ in Backward Elastic deuteron $p$ Scattering at Deuteron Momenta $3.5-6 \mathrm{GeV} / \mathrm{c}$

Phys. Lett. 361 B:21, 1995; JINR-E1-95-263

First Measurement of the Tensor Analyzing Power $T_{20}$ in Inelastic (deuteron, deuteron') X Scattering at $0^{\circ}$ on $p$ and ${ }^{12} \mathrm{C}$ at 4.5 and $5.5 \mathrm{GeV} / c$

JINR-E1-95-357

$T_{20}$ in Inclusive Inelastic (deuteron, deuteron') $\mathrm{X}$ Scattering at $0^{\circ}$ on Protons and ${ }^{12} \mathrm{C}$

Phys. Lett. 387B:37, 1996

Measurement of the Tensor Analyzing Power $T_{20}$ in Inclusive Deuteron Breakup at $\theta \mathrm{GeV} / \mathrm{c}$ on $\mathrm{Hydrogen}$

and Carbon $77: 23,1996$

Polarization Transfer in the ${ }^{12} \mathrm{C}$ (deuteron $\left.\uparrow, p \uparrow\right) \mathrm{X}$ Reaction for Deuteron Momenta between 5.8 and $9.0 \mathrm{GeV} / \mathrm{c}$

Yad. Phys. 61:494, 1998; Phys. At. Nucl. 61:432, 1998; JINR-P1-97-174

Measurement of the Tensor Analyzing Power $T_{20}$ in Reactions $p$ (deuteron, $p$ ) deuteron and $p$ (deuteron, p) $p n$ at $0^{\circ}$ for Incident Deuteron Momenta between $3.5-6.5 \mathrm{GeV} / \mathrm{c}$

Phys. Lett. 391B:22, 1997

Tensor Analyzing Power $T_{20}$ in Backward Elastic deuteron $p$ Scattering and Breakup at $0^{\circ}$ between $3.5-$ 6.5 GeV/c

Kr. Soob. JINR 88:17, 1998

Tensor Analyzing Power $T_{20}$ in Inelastic (deuteron, deuteron') X Scattering at $0^{\circ}$ on $p$ and ${ }^{12} \mathrm{C}$ from 4.5 to 9.0 GeV/c

JINR-P1-98-199

Differential Cross Section, Tensor $A_{y y}$ and Vector $A_{y}$ Analyzing Powers in the ${ }^{12} \mathrm{C}$ (deuteron, $p$ ) X Reaction

at $\theta \mathrm{Gev} / \mathrm{c}$ and $85 \mathrm{mrad}$ Emission Angle of Protons

FERMILAB-CONF-96-118-E; CDF-PUB-TOP-PUBLIC-3679

Top Production at $C D F$

FERMILAB-CONF-96-260-E

Do Measurements of Beauty Production in $p \bar{p}$ Collisions at $\sqrt{s}=1.8 \mathrm{TeV}$

Phys. Lett. 321 B:140, 1994; UM-TH-93-26; JHU-TIPAC-930026; UM-AC-93-20

Closing the Windows on MeV Tau Neutrinos 
Babusci 96

Babusci 98

Bacci 93

Bacci 94

Bacci 94B

Bachler 94

Bachler 94B

Bachler 94C

Bachman 95

Back 98

Back 98B

Back 99

Backenstoss 96

Backenstoss 98

Backenstoss 98B

Backovic 94

Badala 95

Badala 96

Badala 96B

Badala $96 \mathrm{C}$

Badala 97

Badala $97 \mathrm{~B}$

Badala 98

Badala 98B

Badalyan 96

Badalyan 97

Badelek 95

Badertscher 96

Badgett 94

Bagan 95

Baglin 94

Baglin 95

Baglin 95B

Baglin 96

Baglin 96B

Baglin 96C

Baglin 98

Baglin 98B
Phys. Rev. C54:1766, 1996

Quasideuteron Effect with a Polarized $\gamma \uparrow$ Ray Beam

Nucl. Phys. A633:683, 1998; NUCLEX-9801001

Deuteron Photodisintegration with Polarized Photons in the Energy Range $30-50 \mathrm{MeV}$

Astropart. Phys. 2:13, 1994; DAPNIA-SPP-93-16; LMGS-93-80

A Search for Strongly Interacting Massive Particles in the Galactic Halo

Astropart. Phys. 2:117, 1994; DAPNIA-SPP-94-02; LNGS-94-88

Dark Matter Search with Calcium Fluoride Crystals

Nucl. Phys. (PS) 35:159, 1994; LNGS-93-83: GRAN SASSO-93 p. 159

Dark Matter Search with Calcium Fluoride Crystals

Phys. Rev. Lett. 72:1419, 1994

Charged Particles Spectra in Central S+S Collisions at $200 \mathrm{GeV} / \mathrm{c}$ per Nucleon

Nucl. Instr, and Meth. A343:288, 1994

Study of Particle Spectra with an Optically Readout RICH Detector in the NA35 Experiment

2. Phys. C61:551, 1994; MPI-PHE-93-27

An Investigation of Intermittency in Proton-Gold, Oxygen-Gold, Sulphur-Gold and Sulphur-Sulphur Interactions at $200 \mathrm{GeV}$ per Nucleon

Phys. Rev. C52:495, 1995

Measurement of $n p \rightarrow p p \pi^{-}$at $443 \mathrm{MeV}$

Nucl. Phys. A638:57C, 1998; TSUKUBA-97 p. 57C

A $u+A u$ Reactions at the AGS: Experiments E866 and E917

Nucl. Phys. A638:407C, 1998; TSUKUBA-97 p. 407C

An Excitation Function at the AGS: E917 - Probing the Dynamics of Heavy Ion Collisions

NUCLEX-9904005

Fission Hindrance in Hot ${ }^{216} \mathrm{Th}$ : Evaporation Residue Measurements

Phys. Lett. 379B:60, 1996

Contribution of Initial State Interactions to the Three-Nucleon Absorption of Pions by ${ }^{3} \mathrm{He}$

Phys. Rev. C58:3469, 1998

Two Step Processes in Pion Single Charge Exchange on deuteron at $\boldsymbol{T}_{\boldsymbol{\pi}}+=239 \mathrm{MeV}$

Phys. Rev. C58:942, 1998

Total and Partial $\pi^{+}$Absorption Cross Sections on ${ }^{4} \mathrm{He}$ in the $\Delta\left(1232 P_{33}\right)$ Resonance Region

Phys. Rev. C50:1097, 1994

Temperature of Protons in Inelastic (deuteron, $\mathrm{He}, \mathrm{C})+(\mathrm{C}, \mathrm{Ta})$ Collisions at $4.2 \mathrm{~A} \cdot \mathrm{GeV} / \mathrm{c}$

Phys. Rev. Let.t. 74:4779, 1995

Photon-Photon Correlation in ${ }^{36} \mathrm{Ar}+{ }^{27} \mathrm{Al}$ Reaction at $95 \mathrm{MeV} / \mathrm{Nucleon}$

Phys. Rev. C53:1782, 1996

Nuclear Stopping in Heavy-Ion Collisions at $100 \mathrm{MeV} / \mathrm{Nucleon}$ from Inclusive and Exclusive Neutral Pion

Measurements
$\mathrm{Nucl}$. Instr. and Meth. A388:109, 1997; INFN-BE-96-01

Search for $\eta$ Meson Production in Heavy Ion Collisions at $100 \mathrm{MeV} / \mathrm{nucleon}$

INFN-BE-96-03

Neutral Pion and Hard Photon Production in Heavy-Ion Collision around $100 \mathrm{MeV} / \mathrm{nucleon}$

Phys. Rev. C55:2506, 1997

Pion Shadowing as a Tool to Study the Topology of Heavy-Ion Collisions at Intermediate Energies

Phys. Rev. C55:2521, 1997

Measurement of the Space-Time Extent of the Hard-Photon Emitting Source in Heavy-Ion Collisions at $100 \mathrm{MeV} /$ nucleon

Phys. Rev. C57:166, 1998

Search for the Electromagnetic Decay of $\Delta\left(1232 P_{33}\right)$ Resonance in Nuclear Matter

Phys. Rev. Lett. 80:4863, 1998

Subthreshold Production of Low Momentum $K^{+}$in $p+C$ at $1.2 \mathrm{GeV}$

Kr. Soob. JINR 75:27, 1996

Study of the Hadronic Jet Reconstruction Algorithms in $\pi^{-} p$ and $\pi^{-} \mathbf{C}$ Interactions at 40 GeV/c

JINR-P1-97-288

Properties of the hadronic Jets in $\pi^{-} p$ - and $\pi^{-C}$-interactions at $40 \mathrm{GeV} / \mathrm{c}$

Nucl. Phys. (PS) B39:9, 1995

Shadowing in Deuteron and the New $\left[F_{2}^{n} / F_{2}^{p}\right]$ Measurements

Phys. Lett. 392B:278, 1997; PSI-PR-96-34

Experimental Determination of the Kinetic Energy Distribution of $\pi^{-} p$ Atoms in Liquid Hydrogen

CDF-ELECTROWEAK-PUB-PUBLIC-2741; ALBUQUERQUE-94, v. 1, p. 431; FERMILAB-CONF-94-258-E

Measurement of $W^{ \pm}$and $Z^{0}$ Boson Production and Extraction of the Width and Branching Ratios at $C D F$

Phys. Lett. 342B:362, 1995; Phys. Lett. 374B:363, 1996; DESY-94-172; TUM-T31-68-94; UAB-FT-348; HEPPH-9409440 Theoretical Update of the Semileptonic Branching Ratio of $B$ Mesons

Nucl. Phys. A566:371C, 1994; LAPP-EXP-93-06

$\psi(2 S)$ and $J / \psi(1 S)$ Production in $p \mathrm{U}$ and $\mathrm{S} U$ Collisions at $200 \mathrm{GeV} / \mathrm{Nucleon}$

Nucl. Phys. A590:117C, 1995

The Evolution of Cross Section Ratio $\psi(2 S) / J / \psi(1 S)$ from $p$ nucleus to S U Interactions. Direct Photon Emission in Correlation with $\phi(1020)$ and $J / \psi(1 S)$

Phys. Lett. $345 \mathrm{~B}: 617,1995$

$\psi(2 S)$ and $J / \psi(1 S)$ Production in $p \mathrm{Wt}, p \mathrm{U}$ and $\mathrm{S} \mathrm{U}$ Interactions at $200 \mathrm{GeV} / \mathrm{Nucleon}$

Nucl. Phys. A610:306C, 1996

Strangelet Search and Antinuclei Production Studies in $\mathrm{Pb} \mathrm{Pb}$ Collisions

Nucl. Phys. A610:331C, 1996

Intermediate Mass Muon Pair Continuum in $\mathrm{Pb} \mathrm{Pb}$ Collisions at $158 \mathrm{GeV} / \mathrm{c}$

Nucl. Phys. A610:404C, 1996; HEIDELBERG-97 p. 404C

Anomalous $J / \psi$ Suppression in $\mathrm{Pb}+\mathrm{Pb}$ Collisions at $158 \mathrm{~A} \cdot \mathrm{GeV} / \mathrm{c}$

Nucl. Phys. A638:261C, 1998; TSUKUBA-97 p. $261 \mathrm{C}$

Charmonium Production in $\mathrm{Pb}-\mathrm{Pb}$ Interactions at $158 \mathrm{GeV} / \mathrm{c}$ per Nucleon

Nucl. Phys. A638:483C, 1998; TSUKUBA-97 p. $483 \mathrm{C}$

$\phi(1020), \rho$ and $\omega$ Production in Collisions Induced by Deuteron and Heavy Ions around 200 GeV per Nucleon 
Baglin 98C

Bahcall 95

Bahran 95

Bai 94

Bai 94B

Bai 95

Bai 95B

Bai 95C

Bai 96

Bai 96B

Bai 96C

Bai 96D

Bai 97

Bai 98

Bai 98B

Bai $98 \mathrm{C}$

Bai 98D

Bai 98E

Bai $98 \mathrm{~F}$

Bai 98G

Bai 98H

Bai 98I

Bai 98J

Bai $98 \mathrm{~K}$

Bai 98L

Bailey 94

Bailey 98

Baillie 97

Baird 94

Baird 95

Bakatanov 97

Bakatanov 98

Baker 98

Balatz 93

Balatz 94B

Balatz $94 \mathrm{C}$

Balatz 96

Baldin 94
Nucl. Phys. A638:487C, 1998; TSUKUBA-97 p. 487C

NA38/NA50 Results on the Low Mass Dimuon Spectra

Phys. Lett. 348B:121, 1995; IASSNS-AST-94-57 Limits on Electron-Neutrino Oscillations from the GALLEX ${ }^{51} \mathrm{Cr}$ Source Experiment

Phys. Lett. 354B:481, 1995 A Direct Limit on the Heavy Neutrino in Tritium Beta Decay

Phys. Rev. Lett. 74:4599, 1995; SLAC-PUB-6746; BEBC-EP1-95-01 A Direct Measurement of the Pseudoscalar Decay Constant, $f_{D_{s}^{ \pm}}$

Phys. Rev. D52:3781, 1995; SLAC-PUB-6747; BEBC-EP1-95-02 A Direct Measurement of the $D_{s}^{ \pm}$Branching Fraction to $\phi(1020) \pi$

Phys. Rev. D53:20, 1996; SLAC-PUB-95-6930 Measurement of the Mass of the $\tau^{ \pm}$Lepton

Phys. Lett. 355B:374, 1995; Phys. Lett. 363B:267, 1995; BIHEP-EP1-95-03 $A$ Measurement of $J / \psi(1 S)$ Decay Width

BIHEP-EP1-95-04 Amplitudes Analysis of the $\eta(1440)$ in the $J / \psi(1 S)$ Radiative Decay to the $K \bar{K} \pi$ Final State

SLAC-PUB-7147

Measurement of the Pseudoscalar Decay Constant, $f_{D}$

Phys. Rev. Lett. 77:3959, 1996 Structure Analysis of the $f_{J}(1710)$ in the Radiative Decay $J / \psi(1 S) \rightarrow \gamma K^{+} K^{-}$ BIHEP-EPI-96-01

Precision Measurement of $J / \psi(1 S)$ Leptonic Branching Fraction

Phys. Rev. Lett. 76:3502, 1996 Studies of $\xi(2230)$ in $J / \psi(1 S)$ Radiative Decays

Phys. Rev. D56:3779, 1997 A Measurement of the Branching Fraction of $D_{s}^{ \pm}$Inclusive Semileptonic Decay $D_{s}^{+} \rightarrow e^{+} \mathrm{X}$

Phys. Rev. D57:28, 1998 Direct Measurement of $D_{s}^{+} \rightarrow \phi(1020) \mathrm{X}$

Phys. Rev. D57:3854, 1998

Search for $\psi(2 S)$ Production in $e^{+} e^{-}$Annihilations at $4.03 \mathrm{GeV}$

Phys. Rev. D58:092006, 1998; BIHEP-EP1-98-02; UH511-904-98; HEPEX-9806012 Determination of the $J / \psi(1 S)$ Leptonic Branching Fraction via $\psi(2 S) \rightarrow \pi^{+} \pi^{-} J / \psi(1 S)$

Phys. Rev. D58:097101, 1998; BIHEP-EP1-98-01; UH-511-903-98; HEPEX-9806002 Branching Fractions for $\psi(2 S) \rightarrow \gamma \eta^{\prime}$ and $\gamma \eta$

Phys. Rev. Lett. 81:3091, 1998; BIHEP-EP1-98-03; HEPEX-9807001 Study of the $P$-Wave Charmonium State $\chi_{c}(\mathbf{3 4 5 5})$ in $\psi(2 S)$ Decays

BIH EP-EP1-98-05; HEPEX-9812016

Study of the Hadronic Decays of $\chi_{c}$ States

Phys. Rev. Lett. 81:1179, 1998

Experimental Study of $J / \psi(1 S)$ Radiative Decay to $\pi^{0} \pi^{0}$

BIHEP-EP 1-98-06; UH511-923-99; HEPEX-9901022 Charmonium Decays to Axialvector plus Pseudoscalar Mesons

Phys. Lett. 424B:213, 1998; Phys. Lett. 438B:447, 1998 Decays of the $J / \psi(1 S)$ to $\Lambda \bar{\Lambda}, \Lambda \bar{\Lambda} \gamma$ and $\Lambda \bar{\Lambda} \pi^{\circ}$ Final States

Phys. Lett. 429B:188, 1998 Evidence for the Leptonic Decay $D \rightarrow \mu^{ \pm} \nu_{\mu}$

Phys. Lett. 440B:217, 1998 Partial Wave Analysis of $J / \psi(1 S) \rightarrow \gamma K^{+} K^{-} \pi^{0}$

Phys. Rev. Lett. 81:5080, 1998 $\psi(2 S)$ Hadronic Decays to Vector-Tensor Final States

FERMILAB-CONF-94-237-E Measurement of the $B$ Meson Differential Cross-Sections in $p \bar{p}$ Collisions at $\sqrt{s}=1.8 \mathrm{TeV}$ using the Exclusive Decays $B^{ \pm} \rightarrow J / \psi(1 S) K^{ \pm}$and $B^{0} \rightarrow J / \psi(1 S) K^{*}(8 \theta 2)^{\circ}$

Nucl. Phys. A639:197C, 1998; UPTON-97 p. 197C

Kaon Electroproduction on Deuterium

Jour. of Phys. G 23:2161, 1997

Experimental Results at SQM 1997

SLAC-PUB-6601

A Study of $K_{S}, \Lambda$ and $\bar{\Lambda}$ Production in Hadronic $Z^{\circ}$ Decays

SLAC-PUB-95-6932

Measurement of $\alpha_{S}$ from Hadronic Event Observables at the $Z^{0}$ Resonance

Izv. RAN, Fiz. 61:562, 1997

Detection of Muon Groups with Multiplicity $n_{\mu} \geq 1800$ at the Baksan Underground Scintillation Telescope

Phys. At. Nucl. 61:1507, 1998; Yad. Phys. 61:1618, 1998 Detecting Clusters of Muons with a Multiplicity over 1800 and Probing the Composition of Primary Cosmic Rays with Energies of $10^{15}-10^{17} \mathrm{eV}$

Nucl. Phys. A639:189C, 1998; UPTON-97 p. $189 \mathrm{C}$

Associated $\Lambda$ Production at Jefferson Lab

Phys. At. Nucl. 57:238, 1994; Yad. Phys. 57:253, 1994; IFVE-93-6

Study of the Reaction $p$ nucleon $\rightarrow \Sigma\left(1385 P_{13}\right)^{\circ} K^{+}$nucleon at the Energy of $70 \mathrm{GeV}$

Z. Phys. C61:223, 1994; IFVE-93-96

Study of the Diffractive Production of the Baryon States and Search for Cryptoexotic Baryons with Hidden Strangeness

Z. Phys. C61:399, 1994; IFVE-93-97 Search for $N \dot{\phi}(10 \theta 0)$ Baryon

Phys. At. Nucl. 59:1191, 1996; Yad. Phys. 59:1247, 1996

The Investigation of the Quasi-Exclusive Processes of Neutral Meson Production in $p$ nucleon Collisions at $E_{p}=70 \mathrm{GeV}$ in Region of Deep Fragmentation

Nucl. Phys. A566:611C, 1994

Experiment on Relativistic Nuclear Physics in Beams of Dubna Synchrophasotron and Nuclotron

See the legend on page 5 . 
Baldin 95

Barabash 95

Baldin 95

Baldin $95 \mathrm{~B}$

Baldini 98

Baldit 94

Baldoceolin 94

Baldoceolin 94B

Balest 94

Balest 94B

Balest $94 \mathrm{C}$

Balest 95

Balest 96

Balest 97

Balestra 98

Balewski 95

Balewski 96

Balewski 98

Balewski 98B

Balewski 98C

Balkanov 98

Ball $93 \mathrm{C}$

Ball 94

Ball $94 \mathrm{~B}$

Ball $94 \mathrm{C}$

Ball 94D

Ballocchi 94

Ballocchi 98

Baloshin 95

Balysh 94

Balysh 95

Balysh 96

Bannwarth 92

Bantly 95

Bantly 96

Barabash 93

Barabash 94

Barabash 95
Nuovo Cim. 108A:139, 1995

Mass Dependence of Antiproton Production in nucleus-nucleus Collisions at 3.65 GeV/Nucleon

Kr. Soob. JINR 73:41, 1995

Measurement of the Asymmetry of Pions, Kaons and Protons Production by Vector Polarized Deuterons

on Carbon Nuclei
Phys. Lett. 444B:111, 1998

Measurement of the $J / \psi(1 S) \rightarrow$ nucleon nucleon Branching Ratios and Estimate of the Phase of the Strong Decay Amplitude

Phys. Lett. 332B:244, 1994; LYCEN-9426

Study of the Isospin Symmetry Breaking in the Light Quark Sea of the Nucleon from the Drell-Yan Process

Z. Phys. C63:409, 1994; DFPD-94-EP-13

A New Experimental Limit on Neutron-Antineutron Oscillations

Nucl. Phys. (PS) 35:450, 1994; GRAN SASO-93 p. 450; DFPD-94-EP-14

Neutrino Oscillations at Accelerators

Phys. Rev. Lett. 72:2328, 1994; CLNS-94-1267; CLEO-94-2

Measurement of the Branching Fraction for $D^{+} \rightarrow \mathrm{K}^{-} \pi^{+} \pi^{-}$

Phys. Rev. D51:2053, 1995; CLNS-94-1292; CLEO-94-19 $\Upsilon(1 S) \rightarrow \gamma+$ Noninteracting Particles

Phys. Rev. D52:2661, 1995; CLNS-94-1315; CLEO-94-26

Inclusive Decays of $B$ Mesons to Charmonium

Phys. Rev. Lett. 75:3809, 1995; CLNS-95-1347; CLEO-95-11 Measurements of the Decays $\tau^{-} \rightarrow$ hadron ${ }^{-}$hadron ${ }^{+}$hadron ${ }^{-} \nu_{\tau}$ and $\tau^{-} \rightarrow$ hadron ${ }^{-}$hadron $^{+}$hadron $^{-} \pi^{0}$ $\nu_{\tau}$

Phys. Lett. 388B:402, 1996; CLNS-96-1417; CLEO-96-8

Measurement of the $\tau$-Lepton Lifetime

Phys. Rev. Lett. 79:1436, 1997; CLNS-97-1479; CLEO-97-8; HEPEX-9705006

Observation of the Decay $D_{s}^{+} \rightarrow \omega \pi^{+}$

Phys. Rev. Lett. 81:4572, 1998

Production of $\phi(1020)$ and $\omega$ Mesons in Near-Threshold $p p$ Reactions

Nucl. Phys. A581:131, 1995

Differential Cross Section for Elastic $n$ deuteron Scattering and $n$ deuteron Break-Up Quasi Free-Scattering at $67 \mathrm{MeV}$

Phys. Lett. 388B:859, 1996

$\Lambda$ Hyperon Production via the $p p \rightarrow p K^{+} \Lambda$ Reaction $2 \mathrm{MeV}$ above Threshold

Phys. Lett. 420B:211, 1998; FZJ-IKP-I-1997-01; NUCLEX-9803003

Total Cross Section of the Reaction $p p \rightarrow p K^{+} \Lambda$ Close to Threshold

Eur. Phys. J. A2:99, 1998; FZJ-IKP-I-1998-02; NUCLEX-9803008

Low-Energy $\Lambda p$ Scattering Parameters from the $p p \rightarrow p K^{+} \Lambda$ Reaction

Nucl. Phys. A629:164C, 1998; OSAKA-97 p. 164C

Strangeness Production in the $p+p$ Interaction at Threshold at the Experimental Facility COSY-11

Phys. At. Nucl. 61:886, 1998; Yad. Phys. 61:978, 1998

The BAIKAL Deep Underwater Neutrino Experiment: Status Report

Nucl. Phys. A559:511, 1993; Nucl. Phys. A576:640, 1994; ANL-HEP-PR-92-107

Angular Dependence of the Spin Correlation Parameter $A_{o o n n}$ in $n p$ Elastic Scattering between 0.8 and $1.1 \mathrm{GeV}$

Z. Phys. C61:53, 1994; ANL-HEP-PR-93-27: CEN-LNS-PH-93-13

Measurements of the Total Cross Section Difference $\Delta \sigma_{T}$ in $n p$ Transmission between 0.86 and $0.94 \mathrm{GeV}$ Phys. Lett. 320B:206, 1994; ANL-HEP-PR-93-13

Observation of a Narrow Structure in the $p$ Elastic Scattering Observable $A_{00 n n}$ at $\mathrm{T}_{\operatorname{lin}}=2.11 \mathrm{GeV}$

Nucl. Phys. A574:697, 1994

Measurement of the Spin Correlation Parameters $A_{0 o k k}$ and $A_{0 o s k}$ in $n p$ Elastic Scattering at SATURNE II

Z. Phys. C61:579, 1994

Measurements of the Two and Three Spin-Index Observables in $n p$ Elastic Scattering between 0.80 and $1.10 \mathrm{GeV}$

Phys. At. Nucl. 57:1624, 1994; Yad. Phys. 57:1694, 1994

Comparison of Direct $\gamma$ Production in $\bar{p} p$ and $p p$ Reactions and Determination of $\Lambda_{M S}^{(4)}$ and the Gluon Structure Function

Phys. Lett. 436B:222, 1998; CERN-EP-98-111

Direct Photon Cross Sections in Proton-Proton and Antiproton-Proton Interactions at $\sqrt{s}=24.3 \mathrm{GeV}$

Phys. At. Nucl. 58:46, 1995; Yad. Phys. 58:50, 1995

Amplitude Analysis of the $K_{S} K_{S}$ System Produced on Carbon Nucleus by $40 \mathrm{GeV} / c$ Incident $\pi^{-}$Meson

Phys. Lett. 322B:176, 1994

Measurement of the $\beta \beta 2 \nu$ Decay of ${ }^{76} \mathrm{Ge}$

Phys. Lett. 356B:450, 1995

Sub-eV Limit for the Neutrino Mass from ${ }^{76} \mathrm{Ge}$ Double Beta Decay by the HEIDELBERG-MOSCOW Collaboration

hys. Rev. Lett. 77:5186, 1996; NUCLEX-9608001

Double Beta Decay of ${ }^{48} \mathrm{Ca}$

Nucl. Phys. A567:761, 1994; FREI-MEP-92-01

Production of Charged Pions in Neutron-Proton Collisions

FERMILAB-CONF-95-291-E

Observation of the Top Quark

WARSAW-96 p. 855; FERMILAB-CONF-96-363-E

Do Top Quark Cross Section for the 1992 - 1995 Tevatron Run

Phys. At. Nucl. 57:1974, 1994; Yad. Phys. 57:2050, 1994; JINR-P1-93-327

Search for the Prompt Muon Antineutrino from Charm Particle Decays at the IHEP-JINR Neutrino Detector in $70 \mathrm{GeV}$ Proton Beam-Dump Experiment

Jour. of Phys. G 22:487, 1996; ITEP-94-89

Investigation of the $\beta \beta$ Decay of ${ }^{96} \mathrm{Zr}$ to Excited States in ${ }^{88} \mathrm{Mo}$

Z. Phys. A352:231, 1995

Search for $\beta \beta$ Decay of ${ }^{78} \mathrm{Ge}$ to the Exited States of ${ }^{78} \mathrm{Se}$ 
Barabash 95B

Barabash 96

Barabash 96B

Barabash 98

Barabash 98B

Baranko 94

Barate 96

Barate 97

Barate 97B

Barate $97 \mathrm{C}$

Barate 97D

Barate $97 \mathrm{E}$

Barate $97 \mathrm{~F}$

Barate $97 \mathrm{G}$

Barate $97 \mathrm{H}$

Barate 97I

Barate 97J

Barate $97 \mathrm{~K}$

Barate $97 \mathrm{~L}$

Barate $97 \mathrm{M}$

Barate $97 \mathrm{~N}$

Barate 970

Barate 97P

Barate 97Q

Barate 97R

Barate 97S

Barate $97 \mathrm{~T}$

Barate $97 \mathrm{U}$

Barate $97 \mathrm{~V}$

Barate $97 \mathrm{~W}$

Barate 97X

Barate $97 Y$

Barate $97 \mathrm{Z}$

Barate 97ZB

Barate $97 \mathrm{ZC}$

Barate 97ZD

Barate 97ZE
Phys. Lett. 345B:408, 1995

Two Neutrino Double-Beta Decay of ${ }^{100} \mathrm{Mo}$ to the First Excite Level of State in ${ }^{100} \mathrm{Ru}$

Phys. At. Nucl. 59:179, 1996; Yad. Phys. 59:197, 1996; ITEP-95-13

Experimental Constraints on $2 \beta^{+}, K \beta^{+}$, and $2 K$ Processes in ${ }^{130} \mathrm{Ba}$ and on the $K$-Capture in ${ }^{132} \mathrm{Ba}$

JINR-E1-96-308; HEPEX-9611012

Measurement of $x F_{3}, F_{2}$ Structure Functions and Gross-Llewellyn Smith Sum Rule with IHEP-JINR

Neutrino Detector
Nucl. Phys. A629:517C, 1998; OSAKA-97 p. $517 \mathrm{C}$

Investigation of Double Beta Decay of ${ }^{82} \mathrm{Se}$ and ${ }^{86} \mathrm{Zr}$ with Tracking Detector NEMO-2

Pisma Zh. Eksp. Teor. Fiz. 68:104, 1998

Search for Anomalous Carbon Atoms - the Witnesses of Pauli Principle Violation in the Nucleosynthesis Period

COLO-HEP-342; ALBUQUERQUE-94, v. 1, p. 458; SLAC-PUB-6636

Measurement of the Left-Right Asymmetry in Hadronic $Z^{\circ}$ Decays and $A_{c}$ Determination

2. Phys. C74:451, 1997; CERN-PPE-96-168

Inclusive Production of Neutral Pions in Hadronic $Z^{\circ}$ Decays

Z. Phys. C76:191, 1997; CERN-PPE-97-003

The Topology Dependence of Charged Particle Multiplicities in Three-Jet Events

Phys. Lett. 399B:329, 1997; CERN-PPE-97-010 Study of the Muon-Pair Production at Centre-of-Mass Energies from 20 to $136 \mathrm{GeV}$ with the ALEPH Detector

Z. Phys. C76:1, 1997; CERN-PPE-97-002

A Measurement of the QCD Color Factors and a Limit on the Light Gluino

2. Phys. C74:387, 1997; CERN-PPE-97-016

Measurement of the $\tau^{ \pm}$Lepton Lifetime with the Three-Dimensional Impact Parameter Method

Z. Phys. C76:15, 1997; CERN-PPE-97-013

Measurement of the Spectral Functions of Vector Current Hadronic Tau Decays

Phys. Lett. 401B:150, 1997; CERN.PPE-97-017

A Measurement of $R_{b}$ using a Lifetime-Mass Tag

Phys. Lett. 401B:163,1997; CERN-PPE-97-018

A Measurement of $R_{b}$ using Mutually Exclusive Tags

Phys. Lett. 403B:367, 1997; CERN-PPE-97-024

Measurement of the Branching Fraction for $D^{0} \rightarrow K^{-} \pi^{+}$

Phys. Lett. 402B:213, 1997; CERN-P PE-97-026

Search for the $B_{c}$ Meson in Hadronic $Z^{\circ}$ Decays

Phys. Lett. 401B:347, 1997; CERN-PPE-97-025

Measurement of the $W^{ \pm}$Mass in $e^{+} e^{-}$Collisions at Production Threshold

Eur. Phys. J. C1:65, 1998; CERN-PPE-97-069

Three Prong $\tau^{ \pm}$Decays with Charged Kaons

Phys. Lett. 412B:155, 1997; CERN-PPE-97-070

Search for the Standard Model Higgs boson in $e^{+} e^{-}$Collisions at $\sqrt{s}=161,170$ and $172 \mathrm{GeV}$

Phys. Lett. 405B:379, 1997; CERN-PPE-97-041; HEPEX-9706013

Search for Pair-Production of Long-Lived Heavy Charged Particles in $e^{+} e^{-}$Annihilation

Phys. Lett. 405B:191, 1997; CERN-PPE-97-047

Measurement of the Transverse Spin Correlation in the Decay $Z^{0} \rightarrow \tau^{+} \tau^{-}$

Phys. Lett. 412B:173, 1997; CERN-PPE-97-071

Search for the Neutral Higgs Bosons of the MSSM in $e^{+} e^{-}$Collisions at $\sqrt{s}$ from 130 to $172 \mathrm{GeV}$

Phys. Lett. 407B:377, 1997; CERN-PPE-97-056; HEPEX-9706006

Search for Sleptons in $e^{+} e^{-}$Collisions at Centre-of-Mass Energies of 161 and $172 \mathrm{GeV}$

Phys. Lett. 413B:431, 1997; CERN-PPE-97-084; HEPEX-9708013

Searches for Scalar Top and Scalar Bottom Quarks at LEP2

Eur. Phys. J. C2:197, 1998; CERN-PPE-97-111

Measurement of the $b$ Baryon Lifetime and Branching Fractions in $Z^{0}$ Decays

Phys. Lett. 415B:435, 1997; CERN-PPE-97-102

Measurement of the $W^{ \pm}$Pair Cross Section in $e^{+} e^{-}$Collisions at $172 \mathrm{GeV}$

Phys. Lett. 414B:362, 1997; CERN-PPE-97-090; HEPEX-9710026

Updated Measurement of the $\tau^{ \pm}$Lepton Lifetime

Phys. Lett. 418B:419, 1998; CERN-PPE-97-129

Search for Charged Higgs Bosons in $e^{+} e^{-}$Collisions at Centre-of-Mass Energies from 130 to $172 \mathrm{GeV}$

Phys. Lett. 420B:127, 1998; CERN.PPE-97-122; HEPEX-9710009

Searches for Supersymmetry in the Photon(s) Plus Missing Energy Channels at $\sqrt{s}=161$ and $172 \mathrm{GeV}$

Eur. Phys. J. C2:395, 1998; CERN-PPE-97-138

An Upper Limit on the $\tau^{ \pm}$Neutrino Mass from Three- and Five-prong Tau Decays

Eur. Phys. J. C2:417, 1998; CERN-PPE-97-128; HEPEX-9710012

Searches for Charginos and Neutralinos in $e^{+} e^{-}$Collisions at $\sqrt{s}=161$ and $172 \mathrm{GeV}$

Eur. Phys. J. C4:433, 1998; CERN-PPE-97-151; HEPEX-9712013

Search for Supersymmetry with a Dominant R-Parity Violating $L L \bar{E}$ Coupling in $e^{+} e^{-}$Collisions at Center-of-Mass Energies of 130 to $172 \mathrm{GeV}$

Phys. Lett. 420B:196, 1998; CERN-PPE-97-156

Four-Jet Final State Production in $e^{+} e^{-}$Collisions at Centre-of-Mass Energies Ranging from 130 to

$184 \mathrm{GeV}$
Eur. Phys. J. C4:367, 1998; CERN-PPE-97-157

Study of $B_{s}$ Oscillations and Lifetime using Fully Reconstructed $D_{s}^{-}$Decays

Eur. Phys. J. C5:205, 1998; CERN-PPE-97-153

A Measurement of the Semileptonic Branching Ratio $\mathrm{Br}(b$-baryon $\rightarrow p \ell \bar{\nu} \mathrm{X})$ and a Study of Inclusive $\pi^{ \pm}$, $K^{ \pm},(p, \bar{p})$ Production in $Z^{0}$ Decays

Phys. Lett. 422B:369, 1998; CERN-PPE-97-166

Measurement of Triple Gauge-Boson Couplings at $172 \mathrm{GeV}$

Phys. Lett. 422B:384, 1998; CERN-PPE-97-164

Measurement of the $W^{ \pm}$Mass by Direct Reconstruction in $e^{+} e^{-}$Collisions at $172 \mathrm{GeV}$ 
Barate $97 \mathrm{ZF}$

Barate 98

Barate 98B

Barate $98 \mathrm{C}$

Barate 98D

Barate $98 \mathrm{E}$

Barate 98F

Barate 98G

Barate $98 \mathrm{H}$

Barate 98I

Barate 98J

Barate $98 \mathrm{~K}$

Barate 98L

Barate 98M

Barate $98 \mathrm{~N}$

Barate 980

Barate 98P

Barate 98Q

Barate 98R

Barate $98 \mathrm{~S}$

Barate 99

Barate 99B

Barate 99C

Barate 99D

Barate 99E

Barate 99F

Barbarogalti 96

Barberis 96

Barberis 96B

Barberis 97

Barberis 97B

Barberis 98

Barberis 98B

Barberis 98C

Barberis 98D

Barberis $98 \mathrm{E}$

Barberis $98 \mathrm{~F}$

Barbiellini 96
Eur. Phys. J. C4:29, 1998; CERN-PPE-97-167 $K_{S}$ Production in $\tau$ Decays

Phys. Lett. 425B:215, 1998; CERN-EP-98-017

Resonant Structure and Flavour Tagging in the $B \pi^{ \pm}$System using Fully Reconstructed $B$ Decays Phys. Lett. 429B:169, 1998; CERN-EP-98-044

A Measurement of the Inclusive $b \rightarrow s \gamma$ Branching Ratio

Eur. Phys. J. C4:571, 1998; CERN-EP-98-022

Search for Evidence of Compositeness at LEP I

Eur. Phys. J. C4:557, 1998; CERN-EP-98-035

Measurement of the Fraction of Hadronic $Z^{0}$ Decays into Charm Quark Pairs

CERN-EP-98-016

Measurements of the Structure of Quark and Gluon Jets in Hadronic $Z^{\circ}$ Decays

Phys. Lett. 426B:217, 1998; CERN-EP-98-038

Determination of $A_{F B}^{b}$ using Jet Charge Measurements in $Z^{0}$ Decays

Eur. Phys. J. C4:387, 1998; CERN-EP-98-037

Observation of Doubly-Charmed $B$ Decays at LEP

Eur. Phys. J. C4:409, 1998; CERN-EP-98-012

Measurement of the Spectral Functions of Axial-Vector Hadronic $\tau$ Decays and Determination of $\alpha_{s}\left(M_{\tau}^{2}\right)$

Phys. Lett. 429B:201, 1998; CERN-EP-98-053

Single- and Multi-Photon Production in $e^{t} e^{-}$Collisions at a Centre-of-mass Energy of $183 \mathrm{GeV}$

Eur. Phys. J. C6:555, 1999; CERN-EP-98-067

Determination of $\left|V_{u b}\right|$ from the Measurement of the Inclusive Charmless Semileptonic Branching Ratio of $b$ Hadrons

Phys. Lett. 434B:415, 1998; CERN-EP-98-101; HEPEX-9811015

The Forward-Backward Asymmetry for Charm Quarks at the $Z^{0}$

Phys. Lett. 434B:437, 1998; CERN-EP-98-103

A Measurement of the Gluon Splitting Rate into $b \bar{b}$ Pairs in Hadronic $Z^{\circ}$ Decays

Eur. Phys. J. C7:553, 1999; CERN-EP-98-117; HEPEX-9811018

Search for $B$, Oscillations using Inclusive Lepton Events

Phys. Lett. 445B:239, 1998; CERN-EP-98-178

Measurement of Triple Gauge $W^{ \pm} W^{\mp_{\gamma}}$ Couplings at LEP2 using Photonic Events

Phys. Lett. 447B:183, 1999; CERN-EP-98-187

Analysis of Transverse Momentum Correlations in Hadronic $Z^{0}$ Decays

Phys. Lett. 436 B:211, 1998; CERN-EP-98-118

Study of $D^{0}-\bar{D}^{\mathbf{0}}$ Mixing and $D^{0}$ Doubly Cabibbo-Suppressed Decays

Phys. Lett. 433B:176, 1998; CERN-EP-98-077

Search for Sleptons in $e^{+} e^{-}$Collisions at Centre-of-Mass Energies up to $184 \mathrm{GeV}$

Phys. Lett. 440B:419, 1998; Phys. Lett. 447B:355, 1999; CERN-EP-98-145

Searches for the Neutral Higgs Bosons of the MSSM in $e^{+} e^{-}$Collisions at Centre-of-Mass Energies of $181-184 \mathrm{GeV}$

Phys. Lett. 447B:336, 1999; Phys. Lett. 440B:403, 1998; CERN-EP-98-144; HEPEX-9811032

Search for the Standard Model Higgs Boson at the LEP2 Collider near $\sqrt{s}=183 \mathrm{GeV}$

Phys. Lett. 450B:467, 1999; CERN-EP-99-011; HEPEX-9902031

Search for Charged Higgs Bosons in $e^{+} e^{-}$Collisions at $\sqrt{s}=181-184 \mathrm{GeV}$

Phys. Lett. 450B:301, 1999; CERN-EP-99-008

Search for Invisible Higgs Boson Decays in $e^{+} e^{-}$Collisions at Centre-of-Mass Energies up to $184 \mathrm{GeV}$

Phys. Lett. 453B:107, 1999; CERN-EP-99-035

Measurement of W-Pair Production in $e^{+} e^{-}$Collisions at $183 \mathrm{GeV}$

CERN-EP-99-042; HEPEX-9904011

Study of Fermion Pair Production in $e^{+} e^{-}$Collisions at $130-183 \mathrm{GeV}$

CERN-EP-99-026; HEPEX-9903015

Study of $\boldsymbol{\tau}$ Decays Involving Kaons, Spectral Functions and Determination of the Strange Quark Mass

Z. Phys. C10:1, 1999; CERN-EP-99-025; HEPEX-9903014

One-Prong $\tau$ Decays with Kaons

FERMILAB-CONF-96-146-E; CDF-ANAL-TOP-PUBLIC-3687

Top Decays and Mass with CDF

Phys. Lett. 388B:853, 1996; CERN-PPE-96-128

Observation of Vertex Factorization Breaking in Central $p p$ Interactions

Phys. Lett. 397B:339, 1997; PL 410B, 353; CERN-PPE-96-197

A Kinematical Selection of Glueball Candidates in Central Production

Phys. Lett. 413B:217, 1997; CERN-PPE-97-089; HEPEX-9707021

A Study of the Centrally Produced $\pi^{+} \pi^{-} \pi^{+} \pi^{-}$Channel in $p p$ Interactions at $450 \mathrm{GeV} / \mathrm{c}$

Phys. Lett. 413B:225, 1997; CERN-PPE-97-088; HEPEX-9707022

A Study of the $K \bar{K} \pi$ Channel Produced Centrally in $p p$ Interactions at $450 \mathrm{GeV} / c$

Phys. Lett. 422B:399, 1998; HEPEX-9801003

A Study of the Centrally Produced $\pi^{+} \pi^{-} \pi^{0}$ Channel in $p p$ Interactions at $450 \mathrm{GeV} / c$

Phys. Lett. 427B:398, 1998; HEPEX-9803029

A Study of Pseudoscalar States Produced Centrally in $p p$ interactions at $450 \mathrm{GeV} / \mathrm{c}$

Phys. Lett. 432B:436, 1998; HEPEX-9805018

A Study of the Centrally Produced $\phi(1020) \phi(1020)$ System in $p p$ Interactions at $450 \mathrm{GeV} / c$

Phys. Lett. 436B:204, 1998; HEPEX-9807021

A Study of the Centrally Produced $K^{*}(892) \bar{K}^{*}(892)$ and $\phi(1020) \omega$ Systems in $p p$ Interactions at $450 \mathrm{GeV} / \mathrm{c}$

Phys. Lett. 440B:225, 1998; HEPEX-9810003

A Measurement of the Branching Fractions of the $f_{1}(1285)$ and $f_{1}(1420)$ Produced in Central $p p$ Interactions at $450 \mathrm{GeV} / \mathrm{c}$

Phys. Lett. 446B:342, 1999; HEPEX-9812022

A Study of the Centrally Produced Baryon-Antibaryon Systems in $p p$ Interactions at $450 \mathrm{GeV} / c$

INFN-AE-96-02

The Cosmic Ray Positron to Electron Ratio in the Energy Range 0.85 to $14 \mathrm{GeV}$ 
Barbina 97

Bardayan 97

Bardin 94

Bargende 95

Bargholtz 97

Bargholtz 97B

Barish 94

Barish 95

Barish 96

Barish 97

Barish 97B

Barish 98

Barker 94

Barkov 98

Barlag 94

Barmin 94

Barmin 94B

Barmin 96

Barmin 96B

Barnaveli 95

Barnaveli 96

Barnes 94

Barnes 96

Barnes 96B

Barnes 96C

Barnes 97

Barnett 96

Barr 94

Barr 95

Barr 95B

Barr 95C

Barreiro 94

Barrette 94

Barrette 94B

Barrette $94 \mathrm{C}$

Barrette 94D

Barrette 95

Barrette 95B
Nucl. Phys. A612:346, 1997

Measurement of the $\bar{n}$ Fe Absorption Cross Section between 125 and $780 \mathrm{MeV} / \mathrm{c}$

Phys. Rev. C55:820, 1997; LBL-37201

Radioisotope Yields from $1.85 \mathrm{GeV}$ Protons on Mo and 1.85 and $5.0 \mathrm{GeV}$ Protons on Te

Nucl. Phys. B411:3, 1994

Determination of the Electric and Magnetic Form Factors of the Proton in the Time-Like Region

BONN-IR-95-06

Charm Production in $e^{-} p$ Collisions with the ZEUS Detector at HERA

Phys. Lett. 398B:264, 1997

The Inclusive Reaction deuteron + deuteron $\rightarrow{ }^{4} \mathrm{He}+\mathrm{X} 29 \mathrm{MeV}$ above the $2 \pi^{0}$ Threshold

Nucl. Phys. A626:73C, 1997

A Zero-Degree Spectrometer in CELSIUS and the deuteron (deuteron, $2 \pi^{0}$ ) ${ }^{4} \mathrm{He}$ Reaction Close to Thresh-

Phys. Rev. D51:1014, 1995; CLNS-94-1285; CLEO-94-13

Measurements of the $\bar{B} \rightarrow D^{*} \ell^{-} \bar{\nu}$ Branching Fractions and $\left|V_{c b}\right|$

Phys. Rev. Lett. 76:1570, 1996; CLNS-95-1362; CLEO-95-17

Measurements of the $B$ Semileptonic Branching Fraction with Lepton Tags

Phys. Lett. 373B:334, 1996; CLNS-95-1386; CLEO-95-22

A Measurement of $B\left(D^{0} \rightarrow K^{-} \pi^{+} \pi^{0}\right) / B\left(D^{0} \rightarrow K^{-} \pi^{+}\right)$

Phys. Rev. Lett. 79:3599, 1997; CLNS-97-1470; CLEO-97-5; HEPEX-9705005

First Observation of Inclusive $B$ Decays to the Charmed Strange Baryons $\Xi_{c}^{o}$ and $\Xi_{c}^{+}$

Jour. of Phys. G 23:2127, 1997

Search for Strange Quark Matter with the BNL-E864 Spectrometer

Nucl. Phys. A639:423C, 1998; UPTON-97 p. 423C

Strangelet Searches in High Energy Heavy Ion Collisions

Nucl. Instr. and Meth. A351:72, 1994

$B$-Physics Results from Do

Pisma Zh. Eksp. Teor. Fiz. 68:727, 1998

Resonance Behaviour of $K_{S} K_{S}$ System at the Mass about $1775 \mathrm{MeV}$

Phys. Lett. 325B:531, 1994

A Study of the Transverse Polarization of $\Lambda$ and $\bar{\Lambda}$ Hyperons Produced in $\pi^{-}$Cu Interactions at $230 \mathrm{GeV} / c$

Phys. At. Nucl. 57:1835, 1994; Yad. Phys. 57:1905, 1994

Measurement of Average Cross Section of $0.08-0.75 \mathrm{GeV} / \mathrm{c} \bar{p}$ Annihilation on Xe Nuclei

Phys. At. Nucl. 57:1655, 1994; Yad. Phys. 57:1724, 1994

Double Strangeness Production in $\bar{p}$ Xe Annihilation at Low Energy in DIANA Chamber

Phys. At. Nucl. 59:1814, 1996; Yad. Phys. 59:1878, 1996

Heavy Meson Production in $\bar{p}$ Xe Annihilations

Phys. Lett. 370B:233, 1996

New Upper Limits for $H$-Particle Production in $\bar{p}$ Xe Annihilation at Low Energy

Phys. Lett. 346B:178, 1995

On the Behaviour of the Spectra of Primary Cosmic Ray Nuclei in the $10^{18} \mathrm{eV}$ Energy Region (A Signal of Dark Matter? )

Phys. Lett. 369B:372, 1996

New Evidence on the Primary Cosmic Ray Nuclei Spectra Cutoff in the Energy Range $\geq 10^{10} \mathrm{eV}$

Phys. Lett. 331B:203, 1994

Study of the Reaction $\bar{p} p \rightarrow \bar{\Lambda} \Lambda$ below $6 \mathrm{MeV}$ Excess Energy

Phys. At. Nucl. 59:1460, 1996; Yad. Phys. 59:1516, 1996

Antihyperon-Hyperon Production in the Threshold Region at LEAR

Phys. Rev. C54:2831, 1996

Measurement of the $p \bar{p} \rightarrow \bar{\Lambda} \Lambda$ and $p \bar{p} \rightarrow \bar{\Sigma}^{\circ} \Lambda+$ cc Reactions at 1.726 and $1.771 \mathrm{GeV} / c$

Phys. Rev. C54:1877, 1996; CERN-PPE-96-058

Observables in High Statistics Measurements of the Reaction $\bar{p} p \rightarrow \bar{\Lambda} \Lambda$

Phys. Lett. 402B:227, 1997

Measurement of the Reactions $p \bar{p} \rightarrow \bar{\Sigma}^{+} \Sigma^{+}$and $p \bar{p} \rightarrow \bar{\Sigma}^{-} \Sigma^{-}$Close to Threshold

FERMILAB-CONF-96-039-E; CDF-PUB-CDF-PUBLIC-3478

Recent Results in $p \bar{p}$ Collisions at $1.8 \mathrm{TeV}$ from the CDF Collaboration at the Fermilab Tevatron Collider Phys. Lett. 328B:528, 1994

Search for the Decay $K_{L} \rightarrow \pi^{0} \pi^{0} \gamma$

Z. Phys. C65:361, 1995

Measurement of the Branching Ratio of the Double Dalitz Decay $K_{L} \rightarrow e^{+} e^{-} e^{+} e^{-}$and the CP Parity of the $K_{L}$-Meson

Phys. Lett. 351B:579, 1995

A Test of Chiral Perturbation Theory from the Measurement of the Decay $K_{S} \rightarrow \gamma \gamma$

Phys. Lett. 358B:399, 1995

Search for the Decay $K_{L} \rightarrow 3 \gamma$

DESY-94-204

New Results from HERA: on Photoproduction and Diffraction, the Proton Structure Function, Deep Inelastic Scattering at Low $x$, Heavy Flavour Production, Jets and Search for Leptoquarks

Nucl. Phys. A566:411C, 1994

Transverse Energy Production with Si and Au Beams at AGS Energy: Towards Hot and Dense Hadronic Matter

Phys. Rev. C49:1669, 1994

Two Charged Particle and Transverse Energy Correlations in Si + Pb Collisions at $14.8 \mathrm{GeV} / \mathrm{c}$

Phys. Lett. 333B:33, 1994

Evidence for Expansion of a Hot Fireball from Two-Pion Correlations for Si+Pb Collisions at AGS Energy

Phys. Rev. Lett. 73:2532, 1994; SUNY-RHI-94-08

Observation of Anisotropic Event Shapes and Transverse Flow in Ultrarelativistic Au + Au Collisions

Phys. Rev. C51:865, 1995

Electromagnetic Dissociation of Relativistic ${ }^{28} \mathrm{Si}$

Phys. Rev. C51:3309, 1995

Charged-Particle Pseudorapidity Distributions in Au (Al, Cu, Au and $\mathrm{U}$ ) Collisions at $10.8 \mathrm{~A} \mathrm{GeV} / \mathrm{c}$ 
Barret te $95 \mathrm{C}$

Barrette 95C

Barrette 95D

Barrette 95E

Barrette $95 \mathrm{~F}$

Barrette 95G

Barrette 97

Barrette 97B

Barrette 98

Bartalini 94

Bartelt 94

Bartelt 95

Bartelt 96

Bartelt $96 \mathrm{~B}$

Bartelt 97

Bartelt 97B

Bartelt 98

Bartelt $98 \mathrm{~B}$

Barth 97

Baru 96

Barwick 96

Basini 95

Basini 95B

Basova 95

Basova 95B

Bassler 95

Bassompierre 95

Bastid 96

Batalova $9 \pi$

Battle 94

Batusov 96

Batusov 96C

Batyunya 97

Baudis 97

Baudis 98

Bauer 93B

Bauer 94

Bauer 95

Bauer 96
Phys. Rev. C52:956, 1995

Production of Neutron-Rich Isotopes from the Fragmentation of ${ }^{28} \mathrm{Si}$ Projectiles at $P_{l a b}=14.6 \mathrm{GeV} / \mathrm{c}$ per Nucleon

Phys. Rev. C52:2028, 1995

Transverse Energy and Charged Particle Multiplicity in $p$-Nucleus at $14.6 \mathrm{GeV} / c$

Nucl. Phys. A590:259C, 1995

Directed Flow and Particle Production in Au + Au Collisions from Experiment E877 at the AGS

Phys. Rev. C52:2679, 1995

Search for Pion-Neutron Bound States in 14.6 A GeV Si+nucleus Collisions

Phys. Lett. 351B:93, 1995; SUNY-RHI-94-12

Measurement of Pion Enhancement at Low Transverse Momentum and of the $\Delta$ Resonance Abundance in Si Nucleus Collisions at AGS Energy

Phys. Rev. Lett. 78:2916, 1997; CERN-OPEN-97-004; PHYSICS-9702008

Two-Pion Correlations in Au + Au Collisions at $10.8 \mathrm{GeV} / \mathrm{c}$ per Nucleon

Phys. Rev. C55:1420, 1997; Phys. Rev. C56:2336, 1997; NUCLEX-9610006

Energy and Charged Particle Flow in a $10.8 \mathrm{~A} \cdot \mathrm{GeV} / \mathrm{c}$ Au Au Collisions

Nucl. Phys. A638:69C, 1998; TSUKUBA-97 p. 69C

Particle Production and Hydrodynamic Flow: Recent Results from E877 for Au + Au Collisions at AGS Energy

FERMILAB-CONF-94-180-E; CDF-PUB-JET-PUBLIC-2650

Jet Studies at CDF/Do Collaborations

Phys. Rev. Lett. 73:1890, 1994; CLNS-94-1287; CLEO-94-15

Search for Neutrinoless Decays of the $\tau^{ \pm}$Lepton

Phys. Rev. D52:4860, 1995; CLNS-95-1333; CLEO-95-7

Search for $C P$ Violation in $D^{0}$ Decay

Phys. Rev. Lett. 76:4119, 1996; CLNS-96-1395; CLEO-96-5

First Observation of the Decay $\tau^{-} \rightarrow K^{-} \eta \nu_{\tau}$

MANCHESTER-95 p. 41

Charm Meson Spectroscopy Results from CLEO

Phys. Lett. 405B:373, 1997; CLNS-97-1460; CLEO-97-1; HEPEX-9703013

Studies of the Cabbibo-Suppressed Decays $D^{+} \rightarrow \pi^{0} \ell^{+} \nu$ and $D^{+} \rightarrow \eta e^{+} \nu_{e}$

Phys. Rev. Lett. 80:3916, 1998; CLNS-97-1518; CLEO-97-25; HEPEX-9711011

Observation of the Radiative Decay $D^{*}(2010)^{+} \rightarrow D^{+} \gamma$

Phys. Rev. Lett. 82:3746, 1999; CLNS-98-1594; CLEO-98-18; HEPEX-9811042

Measurement of the $B^{-} \rightarrow D^{0} \ell^{-} \nu$ Branching Fractions and Form Factor

Phys. Rev. D59:091303, 1999; CLNS-98-1595; CLEO-98-19; HEPEX-9902005

Search for Baryon and Lepton Number Violating Decays of the $\tau$ Lepton

Phys. Rev. Lett. 78:4007, 1997; GSI-97-19

Subthreshold Production of Kaons and Anti-Kaons in Nucleus-Nucleus Collisions at Equivalent Beam Energies

Phys. Rept. 267:72, 1996; BUDKERIN P-95-36

Experiments with the MD-I Detector at the $e^{+} e^{-}$Collider VEPP-4 in the Energy Region of $\Upsilon$ (unspec) Mesons

Phys. Rev. Lett. 75:390, 1996

Cosmic Ray Positrons at High Energies. A New Measurement.

LNF-95-051-PA; ROME-95 p. 3

Measurement of the Cosmic Ray Muon Spectrum and Charge Ratio in the Atmosphere from Ground Level to Balloon Altitudes

LNF-95-051-PB

Measurement of Electron and Positron Energy Differential Fluxes with MASS2 Apparatus

Yad. Phys. 58:268, 1995; Phys. At. Nucl. 58:223, 1995

Secondary Particles Multiplicity from ${ }^{132} \mathrm{Xe}+\mathrm{Em}$ Collisions at Energies $1 \mathrm{~A} \mathrm{GeV}$

Yad. Phys. 58:474, 1995; Phys. At. Nucl. 58:423, 1995

Secondary Particle Angular Distributions from ${ }^{132} \mathrm{Xe}+\mathrm{Em}$ Collisions at Energies $1 \mathrm{~A} \mathrm{GeV}$

Nucl. Phys. (PS) B39:3, 1995

The Structure Function $F_{2}^{p}\left(x, Q^{2}\right)$ of the Proton and the Hadronic Final State at Very Low $x$

Phys. Lett. 355B:584, 1995; LYCEN-9520

Search for Light Neutral Objects Photoproduced in a Crystal Field and Decaying into $e^{+} e^{-}$Pairs

Nucl. Phys. A622:573, 1997; PCCF-RI-96-04

Out-of-Plane Emission of Nuclear Matter in Au + Au Collisions between 100 and $800 \mathrm{~A} \mathrm{MeV}$

ITEP-97-23

Search for Resonance $X_{2}(1245)$ by the Maximum Likelihood Method

Phys. Rev. Lett. 73:1079, 1994; CLNS-94-1273; CLEO-94-8

Measurement of Cabibbo-Suppressed Decays of the $\tau^{ \pm}$Lepton

Nucl. Phys. B516:3, 1998; JINR-E1-96-252

Measurement of Dalitz Plot Slope Parameters for $K^{+} \rightarrow \pi^{+} \pi^{0} \pi^{0}$ Decay

Phys. At. Nucl. 61:79, 1998; Yad. Phys. 61:86, 1998; JINR-E1-96-218

Emission of Charge Particles in the Annihilation of Stopped Antiprotons on Heavy Photoemulsion Nuclei

Kr. Soob. JINR 84:37, 1997

A Study of the Two-Photon Interactions Tagged at an Average $\left\langle q^{2}\right\rangle$ of $90 \mathrm{GeV}^{2}$

Phys. Lett. 407B:219, 1997

The Heidelberg-Moscow Experiment: Improved Sensitivity for ${ }^{76} \mathrm{Ge}$ Neutrinoless Double Beta Decay

Phys. Rev. D59:022001, 1999; HEPEX-9811045

New Limits on Dark Matter WIMPs from the Heidelberg - Moscow Experiment

Phys. Rev. D50:R13, 1994; LBL-33037; SLAC-PUB-6250

Measurement of the Kaon Content of Three Prong Tau Decays

SLAC-PUB-6518; LBL-35812

Measurement of $\alpha_{s}$ in $e^{+} e^{-}$Annihilation at $E_{c m}=29 \mathrm{GeV}$

FERMILAB-CONF-95-175-E; CDF-PUB-BOTTOM-PUBLIC-3196

Quarkonia Production in $p \bar{p}$ Collisions with $C D F$

FERMILAB-CONF-96-055-E; CDF-PUB-BOTTOM-PUBLIC-3562

$B$ Physics at CDF 
Baumann 98

Baumann 98B

Baur 93

Baur 94B

Baur 95

Baur 96

Baur 97

Bavink 98

Bayukov 94

Bazarko 95

Bazarov 94

Bazarov 95

Bazizi 94

Bazizi 95

Bearden 96

Bearden $96 \mathrm{~B}$

Bearden 96C

Bearden 96D

Bearden 98

Bearden 98B

Bearden 98C

Bearden 98D

Beavis 95

Beavis 95B

Beavis 95C

Beavis 97

Becattini 95

Beck 94

Beck 97

Beckerszendy 94

Beddo 93

Begemannblai 98

Behnke 95

Behrens 97

Beier 94

Beker 93

Beker 94B

Beker 94C
Phys. Rev. C58:1970, 1998; CERN-EP-98-059

Meson-Exchange Enhancement in First-Forbidden $\beta$ Transitions. The Case of ${ }^{50} \mathrm{~K}$ and ${ }^{38} \mathrm{Ca}$

Phys. Lett. 439B:256, 1998; GSI-98-51; NUCLEX-9810011

Longitudinal Momentum Distributions of ${ }^{16} \mathrm{C},{ }^{18} \mathrm{C}$ Fragments after One-Neutron Removal from ${ }^{17} \mathrm{C}$, ${ }^{10} \mathrm{C}$

Nucl. Phys. A566:87C, 1994; CERN-PPE-93-177

First Results of the CERES Electron Pair Spectrometer from $p+B e, p+A u$ and S+Au Collisions

Phys. Lett. 332B:471, 1994; CERN-PPE-94-076

Measurement of Electromagnetically Produced $e^{+} e^{-}$Pairs in Distant S Pt Collisions

Z. Phys. C71:571, 1996; CERN-PPE-95-116

Search for Direct Photons from S Au Collisions at $200 \mathrm{GeV} / \mathrm{u}$

Phys. Lett. 368B:251, 1996

Production of Antihydrogen

Z. Phys. C74:593, 1997

Replay to Comment on Direct Photon Search by the CERES-Collaboration

Nucl. Phys. A631:542C, 1998; GRONINGEN-97 p. 542C

Exclusive near Threshold Two-Pion Production with the MOMO Experiment at COSY

Yad. Phys. 57:421, 1994; Phys. At. Nucl. 57:395, 1994

Nuclear Fragmentation at Limited Energy Transfer in A $\left(\pi, \pi^{\prime} p\right) X$, A ( $\pi$, $\pi^{\prime}$ deuteron)X Reactions at $P_{x}=3.15$ and $1.4 \mathrm{GeV} / c$

Z. Phys. C65:189, 1995; Z. Phys. C66:680, 1995

Determination of the Strange Quark Content of the Nucleon from a Next-to-Leading-Order QCD Analyses of Neutrino Charm Production

Phys. At. Nucl. 57:408, 1994; Yad. Phys. 57:435, 1994

Proton Multiplicity Fluctuations in Narrow Intervals of Azimuthal Angles in p, deuteron, and ${ }^{12} \mathrm{C}$ Inelastic Collisions with Propane and Tantalum Nuclei at $4.2 \mathrm{GeV} / c$ per Nucleon

Yad. Phys. 58:2009, 1995; Phys. At. Nucl. 58:1900, 1995

Factorial Correlators and Proton Multiplicity Fluctuations in Intervals of Azimuthal Angles from Inelastic Collisions of ${ }^{12} \mathrm{C}$ at $4.2 \mathrm{GeV} / c$ per Nucleon with Propane and Tantalum

FERMILAB-CONF-94-300-E

Inclusive $b$ Production at $C D F$ and $D O$

FERMILAB-CONF-95-238-E

Inclusive $b$ Quark and Heavy Quarkonium Production at Do

Phys. Lett. 388B:431, 1996; CERN-PPE-96-107

Mid-Rapidity Protons in $158 \mathrm{~A}$ GeV Pb Pb Collisions

Phys. Rev. Lett. 78:2080, 1997; CERN-PPE-96+163

Collective Expansion in High Energy Heavy Ion Collisions

Nucl. Phys. A610:240C, 1996

Measuring the Space-Time Extent of Nuclear Collisions using Interferometry

Nucl. Phys. A610:175C, 1996

Hadron Distributions - Recent Results from the CERN Experiment NA44

Phys. Rev. C58:1656, 1998; CERN-EP-98-088

High Energy $\mathrm{Pb}+\mathrm{Pb}$ Collisions Viewed by Pion Interferometry

Phys. Rev. C57:837, 1998; TAMU-NA44-97-02; NUCLEX-9711004

Proton and Antiproton Distributions at Mid-Rapidity in Proton-Nucleus and Sulphur-Nucleus Collisions

Nucl. Phys. A638:103C, 1998; TSUKUBA-97 p. $103 \mathrm{C}$

One-, Two- and Three-Particle Hadron Spectra - Recent Results from CERN-SPS Experiment NA44

Nucl. Phys. A638:419C, 1998; TSUKUBA-97 p. 419C

Kaon and Proton Ratios from Central Pb $+\mathrm{Pb}$ Collisions at the CERN SPS

Nucl. Phys. A590:491C, 1995

Antiproton Production at $0^{\circ}$ in Collisions of $11 \mathrm{~A} \mathrm{GeV} / \mathrm{c} \mathrm{Au}$ Beams

Phys. Rev. Lett. 75:3078, 1995

Search for New Metastable Particles Produced in Au Au Collisions at $10.8 \mathrm{~A} \mathrm{GeV/c}$

Phys. Rev. Lett. 75:3633, 1995

Centrality Dependence of Antiproton Production in Au $+\mathrm{Au}$ Collisions

Phys. Rev. C56:1521, 1997

Antiproton Distributions in Au Nucleus Collisions

Nucl. Phys. (PS) B39:153, 1995; MONTREUX-94 p. 153

Measurement of Inclusive Production of Neutral Hadrons from $Z^{0}$ Decays with L3 Detector at LEP

Phys. Lett. 336B:141, 1994

Searching for Dark Matter with the Enriched Ge Detectors of the Heidelberg-Moscow Double Beta Decay Experiment

Phys. Rev. Lett. 78:606, 1997; Phys. Rev. Lett. 79:4515, 1997

Measurement of the $E_{2} / M_{1}$ Ratio in the $N \rightarrow \Delta$ Transition using the Reaction $\gamma \rightarrow p \pi^{0}$

Phys. Rev. D49:2169, 1994

New Magnetic Monopole Flux Limits from IBM Proton Decay Detector

Phys. Rev. D50:104, 1994; ANL-HEP-PR-93-49

Measurement of the Longitudinal Spin Dependent Neutron-Proton Total Cross Section Difference $\Delta \sigma_{L}(n$ p) between $500-800 \mathrm{MeV}$

Phys. Rev, C58:1639, 1998; GSI-98-15; NUCLEX-9803007

Breakup Conditions of Projectile Spectators from Dynamical Observables

Nucl. Phys. (PS) B39:368, 1995

Heavy Quarks and QCD in $e^{+} e^{-}$Collisions

Phys. Rev. Lett. 80:3710, 1998; CLNS-97-1536; CLEO-97-31; HEPEX-9801012

Two-Body $B$ Meson Decays to $\eta$ and $\eta^{\prime}-$ Observation of $B \rightarrow \eta^{\prime} K$

Phil. Transact. of Roy. Soc. of London A346:63,1994

Experimental Results on Atmospheric Neutrinos

Nucl. Phys. A566:115C, 1994; CERN-PPE-93-123

Identifled Particle Interferometry in Heavy-Ion Collisions: Results from the CERN NA44 Experiment

Phys. Rev. Lett. 74:3340, 1995; CERN-PPE-94-119

$m_{T}$ Dependence of Boson Interferometry in Heavy Ion Collisions at the CERN SPS

Z. Phys. C64:209, 1994; CERN-PPE-94-75

Kaon Interferometry in Heavy Ion Collisions at the CERN SPS 
Bekmirzaev 93

Bekmirzaev 94

Bekmirzaev $94 \mathrm{~B}$

Belaga 94

Belaga $94 \mathrm{~B}$

Belaga $94 \mathrm{C}$

Belaga 95

Belaga 95B

Belaga 95D

Belaga 95E

Belaga $95 \mathrm{~F}$

Belaga $95 \mathrm{G}$

Belaga $95 \mathrm{H}$

Belaga 95I

Belaga 96

Beleser 94

Beleser 95

Belforte 93

Belikov 95

Belikov 96

Bella 95

Bellandi 95

Bellemann 98

Belli 96

Bellotti 96

Belogianni 93B

Belogianni 94

Belogianni 95

Belogianni 96

Belolaptikov 97

Belostotsky 94

Belostotsky 97

Belyakov 98

Belz 95

Belz 96

Bel2 96B

Benayoun 96

Yad. Phys. 58:63, 1995; Phys. At. Nucl. 58:58, 1995; JINR-P1-93-464

Rapidity Distributions of $\pi^{-}$Mesons in ( $p$, deuteron, ${ }^{4} \mathrm{He}, \mathrm{C}$ ) $\mathrm{C}$ Interactions at $4.2 \mathrm{GeV} / c \mathrm{per} \mathrm{Nucleon}$

Phys. At. Nucl. 58:1548, 1995; Yad. Phys. 58:1642, 1995; JIN R-P1-94-260

Rapidity Distributions of Protons in ( $p$, deuteron, $\left.{ }^{4} \mathrm{He}, \mathrm{C}\right) \mathrm{C}$ Interactions at $4.2 \mathrm{GeV} / \mathrm{c}$ per $\mathrm{Nucleon}$

Phys. At. Nucl. 58:1721, 1995; Yad. Phys. 58:1822, 1995; JINR-P1-94-376

Rapidity Distribution of $\pi^{-}$Mesons in (deuteron, ${ }^{4} \mathrm{He}, \mathrm{C}$ ) Ta Interactions at $4.2 \mathrm{GeV} / \mathrm{c}$ per $\mathrm{Nucleon}$

Yad. Phys. 58:2014, 1995; Phys. At. Nucl. 58:1905, 1995; JINR-P1-94-285 Coherent Dissociation ${ }^{12} \mathrm{C} \rightarrow 3 \alpha$ in Emulsion Enriched by $\mathrm{Pb}$

Phys. At. Nucl. 57:1437, 1994; Yad. Phys. 57:1510, 1994

A Search for Multiparticle Correlations in Multiple Production Reactions at $E_{0}=200-400 \mathrm{GeV}$

Phys. At. Nucl. 57:1443, 1994; Yad. Phys. 57:1516, 1994 Multiparticle Azimuthal Correlations in Multiple Production Reactions at High Energies

Yad. Phys. 59:869, 1996; Phys. At. Nucl. 59:832, 1996; JINR-P1-95-218

Fragmentation of Carbon into Three Alphas in Propane Chamber at $4.2 \mathrm{GeV} / \mathrm{c}$

Phys. At. Nucl. 59:991, 1996; Yad. Phys. 59:1036, 1996; JINR-P1-95-289 Fragmentation Characteristics of Relativistic Carbon Nucleus at its Interaction with Hydrogen

Phy's. At. Nucl. 59:1198, 1996; Yad. Phys. 59:1254, 1996; JIN R-P1-95-368 A Study of Pair Correlations between $\alpha$-Particles: Fragments of Relativistic Nuclei

Pisma Zh. Eksp. Teor. Fiz. 62:385, 1995; Jetp Lett. 62:395, 1995

Dependence of the Mechanism of the Inelastic Dissociation of the Relativistic Carbon Nuclei into Three $\alpha$-Particles versus its Excitation Energy

Kr. Soob. JIN R $74: 73,1995$

A Study of Multiparticle Azimuthal Correlations in High Energy Interaction

Phys. At. Nucl. 59:1935, 1996; Yad. Phys. 59:2008, 1996; JINR-P1-95-233

A Study of Fragmentation of Relativistic Carbon Nucleus Inelastically Interacting with Propane and Tantalum Nuclei

JINR-P1-95-40

Effective Masses and Relative Angles for $\alpha$-Particle Pairs from Coherent Dissociation Reactions ${ }^{12} \mathrm{C} \rightarrow 3 \mathrm{He}$ at $4.5 \mathrm{GeV} / \mathrm{c}$ per Nucleon

Rad. Meas. 25:271, 1995

A Study of Coherent Dissociation of ${ }^{12} \mathrm{C} \rightarrow 3^{4} \mathrm{He}$ in Usual and Enriched by Pb Emulsions

Phys. At. Nucl. 60:791, 1997; Yad. Phys. 60:885, 1997; JINR-P1-96-144

Search for Coherent Fragmentation of Carbon Nucleus in $2 \mathrm{He}+2$ deuteron and $2 \mathrm{He}+{ }^{3} \mathrm{H}+p$ at $4.2 \mathrm{~A}$ GeV $/ c$

MINR-94-862

First Results of the Troitsk Experiment on the Search for the Electron Antineutrino Rest Mass in Tritium $\beta$-Decay

Phys. Lett. $350 \mathrm{~B}: 263,1995$

Results of the Troitsk Experiment on the Search for the Electron Antineutrino Rest Mass in Tritium Beta Decay

Nuovo Cim. 107A:2085, 1994; FERMILAB-CONF-93-313-E

Measurement of the Elastic, Total and Single Diffraction Cross Sections at Tevatron Energies

Yad. Phys. 58:1993, 1995; Phys. At. Nucl. 58:1886, 1995

Inclusive Prompt Muon Yields in the Proton Beam-Dump Experiment at $70 \mathrm{GeV}$ and an Estimate of the

Charm Production Cross Section
IFVE-97-51

Preliminary Results on Raw Asymmetry in the $\pi^{\circ}$ Production on a Polarized Target at $70 \mathrm{GeV}$

Nucl. Phys. (PS) B40:475, 1995; MONTREUX-94 p. 475

$\tau$ Polarization Measurements using the Pion and the Lepton Channels

Phys. Lett. 343B:410, 1995

On the Behaviour of the Total Cross Section at Superhigh Energies from Cosmic Ray Data

ISKP-12-98-1; NUCLEX-9812011

$P$-Wave Dominance in the $p$ deuteron $\rightarrow{ }^{3} \mathrm{He} \pi^{+} \pi^{-}$Reaction near Threshold Measured with the MOMO Experiment at COSY

Phys. Lett. 387B:222, 1996; Phys. Lett. 389B:783, 1996

Limits on WIMP-129 Xe Inelastic Scattering

Phys. Rev. D53:35, 1996; LNGS-95-02

A Measurement of the Negative Muon Spectrum between 0.3 and $40 \mathrm{GeV} / \mathrm{c}$ in the Atmosphere

Z. Phys. C61:371, 1994; CERN-PPE-93-187

Shape Distortions of Meson Resonances Produced in Hadron Interactions at $p_{T} \geq 2 \mathrm{GeV} / c$

Z. Phys. C64:15, 1994; LPC-94-14

Study of $\phi(1020)$ and $f_{2}(1270)$ Meson Polarization at $p_{T} \geq 2 \mathrm{GeV} / c$ in $\pi-$ Be Interactions at CERN SPS

Z. Phys. C69:235, 1995; CERN-PPE-95-79

Search for Higher Twists in Meson Resonance Production in Hadron Interactions at $p_{T} \geq 2 \mathrm{GeV} / c$

Phys. Lett. 408B:487, 1997; CERN-PPE-96-145

Confirmation of a Soft Photon Signal in Excess of QED Expectations in $\pi p$ Interactions at $280 \mathrm{GeV} / c$

Astropart. Phys. 7:263, 1997; DESY-97-033

The Baikal Underwater Neutrino Telescope: Design, Performance and First Results

WILLIAMSBURG-94 p. 427

The deuteron $\uparrow p \rightarrow p \nmid p n$ Reaction at $2 \mathrm{GeV}$ Energy as the Test of Deuteron Structure and Reaction Mechanism

Phys. Rev. C56:50, 1997; DAPNIA-SPHN-97-08

Measurement of Polarization Observables in deuteron $\uparrow p \rightarrow p \uparrow p n$ Reaction at $T_{d e u t}=2 \mathrm{GeV}$

JINR-P1-98-289

Experimental Investigation of Spectator Particles from Carbon Nuclei Interaction in the Propane Bubble

Chamber in the GeV Area

Phys. Rev. Lett. 74:646, 1995

Two-Body Photodisintegration of the Deuteron up to $2.8 \mathrm{GeV}$

Phys. Rev. Lett. 76:3277, 1996; Phys. Rev. C56:1164, 1997; PRINCETON-HEP-95-12; U'TEXAS-HEP-95-19; HEPEX9603002

Search for the Weak Decay of an H Dibaryon

Phys. Rev. D53:3497, 1996; PRINCETON-HEP-95-11

Search for Diffractive Dissociation of a Long-Lived $H$ Dibaryon

Z. Phys. C72:221, 1996; LPNHE-96-05

A Search for Anomalous Contribution in $e^{+} e^{-} \rightarrow \pi^{0} / \eta \gamma$ Annihilations 
Benck 97

Benck 98

Bender 97

Benjmain 95

Benlloch 94

Bennett 98

Bennhold 98

Benvenuti 93

Berdnikov 94

Berdnikov 94B

Berdnikov 96

Berdnikov 96B

Berdoz 98

Beretvas 95

Beretvas 95B

Beretvas 95C

Beretvas 95D

Berezin 94

Berg 94

Bergfeld 94

Bergfeld 94B

Bergfeld 96

Bergfeld 96B

Bergfeld 97

Bergfeld 98

Bergstrom 96

Bergstrom 97

Bergstrom 98

Bergstrom 99

Berkeland 95

Bernabei 98

Bernabei 99

Bernlohr 98

Bernstein 97

Bertanza 97

Bertin 95

Bertin 96

Bertin 96B

Bertin 96C
Nucl. Phys. A615:220, 1997

Neutron Proton Differential Cross Section Measurements for $E_{n}$ from 28 to $75 \mathrm{MeV}$

Phys. Rev. C58:1558, 1998

Light Charged Particle Production in Neutron-Induced Reactions on Aluminum at $E_{n}=62.7 \mathrm{MeV}$

Phys. Lett. 418B:411, 1998; CERN-OPEN-97-029; SI-97-17A

First Measurement of the Rate $K_{L} \rightarrow \pi \mu^{-} \nu \gamma$

FERMILAB-CONF-95-241-E; CDF-ANAL-ELECTROWEAK-PUB-3191

$W^{ \pm} \gamma$ and $Z^{\circ} \gamma$ Production at the Tevatron

FERMILAB-CONF-94-326-E

CDF top Results in the Dilepton Channel

Phys. Rev. C58:1155, 1998

Light Nuclei Production in Relativistic Au + nucleus Collisions

Nucl. Phys. A639:209C, 1998; UPTON-97 p. 209C

$K^{0} \Sigma^{+}$Photoproduction with SAPHIR

Z. Phys. C63:29, 1994; JINR-E1-93-133; CERN-PPE-94-65

Nuclear Structure Functions in Carbon near $x=1$

Phys. At. Nucl. 57:1536, 1994; Yad. Phys. 57:1610, 1994

VES Study of $\pi(1800)$ Resonance in Diffractive Reactions

Phys. Lett. 337B:219, 1994

Study of the Reaction $\pi^{-} \mathrm{A} \rightarrow K^{+} K^{-} \pi^{-} \mathrm{A}$ at $37 \mathrm{GeV} / \mathrm{c}$

Yad. Phys. 59:43, 1996; Phys. At. Nucl. 59:38, 1996

Knocking out Neutrons by $K^{+}$Mesons from Photoemulsion Light Nuclei at Energy $200 \mathrm{MeV}$

Phys. At. Nucl. 59:380, 1996; Yad. Phys. 59:403, 1996

Inelastic Scattering of $K^{+}$Mesons on Light Nuclei with Emission of Neutron and Proton at $130 \mathrm{MeV}$

Nucl. Phys. A629:433C, 1998; OSAKA-97 p. 433C

Parity Violation in Proton-Proton Scattering

FERMILAB-CONF-95-279-E; CDF-TOP-PUBLIC-3262

CDF Results on TOP

Int. Jour. Mod. Phys. A11:2233, 1996; FERMILAB-PUB-95-311-E; CDF-TOP-PUBLIC-3318 Kinematics of the $t \bar{t}$ Events in $W^{ \pm}+$Jets at CDF

Int. Jour. Mod. Phys. A11:2045, 1996; FERMILA B-PUB-95-310-E; CDF-TOP-PUBLIC-3317 CDF Results on Top

FERMILAB-CONF-95-277-E; CDF-TOP-PUBLIC-3303

Kinematics of the $t \bar{t}$ Events in $W^{ \pm}+($jets) at CDF

Phys. At. Nucl. 57:269, 1994; Yad. Phys. 57:285, 1994

Analysis of Experimental Search of Oscillations Based on Mixing of Three Types of Neutrino

Phys. Rev. Lett. 72:977, 1994

Transverse Momentum Distributions of $\eta$ Mesons in Near-Threshold Relativistic Heavy Ion Reactions

Phys. Lett. 323B:219, 1994; CLNS-94-1269; CLEO-94-4 Study of the Decay $\Lambda_{c}^{+} \rightarrow \Lambda e^{+} \nu$

Phys. Lett. 340B:194, 1994; CLNS-94-1298; CLEO-94-21 Observation of $D_{1}(2420)^{+}$and $D_{2}^{*}(2480)^{+}$

Phys. Rev. Lett. 77:4503, 1996; CLNS-96-1423; CLEO-96-12

A Search for $B^{+} \rightarrow$ hadron ${ }^{+}$hadron $^{-}$hadron $^{+}$(non-resonant)

Phys. Lett. 365B:431, 1996; CLNS-95-1349; CLEO-95-12; HEPEX-9508006

Observation of the $\Xi_{c}^{+}$Charmed Baryon Decays to $\Sigma^{+} K^{-} \pi^{+}, \Sigma^{+} \bar{K}^{*}(892)^{0}$, and $\Lambda K^{-} \pi_{\pi}^{+}{ }_{\pi}^{+}$

Phys. Rev. Lett. 79:2406, 1997; CLNS-97-1489; CLEO-97-13; HEPEX-9706020

First Observation of $\tau^{ \pm} \rightarrow 3 \pi \eta \nu_{\tau}$ and $\tau^{ \pm} \rightarrow f_{1}(1285) \pi \nu_{\tau}$ Decays

Phys. Rev. Lett. 81:272, 1998; CLNS-97-1537; CLEO-97-32

Observation of $B^{+} \rightarrow \omega K^{+}$and Search for Related B Decays Modes

Phys. Rev. C53:R1052, 1996

Measurement of the ${ }^{1} \mathrm{H}\left(\gamma, \pi^{0}\right)$ Cross Section Near Threshold

Phys. Rev. C55:2016, 1997

Measurement of the ${ }^{1} \mathrm{H}\left(\gamma, \pi^{0}\right)$ Cross Section Near Threshold. II. Pion Angular Distributions

Phys. Rev. C57:3203, 1998

Measurement of the ${ }^{2} \mathrm{H}\left(\gamma, \pi^{\circ}\right)$ Reaction Near Threshold

Phys. Rev. C59:2588, 1999

Measurement of the ${ }^{6} \mathrm{Li}\left(\gamma, \pi^{0}\right)$ Reaction Near Threshold

Phys. Rev. Lett. 75:2470, 1995

Precise Optic Measurement of Lamb Shifts in Atomic Hydrogen

Phys. Lett. 436B:379, 1998

New Limits on Particle Dark Matter Search with a Liquid Xenon Target-Scintillator

Phys. Lett. 450B:448, 1999; INFN-AE-98-20

On a Further Search for a Yearly Modulation of the Rate in Particle Dark Matter Direct Search

Astropart. Phys. 8:253, 1998; MPIK-98-001; ASTROPH-9801042

Changes of the Cosmic-Ray Mass Composition in the $10^{14}-10^{18} \mathrm{eV}$ Energy Range

Phys. Rev. C55:1509, 1997; NUCLEX-9610005

Observation of a Unitary Cusp in the Threshold $\gamma p \rightarrow \pi^{0} p$ Reaction

Z. Phys. C73:629, 1997

Measurement of the $K_{S}$ Mean Lifetime from $\pi^{+} \pi^{-}$and $\pi^{0} \pi^{0}$ Decay s using $K_{L}$ Decays to Determine the Acceptance

Phys. Lett. 361B:187, 1995

$E / \iota$ Decays to $K \bar{K} \pi$ in $\bar{p} p$ Annihilation at Rest

Phys. At. Nucl. 59:1312, 1996; Yad. Phys. 59:1371, 1996

$\eta(1440)$ Decays to $K \bar{K} \pi$ in the Reaction $\bar{p} p \rightarrow \eta(1440) \pi \pi$

Phys. At. Nucl. 59:1371, 1996; Yad. Phys. 59:1430, 1996

$\bar{p} \boldsymbol{p}$ Annihilation Cross Section at Very Low Energy

Phys. At. Nucl. 59:1450, 1996; Yad. Phys. 59:1506, 1996

Study of $\phi(1020) \pi \pi$ Final State in $\bar{p} p$ Annihilation at Rest

See the legend on page 5. 
Bertin 96D

Bertin $96 \mathrm{E}$

Bertin $96 \mathrm{~F}$

Bertin 96G

Bertin $96 \mathrm{H}$

Bertin 96I

Bertin 97

Bertin 97B

Bertin $97 \mathrm{C}$

Bertin 97D

Bertin 97E

Bertin 98

Bertin 98B

Bertolot to 94

Bertolotto 95

Bertolotto 96

Bertram 95

Besliu 98

Betker 96

Betsch 98

Beusch 95

Bezzubov 96

Bhang 98

Bhang 98B

Bhat 96

Bhattacharje 96

Bhattacharyy 97

Bhatti 94

Bianchi 94

Bianchi 95

Biasini 95

Biasini 97

Bilger 95

Bilger 97

Bilger 98

Bilger 98B

Binkley 95

Binkley 96
Phys. At. Nucl. 59:1455, 1996; Yad. Phys. 59:1511, 1996

Strangeness Production in $\bar{p}$ deuteron Pontecorvo Reactions at Rest

Phys. At. Nucl. 59:1497, 1996; Yad. Phys. 59:1556, 1996

Antiprotons Stopping Power at Very Low Energies

Phys. Lett. 386B:486, 1996; Phys. Lett. 389 B:781, 1996

Protonium Annihilation into $K_{S} K_{L}$ at Three Different Target Densities

Phys. Lett. 388B:450, 1996; HEPEX-9607006

New Data on OZI Rule Violation in $\bar{p} p$ Annihilation at Rest

Phys. Lett. 385B:493, 1996; Phys. Let.t. 395B:388, 1997

Measurement of the $\eta(1440) \rightarrow K^{ \pm} K_{L} \pi^{\mp}$ Production Rates from $\bar{p} p$ Annihilation at Rest at Three Different Hydrogen Target Densities

Phys. Lett. 369B:77, 1996

$\bar{p} p$ Annihilation Cross Sections at Very Low Energies

Phys. Lett. 403B:177, 1997

New Data on $\Delta\left(1232 P_{\mathbf{3 3}}\right)^{++}$Baryon Production in $\bar{p}$ deuteron Annihilation at Rest

Phys. Lett. 408B:476, 1997

Spin-Parity Analysis of the Final State $\pi^{+} \pi^{-} \pi^{0}$ from $\bar{p} p$ Annihilation at Rest in Hydrogen Targets at Three Densities

Phys. Lett. 400B:226, 1997

A Search for Axial Vectors in $\bar{p} p \rightarrow K^{ \pm} K_{m i s s}^{0} \pi^{\mp} \pi^{+} \pi^{-}$Annihilations at Rest in Gaseous Hydrogen at NTP

Phys. Lett. 410B:344, 1997

Study of $\bar{n} p$ Annihilation in Two Mesons in the Momentum Range between 50 to $400 \mathrm{MeV} / c$ with OBELIX

Phys. Lett. 414B:220, 1997

Study of the $\bar{p} p \rightarrow 2 \pi^{+} 2 \pi^{-}$Annihilation from $S$ States

Phys. Rev. D57:55, 1998

Study of the $f_{0}(1500) / f_{2}(1565)$ Production in the Exclusive Annihilation $\bar{n} p \rightarrow \pi^{+} \pi^{+} \pi^{-}$in Flight

Phys. Lett. 434B:180, 1998

Study of the Isovector Scalar Mesons in the Channel $\bar{p} p \rightarrow K^{ \pm} K_{S} \pi^{\mp}$ at Rest with Initial Angular Moment um

State
Phys. At. Nucl. 57:1503, 1994; Yad. Phys. 57:1578, 1994

Recent Results on Meson Spectroscopy from JETSET

Phys. Lett. 345B:325, 1995; CERN-PPE-94-221 Observation of $\phi(1020) \phi(1020)$ Production in the Reaction $\bar{p} p \rightarrow 4 K^{\ddagger}$ at $1.4 \mathrm{GeV} / c$ Incident $\bar{p}$ Moment um

Phys. At. Nucl. 59:1444, 1996; Yad. Phys. 59:1501, 1996

$\phi(1020) \phi(1020)$ Associated Production in $\bar{p} p$ Annihilation from JETSET

FERMILAB-CON F-95-198-E

Rapidity Gaps between Jets at Do and CDF

Eur. Phys. J. A 1:65, 1998

Jets of Nuclear Matter in He nucleus Inelastic Collisions

Phys. Rev. Lett. 77:3510, 1996

Search for the Production of Pionium Atoms near Threshold

Phys. Lett. 446B:179, 1999; NUCLEX-9810009

Observation of Strong Final-State Effects in $\pi^{+}$Production in $p p$ Collisions at $400 \mathrm{MeV}$

Z. Phys. A350:379, 1995; CERN-PPE-94-086

Measurement of the Polarization of $\Lambda, \bar{\Lambda}, \Sigma^{+}$and $\Xi^{-}$Produced in a $\Sigma^{-}$Beam of $330 \mathrm{GeV} / c$

Yad. Phys. 59:2199, 1996; Phys. At. Nucl. 59:2117, 1996; IFVE-96-36

New Data on the Reaction $p$ nucleon $\rightarrow\left(\Sigma\left(1385 P_{13}\right)^{0} K^{+}\right)$nucleon at $E_{p}=70 \mathrm{GeV}$

Phys. Rev. Lett. 81:4321, 1998

Lifetime Measurement of ${ }^{12} \mathrm{C}_{s},{ }^{28} \mathrm{Si}_{s}$, and $\mathrm{Fe}_{3}$ Hypernuclei

Nucl. Phys. A639:269C, 1998; UPTON-97 p. $269 \mathrm{C}$

Lifetimes of $\Lambda$ Hypernuclei up to $\Lambda F e$

FERMILAB-CONF-96-226-E

Measurement of the $t \bar{t}$ Production Cross Section at Do

FERMILAB-CONF-96-304-EA

Do Papers on QCD Studies with Jets: Inclusive jet cross section at Do

Fizika B6:45, 1997

Heavy Fragments Produced in Relativistic ${ }^{238} \mathrm{U}{ }^{27}$ Al Interactions

FERMILAB-CONF-94-159-E

Inclusive Jet and Two Jet Differential Cross Sections at CDF

Phys. Lett. 325B:333, 1994

Absolute Total Photoproduction Cross Section on Nuclei in the Nucleon Resonance Region

Phys. Rev. C54:1688, 1996; LNF-95-053-P

Total Hadronic Photoabsorption Cross Section on Nuclei in the Nucleon Resonance Region

Nucl. Phys. (PS) B40:331, 1995

Classical Impact Parameter Measurements of the Tau Lifetime

Nucl. Phys. (PS) B55:129, 1997; ESTES PARK-96 p. 129

Study of Tau Decays with Neutral Kaons using the L3 Detector at LEP

Phys. Lett. 363B:41, 1995

Search for the Hypothetical $\pi \rightarrow \mu^{-X}$ Decay

Nucl. Phys. A626:29C, 1997

Meson Production in Light Ion Collisions at CELSIUS

Phys. Lett. 420B:217, 1998

Strangeness Production in the Reaction $p p \rightarrow K^{+} \Lambda p$ in the Threshold Region

Nucl. Phys. A629:141C, 1998; OSAKA-97 p. 141C

A Status-Report on the Search for the $\pi n n$ Resonance $d^{\prime}$

FERMILAB-CONF-95-176-E; CDF-PUB-TOP-GROUP-3188

$t \bar{t}$ Kinematics in $W^{ \pm}+>3$ Jet Events

FERMILAB-CONF-96-325-E

Overview of Kinematic Variables in Top Production 
Bird 94

Bocquet 95

Bird 94

Birnbaum 97

Birsa 94

Bishai 95

Bishai 95B

Bishai 97

Bishai 97B

Bishaj 98

Bisplinghoff 94

Bjorken 96

Blackett 97

Blair 94

Blair 95

Blanford 97

Blank 94

Blank 95

Blank 95B

Blanpied 95

Blanpied 96

Blazey 95

Blazey 96

Blessing 94

Blick 94

Blinov 93

Blinov 94

Blinov 95

Blinov 95B

Bliss 97

Blom 98

Blomquist 96

Blomqvist 96B

Blomqvist 97

Blomqvist 98

Bochna 98

Bock 98

Bock 98B

Bockhorst 94

Bocquet 94

Bocquet 95
Astroph. Jour. 441:144, 1995; ASTROPH-9410067

Detection of a Cosmic Ray with Measured Energy Well beyond the Expected Spectral Cutoff due to Cosmic Microwave Radiation

Phys. Lett. 397 B:143, 1997

An Experimental Limit on Radiative Decays of Solar Neutrinos

Phys. Lett. 405B:389, 1997; Phys. Lett. 339B:325, 1994; CERN-PPE-94-156

High-Precision Measurement of the $\bar{p} p \rightarrow \bar{n} n$ Charge-Exchange Differential Cross Section

Phys. Lett. 350B:256, 1995; CLNS-95-1319; CLEO-95-1

Measurement of the Decay Asymmetry Parameters in $\Lambda_{c}^{+} \rightarrow \Lambda \pi^{+}$and $\Lambda_{c}^{+} \rightarrow \Sigma^{+} \pi^{0}$

Phys. Lett. 369B:186, 1996; CLNS-95-1379; CLEO-95-21

Study of $B \rightarrow J / \psi(1 S) \rho$

Phys. Rev. Lett. 78:3261, 1997; CLNS-96-1449; CLEO-96-20; HEPEX-9701008

Analyses of $D^{+} \rightarrow K_{S} K^{+}$and $D^{+} \rightarrow K_{S} \pi^{+}$

Phys. Rev. D57:3847, 1998; CLNS-97-1513; CLEO-97-20; HEPEX-9710023

Search for the Decay $B \rightarrow D_{s 1}(2538)^{+} \mathrm{X}$

Phys. Rev. Lett. 82:281, 1998; CLEO-98-11; CLNS-98-1574; HEPEX-9809012

First Observation of the Decay $\tau^{-} \rightarrow K^{*}(892)^{-} \eta \nu_{\tau}$

JINR-E1-94-116

On Neutron Generation in Massive Cu-Target at Irradiation with 22 and 44 GeV per Carbon Ions

SLAC-PUB-7311

T884 (MiniMax): A Search for Disoriented Chiral Condensate at the Fermilab Collider

HEPEX-9708032

The Photoproduction of the $b_{1}(1235) \pi$ System

ALBUQUERQUE-94, v. 2, p. 1816; FERMILAB-CONF-94-269-E

The Diphoton Production Rate in $\bar{p} p$ Collisions at $\sqrt{s}=1800 \mathrm{GeV}$

FERMILAB-CONF-95-245-E

Photon Plus Charm and Diphotons at $\sqrt{s}=1.8 \mathrm{TeV}$

Phys. Rev. Lett. 80:3037, 1998; FERMILAB-PUB-97-398-E

Observation of Atomic Antihydrogen

Phys. Rev. C50:2398, 1994

Production Cross Sections and the Particle Stability of Proton-Rich Nuclei from ${ }^{58} \mathrm{Ni}$ Fragmentation

Z. Phys. A352:77, 1995

Partial Charge Changing Cross Sections for Neutron Deficient Isotopes from $58 \mathrm{Ni}$ Fragmentation

Z. Phys. A352:69, 1995

Total Charge Changing Cross Sections for Neutron Dificient Isotopes from ${ }^{58} \mathrm{Ni}$ Fragmentation

Phys. Rev. C52:R455, 1995

New Measurement of deuteron $(\gamma, p) n$ and Spin Problems in Coupled $N \Delta / N N$ Interactions

Phys. Rev. Lett. 76:1023, 1996

Polarized Compton Scattering from the Proton

FERMILAB-CONF-95-094

$\mathrm{QCD}$ at $\mathrm{DO}$ and CDF

FERMILAB-CONF-96-132-E

Preliminary Measurement of the Inclusive Jet and Dijet Cross Section in $\bar{p} p$ Collisions at $\sqrt{s}=1.8$ TeV

ALBUQUERQUE-94,v. 2, p. 1052; FERMILAB-CONF-94-293-E; HEPEX-9410002

Search for Supersymmetric wino and zino States using the Do Detector

Phys. Lett. 334B:234, 1994; IFVE-93-87

Upper Limit for the Branching Ratio of $K_{S} \rightarrow e^{+} e^{-}$Decay

Z. Phys. C70:31, 1996; BUDKERINP-93-54

The Measurement of $R$ in $e^{+} e^{-}$Annihilation at Center-of-Mass Energies between 7.2 and $10.34 \mathrm{GeV}$

Z. Phys. C62:367, 1994; BUDKERINP-93-53

Measurement of Inclusive $\Lambda$ Production in Electron-Positron Interactions at the Upsilon Energies

Z. Phys. C69:215, 1995; BUDKERINP-95-8

Bose-Einstein Correlations in $e^{+} e^{-}$Annihilation in the $\mathbf{\Upsilon}(1 S)$ and Continuum

Yad. Phys. 58:1814, 1995; Phys. At. Nucl. 58:1713, 1995

On the Mechanism of the Reactions He $p \rightarrow{ }^{3} \mathrm{H} p p$ and $\mathrm{He} p \rightarrow{ }^{3} \mathrm{He} p$ with $2.7 \mathrm{GeV} / c \mathrm{He} \mathrm{Particles}$

Phys. Rev. D57:5903, 1998; CLNS-97-1530; CLEO-97-29; HEPEX-9712010

New Limits for Neutrinoless Tau Decays

ASTROPH-9811389

Diffuse Gamma-Ray Emission from Starburst Galaxies and M31

Z. Phys. A353:415, 1996

Precise Pion Electroproduction in the $p\left(e^{-}, e^{-1} \pi^{+}\right) n$ Reaction at $\mathrm{W}=1125 \mathrm{MeV}$

Phys. Rev. Lett. 77:2396, 1996; DAPNIA-SPHN-96-12

Forward-Angle ${ }^{3} \mathrm{He}\left(e, e^{\prime} \pi^{ \pm}\right)$Coincident Electroproduction and the Search for $\Delta$ 's in the Ground State of ${ }^{3} \mathrm{He}$

Nucl. Phys. A626:871, 1997

Pion Electroproduction in the ${ }^{3} \mathrm{He}\left(e^{-}, e^{-} \pi^{+}\right){ }^{3} \mathrm{H}$ Reaction at Intermediate Energies

Phys. Lett. $421 \mathrm{~B}: 71,1998$

Investigation of Short-Range Nucleon-Nucleon Correlation using the Reaction ${ }^{12} \mathrm{C}\left(e^{-}, e^{-1} p p\right)$ in $\mathrm{Close}$ to $4 \pi$ Geometry

Phys. Rev. Lett. 81:4576, 1998; NPL-98-1; NUCLEX-9808001

Measurements of Deuteron Photodisintegration up to $4.0 \mathrm{GeV}$

CERN-EP-98-046

Lower Bound for the Standard Model Higgs Boson Mass from Combining the Results of the Four LEP Experiments

Phys. Rev. Lett. 81:534, 1998

Measurement of the Target Asymmetry of $\eta$ and $\pi^{0}$ Photoproduction on the Proton

Z. Phys. C63:37, 1994

Measurement of the $\gamma p \rightarrow K^{+} \Lambda$ and $\gamma p \rightarrow K^{+} \Sigma^{0}$ at Photon Energies up to $1.47 \mathrm{GeV}$

TSUKUBA-93 p. ?; CERN-PPE-94-047; HEPHY-PUB-599-94

Inclusive Production of Charged Particles and Minijets in $p \bar{p}$ Collisions at $\sqrt{s}=630 \mathrm{GeV}$

Phys. Lett. 366B:441, 1996; CERN-PPE-95-169; HEPHY-PUB-629-95

Inclusive Production of Strange Particles $p \bar{p}$ Collisions at $\sqrt{s}=630 \mathrm{GeV}$ with UA1

See the legend on page 5. 
Bocquet 95B

Bocquet $95 \mathrm{C}$

Bodek 96

Bodmann 94

Bodmann 94B

Boezio 97

Boezio 98

Bogdanov 94

Boggild 94

Boggild 94B

Boggild 95

Boggild 95B

Boggild 95C

Boggild 95D

Boggild 96

Boggild 98

Boggild 99

Bogolyubsky 94

Bogolyubsky 95

Bogolyubsky 97

Bogomolov 94

Bogomolov 95

Boldyshev 95

Bolonkin 95

Bond 94

Bondar 95

Bondarenko 94

Bondarenko 96

Bondarenko 98

Bondarenko $98 \mathrm{~B}$

Bondarenko $98 \mathrm{C}$

Bondarev 94

Bondarev 98

Bonivento 94

Bonutti 97

Bonutti 98

Bonvicini 97
Phys. Lett. 366B:447, 1996; CERN-PPE-95-170; HEPHY-PUB-630-95

A Study of Particle Ratios and Strangeness Suppression in $p \bar{p}$ Collisions at $\sqrt{s}=630$ GeV with UA1

Phys. Lett. 366B:434, 1996; CERN-PPE-95-168

Transverse Momentum Spectra of Charged Particles in $p \bar{p}$ Collisions at $\sqrt{s}=830 \mathrm{GeV}$

FERMILA B-CONF-96-219-E; UR-1465; ER-40685-491

Quarks and Gluons at Hadron Colliders

Phys. Lett. 332B:251, 1994

Neutrino Interactions with Carbon: Recent Measurements and New Tests of $\nu_{e}, \bar{\nu}_{\mu}$ Universality

Phys. Lett. 339B:215, 1994

Determination of the Nuclear Weak Axial Charge Radius of ${ }^{12} \mathrm{C}$ via the ${ }^{12} \mathrm{C}\left(\nu_{e}, e^{-}\right){ }^{12} \mathrm{Nit}_{j . s .}$ Reaction

Astroph. Jour. 487:415, 1997; INFN-AE-97-01

The Cosmic Ray Antiproton Flux between 0.62 and $3.19 \mathrm{GeV}$ Measured near Solar Minimum

INFN-AE-98-06

The Cosmic Ray Proton And Helium Spectra between 0.2 and $200 \mathrm{GeV}$

Phys. At. Nucl. 57:567, 1994; Yad. Phys. 57:601, 1994

Fragmentation of ${ }^{20} \mathrm{Ne},{ }^{40} \mathrm{Ar}$, and ${ }^{56} \mathrm{Fe} N$ Nuclei with Energy of $0.1-0.5 \mathrm{GeV} / \mathrm{Nucleon}$ on Emulsion Nuclei

Nucl. Phys. A566:515C, 1994

Single Particle Spectra from NA44

Phys. Lett. 349B:386, 1995; CERN-PPE-94-177

Directional Dependence of the Pion Source in High-Energy Heavy Ion Collisions

Nucl. Phys. A590:215C, 1995

Recent Results from NA44 and a Review of HBT

Nucl. Phys. A590:483C, 1995

deuteron and deuteron Production in CERN Experiment NA44

Nucl. Phys. A590:523C, 1995

Charged Hadron Distributions in $p$ nucleus and nucleus nucleus Collisions at the CERN SPS

Z. Phys. C69:621, 1996; CERN-PPE-95-157

Low $p_{T}$ Phenomena in $\mathrm{A}+\mathrm{A}$ and $p+\mathrm{A}$ Collisions at Mid Rapidity

Phys. Lett. 372B:339, 1996; CERN-PPE-96-02 Coulomb Effect in Single Particle Distributions

CERN-EP-98-155

Two-Proton Correlations near Midrapidity in $p+\mathrm{Pb}$ and $\mathrm{S}+\mathrm{Pb}$ Collisions at the CERN SPS

Phys. Rev. C59:328, 1999; TAMU-CYCL-9803; NUCLEX-9808002

Charged $K^{ \pm}$and $\pi^{+}$Production at Midrapidity in $p$ nucleus and $\mathrm{S}$ nucleus Collisions

Phys. At. Nucl. 57:2132, 1994; Yad. Phys. 57:2216, 1994

Factorial Moments of Multiplicity Distribution in the Intervals and Clan Model

Yad. Phys. 58:1984, 1995; Phys. At. Nucl. 58:1877, 1995

Characteristics of Charged Particle Groups in $\bar{p} p, p p$, and $K^{-} p$ Interactions at $32 \mathrm{GeV} / c$

Yad. Phys. 60:53, 1997; Phys. At. Nucl. 60:46, 1997

Inclusive Production of the $\rho^{\mathrm{o}}$ and $f_{2}(1270)$ Resonances in $\bar{p} p$ and $p p$ Interactions at $32 \mathrm{GeV} / c$

Kr. Soob. Fiz. 94-1-2:36, 1994

Balloon Studies of the Isotope Composition of the Hydrogen Nucleiof Galactic Cosmic Radiation in the Energy Range $0.8-1.8 \mathrm{GeV} / \mathrm{Nucleon}$

Izv. RAN, Fiz. 59-4:145, 1995

Studies of Deuterium Fluxes in Galactic Cosmic Rays in the Energy Range $0.8-1.8 \mathrm{GeV} / \mathrm{Nucleon}$ with

the Balloon Technic
Phys. At. Nucl. 58:39, 1995; Yad. Phys. 58:43, 1995

Measurement of Photon Linear Polarization by Electron Recoil Asymmetry in the Triplet Photoproduction

Phys. At. Nucl. 58:1535, 1995; Yad. Phys. 58:1628, 1995

Study of $K_{S} K_{L} \pi^{-}$System Produced in $\pi^{-} p$ Interactions at $40 \mathrm{GeV} / c$ Momentum. Observation of $a_{1}(1260)^{-} \rightarrow K^{0} K^{*}(892)^{-}$Decay Mode

Phys. Rev. Lett. 72:13,1994

Measuring Cosmological Parameters with Cosmic Microwave Background Experiments

Phys. Lett. 356B:8, 1995

The $p p \rightarrow p p \pi^{0}$ Reaction near the Kinematic Threshold

Yad. Phys. 57:430, 1994; Phys. At. Nucl. 57:403, 1994

${ }^{12} \mathrm{C} \rightarrow 3{ }^{4} \mathrm{He}$ Dissociation on Hydrogen, Carbon, and Tantalum Nuclei at $4.2 \mathrm{GeV} / \mathrm{c}$ per Nucleon

Phys. At. Nucl. 60:1833, 1997; Yad. Phys. 60:2004, 1997; JINR-P1-96-447

Study of Nucleus-Nucleus Interactions with Complete Destruction of the Target Nucleus with Momentum $4.2 \mathrm{GeV} / \mathrm{c}$ per Nucleon

Phys. At. Nucl. 61:214, 1998; Yad. Phys. 61:263, 1998; JINR-P1-97-99

Properties of the Multiplicity Distributions for $\alpha$ Particles Produced by the Fragmentation of Relativistic Nuclei

JINR-P1-98-292

The Ensemble of Interactions on Carbon and Hydrogen Nuclei Obtained using the 2m Propane Bubble Chamber Exposed to the Beams of Protons and deuteron, ${ }^{4} \mathrm{He},{ }^{12} \mathrm{C}$ Relativistic Nuclei at the Dubna Synchrophasotron

JINR-P1-98-155

Total Destruction of Target Nuclei and Modified Cascade-Evaporation Model of Nucleus-Nucleus Inter-

KOCtions 94 p. 177

Cumulative Particle Production on Beams of Protons and Light Nuclei

DUBNA-96, v. II, p. 136

A-Dependence of Cumulative Particle Production Cross Section

Nuovo Cim. 107A:2377, 1994

DELPHI Results on the Hadronic Decays of the $\tau$-Lepton

Phys. Rev. C55:2998, 1997

Pion-Induced Pion Production in Nuclei

Nucl. Phys. A638:729, 1998

$\pi^{+} \rightarrow \pi^{+} \pi^{ \pm}$on Deuterium at $T_{x+}=283 \mathrm{MeV}$

Phys. Rev. Lett. 79:1221, 1997; CLNS-97-1469; CLEO-97-4; HEPEX-9704010

Search for Neutrinoless $\tau^{ \pm}$Decays Involving $\pi^{0}$ or $\eta$ Mesons 
Bonvicini 97B

Boos 94

Boos 95

Boos 96

Boothby 97

Borderie 96

Bordner 96

Borer 94

Borione 94

Borione 97

Borione 97B

Borisov 94

Borisov 96

Borisov 97

Bormann 97

Bosted 93

Bottino 97

Bottino 98

Botvina 95

Bourdon 95

Bouwhuis 98

Bowler 94

Bowler 95

Boyarinov 94

Brabson 96

Brack 95

Bradamante 94

Bradamante 95

Brady 94

Braghieri 95

Brandenburg 95

Brandenburg 97

Brandenburg 98

Brandt 95

Brandt 97

Brandt 97B

Brandt 97C

Brash 95
Phys. Rev. D57:6604, 1998; CLNS-97-1519; CLEO-97-26; HEPEX-9712008 Study of Semileptonic Decays of $B$ Mesons to Charmed Baryons

Phys. At. Nucl. 57:248, 1994; Yad. Phys. 57:263, 1994 Investigation of the Rations of Hadron's Structure Functions in Inclusive Multiple Processes at High Energies

Z. Phys. A351:209, 1995

Study of Nuclear Density Effects on Secondary Hadrons Many-Particle Correlations

Phys. At. Nucl. 59:463, 1996; Yad. Phys. 59:491, 1996 Invariant Cross Section for Charged-Pion Production in $\bar{p} p$ Collisions at an Incident Momentum of $22.4 \mathrm{GeV} / c$

Astroph. Jour. 491:L35, 1997; ASTROPH-9710168

A New Measurement of Cosmic Ray Composition at the Knee

Phys. Lett. 388B:224, 1996; DAPNIA-SPHN-96-26

Kinematical Properties and Composition of Vaporizing Sources: is Thermodynamical Equilibrium Achieved?

Z. Phys. C72:249, 1996

Experimental Information on the Pion Gluon Distribution Function

Phys, Rev. Lett. 72:1415, 1994; BUHE-93-11

Strangelet Search in S Wt Collisions at $200 \mathrm{~A} \mathrm{GeV} / \mathrm{c}$

Phys. Rev. D49:1171, 1994

Observation of the Shadows of the Moon and Sun using $100 \mathrm{GeV}$ Cosmic Rays

Phys. Rev. D55:1714, 1997

High Statistics Search for Ultrahigh Energy $\gamma$-Ray Emission from Cygnus X-3

EFI-96-43; ASTROPH-9703063

Constraints on Gamma Ray Emission from the Galactic Plane at $300 \mathrm{TeV}$

Izv. RAN, Fiz. 58-12:10, 1994; Bull. Russ. Acad. Sci. Phys. Ser. 58:1957, 1994

Coplanar Emission of Particles in Multiparticle Production at High Energies (PAMIR Experiment)

Phys. Lett. 369B:39, 1996

The Search for Inclusive Electron-Neutrino Oscillations on the Serpukhov $70 \mathrm{GeV}$ Accelerator with the IHEP-JINR Neutrino Detector

Izv. RAN, Fiz. $61: 449,1997$

Mass Composition of Primary Cosmic Rays According to Data on Spectrum of Halo Areas

Jour. of Phys. G 23:1817, 1997

Kaon-, $\Lambda$ - and $\bar{\Lambda}$-Production in $\mathrm{Pb}+\mathrm{Pb}$-Collisions at $158 \mathrm{GeV}$ per Nucleon

Phys. Rev. D49:3091, 1994; SLAC-PUB-6374

Threshold Inelastic Electron Scattering from the Proton at High Momentum Transfer

Phys. Lett. 402B:113, 1997; DFTT-75-96; GEF-TH-19-96; ROM2F-96-63; HEPPH-9612451

Exploring the Supersymmetric Parameter Space by Direct Search for WIMPs

Phys. Lett. 423B:109, 1998; DFTT-49-97; HEPPH-9709292

Pinning Down Neutralino Properties from a Possible Modulation Signal in WIMP Direct Search

- Nucl. Phys. A584:737, 1995

Multifragmentation of Spectators in Relativistic Heavy-Ion Reactions

Nucl. Phys. (PS) B40:203, 1995; MONTREUX-94 p. 203; X-LPNHE-95-02

Study of the $\tau^{ \pm} \rightarrow 3 \pi \pi^{0} \nu_{\tau}$ Decays in ALEPH

Phys. Rev. Lett. 82:3755, 1999; NUCLEX-9810004

Measurement of $T_{20}$ in Elastic Electron-Deuteron Scattering

Phys. Lett. 331B:193, 1994; OUN P-94-04

The Origin of the Spurious $17 \mathrm{keV}$ Neutrino Signal Observed in ${ }^{35} \mathrm{~S} \beta$-Decay

Z. Phys. C68:391, 1995

Investigations into the Origin of the Spurious $17 \mathrm{keV}$ Neutrino Signal Observed in ${ }^{35} \mathrm{~S}$ Beta Decay

Phys. At. Nucl. 57:1379, 1994; Yad. Phys. 57:1452, 1994

Cumulative $p, \bar{p}, \pi^{ \pm}, K^{ \pm}$Production at $97^{\circ}$ in Laboratory System by $10.14 \mathrm{GeV}$ Protons

MANCHESTER-95 p. 494

Study of the $\pi^{0} \pi^{0}$ System in $\pi^{-} p$ Interactions at $18 \mathrm{GeV} / c$

Phys. Rev. C51:929, 1995

Forward Angle $\pi^{ \pm} p$ Elastic Scattering Differential Cross Sections at $T_{\pi}=87$ to $13 \theta \mathrm{MeV}$

CERN-PPE-94-215

Experimental Results on nucleon nucleon Scattering

Phys. Lett. 343B:431, 1995; CERN-PPE-94-211

Determination of the Charged Pion-Nucleon Coupling Constant from $\bar{p} p \rightarrow \bar{n} n$ Differential Cross Section

Phys. Rev. C50:525, 1994

Fragment Mass Dependence of $p_{T}$ at $\mathrm{GeV}$ per Nucleon Energies

Phys. Lett. 363B:46, 1995

Total Cross Section Measurement for the Three Double Pion Photoproduction Channels on the Proton

Phys. Rev. Lett. 75:3804, 1995; CLNS-95-1351; CLEO-95-13

Measurements of the Ratios $B\left(D_{s}^{+} \rightarrow \eta \ell^{+} \nu\right) / B\left(D_{s}^{+} \rightarrow \phi(1020) \ell^{+} \nu\right)$ and $B\left(D_{s}^{+} \rightarrow \eta^{\prime} \ell^{+} \nu\right) / B\left(D_{s}^{+} \rightarrow\right.$ $\left.\phi(1020) \ell^{+} \nu\right)$

Phys. Rev. Lett. 80:2762, 1998; CLNS-97-1485; CLEO-97-11; HEPEX-9706019

A New Measurement of $B \rightarrow D^{*} \pi$ Branching Fractions

Phys. Rev. D58:052003, 1998; CLNS-98-1542; CLEO-98-3; HEPEX-9802022 Continuum Charged $D^{*}$ Spin Alignment at $\sqrt{s}=10.5 \mathrm{GeV}$

JINR-E1-95-502

Further Evidences for Enhanced Nuclear Cross Sections Observed in $44 \mathrm{GeV}$ Carbon Ion Interactions with Copper

Phys. Lett. 421 B:395, 1998; CERN-PPE-97-126; HEPEX-9709015

Cross Section Measurements of Hard Diffraction at the $S p \bar{p} S$-Collider

Nucl. Phys. B519:3, 1997; HEPEX-9712017

Measurements of Inclusive $\bar{\Lambda}$ Production with Large $x_{F}$ at the SPS-Collider

Nucl. Phys. B514:3, 1998; HEPEX-9710004

Measurements of Single Diffraction at $\sqrt{s}=630 \mathrm{GeV}$ : Evidence for a Non-Linear $\alpha_{t}$ of the Pomeron

Phys. Rev. C52:807, 1995

Spin Observables in Elastic Proton Scattering from Polarized ${ }^{3} \mathrm{He}$

See the legend on page 5. 
Brauksiepe 97

Bravar 95

Bravar 96

Bravar 97

Breakstone 95

Breitweg 97

Breitweg 97B

Breitweg $97 \mathrm{C}$

Breitweg 97D

Breitweg $97 \mathrm{E}$

Breitweg 97F

Breitweg $97 \mathrm{G}$

Breitweg $97 \mathrm{H}$

Breitweg 97I

Breitweg 97J

Breitweg $97 \mathrm{~K}$

Breitweg 97L

Breitweg $97 \mathrm{M}$

Breitweg 97N

Breitweg 98

Breitweg 98B

Breitweg 98C

Breitweg 98D

Breitweg $98 \mathrm{E}$

Breitweg $98 \mathrm{~F}$

Breitweg 98G

Breitweg $98 \mathrm{H}$

Breitweg 98I

Breitweg 98J

Breitweg $98 \mathrm{~K}$

Breitweg 98L

Breitweg 99

Bressan 97

Bressi 97

Breton 95

Breuer 94

Breuer 95

Briancon 98

Brient 94
Nucl. Phys. A626:85C, 1997

Threshold Measurements at the Internal Experimental Facility COSY-11

Phys. Rev. Lett. 75:3073, 1995

Analyzing Power Measurement in Inclusive $\Lambda$ Production with a $200 \mathrm{GeV} / \mathrm{c}$ Polarized Proton Beam

Phys. Rev. Lett. 77:2626, 1996; FERMILAB-PUB-96-086-E Single-Spin Asymmetries in Inclusive Charged Pion Production by Transversely Polarized Antiprotons

Phys. Rev. Lett. 78:4003, 1997; FERMILA B-PUB-96-393-E Spin Transfer in Inclusive $\Lambda$ Production by Transversely Polarized Protons at $200 \mathrm{GeV} / \mathrm{c}$

Z. Phys. C69:55, 1995; CERN-PPE-95-102 Inclusive Charged Particle Cross Sections in Full Phase Space from Proton Proton Interactions at ISR Energies

Z. Phys. C74:207, 1997; DESY-97-025; HEPEX-9702015 Comparison of ZEUS Data with Standard Model Predictions for $e^{+} p-e^{+} \mathrm{X}$ Scattering at High $x$ and

Z. Phys. C75:421, 1997; DESY-97-061; HEPEX-9704008

Study of Photon Dissociation in Diffractive Photoproduction at HERA

Z. Phys. C76:599, 1997; DESY-97-147; HEPEX-9708010 Measurement of Inelastic $J / \psi(1 S)$ Photoproduction at HERA

Phys. Lett. 413B:201, 1997; DESY-97-146; HEPEX-9708038

Observation of Isolated High- $E_{T}$ Photons in Photoproduction at HERA

2. Phys. C75:215, 1997; DESY-97-060; HEPEX-9704013

Measurement of Elastic $J / \psi(1 S)$ Photoproduction at HERA

Phys. Lett. 407B:402, 1997; DESY-97-089; HEPEX-9706009

$D^{*}$ Production in Deep Inelastic Scattering at HERA

Phys. Lett. 401B:192, 1997; DESY-97-026; HEPEX-9704011

Differential Cross Sections of $D^{*}$ Photoproduction in $e^{-} p$ Collisions at HERA

Eur. Phys. J. C1:109, 1998; DESY-97-196; HEPEX-9710018

Dijet Cross Sections in Photoproduction at HERA

Eur. Phys. J. C1:81, 1998; DESY-97-184; HEPEX-9709021

Measurement of the Diffractive Structure Function $F_{2}^{D(4)}$ at HERA

Phys. Lett. 407B:432, 1997; DESY-97-135; HEPEX-9707025

Measurement of the Proton Structure Function $\mathrm{F}_{2}$ and $\sigma_{t o t}\left(\gamma^{*} p\right)$ at Low $q^{2}$ and Very Low $x$ at HERA

Eur. Phys. J. C2:61, 1998; DESY-97-191; HEPEX-9710002

Measurement of Jet Shapes in Photoproduction at HERA

Eur. Phys. J. C2:77, 1998; DESY-97-229; HEPEX-9711018

Charged Particles and Neutral Kaons in Photoproduced Jets at HERA

Eur. Phys. J. C2:247, 1998; DESY-97-237; HEPEX-9712020

Measurement of Elastic $\rho^{0}$ Photoproduction at HERA

2. Phys. C76:631, 1997; DESY-97-112; HEPEX-9708007

A Search for Excited Fermions in $e^{+} p$ Collisions at HERA

Eur. Phys. J. C4:591, 1998; DESY-98-018; HEPEX-9802012

High-E $E_{T}$ Inclusive Jet Cross Sections in Photoproduction at HERA

Eur. Phys. J. C5:41, 1998; DESY-98-045; HEPEX-9804013

Diffractive Dijet Cross Sections in Photoproduction at HERA

Eur. Phys. J. C6:239, 1999; DESY-98-050; HEPEX-9805016

Forward Jet Production in Deep Inelastic Scattering at HERA

Phys. Lett. 434 B:214, 1998; DESY-98-069; HEPEX-9806019

Search for Selectrons and Squarks Production in $e^{+} p$ Collisions at HERA

Eur. Phys. J. C6:67, 1999; DESY-98-085; HEPEX-9807008

Measurement of Inclusive $D^{*}(2010)^{ \pm}$and Associated Dijet Cross Sections in Photoproduction at HERA

Eur. Phys. J. C6:43, 1999; DESY-98-084; HEPEX-9807010

Measurement of the Diffractive Cross Section in Deep Inelastic Scattering using ZEUS 1984 Data

Phys. Lett. 437B:432, 1998; DESY-98-089; HEPEX-9807020

Measurement of Elastic $\Upsilon$ Photoproduction at HERA

Eur. Phys. J. C7:609, 1999; DESY-98.121; ANL-HEP-PR-98-120; HEPEX-9809005

ZEUS Results on the Measurement and Phenomenology of $F_{2}$ at Low $\mathrm{x}$ and Low $Q^{2}$

Phys. Lett. 443B:394, 1998; DESY-98-162; HEPEX-9810046 Measurement of Three Jet Distributions in Photoproduction at HERA

Eur. Phys. J. C2:237, 1998; DESY-97-238; HEPEX-9712019

Measurement of the $t$ Distribution in Diffractive Photoproduction at.HERA

Eur. Phys. J. C6:603, 1999; DESY-98-107; ANL-HEP-PR-98-95; HEPEX-9808020

Exclusive Electroproduction of $\rho^{0}$ and $J / \psi(1 S)$ Mesons at HERA

Phys. Lett. 421B:368, 1998; DESY-97-202; HEPEX-9710027

Event Shape Analysis of Deep Inelastic Scattering Events with a Large Rapidity Gap at HERA

Eur. Phys. J. C8:367, 1999; DESY-98-038; HEPEX-9804001

Measurement of Jet Shapes in High $Q^{2}$ Deep Inelastic Scattering at HERA

Nucl. Phys. A625:10, 1997

A New Measurement of the $\bar{p} p \rightarrow \bar{n} n$ Charge Exchange Differential Cross Section at LEAR

Nucl. Phys. B513:555, 1998; DFPD-97-EP-43

New Measurement of the $\pi \rightarrow \mu^{-} \nu \gamma$ Decay

Nucl. Phys. (PS) B39:92, 1995

Precision Measurement of the Proton Polarized Structure Function $g_{1}^{p}$

Phys. Rev. C49:2276, 1994

Excitation Function for ${ }^{4} \mathrm{He}\left(\pi^{+}, p\right.$ p)deuteron Two-Nucleon Absorption across the $\Delta$ Resonance

BONN-IR-95-04

Measurement of $\eta$ Photoproduction on the Proton at Photon Energies around $1150 \mathrm{MeV}$

Phys. At. Nucl. 61:1293, 1998; Yad. Phys. 61:1395, 1998

Experiment AnCor: Search for Scalar Interaction in $\beta$-Decay and $\mu$-Capture

X-LPNHE-94-08

Tau Polarization at LEP 
Brient 97

Brill 96

Brill 97

Brisson 94

Brisson 94B

Britton 94

Brodowski 96

Brohm 95

Brose 94

Brovkin 94

Browder 97

Browder 97B

Browder 98

Brown 94

Brown 96

Brown 96C

Browne 96

Broz 95

Bruins 95

Brummer 96

Brummer 96B

Brune 99

Bruno 94

Bryman 96

Brzychezyk 95

Brzychezyk 98

Buchler 94

Buckleygeer 94

Buckleygeer 95

Budd 94

Buergin 97

Buki 95

Bulekov 94

Bulekov 97

Bulekov 97B

Bulekov 98

Bulgakov 97

Bulten 95
Nucl. Phys. (PS) B55:57, 1997; ESTES PARK-96 p. 57

Review of the Tau Polarization in $Z^{\circ}$ Decays at LEP using $\tau$ Hadronic Decay Channels

Z. Phys. A355:61, 1996; GSI-96-01

Study of the Out-of-Plane Emission of Protons and Light Fragments in Symmetric Heavy-Ion Collisions Z. Phys. A357:207, 1997

Studies of the Out-of-Plane Emission of Pions in Symmetric Heavy-Ion Collisions

GLASGOW-94 p. 635; LAL-94-61; DESY-H 1-09-94-395

Proton Structure Function $F_{2}\left(x, Q^{2}\right)$ at HERA

DESY-94-187

New Results from HERA on Deep Inelastic Scattering at Low $x$, the Proton Structure Function, Jets in Photoproduction, Heavy Flavor Production and Searches for New Particles

Phys. Rev. D49:28, 1994; TRI-P P-93-25

Measurement of the $\pi^{+} \rightarrow e^{+} \nu_{e}$ Branching Ratio

Z. Phys. A355:5, 1996

A Signal of a Narrow $\pi n n$ Resonance in $p p \rightarrow p p \pi^{-} \pi^{+}$

Nucl. Phys. A585:565, 1995

Total Charge-Changing Cross Sections of Stable and Neutron-Deficient Secondary Projectiles around $A=60$

Nuovo Cim. 107A:2227, 1994

High-Statistics Analysis of $\bar{p} p \rightarrow \pi^{0} \pi^{0} \pi^{0}$ at Rest

ITEP-94-58

Observation of the Five-Quark State $E^{--}(1400)$ in the $n 2 \pi^{-}$System

Phys. Rev. D56:5359, 1997; CLNS-97-1484; CLEO-97-10; HEPEX-9706005

A New Upper Limit on the Decay $\eta \rightarrow e^{+} e^{-}$

Phys. Rev. D56:11, 1997; CLNS-96-1422; CLEO-96-11

Search for $B \rightarrow \mu^{-} \bar{\nu}_{\mu} \gamma$ and $B \rightarrow e^{-} \bar{\nu}_{e} \gamma$

Phys. Rev. Lett. 81:1786, 1998; CLNS-98-1544; CLEO-98-4; HEPEX-9804018

Observation of High Momentum $\eta^{\prime}$ Production in $B$ Decay

Phys. Rev. D50:1884, 1994; CLNS-94-1271; CLEO-94-6

Precision Measurement of $D_{s}^{*+}-D_{s}^{+}$Mass Difference

Phys. Rev. C54:3195, 1996; FERMILAB-PUB-96-034-E

Nuclear Dependence of Single-Hadron and Dihadron Production in $p$ A Interactions at $\sqrt{s}=38.3 \mathrm{GeV}$

Phys. Rev. C54:R2105, 1996: MSUCL-1045

Measurement of the $p\left({ }^{8} \mathrm{He},{ }^{6} \mathrm{Li}\right) \boldsymbol{n}$ Reaction in Inverse Kinematics

Phys. Rev. C54:1538, 1996

Measurement of $T_{20}\left(90^{\circ}\right)$ in the $p$ (deuteron $\left.T, \gamma\right){ }^{3} \mathrm{He}$ Reaction below Deuteron Breakup Threshold

PRA-HEP-95-5; NUCLEX-9507002

Measurement of Spin-Dependent Total Cross-Section Difference $\Delta \sigma_{T}$ in Neutron-Proton Scattering at $16 \mathrm{MeV}$

Phys. Rev. Lett. $75: 21,1995$

Measurement of the Neutron Magnetic Form Factor

IC-HEP-96-9

Search for Heavy Right-Handed Electron Neutrinos at HERA

IC-HEP-96-8

Search for Exotics at HERA

Phys. Rev. C60:015801, 1999; NUCLEX-9902010

Total Cross Section of the ${ }^{3} \mathrm{H}(p, n)^{3}$ He Reaction from Threshold to $4.5 \mathrm{MeV}$

Nucl. Phys. A576:138, 1994

Angular and Velocity Analysis of the Three-Fold Events in the Xe $+\mathrm{Cu}$ Reaction at $45 \mathrm{MeV} / \mathrm{u}$

Phys. Rev. D53:558, 1996

Search for Massive Neutrinos in $\pi^{+} \rightarrow \mu^{+} \nu$ Decay

DAPNIA-SPHN-95-11

${ }^{3} \mathrm{He}$ Induced Reactions on $\mathrm{Ag}_{n a t}$ and ${ }^{197} \mathrm{Au}$ at $1.8,3.6$ and $4.8 \mathrm{GeV}$

Phys. Rev. C58:1372, 1998

Diversity of Fragment Sizes in Multifragmentation of Au Nuclei Induced by Relativistic ${ }^{3} \mathrm{He}$ Ions

Nucl. Phys. A570:580, 1994

Photoproduction of Positive Pions from Hydrogen with PHOENICS at ELSA

FERMILAB-CONF-94-336-E

Studies of Prompt Photon Production and Multijet Production at the Tevatron Collider

FERMILA B-CONF-95-316-E; CDF-PUB-JET-PUBLIC-3348

Tests of Perturbative QCD at CDF

FERMILAB-CONF-94-337-E

$W^{ \pm}$- Charge Asymmetry at CDF: Tests of Structure Functions

FREIBURG-EHEP-97-05; HEPEX-9706007

Dijet Production at Photon-Photon Collisions at $\sqrt{s_{e e}}=161$ and $172 \mathrm{GeV}$

Phys. At. Nucl. 58:1271, 1995; Yad. Phys. 58:1353, 1995

The Transversal Momenta of ${ }^{4} \mathrm{He}$ Response Function in the Range of Momentum Transfer $0.75-1.5 \mathrm{fm}^{-1}$ ITEP-94-56

The Characteristics of $\Lambda$-Hyperons Produced in Pion-Nucleus and Pion-Nucleon Interactions at 4 B GeV/c Initial Momenta

Phys. At. Nucl. 61:74, 1998; Yad. Phys. 61:80, 1998; ITEP-97-4

The Characteristics of Leading $\Lambda$ Hyperons Produced by Neutrons at Nuclei

ITEP-97-25

The Correlations of the Longitudinal and Transversal Momenta in Pion-Nucleon and Pion-Nucleus Interactions with Strange Particles Production

ITEP-34-98; HEPEX-9812005

The $\Lambda K$ System Production in the Hadron Interactions

Yad. Phys. 60:1349, 1997; Phys. At. Nucl. 60:1349, 1997

Measurements of deuteron + deuteron $\rightarrow{ }^{3} \mathrm{He} n$ Cross Section at Ultralow Energies using $Z$-Pinch

Phys. Rev. Lett. 74:4775, 1995

Exclusive Electron Scattering from Deuterium at High Momentum Transfer 
Bunyatov 95

Burachas 95

Burkot 96

Burlein 95

Burleson 94

Burmistrov 95

Burnett 96

Burow 94

Burzynski 94

Buschbeck 98

Busetto 96

Buskulic 93D

Buskulic 93P

Buskulic 94

Buskulic 94B

Buskulic 94C

Buskulic 94D

Buskulic $94 \mathrm{E}$

Buskulic $94 \mathrm{~F}$

Buskulic $94 \mathrm{G}$

Buskulic $94 \mathrm{H}$

Buskulic 94I

Buskulic 94J

Buskulic $94 \mathrm{~K}$

Buskulic $94 \mathrm{~L}$

Buskulic 94M

Buskulic $94 \mathrm{~N}$

Buskulic 95

Buskulic 95B

Buskulic $95 \mathrm{C}$

Buskulic 95D

Buskulic $95 \mathrm{E}$

Buskulic 95F

Buskulic $95 \mathrm{G}$

Buskulic $95 \mathrm{H}$

Buskulic 95I

Buskulic 95J

Buskulic 95K

Buskulic 95L
Phys. At. Nucl. 60:935, 1997; Yad. Phys. 60:1045, 1997; JINR-E1-95-398

Cross Section for Production of Charmed Particles in $p$ nucleon Interactions at $70 \mathrm{GeV}$ in Proton Beam Dump Experiment at IHEP-JINR Detector

Phys. At. Nucl. 58:153, 1995; Yad. Phys. 58:195, 1995

A Search for ${ }^{100} \mathrm{Gd}$ Double Beta Decay using GSO Scintillators

Z. Phys. C70:47, 1996; HEPEX-9612015

Nuclear Attenuation of Fast Hadrons Produced in Charged-Current $\nu$ and $\bar{\nu}$ Interactions in Neon

Phys. Rev. C51:88, 1995

Pion Inelastic Scattering to the Low-Lying Positive-Parity States in ${ }^{20} \mathrm{Ne}$

Phys. Rev. C49:2226, 1994

Negative Pion-Nucleus Elastic Scattering at 20 and $40 \mathrm{MeV}$

Kr. Soob. Fiz. 95-3-4:57, 1995

Search for Narrow Dibaryon Resonance States in $p$ deuteron Interaction at the Energy $405 \mathrm{MeV}$

MANCHESTER-95 p. 457

Study of the Radiative Decay $J / \psi(1 S) \rightarrow \gamma \bar{p} p$

DESY-94-215

Photoproduction Physics at HERA

Nucl. Phys. A570:722, 1994; TRI-PP-93-71

The Elastic and Inelastic Scattering of Intermediate Energy Protons on Deuterium at Small Momentum Transfer

PHY-PUB-700-98; STPHY-30-98; HEPEX-9809004

Multiplicity Dependence of Like-Sign and Opposite-Sign Correlations in $\bar{p} p$ Reactions

Nuovo Cim. 109A:1013, 1996; PISA-95 p. 1013

Experimental Results on $B^{0} \bar{B}^{\circ}$ Mixing

Phys. Lett. 307B:194, 1993; Phys. Lett. 325B:537, 1994; CERN-PPE-93-42

Measurement of the $\bar{B}^{0}$ and $B^{-}$Meson Lifetime

Z. Phys. C62:1, 1994; CERN-PPE-93-208; FSU-SCRI-94-61

Production of Charmed Mesons in $Z^{\circ}$ Decays

Phys. Lett. 321 B:168, 1994; CERN-PPE-93-181; FSU-SCRI-94-19

Correlation Measurements in $Z^{0} \rightarrow \tau^{+} \tau^{-}$and the $\tau$ Neutrino Helicity

Phys. Let.t. 322B:275, 1994; CERN-PPE-93-214; RSU-SCRI-94-32 Measurement of the $B_{s}$ Lifetime

Phys. Lett. 322B:441, 1994; CERN-PPE-93-221; FSU-SCRI-94-34

An Investigation of $B^{0}$ and $B$, Oscillation

Z. Phys. C62:179, 1994; CERN-PPE-94-017; FSU-SCRI-94-60

Heavy Flavor Production and Decay with Prompt Leptons in the ALEPH Detector

Z. Phys. C62:539, 1994; CERN-PPE-94-030; FSU-SCRI-94-77

$Z^{\mathbf{0}}$ Production Cross-Sections and Lepton Pair Forward-Backward Asymmetries

Phys. Lett. 332B:219, 1994; CERN-PPE-94-59

$K^{0}$ Production in One Prong $\tau^{ \pm}$Decays

Phys. Lett. 332B:209, 1994; CERN-PPE-94-58

One Prong Tau Decays into Charged Kaons

Phys. Lett. 335B:99, 1994; CERN-PPE-94-084

A Measurement of $A_{F B}^{b}$ in Lifetime Tagged Heavy Flavor $Z^{0}$ Decays

Phys. Lett. 334B:244, 1994

Observation of Monojet Events and Tentative Interpretation

Phys. Lett. 346B:371, 1995; CERN-PPE-94-175

Search for $C P$ Violation in the Decay $Z^{0} \rightarrow \tau^{+} \tau^{-}$

2. Phys. C66:3, 1995; CERN-PPE-94-169

Study of the Four-Fermion Final State at the $Z^{0}$ Resonance

Phys. Lett. 345B:103, 1995; CERN-PPE-94-173

A Study of $D^{*}(2010)^{+} \pi^{-}$Production in Semileptonic $B$ Decay

Z. Phys. C64:361, 1994; CERN-PPE-94-074; FSU-SCRI-95-18

Production of $K^{\circ}$ and $\Lambda$ in Hadronic $Z^{\circ}$ Decays

Z. Phys. C66:355, 1995; CERN-PPE-94-201

Inclusive $\pi^{ \pm}, K^{ \pm}$and $(p, \bar{p})$ Differential Cross Sections at the $Z^{0}$ Resonance

Phys. Lett. 343B:444, 1995; CERN-PPE-94-165; FSU-SCRI-95-22

Measurement of the $b \rightarrow \tau^{-} \bar{\nu}_{\tau}$ X Branching Ratio and an Upper Limit on $B^{-} \rightarrow \tau^{-} \bar{\nu}_{\tau}$

Phys. Lett. 349B:585, 1995; CERN-PPE-95-03

An Upper Limit for the $\nu_{\tau}$ Mass from $\tau^{ \pm} \rightarrow 5 \pi\left(\pi^{0} s\right) \nu_{\tau}$ Decays

Phys. Lett. 355 B:595, 1995; CERN-PPE-95-040

Measurement of the $D^{*}(2010)^{ \pm}$Cross Section in Two Photon Collisions at LEP

Z. Phys. C69:365, 1996; CERN-PPE-95-089

First Measurement of the Quark-to-Photon Fragmentaton Function

Phys. Lett. 369B:151, 1996; CERN-PPE-95-121

$A$ Precise Measurement of the Average $b$ Hadron Lifetime

Phys. Lett. 357 B:685, 1995; CERN-PPE-95-65

Measurements of the $B$ Baryon Lifetime

Phys. Lett. 355B:381, 1995; CERN-PPE-95-18

Test of the Flavor Independence of $\alpha_{S}$

Z. Phys. C70:549, 1996; CERN-PPE-95-128

Measurement of the $\tau^{ \pm}$Lepton Lifetime

Z. Phys. C69:585, 1996; CERN-PPE-95-92

Measurement of $D_{s}^{+}$Meson Production in $Z^{\circ}$ Decays and of the $\bar{B}_{s}$ Lifetime

Phys. Lett. 352B:479, 1995; CERN-PPE-95-44 The Forward-Backward Asymmetry for Charm Quarks at the $Z^{\circ}$ Pole

Z. Phys. C69:183, 1995; CERN-PPE-95-23

Improved $\tau \pm$ Polarization Measurement

Phys. Lett. 357B:699, 1995; CERN-PPE-95-113

Measurement of the Effective $b$ Quark Fragmentation Function at the $Z^{\circ}$ Resonance 
Buskulic 95M

Buskulic 95N

Buskulic 950

Buskulic 95P

Buskulic 95Q

Buskulic 95R

Buskulic 95S

Buskulic 95T

Buskulic 95U

Buskulic 95V

Buskulic 95W

Buskulic 95X

Buskulic 96

Buskulic 96B

Buskulic 96C

Buskulic 96D

Buskulic 96E

Buskulic 96F

Buskulic 96G

Buskulic $96 \mathrm{H}$

Buskulic 96I

Buskulic 96J

Buskulic 96K

Buskulic 96L

Buskulic 96M

Buskulic 96N

Buskulic 960

Buskulic 96P

Buskulic 96Q

Buskulic $96 \mathrm{R}$

Buskulic 96S

Buskulic 96T

Buskulic 96U

Buskulic 96V

Buskulic 96W

Buskulic $96 \mathrm{X}$

Buskulic 96Y

Buskulic $96 \mathrm{Z}$
Z. Phys. C69:15, 1995; CERN-PPE-95-82

Measurement of the Charged Particle Multiplicity Distribution in Restricted Rapidity Intervals

Z. Phys. C70:561, 1996; CERN-PPE-95-127

Tau Leptonic Branching Ratios

Z. Phys. C70:579, 1996; CERN-PPE-95-140

Tau Hadronic Branching Ratios

Phys. Lett. 346B:379, 1995; Phys. Lett. 363B:265, 1995; CERN-PPE-94-209

Michel Parameters and $\tau^{ \pm}$Neutrino Helicity from Decay Correlations in $Z^{0} \rightarrow \tau^{+} \tau^{-}$

Phys. Lett. 346B:389, 1995; CERN-PPE-94-208

Study of the Subjet Structure of Quark and Gluon Jets

Phys. Lett. 384B:353, 1996; CERN-P PE-95-184

Quark and Gluon Jet Properties in Symmetric Three-Jet Events

Phys. Lett. 359B:236, 1995; CERN-PPE-95-094

A Measurement of the $\left|V_{c b}\right|$ from $\bar{B}^{0} \rightarrow D^{*}(2010)+\ell^{-} \bar{\nu}$

Phys. Lett. 361B:221, 1995; CERN-PPE-95-125

Measurement of the $B_{s}$ Lifetime and Production Rate with $D_{s}^{-} \ell^{+}$Combinations in $Z^{\circ}$ Decays

Phys. Lett. 356B:409, 1995; CERN-PPE-95-084

Limit on $B_{s}$ Oscillation using a Jet Charge Method

Z. Phys. C69:393, 1996; CERN-PPE-95-108

Production of Excited Beauty States in $Z^{0}$ Decays

Phys. Lett. 349B:238, 1995; CERN-PPE-95-15

Search for Supersymmetric Particles with $R$ Parity Violation in $Z^{\circ}$ Decays

Phys. Lett. 357B:487, 1995; Phys. Lett. 364B:247, 1995; CERN-PPE-95-096

Measurement of $\alpha_{s}$ from Scaling Violations in Fragmentation Functions in $e^{+} e^{-}$Annihilation

Phys. Lett. 374B:319, 1996; CERN-PPE-96-04

Measurement of $\Lambda$ Polarization from $Z^{\circ}$ Decays

Phys. Lett. 380B:442, 1996; CERN-PPE-96-28

Measurement of the Mass of the $\Lambda_{b}$ Baryon

Z. Phys. C74:263, 1997; CERN-PPE-96-103

A Study of $\tau^{ \pm}$Decays Involving $\eta$ and $\omega$ Mesons

Phys. Lett. 388B:419, 1996; CERN-PPE-96-088

Four Fermion Production in $e^{+} e^{-}$Collisions at Center-of-Mass Energies of 130 and $138 \mathrm{GeV}$

Phys. Lett. 384B:365, 1996; CERN-PPE-96-71

Search for $C P$ Violation in the Decay $Z^{\circ} \rightarrow b \bar{b}$ gluon

Phys. Lett. 384B:414, 1996; CERN-PPE-96-072

Measurement of the $b$ Forward-Backward Asymmetry and Mixing using High-p $p_{T}$ Leptons

Phys. Lett. 385B:445, 1996; CERN-PPE-96-087

Search for Excited Leptons at $130-140 \mathrm{GeV}$

Z. Phys. C73:421, 1997; CERN-PPE-96-085

Transverse Momentum Correlations in Hadronic $Z^{0}$ Decays

Phys. Lett. 384B:427, 1996; CERN-PPE-96-079

Mass Limit for the Standard Model Higgs Boson with the Full LEP I ALEPH Data Sample

Phys. Lett. 384B:333, 1996; CERN-PPE-96-53

A Study of Single and Multi-Photon Production in $e^{+} e^{-}$Collisions at Center-of-Mass Energies of 130 and $136 \mathrm{GeV}$

2. Phys. C71:179, 1996; CERN-PPE-96-052

Four Jet Final State Production in $e^{+} e^{-}$Collisions at Center-of-Mass Energies of 130 and $136 \mathrm{GeV}$

Phys. Lett. 384B:461, 1996; CERN-PPE-96-086

Search for Charginos and Neutralinos with $R$-Parity Violation at $\sqrt{s}=130$ and $136 \mathrm{GeV}$

Phys. Lett. 373B:246, 1996; CERN-PPE-96-10

Search for Supersymmetric Particles in $e^{+} e^{-}$Collisions of Center-of-Mass Energies of 130 and $136 \mathrm{GeV}$

2. Phys. C72:549, 1996; CERN-PPE-96-083

Mass Limit for the Lightest Neutralino

Phys. Lett. 388B:648, 1996; CERN-PPE-96-117

Charm Counting in $B$ Decays

Phys. Lett. 365B:437, 1996; CERN-PPE-95-156

Measurement of $\Lambda_{b}$ Polarization in $Z^{\circ}$ Decays

2. Phys. C73:601, 1997; CERN-PPE-96-092

Production of Orbitally Excited Charm Mesons in Semileptonic $B$ Decays

2. Phys. C75:397, 1997; CERN-PPE-96-102

Improved Measurement of the $B^{0}-\bar{B}^{\circ}$ Oscillation Frequency

Phys. Lett. 384B:471, 1996; CERN-PPE-96-104

Observation of Charmless Hadronic $B$ Decays

Z. Phys. C71:357, 1996; CERN-PPE-96-09

Determination of $\sin ^{2} \theta_{v}$ using Jet Charge Measurements in Hadronic $Z^{0}$ Decays

Phys. Lett. 377 B:205, 1996; : , 1996

Study of the $B_{s} \bar{B}_{s}$ Oscillation Frequency using $D_{s}^{-} \ell^{+}$Combinations in $Z^{0}$ Decays

2. Phys. C71:31, 1996; CERN-PPE-96-014

Improved Measurement of the $\bar{B}^{\circ}$ and $B^{-}$Meson Lifetimes

Phys. Lett. 395B:373, 1997; CERN-PPE-96-150

Measurement of $V_{c b}$, Form Factors and Branching Fractions in the Decays $\bar{B}^{0} \rightarrow D^{*}(2010)^{+} \ell^{-} \bar{\nu}_{\text {and }} \bar{B}^{0}$ $\rightarrow D^{+} \ell^{-} \bar{\nu}$

Z. Phys. C69:379, 1996; CERN-PPE-95-100

Inclusive Production of Neutral Vector Mesons in Hadronic $Z^{0}$ Decays

Phys. Lett. 378B:373, 1996; CERN-PPE-96-045

Measurement of Hadron and Lepton-Pair Production from $e^{+} e^{-}$Annihilation at Center-of-Mass Energies of 130 and $136 \mathrm{GeV}$

Phys. Lett. 384B:439, 1996; CERN-PPE-96-080

Search for Heavy Lepton Pair Production in $e^{+} e^{-}$Collisions at the Centre-of-Mass Energies of 130 and $130 \mathrm{GeV}$

See the legend on page 5. 
Buskulic 97

Buta 95

Buta $95 \mathrm{~B}$

Buta 96

Buta 97

Buta $97 \mathrm{~B}$

Buta $97 \mathrm{C}$

Buta 98

Buta $98 B$

Butler 93

Butler 94

Butler 95

Butler 96

Butsev $9 \vec{t}$

Butterworth 95

Buzzo 97

Byrum 94

Calen 96

Calen 97

Calen 98

Calen 98B

Calen $98 \mathrm{C}$

Camerini 93

Camoana 97

Carlson 94

Carlson 96

Carr 94

Carrel 94

Carter 94

Carter $94 \mathrm{~B}$

Casey 96

Casey 97

Cason 96

Castro 94

Castro 96

Cavallo 97

Cdfcollabora $94 \mathrm{~B}$
Z. Phys. C73:409, 1997; CERN-PPE-96-043

Studies of QCD in $e^{+} e^{-} \rightarrow$ Hadrons at $E_{\mathrm{cm}}=130$ and $136 \mathrm{GeV}$

Nucl. Phys. A584:397, 1995

Azimuthal Correlation Functions and the Energy of Vanishing Flow in Nucleus-Nucleus Collisions

Z. Phys. A 352:355, 1995

On the Transverse Momentum Distribution of Strange Hadrons Produced in Relativistic Heavy Ion Col-

lisions

A610:49C, 1996. NUCLEX-9610007

Particle Production and Flow at SIS Energies

Z. Phys. A359:47, 1997

Proton-Proton Correlations in Central Collisions of Ni Ni at $1.93 \mathrm{~A} \mathrm{GeV}$ and the Space-Time Extent of

the Emission Source

Z. Phys. A 358:73, 1997

Shape of Collective flow in Highly Central Au(150 A MeV) + Au Collisions

Z. Phys. A357:9, 1997

Shadowing Effects and Transverse Momentum Dependence of Particle Emission in Intermediate Energy Heavy Ion Collisions

Phys. Rev. C57:244, 1998; Phys. Rev. C58:603, 1998; NUCLEX-9707001

Stopping and Radial Flow in Central ${ }^{58} \mathrm{Ni}{ }^{58} \mathrm{Ni}$ Collisions between 1 and $2 \mathrm{~A} \mathrm{GeV}$

Nucl. Phys. A646:367, 1999; IRES-98-21; NUCLEX-9812005

Flow Angle from Intermediate Mass Fragment Measurements

Phys. Rev. D49:40, 1994; CLNS-93-1234; CLEO-93-12 Analysis of Hadronic Transitions in $\mathrm{Y}(3 S)$ Decays

Phys. Let t. 324B:255, 1994; CLNS-94-1272; CLEO-94-7

A Measurement of $B\left(D_{s}^{+} \rightarrow \phi(1020) \ell^{+} \nu\right) / B\left(D_{s}^{+} \rightarrow \phi(1020) \pi^{+}\right)$

Phys. Rev. D52:2656, 1995; CLNS-95-1324; CLEO-95-3

Measurement of the Ratio of Branching Fractions $B\left(D^{0} \rightarrow \pi^{-} e^{+} \nu_{e}\right) / B\left(D^{0} \rightarrow K^{-} e^{+} \nu_{e}\right)$

PADUA-96 p. 405; FERMILAB-CONF-96-299-E

Measurement of $\sigma(p \bar{p} \rightarrow t \bar{t})$ at $\sqrt{s}=1.8 \mathrm{TeV}$ by the Do Experiment

JINR-E1-97-195

Emission of Relativistic Heavy Fragments at Wide Angles from the Interaction of $58 \mathrm{GeV}{ }^{16} \mathrm{O}$ Ions with Thick Copper Target

DESY-95-043

Photon Structure as Seen at HERA

Z. Phys. C76:475, 1997; Eur. Phys. J. C5:739, 1998; HEPEX-9801015

Search for Narrow $\bar{p} p$ Resonances in the Reaction $\bar{p} p \rightarrow \bar{p} p \pi^{+} \pi^{-}$

FERMILAB-CONF-94-325-E

Charmonium Production, $b$ Quark and $B$ Meson Production and $b \bar{b}$ Correlations at CDF

Phys. Lett. 366 B:39, 1996

The $p p \rightarrow p p \eta$ Reaction near the Kinematical Threshold

Phys. Rev. Lett. 79:2642, 1997

Measurement of the Quasifree $p+n \rightarrow$ deuteron $+\eta$ Reaction Near Threshold

Phys. Rev. Lett. 80:2069, 1998

Threshold Structure of the Quasifree $p n \rightarrow$ deuteron $\eta$ Reaction

TSL-ISV-98-0198; NUCLEX-9811003

Higher Partial Waves in $p p \rightarrow p p \eta$ near Threshold

Phys. Rev. C58:2667, 1998

Measurement of the Quasifree $p n \rightarrow p n \eta$ Reaction

Nucl. Phys. A552:451, 1993; Nucl. Phys. A572:791, 1994

Threshold Behaviour of the $\pi^{+} \pi^{-}$Invariant Mass in Nuclei

Nucl. Phys. (PS) B55:161, 1997; ESTES PARK-96 p. 161 Studies of $\tau$ Decay Modes with $K$ Mesons

Phys. Rev. C49:3090, 1994

Proton Total Reaction Cross Section for ${ }^{42} \mathrm{Ca},{ }^{44} \mathrm{Ca}$, and ${ }^{48} \mathrm{Ca}$ between 21 and $48 \mathrm{MeV}$

Phys. Rev. D53:3506, 1996; ANL-HEP-PR-95-24 n $p$ Elastic Scattering Spin-Spin Correlation Parameter Measurements between 500 and 800 MeV. III. Mixtures of $C_{S S}, C_{L S}, C_{L L}$, and $C_{N N}$

Nuovo Cim. 107 A:1959, 1994

New Results on Electroweak $b$ Physics from LEP

Phys. Lett. 325B:526, 1994

Observation of Time Correlations in Cosmic Rays

FERMILAB-CONF-94-185-E

Production Asymmetries in $x_{F}$ and $p_{T}^{2}$ for $D^{ \pm}$Mesons

ALBUQUERQUE-94, v. 1, p. 513; FERMILAB-CONF-94-383

Production Asymmetries in $x_{F}$ and $p_{T}^{2}$ for $D^{ \pm}$Mesons

FERMILAB-CONF-96-272-EA

Do Papers on QCD with Vector Bosons Submitted to DPF 98: $p_{T}$ Dependence of Inclusive $Z^{\circ}$ Boson Production

L R-1514

Measurement of the Transverse Momentum of Dielectron Pairs in Proton-Antiproton Collisions

MANCHESTER-95 p. 55

Study of the $\eta \pi^{-}, \eta \pi^{0}$ and $\eta \eta$ Systems in $\pi^{-} p$ Interactions at $18 \mathrm{GeV} / c$

Nuovo Cim. 107 A:1807, 1994

Status of Vector Meson Spectroscopy from DM2 $e^{+} e^{-}$Annihilation Studies in the $1350-2400$ MeV Energy Range

FERMILAB-CONF-96-319-E

Top Production and Decay at CDF

Nucl. Phys. (PS) B55:153, 1997; ESTES PARK-96 p. 153

Study of $\tau^{ \pm}$Decays to $K K \pi$ and $K \pi \pi$ at LEP, with DELPHI

FERMILAB-CONF-94-127-E; CDF-PUB-BOTTOM-PUBLIC-2604

Measurement of the Polarization in the Decay $B^{\circ} \rightarrow J / \psi(1 S) K^{*}(892)^{0}$ in $p \bar{p}$ Collisions at $\sqrt{s}=1.8$ TeV 
Cdfcollabora $94 \mathrm{C}$

Cdfcollabora 94D

Cdfcollabora $94 \mathrm{E}$

Cdfcollabora $94 \mathrm{~F}$

Ceretto 95

Cerutti 95

Cester 94

Chadeeva 97

Chadha 97

Chadha 97B

Chakraborty 94

Chalyshev 98

Chan 98

Chantell 97

Chapkin 96

Charlesworth 95

Checchia 96

Chemakin 98

Chemakin 99

Chen 94

Chen 94B

Chen 96

Chen $96 \mathrm{~B}$

Chen 97

Cheplakov 95

Chernov 95

Chernov 95B

Chernykh 94

Cherry 94

Cherry 94B

Cherry $94 \mathrm{C}$

Cherry 95

Cherry 96

Cherry 97

Chiarelli 96

Chiavassa 94

Chiavassa 94B

Chiavassa $94 \mathrm{C}$
FERMILAB-CONF-94-152-E; CDF-PUB-TOP-PUBLIC-2593

Multi-Jet Analysis for the Top Quark Search at the Fermilab Collider

FERMILAB-CONF-94-154-E; CDF-PUB-ELECTROWEAK-PUBLIC-2676

Production Properties of $Z^{\circ}$ Bosons with Jets in $1.8 \mathrm{TeV} \bar{p} p$ Collisions

FERMILAB-CONF-94-141-E

Measurement of the $B$ Meson Differential Cross-Sections in $p \bar{p}$ Collisions at $\sqrt{s}=1.8 \mathrm{TeV}$ using the Exclusive Decays $B^{ \pm} \rightarrow J / \psi(1 S) K^{ \pm}$and $B^{\mathrm{o}} \rightarrow J / \psi(1 S) K^{*}(892)^{\mathrm{o}}$

CDF-PUB-BOTTOM-PUBLIC-2623; FERMILAB-CONF-94-136-E

$J / \psi(1 S), \psi(2 S) \rightarrow \mu^{+} \mu^{-}$and $B \rightarrow J / \psi(1 S), \psi(2 S)$ Cross Sections

Nucl. Phys. A590:103C, 1995; CERN-PPE-95-80

New Results from NA45/CERES

Nucl. Phys. (PS) B40:71, 1995

Measurement of an Upper Limit on the $\tau^{\ddagger}$ Neutrino Mass at ALEPH

Ann. Rev. Nucl. Part. Sci. 44:329, 1994; FERMILA B-PUB-94-123

Charmonium Formation in $\bar{p} p$ Annihilations

Pisma Zh. Eksp. Teor. Fiz. 66:69, 1997; Jetp Lett. 66:75, 1997; ITEP-97-8

The Contribution of $\left({ }^{3} \mathrm{H}\left({ }^{3} \mathrm{He}\right)+\right.$ nucleon) - Component to the $\alpha$ Particle Wave Function from the Data on the Reactions $p$ ( $\mathrm{He}, p p$ ) ${ }^{3} \mathrm{H}$ and $p$ (He,p n) ${ }^{3} \mathrm{He}$ at the Incident Moment um of $5 \mathrm{GeV} / c$

Phys. Rev. D58:032002, 1998; CLNS-97-1526; CLEO-97-28; HEPEX-9712014

Improved Measurement of the Pseudoscalar Decay Constant $f_{D_{s}}$

Nucl. Phys. (PS) B55:237, 1997; ESTES PARK-96 p. 237 Measurement of Michel Parameters at CLEO

ALBUQUERQUE-94, v. 1, p. 695; FERMILAB-CONF-94-294-E A Search for top top $\rightarrow \ell+$ missing $E_{T}+$ jets. Signature in $p \bar{p}$ Collisions at $\sqrt{s}=1.8$ TeV with the Do

Detector
Nucl. Phys. A639:1C, 1998; UPTON-97 p. 1C

Strangeness Production with Polarized Protons at Saturne

Phys. Rev. D58:072002, 1998

Measurement of the Properties of the $\bar{\Omega}^{+}$and $\Omega^{-}$Hyperons

Phys. Rev. Lett. 79:1805, 1997; ASTROPH-9705246

Limits on the Isotropic Diffuse Flux of Ultrahigh Energy $\gamma$ Radiation

MANCHESTER-95 p. 340

Inclusive Properties of $K^{*}(892)^{ \pm}, \rho^{\circ}, f_{0}(980)$, and $f_{2}(1270)$ in Hadronic Decays of the $Z^{0}$

Nucl. Phys. (PS) B40:341, 1995; MONTREUX-94 p. 341

Measurements of the $\tau^{ \pm}$Lifetime using the Decay Length Method

Nuovo Cim. 109A:999, 1996; PISA-95 p. 999

$D \bar{D}$ and $B \bar{B}$ Mixing

Nucl. Phys. A639:407C, 1998; UPTON-97 p. 407C

Strange Particle Production and an $H$-Dibaryon Search in $p$ nucleus Collisions at the AGS

UCRL-JC-133113; NUCLEX-9902009

Centrality Dependence of $\pi^{-}$Production and Stopping in $p$ nucleus Collisions at $18 \mathrm{GeV} / \mathrm{c}$

Phys. Rev. C49:3200, 1994

Interactions in Hydrogen of Relativistic Neon to Nickel Projectiles: Total Charge-Changing Cross Sections

Nucl. Phys. A569:616, 1994 Cross Section and Recoil Properties of the Products from the Interaction of $240 \mathrm{MeV}{ }^{12} \mathrm{C}$ Ions with $\mathrm{Ag}$

FERMILAB-CONF-96-272-EB

Do Papers on QCD with Vector Bosons Submitted to DPF 98: Isolated Prompt Single Photon and Diphoton Production in $\bar{p} p$ Collisions at $\sqrt{s}=1.8 \mathrm{TeV}$

Phys. Rev. C54:R2114, 1996

Emission of Intermediate Mass Fragments during Fission

Phys. Rev. C56:1536, 1997

Systematics of Isotopic Production Cross Sections from Interactions of Relativistic ${ }^{40} \mathrm{Ca}$ in Hydrogen

Kr. Soob. JINR 70:35, 1995

An Evidence for Collective Phenomenon in Heavy Ion Collisions at $4.2 \mathrm{~A} \mathrm{GeV} / \mathrm{c}$

Rad. Meas. 25:269, 1995

Coherent Dissociation of Oxygen into Four Alphas in Photoemulsion at $4.5 \mathrm{~A} \mathrm{GeV} / \mathrm{c}$

Kr. Soob. JINR 74:83, 1995

Coherent Multifragmentation of Relativistic Nuclei

Kr. Soob. JINR 67:29, 1994

Tensor Analyzing Power $T_{20}$ for deuteron ${ }^{12} \mathrm{C} \rightarrow p \mathrm{X}$ at $\theta_{p}=0^{\circ}$ in the Region of High Internal Momenta

in the Deuteron

Interactions of $10.6 \mathrm{GeV} /$ Nucleon Gold Nuclei in Nuclear Emulsion

Z. Phys. C63:549, 1994

Interactions of $10.6 \mathrm{GeV} / \mathrm{Nucleon}$ Gold Nuclei with Light and Heavy Nuclei in Nuclear Emulsion

Phys. Rev. D50:4272, 1994

Measurements of $525 \mathrm{GeV}$ Pion Interactions in Emulsion

Phys. Rev. C52:2652, 1995 Fragmentation and Multifragmentation of $10.6 \mathrm{~A} \mathrm{GeV}$ Gold Nuclei

Phys. Rev. C53:1532, 1996 Intermittency in ${ }^{197} \mathrm{Au}$ Fragmentation

Phys. At. Nucl. 60:952, 1997; Yad. Phys. 60:1064, 1997

Scaling Properties of Rapidity Distributions in $\pi^{-} A$ Interactions

MANCHESTER-95 p. 62; FERMILA B-CONF-95-292-E

A View from the Top: CDF Results of Top Counting Experiment

Phys. Lett. 322B:270, 1994

Measurement of the $p$ p $\rightarrow p p \eta$ Total Cross Section between 1.265 and $1.5 \mathrm{GeV}$

Phys. Lett. 337B:192, 1994 $\eta$-Meson Production in $p$ deuteron and $p p$ Collisions

Nuovo Cim. 107 A:1195, 1994

Kinetic-Energy Spectrum of $\eta$-Meson Produced on Boron by 1 Gev Protons

See the legend on page 5 . 
Chiavassa 98

Chiba 94

Chiba 96

Chiba 97

Chiba 97B

Chiba 98

Chkhaidze 95

Chkhaidze 96

Chkhaidze 97

Chkhaidze 98

Chklovskaia 95

Choi 94

Choi 95

Choi 96

Chrien 97

Chrien 98

Chudakov 94

Church 97

Cianciolo 95

Cinabro 93B

Cinabro 94

Claes 94

Clajus 95

Clark 97

Clerc 95

Coan 95

Coan 96

Coan 97

Coan 97B

Coan 98

Cobalgrassma 96

Colas 94

Cole 98

Colombo 94

Comfort 98

Comptour 94

Condo 94
Eur. Lett. $41: 365,1998$

The Reaction $p^{12} \mathrm{C} \rightarrow \eta \mathrm{X}$ from $T_{p}=800 \mathrm{MeV}$ to $T_{p}=1500 \mathrm{MeV}$

Nucl. Phys. A583:633C, 1995; KEK-PS 1990-1994 p. 275

Subthreshold Antiproton Productions in $p \mathrm{~A}$, deuteron $\mathrm{A}$ and $\alpha \mathrm{A}$ Reactions

Phys. Rev. D55:2577, 1997; KEK-96-124

Pontecorvo Reactions in Antiproton Annihilation at Rest in Deuterium to $\pi^{0} n, \pi^{0} \Delta\left(1232 P_{33}\right)^{\circ}, \eta n$ and $\eta \Delta\left(1232 P_{33}\right)^{0}$

Phys. Rev. D55:40, 1997; KEK-96-132

Measurement of Monoenergetic Neutrons from $\bar{p}$ deuteron Reaction at Rest

KEK-97-247

Measurement of Monoenergetic $\pi^{\circ}$ and $\eta$ Mesons in Antiproton-Deuterium Annihilation at Rest

Nucl. Instr. and Meth. B143:121, 1998

Four and Five Photon Decay from Positronium and the Lifetime Problem

Jour. of Phys. G 21:1223, 1995

The Study of Angular Distribution of $\pi^{-}$Mesons in Nucleus-Nucleus Interactions at a Momentum of $4.5 \mathrm{GeV} / c$ per Nucleon

Jour. of Phys. G 22:641, 1996

Study of the Inclusive Reaction $\mathrm{Mg} \mathrm{Mg} \rightarrow \pi^{-} \mathrm{X}$ at a momentum of $4.3 \mathrm{GeV} / \mathrm{c}$ per Incident $\mathrm{Nucleon}$

Phys. Lett. 411B:26, 1997; Phys. Lett. 423B:427, 1998; HEPEX-9802021

The Observation of Collective Effects in Central $\mathrm{C}$ Ne and $\mathrm{C} \mathrm{Cu}$ Collisions at a Momentum of $4.5 \mathrm{GeV} / \mathrm{c}$ per Nucleon

Eur. Phys. J. A1:299, 1998; HEPI-97-1; HEPEX-9805028

The Comparison of Characteristics of $\pi^{-}$Mesons Produced in Central Mg Mg Interactions with the Quark Gluon String Model Predictions

Jour. of Phys. G 21:307, 1995

Production Region of Identical Pions at $38 \mathrm{GeV} / c \pi^{-}$Nuclei Interactions with $\mathrm{High} p_{T}$ Particles

Nucl. Phys. A 577:213C, 1994

Positive Photopion Production from ${ }^{12} \mathrm{C},{ }^{13} \mathrm{C}$ using Tagged Photons

Phys. Lett. 355B:406, 1995; KEK-95-27

A Measurement of Bose-Einstein Correlations in $e^{+} e^{-}$Annihilation at TRISTAN

FERMILAB-CONF-96-304-EC

Do Papers on QCD Studies with Jets: The Dijet Mass Spectrum with the Do Detector

Nucl. Phys. A625:251, 1997

Elastic and Inelastic Scattering of $K^{+}$from ${ }^{6} \mathrm{Li}$ and ${ }^{12} \mathrm{C}$

Nucl. Phys. A629:388C, 1998; OSAKA-97 p. 388C

$H$ Particle Searches at Brookhaven

Nuovo Cim. 107A:1971, 1994

Charm Production in the CERN Hyperon Beam

Phys. Rev. C56:2806, 1997; LA-UR-96-1848; NUCLEX-9705002

Measurements of the Reactions ${ }^{12} \mathrm{C}\left(\nu_{\mu}, \mu^{-}\right){ }^{12} \mathrm{Nit}_{g . s}$ and ${ }^{12} \mathrm{C}\left(\nu_{\mu}, \mu^{-}\right) \mathrm{X}$

Nucl. Phys. A590:459C, 1995

Bose-Einstein Correlation of Positive Kaon Pairs in E859 - Extended Results and Model Comparisons

Phys. Rev. Lett. 72:1406, 1994; CLNS-93-1262; CLEO-93-23 Observation of $D^{0} \rightarrow K^{+} \pi^{-}$

Phys. Lett. 340B:129, 1994; CLNS-94-1297; CLEO-94-20 Measurement of the Branching Fraction for $\Upsilon(1 S) \rightarrow \tau^{+} \tau^{-}$

ALBUQUERQUE-94, v. 2, p. 1048; FERMILAB-CONF-94-290-E Search for Squarks and GIuinos in DO

Jour. of Phys. G 21:1363, 1995 Measurement and Calculation of Polarization Transfer Coefficients in the $\operatorname{Reactions}{ }^{2} \mathrm{H}(\vec{p}, \vec{p})^{2} \mathrm{H}$ at $E_{p}=$ $22.5 \mathrm{MeV}$

Phys. Rev. Lett. 79:2754, 1997; Phys. Rev, Lett. 79:345, 1997

Atmospheric Muon Neutrino Fraction above $1 \mathrm{GeV}$

Nucl. Phys. A590:785, 1995

The Influence of Fission on the Fragmentation of Relativistic ${ }^{208} \mathrm{~Pb}$ and ${ }^{238} \mathrm{U}$ Projectiles in Peripheral Collisions

Phys. Lett. 356B:580, 1995; CLNS-95-1332; CLEO-95-6

Measurement of $\alpha_{s}$ from $\tau^{ \pm}$Decays

Phys. Rev. D53:6037, 1996; CLNS-96-1391; CLEO-96-3

Decays of Tau Leptons to Final States Containing $K_{S}$ Mesons

Phys. Rev. D55:7291, 1997; CLNS-96-1450A; CLEO-96-21; HEPEX-9701012 Tau Neutrino Helicity from hadron +- Energy Correlations

Phys. Rev. Lett. 80:1150, 1998; CLNS-97-1516; CLEO-97-23; HEPEX-9710028 Flavor-Speciflc Inclusive $B$ Decays to Charm

Phys. Rev. D59:111101, 1999; CLNS-98-1583; CLEO-98-14; HEPEX-9810043

Search for Exclusive Rare Baryonic Decays of $B$ Mesons

FERMILAB-CONF-96-005-E; CDF-PUB-TOP-PUBLIC-3162

Kinematic Top Analyses at CDF

DAPNIA-SPP-94-01

Charmed Meson Production at LEP

Nucl. Phys. A638:423C, 1998; TSUKUBA-97 p. $423 \mathrm{C}$

Studying Heavy Ion Physics at the AGS using Proton Nucleus Collisions

Nuovo Cim. 107 A:2345, 1994

Hard Scattering in Photoproduction

Nucl. Phys. A639:111C, 1998; UPTON-97 p. $111 \mathrm{C}$

$\left(K_{\text {stop }}^{-}, \pi^{0}\right)$ with the Neutral Meson Spectrometer

Nucl. Phys. A579:596, 1994; DAPNIA-SPHN-94-11

A Study of the $p p \rightarrow p p \pi^{0}$ Reaction at $800 \mathrm{MeV}$ Beam Energy using the DIOGENE Detector at Saturne

Phys. Rev. D49:2164, 1994

Inelastic $\rho^{0}$ Photoproduction in the Reaction $\gamma p \rightarrow \rho^{0} \pi^{+} n$ 
Conrad 95

Conway 96

Conway 97

Cornaz 94

Cornell 96

Cortinagil 97

Cowley 94

Cowley 96

Cowley 97

Cowley 98

Crawford 95

Crawford 96

Crochet 97

Crochet 97C

Cross 95

Cullenvidal 96

Cummings 95

Dabrowska 95B

Dabrowska 97

Dabrowska 98

Daehnick 95

Daehnick 98

Dainton 94

Dam 95

Danagulyan 97

Danagulyan 97B

Danevich 95

Daniel 94

Daniels 94

Danilov 94

Danilova 97

Dardenne 94

Dasgupta 99

Dassie 94

Dassie 98

Dasu 94

Daszewski 95

Datz 97

Daum 95

Daum 95B
FERMILAB-CONF-95-018

Some Highlights of the Recent Fermilab Fixed Target Program of Interest to the Nuclear Physics Community

FERMILAB-CONF-96-181-E

Recent CDF Results

Nucl. Phys. (PS) B55:409, 1997; ESTES PARK-96 p. 409

Physics with Taus at CDF

Phys. Rev. Lett. 72:1152, 1994

Determination of the Gravitational Constant at an Effective Interactions Distance of $12 \mathrm{~m}$

Phys. Rev. Lett. 77:4508, 1996

Investigating the Evolution of Multifragmenting Systems with Fragment Emission Order

Phys. Lett. 401B:9, 1997; GANIL-P-96-37

Proton Elastic Scattering on Light Neutron-Rich Nuclei

Phys. Rev. C50:2449, 1994 Quasifree Knockout in ${ }^{9} \mathrm{Be}(\alpha, 2 \alpha){ }^{5} \mathrm{He}$ at an Incident Energy of $197 \mathrm{MeV}$

Phys. Rev. C54:778, 1996

Inclusive ( $p, \mathrm{He}$ ) Reactions on ${ }^{27} \mathrm{Al},{ }^{59} \mathrm{Co}$ and ${ }^{197} \mathrm{Au}$ at Incident Energies of $120,160 \mathrm{and} 200 \mathrm{MeV}$

Phys. Rev. C55:1843, 1997

Inclusive $\left(p,{ }^{3} \mathrm{He}\right)$ Reactions on ${ }^{59} \mathrm{Co}$ and ${ }^{187} \mathrm{Au}$ at Incident Energies of 120,160 and $200 \mathrm{MeV}$

Phys. Rev. C57:3185, 1998

Rescattering in Knockout Reactions as Manifested in ${ }^{40} \mathrm{Ca}(p, p p)$ at an Incident Energy of $302 \mathrm{MeV}$

Phys. Rev. Lett. 75:624, 1995; CLNS-94-1306; CLEO-94-24

Form Factor Ratio Measurement in $\Lambda_{c}^{+} \rightarrow \Lambda e^{+} \nu_{e}$

Nucl. Phys. A603:303, 1996; DAPNIA-SPHN-96-04

Two-Body Photodisintegration of the Deuteron from 100 to $800 \mathrm{MeV}$

Nucl. Phys. A627:522, 1997; Nucl. Phys. A628:687, ; NUCLEX-9709004

Azimuthal Anisotropies as Stringent Test for Nuclear Transport Models

Nucl. Phys. A624:755, 1997

Onset of Nuclear Matter Expansion in $\mathrm{Au}+\mathrm{Au}$ Collisions

Nucl. Phys. A593:463, 1995

Proton Photoproduction from ${ }^{12} \mathrm{C}$

FERMILAB-CONF-96-304-EI

Do Papers on QCD Studies with Jets: Color Coherence in $p \bar{p}$ Collisions at $\sqrt{s}=1.8$ TeV

TRI-PP-95-65

First Measurement of the Vector Analyzing Power in Muon Capture by Polarized Muonic ${ }^{3}$ He

Z. Phys. C68:65, 1995

Phase Space Dependance of the Correlations among Particles Produced in High Energy Nuclear Collisions

Z. Phys. C73:449, 1997

Transverse Momenta of Helium Fragments in Gold Fragmentation at 10.6 GeV/Nucleon

Nucl. Phys. A633:357, 1998 Comparison of Particle Production in $\mathrm{Pb}(158 \mathrm{GeV} /$ nucleon $) \mathrm{Ag} / \mathrm{Br}$ Collisions with the VENUS and FRITIO F Models

Phys. Rev. Lett. 74:2913, 1995

Differential Cross Sections for $p p \rightarrow p n \pi^{+}$near Threshold

Phys. Lett. 423B:213, 1998

Analyzing Powers in $p \dagger p \rightarrow p n \pi^{+}$and Determination of Pion $S$ and $P$ Wave Amplitudes Near Threshold

RAL-94-012

Results from the H1 Experiment at HERA

Nucl. Phys. (PS) B40:227, 1995

Measurement of the $\tau^{ \pm}$Leptonic Branching Fractions in DELPHI

Phys. At. Nucl. 60:863, 1997; Yad. Phys. 60:965, 1997

Investigation of $(p, x n),(p, p x n)$ and $(p, 2 p)$ Reactions on Tin Isotopes

Yad. Phys, 60:2117, 1997; Phys. At. Nucl. 60:1937, 1997

Investigation of Isomeric Ratios in Simple Photonuclear Reactions on Rb and Te Targets

Phys. Lett. 344B:72, 1995

The Search of $2 \beta$ Decays of ${ }^{116} \mathrm{Cd}$ with Enriched ${ }^{114} \mathrm{CdWtO}$ Crystal Scintillator

Phys. At. Nucl. 57:1738, 1994; Yad. Phys. 57:1809, 1994

Trapping of Antiprotons in Metastable Antiprotonic Helium Atoms: Progress and Prospects

CDF-PUB-BOTTOM-PUBLIC-2731; ALBUQUERQUE-94, v. 1, p. 552; FERMILAB-CONF-94-212-E $J / \psi(1 S), \psi(2 S) \rightarrow \mu^{+} \mu^{-}$and $b \rightarrow J / \psi(1 S), \psi(2 S)$ Cross Sections

ITEP-94-53

Recent ARGUS and CLEO Results on $\tau^{ \pm}$Lepton and charm

Izv, RAN, Fiz, 61:459, 1997

The Knee in the EAS Size Spectrum and the Proton Energy Spectrum of Primary Cosmic Rays

Phys. Rev. C49:2058, 1994

Inclusive Protonlike Production Cross Sections from $0.757 \mathrm{GeV} / \mathrm{Nucleon}$ La+La Collisions

Phys. Rev. Lett. 82:1395, 1999; NUCLEX-9901003

Fusion versus Breakup: Observation of Large Fusion Suppression for ${ }^{\theta} \mathrm{Be}+{ }^{208} \mathrm{~Pb}$

Phys. Rev. D51:2090, 1995; LAL-94-46

Two-Neutrino Double Beta Decay Measurement of ${ }^{100} \mathrm{Mo}$

Nucl. Phys. A636:209, 1998

Double- $\beta$ Decay of ${ }^{82} \mathrm{Se}$

Phys. Rev. D49:5641, 1994; SLAC-PUB-5814; UR-1304; ER-40685-753

Measurement of Kinematic and Nuclear Dependence of $R=\sigma_{L} / \sigma_{T}$ in Deep Inelastic Electron Scattering

Z. Phys. A351:225, 1995

The $Q_{E C}$ Dependence of Evidence for a $17 \mathrm{keV}$ Neutrino from the ${ }^{55}$ Fe Radiative Decay

Phys. Rev. Lett. 79:3355, 1997

Anomalies in the Nuclear Dissociation Cross Sections of ${ }^{208} \mathrm{~Pb}$ at $33 \mathrm{TeV}$

Phys. Lett. 361B:179, 1995; PSI-PR-95-19

Search for a Neutral Particle of Mass $33.9 \mathrm{MeV}$ in Pion Decay

Z. Phys. C66:417, 1995; WU-B-95-03

Determination of the Atmospheric Neutrino Spectra with the Frejus Detector 
David 96

David 96B

David $96 \mathrm{C}$

David 97

David 97B

David 99

Davier 95

Davis $9 \overline{5}$

Debarbaro 94

Debarbaro 95

Deboer 96

Debowski 96

Debowski 97

Debraeckelee 95

Decarlo 94

Decataldo 97

Declais 94

Dedovich 94

Degener 96

Degroot 96

Degtyarenko 93

Degtyarenko 94

Degtyarenko 94B

Degtyarenko 95

Degtyarenko 97

Deinesjones 96

Dejong 98

Dejongh 94

Deloff 95

Delosheros 96

Dementiev 94

Demin 94

Demina 96

Demortier 95

Deoliveirama 96

Depommier 94

Deprospo 94

Derbin 92B
Nucl. Phys. A610:63C, 1996

Flow Studies at $10.8 \mathrm{GeV} /$ nucleon

Nucl. Phys. A610:153C, 1996; NUCLEX-960900

Hadron Yields and Spectra in Au Au Collisions at the AGS

Nucl. Phys. A610:227C, 1996

Two-Particle Correlations in Au Au Collisions at AGS Energy

Nucl. Phys. A622:391, 1997

Backward Yields of Pions, Protons, and Deuterons in Relativistic ${ }^{28} \mathrm{Si} \mathrm{Pb}$ Collisions at $14.8 \mathrm{~A} \mathrm{GeV} / c$

Phys. Rev. C56:3254, 1997; HD-PY-97-07; NUCLEX-9707002

Proton and Pion Production Relative to the Reaction Plane in $\mathrm{Au} \mathrm{Pb}$ Collisions at $11 \mathrm{~A}$ Gev/c AGS Energies

Phys. Rev. C59:884, 1999; NUCLEX-9805006

Directed Flow of Light Nuclei in $\mathrm{Au}+\mathrm{Au}$ Collisions at $10.8 \mathrm{~A} \cdot \mathrm{GeV} / \mathrm{c}$

Nucl. Phys. (PS) B40:395, 1995; LAL-94-81; MONTREUX-94 p. 395

The $\tau^{ \pm}$Leptonic Current: Lifetime, Leptonic Branching Ratios and Michel Parameters

Phys. Rev. C53:2052, 1996; TRI-PP-95-54

The Zero Crossing Angle of the $n \boldsymbol{p}$ Analyzing Power

FERMILAB-CONF-94-246-E

Tests of Structure Functions using Leptons at CDF: the Charge Asymmetry in $W^{ \pm}$-Boson Decays

FERMILAB-CONF-95-164-E; CDF-PUB-EWK-PUBLIC-3190

Tests of Proton Structure Functions using Leptons at CDF and D0: $W^{ \pm}$Charge Asymmetry and Drell-Yan Production

Phys. Lett. 388B:235, 1996

A Deviation in Internal Pair Conversion

Z. Phys. A356:313, 1996

Subthreshold $K^{+}$Production in Proton Nucleus Collisions

Phys. Lett. 413B:8, 1997; NUCLEX-9709002

Subthreshold $K^{+}$Production in Deuteron and Alpha Induced Nuclear Reactions

Phys. Rev. C51:2778, 1995

Radiative Decays of the 16.6 and $16.0 \mathrm{MeV}$ States in ${ }^{8} \mathrm{Be}$ and Tests of the Conservation of the Vector Current in the $A=8$ Multiplet

Nucl. Phys. A566:431C, 1994

Recent Results E735: Search for Quark-Gluon Plasma in $p \bar{p}$ Collisions at $0.3-1.8 \mathrm{TeV}$

Nucl. Phys. A625:494, 1997; NUCLEX-9708001

Search for Exotic Strange Quark Matter in High Energy Nuclear Reactions

Phys. Lett. 338B:383, 1994; LPC-94-42; LAPP-EXP-94-16

Study of Reactor Anti-Neutrino Interaction with Proton at Bugey Nuclear Power Plant

Phys. At. Nucl. 58:808, 1995; Yad. Phys. 58:873, 1995; JINR-P1-94-98

The Properties of Nucleon Clusters Production in ( $p$, deuteron) + Ta and ( $p$, deuteron) + C Interactions over Energy Range from 4.2 to $10 \mathrm{GeV} / c$ per Nucleon

MANCHESTER-95 p. 331

Search for Exotics in Proton-Antiproton Annihilations in Flight

MANCHESTER-95 p. 423

Measurement of the Spin-Dependent Structure Function $g_{1}(x)$ on the Deuteron and the Proton

Z. Phys. A350:263, 1994; ITEP-93-49

The Dependence of Proton Correlations on Integral Characteristics of $e^{ \pm}$nucleus Interactions

Phys. Rev. C50:R541, 1994 Multiple Hadron Production by $14.5 \mathrm{GeV}$ Electron and Positron Scattering from Nuclear Targets

ITEP-94-87

Proton-Pion Correlations in Electron-Nucleus Interactions

ITEP-95-14

Kinematical and Interference Correlations of Pions in $e^{ \pm}-\mathrm{A}$ Interactions

Z. Phys. A357:419, 1997

Proton-Pion and Two-Pion Correlations in e A Interactions

Phys. Rev. C53:3044, 1996

High Multiplicity Lead Lead Interactions at $158 \mathrm{GeV} / \mathrm{c}$ per Nucleon

Nucl. Phys. A628:479, 1998 Fragmentation Cross Sections of Relativistic ${ }^{208} \mathrm{~Pb}$ Projectiles

FERMILAB-CONF-94-245

$B^{+}$and $B^{0}$ Mean Lifetime Measurements

Z. Phys. A353:121, 1995

Observation of Two Enhancements in $M_{\pi-}$ in the Reaction $p$ deuteron $\rightarrow \pi^{-} p p p$ at $1 \mathrm{GeV}$

CERN-PPE-96-111

Low Mass Dilepton Production at the SPS: Probing Hot and Dense Nuclear Matter

Phys. At. Nucl. 57:975, 1994; Yad. Phys. 57:1036, 1994

The Properties of the Exclusive Channel of Diffraction Dissociation in $\bar{p} p$ Interactions at $22.4 \mathrm{GeV} / c$

Nuovo Cim. 107A:2351, 1994

DELPHI Results on the Study of High-Energy Photons in $e^{+} e^{-} \rightarrow f \bar{f}$ Events

PADUA-96 p. 62; FERMILAB-CONF-96-201-E; CDF-PUB-BOTTOM-PUBLIC-3747; CDF-ANAL-BOTTOM-CDFR3747

Quarkonia Production at $\mathrm{CDF}$

FERMILAB-CONF-95-334-E; CDF-PUB-TOP-PUBLIC-3364; RU-95-E-35

Studies of $t \bar{t}$ Production in the Lepton + Jets and All Hadronic Channels

MANCHESTER-95 p. 467

$\eta$ Meson Production in $p$ Nucleus Interactions near Threshold

TRI-PP-94-TT

Observation of Radiative Muon Capture on the Proton

Phys. Rev. D50:6691, 1994

Neutral Strange Particle Production in Neutrino and Antineutrino Charged-Current Interactions on Neon

Yad. Phys. 57:236, 1994; Phys. At. Nucl. 57:222, 1994; LENI-92-1765

A Bound on the Magnetic Moment of the Reactor's Neutrino 
Derbin 96

Deroeck 94

Derrick 93C

Derrick 94

Derrick 94B

Derrick 94C

Derrick 94D

Derrick 94E

Derrick $94 \mathrm{~F}$

Derrick 94G

Derrick $94 \mathrm{H}$

Derrick 94I

Derrick 95

Derrick 95B

Derrick 95C

Derrick 95D

Derrick 95E

Derrick $95 \mathrm{~F}$

Derrick 95G

Derrick 95H

Derrick 95I

Derrick 95J

Derrick $95 \mathrm{~K}$

Derrick $95 \mathrm{~L}$

Derrick 95M

Derrick 95N

Derrick 950

Derrick 95P

Derrick $95 Q$

Derrick 95R

Derrick 96

Derrick 96B

Derrick 96C

Derrick 96D

Derrick $96 \mathrm{E}$

Derrick $96 \mathrm{~F}$

Derrick 96G

Derrick $96 \mathrm{H}$

Derrick 96I
Phys. At. Nucl. 59:2037, 1996; Yad. Phys. 59:2117, 1996

New Limits on Half-Lives of ${ }^{154} \mathrm{Sm},{ }^{180} \mathrm{Gd},{ }^{170} \mathrm{Er}$, and ${ }^{178} \mathrm{Yb}$ with Respect to Double $\beta$ Decay to the Excited $2^{+}$States of Daughter Nuclei

DESY-94-005

Results from the $\mathrm{H} 1$ Experiment at HERA

Phys. Lett. 322B:287, 1994; ANL-HEP-PR-94-14; DESY-93-151

Observation of Direct Processes in Photoproduction at HERA

Z. Phys. C63:391, 1994; DESY-94-032

Measurement of Total and Partial Photon Proton Cross Sections at $180 \mathrm{GeV}$ Center of Mass Energy

Phys. Lett. 338B:483, 1994; DESY-94-117

Comparison of Energy Flows in Deep Inelastic Scattering Events with and without a Large Rapidity Gap

Phys. Lett. 332B:228, 1994; DESY-94-063; ANL-HEP-PR-94-73

Observation of Jet Production in Deep Inelastic Scattering with a Large Rapidity Gap at HERA

Z. Phys. C65:379, 1995; DESY-94-143

Measurement of the Proton Structure Function $F_{2}$ from the 1993 HERA Data

Phys. Lett. 342B:417, 1995; DESY-94-176

Inclusive Jet Differential Cross Section in Photoproduction at HERA

Phys. Lett. 346B:399, 1995; DESY-94-210

Observation of Hard Scattering in Photoproduction Events with a Large Rapidity Gap at HERA

2. Phys. C65:627, 1995; DESY-94-175

A Search for Excited Fermions in Electron-Proton Collisions at HERA

Phys. Lett. 345B:576, 1995; DESY-94-192

Extraction of the Gluon Density of the Proton at Small $x$

Z. Phys. C59:231, 1994; DESY-93-068

Hadronic Energy Distributions in Deep-Inelastic Electron-Proton Scattering

Phys. Lett. 349B:225, 1995; DESY-95-013

Study of $D^{*}(2010)^{ \pm}$Production in $e^{-} p$ Collisions at HERA

Phys. Lett. 348B:665, 1995; DESY-95-033

Dijet Cross Sections in Photoproduction at HERA

Z. Phys. C67:93, 1995; DESY-95-007

Measurement of Multiplicity and Momentum Spectra in the Current Fragmentation Region of the Breit Frame at HERA

Phys. Rev. Lett. 75:1006, 1995; DESY-95-053

Measurement of Charged and Neutral Current $e^{-} p$ Deep Inelastic Scattering Cross Sections at High $Q^{2}$

Z. Phys. C67:81, 1995; DESY-95-016

Jet Production in High $Q^{2}$ Deep-Inelastic $e^{-} p$ Scattering at HERA

Phys. Lett. 350B:120, 1995; DESY-95-052; ANL-HEP-PR-95-52

Measurement of the Cross Section for the Reaction $\gamma p \rightarrow J / \psi(1 S) p$ with ZEUS Detector at HERA

2. Phys. C67:227, 1995; DESY-95-050

Inclusive Transverse Momentum Distributions of Charged Particles in Diffractive Photoproduction at HERA

Z. Phys. C68:29, 1995; DESY-95-084

Neutral Strange Particle Production in Deep Inelastic Scattering at HERA

2. Phys. C68:569, 1995; DESY-95-093

Measurement of the Diffractive Structure Function in Deep Elastic Scattering at HERA

Phys. Lett. 354B:163, 1995; DESY-95-083

Study of the Photon Remnant in Resolved Photoproduction at HERA

Phys. Lett. 356B:129, 1995; DESY-95-115

Diffractive Hard Photoproduction at HERA and Evidence for the Gluon Content of the Pomeron

Phys. Lett. 356B:601, 1995; DESY-95-133

Exclusive $\rho^{\mathbf{O}}$ Production in Deep Inelastic Electron-Proton Scattering at HERA

Z. Phys. C69:607, 1996; DESY-95-193

Measurement of the Proton Structure Function $F_{2}$ at Low $x$ and Low $Q^{2}$ at HERA

Phys. Lett. 363B:201, 1995; DESY-95-182

Measurement of $\alpha_{S}$ from Jet Rates in Deep Inelastic Scattering at HERA

Phys. Lett. 369B:55, 1996; DESY-95-194

Rapidity Gaps between Jets in Photoproduction at HERA

Z. Phys. C70:1, 1996; DESY-95-221

Inclusive Charged Particle Distributions in Deep Inelastic Scattering Events at HERA

Phys. Lett. 377 B:259, 1996; DESY-96-002

Measurement of Elastic $\phi(1020)$ Photoproduction at HERA

Z. Phys. C69:39, 1995; DESY-95-143

Measurement of Elastic $\rho^{0}$ Photoproduction at HERA

Z. Phys. C70:391, 1996; DESY-96-018

Measurement of the Diffractive Cross Section in Deep Inelastic Scattering

Phys. Lett. 384B:401, 1996; DESY-96-094; HEPEX-9605009

Dijet Angular Distributions in Direct and Resolved Photoproduction at HERA

Phys. Lett. 384 B:388, 1996; DESY-96-093

Observation of Events with an Energetic Forward Neutron in Deep Inelastic Scattering at HERA

Z. Phys. C73:73, 1996; DESY-96-159

Measurement of Elastic $\omega$ Photoproduction at HERA

2. Phys. C73:253, 1997; DESY-96-183

Study of Elastic $\rho^{\circ}$ Photoproduction at HERA using the ZEUS Leading Proton Spectrometer

Phys. Lett. 380B:220, 1996; DESY-96-067; HEPEX-9604008

Measurement of the Reaction $\gamma^{*} p \rightarrow \phi(1020) p$ in Deep Inelastic $e^{+} p$ Scattering at HERA

2. Phys. C72:399, 1996; DESY-96-076; HEPEX-9607002

Measurement of the $F_{2}$ Structure Function in Deep Inelastic Scattering using 1994 Data from the ZEUS Detector at HERA

Z. Phys. C73:613, 1997; DESY-96-161

Search for Lepton Flavor Violation in $e^{ \pm} p$ Collisions at $300 \mathrm{GeV}$ Center of Mass Energy

Z. Phys. C72:47, 1996; DESY-96-104; HEPEX-9606014

Study of Charged-Current $e^{-} p$ Interactions at $Q^{2} \geq 200 \mathrm{GeV}^{2}$ with the ZEUS Detector at HERA 
Derwent 94

Derwent 95

Desilva 97

Deswart 94

Dewolf 96

Dewolf $96 \mathrm{~B}$

Deyoung 96

Dheer 95

Dhuga 96

Dias 97

Diehl 95

Distler 98

Dittmann 96

Doeker 95

Dolfini 95

Dombrowski 97

Dominick 94

Donskov 94

Donskov 95

Donzaud 95

Donzaud 98

Dorigo 98

Doroshkevich 94

Doroshkevich 98

Dover 98

Dowd 96

Dowell 94

Dowell 95

Dowell $95 \mathrm{~B}$

Dremin 94

Dress 94

Drndarevic 95

Drochner 96

Drutskoi 93

Dubbs 93

Duboscq 95

Ducret 94

Ducros 94

Dudkin 94
FERMILA B-CONF-94-129-E; CDF-PUB-BOTTOM-PUBLIC-2602

Measurement of Correlated $b$ Quark Cross Sections at CDF

FERMILAB-CONF-95-096-E

Charm and Beauty Results from CDF and Do

Phys. Rev. C56:2451, 1997; NUCLEX-9706005

Double Beta Decays of ${ }^{100} \mathrm{Mo}$ and ${ }^{150} \mathrm{Nd}$

Phys. At. Nucl. 57:1547, 1994; Yad. Phys. 57:1621, 1994; THEF-NYM-94-01; HEPPH-9401279

$\bar{p} p$ Partial-Wave Analysis and nucleon nucleon Potentials

Z. Phys. C72:65, 1996; HEN-373

Nuclear Target Diffraction Dissociation in $\pi^{+}$and $K^{+}$Collisions with $\mathrm{Au}$ and $\mathrm{Al}$ at $250 \mathrm{GeV} / \mathrm{c}$

Z. Phys. C70:233, 1996; HEN-374

Coherent Inelastic Interactions of $\pi^{+}$and $K^{+}$Mesons on $\mathrm{Al}$ and $\mathrm{Au}$ Nuclei at $250 \mathrm{GeV} / \mathrm{c}$

Nucl. Phys. A597:127, 1996

Small Angle Neutron-Neutron Correlation Functions for the ${ }^{18} \mathrm{O}+{ }^{27} \mathrm{Al}$ Reaction at $220 \mathrm{MeV}$

Phys. Rev. C52:1572, 1995

Intermittency and Multifractality of Medium-Energy Particles in $p$ nucleus Interactions at $800 \mathrm{GeV}$

Phys. Rev. C54:2823, 1996

Investigation of Nuclear Charge Symmetry by Pion Elastic Scattering from ${ }^{3} \mathrm{H}$ and ${ }^{3} \mathrm{He}$

Phys. Rev. C55:942, 1997

$\left(\gamma\right.$, deuteron) and $\left(\gamma,{ }^{3} \mathrm{H}\right)$ Reactions on ${ }^{6} \mathrm{Li}$ at Intermediate Photon Energies

FERMILA B-CONF-95-165-E

Search for Anomalous Couplings in $W W$ and $W Z$ Measurements at the Tevatron (Do and CDF results)

Phys. Rev. Lett. 80:2294, 1998

Measurement of Separated Structure Functions in the $p\left(e^{-}, e^{-} p\right) \pi^{0}$ Reaction at Threshold and Chiral Perturbation Theory

FERMILAB-CONF-96-267-E

Production Properties of Jets in $Z^{\circ}$ Boson Events at CDF

BONN-IR-95-23

Measurement of the Diffractive Structure Function of Protons in Deep Inelastic $e^{-} p$ Scattering with the ZEUS Detector

Phys. Rev. C51:3479, 1995

Out-of-Plane Quasielastic Scattering from Deuterium using Polarized Electrons

Nucl. Phys. A619:97, 1997

Low Energy Proton-Proton Scattering near the Interference Minimum using a Windowless Gas Jet Target

Phys. Rev. D50:3027, 1994; CLNS-94-1274; CLEO-94-9 Two Photon Production of Charged Pion and Kaon Pairs

Nuovo Cim. 107 A:1799, 1994

Measurement of the $\omega \rightarrow \eta \gamma$ Decay Rate

MANCHESTER-95 p. 220; KEK-95-158

Recent Results from GAMS

Nucl. Phys. A593:503, 1995

Dissipative Aspects in $200 \mathrm{MeV} / u \mathrm{Kr}$ Induced Reactions

Eur. Phys. J. A1:407, 1998

Low Energy Fission Investigated in Reactions of $750 \mathrm{~A} \cdot \mathrm{MeV}{ }^{238} \mathrm{U}$ Ions on ${ }^{208} \mathrm{~Pb}$. II. Isotopic Distributions CDF-PUB-ELECTROWEAK-PUBLIC-4640; HEPEX-9806022

Observation of $Z^{\circ}$ Decays to $B$ Quark Pairs at the Tevatron Collider

ITEP-94-91

Two Forward Protons Correlations at Small Relative Momentum in $p$ nucleus Interactions

ITEP-98-18

Analysis of Kinematical Correlations in Electron Nucleus Interaction at $5 \mathrm{GeV} / c$

Phys. Rev. C59:2699, 1999; NUCLEX-9811002

Antiproton Production and Antideuteron Production Limits in Relativistic Heavy Ion Collisions from BNL Experiment 864

MANCHESTER-95 p. 497; UMDHEP-E951

Observation of $f_{1}(1285)$ Pion and $\eta^{\prime}$ Pion in the Reaction $\pi^{-} p \rightarrow p \eta \pi^{ \pm} \pi^{-} \pi^{-+}$at $18 \mathrm{GeV} / \mathrm{c}$

WILLIAMSBURG-94 p. 530

Measurements of Quasi-Elastic Pion Single Charge Exchange on ${ }^{3} \mathrm{He}$ at $\boldsymbol{T}_{\boldsymbol{\pi}}=245 \mathrm{MeV}$

Phys. Lett. 344B:91, 1995

Measurements of Quasi-Elastic Pion Single Charge Exchange on ${ }^{3} \mathrm{He}$ at $T_{\pi}=245 \mathrm{MeV}$

Phys. Rev. C51:1551, 1995

Measurement of the ${ }^{3} \mathrm{H}\left(\pi^{+},{ }^{3} \mathrm{He}\right) \pi^{0}$ Differential Cross Section at $T_{x}=142 \mathrm{MeV}$

Phys. Lett. 336B:119, 1994; FNT-AE-93-25; FIAN-TD-15-93

Cumulant to Factorial Moment Ratio and Multiplicity Data

Phys. Rev. D49:4937, 1994

Search for the Decay $\pi^{0} \rightarrow \gamma \mathrm{X}$

Phys. Rev. C52:331, 1995

Emission of Fast Protons in High-Energy Hadron-Emulsion Interactions

Phys. Rev. Lett. 77:454, 1996

Total and Differential Cross Section of $p+p \rightarrow \pi^{+}+$deuteron Reactions Down to $225 \mathrm{keV}$ above Threshold

Phys. Lett. 333B:561, 1994; ITEP-93-52

The Effect of Resonance States on Hyperon Polarization

Phys. Rev. Lett. 72:808, 1994; FERMILAB-PUB-93-226-E

Measurement of the Branching Ratio for $\Xi^{-} \rightarrow \Sigma^{-} \gamma$ Radiative Decay

Phys. Rev. Lett. 76:3898, 1996; CLNS-95-1372; CLEO-95-20

Measurement of the Form Factors for $\bar{B}^{0} \rightarrow D^{*}(2010)+\ell^{-} \bar{\nu}$

Phys. Rev. C49:1783, 1994

Separation of the deuteron $\left(e^{-}, e^{-} p\right)$ Structure Functions up to $0.8 \mathrm{GeV} / c$ Momentum Transfer

DAPNIA-SPP-94-26

Electroweak Physics at Do and CDF

Nucl. Phys. A568:906, 1994

Multiplicities of Secondaries in Nuclear Interactions, Induced by ${ }^{20} \mathrm{Ne},{ }^{40} \mathrm{Ar}$ and ${ }^{56} \mathrm{Fe} \mathrm{Nuclei}$ at $0.1-$ $0.5 \mathrm{GeV} /$ Nucleon 
Duflot 94

Duflot 95

Duijvestijn 99

Duncan 98

Dunne 94

Dutz 96

Dytman 94

Dytman 95

Dzelalija 95

Eden 94

Edsall 97

Edwards 94

Edwards 95

Edwards 95B

Edwards 96

Edwards 97

Edwards 97B

Edwards 98

Efremenko 94

Efremov 97

Egorov 97

Ehrlich 94

Ehrnsperger 95

Eigen 94

Eiseman 94

Eiseman $95 \mathrm{~B}$

Eisenberg 96

Eisenstein 94

Eismont 96

Ejiri 96

Ejiri 98

Elaasar 94

Eliyakutrosh 95

Elliott 96

Ellis 96

Ellison 94

Elmer 96

Elnadi 94
Nucl. Phys. (PS) B40:37, 1995; MONTREUX-94 p. 37; LAL-94-70 $Q C D$ Tests from Tau Decays

Nucl. Phys. (PS) B39:322, 1995; MONTPELLIER-94 p. 322

Measurement of $\alpha_{s}$ and Non-perturbative Terms from $\tau$ Decays

Phys. Rev. C59:776, 1999

$p$-Induced Fission at $190 \mathrm{MeV}$ of $\mathrm{Wt}_{\text {nat }},{ }^{197} \mathrm{Au}, \mathrm{Pb}_{\text {nat }},{ }^{208} \mathrm{~Pb}$ and ${ }^{232} \mathrm{Th}$

Phys. Rev. Lett. 80:4390, 1998

Differential Cross Section of the $p n \rightarrow p p\left({ }^{1} S_{0}\right) \pi^{-}$Reaction Extracted from $p$ deuteron $\rightarrow p p p \pi^{-}$

WILLIAMSBURG-94 p. 729

SLAC Measurement of the Spin-Structure Function of the Neutron

Nucl. Phys. A601:319, 1996

Photoproduction of Positive Pions from Polarized Protons

WILLIAMSBURG-94 p. 518

$p p \rightarrow p n \pi^{+}$Cross Sections Near Threshold

Phys. Rev. C51:2710, 1995

Study of Baryon Resonances through $\gamma p \rightarrow \eta p$ Differential Cross Sections

Phys. Rev. C52:346, 1995

Entropy in Central Au + Au Reactions between 100 and $400 \mathrm{~A} \cdot \mathrm{MeV}$

Phys. Rev. C50:R1749, 1994

Electric Form Factor of the Neutron from ${ }^{2} \mathrm{H}\left(e^{-\uparrow}, e^{-1} n \uparrow\right)^{1} \mathrm{H}$ Reaction at $Q^{2}=0.255 \mathrm{GeV} / c^{2}$

Nucl. Phys. (PS) B55:95, 1997; ESTES PARK-96 p. 95

A Measurement of the $\tau$ Leptonic Branching Fractions

Phys. Rev. Lett. 74:3331, 1995; CLNS-94-1304; CLEO-94-22

Observation of Excited Charmed Baryon States Decaying to $\Lambda_{c}^{+} \pi^{+} \pi^{-}$

Phys. Rev. Lett. 74:2654, 1995

Precise Measurement of Parity Nonconserving Optical Rotation in Atomic Thallium

Phys. Lett. 373B:261, 1996; CLNS-95-1353; CLEO-95-15

Observation of New Decay Modes of the Charmed Strange Baryon $\Xi_{c}(\mathbf{2 4 6 0})^{+}$

Phys. Lett. 368B:39, 1996; Phys. Lett. 380B:493, 1996

The ${ }^{4} \mathrm{He}(p, n){ }^{4} \mathrm{Li}^{*}$ Reaction at 100 and $200 \mathrm{MeV}$ : Implications for the Mass-Four System and for Astrophysics

Phys. Rev. D55:3919R, 1997; CLNS-96-1428; CLEO-96-14

Search for Neutrinoless $\tau^{ \pm}$Decays: $\tau^{ \pm} \rightarrow e^{ \pm} \gamma$ and $\tau^{ \pm} \rightarrow \mu^{ \pm} \gamma$

Phys. Rev. D56:R5297, 1997; CLNS-97-1495; CLEO-97-15; HEPEX-9707029

Search for the Decay $\tau^{-} \rightarrow 4 \pi^{-} 3 \pi^{+}\left(\pi^{\circ}\right) \nu_{\tau}$

Phys. Rev. D59:032003, 1999; CLNS-98-1545; CLEO-98-5; HEPEX-9803010

Measurement of the Mass Splittings between the $b \bar{b} \chi_{b, J}(1 P)$ States

Jetp Lett. 59:77, 1995; Pisma Zh. Eksp. Teor. Fiz. 59:75, 1995; ITEP-94-4

Searches for Narrow Resonance in the $p p \pi^{-}$System

Kr. Soob. JINR 83:5, 1997

Observation of Transversal Handedness in the Diffractive Production of Pion Triples

Nucl. Phys. A621:745, 1997

Beta-Neutrino Angular Correlation in the Decay of ${ }^{18} \mathrm{Ne}$

Nuovo Cim. 107 A:1977, 1994

Recent Results from CLEO-II

Phys. Lett. 348B:619, 1995; UFTP-377-1994

Reanalysis of Hyperon Beta Decay Data on $F / D$

Nucl. Phys. (PS) B40:281, 1995; MONTREUX-94 p. 281; CALT-68-1967

Forbidden $\tau^{ \pm}$Lepton Decays in CLEO II

Phys. Lett. 325B:322, 1994; BNL-49592

$\Xi^{-}$Production in Heavy Ion Collisions at the AGS

Phys. Rev. C52:2289, 1995

Measurement of Low $p_{T} K_{S}$ Production from $14.6 \mathrm{~A} \mathrm{GeV} / c \mathrm{Si}$ Beams on a $\mathrm{Pb}$ Target

Nuovo Cim. 109A:703, 1996. PISA-95 p. 703

Observation of Open-Charm Production in ep Collisions at HERA

Phys. At. Nucl. 57:1680, 1994; Yad. Phys. 57:1751, 1994; ILL-N PL-93-005

Flavor Production in $\bar{p} p$ Reactions at Low Energies

Phys. Rev. C53:2911, 1996

Relative and Absolute Neutron-Induced Fission Cross Section of ${ }^{208} \mathrm{~Pb},{ }^{200} \mathrm{Bi}$, and ${ }^{238} \mathrm{U}$ in the Intermediate Energy Region

Nucl. Phys. A611:85, 1996

Limits on Neutrino-less Double Beta Decay of ${ }^{100} \mathrm{Mo}$

Phys. Lett. 433B:257, 1998

Spin-Isospin Responses of ${ }^{71} \mathrm{Ga}$ for Solar Neutrinos Studied by ${ }^{71} \mathrm{Ga}\left({ }^{3} \mathrm{He}, \mathrm{t \gamma}\right)^{71} \mathrm{Ge}$ Reaction

Phys. Rev. C49:10, 1994

Maximum Azimuthal Anisotropy of Neutrons from $\mathrm{Nb} \mathrm{Nb}$ Collisions at $400 \mathrm{~A} \mathrm{MeV}$ and the $\mathrm{Nuclear}$ Equation of State

Phys. Rev. C51:1295, 1995

Measurement of Proton Total Reaction Cross Sections for ${ }^{58} \mathrm{Ni}$ and ${ }^{80} \mathrm{Ni}$ Including Nonrelativistic and Relativistic Data Analyses

Phys. Lett. 381B:35, 1996

Individual Fragment Yields and Determination of the Critical Exponent $\sigma$

Phys. Lett. 389B:321, 1996

Indications from Precision Electroweak Physics Confront Theoretical Bounds on the Mass of the Higgs Bon

DO-NOTE-2287; UCR-D0-94-20; ALBUQUERQUE-94, v. 1, p. 439; FERMILAB-CONF-94-329-E

Measurement of the $W W \gamma, Z Z \gamma$ and $Z \gamma \gamma$ Couplings at the Fermilab Tevatron

Phys. Rev. Lett. 77:4884, 1996; Phys. Rev. Lett. 78:1396, 1997

$K^{+}$Emission in Symmetric Heavy Ion Reactions at Subthreshold Energies

Nuovo Cim. 107A:1, 1994

Characteristics of Helium Fragments Produced in ${ }^{18} \mathrm{O}$ Emulsion Interactions at $860 \mathrm{GeV} / \mathrm{c}$ 
Elnadi 94B

Elnadi $94 \mathrm{C}$

Elnadi 95

Elnadi $95 \mathrm{~B}$

Elnadi $95 \mathrm{C}$

Elnadi 95D

Elnadi 95E

Elnadi 96

Elnadi $96 \mathrm{~B}$

Elnadi $9 \bar{\imath}$

Elnadi $97 \mathrm{~B}$

Elnadi 98

Elnadi $98 \mathrm{~B}$

Elnaghy 94

Elnaghy 95

Elouadrhiri 94

Elsharkawy 94

Emura 94

Emura 94B

Enik 97

Eno 95

Enomoto 93

Enomoto 94

Enomoto 94B

Enomoto 94C

Ent 94

Eppley 94

Ericson 95

Ermakor 94

Ernst 98

Ero 94

Errede 94

Errede $94 \mathrm{~B}$

Eschrich 98

Eschstruth 94

Eskut 98

Eskut 98B
Nuovo Cim. 107A:13, 1994

Study of Multiparticle Production in the Coherent Production of $\pi^{-}(360 \mathrm{GeV} / \mathrm{c})$ and $\mathrm{K}^{ \pm}(70 \mathrm{GeV} / \mathrm{c})$ Interaction with Emulsion Nucleus

Nuovo Cim. 107 A:31, 1994

Angular Production of Fast and Slow Particles in ${ }^{7}$ Li-Emulsion Interactions at $26 \mathrm{GeV} / \mathrm{c}$

Nuovo Cim. 108A:87, 1995

On Slow-Particle Production in $200 \mathrm{~A} \mathrm{GeV}{ }^{16} \mathrm{O}$-Emulsion Interactions

Nuovo Cim. 108A:281, 1995

Multiplicity Distributions of Produced Particles in $60 \mathrm{~A} \mathrm{GeV}{ }^{16} \mathrm{O}$ Emulsion Interactions and Eclipse Effect

Nuovo Cim. 108A:809, 1995

Nuclear Temperature and Multiplicities of Relativistic He Fragments from ${ }^{16} \mathrm{O}$ Emulsion Interactions at $200 \mathrm{~A} \mathrm{GeV}$

Nuovo Cim. 108A:831, 1995

Multiplicities of Charged Particles in 3.7 A GeV ${ }^{32} \mathrm{~S}$ Fragments Emulsion Interactions

Nuovo Cim. $108 \mathrm{~A}: 935,1995$

Characteristics of Projectile Fragments Produced in ${ }^{24} \mathrm{Mg}$-Emulsion at Dubna Energy

Zh. Eksp. Teor. Fiz. 110:17, 1996

The Anomalous Behaviour of the Doubly Charged Projectile Fragments Produced from ${ }^{18} \mathrm{O} \mathrm{Em}$ Interactions at 60 and $200 \mathrm{~A} \cdot \mathrm{GeV}$

Int. Jour. Nod. Phys. E5:617, 1996

Multiplicity Distributions of Target Fragments in $60 \mathrm{~A} \cdot \mathrm{GeV}{ }^{16} \mathrm{O}-$ Emulsion Interactions

Int. Jour. Mod. Phys. E6:135, 1997

Mechanism of Inelastic Collisions of Oxygen at 3.2 TeV with Different Emulsion Targets

Int. Jour. Mod. Phys. E6:191, 1997

Target-Associated Particles Production in the Interactions of 3.7 and $200 \mathrm{~A} \cdot \mathrm{GeV}$ Sulphur Projectiles with Nuclear Emulsion

Eur. Phys. J. A3:183, 1998

Backward Slow Protons Production in the Inelastic Interactions of ${ }^{6} \mathrm{Li}$ and ${ }^{7} \mathrm{Li}$ Nuclei with Emulsion Nuclei at Dubna Energy

Jour. of Phys. G 24:2265, 1998

Nuclear Multifragmentation of ${ }^{32} \mathrm{~S}$ and ${ }^{28} \mathrm{Si}$ in Emulsion Nuclei

Nuovo Cim. 107A:279, 1994

Correlations in Inelastic Collisions of Relativistic Nuclei with Emulsion at (4.1 - 4.5) A GeV/c

Jour. of Phys. G 21:1539, 1995 Study of Intermittency in Collisions of ${ }^{28} \mathrm{Si}$ and ${ }^{22} \mathrm{Ne}$ with Emulsion

Phys. Rev. C50:R2266, 1994

Measurement of the $\left(e^{-}, e^{-1} p \pi^{-}\right)$Reaction on Nuclei in the Nucleon Resonance Region

Phys. Scr. 50:97, 1994

Inelastic Interactions of ${ }^{6} \mathrm{Li}$ Nuclei in Emulsion at $4.5 \mathrm{~A} \mathrm{GeV} / c$ as a Probe for ${ }^{6} \mathrm{Li}$ Internal Structure

Phys. Rev. Let t. 73:404, 1994; INS-1023

Total Cross Section for Photon Absorption by Two Protons in ${ }^{3} \mathrm{He}$

Phys. Rev. C49:R597, 1994: INS-968

Three Body ${ }^{3}$ He Photodisintegration in the $\Delta\left(1232 P_{33}\right)$ Region

Phys. At. Nucl. 60:567, 1997; Yad. Phys. 60:648, 1997 Specification for Deriving Neutron Electric Polarizability from the Total Cross Section of ${ }^{208} \mathrm{~Pb}$

FERMILA B-CONF-95-095-E Do Search for New Phenomena

Phys. Rev. D50:1879, 1994; KEK-93-107 Measurement of the $D^{*}(2010)^{ \pm}$Cross Section in a Two Photon Process

Phys. Lett. 328B:535, 1994; KEK-93-215; TUAT-HEP-94-1; DPNU-94-11; TIT-HPE-94-01; NWU-HEP-94-01; OCU-IIEP94-1; PU-94-681; KOBE-HEP-94-03

Measurement of the $D^{*}(2010)^{ \pm}$Cross Section using a Soft Pion Analysis in Two-Photon Processes

Phys. Lett. 341B:238, 1994; KEK-94-106; DPNU-94-38; NWU-HEP-94-04; TIT-HPE-94-09; TUAT-HEP-94-04; OC:U. HEP-94-06; PU-94-686; INS-1051; KOBE-HEP-94-05

$K^{\circ} \bar{K}^{\circ}$ Production in Two-Photon Processes at TRISTAN

Phys. Lett. 347B:179, 1995; KEK-94-177; DPNU-94-58; NWU-HEP-94-08; TIT-HPE-94-13; TUAT-HEP-94-08; OCU. HEP-94-10; PU-94-691; INS-1076; KOBE-11EP-94-09

Observation of Excess $\Lambda(\bar{\Lambda})$ Production in Two-Photon Processes at TRISTAN

Nucl. Phys. A578:93, 1994

The (e $e^{-}, e^{-1}$ deuteron) Reaction on ${ }^{4} \mathrm{He},{ }^{8} \mathrm{Li},{ }^{12} \mathrm{C}$

FERMILAB-CONF-94-289-E

Search for New Gauge Bosons at Do

Phys. Rev. Lett. 75:1046, 1995; CERN-TH-95-50

The $\pi$ nucleon nucleon Coupling from High Precision $n p$ Charge Exchange at $162 \mathrm{MeV}$

JINR-P1-94-240

Study of Features in Particle and Event Characteristics in Nucleus-Nucleus Interaction

Nucl. Phys. A639:213C, 1998; UPTON-97 p. 213C

First Results on $\eta^{\prime}$ Photoproduction with SAPHIR

Phys. Rev. C50:2687, 1994; KFK1-93-23-A

${ }_{\mathrm{H}} \mathrm{H}$ (deuteron,2p)n Reaction at $2 \mathrm{GeV}$ Deuteron Energy

FERMILAB-CONF-94-158-E; CDF-PUB-ELECTROWEAK-PUBLIC-2637

Electroweak Boson Pair Production at CDF

FERMILAB-CONF-94-306-E

Electroweak Boson Pair Production at the Tevatron

HEPEX-9811003

Measurement of the $\boldsymbol{\Sigma}^{-}$Charge Radius at Selex

LAL-94-15

Themistocle: Results and Prospects

Phys. Lett. 434 B:205, 1998; CERN-EP-98-073

Search for $\nu_{\mu} \rightarrow \nu_{\tau}$ Oscillation using $\tau^{ \pm}$Decay Modes into a Single Charge Particle

Phys. Lett. 424B:202, 1998

Search for $\nu_{\mu} \rightarrow \nu_{\tau}$ Oscillation 
Espy 96

Espy 97

Etzion 96

Evangelista 97

Evangelista 98

Evans 95

Evans 96

Evans 97

Eyl 95

Faessler 94

Fahley 94

Fan 94

Fan 94B

Fang 94

Fanti 97

Fanti 99

Fanti 99B

Farnham 95

Farrar 97

Fatyga 94

Fatyga 96

Fedorets 94

Feher 96

Feldman 94

Feldman 96

Feliciello 94

Felix 94

Felix 96

Felsher 97

Feltesse 94

Feltham 97

Ferenc 96

Fero 94

Ferrante 95

Ferrer 90

Ferrer 98

Ferroluzzi 96

Fialkowski 94
Phys. Rev. Lett. 76:3667, 1996

Asymmetries for Elastic Scattering of $\pi^{+}$from Polarized ${ }^{3} \mathrm{He}$ and the $\Delta-$ Neutron Spin-Spin Interaction

Phys. Rev. C56:2607, 1997

Asymmetries for Elastic Scattering of $\pi^{ \pm}$from Polarized ${ }^{3} \mathrm{He}$ at $\Delta$ Resonance Energies

SLAC-PUB-96-7170; HEPEX-9606008

Measurement of $R_{b}$ at SLD

Phys. Rev. D56:3803, 1997; HEPEX-9707041

Measurement of the $\bar{p} p \rightarrow K_{S} K_{S}$ Reaction from 0.6 to $1.9 \mathrm{GeV} / c$

Phys. Rev. D57:5370, 1998; CERN-EP-98-011; HEPEX-9802016

Study of the Reaction $\bar{p} p \rightarrow \phi(1020) \phi(1020)$ from 1.1 to $2.0 \mathrm{GeV} / c$

Nucl. Phys. (PS) B40:361, 1995; MONTREUX-94 p. 361

Measurements of Branching Ratios of $\tau$-Leptons to Charged and Neutral Kaons at OPAI

Nuovo Cim. 109A:915, 1996; PISA-95 p. 915

Measurements of the Strong Interaction in $\tau$ Decays

Nucl. Phys. (PS) B55:257, 1997; ESTES PARK-96 p. 257

Charged Current Measurements: a Tau98 Overview

Z. Phys. A352:211, 1995

First Measurement of the Polarization Transfer on the Proton in the Reactions $\left.H\left(e^{-\uparrow}, e^{-\prime} p\right\rceil\right)$ and deuteron $\left(e^{-\uparrow}, e^{-1} p \uparrow\right)$

Nuovo Cim. 107 A:2235, 1994

Hidden and Open $\bar{s} s$ Production in $\bar{p} p$ Annihilation at Rest

AZPH-EXP-94-02; FERMILAB-CONF-94-323-E

Do Quantum Chromodynamics: a Compilation of Results Presented at DPF 1904

Nuovo Cim. 107 A:1825, 1994

The Evidence of $f_{0}(2200)$

Nuovo Cim. 107 A:1829, 1994

The Recent Status of BES at BEBC (Beijing electron positron calorimeter)

FERMILAB-CONF-94-041-E

Exclusive Vector Meson Production in Muon-Nucleus Scattering

Z. Phys. C76:653, 1997

Measurement of the Decay Rate and the Parameter $\alpha_{K^{*}}$ of the Decay $K_{L} \rightarrow \mu^{-} \mu^{-} \gamma$

Phys. Lett. 446B:117, 1999; CERN-EP-98-172

Direct Search for Light Gluinos

Eur. Phys. J. C6:265, 1999

A Measurement of the Transverse Polarization of $\Lambda$-Hyperons Produced in Inelastic $p N$-Reactions at $450 \mathrm{GeV}$ Proton Energy

Phys. Rev. Lett. 75:3598, 1995

Determination of the Electron's Atomic Mass and the Proton/Electron Mass Ratio via Penning Trap Mass Spectroscopy

RU-97-82; TEGERNSEE-97 p. 182; HEPPH-9710395

Experimental and Cosmological Implications of Light Gauginos

Nucl. Phys. A544:137C, 1994

Recent Results from Experiment 814 at Brookhaven

FERMILAB-CONF-96-304-ED

DO Papers on QCD Studies with Jets: Dijet Angular Distributions at Do

Izv. RAN, Fiz. 58-11:132, 1994; Bull. Russ. Acad. Sci. Phys. Ser. 58:1873, 1994

Splitting of $\mathrm{Yt}$ and $\mathrm{Ag}$ Nuclei by ${ }^{12} \mathrm{C}$ Ions with $3.65 \mathrm{~A} \mathrm{GeV}$ Energy

FERMILAB-CONF-96-374-E

Jet Azimuthal Decorrelation Studies with the Do Detector

Phys. Rev. C49:2068, 1994

Neutron Transition Densities for ${ }^{48} \mathrm{Ca}$ from Proton Scattering at 200 and $318 \mathrm{MeV}$

Phys. Rev. C54:R2124, 1996

Compton Scattering, Meson Exchange, and the Polarizabilities of Bound Nucleons

Nuovo Cim. 107 A:2243, 1994

Evidence of a New State at $1860 \mathrm{MeV} / c$ Observed in $\bar{n} p$ Annihilations

Nuovo Cim. 107A:2253, 1994

$\bar{p} p$ Annihilation at Rest into $K_{L} K_{S} \pi^{\circ} \pi^{\circ}$

Phys. Rev. Lett. 76:22, 1996

Study of $\Lambda$ Polarization in $p p \rightarrow p \Lambda K^{+} \pi^{+} \pi^{-} \pi^{+} \pi^{-}$at $27.5 \mathrm{GeV}$

Phys. Rev. C56:38, 1997

Analyzing Power Measurements for the deuteron $\uparrow$ deuteron $\rightarrow$ deuteron $+p+n$ Breakup Reaction at $12 \mathrm{MeV}$

DAPNIA-SPP-94-22

Recent Results from the H1 Collaboration at HERA

Phys. Rev. C55:19, 1997

Spin-Transfer Measurements of the $\pi$ deuteron $\uparrow \rightarrow p \uparrow p$ Reaction at Energies Spanning the $\Delta$ Resonance

Nucl. Phys. A610:523C, 1996; HEIDELBERG-97 p. 523C

Recent Results on Hadron Correlations

SLAC-PUB-6679

Measuring the Left-Right Cross Section Asymmetry in $Z^{0}$ Boson Production by $e^{+} e^{-}$Collisions at the SLC

Nucl. Phys. (PS) B40:299, 1995

Tau Lifetime Measurements with the Impact Parameters Sum Method in 1-1 Events

Phys. Lett. 394B:395, 1997; CERN-PPE-96-157

First Observation of Strong OZI Rule Violation in $\pi$ nucleon Interactions

CERN-EP-98-066

Comprehensive Evidence about a Narrow $p \vec{p}$ State of Mass $2.02 \mathrm{GeV} / \mathrm{c}^{2}$

Phys. Rev. Lett. 77:2630, 1996

Measurement of Tensor Analyzing Powers for Elastic Electron Scattering from a Polarized deuteron Target Internal to a Storage Ring

Z. Phys. C61:313, 1994

New Evidence for a Universal Correlation Term for Like-Sign Particles in Multiple Production 
Filippi 96

Fischbach 94

Fissum 96

Flammang 98

Flattum 96

Flaugher 96

Foka 95

Ford 94

Fortsch 98

Fortune 94

Fortune 97

Foxford 96

Frabetti 93C

Frabetti 94

Frabetti 94B

Frabetti $94 \mathrm{C}$

Frabetti $94 \mathrm{D}$

Frabetti $94 \mathrm{E}$

Frabetti $94 \mathrm{~F}$

Frabetti $94 \mathrm{G}$

Frabetti $94 \mathrm{H}$

Frabetti 94I

Frabetti 94J

Frabetti 95

Frabetti 95B

Frabetti 95C

Frabetti 95D

Frabetti $95 \mathrm{E}$

Frabetti $95 \mathrm{~F}$

Frabetti $95 \mathrm{H}$

Frabetti 95I

Frabetti 96

Frabetti 96B

Frabetti 97

Frabetti $97 \mathrm{~B}$

Frabetti 97C

Frabetti 98

Frabetti 98B
MANCHESTER-95 p. 337

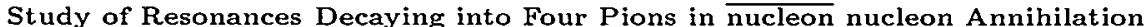

Phys. Rev. Lett. 73:514, 1994 New Geomagnetic Limits on the Photon Mass and on Long-Range Forces Coexisting with Electromagnetism

Phys. Rev. C53:1278, 1996

Inclusive Positive Pion Photoproduction

Phys. Rev. C58:916, 1998

Analyzing Power and Cross Sections of $p p \rightarrow p n \pi+$ Near Threshold

PADUA-96 p. 537; FERMILAB-CONF-96-199-E

A Preliminary Measurement of the $W^{ \pm}$Boson Mass using $W^{-} \rightarrow e^{-} \bar{\nu}_{e}$ Decays at $D^{0}$

FERMILAB-CONF-96-225-E

High $E_{T}$ Jet Cross Sections at CDF

Nucl. Phys. A583:687C, 1995

Strange Particle Production in Nuclear Collisions

Nucl. Phys. (PS) B40:191, 1995; MONTREUX-94 p. 191; COLO-HEP-352

Tau Decays to 3 hadron $\left(\pi^{\circ}\right) \nu$ and 5 hadron $\left(\pi^{\circ}\right) \nu$ from CLEO

Nucl. Phys. A643:55, 1998

The $p p \rightarrow \pi^{+}$deuteron Reaction Close to Threshold at COSY

Phys. Rev. C50:306, 1994

Energy Dependence of ${ }^{58} \mathrm{Ni}\left(\pi^{+}, \pi^{-}\right){ }^{58} \mathrm{Zn}$ Double Charge Exchange

Phys. Rev. C55:2468, 1997

Energy Dependence of Cross Section for Pion Double Charge Exchange on ${ }^{60} \mathrm{Ni},{ }^{62} \mathrm{Ni},{ }^{64} \mathrm{Ni}$

Phys. Rev. C54:749, 1996; DAPNIA-SPHN-96-07; INC-40007-105 $4 \pi$ Studies of the $1.8-4.8 \mathrm{GeV}^{3} \mathrm{He}+\mathrm{Ag}_{\text {nat }},{ }^{197} \mathrm{Au}$ Reactions. II. Multifragmentation

Phys. Rev. Lett. 72:961, 1994; FERMILA B-PUB-93-323-E

An Observation of an Excited State of the $\Lambda_{c}^{+}$Baryon

Phys. Lett. 328B:187, 1994; FERMILAB-PUB-94-049-E

Measurement of the Form Factors for the Decay $D_{s}^{+} \rightarrow \phi(1020) \mu^{+} \nu$

Phys. Lett. 328B:193, 1994; FERMILA B-PUB-94-079-E

First Observation of the $\Sigma^{-} \pi^{+} \pi^{+}$Decay Mode of the $\Lambda_{c}$ Baryon and Its Branching Ratio Relative to the $\Sigma^{+} \pi+\pi^{-}$Mode

Phys. Rev. D50:R2953, 1994; FERMILAB-PUB-94-071-E Search for $C P$ Violation in Charm Meson Decay

Phys. Let t. 331B:217, 1994; FERMILA B-PUB-94-076-E Analysis of Three $D \rightarrow$ kaon $\pi \pi$ Dalitz Plots

Phys. Rev. Lett. 72:324, 1994; FERMILAB-PUB-93-249-E Measurement of the Masses and Widths of $L=1$ Charm Mesons

Phys. Lett. 321B:295, 1994; FERMILA B-PUB-93-361-E

A Measurement of the Cabibbo-Suppressed Decays $D^{0} \rightarrow \pi^{-} \pi^{+}$and $D^{0} \rightarrow K^{-} K^{+}$

Phys. Lett. 323B:459, 1994; FERMILAB-PUB-93-332-E

Precise Measurements of the $D^{\circ}$ and $D^{+}$Meson Lifetimes

Phys. Lett. 338B:106, 1994; FERMILAB-PUB-94-248-E Observation and Mass Measurement of $\Omega_{c} \rightarrow \Sigma^{+} K^{-} K^{-} \pi^{+}$

Phys. Lett. 340B:254, 1994; FERMILA B-PUB-94-272-E Branching Ratios of the Decays $D^{\circ} \rightarrow \bar{K}^{\circ} K^{\circ}$ and $D^{\circ} \rightarrow K_{S} K_{S} K_{S}$

Phys. Lett. 346B:199, 1995; FERMILA B-PUB-94-417-E Charm Meson Decay into the Final States $K_{S} K^{+}$and $K_{S} K^{*}(892)^{+}$

Phys. Lett. 351B:591, 1995; FERMILAB-PUB-95-032-E

Analysis of the $D^{+}, D_{s}^{+} \rightarrow K^{+} K^{-} \pi^{+}$Dalitz Plots

Phys. Lett. 354B:486, 1995; FERMILAB-PUB-95-114-E Study of Charged Hadronic Four Body Decays of the $D^{\circ}$ Meson

Phys. Lett. 357B:678, 1995; FERMILAB-PUB-95-163-E First Measurement of the Lifetime of the $\Omega_{c}$

Phys. Let t. 359B:403, 1995; FERMILAB-PUB-95-262-E

Doubly and Singly Cabibbo Suppressed Charm Decays into the $K^{+} \pi^{-} \pi^{+}$Final State

Phys. Lett. 363B:259, 1995; FERMILAB-PUB-95-318-E

Search for the Decay of $D^{+}$and $D_{s}^{+}$Mesons to Three Charged Kaons

Phys. Lett. 365B:461, 1996; FERMILAB-PUB-95-319-E

Study of Higher Mass Charm Baryons Decaying to $\Lambda_{c}^{+}$

Phys. Lett. 364B:127, 1995; FERMILAB-PUB-95-338-E

Analysis of the Decay Mode $D^{0} \rightarrow K^{-} \mu^{+} \nu_{\mu}$

Phys. Lett. 370B:222, 1996; FERMILAB-PUB-95-410-E

Charm-Anticharm Asymmetries in High Energy Photoproduction

Phys. Lett. 382B:312, 1996; FERMILAB-PUB-96-139-E

Analysis of the Cabibbo Suppressed Decay $D^{0} \rightarrow \pi^{-} \ell^{+} \nu$

Phys. Lett. 391B:235, 1997; FERMILAB-PUB-96-388-E

Observation of the Vector Meson Cabibbo Suppressed Decay $D^{+} \rightarrow \rho^{0} \mu^{+} \nu$

Phys. Lett. 398B:239, 1997; FERMILAB-PUB-97-029-E

Search for Rare and Forbidden Decays of the Charmed Meson $D^{+}$

Phys. Lett. 401B:131, 1997; FERMILAB-PUB-97-006-E

Study of Charged Hadronic Five-body Decays of the $D^{+}$and $D_{3}^{+}$Mesons

Phys. Lett. 407B:79, 1997; FERMILAB-PUB-97-045-E

Analysis of the $D^{+}, D_{s}^{+} \rightarrow \pi^{-} \pi^{+} \pi^{+}$Dalitz Plots

Phys. Lett. 426 B:403, 1998; FERMILA B-PUB-98-090-E

Observation of a Narrow State Decaying into $\Xi_{c}(2480)^{\circ} \pi^{+}$

Phys. Lett. 427B:211, 1998; FERMILAB-PUB-98-068-E

A New Measurement of the Lifetime of the $\Xi_{c}(2460)^{+}$ 
Franczuk 99

Frascaria 94

Frey 96

Freyberger 94

Freyberger 96

Friedman 97

Friese 97

Frisch 94

Frisch 95

Frlez 97

Frolov 99

Frommberger 94

Frommhold 94

Fu 96

Fuchs 94

Fuchs 96

Fuess 94B

Fuess 94C

Fuess 95

Fujikawa 98

Fujino 95

Fujiwara 94

Fukuda 94

Fukuda 96

Fukuda 96B

Fukuda 98

Fukuda 98B

Fukuda 98C

Fukuda 98D

Fukuda $98 \mathrm{E}$

Fukuda $98 \mathrm{~F}$

Fukuda 98G

Fukuda 98H

Furget 98

Furtjes 96

Furutani 94

Gabrielse 95
Phys. Lett. 450B:332, 1999

${ }^{2} \mathrm{C}(\gamma \uparrow, p n)$ Photon Asymmetry for $E_{\gamma}=180-340 \mathrm{MeV}$

Phys. Rev. C50:R537, 1994; IPNO-DRE-94-09

Total deuteron deuteron $\rightarrow \alpha \eta$ Cross Sections near Threshold

BONN-IR-96-03

Measurement of the Proton Structure Function $F_{2}\left(x, Q^{2}\right)$ in Radiative $e^{-} p$ Scattering with the ZEUS Detector

FIFT-HEP-94-1

Charm Semileptonic Decays at $e^{+} e^{-}$Machines

Phys. Rev. Lett. 77:2147, 1996; Phys. Rev. Lett. 76:3065, 1996; CLNS-96-1389; CLEO-96-1

Limits on Flavor Changing Neutral Currents in $D^{\circ}$ Meson Decays

Phys. Rev. C55:1304, 1997

$K^{+}$nucleus Reaction and Total Cross Sections: New Analysis of Transmission Experiments

Jour. of Phys. G 23:1837, 1997

$\phi(1020)$ Production in $158 \mathrm{GeV} / \mathrm{u} \mathrm{Pb}+\mathrm{Pb}$ Collisions

FERMILAB-CONF-94-044-E

CDF Electroweak Studies and the Search for the Top Quark

FERMILAB-CONF-95-129-E

A New Measurement of the $W^{ \pm}$Mass

Phys, Rev, C57:3144, 1998; UVA-IN P P-97-06; HEPEX-9712024

Differential Cross Sections for Pion Charge Exchange on the Proton at 27.5 MeV

Phys. Rev. Lett. 82:45, 1999; HEPEX-9808024

Electroproduction of the $\Delta\left(1232 P_{33}\right)$ Resonance at High Momentum Transfer

Phys. Lett. 339B:17, 1994

Measurement of the Left-Right Asymmetry in the Electrodisintegration of the Deuteron

Z. Phys. A350:249, 1994

Photofission of ${ }^{238} \mathrm{U}$ at Intermediate Energies: Absolute Cross Sections and Fragments Mass Distributions

Phys. Rev. Lett. 79:3125, 1997; CLNS-96-1397; CLEO-96-6

Observation of Exclusive $B$ Decays to Final States Containing a Charmed Baryon

Phys. Rev. D53:11, 1996; JULICH-94 p. 125

Direct Measurement of the Branching Ratio for the Decay of the $\eta$ Meson into Two Photons

Phys. Lett. 368B:20, 1996

Neutral Pion Photoproduction from the Proton near Threshold

ALBUQUERQUE-94, v. 1, p. 447; FERMILAB-CONF-94-283-E

Electroweak Boson Pair Production in $p \bar{p}$ Collisions at $\sqrt{s}=1800 \mathrm{GeV}$

Nucl. Phys. B414:3, 1994

Diffraction Dissociation in $\pi^{+} p$ Interaction at $147 \mathrm{GeV} / \mathrm{c}$

FERMILAB-CONF-95-072-E

$W W$ and $W Z$ Production at the Tevatron

NUCLEX-9806001

A New Measurement of the Partial $0^{+} \rightarrow 0^{+}$Half Life of ${ }^{10} \mathrm{C}$ with GAMMASPHERE

OHSTPY-HEP-E-95-009; DOE-ER-01545-648

Charmed Baryon Production and Decay: Recent Results from CLEO and E687

Nucl. Phys. A.577:43C, 1994

Spin-Isospin Excitations in Nuclei via $\left({ }^{3} \mathrm{He},{ }^{3} \mathrm{H}\right)$ Reactions at $450 \mathrm{MeV}$

Phys. Lett. 335B:237, 1994

Atmospheric $\nu_{\mu} / \nu_{e}$ Ratio in the Multi-GeV Energy Range

Phys. Lett. 388B:397, 1996; ICRR-373-96-24; TOHOKU-HEP-96-04; KEK-96-98; TKU-PAP-96-02; OULNS-96-02; TITHPE-96-09; KOBE-AP-96-01; INS-1159

Study of Neutron Background in the Atmospheric Neutrino Sample in KAMIOKANDE

Phys. Rev. Lett. 77:1683, 1996; ICRR-372-96-24; KEK-96-94; TOHOKU-HEP-96-03; INS-1155; TKU-PAP-96-01; TITHPE-96-08; OULNS-96-03

Solar Neutrino Data Covering Solar Cycle 22

Phys. Lett. 436B:33, 1998; ICRR-418-98; HEPEX-9805006

Study of the Atmospheric Neutrino Flux in the Multi-GeV Energy Range

Phys. Rev. Lett. 81:4279, 1998; Phys. Rev. Lett. 81:1158, 1998; ICRR-417-98-13; CSUDH-HEP 98-02; KEK-98-40; SBHEP98-2; NGTHEP-98-02; OULNS-98-01; TKU-PAP-98-02; TIT-HPE-98-05; UWSEA-PUB 98-02; HEPEX-9805021 Measurements of the Solar Neutrino Flux from Super-Kamiokande's First 300 Days

Phys. Rev. Lett. 81:1562, 1998; BU-98-17; HEPEX-9807003 Evidence for Oscillation of Atmospheric Neutrinos

Phys. Rev. Lett. 82:2430, 1999; ICRR-REPORT-442-98-38; HEPEX-9812011

Measurement of the Solar Neutrino Spectrum using Neutrino-Electron Scattering

Phys. Rev. Lett. 82:2644, 1999; ICRR-440-98-36; HEPEX-9812014

Measurement of the Flux and Zenith-Angle Distribution of Upward Through-Going Muons by SuperKamiokande

Phys. Lett. 433B:9, 1998

Measurement of a Small Atmospheric $\nu_{\mu} / \nu_{e}$ Ratio

Phys. Rev. Lett. 82:1810, 1999; ICRR-REPORT-441-98-37; HEPEX-9812009

Constraints on Neutrino Oscillation Parameters from the Measurement of Day-Night Solar Neutrino Fluxes at Super-Kamiokande

Phys. Rev. C58:1306, 1998

Cascade Hypernuclei in the $\left(K^{-}, K^{+}\right)$Reaction on ${ }^{12} \mathrm{C}$

Nucl. Phys. A631:747C, 1998; GRONINGEN-97 p. 747C

Measurement of Tensor Polarization Observables in the $p$ ( $p$, deuteron) $\pi^{+}$Reaction between 580 and $1300 \mathrm{MeV}$

Nuovo Cim. 109A:641, 1996; PISA-95 p. 641

Production of Excited States

Phys. Rev. C50:1561, 1994

Exclusive Pion Production from Few Nucleon Systems in the Region of the $\Delta\left(1232 P_{33}\right)$ Resonance

Phys. Rev. Lett. 74:3544, 1995

Special Relativity and the Single Antiproton: Fortyfold Improved Comparison of $\bar{p}$ and $p$ Charge-to-Mass Ratios 
Gaff 95

Gaitinov 94

Gallas 9.5

Gallas 96

Gallas 96B

Gankhuyag 98

Ganz 96

Gao 94

Gao $94 B$

Garbincius 94

Garbincius 95

Garcilazo 95

Gardner 95

Garsevanishy 98

Garson 94

Gaspero 94

Gaspero 95

Gaspero $95 \mathrm{~B}$

Gaspero 96

Gauzzi 94

Gazdzcki 94

Gazdzicki 95

Gazdzicki 96

Geer 93

Geer 94

Geer 95

Geer 95B

Geer 98

Geer 98B

Geiger 98

Gelderloos 96

Gelovani 98

Genser 94

Gentile 95

Georgadze 95

Gerdes 94

Gerdes 96

Gerhards 96
Phys. Rev. C52:2782, 1995

Two-Proton Correlations for ${ }^{16} \mathrm{O}+{ }^{187} \mathrm{Au}$ Collisions at $E / A=200 \mathrm{MeV}$

JINR-E1-94-201

Cascades of Nuclear Interactions in Liquid Hydrogen

Phys. Rev. D52:6, 1995

Search for Neutral Weakly Interacting Massive Particles in the Fermilab Tevatron Wide Band Neutrino Beam

FERNIILAB-CONF-96-114-E; DO-NOTE-2905

New Particle Searches at the Tevatron (I)

FERMILAB-CONF-96-304-EF

Do Papers on QCD Studies with Jets: A Study of Multijet Production Ratios in $p \bar{p}$ Collisions at Do

JINR-P2-98-26

Description of $\pi^{-}$-Meson and Proton Characteristics in $n p$-Interactions at $P_{n}=1.25-5.1 \mathrm{GeV} / c$ within

the Framework of FRITIOF Model

Phys. Lett. 389B:4, 1996; GSI-96-40

Search for $e^{+} e^{-}$Pairs with Narrow Sum-Energy Distributions in Heavy-Ion Collisions

Phys. Rev. C50:R546, 1994

Measurement of the Neutron Magnetic Form Factor from Inclusive Quasielastic Scattering of Polarized Electrons from Polarized ${ }^{3} \mathrm{He}$

WILLIANSBURG-94 p. 693

Measurement of the Neutron Magnetic Form Factor from Inclusive Quasielastic Scattering of Polarized

Electrons from Polarized ${ }^{3} \mathrm{He}$

Nuovo Cim. 107A:1989,1994; FERMILAB-CONF-93-238

Recent Fermilab Results on Hadroproduction of Heavy Flavors

FERMILAB-CONF-95-041

Charm Photoproduction Dynamics

Phys. Rev. C52:49, 1995

Pion Photoproduction on the Deuteron: the Reaction $\gamma$ deuteron $\rightarrow \pi^{0}$ deuteron

FERMILAB-CONF-95-133-E

Charm Production Physics from Fermilab Fixed Target Experiments

Phys. At. Nucl. 61:1356, 1998; Yad. Phys. 61:1460, 1998

Analysis of Secondary Charged Particles Produced in Hadron-Nucleus and Nucleus-Nucleus Interactions in Terms of Multidimensional KNO-Scaling

Phys. Rev. C49:2516, 1994

Tensor Polarization in Elastic Electron Deuteron Scattering in the Momentum Transfer Range $3.8 \leq Q \leq$ $4.6 \mathrm{fm}^{-1}$

Phys. At. Nucl. 57:1821, 1994; Yad. Phys. 57:1891, 1994

New Results Obtained by the Rome Data on $\bar{p}$ Annihilation at Rest

Phys. Lett. 358B:146, 1995

Study of the Pionic Correlations in $\bar{p} n \rightarrow \pi^{+} 2 \pi^{-}$Annihilation at Rest

Nucl. Phys. A588:861, 1995; Nucl. Phys. A614:565, 1997

Study of the Pionic Correlations in $\bar{p} n \rightarrow 2 \pi^{+} 3 \pi^{-}$Annihilation at Rest

MANCHESTER-95 p. 557

Study of the Pionic Correlations in $\bar{p} n$ Annihilation at Rest

Nuovo Cim. 107 A:2383, 1994

Measurement of the Cross-Section for $e^{+} e^{-} \rightarrow$ Hadrons near the Nucleon-Antinucleon Threshold by the

FENICE Experiment

Nucl. Phys. A566:503C, 1994

New Data on the Strangeness Enhancement in Central Nucleus-Nucleus Collisions at 200 A GeV

2. Phys. C65:215, 1995

Pion Multiplicity in Nuclear Collisions

Z. Phys. C71:55, 1996; IKF-HENPG-8-95

Strangeness in Nuclear Collisions

Phys. Rev. Lett. 72:1596, 1994; FERNILAB-PUB-93-315-E

Search for Antiproton Decay at the Fermilab Antiproton Accumulator

CDF-PUB-CDF-PUBLIC-2787; FERMILAB-CONF-94-275-E

Physics at the Fermilab Tevatron Proton-Antiproton Collider

Phys. Rev. C52:334, 1995

Charge-Changing Fragmentation of $10.6 \mathrm{GeV} / \mathrm{Nucleon}{ }^{197} \mathrm{Au} N u c l e i$

FERMILAB-CONF-95-225-E

Multijet Events at the Tevatron Proton-Antiproton Collider

FERMILAB-PUB-98-383-A; UF-IFT-HEP-98-34; ASTROPH-9812025 A New Limit on the Antiproton Lifetime

FERMILAB-PUB-98-265-A; UF-IFT-HEP-98-13; ASTROPH-9809101

The Cosmic Ray Antiproton Spectrum and a Limit on the Antiproton Lifetime

Nucl. Phys. A629:27 TC, 1998; OSAKA-97 p. $277 \mathrm{C}$

First Results from the HERMES Experiment

Phys. Rev, C54:3056, 1996

Classical Tests for Statistical Evaporation at $680 \mathrm{MeV}{ }^{40} \mathrm{Ar}+\mathrm{Ag}_{n a t}$

HEPPH-9803240

Study of Fractality and Chaoticity in Central 4.5 A.GeV/c C Cu Collisions

FERMILAB-CONF-94-288

Search for Top with Do Detector in Dilepton Channel

Nucl. Phys. (PS) B40:171, 1995

$\tau$ Decays with One Charged Particle and $\pi^{0}$ 's

Phys. At. Nucl. 58:1093, 1995; Yad. Phys. 58:1170, 1995

The Research of $2 \beta$ Decays of ${ }^{116} \mathrm{Cd}$ with Enriched ${ }^{114} \mathrm{CdWtO} 4$ Crystal Scintillator

ALBUQUERQUE-94, v. 1, p. 803; FERMILAB-CONF-94-328-E

Measurement of Correlated $b$ Quark Cross Sections at CDF

FERMILAB-CONF-96-342-E

Top Quark Physics Results from CDF and Do

Nuovo Cim. 109A:695, 1996; PISA-95 p. 695

Charmonium Production in ep Collisions at HERA 
Germain 97

Gerschel 95

Geso 98

Ghosh 94

Ghosh 94B

Ghosh 94C

Ghosh 94D

Ghosh 94E

Ghosh $94 \mathrm{~F}$

Ghosh 95

Ghosh 95B

Ghosh 95C

Ghosh 96

Ghosh 96B

Ghosh 97

Ghosh 97B

Ghosh 97C

Ghosh 97D

Ghosh 98

Ghosh 98B

Giannetti 94

Gibaut 94

Gibaut 94B

Gibaut 95

Gibbons 95

Gibbons 95B

Gibbons 95C

Gibbons 96

Gibbons 97

Gilkes 94

Gillitzer 96

Giokaris 96

Girone 94

Girone 95

Glagolev 94

Glagolev 94 B

Glagolev 95

Glagolev 95B
Nucl. Phys. A620:81, 1997

High Transverse Momentum Proton Emission in Ar Ta Collisions at $84 \mathrm{MeV} / \mathrm{u}$

Nucl. Phys. A583:643C, 1995; TAORMINA-95 p. 643C

Vector Meson Production in Ultra-Relativistic Heavy Ion Collisions at CERN

Phys. Rev. C58:3742, 1998

Measurements of Spin Observables for the $p{ }^{4} \mathrm{He}$ Elastic Scattering at $800 \mathrm{MeV}$

Phys. Rev, D49:3113, 1994

Multihadron Production in High-Energy Interactions and Intermittency

Mod. Phys. Lett. A9:591, 1994

Evidence of Intra- and Intergroup Azimuthal Correlations in Nuclear Interactions at Few GeV/c

Nuovo Cim, 107A:533, 1994

Fractal Study of Pionization in $p-\mathrm{AgBr}$ Interaction at $400 \mathrm{GeV} / \mathrm{c}$

Nuovo Cim. 107A:1517, 1994

Analysis of $p_{T}$ Spectrum of Projectile Fragments in Heavy-Ion Interactions. Identification of Collective Flow of Nuclear Matter

Phys. Rev. C49:R1747, 1994

Intermittency and Fragmentation of Target Residue in High-Energy Nuclear Interactions

Phys. Rev. C49:3219, 1994

Intermittency and Correlations in ${ }^{16} \mathrm{O}+\mathrm{Ag} / \mathrm{Br}$ Interactions at $2.1 \mathrm{GeV} / \mathrm{Nucleon}$

Plyys. Rev. C52:2092, 1995

Factorial Correlator Study in ${ }^{32} \mathrm{~S} \mathrm{Ag} / \mathrm{Br}$ Interaction at $200 \mathrm{~A} \mathrm{GeV}$

Phys. Rev. D51:1406, 1995

Multiparticle Data in High Energy Hadronic Interaction with Nuclei and Koba-Nielsen-OlsenGolokhvastov Scaling

Eur. Lett. 29:521, 1995

Evidence of Intermittency in Compound Multiplicity and High-Energy Hadron-Nucleus Interactions

Z. Phys. C71:243, 1996

Fluctuation Study of Pionization in Ultrarelativistic Nucleus-Nucleus Interaction

Fizika B5:135, 1996

Measurement of Charge of Projectile Fragments $(1 \leq Z \leq 3)$ in $60 \mathrm{~A} \cdot \mathrm{GeV} / \mathrm{c}^{16} \mathrm{O}$-Emulsion Interaction A Search for Fractionally Charged Fragment

Phys. Rev. C56:2879, 1997

Evidence of Scaling of Void Probability in A A Interactions at Few GeV Energy

Z. Phys. C73:269, 1997

Fluctuation and Fractality Study of Target Residue in ${ }^{32} \mathrm{~S} \mathrm{AgBr}$ Interaction at $200 \mathrm{~A} \cdot \mathrm{GeV}$

Fizika B6:37, 1997

Study of Multiplicity Distribution in Full Phase Space in Ultra Relativistic Nuclear Collision

Fizika B6:141, 1997

Transverse Momentum Spectrum of Ultra-Relativistic Li Projectile Fragments $(Z=3)$ in $60 \mathrm{~A} \cdot \mathrm{GeV} / \mathrm{c}$ Oxygen-Emulsion Interaction

Phys. Rev. C58:3553, 1998 Evidence of Multifractal Nature of Target-Evaporated Slow Particles Produced in Ultrarelativistic Heavy Ion Interactions

Eur. Lett. $41: 372,1998$

Evidence of Non-Thermal Phase Transition in Ultrarelativistic Heavy-Ion Interactions

FERMILAB-CONF-94-151-E

Evidence for Color Coherence in Jet Events

Phys. Rev. Lett. 73:934, 1994; CLNS-94-1284; CLEO-94-12

Study of the Five Charged Pion Decay of the $\tau^{ \pm}$Lepton

Mod. Phys. Lett. A9:675, 1994; VPI-IHEP-94-2; CMU-HEP-94-03; DOE-ER-40682-57

Recent Results on Semimuonic Decays of Charm Mesons from Fermilab Experiment E653

Phys. Rev. D53:4734, 1996; CLNS-95-1354; CLEO-95-16 Measurements of $B \rightarrow D_{s}^{+} \mathrm{X}$ Decays

CLNS-95-1323; CLEO-95-2

A Study of Jet Production in the Four Flavor Continuum and a Test of QCD

UR-1427

Preliminary Results for Exclusive $b \rightarrow u \ell \nu$ Decays from CLEO

Phys. Rev. D55:6625, 1997; FERMILAB-PUB-95-392-E

$C P$ and $C P T$ Symmetry Test from the Two Pion Decays of the Neutral Kaon with the FNAL E731 Detector

Phys. Rev. Lett. 77:810, 1996; CLNS-96-1394; CLEO-96-4

Observation of an Excited Charmed Baryon Decaying into $\Xi_{c}^{0} \pi^{+}$

Phys. Rev. D56:3783, 1997; CLNS-96-1454; CLEO-96-23; HEPEX-9703006

The Inclusive Decays $B \rightarrow D X$ and $B \rightarrow D^{*} X$

Phys. Rev. Lett. 73:1590, 1994; LBL-36121

The Determination of Critical Exponents from the Multifragmentation of Gold Nuclei

Z. Phys. A354:3, 1996

Subthreshold Production of Energetic $\pi^{-}$in Relativistic Ne $\mathrm{Na}$ and Ni Ni Collisions

Nuovo Cim. 109A:747, 1996; PISA-95 p. 747

CDF Top-Quark Kinematical Analyses

Nuovo Cim. 107A:1841, 1994

Study of Vector-Vector Final States Centrally Produced in $p$ p Interactions at $300 \mathrm{GeV} / \mathrm{c}$

Nucl. Phys. (PS) B40:153, 1995

Global Analysis of Tau Hadronic Branching Ratios in ALEPH

Pisma Zh. Eksp. Teor. Fiz. 59:316, 1994; Jetp Lett. 59:336, 1994

Yield of Mirror Nucleus ${ }^{3} \mathrm{H},{ }^{3} \mathrm{He}$ and ${ }^{7} \mathrm{Li},{ }^{7} \mathrm{Be}$ in the Interactions of Relativistic Oxygen Nucleus with Proton

Phys. At. Nucl. 58:1892, 1995; Yad. Phys. 58:2000, 1995; JINR-P1-94-274

Final State Interaction in ${ }^{3} \mathrm{He} p$ Collisions

Yad. Phys. 58:2005, 1995; Phys. At. Nucl. 58:1896, 1995

Formation of Helium Nuclei in Oxygen-Proton Interactions at Relativistic Energies

Kr. Soob. JINR 73:51, 1995

Space Correlations and Final State Interaction in the Deuteron and Helium Nuclei Break-Up Reactions

See the legend on page 5 . 
Glagolev 96

Glagolev 97

Glagolev 98

Glagolev 98B

Glenn $9 i$

Glushkov 95

Glushkov 97

Gninenko 98

Godang 97

Godang $97 \mathrm{~B}$

Goetz 94

Gogiberidze 98

Golden 92

Golden 96

Goldenbaum 96

Gollwitzer 94

Golovkin 94

Golovkin 95

Golovkin 96

Golovkin 97

Golovkin $97 \mathrm{~B}$

Golutvin 94

Gomez 93

Gomezycadena 94

Gonin 94

Gordeev 94

Gordeev 96

Gordeev 97

Gorichev 93

Gorringe 98

Goshaw 96

Goshaw $96 \mathrm{~B}$

Gothe 95

Gotta 94

Goulianos 94

Goulianos 96

Goy 96

Goy 98
Phys. At. Nucl. 59:2125, 1996; Yad. Phys. 59:2207, 1996 Spatial Correlations in Deuteron Breakup with Allowance for Final-State Interaction

Phys. Rev. C59:405, 1999; JINR-E1-97-214 Delta Isobar Production in the ${ }^{4} \mathrm{He} p \rightarrow{ }^{3} \mathrm{H} p n \pi+$ Reaction

Kr. Soob. JINR 89:37, 1998

Self-Similarity Properties of the Light Nuclei Collisions. Experimental Results of the Fragmentation Reactions Investigation

DUBNA-96, v. I, p. 185

Delta Isobar Production in the ${ }^{4} \mathrm{He} p \rightarrow{ }^{3} \mathrm{H} p n \pi$ Reaction

Phys. Rev. Lett. 80:2289, 1998; CLNS-97-1514; CLEO-97-21; HEPEX-9710003 Search for Inclusive $b \rightarrow s \ell^{+} \ell^{-}$

Phys. At. Nucl. 58:1186, 1995; Yad. Phys. 58:1265, 1995

Irregularity of Muon Component of EAS with Energies above $5 \times 10^{18} \mathrm{eV}$

Izv. RAN, Fiz. 61:516, 1997

Lateral Distribution of Charged Particles in EAS with $E_{0}=10^{17}-5 \times 10^{18} \mathrm{eV}$

Phys. Lett. $427 \mathrm{~B}: 206,1998$

Limits on $\nu_{\mu}\left(\nu_{e}\right) \rightarrow \nu_{\tau}$ Oscillations and $\nu_{\tau}$ Magnetic Moment from Neutrino-Electron Scattering

Phys. Rev. Lett. 79:3829, 1997; CLNS-97-1467; CLEO-97-3; HEPEX-9703009

Limit on the Two-Photon Production of the Glueball Candidate $f_{J}(\mathbf{2 2 2 0})$ at CLEO

Phys. Rev. Lett. 80:3456, 1998; CLNS-97-1522; CLEO-97-27; HEPEX-9711010

Observation of Exclusive Two-Body $B$ Decays to Kaons and Pions

Nucl. Phys. A574:467, 1994

Precise Relative Cross Sections for $n p$ Scattering

Phys. Lett. 430B:368, 1998

Study of Multiparticle Spikes in Central 4.5 A GeV/c C Cu Collisions

Astroph. Jour. 436:769, 1994; LNF-92-O52-P

Observation of Cosmic Ray Electrons and Positrons using an Imaging Calorimeter

Astrophys. Lett 453:L103, 1996; INFN-AE-95-22

Measurement of the Positron to Electron Ratio in the Cosmic Rays above $5 \mathrm{GeV}$

Phys. Rev. Lett. 77:1230, 1996

Heating of Nuclei with Energetic Antiprotons

Phys. At. Nucl. 57:1722, 1994; Yad. Phys. 57:1793, 1994

Charmonium Formation in $\bar{p} p$ Annihilations by Experiment E760

Z. Phys. C68:585, 1995; IFVE-94-78

Study of Coherent Diffractive Production Reactions of $p \mathrm{C} \rightarrow Y^{0} K+C$ Type and Observation of the New Baryonic States $X(2050) \rightarrow \Sigma\left(1385 P_{13}\right)^{0} K^{+}$and $X(2000) \rightarrow \Sigma^{0} K^{+}$

Phys. At. Nucl, 59:1336, 1996; Yad. Phys. 59:1395, 1996; IFVE-95-144

Study of Diffractive Production Processes in Proton-Nucleon and Proton-Nucleus Interactions and Search for Exotic Baryons

Phys. At. Nucl. 59:1640, 1996; Yad. Phys. 59:1695, 1996

Production of $\pi^{\circ}, \eta$ and $\omega$ Mesons in Deep Fragmentation Region by $70 \mathrm{GeV}$ Protons

Z. Phys. A359:435, 1997; IFVE-97-20

Study of the OZI Selection Rule in Hadronic Processes

Z. Phys. A359:327, 1997; IFVE-97-21

Study of Quasiexclusive Neutral Meson Production in $p$ nucleon Interactions at $E_{p}=70 \mathrm{GeV}$ in the Deep Fragmentation Region

DESY-94-168

$\tau^{ \pm}$Decays

Phys. Rev. D49:4348, 1994; SLAC.PUB-5813

Measurement of the A-Dependence of Deep-Inelastic Electron Scattering

CERN-PPE-94-13

Experimental Results on $\tau^{ \pm}$Physics

Nucl. Phys. A566:601C, 1994

Baryon Distributions and Meson Production in Au+Au at $11.6 \mathrm{GeV} / \mathrm{c}$ First Particle Spectra from E886

Pisma Zh. Eksp. Teor. Fiz. 59:565, 1994; Jetp Lett. 59:589, 1994

Analysis of the Results of Investigations the Conversion Probability of the Muonium to Antimuonium and Background Processes on Separated "Surface" Muon Beam of the JINR LNP Phasotron

Kr. Soob. JINR 78:15, 1996

The New Limit of the Probability of Muonium Antimuonium Conversionin SPINP-JINR Experiment

Yad. Phys. 60:1291, 1997; :

New Method of Investigating of the Muonium-to-Antimuonium Conversion

Nucl. Phys. A578:586, 1994; ITEP-93-53

Slow Particles Production in Interactions of Neutrinos with Heavy Nuclei of Photoemulsion

Phys. Rev. C58:1767, 1998

Isotope Dependence of Radiative Muon Capture on the ${ }^{58} \mathrm{Ni},{ }^{80} \mathrm{Ni},{ }^{82} \mathrm{Ni}$ Isotopes

FERMILAB-CONF-96-209-E; CDF-PUB-ELECTROWEA K-PUBLIC-3802

$W^{ \pm}$and $Z^{0}$ Boson Production with Jets and Inclusive Jet Production in $p \bar{p}$ Collisions at $\sqrt{s}=1.8 \mathrm{TeV}$

FERMILAB-CONF-96-314-E

Dijet Production from $\bar{p} p$ Collisions at $1.8 \mathrm{TeV}$

Phys. Lett. 355B:59, 1995

Measurement of the Elastic $\pi^{0}$ Photoproduction Cross Sections on ${ }^{12} \mathrm{C}$ and ${ }^{13} \mathrm{C}$

Phys. Rev. C51:469, 1995; PSI-PR-94-18

Negative Pion Absorption at Rest in ${ }^{3} \mathrm{He}$

FERMILAB-CONF-94-266-E

Universality of Particle Multiplicities

FERMILAB-CONF-96-233-E; CDF-PUB-JETS-PUBLIC-3821; RU-96-E-29

New Evidence for Rapidity Gaps between Jets and Diffractive $W^{ \pm}$and Dijet Production at CDF

MANCHESTER-95 p. 479

Production of $B^{*}$ and $B^{* *}$ in Hadronic $Z^{\circ}$ Decays in the ALEPH Detector

Phys. Rev. C57:420, 1998

Measurement of the Analyzing Power $A_{y o}$ for the Reaction $p$ ( $p$, deuteron) $\pi^{+}$between 1000 and $1300 \mathrm{MeV}$ 
Grab 96

Graf 94

Grafter 98

Grannis 94

Grassmann 95

Grassmann 95B

Grater 98

Grechko 94

Greenlee 95

Greenlee 96

Greenshaw 94

Greenwood 96

Gregoire 97

Gress 98

Gribushin 95

Gridnev 95

Grigalashvil 98

Grigoriev 96

Grimani 95

Gronberg 95

Gronberg 97

Grosnick 97

Grossopilche 98

Grunewald 93

Grzonka 97

Grzonka 98

Gu 94

Gu 96

Gu 99

Guglielmo 96

Gulamov 95

Gulamov 96

Gulamov 96B

Gunther 95

Gutierrez 94

Gutierrez 96

Haas 94
Nucl. Phys. (PS) B50:192, 1996; STRASBOURG-95 p. 192

Results on $J / \psi(1 S)$ Production at HERA

FERMILAB-CONF-94-021-E

Production of $W^{ \pm}$and $Z^{\circ}$ Bosons at Do

Phys. Lett. 420B:37, 1998

The ${ }^{4} \mathrm{He}\left(\pi^{+}, \pi^{-}\right)$Reaction at Low Energies

FERMILAB-PUB-94-277

Search for the Top Quark: Results from the Do Experiment

FERMILAB-CONF-95-060-E

Kinematic Top Analyses at CDF

Nucl. Phys. (PS) 39BC:348, 1995; FERMILAB-CONF-94-299-E; MONTPELLIER-94 p. 348

Kinematical Evidence for Top Pairs at the Tevatron

Phys. Rev. C58:1576, 1998

Energy Dependence of the ${ }^{4} \mathrm{He}\left(\pi^{+}, \pi^{-}\right)$Total Cross Section

Phys. Lett. 343B:41, 1994; Jetp Lett. 59:325, 1994; Pisma Zh. Eksp. Teor. Fiz. 59:305, 1994; ITEP-94-25

Determination of the Momentum Distribution of the Deuteron-Deuteron Relative Motion of the $\alpha$-Particle from Data on the Reaction ${ }^{4} \mathrm{He} p \rightarrow p$ deuteron deuteron for Incident $\alpha$-Particle Momentum $2.7 \mathrm{GeV} / c$

FERMILAB-CONF-95-103-E

Observation of the TOP Quark

FERMILAB-CONF-96-244-E

Search for a Fourth Generation Charge -1/3 Quark via Flavor Changing Neutral Currents

DESY-94-112A; DESY-94-112B; DESY-94-112C; DESY-94-112D; MORIOND-94 p. 3; MORIOND-94 p. 163; MORIOND94 p. 295; MORIOND-94 p. 85; DESY-94-112

New Results from H1 Experiment at HERA on Jets, the Proton Structure Function, Rapidity Gap Events, Charged Current Cross Section and Searches for New Particles

Phys. Rev. D53:6054, 1996

Results of a Two-Position Reactor Neutrino-Oscillation Experiment

Nucl. Phys. (PS) B55:419, 1997; ESTES PARK-96 p. 419

Search for $\nu_{\mu} \rightarrow \nu_{\tau}$ Oscillations in the CHORUS Experiment

Izv. RAN, Fiz. 61:511, 1998

Investigation of PCR Energy Spectrum with the Tunka Array Detecting the EAS Cherenkov Radiation

Phys. Rev. D53:4723, 1996; FERMILAB-PUB-95-298-E

Production of $J / \psi(1 S)$ and $\psi(2 S)$ Mesons in $\pi^{-}$Be Collisions at $515 \mathrm{GeV} / c$

Yad. Phys. 58:461, 1995; Phys. At. Nucl. 58:410, 1995

Test of Charge Symmetry in $\pi^{-}$deuteron and $\pi^{+}$deuteron Elastic Scattering at Energy $T=417 \mathrm{MeV}$

IOP-H E-98-13; HEPEX-9803016

A-Dependencies of Neutral Strange Particle Yields at $40 \mathrm{GeV} / c \bar{p}$ nucleus Collisions

Yad. Phys. 59:2187, 1996; PAN 59 p. 5

Observation of an Anomaly in the Invariant Mass of Two $K_{S}$ Mesons near $1245 \mathrm{MeV}$

LNF-95-051-P

Differential Flux Measurement of Atmospheric Pion, Muon, Electron and Positron Energy Spectra at Balloon Altitudes

Phys. Rev. Lett. 75:3232, 1995; CLNS-95-1346; CLEO-95-10; HEPEX-9508001

Observation of the Isospin Violating Decay $D_{s}^{*+} \rightarrow D_{s}^{+} \pi^{0}$

Phys. Rev. D57:33, 1998; CLNS-97-1477; CLEO-97-7; HEPEX-9707031

Measurements of the Meson-Photon Transition Form Factors of Light Pseudoscalar Mesons at Large Momentum Transfer

Phys. Rev. D55:1159, 1997; ANL-HEP-PR-96-36

Measurement of the Differences in the Total Cross-Section for Antiparallel and Parallel Longitudinal Spins and a Measurement of Parity Nonconservation with Incident Polarized Protons and Anti-Protons at $200 \mathrm{GeV} / \mathrm{c}$

HEPEX-9810015

Combined Limits on First Generation Leptoquarks from the CDF and Do Experiments

UMI-95-26828; RX-1441 (CAL-TECH)

Measurement of the Tau-Pair Cross Section and Charge Asymmetry at the $Z^{\circ}$ Resonance

Nucl. Phys. A626:41C, 1997

First Results from COSY

Nucl. Phys. A631:262C, 1998; GRONINGEN-97 p. 262C

Strangeness Production at COSY

Phys. Rev. Lett. 72:3000, 1994

Measurement of the Branching Ratio and a Study of $C P$ for the Leptonic Decay $K_{L} \rightarrow e^{+} e^{-} e^{+} e^{-}$

Phys. Rev. Lett. 76:4312, 1996

First Evidence for the Decay $K_{L} \rightarrow e^{+} e^{-} \mu^{+} \mu^{-}$

Phys. Lett. 449B:361, 1999; BIHEP-EP1-98-04; HEPEX-9812027

On Recent Data of Branching Ratios for $\psi(2 S) \rightarrow J / \psi(1 S) \pi \pi$ and $\psi(2 S) \rightarrow J / \psi(1 S) \eta$

FERMILAB-CONF-96-245-E

Measurement of $W^{ \pm}+1 \mathrm{Jet} / W^{ \pm}+0 \mathrm{~J} e t s$ Cross Sections

Phys. At. Nucl. 58:1555, 1995; Yad. Phys. 58:1649,1995

The Investigations of Intermittency in ${ }^{12} \mathrm{C},{ }^{16} \mathrm{O}$, and ${ }^{22} \mathrm{Ne}$ Nucleus Interactions at Momentum $4.5 \mathrm{GeV} / \mathrm{c}$ per Nucleon with Nuclear Emulsion

Phys. At. Nucl. 59:997, 1996; Yad. Phys. 59:1042, 1996

Study of Total Disintegration of Relativistic Oxygen Nuclei in Interactions with Proton

Phys. At. Nucl. 59:1924, 1996; Yad. Phys. 59:1997, 1996

Effect of Factorization in $\pi^{-} N$ and $\pi^{-} \mathrm{C}$ Collisions with a Production of Leading Pions at $40 \mathrm{GeV} / \mathrm{c}$

Nucl. Phys. A590:487C, 1995

Antibaryon Production in ${ }^{32} \mathrm{~S}+$ Nucleus Collisions at $200 \mathrm{GeV} / \mathrm{Nucleon}$

Nuovo Cim. 107 A:1847, 1994

Light-Meson Spectroscopy in Fermilab Experiment E690

MANCHESTER-95 p. 408

Spin Parity Analysis of the Centrally Produced $K_{S} K^{ \pm} \pi^{\mp}$ System at $800 \mathrm{GeV}$

DESY-94-160

Recent Results from $e^{-} p$ Scattering at HERA 
Hacket: 96

Hadley 95

Haeberli $9 \tau$

Hafidouni 94

Hager 95

Hagley 94

Hagner 95

Hagopian 94

Haguenauer 94

Hahn 94

Hahn 99

Hall 96

Halling 96

Hamagaki 94

Hamasaki 97

Hamilton 94

Hammache 97

Hampel 98

Hanai 95

Hanai 97

Hanna 9-4

Hansen 94

Hansen 95

Hara 94

Harakeh 94

Hardie 97

Harnew 94

Harr 97

Harris 94

Harris $94 \mathrm{~B}$

Harris 95

Harris $95 \mathrm{~B}$

Harter 97

Harton 95

Hartouni 94

Harty 95

Harty 98

Hasan 94

Hasan 96
Phys. Rev. C53:R1047, 1996

Reaction Mechanisms in ${ }^{12} \mathrm{C}(\gamma, p p)$ Near $200 \mathrm{MeV}$

FERMILAB-CONF-95-352-E

Observation of the Top Quark with the Do Detector

Phys. Rev. C55:597, 1997

Proton-Proton Spin Correlation Measurements at $200 \mathrm{MeV}$ with an Internal Target in a Storage Ring

Nucl. Instr. and Meth. A339:570, 1994; CRN-94-20

Correction of Measured Multiplicity Distributions by the Simulated Annealing Method

Nucl. Phys. A595:287, 1995

Elastic Scattering of 58 and $75 \mathrm{MeV}$ Photons by ${ }^{12} \mathrm{C}$ and ${ }^{18} \mathrm{O}$ and Electromagnetic Polarizabilities of the Bound Nucleon

Phys. Rev. Lett. $72: 1172,1994$

Separated Oscillatory Field Measurement of Hydrogen $2 S_{1 / 2}-2 P_{3 / 2}$ Fine Structure Interval

Phys. Rev. D52:1343, 1995

Experimental Search for the Neutrino Decay $\nu \rightarrow \nu+e^{+}+e^{-}$and Limits on Neutrino Mixing

FERMILAB-CONF-94-331-E

Search for Supersymmetry and Leptoquark States at FNAL

Nuovo Cim. 107A:2093, 1994

The Real Part of the Elastic Scattering Amplitude at the $S \bar{p} p S$ and Predictions at LHC and SSC

Phys. Rev. C53:1074, 1996; TRI-PP-94-103; TAUP-2217-94

Pion Absorption on ${ }^{3} \mathrm{He}$ at Low Energies

Phys. Rev. Lett. 82:2258, 1999

Analyzing Powers and Partial Wave Decomposition of $\left.p n \rightarrow p p\left({ }^{1}\right) S_{0}\right) \pi^{-}$at Low Energies

FERMILAB-CONF-96-151-E

Do Top Analyses: Recent Results on the Properties of the Sixth Quark

Nuovo Cim. 109A:617, 1996; PISA-95 p. 617

Asymmetries in Charm Production in Fixed-Target Experiments

Nucl. Phys. A566:27C, 1994

Semiinclusive Hadron Spectra with Beams of Si and Au at the AGS

Phys. Lett. 407B:185, 1997; KEK-97-9

Measurement of the Proton-Antiproton Pair Production from Two-Photon Collisions at TRISTAN

ALBUQUERQUE-94. v. 1, p. 532; FERMILAB-CONF-94-209-E

Photon-Charm Production in $p \bar{p}$ Collisions at $\sqrt{s}=1.8 \mathrm{TeV}$

Phys. Rev. Lett. 80:928, 1998; CSNSM-97-21; NUCLEX-9712003

New Measurement and Analysis of the ${ }^{7} \mathrm{Be}(p, \gamma){ }^{8}$ Bor Cross Section

Phys. Lett. 436 B:158, 1998

Veriflcation Tests of the GALLEX Solar Neutrino Detector with ${ }^{71}$ Ge Produced In-Situ from the $\beta$-Decay of ${ }^{71} \mathrm{As}$

Nucl. Phys. (PS) B40:431, 1995; MONTREUX-94 p. 431

A Measurement of $\tau^{ \pm}$Polarization using the VENUS Detector at TRISTAN

Phys. Let t. 403B:155, 1997; KEK-96-171; KOBE-HEP-97-01; NGTHEP-97-01; OULNS-96-05; TMU-HEP-97-20; IIEPEX 9703003

Measurement of $\tau$ Polarization in $e^{+} e^{-}$Annihilation at $\sqrt{s}=58 \mathrm{GeV}$

Nucl. Phys. A577:173C, 1994

Isospin Splitting in Analog Giant Resonances Excited by Single Pion Charge Exchange Reactions

WILLIAMSBURG-94 p. 697

Measurement of the Transverse-Longitudinal Asymmetry in Inclusive Spin-Dependent Quasielastic Electron Scattering from Polarized ${ }^{3} \mathrm{He}$

Phys. Rev. Let t. 74:654, 1995

Transverse-Longitudinal Asymmetry in the Quasielastic ${ }^{3} \mathrm{He} \uparrow\left(e^{-\uparrow}, e^{-\prime}\right)$ Reaction

FERMILAB-CONF-94-088-E

Electroweak and Bottom Physics at the Tevatron Collider

Nucl. Phys. A577:57C, 1994

The $\left({ }^{3} \mathrm{He},{ }^{3} \mathrm{H} \bar{p}\right)$ Reactions: a Means to Study the Microscopic Structure of Spin-Isospin Modes

Phys. Rev. C56:20, 1997

Kinematically Complete Measurement of $p p \rightarrow p n \pi^{+}$near Threshold

DESY-94-023

Exotic Particle Searches, Photoproduction and Diffraction in DIS at HERA

Phys. Lett. 401B:176, 1997; HEPEX-9703002

Pseudorapidity Distribution of Charged Particles in $\bar{p} p$ Collisions at $\sqrt{s}=630 \mathrm{GeV}$

FERMLAB-CONF-94-101-E; CDF-PUB-JET-PUBLIC-2567

Photon production at $\mathrm{CDF}$ and $\mathrm{DO}$

A LBUQUERQUE-94, v. 2, p. 1146; FERMILAB-CONF-94-222-E; CDF-PUB-EXOTIC-PUBLIC-2714

Search for New Particles Decaying to Dijets in $p \bar{p}$ Collisions at $\sqrt{s}=1.8 \mathrm{TeV}$

FERMILAB-CONF-95-024-E

Search for Exotic Particles at CDF

FERMILAB-CONF-95-152-E; CDF-PUB-EXOTIC-PUBLIC-3192

Search for New Particles Decaying to Dijets, $b \bar{b}$, and $t \bar{t}$ at CDF

Phys. Lett. 401B:229, 1997

Two Neutral Pion Photoproduction off the Proton between Threshold and $800 \mathrm{MeV}$

Nucl. Phys. (PS) B40:463, 1995; MONTREUX-94 p. 463

$\tau$ Polarization Measurements at LEP using $\tau^{ \pm} \rightarrow \rho \nu$ and $\tau^{ \pm} \rightarrow a_{1} \nu$

Phys. Rev. Lett. 72:1322, 1994; Phys. Rev. Lett. 72:2821, 1994

Precise Measurement of the $\Lambda$ and $\bar{\Lambda}$ Masses and a Test of $C P T$ Invariance

Phys. Rev. C51:1982, 1995

${ }^{12} \mathrm{C}(\gamma, p){ }^{11}$ Bor Cross Section from 80 to $157 \mathrm{MeV}$

Phys. Rev. C57:123, 1998

Investigation of Multiparticle Final States in ${ }^{12} \mathrm{C}$ Photoreactions

Phys. Lett. 334B:215, 1994

Amplitudes for $\bar{p} p \rightarrow \pi \pi$ from 0.36 to $2.5 \mathrm{GeV} / c$

MANCHESTER-95 p. 334

Light Quark Resonances Formed in $p \bar{p}$ Annihilations in Flight at Fermilab 
Hasan $96 \mathrm{~B}$

Hasegawa 95

Hasegawa 95B

Hasegawa 96

Hasegawa 96B

Hashimoto 94

Hasinoff 94

Hatano 95

Hauger 94B

Hauger 96

Hauger 96B

Hauger $96 \mathrm{C}$

Hauger 97

Ilauger 98

Hauser 98

Hausser 95

Hawk 94

Hawker 98

Hayano 94

Hayano 94B

Hayashida 94

Hayashida 95

Hayashida 96

He 94

He 97

He 97B

Hedin 94

Heilbronn 98

Heimberg 96

Heinson 94

Heinson 96

Heltsley 95

Heltsley 97

Hemmick 94

Hempen 98

Herrick 95

Hess 98
Phys. Lett. 388B:376, 1996; BRU.PH-213

A Search for $f_{J}(2220)$ in $\bar{p} p \rightarrow \pi^{+} \pi^{-}$

Phys. Rev. Lett. 74:224, 1995; INS-1037

Core-Excited States of ${ }^{12} C_{s}$ Hypernuclei Formed in the $\left(\pi^{+}, K^{+}\right)$Reaction

Nucl. Phys. (PS) 39BC:121, 1995; SLAC-PUB-6647; TOHOKU-HEP-03

A Search for Jet Handedness in Hadronic $Z^{\circ}$ Decays

Phys. Rev. C53:1210, 1996

Spectroscopic Study of ${ }^{10} \mathrm{Bor}_{s},{ }^{12} \mathrm{C}_{s},{ }^{28} \mathrm{Si}_{s},{ }^{89} \mathrm{Yt}_{s},{ }^{139} \mathrm{La}_{s}$, and ${ }^{208} \mathrm{~Pb}_{s}$ by the $\left(\pi^{+}, K^{+}\right)$Reaction

Nucl. Phys. B474:141, 1996

A Study on Hadron Interactions through Observation of Cosmic-Ray Families of Visible Energy Greater than $500 \mathrm{TeV}$ in High Mountain Emulsion Chambers

Phys. Rev. C49:420, 1994

Projectile Rapidity Pions in $775 \mathrm{MeV} / \mathrm{Nucleon}{ }^{139} \mathrm{La}+{ }^{12} \mathrm{C}$ and ${ }^{139} \mathrm{La}+{ }^{139} \mathrm{La}$ Reactions

TRI-PP.94-73

Measurement of the Induced Pseudoscalar Coupling via Radiative Muon Capture on Hydrogen

Phys. Rev. D52:6219, 1995

Relative Abundance of ${ }^{3} \mathrm{He}$ and ${ }^{4} \mathrm{He}$ in Cosmic Rays near $10 \mathrm{GeV}$

FERMILAB-CONF-94-235-E

Properties of Jets in $Z^{0}$ Events from $1.8 \mathrm{TeV} \bar{p} p$ Collisions

Phys. Rev. Lett. 78:2535, 1996

The Energy Dependence of Flow in Ni Induced Collisions from $400 \mathrm{~A}$ to $1970 \mathrm{~A} \mathrm{MeV}$

Phys. Rev. Lett. 76:3911, 1996

In-Plane Retardation of Collective Expansion in $\mathrm{Au}+\mathrm{Au}$ Collisions

Phys. Rev. Lett. 77:235, 1996

Dynamics of the Multifragmentation of $1 \mathrm{~A} \cdot \mathrm{GeV}$ Gold on Carbon

Phys. Rev. Lett. 78:4165, 1997

Pion Flow and Antiflow in $1.15 \mathrm{~A} \mathrm{GeV} \mathrm{Au}+\mathrm{Au}$

Phys. Rev. C57:764, 1998

Multifragmentation of the Remnant Produced in the Reaction of $1 \mathrm{~A} \mathrm{GeV} \mathrm{Au}$ with C

Phys. Rev. C58:1869, 1998

New Precision Measurement of the Pionic Deuterium s-Wave Strong Interaction Parameters

Phys. Lett. 343B:36, 1995

Elastic Scattering of Polarized Protons from Polarized ${ }^{3} \mathrm{He}$

ALBUQUERQUE-94, v. 2, p. 1813; FERMILAB-CONF-94-226-E

A Study of Parton Distribution Functions with the Use of Photon + Jet Event Kinematics in $p \bar{p}$ Collisions at $\sqrt{s}=1.8 \mathrm{TeV}$

Phys. Rev. Lett. 80:3715, 1998; HEPEX-9803011

Measurements of the Light Anti-Quark Flavor Asymmetry in the Nucleon Sea

Nucl. Phys. A 566:439C, 1994

Rapidity Distributions of Antiprotons in Si $+\mathrm{A}$ and $\mathrm{Au}+\mathrm{A}$ Collisions

Phys. Rev. Lett. 73:1485, 1994; Phys. Rev. Lett. 73:3181, 1994; INS-1029

Laser Studies of the Decay Chain of Metastable Antiprotonic Helium Atoms

Phys. Rev. Lett. 73:3491, 1994; ICRR-324-94-19

Observation of a Very Energetic Cosmic Ray Well beyond the Predicted $2.7 \mathrm{~K}$ Cutoff in the Primary Energy Spectrum

Jour. of Phys. G 21:1101, 1995; ICRR-337-95-3

Muons $\geq 1 \mathrm{GeV}$ in Large Extensive Air Showers of Energies between $10^{16.5} \mathrm{eV}$ and $10^{19.5} \mathrm{eV}$ Observed

at Akeno
Phys. Rev. Lett. 77:1000, 1996

Possible Clustering of the Most Energetic Cosmic Rays within a Limited Source Angle Observed by the Akeno Giant Air Shower Array

Z. Phys. A348:105, 1994

Nuclear and Electromagnetic Fragmentation of $2.25 \mathrm{TeV}{ }^{197} \mathrm{Au}$ Nuclei

Nucl. Phys. A620:214, 1997

The Correlation Functions and Emission Time Scales for Light Particles and Light Fragments in ${ }^{40} \mathrm{Ar}$

${ }^{107} \mathrm{Au}$ Reactions at $25 \mathrm{MeV} / \mathrm{Nucleon}$

Phys. Rev. Lett. 79:3134, 1997

Search for a Dirac Magnetic Monopole in High Energy Nucleus-Nucleus Collisions

FERMILAB-CONF-94-415-E

$B$ Physics Results from Do

Phys. Rev. C58:3451, 1998

Neutron Yields from $435 \mathrm{MeV} /$ Nucleon $\mathrm{Nb}$ Stopping in $\mathrm{Nb}$ and $272 \mathrm{MeV} / \mathrm{Nucleon} \mathrm{Nb}$ Stopping in $\mathrm{Nb}$ and Al

Phys. Rev. Lett. 77:1012, 1996

Measurement of the $p \uparrow p \rightarrow$ deuteron $\pi^{+}$Cross Section and Analyzing Power at Threshold

Phys. Rev. D51:985, 1995; PRINCETON-HEP-94-15

Measurement of the Branching Ratio for the Rare Decay $K^{0} \rightarrow \mu^{+} \mu^{-}$

FERMILA B-CONF-96-009-E; UCR-D0-96-01; DO-NOTE-2830

Top Quark Physics at the Do Experiment

Nucl. Phys. (PS) B40:413, 1995; CLNS-94-1303; MONTREUX-94 p. 413

Hadronic Decay Modes of the Tau Lepton: a TAU 04 Review

Nucl. Phys. (PS) B55:79, 1997; ESTES PARK-96 p. 79

Experimental Tests of Lepton Universality in $\tau$ Decay from CLEO

Nucl. Phys. A566:435C, 1994

Low Pt Pion Enhancement in ${ }^{28} \mathrm{Si}+\mathrm{Pb}$ Collisions at $14.6 \mathrm{~A} \mathrm{GeV} / \mathrm{c}$

Phys. Rev. C57:484, 1998

Measurement and Calculation of the Polarization Transfer Parameter $K_{y}^{y^{\prime}}$ in $n$ deuteron Scattering at 15.0, $17.0,18.0$, and $25.8 \mathrm{MeV}$

Phys. Rev. C52:744, 1995

Elastic Scattering and Quasielastic Transfer for ${ }^{32} \mathrm{~S}{ }^{90} \mathrm{Mo},{ }^{100} \mathrm{Mo}$ at $E_{\mathrm{lab}}=180 \mathrm{MeV}$

ASTROPH-9812341

The Time Structure of Cerenkov Images Generated by TeV Gamma Rays and by Cosmic Rays 
Hessling 95

Heuring 95

Heuring 96

Hibou 98

Hibou 99

Hicks 97

Hiddemann 95

Hill 95

Hindi 94

Hirzebruch 95

Hlavacova 97

Hocker 94

Hof 95

Hof 96

Hoffmann 94

Hoffmann 98

Hoffmannroth 97

Hofmann 94

Hoft un 95

Holme 97

Holtrop 97

Holzmann 96

Holzmann 97

Honda $9 \bar{\imath}$

Hong 97

Horikawa 94

Horn 96

Hosoda 94

Hotta 98

Howell 98

Hsi 98

Hsiung 94

Htun 98

Hu 98

Huang 94

Huang 96

Huber 97

Hubler 95
Nucl. Phys. (PS) B39:128, 1995; MONTREUX-94 p. 128

Jets in Photoproduction at HERA

FERMILAB-CONF-95-161-E

Jet Correlation Studies as a Function of Rapidity Interval

FERMILAB-CONF-96-162-E; HEPEX-9607001

Jet Decorrelation and Jet Shapes at the Tevatron

Phys. Lett. 438B:41, 1998; IRES-98-05; NUCLEX-9802002 Comparison of $\eta$ and $\eta^{\prime}$ Production in the $p p \rightarrow p p \eta\left(\eta^{\prime}\right)$ Reactions Near Threshold

Phys. Rev. Lett. 83:492, 1999; IRES-99-07; NUCLEX-9903003

Near-Threshold Production of $\omega$ Mesons in the $p p \rightarrow p p \omega$ Reaction

Phys. Rev. C55:R12, 1997 Spin Asymmetries from ${ }^{16} \mathrm{O}\left(\gamma, p \pi^{-}\right)$Near $\Delta$ Resonance Energies

Jour. of Phys. G $21: 639,1995$

Limits on Neutrino Masses from the Tritium $\beta$ Spectrum

Phys. Rev. Let t. 75:2654, 1995

Alternative Analysis of the LSND Neutrino Oscillation Search Data on $\bar{\nu}_{\mu} \leftrightarrow \bar{\nu}_{e}$

Phys. Rev. C49:3289, 1994

Search for a $17 \mathrm{KeV}$ Neutrino in the Internal Bremsstrahlung Spectrum of ${ }^{125} \mathrm{I}$

Phys. Rev. C51:2085, 1995

Charge Changing Interactions of ${ }^{197} \mathrm{Au}$ at $10 \mathrm{GeV} / \mathrm{Nucleon}$ in Collisions with Targets from $\mathrm{H}$ to $\mathrm{Pb}$

Yad. Phys. 60:464, 1997; Phys. At. Nucl. 60:391, 1997; JINR-P1-96-122 Study of the deuteron $p \rightarrow p \pi^{+} n n$ Reaction

BONN-IB-94-17

Test of $C P$ Invariance in $Z^{0} \rightarrow \tau^{+} \tau^{-}$Decay and Determination of the Weak Dipole Moment of $\tau$ Leptons

ROME-95 p. 15; LNF-95-051-PD

Cosmic Ray Antiproton Measurements in the $4-19 \mathrm{GeV}$ Energy Range using the NMSU/WIZARD Matter Antimatter Superconducting Spectrometer 2 (MASS2)

Astrophys. Lett 467:L33, 1996; LNF-96-010-P

Measurement of Cosmic Ray Antiprotons from 3.7 to $19 \mathrm{GeV}$

Phys. Rev. C49:630, 1994

Spin Correlation Measurements for $p \nmid p \uparrow$ Elastic Scattering at $497.5 \mathrm{MeV}$

Phys. At. Nucl. 61:926, 1998; Yad. Phys. 61:1018, 1998 SUSY at LEP 2

Phys. Rev. Lett. 78:4697, 1997; DAPNIA-SPHN-97-03 Break-up and Coherent Photoproduction of $\eta$ Mesons on the Deuteron

Phys. Rev. C49:2555, 1994

Fission of Heavy Nuclei Induced by Stopped Antiprotons. I. Inclusive Characteristics of Fission Fragments FERMILAB-CONF-95-009-E

The Do Detector at Fermilab: Recent Results and Future Plans

Jour. of Phys. G 23:1851,1997

Production of Multi-Strange Particles in $\mathrm{Pb}-\mathrm{Pb}$ Interactions at $158 \mathrm{~A} \cdot \mathrm{GeV} / \mathrm{c}$

Phys. Rev. C58:3205, 1998; NUCLEX-9709003

A Measurement of the Interference Structure Function, $R_{L T}$, for the ${ }^{12} \mathrm{C}\left(e^{-}, e^{-1} p\right){ }^{11}$ Bor Reaction in the Quasielastic Region

Phys. Lett. 366B:63, 1996; Phys. Lett. 375B:359, 1996; GSI-PREPRINT-95-67 Pion Reabsorption in Heavy Ion Collisions Interpreted in Terms of the $\Delta$ Capture Process

Phys. Rev. C56:2920, 1997; GSI-97-46 Contribution of $\pi^{\circ}$ and $\eta$ Dalitz Decays to the Dilepton Invariant-Mass Spectrum in 1 A-GeV Heavy Ion Collisions

Phys. Rev. D56:3833, 1997 Characteristics of Muonic and Electromagnetic Components Far from the Core of Giant Air Showers above $10^{18} \mathrm{eV}$

Phys. Lett. 407B:115, 1997; NUCLEX-9706001 Abundance of $\Delta$ Resonances in ${ }^{58} \mathrm{Ni}+{ }^{58} \mathrm{Ni}$ Collisions between 1 and $2 \mathrm{~A} \cdot \mathrm{GeV}$

Nucl. Phys. A577:313C, 1994

The Present Status of the Nucleon Spin Structure Functions

Phys. Rev. Lett. 77:2408, 1996; TASCC-P-96-1; NUCLEX-9608004

Pionic Fusion of Heavy Ions

Phys. Lett. 331B:211, 1994; KEK-93-194

A Study of Single Photon Production in $e^{+} e^{-}$Annihilation at $\sqrt{s}=58 \mathrm{GeV}$

Nucl. Phys. A645:492, 1999; NUCLEX-9810016

Measurement of the ${ }^{6} \mathrm{Li}\left(e, e^{\prime} p\right)$ Reaction Cross Sections at Low Momentum Transfer

Phys. Lett. 444B:252, 1998

Toward a Resolution of the Neutron-Neutron Scattering-Length Issue

Phys. Rev. C58:13, 1998

Sideways Peaked Angular Distributions in hadron Induced Multifragmentation: Shock Waves, Geometry, or Kinematics?

FERMILAB-CONF-94-214-E

$C P$ Violation Measurements in Neutral Kaon System at Fermilab

Phys. Rev. C59:336, 1999; NUCLEX-9811013

Neutrons from Multiplicity-Selected La $\mathrm{La}$ and $\mathrm{Nb} \mathrm{Nb}$ Collisions at $400 \mathrm{~A} \cdot \mathrm{MeV}$ and La La Collisions at $250 \mathrm{~A} \cdot \mathrm{MeV}$

Phys. Rev. D58:111101, 1998; FERMILA B-PUB-98-216-E; HEPEX-9807025 Search for Muonic Decays of the Antiproton at the Fermilab Antiproton Accumulator

Phys. Rev. C49:314, 1994; LBL-34247

Mass and Transverse Momentum Dependence of Dielectron Production in $p$ deuteron and $p+p$ Collisions at $4.9 \mathrm{GeV}$

MANCHESTER-95 p. 518

Recent Progress on $\psi(2 S)$ and $J / \psi(1 S)$ Physics from BES Collaboration

Nucl. Phys. A624:623, 1997

Multinucleon Contributions to the ${ }^{12} \mathrm{C}\left(\pi^{+}, p p\right)$ Reaction at 100 and $165 \mathrm{MeV}$ Incident Pion Energies

Phys. Rev. D51:4005, 1995

Determination of the Gravitational Constant with a Lake Experiment: New Constraints for NonNewtonian Gravity 
Huehn 94

Huffman 96

Hughes 95

Hui 94

Hui 95

Hunger 97

Iazzi 96

Ichihara 94

Igarashi 95

Ignatovich 95

Imlay 98

Incandela 95

Incandela 95B

Irmscher 94

Isbert 94

Ishibashi 94

Ishida 95

Ishida 97

Ito 98

Itoh 94

Itoh 95

Itow 94

Ivanov 94

Ivanov 95

Ivanshin 94

Iwasaki 94

Iwasaki 97

Jackson 96

Jahnke 96

Jain 94

Jain $94 \mathrm{~B}$

Jain 95

Jain 96

Jakobs 94

Jakobsson 97

Jakobsson 97B

Jakobsson 98
ALBUQUERQUE-94, v. 1, p. 811; FERMILAB-CONF-94-319-E

Bottom-Quark Production from Muon-Jet and Dimuon Events in $p \bar{p}$ Interactions at $\sqrt{s}=1.8 \mathrm{TeV}$

FERMILAB-CONF-96-312-E; CDF-PUB-BOTTOM-PUBLIC-3858

Recent Beauty Results from CDF and the Run II Upgrades

CERN-PPE-95-178

Nucleon Spin Structure from Polarized Deep Inelastic Muon-Nucleon Scattering at CERN

Phys. Rev. C49:83, 1994

Pion Double Charge Exchange on $\mathrm{Se}_{\text {nat }}$

Phys. Rev. C51:3169, 1995

Pion Double Charge Exchange on the Even Selenium Isotopes

Nucl. Phys. A620:385, 1997

Compton Scattering by the Proton Through $\theta_{\mathrm{cms}}=75^{\circ}$ and $90^{\circ}$ in the $\Delta$ Resonance Region

MANCHESTER-95 p. 99

Meson Spectroscopy with Antineutrons

Nucl. Phys. A577:93C, 1994

Excitation of Spin-Dipole States by the ${ }^{12} \mathrm{C}\left({ }^{12} \mathrm{C},{ }^{12} \mathrm{Nit}\right){ }^{12}$ Bor Reaction at $E / A=135 \mathrm{MeV}$

Phys. Rev. C52:755, 1995

Photon Scattering from ${ }^{12} \mathrm{C}$ and ${ }^{4} \mathrm{He}$ Nuclei near the $\Delta\left(1232 P_{33}\right)$ Resonance

Jetp Lett. 62:1, 1995; Pisma Zh. Eksp. Teor. Fiz. 62:3, 1995

Additional Result on Lifetime of Neutron from Experiments on Storage of Ultracold Neutrons

Nucl. Phys. A629:531C, 1998; OSA KA-97 p. 531C

New Results on $\nu_{e}$ Carbon Scattering and $\nu_{\mu}$ Carbon Scattering at LSND

Nuovo Cim. 109A:741, 1996; FERMILAB-CONF-95-237-E; CDF-PUB-TOP-PUBLIC-3273

CDF Top Quark Production and Mass

FERMILAB-CONF-95-162-E; CDF-PUB-TOP-3209

Top Decay to Lepton + Jets: CDF $B$ Tags and Cross Section

Nucl. Phys. A 566:347C, 1994

Search for Direct Thermal Photons in the NA45/CERES Experiment

Nucl. Phys. A578:525, 1994; DAPNIA-SPHN-93-64

Two-Body Photodisintegration of ${ }^{3} \mathrm{He}$ between 200 and $800 \mathrm{MeV}$

KEK.PS 1990-1994 p. 283

Measurement of Neutron-Production Double-Differential Cross Sections for Incident Protons of $0.8,1.5$ and $3 \mathrm{GeV}$

MANCHESTER-95 p. 451; KEK-95-159

Study of the $\pi^{\circ} \pi^{\circ}$ System below $1 \mathrm{GeV}$ Region in the $p$ pentral Collision Reaction at $450 \mathrm{GeV} / \mathrm{c}$

Progr. of Theor. Phys. 98:621, 1997; KEK-97-118; NUP-A-97-16; HEPPH-9705437

Analysis of a $K \pi$-Scattering Phase Shift and Evidence for the $\kappa(900)$ Meson

Phys. Rev. C58:2366, 1998

Observation of $K^{-} p$ Atom $\gamma^{\prime} s$

Phys. Lett. 345B:335, 1995; KEK-94-162; NWU-HEP-94-07; DPNU-94-59; TIT-HPE-94-013; TUAT-HEP-94-07; OCUHEP-94-07; PU-94-692; INS-1077; KOBE-HEP-94-06

Measurement of Inclusive Particle Spectra and Test of MLLA Prediction in $e^{+} e^{-}$Annihilation at $\sqrt{s}=$ $58 \mathrm{GeV}$

Phys. Lett. 368B:299, 1996; KEK-95-179; NWU-HEP-95-03; DPNU-95-22; TUAT-HEP-95-01; PU-95-697; HEPEX9512010

Observation of Highly Virtual Photon-Photon Collisions to Hadrons at TRISTAN

Nuovo Cim. 107A:2415, 1994

Search for the $H$ Dibaryon by Scintillating Track Detector

Phys. At. Nucl. 58:1650, 1995; Yad. Phys. 58:1750, 1995; MINR-94-0871

Study of $\left(\gamma, p_{f}\right)$ Reaction on ${ }^{235} \mathrm{U},{ }^{238} \mathrm{U}$, and ${ }^{237} \mathrm{~Np}$ Nuclei with Tagged Photons in $60-240 \mathrm{MeV}$ Energy Range

Z. Phys. A352:191, 1995

Fission Probabilities of $\mathrm{Cu}, \mathrm{Nb}, \mathrm{Ag}, \mathrm{Ho}, \mathrm{Au}$ and Bi Nuclei Excited by Stopped Antiprotons

Nuovo Cim. 107A:2855, 1994; JINR-E1-93-155

Evidence for a New $0^{-} S$ Resonance in the Diffractively Produced $3 \pi$ System

Phys. Lett. 341B:99, 1994; KEK-94-109; NWU-HEP-94-05; TUAT-HEP-94-06; DPNU-94-41; TIT-HPE-94-08; OCU-HEP94-08; PU-94-687; INS-1063; KOBE-HEP-94-07

Measurement of Inclusive Electron Cross Section in $\gamma \gamma$ Collisions at TRISTAN

Phys. Rev. Lett. 78:3067, 1997; KEK-96-173

Observation of Kaonic Hydrogen $K_{\alpha} X$ Ray

MANCHESTER-95 p. 393

Preliminary Measurements of the $B^{0}$ and $B^{+}$Lifetimes at SLD

Phys. At. Nucl. 59:1567, 1996; Yad. Phys. 59:1625, 1996

Production and Decay of Hot Nuclei Following Antiproton Annihilation at Rest and in Flight

Phys. Rev. C50:1085, 1994

Intermediate Mass Fragment Emission by ${ }^{107}$ Au Projectiles at Relativistic Energy in Nuclear Emulsion

Phys. Rev. C49:3320, 1994

Collective Flow by the Azimuthal Correlation of Projectile Fragments in Relativistic Heavy-Ion Collisions

Phys. Rev. Lett. 74:1534,1995

Nuclear Collective Flow in ${ }^{197} \mathrm{Au}$ Emulsion Interactions at $10.6 \mathrm{~A} \mathrm{GeV}$

Phys. Lett. 366B:32, 1996; UB-HEX-95-05

Collective-Flow Correlations in ${ }^{187} \mathrm{Au}$ Emulsion Interactions at $10.6 \mathrm{~A} \mathrm{GeV}$

Int. Jour. Mod. Phys. A9:2903, 1994; M PI-PHE-94-04

The Physics Results of the UA2 Experiment at the CERN $p \bar{p}$ Collider

Phys. Rev. Lett. 78:3828, 1997

Gross and Fine Structure of Pion Production Excitation Functions in $p$ Nucleus and Nucleus Nucleus Reactions

Nucl. Phys. A626:55C, 1997

Pion Production Experiments with Slowly Ramped Beams at Storage Rings

Nucl. Phys. A630:184C, 1998; GATLINBURG-97 p. 184C

Excitation Function Experiments at Storage Rings with the Ultimate Goal to Probe the Liquid-Gas Phase Transition 
James 96

Janot 94

Janousch 97

Jansen 94

Jaques 96

Jaros 95

Jeckelmann 94

Jensen 94

Jeon 95

Jesik $9 \cdot 1$

Jessop 97

Jessop $97 \mathrm{~B}$

Jessop 98

Jilany 9.4

Jilany 95

Jin 96

Jipa 95

Joensson 94

Joffeminor 96

Johansson 98

Johansson $98 \mathrm{~B}$

Johns 94

Johnson 96

Jokinen 98

Jones 94

Jones $94 \mathrm{~B}$

Jones $94 \mathrm{C}$

Jones 94D

Jones $9 . \overline{5}$

Jonkmans 94

Jonkmans 96

Joram 95

Joram $95 \mathrm{~B}$

Jordan 94

Jordan 96
MANCHESTER-95 p. 106

Charm Physics from Fermilab E791

Nucl. Phys. (PS) B38:264, 1995; EILAT-94 p. 264; LAL-94-59

Searches for New Particles at LEP

Phys. Let t, 4148:237, 1997; ETHZ-IPP-PR-97-03 Destructive Interference of $s$ and $p$ Waves in $180^{\circ} \pi^{-} p$ Elastic Scattering

Phys. Rev. Lett. 74:3118, 1995; FER.IILAB-PUB-94-403; LA-UR-94-3812 Measurement of the Bottom-Quark Production Cross Section in $800 \mathrm{GeV} / \mathrm{c}$ Proton-Gold Collisions

PADUA-96 p. 221; FERMILAB-CONF-96-246-E

Jet Angular Decorrelation and Color Coherence in $p \bar{p}$ Collisions at $\sqrt{3}=1.8 \mathrm{TeV}$

SLAC-PUB-95-6810

A Search for Millicharged Particles at SLAC

Phys. Lett. 335 B:326, 1994; ETHZ-IPP-PR-94-9 The Mass of the Negative Pion

FERMILAB-CONF-94-339-E

Evidence for Top Quark Production in $1.8 \mathrm{TeV} p \bar{p}$ Collisions

Phys. Rev. Lett. 75:1443, 1995; Phys. Rev. Lett. 76:159, 1996 Search for Magnetic Monopoles Trapped in Matter

Phy's. Rev. Lett. 74:495, 1995; FERMILAB-PUB-94-095-E Bottom Production in $\pi^{-}$Be Collisions at $515 \mathrm{GeV} / c$

Phys. Rev. Let t. 79:4533, 1997; CLNS-96-1455; CLEO-96-24; HEPEX-9702013 Measurement of the Decay Amplitudes and Branching Fractions of $B \rightarrow J / \psi(1 S) K^{*}(892)$ and $B \rightarrow J / \psi(1 S)$ $K$ Decays

Phys. Rev. D58:052002, 1998; CLNS-97-1515; CLEO-97-22; HEPEX-9801010 Measurement of the Branching Ratios for the Decays of $D_{s}^{+} \rightarrow \eta \pi^{+}, \eta^{\prime} \pi^{+}, \eta^{+}$, and $\eta^{\prime} \rho^{+}$

Phys. Rev. Lett. 82:492, 1999; CLNS-98-1581; CLEO-98-13; IIEPEX-9810036 Observation of Two Narrow States Decaying into $\Xi_{c}(2460)^{+\gamma}$ and $\Xi_{c}(2460)^{0} \gamma$

Nucl. Phys. A579:627, 1994

Inelastic Interactions of $4.5 \mathrm{GeV} / c$ per Nucleon ${ }^{28} \mathrm{Si}$ with Emulsion Nuclei

Int. Jour. Mod. Phys. E4:815, 1995 Interactions of Relativistic ${ }^{24} \mathrm{Mg}$ Nuclei with Emulsion Nuclei at $4.5 \mathrm{GeV} / \mathrm{c}$ per Nucleon

MANCHESTER-95 p. 113

Study of $f_{J}(\mathbf{2 2 2 0})$ in $J / \psi(1 S)$ Radiative Decays and Glueball

Nuovo Cim. 108A:1271, 1995; Nuovo Cim. 109A:603, 1996

On the Participants from Projectile and Target Nuclei in Relativistic Nuclear Collisions

Nucl. Phys. A566:5C, 1994 First Measurement of Proton Structure Function $F_{2}$ at HERA

FERMILAB-CONF-96-272-EC Do Papers on QCD with Vector Bosons Submitted to DPF 96: a Measurement of the Ratio of $W^{ \pm}+1 J$ et $\rightarrow$ $W^{ \pm}+$oJets Cross Sections and Comparisons to QCD

Nucl. Phys. A641:389, 1998 Measurement of the Exclusive deuteron $\left(p, e^{+} e^{-}\right){ }^{3} \mathrm{He}$ and deuteron $(p, \gamma){ }^{3} \mathrm{He}$ Reactions at 98 and $176 \mathrm{MeV}$

Nucl. Phys. A631:331C, 1998; GRONINGEN-97 p. 331C

Experimental Studies of Threshold Meson Production at CELSIUS

FERIIILAB-CONF-94-365-E

Recent Results from Fermilab E687: Charm Particle Decays, Lifetimes and Photoproduction Dynamics: A Compilation of the Results Presented at DPF 1994

Phys. Rev. C54:2714, 1996 Observables in Muon Capture on ${ }^{23} \mathrm{Na}$ and the Effective Weak Couplings $\tilde{g_{a}}$ and $\tilde{g_{p}}$

Eur. Phys. J. A3:271, 1998; CERN-EP-98-201 $\beta$-Decay of the $M_{T}=-1$ Nucleus ${ }^{58} Z_{n}$ Studied by Selective Laser Ionization

CALT-68-1920

A Measurement of the Mass of the Tau Lepton

Nuovo Cim. $107 \mathrm{~A}: 2007,1994$

Exclusive Beauty States and their Lifetimes from the OPAL Experiment

Z. Phys. C62:575, 1994

Determination of the Parton Distributions and Structure Functions of the Proton from Neutrino and Antineutrino Reactions on Hydrogen and Deuterium

Z. Phys. C62:601, 1994

Determination of the Ratio $r_{v}=d_{v} / u_{v}$ of the Valence Quark Distributions in the Proton from Neutrino and Antineutrino Reactions on Hydrogen and Deuterium

Phys. Rev. C52:1520, 1995

Measurement of the Spin-Dependent Asymmetry in ${ }^{3} \mathrm{He}\left(e^{-}, e^{-\prime}\right)$ Inelastic Scattering at Low Energy Transfer.

TRI-PP-94-75

Radiative Muon Capture on the Proton

Phys. Rev. Lett. 77:4512, 1996

Radiative Muon Capture on Hydrogen and the Induced Pseudoscalar Coupling

Phys. Rev. C51:2144, 1995

Low-Energy Differential Cross-Sections of Pion Proton $\left(\pi^{ \pm} p\right)$ Scattering. I. The Isospin Even Forward Scattering Amplitude at $T_{\pi}=32.2$ and $44.6 \mathrm{MeV}$

Phys. Rev. C51:2159, 1995

Low-Energy Differential Cross-Sections of Pion Proton $\left(\pi^{ \pm} p\right)$ scattering. II. Phase Shifts at $T_{\pi}=32.7$, 45.1 , and $68.6 \mathrm{MeV}$

WILLIANSBURG-94 p. 776 deuteron $\left(e^{-}, e^{-} p\right)$ Longitudinal-Transverse Separation

Phys. Rev. Lett. 76:1579, 1996

Measurement of the Longitudinal, Transverse, and Longitudinal-Transverse Structure Functions in the deuteron $\left(e^{-}, e^{-} p\right) n$ Reaction 
Julien 94

Jun 96

Jung 94

Jung 95

Jungmann 98

Jungst 94

Justice 94

Justice 95

Justice 97

Kabana 97

Kagarlis 94

Kagarlis 98

Kalırimanis 97

Kaimi 97

Kalantarnaye 98

Kalmakhelid $\% 97$

Kalmykov 95

Kaloshin 94

Kamel 96

Kamon 94

Kamon 96

Kampert 94

Kampert 97

Ḱanda 95

Kaneta 97

Kaplan 96

Karchin 95

Karen 94

Karle 95

Karisson 96

Karnaukhov 93

Karnaukhov 95

Karnaukhov 95B

Karnaukhov $95 \mathrm{C}$

Karnaukhov 96

Karnaukhov 96C

Karnaukhov 98

Karnaukhov 98B

Kartasheva 96
Z. Phys. A347:181, 1994

Search for an Enhanced Production of Low Energy Pions in $\mathrm{C}\left(p, \pi^{+}\right) \mathrm{X}, \mathrm{Cu}\left(p, \pi^{+}\right) \mathrm{X}, \mathrm{and}_{\mathrm{Cu}}\left(p, \pi^{\circ}\right) \mathrm{X}$ Reactions for $300 \leq T_{p} \leq 400 \mathrm{MeV}$

FERMILAB-CONF-96-304-EH

DO Papers on QCD Studies with Jets: Azimuthal Decorrelation of Jets Widely Separated in Rapidity at DO

FERMILAB-CONF-94-334-E

$W^{ \pm}$Mass Measurement from Do and CDF Experiments at Tevatron

Nucl. Phys. (PS) B39:73, 1995; MONTREUX-94 p. 73

Physics at HERA

UHD-PI-MY-04-98; NUCLEX-9805004

Results from Searching for Muonium to Antimuonium Conversion

BONN-IR-95-19

Measurement of the Reactions $\gamma p \rightarrow K^{+} \Lambda$ and $\gamma p \rightarrow K^{+} \Sigma^{\circ}$ up to 2 GeV with SAPHIR at ELSA

Phys. Rev. C49:5, 1994

Electromagnetic Dissociation of ${ }^{238} \mathrm{U}$ at $120 \mathrm{MeV} / \mathrm{Nucleon}$

Nucl. Phys. A590:549C, 1995

Observation of Collective Effects in $\Lambda$ at $2 \mathrm{GeV} / \mathrm{Nucleon}$

Phys. Lett. 440B:12, 1998; NUCLEX-9708005

$\Lambda$ Flow in $2 \mathrm{~A} \mathrm{GeV} \mathrm{Ni} \mathrm{Cu} \mathrm{Collisions}$

Jour. of Phys. G 23:2135, 1997

Recent Results from Experiment NA52 at the CERN SPS

Phys. Rev. C43:1219, 1994

Double Charge Exchange in ${ }^{93} \mathrm{Nb}\left(\pi^{+}, \pi^{-}\right){ }^{93} \mathrm{Tc}$ at $T_{\pi}=164,230$ and $294 \mathrm{MeV}$

NUCLEX-9811010

Subthreshold $\rho^{0}$ Photoproduction on ${ }^{3} \mathrm{He}$

Phys. Rev. C55:2533, 1997; NUCLEX-9702003

Pion-Nucleus Elastic Scattering on ${ }^{12} \mathrm{C},{ }^{40} \mathrm{Ca},{ }^{00} \mathrm{Zr}$, and ${ }^{208} \mathrm{~Pb}$ at 400 and $500 \mathrm{MeV}$

Z. Phys. C75:619, 1997; CERN-PPE-97-007

Multiplicity Dependence of the Pion Source in $\mathrm{S}+A$ Collisions at the CERN SPS

Nucl. Phys. A631:242C, 1998; GRONINGEN-97 p. 242C Results of Recent Bremsstrahlung Measurements on Few-Body Systems at KVI

Izv. RAN, Fiz. 61:462, 1997

Investigation of Experimental $\gamma$-Families by the Methods of Factorial Moments and Fractal Dimensions

Phys. At. Nucl. 58:1563, 1995; Yad. Phys. 58:1657, 1995 Longitudinal Development of EAS using the Results of Cerenkov Light Study

Phys. At. Nucl. 57:2207, 1994; Yad. Phys. 57:2298, 1994; ISU-IAP-TH-94-01; HEP PH-9402220

First Estimates of the $(\alpha+\beta)^{\pi}$ from Two Photon Experiments

Phys. Lett. 368B:291, 1996

Direct Electron-Pair Production by $6.4 \mathrm{TeV}^{32} \mathrm{~S}$ Emulsion Interactions

FERMILAB-CONF-94-270-E

Search for SUSY at CDF

FERMILAB-CONF-96-106-E; CDF-PUB-EXOTIC-PUBLIC-3667; HEPEX-9605006

New Particle Searches at Tevatron (II)

IKP-MS-94-0101

Electromagnetic Probes of Hot and Dense Nuclear Matter

2. Phys. C74:587, 1997; HEPPH-9703440

Comment on Search for Direct Photons from S Au Collisions at $200 \mathrm{GeV} / \mathrm{u}$ by CERES Collaboration

Phys. Rev. D52:4872, 1995

Study of Two Particle Azimuthal Correlations in $e^{+} e^{-}$Annihilation at $\sqrt{s}=58 \mathrm{GeV}$

Jour. of Phys. G 23:1865, 1997

Particle Ratios from Central Pb+Pb Collisions at the CERN SPS

MANCHESTER-95 p. 405; IIT-HEP-95-5; HEPEX-9510002

Production of Charm, Charmonium, and Beauty in $800 \mathrm{GeV}$ Proton Nucleon Collisions

FERMILA B-CON F-95-053-E

Current Issues in Open Charm Hadroproduction and New Preliminary Results from Fermilab E768

ALBUQUERQUE-94,v. 1, p. 861; FERMILA B-CONF-94-216-E Measurement of the Polarization in the Decay $B^{0} \rightarrow J / \psi(1 S) K^{*}(892)^{\circ}$ in $p \bar{p}$ Collisions at $\sqrt{s}=1.8$ TeV

Pliys. Lett. $347 \mathrm{~B}: 161,1995$

Search for Isotropic $\gamma$ Radiation in the Cosmological Window between 65 and $200 \mathrm{TeV}$

Nuovo Cim. 109A:675, 1996; PISA-95 p. 675 A Review of $R_{b}$ at LEP

Phys. At. Nucl. 57:790, 1994; Yad. Phys. 57:841, 1994; JINR-93-121

About Existence of a Baryon with the $3.52 \mathrm{GeV} / \mathrm{c}^{2}$ Mass

JINR-P1-95-187

Evidence of Existence of the Strange Meson with Mass of $1627 \mathrm{MeV} / \mathrm{c}^{2}$

JINR-P1-95-293

Peculiarities of Mesonic Structure $K(1627) \rightarrow K_{S} \pi^{+} \pi^{-}$, Methods of their Observation

Phys. At. Nucl. 58:796, 1995; Yad. Phys. 58:860, 1995

Peculiarity of the Baryonic Structure with $3.52 \mathrm{GeV} / \mathrm{c}^{2}$ Mass

JINR-E1-96-50

"Thermal" Multifragmentation Induced in Gold Target by Relativistic Protons

JIN R-P 1-96-76

Processes with the Formation of Exotic Mesonic Structure K(1627)

Phys. At. Nucl. 61:203, 1998; Yad. Phys. 61:252, 1998

A Narrow Structure with $M \approx 1.63 \mathrm{GeV} / c^{2}$ in the Mass Spectrum of the $K_{S} \pi^{+} \pi^{-} \mathrm{System}$

JIN R-P1-98-169

Analysis of Exotic Structure $K^{*}(1630) \rightarrow K_{S} \pi^{+}$

IFVE-96-45

Preliminary Results of Data Handling at SIGMA-AYAKS Experiment on Search of Dibaryons with Separated Color in $\pi$ Be Interactions at Momentum $43 \mathrm{GeV} / c$

See the legend on page 5 . 
Kartasheva 97

Kartashera 97

Kasahara 97

Kass 97

Kaufman 94

Kaufman 94B

Kaufman $94 \mathrm{C}$

Kaufman 95

Kaufman 96

Kaufman 97

Kaufman 97B

Kaulard 98

Kawasaki 95

Kawasumi 96

Keith 94

Keith 96

Kelly 96

Keranen 96

Kermani 98

Kermani 98B

Kermani $98 \mathrm{C}$

Kernan 95

Kester 95

Kester 95B

Keup 94

Khan 95

Kiesling 94

Kikuchi 98

$\operatorname{Kim} 94$

Kim 95

Kim 95B

Kim $95 \mathrm{C}$

Kim 98

Kim 98B

Kimure 94

King 94

Kingler 98

Kinson 91
Yad. Phys. 62:686, 1999; Phys. At. Nucl. 62:638, 1999; IFVE-97-47 Search for Process of Double Color Charge Exchange of Pions with Momentum of $43 \mathrm{GeV} / \mathrm{c}$ on the Protons from Be Nucleus

Phys. Rev. D55:5282, 1997; ANL-HEP-PR-97-59; HEPEX-9612004 Study of Cosmic Ray Composition in the Knee Region using Multiple Muon Events in the Soudan 2 Detector

Nucl. Phys. (PS) B55:145, 1997; ESTES PARK-96 p. 145

Three Prong $\tau$ Decays with Charged Kaons in the Final State

Nucl. Phys. A566:269C, 1994

Recent Results from E850 using Si Beams at $14.6 \mathrm{~A} \mathrm{GeV} / c$

Nucl. Phys. A566:443C, 1994

Backward Emission of Protons in Au+Au Collisions at $11.7 \mathrm{~A} \mathrm{GeV} / \mathrm{c}$

Nucl. Phys. A566:457C, 1994

Kaon Production in $\mathrm{Si}+\mathrm{Al}$ and $\mathrm{Si}+\mathrm{Au}$ Collisions at $14.6 \mathrm{~A} \mathrm{GeV} / \mathrm{c}$

Nucl. Phys. A590:179C, 1995

Recent Results from Experiment 859 at the BNL AGS

Nucl. Phys. A610:213C, 1996

The Centrality Dependence of the Source Size for Au Au Collisions at the AGS

Phys. Rev. C55:2604, 1997

Baryon Emission at Target Rapidities in Si Al, Cu, Au Collisions at $14.6 \mathrm{~A} \cdot \mathrm{GeV} / \mathrm{c}$ and Au Au Collisions at $11.7 \mathrm{~A} \cdot \mathrm{GeV} / \mathrm{c}$

Phys. Rev. C56:1544, 1997

Two-Particle Rapidity Correlations from the Bose-Einstein Effect in Central ${ }^{28}$ Si Au Collisions at 14.6 $\mathrm{A} \cdot \mathrm{GeV} / \mathrm{c}$ and Intermittency

Phys. Lett. 422 B:334, 1998

Improved Limit on the Branching Ratio of $\mu^{-} \rightarrow e^{+}$Conversion on Titanium

Phys. Lett. 348 B:623, 1995

The Elastic $\bar{p} p$ Differential Cross Section Measurements at the CERN $S p \bar{p} S$ and the Real Part of the Scattering Amplitude

Phys. Rev. D53:3534, 1996

Simultaneous Observation of Families and Accompanied Air Showers at Mt. Chacaltaya

WILLIAMSBURG-94 p. 439

Measurement of the Polarized Neutron-Polarized ${ }^{3}$ He Total Cross Section

Phys. Rev. C54:477, 1996; NUCLEX-9607011 Measurements of the Total Cross Section for the Scattering of Polarized Neutrons from Polarized ${ }^{3}$ He

FERMILAB-CONF-96-236-EA Do Papers on Electroweak Physics Submitted to DPF 96: Study of the $W W \gamma$ and $W W Z^{\circ}$ Interactions at Do

Nuovo Cim. $109 \mathrm{~A}: 821,1996$; PISA-95 p. 821

Production of Onia in Hadronic $Z^{0}$ Decays

Phys. Rev. C58:3419, 1998

Exclusive Measurements of $\pi^{ \pm} p \rightarrow \pi^{+} \pi^{ \pm} n$ Near Threshold

Phys. Rev. C58:3431, 1998

$\pi \pi \rightarrow \pi \pi$ Cross Sections Near Threshold

DUBNA-96, v. I, p. 216

$\pi^{+} \pi^{-} \rightarrow \pi^{+} \pi^{-}$Cross Sections Near Threshold

Nucl. Phys. B437:243, 1995

Update Limits on the $\nu_{e}$ Mass and Large Angle Oscillations from SN1987A

Phys. Lett. 344B:79, 1995

Two Nucleon Knock-out Investigated with the SemiInclusive ${ }^{12} \mathrm{C}\left(e^{-}, e^{-\prime} p\right)$ Reaction

Phys. Rev. Lett. 74:1712, 1995

Short-Range Nucleon-Nucleon Correlations Investigated with the Reaction ${ }^{12} \mathrm{C}\left(e^{-}, e^{-1} p p\right)$

FERMILAB-CONF-94-282-E

A Measurement of the Mass of the $W \pm$ Vector Boson at CDF

Nuovo Cim. 108A:147, 1995

Study of Disintegrations Caused by $4.5 \mathrm{GeV} / \mathrm{c}$ Nucleon Carbon Nuclei in Nuclear Emulsion

DESY-94-137; MPI-PHE-94-20 Physics from the First Year of $\mathrm{H1}$ at HERA

Eur. Phys. J. A3:213, 1998 Further Measurement of the ${ }^{7} \operatorname{Be}(p, \gamma)^{8}$ Bor Cross Section at Low Energies with the Coulomb Dissociation of ${ }^{8}$ Bor

FERMILAB-CONF-94-153-E; CDF-PUB-ELECTROWEAK-PUBLIC-2632

The Measurement of the $W \pm$ Boson Mass from CDF

FERMILAB-CONF-95-240-E

Measurement of the $W \pm$ Boson Mass from CDF

FERMILAB-CONF-95-111-E

Observation of Top Quark Production using Kinematic Techniques

FERMILAB-CON F-95-276-E; CDF-PUB-TOP-PUBLIC-3302; D0-PUB-TOP-PUBLIC-2693

Observation of the Top Quark

Phy's. Rev. Lett. 81:3595, 1998; HEPEX-9808015

A measurement of $\alpha_{s}\left(Q^{2}\right)$ from the Gross-Llewellyn Smith Sum Rule

Phys. Lett. $420 \mathrm{~B}: 233,1998$

Measurement of $\alpha_{s}$ using NLLA $+O\left(\alpha_{s}^{2}\right)$ in $e^{+} e^{-}$Annihilation at $\sqrt{s}=58.0 \mathrm{GeV}$

Phys. Lett. 336B:303, 1994

Search for Parity Nonconservation in the Compound Nucleus Reaction via an Isobaric Analog Resonance using a Thick Target

ALBUQUERQUE-94, v. 1, p. 394; SLAC-PUB-6653

A Measurement of the Left-Right, Forward-Backward Asymmetry for Charm Quarks using $D^{*}(2010)^{+}$and $D^{*}(2010)^{-}$Mesons

Nucl. Phys. A634:325, 1998

Search for Very Light Lambda Hypernuclei in Proton Induced Reactions

Nucl. Phys. A544:321C, 1992; CERN-PRE-91-94; GATLINBURG-94 p. 321

Strange Particle Production in Sulphur Tungsten Interactions at $200 \mathrm{GeV} / \mathrm{c}$ per Nucleon 
Kirichenko 95

Kirk 94

Kirk 98

Kiselev 96

Kiselevich 94

Kiselevich 95

Kishimoto 94

Kishimoto 95

Kitching 97

Klima 95

Klima 95B

Klima 98

Klimenko 98

Klimenko 98B

Kloet 96

Kniehl 94

Knott 97

Kobayashi 91

Kobayashi 94

Kobel 96

Kodama 94

Kodama 95

Kodama 96

Kohler 94

Kojima 97

Kolata 98

Kolb 94

Kondashov 94

Kondashov 95

Konigsberg 94

Kopecky 95

Kopenkin 94

Kopenkin 94B

Kopp 94

Kopp 95

Koptev 95

Koreshev 96

Kormanyos 95

Kornmayer 95
Phys. At. Nucl. 58:10, 1995; Yad. Phys. 58:12, 1995

Energy and Angular Correlations in the $\gamma^{16} \mathrm{O} \rightarrow 4 \mathrm{He}$ Reaction

Nuovo Cim. 107A:1857, 1994; CERN-PPE-93-133

First Results from Experiment WA91 at the CERN OMEGA Spectrometer

Phys. At. Nucl. 62:398, 1999; Yad. Phys. 62:439, 1999; HEPEX-9803024 A Search for Non $q \bar{q}$ Mesons at the CERN OMEGA Spectrometer

ITEP-96-56

Subthreshold $\mathrm{K}^{+}$Mesons Production in $p$ Nucleus Collisions

Phys. At. Nucl. 57:2140, 1994; Yad. Phys. 57:2225, 1994

The Investigation of Inelastic Interactions of Pions with Ne Nuclei at $6.2 \mathrm{GeV} / c$ in Terms of Four-Velocity

Phys. At. Nucl. 58:419, 1995; Yad. Phys. 58:470, 1995; ITEP-93-96

Multiplicity of Secondary Particles in the $\pi^{-}$Ne Interactions at $6.2 \mathrm{GeV} / c$ with Strange Particles in the Final State

Nucl. Phys. A $577: 263 \mathrm{C}, 1994$

Spin Polarization and Weak Decay of Polarized $\Lambda$ Hypernuclei

Phys. Rev. C51:2233, 1995

$\Lambda$-Nucleon Interaction in Nuclei Probed by the Quasifree ${ }^{12} \mathrm{C}(\pi, K)$ Reaction

Phys. Rev. Lett. 79:4079, 1997; BNL-64628; PRINCETON-HEP-97-10; TRI-PP-97-26; KEK-97-113; HEPEX-9708011 Observation of the Decay $\mathrm{K}^{+} \rightarrow \pi^{+} \gamma \gamma$

FERMILAB-CONF-95-102-E

Observation of the Top Quark

FERMILAB-CONF-95-303-E

Top Physics at Do

FERMILAB-CONF-98-137-E; HEPEX-9804017

Do Findings on the Top Quark

Phys. At. Nucl. 61:1310, 1998; Yad. Phys. 61:1412, 1998

Double Beta Decay of ${ }^{86} \mathrm{Zr}$ to the Ground and Excited States of ${ }^{86} \mathrm{Mo}$

Phys. At. Nucl. 61:1129, 1998; Yad. Phys. 61:1229, 1998

Search for Annual and Daily Dark Matter Modulations with Ge Detectors at Baksan

Phys. Rev. D53:6120, 1996

Amplitude Analysis of the $\overline{\text { nucleon }}$ nucleon $\rightarrow \pi^{-} \pi^{+}$Reaction

DESY-94-136-B

Inclusive Photoproduction of Hadrons at HERA

Phys. Rev. C56:398, 1997

Interactions of Relativistic ${ }^{36} \mathrm{Ar}$ and ${ }^{40} \mathrm{Ar}$ Nuclei in Hydrogen: Isotopic Production Cross Sections

Nucl. Phys. A569:791, 1994; KEK-91-135

Energy Dependent Measurements of the $p$ p Analyzing Power and Narrow Dibaryon Resonances

Nucl. Phys. A586:457, 1995; KEK-94-4

Neutrinoless Double Beta Decay of ${ }^{160} \mathrm{Gd}$

MANCHESTER-95 p. 402

Charm Physics with the OPAL Detector

Phys. Lett. 336B:605, 1994

Measurement of $\Gamma\left(D^{0} \rightarrow K^{-} \mu^{+} \nu\right) / \Gamma\left(D^{0} \rightarrow \mu^{+} \mathrm{X}\right)$ using a $D^{*}$ Tag

Phys. Lett. 345B:85, 1995

Upper Limits for Charm Hadron Decays to Two Muons Plus Hadrons

Phys. Lett. 382B:299, 1996; DPNU-96-33; HEPEX-9606017

Measurement of $B\left(D_{s}^{+} \rightarrow \mu^{+} \nu_{\mu}\right) / B\left(D_{s}^{+} \rightarrow \phi(1020) \mu^{+} \nu_{\mu}\right)$ and Determination of the Decay Constant $f_{D_{s}}$

Phys. Rev. C49:1715, 1994; TRI-P P-93-81

Measurements of $i T_{11}$ in $\pi$ deuteron Elastic Scattering at $49 \mathrm{MeV}$

Phys. Lett. 400B:395, 1997; KEK-96-177

A Measurement of the Photon Structure Function $F_{2}^{\gamma}$ at $Q^{2}=6.8 \mathrm{GeV}^{2}$

Phys. Rev. Lett. 81:4580, 1998

Sub-barrier Fusion of ${ }^{8} \mathrm{He}$ with ${ }^{200} \mathrm{Bi}$

Phys. Rev. C49:2586, 1994

${ }^{3} \mathrm{He}(\gamma, p$ deuteron $)$ Cross Section with Tagged Photons below the $\Delta$ Resonance

Nuovo Cim. 107A:1903, 1994; Sov. Phys. Doklady 39:420, 1994; Doklady RAN 336:613, 1994

Partial-Wave Analysis of $\pi^{-} p \rightarrow \pi^{0} \pi^{0} n$ Reaction at $38 \mathrm{GeV} / c$ Momentum in the Mass Range up to $1.9 \mathrm{GeV}$

Phys. At. Nucl. 59:1624, 1996; Yad. Phys. 59:1680, 1996; IFVE-95-137

Further Study of the $\pi^{\circ} \pi^{\circ}$ System Produced in $38 \mathrm{GeV} / c \pi^{-} p$ Charge Exchange Collisions

ALBUQUERQUE-94, v. 1, p. 681; FERMILAB-CONF-94-314-E

Evidence for top top Production at the Tevatron: Statistical Signiflcance and Cross Section

Phys. Rev. Lett. 74:2427, 1995

New Measurement of the Charge Radius of the Neutron

Izv. RAN, Fiz. 58-12:13, 1994; Bull. Russ. Acad. Sci. Phys. Ser. 58:1960, 1994

Alignment of High Energy Fluxes in $\gamma$ hadron Families and Role of the Hadron Component

Phys. Rev. D52:2766, 1995; SU-ITP-94-25; HEPPH-9408247

Alignment in Gamma-Hadron Families of Cosmic Rays

FERMILAB-CONF-94-155-E; CDF-PUB-EXOTIC-PUBLIC-2634

Search for $W^{\prime \pm} \rightarrow e^{ \pm} \nu$ in $p \bar{p}$ Collisions at $\sqrt{s}=1800 \mathrm{GeV}$

Int. Jour. Mod. Phys. A10:4413, 1996; FERMILA B-PUB-95-264-E Properties of the $W^{ \pm}$Boson from the Fermilab Tevatron

Jetp Lett. 61:877, 1995; Pisma Zh. Eksp. Teor. Fiz. 61:865, 1995 Measurement of $\pi^{ \pm}$and $K^{ \pm}$Life-Time

Phys. Rev. Lett. 77:4294, 1996; FERMILAB-PUB-96-093-E

Production of Charmonium States in $\pi^{-}$Be Collisions at $515 \mathrm{GeV} / \mathrm{c}$

Phys. Rev. C51:669, 1995 Quasielastic $K^{+}$Scattering

Jour. of Phys. G 21:439, 1995

High-Energy Cosmic Ray Neutrons at Sea Level 
Korolko 95

Kudomi 98

Korolko 95

Korsheninnik 97

Kostin 96

Kotlinski 98

Kotte 95

Kotwal 94

Kotwal 94B

Kotwal 95

Kotwal 96

Kounine 95

Kounine $9 \overline{7}$

Kovacs 94

Kovacs 94B

Kowitt 94

Kozlov 98

Kramer 94

Krane 96

Krasny 93B

Kraus 97

Kraus 98

Krawczynski 96

Krcmar 98B

Kretschmer 94

Kretschmer 94B

Kreutz 95

Kriss 99

Kriznic 94

Krolak 94

Krusche 95

Krusche 95B

Krusche 95C

Krusche 97

Kruse 96

Kubota 94

Kubota 95

Kubota 96

Kubota $96 \mathrm{~B}$

Kudomi $9 \dot{8}$
Nucl. Phys. (PS) B40:275, 1995

Determination of the Michel Parameter $\eta$ from the Muon Spectrum

Nucl. Phys. A617:45, 1997

Scattering of Radioactive Nuclei ${ }^{6} \mathrm{He}$ and $H_{3}$ by Protons: Effects of Neutron Skin and Halo in ${ }^{8} \mathrm{He},{ }^{8} \mathrm{He}$, and ${ }^{11} \mathrm{Li}$

MANCHESTER-95 p. 411

Inclusive Resonance Production in $p p$ and $\bar{p} p$ Interactions at $32 \mathrm{GeV} / c$

Eur. Phys. J. A 1:435, 1998; PSI-PR-98-01

Initial State Interaction in the $\left(\pi^{+}, 3 p\right)$ Reaction on $\mathrm{Nit}, \mathrm{Ar}$ and $\mathrm{Xe}$

Phys. Rev. C51:2686, 1995

Interplay of Collective Flow Phenomena and Velocity Correlations of Intermediate-Mass Fragments in Collisions of $\mathrm{Au} \mathrm{Au}$ at $E=(100-400) \mathrm{A} \mathrm{MeV}$

FERMILAB-CONF-94-251-E

Structure Functions and Structure Function Ratio $F_{2}^{(n)} / F_{2}^{(p)}$ at Low $x_{B j}$ and $Q^{2}$ in Inelastic Muon Scattering

FERMILAB-CONF-94-345-E

Recent Results from E685

FERMILAB-CONF-95-046-E

Proton and Deuteron Structure Functions in Muon Scattering at $470 \mathrm{GeV}$

FERMILAB-CONF-96-236-EE

Do Papers on Electroweak Physics Submitted to DPF 96: Measurement of the $W^{t}$ Boson Mass

Nucl. Phys. (PS) B40:437, 1995

Neutral Couplings of the $\tau^{ \pm}$Lepton

Nucl. Phys. (PS) B55:49, 1997; ESTES PARK-96 p. 49

Polarization Measurement using Leptonic $\tau^{ \pm}$Decays

ALBUQUERQUE-94,v.2, p. 1653; FERMILAB-CONF-94-215-E; CDF-PUB-JET-PUBLIC-2726

Testing QCD with Jet Physics at CDF

FERMILAB-CONF-94-144-E

Measurement of the SS - OS Dijet Cross Section Ratio

Phys. Rev. Lett. 72:1318, 1994; LBL-34790; FERMILAB-PUB-93-344

Production of $J / \psi(1 S)$ at Large $x_{F}$ in $800 \mathrm{GeV} p$ Copper and $p$ Beryllium Collisions

Phys. At. Nucl. 61:1268, 1998; Yad. Phys. 61:1370, 1998; HEPEX-9811020

Modern Status of Neutrino Experiments at the Underground Neutrino Laboratory of Kurchatov Institute Near Krasnoyarsk Nuclear Reactor

DESY-94-136-A

Direct Photon Production at LEP and HERA

FERMILAB-CONF-96-304-EB

Do Papers on QCD Studies with Jets: The Inclusive Jet Cross Section at $\sqrt{s}=630 \mathrm{GeV}$ at Do

Nuovo Cim. 107A:2109, 1994; LPNHE-93-09

Results from the H1 Experiment at HERA

Phys. Rev. Let t. 79:3834, 1997

Beam Asymmetry of Coherent $\pi^{0}$ Photoproduction on ${ }^{4} \mathrm{He}$ in the $\Delta(1232)$ Range Measured using Coherent Bremsstrahlung

Phys. Lett. 432B:45, 1998

Angular and Polarization Dependence of Compton Scattering from ${ }^{4} \mathrm{He}$ in the $\Delta$-Resonance Region

ASTROPH-9611044

Search for TeV Counterparts of Gamma-Ray Bursts with the HEGRA Experiment

Phys. Lett. 442B:38, 1998; IRB-ZEF-98-01; NUCLEX-9801005

Search for Solar Axions using ${ }^{57} \mathrm{Fe}$

Phys. Lett. 328B:5, 1994

Measurement of Polarization Transfer in p-p Scattering for a Test of Nucleon-Nucleon Potentials

Nucl. Phys. A577:421C, 1994

Neutrino-Nucleus Reactions with KARMEN

Z. Phys. C65:67, 1995

Determination of the Branching Ratios $\Gamma\left(K_{L} \rightarrow 3 \pi^{0}\right) / \Gamma\left(K_{L} \rightarrow \pi^{+} \pi^{-} \pi^{0}\right)$ and $\Gamma\left(K_{L} \rightarrow 3 \pi^{0}\right) / \Gamma\left(K_{L} \rightarrow \pi\right.$ $e^{-} \nu_{e}$ )

Phys. Rev. C59:1480, 1999

Pion-Proton Integral Cross Sections at $T_{\pi}=40$ to $284 \mathrm{MeV}$

Nuovo Cim. 107 A:2359, 1994

Recent Two-Photon Results from ARGUS

Phys. Lett. 320 B:407, 1994

A Limit on the Lepton Family Number Violating Process $\pi^{0} \rightarrow \mu^{ \pm} e^{-+}$

Phys. Rev. Lett. 74:3736, 1995

Near Threshold Photoproduction of $\eta$ Mesons off the Proton

Phys. Lett. 358B:40, 1995; Phys. Lett. 376B:331, 1996

Near Threshold Photoproduction of $\eta$ Mesons from the Deuteron

Z. Phys. A351:237, 1995

Mass and $\Gamma_{3 \pi_{0}} / \Gamma_{\gamma \gamma}$ Decay Branching Ratio of the $\eta$ Meson from the $p(\gamma, \eta) p$ Reaction

Phy's. Lett. 397B:171, 1997

Constraining $N\left(1535 S_{11}\right)$ Parameters from the $\eta$ Photoproduction

FERMILAB-CONF-96-238-E; CDF-DOC-TOP-CDFR-3825

Production and Decays of Top Quark Pairs in the Single Lepton and Dilepton Channels at CDF

Phy's. Rev. Lett. 72:1972, 1994; CLNS-94-1266; CLEO-94-1

Observation of a New Charmed Strange Meson

Phy's. Rev. D54:2994, 1996; CLNS-95-1363; CLEO-95-18

Measurements of the Inclusive Semielectronic $D^{\circ}$ Branching Fraction

Phy's. Rev. D53:6033, 1996; CLNS-95-1327; CLEO-95-4

The Inclusive Decay $B \rightarrow \eta \mathrm{X}$

Nucl. Phys. A602:327, 1996

Search for Neutron-Rich $\Lambda$ Hypernuclei in the $\left(K_{\text {stopped }}^{-}, \pi^{+}\right)$Reaction

Nucl. Phys. A629:527C, 1998; OSAKA-97 p. 527C

Double $\beta$-Decays and ELEGANT V and VI at Oto Cosmo Observatory 
Kuehn 94

Kugler 94

Kuhlen 94

Kuhlmann 94

Kuhlmann 94B

Kuhn 94

Kulakov 95

Kulakov 97

Kulakov 98

Kulessa 98

Kulessa 98B

Kulik 94

Kume 94

Kunde 95

Kunde 97

Kuno 94

Kuplennikov 94

Kuplennikov 95

Kuzichev 92

Kuzichev 93

Kuznetsov 94

Kuznetsov 95

Kuznetsov 96

Kuznetsov 97

Kuznetsov 97B

Kuznetsov 97C

Kwiatkowski 95

Kwiatkowski 98

Kwiatkowski $98 \mathrm{~B}$

Laasanen 96

Labarga 94

Lach 94

Ladygin 97

Laforest 99

Lamanna 94

Lamanna 96
Phys. Lett. 334 B:298, 1994

The Measurement of the Polarization Transfer Coefficient in the (deuteron $\uparrow, p \uparrow$ ) Reaction at a Fixed Proton Momentum4.5 GeV/c and a Deuteron Momentum Range 6.0 - $8.0 \mathrm{GeV} / \mathrm{c}$

Phys. Lett. 335B:319, 1994

Emission of Nucleons and Light Fragments Relative to the Reaction Plane in $\mathrm{Bi}+\mathrm{Pb}$ Collisions at $1 \mathrm{GeV} / u$

DESY-94-191; MPI-PHE-94-23

New Experimental Results at HERA

FERMILAB-CONF-94-148-E; CDF-PUB-JET-PUBLIC-2605

Precision Measurement of the Prompt Photon Cross Section in $p \bar{p}$ Collision at $\sqrt{s}=1.8 \mathrm{TeV}$

FERMILAB-CONF-94-014-E

Direct Photon Plus Charm Quark Production at CDF

Nucl. Phys. A569:175C, 1994

Relativistic Coulomb Excitation of the 2 Phonon GDR in ${ }^{208} \mathrm{~Pb}$

Rad. Meas. 25:261, 1995

Investigation of Neutron Emission from Relativistic Heavy Ion Interactions by Nuclear Tracks

Kr. Soob. JINR 84:45, 1997

Cluster and Single-Particle Distributions in Nucleus-Nucleus Interactions

JINR-E1-98-51

On Different Experimental Behaviour of Fast Secondary Particles Produced in ${ }^{12} \mathrm{C}$ Interactions at Relativistic Energies as Studied with Radiochemistry and in a Propane Chamber

Phys. Lett. 427B:403, 1998

Production of Heavy Hypernuclei in the $p$ Be Reaction and Determination of their Lifetime for Fission Induced by $\Lambda$ Decay

Nucl. Phys. A639:283C, 1998; UPTON-97 p. 283C

Measurement of the Lifetime of Heavy Hypernuclei Produced in the Bombardment of Bi with Protons

Nuovo Cim. 107A:2435, 1994

Observation of the $X(1740) \rightarrow K_{S} K_{S}$ Decay

Nucl. Phys. A577:405C, 1994

Double Beta Decays of ${ }^{110} \mathrm{Cd}$

Phys. Rev. Lett. 74:38, 1995; GSI-94-46

Fragment Flow and the Multifragmentation Phase Space

Phys. Rev. C55:990, 1997

Azimuthal $2 \mathrm{He}$ Correlations and Projectile-Residue Distributions Selected by $\boldsymbol{n}$ and charged-Particle Multiplicity Measurements

Nucl. Phys. (PS) 37A:87, 1994; KEK-94-10 Search for Time Reversal Violation in $K^{+}$Decays

Phys. At. Nucl. 57:721, 1994; Yad. Phys. 57:771, 1994

Examination of the Cross Sections ${ }^{4} \mathrm{He}\left(e^{-}, e^{-t}\right)$ Reaction in the Quasifree, Dip and $\Delta\left(1232 P_{33}\right)$ Resonance Region

Phys. At. Nucl. 58:891, 1995; Yad. Phys. 58:963, 1995

Pion-Electroproduction Peak in the Energy Spectrum of the Reaction ${ }^{4} \mathrm{He}\left(e^{-}, e^{-1}\right)$

Yad. Phys. 57:1910, 1994; Phys. At. Nucl. 57:1840, 1994; ITEP-92-46

The Antiproton-Nuclei Annihilation Cross Section at the Momentum Range from $0.70 \mathrm{GeV} / \mathrm{c}$ to 2.50 $\mathrm{GeV} / \mathrm{c}$

Nucl. Phys. A576:581, 1994; ITEP-93-94

The Antiproton-Nuclei Annihilation Cross Section at the Momentum Range from 0.70 to $2.50 \mathrm{GeV} / \mathrm{c}$

Pisma Zh. Eksp. Teor. Fiz. 60:311, 1994; Jetp Lett. 60:315, 1994

New Measurement of the Antineutrino Spin Asymmetry in Beta Decay of the Neutron and Restriction for Mass of $W_{R}$

Phys. Rev. Lett. 75:794, 1995

Measurements of the Antineutrino Spin Asymmetry in Beta Decay of the Neutron and Restrictions on the Mass of a Right-Handed Gauge Boson

Kr. Soob. JINR 75:51, 1996

Investigation of Processes of Total Disintegration of Nuclei in Nucleus-Nucleus Collisions with Impulse $4.2 \mathrm{GeV} / \mathrm{c}$

Kr. Soob. JINR 84:57, 1997

The Coulomb Interaction of Charged Pions in $\mathrm{C} \mathrm{C}$ - and $\mathrm{C}$ Ta-Collisions at $4.2 \mathrm{~A} \cdot \mathrm{GeV} / \mathrm{c}$

JIN R-E1-97-178

On the Possibility of Experimental Investigation of Extreme States of Nuclear Matter. The Invariant Inclusive Spectra of Secondary Particles Production in $\pi^{-}, p$, deuteron, ${ }^{4} \mathrm{He},{ }^{12} \mathrm{C}+\mathrm{C}$ Interactions

Phys. Rev. C58:351, 1998; JINR-E1-97-342 Study of Single-Particle Correlation in Events with the Total Disintegration of Nuclei

Phys. Rev. Lett. 74:3756, 1995; CEA-DAPNIA-SPHN-94-54 Multifragmentation in the $4.8 \mathrm{GeV}{ }^{3} \mathrm{He}+\mathrm{Ag}_{\text {nat }},{ }^{197} \mathrm{Au}$ Reactions

Nucl. Phys. A630:168C, 1998; GATLINBURG-97 p. 168C

Multifragmentation: Thermal vs. Dynamic Effects

Phys. Lett. 423B:21, 1998

Heating Nuclear Matter with $\mathrm{GeV}{ }^{3} \mathrm{He}$ Beams

FERMILAB-CONF-96-198-E; CDF-PUB-BOTTOM-PUBLIC-3759

Measurement of the $B$ Meson Differential Cross Section in $p \bar{p}$ Collisions at $\sqrt{s}=1.8 \mathrm{TeV}$

Nuovo Cim. 107 A:2123, 1994

Results from ZEUS on Deep Inelastic Scattering and Photoproduction at HERA

FERMILAB-CONF-94-031

Hyperon Polarization, Crystal Channeling and E781 at Fermilab

Nucl. Instr. and Meth. A404:129, 1998; DAPNIA-SPHN-97-51

Analyzing Powers for the Inclusive Reaction of Deuterons on Carbon at Energies between 0.175 and $1.6 \mathrm{GeV}$

Phys. Rev. C59:2567, 1999

Dependence of Projectile Fragmentation on Target $N / Z$

Nucl. Phys. B434:479, 1995; CERN-PPE-94-137

A High-Statistics Measurement of the $\bar{p} p \rightarrow \bar{n} n$ Charge-Exchange Reaction at $875 \mathrm{MeV} / c$

Phys. At. Nucl. 59:1344, 1996; Yad. Phys. 59:1403, 1996

nucleon nucleon $\rightarrow$ nucleon nucleon: a 1995 Update

See the legend on page 5 . 
Lamberti 95

Lambrecht 94

Lamoureux 95

Land berg 96

Landsberg 89

Landsberg $94 \mathrm{~B}$

Landsberg $94 \mathrm{C}$

Landsberg 95

Landsberg 96

Lange 98

Laue 99

Lauret 98

Laymon 96

Leber 96

Lecolley 95

Lecolley 96

Lecolley 97

Lecompte 94

Lecompte 96

Ledoux 98

Lee 94

Lee 95

Legoff 94

Legoff 97

Legrain 97

Legrand 97

Lehmann 97

Lehmann $97 \mathrm{~B}$

Lehmann 98

Leinberger 96

Leinberger 97

Leitch 94

Leitch 95

Lenz 98

Leone 94

Leone 96

Leone 96B

Leone $96 \mathrm{C}$

Lepekhin $94 \mathrm{~B}$
Nucl. Phys. (PS) B39:41, 1995; MONTREUX-94 p. 41

Exclusive Vector Meson Production at HERA

Z. Phys. A350:115, 1994

Energy Dependence of Collective Flow of Neutrons and Protons in ${ }^{187} \mathrm{Au}+{ }^{187} \mathrm{Au}$ Collisions

FERMILAB-CONF-95-174-E; CDF-PUB-JET-PUBLIC-3186

Photon Production at CDF and Do

Phys. Rev. D53:2839, 1996

Test of the OZI Rule in Hadroproduction of $\phi(1020) \phi(1020)$ and $\phi(1020) K^{+} K^{-}$

Yad. Phys. 57:47, 1994; Phys. At. Nucl. 57:42, 1994; IFVE-89-54

Exotic Hadrons

Nuovo Cim. 107A:2441, 1994; Phys. At. Nucl. 57:1954, 1994; Yad. Phys. 57:2030, 1994; IFVE-94-7

Search for Exotic Baryons with the SPHINX Facility

Usp. Fiz. Nauk 164:1129, 1994

Exotic Baryons

FERMILAB-CONF-95-061-E

Search for Anomalous $Z^{0} Z^{0} \gamma$ and $Z^{0} \gamma \gamma$ Couplings with Do

FERMILAB-CONF-96-236-EB

DO Papers on Electroweak Physics Submitted to DPF 98: Recent $D^{\circ}$ Results of $Z^{\circ} \gamma$ Production

Phys. Rev. Lett. 80:1597, 1998

Determination of the $\pi^{ \pm} p \rightarrow \pi^{ \pm} \pi^{+} n$ Cross Section near Threshold

Phys. Rev. Let t. 82:1640, 1999; NUCLEX-9901005

Medium Effects in Kaon and Antikaon Production in Nuclear Collisions at Subthreshold Beam Energies

Phys. Rev. C57:1051, 1998

Dynamics of Radial Collective Energy in Near Central Collisions for $1 \mathrm{~A} \mathrm{GeV} \mathrm{Au} \mathrm{C}$

Phys. Rev. C53:1167, 1996

Pion Elastic and Inelastic ${ }^{2}(1)+$ Scattering on ${ }^{58} \mathrm{Ni},{ }^{60} \mathrm{Ni},{ }^{62} \mathrm{Ni},{ }^{64} \mathrm{Ni}$ at $T_{\pi}=180 \mathrm{MeV}$

Phys. Lett. 369B:69, 1996

Measurement of the Decay Rate of the Radiative $K_{e 3}^{0}$ Decay

Nucl. Phys. A583:379C, 1995; CEA-DAPNIA-SPHN-94-38; LPCC-94-07

Kaon Production in Nucleus-Nucleus Collisions at $92 \mathrm{MeV}$ per Nucleon

LPCC-96-01

Reaction Mechanism in Highly Fragmented $\mathrm{Pb}+\mathrm{Au}$ Collisions at $2 \theta \mathrm{MeV} / \mathrm{u}$

Nucl. Phys. A620:327, 1997

Highest Temperatures Sustainable in Heavy Nuclei Produced in Ar Au Collisions at $60 \mathrm{~A} \mathrm{MeV}$

FERMILAB-CONF-94-134-E; ILL-P-94-05-037

Measurement of the $B$ Cross Section at $C D F$ via $B$ Semileptonic Decays

FERMILAB-CONF-96-021-E; ANL-HEP-CP-96-04; CDF-PUB-TOP-PUBLIC-3465

Top Decay Physics at CDF and Measurement of the CKM Element $V_{t b}$

Phys. Rev. C57:2375, 1998

Formation and Decay of Hot Nuclei in $475 \mathrm{MeV}, 2 \mathrm{GeV} p$ and $2 \mathrm{GeV}{ }^{3} \mathrm{He}-$ Induced Reactions on $\mathrm{Ag}, \mathrm{Bi}$, A $u$ and $U$

Phys. Lett. 323B:227, 1994

Spin-Parity Analysis of the $f_{1}(1285) \pi^{-}$System in the Reaction $\pi^{-} p \rightarrow f_{1}(1285) \pi^{-} p$ at $18 \mathrm{GeV} / \mathrm{c}$

Phys. Rev. C51:2770, 1995

Experimental Search for a $17 \mathrm{keV}$ Neutrino in the Internal Bremsstrahlung Spectrum of ${ }^{71} \mathrm{Ge}$

Phys. Rev. C50:2278, 1994

Short Range Interaction of Nucleons Inside the Nucleus via ${ }^{4} \mathrm{He}\left(e^{-}, e^{-t} p\right)$ Reactions

Phys. Rev. C55:1600, 1997

Anomaly in the Transverse Longitudinal Ratio for $\mathrm{He}\left(e^{-}, e^{-} p\right) \mathrm{X}$ Reaction at $260 \mathrm{MeV} / c$ Recoil Momentum

Phys. Rev. C59:1464, 1999; DAPNIA-SPHN-97-46

Kaon Production in Nucleus Nucleus Collisions at $92 \mathrm{MeV}$ per Nucleon

Nucl. Phys. A625:307, 1997; NUCLEX-9704005

$\mathrm{K}^{+}$Production in the Reaction ${ }^{58} \mathrm{Ni}{ }^{58} \mathrm{Ni}$ at Incident Energies from 1 to $2 \mathrm{~A} \mathrm{GeV}$

Phys. Rev. C55:2931, 1997; PSI-PR-97-05

Pion Absorption on ${ }^{3} \mathrm{He}$ and ${ }^{4} \mathrm{He}$ with Emission of Three Energetic Protons

Phys. Rev. C56:1872, 1997; PSI-PR-97-16

Multinucleon Pion Absorption on ${ }^{4} \mathrm{He}$ into the $p p p n$ Final State

PSI-PR-98-30

Total Cross Sections of the Charge Exchange Reaction $\left(\pi^{+}, \pi^{0}\right)$ on deuteron, ${ }^{3} \mathrm{He}$, and ${ }^{4} \mathrm{He}$ across the $\Delta(1232)$ Resonance

Phys. Lett. 394B:16, 1997; UCY-PHY-96-13; NUCLEX-9610001

New Results on $e^{+} e^{-}$Line Emission in $U+$ Ta Collisions

Eur. Phys. J. A1:249, 1998; NUCLEX-9704007

First Energy and Angle Differential Measurements of $e^{+} e^{-}$Pairs Emitted by Internal Pair Conversion of Excited Heavy Nuclei

Phys. Rev. Lett. 72:2542, 1994; FERMILAB-PUB-94-012-E

Nuclear Dependence of Neutral $D$-Meson Production by $800 \mathrm{GeV} / \mathrm{c}$ Protons

Phy.s. Rev. D52:4251, 1995; FERMILAB-PUB-95-047-E

Nuclear Dependence of $J / \psi(1 S)$ Production by $800 \mathrm{GeV} / \mathrm{c}$ Protons Near $x_{F}=0$

Phy's. Lett. 416B:50, 1998

A New Determination of the Mass of the Charged Pion

INFN-PI-AE-94-007

Search for the Top Quark at CDF in Events with Two Charged Leptons, Neutrinos and Hadronic Jets

MANCHESTER-95 p. 135; FERMILAB-CONF-95-332-E

Kinematics of Top Decays from CDF

PADUA-96 p. 435; FERMILAB-CONF-96-195-E; HEPEX-9608002

New Observations of Top at CDF

FERMILAB-CONF-96-207-E

Top Quark Results at CDF

Pisma Zh. Eksp. Teor. Fiz. 59:312, 1994; Jetp Let.t. 59:332, 1994

Some Peculiarities of Transverse Momentum Distribution of Relativistic ${ }^{\circ} \mathrm{Li}$ Fragments in Photoemulsion 
Lepekhin 95

Lepekhin 98

Levine 97

Levy 95

Lewis 94

Lewis $94 \mathrm{~B}$

Lewis 96

Li 95

Liang 97

Liaud 97

Lidemarteau 94

Lindner 98

Lips 94

Lips 94B

Lips 94C

Lisa 95

Lissauer 94

Lissauer 94B

Lissauer $94 \mathrm{C}$

Litvintsev 94

Liu 93

Liu 96

Liu 98

Llope 95

Loginov 98

Lohmann 97

Lohr 95

Lolos 96

Lolos 98

Long 98

Longacre 93

Longacre 95

Longley 95

Loomis 96

Loomis $96 \mathrm{~B}$

Lopatin 94

Lorstad 97

Losecco 93
Yad. Phys. 58:881, 1995; Phys. At. Nucl. 58:816, 1995

Fragmentation of ${ }^{\circ} \mathrm{Li}$ Nuclei with a Momentum of $4.5 \mathrm{GeV} / \mathrm{c}$ per Nucleon in Photoemulsion

Eur. Phys. J. A1:137, 1998

Yields and Transverse Momenta of the ${ }^{8} \mathrm{Li}$ Fragments in the Emulsion at $4.5 \mathrm{GeV} / \mathrm{c}$ per Nucleon

Phys. Rev. Lett. 78:424, 1997

Measurement of the Electromagnetic Coupling at Large Momentum Transfer

DESY-95-003; HEPPH-9501346

The Energy Behaviour of Real and Virtual Photon Photon Cross Sections

FERMILAB-CONF-94-128-E; CDF-PUB-BOTTOM-PUBLIC-2603

Mass and Lifetime Measurements with Exclusive $B$ Reconstruction at CDF

FERMILAB-CONF-94-274-E

Measurement of the $b$ Cross Section at CDF via $b$ Semileptonic Decays

FERMILAB-CONF-96-415-E; CDF-PUB-BOTTOM-PUBLIC-3921

Fully Reconstructed $B$ Decay Results from CDF

Phys. Lett. 355B:394, 1995

A Determination of $\alpha_{S}$ in $e^{+} e^{-}$Annihilation at $\sqrt{s}=57.3 \mathrm{GeV}$

Phys. Lett. 411B:244, 1997

${ }_{12} \mathrm{C}\left(\gamma, \pi^{+} n\right)$ and ${ }^{12} \mathrm{C}\left(\gamma, \pi^{+} p\right)$ Reactions across the $\Delta$ Resonance Region

Nucl. Phys. A612:53, 1997

The Measurement of the $\beta$ Asymmetry in the Decay of Polarizes Neutrons

FERMILAB-CONF-94-332

Status of the DO Top Search

JERUSALEM-97 p. 1007; HEPPH-9801311

New Results of the HEGRA Air Shower Detector Complex

Nucl. Phys. A583:585C, 1995; HIRSCHEGG-94 p. 21

Multifragmentation Induced by Relativistic $\alpha$ Projectiles

Phys. Lett. 338B:141, 1994

Evidence for Simultaneous Breakup in Reactions with Relativistic $\alpha$-Projectile

Phys. Rev. Lett. 72:1604, 1994; JULICH-94 p. 476

Multifragmentation Induced by Relativistic ${ }^{4} \mathrm{He}$-Projectiles

Phys. Rev. Lett. 75:2662, 1995; LBL-35504

Radial Flow in Au Au Collisions at $\mathrm{E}=(0.25-1.15) \mathrm{A} \cdot \mathrm{GeV}$

Nucl. Phys. A566:451C, 1994

Low $p_{T}$ Photon Production in Proton-Nucleus Collisions at $18 \mathrm{GeV} / \mathrm{c}$

Phys. Rev. C50:1077, 1994

Production of Light Nuclei in Relativistic Heavy-Ion Collisions

Phys. Rev. C50:3047, 1994

Centrality Dependence of Longitudinal and Transverse Baryon Distributions in Ultrarelativistic Nuclear Collisions

Phys. At. Nucl. 57:1542, 1994; Yad. Phys. 57:1616, 1994

Recent ARGUS Results on Charmed Hadrons Production

Phys. Rev. D49:4339, 1994; KEK-93-147

Measurements of Cross-Section and Asymmetry for $e^{+} e^{-} \rightarrow b \bar{b}$ and Heavy Quark Fragmentation at KEK TRISTAN

Phys. Rev. C53:1711, 1996

Test of a Density-Dependent Interaction using in-Plane ${ }^{28} \mathrm{Si}(p, p)^{28} \mathrm{Si}$ Polarization Transfer Measurements

Nucl. Phys. A630:549C, 1998; GATLINBURG-97 p. 549C

Collective Flow and Particle Spectra in Relativistic Heavy Ion Collisions

Phys. Rev. C52:1900, 1995

The Sphericity of Central Heavy-Ion Reactions

Pisma Zh. Eksp. Teor. Fiz. 67:730, 1998; Jetp Lett. 67:770, 1998

Study of the reaction deuteron $\left(e^{-}, p p\right) e^{-t} \pi^{-}$on Tensor Polarized Deuterium Target at Large Proton Momentum

Nucl. Phys. (PS) B55:101, 1997; ESTES PARK-96 p. 101

Measurement of the Branching Ratios of the $\tau$ into Leptons with the L3 Detector at LEP

DESY-95-042

Results from the ZEUS Experiment at HERA

Phys. Rev. C54:211, 1996

Role of Quasideuteron Absorption in the ${ }^{6} \mathrm{Li}\left(\pi^{+}, p p\right)$ Reaction at $T_{\star}+=100,165 \mathrm{MeV}$

Phys. Rev. Lett. 80:241, 1998

Evidence for $\rho^{0}$ Mass Modiflcation in the ${ }^{3} \mathrm{He}\left(\gamma, \rho^{\circ}\right) p p n$ Reaction

Phys. Rev. C57:3191, 1998

Spin Isovector Giant Resonances Induced by $(n, p)$ Reactions on Heavy Nuclei

Nucl. Phys. A566:167C, 1994; BNL-49446

Results from E810 Concerning Strange Particles and Strangelet Search

Nucl. Phys. A590:477C, 1995

$H^{\mathrm{o}}$ Candidates from the Decay $\mathrm{H}^{\mathrm{o}} \rightarrow \Sigma^{-}$, Observed in Heavy Ion Collisions with 14.6 A GeV/c Si Beam on $\mathrm{Pb}$ Target

Phys. Rev. D52:2760, 1995; ANL-HEP-PR-94-45

Ultrahigh Energy Cosmic Ray Composition from Surface Air Shower and Underground Muon Measure-

ments at Soudan 2
FERMILAB-CONF-96-179-E; CDF-PUB-EXOTIC-PUBLIC-3744

Charged Higgs and SUSY Searches at CDF

FERMILAB-CONF-96-232-E

Search for Charged Higgs Bosons in CDF

Nucl. Phys. A567:882, 1994

Measurement of the Spin Rotation Parameters $R$ and $A$ in $\pi p$ Elastic Scattering and a Test of $\pi n$ Partial-Wave Analyses in the Region of Low-Lying $P_{11}, S_{11}$ and $D_{13}$ Resonances

Kr. Soob. JIN R 81:95, 1997

Analysis of Transverse Mass Dependence of Bose-Einstein Correlation Radii using the Delphi Data

Astroph. Jour. 425:217, 1994; UNDPDK-93-02

Search for Gamma Ray Burst Correlation with Neutrinos 
Losecco 95

Losecco 98

Lott 97

Lou 96

Lourenco 93

Lourenco 94

Lourenco 95

Loverre 96

Lowe 94

Lowe 96

Lowe 98

Lubinski 94

Lubinski 98

Lucchesi 96

Lucherini 94

Ludlam 94

Ludovici 96

Luescher 98

Luke 93

Lyakhno 96

Lynen 98

Lys 96

Lyuboshitz 95

$\mathrm{Na} 97$

Maccormick 97

Macgibbon 95

Macgregor 98

Macher 98

Machner 96

Mack 95

Mackenzie 96

Maeda 94

Maeno 95

Maeshima 94

Maeshima 96

Maghakian 94

Maity 95

Makins 94

Makoff 93
Phys. Lett. 342 B:392, 1995

Limits on Cold Dark Matter from Underground Neutrino

UND-PDK-98-05; UNDPDK-98-05; HEPEX-9811008

Bounds on $\nu_{\mu}$ Oscillations from Atmospheric Neutrinos

GANIL-P-97-37

Neutron Multiplicity Distributions for $200 \mathrm{MeV}$ Proton-, Deuteron- and ${ }^{4}$ He-induced Spallation Reactions on Thick $\mathrm{Pb}$ Targets

Nucl. Phys. A604:219, 1996

Fragment Emission from Modestly Excited Nuclear Systems

Nucl. Phys. A566:77C, 1994; LIP-PHYS-93-02

$J / \psi(1 S), \psi(2 S)$ and Muon Pair Production in $p-W t$ and $S-U$ Collisions

LIP-PHYS-94-01

Dimuon Production in $p-\mathrm{Wt}, p-\mathrm{U}$ and $\mathrm{S}-\mathrm{U}$ Collisions at $200 \mathrm{GeV} / \mathrm{Nucleon}$

CERN-PPE-95-72; LIP-95-03

Recent Results on Dimuon Production from the NA38 Experiment

Phys. Lett. 370B:156, 1996

Limits on $\nu_{\mu}$ Oscillations from the Measurement of the Ratio of $0 \mu^{ \pm}$to $1 \mu^{ \pm}$Events at the CERN Narrow Band Neutrino Beam

Nuovo Cim. 107 A:2183,1994

Results from SMC on the Deuteron Spin Structure Function

Phys. Rev. C54:R15, 1996

Strangelet Search and Light Nucleus Production in Relativistic Si Pt and Au Pt Collisions

Nucl. Phys. A639:371C, 1998; UPTON-97 p. 371C

$H$-Dibaryon Search via the $\left(K^{-}, K^{+}\right)$Reaction using a Diamond Target (BNL-E885)

Phys. Rev. Lett. 73:3199, 1994

Neutral Halo in Heavy Nuclei from Antiproton Absorption

Phys. Rev. C57:2962, 1998

Composition of the Nuclear Periphery from $\bar{p}$ Absorption

MANCHESTER-95 p. 142; FERMILAB-CONF-95-321-E

CDF Results on $B$ Lifetimes and Mixing

Nuovo Cim. 107A:2271, 1994

The $\phi(1020) \pi^{+} / \omega \pi^{+}$Ratio from $\bar{n} p$ Annihilations

Nucl. Phys. A566:183C, 1994

Particle Spectra and Correlations from Experiment 814

Nuovo Cim. 109A:651, 1996; PISA-95 p. 651

Heavy-Flavour Production Asymmetry

Phys. Lett. 434B:407, 1998

Search for $\beta \beta$ Decay in ${ }^{136} \mathrm{Xe}$ : New Results from the Gotthard Experiment

Phys. Lett. 321B:88, 1994; UCSD-PTH-93-25; UTPT-93-21; CMU-HEP-93-13; DOE-ER-40682-37 Extracting $\left|V_{b c}\right|, m_{c}$ and $m_{b}$ from Inclusive $D$ and $B$ Decays

Phys. At. Nucl. 59:14, 1996; Yad. Phys. 59:18,1996

Measurement of Angular Dependence of the Asymmetry of the Cross Section for ${ }^{4} \mathrm{He}(\gamma, p)^{3} \mathrm{H}$ and ${ }^{4} \mathrm{He}(\gamma$, n) ${ }^{3} \mathrm{He}$ Reactions Induced by 40,60 and $80 \mathrm{MeV}$ Linearly Polarized Photons

Nucl. Phys. A630:176C, 1998; GATLINBURG-97 p. 176C

Present Status of the Caloric Curve of Nuclei

FERMILAB-CONF-96-409-E

Top Nass Measurements at CDF

Kr. Soob. JINR 74:109,1995

Determination of the Sizes of the Pion Emission Region in $n p$ Interaction at $P_{n}=(5.2 \pm 0.16) \mathrm{GeV} / c \mathrm{using}$ the Interference Correlation Methods for Identical Particles

Phys. Rev. C55:588, 1997

Measurement of $p$ (deuteron, $\gamma){ }^{3} \mathrm{He}$ and deuteron $(p, \gamma){ }^{3} \mathrm{He}$ at Very Low Energies

Phys. Rev. C55:1033, 1997 Total Photoabsorption Cross Section for ${ }^{4} \mathrm{He}$ from 200 to $800 \mathrm{MeV}$

Phys. Rev. C52:2097, 1995

Measurement of the Electric and Magnetic Polarizabilities of the Proton

Phys. Rev. Lett. 80:245, 1998

Mechanisms in the ${ }^{12} \mathrm{C}(\gamma, p n)$ and $(\gamma, p p)$ Reactions

DUBNA-96, v. II, p. 101

Pion Production Close to Threshold with the GEM Detector at COSY

Phys. At. Nucl. 59:1606, 1996; Yad. Phys. 59:1663,1996

The COSY Accelerator and First Pion Production Studies with the GEM Detector System

Phys. Rev. C52:291, 1995

Proton Scattering by ${ }^{206} \mathrm{~Pb},{ }^{207} \mathrm{~Pb},{ }^{208} \mathrm{~Pb}$ at $650 \mathrm{MeV}$ : Phenomenological Analysis

Phys. Rev. C54:R6, 1996

Quasifree $\pi^{+}$Production Studied using the ${ }^{12} \mathrm{C}\left(\gamma, \pi^{+} n\right)^{11}$ Bor Reaction in the $\Delta$ (1232) Resonance Region

Nucl. Phys. A577:277C, 1994

Quasi-Free $K^{+}$Photoproduction in ${ }^{12} \mathrm{C}$

Phys. Lett. 351B:574, 1995

A Search for Massive Neutral Bosons in Orthopositronium Decay

ALBUQUERQUE-94, v. 2, p. 1141; FERMILAB-CONF-94-227-E; CDF-PUB-EXOTIC-PUBLIC-2722

Search for $Z^{\prime}$ and $W^{\prime} \pm$ at $\mathrm{CDF}$

FERMILAB-CONF-96-412-E

New particle searches at CDF

ALBUQUERQUE-94,v. 2, p. 1810; FERMILAB-PUB-94-239-E

A Precision Measurement of the Prompt Photon Cross Section in $p \bar{p}$ Collisions at $\sqrt{s}=1.8$ TeV

Z. Phys. C65:75, 1995

Clan Structure and Intermittency in Proton-Nucleus Interactions at High Energy

Phys. Rev. Lett. 72:1986, 1994

Momentum Transfer Dependence of Nuclear Transparency from Quasielastic ${ }^{12} \mathbf{C}\left(e^{-}, e^{-\prime} p\right) R^{2 a c t i o n}$

Phys. Rev. Lett. 75:2069, 1995; Phys. Rev. Lett. 70:1591, 1993

Study of the Decay $K_{L} \rightarrow \pi^{ \pm} \pi^{0} e^{\mp} \bar{\nu}(\nu)$ 
Malik 95

Malinin 96

Mallot 94

Malvezzi 96

Manly 94

Mar 96

Mardor 97

Mardor 98

Margetis 95

Marie 98

Markaryan 95

Markiewicz 95

Markosky 95

Marques 96

Marti 95

Martirena 94

Martoff 96

Maruyama 96

Masera 95

Masoni 94

Mass 95

Matorras 95

Matsunaga 98

Matsuoka 95

Matthews 94

Matthews 95

Matthews 95 B

Mauceli 97

Maur 94

May 97

Mazzoni 94

Mcaninch 94

Mecarthy 95

Mccarthy 96

Mcfarland 95

Mcfarland 96

Mcfarland 97

Mcgaughey 94

Mcgeorge 95
Z. Phys. C67:239, 1995

Negative Binomial Distributions in Different Pseudorapidity Windows in 800 GeV Proton Nucleon Interactions

MANCHESTER-95 p. 349

Neutral Hadron Production from $Z^{0}$ Decay

CERN-PPE-94-06

Deep Inelastic Lepton Scattering

Nuovo Cim. 109A:727, 1996; PISA-95 p. 727

Charm Lifetime

SLAC-PUB-6668

A Preliminary Measurement of the Average $B$ Hadron Lifetime

Phys. Rev. D53:6017, 1996; SLAC-PUB-7046

An Improved Search for Elementary Particles with Fractional Electric Charge

Phys. Lett. 437B:257, 1998; NUCLEX-9710002

Measurement of Quasi-Elastic ${ }^{12} \mathrm{C}(p, 2 p)$ Scattering at High Momentum Transfer

Phys. Rev. Lett. 81:5085, 1998

Nuclear Transparency in Large Momentum Transfer Quasielastic Scattering

Nucl. Phys. A590:355C, 1995

First Results from $\mathrm{NA49}$ on $\mathrm{Pb}+\mathrm{Pb}$ Collisions at $158 \mathrm{GeV} / \mathrm{Nucleon}$

Phys. Rev. C58:256, 1998; GANIL-P-98-13

Experimental Determination of Fragment Excitation Energies in Multifragmentation Events

YERE-1431(1)-95

Source of Intermediate-Mass Fragment Emission, $4 \leq Z_{f} \leq 10$, in the Interactions of $3 \mathrm{GeV}$ Electrons with ${ }^{207}$ Au Nuclei

Nucl. Phys. (PS) 39BC:141, 1995; SLAC.PUB-6659

$A$ Test of the Flavor Independence of Strong Interactions

FERMILAB-CONF-95-137-E

Measurement of Heavy Quark Production at Do

Phys. Lett. 381B:407, 1996

Neutrons from the Breakup of ${ }^{18} \mathrm{C}$

Nucl. Phys. (PS) B39:131, 1995; MONTREUX-94 p. 131

Differences between Quark and Gluon Jets in DELPHI at LEP

SLAC-439

A Calorimetric Measurement of the Strong Coupling Constant in Electron-Positron Annihilation at a Center-of-Mass Energy of $91.6 \mathrm{GeV}$

Phys. Rev. C54:2767, 1996

Reaction ${ }^{13} \mathrm{C}(n, p){ }^{13}$ Bor at $118 \mathrm{MeV}$

Phys. Lett. 393B:295, 1997; INS-1180

A Step-Like Rise in the ${ }^{4} \mathrm{He}(\gamma, p n)$, deuteron Cross Section Near the Pion Production Threshold

Nucl. Phys. A590:93C, 1995

Dimuon Production below Mass $3.1 \mathrm{GeV} / \mathrm{c}^{2}$ in $p \mathrm{Wt}$ and $\mathrm{S} \mathrm{Wt}$ Interactions at $200 \mathrm{GeV} / \mathrm{c} / \mathrm{A}$

Nuovo Cim. 107A:2279, 1994

Meson Spectroscopy with $S$ - and $P$-Wave-Dominant Initial-State Selection in $\bar{p} p$ Annihilation

BONN-IR-95-07

Evidence for $J / \psi(1 S)$ Mesons with the Help of Neural Networks in Photoproduction Events at HERA

Nucl. Phys. (PS) B40:487, 1995; MONTREUX-94 p. 487

$\tau^{ \pm}$Polarization Measurements using Inclusive Methods

Phys. Rev. Lett. 81:4052, 1998; RESCEU-20-98; UT-ICEPP-98-02; ASTROPH-9809326

Measurement of Low-Energy Cosmic Ray Antiprotons at Solar Minimum

Phys. Lett. 359B:39, 1995

Search for Deeply-Bound Pionic States using ( $p,{ }^{2} \mathrm{He}$ ) Reactions at $390.6 \mathrm{MeV}$

WILLIAMSBURG-94 p. 538

Elastic Scattering of Pions from ${ }^{3} \mathrm{H}$ and ${ }^{3} \mathrm{He}$ into the Backwards Hemisphere

Phys. Rev. C51:2534, 1995

Elastic Scattering of Pions from ${ }^{3} \mathrm{H}$ and ${ }^{3} \mathrm{He}$ into the Backward Hemisphere

Phys. Rev. Lett. 75:2803, 1995

New Measurement of the $C P$ Violation Parameter $\eta_{+-\gamma}$

Phys. Rev. D56:6081, 1997

Search for Gravitational Radiation from Supernova 1983J

BONN-IR-94-06

A Study of Production of $\Lambda$ Baryons and the Correlative Production of Strange Particles with the OPAL Detector in the Range of the $Z^{0}$ Pole

Phys. Rev. Lett. 78:4343, 1997

First Observation of the $p_{\Lambda} \rightarrow s_{\Lambda} \gamma$-Ray Transition in ${ }_{\Lambda}^{13} \mathrm{C}$

Nucl. Phys. A566:95C, 1994

Dimuon and Vector-Meson Production in $p \mathrm{Wt}$ and $\mathrm{S} W \mathrm{t}$ Interactions at $200 \mathrm{GeV} / \mathrm{c} / \mathrm{Nucleon}$

Phys. Rev. C50:589, 1994

Analyzing Power in Neutron-Deuteron Elastic Scattering at $E_{1 \mathrm{ab}}=3 \mathrm{MeV}$

Nucl. Phys. (PS) B39:13, 1995; MONTREUX-94 p. 13

Test of Parton Distribution Functions at the Tevatron Collider

MANCHESTER-95 p. 149

A Test of Soft QCD at Do

Phys. Rev. Lett. 75:3993, 1995; FERMILA B-PUB-95-153

A Limit on $\nu_{\mu}\left(\bar{\nu}_{\mu}\right) \rightarrow \nu_{\tau}\left(\bar{\nu}_{\tau}\right)$ Oscillations from a Precision Measurement of Neutrino-Nucleon Neutral Current Interactions

FERMILAB-CONF-96-227-E

Updated Electroweak Measurements from Neutrino - Nucleon Deeply Inelastic Scattering at CCFR

Eur. Phys. J. C1:509, 1998; FERMILAB-PUB-97-001-E; HEPEX-9701010

A Precision Measurement of Electroweak Parameters in Neutrino-Nucleon Scattering

Phys. Rev. D50:3038, 1994; FERMILAB-PUB-94-038-E

Cross Sections for the Production of High-Mass Muon Pairs from $800 \mathrm{GeV}$ Proton Bombardment of ${ }^{2} \mathrm{H}$

Phys. Rev. C51:1967, 1995

$(\gamma, 2 n)$ Reaction in ${ }^{12} \mathrm{C}$

See the legend on page 5 . 
Mckinzie 94

Medinatanco 98

Meier 94

Melese 94

Melese 95

Melese 96

Melese $96 \mathrm{~B}$

Melese $96 \mathrm{C}$

Menichetti 94

Menze 94

Menzione 95

Mercer 94

Mercer 97

Mertz 99

Meschi 96

Meshcheryako 97

Messchendorp 98

Meyer 98

Meyer 98B

Meyerhoff 94

Miao 96

Michael 96

Michotte 96

Mielke 94

Mikhailichen 98

Mikhajlichen 97

Milbrath 97

Miller 9.5

Miller 98

Minaenko 94

Minowa 98

Mirazita 97

Mishra 94

Miskowiec 94

Miskowiec 95

Mitchell 94

Mitchell 96
Phys. Rev, C49:2054, 1994

Interference Effects in Non-analog Pion Double Charge Exchange

ASTROPH-9810366

The Energy Spectrum Observed by the AGASA Experiment and the Spatial Distribution of the Sources of Ultrahigh-Energy Cosmic Rays

Phys. Rev. C49:320, 1994

Vector Analyzing Power Measurement of Pion Scattering from Polarized ${ }^{7} \mathrm{Li}$ in the Region of the $\triangle\left(1232 P_{33}\right)$ Resonance

FERMILA B-CONF-94-271-E

Search for Diffractive $W^{ \pm \prime} s$ in CDF

FERMILAB-CONF-95-109-E

Probing Pomeron Structure at Fermilab

PADUA-96 p. 360; FERMILAB-CONF-96-205-E; RU-96-E-24; CDF-PUB-EXOTIC-PUBLIC-3788

Search for Centauro Events at CDF

PADUA-96 p. 622; WARSAW-96 p. 693; FERMILAB-CONF-96-197-E; RU-96-E-22; CDF-PUB-MIN-BIAS-PUBLIC-3770 Hard Diffraction at CDF

FERMILAB-CONF-96-231-E; CDF-PUB-MIN-BIAS-PUBLIC-3812; RU-96-E-30

Diffractive Dijet Search with Roman Pots at CDF

Nuovo Cim. 107A:2013, 1994

New Results from E760. Study of Charmonium States at the Fermilab Accumulator

WILLIAMSBURG-94 p. 713

Measurement of $\gamma p \rightarrow K^{+} \Lambda$ and $\gamma p \rightarrow K^{+} \Sigma^{0}$ at Proton Energies up to $2.1 \mathrm{GeV}$

FERMILAB-CON F-95-340-E

Top Quark Physics

Phys. Rev. C49:3104, 1994

Multipole Decomposition of the ${ }^{16} \mathrm{O}(p, n){ }^{16} \mathrm{Fl}$ and ${ }^{18} \mathrm{O}(p, n){ }^{18} \mathrm{Fl}$ Reactions at $494 \mathrm{MeV}$

Phys. Rev. C55:946, 1997

Suggested Correction to ${ }^{6} \mathrm{He},{ }^{6} \mathrm{Li},{ }^{7} \mathrm{Li}$ and ${ }^{7} \mathrm{Be}$ Production Cross Sections in He He Reactions between 60 and $180 \mathrm{MeV}$

NUCLEX-9902012

Search for Quadrupole Strength in the Electro-Excitation of the $\Delta\left(1232 P_{33}\right)^{+}$

Nucl. Phys. (PS) 50:77, 1996; STRASBOURG-95 p. 77; FERMILAB-CONF-96-013-E; CDF-PUB-BOT'POM-PUBLIC3444

$\mathrm{B}$ Physics at $\mathrm{CDF}$

Phys. At. Nucl. 60:1265, 1997; Yad. Phys. 60:1400, 1997

On Interpretation of Results of Experimant PS-170 on the Proton Electromagnetic Form Factor near $p \vec{p}$ Threshold

Nucl. Phys. A631:618C, 1998; GRONINGEN-97 p. 618C

Measurements of the Virtual Bremsstrahlung Yields in the $p+p$ System

UHD-PI-MY-9715; HEPEX-9805012

Increased Sensitivity to Possible Muonium to Antimuonium Conversion

Phys. Rev. Lett. 81:3096, 1998; 1UCF-98-7; NUCLEX-9807002

Dependence of $p \uparrow p \uparrow \rightarrow p p \pi^{\circ}$ near Threshold on the Spin of the Colliding Nucleons

Phys. Lett. 327 B:201, 1994

First Measurement of the Electric Formfactor of the Neutron in the Exclusive Quasielastic Scattering of Polarized Electrons from Polarized ${ }^{3} \mathrm{He}$

FERMILAB-CONF-96-269-E; CDF-PUB-BOTTOM-PUBLIC-3844

Fully Reconstructed $B$-Meson Decays using $J / \psi(1 S)$ and $\psi(2 S)$

Phys. Lett. 382B:29, 1996

$K^{+}$Elastic Scattering from $\mathrm{C}$ and ${ }^{6} \mathrm{Li}$ at $715 \mathrm{MeV} / \mathrm{c}$

Phys. Lett. 381 B:402, 1996 New Limits for the ${ }^{19} \mathrm{Ne}(p, \gamma)^{20} \mathrm{Na}$ Astrophysical Reaction Rate from Direct Measurements using Radioactive Beams

Jour. of Phys. G 20:637, 1994 Cosmic Ray Hadron Flux at Sea Level up to $15 \mathrm{TeV}$

ITEP-26-98

The Fourdimentional Characteristics and Automodel Properties of the Pion Jets in the $\pi+p$ Interaction at Momentum $4.2 \mathrm{GeV} / \mathrm{c}$

Phys. At. Nucl. 60:571, 1997; Yad. Phys. 60:653, 1997 The Nuclear Transparency in Soft Processes

Phys. Rev. Lett. 80:452, 1998; Phys. Rev. Lett. 82:2221, 1999; NUCLEX-9712006

A Comparison of Polarization Observables in Electron Scattering from the Proton and Deuteron

Phys. Rev. Lett. 74:502, 1995 Measurement of Quasielastic ${ }^{3} \mathrm{He}(p, p n)$ Scattering from Polarized ${ }^{3} \mathrm{He}$ and the Three-Body Ground State Spin Structure

Phys. Rev. C57:1756, 1998

Spin Dependent Scattering of Deeply Bound Nucleons

2. Phys. C62:15, 1994; IFVE-93-98

Investigation of $\rho^{\mathrm{o}}$ Meson Spin Alignment in $\bar{p} p$ Interactions at $32 \mathrm{GeV} / c$

Phys. At. Nucl. 61:1117, 1998; Yad. Phys. 61:1217, 1998 Cryogenic Dark Matter Search and Solar Axion Search at the University of Tokyo

Phys. Lett. 407 B:225, 1997

Total Hadronic Photoabsorption on Carbon and Lead in the Shadowing Threshold Region

Phys. Rev. D50:R9, 1994; FERMILAB-PUB-94-083-E

Search for the Decay $D^{0} \rightarrow \mu^{+} \mu^{-}$

Phys. Rev. Lett. 72:3650, 1994; GSI-94-28

Observation of Enhanced Subthreshold $K+$ Production in Central Collisions between Heavy Nuclei

Nucl. Phys. A590:473C, 1995 Pion-Pion Correlations in Au+Au Collisions at AGS Energy

Nucl. Phys. A566:415C, 1994 Charged Hadron Distributions in $200 \mathrm{GeV} / \mathrm{A} \mathrm{S}+\mathrm{Au}$ Collisions: a Look at Stopping

Phys. Rev. Lett. 76:3057, 1996 Measurement of $0.25-3.2 \mathrm{GeV}$ Antiprotons in the Cosmic Radiation 
Miura 98

Miyabayashi 95

Miyajima 94

Moftah 97

Mohr 98

Molinari 94

Molinari 96

Montanet 95

Montgomery 96

Moore 97

Morandin 94

Morelos 93C

Morgan 94

Mori 95

Morita 95

Moriyama 95

Moriyama 98

Morlet 94

Morley 95

Moriey 96

Moroni 94

Morrison 98

Morsch 94

Mosca 94

Moskal 98

Moutoussi 98

Movillaferna 97

Muccifora 98

Mueller 94

Mueller 97

Muheim 93B

Mukha 95

Mukherjee 95

Mukhopadhyay 95

Muller 97

Munakata 97

Muntz 95

Muntz 97

Muramatsu 94
Phys. Rev. D57:5345, 1998; KEK-97-210; KEK-PREPRINT-97-210; KOBE-HEP-97-03; NGTHEP-97-3; OULNS-97-02 Precise Measurement of the $e^{+} e^{-} \rightarrow \mu^{+} \mu^{-}$Reaction at $\sqrt{s}=57.77 \mathrm{GeV}$

Phys. Lett. 347B:171, 1995; DPNU-94-47; NWU-HEP-94-06; KEK-94-152; TUAT-HEP-94-05; TIT-HPE-94-11; OCUHEP-94-09; PU-94-688; INS-1074; KOBE-HEP-94-08

Measurement of the Total Hadronic Cross Section and Determination of $\gamma Z^{0}$ Interference in $e^{t} e^{-}$ Annihilation

IEEE Trans. on Nucl. Sci. 41:835, 1994; KEK-94-21

Search for Double $\beta$-Decay Products of ${ }^{136}$ Xe in Liquid Xenon

Phys. Lett. 395B:157, 1997

Muon Capture in ${ }^{28} \mathrm{Si}$ and $g_{p} / g_{a}$

Phys. Rev. C59:1790, 1999; NUCLEX-9812006

Absolute Measurement of the $5 / 2^{+}$Resonance of ${ }^{36} \operatorname{Ar}(p, \gamma)^{37} K$ at $E_{p}=918 \mathrm{keV}$

WILLIAMSBURG-94 p. 709

Scattering of Photons by the Proton in the $\Delta\left(1232 P_{33}\right)$-Resonance Region

Phys. Lett. 371 B:181, 1996

Scattering of Photons by the Proton through $\theta_{c m s}=90^{\circ}$ in the $\Delta$-Resonance Region

Nucl. Phys. (PS) B39:281, 1995

Scalar Mesons

FERMILAB-CONF-96-043-E

The Top Quark and More: Some Highlights of Physics from the Do Experiment

Nucl. Phys. (PS) B55:231, 1997; ESTES PARK-96 p. 231

Measurement of the Michel Parameters and the Chirality Parameter in $\tau^{ \pm}$Decays from $e^{+} e^{-} \rightarrow \tau^{+} \tau^{-}$

Nuovo Cim. 107A:1875, 1994; DFPD-93-EP-64

First Measurement of the Neutron Electromagnetic Form-Factors in the Time-Like Region

Phys. Rev. D52:3777, 1995; FERMILAB-PUB-93-331-E

$p_{T}$ and $x_{F}$ Dependence of the Polarization of $\Sigma^{+}$Hyperons Produced by $800 \mathrm{GeV} / \mathrm{c}$ Protons

Jour. of Phys. G 20:A1, 1994; DPDG-94-01; RAL-94-061

A Compilation of Data on Two-Photon Reactions Leading to Hadron Final State

Phys. Rev. C51:2611, 1995

${ }_{12} \mathrm{C}(\gamma, p){ }_{11}^{11}$ Bor Cross Section from 44 to $88 \mathrm{MeV}$

Phys. Rev. Lett. 72:1180, 1995

First Observation of Laser-Induced Resonant Annihilation in Metastable Antiprotonic Helium Atoms

Phys, Lett. 347B:152, 1995

The $17 \mathrm{KeV}$ Neutrino and the Search for Anomalous $\gamma$ Rays in ${ }^{35} \mathrm{~S}$ Decay

Phys. Lett. 434B:147, 1998; RESCEU-23-98; HEPEX-9805026

Direct Search for Solar Axions by using Strong Magnetic Field and $X$-ray Detectors

Nucl. Phys. A577:155C, 1994

Recent Results on the Isoscalar Spin Response in ${ }^{40} \mathrm{Ca}$ and ${ }^{12} \mathrm{C}$

Phys. Lett. 355B:52, 1995

Saturation of Deposition Energy in Relativistic ${ }^{3} \mathrm{He}$-Induced Reactions

Phys. Rev. C54:737, 1996; DAPNIA-SPHN-96-06; INC-40007-104

$4 \pi$ Studies of the $1.8-4.8 \mathrm{GeV}^{3} \mathrm{He}+\mathrm{Ag}_{\text {nat }},{ }^{197} \mathrm{Au}$ Reactions. I. Energy Deposition

Nuovo Cim. 107 A:2025, 1994

New Results on Heavy-Flavor Photoproduction from the E687 Experiment at FNAL

Phys, Rev. C59:221, 1999; NUCLEX-9811012

Quasielastic ${ }^{12} \mathrm{C}\left(e, e^{\prime} p\right)$ Reaction at High Momentum Transfer

Z. Phys. A350:167, 1994; Z. Phys. A353:349, 1994

Study of Elastic ${ }^{4} \mathrm{He}$-Scattering from deuteron and ${ }^{12} \mathrm{C}$ at $E=4.2 \mathrm{GeV}$

DAPNIA-SPP-94-12

Review of Particle Dark Matter Experiments (Direct Detection)

Phys, Rev. Lett. 80:3202, 1998; FZJ-IKP-I-1997-02; NUCLEX-9803002

$\eta^{\prime}$ Production in Proton-Proton Scattering Close to Threshold

MANCHESTER-95 p. 156; IC-HEP-95-11

Quark and Gluon Jet Properties Measured at LEP

Eur. Phys. J. C1:461, 1998; PITHA-97-27; HEPEX-9708034

A Study of Event Shapes and Determinations of $\alpha_{s}$ using Data of $e^{+} e^{-}$Annihilations at $\sqrt{s}=22$ to $44 \mathrm{GeV}$

NUCLEX-9810015

Photoabsorption on Nuclei in the Shadowing Threshold Region

Nucl. Instr. and Meth. A351:59, 1994; FERMILAB-CONF-94-252-E

$B$ Physics with CDF: Recent Results and Future Prospects

Phys. Rev. Lett. 78:3824, 1997; OAP-740; NUCLEX-9702004

Measurement of the Proton's Neutral Weak Magnetic Form Factor

Phys. Rev. D49:3767, 1994; HEPSY-93-3

Three Model Dependent Estimates of $\operatorname{Br}\left(D_{s}^{+} \rightarrow \phi(1020) \pi^{+}\right)$

Phys. Lett. 367B:65, 1996; CERN-PPE-95-150

Observation of the ${ }^{11} \mathrm{Li}(\beta$ deuteron $)$ Decay

Z. Phys. A352:417, 1995

Intermediate Mass Fragment Production in $\left({ }^{4} \mathrm{He}{ }^{27} \mathrm{Al}\right)$ Reaction at Low Energies

Nuovo Cim. 108A:775, 1995; UB-HEX-95-02

Two and Three Particle Correlations in Gold Emulsion Interactions at $10.6 \mathrm{~A} \mathrm{GeV}$

Nucl. Phys. (PS) B55:333, 1997; ESTES PARK-96 p. 333

Hadronic Decay Structure and $\nu_{\tau}$ Helicity in $\tau^{-} \rightarrow \pi^{-} \pi^{-} \pi^{+} \nu_{\tau}$ with the OPAL Detector at LEP

Phys. Rev. D56:23, 1997

Large-Scale Anisotropy of the Cosmic-Ray Muon Flux in Kamiokande

Z. Phys. A352:175, 1995

Properties of High Energy Pions Emitted from Heavy Ion Collisions at $1 \mathrm{GeV} /$ Nucleon

Z. Phys. A357:399, 1997

Pion Production in Mass-Symmetric Heavy Ion Collisions at $0.8-1.8 \mathrm{~A} \cdot \mathrm{GeV}$

Phys. Lett. 332B:477, 1994; KEK-94-13; NWU-HEP-94-02; DPNU-94-18; INS-1031; KOBE-HEP-94-04; OCU-HEP-94-02; PU-94-683; TIT-HPE-94-03; TUAT-HEP-94-02

Measurement of the Photon Structure Function $F_{2}^{\gamma}$ and Jet Production at TRISTAN 
Musolino 95

Nadasen 99

Nagae 94

Nagae 95

Nagae 98

Nagle 97

Nakamura 94

Nakano 94

Nakaya 94

Nakazawa 98

Naples 94

Naples 99

Narain 96

Narayanan 96

Nassalski 94

Nemati 96

Nemati 97

Neuberger 96

Neuberger $96 \mathrm{~B}$

Nguyen 94

Niculescu 98

Niinikoski 94

Niizeki 94

Nikolic 97

Nikulin 96

Nilsen 94

Nilsen 95

Nilsson 95

Nilsson 96

Nisius 95

Nodulman 94

Nodulman 96

Nodulman $96 \mathrm{~B}$

Nomachi 98

Nomofilov 94

Nomura 97

Norman 94

Norman 96

Notheisen $\cdot 96$
MANCHESTER-95 p. 482; LAL-95-84

Exclusive $\Lambda_{b}$ Reconstruction

Phys. Rev. C59:760, 1999

(He, $2 \mathrm{He}$ ) Cluster Knockout Reaction on ${ }^{9} \mathrm{Be}$ and ${ }^{12} \mathrm{C}$ at $580 \mathrm{MeV}$

Phys. Lett. 335 B:330, 1994; INS-1020

Two Nucleon Pion Absorption in ${ }^{4} \mathrm{He}$ at $1 \mathrm{GeV} / \mathrm{c}$

INS- 1096

$\left(\pi^{+}, K^{+}\right)$Spectroscopy with the SKS Spectrometer

Phys. Rev. Lett. 80:1605, 1998

Observation of a $\frac{4}{2} \mathrm{He}$ Bound State in the ${ }^{4} \mathrm{He}\left(\mathrm{K}^{-}, \pi^{-}\right)$Reaction at $600 \mathrm{MeV} / \mathrm{c}$

Jour. of Phys. G 23:2145, 1997

Antiproton Production and Antideuteron Limits from Experiment E864

Phys. Rev. A49:4457, 1994; CERN-PRE-93-069; INS-1013

Delayed Annihilation of Antiproton in Helium Gas

Phys. Lett. 340B:135, 1994; KEK-94-101

Measurement of the Forward-Backward Asymmetries for Charm- and Bottom-Quark Pair Productions at $\langle\sqrt{3}\rangle=58 \mathrm{GeV}$ with Electron Tagging

Phys. Rev. Lett. 73:2169, 1994

Measurement of the Branching Ratio of $K_{L} \rightarrow e^{+} e^{-} \gamma \gamma$

Nucl. Phys. A639:345C, 1998; UPTON-97 p. 345C

Strangeness -2 Studies at KEK

Phys. Rev. Lett. 72:2341, 1994; FERMILAB-PUB-94-028-E

A-Dependence of Photoproduced Dijets

Phys. Rev. D59:031101, 1999; HEPEX-9809023

A High Statistics Search for $\nu_{e}\left(\bar{\nu}_{e}\right) \rightarrow \nu_{\tau}\left(\bar{\nu}_{\tau}\right)$ Oscillations

FERMILAB-CONF-96-192-E

Top Production at Do

FERMILAB-CONF-96-236-ED

Do Papers on Electroweak Physics Submitted to DPF 96: Muon Charge Asymmetry from $W^{ \pm}$Decays at the Do Detector

Nucl. Phys. A5 7 7:325C, 1994

New Results on the Gottfried Sum and $\bar{d} \neq \bar{u}$ in the Nucleon Sea

Phys. Rev. D55:5273, 1997; CLNS-96-1435; CLEO-96-18

Measurement of the Direct Photon Spectrum in $\Upsilon(1 S)$ Decays

Phys. Rev. D57:5363, 1998; CLNS-97-1503; CLEO-97-19; CLEO-CONF-97-12; EPS-ABSTRACT-338; HEPEX-9708033 Search for Color-Suppressed $B$ Hadronic Decay Processes with CLEO

PADUA-96 p. 574; FERMILAB-CONF-96-203-E

Double Boson Production at CDF

FERMILAB-CONF-96-153-E

Electroweak Measurements from $\mathrm{CDF}$ and Do

FERMILAB-CONF-94-187-E

Search for $D^{+} \rightarrow \pi^{+} \mu^{+} \mu^{-}$

Phys. Rev. Lett. $81: 1805,1998$

Longitudinal and Transverse Cross Sections in $p\left(e^{-}, e^{-}, K^{+}\right) \Lambda$ Reaction

CERN-PPE-94-05

The SMC Experiment and First Results on the Longitudinal Deuteron Spin Asymmetry

Nucl. Phys. A577:37C, 1994

Spin-Isospin Excitation in Sd-Shell Nuclei Studied by the (deuteron, ${ }^{2} \mathrm{He}$ ) Reaction at $E_{\text {doutoron }}=270 \mathrm{MeV}$ LAL-97-78

A Combined Upper Limit on the $\tau$ Neutrino Mass with the ALEPH Detector

Phys. Rev. C54:1732, 1996; DAPNIA-SPIIN-96-15

The deuteron $\uparrow p \rightarrow{ }^{3} \mathrm{He} \pi^{0}$ Reaction Near Threshold

Phys. Rev. C50:1065, 1994

Charge-Pickup by Heavy Relativistic Nuclei

Phys. Rev. C52:3277, 1995

Fragmentation Cross Sections of Relativistic ${ }^{84} \mathrm{Kr}$ and ${ }^{109} \mathrm{Ag}$ Nuclei in Targets from Hydrogen to Lead

Eur. Lett. 30:19, 1995

Neutron Momentum Distributions from "Core Break-Up" Reactions of Halo Nuclei

N'ucl. Phys. A598:418, 1996; GSI-96-04

${ }^{8} \mathrm{He}$ and Neutron Momentum Distributions from ${ }^{8} \mathrm{He}$ in Nuclear Break-up Reactions at $240 \mathrm{MeV} / \mathrm{u}$

Nucl. Phys. (PS) B39:124, 1995; MONTREUX-94 p. 124

Jet Studies in Deep Inelastic $e^{-} p$ Scattering at HERA

FERMILAB-CONF-94-317-E

Electroweak Physics from the Tevatron

FERMILAB-CONF-96-337-E

Direct Photons at $\mathrm{CDF}$

FERMILAB-CONF-96-326-E

Diboson Production at the Tevatron

Niucl. Phys. A629:213C, 1998; OSAKA-97 p. 213C

First Experiment at RCNP on $p$-Bremsstrahlung at $400 \mathrm{MeV}$

Phys. Lett. 325B:327, 1994; JINR-E1-93-405

Measurement of Polarization Transfer and the Tensor Analyzing Power in Polarized Deuteron Break-up with Deuteron Momenta up to $9 \mathrm{GeV} / \mathrm{c}$

Phys. Lett. 408B:445, 1997

Experimental Search for the Decay Mode $K_{L} \rightarrow \pi^{+} \pi^{-} e^{+} e^{-}$

ALBUQUERQUE-94, v. 2, p. 1165; FERMILAB-CONF-94-267-E; HEPEX-9409008

Search for First and Second Generation Leptoquarks at Do

MANCHESTER-95 p. 414

Preliminary Results from Second Phase of the WA91 Experiment

MANCHESTER-95 p. 470

Neutral Meson Production in Ultrarelativistic Proton Nucleus Collisions 
Noumi 95

Novikov 94

Numao 95

Oakden 94

Ochiishi 95

Odaka 98

Odyniec 97

Ogilvie 97

Ogilvie 98

Ohl 96

Ohm 97

Ohnishi 98

Ohyama 96

Okamura 94

Okonov 94

Olsson 98

Oneill 95

Oshima 94

Outa 98

Oyama 97

Oyama 98

Oyama 98B

Ozawa 94

Padilla 95

Paic 94

Pairsuwan 95

Pak 96

Palano 95

Palano 96

Palarczyk 98

Panafiotou 96

Panafiotou 98

Panafiotou 98B

Panafiotou 98C

Panagiotou 94

Panagiotou 95

Panagiotou 96

Panagiotou 96B

Panagiotou 97

Phys, Rev, C52:2936, 1995; KEK-95-10

Hypernuclear Weak Decay of ${ }^{12} \mathrm{C}_{\Lambda}$ and ${ }^{11} \mathrm{Bor}_{\Lambda}$

Phys. Lett. 331B:433, 1994; CERN-TH-7137-94

The Values of $m_{t}$ and $\alpha_{S}$ Derived from the Nonobservation of Electroweak Radiative Corrections at LEP. Global Fit

Phys. Rev. D52:4855, 1995; TRI-P P-95-17

A New $\pi+$ Lifetime Measurement

Nucl. Phys. A574:731, 1994; DTP.93-60

Amplitude Analysis of Data on $\bar{p} p \longrightarrow \pi \pi$ at Low-Energy

KEK-95-37

Measurements of Fragments Produced in Target Multifragmentation using Bragg Curve Counters

Phys. Rev. Lett. 81:2428, 1998; KEK-98-47; NGTHEP-98-2; KOBE-HEP-98-01

Measurements of the Running of Effective QED Coupling at Large Momentum Transfer in the Space-Like Region

Jour. of Phys. G 23:1827, 1997

$\Xi\left(\Omega^{-}\right)$Production in $\mathrm{Pb}+\mathrm{Pb}$ Collisions at $158 \mathrm{GeV} / \mathrm{c}$

Jour. of Phys. G 23:1803, 1997

Strangeness Production at the AGS

Nucl. Phys. A630:571C, 1998; GATLINBURG-97 p. 571C

Kaon Production in Au+ Au Collisions at the AGS

FERMILAB-CONF-96-110-E; CDF-PUB-BOTTOM-PUBLIC-3676

Onia and Heavy Flavor Production at the Tevatron Collider

Phys. Rev. C55:3062, 1997

$\Lambda$-Hyperon Lifetime in Very Heavy Hypernuclei Produced in the $p$ Interaction

Phys. Lett. 438B:27, 1998

Measurement of a Complete Set of Analyzing Powers for Deuteron Elastic Scattering from $40 \mathrm{Ca}$ at $270 \mathrm{MeV}$ and an Investigation of Tensor Potentials

Z. Phys. C75:209, 1996; KEK-96-152; HUPD-9621; KOBE-HEP-96-04; KUNS-1426; OULNS-96-04 $D^{*}(2010)^{ \pm}$Inclusive Production in Two-Photon Process at $\sqrt{s}=58 \mathrm{GeV}$ in TRISTAN

Nucl. Phys. A577:89C, 1994 Study of the Spin-Dipole State via the (deuteron, ${ }^{2} \mathrm{He}$ ) Reaction at $270 \mathrm{MeV}$

Nucl. Phys. A583:711C, 1995; JINR-E1-94-387

Highly Excited Matter Probed with Strangeness in Nucleus-Nucleus Collisions at JINR

Nucl. Phys. A631:416C, 1998; GRONINGEN-97 p. 416C Measurements of $n p$ Scattering at 96 and $162 \mathrm{MeV}$

Phys. Lett. 351 B:87, 1995; OAP-731 $A$-Dependence of Nuclear Transparency in Quasielastic A $\left(e^{-}, e^{-t} p\right)$ at $\mathrm{High} q^{2}$

FERMILAB-CONF-94-285-E

Search for $W^{ \pm}$Pair Production with Dilepton Decay Modes at Do

Nucl. Phys. A639:251C, 1998; UPTON-97 p. 251C Mesonic and Non-Mesonic Decay Widths of ${ }_{\Lambda}^{4} \mathrm{H}$ and ${ }_{\Lambda}^{4} \mathrm{He}$

HEPEX-9706008

Experimental Study of High Energy Atmospheric Neutrinos in Kamiokande

Phys. Rev. Lett. 81:2016, 1998

Measurement of the FIux and Zenith-Angle Distribution of Upward Through-Going Muons in Kamiokande II + III

Phys. Rev. D57:R6594, 1998

Examination of Neutrino Oscillations using High-Energy Atmospheric Neutrino Data from Kamiokande

Phys. Lett. 334B:18, 1994

Interaction Cross Sections and Radii of the Mass $\mathrm{Number} A=17$ Isobar $\left({ }^{17} \mathrm{Nit},{ }^{17} \mathrm{Fl},{ }^{17} \mathrm{Ne}\right)$

Nucl. Phys. (PS) B39:161, 1995

Measurement of Scaling Violations in Fragmentation Functions in $e^{+} e^{-}$Annihilation

Nuovo Cim. 107 A:2141, 1994

Structure Function Ratios $F_{2}^{\mathrm{Li}} / F_{2}^{\text {deuteron }}$ and $F_{2}^{\mathrm{C}} / F_{2}^{\text {deuteron }}$ at Low $x_{\mathrm{Bj}}$

Phys. Rev, C52:2552, 1995

Analyzing Power for the ${ }^{2} H(p \nmid, p n){ }^{1} H$ Reaction at $200 \mathrm{MeV}$

Phys. Rev, C54:1681, 1996

Radial Flow in ${ }^{40} \mathrm{Ar}{ }^{45} \mathrm{Sc}$ Reactions at $E=35-115 \mathrm{MeV} /$ Nucleon

Nucl. Phys. (PS) 39BC:287, 1995; IN FN-AE-94-25; MONTPELLIER-94 p. 287

Glueballs and Hybrids: an Experimental Review

MANCHESTER-95 p. 171

Recent Results from JETSET

Phys. Rev. C58:645, 1998 Cross Sections and Analyzing Power for the $(p, n)$ Reaction on ${ }^{3} \mathrm{He}$ and ${ }^{4} \mathrm{He}$ at $200 \mathrm{MeV}$

Nucl. Phys. A610:188C, 1996 Hadron Yields and Hadron Spectra from the NA48 Experiment

Nucl. Phys. A638:91C, 1998 Recent Results on Central Pb + Pb Collisions from Experiment NA49

Nucl. Phys. A638:431C, 1998 $\phi(1020)$ Emission in Central $\mathrm{Pb}+\mathrm{Pb}$ Collisions at $158 \mathrm{GeV} / \mathrm{u}$

Nucl. Phys. A638:463C, 1998 Directed and Elliptic Flow in $158 \mathrm{GeV} / \mathrm{Nucleon} \mathrm{Pb}+\mathrm{Pb}$ Collisions

Nucl. Phys. A566:35C, 1994 Hadron Production in S+Ag and S+Au Collisions at $200 \mathrm{GeV} / \mathrm{Nucleon}$

Nucl. Phys. A590:197C, 1995

Recent Results from NA35

Nucl. Phys. A610:76C, 1996 Stopping and Collective Effects at SPS Energies

Nucl. Phys. A610:248C, 1996

Particle Correlations in $\mathrm{Pb} \mathrm{Pb}$ Collisions at the CERN-SPS - Results from the NA49 Experiment

Z. Phys. C73:443, 1997

A New Coulomb Correction Method for Bose-Einstein Correlations, Based on the $\pi^{+} \pi^{-}$Correlation Measurements

See the legend on page 5 
Panagiotou 98

Pantaleone 94

Panvini 97

Papadimitrio 94

Papadimitrio 95

Papadimitrio 95B

Papadimitrio 96

Papadimitrio $96 \mathrm{~B}$

Papandreou 95B

Papini 95

Parashar 94

Parashar 94B

Parashar 95

Parashar $95 \mathrm{~B}$

Parashar $95 \mathrm{C}$

Park 94

Park 94B

Park 95

Park 95B

Partlan 95

Passalacqua 97

Pasyuk 96

Pate 9.1

Paul 96

Paulini 96

Pavlik 98

Pawlowski 97

Payne 94

Peaslee 94

Peise 96

Pellegrino 97

Peng 98

Perasso 96

Perepelitsa 96

Perilloisaac 98

Perkins 96

Peryshkin 95

Peterson 94

Peterson 95

Petratos 93
Eur. Phys. J. A2:383, 1998

Spectator Nucleons in $\mathrm{Pb} \mathrm{Pb}$ Collisions at $158 \mathrm{~A} \cdot \mathrm{GeV}$

Phys. Rev. D49:R2152, 1994

Constraints on Three-Neutrino Mixing from Atmospheric and Reactor Data

Nucl. Phys. (PS) B55:41, 1997; ESTES PARK-96 p. 41

Neutral Current Couplings of Leptons to Polarized $Z^{\circ}$ in the SLD Experiment

FERMILAB-CONF-94-221-E; CDF-PUB-BOTTOM-PUBLIC-2751 $\Upsilon$ Production at CDF

CDF-PUB-BOTTOM-PUBLIC-3211; FERMILAB-CONF-95-128-E Production of Heavy Quark States at CDF

FERMILAB-CONF-95-227-E; CDF-PUB-BOTTOM-PUBLIC-3240 $\Upsilon$ Production at CDF

FERMILAB-CON F-96-135-E; CDF-PUB-BOTTOM-PUBLIC-3689 Quarkonia Production at $C D F$ and $D 0$

FERMILAB-CONF-96-402-E; CDF-PUB-BOTTOM-PUBLIC-3722 $Q$ uarkonia Production at CDF

Phys. Rev. C51:2862, 1995; TRI-PP-95-10

${ }^{6} \mathrm{Li}\left(\pi^{+}, p p\right){ }^{4} \mathrm{He}_{(\mathrm{g.s.})}$ Reaction at 100 and $165 \mathrm{MeV}$ Incident Pion Energies

LNF-95-051-PQ

Atmospheric Proton and Deuterium Energy Spectra Determination with the MASS2 Experiment

Phys. Rev. D50:3553, 1994

Correlations in High-Energy Interactions

Nuovo Cim. 107A:1419,1994

Relation between Short-Range Correlations and Intermittency

Nuovo Cim. 108A:789, 1995

Intermittency and Fractal Behaviour of Shower Particles at High Energy

Jour. of Phys. G 21:173, 1995

One-Dimensional and Two-Dimensional Multifractals in Varying Large Bin Sizes

Nuovo Cim. 108A:489, 1995

Multifractal Moments in High-Energy Interactions

SLAC -435

A Measurement of the Left-Right Cross Section Asymmetry in $Z^{0}$ Production with Polarized $e^{+} e^{-}$ Collisions

ALBUQUERQUE-94, v. 2, p. 1169; FERMILAB-CONF-94-228-E; CDF-PUB-EXOTIC-PUBLIC-2734

Search for Second Generation Leptoquarks at CDF

FERMILAB-CONF-95-155-E; CDF-PUB-EXOTIC-PUBLIC-3199

Search for New Phenomena in CDF. 1. Z-prime, W-prime and Leptoquarks

Nucl. Phys. (PS) B40:381, 1995; LAL-94-87; MONTREUX-94 p. 381

1 Prong $\tau^{ \pm}$Decays into Kaons in ALEPH

Phys. Rev. Lett. 75:2100,1995

Fragment Flow in Au Au Collisions

Nucl. Phys. (PS) B55:435, 1997; ESTES PARK-96 p. 435 Direct Bounds on the Tau Neutrino Mass from LEP

Phys. Rev. C55:1026, 1997; LA-UR-95-3656; NUCLEX-9611001 $\pi^{+}$deuteron $\rightarrow p p$ Reaction between 18 and $44 \mathrm{MeV}$

WILLIAMSBURG-94 p. 542

Two-Nucleon Processes in Pion-Induced Double Charge Exchange in ${ }^{4} \mathrm{He}\left(\pi^{+}, \pi^{-} p\right) 3^{p}$ Reaction

Nuovo Cim. 109A:735, 1996; PISA-95 p. 735 Measurement of the Lifetime of $\Omega_{c}$

Nuovo Cim. 109A:631, 1996; PISA-95 p. 631; FERMILAB-CONF-95-253-E; CDF-PUB-BOTTOM-PUBLIC-3281 Highlights of $B$ Physics at CDF

Phys. Rev. C57:2416, 1998

${ }^{27}$ Al $(n, x \gamma)$ Reactions for Neutron Energies from 3 to $400 \mathrm{MeV}$

Z. Phys. A357:387, 1997

Characterization of Reaction Mechanisms in the ${ }^{40} \mathrm{Ca}{ }^{40} \mathrm{Ca}$ Reaction at $E_{l a b}=35 \mathrm{MeV} / \mathrm{Nucleon}$

Nucl. Instr. and Meth. A351:19, 1994

Recent $B$ Physics Results from CLEO-II

Phys. Rev. C49:2271, 1994

Energy Dependence of Multifragmentation in ${ }^{84} \mathrm{Kr}+{ }^{187} \mathrm{Au}$ Collisions

Phys. Lett. $384 \mathrm{~B}: 37,1996$

Compton Scattering by the Proton through $\theta=75^{\circ}$ in the $\Delta$-resonance Region

Phys. Rev. Lett. 78:4011, 1997

Deuteron Electrodisintegration in the $\triangle$-Resonance Region

Phys. Rev. D58:092004, 1998; FERMILAB-PUB-98-108-E; HEPEX-9804288

$\bar{d} / \bar{u}$ Asymmetry and the Origin of the Nucleon Sea

Nuovo Cim. 109A:841, 1996; PISA-95 p. 841

Charm Semi-Leptonic Decays and Measurements of the Form Factors

MANCHESTER-95 p. 460

Narrow $\bar{p} p$ State at $2.02 \mathrm{GeV} / c^{2}$

Phys. Rev. Lett. 81:2416, 1998; HEPEX-9806147 Search for Strange Matter by Heavy Ion Activation

FERMILAB-CONF-96-304-EJ

$D^{\circ}$ Papers on QCD Studies with Jets: Rapidity Gaps in Hard Processes at $D^{\circ}$

Nucl. Phys. (PS) B39:353, 1995

The Search for the Top Quark at Do

Nucl. Phys. A577:161C, 1994

Isoscalar Spin Response of Nuclei

Phys. Rev. C52:33, 1995

$500 \mathrm{MeV}$ Pion Single-Charge Exchange on Deuterium

Nuovo Cim. 107A:2197, 1994; SLAC-PUB-6327

SLAC Measurements of the Neutron Spin-Structure Function. 
Petratos 98

Petrovici 95

Pfaff 96

Pham 95

Phillips 96

Piccinini 94

Piekarz 93B

Pienkowski 94

Pienkowski 97

Piepke 94

Pillai 96

Pinder 96

Plotzke 98

Plunkett 94

Pocanic 94

Pochodzalla 95

Podobrin 95

Poggi 95

Polikanov 94

Polster 95

Popescu 98

Pordes 96

Porter 97

Porter 98

Posthaus 94

Prakhov 96

Price 95

Prinz 98

Procario 93

Proff 99

Prokofiev 95

Prokoshkin 94

Prokoshkin 96

Prokoshkin 96B

Prokoshkin 96C

Prokoshkin 96D

Prokoshkin 96E
Nucl. Phys. A631:507C, 1998; GRONINGEN-97 p. 507C Measurements of the Neutron Spin Structure Functions using the SLAC 50 GeV Fixed Target Facility and a Polarized ${ }^{3} \mathrm{He}$ Target

Phys. Rev. Lett. 74:5001, 1995

Cluster Formation during Expansion of Hot and Compressed Nuclear Matter Produced in Central Collisions of $\mathrm{Au}+\mathrm{Au}$ at $150 \mathrm{~A} \cdot \mathrm{MeV}$

Phys. Rev. C53:1753, 1996 Fragmentation of ${ }^{78} \mathrm{Kr}$ Projectiles

Phys. Rev. C51:526, 1995

Fragmentation and Splitting of Gamow-Teller Resonances in Sn $\left({ }^{3} \mathrm{He},{ }^{3} \mathrm{H}\right)$ Sb Charge-Exchange Reactions,

FERMILAB-CONF-96-275-E; CDF-PUB-JET-PUBLIC-3760

$W^{ \pm}$and $Z^{\circ}$ Boson Plus Jets Production at CDF

Nuovo Cim. 107A:2287, 1994

First Results From OBELIX on Reactions with Detected Neutral Pions in the Final State

ALBUQUERQUE-94, v. 2, p. 1192; FERMILAB-CONF-94-330-E; FSU-HEP-940816

A Study of Events with Large Total Transverse Energy Produced in $p \bar{p}$ Collisions at $\sqrt{s}=1.8$ TeV

Phys. Lett. 336B:147, 1994; GANIL-P-94-10

Hot Nuclei in Reactions Induced by $475 \mathrm{MeV}, 2 \mathrm{GeV}{ }^{1} \mathrm{H}$ and $2 \mathrm{GeV}{ }^{3} \mathrm{He}$

Phys. Rev. C56:1909, 1997

Neutron Multiplicity Distributions for 1.94 to $5 \mathrm{GeV} / c$ Proton, Antiproton, Pion, Kaon, and DeuteronInduced Spallation Reactions on Thin and Thick Targets

Nucl. Phys. A577:493, 1994

Investigation of the $\beta \beta$ Decay of ${ }^{116} \mathrm{Cd}$ into Exited States of ${ }^{116} \mathrm{Sn}$

FERMILAB-CONF-96-279-E; HEPEX-9608006

A Search for Neutral Heavy Vector Gauge Bosons in $\bar{p} p$ Collisions at $\sqrt{s}=1.8 \mathrm{TeV}$

MANCHESTER-95 p. 178

The Status of the Light Quark Spectroscopy of $J^{P C}=2^{-+}$Objects

Phys. Lett. 444B:555, 1998

Photoproduction of $\eta^{\prime}$-Mesons with the $4 \pi$-Detector SAPHIR

FERMILAB-CONF-94-045-E

Jet Physics and QCD Tests at the Tevatron Collider

Phys. Rev. Lett. 72:1156, 1994

Reaction $\pi^{+} p \rightarrow \pi^{+} \pi^{0} p$ Near Threshold and Chiral Symmetry Breaking

Phys. Rev. Lett. 75:1040, 1995

Probing the Nuclear Liquid-Gas Phase Transition

Nucl. Phys. (PS) 39BC:373, 1995; IEKP-KA-94-13

Measurement of $B^{*}, B_{s}$ and $\Lambda_{b}$ Production in $Z^{0}$ Decays

Nucl. Phys. A586:755, 1995

Evidence for Collective Expansion in Light-Particle Emission Following Au Au Collisions at 100 , 150 and $250 \mathrm{~A} \mathrm{MeV}$

2. Phys. A350:221, 1994

Electromagnetic and Nuclear Fission of ${ }^{238} \mathrm{U}$ in the Reaction of 100,500 and $1000 \mathrm{~A} \mathrm{MeV} 208 \mathrm{~Pb}$ with $238 \mathrm{U}$

Phys. Rev. C51:1167, 1995

Light Particle Emission Induced by Stopped Antiprotons in Nuclei: Energy Dissipation and Neutron-toProton Ratio

Phys. Rev. C58:270, 1998

Sensitivity of Two Fragment Correlation Functions to Initial State Momentum Correlations

MANCHESTER-95 p. 185; FERMILAB-CONF-95-384-E

A Study of the Charmonium Spectrum through Proton Antiproton Annihilation: Results and Prospects at Fermilab

Phys. Rev. Lett. 79:1229, 1997; LBNL-39957; NUCLEX-9703001

Dielectron Cross Section Measurements in Nucleus-Nucleus Reactions at $1.0 \mathrm{~A} \cdot \mathrm{GeV}$

Nucl. Phys. A638:499C, 1998; TSUKUBA-97 p. 499C

Dielectron Production in $A-A$ Reactions at $1.0 \mathrm{~A} \cdot \mathrm{GeV}$

BONN-IB-94-03

Determination of an upper Limit on the Mass of $\nu_{\tau}$ 's using the OPAL Detector / Bestimmung einer oberen Grenze fur die Masse des $\tau$-Neutrinos mit dem OPAL Detector

MANCHESTER-95 p. 377

Study of the $\bar{p} p \rightarrow K^{+} K^{-} \pi^{\text {o }}$ Reaction in Antiproton

Phys. Rev. C51:R2283, 1995

$\eta$ Meson Photoproduction on Hydrogen near Threshold

Phys. Rev. Lett. 81:1175, 1998; SLAC-PUB-7762; HEPEX-9804008

Search for Millicharged Particles at SLAC

Phys. Rev. Lett. 73:1472, 1994; CLNS-93-1264; CLEO-93-24

Observation of Inclusive $B$ Decays to the Charmed Baryons $\Sigma_{c}(2455)^{++}$and $\Sigma_{c}(2455)^{0}$

Nucl. Phys. A646:67, 1999; NUCLEX-9901002 Electromagnetic Polarizabilities of Nucleons bound in ${ }^{40} \mathrm{Ca},{ }^{16} \mathrm{O}$ and ${ }^{4} \mathrm{He}$

Yad. Phys. 58:1740, 1995; Phys. At. Nucl. 58:1640, 1995 Investigation of the Vector Analyzing Power $i T_{11}$ from Deuteron Breakup Reaction $\pi^{+}$deuteron $\rightarrow p$ at

$350-450 \mathrm{MeV}$
Phys. At. Nucl. 58:606, 1995; Yad. Phys. 58:662, 1995; IFVE-94-42

Analysis of $D$-Wave in $\pi^{-} p \rightarrow a_{2}(1320)^{\circ} n$ Reaction

Phys. Dokl. (Transl. of DANS) 41:247, 1996; MANCHESTER-95 p. 439

Observation of a Narrow $1650 \mathrm{MeV}$ Meson Decaying into $\omega \eta$

MANCHESTER-95 p. 442

Measurement of the Rate Radiative $\omega \rightarrow \pi^{0} \pi^{0} \gamma$ Decay

MANCHESTER-95 p. 445

Observation of a Spin-4 Meson Decaying into $\eta \pi^{0}$

Phys. At. Nucl. 59:1333, 1996; Yad. Phys. 59:1392, 1996

$a_{4}(2040)^{\circ}$ Meson Decaying into $\eta \pi^{\circ}$

Doklady RAN 348:481, 1996

Observation of Narrow Meson with $1650 \mathrm{MeV}$ Mass Decaying into $\boldsymbol{\omega} \eta$

See the legend on page 5 . 
Protopopescu 96

Prout 94

Prout 95

Prout 96

Pruneau 98

Przewoski 98

Pukhaeva 96

Punjabi 94

Punjabi 95

Purohit 94

Purohit 94B

Qi 95

Qin 95

Quednau 96

Quenby 95

Quigley 97

Quintas 94

Quraan 98

Raab 9.5

Ragan 9.5

Rahm 98

Raja 94

Rajagopalan 96

Ram 93

Ramberg 94

Ramillien 95

Rapaport 94

Rappenecker 95

Rathmann 98

Raue 95

Ravndal 94

Rawoolsulliv 94

Raywood 97

Razen $9 T$

Reinhold 98

Reisdorf 97

Resag 96

Ressing 95
Nuovo Cim. 109A:755, 1996; PISA-95 p. 755

Observation of the Top Quark

Nucl. Phys. A577:233C, 1994 Spin-Longitudinal and Spin-Transverse Cross Sections for $\Delta$ Production in the ${ }^{12} \mathrm{C}(p, n)$ Reaction

Phys. Rev. C52:228, 1995 Cross Sections and Analyzing Powers for Quasielastic Scattering at 795 and $495 \mathrm{MeV}$ using the ( $p$, $n$ ) Reaction

Phys. Rev. Lett. 76:4488, 1996

Spin Decomposition of the $\Delta$ Resonance Cross Section using the ${ }^{12} C(\vec{p}, \vec{n})$ Reaction at $E_{p}=795 \mathrm{MeV}$

Nucl. Phys. A638:81C, 1998; TSUKUBA-97 p. $81 \mathrm{C}$

Strangelet Searches, Light Nuclei and $\bar{p}$ Production Measurements by AGS E884

Phys. Rev. C58:1897, 1998

$p-p$ Analyzing Power and Spin Correlation Measurements between 250 and $450 \mathrm{MeV}$ at $7^{\circ} \leq \Theta \leq 90^{\circ}$ with an Internal Target in a Storage Ring

MANCHESTER-95 p. 563

$K K$ Bose-Einstein Correlations

WILLIAMSBURG-94 p. 419

$T_{20}$ and $k_{0}$ in Deuteron Backward Elastic Scattering

Phys. Lett. 350B:178, 1995

Measurement of Polarization Transfer $\kappa_{0}$ and Tensor Analyzing Power $T_{20}$ in the Backward Elastic deuteron p Scattering

HEP-94-13; FERMILAB-CONF-94-186-E

$D^{\circ}-\bar{D}^{0}$ Mixing and Doubly Cabibbo Suppressed Decays of the $D^{+}$

FERMILA B-CONF-94-408-E; USCHEP-94-01 Preliminary Results on the Decays $D^{+} \rightarrow K^{+} \pi^{+} \pi^{-}, D^{+} \rightarrow K^{+} K^{+} K^{-}$

Nucl. Phys. (PS) B40:387, ; MONTREUX-94 p. 387

Tau Lepton Experiment at BES Detector

Nucl. Phys. A587:252, 1995

Tensor and Vector Analyzing Powers of the $p$ (deuteron, $p p$ )n Reaction

Nucl. Phys. A606:538, 1996; GANIL-P-96-10

Decay Patterns of Target-Like and Projectile-Like Nuclei Produced in ${ }^{84} \mathrm{Kr}+{ }^{107} \mathrm{Au}, \mathrm{U}$ Reactions at $E / A=$

$150 \mathrm{MeV}$
Phys. Lett. 351B:70, 1995

Results from the First Stage of a UK Galactic Dark Matter Search using Low Background Sodium Iodide Detectors

Nucl. Phys. (PS) B55:223, 1997; ESTES PARK-96 p. 223

Tau Michel Parameters and Neutrino Helicity at SLD

ALBUQUERQUE-94, v. 1, p. 435; FERMILAB-CONF-94-341-E

Measurement of $W^{ \pm}$and $Z^{0}$ Production Cross-Sections in $p \bar{p}$ Collisions at $\sqrt{s}=1.8 \mathrm{TeV}$

Phys. Rev. C57:2118, 1998

Final State Interactions in deuteron $\left(\gamma, p p \pi^{-}\right)$Near the $\Delta\left(\mathbf{1 2 3 2} P_{33}\right)$ Resonance

Nucl. Phys. (PS) B40:255, 1995

Michel Parameters and $\tau^{ \pm}$Neutrino Helicity from Spin/Energy Correlations in $Z^{0} \rightarrow \tau^{+} \tau^{-}$at ALEPH

FERMILAB-CONF-95-150-E; CDF-PUB-BOTTOM-PUBLIC-3173; MCGILL-95-32 Rare $B$ Decays and Time Dependent Mixing at CDF

Phys. Rev. C57:1077, 1998

$n$ p Scattering Measurements at $162 \mathrm{MeV}$ and the $\pi$ nucleon nucleon Coupling Constant

FERMILAB-CONF-94-327-E

Search for Top in Do using the Electron + Jets Channel with Soft $\mu$ Tagging

FERMILAB-CONF-96-236-EF

Do Papers on Electroweak Physics Submitted to DPF 96: Measurement of $m\left(W^{ \pm}\right)$using the Transverse Mass Ratio of $W^{ \pm}$and $Z^{0}$

Phys. Rev. D49:3120, 1994; TRI-PP-93-14

An Experimental Search for a New Light Baryon

Phys. Lett. 338B:403, 1994; FERMILAB-PUB-94-015

Polarization of $\Lambda$ and $\bar{\Lambda}$ Produced by $800 \mathrm{GeV}$ Protons

Nucl. Phys. A587:802, 1995

Sideward Flow in $\mathrm{Au} \mathrm{Au}$ Collisions at $400 \mathrm{~A} \mathrm{MeV}$

Nucl. Phys. A577:83C, 1994

Charge-Exchange Spin-Dipole and Quasifree Excitations in $p$-Shell Nuclei

Nucl. Phys. A590:763, 1995; DAPNIA-SPHN-95-09

Cross Sections and Asymmetries for the $p\left(p, \pi^{\circ}\right) p p$ Reaction from Threshold to $1 \mathrm{GeV}$

Phys. Rev. C58:658, 1998

Complete Angular Distribution Measurements of $p p$ Spin Correlation Parameters $A_{x x}, A_{y y}$, and $A_{x z}$ and Analyzing Power $A_{y}$ at $187.4 \mathrm{MeV}$

Phys. Rev. C52:R445, 1995

Study of the Isospin Response of the ${ }^{4} \mathrm{He}$ Continuum using the ${ }^{4} \mathrm{He}\left(p, p^{\prime} \mathrm{X}\right)$ Reaction

Nuovo Cim. 107 A:2305, 1994

$\bar{p} p$ Annihilation in Flight

Phys. Rev. C49:627, 1994

Pion Elastic Scattering on ${ }^{28} \mathrm{Si}$ at $T_{\pi}=400 \mathrm{MeV}$

Phys. Rev. C55:2492, 1997

Search for Deeply Bound Pionic States in ${ }^{208} \mathrm{~Pb}$ Via Radiative Atomic Capture of $\pi^{-}$

Nucl. Phys. A626:63C, 1997

$p p \rightarrow$ deuteron $\pi+$ Precise Angular Distributions Close to Threshold from 0.3 to $5 \mathrm{MeV}$

Phys. Rev. C58:247, 1998; GSI-98-13; NUCLEX-9803014

Projectile Fragmentation of ${ }^{129} \mathrm{Xe}$ at $E_{l a b}=790 \mathrm{~A} \mathrm{MeV}$

Nucl. Phys. A612:493, 1997; NUCLEX-9610009

Central Collisions of $\mathrm{Au}$ on $\mathrm{Au}$ at 150,250 and $400 \mathrm{~A} \cdot \mathrm{MeV}$

HADRON $-95^{*}$ p. 374

Study of $f_{0}(1500)$ Decay into $\Sigma \Sigma$ in $\bar{p} p$ Annihilation at Rest

Nucl. Phys. (PS) B39:378, 1995 Contributions to $Q C D$ from ARGUS 
Retyk 97

Retzlaff 94

Reyes 97

Richard 94

Richichi 98

Richichi 98B

Richter 94

Richter 96

Rijssenbeek 96

Riley 99

Rimondi 94

Rivet 96

Rivet 98

Roberts 94

Roblin 95

Rohdjeb 98

Rohrich 96

Roland 94

Rolli 96

Romaides 94

Romosan 96

Roney 97

Rosati 94

Roser 95

Ross 95

Rosselet 96

Rouge 95

Rouge 97

Rubchn 95

Rubehn 96

Rubchn 96B

Rubehn $96 \mathrm{C}$

Ruckstuhl 95

Ruijter 96

Ruiz 97

Rusch 94

Rusek 95

Ruth 94

Ryabchikov 96
Jour. of Phys. G 23:1845, 1997

$\Xi$ Hyperon Production in Sulphur-Gold Interactions at $200 \mathrm{GeV} /$ nucleon

Phys. Rev. C49:1263, 1994

Threshold Electrodisintegration in the $A=3$ System

Phys. Rev. Lett. 81:4079, 1998; FERMILAB-PUB-97-223-E

Partial Wave Analysis of the Centrally Produced $K_{S} K_{S}$ System at $800 \mathrm{GeV} / \mathrm{c}$

LAL-94-50

Search for Heavy Neutral Higgs Bosons

CLNS-98-1573; CLEO-98-10; HEPEX-9810026

Study of 3-prong Hadronic $\tau$ Decays with Charged Kaons

Phys. Rev. D59:052003, 1999; CLNS-98-1576; CLEO-98-12; HEPEX-9808008

$\boldsymbol{\Upsilon}(1 S)$ Dipion Transitions at Energies Near the $\boldsymbol{\Upsilon}(4 S)$

Phys. Rev. C49:1001, 1994

Preequilibrium $(p, p)$ Measurements and Calculations for ${ }^{\circ 0} \mathrm{Zr}$ and Neighboring Nuclei for Incident Energies up to $200 \mathrm{MeV}$

Phys. Rev. C54:1756, 1996

Inclusive $\left(p, p^{\prime}\right)$ Reactions on Nuclei in the Mass Range 115 to 181 at Incident Energies from 120 to $200 \mathrm{MeV}$

FERMILAB-CONF-96-365-E

$W^{ \pm}$Mass from the Tevatron

Phys. Rev. C59:1780, 1999; UCI-98-37; HEPEX-9904001

Neutrino-Induced Deuteron Disintegration Experiment

FERMILAB-CONF-94-404-E

Study of the Structure of the Events Produced in Soft $\bar{p} p$ Interactions at $\sqrt{s}=1800 \mathrm{GeV}$

Phys. Lett. 388B:219, 1996; DAPNIA-SPHN-96-25 Vaporization Events from Binary Dissipative Collisions

Phys. Lett. 430B:217, 1998 Independence of Fragment Charge Distributions of the Size of Heavy Multifragmenting Sources

Phys. Rev. D50:1874, 1994

Search for the Decay $K_{L} \rightarrow \pi^{0} \pi^{0} \gamma$

Nucl. Phys. (PS) B39:95, 1995

Measurement of the Spin Structure Function $g_{1}^{n}$ of the Neutron

Phys. Rev. C57:2111, 1998

Elastic $p$ deuteron Scattering with $200-300 \mathrm{MeV}$ Protons

MANCHESTER-95 p. 420

Strangeness Production in Antiproton Proton Annihilations in Flight

Nucl. Phys. A566:527C, 1994

Rapidity and Transverse Momentum Dependence of the Two- $\pi^{-}$Correlation Function in $200 \mathrm{GeV} / \mathrm{Nucleon}$ $\mathrm{S}+$ nucleus Collisions

FERMILAB-CONF-96-141-E; CDF-DOC-PUBL-3703

Top Mass Measurement at CDF

Phys. Rev. D50:3608, 1994 Second Tower Experiment: Further Evidence for Newtonian Gravity

Phys. Rev. Lett. 78:2912, 1997; NEVIS-1529; HEPEX-9611013

A High Statistics Search for $\nu_{\mu}\left(\bar{\nu}_{\mu}\right) \rightarrow \nu_{e}\left(\bar{\nu}_{e}\right)$ Oscillations in the Small Mixing Angle Regime

Nucl. Phys. (PS) B55:67, 1997; ESTES PARK-96 p. 67 Review of Recent Neutral Current Results

Nucl. Phys. A566:597C, 1994

Particle Production in $p+A$ Collisions at $14.6 \mathrm{GeV} / \mathrm{c}$

FERMILAB-CONF-95-158-E; CDF-PUB-TOP-GROUP-3204

Top Decays in the Dilepton Channel at CDF

Nucl. Phys. (PS) B39:381, 1995

Recent CLEO B Results

Nucl. Phys. A610:256C, 1996

Interferometry Results from the CERN WA98 Experiment

X-LPNHE-95-01

Results on $\tau$ Neutrino from Colliders

Nucl. Phys. (PS) B55:189, 1997; ESTES PARK-96 p. 189

$r^{ \pm}$Decays Involving $\eta$ and $\omega$ Mesons in ALEPH

Z. Phys. A353:197, 1995; GSI-95-28 Electromagnetic Fission of ${ }^{238} \mathrm{U}$ at 600 and $1000 \mathrm{MeV}$ per Nucleon

Phys. Rev. C53:993, 1996

Charge Pickup of ${ }^{238} \mathrm{U}$ at Relativistic Energies

Phys. Rev. C53:3143, 1996

Total and Nuclear Fission Cross Section of ${ }^{238} \mathrm{U}$ at Relativistic Energies

Phys. Rev. C54:3062, 1996

Scaling Laws in ${ }^{3} \mathrm{He}$ Induced Nuclear Fission

Nucl. Phys. (PS) B40:371, 1995; NIKHEF-H-94-33; MONTREUX-94 p. 371

Tau Decays into Kaons in DELPHI

Phys. Rev. C54:3076, 1996

Angular Distributions for the ${ }^{12} \mathrm{C}(\gamma, p){ }^{11}$ Bor Reaction

Nucl. Phys. (PS) B55:249, 1997; ESTES PARK-96 p. 249

Tau Decays to Hadronic States with $\pi^{0}$

Phys. Rev. C49:901, 1994

Charge Correlations and Transverse Momenta Observed in Multifragmentation of $1 \mathrm{GeV} / \mathrm{Nucleon}$ Au Projectiles

Phys. Rev. C52:1580, 1995

Search for $p$ Dibaryon-Nucleus Bound States in Relativistic $\mathrm{Au}+\mathrm{Pt}$ Collisions

Phys. Rev. Lett. 72:617, 1994

First Measurement of the Reaction ${ }^{3} \mathrm{He}(\gamma \uparrow, p) \mathrm{X}$ with Polarized Photons

MANCHESTER-95 p. 192

Study of Exotic Mesons on the VES Setup

See the legend on page 5. 
Rybicki 95

Ryckbosch 94

Rzehorz 96

Sadovsky 94

Sagara 94

Sahu 95

Sahu 95B

Saito 94

Sakaguchi 98

Sakai 94

Sakai 98

Sakamoto 96

Sakamoto 99

Sakemi 94

Sakemi 95

Sakuda 93

Sakyumoto 94

Salgado 96

Sampsonidis 95

Sampsonidis 95B

Sams 95

Sanchez $9 \pi$

Sangster 95

Sansoni 95

Sansoni 96

Sapienza 98

Sapozhnikov 94

Sapozhnikov 94B

Sapozhnikov 95

Sarkisyan 95

Sarsa 96

Sarsa 97

Sato 98

Saunders 96

Savinov 95

Sawada 97

Sawafta 98

Schafer 95

Schellman 94

Schenk 96
Phys. Lett. 353B:547, 1995; BRU-PH-201

Momentum Correlations of Charmed Pairs Produced in $\pi^{-} \mathrm{Cu}$ Interactions at $230 \mathrm{GeV} / \mathrm{c}$

Nucl. Phys. A568:52, 1994

Absorption of Intermediate-Energy Photons by Multi-Particle Clusters in ${ }^{8} \mathrm{Li}$

Eur. Lett. 34:103, 1996

Evidence for Pion Absorption on Four Nucleons

Phys. At. Nucl. 57:1526, 1994; Yad. Phys. 57:1600, 1994 New GAMS Results on Meson Spectroscopy

Phys. Rev. C50:576, 1994

Energy Dependence of Analyzing Power $A_{y}$ and Cross Section for $p$ +deuteron Scattering below $18 \mathrm{MeV}$

Phys. Lett. 346B:208, 1995; KEK-94-184; SAGA-HE-75

A. High $Q^{2}$ Measurement of the Photon Structure Function $F_{2}^{(\gamma)}$

KEK-95-18

A High $Q^{2}$ Measurement of the Photon Structure Function $F_{2}^{(\gamma)}$

Phys. Rev. C49:3211, 1994

Composite Particle Production in Relativistic Au+Pt, Si+Pt and $p+\mathrm{Pt}$ Collisions

Phys. Rev. C57:1749, 1998

Elastic Scattering of Polarized Protons from ${ }^{38} \mathrm{Ni}$ at $E_{p}=192,295$, and $400 \mathrm{MeV}$

Nucl. Phys. A577:111C, 1994

Measurement of Quasi-Elastic Scattering and $D_{N N}\left(0^{\circ}\right)$ with $(p, n)$

Nucl. Phys. A631:757C, 1998; GATLINBURG-97 p. 757C Cross Sections and Complete Polarization Transfer Measurements for the deuteron $(p, n) p p$ Reaction at $346 \mathrm{MeV}$

Phys. Lett. 367B:60, 1996

Measurement of the Vector and Tensor Analyzing Power for the deuteron $p$ Elastic Scattering at $E_{d e u t}=$ $270 \mathrm{MeV}$

Phys. Rev. C59:1497, 1999

Mass Yield Features $\left(\gamma, \pi^{+}\right)$and $\left(\gamma, \pi^{-} x n\right)$ Reactions for $x=0-\theta$ on Complex Nuclei in the Delta Region

Nucl. Phys. A577:33C, 1994

Spin Excitation Strength from Proton Inelastic Scattering at Zero

Phys. Rev. C51:3162, 1995

Zero-Degree Proton Inelastic Scattering to the $1^{+}, T=0$ and $T=1$ States in ${ }^{12} \mathrm{C}$

Nuovo Cim. 107A:2389, 1994; KEK-93-124

New Results from TRISTAN Experiments

FERMILAB-CONF-94-265-E

Test of Structure Functions using Lepton Pairs: $W^{ \pm}$-Charge Asymmetry at CDF

MANCHESTER-95 p. 503

Nuclear Effects in Vector Meson Production by Lepton Scattering off Nuclei

Phys. Rev. C51:3304, 1995

Fragmentation Cross Sections of ${ }^{16} \mathrm{O},{ }^{24} \mathrm{Mg}$ and ${ }^{32} \mathrm{~S}$ Projectiles at $3.65 \mathrm{GeV} /$ Nucleon

Rad. Meas. 25:231, 1995 Projectile Fragmentation of ${ }^{16} \mathrm{O}$ and ${ }^{32} \mathrm{~S}$ at $3.65 \mathrm{GeV} / \mathrm{n}$ on Several Targets

Phys. Rev. C51:1945, 1995

Quasifree (deuteron $\uparrow,{ }^{2} \mathrm{He}$ ) Data

Nucl. Phys. (PS) B55:33, 1997; ESTES PARK-96 p. 33

Measurement of the Transverse Spin Correlations in the Decay $Z^{0} \rightarrow \tau^{+_{\tau}-}$

Phys. Rev. C51:1280, 1995

Correlations of Intermediate Mass Fragments from $\mathrm{Fe}+\mathrm{Ta}, \mathrm{Au}$, and $\mathrm{Th}$ Collisions

Nuovo Cim. 109A:827, 1996; FERMILAB-CONF-95-263-E; CDF-PHYS-BOTTOM-PUBLIC-3270 Quarkonia Production at Fermilab

Nucl. Phys. A610:373C, 1996; FERMILAB-CONF-96-221-E; CDF-PUB-BOTTOM-PUBLIC-3804 Quarkonia Production at CDF

Nucl. Phys. A630:215C, 1998; GATLINBURG-97 p. 215C

Hard Photon and Energetic Proton Emission in Heavy Ion Collisions at Intermediate Energy

Nuovo Cim. 107A:2315, 1994

Study of the OZI-Rule Violation in Antiproton Annihilation in Deuterium at Rest

JINR-E15-94-501

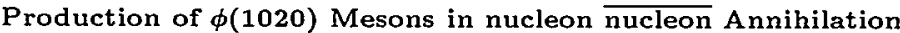

JINR-E15-95-544

Production of $\phi(1020)$-Mesons in $\overline{\text { nucleon }}$ nucleon Annihilation and Polarized Strangeness in the Nucleon

Phys. Lett. 347B:439, 1995; TBILISI-HE-7-94-E

On Dynamics of Pseudorapidity Fluctuations in Central $\mathrm{C} \mathrm{Cu}$ Collisions at $4.5 \mathrm{~A} \mathrm{GeV} / \mathrm{c}$

Phys. Lett. 386B:458, 1996

Searching for Annual Modulation of WIMPs with NaI Scintillators

Phys. Rev. D56:1856, 1997

Results of a Search for Annual Modulation of WIMP Signals

Nucl. Phys. A639:279C, 1998; UPTON-97 p. 279C

$\pi^{-}$Mesonic Weak Decay width of ${ }_{\Lambda}^{12} \mathrm{C}$

Phys. Rev. C53:1745, 1996

Reaction and Total Cross Sections for Low Energy $\pi^{+}$and $\pi^{-}$on Isospin Zero Nuclei

HEPEX-9507005

A Measurement of the Form Factors of Light Pseudoscalar Mesons at a Large Momentum Transfer

Nucl. Phys. A615:277, 1997; KEK-96-155

Differential Cross Sections for $n p \rightarrow$ deuteron $X$ at 1 and $2 \mathrm{GeV}$

Nucl. Phys. A639:103C, 1998; UPTON-97 p. 103C

Recent Experimental Results in $\Sigma$ Hypernuclei

Phys. Rev. A51:838, 1995

Vacuum Polarization as a Test of $C$ and $C P T$ Invariance

FERMILAB-CONF-94-219-E

Nuclear $A$ Dependence of Exclusive Vector Meson Production in Muon Scattering

MANCHESTER-95 p. 352

Baryon Production Measurement from OPAL 
Schmid 94

Schmid 95

Schmid 96

Schmid 96B

Schmid 97

Schmid 97B

Schmidtler 95

Schmidtsoren 98

Schmitt 96

Schmitt 96B

Schmitt 97

Schreckenbac 95

Schroeter 94

Schub 95

Schumm 95

Schuttauf 96

Schutz 97

Schutz 98

Schwab 98

Schwalb 94

Schwiening 95

Schwingenheu 95

Sciolla 96

Sedlar 99

Seligman 97

Seligman 97B

Seligman $97 \mathrm{C}$

Selke 96

Semenov 94

Semenov 96

Semenov 96B

Setze 96

Setzu 98

Severijns 93

Sewerin $98 \mathrm{~B}$

Seyboth 97

Shakhbazyan 94

Shakhbazyan 95

Shakhbazyan 96
Nucl. Phys. A569:689, 1994

Absolute Probabilities of Fission Induced by Stopped Antiprotons

Phys. Rev. C52:R1732, 1995

Polarized Proton Capture by Deuterium and the deuteron $(p, \gamma){ }^{3} \mathrm{He}$ Astrophysical $S$ Factor

Phys. Rev. Lett. 76:3088, 1996; Phys. Rev. Lett. 77:586, 1996; CEBAF-TH-95-19

Effects of Non-Nucleonic Degrees of Freedom in the deuteron $(p \uparrow, \gamma){ }^{3} \mathrm{He}$ and $p$ (deuteron $\left.\uparrow, \gamma\right){ }^{3} \mathrm{He}$ Reactions

Phys. Rev. C53:35, 1996

$T_{20}$ Measurements for ${ }^{1} H$ (deuteron $\left.T, \gamma\right){ }^{3} \mathrm{He}$ and the $P$-Wave Component of the Nucleon-Nucleon Force

Z. Phys. A359:27, 1997

Probability of Fission Induced by $1.2 \mathrm{GeV}$ Antiprotons

Phys. Rev. C55:2965, 1997

Neutrons in Coincidence with Fission of U238 Induced by Stopped Antiprotons

Nucl. Phys. (PS) B40:265, 1995

Determination of the Michel Parameters $\chi$ and $\delta$ in Leptonic $\tau^{ \pm}$Decays

Nucl. Phys. A638:471C, 1998; TSUKUBA-97 p. $471 \mathrm{C}$

Three Pion Correlations in Heavy Ion Collisions at the CERN SPS (NA44)

MANCHESTER-95 p. 199

Observation of $\Omega_{c}$ Decays in the Hyperon Beam Experiment WA89

Nuovo Cim. 109A:851, 1996; PISA-95 p. 851

Beauty Semi-Leptonic Branching Ratios

Phys. Rev. C56:1687, 1997

Deuteron Threshold Electrodisintegration at High Momentum Transfer

Phys. Lett. 349 B:427, 1995

A New Measurements of the Beta Emission Asymmetry in Free Decay of Polarized Neutrons

Z. Phys. A350:101, 1994

Subthreshold Antiproton and $K^{-}$Production in Heavy Ion Collisions

Phys. Rev. D52:1307, 1995; Phys. Rev. D53:570, 1996; FERMILAB-PUB-95-058-E

Measurement of $J / \psi(1 S)$ and $\psi(2 S)$ Production in $800 \mathrm{GeV} / \mathrm{c}$ Proton-Gold Collisions

Nucl. Phys. (PS) B40:497, 1995; MONTREUX-94 p. 497

Precise Measurements of the Left-Right Cross Section Asymmetry in $Z^{0}$ Boson Production by $e^{+} e^{-}$ Collisions

Nucl. Phys. A607:457, 1996; GSI-96-26; NUCLEX-9606001

Universality of Spectator Fragmentation at Relativistic Bombarding Energies

Nucl. Phys. A622:404, 1997; GANIL-P-97-15

Hard Photons and Neutral Pions as Probes of Hot and Dense Nuclear Matter

Nucl. Phys. A630:126C, 1998; GRONINGEN-97 p. 126C

Thermalizing Nuclear Matter Probed by Hard Photons

Eur. Phys. J. A2:179, 1998

Fission of Highly Excited Fragments from Collisions of $750 \mathrm{~A} \cdot \mathrm{MeV}{ }^{238} \mathrm{U}$ Ions on ${ }^{208} \mathrm{~Pb}$

Phys. Lett. 321B:20, 1994

Mass Dependence of $\pi^{0}$-Production in Heavy Ion Collisions at $1 \mathrm{~A} \cdot \mathrm{GeV}$

BONN-IR-95-13

Eine Stude zur Produktion und Bose - Einstein - Korrelation neutraler Kaonen in Hadronischen $Z^{\circ}$ Zerfallen mit dem OPAL - Detektor

Phys. Rev. Lett. 74:4376, 1995

$C P T$ Tests in the Neutral Kaon System

MANCHESTER-95 p. 485

Observation of Excited $B$ Hadron in DELPHI

Phys. Rev. C59:789, 1999

Gamow-Teller Strength in $(n, p)$ Charge Exchange on ${ }^{31} \mathrm{Ph}$

HEPEX-9701017

A New Determination of $\alpha_{s}$ from Neutrino-Nucleon Scattering

NEVIS-292

A Next-to-leading Order QCD Analysis of Neutrino Iron Structure Function at the Tevatron

Phys. Rev. Lett. 79:1213, 1997

Improved Determination of $\alpha_{s}$ from Neutrino-Nucleon Scattering

Phys. Lett. 369B:207, 1996

Compton Scattering from ${ }^{4} \mathrm{He}$ in the $\Delta(1232)$ Resonance Region

ITEP-94-3

Recent ARGUS Results on Charm Physics

Yad. Phys. 59:1573, 1996; Phys. At. Nucl. 59:1514, 1996; ITEP-96-18

Hadrons with Heavy Quarks (Experimental Review)

Nucl. Phys. (PS) 50:183, 1996; ITEP-96-19

ARGUS Results on Charmed Hadrons

Phys. Lett. 388B:229, 1996

Veriflcation of the Space-Star Anomaly in $n$ deuteron Breakup

Phys. Lett. 420B:205, 1998

Measurement of the Decay $K_{L} \rightarrow e^{+} e^{-} \gamma \gamma$

Phys. Rev. Lett. 70:4047, 1993; Phys. Rev. Lett. 73:611, 1994

Limits on Right-Handed Charged Weak Currents from a Polarization Asymmetry Correlation Experiment with 107 In

FZJ-IKP-1-98-3; NUCLEX-9811004

Comparison of $\Lambda$ and $\Sigma^{0}$ Threshold Production in Proton-Proton Collisions

Jour. of Phys. G 23:1787, 1997

Strange Particle Production in Ultrarelativistic Heavy Ion Collisions at the CERN SPS

Nuovo Cim. 107 A:2459, 1994

Evidence for Light dibaryon $(S=-2)$ and Heavy dibaryon $(S=-2)$, dibaryon $(S=-2)+$ Stable $S=-2$ Dibaryons

Kr. Soob. JINR 69:61, 1995

An Evidence for the Exited State of the $S=-2$ Stable Light Dibaryon

MANCHESTER-95 p. 509

Evidence for the Excited States of the $S=-2$ Stable Light Dibaryon

See the legend on page 5 . 
Shaoshun 95

Shaoshun 98

Sharov 96

Shaw 9.5

Shelkov 93

Shelkov 97

Sherif 95

Shevchenko 94

Shigaki 95

Shimizu 95

Shin 98

Shiozawa 98

Shirai 94

Shivpuri 94

Shivpuri $94 \mathrm{~B}$

Shivpuri $94 \mathrm{C}$

Shivpuri 95

Shivpuri $95 \mathrm{~B}$

Shmakov 95

Shochet 94

Siebert 94

Sigg 95

Simic 95

Simic 96

Simic 98

Simon 96

Sims 96

Sinclair 98

Sinervo 95

Sinervo 96

Singh 94

Singh $94 \mathrm{~B}$

Singh $94 \mathrm{C}$

Singh 96

Singh 97

Singh 98

Singh $98 \mathrm{~B}$

Singovsky. 94
Phys. Lett. 353B:391, 1995

The Study of the Azimuthal Structure of Particles Produced in $p$ Collisions at $400 \mathrm{GeV} / \mathrm{c}$

Phys. Lett. 416B:216, 1998

The Measurement of Entropy Indices in $p$ Collisions at $400 \mathrm{GeV} / c$

Kr. Soob. JINR 77:13, 1996

Measurements of the $n \uparrow p \uparrow$ Total Cross Section Differences for Pure Helicity States at $1.20,2.50$ and

3.86 GeV
Phys. Rev. C52:199, 1995

Negative Pion Photoproduction from ${ }^{15} \mathrm{Nit}$ in the Region of the Delta Resonance

Phys. Rev. D50:4265, 1994; CLEO-93-19; CLNS-93-1250

Measurement of Two-Photon Production of the $\chi_{c 2}(1 P)$

Nucl. Phys. (PS) B55:195, 1997; ESTES PARK-96 p. 195

Tau Decays Involving $\eta$ and $\tau^{ \pm} \rightarrow f_{1}(1285) \pi \nu_{\tau}$ from CLEO

Phys. Scr. 51:431, 1995 Central Collisions of $4.5 \mathrm{GeV} / \mathrm{c}$ per Nucleon ${ }^{16} \mathrm{O}$ and ${ }^{28} \mathrm{Si} N u c l e i$ in Nuclear Emulsion

CALT-68-1977

Search for Rare $Z^{0}$ Decays at LEP

Nucl. Phys. A590:519C, 1995

Study of Hadron Production in Au Au Collisions at 11 A GeV/c with the AGS-E868 Forward Spectrometer

Phys. Rev. C52:1193, 1995

Analyzing Power of $p+$ deuteron Scattering below the Deuteron Breakup Threshold

Phys. Rev. Lett. 81:1576, 1998; GSI-PREPRINT-98-35; NUCLEX-9807003

Enhanced Out-of-Plane Emission of $K^{+}$Mesons Observed in Au Au Collisions at $1 \mathrm{~A} \cdot \mathrm{GeV}$

Phys. Rev. Lett. 81:3319, 1998; ICRR-419-98-15; SBHEP98-4; UCI-98-12; KEK-98-46; LSU-HEPA-4-98; UMD 98-115; OULNS 98-03; TKU-PAP-98-04; TIT-HPE-98-07; UWSEA PUB 98-05; HEPEX-9806014

Search for Proton Decay via $p \rightarrow e^{+} \pi^{0}$ in a Large Water Cherenkov Detector

Phys. Rev. Let t. 72:3313, 1994; KEK-93-189; HUPD-9331; KOBE-HEP-94-02; KUNS-1243; OUHEP-94.2; OULNS-93-08; TMU-HEP-94-2

Search for a Light Scalar Top in $e^{+} e^{-}$Reaction at $E_{\mathrm{cm}}=58 \mathrm{GeV}$

Phys. Rev. D 49:219,1994

Target and Energy Dependence of Intermittency and Multifractality in Interactions at Cosmic Ray Energies

Z. Phys. C62:609, 1994

Nuclear Effects in Multiparticle Production in $800 \mathrm{GeV}$ and $400 \mathrm{GeV}$ Proton-Nucleus Interactions

Phys. Rev. D50:287, 1994

Fractal Behaviours in Proton Nucleus Interactions at $800 \mathrm{GeV}$

Phys. Rev. C51:1367, 1995

Multifractal Structure of Medium Energy Particles in $p$ Ag-Br Interactions at $800 \mathrm{GeV}$

2. Phys. C66:403,1995

Fractals in One and Two Dimensions of Medium Energy Particles in $800 \mathrm{GeV} p$ Ag-Br Interactions

Yad. Phys. 58:1735, 1995; Phys. At. Nucl. 58:1635, 1995

On the Time Scale of Multifragment Emission in ${ }^{4} \mathrm{He}+\mathrm{Au}$ Collisions at $E / A=3.65 \mathrm{GeV} / \mathrm{Nucleon}$

FERMILAB-CONF-94-276-E

Physics at the Fermilab Collider

Nucl. Phys. A567:819, 1994

High Resolution Study of Hyperon-Nucleon Interactions by Associated Strangeness Production in $p$ Collisions

Phys. Rev. Lett. $75: 3245,1995$; ETIIZ-IPP.PR-95-4

The Strong Interaction Shift and Width of the $1 \mathrm{~s}$ Level in Pionic Hydrogen

Phys. Rev. C52:356, 1995

Centrality Dependence of Pion and Proton Spectra in C+C and $\mathrm{C}+\mathrm{Ta}$ Interactions at $4.2 \mathrm{GeV} / \mathrm{c}$ per Nucleon

Phys. Rev. C54:1887, 1996

Two Particle Rapidity Correlations in C Ta Interactions at $4.2 \mathrm{~A} \mathrm{GeV/c}$

Phys. Rev. C58:2585, 1998; IF-10-98; NUCLEX-9804005

Proton Stopping in C C, deuteron C, C Ta and deuteron Ta at $4.2 \mathrm{~A} \mathrm{GeV/c}$

Phys. Rev. C53:30, 1996 Absolute $p p$ Elastic Cross Sections from 492 to $793 \mathrm{MeV}$ using $\mathrm{CH}_{2}$ Target

Phys. Lett. $442 \mathrm{~B}: 43,1996$

The ${ }^{4} \mathrm{He}(\gamma, n)$ Reaction: a Potential Testing Ground for the Alpha-Particle Wave Function

GLAS-PPE-98-01; HEPEX-9805010

Dynamics of Multijet Processes in Photoproduction

FERMILAB-CONF-95-371-E; UTPT-95-25; CDF-PUB-TOP-PUBLIC-3411

The Discovery of the Top Quark

FERMILAB-CONF-96-223-E

Top Quark Studies at Hadron Colliders

Z. Phys. A348:99, 1994

Production of Helium Fragments in ${ }^{197}$ Au-Emulsion Collisions

Phys. Rev, C50:2508, 1994

Multifractal Analysis of ${ }^{187}$ Au-Emulsion Collisions at $10.6 \mathrm{~A} \cdot \mathrm{GeV}$

Nucl. Phys. A570:819, 1994

Some General Characteristics of ${ }^{28} \mathrm{Si}$-Emulsion Interactions at $4.5 \mathrm{GeV} / \mathrm{c}$ per Nucleon

Phys. Rev. C54:3185, 1996

Target and Projectile Fragmentations in ${ }^{208} \mathrm{~Pb}$-Emulsion Collisions at $180 \mathrm{~A} \mathrm{GeV}$

Jour. of Phys. G 23:1655,1997

Azimuthal Correlation of Multifragments of ${ }^{208} \mathrm{~Pb}$ Nuclei at $160 \mathrm{~A} \cdot \mathrm{Gev}$

FERMILAB-CONF-98-209-E; HEPEX-9807022

Discovery of the $B_{c}$ Meson

Int. Jour. Mod. Phys. E7:341, 1998

On $Z=2$. Projectile Fragments Emitted in the Interactions of $3.7 \mathrm{~A} \cdot \mathrm{GeV}{ }^{28} \mathrm{Si}$ in $\mathrm{Nuclear}$ Emulsion

Nuovo Cim. 107A:1911, 1994

The $\eta \eta$ Systems Produced in $450 \mathrm{GeV} / c$ Central $p$ Collisions 
Sivertz 95

Skalsey 95

Skank 96

Skarha 94

Skarha 95

Skrzypczak 98

Slaughter 96

Slegt 95

Slypen 95

Slypen 96

Smith 94

Smith $94 \mathrm{~B}$

Smith 94C

Smith 96

Smith 98

Smith $98 \mathrm{~B}$

Snihur 96

Snowdenifft 95

Sobchak 95

Sobchak 95B

Sobie 97

Sollfrank 94

Solomey 98

Soni 94

Sonnadara 94

Sopczak 98

Sosin 94

Spaan 96

Spadafora 94

Spaltro 98

Spanier 94

Spanier 96

Spanier $96 \mathrm{~B}$

Speer 96

Spencer 95

Spengler 94

Spentzouris 94

Sphicas 94
Nucl. Phys. (PS) B39:179, 1995; MONTPELLIER-94 p. 179

QCD Studies in $e^{+} e^{-}$Interactions at CLEO

Phys. Rev. D51:6292, 1995

Search for Exotic Decays from Low-energy $e^{+} e^{-}$Direct Annihilation

Nucl. Phys. A610:297C, 1996

Strangelets, Antimatter and Coalescence: First Results from BNL E864

FERMILAB-CONF-94-224-E

$B$-Meson Lifetimes at CDF

FERMILAB-CONF-95-135-E

CDF Results on $B$ Decays

Phys. Rev. Lett. 80:4136, 1998; LBNL-41016; NUCLEX-9711001

Directed and Elliptic Flow in $158 \mathrm{GeV} / \mathrm{Nucleon} \mathrm{Pb}+\mathrm{Pb}$ Collisions.

Nuovo Cim. 109A:607, 1996; PISA-95 p. 607

Hadroproduction and Photoproduction of Beauty and Charm in Fixed-Target Experiments

Nucl. Phys. A590:469C, 1995

Momentum Distributions and Interferometry Analysis of Negatively Charged Hadrons from $200 \mathrm{~A} \mathrm{GeV}$ $\mathrm{S}+$ A u Reactions

Phys. Rev. C51:1303, 1995

Proton and Deuteron Production in Neutron-Induced Reactions on Carbon at $E_{n}=42.5,62.7$, and $72.8 \mathrm{MeV}$

Phys. Rev. C53:1309, 1996

Triton and Alpha-Particle Production in Neutron-Induced Reactions on Carbon at $E=42.5,62.7$ and $72.8 \mathrm{MeV}$

Nucl. Phys. (PS) B40:351, 1995; MONTREUX-94 p. 351; COLO-HEP-350

Studies of Tau Decay Modes with $K^{\mathbf{0}}$ Mesons from CLEO

Nuovo Cim. 107A:2163, 1994

Measurements of the Magnetic Moment of the $\Sigma^{+}$and $\bar{\Sigma}^{-}$Hyperons and Hyperon Radiative Decays

Nuovo Cim. 107A:2221, 1994

Polarization of $\boldsymbol{\Sigma}^{+}$and $\overline{\boldsymbol{\Sigma}}^{-}$Hyperons Produced in High-Energy Proton-Nucleus Collisions

Phys. Lett. 379B:299, 1996

Improved Dark Matter Limits from Pulse Shape Discrimination in a Low Background Sodium Iodide Detector at the Boulby Mine

COLO-HEP-395; HEPEX-9803028

First Observation of Five Charmless Hadronic $B$ Decays

Phys. Rev. C58:3484, 1998

Analyzing Powers for $\pi^{ \pm} p$ Elastic Scattering between 87 and $263 \mathrm{MeV}$

FERMILAB-CONF-96-304-EE

Do Papers on QCD Studies with Jets: Subject Structure of Jets at Do

Phys. Rev. Lett. 74:4133, 1995

Limits on Dark Matter using Ancient Mica

Phys. At. Nucl. 59:1928, 1996; Yad. Phys. 59:2001, 1996; JINR-P1-95-329

Study of the ${ }^{4}$ He $p$ Pionless Reactions

Yad. Phys. 58:1017, 1995; Phys. At. Nucl. 58:944, 1995

Invariant Differential Cross Sections on Nucleons in ${ }^{4} \mathrm{He} p$ Interactions

Nucl. Phys. (PS) B55:87, 1997; ESTES PARK-96 p. 87

Measurements of the Leptonic Branching Ratios with OPAL

Z. Phys. C61:659, 1994; TPR-93-14

Chemical Freeze-out Conditions in Central S-S Collisions at $200 \mathrm{~A} \cdot \mathrm{GeV}$

Nucl. Phys. A639:287C, 1998; UPTON-97 p. 287C

Semi-Leptonic Decays of the $\Xi^{0}$ Hyperon

Z. Phys. A348:311, 1994

Some Characteristics of High Multiplicity ( $\geq 45$ ) Proton-AgBr Interactions at $800 \mathrm{GeV}$

Nucl. Phys. A569:149C, 1994

Electromagnetic Dissociation of Relativistic Si via Giant Dipole Resonance

Yad. Phys. 61:1030, 1998; Phys. At. Nucl. 61:938, 1998

Searches for Higgs Bosons at LEP 2

Nucl. Phys. A574:474, 1994

Intermediate-Mass Fragments in ${ }^{14} \mathrm{Nit}+{ }^{159} \mathrm{~Tb} / \mathrm{Ag}_{\mathrm{nat}} / \mathrm{Cu}_{\text {nat }}$ Reactions at $22 \mathrm{MeV} / u$

MANCHESTER-95 p. 206

Measurements of Form Factor Ratios and Shapes in $D$ and $B$ Meson Decays

TSUKUBA-93 p. 97; FERMILAB-CONF-94-016-E

Study of Associated Gauge Boson Production a D0: $W^{ \pm} \gamma$ Production

Phys. Rev. Lett. 81:2870, 1998

$q$ and $p_{m}$ Dependence of the ${ }^{3} \mathrm{He}\left(e^{-}, e^{-1}\right.$ deuteron) $p$ Reaction

Nuovo Cim. 107A:2321, 1994

Partial-Wave Analysis of $\pi \pi \eta$ in $\bar{p} p$ Annihilation at Rest

MANCHESTER-95 p. 213

Spectroscopy of Scalar Mesons

Phys. At. Nucl. 59:1319, 1996; Yad. Phys. 59:1378, 1996

Observation of $\eta(1440)$ Decay in $\pi \pi \eta$ in the Reaction $\bar{p} p \rightarrow \eta(1440) \pi \pi$

FERMILAB-CON F-96-320-E

Search for $B_{c}^{+} \rightarrow J / \psi(1 S) \pi^{+}$and the $B$ Rare Decays $B^{0} \rightarrow \mu^{+} \mu^{-}$and $B_{s} \rightarrow \mu^{+} \mu^{-}$at CDF

Phys. Rev. Lett. 74:3323, 1995; FERMILA B-PUB-95-043-E

A Measurement of the Branching Ratio and Form-Factor of $K_{L} \rightarrow \mu^{+} \mu^{-} \gamma$

Nuovo Cim. 107 A:2045, 1994

New Results on Charmed Baryons from ARGUS

FERMILA B-CONF-94-220-E

Structure Functions and Structure Functions Ratio $F_{2}^{n} / F_{2}^{p}$ at Small $x_{B_{j}}$ and $Q^{2}$ in Muon-Nucleon Scattering

Nucl. Instr. and Meth. A351:68, 1994

Status of the Search for the Top Quark 
Spurio 98

Stahl 95

Stasko 94

Stefanini 95

Steinkamp 94

Stephans 97

Stephens 95

Stephenson 97

Steyn 99

Stichelbaut 96

Stocker 95

Stoeff 95

Stotzer 97

Strassburger 94

Strausz 97

Streets 95

Strohmer 94

Stroot 95

Strovink 95

Strovink 96

Strugalskago 94

Strugalskago 96

Strugalski 96

Stuart 94

Stuart 98

Stugu 95

Su 94

Su 96

Suft 98

Sugaya 97

Sugimoto 95

Suleimanov 98

Suleimanov 98B

Summerer 95

Summerer 95B

Suzuki 95

Svec 96

Swain 97

Swain $97 \mathrm{~B}$
DFUB-98-17; HEPEX-9808001

Low Energy Atmospheric $\nu_{\mu}$ in MACRO

Nucl. Phys. (PS) B40:505, 1995; MONTREUX-94 p. 505

$C P$ Violation in the Decay $Z^{0} \rightarrow \tau^{+} \tau^{-}$

Phys. Rev. Lett. 72:973, 1994

Radiative Decay of $\Delta\left(1232 P_{33}\right)$ Resonance: Analyzing Powers for $\pi^{-} p \rightarrow \gamma n$

Phys. Rev. C52:R1727, 1995

Signatures of Multiphonon and Neutron Transfer Couplings in the Fusion of ${ }^{38} \mathrm{~S},{ }^{32} \mathrm{~S}+{ }^{110} \mathrm{Pd}$

Nuovo Cim. 107A:2329, 1994

Results from $\bar{p} p \rightarrow \phi(1020) \phi(1020)$

Jour. of Phys. G 23:1895, 1997

Strange, Anti-, and Anti-Strange Baryons at AGS Energies

LNF-95-051-PR

Ground Level Cosmic Ray Observations

Phys. Rev. Lett. 78:1636, 1997

Effective Isovector $N N$ Interaction Strengths Determined from the ${ }^{28} \operatorname{Si}\left(\vec{p}, \vec{p}^{\prime}\right)^{28} S i\left(\theta^{-}, T=1\right)$ Reaction

Phys. Rev. C59:2097, 1999

He-Clustering Probabilities Extracted from the ${ }^{12} \mathrm{C}$ ( $\mathrm{He}, 2 \mathrm{He}$ ) ${ }^{8} \mathrm{Be}$ Reaction at $200 \mathrm{MeV}$

Mod. Phys. Lett. A11:2809, 1996

Study of $e^{+} e^{-}$Interactions at Center-of-Mass Energies of 130 and $136 \mathrm{GeV}$

Nucl. Phys. A590:271C, 1995

Physics in the Baryon-Rich Regime

Phys. Rev. Lett. 75:3237, 1995

Anomalous Structure in the $\beta$-Decay of Gaseous Molecular Tritium

Phys. Rev. Lett. 78:3646, 1997

Search for the dibaryon $(S=-2)$ Dibaryon in ${ }^{3} \mathrm{He}\left(K^{-}, K^{+}\right)$dibaryon $(S=-2) n$

Nuovo Cim. 107 A:2339, 1994

Antiproton Annihilation at Rest in Liquid Deuterium into $\pi^{-} \pi^{0} \pi^{0} p_{s}$

Phys. Rev. D55:4566, 1997 Evidence for Supersymmetric Dark Matter Annihilations into $\gamma$ Rays

FERMILAB-CONF-95-116-E

Jet Shapes at DO and CDF

Nuovo Cim. 107 A:2055, 1994

A Test of the Flavor Independence of the Strong Interaction for Five Flavors

Nucl. Phys. (PS) B39:284, 1995

Scalar and Exotics Mesons at GAMS

FERMILAB-CONF-95-189-E; D0-2611

Do Top Quark Mass Analysis

FERMILAB-CONF-96-336-E

Do Top Quark Mass Analysis

JINR-E1-94-296

Observations of Fast Hadron Passages through Intranuclear Matter

JINR-E1-96-330

On Pion-Production Intensities in Hadron-Nucleus Collisions

JIN R-E1-96-331

Observations and Determinations of the Hadron Mean Free Paths for Particle-Producing Collision Reactions in Intranuclear Matter

Phys. Rev. D49:3098, 1994

Forward Backward Charge Asymmetry of Quark Pairs Produced at the KEK TRISTAN $e^{+} e^{-}$Collider

Phys. Rev. D58:032003, 1998

Measurements of the $\Delta(1232)$ Transition Form Factor and the Ratio $\sigma_{n} / \sigma_{p}$ from Inelastic Electron-Proton and Electron-Deuteron Scattering

Nucl. Phys. (PS) B40:289, 1995

Upper Limit on the Decays $\tau^{ \pm} \rightarrow e^{ \pm} \gamma$ and $\tau^{ \pm} \rightarrow \mu^{ \pm} \gamma$ using Data from DELPHI

Phys. Rev. D50:3614, 1994; Phys. Rev. D51:3135, 1995 New Tests of the Universality of Free Fall

Nuovo Cim. 109A:663, 1996; SLAC-PUB-95-7000; PISA-95 p. 663

Production of Charm and Beauty in $e^{+} e^{-}$with Polarized Electron Beam

Phys. Lett. 425B:19, 1998

Polarization Transfer Observables in $\pi$ deuteron Elastic Scattering

Nucl. Phys. A634:115, 1998; KEK-97-92

Subthreshold Antiproton Productions in $p \mathbf{A}$, deuteron $\mathbf{A}$ and $\alpha \mathbf{A}$ Reactions

Phys. Lett. 369B:86, 1996; KEK-95-171

New Limits on the Masses of the Selectron and Photino

JINR-E1-98-328; HEPEX-9901016

Centrality of Collisions and Total Disintegration of Nuclei

Kr. Soob. JINR 91:41, 1998

Inclusive Spectra of Protons and $\pi^{-}$Mesons Emitted in ${ }^{4} \mathrm{He} \mathrm{C}$ and ${ }^{12} \mathrm{C} \mathrm{C}$ Interactions with Total Disintegration of Nuclei

Phys. Rev. C52:1106, 1995

Charge-Pickup Processes in Relativistic Heavy-Ion Reactions

Z. Phys. A352:163, 1995; GSI-95-19

Few Neutron Removal from ${ }^{238} U$ at Relativistic Energies

Phys. Rev. Lett. 75:3241, 1995; RIKEN-AF-NP-204

Neutron Skin of Na Isotopes Studied via their Interaction Cross Section

Phys. Rev. D53:2343, 1996; MCGILL-95-55; HEPPH-9511205

Study of $\sigma(750)$ and $\rho^{\circ}$ Production in Measurements of $\pi$ nucleon $\uparrow \rightarrow \pi^{+} \pi^{-}$nucleon on a Polarized Target at $5.88,11.85$, and $17.2 \mathrm{GeV} / \mathrm{c}$

Phys. Rev. D58:093006, 1998; HEPPH-9712420

First Determination of the Quark Mixing Matrix Element $V_{t b}$ Independent of Assumptions of Unitarity

Phys. Rev. D55:R1, 1997 Constraints on the $\tau$ Neutrino Mass and Mixing from Precise Measurements of $\tau$ Decay Rates 
Syed 95

Sykora 95

Synder 95

Tacik 98

Taddeucci 94

Taddeucci 97

Tadokoro 94

Takahashi 95

Takashimizu 96

Takeda 98

Takeda 98B

Taketani 94

Takeuchi 98

Tanaka 94

Tannenbaum 94

Tao 95

Tarazi 96

Tariq 94

Tariq 95

Tartarelli 96

Tatischeff 97

Tatischeff 98

Tauchi 94

Taylor 97

Tedeschi 94

Tedeschi $94 \mathrm{~B}$

Temnikov 96

Templon 97

Terranova 94

Tesch 95

Theunissen 94

Thompson 95

Thompson 97

Thompson 98

Thomson 94

Thurmankeup 96

Tikhomirov 96
Nucl. Phys. (PS) B39:157, 1995; MONTREUX-94 p. 157

Gluon Coherence and String Effect in $Z^{0}$ Decays

Phys. Rev. C51:2765, 1995

No Evidence for a $17 \mathrm{keV}$ Neutrino in the Electron-Capture Decay ${ }^{55} \mathrm{Fe}$

FERMILAB-CONF-95-341-E

Measurement of the Top Quark Mass and Kinematic Properties with the Do Detector

Phys. Rev. C57:1295, 1998

Pion Absorption in ${ }^{12} \mathrm{C}$

Nucl. Phys. A577:105C, 1994

Nuclear Isovector Spin Responses from $(p, n)$ Reactions at $494 \mathrm{MeV}$

Phys. Rev. C55:1551, 1997

Total Cross Sections for Production of ${ }^{7} \mathrm{Be},{ }^{22} \mathrm{Na}$ and ${ }^{24} \mathrm{Na}$ in $p+{ }^{7} \mathrm{Li}$ and $p+{ }^{27} \mathrm{Al}$ Reactions at 495 and $795 \mathrm{MeV}$

Nucl. Phys. A575:333, 1994

The $\left(e^{-}, e^{-1} p\right)$ Coincidence Cross Section for ${ }^{12} \mathrm{C}$ at Transfer Energy of $40 \mathrm{MeV}$

Phys. Rev. C51:2542, 1995; INS-1092; KUNS-1321

$\pi^{-12} \mathrm{C}$ Elastic Scattering above the $\Delta\left(1232 P_{33}\right)$ Resonance

Phys. Lett. 381B:372, 1996; Phys. Lett. 384B:481, 1996; KEK-96-19

Measurement of $D^{*}(2010)^{ \pm}$Production in Two Photon Processes at TRISTAN

Phys. Rev. Lett. 81:1163, 1998

Extension of the Cosmic-Ray Energy Spectrum beyond the Predicted Greisen-Zatsepin-Kuz'min Cutoff

Astropart. Phys. 10:303, 1999; ASTROPH-9807045

The Anisotropy of Cosmic Ray Arrival Directions around $10^{18} \mathrm{eV}$

FERMILAB-CONF-94-017-E

Lepton Charge Asymmetry from $W^{ \pm}$Decay and Search for New Gauge Bosons at Do

Phys. Lett. 443B:409, 1998; KUNS-1537; HEPEX-9810018

Observation of the decay mode $K_{L} \rightarrow \pi^{+} \pi^{-} e^{+} e^{-}$

Nucl. Phys. A583:581C, 1995; KEK-94-70

Energy Spectra and Angular Distribution of Intermediate Mass Fragments Emitted in $\mathrm{Au} / \mathrm{Ag}(p, \mathrm{X})$ Reactions with $E_{p}=12 \mathrm{GeV}$ (The first result of the KEK - $12 \mathrm{GeV}-$ PS Experiments: E288)

Mod. Phys. Lett. A9:89, 1994

Negative Binomial Fits to Multiplicity Distributions from Central ${ }^{16} \mathrm{O}+\mathrm{Cu}$ Collisions at $14.6 \mathrm{GeV} / \mathrm{c}$ and their Implication for "Intermittency"

Z. Phys. C70:387, 1996; SLAC-PUB-95-6737

Precision Measurement of $R=\sigma_{L} / \sigma_{T}$ on Hydrogen, Deuterium and Beryllium Targets in Deep Inelastic Electron Scattering

FERMILAB-CONF-96-236-EC

Do Papers on Electroweak Physics Submitted to DPF 96: $W^{ \pm}$and $Z^{\circ}$ Production Cross Sections at Do

Nuovo Cim. 107A:2687, 1994

Angular Distribution of Slow and Relativistic Charged Particles Produced in Silicon and Carbon Emulsion Interactions at $4.5 \mathrm{~A} \mathrm{GeV} / c$

Int. Jour. Mod. Phys. E4:347, 1995

Some Features of Inelastic Interactions of ${ }^{28} \mathrm{Si}$ and ${ }^{12} \mathrm{C}$ Ions in Nuclear Emulsion at $4.5 \mathrm{~A} \cdot \mathrm{GeV} / \mathrm{c}$

FERMILAB-CONF-96-099-E; CDF-PUB-TOP-PUBLIC-3664 CDF Top Physics

Phys. Rev. Lett. 79:601, 1997

Evidence for Narrow Baryon Resonances in Inelastic $p$ p Scattering

DUBNA-96, v. II, p. 29

Are the Low Masses Narrow Baryons, Recently Observed, Exotic Multiquark States?

KEK-94-67

Inclusive Jet Measurements in $\gamma \gamma$ Collisions at TRISTAN

Nucl. Phys. (PS) B55:285, 1997; ESTES PARK-96 p. 285

Measurement of the $\tau$ Anomalous Magnetic Moment

Phys. Rev. Lett. 73:408, 1994

Exclusive Photodisintegration of ${ }^{3} \mathrm{He}$ with Polarized Photons

WILLIAMSBURG-94 p. 721

Exclusive Photodisintegration of ${ }^{3} \mathrm{He}$ with Polarized Photons

MANCHESTER-95 p. 325

Study of $\iota$ Decays to $K \bar{K} \pi$ in $\bar{p} p$ Annihilation at Rest

Phys. Lett. 413B:253, 1997; NUCLEX-9702001

Study of Continuum Nuclear Structure of ${ }^{12} \mathrm{C}$ via $\left(p, p^{\prime} \mathrm{X}\right)$ at Intermediate Energies

Phys. Scr. 49:267, 1994

Total Nuclear Photoabsorption Cross Section in the Range $0.2-1.0 \mathrm{GeV}$ for Nuclei throughout the Periodic Table

BONN-IR-95-27

Measurement of the Fragmentation Functions of Charged Hadrons and Search for Lepton Number Violating $Z^{\circ}$ Decays

WILLIAMSBURG-94 p. 768

Measurement of the Asymmetry in the Electro-Desintegration of Tensor Polarized Deuterons at the Novosibirsk VEPP-3 Ring

FERMILAB-CONF-95-106-E

Observation of the Top Quark

Phys. Rev. Lett. 79:1630, 1997; HEPEX-9705011

Evidence for Exotic Meson Production in the Reaction $\pi^{-} p \rightarrow \eta \pi^{-} p$ at $18 \mathrm{GeV} / \mathrm{c}$

Phys. Rev. Lett. 81:5760, 1998

Observation of a New $J^{P C}=1^{-+}$Exotic State in the Reaction $\pi^{-} p \rightarrow \pi^{+} \pi^{-} \pi^{-} p$ at $18 \mathrm{GeV} / \mathrm{c}$

Phys. Lett. 337B:411, 1994

Measurement of the Amplitude of the $C P$ Conservating Decay $K_{S} \rightarrow \pi^{+} \pi^{-} \pi^{0}$

FERMILAB-CONF-96-210-E; CDF-PUB-ELECTROWEAK-PUBLIC-3765

$W^{ \pm} / Z^{0}$ at $\mathrm{CDF}$

MANCHESTER-95 p. 560

Low $p_{T} \pi^{0}$ and $\eta$ Production in $p$ Be Interactions at $450 \mathrm{GeV}$ 
Timm 94

Timmermans 94

Tincknell 96

Tkaczyk 96

Toback 96

Todorovic 98

Tokushuku 98

Tollestrup 94

Tomizaw a 94

Towers 97

Tran 98

Traneus 97

Tretyak 95

Tretyakova 94

Tretyakova 95

Trinder 97

Troyan 94

Troyan 94B

Troyan 96

Troyan 98

Troyan 98B

Tsang 97

Tserruya 95

Tsinoev 98

Tsunoda 95

Tuccillo 94

Tuve 97

Tuve 99

Uehara 93

Uehara 95

Ueno 96

Ukegawa 95

Unal 95

Urheim 94

Urheim 97

Uribe 94
Phys. Rev. D51:4638, 1995; FERMILA B-PUB-94-346-E Measurement of the Branching Ratio and Asymmetry Parameter for the $\Sigma^{+} \rightarrow p \gamma$ Radiative Decay

Phys. Rev. C50:48, 1994; THEF-NYM-93-02 Antiproton-Proton Partial Wave Analysis below $925 \mathrm{MeV}$

Phys. Rev. C54:1918, 1996 Low Transverse Momentum Photon Production in Proton Nucleus Collisions at $18 \mathrm{GeV} / \mathrm{c}$

FERMILAB-CONF-96-425-E; HEPEX-9611009 Quarkonium Production in $p \bar{p}$ Collisions at the Tevatron

FERMILAB-CONF-96-240-E; CDF-PUB-EXOTIC-PUBLIC-3837 The Diphoton Missing $E_{T}$ Distribution at CDF

Eur. Phys. J. A2:295, 1998 Production of Fragments with $Z \leq 8$ in Interaction of $12.7 \mathrm{GeV}{ }^{4} \mathrm{He} \mathrm{U}, \mathrm{Pb}, \mathrm{Au}$ and Ag: Track Detector Study

Nucl. Phys. A638:305C, 1998; TSUKUBA-97 p. 305C Recent Results from HERA.

FERMILAB-CONF-94-419-E

The Top ... is it There? A Survey of the CDF and Do Experiments

Phys. Lett. 328B:264, 1994; KEK-94-20

$\Lambda$ Hyperon Production at Backward Angles in the Reaction $\pi^{-}{ }^{8} \mathrm{Li} \rightarrow \Lambda \mathrm{X}$ at $4 \mathrm{GeV} / \mathrm{c}$

Nucl. Phys. (PS) B55:137, 1997; ESTES PARK-96 p. 137 Tau Decays Involving Neutral Kaons at OPAL

Phys. Lett. 445B:20, 1998 Measurement of $\gamma p \rightarrow K^{+} \Lambda$ and $\gamma p \rightarrow K^{+} \Sigma^{0}$ at Photon Energies up to $2 \mathrm{GeV}$

Nucl. Phys. A613:267, 1997

First Measurement of the Exclusive $\left(p, e^{+} e^{-}\right)$Reaction

KEK-94-189

Tables of the Results of $2 \beta$ Decays Research

KOSICE-94 p. 110

Factorial Moments in Central $\mathrm{Si}+\mathrm{Ag}, \mathrm{Br}$ Interactions at $14.6 \mathrm{~A} \cdot \mathrm{GeV} / \mathrm{c}$

Rad. Meas. 25:197, 1995 Investigation of Nucleus-Nucleus Interactions by Means of Emulsions Exposed in Pulsed Magnetic Fields of 30-50 $\mathrm{T}$ at the Nuclotron

Phys. Lett. 415B:211, 1997; GANIL-P-97-24

${ }^{40} \mathrm{Ti} \beta$ Decay and the Neutrino Capture Cross Section of ${ }^{40} \mathrm{Ar}$

Kr. Soob. JINR 67:67, 1994

Narrow Dibaryon Resonances with Isotopic Spin $I=2$

Kr. Soob. JINR 68:51, 1994

Energy Spectra of $\gamma$ Quanta from deuteron Propane Interactions at Momentum $P_{d}=1.25 \mathrm{GeV} / \mathrm{c} \mathrm{per}$ Nucleon

Kr. Soob. JINR 80:73, 1996

Narrow Resonances in the System of Two $\pi^{-}$Mesons

Kir. Soob. JINR 91:33, 1998

The Evidence of $\sigma\left[0^{+}\left(0^{++}\right)\right]$Meson at a Mass of $M_{\pi^{+} \pi^{-}}=(759 \pm 5) \mathrm{MeV} / \mathrm{c}^{2}$ Observed in $\pi^{+} \pi^{-}$Combinations from the Reaction $n p \rightarrow n p \pi^{+_{\pi^{-}}}$at an Incident Moment um of $P_{n}=(5.20 \pm 0.16) \mathrm{GeV} / \mathrm{c}$

JIN R-P1-98-325

Search and Investigation of the Narrow Resonances with Isotopic Spin $I=2$ in $n p$ Interactions at the Momentum of Incident Neutrons $P_{n}=(5.20 \pm 0.13) \mathrm{GeV} / c$

Phys. Rev. C55:557, 1997

Fragment Multiplicity Dependent Charge Distributions in Heavy Ion Collisions

Nucl. Phys. A590:127C, 1995; CERN-PPE-95-52

Summary of Low Mass Dilepton and Direct Photon Results

Phys. At. Nucl. 61:1255, 1998; Yad. Phys. 61:1357, 1998

Search for P-and CP-Violation in M1 $\gamma$-Transition of ${ }^{119} \mathrm{Sn}$ with Mossbauer Polarimetry Technique

Eur. Lett. 30:273, 1995

A Search for Back-to-Back $e^{+} e^{-}$Pairs in the Spontaneous-Fission Disintegration of ${ }^{252} \mathrm{Cf}$

Nucl. Phy's. A580:253, 1994

Measurement of the Analyzing Power $A_{y}$ in Neutron Proton Radiative Capture at $E_{n}=68 \mathrm{MeV}$

Phys. Rev. C56:1057, 1997

Neutron Production at $0^{\circ}$ from the ${ }^{40} \mathrm{Ca} p$ Reaction at $E_{l a b}=357 \mathrm{~A}$ and $565 \mathrm{~A} \mathrm{MeV}$

Phys. Rev. C59:233, 1999

Neutron Production in Coincidence with Fragments from the ${ }^{40} \mathrm{Ca}+p$ Reaction at $E_{l a b}=357 \mathrm{~A}$ and $565 \mathrm{~A} \mathrm{MeV}$

Z. Phys. C63:213, 1994; KEK-93-142

Measurement of Open Charm Production in Two-Photon Processes with Detection of Electron-Inclusive Events

Z. Phys. C69:597, 1996; KEK-95-98; IICMT-9501; KOBE-HEP-95-05; KUNS-1358; OUHEP-95-2; OULNS-95-01; TMU. H EP-95-11

Study of Inclusive Baryon-Antibaryon Pair Production of $p$ or $\Lambda$ in Two Photon Processes

Phys. Lett. 381B:365, 1996; KEK-96-9

Measurement of the Forward-Backward Asymmetry in $e^{+} e^{-} \rightarrow b \bar{b}$ and the $b$-quark Branching Ratio to Muons at TRISTAN using Neural Networks

FERMILAB-CONF-95-234-E; CDF-PUB-BOTTOM-PUBLIC-3267

Measurement of the $B^{-}$and $\bar{B}^{\circ}$ Meson Lifetimes using Semileptonic Decays

Nucl. Phys. (PS) B39:343, 1995; MONTPELLIER-94 p. 343

Evidence for Top Quark Production in $\bar{p} p$ Collisions at $\sqrt{3}=1.8 \mathrm{TeV}$

ALBUQUERQUE-94, v. 1, p. 619; CALT-68-1945; HEPEX-9408003

New results from CLEO-II on Hadronic Decays of the Tau Lepton

Nucl. Phys. (PS) B55:359, 1997; ESTES PARK-96 p. 359

The Hadronic Current in $\tau^{ \pm}$Lepton Decay to Two Pseudoscalar Mesons

Phys. Rev. D49:4373, 1994; UMHEP-PUB-401; NEVIS-1497; FERMILAB-PUB-93-356

Pion-Pion Correlations at Low Relative Momentum Produced in $p$ p Collisions at $27.5 \mathrm{GeV} / c$ 
Valls 96

Van 99

Vandegriff 96

Vandenbrandt 95

Vandenbrink 95

Vandenbrink 97

Vandergrinte 98

Vandewicle 94

Vangeert 98

Vanleeuwe 96

Vanleeuwe 98

Vanleeuwe 98B

Varelas 96

Vartapetyan 95

Vasilevsky 95

Vasiliev 95

Vassiliadis 95

Vassiliadis 96

Vassiliadis 98

Vassiliadis $98 \mathrm{~B}$

Vavilov 94

Vavilov 94B

Vavilov 94C

Vavilov 95

Vavilov 95B

Vavilov $95 \mathrm{C}$

Vavilov 96

Velasco 95

Velissaris 94

Venables 97

Verma 94

Vetter 95

Victorov 95

Victorov $95 \mathrm{~B}$

Vidyakin 94

Vignaud 95
FERMILAB-CONF-96-171-E; CDF-DOC-TOP-PUBLIC-3737

Recent Top Quark Physics Results at Tevatron

Phys. Rev. C59:1488, 1999 Zero-Degree Differential Cross Section and $D_{N N}$ Values for the ${ }^{17} \mathrm{O},{ }^{18} \mathrm{O}(\vec{p}, \vec{n}){ }^{17} \mathrm{Fl},{ }^{18} \mathrm{Fl}$ Reactions at $E_{p}$ $=118 \mathrm{MeV}$

Phys. Lett. 365B:418, 1996; NUCLEX-9510003

A Search for Stable Strange Quark Matter Nuggets in Helium

Phys. Rev. D52:4868, 1995

Evidence for Virtual Compton Scattering from the Proton

Phys. Rev. Lett. 74:3561, 1995

Neutral-Pion Electroproduction on the Proton near Threshold

Nucl. Phys. A612:391, 1997

Electroproduction of Neutral Pions on the Proton

Phys. At. Nucl. 61:1307, 1998; Yad. Phys. 61:1409, 1998

The Search for the Electric Dipole Moment of the Neutron

Phys. Rev. C50:2935, 1994

Experiment Survey of the (deuteron, ${ }^{3} \mathrm{H}$ ) Reaction at $E_{\text {deuteron }}=200 \mathrm{MeV}$

Phys. At. Nucl. 61:1274, 1998; Yad. Phys. 61:1376, 1998

Results on the First Nuclear Orientation Experiment

DAPNIA-SPHN-95-43

High Missing-Momentum Components in ${ }^{4} \mathrm{He}$ Studied with the $\left(e^{-}, e^{-9} p\right)$ Reaction

Phys. Rev. Lett. 80:2543, 1998; DAPNIA-SPHN-97-38

High Missing-Momentum Components in the ${ }^{4} \mathrm{He}\left(e^{-}, e^{-1} p\right){ }^{3} \mathrm{H}$ Reaction

Nucl. Phys. A631:593C, 1998; GRONINGEN-97 p. 593C

The ${ }^{4} \mathrm{He}\left(e^{+}, e^{+} p\right)$ Cross Section at Large Missing Energy

FERMILAB-CONF-96-166-E

Jet Studies at all Rapidities from Do and CDF

Phys. At. Nucl. 58:718, 1995; Yad. Phys. 58:779, 1995

Electron Scattering by ${ }^{12} \mathrm{C}$ Nuclei and the Coulomb Sum Rule at Momentum Transfers $|Q| \leq 700 \mathrm{MeV} / \mathrm{c}$

Kr. Soob. JINR 72:75, 1995

On Iteration Method of the Partial Wave Analysis of the 3i-Systems

Pisma Zh. Eksp. Teor. Fiz. 61:353, 1995; Jetp Lett. 61:358, 1995

Experimental Search for Double Beta Decay of ${ }^{8 \mathrm{~B}} \mathrm{Zr}$ to Exited Levels of ${ }^{96} \mathrm{Mo}$

Nucl. Phys. A590:139C, 1995

First Results from the 1994 Lead Beam Run of WA97

Nucl. Phys. A610:165C, 1996

WA97 Results on Strangeness Production in Lead-Lead Collisions at $158 \mathrm{~A} \mathrm{GeV} / \mathrm{c}$

Nucl. Phys. A638:115C, 1998; TSUKUBA.97 p. 115C

$\Lambda, \Xi$ and $\omega$ Production in $\mathrm{Pb}-\mathrm{Pb}$ Collisions at $158 \mathrm{~A} \cdot \mathrm{GeV} / \mathrm{c}$

Nucl. Phys. A630:582C, 1998; GATLINBURG-97 p. 582C

Strange and Multistrange Baryon Production in $\mathrm{Pb}+\mathrm{Pb}$ Collisions at $158 \mathrm{~A} \cdot \mathrm{GeV} / \mathrm{c}$

Phys. At. Nucl. 57:1376, 1994; Yad. Phys. 57:1449, 1994; IFVE-94-6

Study of the Reaction $p$ nucleon $\rightarrow\left[\Sigma^{\circ} K^{+}\right]$nucleon at $E_{p}=70 \mathrm{GeV}$

Phys. At. Nucl. 57:1970, 1994; Yad. Phys. 57:2046, 1994; IFVE-93-138

Investigation of Diffraction Process $p$ nucleon $\rightarrow p p \bar{p}$ nucleon and $p$ nucleon $\rightarrow p p \bar{p} \pi^{0}$ nucleon at $E_{p}=70 \mathrm{GeV}$

Phys. At. Nucl. 57:227, 1994; Yad. Phys. 57:241, 1994; IFVE-93-5

Search for Heavy Pentaquark Exotic Baryons with Hidden Strangeness in the Reaction $p$ nucleon $\rightarrow(p$ $\phi(1020))$ nucleon and $p$ nucleon $\rightarrow\left(\Lambda\left(1520 D_{03}\right) K^{+}\right)$nucleon at $E_{p}=70 \mathrm{GeV}$

Phys. At. Nucl. 58:1342, 1995; Yad. Phys. 58:1426, 1995

Study of Coherent Diffractive Reactions $p \mathrm{C} \rightarrow\left[\Sigma\left(1385 P_{13}\right)^{0} K^{+}\right] \mathrm{C}$ and $p \mathrm{C} \rightarrow\left[\Sigma^{0} K^{+}\right] \mathrm{C}$ and Search for Exotic Baryons

Phys. At. Nucl. 59:1186, 1996; Yad. Phys. 59:1242, 1996; IFVE-95-143

Study of Quark Line Selection Rule (OZI Rule) in Hadron Processes. 3. Study of the OZI Rule in Production of $\phi(\mathbf{1 0 2 0})$ and $\omega$ Mesons in Diffractive $p$ nucleon Interactions at the Energy of $70 \mathrm{GeV}$

Phys. At. Nucl. 59:1712, 1996; Yad. Phys. 59:1770, 1996; IFVE-95-138

Further Study of the Quasi-Exclusive Reaction of the Neutral Meson Production by 70 GeV Protons in $p$ nucleon Interactions in the Deep Fragmentation Region

Phys. At. Nucl. 59:1921, 1996; Yad. Phys. 59:1994, 1996; IFVE-96-6

$K^{\circ}$ Meson Production in $p N$ Interactions at $E_{p}=70 \mathrm{GeV}$ in Deep Fragmentation Region

Nucl. Phys. (PS) B39:88, 1995

Results on the Spin Structure Functions $g_{1}$ and $g_{2}$ for the Proton

Phys. Lett. 331B:227, 1994; KEK-94-28; AMY-94-1 Measurements of Cross Section and Charge Asymmetry for $e^{+} e^{-} \rightarrow \mu^{+} \mu^{-}$and $e^{+} e^{-} \rightarrow \tau^{+} \tau^{-}$at $\sqrt{s}=$ $57.8 \mathrm{GeV}$

Jour. of Phys. G 23:1857, 1997

Strangeness Production in the WA94 Experiment at CERN

Phys. Rev. C50:2963, 1994

Scaling of Multiplicity Fluctuations in $800 \mathrm{GeV}$ Proton Nucleus Interactions

Phys. Rev. Lett. $74: 2658,1995$

Precise Test of Electroweak Theory from a New Measurement of Parity Nonconservation in Atomic Thal-

Phys. At. Nucl. 59:1184, 1996; Yad. Phys. 59:1239, 1996; IFVE-95-107

Study of Quark Line Selection Rule (OZI Rule) in Hadron Processes. 2. Search for the OZI Suppressed Decay $B / b_{1}(1235) \rightarrow \phi(1020) \pi^{0}$

Phys. At. Nucl. 59:1175, 1996; Yad. Phys, 59:1229, 1996; IFVE-95-106

Study of Quark Line Selection Rule (OZI Rule) in Hadron Processes. 1. Charge-Exchange OZI Suppressed Reaction $\pi^{-} p \rightarrow \phi(1020) n$ at $P_{x-}=32 \mathrm{GeV} / c$

Pisma Zh. Eksp. Teor. Fiz. 59:364, 1994; Jetp Lett. 59:390, 1994

Limits on the Neutrino Oscillation Parameters

DAPNIA-SPP-95-06

The Solar Neutrino Problem after the GALLEX Artificial Neutrino Source Experiment 
Vilain 93B

Vilain 94

Vilain $94 \mathrm{~B}$

Vilain $94 \mathrm{C}$

Vilain $94 \mathrm{D}$

Vilain 95

Vilain $95 \mathrm{~B}$

Vildanova 94

Vildanova $94 \mathrm{~B}$

Villa 96

Vishnevskaya 98

Vishnevsky 94

Viyogi 94

Vlasov 95

Vlasova 96

Vo 94

Voevodsky 98

Vogelsang 97

Voloshin 95

Voloshin 98

Vonach 97

Vonbusch 94

Vorobiev 94

Vorobiev 94B

Vorobiev 95

Vorobiev 95B

Vorobiev 96

Vorobiev 98

Vossnack 94

Vyrodov 95

Vyrodov 98

Waddington 94

Wagner 95

Wakasa 95

Wakasa 98

Waldi 96

Walker 94

Wallace 94
Phy's. Lett. 320B:203, 1994; CERN-PPE-93-186

Flavour Universality of Neutrino Couplings with the $Z^{\circ}$

Phys. Lett. 335B:246, 1994; CERN-PPE-94-124

Precision Measurement of Electroweak Parameters from the Scattering of Muon Neutrinos on Electrons Z. Phys. C64:539, 1994; CERN-PPE-94-138

Search for Muon to Electron Neutrino Oscillations

Phys. Lett. 345B:115, 1995; CERN-PPE-94-190

Experimental Study of Electromagnetic Properties of the Muon Neutrino in Neutrino Electron Scattering

Phys. Lett. 332B:465, 1994; CERN-PPE-94-080

Constraints on Additional $Z^{0}$ Bosons Derived from Neutrino Electron Scattering Measurements

Phys. Lett. 343B:453, 1995; Phys. Lett. 351B:387, 1995

Search for Heavy Isosinglet Neutrinos

Phys. Lett. 364B:121, 1995; CERN-PPE-96-1; CERN-PPE-96-01

A Precise Measurement of the Cross Section of the Inverse Muon Decay $\nu_{\mu} e^{-} \rightarrow \mu^{-} \nu_{e}$

Izv. RAN, Fiz. 58-12:79, 1994

The Primary Cosmic Ray Energy Spectrum and Its Peculiarities at Energies above $10^{18} \mathrm{eV}$ According the Size Spectrum Data at Tein-Shan Level

Phys. At. Nucl. 57:2145, 1994; Yad. Phys. 57:2231, 1994

Peculiarities of the Electron Size Spectrum of Extensive Air Showers at the Mountain Level and their Relation to the Energy Spectrum of Primary Cosmic Rays above the Energy $10^{17} \mathrm{eV}$

MANCHESTER-95 p. 417

$\bar{p} p$ Annihilation into $K_{S} K_{L}:$ A Study of the ${ }^{3} S_{1}$ Initial State

Izv. RAN, Fiz. 61:500, 1998

The Primary Cosmic Ray Composition in the Knee Region of the Energy Spectrum $\left(10^{15}-10^{17} \mathrm{eV}^{15}\right.$ According to MSU EAS Array Data

Phys. At. Nucl. 57:985, 1994; Yad. Phys. 57:1046, 1994

Polarization of $\Lambda$-Hyperons in $n \mathrm{C}$ and $n \mathrm{~Pb}$ Interactions at $4-10 \mathrm{GeV} / \mathrm{c}$

Nucl. Phys. A566:623C, 1994

Photon Multiplicity Measurements in Nucleus-Nucleus Collisions at $200 \mathrm{~A} \mathrm{GeV}$

Phys. At. Nucl. 58:613, 1995; Yad. Phys. 58:669, 1995 Analysis of Cumulative Particle Correlation Data

Pisma Zh. Eksp. Teor. Fiz. 64:237, 1996

Polarization of Leading $\Lambda$-Hyperons Produced by Neutrons on Nuclei

Phys. Rev. C49:1551, 1994

Search for Resonance in Multiphoton Final States from Low-Energy $e^{+} e^{-}$Scattering

Izv. RAN, Fiz. 61:496, 1998

The First Results of Joint Registration of EAS with Energies more than $10^{14} \mathrm{eV}$ by the Underground Telescope and the Andyrchi Surface Array

Jour. of Phys. G 23:A1, 1997

A Compilation of Data on Single and Double Prompt Photon Production in Hadron-Hadron Interactions

Nucl. Phys. A590:605C, 1995

$d N_{\mathrm{ch}} / d \eta$ Distributions in $\mathrm{Au} \mathrm{Al}, \mathrm{Cu}, \mathrm{Au}$, and $\mathrm{U}$ Collisions at $10.8 \mathrm{~A} \mathrm{GeV} / \mathrm{c}$ and $E_{t}$ per Charged Particle

Nucl. Phys. A638:455C, 1998; TSUKUBA-97 p. 455C

Anisotropic Flow of Identifled Particles in $\mathrm{Au}+\mathrm{Au}$ Collisions at AGS Energy

Phys. Rev. C55:2458, 1997

Spallation Reactions in ${ }^{27} \mathrm{Al}$ and ${ }^{56} \mathrm{Fe}$ Induced by $800 \mathrm{MeV}$ Protons

Phys. Lett. 325B:300, 1994

Measurement of the Decay $e^{+} e^{-} \rightarrow 4 \gamma$ at Rest

Phys. At. Nucl. 57:1, 1994; Yad. Phys. 57:3, 1994 Quasifree Scattering of $1.0 \mathrm{GeV}$ Protons on ${ }^{23} \mathrm{Na},{ }^{28} \mathrm{Si},{ }^{29} \mathrm{Si},{ }^{30} \mathrm{Si},{ }^{31} \mathrm{Ph},{ }^{34} \mathrm{~S}$, and ${ }^{39} \mathrm{KK}$

Jetp Lett. 59:510, 1994; Pisma Zh. Eksp. Teor. Fiz. 59:486, 1994 Search for Exotic Long Range Force Violating $T$ - and $P$-Invariance

Phys. At. Nucl. 58:1817, 1995; Yad. Phys. 58:1923, 1995 Investigation of ${ }^{90} \mathrm{Zr}$ and ${ }^{208} \mathrm{~Pb}$ Deep Hole States Structure by Means of Quasielastic Proton Scattering at $1.0 \mathrm{GeV}$

Nucl. Phys. (PS) B40:513, 1995; MONTREUX-94 p. 513

Searches for Lepton Flavour Changing Neutral Currents using $\tau^{ \pm}$

Nuovo Cim. 109A:939, 1996; PISA-95 p. 939

Measurements of $\tau$ Polarization at LEP

Phys. At. Nucl. 61:771, 1998; Yad. Phys. 61:855, 1998

Searches for a Narrow Resonance in the $p$ p $\pi^{-}$System

Nucl. Phys. A566:535C, 1994

Kaon Correlation Systematics in E859

Pisma Zh. Eksp. Teor. Fiz. 61:161, 1995; Jetp Lett. 61:163, 1995

Precision Measurement of the $\bar{\nu}_{e}+p \rightarrow e^{+} n$ Reaction Cross Section at Bugey Reactor (France)

Phys. At. Nucl. 61:760, 1998; Yad. Phys. 61:844, 1998 Results of Inverse $\beta$-Decay at Proton Reaction Measurements by Means of Modernized Detector "Deuteron"

Nucl. Phys. A566:427C, 1994

Interactions of $10.6 \mathrm{GeV} / \mathrm{Nucleon}$ Gold Nuclei in Targets from $p$ to $\mathrm{Pb}$

FERMILAB-CONF-95-062-E; ANL-HEP-CP-95-20 $C D F$ Results on $Z^{0} \gamma$ Production

Phys. Rev. C51:R2871, 1995

Spin-Flip Strength in the Continuum and Effective Tensor Interactions via Polarization Transfer Coeffcients $D_{N N}$ at $0^{\circ}$ for $(p, n)$ Reactions at $295 \mathrm{MeV}$

Phys. Lett. 426B:257, 1998

Strength Observed in Gamow-Teller Resonance Plus Continuum in the ${ }^{27} \mathrm{Al}(\vec{p}, \vec{n})$ Reaction at $295 \mathrm{MeV}$

Nuovo Cim. 109A:905, 1996; PISA-95 p. 905

Results on the Lorentz Structure of $\tau$ Decays

Phys. Rev. D49:5671, 1994; SLAC-PUB-5815; UR-1305; ER-40685-754

Measurements of the Proton Elastic Form-Factors for $1 \mathrm{GeV} / c^{2} \leq Q^{2} \leq 3 \mathrm{GeV} / \mathrm{c}^{2}$ at SLAC

FERMILAB-CONF-94-184-E

Beam Flavor Dependence in the Hadroproduction of $D^{ \pm}$and $D_{s}^{ \pm}$Mesons 
Wallace 95

Wang 94

Wang 94B

Wang 94C

Wang 94D

Wang 94E

Wang 95

Wang 95B

Wang 95C

Wang 95D

Wang 96

Wang 96B

Wang 97

Wang 97B

Wang 97D

Wang 97E

Wang $97 \mathrm{~F}$

Wang 98

Wang 98B

Wang $98 \mathrm{C}$

Wang 98D

Ward 95

Warner 94

Warner 96

Warren 98

Watkins 95

Watson 94

Watts 94

Watts 96

Weaver 94

Webb 98

Webber 98

Weber 94

Weber 96

Weber 97

Weerts 94

Weigang 98

Weinstein 95

Weiss 94

Phys. Rev. Lett. 74:3732, 1995

Precision Measurement of the $\Omega^{-}$Magnetic Moment

Nucl. Phys. A566:379C, 1994

Production of $\phi(1020)$ Mesons in Central Si+Au Collisions at $14.6 \mathrm{~A} \mathrm{GeV} / \mathrm{c}$

Phys. Rev. C50:2438, 1994

$(p, n)$ Quasifree Excitations in $p$-Shell Nuclei at $186 \mathrm{MeV}$

Phys. Rev. D49:5785, 1994

Intermittency Exponents in $p$ p Collisions at $400 \mathrm{GeV} / \mathrm{c}$

Phys. Lett. 359B:397, 1994

Factorial Correlators and Cumulant Correlators in $p$ p Collisions at $400 \mathrm{GeV} / \mathrm{c}$

Phys. Lett. 321 B:431, 1994; China Phys. Lett. 11:657, 1994

Factorial Cumulant Moments and Correlations in $p$ p Collisions at $400 \mathrm{GeV} / \mathrm{c}$

Nucl. Phys. A590:539C, 1995

Multiplicity Dependence of the Mass of $\phi(1020)$ Mesons from Si Au Collisions at $14.6 \mathrm{~A} \mathrm{GeV} / \mathrm{c}$

Phys. Lett. 344B:447, 1995

Fractal Analysis of $p$ Interactions at $400 \mathrm{GeV} / \mathrm{c}$

Z. Phys. C68:415, 1995

Factorial Cumulant Moments and Correlation Integrals in $p p$ Collisions at $400 \mathrm{GeV} / c$

Phys. Rev. Lett. 74:2646, 1995; LBL-36077

Light Fragment Production and Power Law Behaviour in $\mathrm{Au}+\mathrm{Au}$ Collisions

MANCHESTER-95 p. 227

CDF Heavy Flavour Spectroscopy of $b$ and $c$ Hadrons

SLAC-PUB-7312

Semileptonic Branching Fraction and Hadronic Width of the $B$ Mesons in Light of the New CLEO Observation of $b \rightarrow B$ X

Phys. Rev. D56:2544, 1997; FERMILA B-PUB-97-057

A Precise Measurement of the $\Sigma^{0}$ Mass

Phys. Lett. 398B:420, 1997

Angular Dependence of Particle Correlations in $p p$ Collisions at $400 \mathrm{GeV} / \mathrm{c}$

Phys. Rev. D56:1668, 1997

Cumulant to Factorial Moment Ratio of Multiplicity Distributions of $p$ Interactions at $400 \mathrm{GeV} / c$

Phys. Rev. Lett. 79:4096, 1997

Anisotropy of Subthreshold $K^{+}$Emission in Heavy Ion Reactions

Phys. Lett. 393B:290, 1997

Time Dependence of Multifragmentation in Light-Ion-Induced Reactions

Phys. Lett. 427B:385, 1998

Characteristics of Charged-Particle Groups Produced in $p p$ Collisions at $400 \mathrm{GeV} / \mathrm{c}$

Phys. Rev. D57:3036, 1998

Chaotic Behaviour of Multiparticle Production in $p$. Collisions at $400 \mathrm{GeV} / \mathrm{c}$

Phys. Lett. 438B:353, 1998

Non-Thermal Phase Transition in $p$ Collisions at $400 \mathrm{GeV} / c$ ?

Phys. Lett. 441B:473, 1998

Bunching Parameters and Correlators in $400 \mathrm{GeV} / c p p$ Collisions

Nucl. Phys. (PS) B39:134, 1995; MONTREUX-94 p. 134

Properties of Quark and GIuon Jets

Phys. Rev. C49:1534, 1994

${ }^{4} \mathrm{He}\left({ }^{4} \mathrm{He},{ }^{3} \mathrm{He}\right){ }^{5} \mathrm{He}_{\text {g.s. }}$. Reaction at $118 \mathrm{MeV}$, and Its Distorted Wave Born Approximation Interpretation

Phys. Rev. C54:1700, 1996

Total Reaction and $2 n$ Removal Cross Sections of $20-60 \mathrm{~A} \mathrm{MeV}{ }^{4} \mathrm{He},{ }^{8} \mathrm{He},{ }^{8} \mathrm{He},{ }^{8} \mathrm{Li},{ }^{7} \mathrm{Li},{ }^{8} \mathrm{Li},{ }^{8} \mathrm{Li},{ }^{11} \mathrm{Li}$,

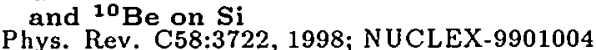

Induced Proton Polarization for $\pi^{\circ}$ Electroproduction at $Q^{2}=0.126(\mathrm{GeV} / \mathrm{c})^{2}$ around the $\Delta$ Resonance

Nucl. Phys. (PS) B40:247, 1995

Measurement of the Tau Leptonic Branching Ratios by the OPAL

Nucl. Phys. A577:79C, 1994

The Spin-Dipole Resonance in $(p, n)$ on ${ }^{18} \mathrm{O}$ and ${ }^{40} \mathrm{Ca}$

CDF-PUB-TOP-PUBLIC-2773; ALBUQUERQUE-94, v. 1, p. 675; FERMILAB-CONF-94-247-E

CDF top Results in the $\ell+$ Jets Channel

Phys. Rev. C55:1832, 1997; INS-1142

Single and Double Delta Production in the ${ }^{3} \mathrm{He}\left(\gamma, \pi^{+} \pi^{-}\right)$Reaction at $380 \mathrm{MeV} \leq E_{\gamma} \leq 700 \mathrm{MeV}$

Phys. Rev. Lett. 72:3758, 1994

Limit on the Branching Ratio of $K_{L} \rightarrow \pi^{0} \nu \bar{\nu}$

Phys. Rev. Lett. 82:884, 1999; ASTROPH-9803165

A Search for Time Variation of the Fine Structure Constant

Phys. Rev. C58:3539, 1998

Measurement of Charge Changing and Isotopic Cross Sections at $600 \mathrm{MeV} /$ nucleon from the Interactions of 30 Separate Beams of Relativistic Nuclei from ${ }^{10}$ Bor to ${ }^{55} \mathrm{Mn}$ in a Liquid Hydrogen Target

Nucl. Phys. A578:659, 1994; GSI-94-29

Longitudinal Momenta and Production Cross Sections of Isotopes Formed by Fragmentation of a $500 \mathrm{~A} \cdot \mathrm{MeV}{ }^{86} \mathrm{Kr}$ Beam

Nuovo Cim. 109A:925, 1996 ; PISA-95 p. 925

Measurements of the $\tau$ Lepton Lifetime

Nucl. Phys. (PS) B55:107, 1997; ESTES PARK-96 p. 107

Review of $\tau^{ \pm}$Lifetime and Leptonic Branching Ratio Measurements

FERMILAB-CONF-94-035-E; D0-NOTE-1956

Studies of Jet Production with the Do Detector

Phys. Rev. C58:3560, 1998

Measurement of Collectivity of Collective Flow in Relativistic Heavy-Ion Collisions using Particle Group Correlations

Nucl. Phys. (PS) B40:163, 1995

A Precision Measurement of the Branching Fraction $B\left(\tau^{ \pm} \rightarrow h^{ \pm} \pi^{0} \nu_{\tau}\right)$

Phys. Rev. C49:2569, 1994

Measurement of Low Energy $K^{+}$Total Cross Sections on $N=Z$ Nuclei

See the legend on page 5 . 
Wenzel 96

Wenzel 96B

Werlen 99

Wermes 94

Wermes 97

Wessler 95

Weygand 96

White 94

White $94 \mathrm{~B}$

White 95

White 96

Whitmore 94

Wiaux 98

Wibig 98

Wichmann 96

Widmann 94

Wiedner 94

Wieser 96

Wietfeldt 95

Wilburn 95

Wilburn $9 \pi$

Wilk 95

Williams 94

Williams 97

Williamson 97

Willis 97

Willmann 98

Wilson 94

Wilson 96

Wilson 97

Wimpenny 95

Wimpenny $95 \mathrm{~B}$

Wischnewski 94

Wislicki 96

Wiss 96

Wissink 94

Wissmann 94

Witzmann. 94
PADUA-96 p. 343; FERMILAB-CONF-96-186-E; CDF-PUB-EXOTIC-PUBLIC-3745

Search for New Exotic Particles at CDF

FERMILAB-CONF-96-204-E; CDF-PHYS-BOTTOM-CDFR-3734

Measurement of the Average Lifetime of $B$ Hadrons Produced in $p \bar{p}$ Collisions at $\sqrt{s}=1.8 \mathrm{TeV}$

Phys. Let t. 452B:201, 1999; CERN-EP-99-021

A New Determination of $\alpha_{s}$ using Direct Photon Production Cross Sections in $p p$ and $\bar{p} p$ Collisions at $\sqrt{s}=24.3 \mathrm{GeV}$

Nucl. Phys. (PS) B40:213, 1995; MONTREUX-94 p. 213; BONN-HE-94-11

Branching Fractions and Hadronic Structure Functions in $\tau^{\ddagger} \rightarrow 3$ hadron $\left(\pi^{0}\right) \nu_{\tau}$ Decays

Nucl. Phys. (PS) B55:313, 1997; ESTES PARK-96 p. 313

$C P$ Tests and Dipole Moments in $\tau^{ \pm}$-Pair Production Experiments

Phys. Rev. C51:2575, 1995 Polarization Observables in $\pi$ deuteron Elastic Scattering: Analyzing Power $\tau_{22}$ and $i T_{11}$ in Forward Hemisphere

MANCHESTER-95 p. 241

Study of $\pi^{+} \pi^{-} \pi^{-}$Interactions at $18 \mathrm{GeV} / c$

FERMILAB-CONF-94-036-E

Search for Supersymmetry and Leptoquark States in Do

Phys. Rev. D49:58, 1994; BNL-49059

Comparison of 20 Exclusive Reactions at Large $t$

Nucl. Phys. (PS) B40:311, 1995

Measurement of the Tau Lepton Lifetime at CLEO II

Phys. Rev. D53:6658, 1996

Search for the decays $\eta \rightarrow \mu e$ and $\eta \rightarrow e^{+} e^{-}$

Z. Phys. C62:199, 1994

Inclusive Charged Pion Production in Hadron-Nucleus Interactions at 100 and $320 \mathrm{GeV} / \mathrm{c}$

Phys. At. Nucl. 61:1301, 1998; Yad. Phys. 61:1403, 1998

Muon Capture on ${ }^{1} 1 B$ : Pseudoscalar Coupling and the Hyperfine Effect

Jour. of Phys. G 24:2037, 1998; ZPK-UL-980923; HEPPH-9809494

Proton-Nucleus Cross Sections at High Energies

Z. Phys. A355:169, 1996

Photoemission of Protons from ${ }^{4} \mathrm{He}$ in the $\Delta$ Resonance Region

Phys. Rev. A51:2870, 1995; CERN-PPE-94-213

Phase and Density Dependence of the Delayed Annihilation of Metastable Antiprotonic Helium Atoms in Gas, Liquid and Solid Helium

Nuovo Cim. 107A:2589, 1994

Test of Chiral Perturbation Theory in $\eta$ Decays

Phys. Rev. C54:1930, 1996

Measurement of the $\pi^{+} p \uparrow$ Analyzing Power at $68.3 \mathrm{MeV}$

Phys. Rev. C52:1028, 1995

Further Studies on the Evidence for a $17 \mathrm{keV}$ Neutrino in a ${ }^{14} \mathrm{C}$-Doped Germanium Detector

Phys. Rev. C52:2351, 1995

Measurements of $\Delta \sigma_{T}$ in Polarized Neutron Polarized Proton Scattering

Act a Phys. Austriaca., New Ser./Few Body 24:27, 1998; LA-UR-97-4376; NUCLEX-9710003

Measurements at Low Energies of the Polarization-Transfer Coefficient $K_{y}^{y^{\prime}}$ for the reaction ${ }^{3} \mathrm{H}(p \uparrow, n \uparrow)$ ${ }^{3} \mathrm{He}$ at $0^{\circ}$

Acta Phys. Polon. B27:2649, 1996; SINS-PVIII-1995-25; IF-PUK-5-1995; HEPPH-9511427

Surprising Results from Cosmic Rays

FERMILAB-CONF-94-368-E

Top Quark Kinematics and Mass Determination

Phys. Rev. C55:2132, 1997

Fragment Distributions for Highly Charged Systems

Phy's. Rev. C56:3152, 1997 Quasielastic Electron Scattering from ${ }^{40} \mathrm{Ca}$

Phy's. Lett. 406 B:14, 1997; IPNO-DRE-97-09; NUCLEX-9703002 $\eta$-Helium Quasi-Bound States

Phys. Rev. Lett. 82:49, 1999; UHD-PI-MY-9812; HEPEX-9807011 New Bounds from Searching for Muonium to Antimuonium Conversion

Nucl. Phys. A566:387C, 1994 Measurements of Dilepton Production in Nucleon-Nucleon Interactions

MANCHESTER-95 p. 430

Measurement of Fragmentation at LEP

Phy's. Rev. C57:1865, 1998; NUCLEX-9708002 Inclusive Dielectron Cross Sections in $p+p$ and $p+$ deuteron Interactions at Beam Energies from 1.04 to $4.88 \mathrm{GeV}$

FERMILAB-CONF-95-016-E

Search for the Top Quark in Dilepton Decay Modes at Do

FERMILAB-CONF-95-260-E

Results on Top Quark Production from Do

PYLOS-93 p. 213; DESY-94-049; BAIKAL-93-15 The Lake Baikal Telescope NT-36: A First Deep Underwater Multistring Array

MANCHESTER-95 p. 427

Polarization of Valence Quarks and Light Quark Sea in the Nucleon

Nuovo Cim. 109A:895, 1996; PISA-95 p. 895 Hadronic Charm Decays

Nucl. Phys. A577:27C, 1994

Studies of Spin Observables in $\left(p \uparrow, p \uparrow^{\prime}\right)$ and $\left(p \uparrow, p^{\prime} \gamma\right)$ Reactions at Intermediate Energies

Phys. Lett. 335B:119, 1994 Elastic and Inelastic Photon Scattering from ${ }^{12} \mathrm{C}$

Nucl. Phys. A577:319C, 1994

Results of SMC on the Spin-Dependent Structure Function $q_{1}^{p}$ of the Proton 
Wolbers 94

Wolf 94

Wolf 98

Wolinski 94

Womersley 95

Woo 97

Woods 96

Wormser 94

Wosiek 94

Wright 98

Wurzinger 94

Wurzinger 95

Wurzinger 96

Wurzinger 96B

Wurzinger 98

Xi 97

Xu 94

Xu 95

$\mathrm{Xu} 95 \mathrm{~B}$

Yabuki 94

Yamagata 94

Yamartino 94

Yamaya 95

Yamazaki 95

Yamazaki 96

Yamazaki 98

Yang 96

Yang 96B

Yasin 95

Yasinelbakry 95

Yasuda 96

Yasumi 94

Yau 98

Ye 96

Ye 97

Yeomans 94

Yeomans 95

Yokkaich 98

Yonnet 98
MERIBEL LES ALLUES-94 p. 209; FERMILAB-CONF-94-139-E

Structure Functions and $\sigma_{n} / \sigma_{p}$ Measured in $465 \mathrm{GeV} / \mathrm{c}$ Muon-Nucleon Interactions

Nucl. Phys, (PS) 38:107, 1995; EILAT-94 p. 107; DESY-94-178

Deep Inelastic Structure Functions from HERA

Phys. Rev. Lett. 80:5281, 1998; GSI-98-28

Multistep Production of $\eta$ and Hard $\pi^{\circ}$ Mesons in Subthreshold Au Au Collisions

FERMILA B-CONF-94-149-E; CDF-ANAL-EXOTIC-PUBLIC-2621 Search for Supersymmetry at CDF

FERMILAB-CONF-95-364-E

QCD at the Tevatron: Recent QCD Results from the CDF and Do Experiments

Phys. Rev. Lett. 80:456, 1998; NUCLEX-9712001

Measurement of the Induced Proton Polarization $P_{n}$ in the ${ }^{12} \mathrm{C}\left(e^{-}, e^{-1} \vec{p}\right)$ Reaction

Phys. Rev. D54:6610, 1996

Polarization of $\Xi^{-}$and $\Omega^{-}$Hyperons Produced from Neutral Beams

Nuovo Cim. 107A:2073, 1994

LEP Measurement on Production, Mass, Lifetime of Beauty Particles

Nucl. Phys. A566:593C, 1994

A Study of Correlation Integrals in Proton-Nucleus and Nucleus-Nucleus Collisions

Phys. Rev. C57:373, 1998

Measurement of the Induced Pseudoscalar Coupling using Radiative $\mu^{-}$Capture on $p$

WILLIAMSBURG-94 p. 514

Near-Threshold Supression of $\omega$ Production in the $p$ deuteron $\rightarrow{ }^{3}$ He $\omega$ Reaction

Phys. Rev. C51:R443, 1995; CEN-LNS-PH-94-11

Near-Threshold Production of $\omega$ Mesons in the $p$ deuteron $\rightarrow{ }^{3} \mathrm{He} \omega$ Reaction

MANCHESTER-95 p. 464

Meson Production in the $1 \mathrm{GeV} / c^{2}$ Mass Region in the $p$ deuteron $\rightarrow{ }^{3} \mathrm{He} \mathrm{X}$ Reaction

Phys. Lett. 374B:283, 1996

Observation of $\eta^{\prime}$ and $\phi(1020)$ Meson Production Very Close to Threshold in the $p$ deuteron $\rightarrow{ }^{3}$ He $X$ Reaction

Phys. Lett. 445B:423, 1999; TSL-ISV-98-0197; NUCLEX-9810010

Study of the ABC Enhancement in the deuteron $\uparrow$ deuteron $\rightarrow \alpha X^{0}$ Reaction

Z. Phys. A359:397, 1997; Eur. Phys. J. A1:235, 1998; GSI-97-20; NUCLEX-9703004

Breakup Temperature of Target Spectators in Au Au Collisions at $E / A=1000 \mathrm{MeV}$

Nucl. Phys. A566:585C, 1994 Pion Interferometry in ${ }^{28} \mathrm{Si}+\mathrm{Pb}$ Central Collisions

Phys. Rev. C52:R1161, 1995 (deuteron, ${ }^{2} \mathrm{He}$ ) Reactions at $E_{\text {deuteron }}=125.2 \mathrm{MeV}$

Phys. Rev. C52:2859, 1995 Radiative Capture of Polarized Neutrons by Polarized Protons at $T_{n}=183 \mathrm{MeV}$

Jour. of Phys. Soc. Jap. 64:435, 1995; KEK-94-183; KUNS-1315; OUHEP-95-1; KOBE-HEP-95-01; OULNS-94-03; IIUPD-9501; TMU-HEP-95-10 Study of $\pi^{+} \pi^{-}$Pair Production in a Two-Photon Process at TRISTAN

Phys. Rev. C50:2606, 1994

Successful Description of Elastic Scattering of ${ }^{3} \mathrm{He}$ Particles at $150 \mathrm{MeV} / \mathrm{Nucleon}$ with the Single Folding Potential Model

SLAC-426

A Measurement of the $e^{+} e^{-}$Decay Width of the $Z^{0}$

Phys. Rev. C51:493, 1995

Dipole Strength in ${ }^{11} \mathrm{Be}$ from Electroproduction of Charged Pions

Phys. Rev. C52:R1157, 1995

The ${ }^{12} \mathbf{C}\left(\gamma, K^{+}\right)$Reaction in the Threshold Region

CERN-PPE-96-160

High Precision Structural Studies of the Antiprotonic Helium Atom ${ }^{4}$ He by Observing Laser Resonances with $\Delta v=\Delta(n-l-1)=2$

Nucl. Phys. A629:338C, 1998; OSAKA-97 p. 338C

Observation of a Pion Mass Shift in the Deeply Bound Pionic 1s and 2p States in ${ }^{207} \mathrm{~Pb}$

Jour. of Phys. G 22:775, 1996; UR-1460; ER-40685-889; HEPEX-9605005

A Measurement of $R=\sigma_{L} / \sigma_{T}$ in Deep Inelastic Neutrino Nucleon Scattering at the Tevatron

MANCHESTER-95 p. 476

Charmed Baryon Results from CLEO

Nuovo Cim. 108A:1041, 1995

Projectile Multifragments Break-Up and the Electromagnetic-Field Effect of Target Nucleus

Nuovo Cim. 108A:929, 1995

Further Study of $N$ uclear Transparency for ${ }^{12} \mathrm{C}$ and ${ }^{16} \mathrm{O}$ in Emulsion Interaction at $4.5 \mathrm{GeV} / c$

FERMILAB-CONF-96-239-E

Double Boson Production at Do

Phys. Lett. 334B:229, 1994

The Mass of the Electron-Neutrino from Electron Capture in ${ }^{163} \mathrm{Ho}$

Eur. Phys. J. A 1:241, 1998

The Angular Distribution of the ${ }^{12} \mathrm{C}(\gamma, p n)$ Reaction for $E_{\gamma}=120-150 \mathrm{MeV}$

MANCHESTER-95 p. 346

Measurement of $\eta$ Production in Two and Three Jet Events from Hadronic $Z^{\circ}$ Decays at LEP

Phys. Rev. D55:5641, 1997

Fractal Structure of Multiparticles Produced in $p$ p Collisions at $400 \mathrm{GeV} / \mathrm{c}$

Phys. Rev. C49:2898, 1994

Analyzing Power Measurements in Pion Deuteron Absorption at Low Energies

Phys. Rev. C52:2526, 1995

Analyzing Power Measurements in Pion Deuteron Breakup at Intermediate Energies

Nucl. Phys. A638:435C, 1998; TSUKUBA-97 p. $435 \mathrm{C}$

Measurements of $\phi(1020)$ Meson Decays in Nuclear Matter at KEK-PS

Nucl. Phys. A637:63, 1998; IPNO-DRE-98-04

Analyzing Powers of One and Two Delta Production in Inelastic $p$ p Reactions at Energies between 1.5 and $2.1 \mathrm{GeV}$

See the legend on page 5 . 
Yoshida 95

Yoshimura 95

Youdin 96

Yu 94

Yu 94B

Yu 95

Yu 95B

Yu 96

Yu 96B

Yu 96C

Zabrodin 95

Zabrodin 97

Zachariadou 96

Zaetz 95

Zaitsev 94

Zaitsev 96

Zarubin 94

Zatsepin 94

Zatsepin 94B

Zatsepin 97

Zeitlin 97

Zejma 97

Zenoni 94

Zeps 98

Zghiche 94

Zhang 95

Zhang $95 \mathrm{~B}$

Zhang $95 \mathrm{C}$

Zhang 97

Zhao 94

Zhao 98

Zhou 98

Zhumanov 94

Zlomanczuk 98

Zlomanczuk $98 \mathrm{~B}$

Zou 94

Zou 96
Astropart. Phys. 3:105, 1995; ICRR-325-94-20

The Cosmic Ray Energy Spectrum above $3 \times 10^{18} \mathrm{eV}$ Measured by the Akeno Giant Air Shower Array

Phys. Rev. Lett. 75:3792, 1995; KEK-94-202; UT-ICEPP-95-02; KOBE-HEP-95-04

Observation of Cosmic Ray Antiprotons at Energies below $500 \mathrm{MeV}$

Phys. Rev. Lett. 77:2170, 1996

Limits on Spin - Mass Couplings within the Axion Window

FERMILAB-CONF-94-167

Recent QCD Results from the Tevatron $\bar{p} p$ Collider at $\sqrt{s}=1.8 \mathrm{TeV}$

FERMILA B-CONF-94-241-E

A Measurement of the $b \bar{b}$ Cross Section at CDF

FERMILAB-CONF-95-146-E

$W^{ \pm} / Z^{0}+$ Jets Production at the Tevatron $\bar{p} p$ Collider

FERMILAB-CONF-95-178-E

$W^{ \pm} / Z^{0}+$ Jets Production at the Tevatron

Phy's. Rev. C53:1725, 1996

Differential Cross Sections of the ${ }^{12} \mathrm{C}{ }^{13} \mathrm{C}(p, p){ }^{12} \mathrm{C}^{13} \mathrm{C}$ and ${ }^{12} \mathrm{C}{ }^{13} \mathrm{C}(p, n){ }^{12} \mathrm{Nit}$ (3) Nit Reactions Near $180^{\circ}$

PADUA-96 p. 28; FERMILAB-CONF-96-220-E; CDF-PUB-BOTTOM-PUBLIC-3768

$B$ Hadron Production and $b \bar{b}$ Correlation at CDF

FERMILAB-CONF-96-304-EG

DO Papers on QCD Studies with Jets: Search for Double Parton Scattering in the 4 Jet Channel

Phys. Rev. D52:1316, 1995

Inclusive Spectra of Charged Particles in $p p$ and $\bar{p} p$ Interactions at $32 \mathrm{Gev} / \mathrm{c}$

Phys. Rev. C55:1617, 1997; DAPNIA-SPHN-97-05

Total Cross Section Measurement of the $\gamma n \rightarrow p \pi^{-} \pi^{0}$ Reaction

Nuovo Cim. 109A:815, 1996; PISA-95 p. 815

Search for Exclusive Hadronic Charmless b Decays at LEP

Z. Phys. C66:583, 1995

Spin Alignment and Parity Violation Effects in $\rho$ Production in Neutrino and Antineutrino ChargedCurrent Interactions

Nuovo Cim. 107A:1941,1994

Latest Results form the VES Experiment

Phys. At. Nucl. 59:1617, 1996; Yad. Phys. 59:1674, 1996

Search for Exotic Mesons in Diffractive Reactions

JULICH-94 p. 497

Cumulative Pion Production in Fragmentation of $4.5 \mathrm{~A} \cdot \mathrm{GeV} / \mathrm{c}$ Carbon Nuclei

Phy's. At. Nucl. 57:645, 1994; Yad. Phys. 57:684, 1994

Energy Spectra and Composition of Primary Cosmic Rays in the Energy Region above $10 \mathrm{TeV}$ per Nucleus

Izv. RAN, Fiz. 58-12:119, 1994; Bull. Russ. Acad. Sci. Phys. Ser. 58:2050, 1994

Energy Spectrum of PCR Nucleons at Energies 20-400 TeV and Charmed-Particle Production According to the MSU Muon Experiment Data

Izv. RAN, Fiz. 61:559, 1997

The Results of Primary Nucleon Spectrum and Charm Production Investigation According to the MSU Muon Experimental Data

Phys. Rev. C56:388, 1997 Heavy Fragment Production Cross Sections from $1.05 \mathrm{GeV} /$ nucleon ${ }^{58} \mathrm{Fe}$ in $\mathrm{C}, \mathrm{Al}, \mathrm{Cu}, \mathrm{Pb}$, and $\mathrm{CH}_{2}$ Targets

Phys. Rev. C55:42, 1997

Cross Sections and Analyzing Powers $A_{y}$ in the Breakup Reaction ${ }^{2} H(p \uparrow, p p) n$ at $65 \mathrm{MeV}$ : Star configurations

Phys. At. Nucl. 57:1478, 1994; Yad. Phys. 57:1554, 1994

The Case of $\mathrm{AX}$

Nucl. Phys. A639:261C, 1998; UPTON-97 p. $261 \mathrm{C}$

The Weak Decays of Light Hypernuclei

Nucl. Phys. A572:513, 1994; Nucl. Phys. A584:757, 1995; DAPNIA-SPHN-93-25

Longitudinal and Transverse Responses in Quasielastic Electron Scattering from ${ }^{208} \mathrm{~Pb}$ and ${ }^{4} \mathrm{He}$

Phys. Lett. 352B:169, 1995

Star Integral and Multidimension Intermittency in $400 \mathrm{GeV} / \mathrm{c} p$ p Collisions

Nucl. Phys. A590:557C, 1995

Energy Flow and Particle Spectra with Respect to the Reaction Plane for $\mathrm{Au}+\mathrm{Au}$ Collisions at AGS Energies

Phys. Rev. C52:2643, 1995 Neutrons from Multiplicity-Selected Au-Au Collisions at 150 A, 250 A, 400 A, and 650 A MeV

Phys. Rev. C56:1918, 1997 Complex Fragment Emission in the $200 \mathrm{MeV}{ }^{4} \mathrm{He} \mathrm{Ag},{ }^{107} \mathrm{Au}$ Reactions

TRI-PP-94-31

A Preliminary Result of the Second $n p$ Charge Symmetry Breaking Experiment at TRIUMF

Phys. Rev. C57:2126, 1998

Precision Measurement of Charge Symmetry Breaking in $n p$ Elastic Scattering at $347 \mathrm{MeV}$

Phys. Rev. Lett. 82:687, 1998; NUCLEX-9809002

Tensor A nalyzing Powers for Quasielastic Electron Scattering from Deuterium

Phys. At. Nucl. 57:1389, 1994; Yad. Phys. 57:1462, 1994 Rapidity and Azimuthal Correlations in Collisions of the Ne Nuclei with the Emulsion Nuclei at 4.1 $\mathrm{A} \mathrm{GeV} / c$ and $\pi^{-} p$ Collisions at $40 \mathrm{GeV} / c$

Phys. Lett. 436 B:251, 1998

Pionic $D$-Wave Effects in $p p \rightarrow p p \pi^{0}$ Near Threshold

Nucl. Phys. A631:622C, 1998; GRONINGEN-97 p. 622C Proton-Proton Bremsstrahlung at $310 \mathrm{MeV}$

Phys. Lett. 329B:519, 1994 Search for $C P$ Violation in the Decay $K_{S} \rightarrow \pi^{+} \pi^{-} \pi^{0}$

Phys. Lett. 369B:362, 1996

Measurement of the Amplitude of the CP Conserving Decay $K_{S} \rightarrow \pi^{+} \pi^{-} \pi^{\circ}$ 
Zybalov 95

Phys. At. Nucl. 58:894, 1995; Yad. Phys. 58:966, 1995 Proton Polarization in the Inclusive Photodisintegration of ${ }^{3} \mathrm{He}$

Zylberajch 94 DAPNIA-SPP-94-27

Recent Developments in Microlensing Experiments 
This index lists papers by beam, target, and beam momentum. The ordering is by beam particle, then by target particle, then beam momentum.

Particle names follow the ordering in the Particle Vocabulary of this compilation: Gauge bosons, leptons, mesons, baryons, atoms, and nuclei; and within each group the ordering is mainly by increasing mass. Within mesons and baryons, however, we further categorize these particles as having only light quarks, or as having as the heaviest quark, a strange, charmed, bottom, or top quark. To simplify searching in this Index, a short "Table of Contents" of the full Index comes first.

For a given beam, target, and momentum, papers are ordered by year (most recent first), then first author name. For the full reference, see the ID/Reference/Title Index.

In most cases, we give both the equivalent lab momentum for scattering on a fixed target and, in parentheses, the total c.m. energy $E_{\mathrm{cm}}$. However, for colliding beam experiments, we usually only give $E_{\mathrm{cm}}$. When a range of momenta are studied, we list the range, e.g. " $50-70$," ordered by its lower end. For some experiments, such as neutrino experiments, the listed range is only approximate.

A question mark means that the indicated information is unknown to us, usually because it was not given in the paper.

\section{Illustrative Key}

Beam and Target: see the Particle Vocabulary for nomenclature.

Lab Momentum: in $\mathrm{GeV} / c$ (not listed for colliding beam experiments).

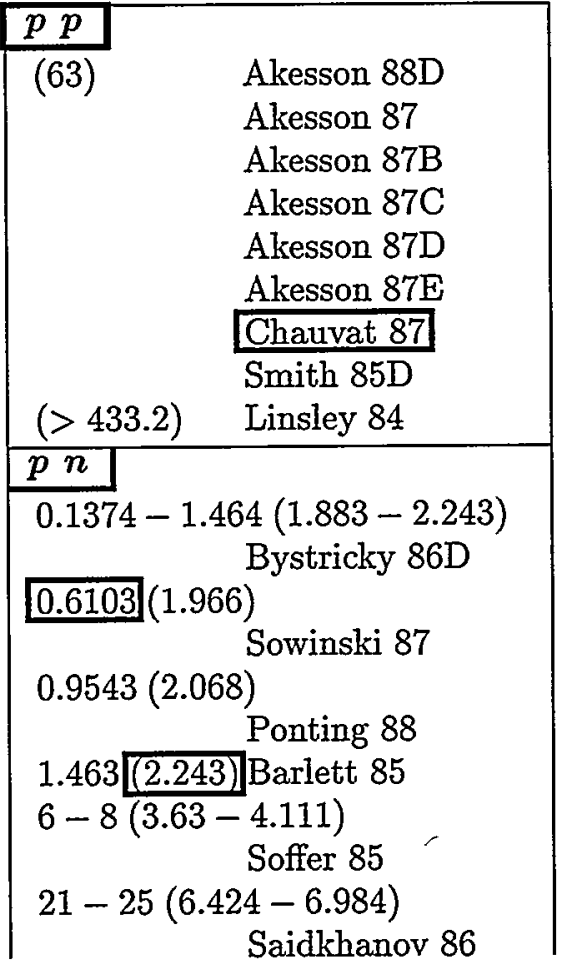

Document ID: see the ID/Reference/Title Index for the full reference.

CM Energy $\boldsymbol{E}_{\mathrm{cm}}$ : in $\mathrm{GeV}$, listed in parentheses. 
This is a short "Table of Contents" of the full Beam/Target/Momentum Index

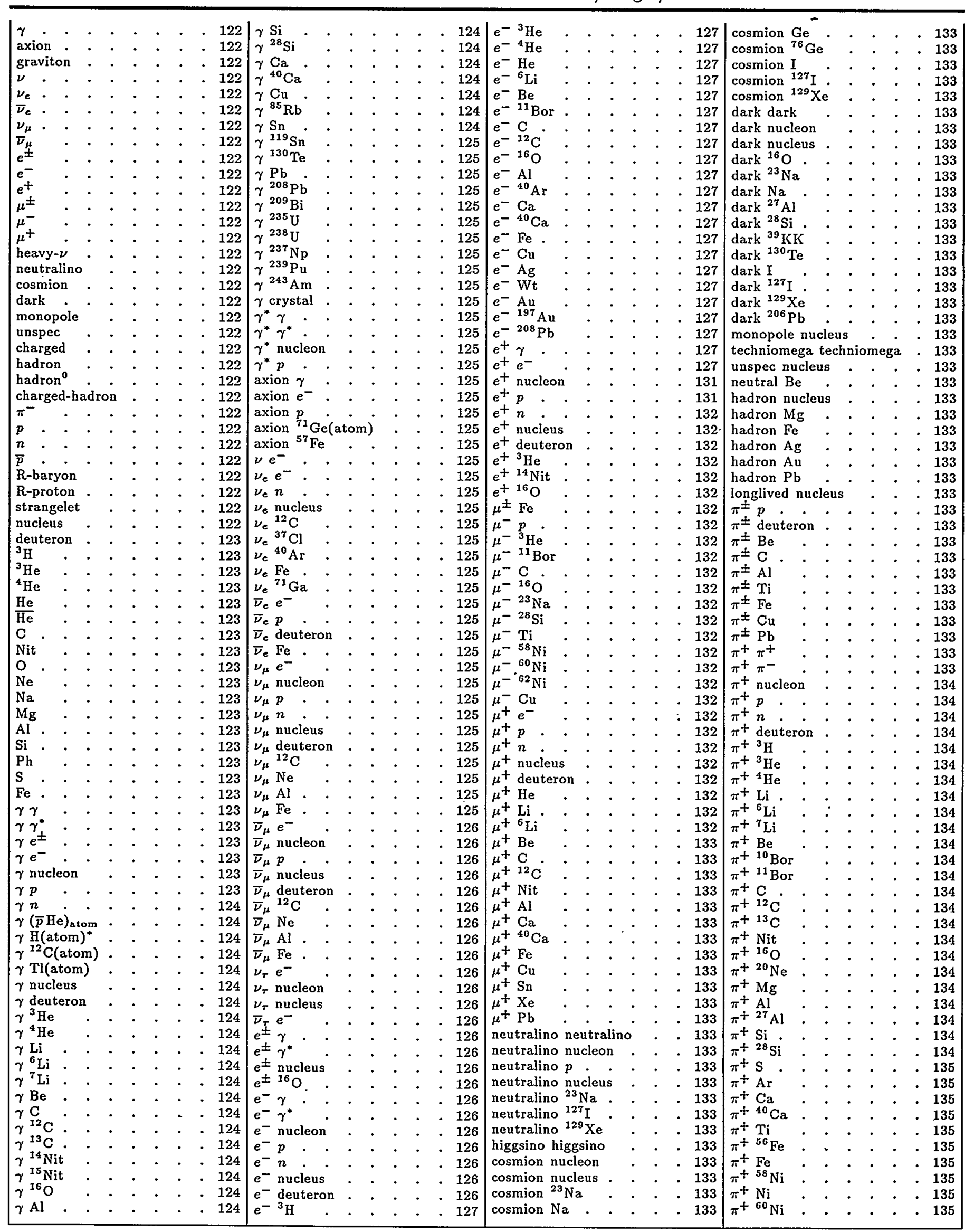


This is a short "Table of Contents" of the full Beam/Target/Momentum Index

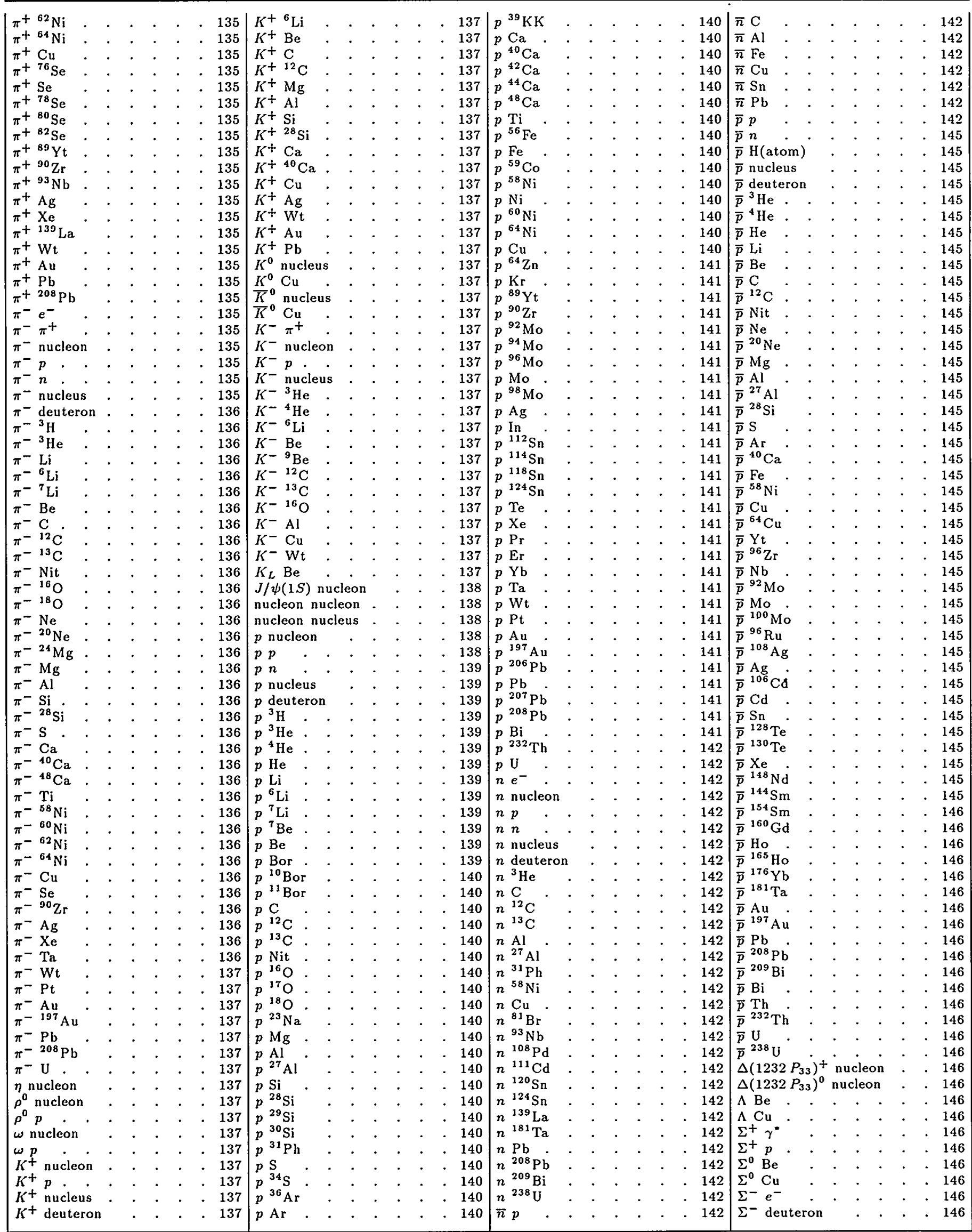


This is a short "Table of Contents" of the full Beam/Target/Momentum Index

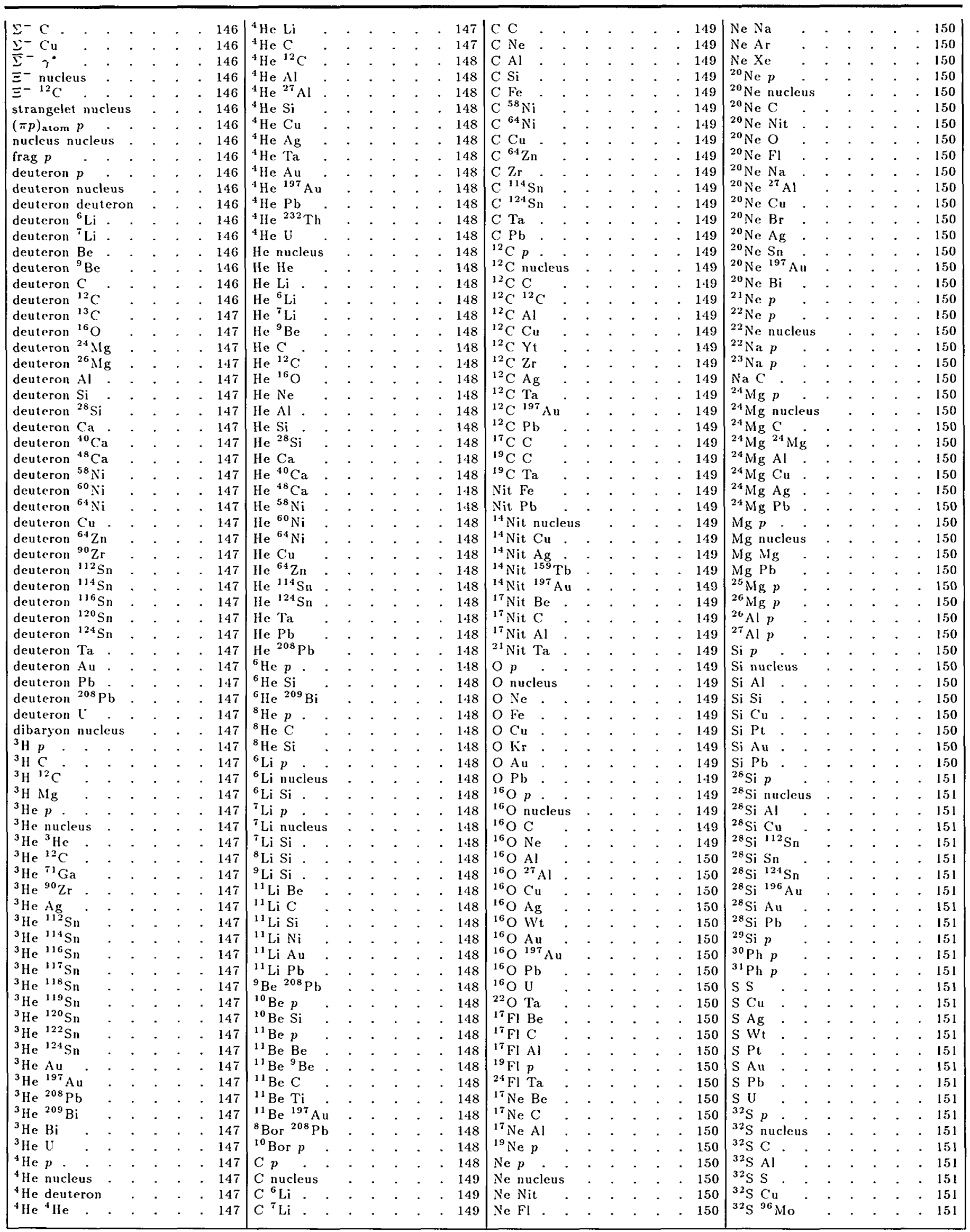


This is a short "Table of Contents" of the full Beam/Target/Momentum Index

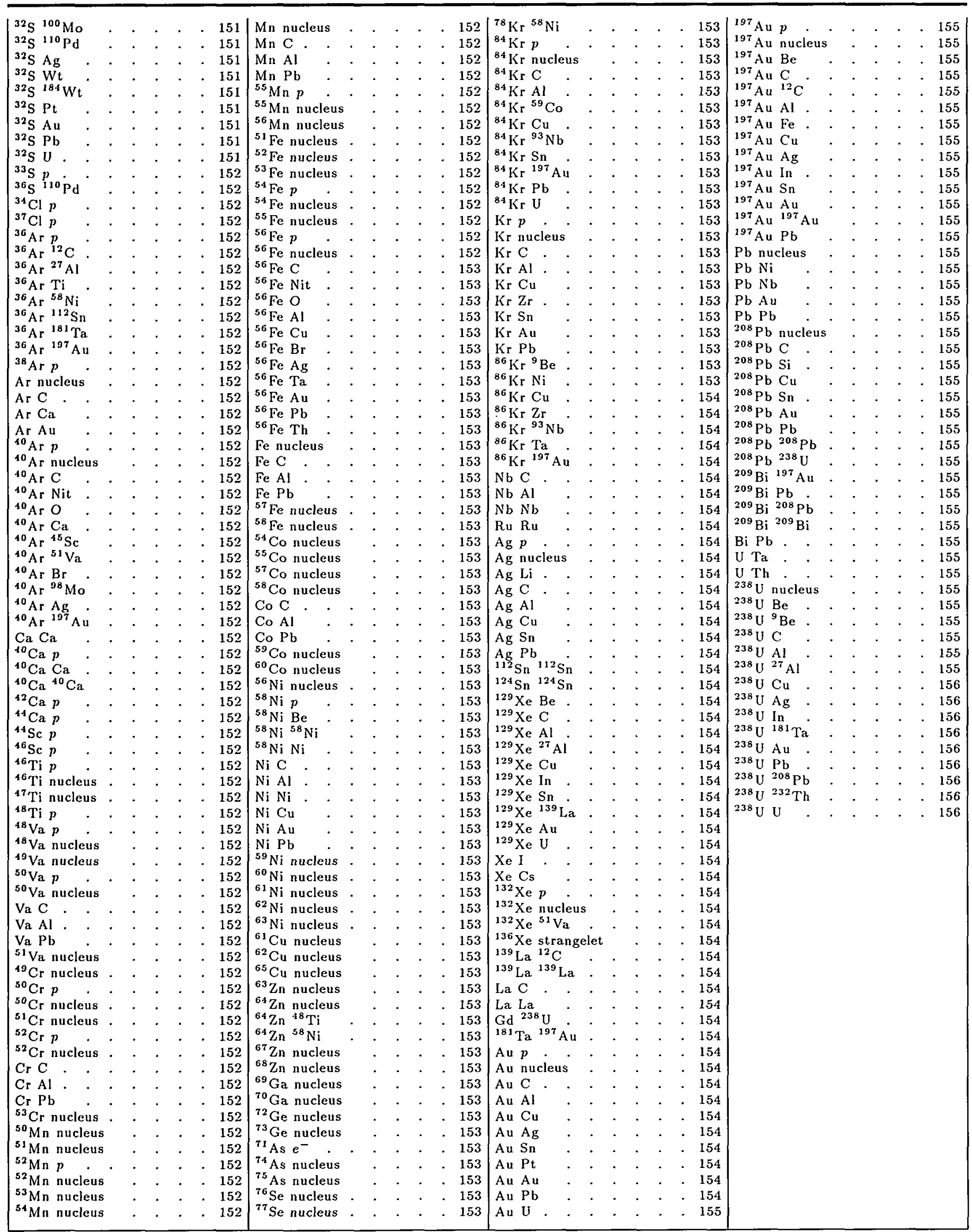




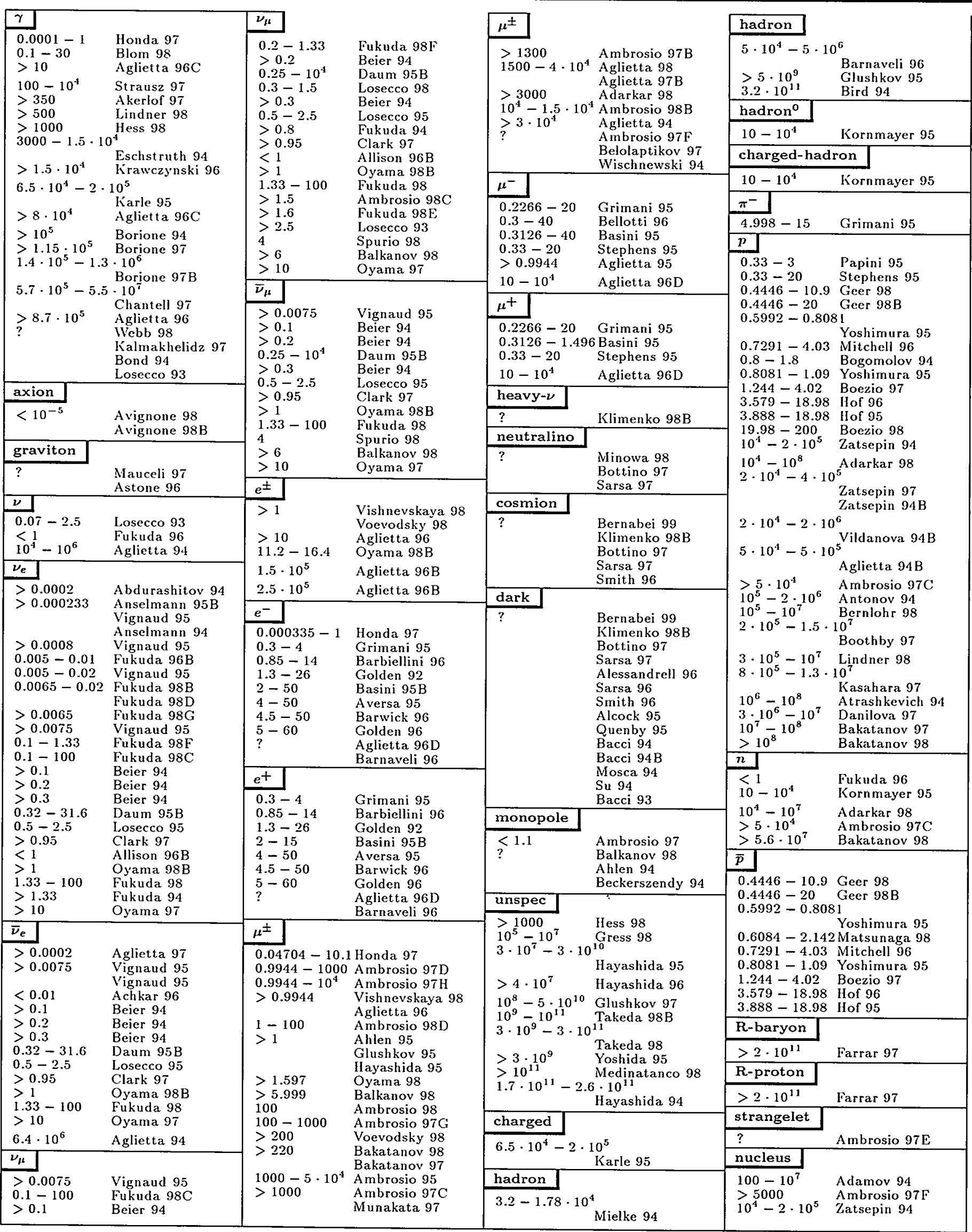




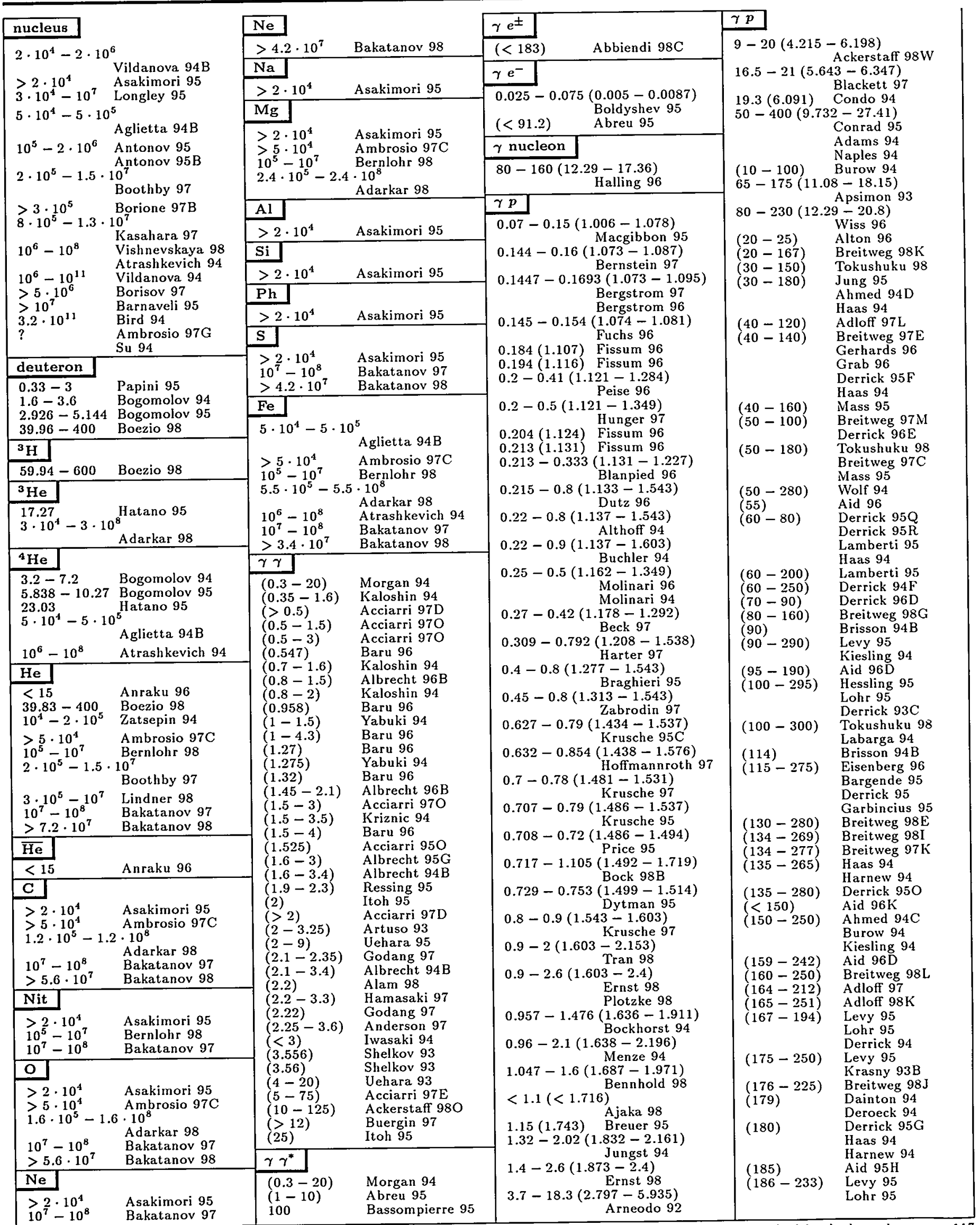

Entries are in order of beam name, then target name, then beam momentum. Particle names are ordered as described in the legend on page 117 Entries are in order of beam name, then target. name, then beam momentum. Particle names are ordered as a 118 . A few chemical symbols for and as listed in the Particle Vocabulary. See also the Table of Contents of this Index beginning on the page then $E_{\mathrm{cm}}$ in $\mathrm{GeV}$ follows in parentheses. For certain initial states only $E_{\mathrm{cm}}$ (in parentheses) is given. 


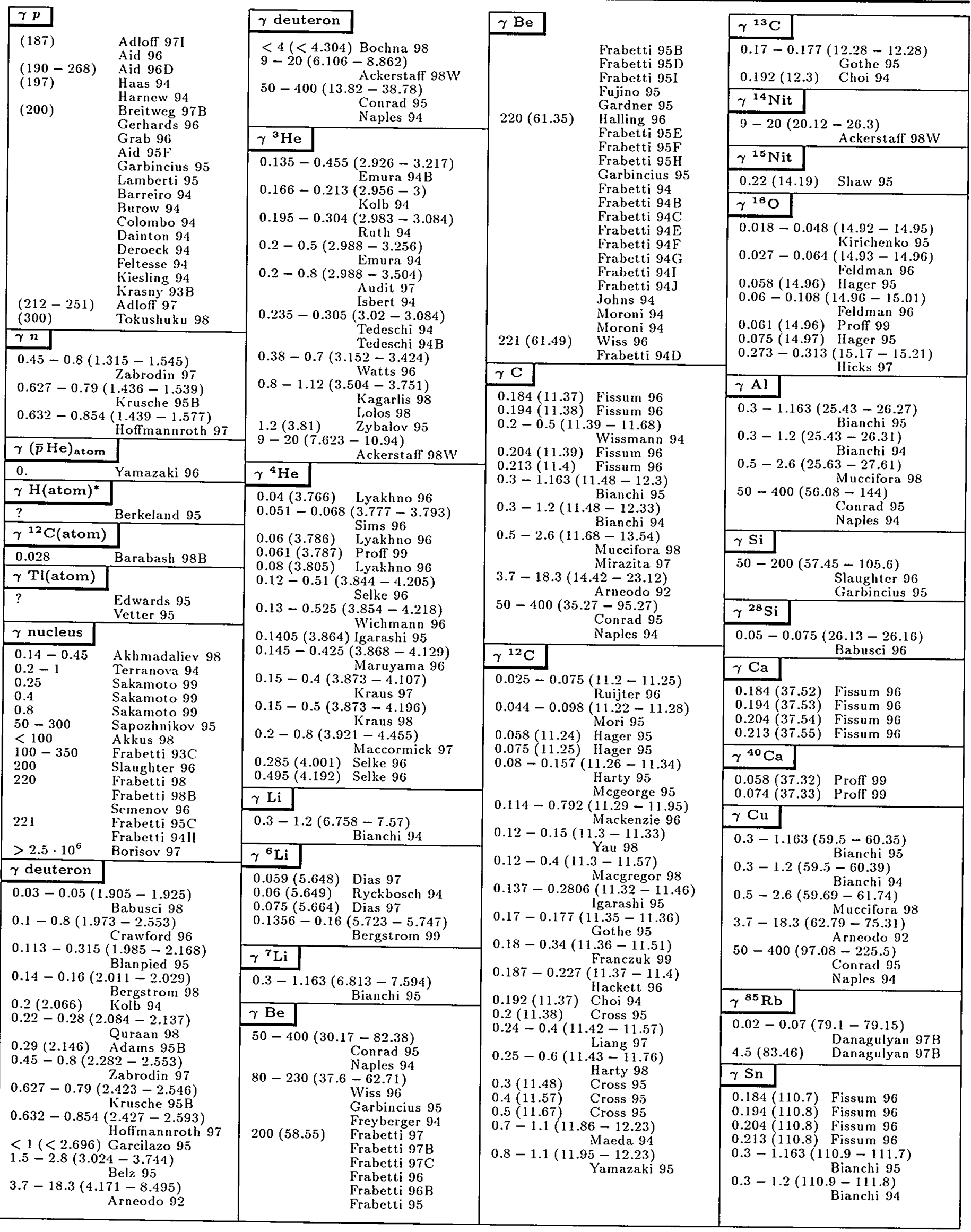




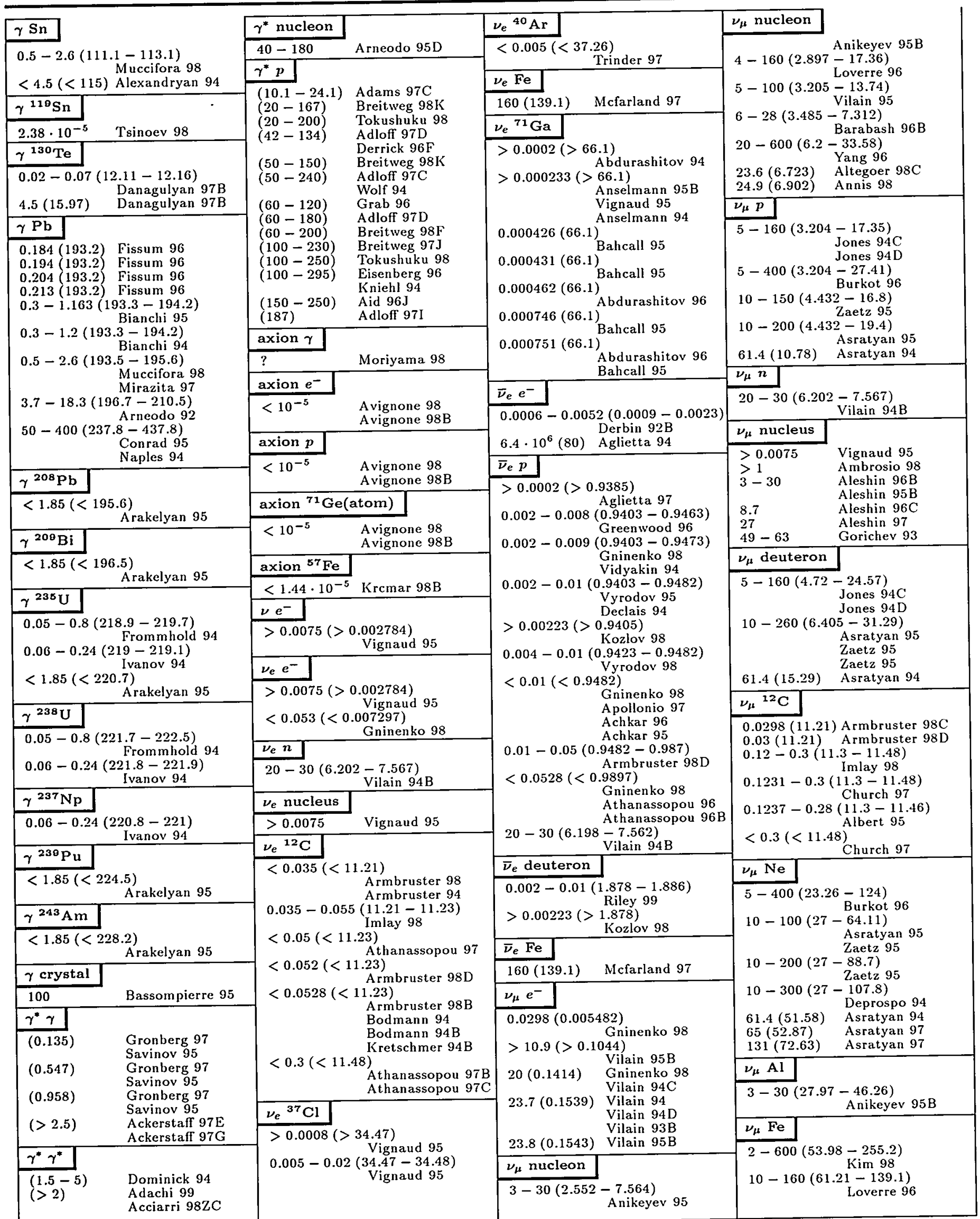

Entries are in order of beam name, then target name, then beam momentum. Particle names are ordered as described in the legend on page 117

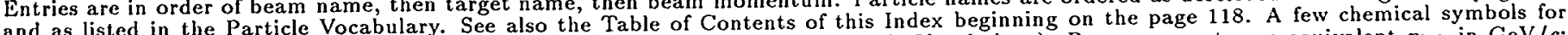

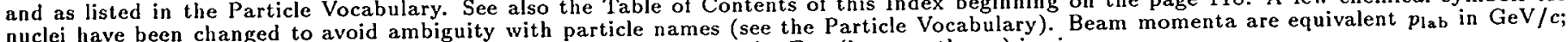
then $E_{\mathrm{cm}}$ in GeV follows in parentheses. For certain initial states only $E_{\mathrm{cm}}$ (in parentheses) is given. 


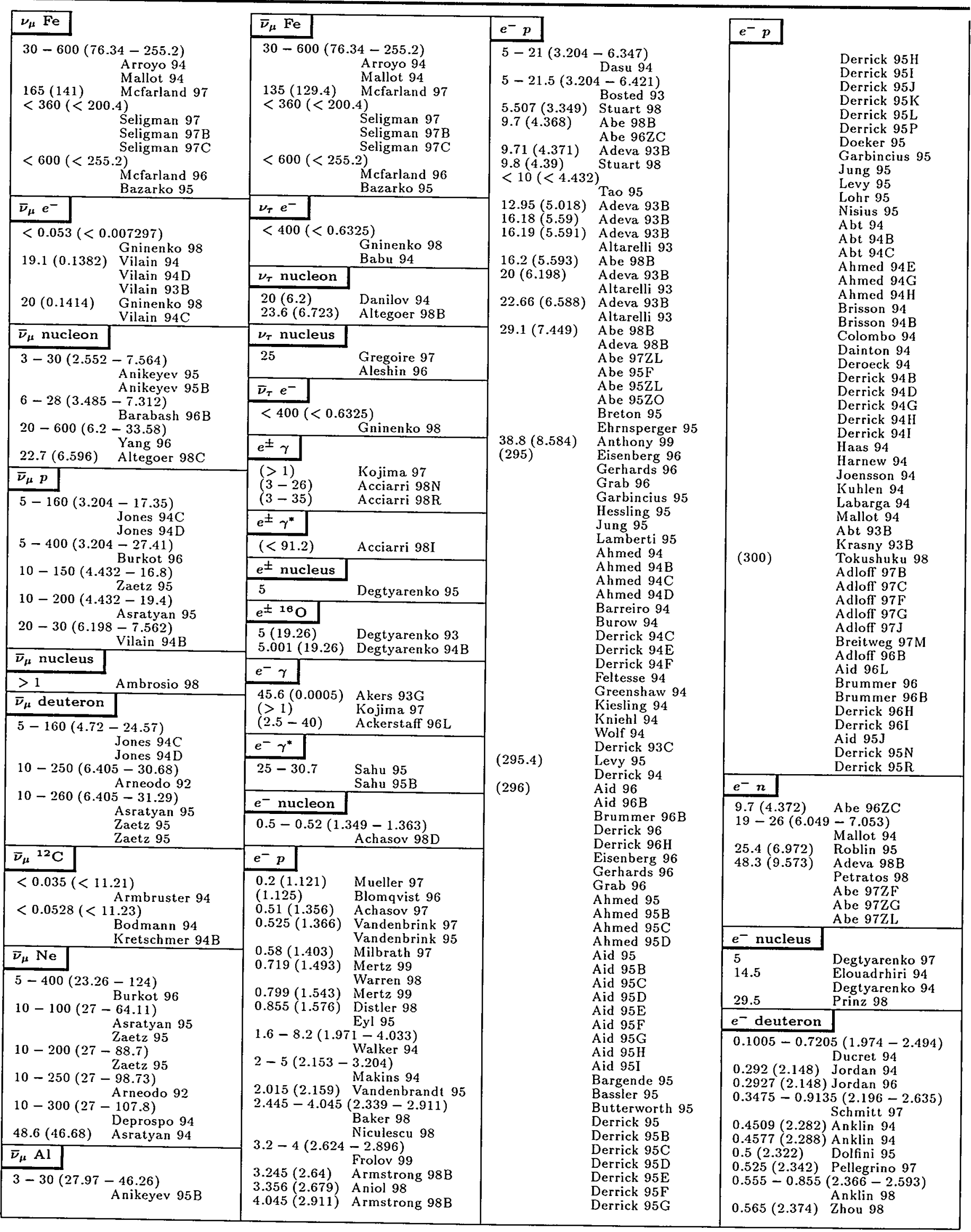




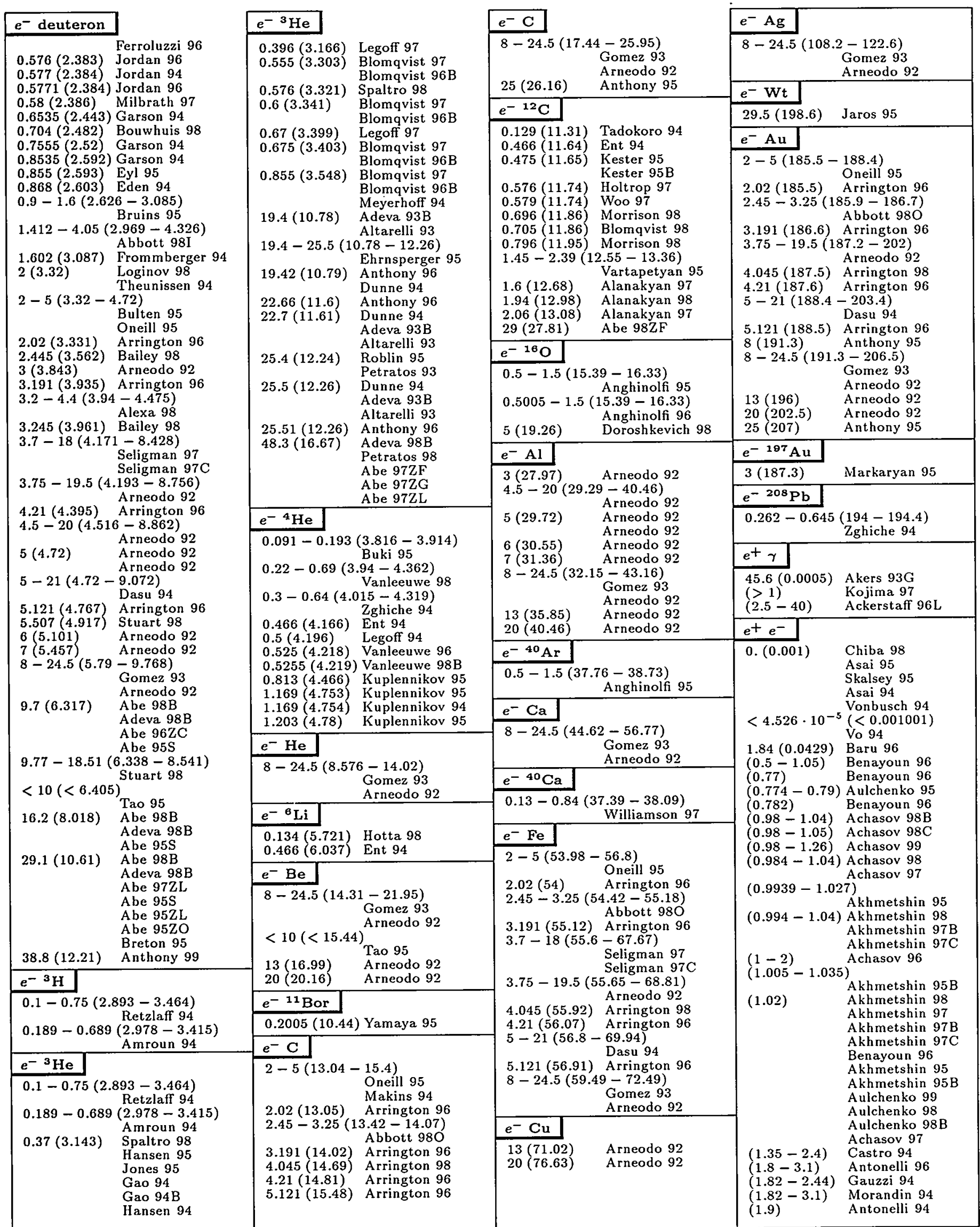

Entries are in order of beam name, then target name, then beam momentum. Particle names are ordered as described in the legend on page 117 and as listed in the Particle Vocabulary. See also the Table of Contents of this Index beginning on the page 118. A few chemical symbols for nuclei have been changed to avoid ambiguity with particle names (see the Particle Vocabulary). Beam momenta are equivalent $p_{1 a b}$ in GeV/c; then $E_{\mathrm{cm}}$ in $\mathrm{GeV}$ follows in parentheses. For certain initial states only $E_{\mathrm{cm}}$ (in parentheses) is given. 


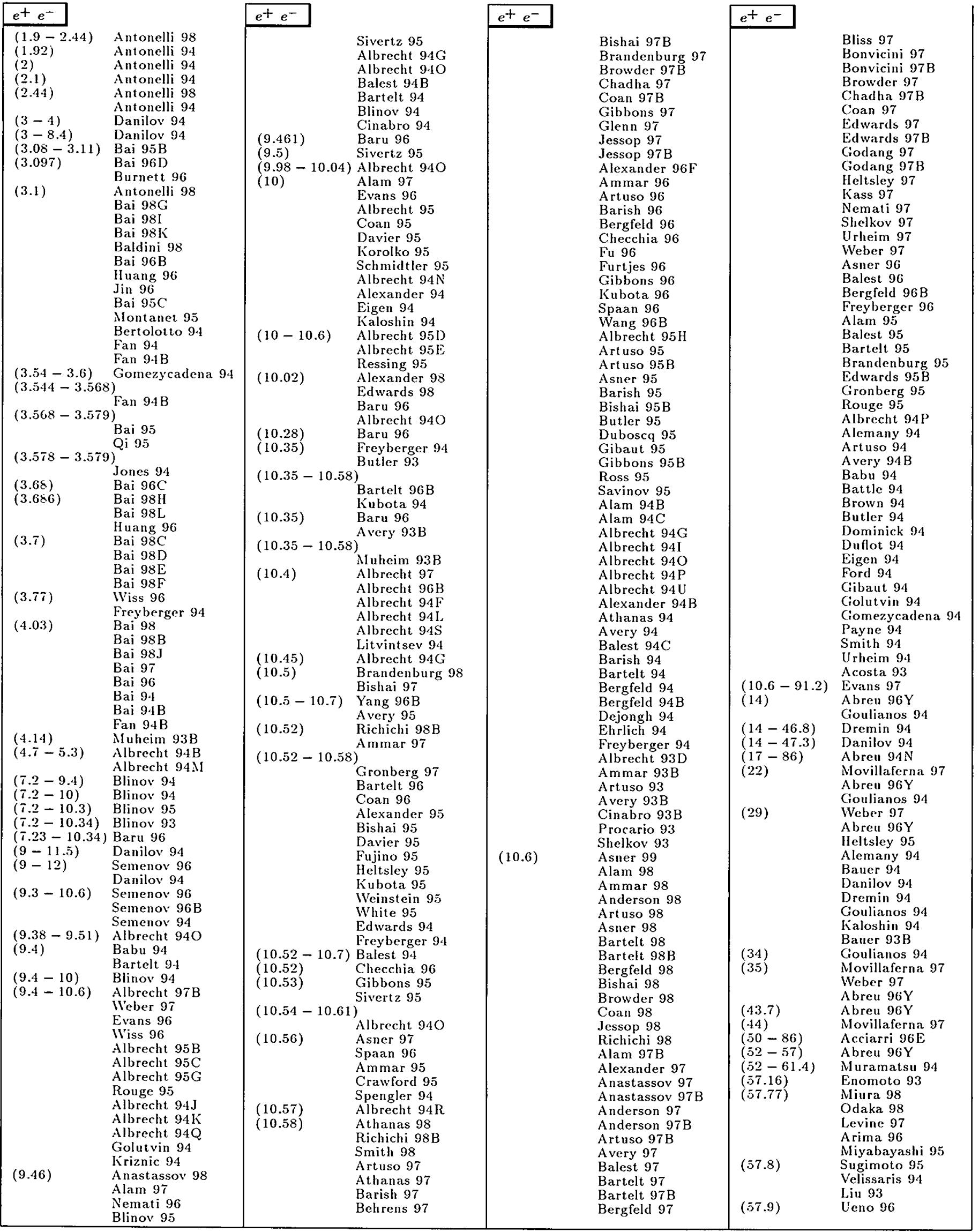




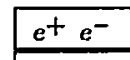

(58)

(58)

Nakano 94

Adachi 99

A hn $98 \mathrm{C}$

Aoki 98

Kim 98B

Hamasaki 97

Hanai 97

Kojima 97

Ohyama 96

Takashimizu 96

A be $95 \mathrm{ZJ}$

Aso 95

Aso $95 \mathrm{~B}$

Choi 95

Hanai 95

Itoh 95

Kanda 95

Li 95

Sahu 95

Sahu 95B

Uehara 95

Enomoto $94 \mathrm{~B}$

Enomoto $94 \mathrm{C}$

Hosoda 94

Itoh 94

Iwasaki 94

Shirai 94

Stuart 94

Tauchi 94

Yabuki 94

Sakuda 93

Uehara 93

(58.1) Enomoto 94

(81.2)

Acciarri 96E

Acciarri 980

$(88-95) \quad$ Akers $94 \mathrm{~F}$

(88.2 - 94.2) Acciarri 97V

Acciarri 95F

Acciarri 95G

A kers 95D

Behnke 95

Bella 95

Buskulic 95B

Buskulic 95E

Matorras 95

Rouge 95

Abreu $94 \mathrm{E}$

Abreu 94F

A breu $94 \mathrm{H}$

Abreu $94 \mathrm{M}$

Acciarri 94

Acciarri 94D

Acciarri 94F

Akers 94D

Akers $94 \mathrm{G}$

Buskulic 94D

Buskulic 94G

Duflot 94

Abreu $93 \mathrm{G}$

Luke 93

Vilain 93B

(88.2 - 94.22) Kramer 94

$(88.24-94.23)$

Gruncwald 93

(88.25 - 93.7) Akers $94 \mathrm{M}$

$(88.25-94.25)$

$(88.28-94.28)$

Buskulic 95D

Akers 95B

$(88.28-95.04)$

Bella 95

Akers $95 \mathrm{~K}$

(88.3 - 94.3) Behnke 95

Abreu 94D

Abreu 93L

Buskulic 93D

Buskulic 93P

(88.4 - 90.4) Ackerstaff 97C

$(88.4-93.7)$ Acciarri $96 \mathrm{M}$

$(88.4-93.9)$ Buskulic 94K

(88.5 - 93.7) Acciarri 95B

Adam 93

(88.5 - 93.8) Acciarri $94 \mathrm{E}$

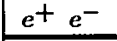

$(88.56-93.75)$

89.2-93.2) Acciarri 98

$\begin{array}{ll}(89.37) & \text { Ackerstaff } 97 \mathrm{G} \\ \text { Barate } 98 \mathrm{~K}\end{array}$

89.45) Alexander 96S

(89.46) Abreu $98 \mathrm{~L}$

$(89.48-91.26)$

Abreu 96

Acciarri 98V
Acciarri $96 \mathrm{E}$

(89.52) Alexander $95 \mathrm{H}$

$(89.52-92.44)$

(89.55) Akers $95 \mathrm{X}$

(90) Buskulic 95C

$(90.5-91.5)$ Alexander 98B

$(90.95-91.45)$

Akers $94 \mathrm{E}$

Acciarri $98 \mathrm{~S}$

Acciarri 98T

Ackerstaff $98 \mathrm{~V}$

Acciarri 97D

Acciarri 97R

Ackerstaff $97 \mathrm{E}$

Abreu 95E

Abreu $95 \mathrm{~F}$

Dremin 94

$(91-91.5) \quad$ Buskulic $95 \mathrm{G}$

Kramer 94

(91.05-91.4) Ackerstaff 97C

(91.2) Abbiendi 99

Abbiendi 99B

Abbiendi 99C

Abbiendi 99D

A be $99 \mathrm{C}$

Abreu $99 \mathrm{C}$

Abreu 99D

Abreu $99 \mathrm{~K}$

Barate 99E

Barate 99F

Abbiendi 98D

Abbiendi 98E

Abbiendi $98 \mathrm{~F}$

Abbiendi $98 \mathrm{H}$

Abbiendi $98 \mathrm{I}$

Abbiendi 98K

Abbiendi 980

Abe $98 \mathrm{H}$

A be $98 \mathrm{~L}$

Abreu 98

Abreu 98B

Abreu $98 \mathrm{C}$

Abreu 98D

Abreu $98 \mathrm{E}$

A breu $98 \mathrm{H}$

Abreu 98L

Abreu $98 \mathrm{M}$

Abreu 980

Abreu $98 \mathrm{Q}$

A breu $98 \mathrm{~S}$

Acciarri 98C

Acciarri 98D

Acciarri 98E

Acciarri 98G

Acciarri 98H

Acciarri 98I

Acciarri 98L

Acciarri 98P

Acciarri 98X

Acciarri $98 \mathrm{Y}$

Acciarri 98ZC

Acciarri 98ZE

Ackerstaff 98

A ckerstaff $98 \mathrm{~B}$

Ackerstaff $98 \mathrm{D}$

Ackerstaff 98F

Ackerstaff $98 \mathrm{H}$

Ackerstaff 98I

Ackerstaff $98 \mathrm{~J}$

Ackerstaff 98K

Ackerstaff 98P

Ackerstaff $98 \mathrm{Q}$

Ackerstaff $98 \mathrm{~S}$

Ackerstaff $98 \mathrm{~T}$

Barate 98

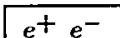

$e^{+} e^{-}$

arate $98 \mathrm{~B}$

Barate 98C

Barate 98D

Barate 98E

Barate 98F

Barate 98G

Barate $98 \mathrm{H}$

Barate 98J

Barate $98 \mathrm{~K}$

Barate 98L

Barate $98 \mathrm{M}$

Barate 980

Barate 98P

Abe $97 \mathrm{~B}$

Abe $97 \mathrm{E}$

A be $97 \mathrm{G}$

Abe $97 \mathrm{~J}$

Abe 97T

Abe $97 \mathrm{U}$

Abreu 97B

Abreu 97D

Abreu 97E

Abreu 97F

Abreu 97G

Abreu 97H

Abreu 97I

Abreu 97J

Abreu $97 \mathrm{~K}$

Abreu 970

Acciarri $97 \mathrm{~F}$

Acciarri 97G

Acciarri 97I

Acciarri $97 \mathrm{M}$

Acciarri 970

Acciarri 97P

Acciarri 97W

Acciarri 97ZB

Ackerstaff $97 \mathrm{D}$

Ackerstaff $97 \mathrm{~F}$

Ackerstaff $97 \mathrm{H}$

Ackerstaff 97I

Ackerstaff 97K

Ackerstaff $97 \mathrm{~L}$

Ackerstaff $97 \mathrm{M}$

Ackerstaff $97 \mathrm{~N}$

Ackerstaff 970

Ackerstaff $97 \mathrm{R}$

Ackerstaff $97 \mathrm{X}$

Ackerstaff $97 \mathrm{Y}$

Ackerstaff 97ZB

Ackerstaff 97ZE

Ackerstaff 97ZF

Ackerstaff $972 \mathrm{H}$

Adam 97

Alemany 97

Alvaro 97

Barate 97

Barate $97 \mathrm{C}$

Barate 97D

Barate $97 \mathrm{E}$

Barate $97 \mathrm{~F}$

Barate $97 \mathrm{G}$

Barate $97 \mathrm{H}$

Barate 97I

Barate $97 \mathrm{~K}$

Barate $97 \mathrm{~N}$

Barate 97R

Barate $97 \mathrm{~T}$

Barate $97 \mathrm{~W}$

Barate $972 \mathrm{~B}$

Barate $972 \mathrm{C}$

Barate 972F

Batyunya 97

Biasini 97

Brient 97

Camoana 97

Cavallo 97

Edsall 97

Kounine 97

Lohmann 97

Lorstad 97

Moore 97

Muller 97

Panvini 97

Passalacqua 97

Quigley 97

Roney 97

Rouge 97

Ruiz 97

Sanchez 97

Sobie 97

Taylor 97

Towers 97

Weber 97

Wermes 97

Abe $96 \mathrm{~T}$

Abe $96 \mathrm{U}$

A be $96 \mathrm{~V}$

Abe $96 \mathrm{~W}$

Abe $96 \mathrm{Y}$

Abe 962

Abe $962 \mathrm{~B}$

Abe 96ZE

Abe $962 \mathrm{~F}$

Abe $96 \mathrm{ZG}$

Abreu 96B

Abreu 96C

Abreu 96E

Abreu 96F

Abreu 96H

Abreu 96I

Abreu 96J

Abreu $96 \mathrm{~K}$ 


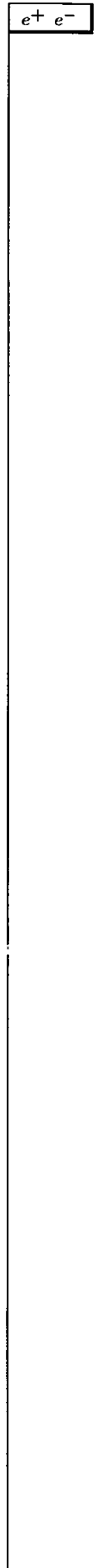

Buskulic $96 \mathrm{~F}$ Buskulic 96 F Buskulic $96 \mathrm{H}$ Buskulic 96I Buskulic $96 \mathrm{~N}$ Buskulic 960 Buskulic $96 \mathrm{P}$ Buskulic 96Q Buskulic 96R Buskulic 96S Buskulic 96T Buskulic $96 \mathrm{U}$ Buskulic 96V

Buskulic 96W

Buskulic 96X

Chapkin 96

Checchia 96

Etzion 96

Evans 96

Furtjes 96

Goy 96

Jackson 96

Karlsson 96

Keranen 96

Kobel 96

Ludovici 96

Malinin 96

Moutoussi 96

Pukhaeva 96

Schenk 96

Sciolla 96

Semenov 96

Su 96

Vorobiev 96

Wilson 96

Ye 96

Zachariadou 96

A be $95 \mathrm{~V}$

$A$ be $95 \mathrm{ZG}$

A be $95 \mathrm{ZM}$

A be $95 \mathrm{ZN}$

Abe $95 \mathrm{ZP}$

Abe $95 \mathrm{ZQ}$

Abreu 95

Abreu $95 \mathrm{~B}$

Abreu 95C

Abreu 95D

Abreu 95G

Abreu 95H

Abreu 95I

Abreu 95 J

Abreu $95 \mathrm{~K}$

Abreu $95 \mathrm{~L}$

Abreu $95 \mathrm{M}$

Abreu 95N

Abreu 950

Abreu 95P

Abreu 95Q

Abreu 95R

Abreu 95S

Abreu 95T

Abreu $95 \mathrm{U}$

Abreu $95 \mathrm{~V}$

Abreu 95X

Acciarri 95

Acciarri 95C

Acciarri 95D

Acciarri 95E

Acciarri 95

Acciarri 95I

Acciarri 95J

Acciarri $95 \mathrm{M}$

Acciarri 950

Acciarri 95P

Adam 95

Adam 95B

A kers 95

Akers 95C

A kers $95 \mathrm{E}$

Akers $95 \mathrm{~F}$

Akers $95 \mathrm{G}$

A kers 95H

Akers 95I

Akers 95J
A kers $95 \mathrm{~L}$

A kers $95 \mathrm{M}$

A kers $95 \mathrm{~N}$

A kers 950

A kers $95 \mathrm{P}$

A kers $95 \mathrm{O}$

A kers $95 \mathrm{R}$

A kers $95 \mathrm{~S}$

Akers 95T

A kers $95 \mathrm{U}$

Akers $95 \mathrm{~V}$

A kers $95 \mathrm{~W}$

Alcaraz 95

Alexander 95B

Alexander 95C

Alexander 95D

Alexander $95 \mathrm{E}$

Alexander 95F

Alexander 95G

Alexander 95I

Andreazza 95

Baird 95

Becattini 95

Behnke 95

Biasini 95

Bourdon 95

Buskulic 95

Buskulic 95F

Buskulic 95 $\mathrm{H}$

Buskulic 95I

Buskulic 95J

Buskulic 95K

Buskulic 95L

Buskulic 95

Buskulic 95N

Buskulic 950

Buskulic 95P

Buskulic 95Q

Buskulic 95R

Buskulic 95S

Buskulic 95T

Buskulic 95U

Buskulic $95 \mathrm{~V}$

Buskulic 95W

Buskulic $95 \mathrm{X}$

Cerutti 95

Charlesworth 95

Dam 95

Davier 95

Duflot 95

Evans 95

Ferrante 95

Gentile 95

Girone 95

Harton 95

Heltsley 95

Kounine 95

Marti 95

Musolino 95

Padilla 95

Park 95B

Podobrin 95

Raab 95

Rouge 95

Ruckstuhl 95

Schwiening 95

Stahl 95

Stugu 95

Syed 95

Tesch 95

Vorobiev 95B

Ward 95

Watkins 95

A be $94 \mathrm{U}$

A be $94 \mathrm{~V}$

Abe $94 \mathrm{~W}$

A be $94 \mathrm{ZB}$

A be $94 \mathrm{ZI}$

Abreu 94

Abreu 94I

Abreu 94J

Abreu $94 \mathrm{~K}$

Abreu 94 L

Abreu $94 \mathrm{M}$ $e^{+} e^{-}$

Abreu $94 \mathrm{~N}$

Abreu $94 \mathrm{O}$

Abreu $94 \mathrm{Q}$

Abreu 94R

Abreu 94S

Acciarri 94B

Acciarri 94C

A kers $94 \mathrm{C}$

A kers $94 \mathrm{H}$

Akers 94I

Akers 94J

A kers 94L

A kers $94 \mathrm{~N}$

Akers 940

Akers 94P

Akers 94Q

Akers $94 \mathrm{R}$

Akers $94 \mathrm{~S}$

Alemany 94

Baird 94

Baranko 94

Bonivento 94

Brient 94

Buskulic 94

Buskulic 94B

Buskulic 94C

Buskulic 94F

Buskulic 94H

Buskulic 94I

Buskulic 94J

Buskulic 94L

Buskulic 94M

Buskulic 94N

Carr 94

Colas 94

Danilov 94

Dejongh 94

Demin 94

Dremin 94

Fero 94

Golutvin 94

Gomezycadena 94

Hocker 94

Janot 94

Jones $94 \mathrm{~B}$

King 94

Kramer 94

Manly 94

Maur 94

Posthaus 94

Richard 94

Shevchenko 94

Strohmer 94

Wermes 94

Wormser 94

A kers 93F

A kers 93G

A kers $93 \mathrm{H}$

Akers 93J

Buskulic 93P

(91.22) Abe 98K

Alexander $96 \mathrm{~S}$

Hasegawa 95B

Markiewicz 95

(91.24) Alexander $95 \mathrm{H}$

$(91.24-91.28)$

Abe 95ZR

(91.25) Abreu 96Y

Dremin 94

Acciarri $98 \mathrm{~V}$

Schumm 95

A be $94 \mathrm{C}$

$\begin{array}{ll}\text { (91.27) Abe } 96 \mathrm{O} \\ & \text { Buskulic } 94 \mathrm{E}\end{array}$

(91.28) Buskulic 94E

Abe $96 \mathrm{X}$

Abreu 94B

Abreu $94 \mathrm{G}$

$(91.3)$

A kers 93D

Abreu 94Q

Acciarri 94D

A be $95 \mathrm{Z}$

Abe 95ZD

$e^{+} e^{-}$

$(91.56)$
$(91.6)$
$(91.8-94)$
$(92.26)$
$(92.28)$
$(92.94)$

A be $95 \mathrm{ZF}$

Abe $942 \mathrm{C}$

ino 94

Park 94

Martirena 94

Ackerstaff $97 \mathrm{C}$

Barate 98K

Abreu 94C

A breu $98 \mathrm{M}$

Alexander $95 \mathrm{H}$

(93)

$(93.1)$

$(<120)$

Acciarri 96

Alexander $96 \mathrm{~S}$

Acciarri $98 \mathrm{~V}$

Danilov 94

Abreu 99I

Abreu $99 \mathrm{~K}$

Acciarri $98 \mathrm{M}$

Acciarri 980

Acciarri 97E

Ackerstaff $97 \mathrm{~S}$

Barate $97 \mathrm{M}$

Barate $97 \mathrm{Q}$

Barate $97 \mathrm{U}$

Barate 97Z 
$e^{+} e^{-}$

$(133-136)$
$(133-140)$
$(136)$

(136.2)

(136.3) Ackerstaff 97U

Abreu 98F

Acciarri 98B

Acciarri 98J

Acciarri 97V

Acciarri $95 \mathrm{~K}$

(140) Acciarri 97E

(140.1) Ackerstaff 97R

(140.2) Acciarri 98B

(161)

Acciarri 95K

Abreu $99 \mathrm{H}$

Abreu 99I

Abreu $99 \mathrm{~K}$

Acciarri 99

Barate 99B

Barate $99 \mathrm{C}$

Abbiendi $98 \mathrm{~B}$

Abbiendi $98 \mathrm{~N}$

Abreu $98 \mathrm{~K}$

Abreu $98 \mathrm{~N}$

Abreu 98T

Acciarri $98 \mathrm{~K}$

Acciarri $98 \mathrm{M}$

Acciarri 980

Acciarri 98U

Acciarri 98ZF

Ackerstaff $98 \mathrm{E}$

Ackerstaff $98 \mathrm{G}$

Ackerstaff 980

Barate $98 \mathrm{~N}$

Bock 98

Hoffmann 98

Abreu $97 \mathrm{M}$

Abreu 97N

Abreu 97R

Acciarri 97C

Acciarri 97E

Acciarri $97 \mathbf{H}$

Acciarri 97L

Acciarri 97X

Acciarri 97Y

Acciarri $97 \mathrm{Z}$

Ackerstaff $97 \mathrm{~J}$

Ackerstaff 97S

Ackerstaff 97V

Ackerstaff $97 \mathrm{Z}$

\begin{tabular}{|c|c|c|}
\hline$e^{+} e^{-}$ & & $e^{+} e^{-}$ \\
\hline $\begin{array}{l}(170-172) \\
(170.3)\end{array}$ & 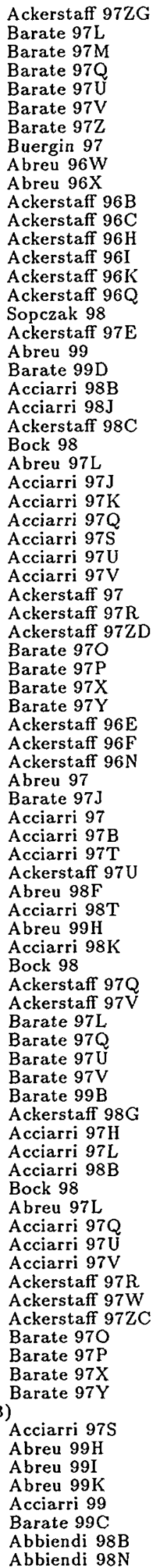 & $\begin{array}{l}(172.4) \\
(181) \\
(181-184) \\
(182) \\
(182.6) \\
(182.7)\end{array}$ \\
\hline
\end{tabular}

Abreu $98 \mathrm{~K}$

Abreu 98N

Abreu 98T

Acciarri 98K

Acciarri $98 \mathrm{M}$

Acciarri 980

Acciarri 98U

Acciarri 98ZF

Ackerstaff $98 \mathrm{E}$

Ackerstaff 980

Barate $98 \mathrm{~N}$

Bock 98

Hoffmann 98

Abreu $97 \mathrm{C}$

Abreu $97 \mathrm{M}$

Abreu $97 \mathrm{~N}$

Abreu 97P

Abreu $97 \mathrm{Q}$

Abreu 97R

Acciarri 97C

Acciarri $97 \mathrm{~N}$

Acciarri 97X

Acciarri 97Y

Acciarri $97 \mathrm{Z}$

Ackerstaff 970

Ackerstaff 97S

Ackerstaff $97 \mathrm{~V}$

Ackerstaff 97Z

Ackerstaff 97ZG

Barate $97 \mathrm{~L}$

Barate $97 \mathrm{M}$

Barate 97Q

Barate $97 \mathrm{U}$

Barate $97 \mathrm{~V}$

Barate 97Z

Barate 97ZD

Buergin 97

Abreu 96X

Barate 97S

Barate 97ZE

Abreu 99

Barate 99D

Ackerstaff 98C

Acciarri 97 J

Ackerstaff $97 \mathrm{ZD}$

Ackerstaff 97P

Ackerstaff 97T

Acciarri 98B

Acciarri 98J

Acciarri 97K

Acciarri 970

Acciarri 97U

Acciarri 97V

Ackerstaff 97R

Ackerstaff $97 \mathrm{U}$

Ackerstaff 97W

Ackerstaff 97ZC

Barate 970

Barate 97P

Barate 97X

Barate 97Y

Acciarri $97 \mathrm{~T}$

Abreu 98F

Barate $98 \mathrm{Q}$

Barate 99

Barate 99B

Abbiendi 98P

Barate 98R

Barate $98 Q$

Ackerstaff 98R

Abreu 99J

Acciarri 98Q

Barate $99 \mathrm{C}$

Barate 99D

Abreu 98F

Acciarri 98F

Acciarri 98J

Acciarri 98ZD

Ackerstaff 98C

Abreu 99B

Abreu $99 \mathrm{E}$

Abreu 99I

Abreu $99 \mathrm{~K}$

Acciarri 99
Abbiendi 98

Abbiendi 98C

Abbiendi 98G

Abbiendi 98J

Abbiendi $98 \mathrm{~L}$

Abbiendi $98 \mathrm{M}$

Abbiendi $98 \mathrm{~N}$

Abbiendi 98R

Abreu 98J

Abreu 98N

Abreu 98Q

Abreu 98 T

Abreu $98 \mathrm{~V}$

Acciarri 98K

Acciarri 98M

Acciarri 980

Acciarri 98S

Acciarri 98T

Acciarri 98U

Acciarri 98W

Acciarri $98 \mathrm{Z}$

Acciarri 98ZC

Acciarri 98ZF

Ackerstaff $98 \mathrm{E}$

Ackerstaff $98 \mathrm{~N}$

Ackerstaff $98 \mathrm{U}$

Ackerstaff $98 \mathrm{~V}$

Barate 98I

Barate $98 Q$

Barate $98 \mathrm{~S}$

Sopczak 98

(184) Barate 98N

Barate 980

Barate 972

\section{$e^{+}$nucleon}

$0.5-0.52(1.349-1.363)$

Achasov 98D

\begin{tabular}{|l|}
\hline$e^{+} p$ \\
\hline $0.51(1.356) \quad$ Achasov 97
\end{tabular}

$27.5(7.245) \quad$ Ackerstaff $98 \mathrm{~L}$

Ackerstaff $98 \mathrm{M}$

Ackerstaff $98 \mathrm{~W}$

27.57 (7.254) Airapetyan 98

(296) Derrick 96 H

Frey 96

Aid 95B

Aid $95 \mathrm{G}$

$(300)$

Breitweg 99

Adloff 98

Adloff 98B

Adloff 98C

Adloff 98D

Adloff $98 \mathrm{E}$

Adloff $98 \mathrm{~F}$

Adloff 98G

Adloff $98 \mathrm{H}$

Adloff $98 \mathrm{I}$

Adloff 98J

Adloff $98 \mathrm{~L}$

Breitweg 98

Breitweg 98B

Breitweg 98C

Breitweg 98D

Breitweg 98E

Breitweg 98F

Breitweg 98G

Breitweg 98H

Breitweg 98J

Breitweg 98K

Breitweg $98 \mathrm{~L}$

Sinclair 98

Adloff 97

Adloff $97 \mathrm{~B}$

Adloff 97C

Adloff 97D

Adloff 97E

Adloff $97 \mathrm{~F}$

Adloff $97 \mathrm{G}$

Adloff $97 \mathrm{H}$

Adloff 97I

Adloff $97 \mathrm{~K}$

Adloff 97L

Entries are in order of beam name, then target name, then beam momentum. Particle names are ordered as described in the legend on page 117 and as listed in the Particle Vocabulary. See also the Table of Contents of this Index beginning on the page 118. A few chemical symbols for nuclei have been changed to avoid ambiguity with particle names (see the Particle Vocabulary). Beam momenta are equivalent $p_{1 a b}$ in GeV/c; then $E_{\mathrm{cm}}$ in $\mathrm{GeV}$ follows in parentheses. For certain initial states only $E_{\mathrm{cm}}$ (in parentheses) is given. 


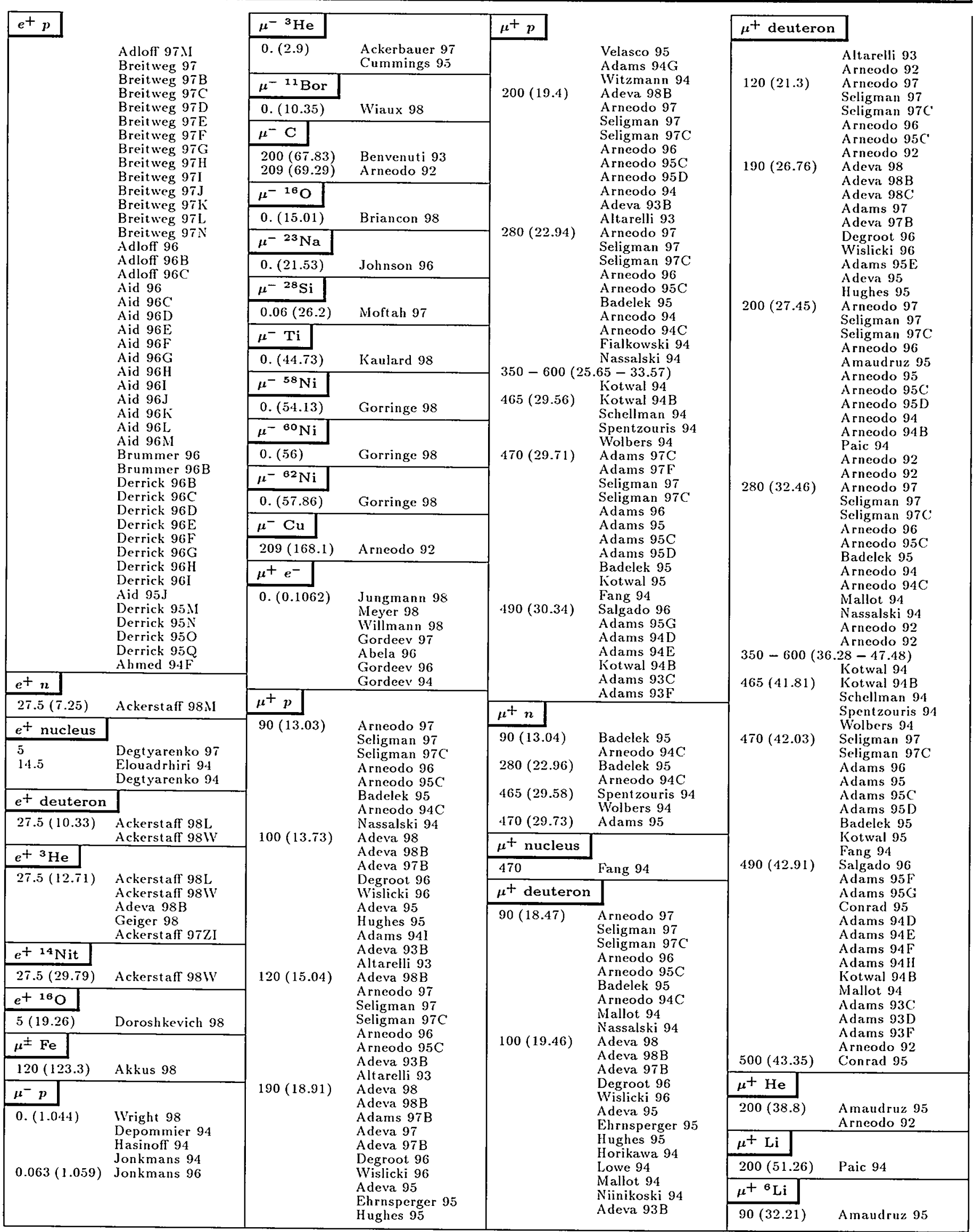




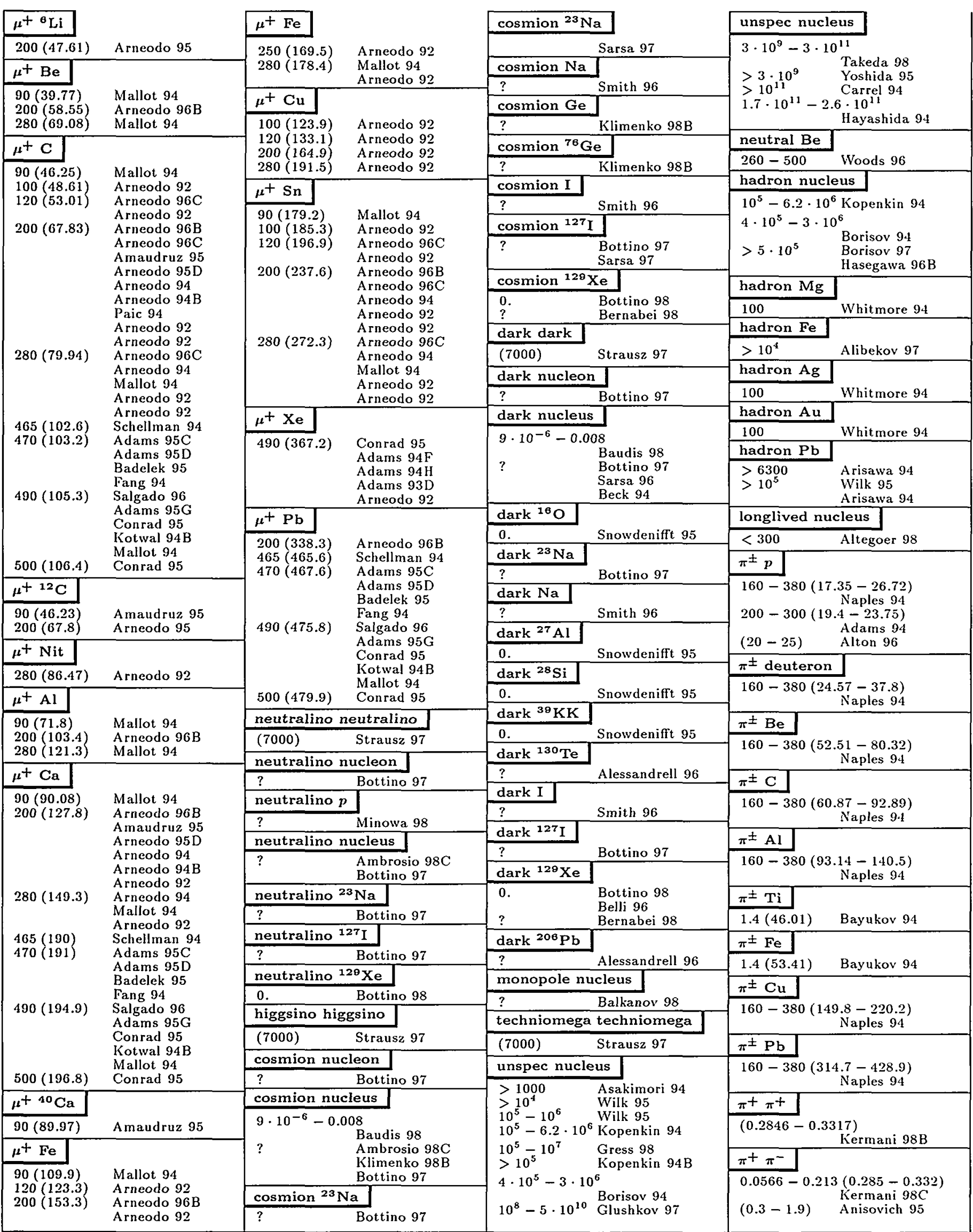

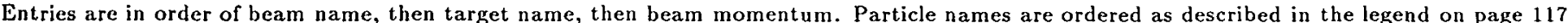

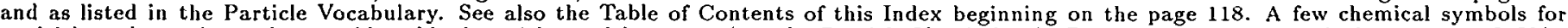

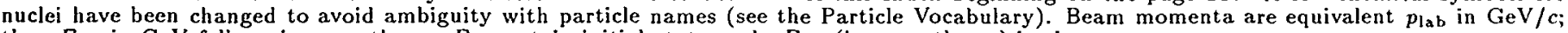
then $E_{\mathrm{cm}}$ in $\mathrm{GeV}$ follows in parentheses. For certain initial states only $E_{\mathrm{cm}}$ (in parentheses) is given. 


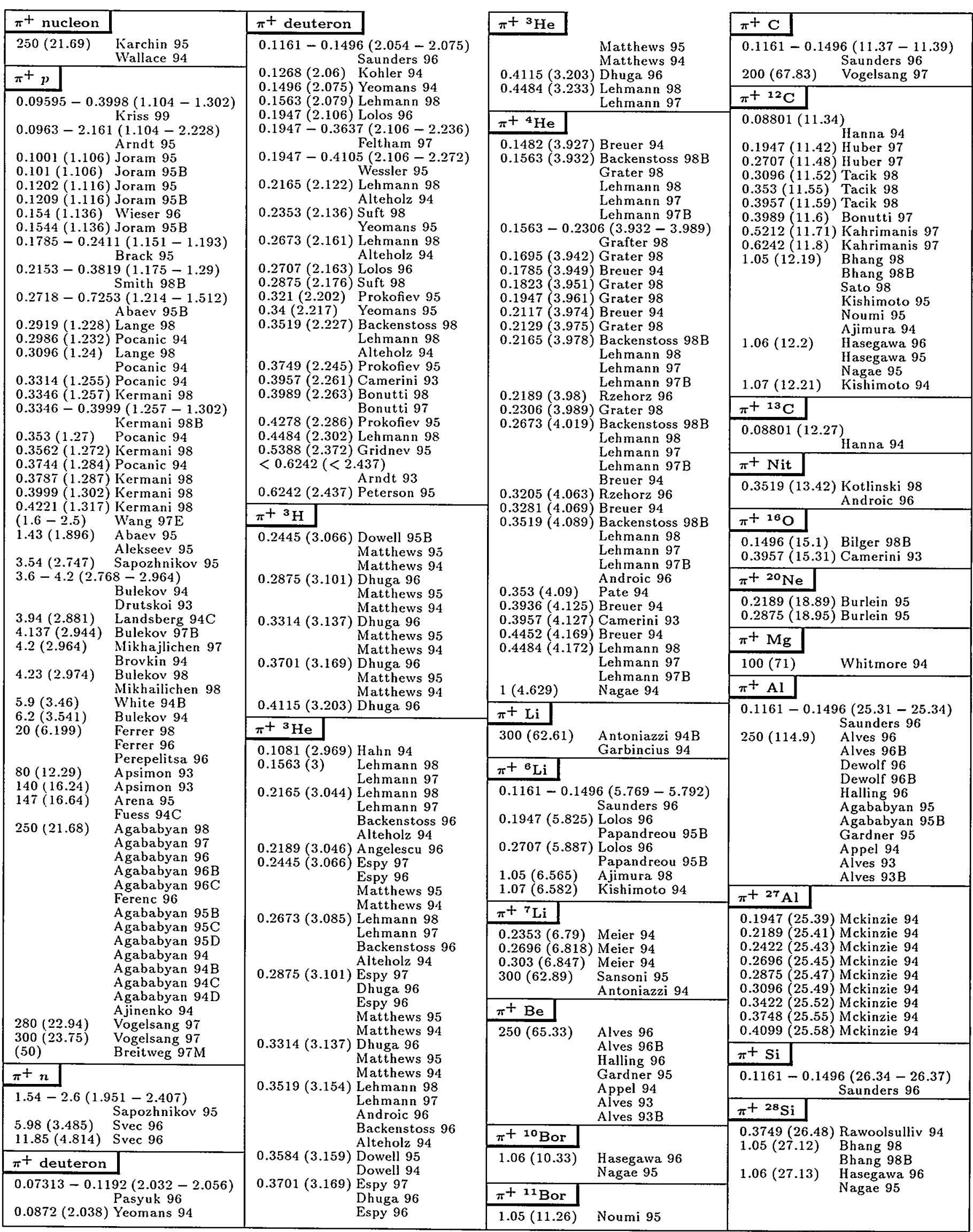




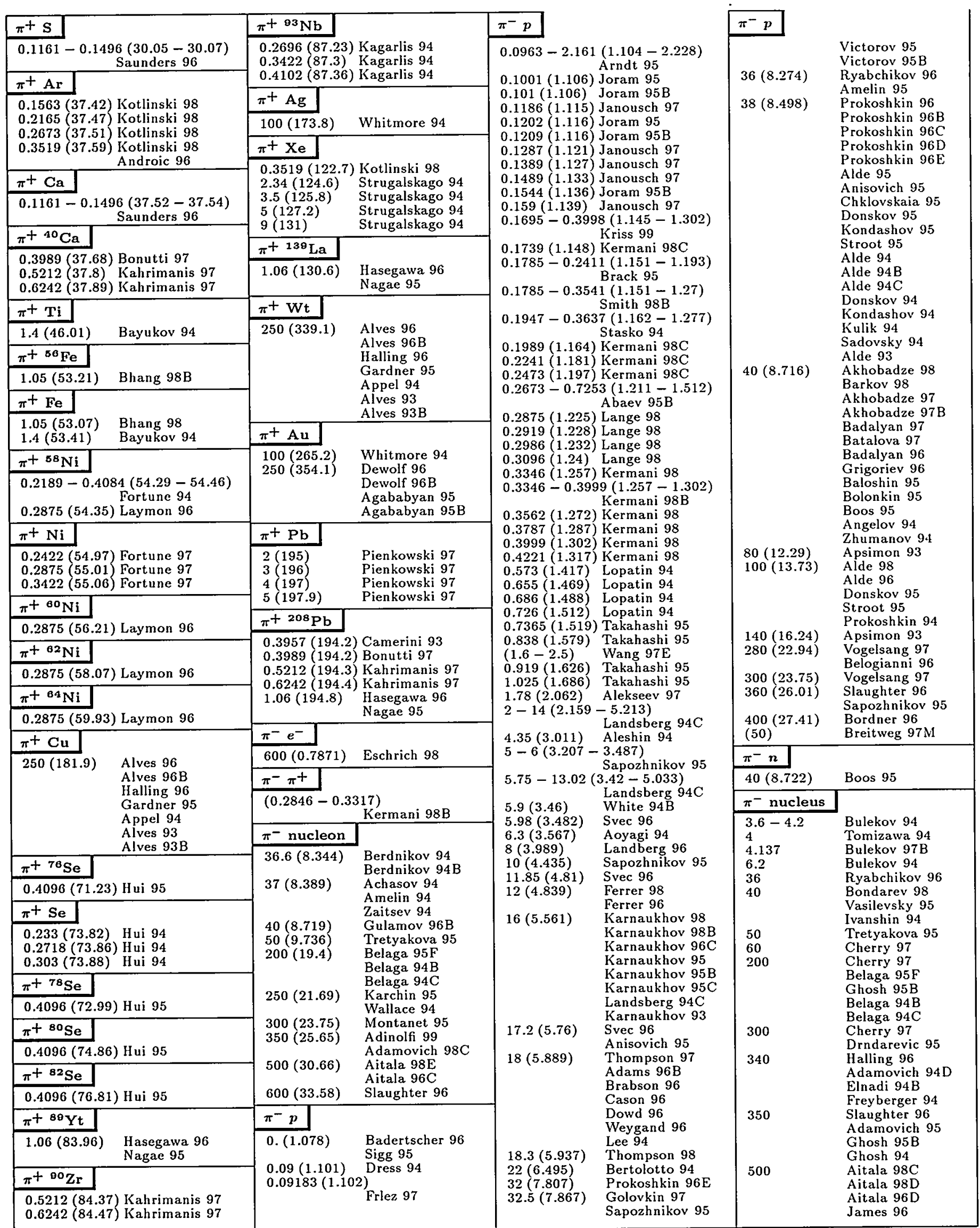

Entries are in order of beam name, then target name, then beam momentum. Particle names are ordered as described in the legend on page 117 and as listed in the Particle Vocabulary. See also the Table of Contents of this Index beginning on the page 118. A few chemical symbols for nuclei have been changed to avoid ambiguity with particle names (see the Particle Vocabulary). Beam momenta are equivalent plab in GeV/c; then $E_{c m}$ in $\mathrm{GeV}$ follows in parentheses. For certain initial states only $E_{\mathrm{cm}}$ (in parentheses) is given. 


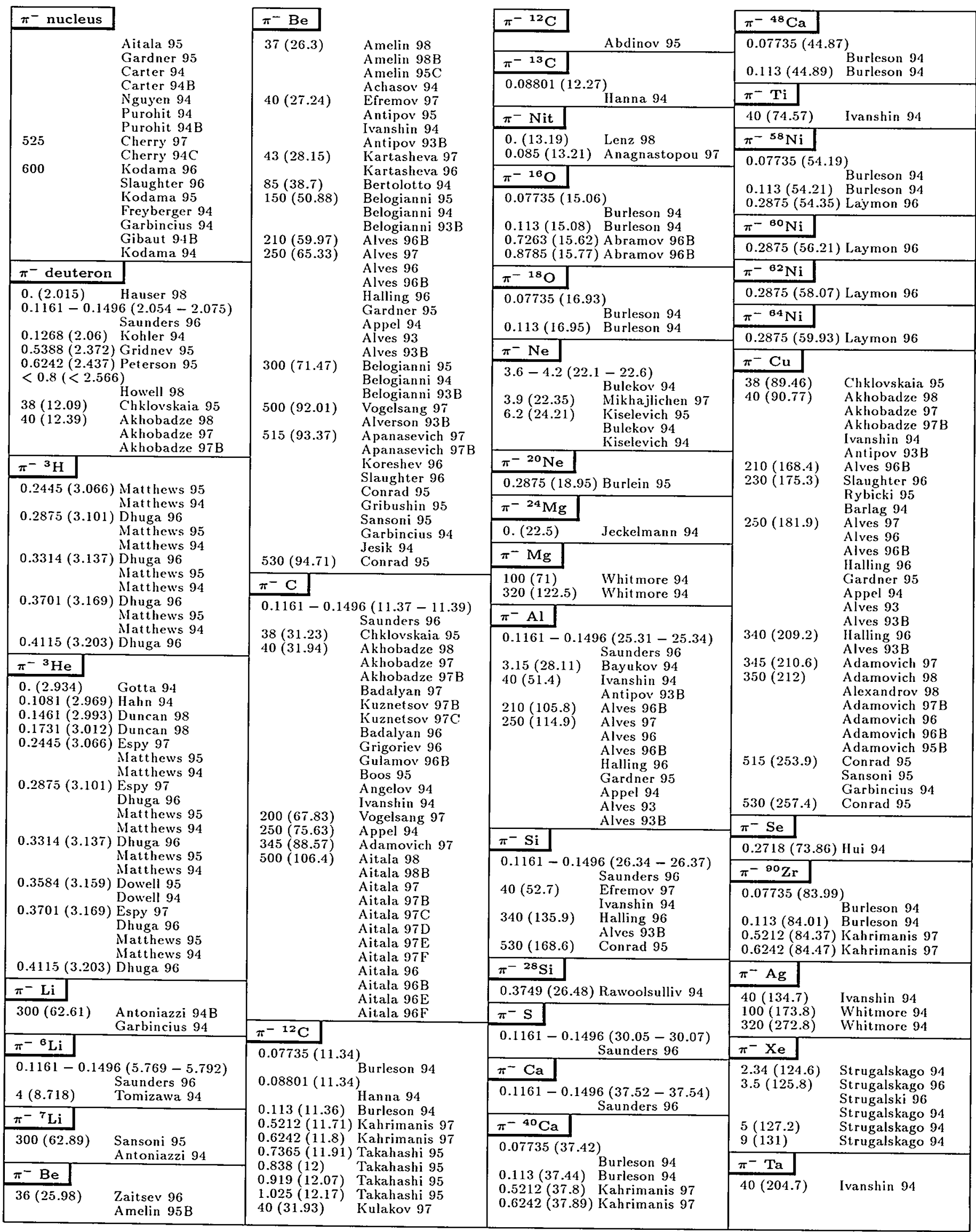




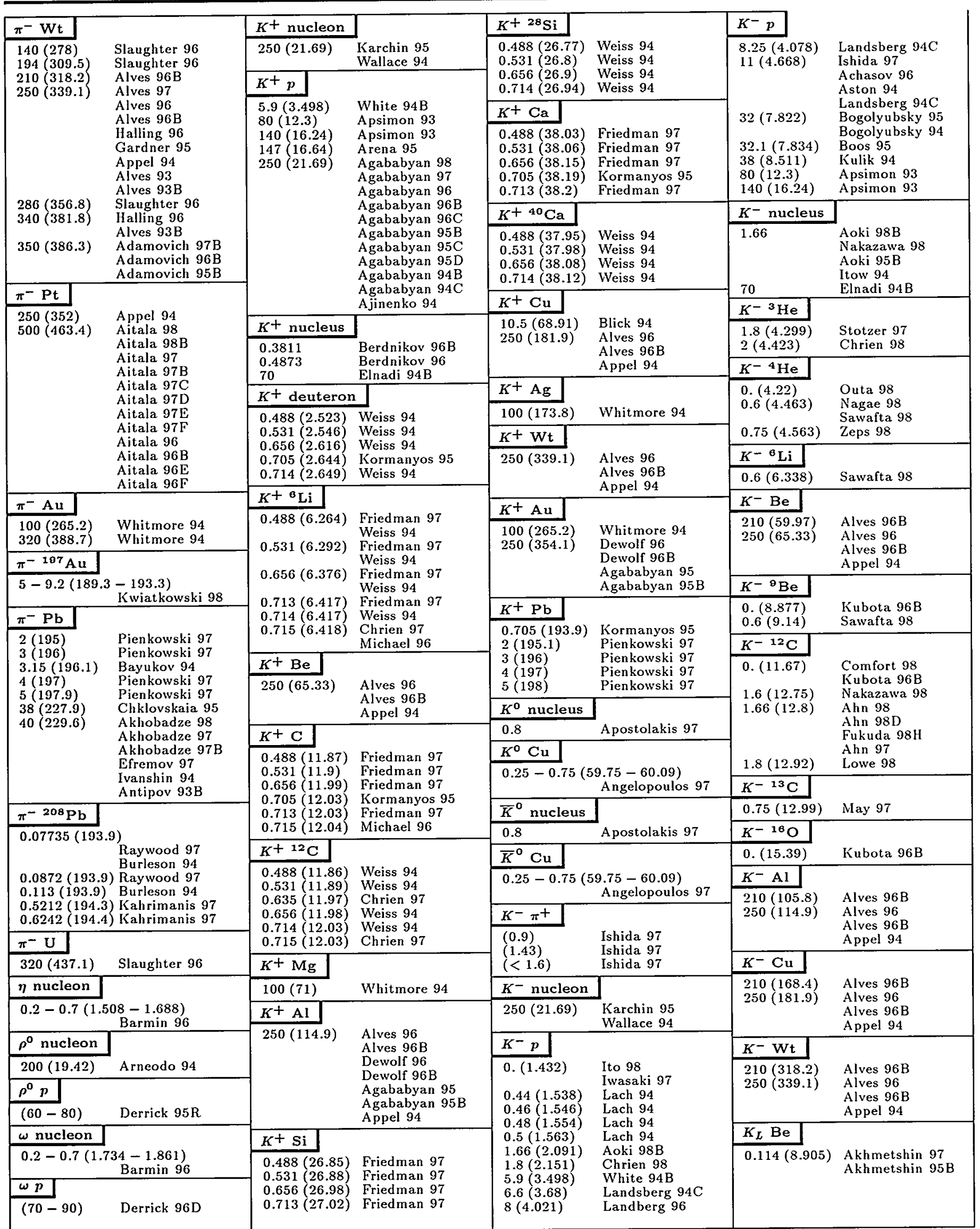

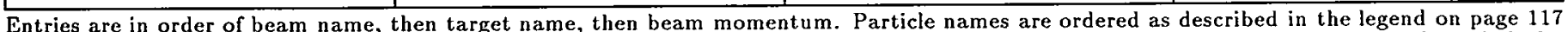

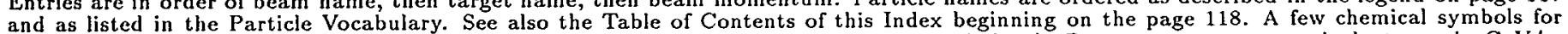

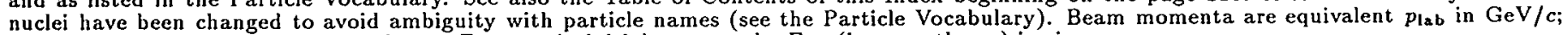
then $E_{\mathrm{cm}}$ in $\mathrm{GeV}$ follows in parentheses. For certain initial states only $E_{\mathrm{cm}}$ (in parentheses) is given. 


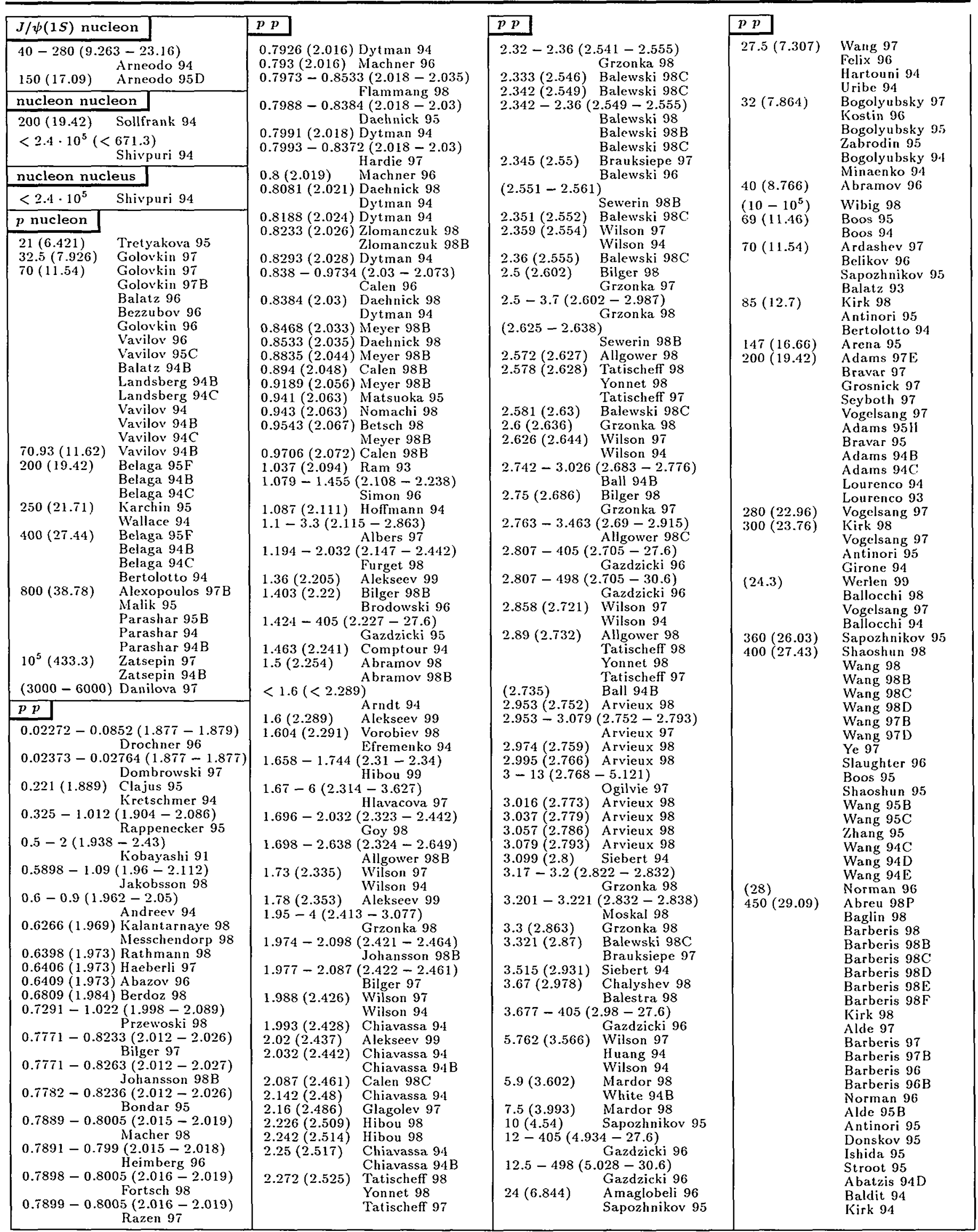




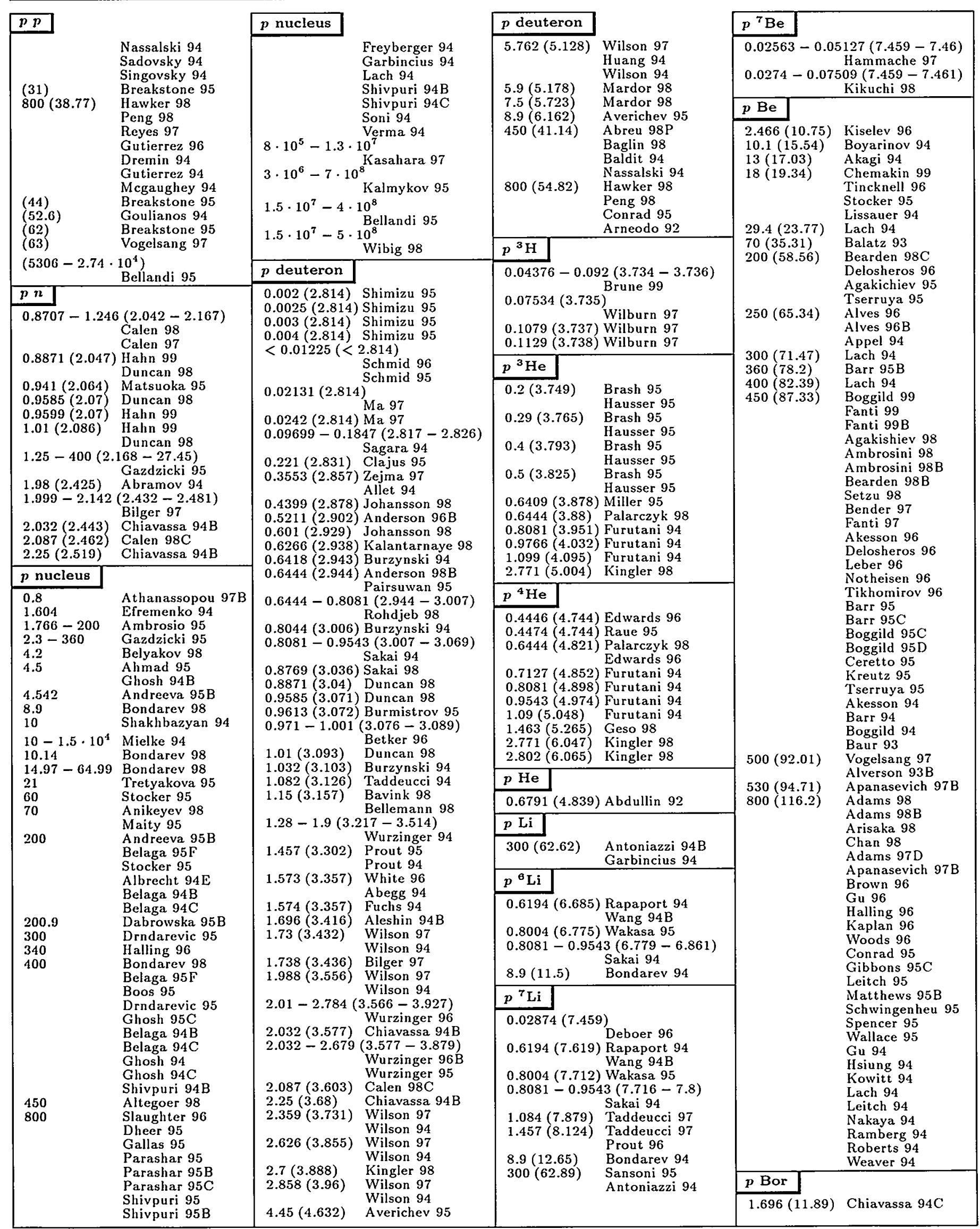

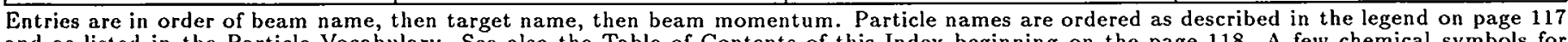

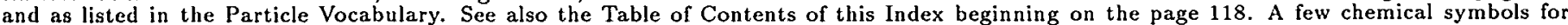

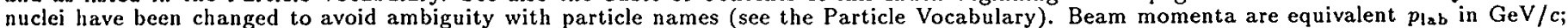
then $E_{\mathrm{cm}}$ in $\mathrm{GeV}$ follows in parentheses. For certain initial states only $E_{\mathrm{cm}}$ (in parentheses) is given. 


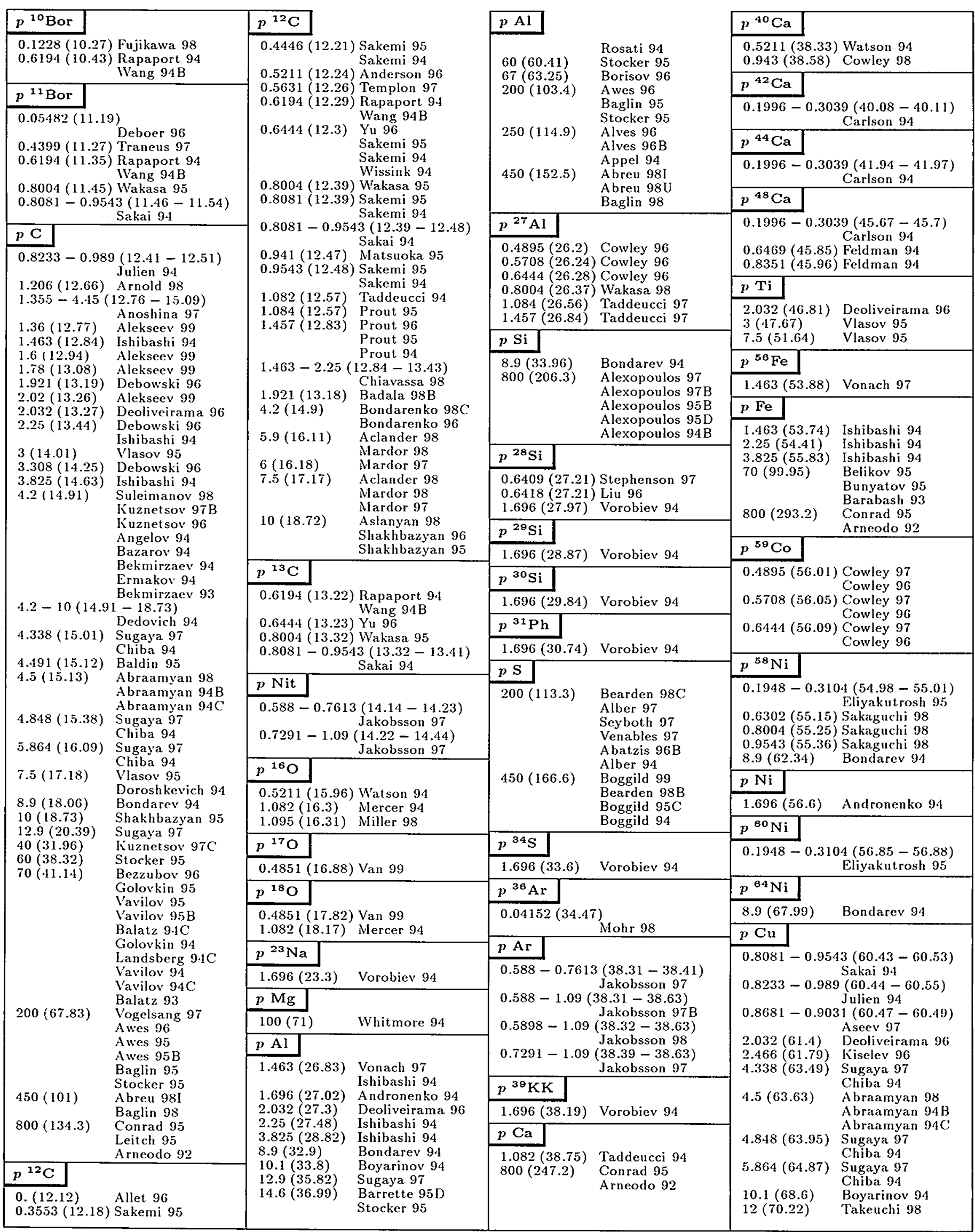




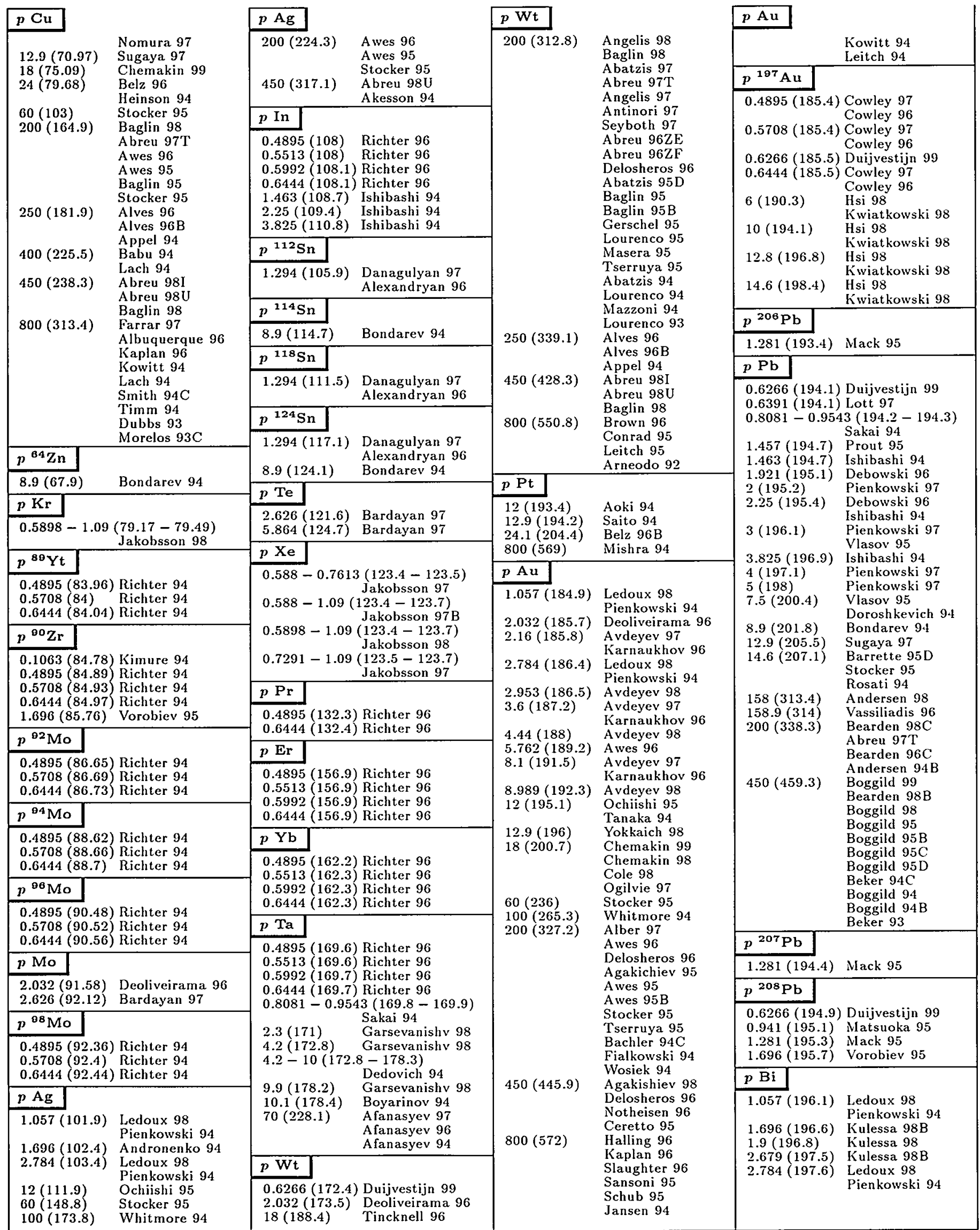

Entries are in order of beam name, then target name, then beam momentum. Particle names are ordered as described in the legend on page 117 and as listed in the Particle Vocabulary. See also the Table of Contents of this Index beginning on the page 118 . A few chemical symbols for nuclei have been changed to avoid ambiguity with particle names (see the Particle Vocabulary). Beam momenta are equivalent $p_{1 a b}$ in GeV/c; then $E_{\mathrm{cm}}$ in $\mathrm{GeV}$ follows in parentheses. For certain initial states only $E_{\mathrm{cm}}$ (in parentheses) is given. 


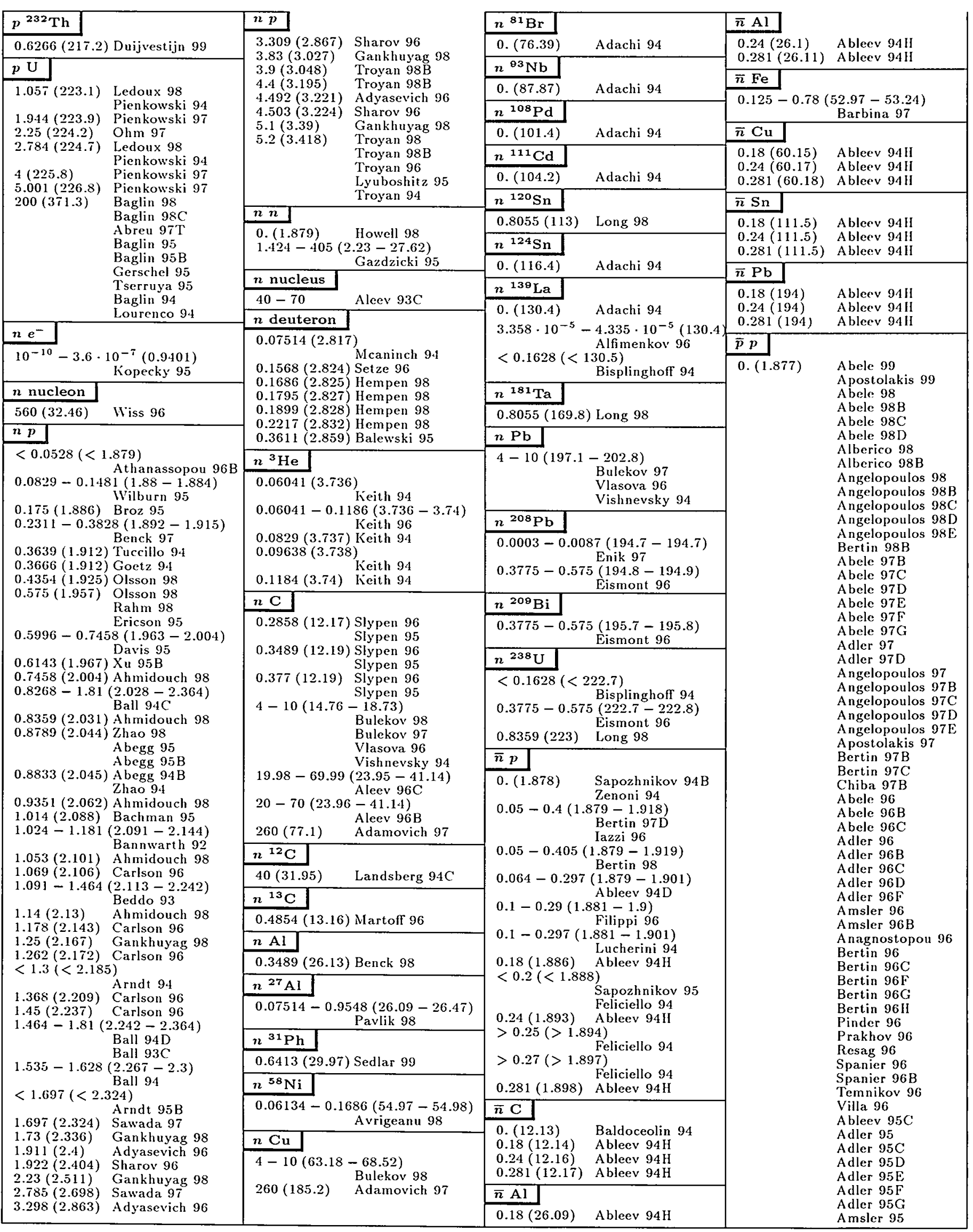




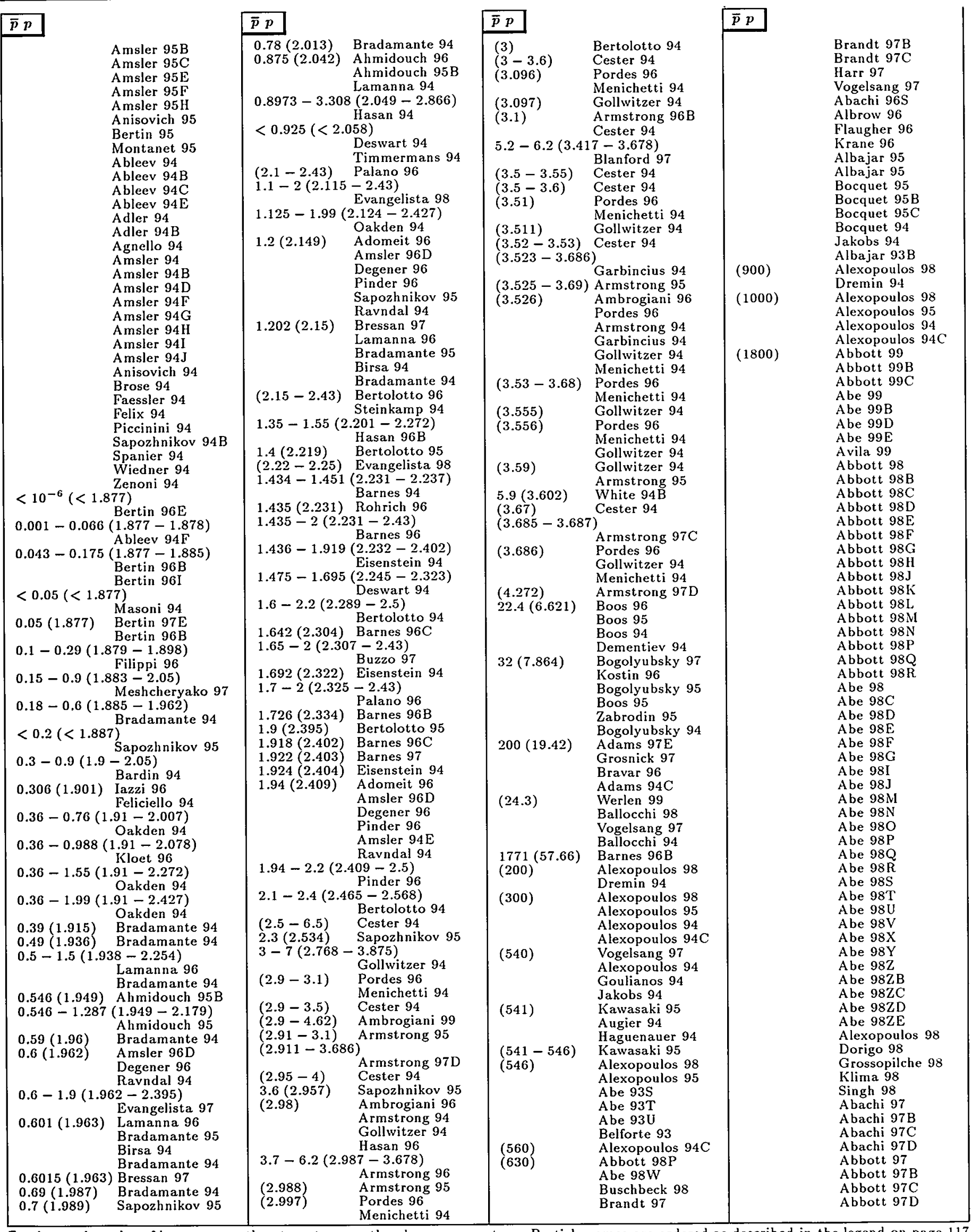

Entries are in order of beam name, then target name, then beam momentum. Particle names are ordered as described in the legend on page 117 and as listed in the Particle Vocabulary. See also the Table of Contents of this Index beginning on the page 118 . A few chemical symbols for nuclei have been changed to avoid ambiguity with particle names (see the Particle Vocabulary). Beam momenta are equivalent $p_{\text {lab in }}$ GeV/c; then $E_{\mathrm{cm}}$ in $\mathrm{GeV}$ follows in parentheses. For certain initial states only $E_{\mathrm{cm}}$ (in parentheses) is given. 


\begin{tabular}{|c|c|c|c|c|c|c|c|}
\hline$\overline{\bar{p} p}$ & & $\overline{\bar{p} p}$ & & $\bar{p} p$ & & $\overline{\bar{p} p}$ & \\
\hline
\end{tabular}




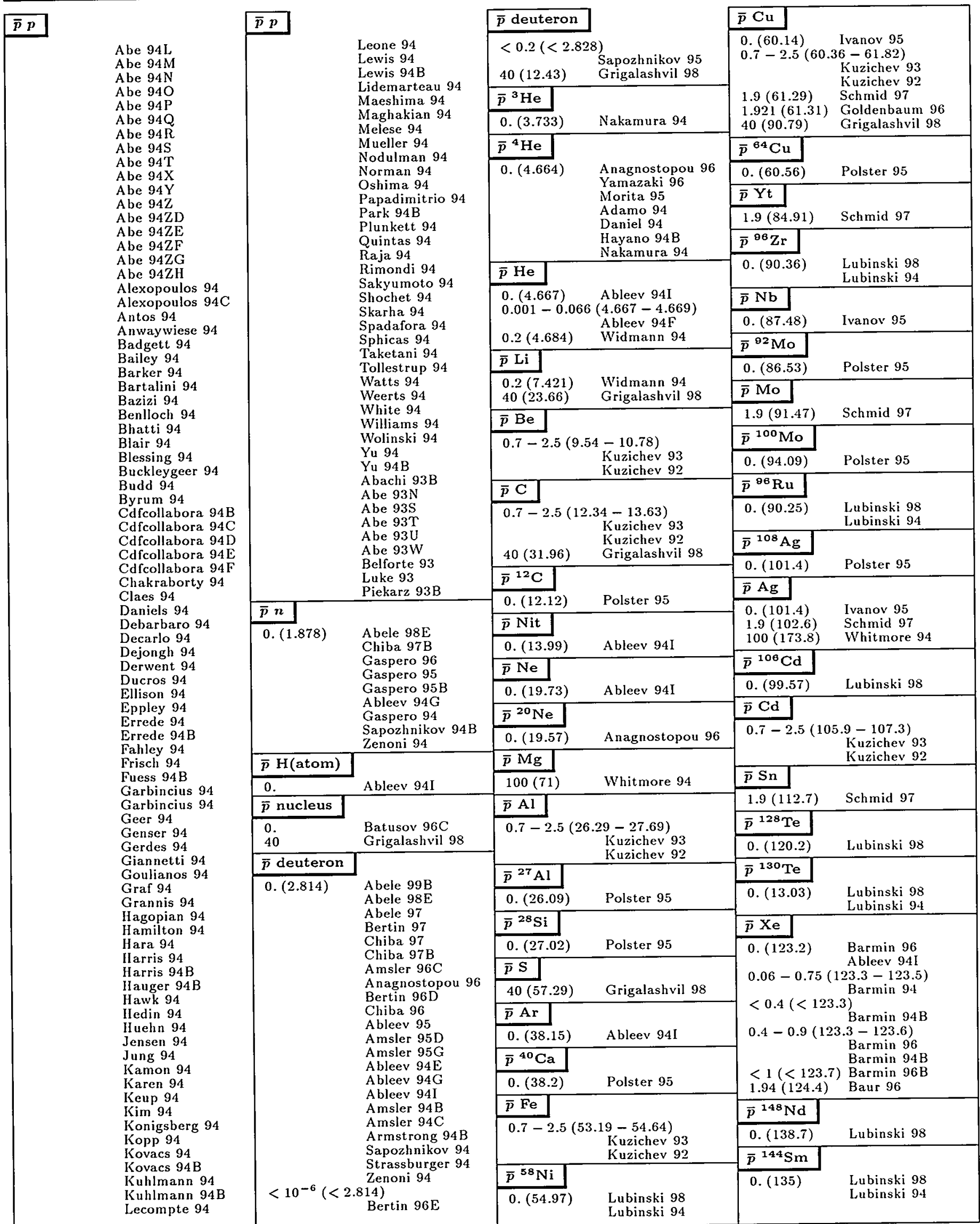

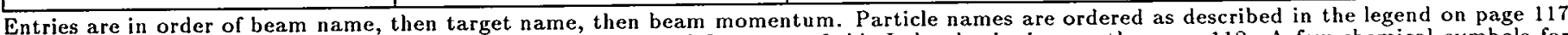

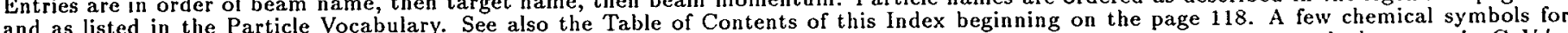

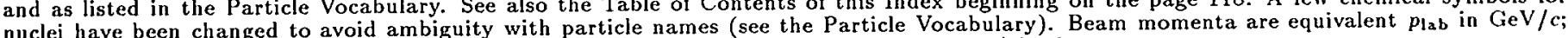
then $E_{\mathrm{cm}}$ in $\mathrm{GeV}$ follows in parentheses. For certain initial states only $E_{\mathrm{cm}}$ (in parentheses) is given. 


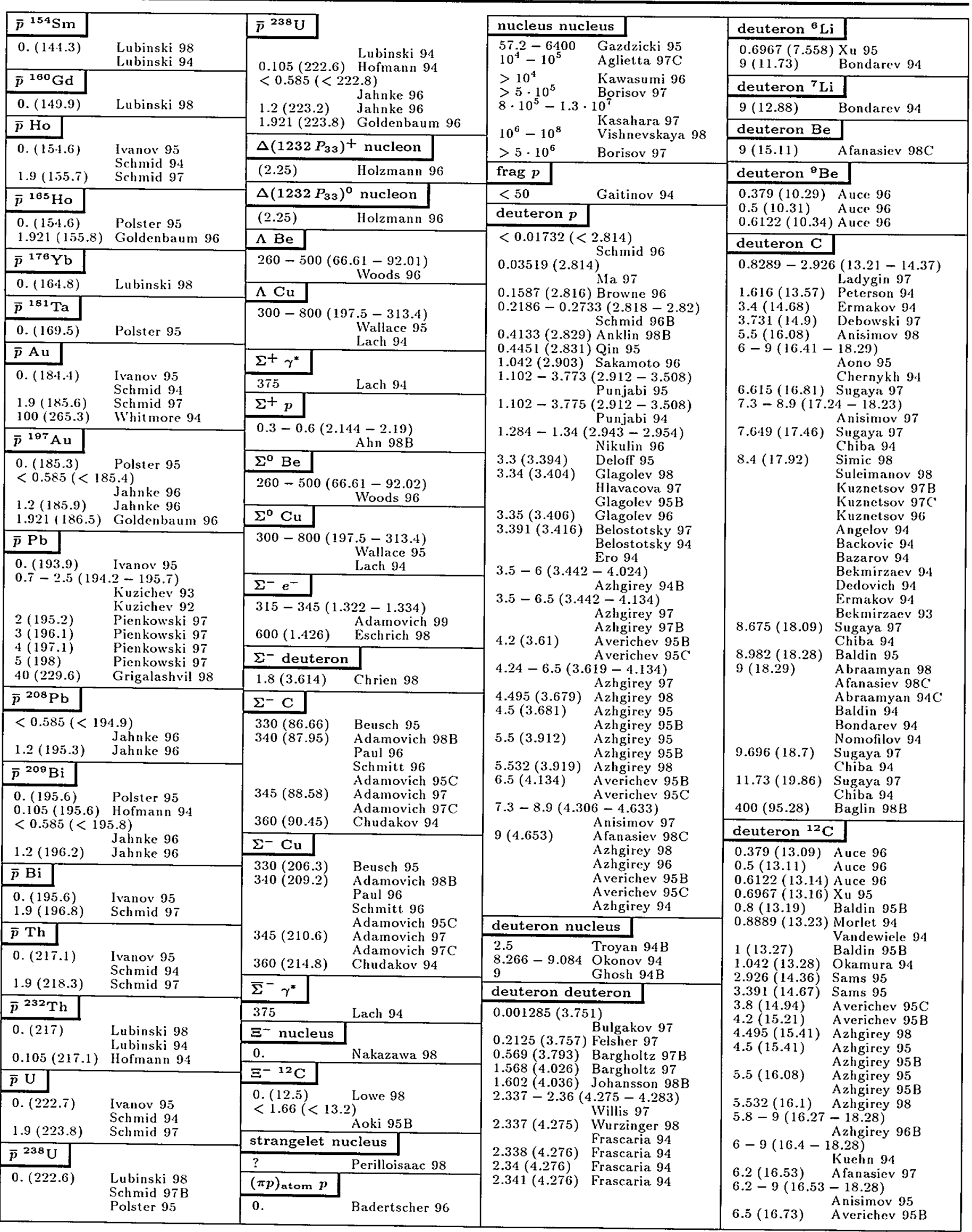




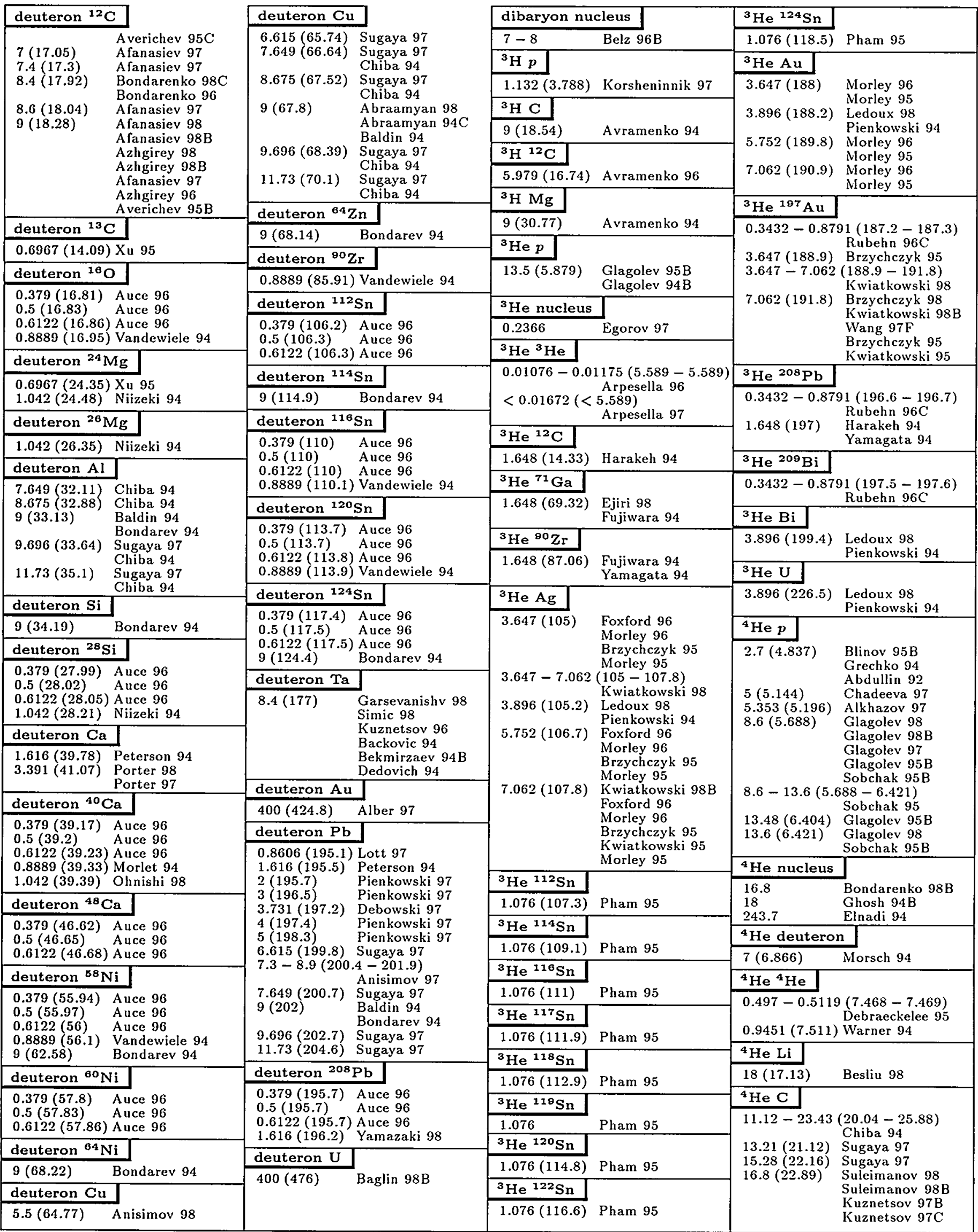

Entries are in order of beam name, then target name, then beam momentum. Particle names are ordered as described in the legend on page 117 and as listed in the Particle Vocabulary. See also the Table of Contents of this Index beginning on the pagè 118. A few chemical symbols for nuclei have been changed to avoid ambiguity with particle names (see the Particle Vocabulary). Beam momenta are equivalent $p_{\text {lab }}$ in GeV/c; then $E_{\mathrm{cm}}$ in $\mathrm{GeV}$ follows in parentheses. For certain initial states only $E_{\mathrm{cm}}$ (in parentheses) is given. 


\begin{tabular}{|c|c|c|c|c|c|c|c|}
\hline \multirow[t]{2}{*}{${ }^{4} \mathrm{He} \mathrm{C}$} & \multirow{6}{*}{$\begin{array}{l}\text { Bekmirzaev } 94 \\
\text { Ermakov } 94 \\
\text { Bekmirzaev } 93 \\
\text { Sugaya } 97 \\
\text { Abraamyan } 98 \\
\text { Abraamyan } 92 \\
\text { Sugaya } 97 \\
\text { Sugaya } 97\end{array}$} & He He & \multirow[b]{2}{*}{ Cowley 94} & \multirow{3}{*}{\multicolumn{2}{|c|}{$\begin{array}{ll}\mathrm{He}^{58} \mathrm{Ni} & \\
0.9904(57.88) & \text { Auce } 94 \\
1.103(57.9) & \text { Auce } 94 \\
1.214(57.94) & \text { Auce } 94 \\
18(70.14) & \text { Bondarev 94 }\end{array}$}} & \multirow{2}{*}{\multicolumn{2}{|c|}{ 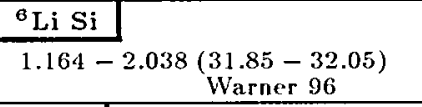 }} \\
\hline & & $1.228(7.555)$ & & & & & \\
\hline \multirow{4}{*}{$\begin{array}{l}17.33(23.15) \\
18(23.46) \\
19.37(24.09) \\
23.43(25.88)\end{array}$} & & \multirow{3}{*}{$18(17.13)$} & \multirow{3}{*}{$\begin{array}{l}\text { Anikina } 98 \\
\text { Chkhaidze } 95 \\
\text { Albaaj } 94 \\
\end{array}$} & & & \multicolumn{2}{|l|}{${ }^{7} \mathrm{Li} p$} \\
\hline & & & & \multirow{2}{*}{\multicolumn{2}{|c|}{$\mathrm{He}^{60} \mathrm{Ni}$}} & \multicolumn{2}{|c|}{$2.556(7.519) \quad$ Cortinagil 97} \\
\hline & & & & & & \multicolumn{2}{|l|}{${ }^{7} \mathrm{Li}$ nucleus } \\
\hline & & \multicolumn{2}{|l|}{$\mathrm{He}^{6} \mathrm{Li}$} & $0.8833(59.72$ & Auce 94 & \multirow{4}{*}{$\begin{array}{l}20.93 \\
26.74 \\
28.7 \\
31.76 \\
37.46 \\
\end{array}$} & \multirow{4}{*}{$\begin{array}{l}\text { Elnadi } 98 \\
\text { Yasin } 95 \\
\text { Elnaghy } 94 \\
\text { Elnadi } 94 \mathrm{C} \\
\text { Yasin } 95 \\
\end{array}$} \\
\hline \multirow{3}{*}{$\begin{array}{l}{ }^{4} \mathrm{He}{ }^{12} \mathrm{C} \\
7(17.78) \\
16.8(22.88)\end{array}$} & \multirow{4}{*}{$\begin{array}{l}\text { Morsch 94 } \\
\text { Bondarenko 98C } \\
\text { Bondarenko } 96 \\
\end{array}$} & $18(15.83)$ & Bondarev 94 & $\begin{array}{l}0.9904(59.74 \\
1.103(59.77)\end{array}$ & $\begin{array}{l}\text { Auce } 94 \\
\text { Auce } 94\end{array}$ & & \\
\hline & & $\mathrm{He}^{7} \mathrm{Li}$ & & & & & \\
\hline & & $18(17.21)$ & Bondarev 94 & $\mathrm{He}^{64} \mathrm{Ni}$ & & & \\
\hline $4 \mathrm{He}$ & & $\mathrm{He}{ }^{9} \mathrm{Be}$ & & $18(75.89)$ & Bondarev 94 & ${ }^{7} \mathrm{Li} \mathrm{Si}$ & \\
\hline $17.74(39.45)$ & vich 94 & $1.228(12.25)$ & Cowley 94 & $\mathrm{He} \mathrm{Cu}$ & & $1.358-2.378($ & $\begin{array}{l}(32.79-33.02) \\
\text { Warner } 96\end{array}$ \\
\hline & Adyasevich $94 \mathrm{~B}$ & He $\mathrm{C}$ & & $18(75.46)$ & Jipa 95 & ${ }^{8} \mathrm{Li} \mathrm{Si}$ & \\
\hline $\begin{array}{l}19.37(40.46) \\
23.43(42.87)\end{array}$ & Sugaya 97 & $7.447(18.04)$ & Debowski 97 & $\mathrm{He}^{64} \mathrm{Zn}$ & Iamyand 94 & $1.553-2.717$ & $\begin{array}{l}(33.74-33.99) \\
\text { Warner } 96\end{array}$ \\
\hline${ }^{4} \mathrm{He}^{27} \mathrm{Al}$ & & $16.8(22.89)$ & $\begin{array}{l}\text { Aslanyan } 96 \\
\text { Kuznetsov } 96\end{array}$ & $18(75.81)$ & Bondarev 94 & ${ }^{8} \mathrm{Li} \mathrm{Si}$ & \\
\hline $\begin{array}{l}0.6125(28.92) \\
0.6714(28.93)\end{array}$ & Mukherjee 95 & & $\begin{array}{l}\text { Angelov } 94 \\
\text { Backovic } 94\end{array}$ & $\mathrm{He}^{114} \mathrm{Sn}$ & & $1.747-3.057$ & $\begin{array}{l}(34.68-34.95) \\
\text { Warner } 96\end{array}$ \\
\hline & & $10(20.40)$ & $\begin{array}{l}\text { Anwmaidze 95 } \\
\text { Chkhaide }\end{array}$ & $18(123.2)$ & Domidarer $3 x_{4}$ & ${ }^{11} \mathrm{Li} \mathrm{Be}$ & \\
\hline He & & & Jipa 95 & $\mathrm{He}^{124} \mathrm{Sn}$ & & $2.531(18.78)$ & Nilsson 95 \\
\hline $0.1163-1.359$ & Warner 96 & & $\begin{array}{l}\text { Abraamyan } 94 \mathrm{C} \\
\text { Bondarev } 94\end{array}$ & $\begin{array}{l}0.7491(119.3 \\
0.8833(119.3\end{array}$ & $\begin{array}{l}\text { Auce } 94 \\
\text { Auce } 94\end{array}$ & ${ }^{11} \mathrm{Li} \mathrm{C}$ & \\
\hline${ }^{4} \mathrm{He} \mathrm{Cu}$ & & $\mathrm{He}{ }^{12} \mathrm{C}$ & & $0.9904(119.4$ & Auce 94 & $8.521(22.99)$ & Nilsson 95 \\
\hline $11.12(70.05)$ & Sugaya 97 & $0.7491(14.96)$ & Auce 94 & $1.214(119.4)$ & Auce 94 & ${ }^{11} \mathrm{Li} \mathrm{Si}$ & \\
\hline $11.12-23.43$ & $\begin{array}{l}(70.05-79.54) \\
\text { Chiba } 94\end{array}$ & $\begin{array}{l}0.8833(14.99) \\
0.9904(15.01)\end{array}$ & $\left\{\begin{array}{l}\text { Auce } 94 \\
\text { Auce } 94\end{array}\right.$ & $18(132.7)$ & Bondarev 94 & $2.135-3.736$ & $(36.57-36.88)$ \\
\hline $13.21(71.72)$ & Sugaya $9 \bar{T}$ & $1.103(15.03)$ & Auce 94 & He Ta & & & Warner 96 \\
\hline $15.28(73.35)$ & Sugaya 97 & $1.214(15.05)$ & Auce 94 & $16.8(185)$ & Garsevanishy 98 & ${ }^{11} \mathrm{Li} \mathrm{Ni}$ & \\
\hline $\begin{array}{l}17.33(74.95) \\
18(75.46)\end{array}$ & $\begin{array}{l}\text { Sugaya } 9 \overline{7} \\
\text { Abraamyan } 98\end{array}$ & $\begin{array}{l}1.237(15.06) \\
2.159(15.34)\end{array}$ & $\begin{array}{l}\text { Steyn } 99 \\
\text { Nadasen } 99\end{array}$ & & Backovic 94 & $2.531(65.19)$ & Nilsson 95 \\
\hline & Besliu 98 & $\mathrm{He}^{16} \mathrm{O}$ & & He Pb & & ${ }^{11} \mathrm{Li} \mathrm{Au}$ & \\
\hline $19.37(76.51)$ & Sugaya 97 & $0.7491(18.69)$ & Auce 94 & $\begin{array}{l}1.441(201.2) \\
18(210.6)\end{array}$ & $\begin{array}{l}\text { Debowski } 96 \\
\text { Jipa } 95\end{array}$ & $2.531(194)$ & Nilsson 95 \\
\hline $23.43(79.54)$ & Sugaya $9 \bar{T}$ & $0.8833(18.71)$ & Auce 94 & & Bondarev 94 & ${ }^{11} \mathrm{Li} \mathrm{Pb}$ & \\
\hline${ }^{4} \mathrm{He} \mathrm{Ag}$ & & $\begin{array}{l}0.9904(18.73) \\
1.103(18.76)\end{array}$ & $\begin{array}{l}\text { Auce } 94 \\
\text { Auce } 94\end{array}$ & $\mathrm{He}^{208} \mathrm{~Pb}$ & & $8.521(206.2)$ & Nilsson 95 \\
\hline $1.237(104.4)$ & Zhang 97 & $1.214(18.78)$ & Auce 94 & $\begin{array}{l}0.7491(197.6 \\
0.8833(197.6\end{array}$ & $\begin{array}{l}\text { Auce } 94 \\
\text { Auce 94 }\end{array}$ & ${ }^{9} \mathrm{Be}^{208} \mathrm{~Pb}$ & \\
\hline$\frac{16(115.8)}{{ }^{4} \mathrm{He} \mathrm{Ta}}$ & vic 98 & $\begin{array}{l}\mathrm{He} \mathrm{Ne} \\
18(32.52)\end{array}$ & Jipa 95 & $\begin{array}{l}0.9904(197.6 \\
1.103(197.6)\end{array}$ & $\begin{array}{l}\text { Auce } 94 \\
\text { Auce } 94\end{array}$ & $0.7668-0.926$ & $\begin{array}{l}\text { (202.2-202.2) } \\
\text { Dasgupta } 99\end{array}$ \\
\hline $16.8(185)$ & Bekmirzaev $94 \mathrm{~B}$ & He A.l & & $1.214(197.7)$ & & ${ }^{10} \mathrm{Be} p$ & \\
\hline${ }^{4} \mathrm{He} \mathrm{Au}$ & & $18(39.62)$ & $\mathrm{Jip}$ & ${ }^{6} \mathrm{He} p$ & & $3.377(10.31)$ & Cortinagil 97 \\
\hline $\begin{array}{l}1.237(187.4) \\
16(199.3)\end{array}$ & $\begin{array}{l}\text { Zhang } 9 \bar{t} \\
\text { Todorovic } 98\end{array}$ & $\mathrm{He} \mathrm{Si}$ & $B_{0}>>>$ & $\begin{array}{l}1.689(6.563) \\
2.223(6.588)\end{array}$ & $\begin{array}{l}\text { Cortinagil } 97 \\
\text { Korsheninnik } 97\end{array}$ & ${ }^{10} \mathrm{Be} \mathrm{Si}$ & \\
\hline${ }^{4} \mathrm{He}^{187} \mathrm{Au}$ & & $18(40.75)$ & Bondarev 94 & $\begin{array}{l}2.559(6.607) \\
8.161(7.119)\end{array}$ & $\begin{array}{l}\text { Brown 96C } \\
\text { Alkhazov } 97\end{array}$ & $1.941-3.398$ & $\begin{array}{l}(35.63-35.92) \\
\text { Warner } 96\end{array}$ \\
\hline $4-14.4(189.8$ & $8-198.7$ ) & $\mathrm{He}^{28} \mathrm{Si}$ & & ${ }^{8} \mathrm{He} \mathrm{Si}$ & & ${ }^{11} \mathrm{Be} p$ & \\
\hline $6.768(191.9)$ & $\begin{array}{l}\text { Lips } 94 \mathrm{C} \\
\text { Lips } 94\end{array}$ & $\begin{array}{l}0.7491(29.88) \\
0.8833(29.9)\end{array}$ & $\begin{array}{l}\text { Auce } 94 \\
\text { Auce } 94\end{array}$ & $1.164-2.038$ & $\begin{array}{l}(31.85-32.05) \\
\text { Warner } 96\end{array}$ & $3.378(11.23)$ & Cortinagil 97 \\
\hline $17.74(201.7)$ & $\begin{array}{l}\text { Lips } 94 \\
\text { Lips } 94 \mathrm{~B}\end{array}$ & $\begin{array}{l}0.9904(29.92) \\
1.103(29.95)\end{array}$ & $\begin{array}{l}\text { Auce } 94 \\
\text { Auce } 94\end{array}$ & ${ }^{6} \mathrm{He}^{209} \mathrm{Bi}$ & & ${ }^{11} \mathrm{Be} \mathrm{Be}$ & \\
\hline $17.94(201.9)$ & Shmakov 95 & $1.214(29.98)$ & Auce 94 & $0.4612-0.57$ & & $3.073(18.84)$ & Nilsson 95 \\
\hline${ }^{4} \mathrm{He} \mathrm{Pb}$ & & $\mathrm{He} \mathbf{C a}$ & & & Kolata 98 & ${ }^{11} \mathrm{Be}^{9} \mathrm{Be}$ & \\
\hline $1.281(196.9)$ & Lott 97 & $6.77(44.55)$ & Porter 98 & ${ }^{8} \mathrm{He} p$ & & $3.073(18.83)$ & Anne 94 \\
\hline $17.74(210.4)$ & $\begin{array}{l}\text { Todorovic } 98 \\
\text { Adyasevich } 94\end{array}$ & & Porter 97 & $10.46(8.973)$ & Alkhazov 97 & ${ }^{11} \mathrm{Be} \mathrm{C}$ & \\
\hline $18(2106)$ & Adyasevich $94 \mathrm{~B}$ & $\mathrm{He}^{40} \mathrm{Ca}$ & & ${ }^{8} \mathrm{He} \mathrm{C}$ & & $11.37(23.93)$ & Nilsson 95 \\
\hline $\begin{array}{l}18(210.6) \\
>2 \cdot 10^{4}(>2\end{array}$ & $\begin{array}{l}\text { Beshiu } 98 \\
785)\end{array}$ & $\begin{array}{l}0.7491(41.06) \\
0.8833(41.08)\end{array}$ & $\left\{\begin{array}{l}\text { Auce } 94 \\
\text { Auce } 94\end{array}\right.$ & $5.683(19.76)$ & Nilsson 96 & ${ }^{11} \mathrm{Be} \mathrm{Ti}$ & \\
\hline & A panasenko 94 & $\begin{array}{l}0.9904(41.11) \\
1.103(41.13)\end{array}$ & $\begin{array}{l}\text { Auce } 94 \\
\text { Auce } 94\end{array}$ & ${ }^{8} \mathrm{He} \mathrm{Si}$ & & $3.073(55.23)$ & $\begin{array}{l}\text { Nilsson } 95 \\
\text { Anne } 94\end{array}$ \\
\hline${ }^{4} \mathrm{He}^{232} \mathrm{Th}$ & & $1.214(41.16)$ & Auce 94 & $1.553-2.717$ & $\begin{array}{l}(33.74-33.99) \\
\text { Warner } 96\end{array}$ & ${ }^{11} \mathrm{Be}^{197} \mathrm{Au}$ & \\
\hline $1.237(220)$ & Chen 96B & $\mathrm{He}^{48} \mathrm{Ca}$ & & ${ }^{6} \mathrm{Li} p$ & & $3.073(195)$ & Anne 94 \\
\hline${ }^{4} \mathrm{He} \mathrm{U}$ & & $\begin{array}{l}0.7491(48.51) \\
0.8833(48.54)\end{array}$ & $\left\{\begin{array}{l}\text { Auce } 94 \\
\text { Auce } 94\end{array}\right.$ & $6.804(6.974)$ & Webber 98 & ${ }^{8}$ Bor ${ }^{208} \mathrm{~Pb}$ & \\
\hline$\frac{16(237.6)}{\text { He nucleus }}$ & ovic 98 & $0.9904(48.56)$ & Auce 94 & ${ }^{6} \mathrm{Li}$ nucleus & & $0.8813(201.3)$ & Kikuchi 98 \\
\hline $16.51-18.15$ & Okonov 94 & $\begin{array}{l}1.103(48.39) \\
1.214(48.62)\end{array}$ & $\begin{array}{l}\text { Auce } 94 \\
\text { Auce 94 }\end{array}$ & 27 & Lepekhin 98 & ${ }^{10}$ Bor $p$ & \\
\hline $\mathrm{He} \mathrm{He}$ & & $\mathrm{He}^{58} \mathrm{Ni}$ & & & Elsharkawy 94 & $11.26(10.73)$ & Webber 98 \\
\hline $0.68-1.098$ & $7.487-7.535)$ & $0.7491(57.83)$ & A & 27.22 & $\begin{array}{l}\text { Lepekhin 94B } \\
\text { Elnadi } 98\end{array}$ & $\mathbf{C p}$ & \\
\hline & $\mathrm{M}$ & $0.8833(57.8)$ & & 21.22 & & $23.79(13.24)$ & Kulakov 98 \\
\hline
\end{tabular}




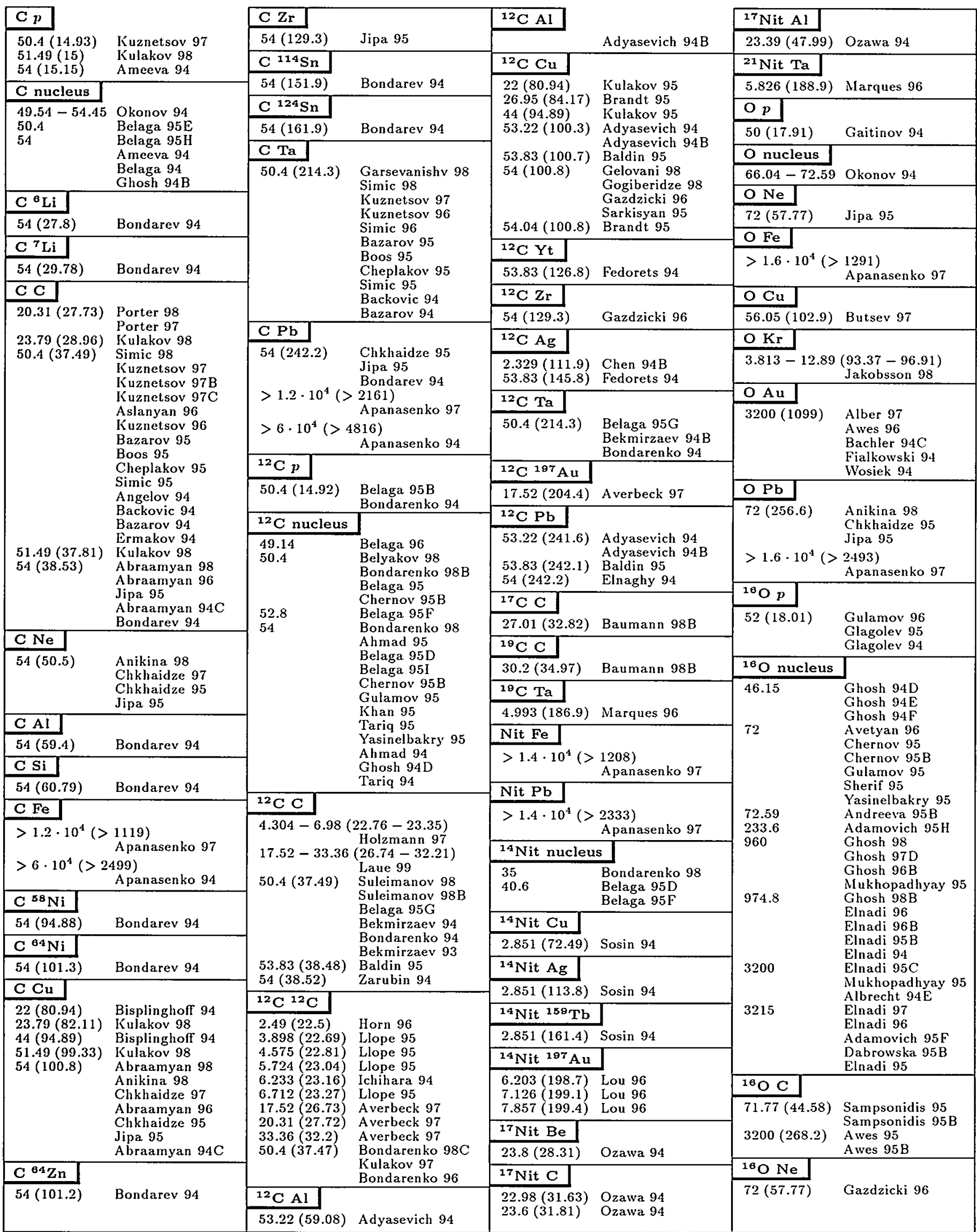

Entries are in order of beam name, then target name, then beam momentum. Particle names are ordered as described in the legend on page 117 and as listed in the Particle Vocabulary. See also the Table of Contents of this Index beginning on the page 118 . A few chemical symbols for nuclei have been changed to avoid ambiguity with particle names (see the Particle Vocabulary). Beam momenta are equivalent $p_{\text {lab }}$ in GeV/c; then $E_{\mathrm{cm}}$ in $\mathrm{GeV}$ follows in parentheses. For certain initial states only $E_{\mathrm{cm}}$ (in parentheses) is given. 


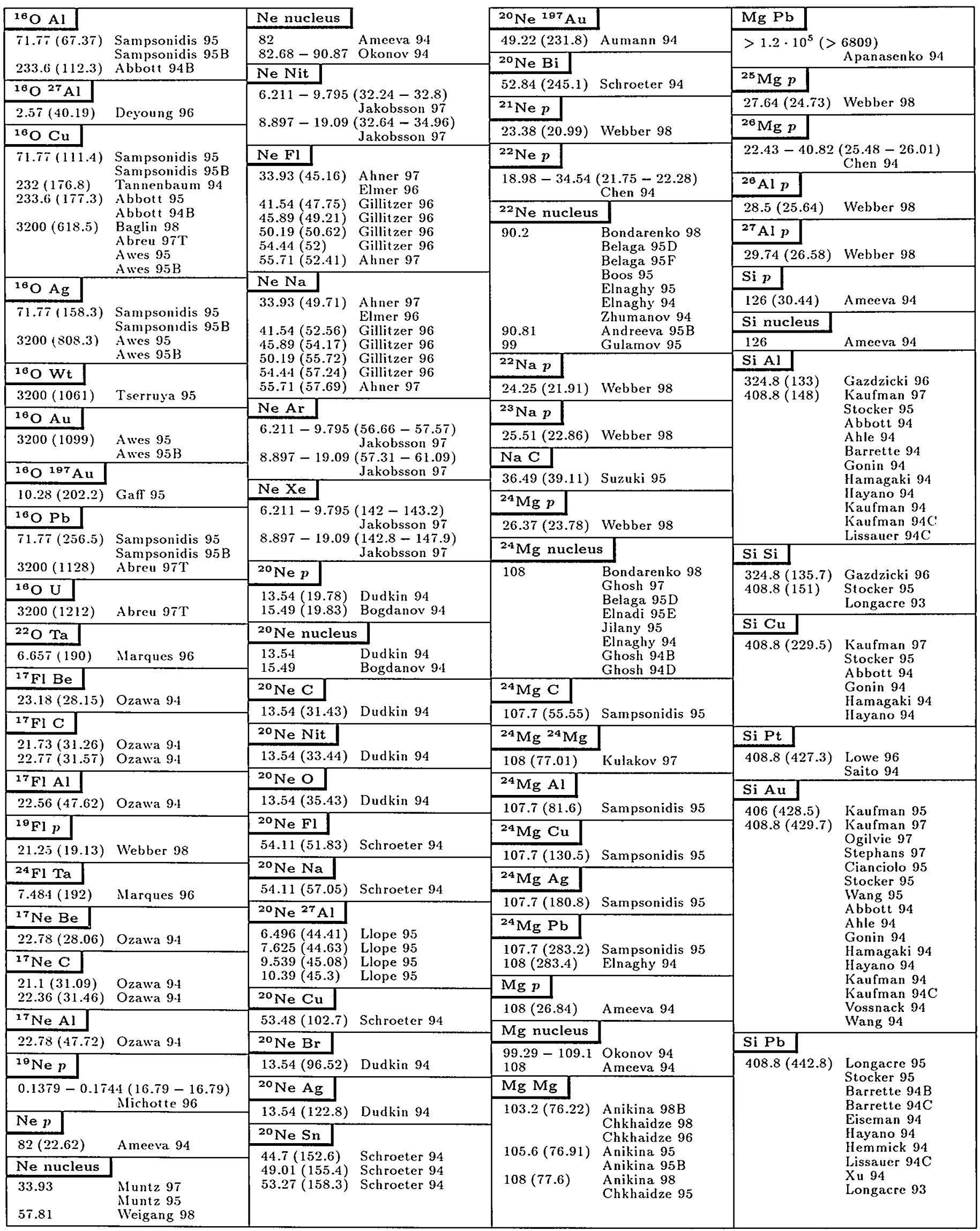




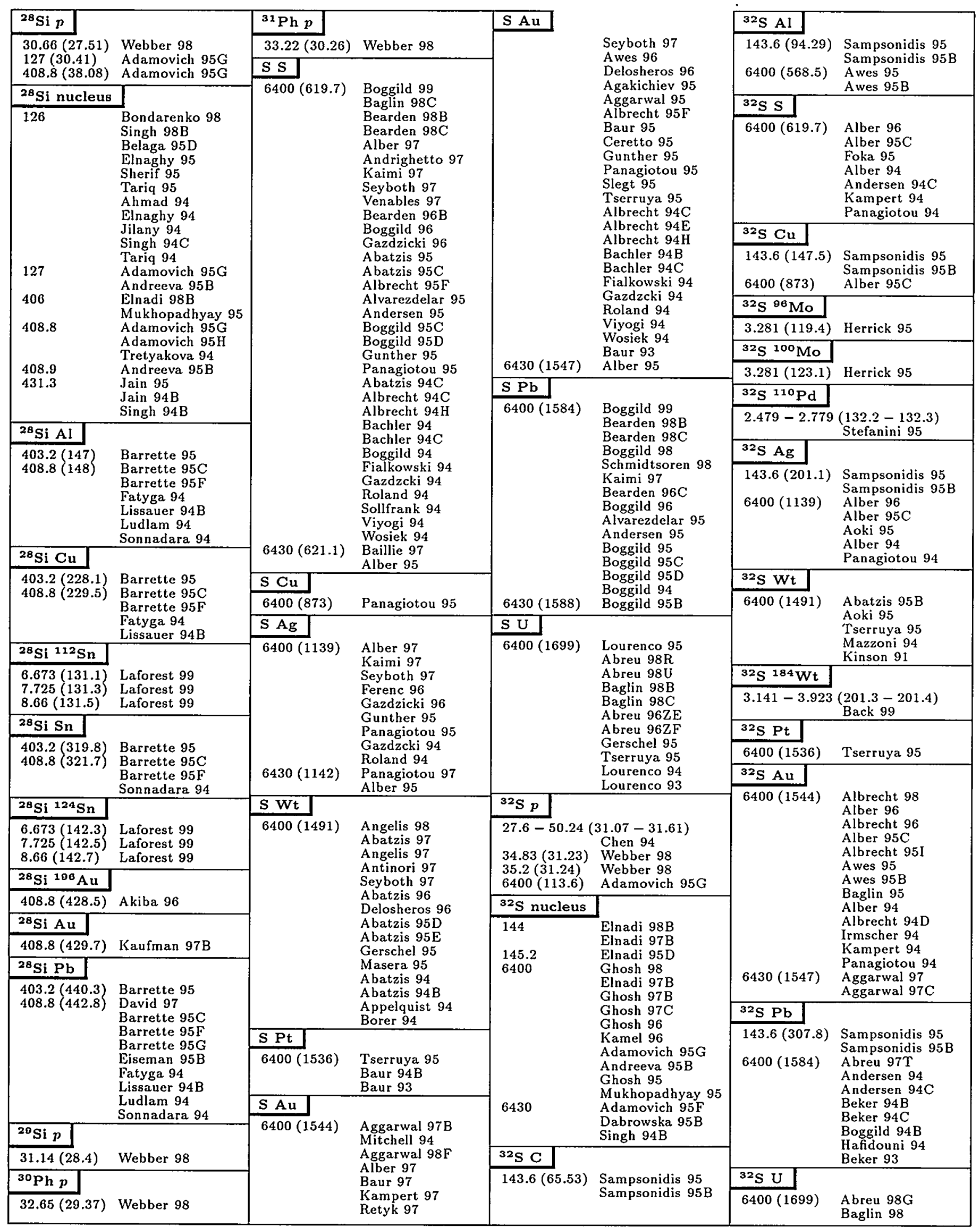

Entries are in order of beam name, then target name, then beam momentum. Particle names are ordered as described in the legend on page 117 and as listed in the Particle Vocabulary. See also the Table of Contents of this Index beginning on the page 118. A few chemical symbols for nuclei have been changed to avoid ambiguity with particle names (see the Particle Vocabulary). Beam momenta are equivalent plab in GeV/c; then $E_{\mathrm{cm}}$ in $\mathrm{GeV}$ follows in parentheses. For certain initial states only $E_{\mathrm{cm}}$ (in parentheses) is given. 


\begin{tabular}{|c|c|c|c|c|}
\hline${ }^{32} \mathrm{~S} \mathrm{U}$ & \multirow{4}{*}{$\begin{array}{l}\text { Abreu } 97 \mathrm{~T} \\
\text { Baglin } 95 \\
\text { Baglin } 95 \mathrm{~B} \\
\text { Baglin } 94\end{array}$} & \multirow[b]{2}{*}{ Webber 98} & \multirow{3}{*}{$\begin{aligned}{ }^{40} \mathrm{Ca} \mathrm{Ca} & \\
7.762-12.37 & (74.99-75.59) \\
& \text { Holzmann } 97\end{aligned}$} & \multirow{2}{*}{$\begin{array}{l}\text { Cr C } \\
52.34(63.77) \quad \text { Blank 95B }\end{array}$} \\
\hline & & & & \\
\hline & & \begin{tabular}{l|l} 
Ar nucleus & \\
75.32 & Weigang 98 \\
\end{tabular} & & \multirow{2}{*}{\begin{tabular}{l|} 
Cr Al \\
$52.34(81.01) \quad$ Blank 95B
\end{tabular}} \\
\hline & & Weigang 98 & \multirow{2}{*}{$\frac{{ }^{40} \mathrm{Ca}{ }^{40} \mathrm{Ca}}{10.31(75.22)}$ Pawlowski 97} & \\
\hline${ }^{33} \mathrm{~S} p$ & \multirow[b]{2}{*}{ Webber 98} & Ar C & & \multirow{2}{*}{$\begin{array}{l}\mathrm{Cr} \mathrm{Pb} \\
52.34(259.1) \quad \text { Blank } 95 \mathrm{~B}\end{array}$} \\
\hline $35.4(32.12)$ & & Brady 94 & \multirow{2}{*}{$\begin{array}{l}{ }^{42} \mathrm{Ca} p \\
49.78(40.62) \quad \text { Webber } 98 \\
\end{array}$} & \\
\hline${ }^{36} \mathrm{~S}{ }^{110} \mathrm{Pd}$ & \multirow{3}{*}{$\begin{array}{c}(135.9-136) \\
\text { Stefanini } 95\end{array}$} & \multirow{4}{*}{ 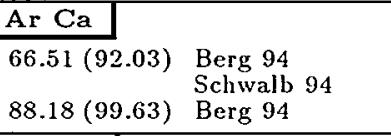 } & & $52.34(259.1)$ Blank 95 B \\
\hline $2.673-3.034$ & & & ${ }^{44} \mathrm{Ca} p$ & $\begin{array}{ll}53 \mathrm{Cr} \text { nucleus } \\
58.63 & \text { Brohm } 95\end{array}$ \\
\hline 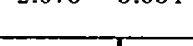 & & & $52.21(42.49) \quad$ Webber 98 & ${ }^{50} \mathrm{Mn}$ nucleus \\
\hline${ }^{34} \mathrm{Cl} p$ & & & ${ }^{44} \mathrm{Sc} p$ & Brohm 95 \\
\hline $36.11(33.04)$ & Webber 98 & $\frac{\text { Ar Au }}{134(2226)}$ & $51.98(42.48)$ Webber 98 & ${ }^{51} \mathrm{Mn}$ nucleus \\
\hline${ }^{37} \mathrm{Cl} p$ & & $\frac{13.4(222.6)}{40}$ & & Brohm 95 \\
\hline $40.12(35.89)$ & Webber 98 & ${ }^{40} \mathrm{Ar} p$ & $-s c p$ & ${ }^{52} \mathrm{Mn} p$ \\
\hline${ }^{36} \mathrm{Ar} p$ & & $30.35(38.46) \quad$ Bogdanov 94 & $54.41(44.35)$ Webber 98 & $59.9(49.88)$ \\
\hline $31.05-56.52$ & $(34.8-35.33)$ & $34.5-62.8(38.53-39.07)$ & ${ }^{46} \mathrm{Ti} p$ & ${ }^{52} \mathrm{Mn}$ nucleus \\
\hline $01.00-30.02$ & $\begin{array}{l}(34.0-50.33) \\
\text { Chen } 94\end{array}$ & $\begin{array}{ll} & \text { Chen 94 } \\
35.32(38.54) & \text { Ḱnot t 97 }\end{array}$ & $54.17(44.35) \quad$ Webber 98 & Brohm 95 \\
\hline $32.26(34.82)$ & Knott 97 & $40 \mathrm{anm}$ & ${ }^{46} \mathrm{Ti}$ nucleus & Brohm 95 \\
\hline $\begin{array}{l}39.03(34.95) \\
41.28(35)\end{array}$ & $\begin{array}{l}\text { Webber } 98 \\
\text { Kinott } 97\end{array}$ & ${ }^{40}$ Ar nucleus & $63.95 \quad$ Brohm 95 & ${ }^{53} \mathrm{Mn}$ nucleus \\
\hline $51.9(35.23)$ & Knott 97 & $\begin{array}{ll}30.35 & \begin{array}{l}\text { Bogdanov } 94 \\
\text { Dudkin } 94\end{array} \\
\end{array}$ & $47 \mathrm{Ti}$ nucleus & $\begin{array}{ll}61.05 & \text { Brohm 95 } \\
70.29 & \text { Brohm 95 } \\
\end{array}$ \\
\hline${ }^{36} \mathrm{Ar}{ }^{12} \mathrm{C}$ & & ${ }^{40} \mathrm{ArC}$ & \begin{tabular}{l|l}
62.91 & Brohm 95 \\
$48 \mathrm{Ti}$ &
\end{tabular} & ${ }^{54} \mathrm{Mn}$ nucleus \\
\hline $15.27(45.53)$ & Legrain 97 & $30.35(50.88)$ Dudkin 94 & Tip & Brohin 95 \\
\hline & Lecolley 95 & ${ }^{40}$ Ar Nit & $56.59(46.21) \quad$ Webber 98 & Brohm 95 \\
\hline${ }^{36}$ Ar ${ }^{27} \mathrm{Al}$ & & $30.35(53.03)$ Dudkin 94 & ${ }^{48} \mathrm{Va} p$ & Mn nucleus \\
\hline $11.69(59.52)$ & Angelique 97 & ${ }^{40} \mathrm{ArO}$ & $56.34(46.21) \quad$ Webber 98 & Blank 95 \\
\hline $11.69-15.53$ & $\begin{array}{l}\text { Buta } 97 \mathrm{C} \\
(59.52-60.13)\end{array}$ & & $48 \mathrm{Va}$ nucleus & $\mathrm{MnC}$ \\
\hline $10.00-1.00$ & Buta 95 & $30.35(55.16) \quad$ Dudkin 94 & Va nucleus & $54.74(66.49)$ Blank 95B \\
\hline $12.94(59 . \overline{7})$ & $\begin{array}{l}\text { Angelique } 97 \\
\text { Buta } 97 \mathrm{C}\end{array}$ & ${ }^{40} \mathrm{Ar} \mathrm{Ca}$ & $\begin{array}{ll}57.38 & \text { Brohm 95 } \\
66.33 & \text { Brohm 95 } \\
\end{array}$ & Mn Al \\
\hline $14.1(59.89)$ & Angelique 97 & 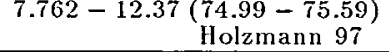 & ${ }^{48} \mathrm{Va}$ nucleus & $54.74(83.77) \quad$ Blank 95B \\
\hline $\begin{array}{l}14.74(59.99) \\
15.53(60.13)\end{array}$ & $\begin{array}{l}\text { Angelique } 9 \bar{t} \\
\text { Badala } 98\end{array}$ & ${ }^{40} \mathrm{Ar}{ }^{45} \mathrm{Sc}$ & $56.58 \quad$ Brohm 95 & $\mathrm{Mn} \mathrm{Pb}$ \\
\hline & Angelique 97 & $1.615-2.93(79.18-79.22)$ & $65.33 \quad$ Brohm 95 & $54.74(262.3) \quad$ Blank $95 \mathrm{~B}$ \\
\hline & $\begin{array}{l}\text { Badala } 97 \\
\text { Badala } 97 \mathrm{~B}\end{array}$ & $\begin{array}{l}\text { Pak } 96 \\
\end{array}$ & ${ }^{50} \mathrm{Va} p$ & ${ }^{55} \mathrm{Mn} p$ \\
\hline & $\begin{array}{l}\text { Buta } 97 \mathrm{C} \\
\text { Badala } 96\end{array}$ & $\begin{array}{ll}6.114(19.48) & \text { Llope } 95 \\
10.31(79.9) & \text { Llope } 95\end{array}$ & $58(48) \quad$ Webber 98 & $63.54(52.68)$ Webber 98 \\
\hline & Badala $96 \mathrm{~B}$ & $\begin{array}{ll}15.25(80.73) & \text { Llope } 95 \\
17.25(81.15) & \text { Llope } 95\end{array}$ & ${ }^{50}$ Va nucleus & ${ }^{55} \mathrm{Mn}$ nucleus \\
\hline & Badala $96 \mathrm{C}$ & $19.08(81.56)$ Llope 95 & $\frac{56.48}{4} \quad$ Brohm 95 & $60.81 \quad$ Brohm 95 \\
\hline${ }^{36} \mathrm{Ar} \mathrm{Ti}$ & Dacala 95 & ${ }^{40} \mathrm{Ar}{ }^{51} \mathrm{Va}$ & $\frac{}{50.71(62,7)}$ Blant $95 \mathrm{~B}$ & ${ }^{56} \mathrm{Mn}$ nucleus \\
\hline $15.27(80.02)$ & Legrain 97 & $11.59(85.75)$ Sapienza 98 & $\mathrm{VaAl}$ & Brohm 95 \\
\hline & Lecolley 95 & ${ }^{40} \mathrm{Ar} \mathrm{Br}$ & Va Al & ${ }^{51} \mathrm{Fe}$ nucleus \\
\hline${ }^{36} \mathrm{Ar}^{58} \mathrm{Ni}$ & & $30.35(118.7)$ Dudkin 94 & $50.71(79.84)$ Blank 95B & Brohm 95 \\
\hline $11.36(88.7)$ & Borderie 96 & $40 \mathrm{Ar}^{88} \mathrm{Mg}$ & $\mathrm{Va} \mathrm{Pb}$ & ${ }^{52}$ Fe nucleus \\
\hline $1000(0) 10)$ & Rivet 96 & ${ }^{40} \mathrm{Ar}{ }^{88} \mathrm{Mo}$ & $50.71(257.5)$ Blank 95B & Brohm 95 \\
\hline $13.63(89.19)$ & $\begin{array}{l}\text { Borderie } 96 \\
\text { Rivet } 96\end{array}$ & $10.61(129.6)$ Sapienza 98 & ${ }^{51} \mathrm{Va}$ nucleus & ${ }^{53}$ Fe nucleus \\
\hline $14.56(89.4)$ & Borderie 96 & ${ }^{40} \mathrm{Ar} \mathrm{Ag}$ & $57.77 \quad$ Brohm 95 & Brohm 95 \\
\hline $15.53(89.64)$ & $\begin{array}{l}\text { Rivet } 96 \\
\text { Badala } 96\end{array}$ & $7.151(138.2)$ Gelderloos 96 & ${ }^{49} \mathrm{Cr}$ nucleus & ${ }^{54} \mathrm{Fe} p$ \\
\hline & Badala $96 \mathrm{~B}$ & $30.35(145.4)$ Dudkin 94 & Brohm 95 & $62.69(51.79)$ Webber 98 \\
\hline & $\begin{array}{l}\text { Badala } 96 \mathrm{C} \\
\text { Borderie } 96\end{array}$ & ${ }^{40} \mathrm{Ar}{ }^{197} \mathrm{Au}$ & ${ }^{50} \mathrm{Cr} p$ & ${ }^{54} \mathrm{Fe}$ nucleus \\
\hline & Rivet 96 & $8.69(222.4)$ & $57.75(47.99) \quad$ Webber 98 & $64.42 \quad$ Brohm 95 \\
\hline${ }^{36} \mathrm{Ar}{ }^{112} \mathrm{Sn}$ & & $\mathrm{Ca} \mathrm{Ca}$ & ${ }^{50} \mathrm{Cr}$ nucleus & $73.51 \quad$ Brohm 95 \\
\hline $15.53(140.4)$ & Badala $97 \mathrm{~B}$ & $67.73(92.53)$ Porter 98 & Brohm 95 & ${ }^{55} \mathrm{Fe}$ nucleus \\
\hline & $\begin{array}{l}\text { Badala } 96 \\
\text { Badala } 96 \mathrm{~B}\end{array}$ & ${ }^{40} \mathrm{Ca} p$ & \begin{tabular}{l|l}
67.93 & Brohm 95 \\
${ }^{51} \mathrm{Cr}$ nucleus
\end{tabular} & $\begin{array}{l}\text { Brohm } 95 \\
\text { Brohm } 95\end{array}$ \\
\hline & Badala $96 \mathrm{C}$ & $34.5-62.8(38.53-39.07)$ & 59.6 & ${ }^{56} \mathrm{Fe} p$ \\
\hline${ }^{36} \mathrm{Ar}{ }^{181} \mathrm{Ta}$ & & Chen 94 & Brohm 95 & $59.39(53.58)$ Bogdanov 94 \\
\hline $15.27(204.9)$ & $\begin{array}{l}\text { Legrain } 97 \\
\text { Lecolley } 95\end{array}$ & $\begin{array}{ll}35.55(38.55) & \text { Chen } 97 \\
35.61(38.55) & \text { Tuve } 99\end{array}$ & ${ }^{52} \mathrm{Cr} p$ & $\begin{array}{ll} & \text { Dudkin 94 } \\
66.16(53.67) & \text { Webber } 98\end{array}$ \\
\hline $15.44(204.9)$ & Germain 97 & $\begin{array}{ll} & \text { Tuve } 97 \\
46.85(38.75) & \text { Tuve } 99\end{array}$ & $44.33-80.56(49.64-50.17)$ & $97.94(54.13)$ Zeitlin 97 \\
\hline${ }^{36} \mathrm{Ar}{ }^{187} \mathrm{Au}$ & & Chen 97 & $60.15(49.85) \quad$ Webber 98 & $140(54.79) \quad \mathrm{Am}$ \\
\hline $15.53(220.7)$ & Badala $97 \mathrm{~B}$ & Tuve 97 & $52 \mathrm{Cr}$ nucleus & ${ }^{58} \mathrm{Fe}$ nucleus \\
\hline & Badala 96 & $56.62(38.94)$ Chen 9 & Cr nucieus & Bogdanov 94 \\
\hline & $\begin{array}{l}\text { Badala } 96 \mathrm{~B} \\
\text { Badala } 96 \mathrm{C} \\
\text { Holzmann } 96\end{array}$ & & Brohm 95 & $\begin{array}{l}\text { Dudkin } 94 \\
\text { Brohm } 95 \\
\text { Brohm } 95\end{array}$ \\
\hline
\end{tabular}




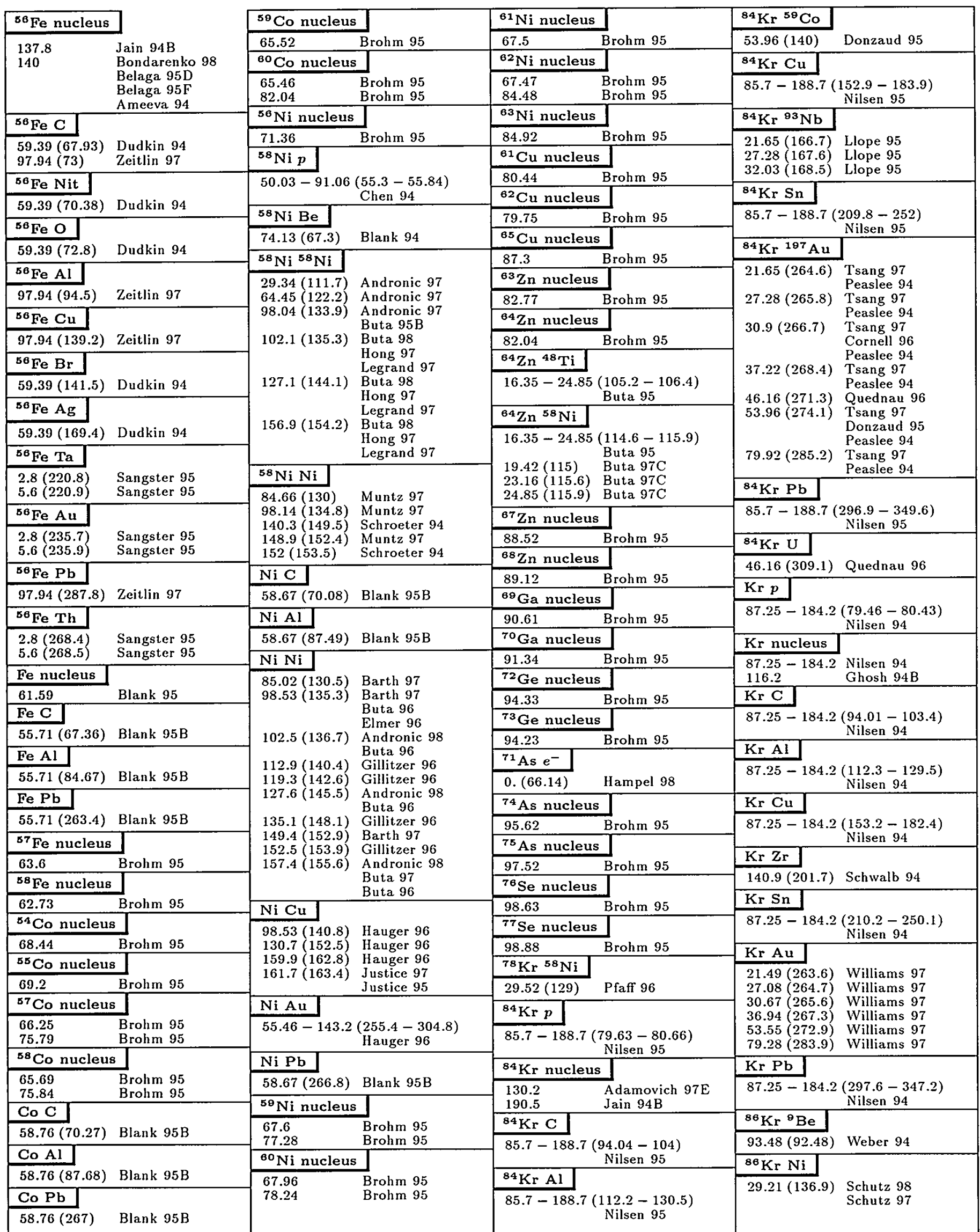

Entries are in order of beam name, then target name, then beam momentum. Particle names are ordered as described in the legend on page 117 and as listed in the Particle Vocabulary. See also the Table of Contents of this Index beginning on the page 118. A few chemical symbols for nuclei have been changed to avoid ambiguity with particle names (see the Particle Vocabulary). Beam momenta are equivalent $p_{\text {lab }}$ in GeV/c; then $E_{\mathrm{cm}}$ in $\mathrm{GeV}$ follows in parentheses. For certain initial states only $E_{\mathrm{cm}}$ (in parentheses) is given. 


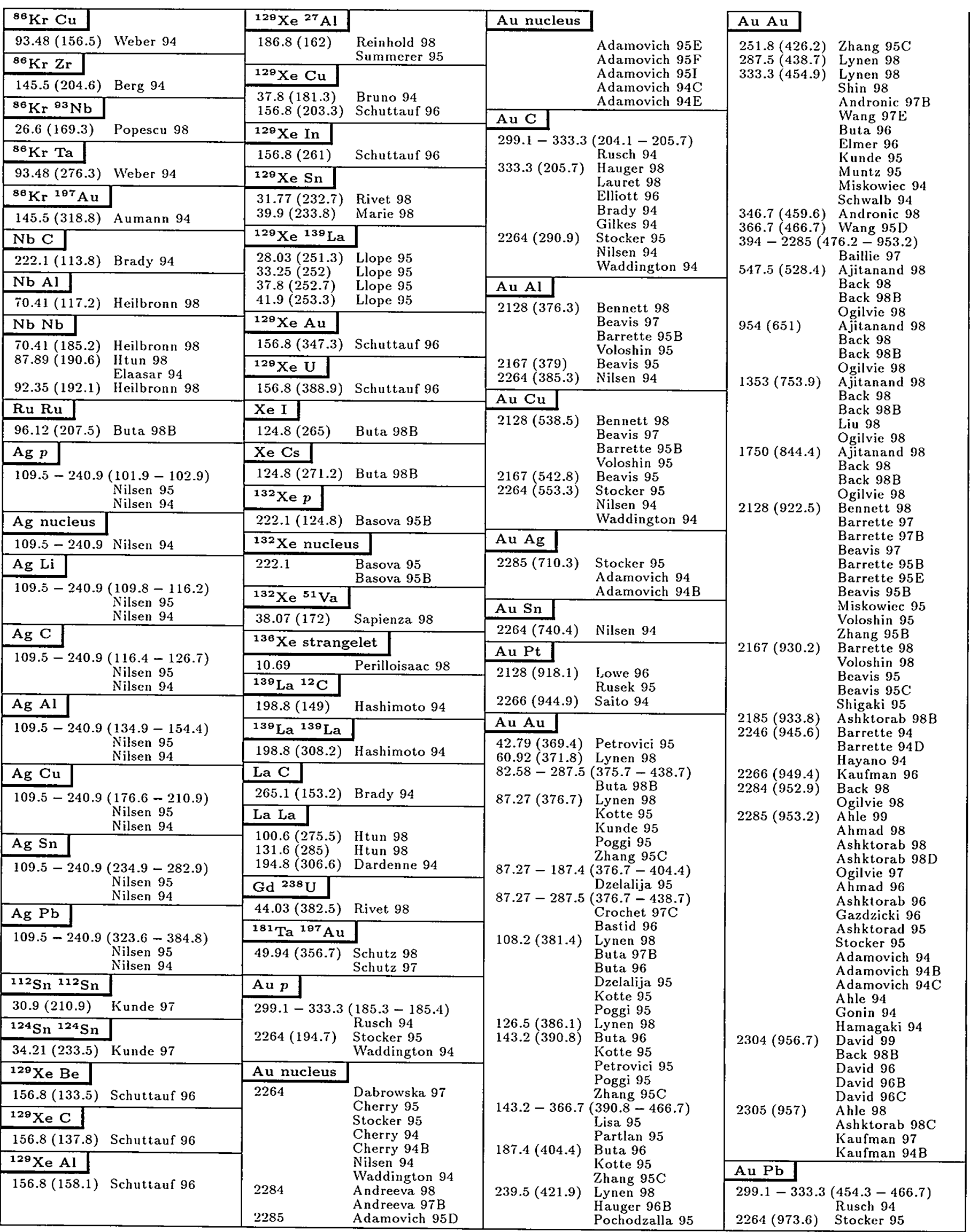




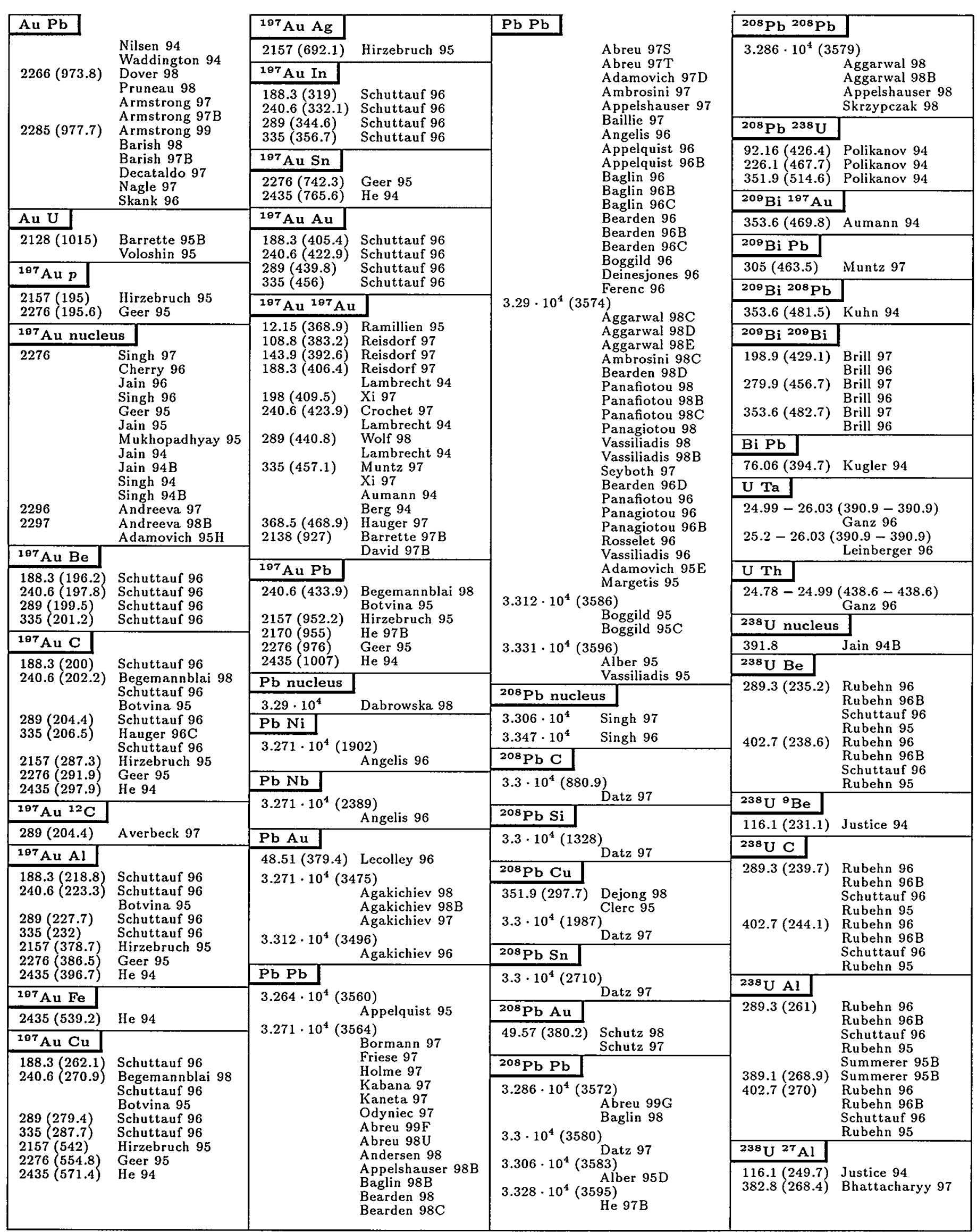

Entries are in order of beam name, then target name, then beam momentum. Particle names are ordered as described in the legend on page 117 and as listed in the Particle Vocabulary. See also the Table of Contents of this Index beginning on the page 118. A few chemical symbols for nuclei have been changed to avoid ambiguity with particle names (see the Particle Vocabulary). Beam momenta are equivalent $p_{\text {lab }}$ in GeV/c; then $E_{\mathrm{cm}}$ in $\mathrm{GeV}$ follows in parentheses. For certain initial states only $E_{\mathrm{cm}}$ (in parentheses) is given. 


\begin{tabular}{|c|c|}
\hline \multicolumn{2}{|l|}{${ }^{238} \mathrm{UCu}$} \\
\hline $\begin{array}{l}389.1(325.1) \\
402.7(327.2)\end{array}$ & $\begin{array}{l}\text { Justice } 94 \\
\text { Rubehn } 96 \\
\text { Rubehn } 96 \mathrm{~B} \\
\text { Schuttauf } 96 \\
\text { Rubehn } 95 \\
\text { Summerer } 95 \mathrm{~B} \\
\text { Clerc } 95 \\
\text { Summerer } 95 \mathrm{~B} \\
\text { Rubehn } 96 \\
\text { Rubehn } 96 \mathrm{~B} \\
\text { Schuttauf } 96 \\
\text { Rubehn } 95\end{array}$ \\
\hline \multicolumn{2}{|l|}{${ }^{238} \mathrm{U} \mathrm{Ag}$} \\
\hline $116.1(331)$ & Justice 94 \\
\hline \multicolumn{2}{|l|}{238 U In } \\
\hline $289.3(372.2)$ & $\begin{array}{l}\text { Rubehn } 96 \\
\text { Rubehn } 96 \mathrm{~B} \\
\text { Schuttauf } 96 \\
\text { Rubehn } 95 \\
\text { Rubehn } 96 \\
\text { Rubehn } 96 \mathrm{~B} \\
\text { Schuttauf } 96 \\
\text { Rubehn } 95\end{array}$ \\
\hline \multicolumn{2}{|l|}{${ }^{238} \mathrm{U}^{181} \mathrm{Ta}$} \\
\hline \multicolumn{2}{|c|}{$\begin{array}{r}24.76-25.1(390.9-390.9) \\
\text { Ahmad } 97 \\
\text { Ahmad } 95 \mathrm{~B} \\
25.08-25.41(390.9-390.9) \\
\text { Ahmad } 97 \\
\text { Ahmad } 95 \mathrm{~B} \\
25.48-25.83(390.9-390.9) \\
\text { Ahmad } 97 \\
\text { Ahmad } 95 \mathrm{~B} \\
\end{array}$} \\
\hline \multicolumn{2}{|c|}{${ }^{238} \mathrm{UAu}$} \\
\hline $289.3(465.4)$ & $\begin{array}{l}\text { Rubehn } 96 \\
\text { Rubehn } 96 \mathrm{~B} \\
\text { Schuttauf } 96 \\
\text { Rubehn } 95 \\
\text { Rubehn } 96 \\
\text { Rubehn } 96 \mathrm{~B} \\
\text { Schuttauf } 96 \\
\text { Rubehn } 95 \\
\end{array}$ \\
\hline \multicolumn{2}{|l|}{${ }^{238} \mathrm{U} \mathrm{Pb}$} \\
\hline $\begin{array}{l}289.3(476.6) \\
389.1(509.2)\end{array}$ & $\begin{array}{l}\text { Summerer } 95 \mathrm{~B} \\
\text { Summerer } 95 \mathrm{~B}\end{array}$ \\
\hline \multicolumn{2}{|l|}{${ }^{238} \mathrm{U}{ }^{208} \mathrm{~Pb}$} \\
\hline $333.2(491.7)$ & $\begin{array}{l}\text { Donzaud } 98 \\
\text { Schwab } 98\end{array}$ \\
\hline \multicolumn{2}{|c|}{${ }^{238} \mathrm{U}^{232} \mathrm{Th}$} \\
\hline \multicolumn{2}{|c|}{$\begin{array}{r}24.74-25.1(438.5-438.5) \\
\text { Ahmad } 97 \\
\text { Ahmad } 95 \mathrm{~B} \\
24.76-25.1(438.5-438.5) \\
\text { Ahmad } 95 \mathrm{~B} \\
25.08-25.41(438.5-438.5) \\
\text { Ahmad } 95 \mathrm{~B} \\
25.48-25.83(438.5-438.5) \\
\text { Ahmad } 95 \mathrm{~B} \\
\end{array}$} \\
\hline${ }^{238} \mathrm{U} U$ & \\
\hline $\begin{array}{l}116.1(457.5) \\
289.3(509.9)\end{array}$ & $\begin{array}{l}\text { Justice } 94 \\
\text { Rubehn } 96 \\
\text { Rubehn } 96 \mathrm{~B} \\
\text { Schuttauf } 96 \\
\text { Rubehn } 95 \\
\text { Rubehn } 96 \\
\text { Rubehn } 96 \mathrm{~B} \\
\text { Schuttauf } 96 \\
\text { Rubehn } 95\end{array}$ \\
\hline
\end{tabular}


This index lists papers by complete reactions: beam, target, final state, and momentum. There are three kinds of "reactions" in the data base: particle fluxes, particle decays, and collisions of two particles. Fluxes go first here, then collisions. Decays are indexed in the Particle/Decay Index.

In each class the ordering is by beam particle name, then by target particle name. For a given beam and target, reactions are further divided into two classes, inclusive and exclusive, with inclusive first. In each inclusive or exclusive sample, ordering is by the final state multiplicity with separation of semileptonic from nonleptonic final states, with semileptonic first. Reactions are further ordered by names of the final state particles, taking into account the weak and strong isospin multiplet structures.

Particle names follow the ordering in the Particle Vocabulary of this compilation: Gauge bosons, leptons, mesons, baryons, atoms, and nuclei; and within each group the ordering is mainly by increasing mass. Within mesons and baryons, however, we further categorize these particles as having only light quarks, or as having as the heaviest quark, a strange, charmed, bottom, or top quark. To simplify searching in this Index, a short "Table of Contents" of the full Index comes first.

The beam momentum is the equivalent lab momentum in $\mathrm{GeV} / c$. For most colliding beam experiments and for reactions above $2 \mathrm{TeV} / c$ equivalent lab momentum, we give instead the c.m. energy $E_{\mathrm{cm}}$ in $\mathrm{GeV}$. C.m. energies are enclosed in parentheses. For a given beam momentum, papers are ordered by year (most recent first), then first author name.

When a range of momenta were studied, we list the range, e.g. " $50-70$," ordered by the lower end. For some experiments, such as neutrino experiments, the listed range is only approximate.

A question mark means that information is unknown, usually because it was not given in the paper.

\section{Illustrative Key}

Initial State of the Reaction: see the Particle Vocabulary for nomenclature.

Final State of the Reaction: see the Particle Vocabulary for nomenclature.

Document ID: see the ID/Reference/Title Index for the full reference.

Lab Momentum: in $\mathrm{GeV} / \mathrm{c}$ or (in parentheses) the c.m. energy $E_{\mathrm{cm}}$, in $\mathrm{GeV}$.

Data Descriptor: brief description of the data measured; see the Data Descriptor Vocabulary for nomenclature.

\begin{tabular}{|c|c|c|}
\hline $\bar{p}$ & & \\
\hline $\begin{array}{c}J / \psi \mathrm{X} \\
80-190 \\
n^{ \pm \mathrm{Y}}\end{array}$ & Sokoloff 86 & cs \\
\hline 20 & Butler 86 & - \\
\hline$D_{s}^{-\mathrm{X}}$ & Anjos 89B & cs \\
\hline $\begin{array}{l}D_{s}^{+} \mathrm{X} \\
\quad 100-260\end{array}$ & $\begin{array}{l}\text { Anjos 89B } \\
\text { Anjos 87C }\end{array}$ & $\begin{array}{c}\operatorname{cs} \\
-\end{array}$ \\
\hline $\begin{array}{c}D^{*}(2010)^{0} \mathrm{X} \\
20\end{array}$ & Abe 86 & $\mathrm{cs}$ \\
\hline $\begin{array}{c}\bar{D}^{*}(2010)^{0} \mathrm{X} \\
20\end{array}$ & Abe 86 & $\operatorname{cs}$ \\
\hline $\begin{array}{l}D^{*}(2010)+X \\
\quad 20 \\
\quad(40-160)\end{array}$ & $\begin{array}{l}\text { Abe } 86 \\
\text { Sliwa } 83\end{array}$ & angp, cs, pt \\
\hline $\begin{array}{l}D^{*}(2010)-\mathrm{X} \\
20\end{array}$ & & CS \\
\hline
\end{tabular}


This is a short "Table of Contents" of the full Reaction/Momentum/Data-Descriptor Index

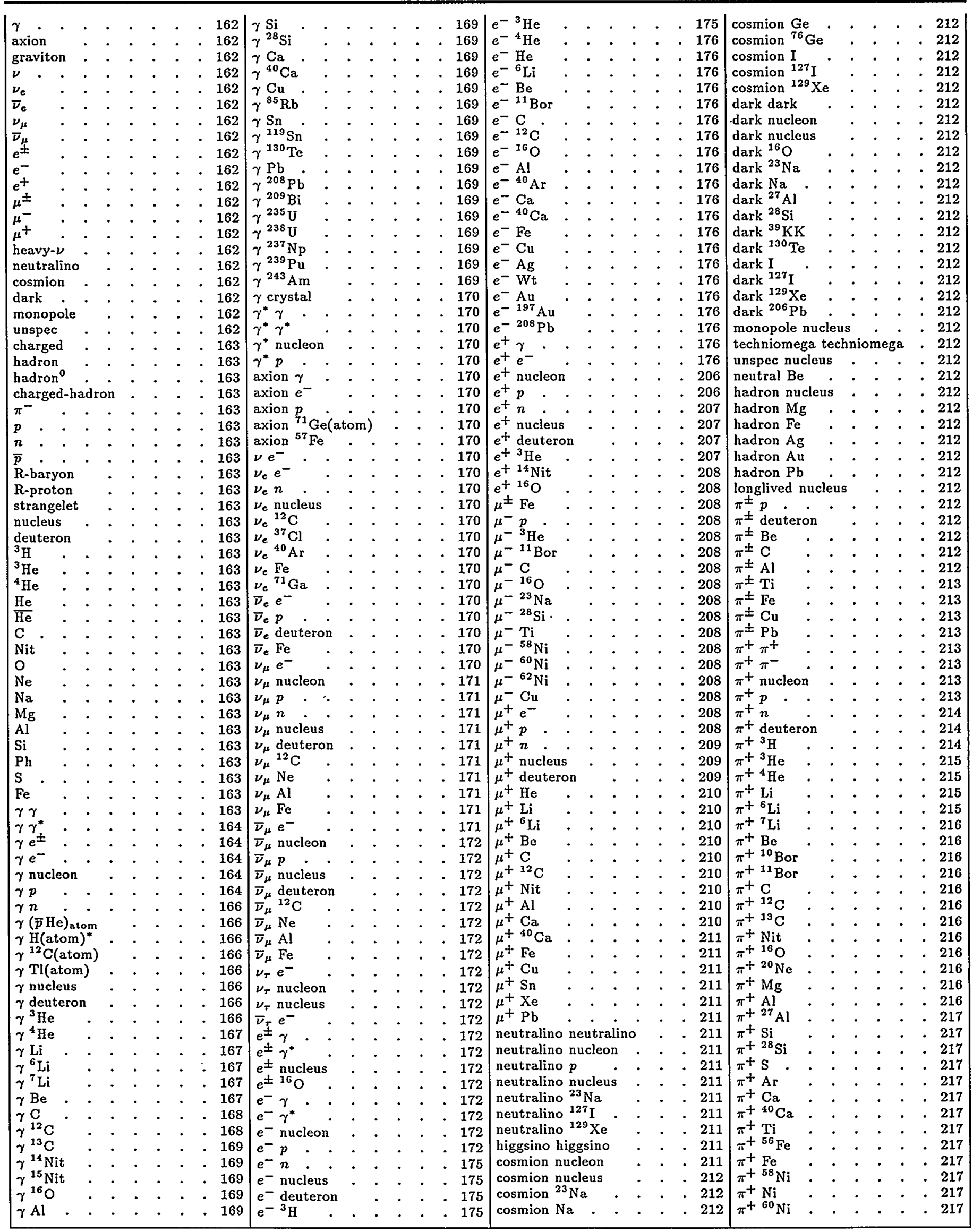


This is a short "Table of Contents" of the full Reaction/Momentum/Data-Descriptor Index

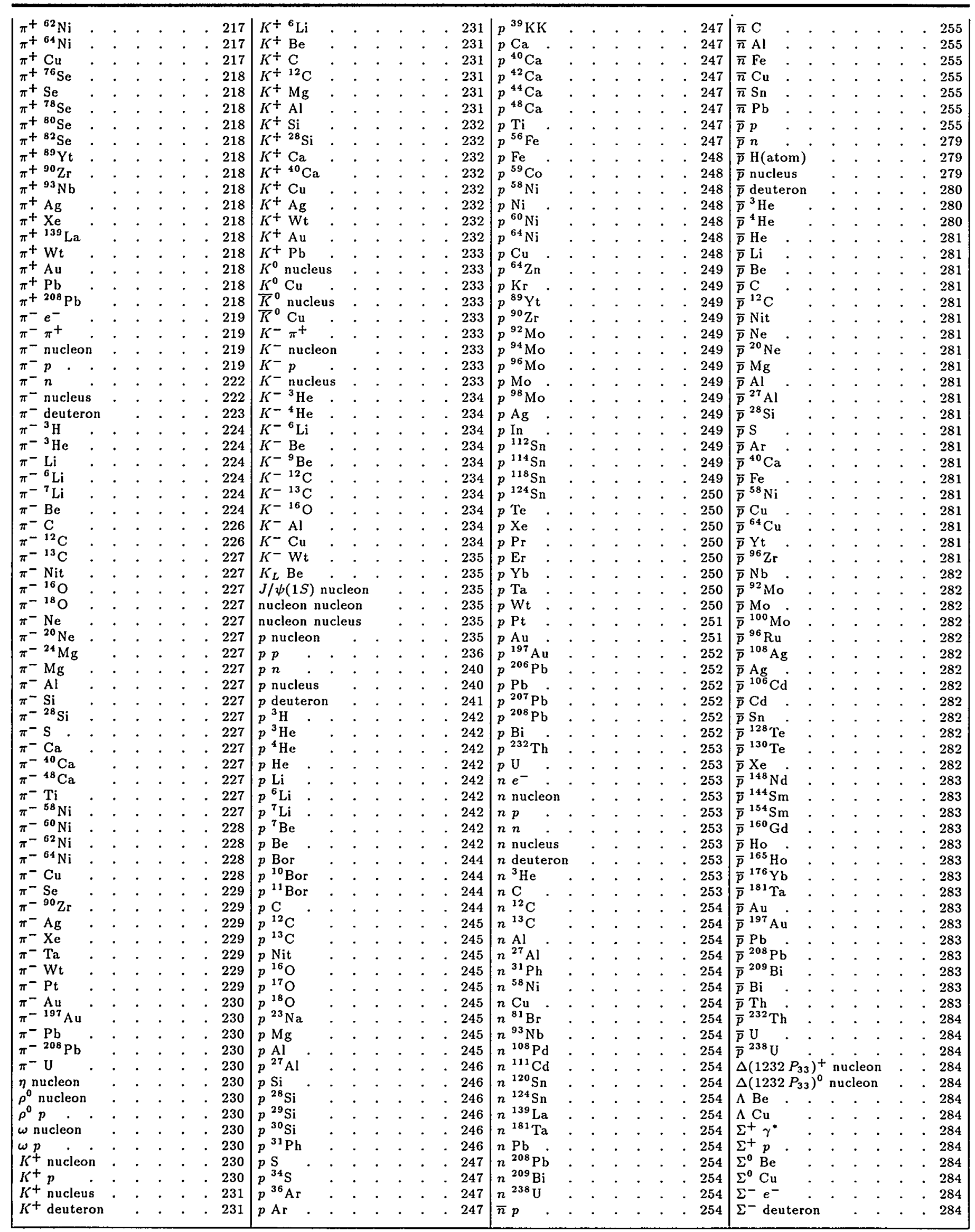


This is a short "Table of Contents" of the full Reaction/Momentum/Data-Descriptor Index

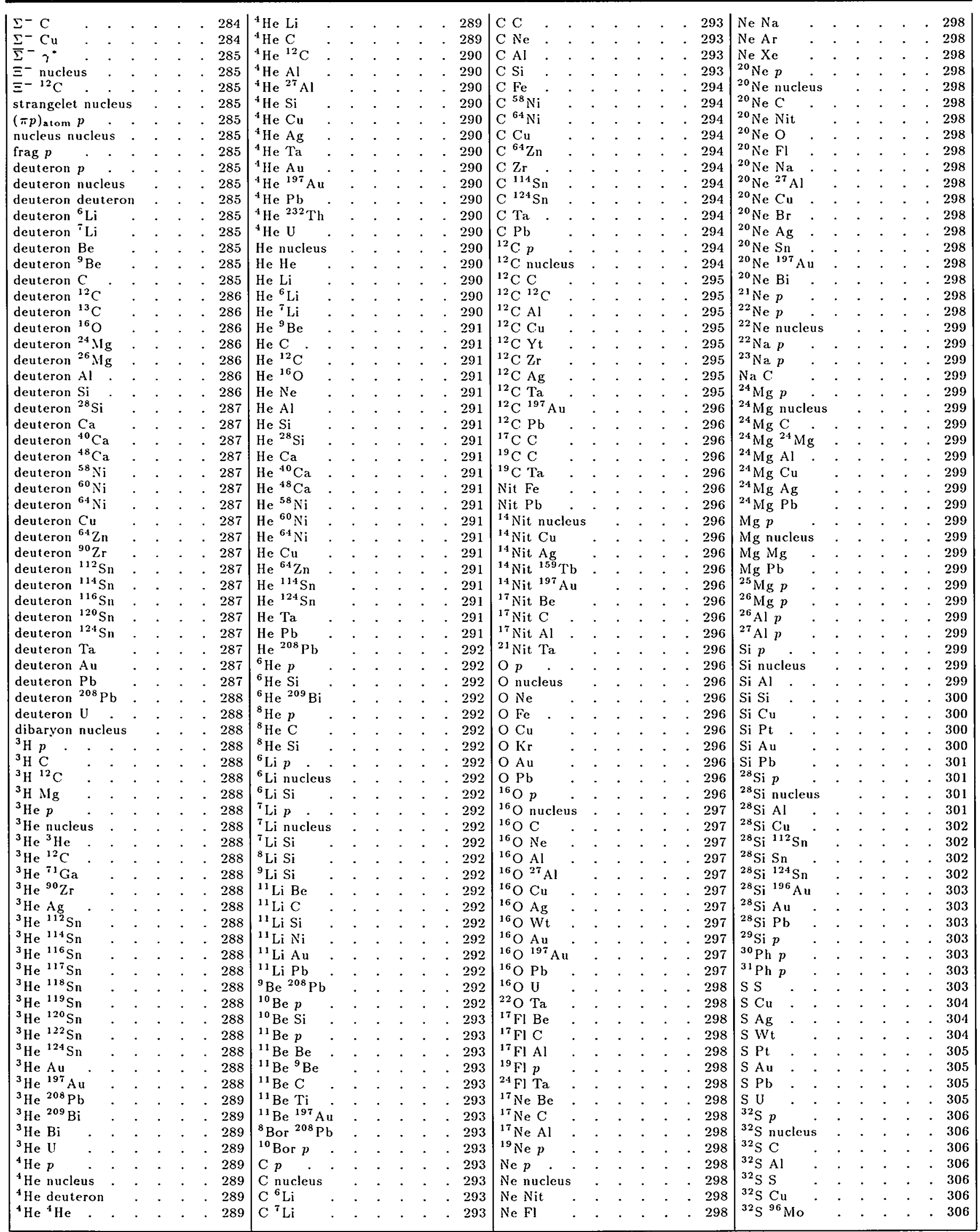


This is a short "Table of Contents" of the full Reaction/Momentum/Data-Descriptor Index

\begin{tabular}{|c|c|c|c|c|c|c|c|c|c|c|c|c|c|c|c|c|c|c|c|c|}
\hline${ }^{32} \mathrm{~S}{ }^{100} \mathrm{Mo}$ & & & & . 306 & Mn nucleus & $\cdot$ & & & & 310 & ${ }^{78} \mathrm{Kr}{ }^{58} \mathrm{Ni}$ & & • & & 312 & ${ }^{197} \mathrm{Aup}$ & & & & . 317 \\
\hline${ }^{32} \mathrm{~S}^{110} \mathrm{Pd}$ & 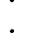 & . & . & . 306 & $\mathrm{MnC}$. & • & & & . & 310 & ${ }^{84} \mathrm{Kr} p$ & $\bullet$ & . & $\cdot$ & 312 & ${ }^{197} \mathrm{Au}$ nucleus & & . & . & . 317 \\
\hline${ }^{32} \mathrm{~S} \mathrm{Ag}$ & $\cdot$ & . & . & .306 & $\mathrm{Mn} \mathrm{Al}$ & . & & - & . & 310 & ${ }^{84} \mathrm{Kr}$ nucleus & . & . & ${ }^{\circ}$ & . 312 & ${ }^{197} \mathrm{~A} u \mathrm{Be}$ & & . & . & . 318 \\
\hline${ }^{32} \mathrm{~S} \mathrm{Wt}$ & . & . & . & . 307 & $\mathrm{Mn} \mathrm{Pb}$ & . & & & . & 310 & ${ }^{84} \mathrm{Kr} \mathrm{C}$ & . & . & . & . 312 & ${ }^{197} \mathrm{AuC}$ & & . & . & . 318 \\
\hline${ }^{32} \mathrm{~S}{ }^{184} \mathrm{Wt}$ & . & . & . & . 307 & ${ }^{55} \mathrm{Mn} p$ & $\cdot$ & & & . & 310 & ${ }^{84} \mathrm{Kr} \mathrm{Al}$ & . & . & - & . 312 & ${ }^{197} \mathrm{Au}{ }^{12} \mathrm{C}$ & & & . & . 318 \\
\hline${ }^{32} \mathrm{~S} \mathrm{Pt}_{\mathrm{t}}$ & . & . & . & . 307 & ${ }^{55} \mathrm{Mn}$ nucleu & & & . & . & 310 & ${ }^{84} \mathrm{Kr}{ }^{59} \mathrm{Co}$ & $\cdot$ & . & . & . 312 & ${ }^{197} \mathrm{Au} \mathrm{Al}$ & & & . & . 318 \\
\hline${ }^{32} \mathrm{~S} \mathrm{Au}$ & . & . & . & . 307 & ${ }^{56} \mathrm{Mn}$ nucleu & & & . & . & 310 & ${ }^{84} \mathrm{Kr} \mathrm{Cu}$ & . & . & 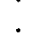 & . 312 & ${ }^{197} \mathrm{Au} \mathrm{Fe}$ & & & . & . 318 \\
\hline${ }^{32} \mathrm{~S} \mathrm{~Pb}$ & . & . & . . & . 307 & ${ }^{51} \mathrm{Fe}$ nucleus & & & $\theta$ & . & 310 & ${ }^{84} \mathrm{Kr}{ }^{93} \mathrm{Nb}$ & . & . & . & . 312 & ${ }^{197} \mathrm{Au} \mathrm{Cu}$ & & & . & . 318 \\
\hline${ }^{32} \mathrm{~S} \mathrm{U}$ & 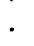 & . & . . & . 307 & ${ }_{53}^{52} \mathrm{Fe}$ nucleus & & & & . & 310 & ${ }^{84} \mathrm{Kr} \mathrm{Sn}$ & . & . & . & 312 & ${ }^{197} \mathrm{Au} \mathrm{Ag}$ & & & . & . 318 \\
\hline${ }^{33} \mathrm{~S} p$ & $\cdot$ & . & . . & . 308 & ${ }^{53} \mathrm{Fe}$ nucleus & & & & . & 310 & ${ }^{84} \mathrm{Kr}{ }^{197} \mathrm{Au}$ & . & . & $\cdot$ & . 312 & ${ }^{197} \mathrm{Au} \mathrm{In}$ & & & . . & . 318 \\
\hline${ }^{36} \mathrm{~S}{ }^{110} \mathrm{Pd}$ & . & . & . . & . 308 & ${ }^{54} \mathrm{Fe} p$ & . & & & . & 310 & ${ }^{84} \mathrm{Kr} \mathrm{Pb}$ & . & . & . & . 312 & ${ }^{197} \mathrm{Au} \mathrm{Sn}$ & & . & . . & . 318 \\
\hline${ }^{34} \mathrm{Cl} p$ & . & & . & . 308 & ${ }^{54} \mathrm{Fe}$ nucleus & & & & . & 310 & ${ }^{84} \mathrm{Kr} \mathrm{U}$ & . & 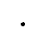 & . & . 312 & ${ }^{197} \mathrm{Au} \mathrm{Au}$ & & & . & . 318 \\
\hline${ }^{37} \mathrm{Cl} p$ & . & . & . & . 308 & ${ }^{55} \mathrm{Fe}$ nucleus & & & & . & 310 & $\operatorname{Kr} p$ & . & . & . & . 312 & ${ }^{197} \mathrm{Au}{ }^{197} \mathrm{Au}$ & & & . & . 318 \\
\hline${ }^{36} \mathrm{Ar} \mathrm{p}$ & . & . & . & . 308 & ${ }^{56} \mathrm{Fe} p$ & - & & • & . & 310 & Kr nucleus & 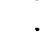 & . & . & . 313 & ${ }^{197} \mathrm{Au} \mathrm{Pb}$ & & . & . & . 319 \\
\hline${ }^{36} \mathrm{Ar}{ }^{12} \mathrm{C}$ & 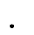 & . & . & .308 & ${ }^{56} \mathrm{Fe}$ nucleus & & & & . & 310 & $\mathrm{KrC}$. & . & . & r & . 313 & $\mathrm{~Pb}$ nucleus & & & . & . 319 \\
\hline${ }^{36} \mathrm{Ar}{ }^{27} \mathrm{Al}$ & ${ }^{\circ}$ & . & . & . 308 & ${ }^{56} \mathrm{Fe} \mathrm{C}$ & - & & & . & 310 & $\mathrm{Kr} \mathrm{Al}$ & . & . & 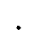 & . 313 & $\mathrm{~Pb} \mathrm{Ni}$. & & & . & . 319 \\
\hline${ }^{36} \mathrm{Ar} \mathrm{Ti}$ & . & . & . & . 308 & ${ }^{56} \mathrm{Fe}$ Nit & . & . & - & . & 310 & $\mathrm{Kr} \mathrm{Cu}$ & . & . & . & . 313 & $\mathrm{~Pb} \mathrm{Nb}$ & . & . & . & . 319 \\
\hline${ }^{36} \mathrm{Ar}{ }^{58} \mathrm{Ni}$ & . & . & . & . 308 & ${ }^{56} \mathrm{Fe} \mathrm{O}$ & . & & & . & 310 & $\mathrm{Kr} \mathrm{Zr}$ & . & . & . & . 313 & $\mathrm{~Pb} \mathrm{Au}$ & . & . & . & . 319 \\
\hline${ }^{36} \mathrm{Ar}{ }^{112} \mathrm{Sn}$ & . & . & . & . 308 & ${ }^{56} \mathrm{Fe} \mathrm{Al}$ & . & & & . & 310 & Kr Sn & & . & . & . 313 & $\mathrm{~Pb} \mathrm{~Pb}$ & & & 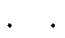 & 319 \\
\hline${ }^{36} \mathrm{Ar}{ }^{181} \mathrm{Ta}$ & . & . & . & . 308 & ${ }^{56} \mathrm{Fe} \mathrm{Cu}$ & . & & . & . & 310 & $\mathrm{Kr} \mathrm{Au}$ & . & . & . & . 313 & ${ }^{208} \mathrm{~Pb}$ nucleus & & & . & . 320 \\
\hline${ }^{36} \mathrm{Ar}{ }^{197} \mathrm{Au}$ & . & . & . & . 308 & ${ }^{56} \mathrm{Fe} \mathrm{Br}$ & . & & - & . & 310 & $\mathrm{Kr} \mathrm{Pb}$ & 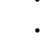 & . & . & . 313 & ${ }^{208} \mathrm{~Pb} \mathrm{C}$ & & . & . & . 321 \\
\hline${ }^{38} \mathrm{Ar} p$ & . & . & . & . 308 & ${ }^{56} \mathrm{Fe} \mathrm{Ag}$ & . & & & . & 310 & ${ }^{86} \mathrm{Kr}{ }^{9} \mathrm{Be}$ & . & . & . & . 313 & ${ }^{208} \mathrm{~Pb} \mathrm{Si}$ & & . & . & . 321 \\
\hline Ar nucleus & . & . & . & . 308 & ${ }^{56} \mathrm{Fe} \mathrm{Ta}$ & . & & . & . & 310 & ${ }^{86} \mathrm{Kr} \mathrm{Ni}$ & . & . & . & .313 & ${ }^{208} \mathrm{~Pb} \mathrm{Cu}$ & . & & . & . 321 \\
\hline ArC & . & . & . & . 308 & ${ }^{56} \mathrm{Fe} \mathrm{Au}$ & . & . & . & . & 310 & ${ }^{86} \mathrm{Kr} \mathrm{Cu}$ & . & . & 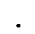 & . 313 & ${ }^{208} \mathrm{~Pb} \mathrm{Sn}$ & & & . & . 321 \\
\hline Ar $\mathrm{Ca}$ & . & . & . & . 308 & ${ }^{56} \mathrm{Fe} \mathrm{Pb}$ & . & & . & . & 310 & ${ }^{86} \mathrm{Kr} \mathrm{Zr}$ & . & . & . & . 313 & ${ }^{208} \mathrm{~Pb} \mathrm{Au}$ & & & . & . 321 \\
\hline Ar Au & . & . & . . & . 308 & ${ }^{56} \mathrm{Fe} \mathrm{Th}$ & . & & & . & 310 & ${ }^{86} \mathrm{Kr}^{93} \mathrm{Nb}$ & . & . & . & . 313 & ${ }^{208} \mathrm{~Pb} \mathrm{~Pb}$ & & & & . 321 \\
\hline${ }^{10} \mathrm{Ar} p$ & . & . & . & . 308 & Fe nucleus & . & . & - & . & 310 & ${ }^{86} \mathrm{Kr} \mathrm{Ta}$. & . & . & . & . 313 & ${ }^{208} \mathrm{~Pb}{ }^{208} \mathrm{~Pb}$ & & & . & . 321 \\
\hline${ }^{40} \mathrm{Ar}$ nucleus & & . & . & . 309 & $\mathrm{FeC}$ & 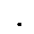 & . & • & . & 310 & ${ }^{86} \mathrm{Kr}{ }^{197} \mathrm{Au}$ & . & . & . & . 313 & ${ }^{208} \mathrm{~Pb}{ }^{238} \mathrm{U}$ & & & . & . 321 \\
\hline${ }^{40} \mathrm{Ar} \mathrm{C}$ & . & . & . & . 309 & Fe Al & . & . & . & . & 310 & $\mathrm{Nb} \mathrm{C}$ & . & . & . & . 313 & ${ }^{209} \mathrm{Bi}^{197} \mathrm{Au}$ & & . & . & . 321 \\
\hline${ }^{40} \mathrm{Ar} \mathrm{Nit}$ & . & . & . & . 309 & $\mathrm{Fe} \mathrm{Pb}$ & . & . & . & . & 310 & $\mathrm{Nb}$ Al & - & . & . & . 313 & ${ }^{209} \mathrm{Bi} \mathrm{Pb}$ & . & & . & . 321 \\
\hline${ }^{40} \mathrm{ArO}$ & . & . & . & . 309 & ${ }^{57} \mathrm{Fe}$ nucleus & & • & . & . & 310 & $\mathrm{Nb} \mathrm{Nb}$ & . & . & . & . 313 & ${ }^{209} \mathrm{Bi}{ }^{208} \mathrm{~Pb}$ & . & . & . & . 321 \\
\hline${ }^{10} \mathrm{Ar} \mathrm{Ca}$ & . & . & . & . 309 & ${ }^{58} \mathrm{Fe}$ nucleus & & & & . & 310 & Ru Ru & . & . & . & .313 & ${ }^{209} \mathrm{Bi}{ }^{209} \mathrm{Bi}$ & & . & . & . 321 \\
\hline${ }^{40} \mathrm{Ar}{ }^{45} \mathrm{Sc}$ & . & . & . & . 309 & ${ }^{54}$ Co nucleus & & & . & . & 310 & Ag $p$ & . & . & . & . 313 & $\mathrm{Bi} \mathrm{Pb}$ & & & . & . 321 \\
\hline${ }^{40} \mathrm{Ar}{ }^{51} \mathrm{Va}$ & . & . & . & . 309 & ${ }^{55} \mathrm{Co}$ nucleus & & . & - & . & 310 & Ag nucleus & & . & . & . 313 & U Ta & & & . & . 321 \\
\hline${ }^{10} \mathrm{Ar} \mathrm{Br}$ & . & . & . & . 309 & ${ }^{57}$ Co nucleus & & . & . & . & 310 & $\mathrm{Ag} \mathrm{Li}$ & & . & . & . 313 & U Th & . & & . & . 321 \\
\hline${ }^{10} \mathrm{Ar}{ }^{98} \mathrm{Mo}$ & . & . & . & . 309 & ${ }^{58} \mathrm{Co}$ nucleus & & & 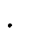 & . & 310 & $\mathrm{Ag} \mathrm{C}$ & & . & 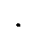 & . 313 & ${ }^{238}$ U nucleus & & & . & . 321 \\
\hline${ }^{40} \mathrm{Ar} \mathrm{Ag}$ & . & . & . & . 309 & Co $\mathrm{C}$ & . & . & . & . & 310 & $\mathrm{Ag} \mathrm{Al}$ & . & . & 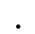 & . 313 & ${ }^{238} \mathrm{U}$ Be. & & 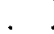 & . & . 321 \\
\hline${ }^{10} \mathrm{Ar}{ }^{107} \mathrm{Au}$ & . & . & . & . 309 & Co Al & . & . & . & . & 311 & $\mathrm{Ag} \mathrm{Cu}$ & . & . & . & . 313 & ${ }^{238} \mathrm{U}^{9} \mathrm{Be}$ & & & . & . 321 \\
\hline $\mathrm{Ca} \mathrm{Ca}$ & . & . & . & . 309 & $\mathrm{Co} \mathrm{Pb}$ & . & . & . & . & 311 & $\mathrm{Ag} \mathrm{Sn}$ & . & . & r & . 313 & ${ }^{238} \mathrm{U} \mathrm{C}$ & & & . & . 321 \\
\hline${ }^{10} \mathrm{Ca} p$ & . & . & . & . 309 & ${ }^{59} \mathrm{Co}$ nucleus & & & & . & 311 & $\mathrm{Ag} \mathrm{Pb}$ & . & . & . & . 313 & ${ }^{238} \mathrm{U} \mathrm{Al}$ & & & . & . 321 \\
\hline${ }^{10} \mathrm{Ca} \mathrm{Ca}$ & . & . & . & . 309 & ${ }^{60} \mathrm{Co}$ nucleus & & & $\cdot$ & . & 311 & ${ }^{112} \mathrm{Sn}{ }^{112} \mathrm{Sn}$ & & $\cdot$ & . & . 313 & ${ }^{238} \mathrm{U}^{27} \mathrm{Al}$ & & & . & . 321 \\
\hline${ }^{10} \mathrm{Ca}{ }^{40} \mathrm{Ca}$ & . & . & . & . 309 & ${ }^{56} \mathrm{Ni}$ nucleus & & - & - & . & 311 & ${ }^{124} \mathrm{Sn}{ }^{124} \mathrm{Sn}$ & 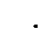 & . & . & . 313 & ${ }^{238} \mathrm{UCu}^{\mathrm{Cu}}$. & & & . & . 322 \\
\hline${ }^{42} \mathrm{Ca} p$. & . & . & . & . 309 & ${ }^{58} \mathrm{Ni} p$ & . & & & . & 311 & ${ }^{129} \mathrm{Xe} \mathrm{Be}$ & & . & . & . 313 & ${ }^{238} \mathrm{U} \mathrm{Ag}$ & & & . & . 322 \\
\hline${ }^{44} \mathrm{Ca} p$ & . & . & . & . 309 & ${ }^{58} \mathrm{Ni} \mathrm{Be}$ & . & . & • & . & 311 & ${ }^{129} \mathrm{Xe} \mathrm{C}$ & & r & . & . 313 & ${ }^{238} \mathrm{U}$ In & & & . & . 322 \\
\hline${ }^{44} \mathrm{Sc} p$ & . & . & . & . 309 & ${ }^{58} \mathrm{Ni}{ }^{58} \mathrm{Ni}$ & . & & 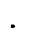 & . & 311 & ${ }^{129} \mathrm{Xe} \mathrm{Al}$ & & . & $\bullet$ & . 313 & ${ }^{238} \mathrm{U}^{181} \mathrm{Ta}$ & & & & . 322 \\
\hline${ }^{46} \mathrm{Sc} p$ & . & . & . & . 309 & ${ }^{58} \mathrm{Ni} \mathrm{Ni}$ & 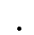 & & & . & 311 & ${ }^{129} \mathrm{Xe}^{27} \mathrm{Al}$ & & 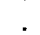 & . & 313 & ${ }^{238} \mathrm{U} A u$ & & & & 322 \\
\hline${ }^{16} \mathrm{Ti} p$ & . & . & . & . 309 & $\mathrm{NiC}$ & . & . & • & . & 311 & ${ }^{129} \mathrm{Xe} \mathrm{Cu}$ & & . & . & . 313 & ${ }^{238} \mathrm{UPb}$ & & & . & . 322 \\
\hline${ }^{16} \mathrm{Ti}$ nucleus & & . & . & . 309 & $\mathrm{Ni} \mathrm{Al}$ & . & & - & . & 311 & ${ }^{129} \mathrm{Xe} \mathrm{In}$ & & . & . & . 313 & ${ }^{238} \mathrm{U}^{208} \mathrm{~Pb}$ & & & & 322 \\
\hline${ }^{47} \mathrm{Ti}$ nucleus & & & . & . 309 & $\mathrm{Ni} \mathrm{Ni}$ & . & & & . & 311 & ${ }^{129} \mathrm{Xe} \mathrm{Sn}$ & & $\cdot$ & 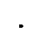 & . 314 & ${ }^{238} \mathrm{U}{ }^{232} \mathrm{Th}$ & & & & 322 \\
\hline${ }^{48} \mathrm{Ti} p$ & $\cdot$ & . & . & . 309 & $\mathrm{Ni} \mathrm{Cu}$ & . & . & 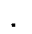 & . & 311 & ${ }^{129} \mathrm{Xe}^{139} \mathrm{La}$ & & r. & 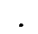 & . 314 & ${ }^{238} \mathrm{U} U$ & & & . & . 322 \\
\hline${ }^{48} \mathrm{Va} p$ & . & . & . & . 309 & $\mathrm{Ni} A u$ & r. & - & $\cdot$ & . & 311 & ${ }^{129} \mathrm{Xe} \mathrm{Au}$ & & . & . & . 314 & & & & & \\
\hline${ }^{18} \mathrm{Va}$ nucleus & & & . & . 309 & $\mathrm{Ni} \mathrm{Pb}$ & 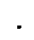 & • & & . & 312 & ${ }^{129} \mathrm{Xe} \mathrm{U}$ & & • & 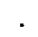 & . 314 & & & & & \\
\hline${ }^{49} \mathrm{Va}$ nucleus & & $\cdot$ & . & . 309 & ${ }^{59} \mathrm{Ni}$ nucleus & & • & - & . & 312 & Xe I . & & $\cdot$ & ${ }^{\circ}$ & . 314 & & & & & \\
\hline${ }^{50} \mathrm{Va} p$ & . & . & . & . 309 & ${ }^{60} \mathrm{Ni}$ nucleus & & $\cdot$ & $\cdot$ & . & 312 & $\mathrm{Xe} \mathrm{Cs}$ & 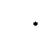 & . & . & . 314 & & & & & \\
\hline${ }^{50} \mathrm{Va}$ nucleus & & & . & . 309 & ${ }^{61} \mathrm{Ni}$ nucleus & & & & . & 312 & ${ }^{132} \mathrm{Xe} p$ & & 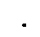 & & . 314 & & & & & \\
\hline $\mathrm{Va} C$ & . & . & . & . 309 & ${ }^{62} \mathrm{Ni}$ nucleus & & & & . & 312 & ${ }^{132} \mathrm{Xe}$ nucleus & & 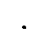 & 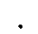 & . 314 & & & & & \\
\hline Va Al & . & $\cdot$ & . & . 309 & ${ }^{63} \mathrm{Ni}$ nucleus & & & & . & 312 & ${ }^{132} \mathrm{Xe}^{51} \mathrm{Va}$ & & . & . & . 314 & & & & & \\
\hline $\mathrm{Va} \mathrm{Pb}$ & . & . & . & . 309 & ${ }^{61} \mathrm{Cu}$ nucleus & & . & & . & 312 & ${ }^{136} \mathrm{Xe}$ strangele & & • & & . 314 & & & & & \\
\hline${ }^{51} \mathrm{Va}$ nucleus & & & . & . 309 & ${ }^{62} \mathrm{Cu}$ nucleus & & & & . & 312 & ${ }^{139} \mathrm{La}{ }^{12} \mathrm{C}$ & & & ${ }^{\circ}$ & . 314 & & & & & \\
\hline${ }^{49} \mathrm{Cr}$ nucleus & & . & . & . 309 & ${ }^{65} \mathrm{Cu}$ nucleus & & & & 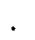 & 312 & ${ }^{139} \mathrm{La}{ }^{139} \mathrm{La}$ & & $\cdot$ & . & . 314 & & & & & \\
\hline${ }^{50} \mathrm{Cr} p$ & . & . & . & . 309 & ${ }^{63} \mathrm{Zn}$ nucleus & & & & . & 312 & La C & & . & 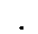 & . 314 & & & & & \\
\hline${ }^{50} \mathrm{Cr}$ nucleus & & & & . 309 & ${ }^{64} \mathrm{Zn}$ nucleus & & & & . & 312 & La La & & . & & . 314 & & & & & \\
\hline${ }^{51} \mathrm{Cr}$ nucleus & & . & . & . 309 & ${ }^{64} \mathrm{Zn}^{48} \mathrm{Ti}$ & & & & $\cdot$ & 312 & $\mathrm{Gd}^{238} \mathrm{U}$ & & r. & . & . 314 & & & & & \\
\hline${ }^{52} \mathrm{Cr} p$ & $\cdot$ & $\cdot$ & . & 309 & ${ }^{64} \mathrm{Zn}^{58} \mathrm{Ni}$ & & & & $\cdot$ & 312 & ${ }^{181} \mathrm{Ta}{ }^{197} \mathrm{Au}$ & & 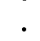 & . & . 314 & & & & & \\
\hline${ }^{52} \mathrm{Cr}$ nucleus & & & . & 309 & ${ }^{67} \mathrm{Zn}$ nucleus & & & & . & 312 & Au $p$ & & - & $\cdot$ & . 314 & & & & & \\
\hline $\mathrm{Cr} \mathrm{C}$. & . & . & . & . 309 & ${ }^{68} \mathrm{Zn}$ nucleus & & & & $\cdot$ & 312 & Au nucleus & & 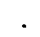 & . & . 314 & & & & & \\
\hline $\mathrm{Cr} \mathrm{Al}$ & 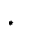 & . & . & . 309 & ${ }^{69} \mathrm{Ga}$ nucleus & & & & . & 312 & $\mathrm{AuC}$ & & . & . & . 315 & & & & & \\
\hline $\mathrm{r} \mathrm{Pb}$ & . & . & . & . 310 & ${ }^{70} \mathrm{Ga}$ nucleus & & $\cdot$ & & 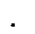 & 312 & $\mathrm{Au} \mathrm{Al}$ & & • & & . 315 & & & & & \\
\hline Cr nucleus & & & . & . 310 & ${ }^{72} \mathrm{Ge}$ nucleus & & & & . & 312 & $\mathrm{AuCu}$ & & & & . 315 & & & & & \\
\hline${ }^{50} \mathrm{Mn}$ nucleus & & . & . & . 310 & ${ }^{73} \mathrm{Ge}$ nucleus & & & & . & 312 & $\mathrm{AuAg}$ & & 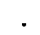 & . & . 315 & & & & & \\
\hline${ }^{51} \mathrm{Mn}$ nucleus & & . & . & . 310 & ${ }^{71}$ As $e^{-}$ & & & & 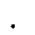 & 312 & $\mathrm{AuS} \mathrm{Sn}$ & & . & . & . 315 & & & & & \\
\hline${ }^{52} \mathrm{Mn} p$ & & & . & . 310 & ${ }^{74}$ As nucleus & & & & & 312 & $\mathrm{Au} \mathrm{Pt}$ & & - & & . 315 & & & & & \\
\hline${ }^{52} \mathrm{Mn}$ nucleus & & r & . & . 310 & ${ }^{75}$ As nucleus & & & & & 312 & $\mathrm{Au} \mathrm{Au}$ & & 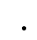 & & . 315 & & & & & \\
\hline${ }^{53} \mathrm{Mn}$ nucleus & & & & . 310 & ${ }^{76}$ Se nucleus & & & & 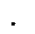 & 312 & $\mathrm{AuPb}$ & & $\cdot$ & . & . 317 & & & & & \\
\hline${ }^{54} \mathrm{Mn}$ nucleus & & & . & . 310 & ${ }^{77}$ Se nucleus & & & & & 312 & $A \cup U$ & & . & & . 317 & & & & & \\
\hline
\end{tabular}




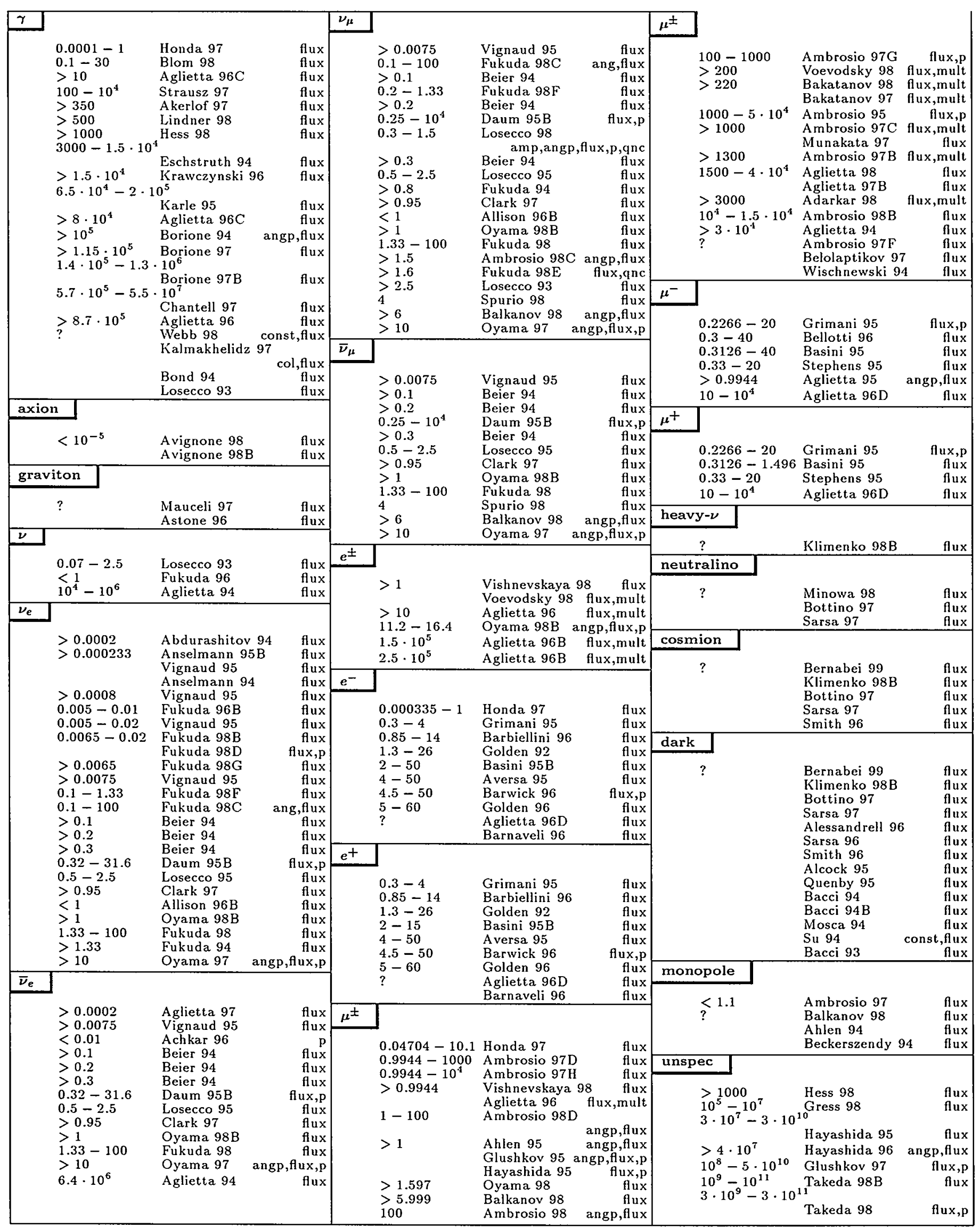


unspec

$\gamma \gamma \rightarrow \operatorname{charm} \mathrm{X}$

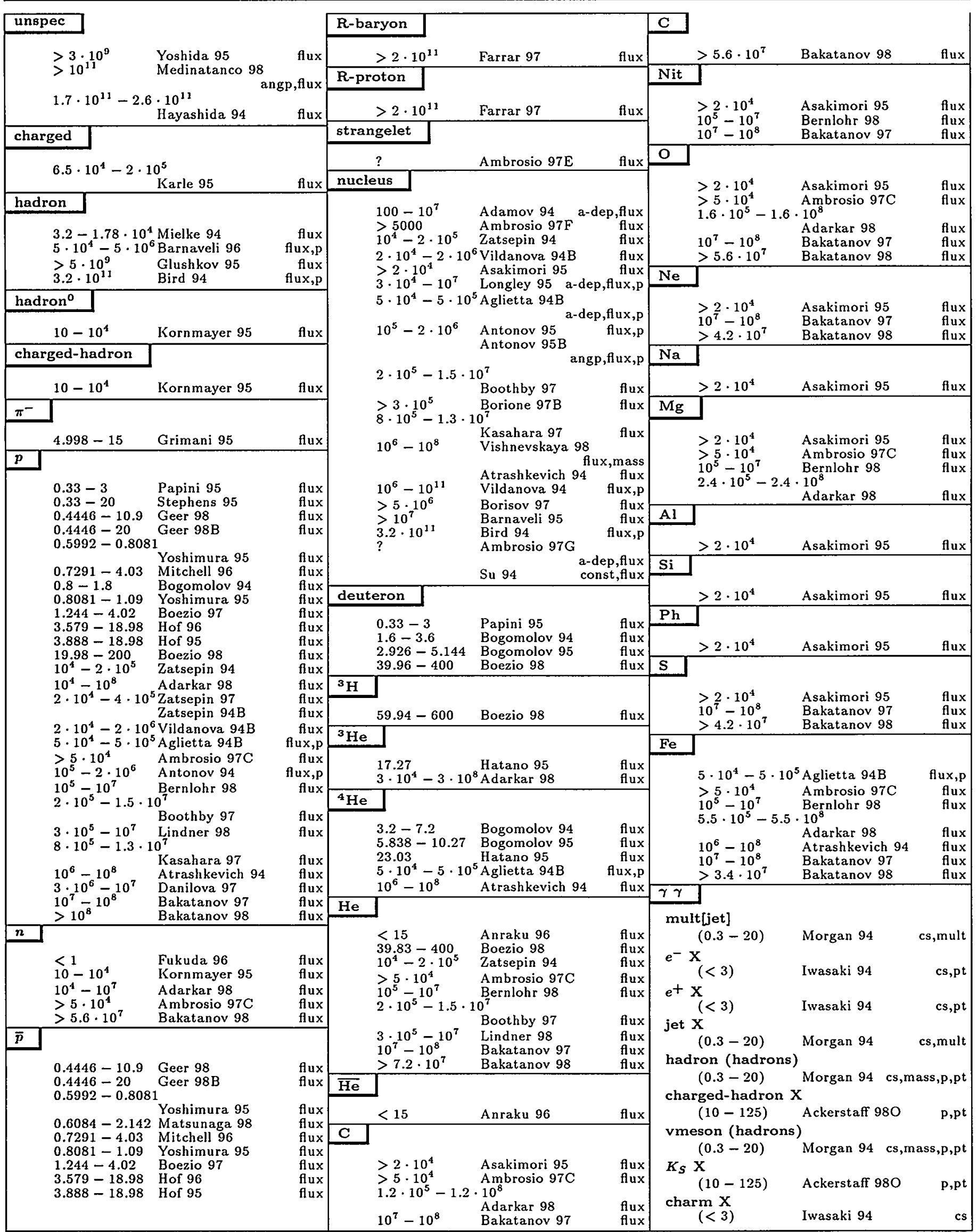

Entries are in order of beam name, then target name, then multiplicity of final state. Particle names are ordered as described in the legend on page 157 and as listed in the Particle Vocabulary. See also the Table of Contents of this Index beginning on the page 158. A few chemical symbols for nuclei have been changed to avoid ambiguity with particle names (see the Particle Vocabulary). Beam momenta are $p_{\text {lab }}$ in GeV/c, or in parentheses $E_{\mathrm{cm}}$ in $\mathrm{GeV}$. 


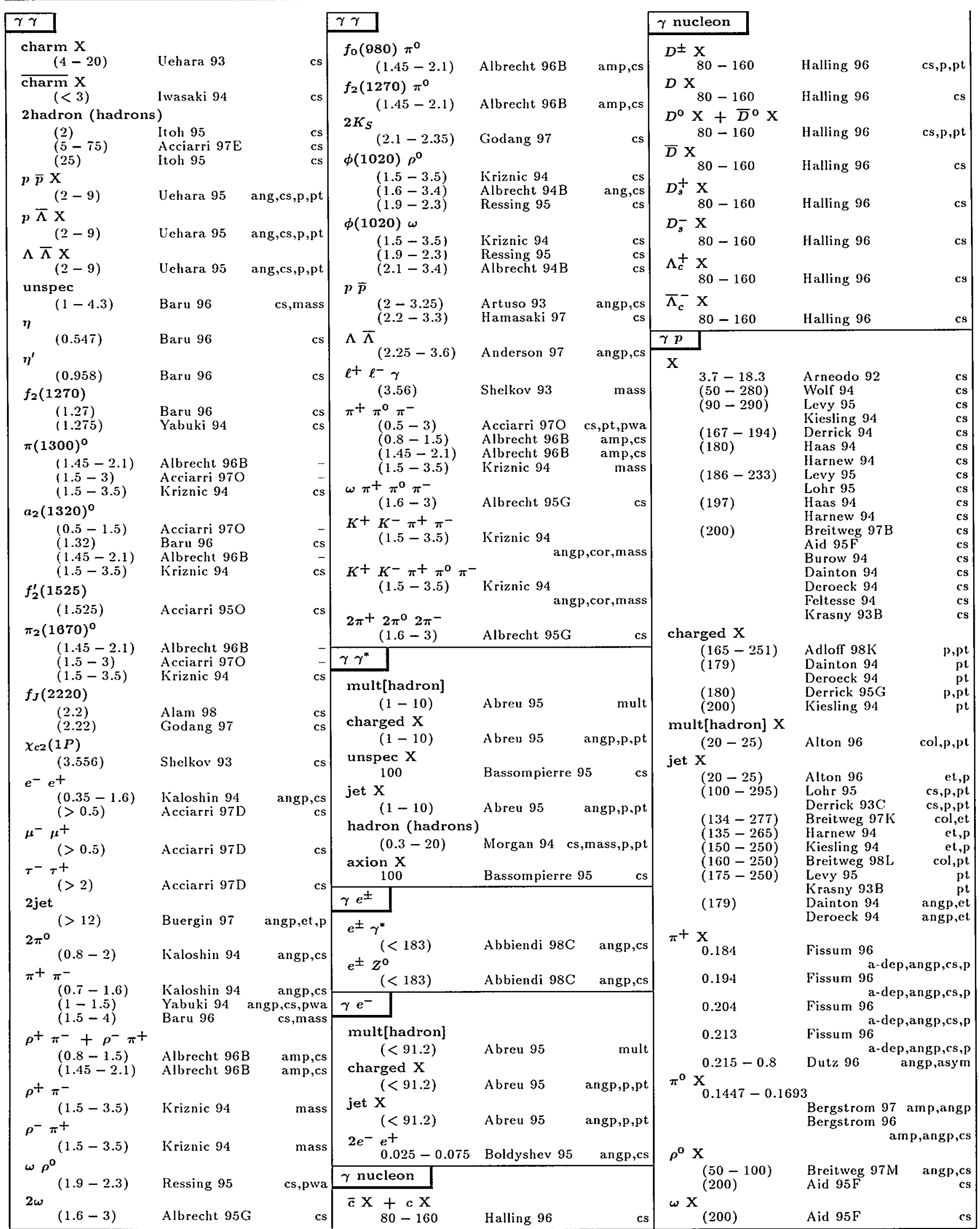




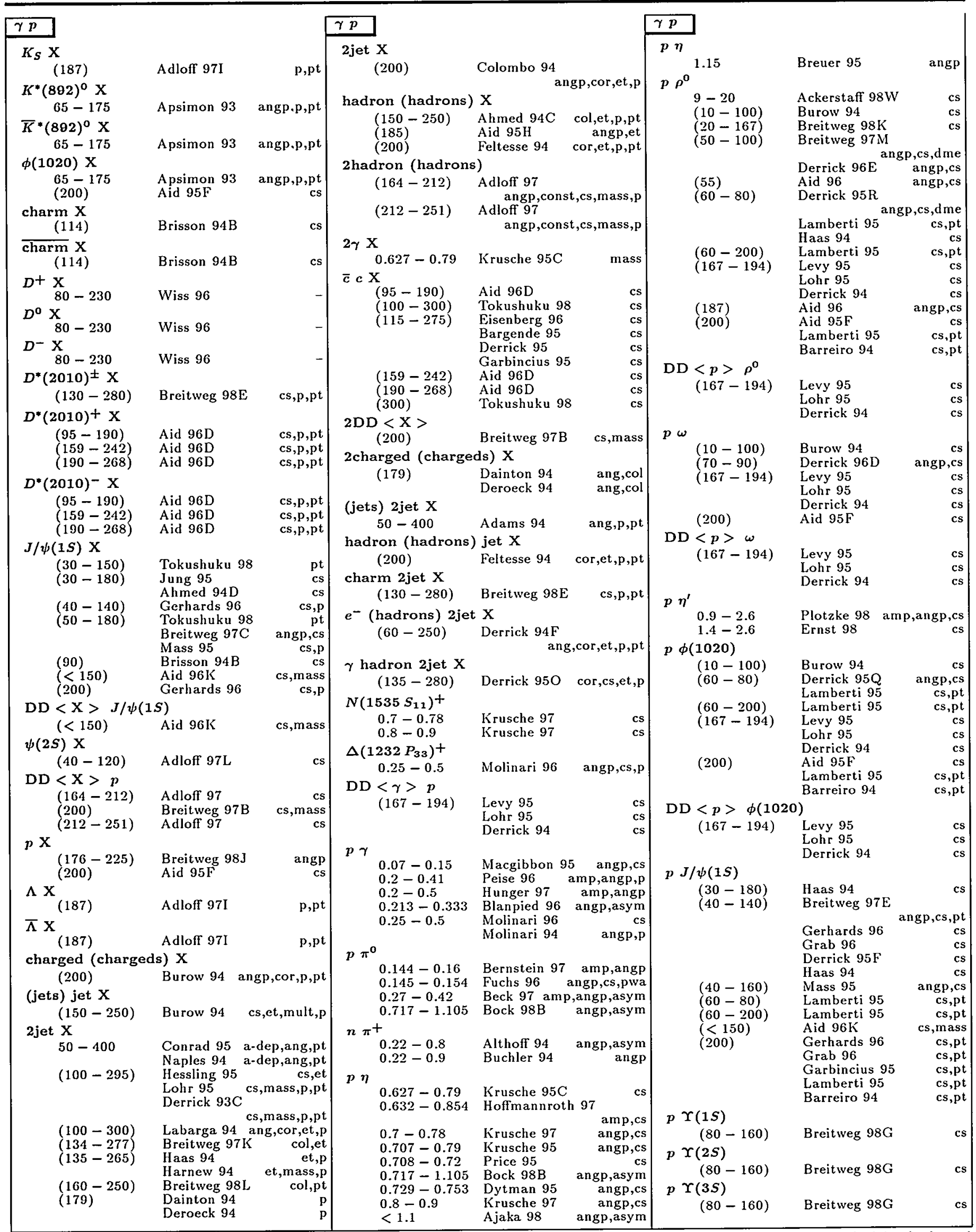

Entries are in order of beam name, then target name, then multiplicity of final state. Particle names are ordered as described in the legend on page 157 and as listed in the Particle Vocabulary. See also the Table of Contents of this Index beginning on the page 158 . A few chemical symbols for nuclei have been changed to avoid ambiguity with particle names (see the Particle Vocabulary). Beam momenta are $p_{1 a b}$ in GeV/c, or in parentheses $E_{\mathrm{cm}}$ in $\mathrm{GeV}$. 


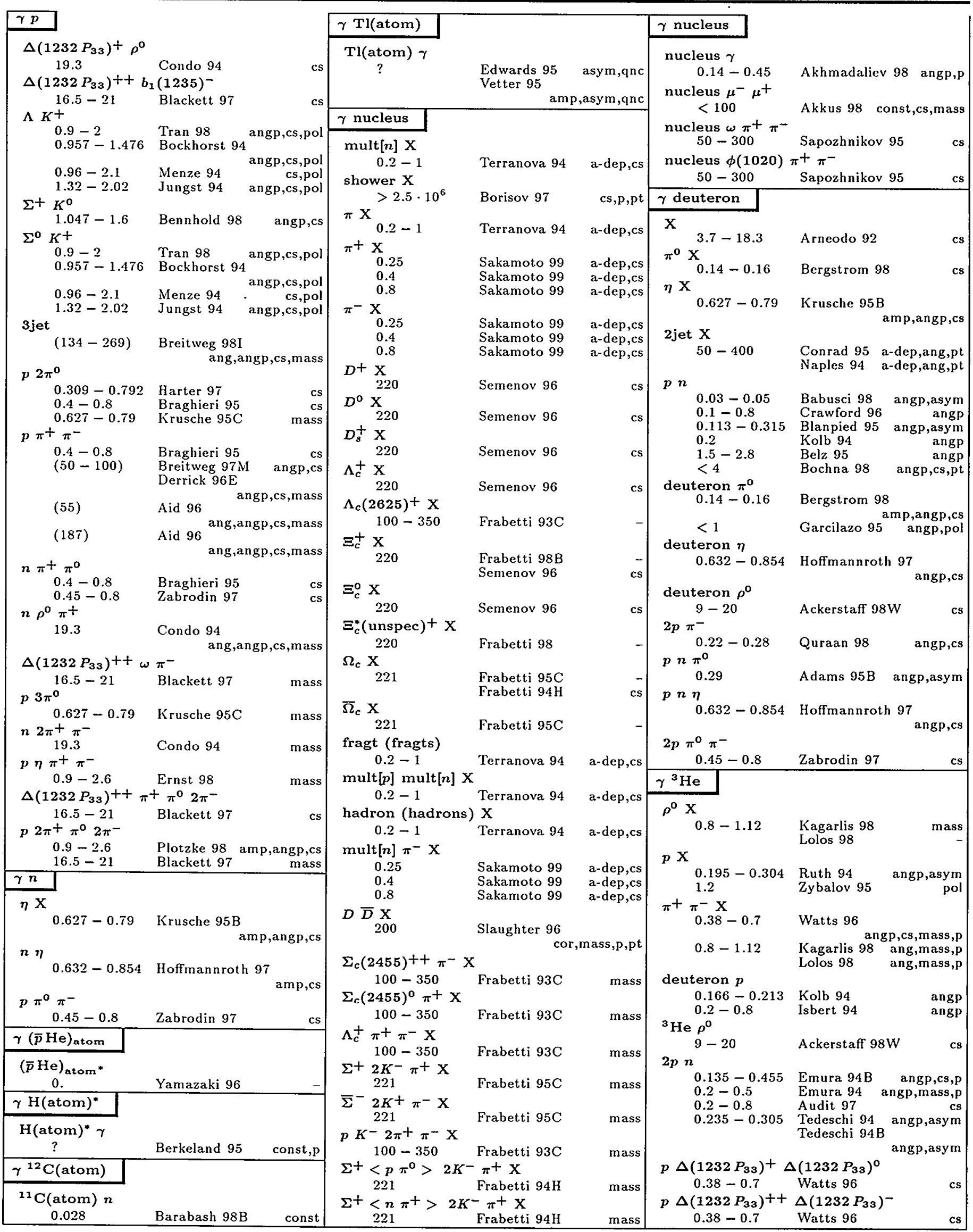




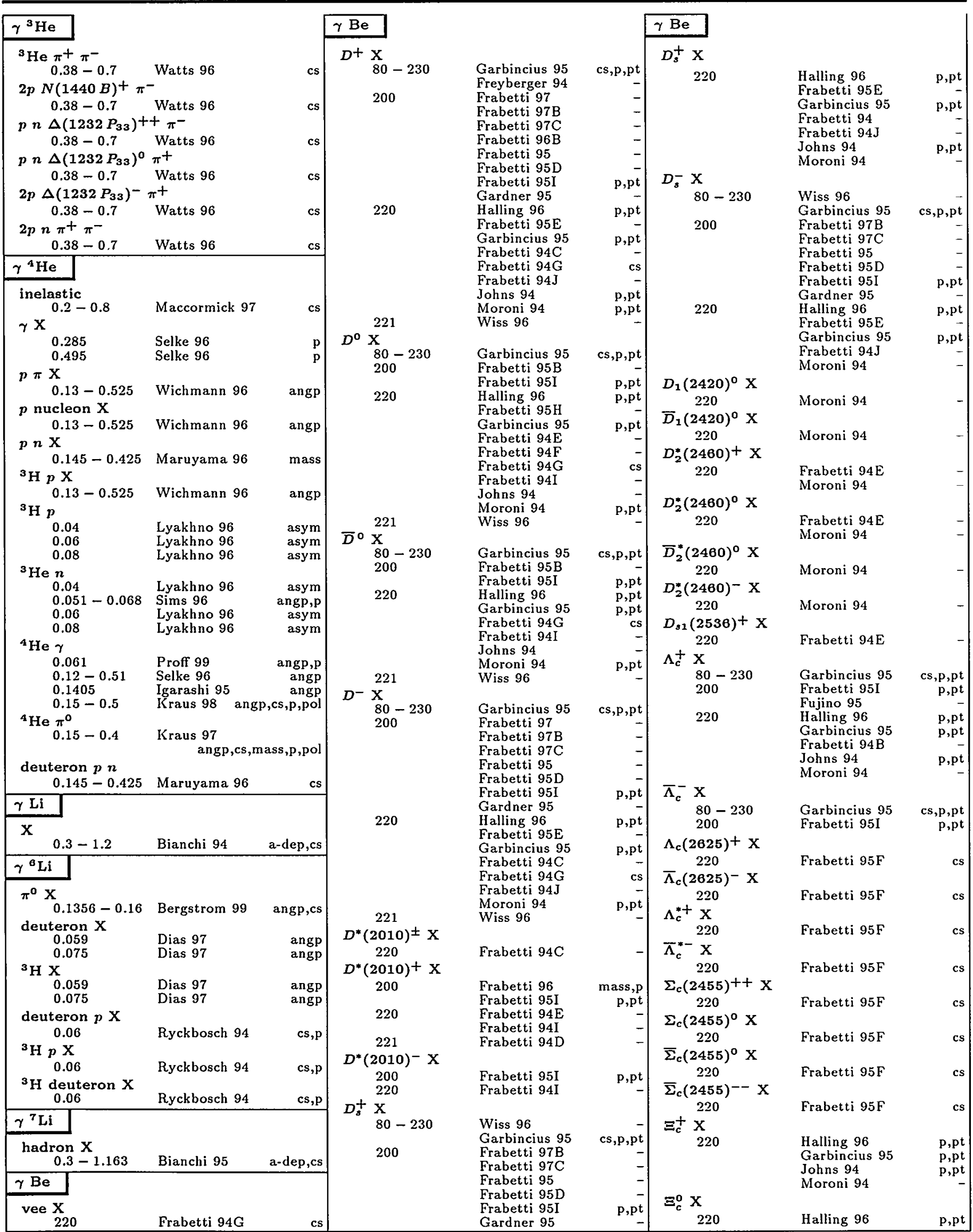

Entries are in order of beam name, then target name, then multiplicity of final state. Particle names are ordered as described in the legend on page 157 and as listed in the Particle Vocabulary. See also the Table of Contents of this Index beginning on the page 158. A few chemical symbols for nuclei have been changed to avoid ambiguity with particle names (see the Particle Vocabulary). Beam momenta are $p_{\text {lab }}$ in GeV/c, or in parentheses $E_{\mathrm{cm}}$ in $\mathrm{GeV}$. 


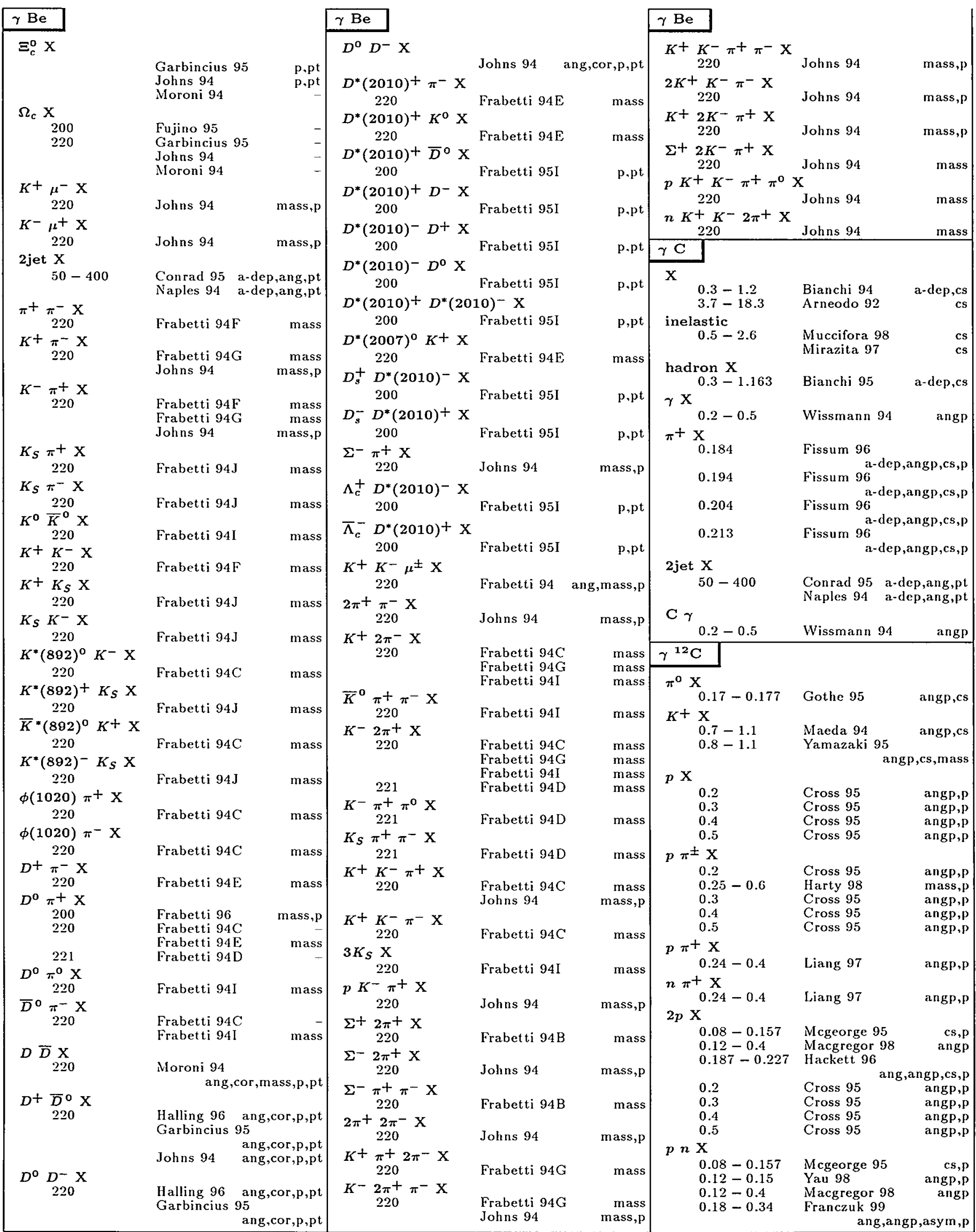




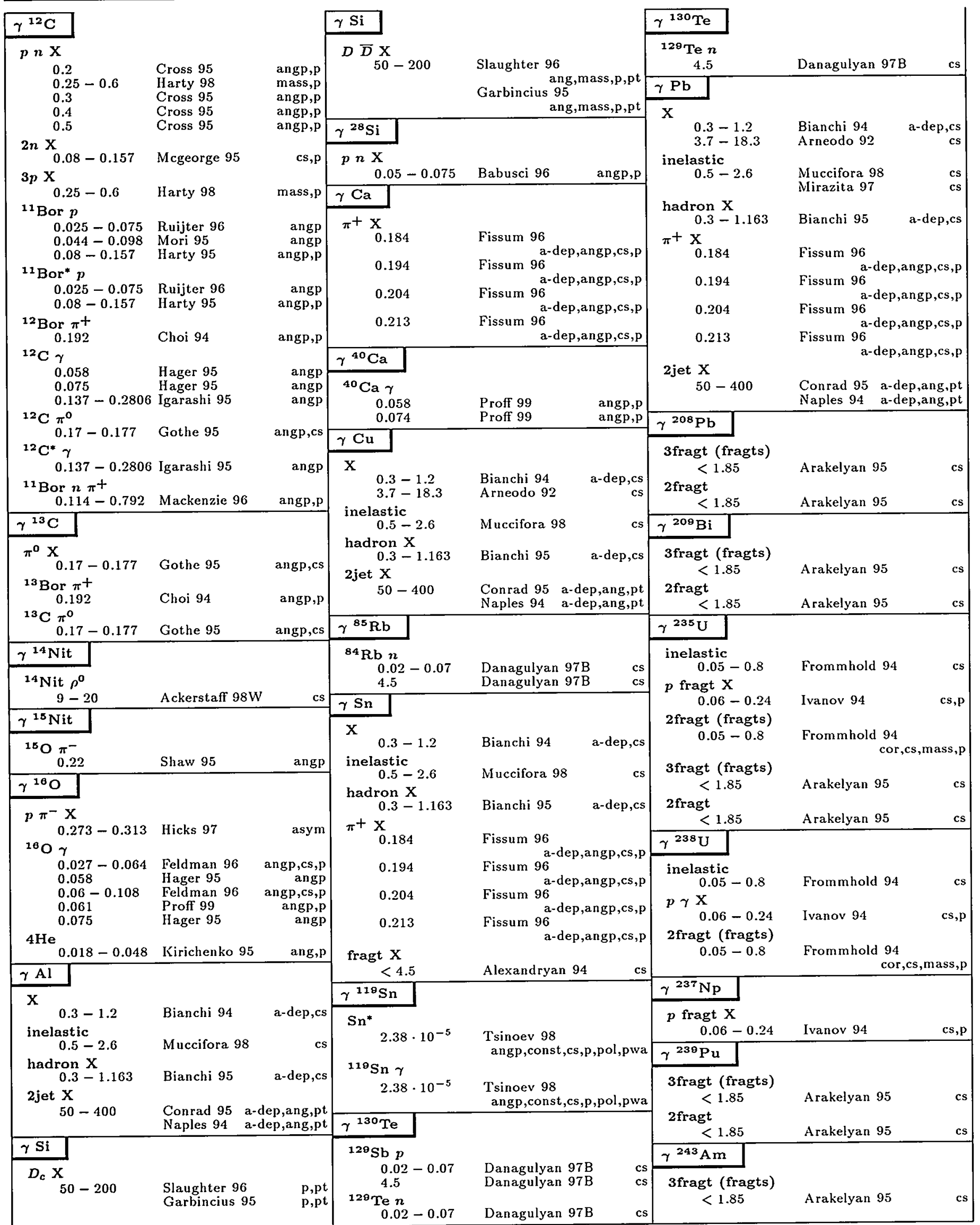

Entries are in order of beam name, then target name, then multiplicity of final state. Particle names are ordered as described in the legend Entries are in order of beam name, then target name, the also the Table of Contents of this Index beginning on the page 158. A few chemical symbols for nuclei have been changed to avoid ambiguity with particle names (see the Particle Vocabulary). Beam momenta are $p_{l a b}$ in GeV/c, or in parentheses $E_{c m}$ in $\mathrm{GeV}$. 
$\gamma^{243} \mathrm{Am} \rightarrow 2 \mathrm{fragt}$

$\nu_{\mu} e^{-} \rightarrow e^{-} \nu_{\mu}$

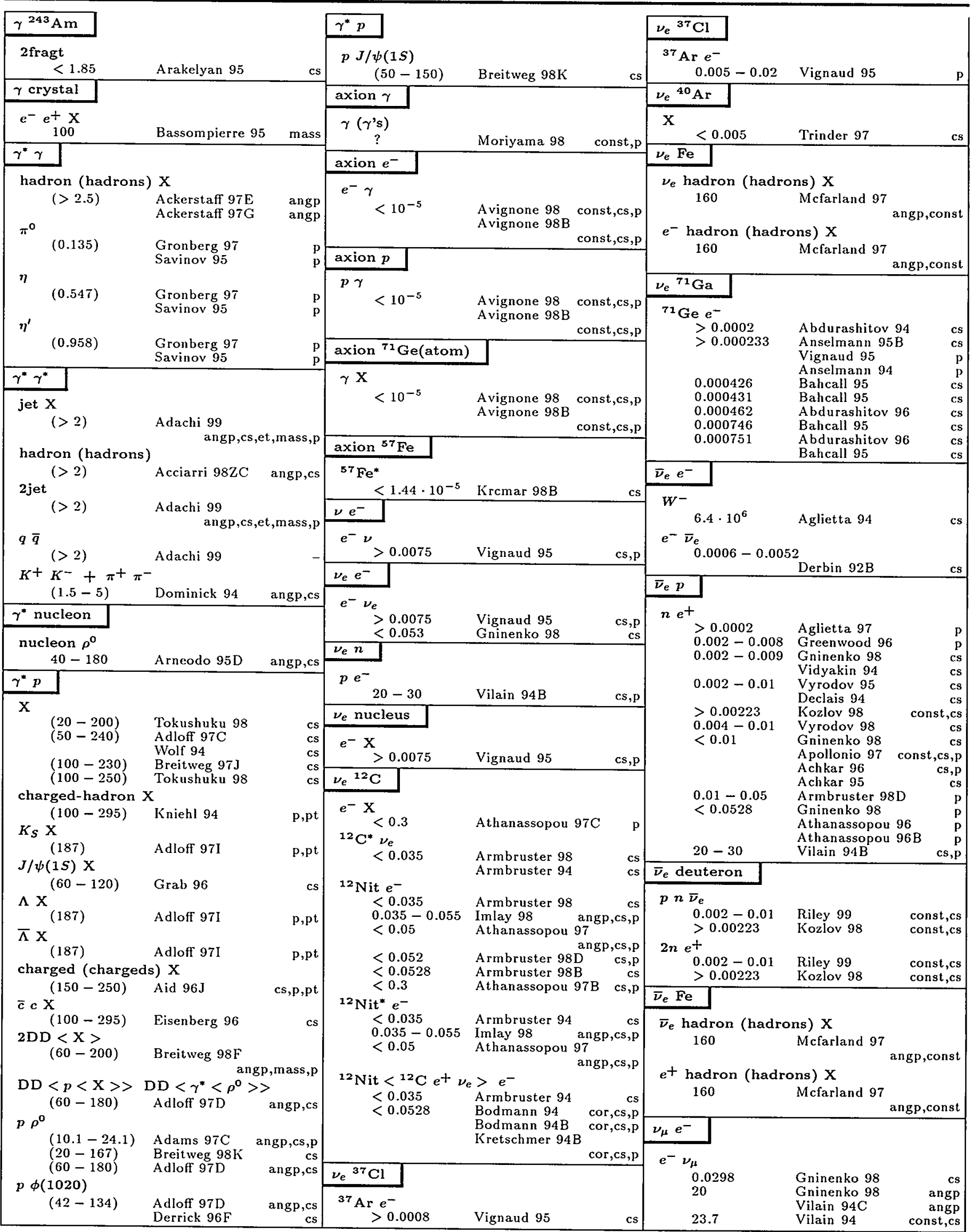




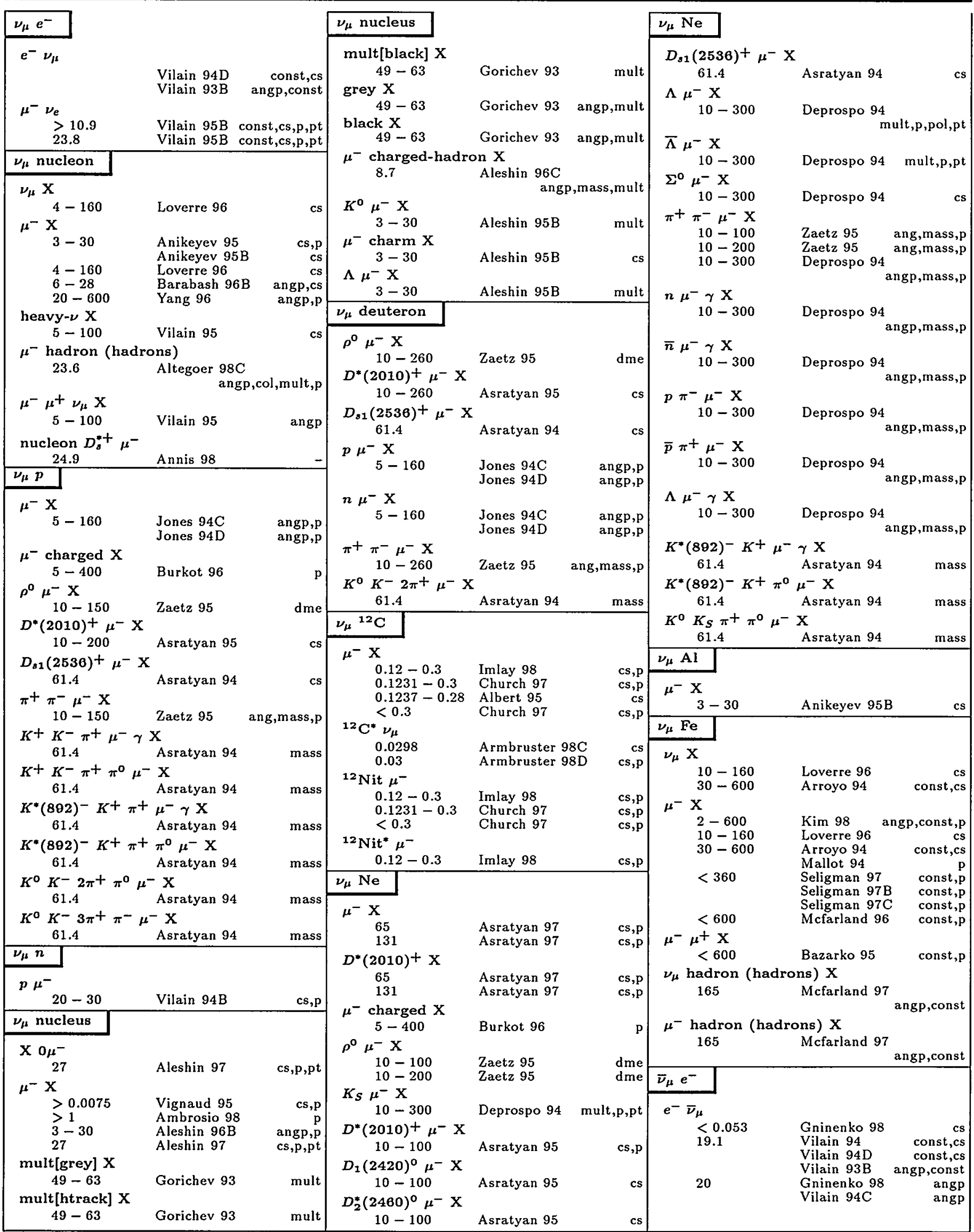

Entries are in order of beam name, then target name, then multiplicity of final state. Particle names are ordered as described in the legend on page 157 and as listed in the Particle Vocabulary. See also the Table of Contents of this Index beginning on the page 158. A few chemical symbols for nuclei have been changed to avoid ambiguity with particle names (see the Particle Vocabulary). Beam momenta are $p_{\text {lab }}$ in GeV/c, or in parentheses $E_{\mathrm{cm}}$ in $\mathrm{GeV}$. 
$\bar{\nu}_{\mu}$ nucleon $\rightarrow \mu^{+} \mathrm{X}$

$e^{-} p \rightarrow \nu_{e} \mathrm{X}$

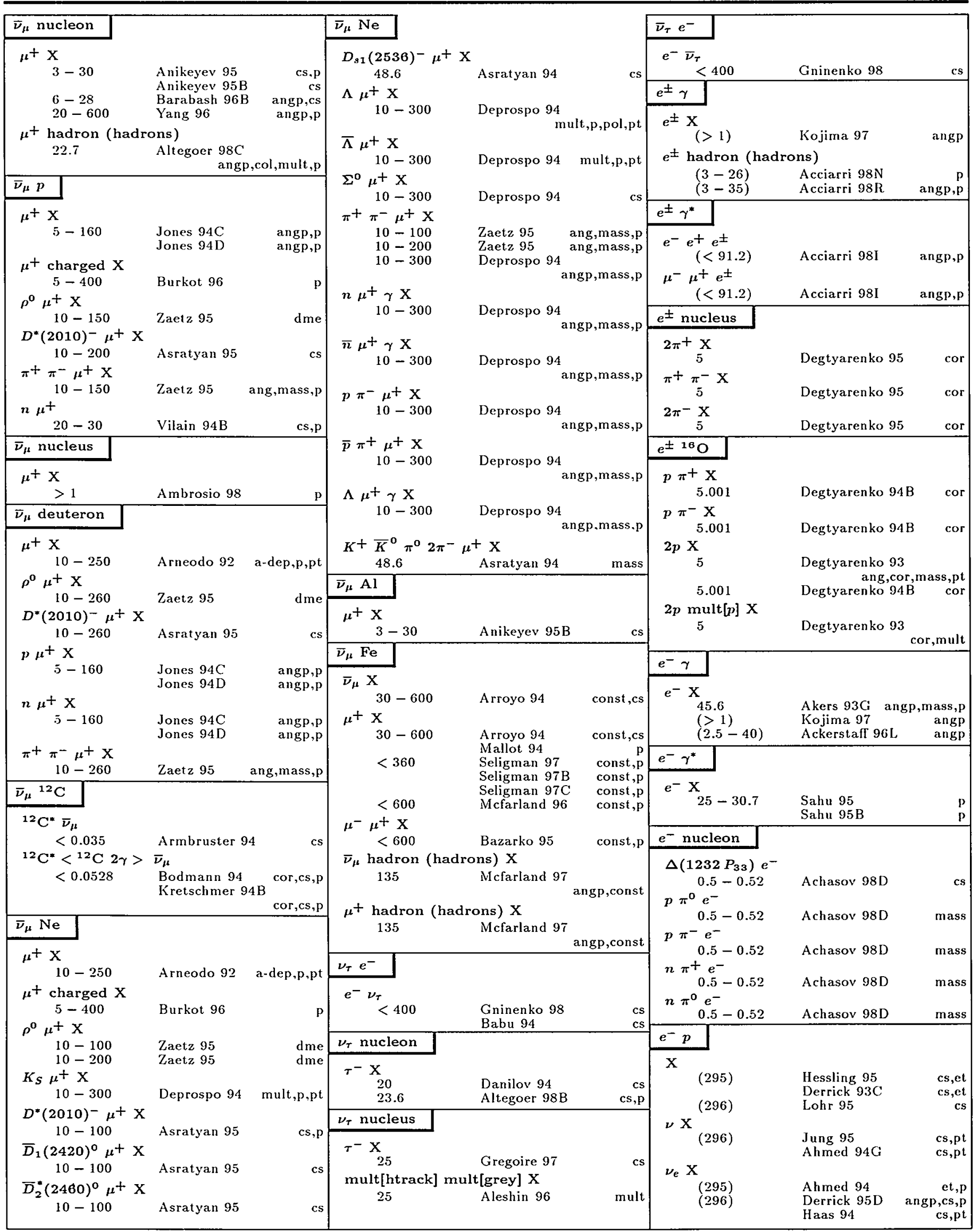




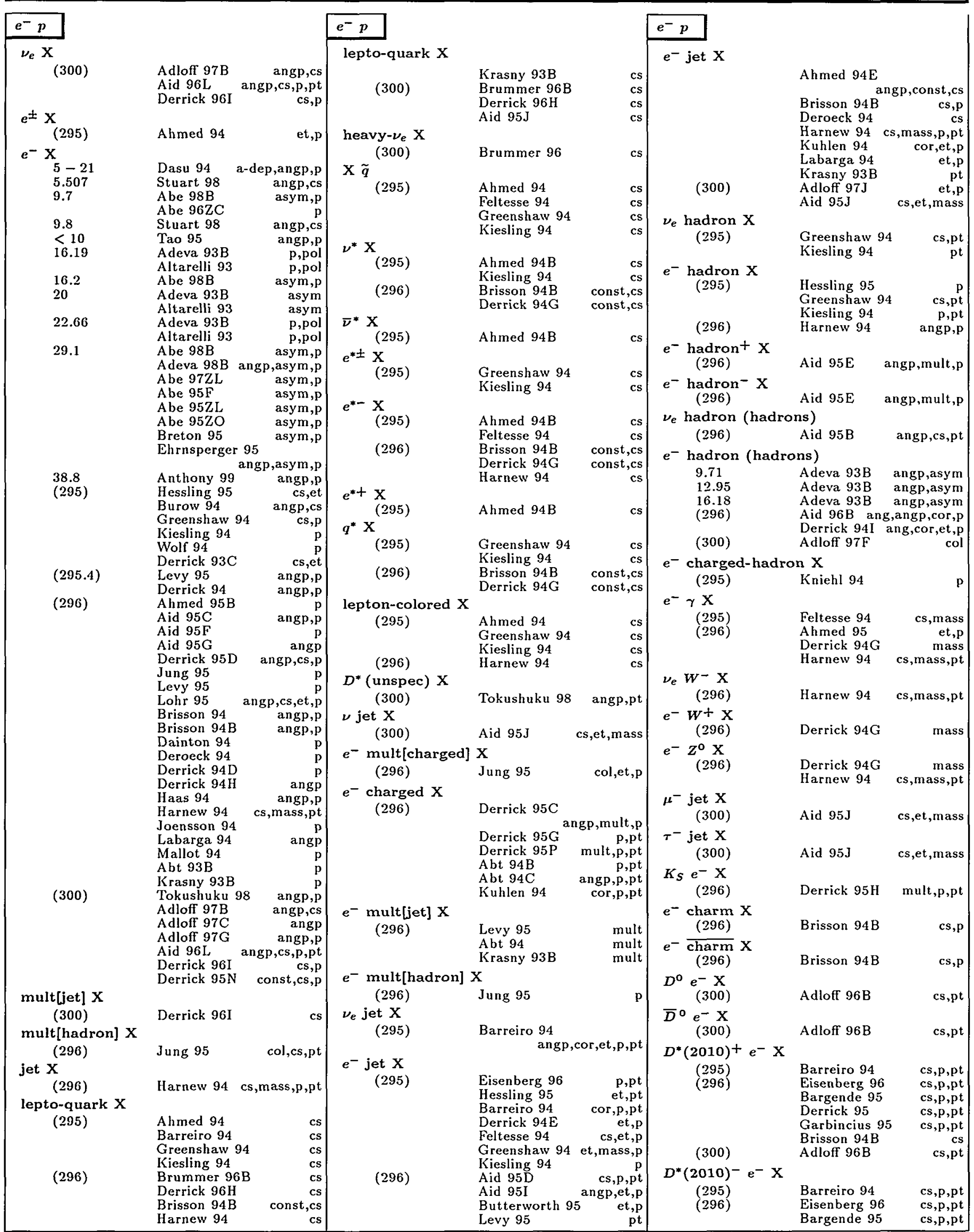

Entries are in order of beam name, then target name, then multiplicity of final state. Particle names are ordered as described in the legend on page 157 and as listed in the Particle Vocabulary. See also the Table of Contents of this Index beginning on the page 158. A few chemical symbols for nuclei have been changed to avoid ambiguity with particle names (see the Particle Vocabulary). Beam momenta are $p$ lab in GeV/c, or in parentheses $E_{\mathrm{cm}}$ in $\mathrm{GeV}$. 


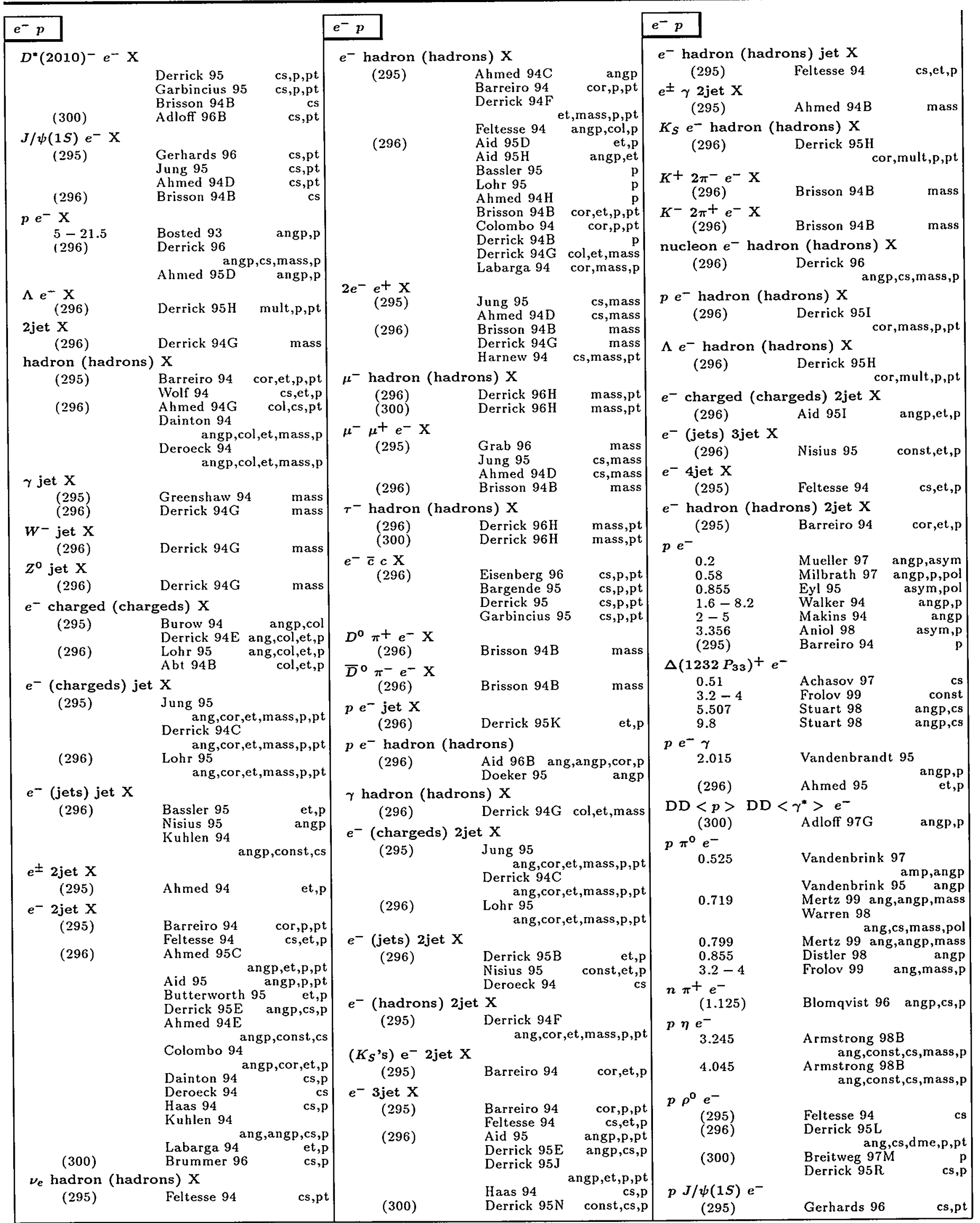


$e^{-3} \mathrm{He} \rightarrow 3 p \pi^{-} e^{-}$

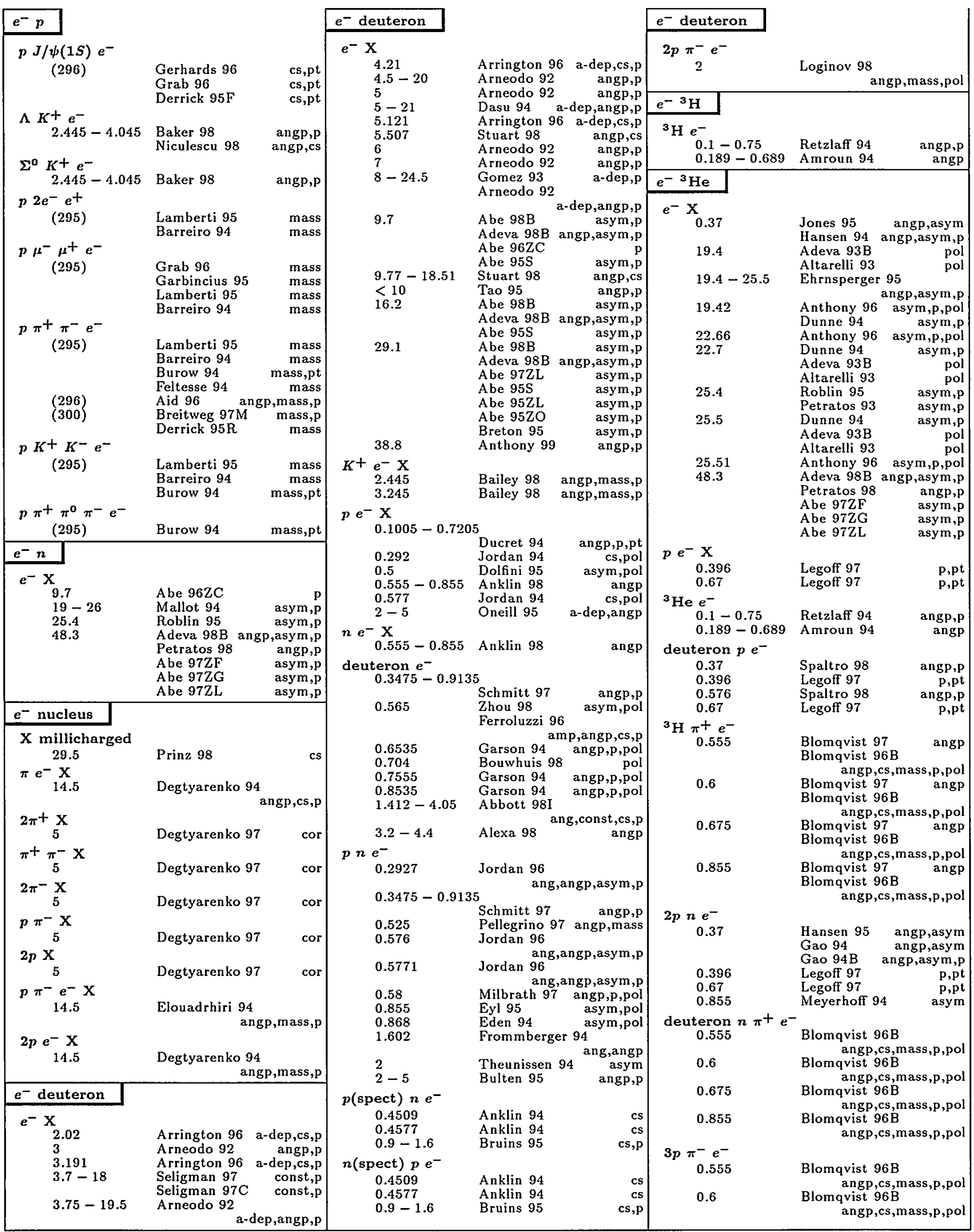

Entries are in order of beam name, then target name, then multiplicity of final state. Particle names are ordered as described in the legend on page 157 and as listed in the Particle Vocabulary. See also the Table of Contents of this Index beginning on the page 158 . A few chemical symbols for nuclei have been changed to avoid ambiguity with particle names (see the Particle Vocabulary). Beam momenta are $p_{\text {lab }}$ in GeV/c, or in parentheses $E_{\mathrm{cm}}$ in $\mathrm{GeV}$. 


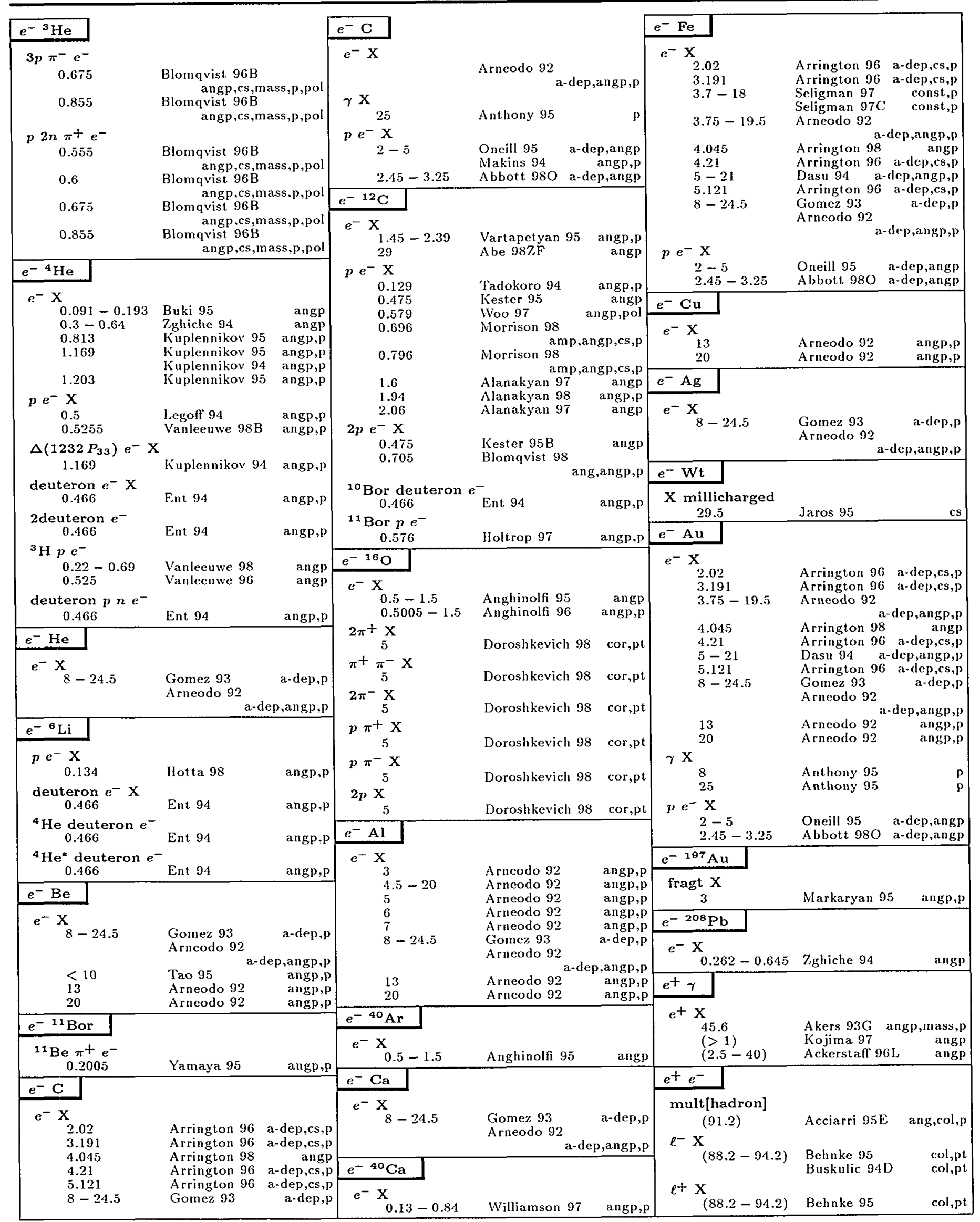




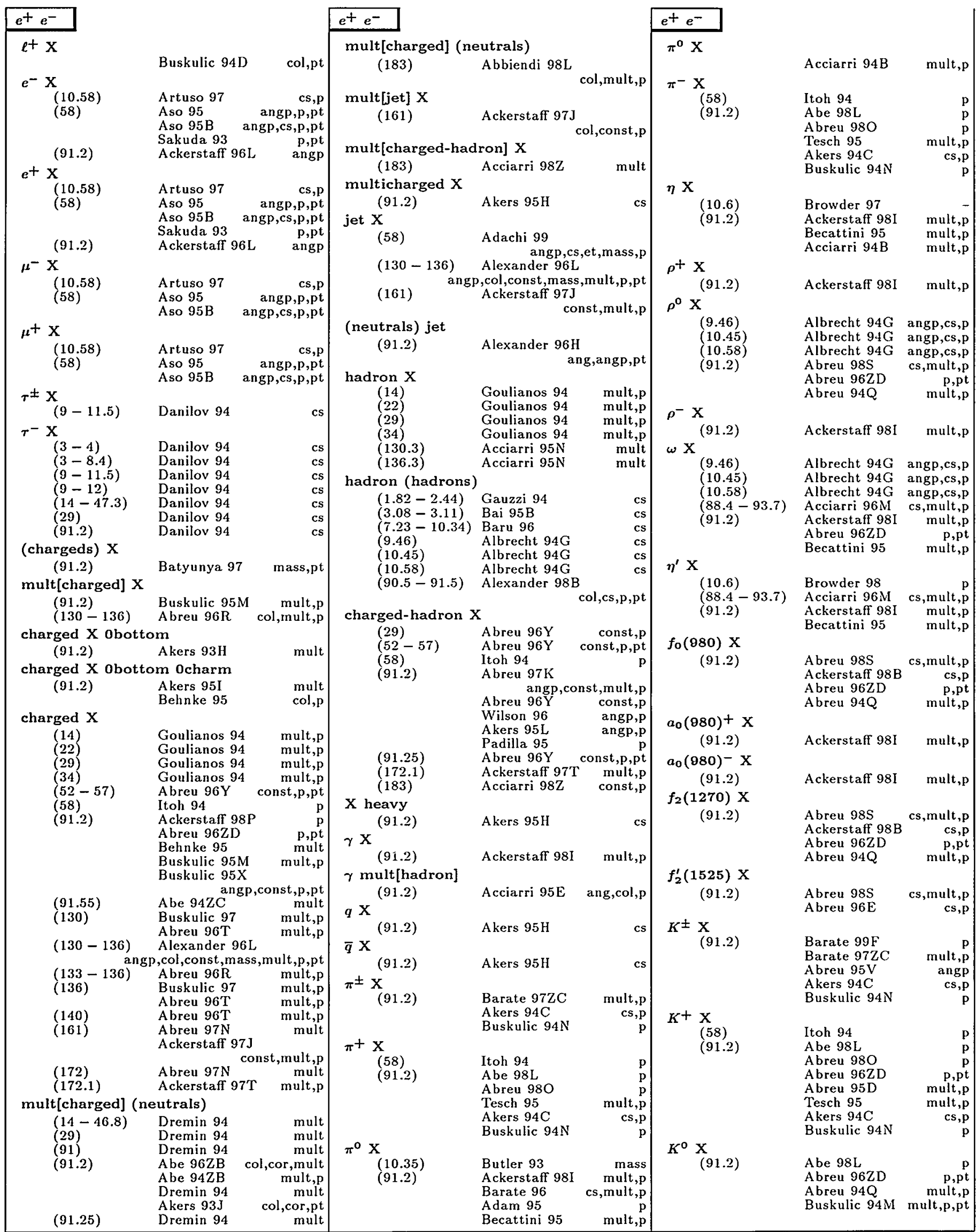

Entries are in order of beam name, then target name, then multiplicity of final state. Particle names are ordered as described in the legend on page 157 and as listed in the Particle Vocabulary. See also the Table of Contents of this Index beginning on the page 158. A few chemical symbols for nuclei have been changed to avoid ambiguity with particle names (see the Particle Vocabulary). Beam momenta are $p_{\text {lab }}$ in GeV/c, or in parentheses $E_{c m}$ in $\mathrm{GeV}$. 


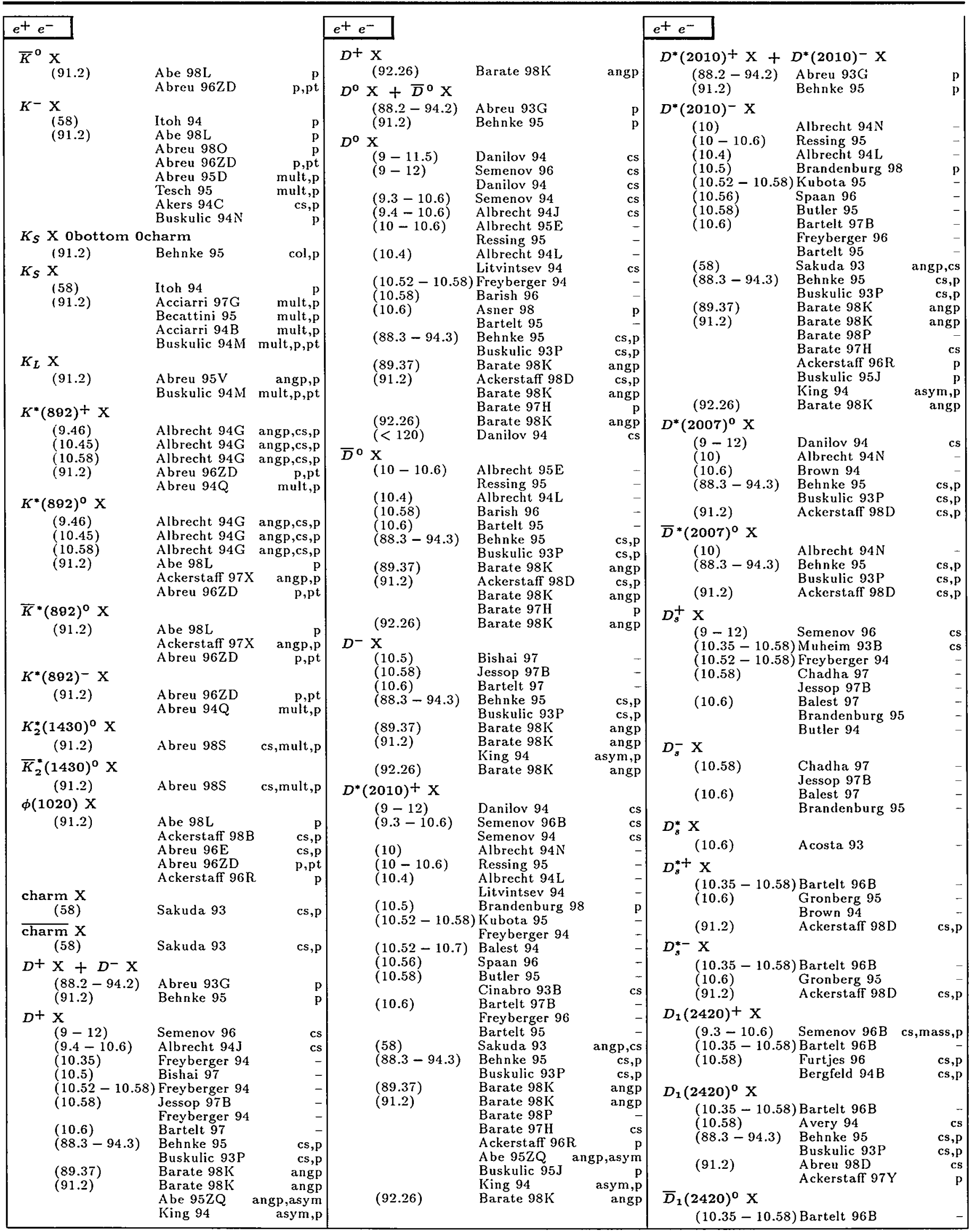




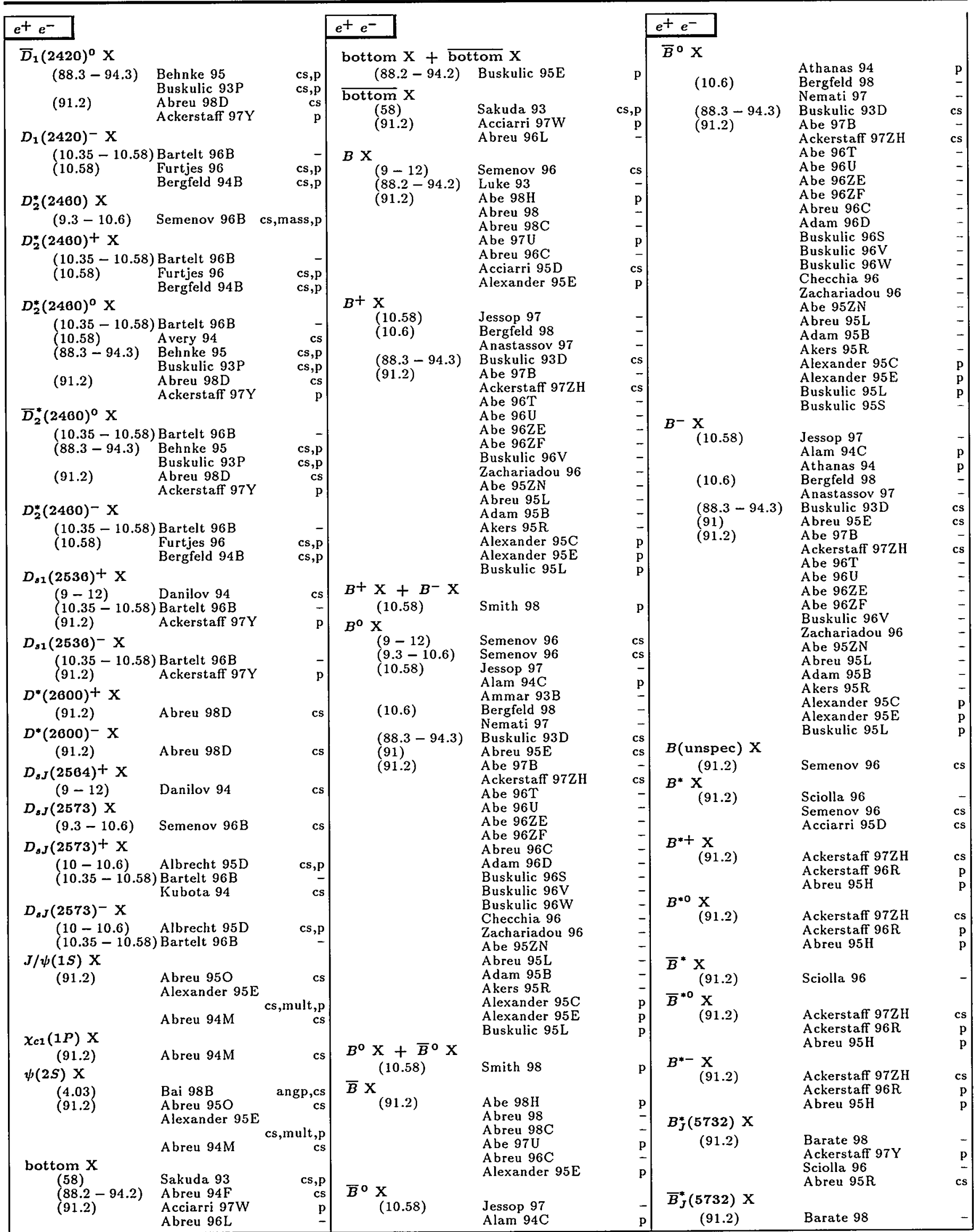

Entries are in order of beam name, then target name, then multiplicity of final state. Particle names are ordered as described in the legend on page 157 and as listed in the Particle Vocabulary. See also the Table of Contents of this Index beginning on the page 158. A few chemical symbols for nuclei have been changed to avoid ambiguity with particle names (see the Particle Vocabulary). Beam momenta are plab in GeV/c, or in parentheses $E_{\mathrm{cm}}$ in $\mathrm{GeV}$. 


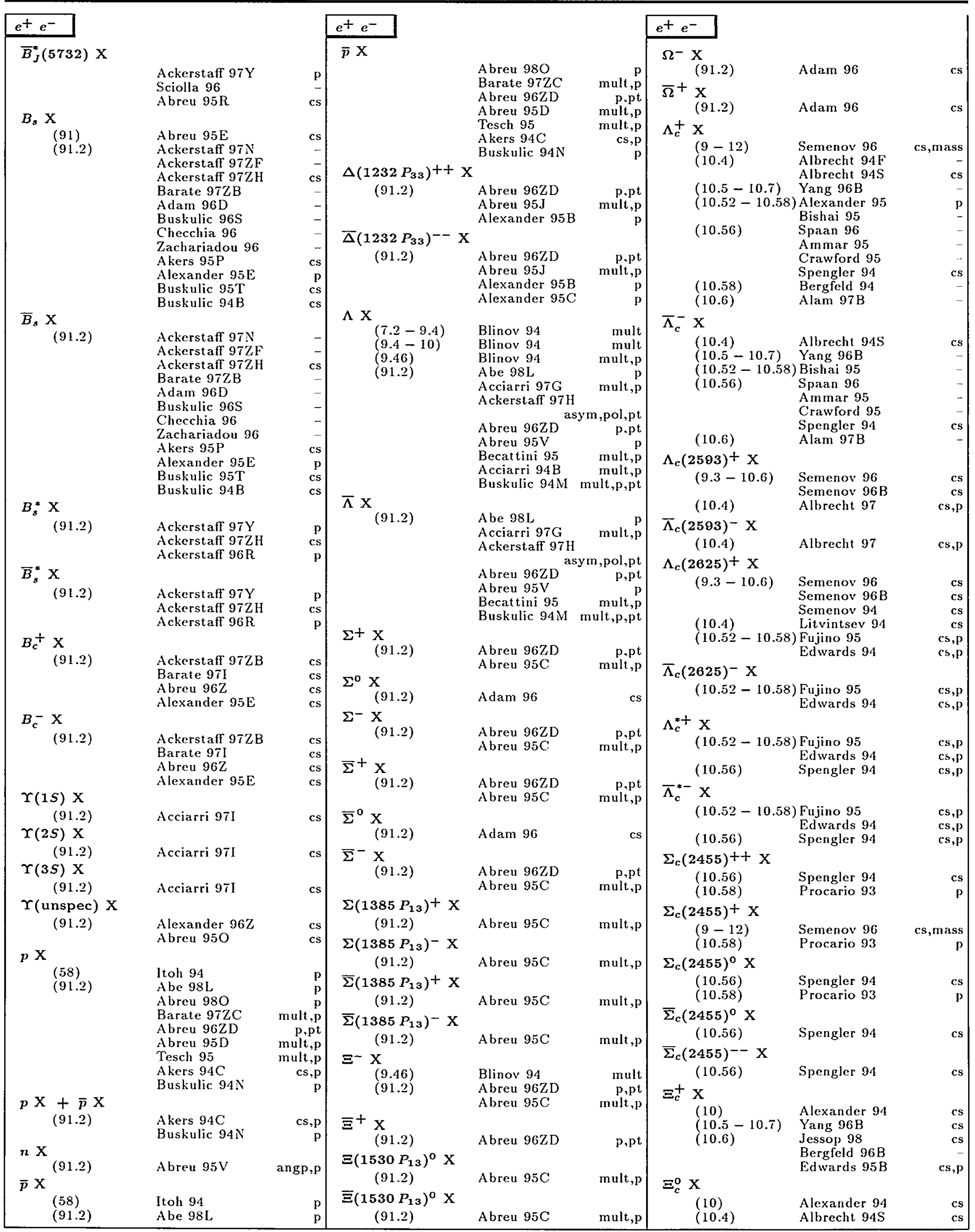




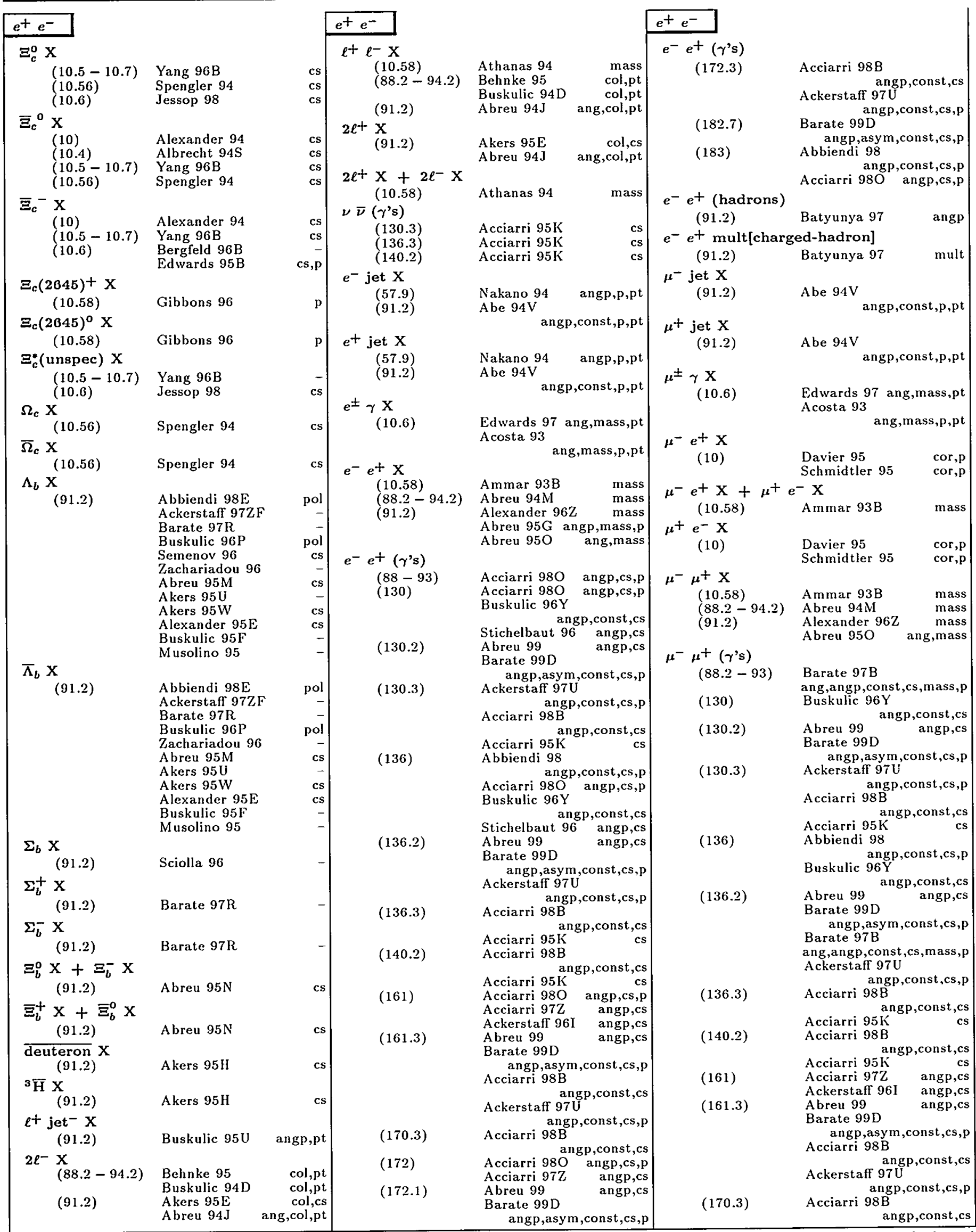

Entries are in order of beam name, then target name, then multiplicity of final state. Particle names are ordered as described in the legend on page 157 and as listed in the Particle Vocabulary. See also the Table of Contents of this Index beginning on the page 158 . A few chemical symbols for nuclei have been changed to avoid ambiguity with particle names (see the Particle Vocabulary). Beam momenta are plab in GeV/c, or in parentheses $E_{\mathrm{cm}}$ in $\mathrm{GeV}$. 
$e^{+} e^{-} \rightarrow \mu^{-} \mu^{+}\left(\gamma^{\prime} \mathrm{s}\right)$

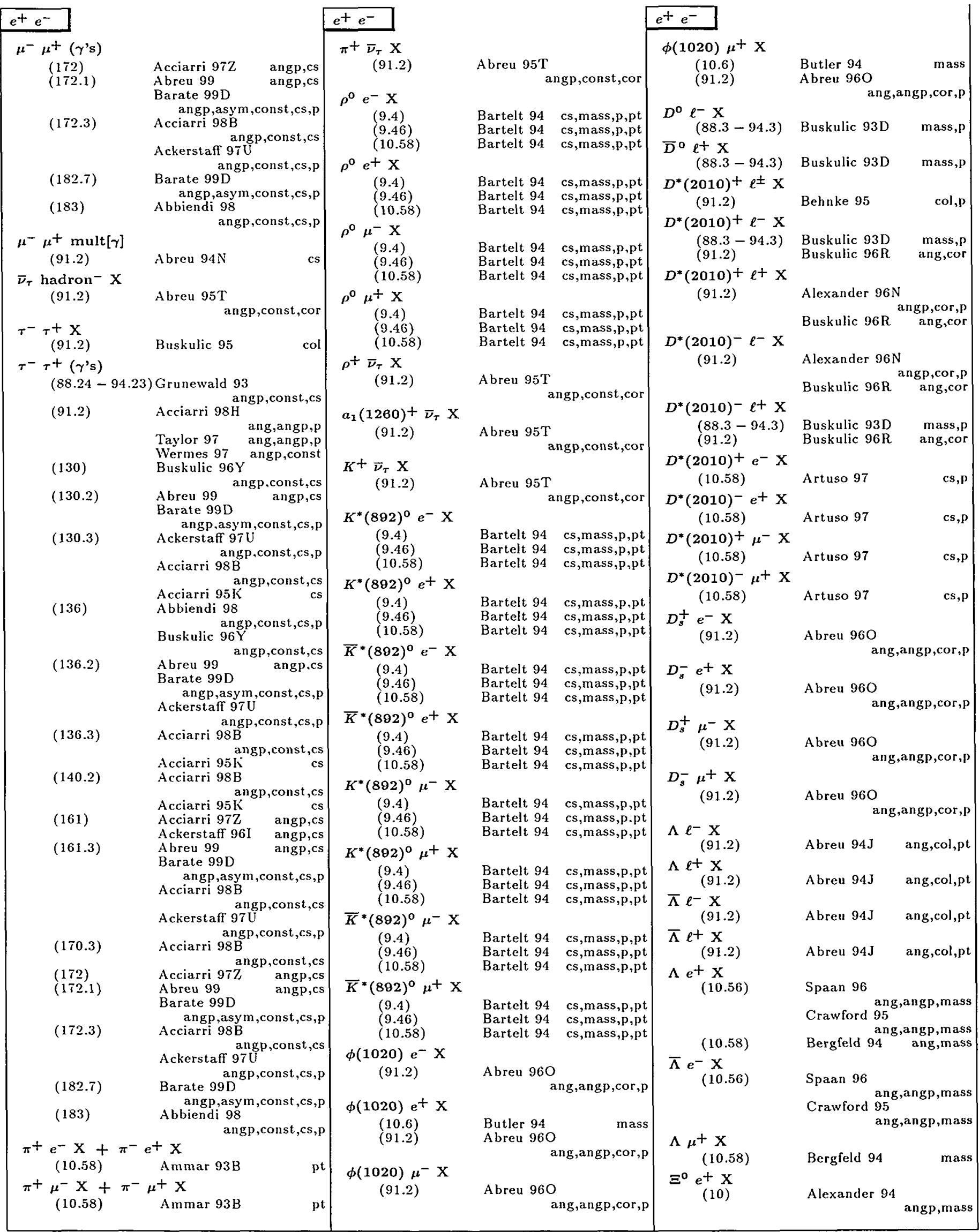




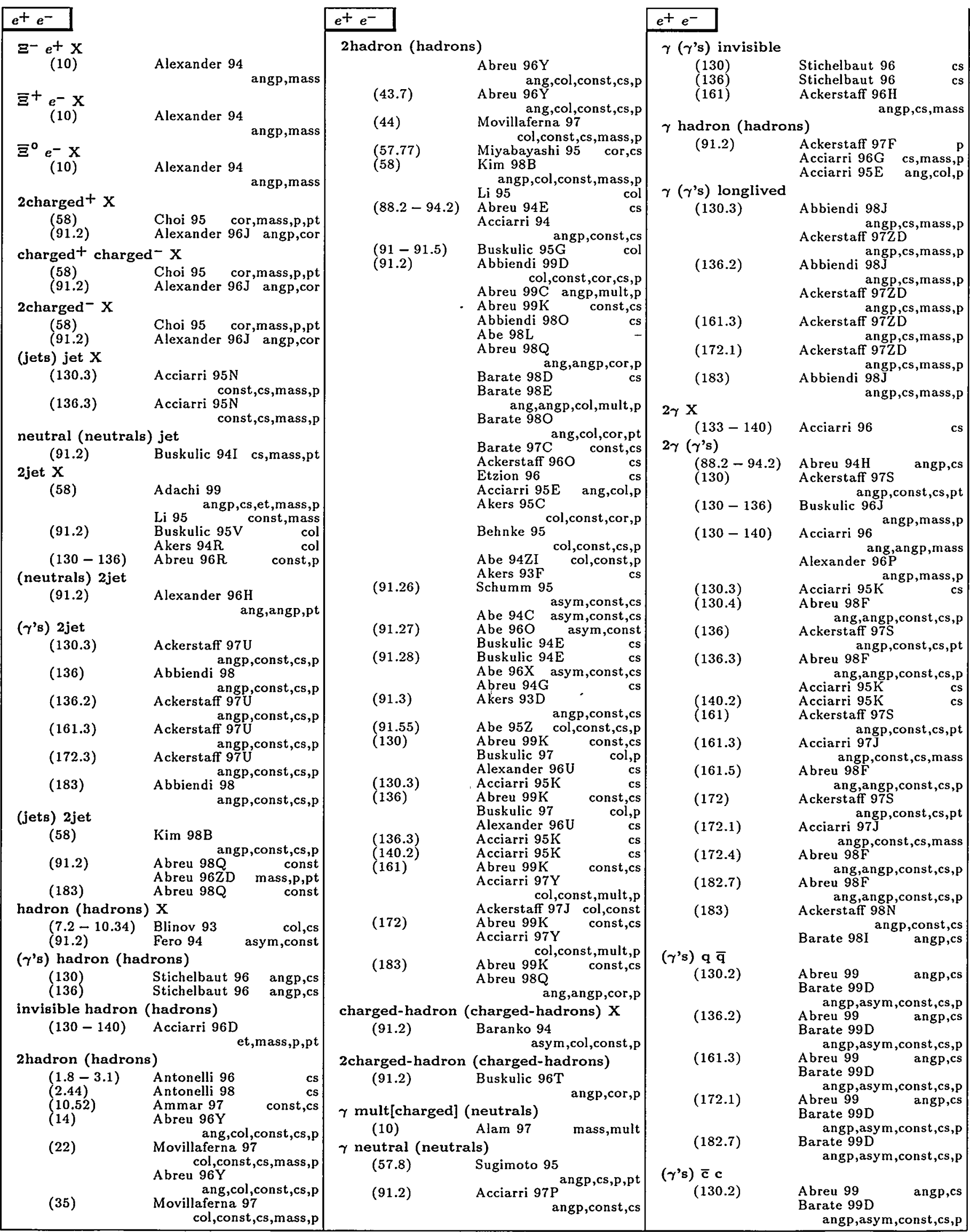

Entries are in order of beam name, then target name, then multiplicity of final state. Particle names are ordered as described in the legend on page 157 and as listed in the Particle Vocabulary. See also the Table of Contents of this Index beginning on the page 158 . A few chemical symbols for nuclei have been changed to avoid ambiguity with particle names (see the Particle Vocabulary). Beam momenta are $p_{l a b}$ in GeV/c, or in parentheses $E_{\mathrm{cm}}$ in $\mathrm{GeV}$. 


\begin{tabular}{|c|c|c|c|c|c|c|c|}
\hline \multirow{2}{*}{$\begin{array}{r}e^{+} e^{-} \\
\left(\gamma^{\prime} \mathrm{s}\right) \overline{\mathrm{c}} \mathrm{c} \\
(136.2)\end{array}$} & & \multicolumn{3}{|l|}{$e^{+} e^{-}$} & \multicolumn{3}{|c|}{$e^{+} e^{-}$} \\
\hline & & $2 \eta \times(10.58)$ & & $\mathrm{cs}, \mathrm{mass}$ & $K^{\mathbf{0}} \underset{(10.58)}{\omega}$ & Smith 98 & cs,mass \\
\hline & Barate $99 \mathrm{D}$ angp, cs & $\rho^{0} \pi^{-X}$ & & & $K^{+} \eta^{\prime} \times$ & & 年, \\
\hline$(161.3)$ & $\begin{array}{rr}\text { angp,asym,const,cs,p } \\
\text { Abreu } 99\end{array}$ & (91) & Abreu $95 \mathrm{E}$ & mass & $(10.58)$ & Smith 98 & $\mathrm{cs}, \mathrm{mass}$ \\
\hline & $\begin{array}{l}\text { Barate 99D } \\
\quad \text { angp,asym,const,cs,p }\end{array}$ & $\rho^{+} \eta \mathrm{X}$ & & & $\begin{array}{l}K^{0} \eta^{\prime} \mathrm{X} \\
(10.58)\end{array}$ & & \\
\hline$(172.1)$ & Abreu 99 angp,cs & $\rho^{0} \eta \mathrm{X}$ & Smith 98 & $\mathrm{cs}$, mass & $K+a_{1}(1260)-\mathrm{X}$ & smith 98 & cs, mass \\
\hline & $\begin{array}{l}\text { Barate } 99 \mathrm{D} \\
\text { angp,asym, const,cs,p }\end{array}$ & $(10.58)$ & Smith 98 & $\mathrm{cs}, \mathrm{mass}$ & $(91)$ & Abreu $95 \mathrm{E}$ & mass \\
\hline$(182.7)$ & $\begin{array}{l}\text { Barate } 99 \mathrm{D} \\
\quad \text { angp,asym,const,cs,p }\end{array}$ & $\omega \pi^{+} \underset{(10.58)}{\mathrm{X}}$ & Smith 98 & $\mathrm{cs}, \mathrm{mass}$ & $2 K+\underset{(91.2)}{\mathrm{X}}$ & Abreu $96 \mathrm{II}$ & \\
\hline $\bar{b} b \times$ & & $\omega \pi^{0} \mathrm{X}$ & & & & angp & or,cs, mass \\
\hline$(91.2)$ & $\begin{array}{l}\text { Abreu } 95 \mathrm{M} \\
\text { Akers } 95 \mathrm{~W}\end{array}$ & $\omega n \mathrm{x}^{(10.58)}$ & Smith 98 & cs,mass & $K^{+} \underset{(91)}{K^{-} \mathrm{X}}$ & Abreu $95 \mathrm{E}$ & mass \\
\hline$\left(\gamma^{\prime} \mathrm{s}\right) \overline{\mathrm{b}} \mathrm{b}$ & & $(10.58)$ & Smith 98 & cs,mass & (91.2) & Abreu 9611 & manos \\
\hline$(130.2)$ & $\begin{array}{ll}\text { Abreu } 99 & \text { angp,cs } \\
\text { Barate 99D } & \end{array}$ & $\omega \rho^{+} \mathrm{X}$ & 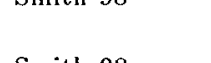 & cositasos & & $\begin{array}{r}\text { angp } \\
\text { Ackerstaff } 96\end{array}$ & $r, c s$, mass \\
\hline$(130.3)$ & $\begin{array}{l}\text { angp,asym,const,cs,p } \\
\text { Ackerstaff } 97 \mathrm{U}\end{array}$ & $\omega \rho^{\circ} \mathrm{X}^{(10.58)}$ & Smith 98 & $\mathrm{cs}$, mass & & Akers 95J & $\begin{array}{r}\text { engp, mass } \\
\text { cs,p }\end{array}$ \\
\hline (136) & 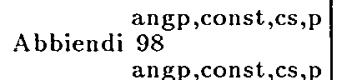 & $2 \omega \mathrm{X}^{(10.58)}$ & Smith 98 & $\mathrm{cs}$, mass & $2 K^{-} \underset{(91.2)}{\mathrm{X}}$ & Abreu 96H & csmass \\
\hline$(136.2)$ & $\begin{array}{cr}\text { Abreu } 99 & \text { angp,cs } \\
\text { Barate } 99 \mathrm{D} & \\
\text { angp,asym,const,cs,p } \\
\text { Ackerstaff } 97 \mathrm{U} \\
\text { angp,const,cs,p }\end{array}$ & $\begin{array}{l}\eta^{\prime} \pi^{+} \mathrm{X} \\
(10.58) \\
\eta^{\prime} \pi^{\circ} \mathrm{X}\end{array}$ & $\begin{array}{l}\text { Smith } 98 \\
\text { Smith } 98\end{array}$ & $\mathrm{cs}$, mass & $2 K_{S} \underset{(91.2)}{\mathrm{X}}$ & $\begin{array}{l}\text { angp, } \\
\text { Abreu } 96 \mathrm{H} \\
\text { angp,c } \\
\text { A kers } 95 \mathrm{~J}\end{array}$ & r,cs, mass \\
\hline$(161.3)$ & $\begin{array}{l}\text { Abreu } 99 \\
\text { Barate } 99 \mathrm{D} \\
\text { angp,asym,const,cs,p } \\
\text { Ackerstaff } 97 \mathrm{U}\end{array}$ & $\begin{array}{c}(10.58) \\
\eta^{\prime} \rho^{+} \mathrm{X} \\
(10.58)\end{array}$ & $\begin{array}{l}\text { Smith } 98 \\
\text { Smith } 98\end{array}$ & cs,mass & & $\begin{array}{r}\text { const,cor,cs, m } \\
\text { Schwiening } 95 \\
\text { Buskulic 94M } \\
\text { ang, }\end{array}$ & $\begin{array}{r}\text { cor, }, \mathrm{p} \\
\text { or, mult, } \mathrm{p}\end{array}$ \\
\hline$(172.1)$ & $99 \begin{array}{r}\text { angp,const,cs,p } \\
\text { angp,cs }\end{array}$ & $\eta^{\prime} p^{0} \mathrm{X}$ & & & $(92.2$ & $\begin{array}{l}\text { Maur 94 } \\
\text { Abreu 94C }\end{array}$ & $\begin{array}{r}\text { cor, } \mathrm{p} \\
\text { cor, mass }\end{array}$ \\
\hline & $\begin{array}{l}\text { Barate 99D } \\
\text { ang asym,const, } \mathrm{cs}, \mathrm{p}\end{array}$ & $\eta^{\prime} \omega \mathrm{X}^{(10.58)}$ & Smith 98 & $\mathrm{cs}$,mass & $\bar{K}^{*}(892)^{0} \pi^{-} \mathrm{X}$ & & \\
\hline$(172.3)$ & Ackerstaff $97 \mathrm{U}$ & $2 n^{\prime} \mathrm{x}^{(10.58)}$ & Smith 98 & cs,mass & $(91)$ & Abreu $95 \mathrm{E}$ & mass \\
\hline$(182.7)$ & $\begin{array}{l}\text { Barate } 99 \mathrm{D} \\
\text { angp, asym,const,cs,p }\end{array}$ & $2 \eta^{\prime} \times(10.58)$ & Smith 98 & $\mathrm{cs}$, mass & $\begin{array}{c}(10.58) \\
0.48\end{array}$ & Smith 98 & cs,mass \\
\hline$(183)$ & $\begin{array}{r}\text { Abbiendi } 98 \\
\text { angp, const, cs, } \mathrm{p}\end{array}$ & $K^{ \pm} \underset{(10.6)}{\text { (neutrals) }} \mathrm{X}$ & Battle 94 & mass & $\begin{array}{c}K^{*}(892)^{0} \eta \mathrm{X} \\
(10.58)\end{array}$ & Smith 98 & cs,mass \\
\hline$\pi^{0} \underset{(10.6)}{\text { charged }}+\mathrm{X}$ & Urheim 94 & $K^{ \pm} \pi_{(10.6)}^{0} \mathrm{X}$ & Battle 94 & mass & $\begin{array}{c}K^{*}(882)^{+} \omega \mathrm{X} \\
(10.58)\end{array}$ & Smith 98 & cs,mass \\
\hline$\pi^{0} \underset{(10.6)}{\text { charged }^{-} \mathrm{X}}$ & Urheim 94 & $K^{+} \pi^{-} \mathrm{X}$ & $\begin{array}{l}\text { Albrecht } 94 \mathrm{G} \\
\text { Albrecht } 94 \mathrm{~N}\end{array}$ & mass & $\begin{array}{c}K^{*}(892)^{\circ} \omega \mathrm{X} \\
(10.58)\end{array}$ & Smith 98 & cs,mass \\
\hline $\begin{array}{c}\pi^{+} \text {jet } \mathrm{X} \\
(91.2)\end{array}$ & Barate $97 \mathrm{H}$ & $(10.4)$ & $\begin{array}{r}\text { Albrecht } 94 \mathrm{~N} \\
\text { ang }\end{array}$ & $\begin{array}{r}\text { mass } \\
p, \text { mass }, p\end{array}$ & $\begin{array}{c}K^{*}(892)+\eta^{\prime} \mathrm{X} \\
(10.58)\end{array}$ & Smith 98 & $\mathrm{cs}, \mathrm{mass}$ \\
\hline $\begin{array}{l}\pi^{-} \text {jet } \mathrm{X} \\
\quad(91.2) \\
2 \pi^{+} \mathrm{X}\end{array}$ & Barate $97 \mathrm{H}$ & $\begin{array}{l}(10.58) \\
(88.3-94.3) \\
(91)\end{array}$ & $\begin{array}{l}\text { Barish } 96 \\
\text { Behnke } 95 \\
\text { Buskulic 93P } \\
\text { Abreu } 95 \mathrm{E}\end{array}$ & $\begin{array}{r}\text { mass } \\
\text { mass,p } \\
\text { mass,p } \\
\text { mass }\end{array}$ & $\begin{array}{c}K^{*}(892)^{0} \eta^{\prime} \mathrm{X} \\
(10.58) \\
\phi(1020) \pi^{+} \mathrm{X}\end{array}$ & Smith 98 & $\mathrm{cs}, \mathrm{mass}$ \\
\hline $\begin{array}{l}(7.2-10.3) \\
(91.2)\end{array}$ & $\begin{array}{l}\text { Blinov } 95 \quad \text { cor,mass } \\
\text { Alexander } 96 \mathrm{~J} \\
\text { ang,cor,mass,p }\end{array}$ & $K^{-} \pi^{+} \mathrm{X}$ & Albrecht $94 N$ & mass & $\begin{array}{l}(10.58) \\
(10.6)\end{array}$ & $\begin{array}{l}\text { Smith } 98 \\
\text { Brown } 94\end{array}$ & $\begin{array}{r}\mathrm{cs}, \text { mass } \\
\text { mass }\end{array}$ \\
\hline$(91.28)$ & Abreu $94 \mathrm{~B}$ cor,mass & $(10.4)$ & $\begin{array}{l}\text { Albrecht } 94 \mathrm{~L} \\
\text { an }\end{array}$ & mass,p & $\phi(1020) \pi^{0} \mathrm{X}$ & & \\
\hline$(172)$ & $\begin{array}{l}\text { Abreu } 97 \mathrm{C} \\
\quad \text { angp,const,cor, mass }\end{array}$ & $(10.52-$ & $\begin{array}{l}\text { Litvintsev } 94 \\
\text { Balest } 94\end{array}$ & $\begin{array}{l}\text { mass } \\
\text { mass }\end{array}$ & $\begin{array}{l}(9.4-10.6) \\
(10.58)\end{array}$ & $\begin{array}{l}\text { Albrecht } 94 \mathrm{~J} \\
\text { Smith } 98\end{array}$ & $\begin{array}{l}\text { cs,mass } \\
\text { cs,mass }\end{array}$ \\
\hline$\pi^{+} \pi_{(9.46)}^{-\mathrm{X}}$ & it $94 \mathrm{G}$ & $(10.58)$ & $\begin{array}{l}\text { Barish } 96 \\
\text { Alam } 94 \mathrm{C}\end{array}$ & $\begin{array}{r}\text { mass } \\
\mathrm{cs}, \mathrm{mass}\end{array}$ & $\begin{array}{c}\phi(1020) \eta \mathrm{X} \\
(10.58)\end{array}$ & Smith 98 & cs,mass \\
\hline $\begin{array}{l}(10.35) \\
(91) \\
(91.2)\end{array}$ & $\begin{array}{ll}\text { Butler 93 } & \text { mass } \\
\text { Abreu 95E } & \text { mass } \\
\text { Alexander 96 } & \end{array}$ & $\begin{array}{l}(10.6) \\
(88.3-94.3)\end{array}$ & $\begin{array}{l}\text { Avery } 94 \\
\text { Brown } 94 \\
\text { Behnke } 95\end{array}$ & $\begin{array}{r}\text { mass } \\
\text { mass } \\
\text { mass, } \mathrm{p}\end{array}$ & $\begin{array}{c}\phi(1020) \rho^{+} \mathrm{X} \\
(10.58)\end{array}$ & Smith 98 & $\mathrm{cs}$, mass \\
\hline & ang,cor, mass, $p$ & & Buskulic 93P & mass, $p$ & $\phi(1020) \rho^{0} \mathrm{X}$ & & \\
\hline $\begin{array}{l}(91.28) \\
(91.3)\end{array}$ & $\begin{array}{c}\text { Abreu 94B } \quad \text { cor,mass } \\
\text { Abreu } 94 \mathrm{Q}\end{array}$ & $K_{S} \pi$ & & & $(9.4-10.6)$ & & cs, mass \\
\hline & $\begin{array}{l}\text { Abreu } 97 \mathrm{C} \\
\text { angp,const,cor,mass }\end{array}$ & $\begin{array}{l}(9.46) \\
(91.3)\end{array}$ & $\begin{array}{l}\text { Albrecht } 94 \mathrm{G} \\
\text { Abreu } 94 \mathrm{Q}\end{array}$ & $\begin{array}{r}\operatorname{mass} \\
\text { mass,p }\end{array}$ & rat & $\begin{array}{l}\text { Ressing 95 } \\
\text { Smith 98 }\end{array}$ & $\begin{array}{r}c s \\
\text { cs,mass }\end{array}$ \\
\hline $2 \pi^{-} \underset{(\tau .2-10.3)}{X}$ & Blinov 95 & $K_{S} \pi^{-\mathrm{X}}$ & Abreu 94Q & mass,p & $\begin{array}{l}\phi(1020) \omega X \\
(10-10.6)\end{array}$ & Ressing 95 & $\operatorname{cs}$ \\
\hline$(91.2)$ & $\begin{array}{c}\text { Alexander } 96 \mathrm{~J} \\
\text { ang,cor,mass,p }\end{array}$ & $K^{+} \eta \mathrm{X}$ & Smith 98 & cs,mass & $\begin{array}{c}\phi(1020) \eta^{\prime} \mathrm{X} \\
(10.58)\end{array}$ & Smith 98 & cs,mass \\
\hline $\begin{array}{l}(91.28) \\
(172)\end{array}$ & $\begin{array}{l}\text { Abreu } 94 \mathrm{~B} \quad \text { cor,mass } \\
\text { A breu } 97 \mathrm{C}\end{array}$ & $K^{0} \eta \mathrm{X}$ & 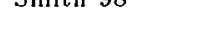 & cs, mass & $K^{+} \phi(1020) \mathrm{X}$ & & \\
\hline & angp,const,cor,mass & $(10.58)$ & Smith 98 & cs,mass & $(10.58)$ & Smith 98 & cs, mass \\
\hline$\eta \pi^{+}+\underset{(10.58)}{\mathrm{x}}$ & Smith 98 & $\begin{array}{r}K^{-} \\
\rho^{0} \mathrm{X} \\
(91)\end{array}$ & Abreu $95 \mathrm{E}$ & mass & $\begin{array}{c}K^{0} \phi(1020) \mathrm{X} \\
(10.58)\end{array}$ & Smith 98 & cs,mass \\
\hline$\eta \pi^{0} \underset{(10.58)}{X}$ & Smith 98 & $K+\underset{(10.58)}{\omega \mathrm{X}}$ & Smith 98 & $\mathrm{cs}$, mass & $\begin{array}{c}K^{-} \phi(1020) \mathrm{X} \\
(91)\end{array}$ & Abreu 95E & mass \\
\hline
\end{tabular}




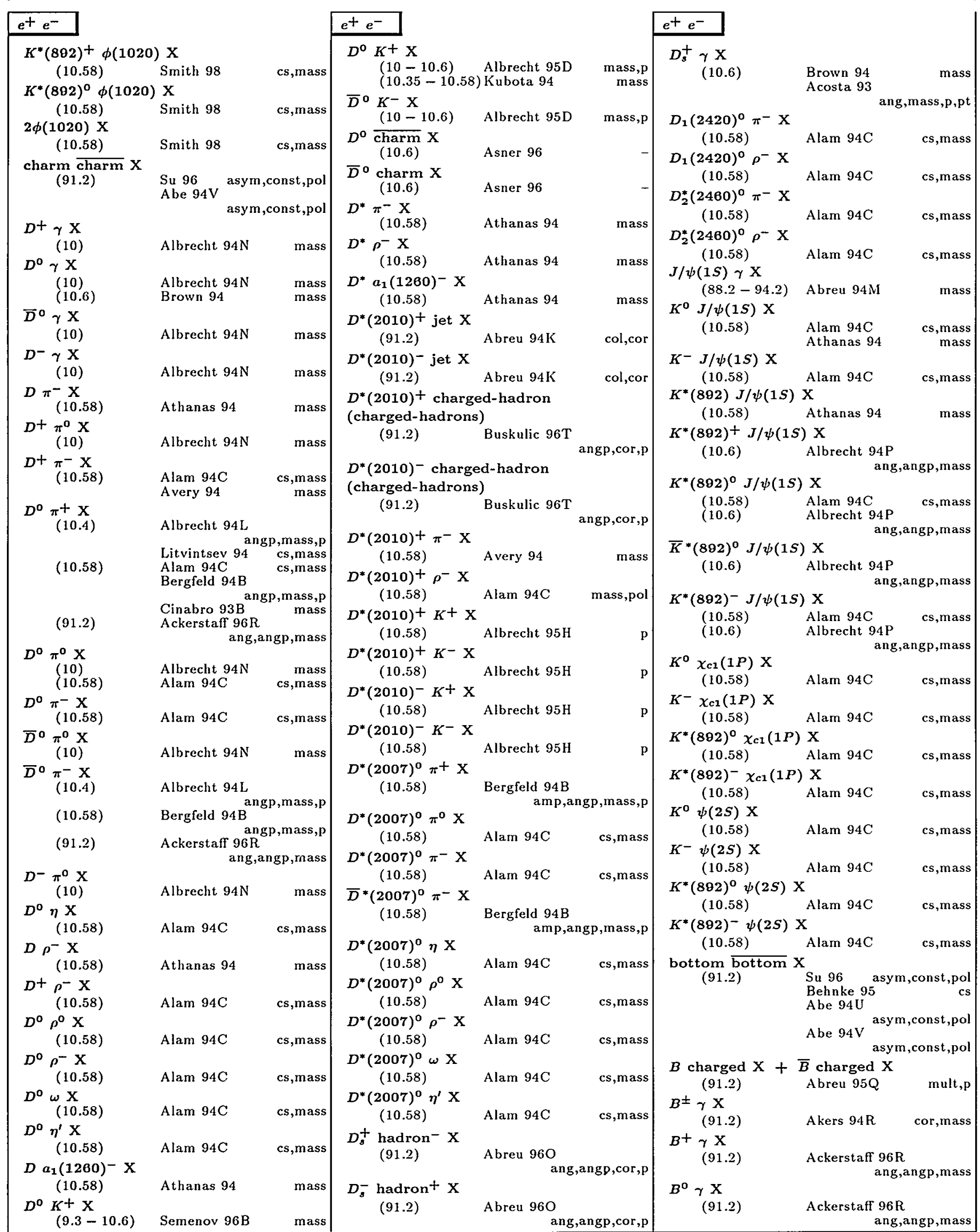

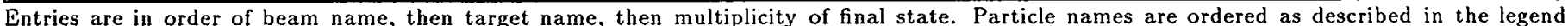

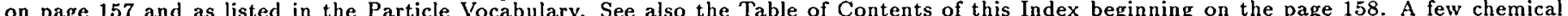

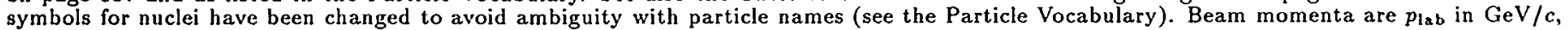
or in parentheses $E_{\mathrm{cm}}$ in $\mathrm{GeV}$. 


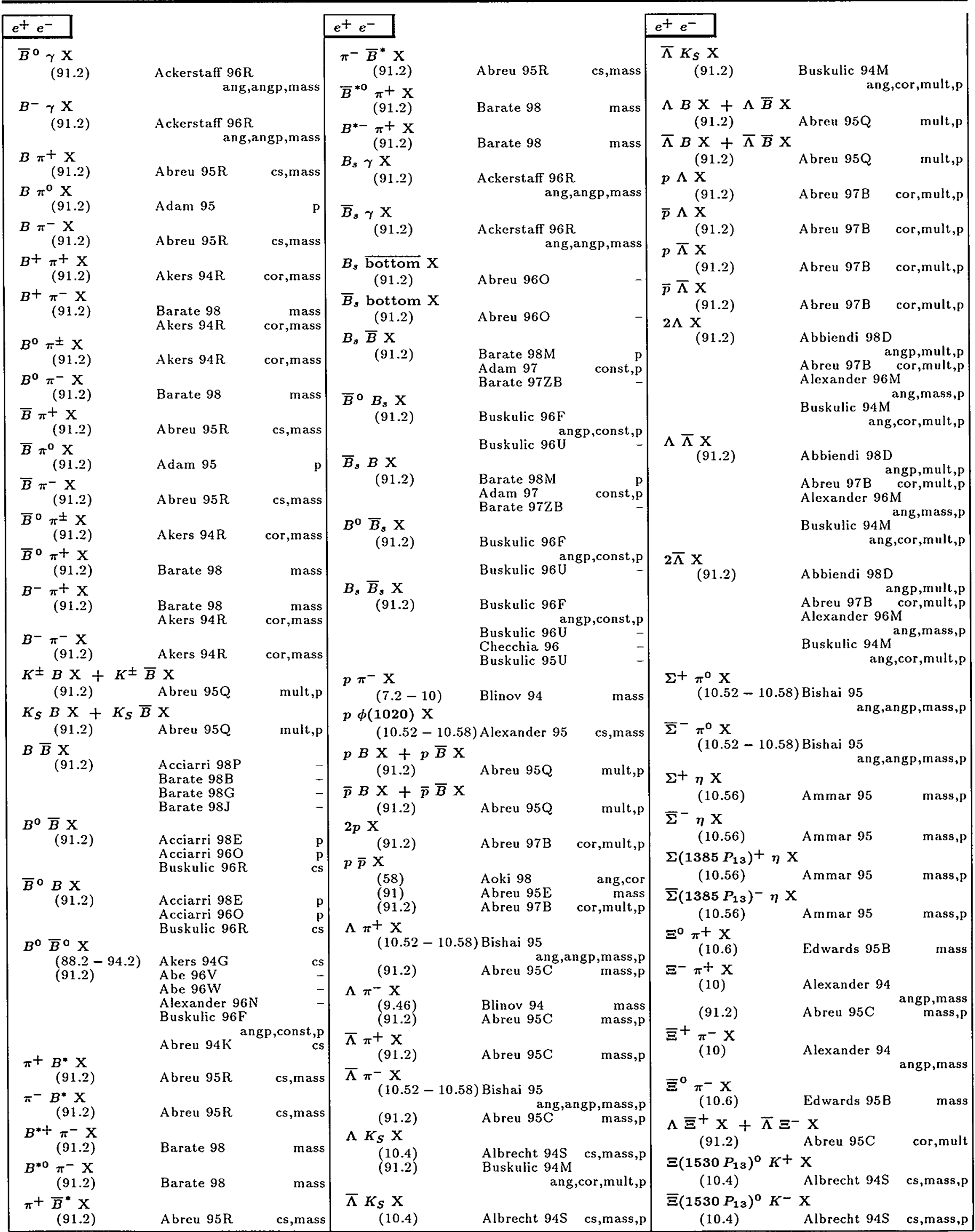




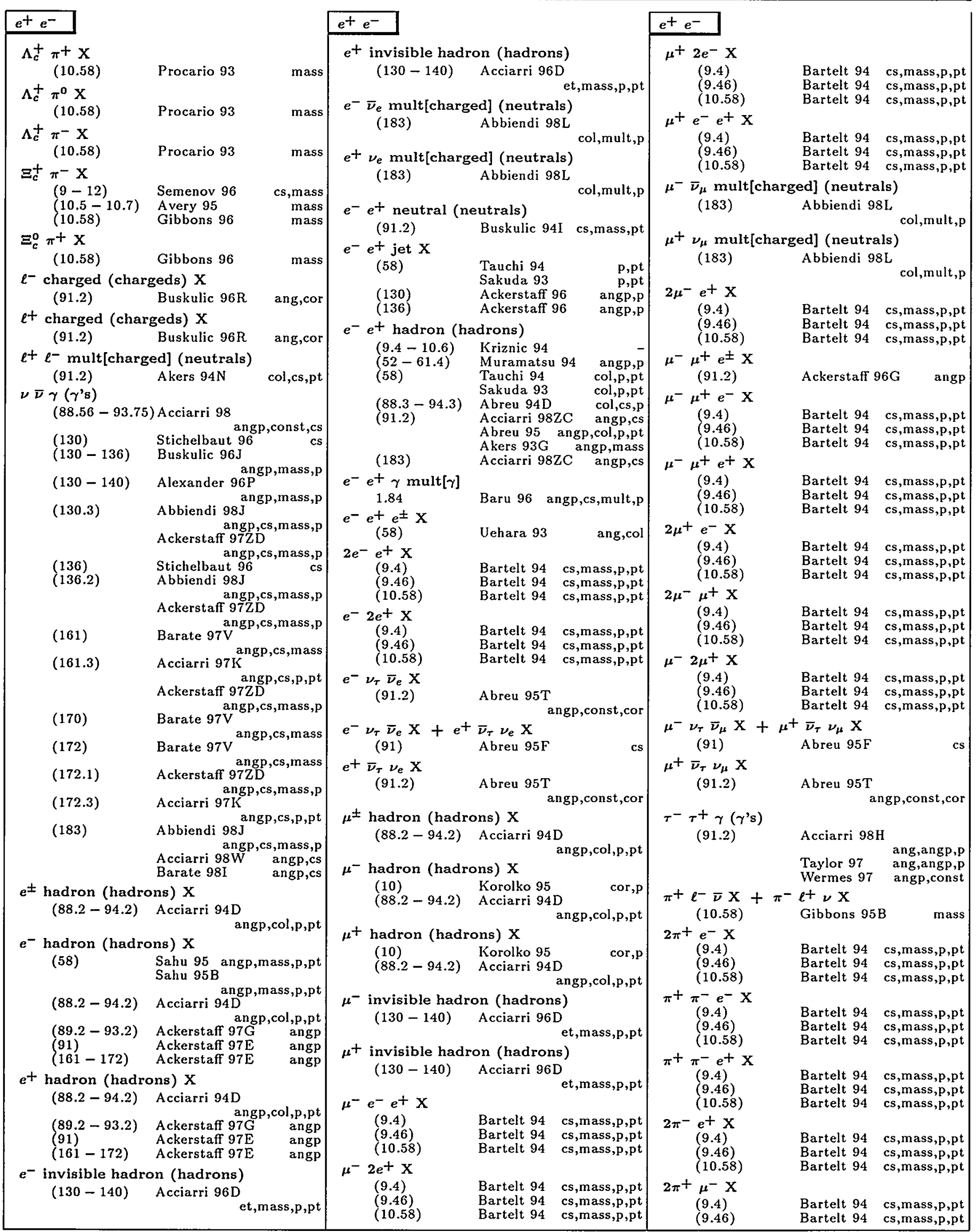

Entries are in order of beam name, then target name, then multiplicity of final state. Particle names are ordered as described in the legend on page 157 and as listed in the Particle Vocabulary. See also the Table of Contents of this Index beginning on the page 158. A few chemical symbols for nuclei have been changed to avoid ambiguity with particle names (see the Particle Vocabulary). Beam momenta are $p_{\text {lab }}$ in GeV/c, or in parentheses $E_{c m}$ in $\mathrm{GeV}$. 
$e^{+} e^{-} \rightarrow\left(\gamma^{\prime} s\right)$ 2hadron (hadrons)

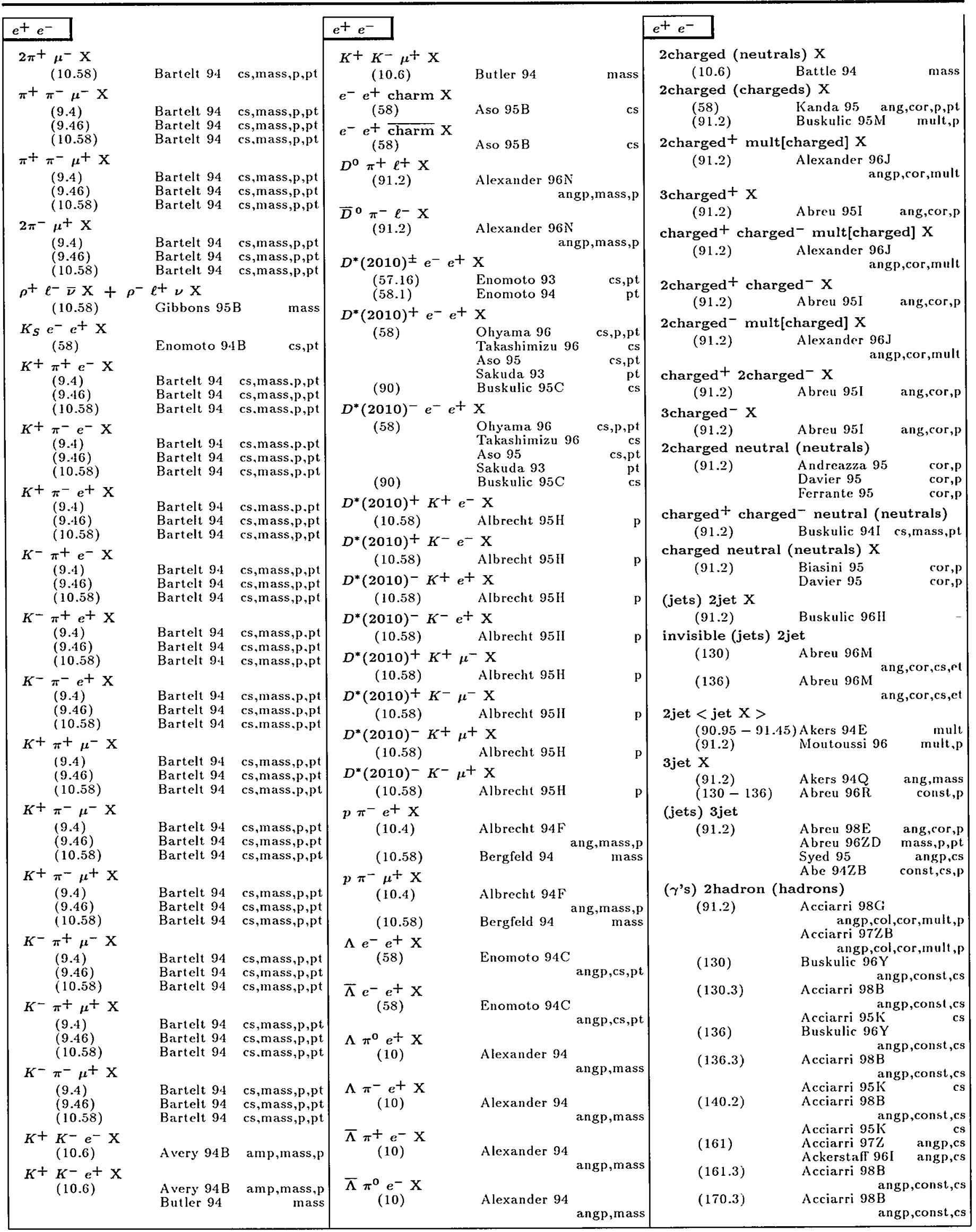




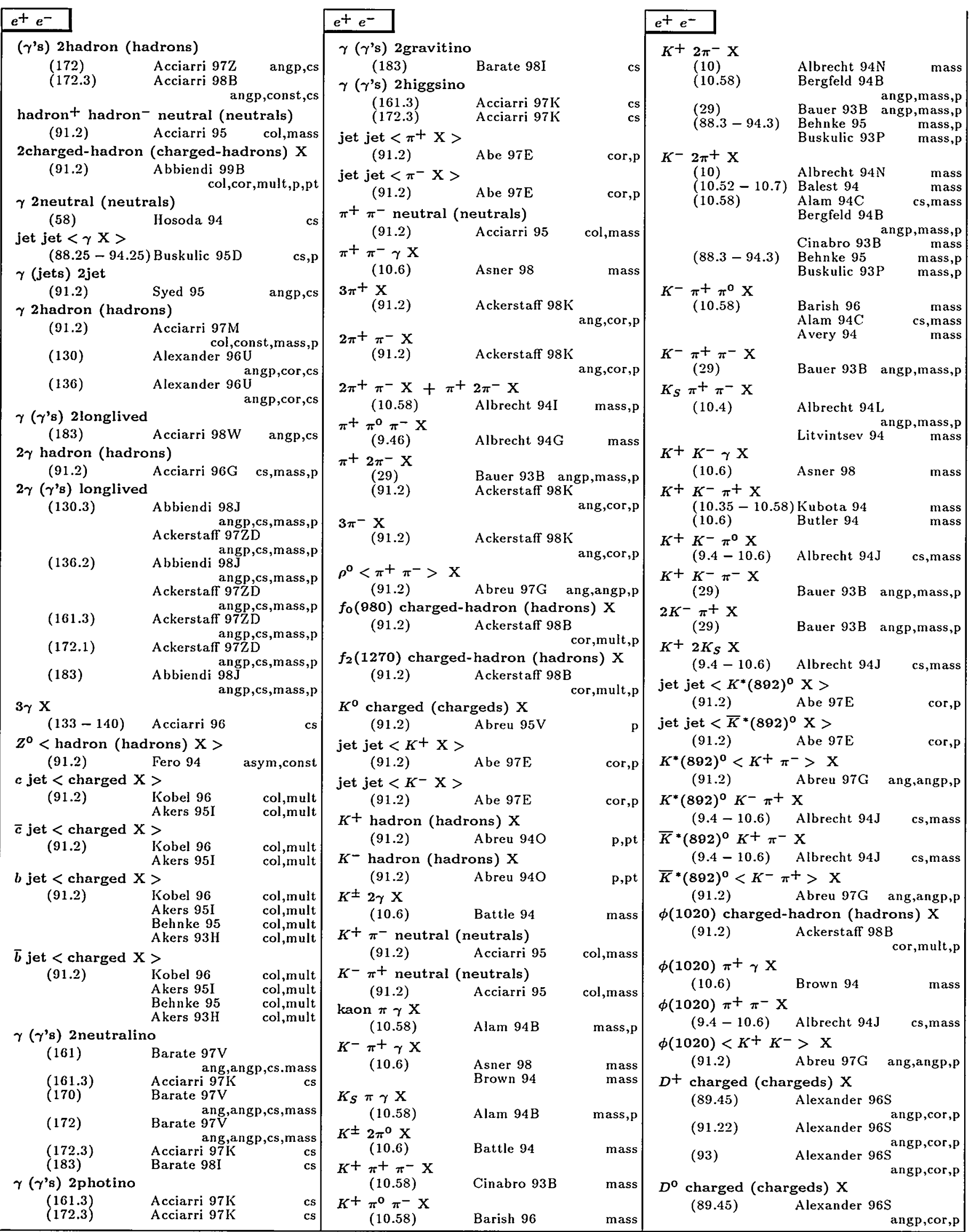




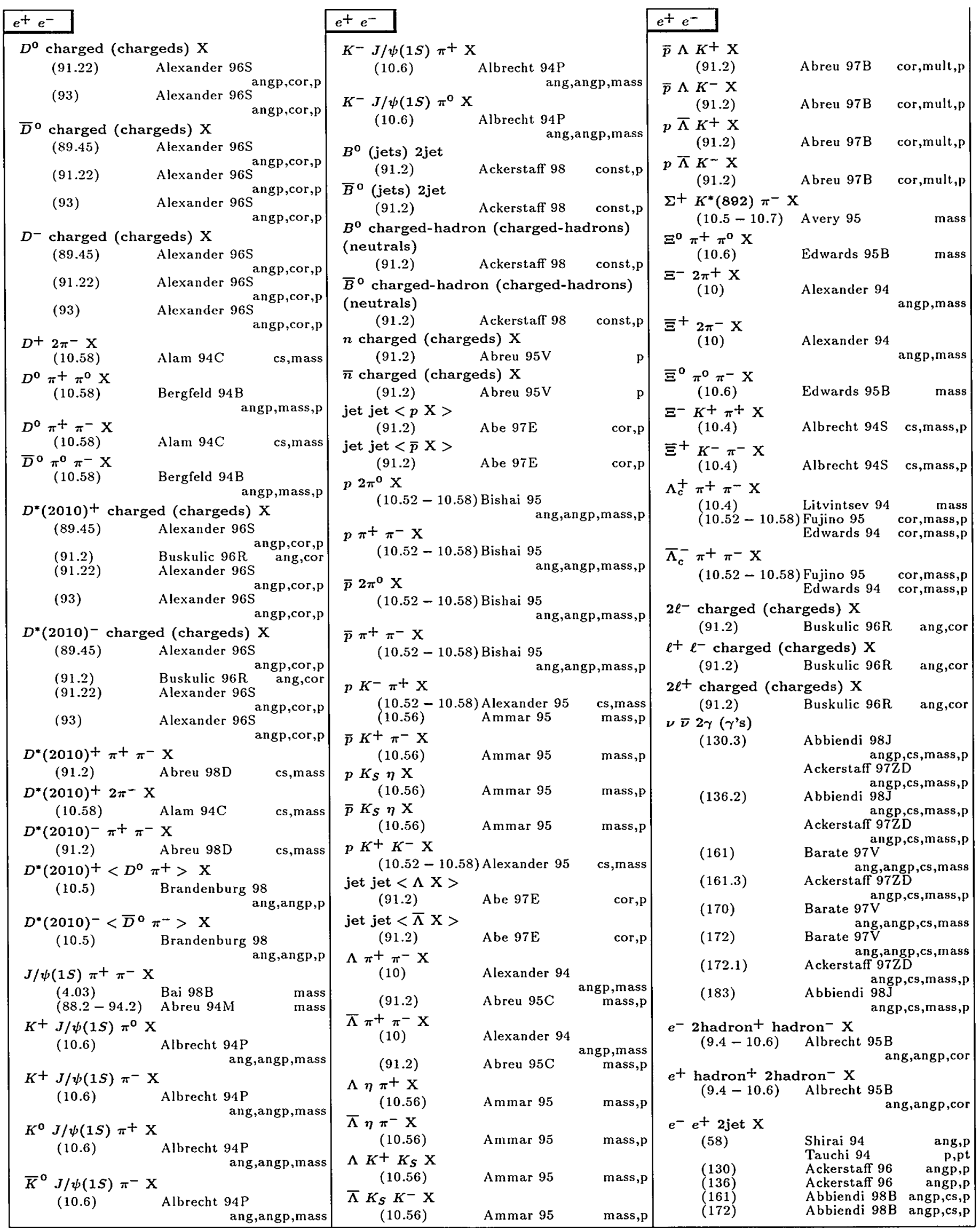




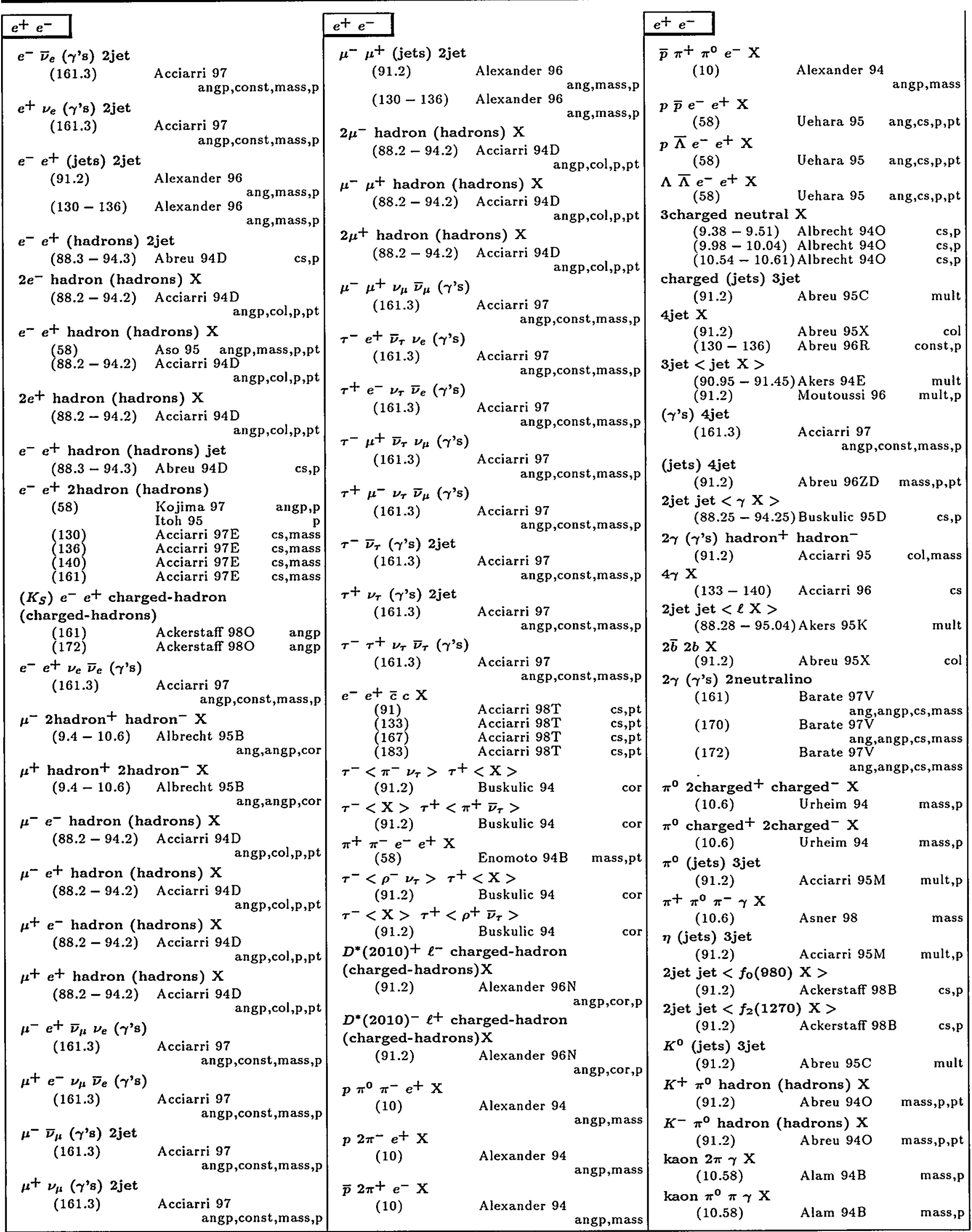

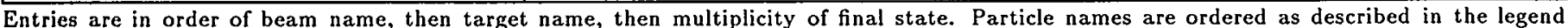

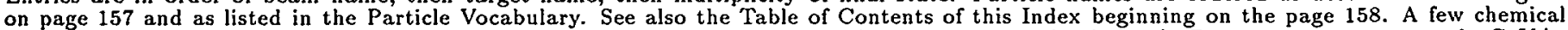

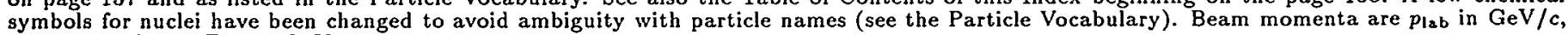
or in parentheses $E_{c m}$ in $\mathrm{GeV}$. 


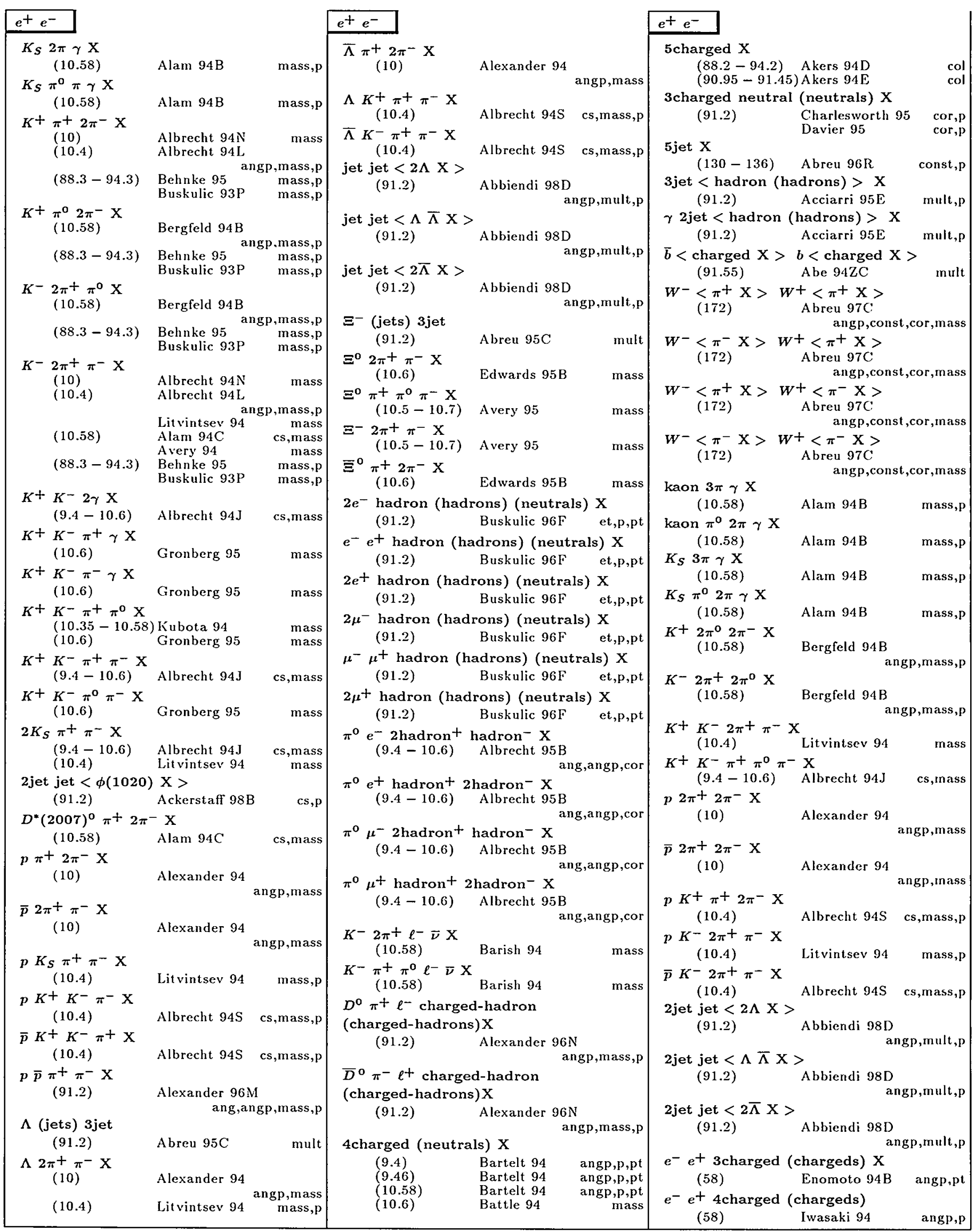




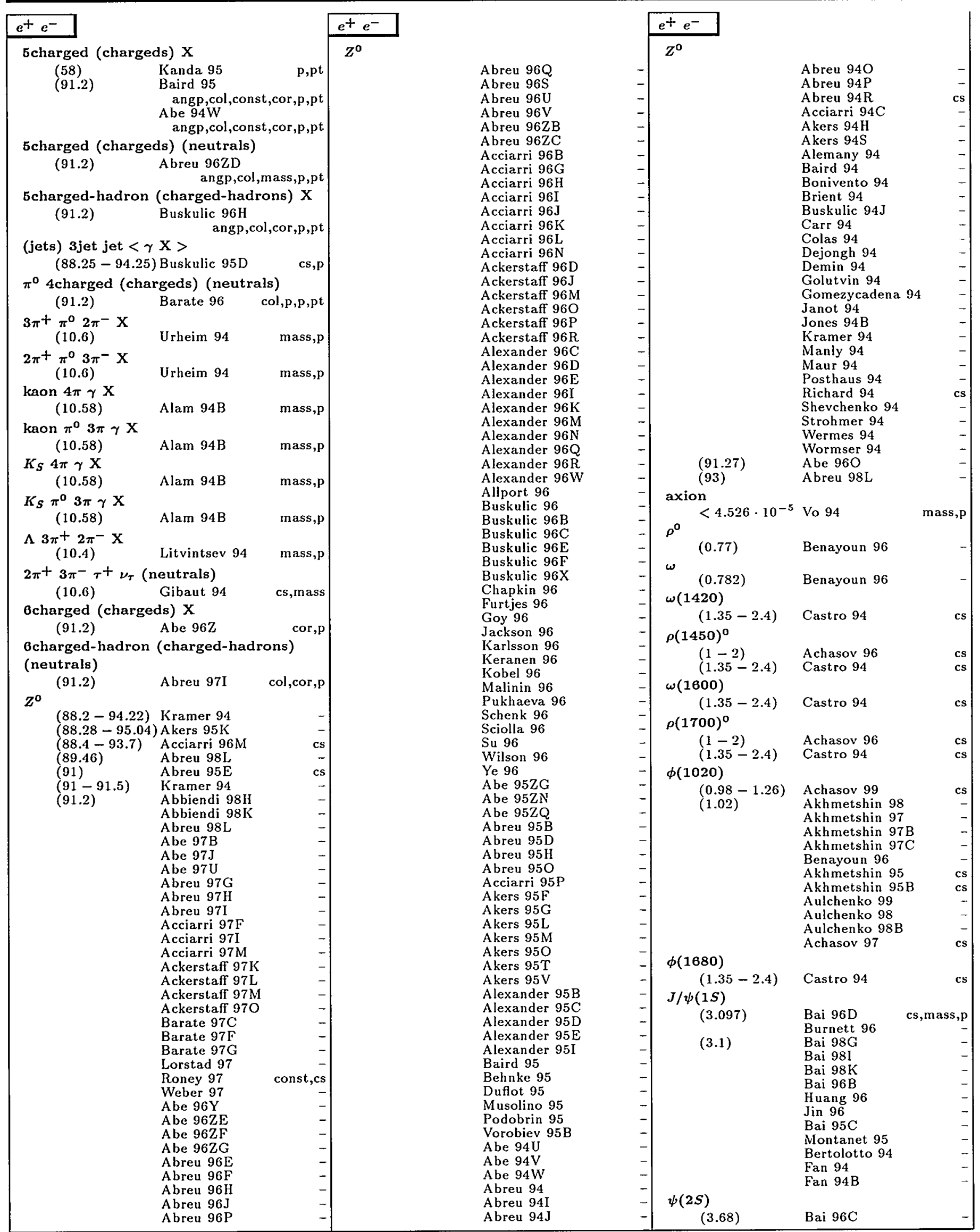

Entries are in order of beam name, then target name, then multiplicity of final state. Particle names are ordered as described in the legend on page 157 and as listed in the Particle Vocabulary. See also the Table of Contents of this Index beginning on the page 158 . A few chemical symbols for nuclei have been changed to avoid ambiguity with particle names (see the Particle Vocabulary). Beam momenta are $p_{\text {lab }}$ in GeV/c, or in parentheses $E_{\mathbf{c m}}$ in $\mathrm{GeV}$. 


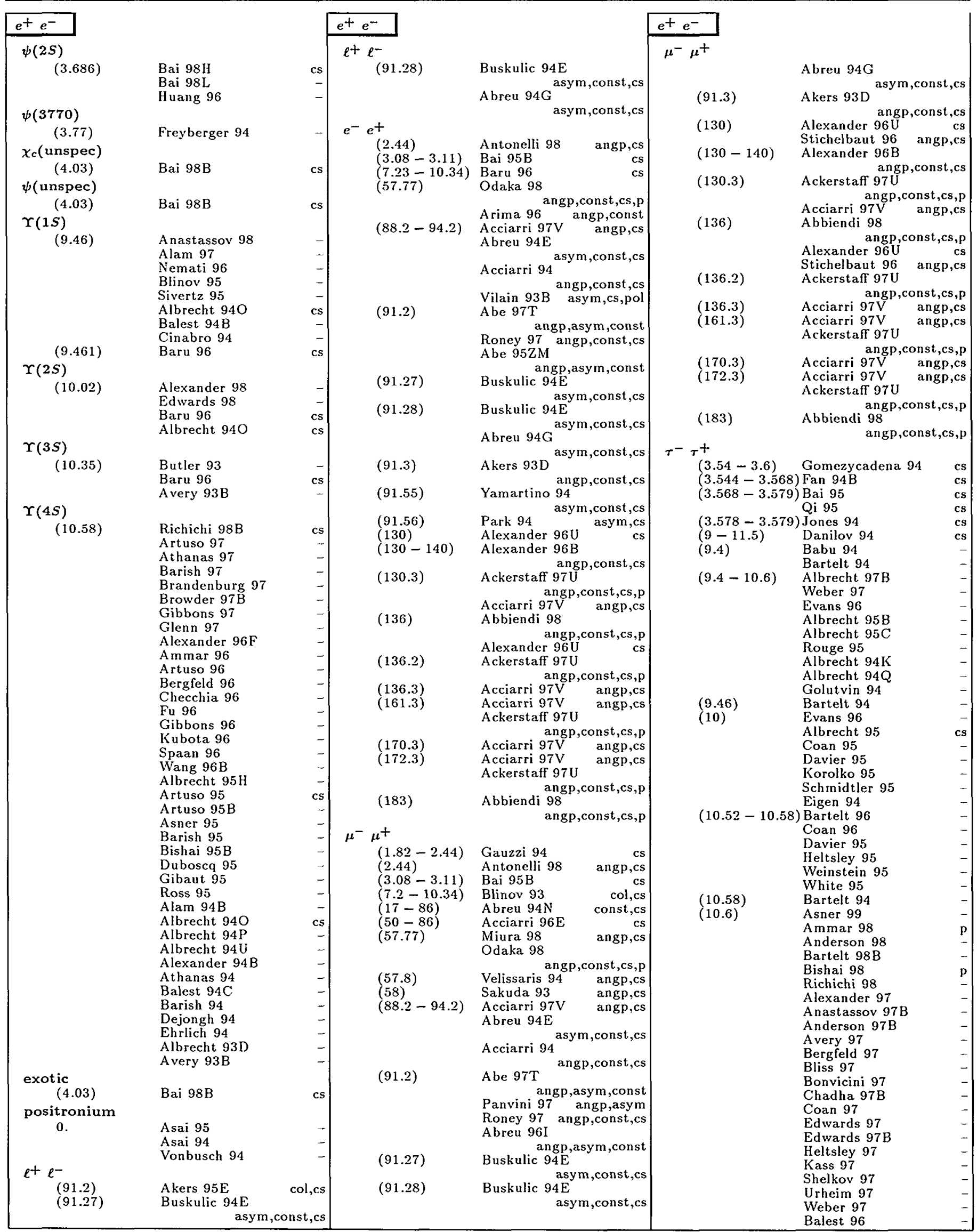




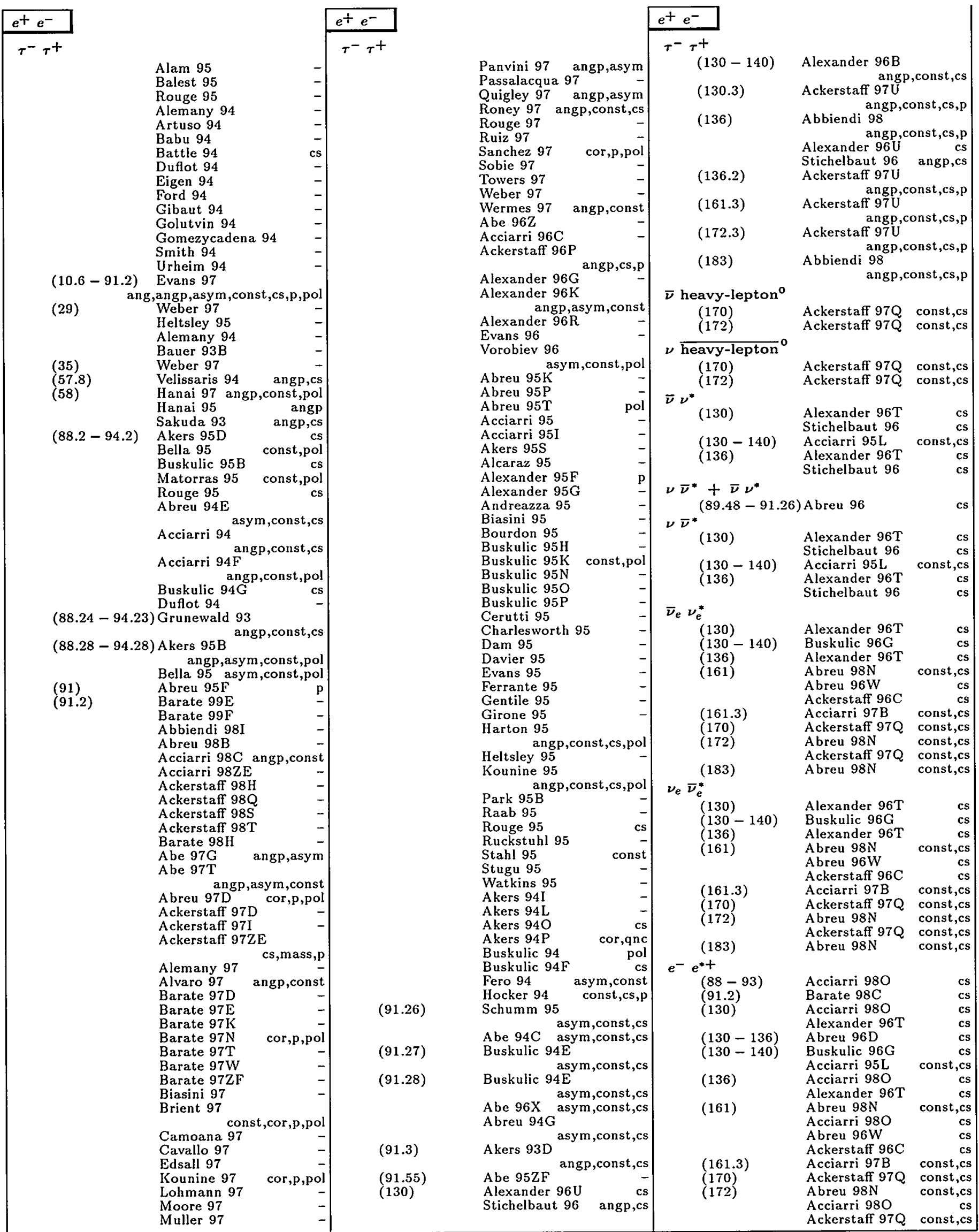

Entries are in order of beam name, then target name, then multiplicity of final state. Particle names are ordered as described in the legend on page 157 and as listed in the Particle Vocabulary. See also the Table of Contents of this Index beginning on the page 158. A few chemical symbols for nuclei have been changed to avoid ambiguity with particle names (see the Particle Vocabulary). Beam momenta are $p_{\text {lsb }}$ in GeV/c, or in parentheses $E_{\mathrm{cm}}$ in $\mathrm{GeV}$. 


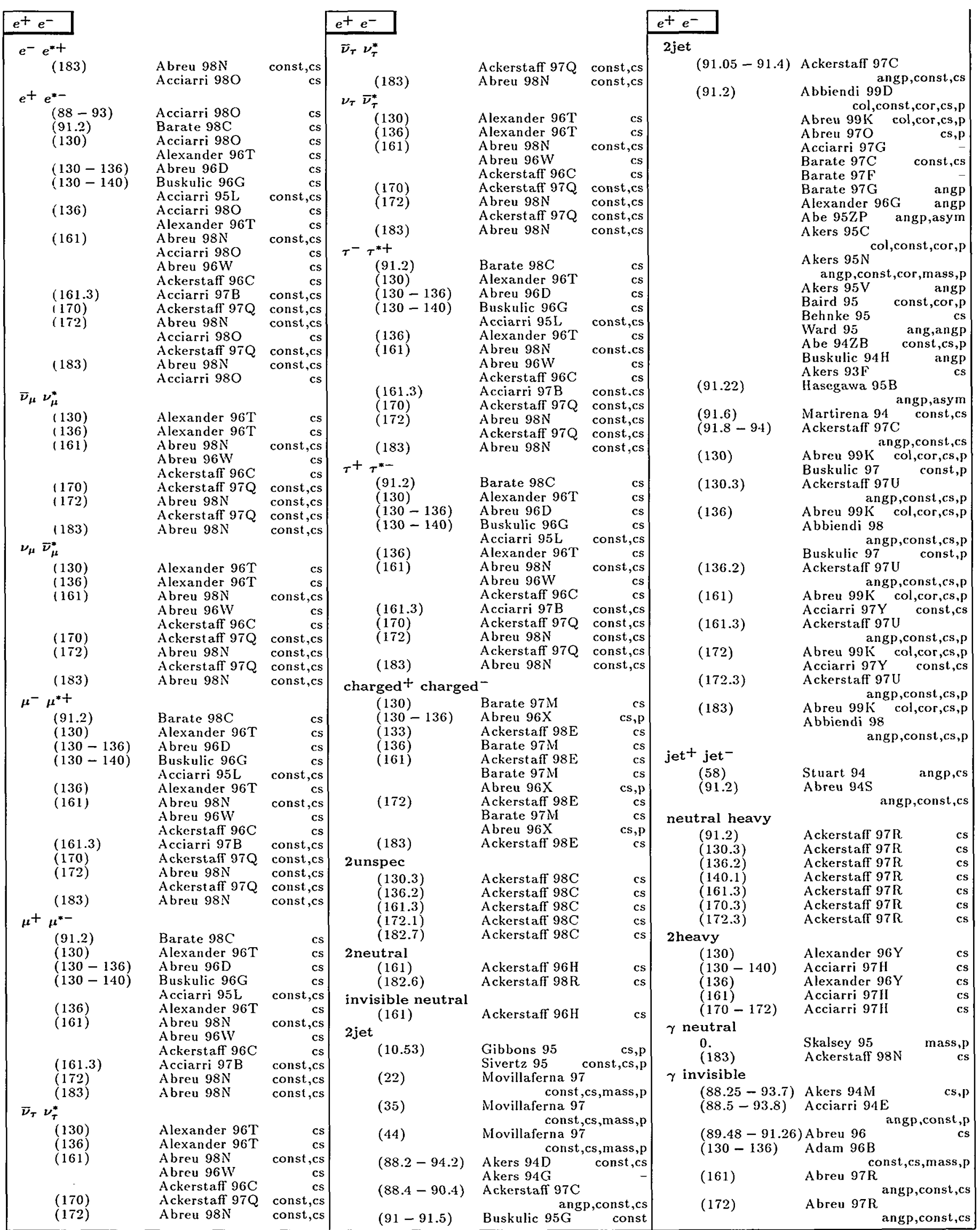




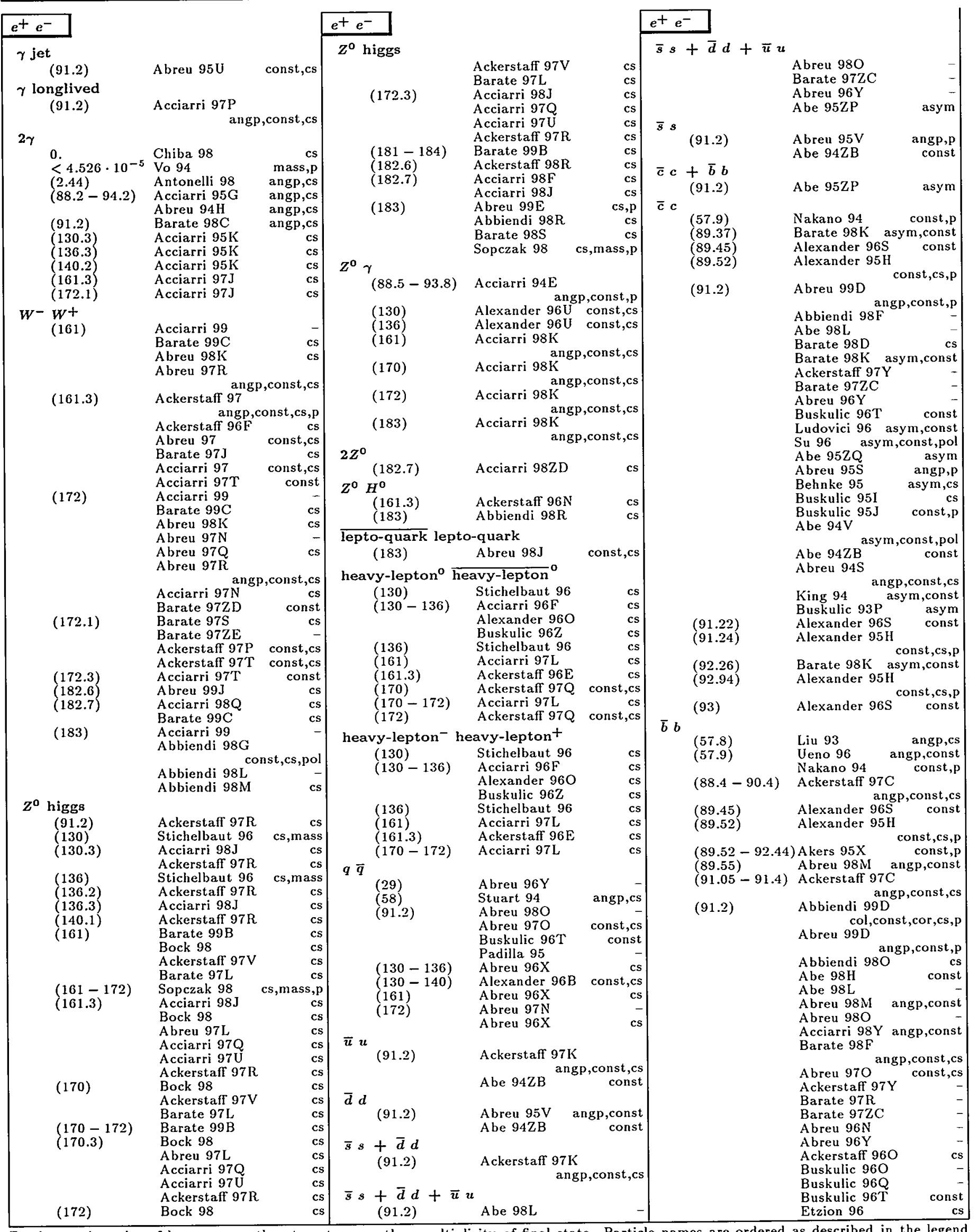

Entries are in order of beam name, then target name, then multiplicity of final state. Particle names are ordered as described in the legend on page 157 and as listed in the Particle Vocabulary. See also the Table of Contents of this Index beginning on the page 158. A few chemical symbols for nuclei have been changed to avoid ambiguity with particle names (see the Particle Vocabulary). Beam momenta are $p_{\mid a b}$ in GeV/c, or in parentheses $E_{\mathrm{cm}}$ in $\mathrm{GeV}$. 
$e^{+} e^{-} \rightarrow \bar{b} b$

$e^{+} e^{-} \rightarrow H^{+} H^{-}$

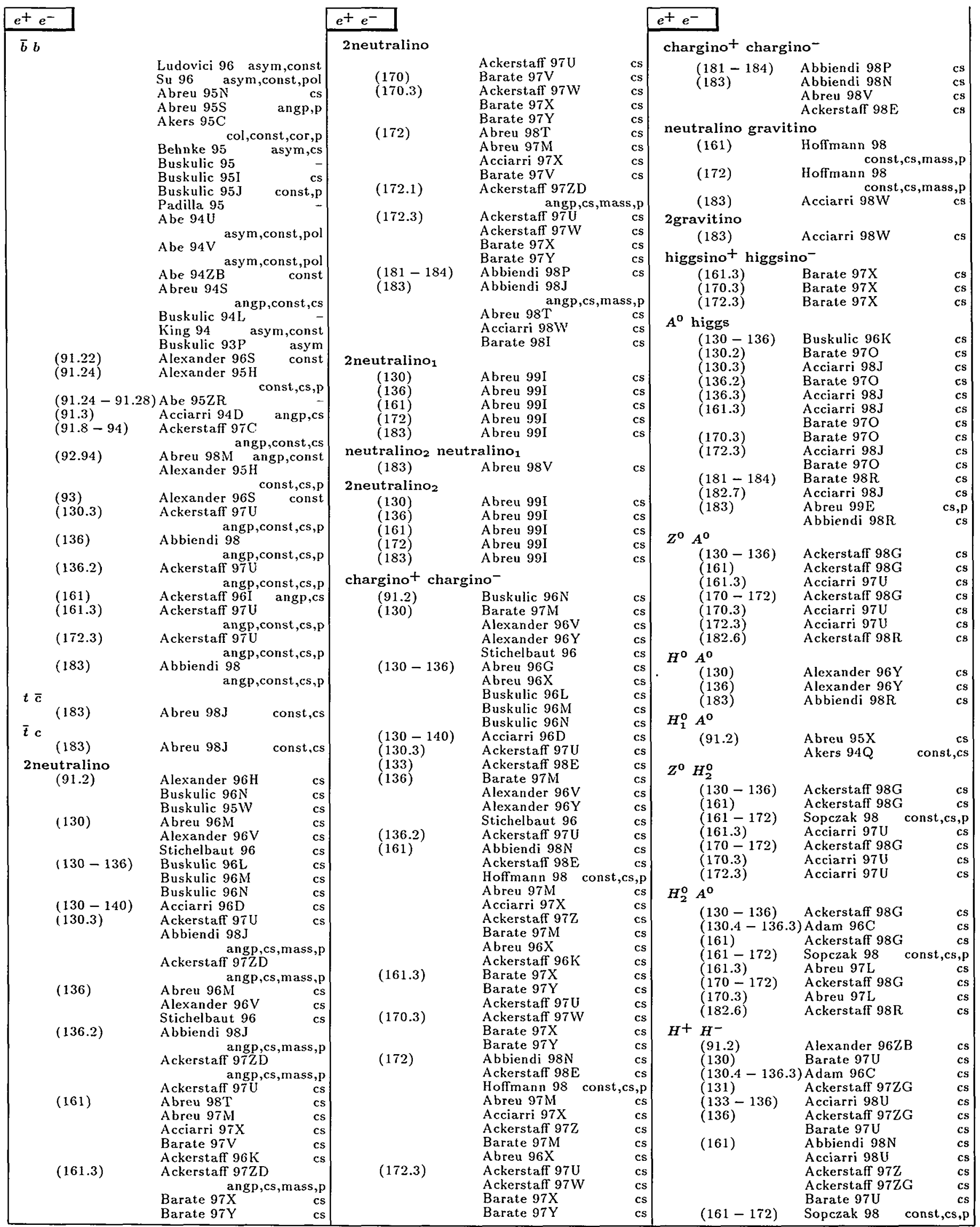




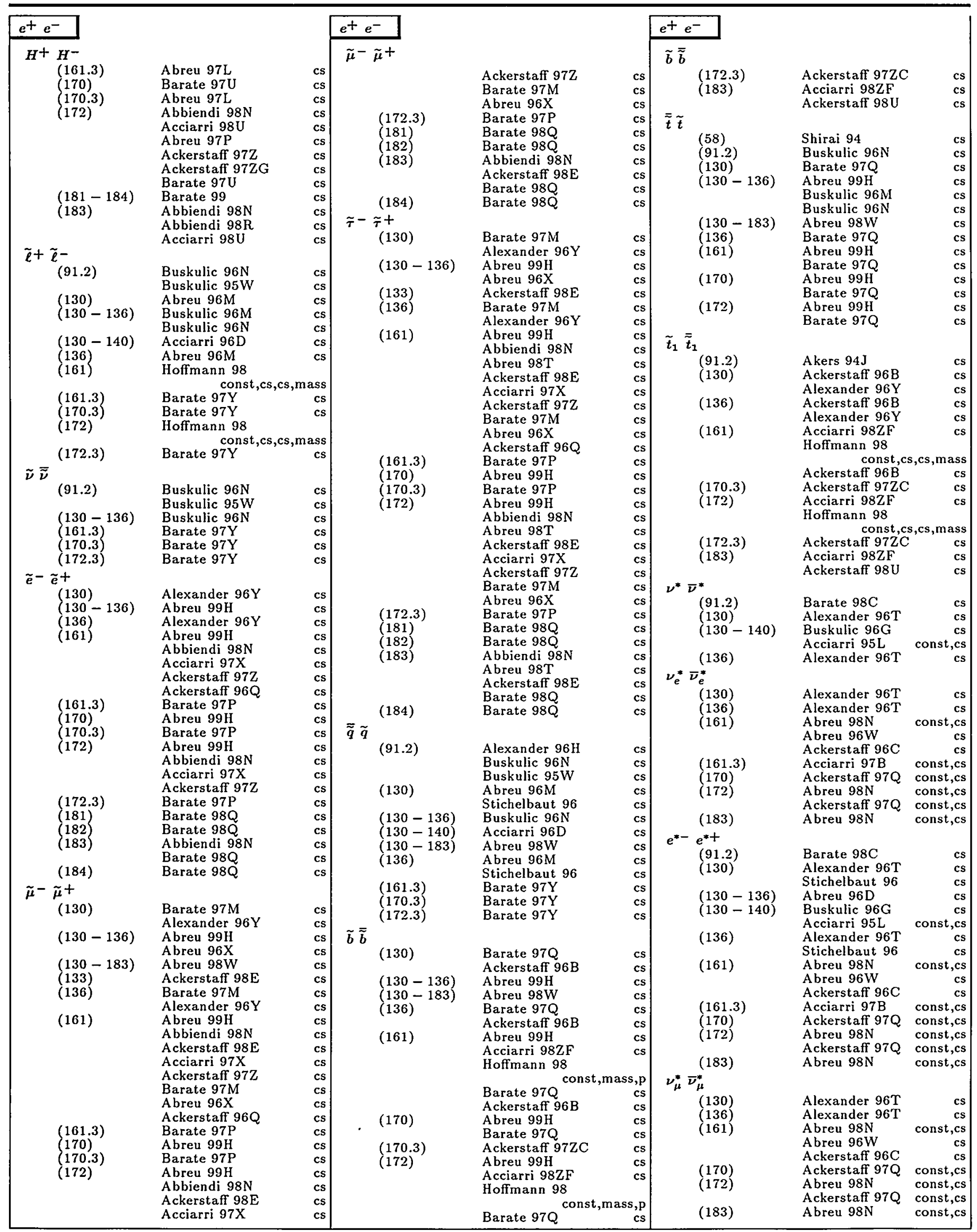

Entries are in order of beam name, then target name, then multiplicity of final state. Particle names are ordered as described in the legend on page 157 and as listed in the Particle Vocabulary. See also the Table of Contents of this Index beginning on the page 158. A few chemical symbols for nuclei have been changed to avoid ambiguity with particle names (see the Particle Vocabulary). Beam momenta are $p_{\text {lab }}$ in GeV/c, or in parentheses $E_{\mathrm{cm}}$ in $\mathrm{GeV}$. 


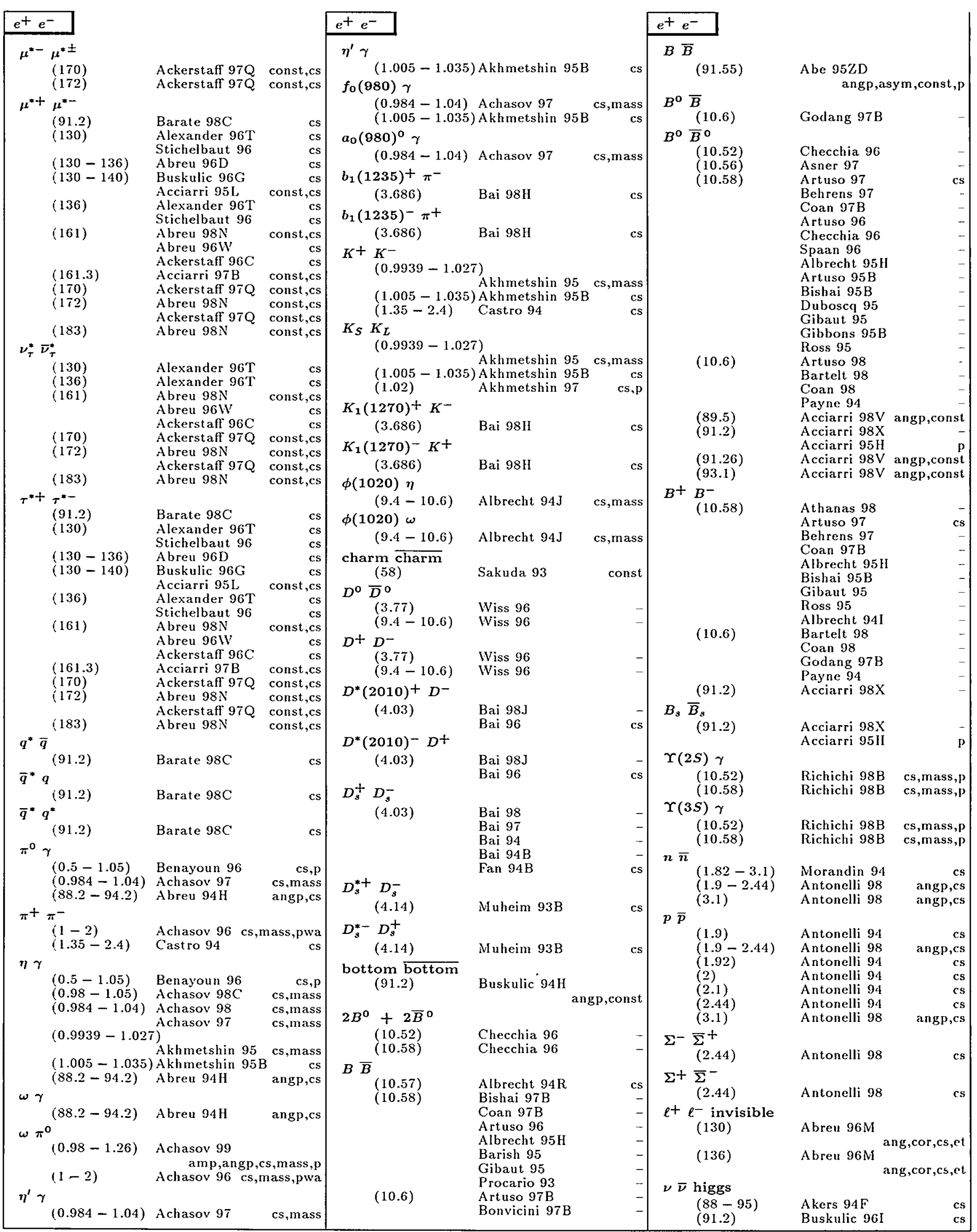




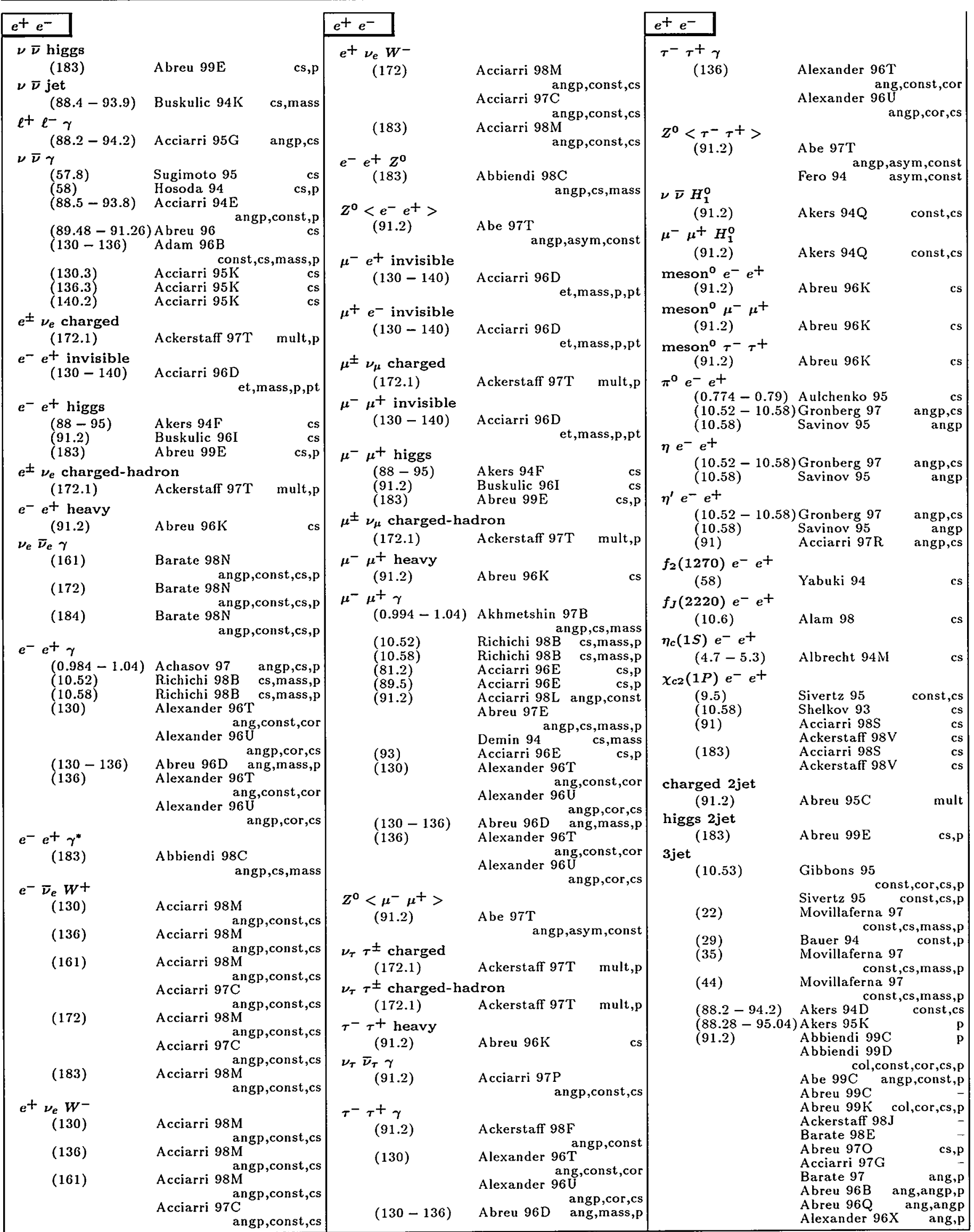

Entries are in order of beam name, then target name, then multiplicity of final state. Particle names are ordered as described in the legend on page 157 and as listed in the Particle Vocabulary. See also the Table of Contents of this Index beginning on the page 158 . A few chemical symbols for nuclei have been changed to avoid ambiguity with particle names (see the Particle Vocabulary). Beam momenta are $p_{l a b}$ in GeV/ $c$, or in parentheses $E_{\text {cm }}$ in $\mathrm{GeV}$. 


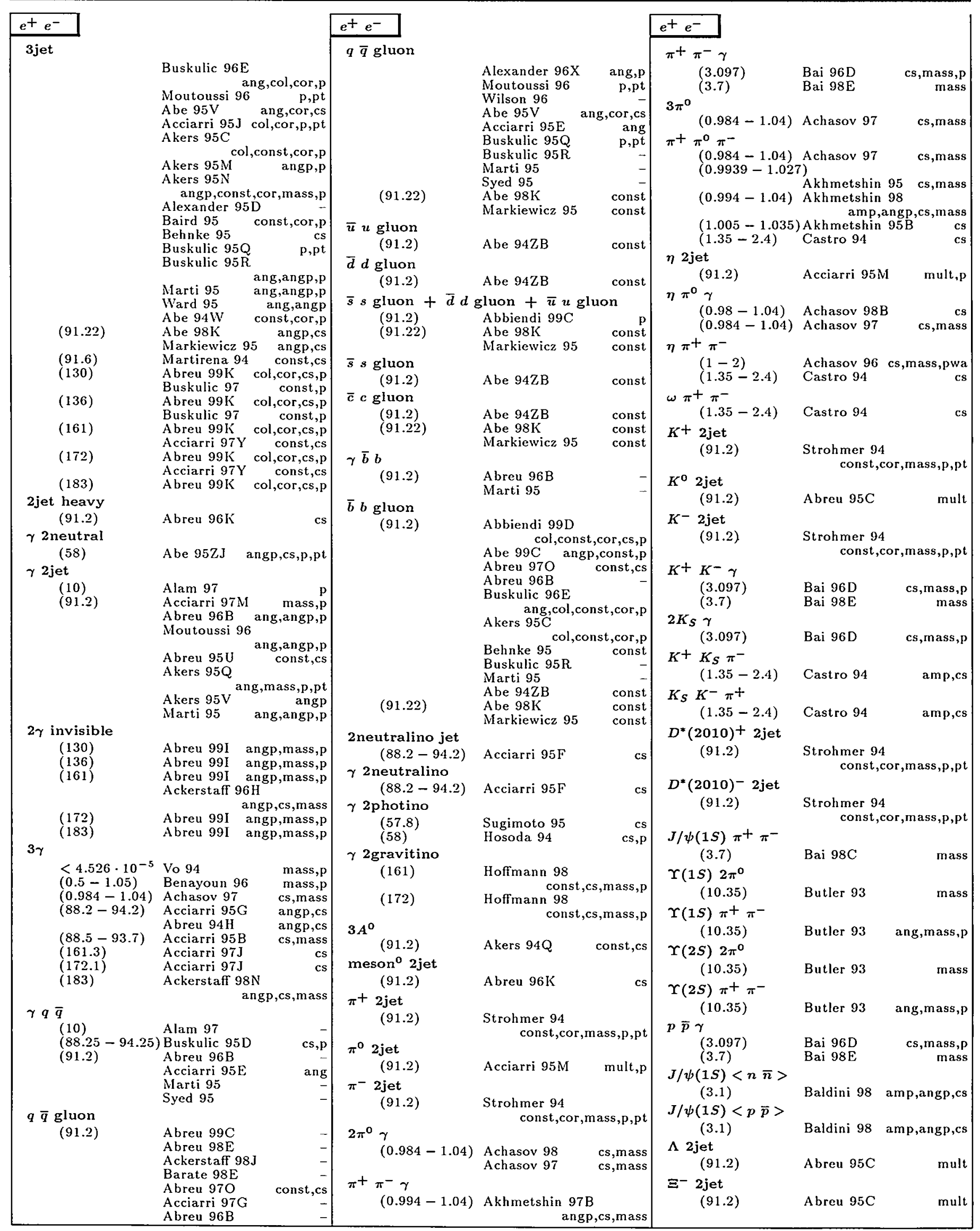




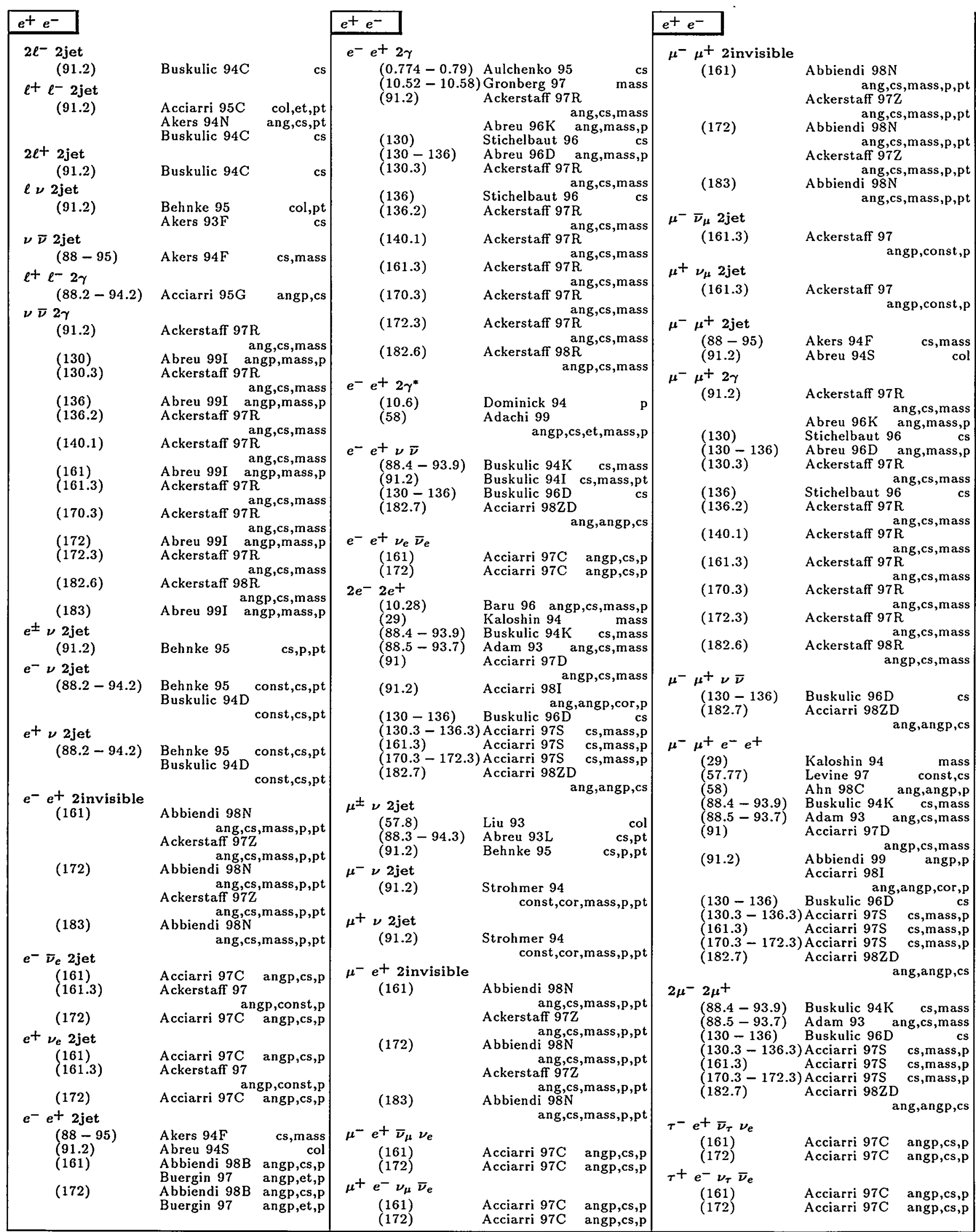

Entries are in order of beam name, then target name, then multiplicity of final state. Particle names are ordered as described in the legend on page 157 and as listed in the Particle Vocabulary. See also the Table of Contents of this Index beginning on the page 158. A few chemical symbols for nuclei have been changed to avoid ambiguity with particle names (see the Particle Vocabulary). Beam momenta are $p_{l a b}$ in GeV/c, or in parentheses $E_{\mathrm{cm}}$ in $\mathrm{GeV}$. 


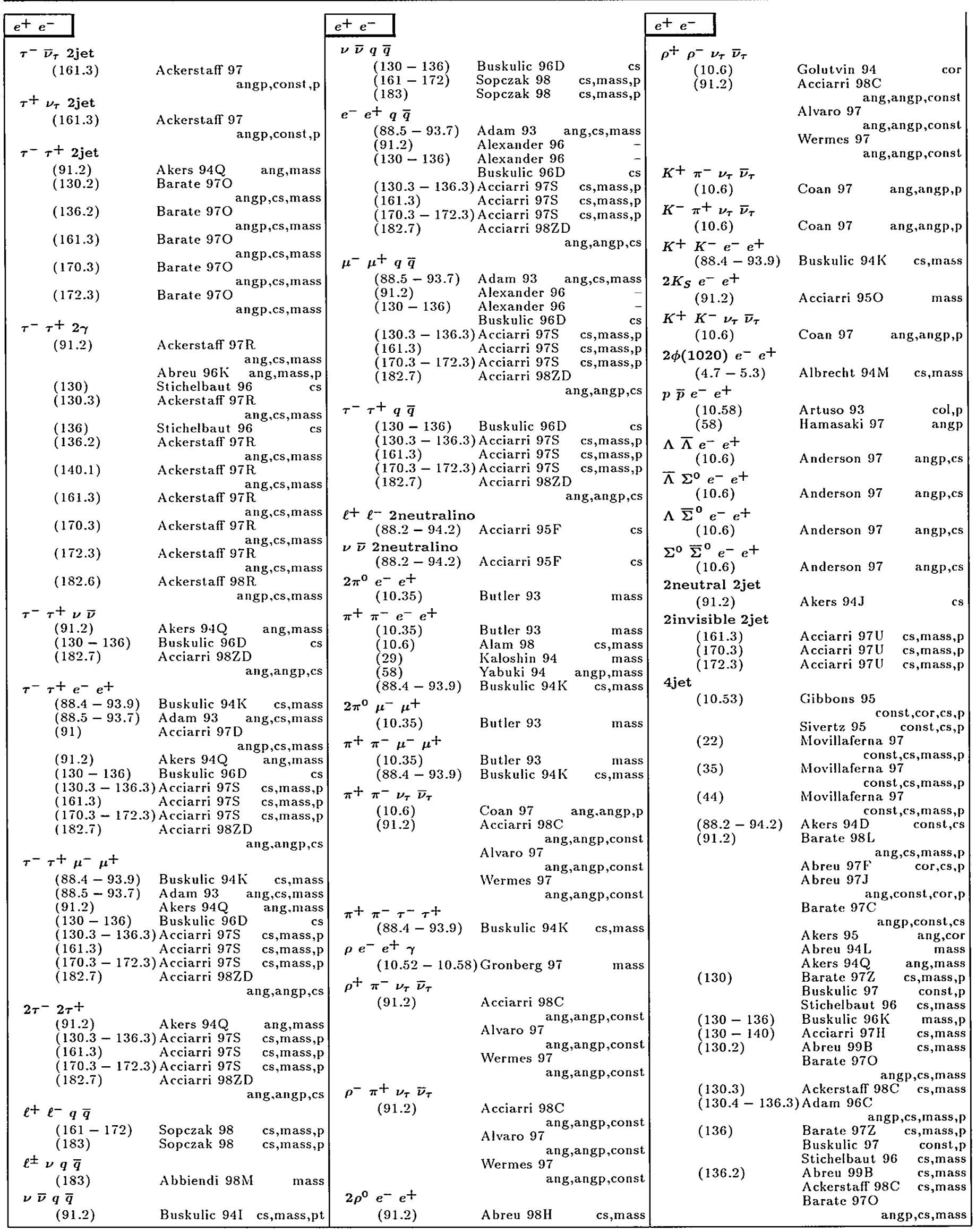




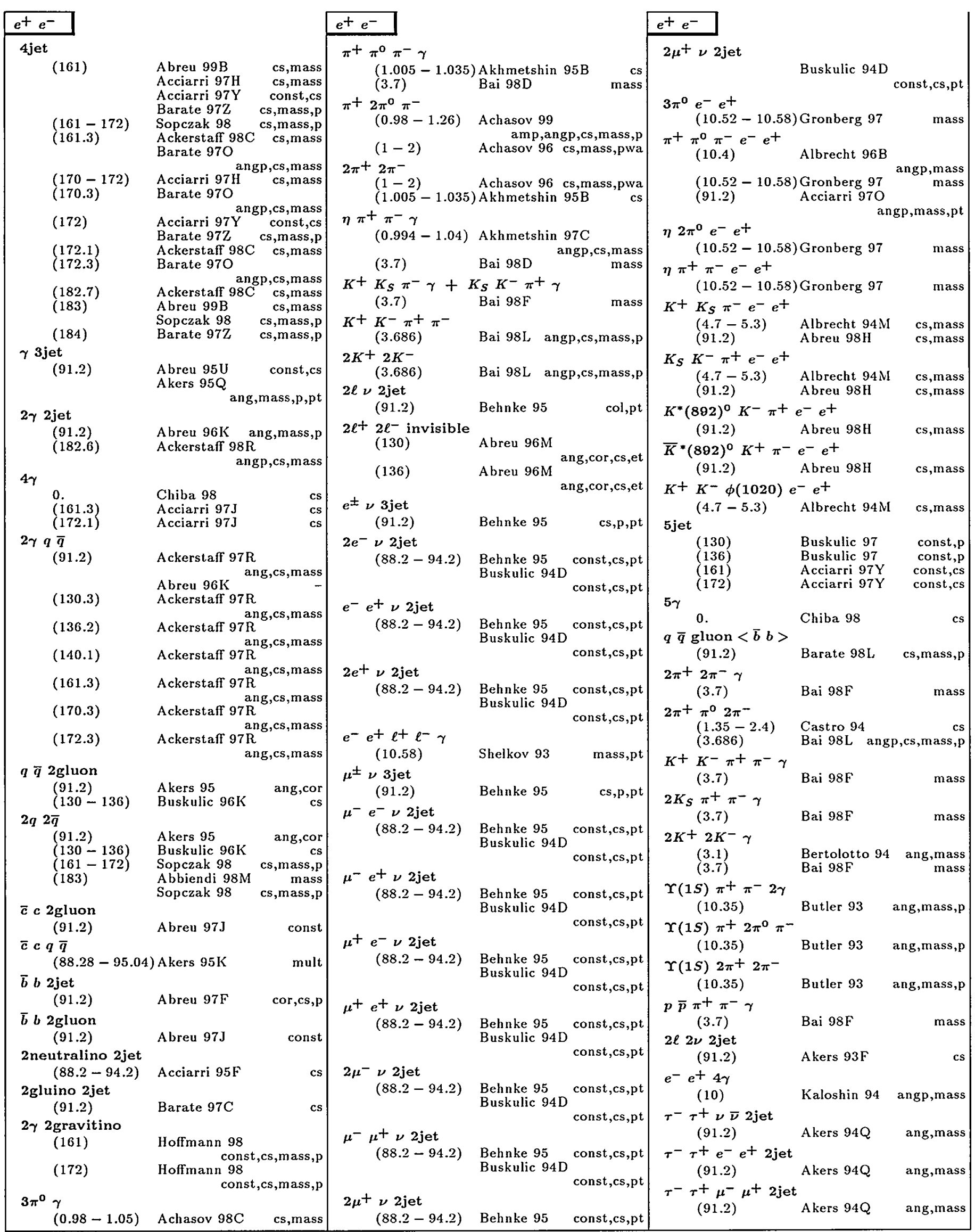

Entries are in order of beam name, then target name, then multiplicity of final state. Particle names are ordered as described in the legend on page 157 and as listed in the Particle Vocabulary. See also the Table of Contents of this Index beginning on the page 158 . A few chemical symbols for nuclei have been changed to avoid ambiguity with particle names (see the Particle Vocabulary). Beam momenta are $p_{l a b}$ in GeV/c, or in parentheses $E_{\mathrm{cm}}$ in $\mathrm{GeV}$. 


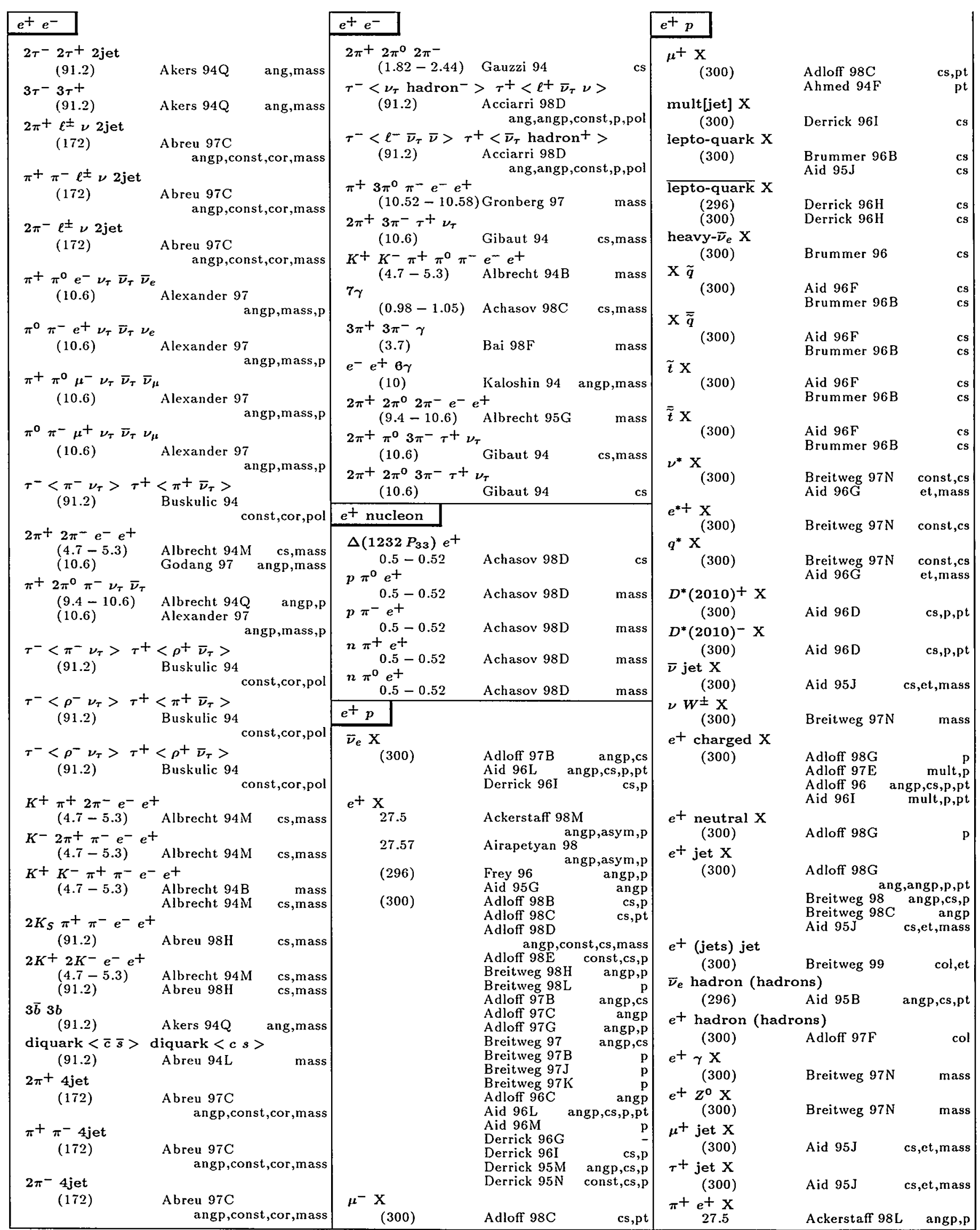




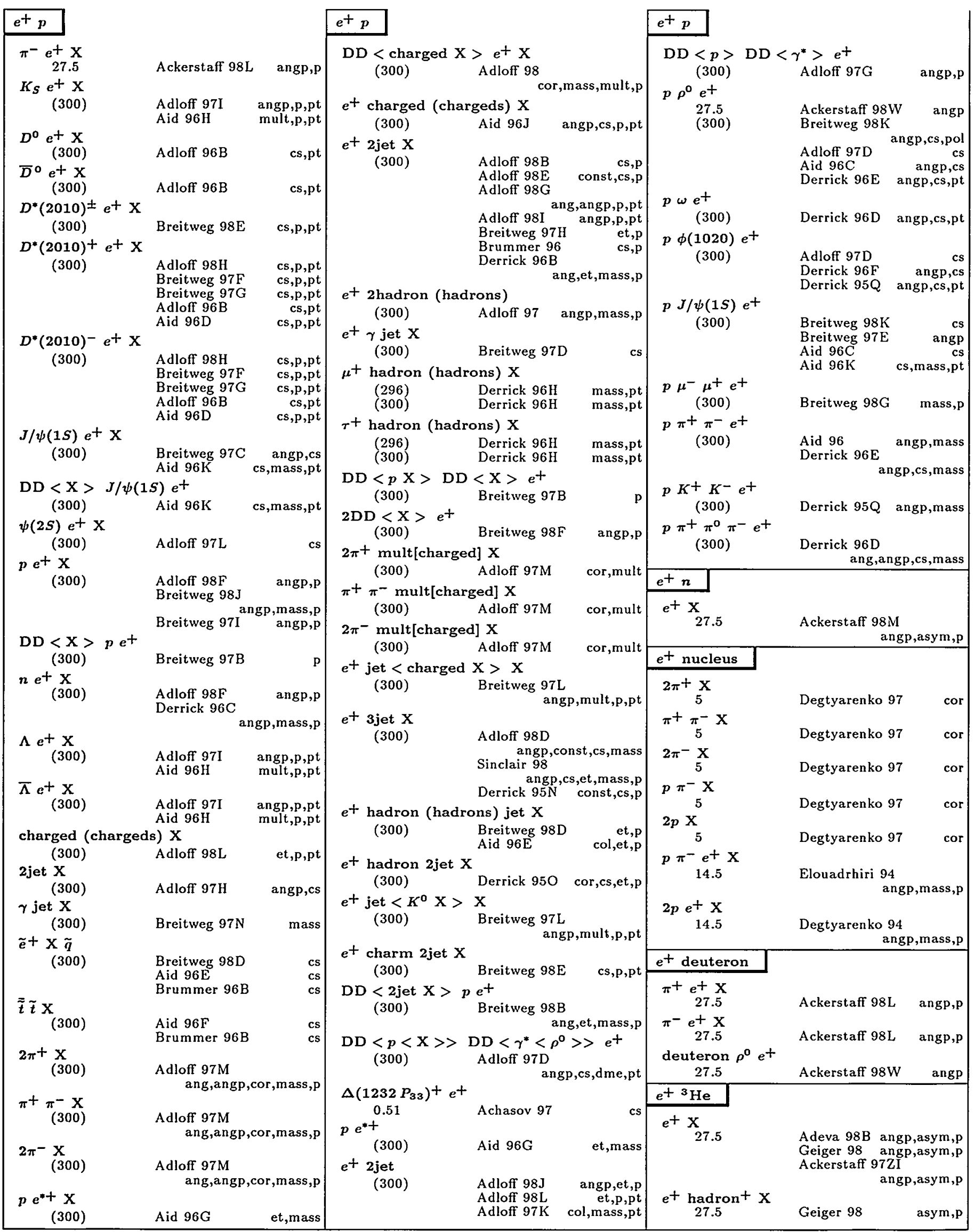

Entries are in order of beam name, then target name, then multiplicity of final state. Particle names are ordered as described in the legend on page 157 and as listed in the Particle Vocabulary. See also the Table of Contents of this Index beginning on the page 158 . A few chemical symbols for nuclei have been changed to avoid ambiguity with particle names (see the Particle Vocabulary). Beam momenta are $p_{l a b}$ in GeV/c, or in parentheses $E_{\mathrm{cm}}$ in $\mathrm{GeV}$. 


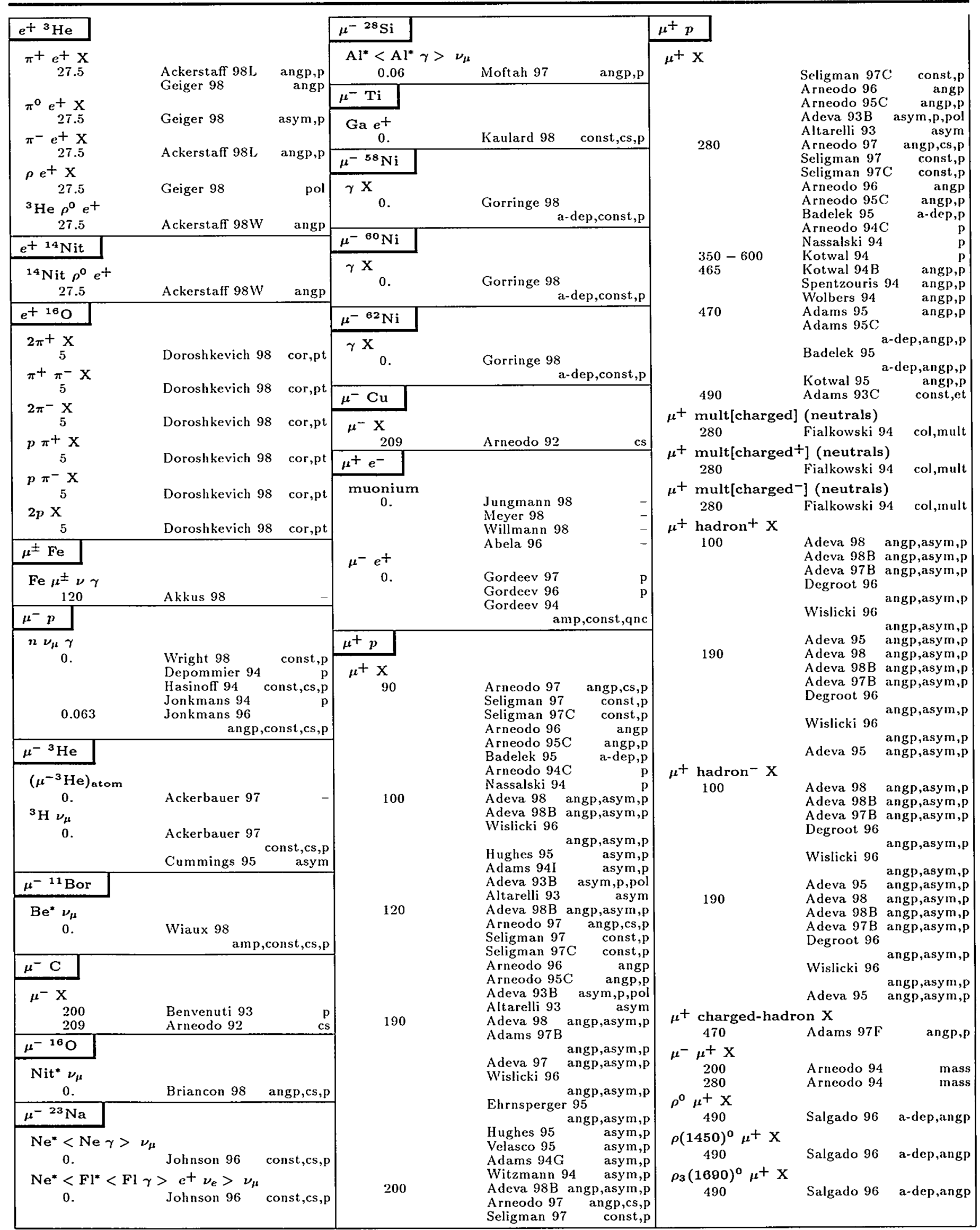




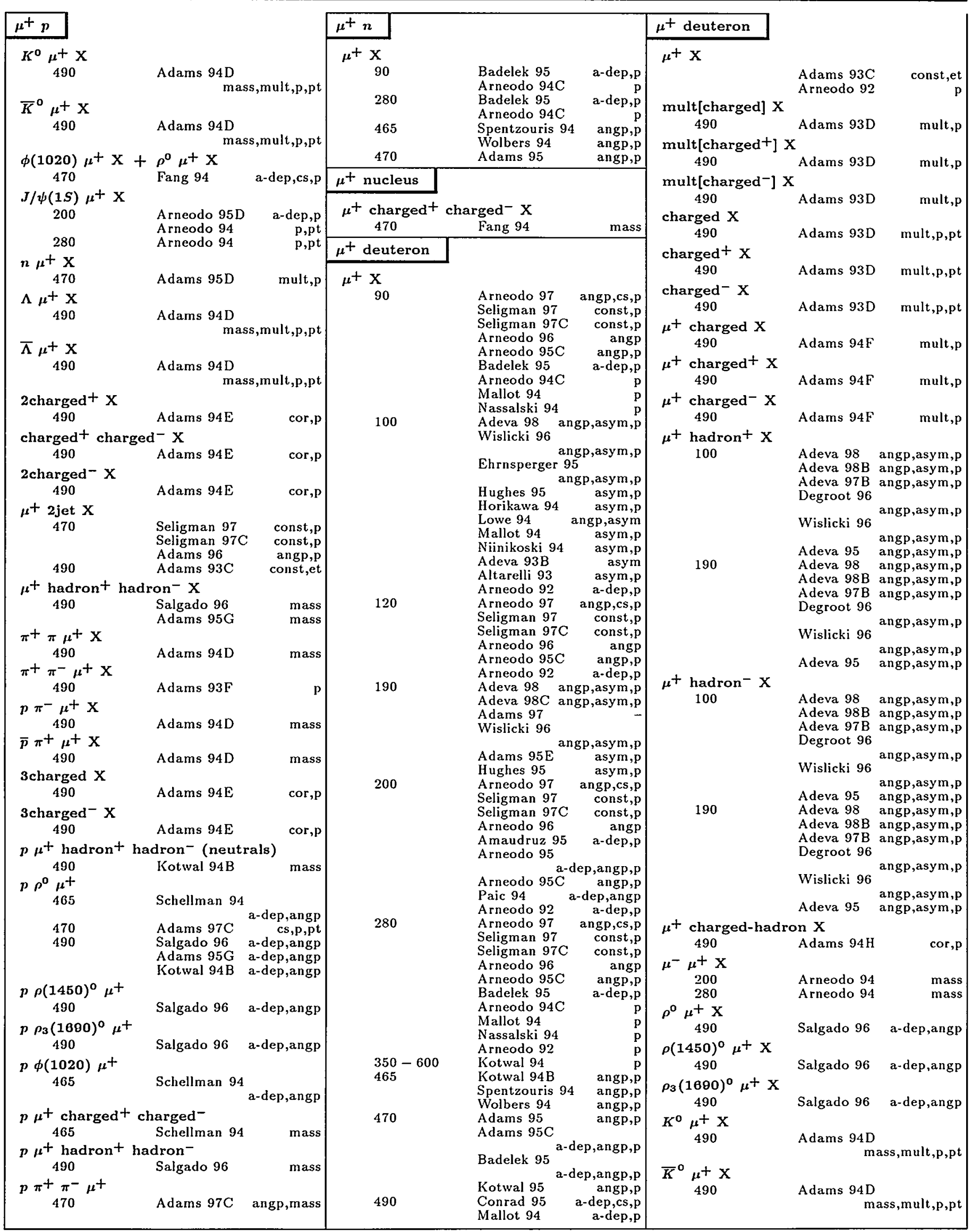




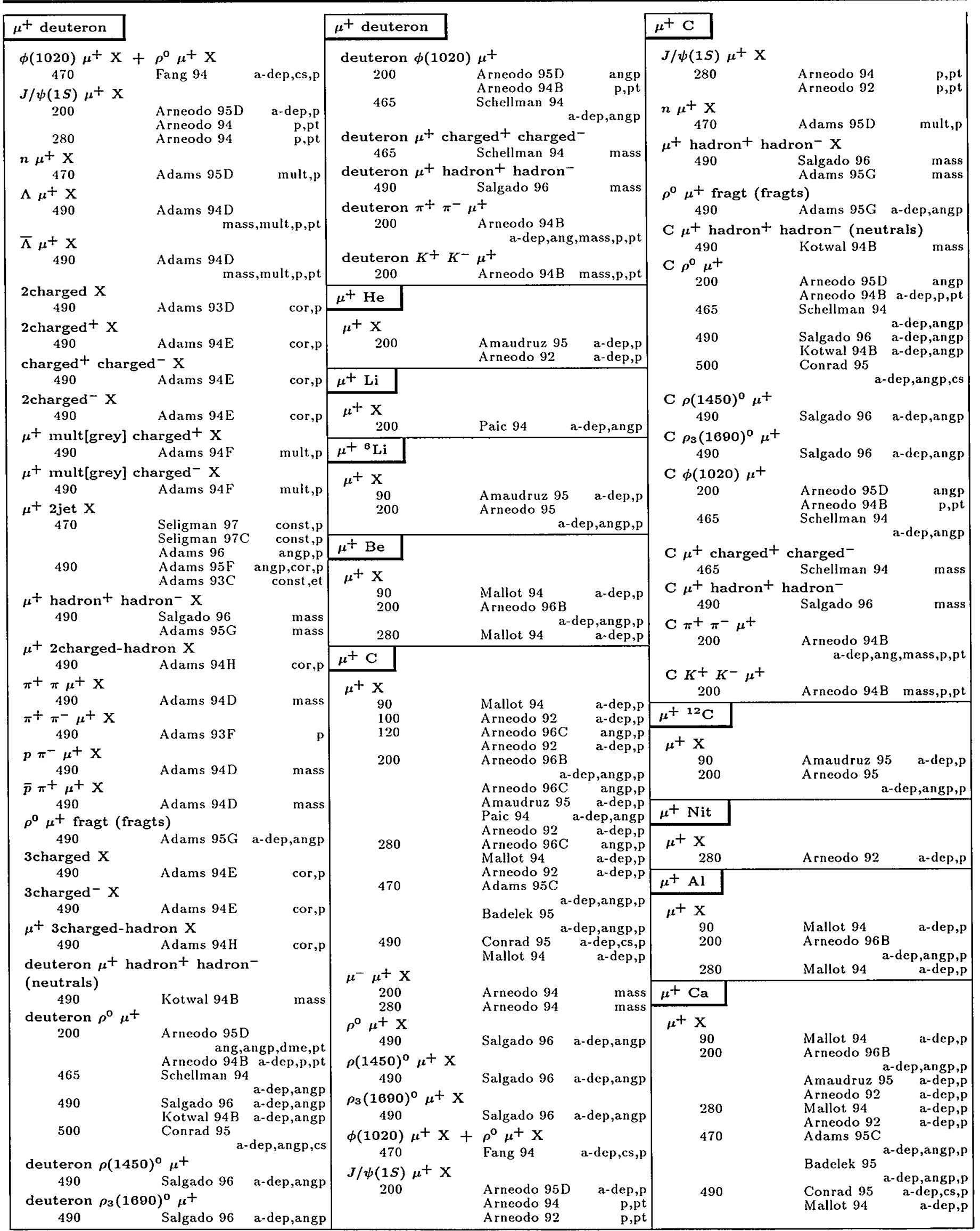




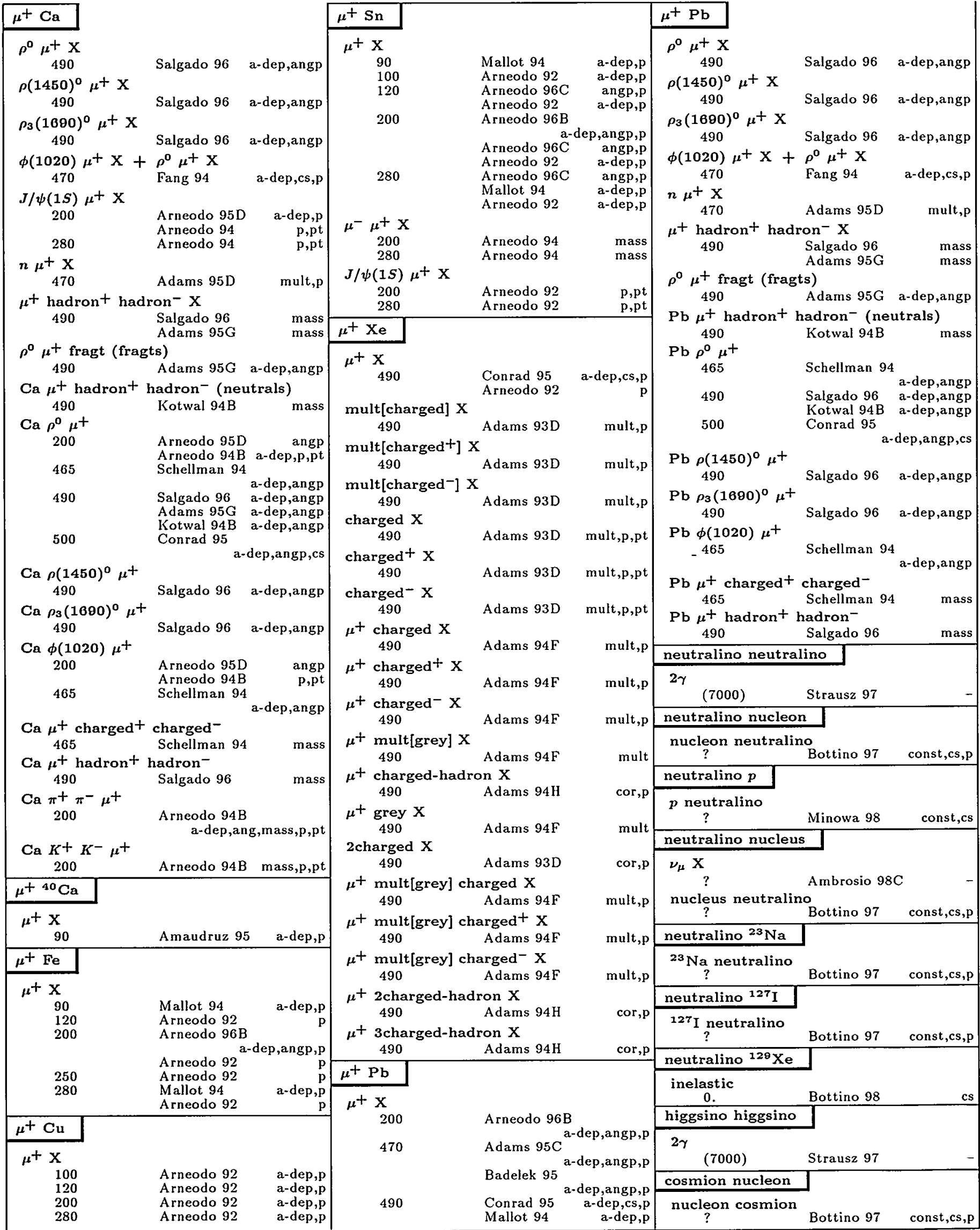

Entries are in order of beam name, then target name, then multiplicity of final state. Particle names are ordered as described in the legend on page 157 and as listed in the Particle Vocabulary. See also the Table of Contents of this Index beginning on the page 158. A few chemical symbols for nuclei have been changed to avoid ambiguity with particle names (see the Particle Vocabulary). Beam momenta are $p_{\text {lab }}$ in GeV/c, or in parentheses $E_{\mathrm{cm}}$ in $\mathrm{GeV}$. 
cosmion nucleus $\rightarrow \mathrm{X}$

$\pi^{ \pm} \mathrm{Al} \rightarrow 2$ jet $\mathrm{X}$

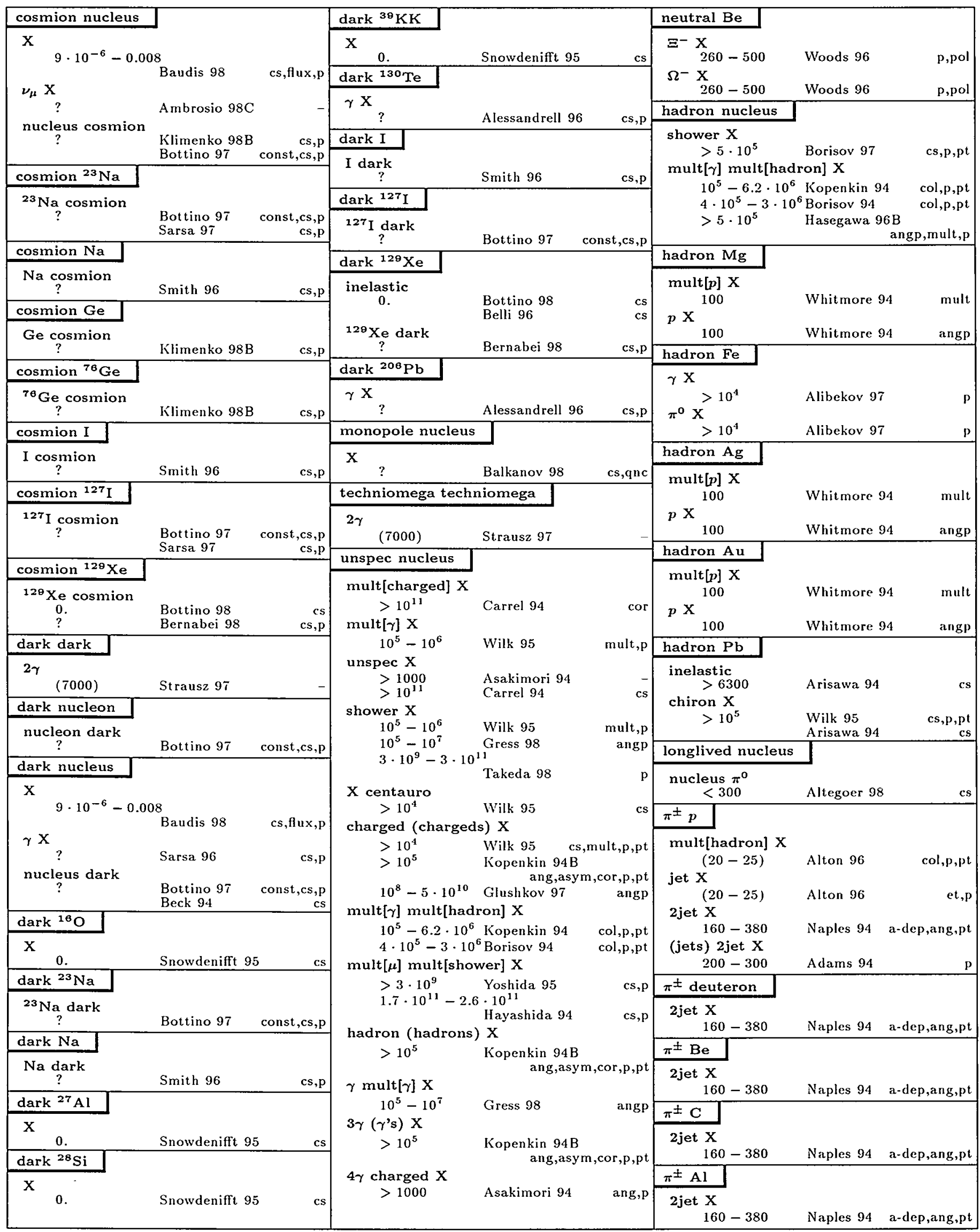




\begin{tabular}{|c|c|c|}
\hline \multicolumn{3}{|l|}{$\pi^{ \pm} \mathbf{T i}$} \\
\hline \multicolumn{3}{|l|}{$\begin{array}{c}p \pi^{ \pm} \mathrm{X} \\
1.4 \\
\text { deuteron } \pi^{ \pm} \mathrm{X} \\
1.4\end{array}$} \\
\hline \multicolumn{3}{|l|}{$\pi^{ \pm} \mathrm{Fe}$} \\
\hline $\begin{array}{c}p \pi^{ \pm} \mathrm{X} \\
1.4 \\
\text { deuteron } \pi^{ \pm} \mathrm{X} \\
1.4\end{array}$ & $\begin{array}{l}\text { Bayukov } 94 \\
\text { Bayukov } 94\end{array}$ & $\begin{array}{l}\text { angp,cor,p } \\
\text { angp,cor,p }\end{array}$ \\
\hline \multicolumn{3}{|l|}{$\pi^{ \pm} \mathrm{Cu}$} \\
\hline \multicolumn{3}{|l|}{$\begin{array}{l}\text { 2jet } X \\
\quad 160-380\end{array}$} \\
\hline \multicolumn{3}{|l|}{$\pi^{ \pm} \mathrm{Pb}$} \\
\hline \multicolumn{3}{|l|}{$\begin{array}{l}\text { 2jet } X \\
\quad 160-380\end{array}$} \\
\hline$\pi^{+} \pi^{+}$ & & \\
\hline $2 \pi^{+}{ }_{(0.2846-0 .}$ & & \\
\hline
\end{tabular}

\begin{tabular}{|l}
$\pi^{+} \pi^{-}$ \\
\hline$\pi^{+} \pi^{-}$
\end{tabular}

$(0.05659-0.2128)$

$\begin{array}{rlr}\text { Kermani } 98 \mathrm{C} & \text { cs } \\ \text { Anisovich 95 } & \text { angp,pwa }\end{array}$

$\pi^{+}$nucleon

$D^{ \pm} \mathrm{X}$

$250 \quad$ Wallace 94

$D^{+} \mathrm{X}+D^{-} \mathrm{X}$

$250 \quad$ Karchin 95

$D^{0} \mathrm{X}+\bar{D}^{\circ} \mathrm{x}$

$$
250
$$

$D_{s}^{ \pm} \mathrm{X}$

$250 \quad$ Wallace 94

$D_{s}^{+} \mathrm{X}+D_{s}^{-} \mathrm{X}$

$250 \quad$ Karchin 95

$K^{*}(892)^{\circ} K^{-} \mathrm{X}+\bar{K}^{*}(892)^{\circ} K^{+} \mathrm{X}$

$250 \quad$ Karchin 95

Wallace 94

$\phi(1020) \pi^{+} \mathrm{X}+\phi(1020) \pi^{-} \mathrm{X}$

$250 \quad$ Karchin 95

Wallace 94

$K^{+} 2 \pi^{-} \mathrm{X}+K^{-2} \pi^{+} \mathrm{X}$

$250 \quad$ Karchin 95

Wallace 94

$\pi^{+} p$

$\mathbf{x}$

$0.09595-0.3998$

Kriss 99

mult[charged] $\mathbf{X}$

147
250

charged $\mathrm{X}$

250

charged $+\mathrm{X}$

250

charged $^{-} \mathrm{X}$

$$
250
$$

$\gamma \mathrm{X}$

$$
280
$$

300

$\pi^{+} \mathrm{x}$

0.1001
Kriss 99
Breitweg $97 \mathrm{M}$

Arena 95

Agababyan 98

col,cor,mult,p,pt

Agababyan 94

Agababyan $94 \mathrm{D}$ cs, mult,pt

Agababyan $96 \mathrm{~B}$

Agababyan 96B

Vogelsang 97

Vogelsang 97

Joram 95 $\pi^{+} p$
$\pi^{+} \mathrm{X}$
0.

$$
\text { cs } \underset{250}{2 \text { 2charged }}+\mathrm{X}
$$

charged ${ }^{+}$charged $^{-} X$

mass

mass

mass 2charged $-\mathrm{X}$

mass
0.101
0.1202
0.1209

0.1209

0.1544

$\pi-\mathrm{X}$

$K^{*}(892)^{\circ} \mathrm{X}$

80

$\bar{K}^{*}(892)^{0} \mathrm{X}$

80

$\phi(1020) \mathrm{X}$

80

140

$1 \mathrm{X}$

$3.6-4.2$

6.2

mult [char

$$
250
$$

0

charged
250

$$
250
$$

2charged $\mathrm{X}$

250

$$
250
$$

$$
250
$$

Joram 95B

Joram 95

Joram 95B

Joram 95B

Agababyan 97

Apsimon 93

Apsimon 93

Apsimon 93

Apsimon 93

Apsimon 93

Apsimon 93

angp,p,pt

Bulekov 94

Bulekov 94

$\mathrm{p}, \mathrm{pol}, \mathrm{pt}$

p,pol,pt

mult [charged-] $X$

Agababyan 96B

col,cor,p,pt $x$

Agababyan 94 cor,p,pt A gababyan $94 \mathrm{D}$

ang,col, $p, p t$

ang,cor,

Agababyan 94B ang,col

Agababyan $94 \mathrm{C}$ col,cor

Agababyan $96 \mathrm{~B}$

ol, cor,p,pt

ang,cor, $p$

Agababyan 94B ang,col

Agababyan $94 \mathrm{C}$ col,cor

Ajinenko 94 ang,col,cor,mass,pt

Agababyan 96B

Agababyan 95D

Agababyan $94 \mathrm{C}$ col,cor

Ajinenko 94

ang,col,cor,mass,pt

Agababyan $96 \mathrm{~B}$

Agababyan $95 \mathrm{D}$

col,cor,p,pt

ang,cor, $p$

Agababyan $94 \mathrm{~B}$ ang,col

Agababyan $94 \mathrm{C}$ col,cor

Ajinenko 94

ang,col,cor,mass,pt

Ferenc 96

Agababyan 97

Agababyan $96 \mathrm{C}, \mathrm{Cor}, \mathrm{p}, \mathrm{pt}$ angp,cor,p,pt

Agababyan 95B

Agababyan $95 \mathrm{C}$,cor,p,pt

ang,cor,p,pt

2charged (chargeds) $X$

$$
250 \text { Agababyan } 96
$$

3charged $\mathrm{X}$

$$
250
$$

3charged $+\mathrm{X}$

250

col,cor,p,pt

Agababyan 94B ang,col

Agababyan 94C col,cor

Agababyan 94B ang,col
Agababyan 95D

Agababyan 95D

Agababyan ang,cor,p

$\pi+p$

3charged $^{+} X$

3charged $+X+$ 3charged $^{-} \mathrm{X}$

Agababyan $94 \mathrm{C}$ col,cor

2charged + charged $-\mathrm{X}$

250 Agababyan 94B ang,col Ajinenko 94 cor, mass

charged $^{+}$2charged $^{-} \mathrm{X}$

250 Agababyan 94B ang,col Ajinenko 94 cor, mass

3charged $^{-} \mathrm{X}$ 250

Agababyan 94B ang,col Ajinenko 94 cor,mass

$\Lambda K \pi \mathrm{X}$ $3.6-4.2$

Drutskoi 93 mass,pol

$\boldsymbol{\Sigma}^{+} \boldsymbol{K} \pi \mathrm{X}$

$3.6-4.2$

4charged $\mathrm{X}$

250

Drutskoi 93 mass,pol

Agababyan 94B ang,col Agababyan $94 \mathrm{C}$ col,cor

4charged $+x+4$ charged $^{-} \mathrm{X}$ 250

Agababyan 94C col,cor

4charged $+\mathrm{X}$ 250

Agababyan $94 \mathrm{~B}$ ang,col 3charged $^{+}$charged $^{-} \mathrm{X}$

$$
250 \text { Agababyan 94B ang,col }
$$

2charged + 2charged $^{-} \mathrm{X}$

$$
250 \text { Agababyan 94B ang,col }
$$

charged $^{+}$3charged $^{-} \mathrm{X}$

$$
250 \quad \text { Agababyan 94B ang,col }
$$

4charged ${ }^{-} X$

250

5charged $\mathrm{X}$

250

Agababyan 94B ang,col

charged $+\mathrm{X}$ 250 Agababyan $94 \mathrm{C}$ col,cor

Agababyan 94B ang,col 4charged ${ }^{+}$charged $^{-} \mathrm{X}$ 250 Agababyan 94B ang,col 3charged + 2charged $^{-} \mathrm{X}$

250 Agababyan 94B ang,col

2charged + 3charged ${ }^{-} \mathrm{X}$

250 Agababyan 94B ang,col

charged $^{+} 4$ charged $^{-} \mathrm{X}$

$$
250 \quad \text { Agababyan } 94 \mathrm{~B} \text { ang,col }
$$

5charged ${ }^{-} \mathrm{X}$ 250

$\mathrm{DD}\left\langle\boldsymbol{p}>\boldsymbol{\pi}^{+}\right.$

147

Agababyan $94 \mathrm{~B}$ ang,col

$\mathrm{DD}<\pi^{+}>p$ 147

$$
p \pi^{+}
$$

Fuess 94C

angp,cs

$0.0963-2.16$

0.101

0.1209

0.154

0.1544

$0.1785-0.2411$

$0.2153-0.3819$

$0.2718-0.7253$

1.43

Fuess 94C

angp,cs

Arndt 9

Joram 95B

Joram 95B

Wieser 96

Joram $95 \mathrm{~B}$

angp,cs,pwa angp,pwa

angp,pwa

angp,pol

Brack 95

angp,pwa

95 angp
Smith $98 \mathrm{~B}$ angp,asym,p

Abaev 95B

Abaev 95

angp,asym,pol

White $94 \mathrm{~B}$

angp White $94 \mathrm{~B}$, asym, pol Fuess 94C angp

entries are in order of beam name, then target name, then multiplicity of final state. Particle names are ordered as described in the legend

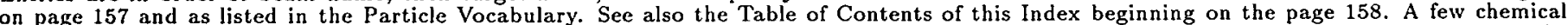

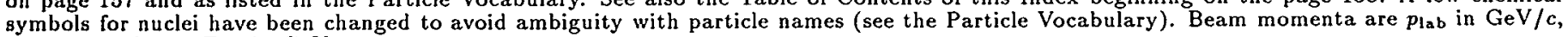
or in parentheses $E_{\mathrm{cm}}$ in $\mathrm{GeV}$. 


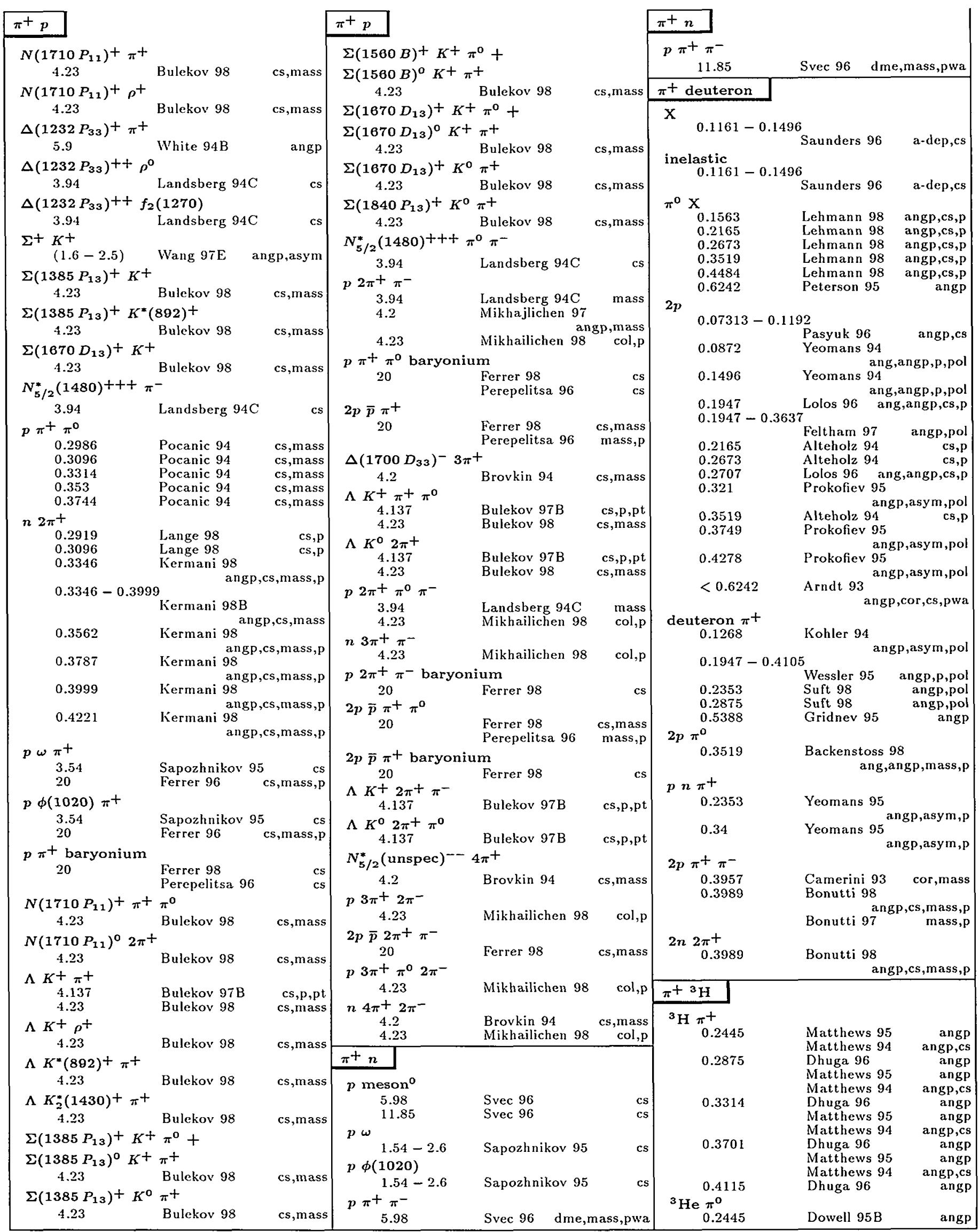




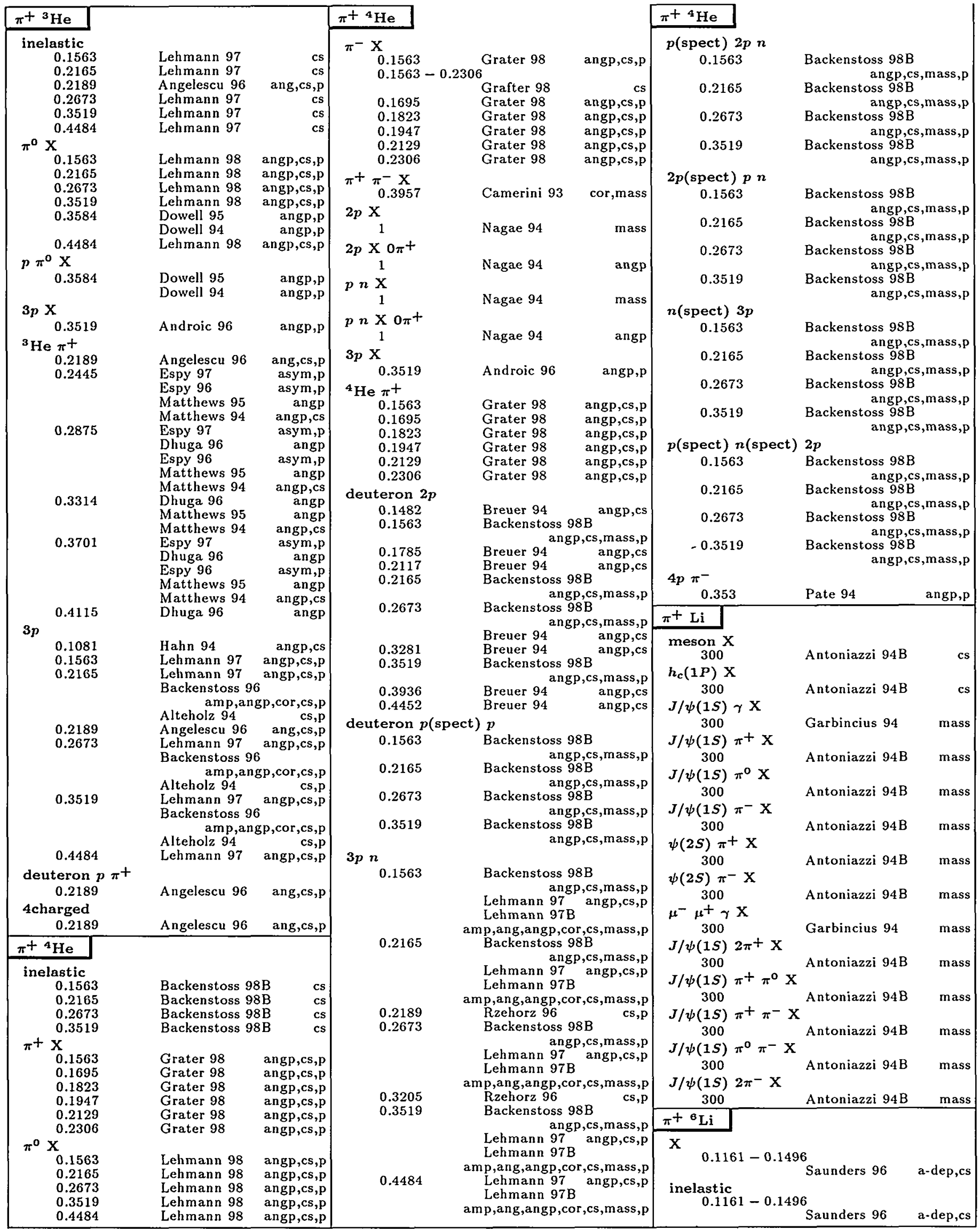

Entries are in order of beam name, then target name, then multiplicity of final state. Particle names are ordered as described in the legend on page 157 and as listed in the Particle Vocabulary. See also the Table of Contents of this Index beginning on the page 158. A few chemical symbols for nuclei have been changed to avoid ambiguity with particle names (see the Particle Vocabulary). Beam momenta are $p_{\text {lab }}$ in GeV/c, or in parentheses $E_{\mathrm{cm}}$ in $\mathrm{GeV}$. 
$\pi^{+6} \mathrm{Li} \rightarrow \Lambda \mathrm{X}$

$\pi^{+} \mathrm{Al} \rightarrow D^{*}(2010)^{ \pm} \mathrm{X}$

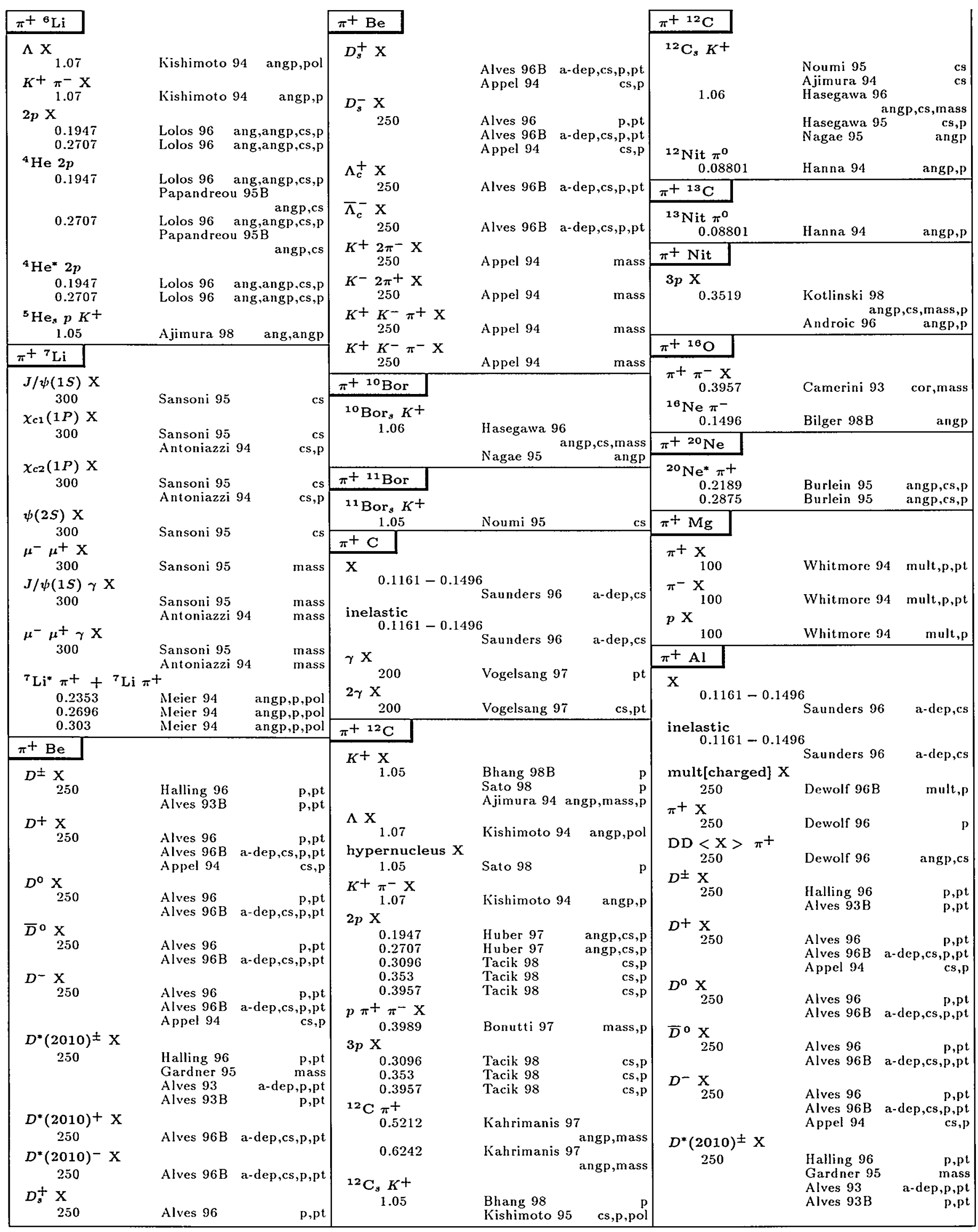




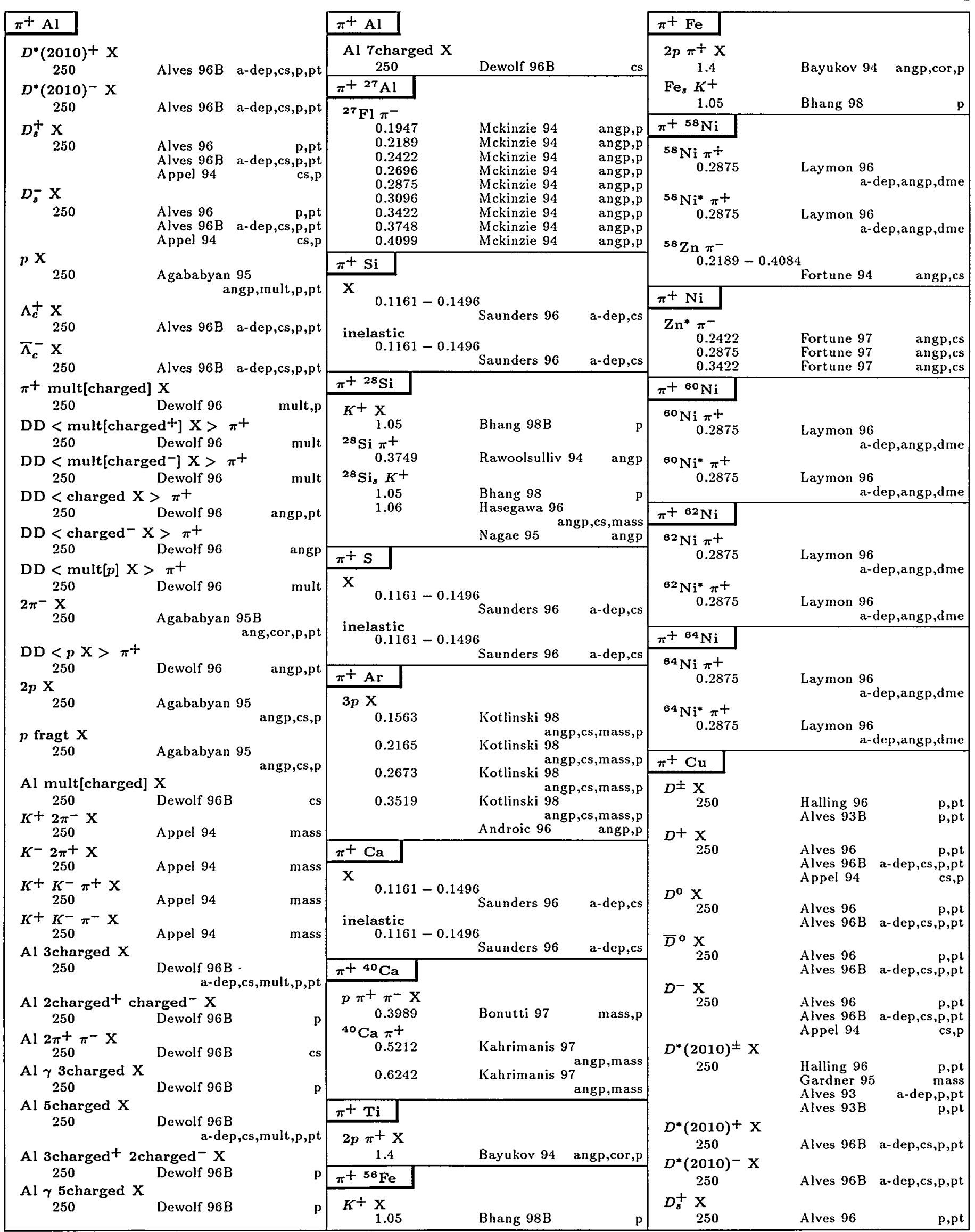

Entries are in order of beam name, then target name, then multiplicity of final state. Particle names are ordered as described in the legend on page 157 and as listed in the Particle Vocabulary. See also the Table of Contents of this Index beginning on the page 158. A few chemical symbols for nuclei have been changed to avoid ambiguity with particle names (see the Particle Vocabulary). Beam momenta are $p_{l a b}$ in GeV/c, or in parentheses $E_{\mathrm{cm}}$ in $\mathrm{GeV}$. 


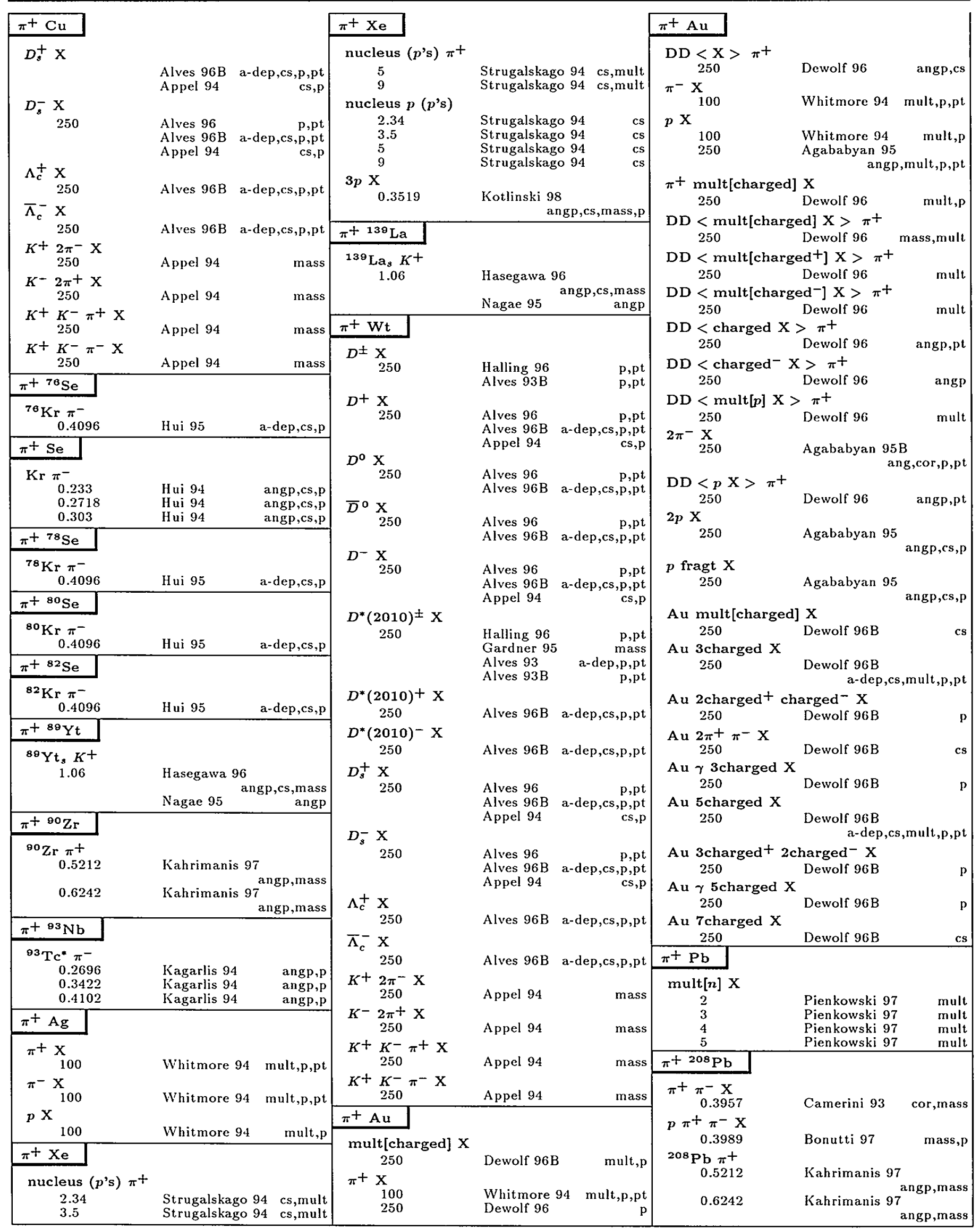




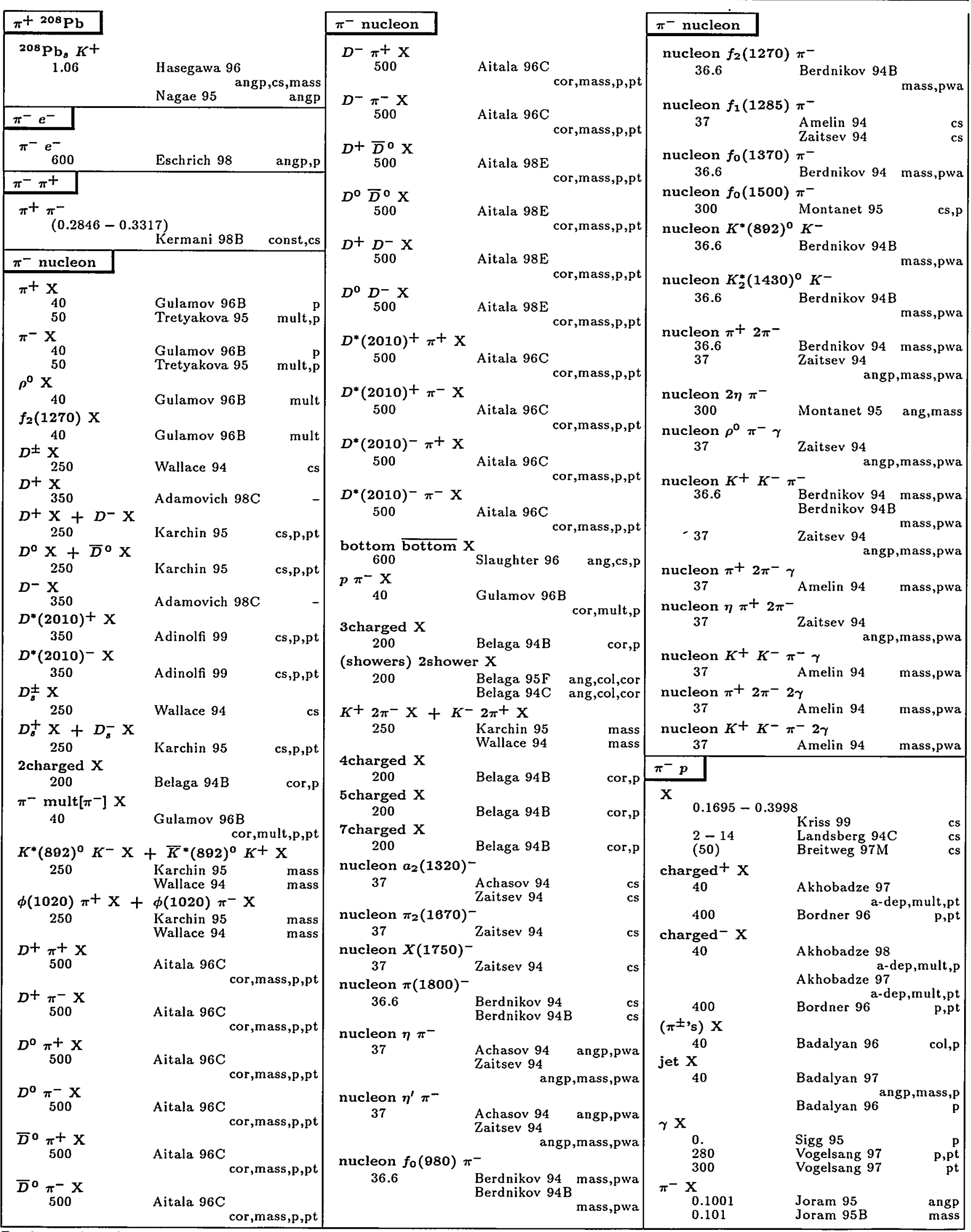

Entries are in order of beam name, then target name, then multiplicity of final state. Particle names are ordered as described in the legend on page 157 and as listed in the Particle Vocabulary. See also the Table of Contents of this Index beginning on the page 158 . A few chemical symbols for nuclei have been changed to avoid ambiguity with particle names (see the Particle Vocabulary). Beam momenta are $p_{\text {lab }}$ in GeV/c, or in parentheses $E_{\mathrm{cm}}$ in $\mathrm{GeV}$. 


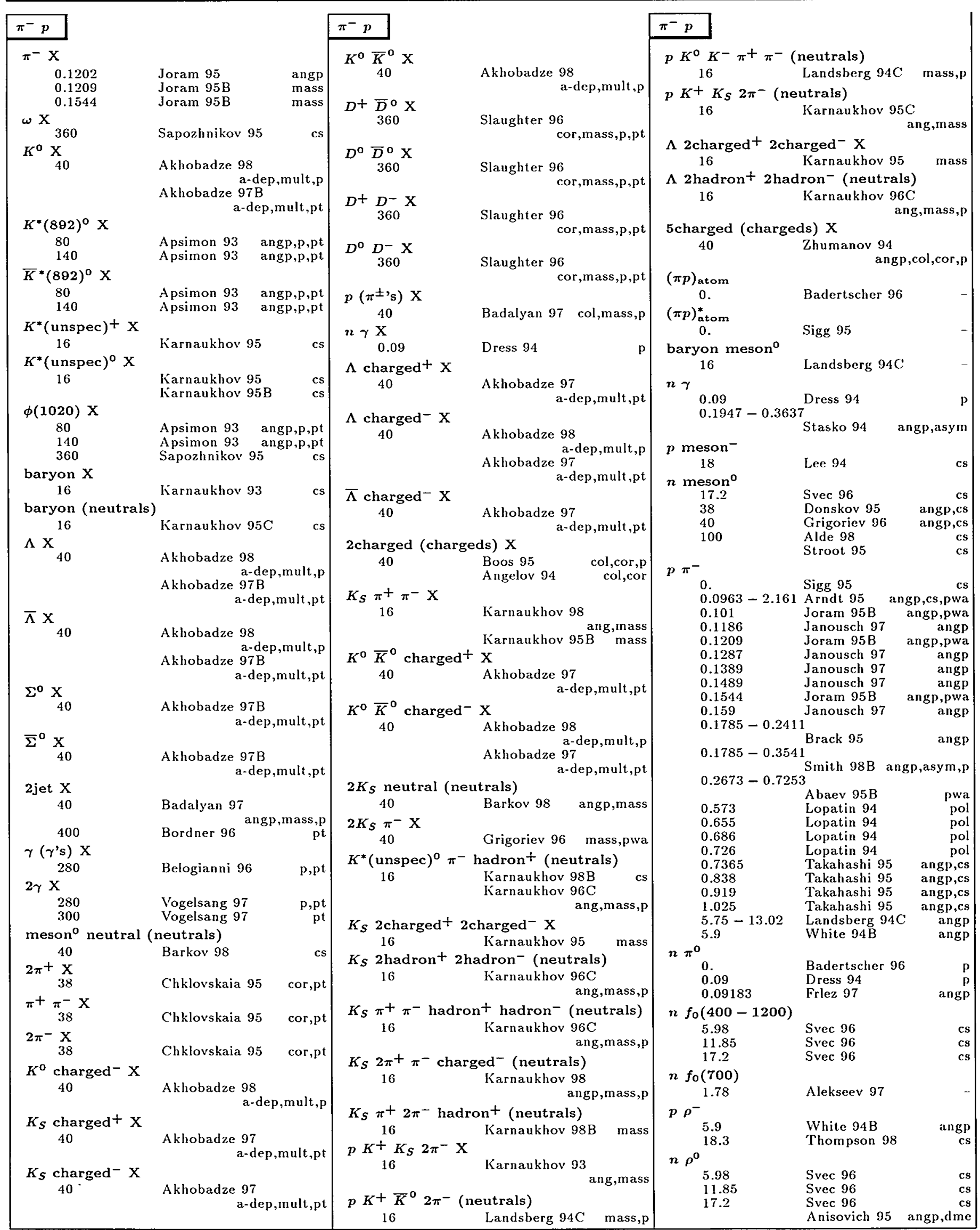




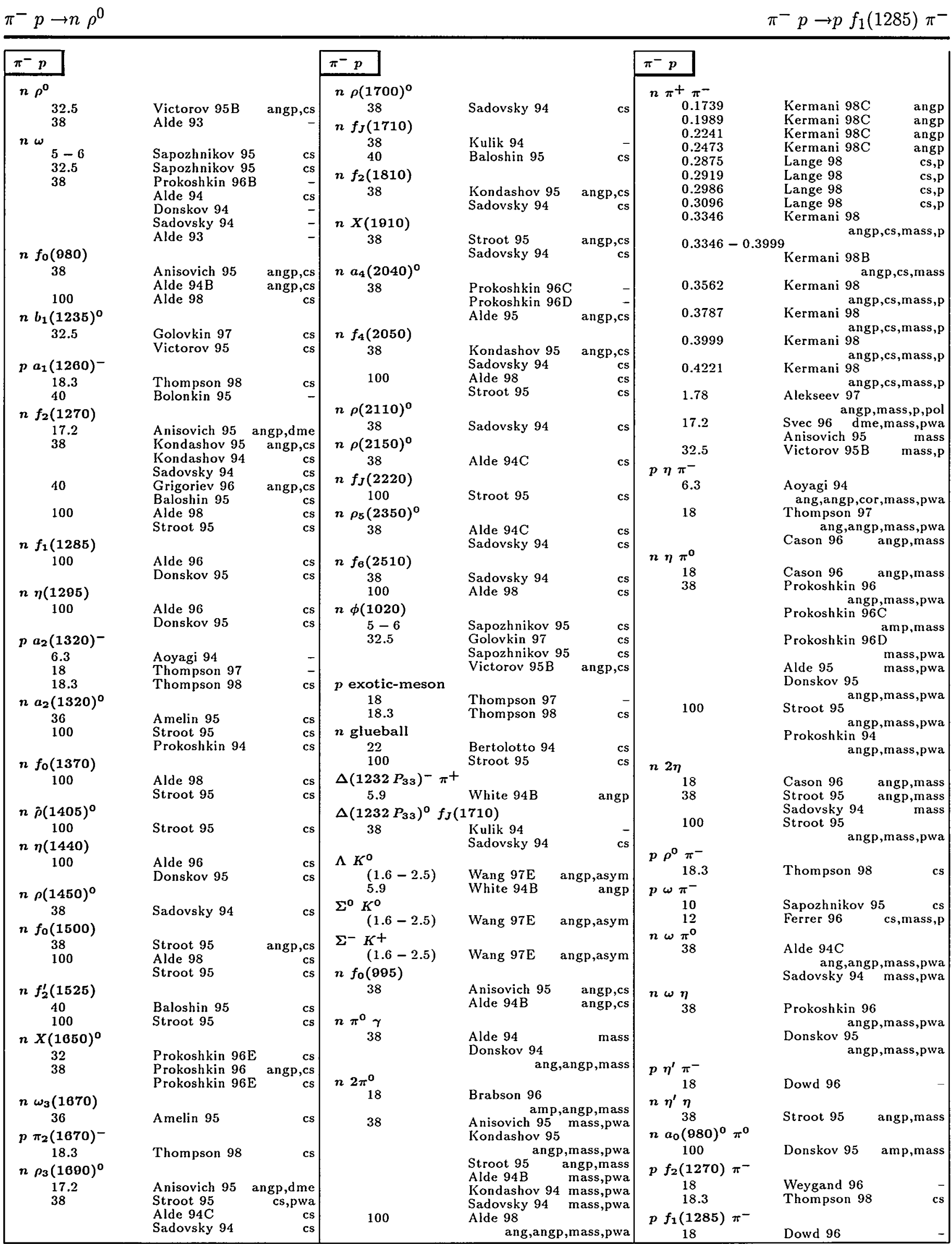




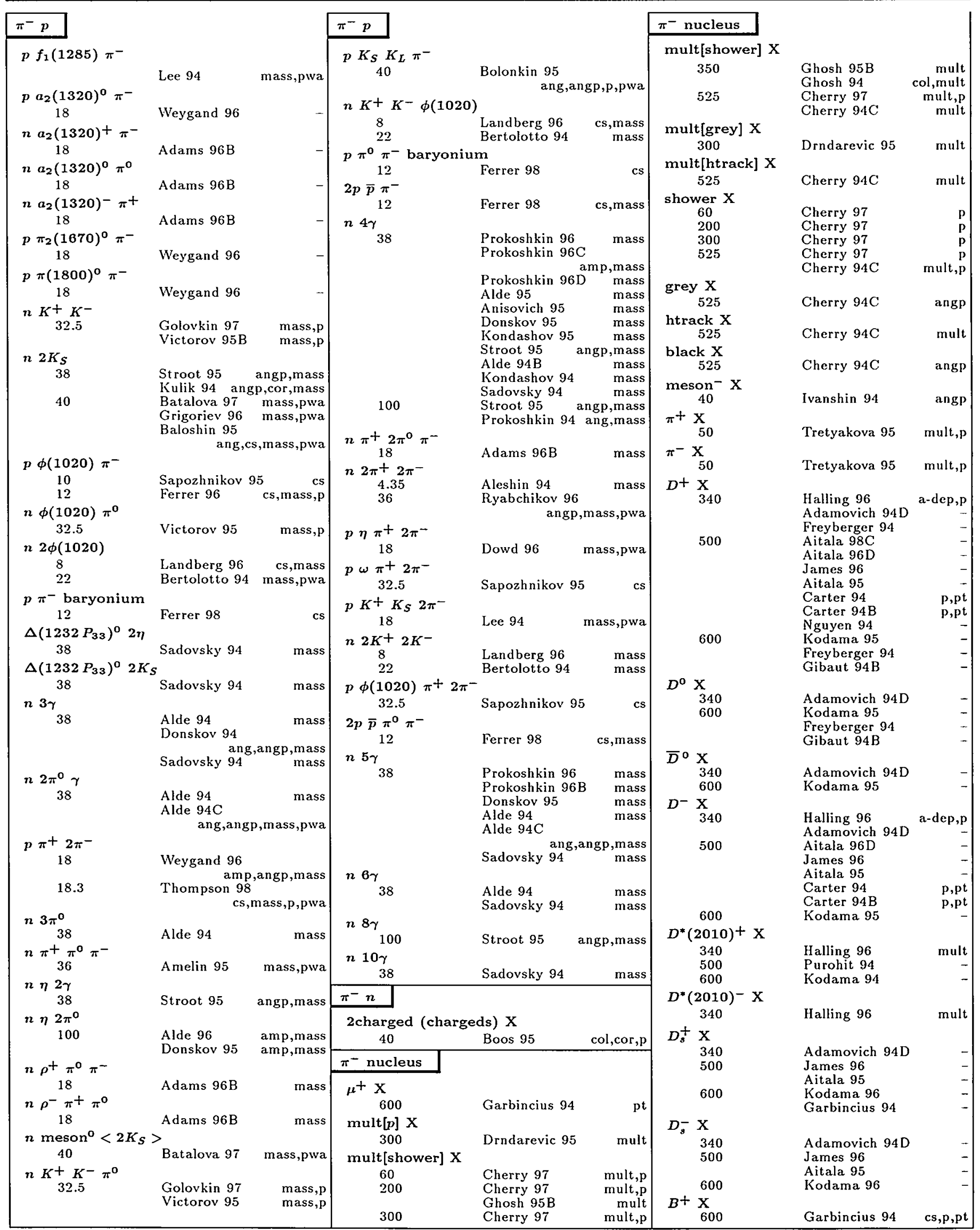




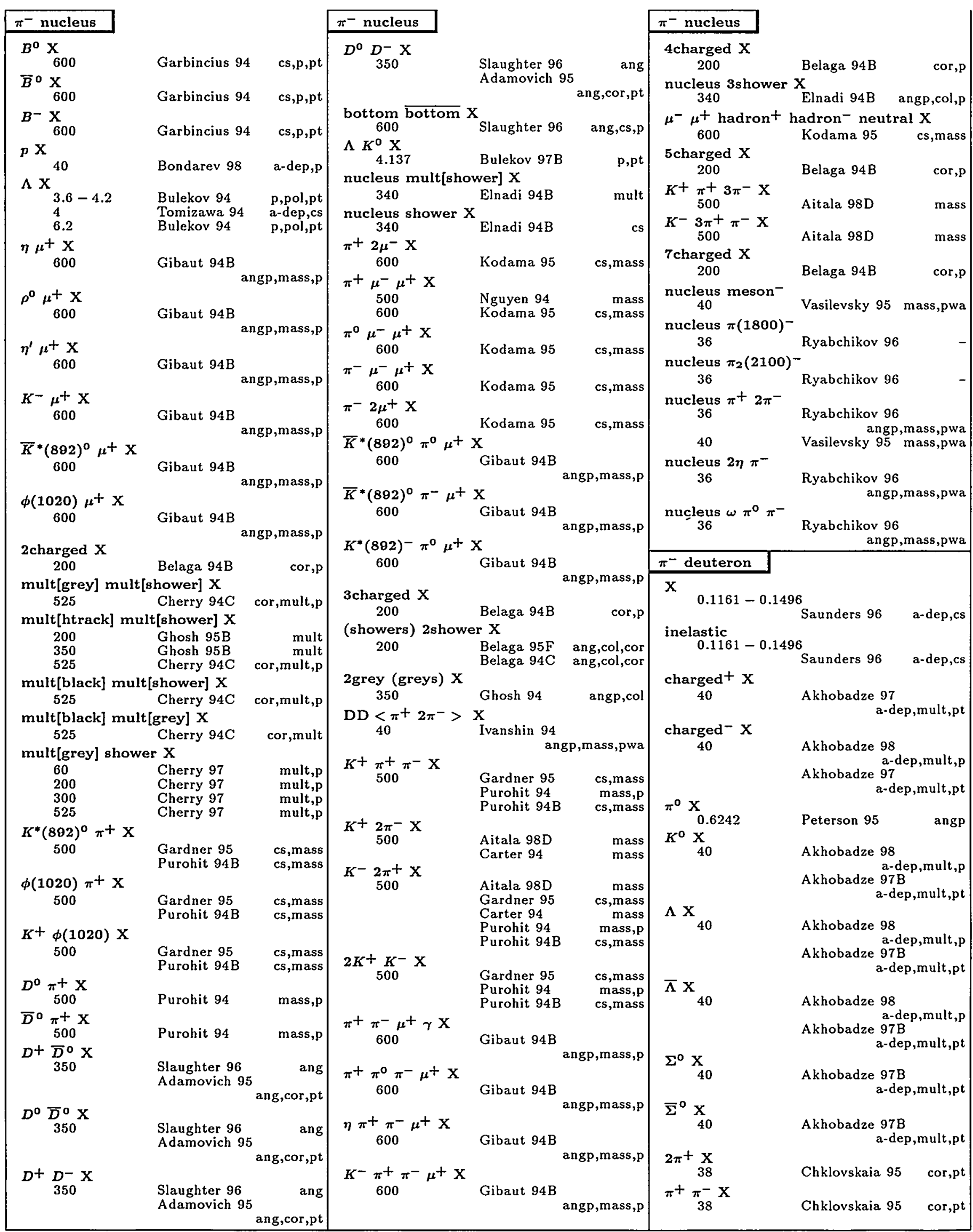




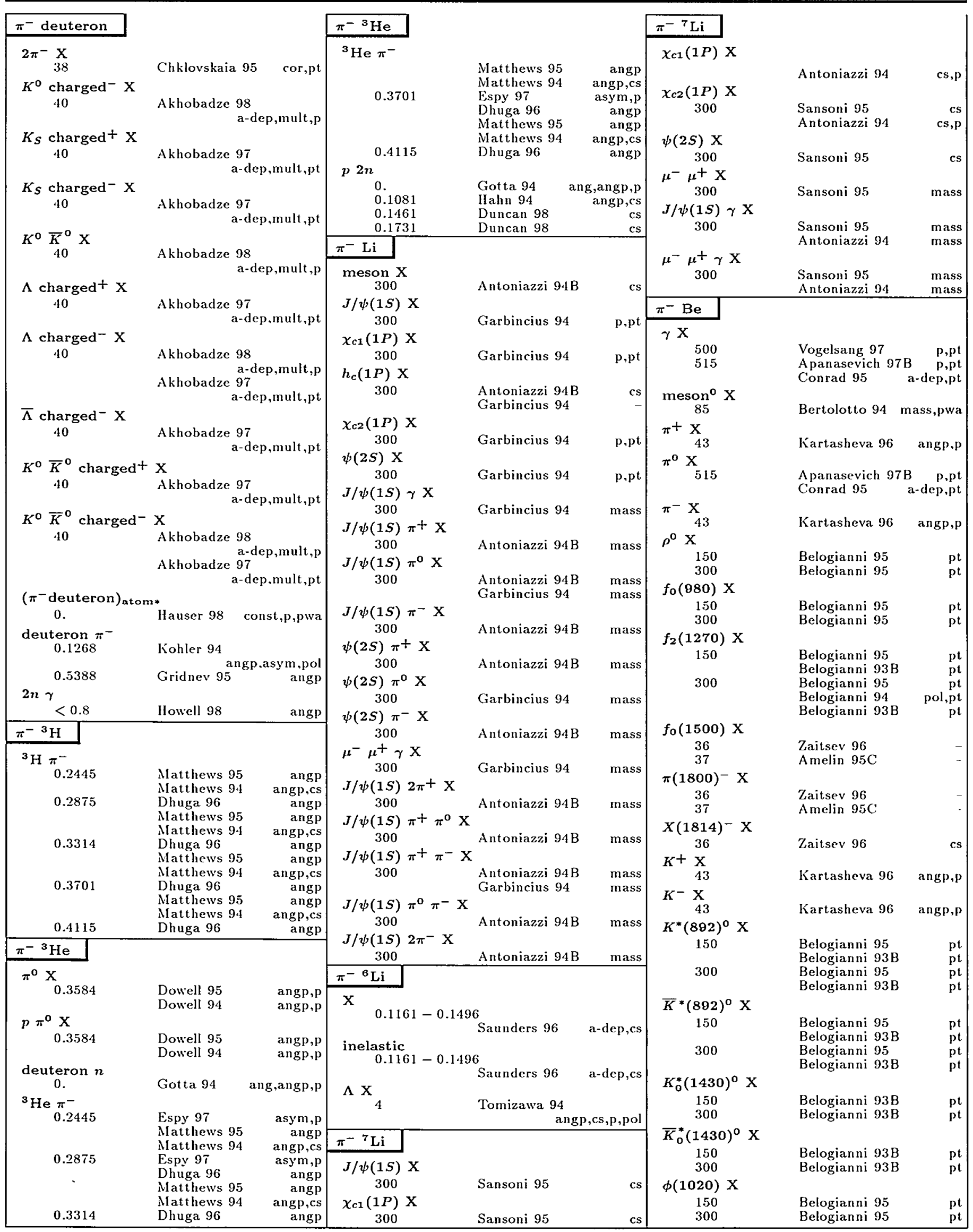




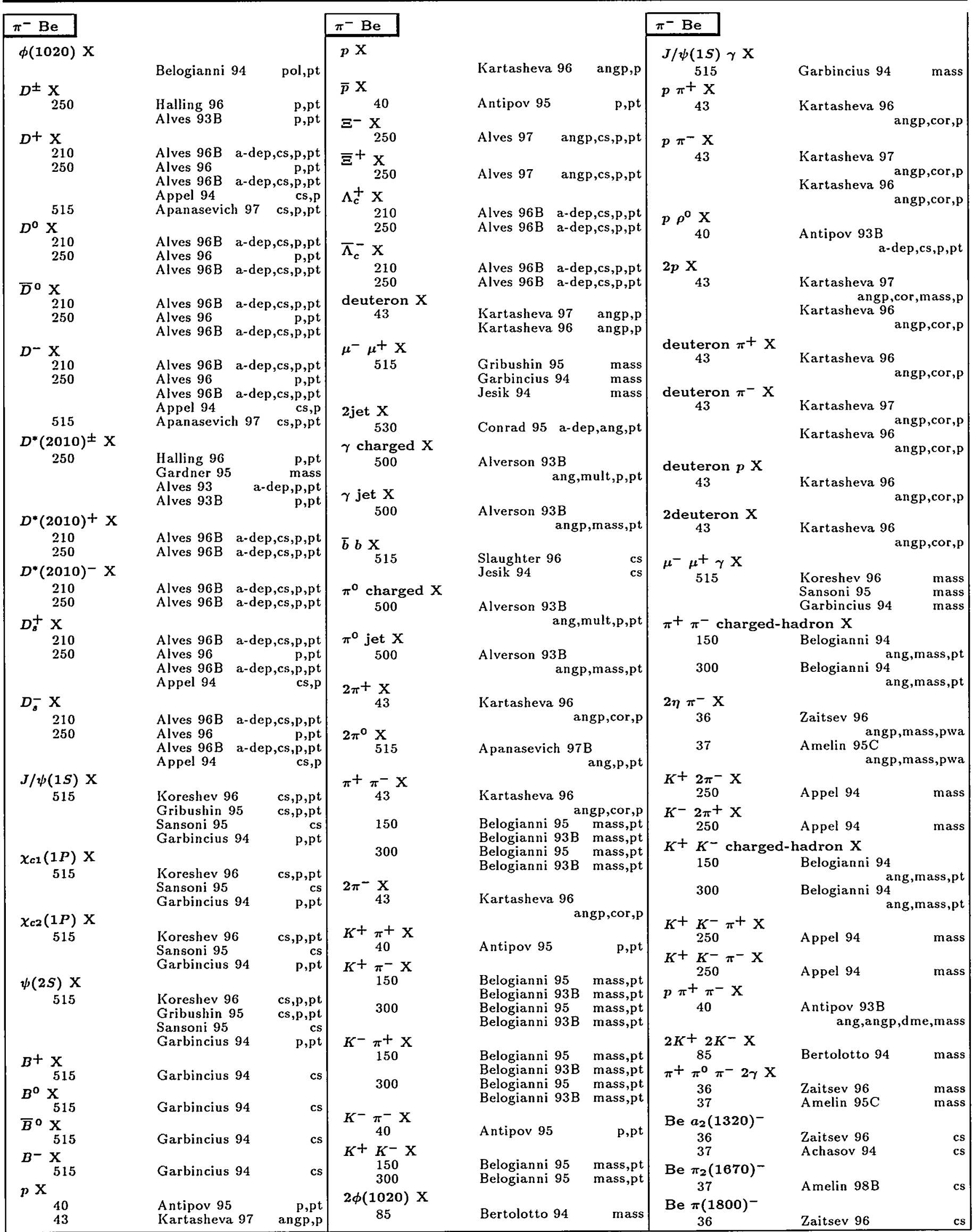

Entries are in order of beam name, then target name, then multiplicity of final state. Particle names are ordered as described in the legend on page 157 and as listed in the Particle Vocabulary. See also the Table of Contents of this Index beginning on the page 158. A few chemical symbols for nuclei have been changed to avoid ambiguity with particle names (see the Particle Vocabulary). Beam momenta are $p_{\text {lab }}$ in GeV/c, or in parentheses $E_{\mathrm{cm}}$ in $\mathrm{GeV}$. 
$\pi^{-} \mathrm{Be}$

Be $\pi(1800)^{-}$

$\mathrm{Be} a_{4}(2040)^{-}$ 37

Be exotic-meson 36

$\mathrm{Be} \eta \pi^{-}$ 36

Be $\eta^{\prime} \pi^{-}$

36

Be $f_{0}(980) \pi^{-}$ 36

$\mathrm{Be} f_{2}(1270) \pi^{-}$

36
$\operatorname{Be} K^{*}(892)^{\circ} K^{-}$

Be $K_{2}^{*}(1430)^{\circ} K^{-}$

Be $\pi^{+} 2 \pi-$

36 Zaitsev 96

40

Amelin 95B

Amelin 95B

Amelin 98B

Zaitsev 96

Zaitsev 96

A melin 98

Zaitsev 96 angp,pwa

Zaitsev 96

Zaitsev 96 mass,pwa

Zaitsev 96

Zaitsev 96

Zaitsev 96

$\mathrm{DD} \underset{40}{<\pi^{+}} 2 \pi^{-}>\underset{\text { Ivanshin } 94}{\mathrm{Be}}$

$\operatorname{Be} \omega \pi^{0} \pi^{-}$

Amelin $98 \mathrm{~B}$

angp,mass,pwa

$\mathrm{Be} K^{+} K^{-} \pi^{-}$

Be $\pi^{+} \pi^{\circ} 2 \pi^{-}$

Be $\pi_{36}^{+} \pi^{0} 2 \pi$

Be $\eta \pi^{+}+2 \pi^{-}$

Be $\pi^{+}+2 \pi^{-2 \gamma}$

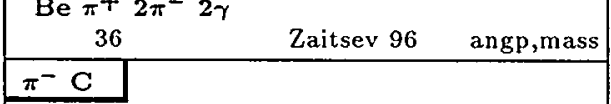

$\mathrm{X}$

$0.1161-0.1496$ Saunders $96 \quad$ a-dep,cs

inelastic

$0.1161-0.1496$

charged $+x$

40

charged- $\mathrm{X}$

40

$\left(\pi^{ \pm}, s\right) \mathrm{X}$

40

jet $\mathrm{X}$

40

${ }^{\gamma} \times{ }_{200}$

$\pi+\underset{40}{\mathrm{X}}$

$\pi^{-} \mathrm{X}$

$\rho^{\circ} \mathrm{X}$

$f_{2}(1270) X$

40

$K^{\circ} \mathrm{X}_{40}$

Saunders $96 \quad$ a-dep,cs angp,mass,pwa

Zaitsev $96 \quad$ mass,pwa

Zaitsev 96 angp,mass

Zaitsev 96

40

Akhobadze 97

a-dep, mult,pt

Akhobadze 98 a-dep,mult,p

Akhobadze 97

a-dep,mult,pt

$\boldsymbol{\Sigma}_{c}(\mathbf{2 4 5 5})++\mathbf{X}$

Badalyan 97
Badalyan 96

Vogelsang 97

Gulamov $96 \mathrm{~B}$

Gulamov 96B

Gulamov $96 \mathrm{~B}$

Gulamov $96 \mathrm{~B}$

Akhobadze 98
$\bar{\Sigma}^{0} \mathrm{X}$

$\Xi^{-} \mathrm{x}$

$\Lambda_{c}^{+} \mathrm{X}$

500

500
$2_{c}(2455)^{\circ} \mathrm{X}$

$\pi^{-} \mathrm{C}$

$K^{0} \mathrm{X}$

$D^{+} \mathrm{X}$

500

$D^{\circ} \mathrm{X}$

$\bar{D}^{\circ} \mathrm{X}$

500

$-\mathrm{X}$

500

$D^{*}(2010)+\mathrm{X}$

500

$D^{*}(2010)-\mathrm{X}$

$D_{s}^{+} \mathrm{X}$

250
500

$D_{s}^{-} \mathrm{X}$

500

$\mathrm{X}_{40}$

$\bar{\Lambda} \mathrm{X}_{40}$

$\Sigma^{\circ} \mathrm{X}$

40

0

500
${ }^{2}(2455)^{0} \mathrm{X}$

exotic-nucleon $\mathrm{X}$ 500

mult $[p]$ mult $\left[\pi^{-}\right] X$

40

2 jet $X$

40

$2 \gamma \mathrm{X}$

200

$\pi^{-} \operatorname{mult}\left[\pi^{-}\right] \mathrm{X}$

40

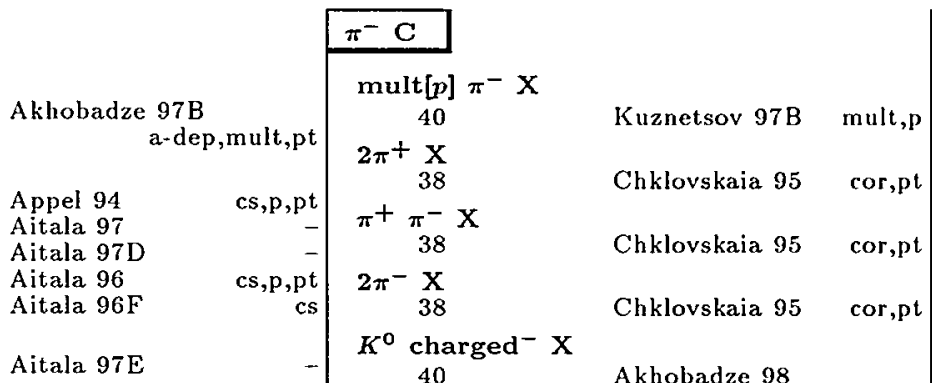

a-dep,mult,p

Aitala $97 \mathrm{E}$

Aitala $97 \mathrm{~F}$

Appel 94

Aitala 97

Aitala $97 \mathrm{D}$

Aitala 96

$\mathrm{cs}, \mathrm{p}, \mathrm{pt}$

$K_{S}$ charged $+\mathrm{X}$

40

Akhobadze 97

$K_{S}$ charged- $\mathrm{X}$ 40

a-dep, mult,pt

Aitala $96 \mathrm{~F}$

$K^{\mathrm{o}} \bar{K}^{\mathrm{o}} \mathrm{X}$

Aitala $97 \mathrm{E}$

Aitala $96 \mathrm{E}$

Aitala $97 \mathrm{E}$

Aitala $96 \mathrm{E}$

Appel 94

Aitala 98

Aitala $98 \mathrm{~B}$

Aitala $97 \mathrm{~B}$

Aitala $97 \mathrm{C}$

Appel 94

Aitala 98

Aitala $97 \mathrm{~B}$

Aitala $97 \mathrm{C}$

Akhobadze 98

a-dep,mult, $p$

Akhobadze 97B

a-dep,mult,pt

Akhobadze 98 a-dep,mult,

Akhobadze 97B

a-dep, mult,pt

Akhobadze 97B

a-dep,mult,pt

Akhobadze 97B

a-dep,mult,pt

Adamovich 97

a-dep,cs,p,pt

$2 K_{S} \mathrm{X}$

$p$ ( $\pi^{ \pm}$'s) $\mathrm{X}$

40

$p$ mult $[p] \mathrm{X}$

40
$-\quad \mathrm{X}$

40

$\Lambda$ charged ${ }^{+} \mathrm{X}$

40

$\Lambda$ charged $^{-} \mathrm{X}$

40

$\bar{\Lambda}$ charged $^{-} \mathrm{X}$

40

2charged (chargeds)

40 Boos 95

$K^{+} 2 \pi^{-} \mathrm{X}$

Angelov 94

col,cor

$K-2 \pi+\mathrm{X}$

Appel 94

$250 \quad$ Appel 94

$K^{\circ} \bar{K}^{0}$ charged $+\mathrm{X}$

$40 \quad$ Akhobadze 97

$K^{\mathbf{0}} \bar{K}^{\mathrm{o}}$ charged $^{-} \mathrm{X}$

40

Aitala $96 \mathrm{~B}$

cs,p,pt

Aitala $96 \mathrm{~B}$

cs,p,pt

$K^{+} K^{-} \pi^{+} \mathrm{X}$

Aitala $96 \mathrm{~B}$

$c s, p, p t$

$K^{+} K^{-} \pi^{-} \mathrm{X}$

Aitala $97 \mathrm{C}$

250
$p \phi(1020) \pi^{-} \mathrm{X}$

$500 \quad$ Aitala $97 \mathrm{C}$

Kuznetsov 97C

DD $<\pi^{+} 2 \pi^{-}>\mathrm{C}$

$40 \quad$ Ivanshin 94

Badalyan 97

angp,mass, $\mathrm{p}$

Vogelsang 97

cs,pt $\quad\left(\pi^{+}, \mathrm{s}\right)\left(\pi^{-\prime} \mathrm{s}\right) \mathrm{X}$

Kulakov 97

angp

Gulamov $96 \mathrm{~B}$

cor,mult,p,pt mult $\left[\pi^{+}\right]$mult $\left[\pi^{-}\right] \mathbf{X}$

Abdinov 95 mult,p,pt 


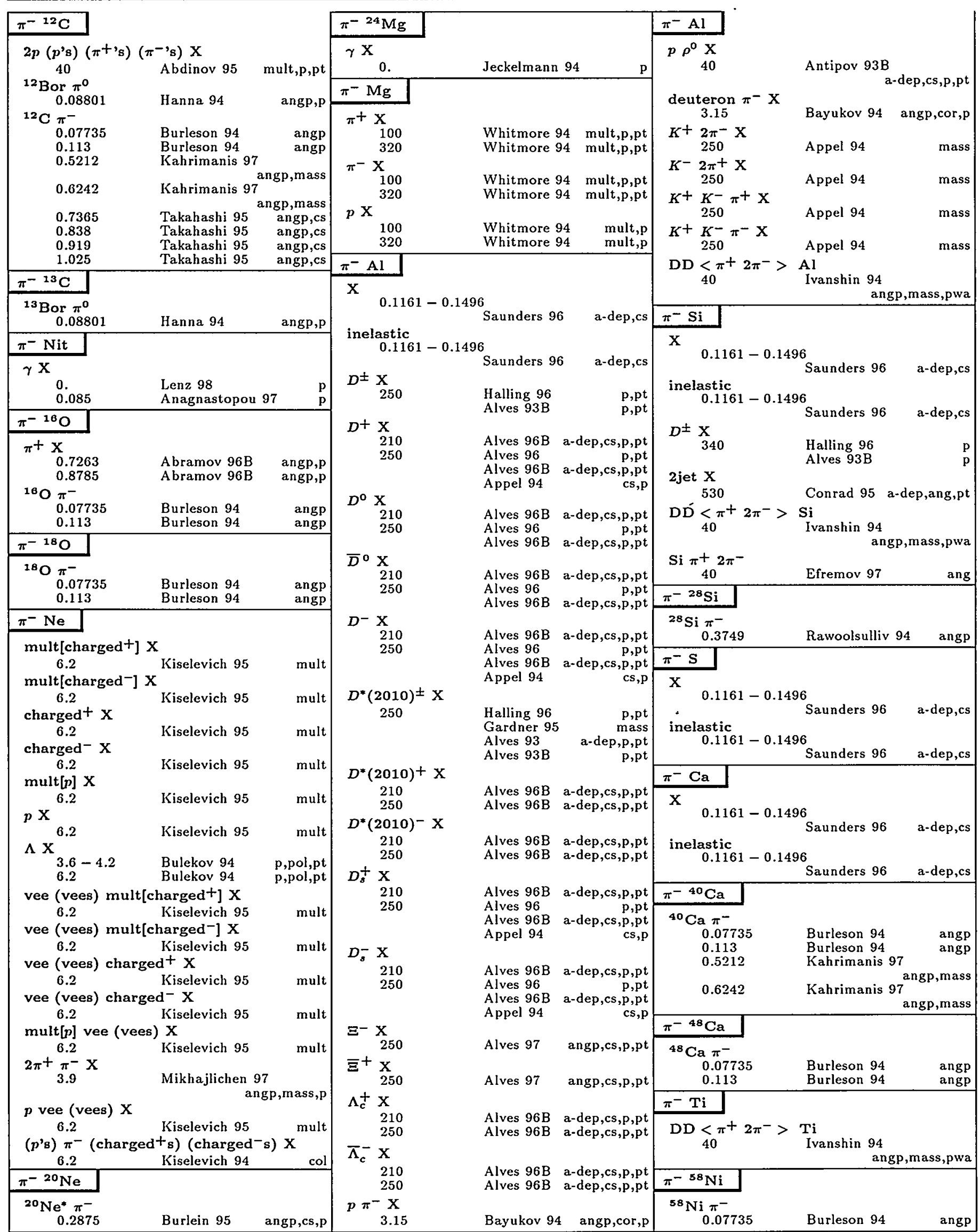

Entries are in order of beam name, then target name, then multiplicity of final state. Particle names are ordered as described in the legend on page 157 and as listed in the Particle Vocabulary. See also the Table of Contents of this Index beginning on the page 158. A few chemical symbols for nuclei have been changed to avoid ambiguity with particle names (see the Particle Vocabulary). Beam momenta are $p_{\mathrm{lab}}$ in GeV/c, or in parentheses $E_{c m}$ in $\mathrm{GeV}$. 


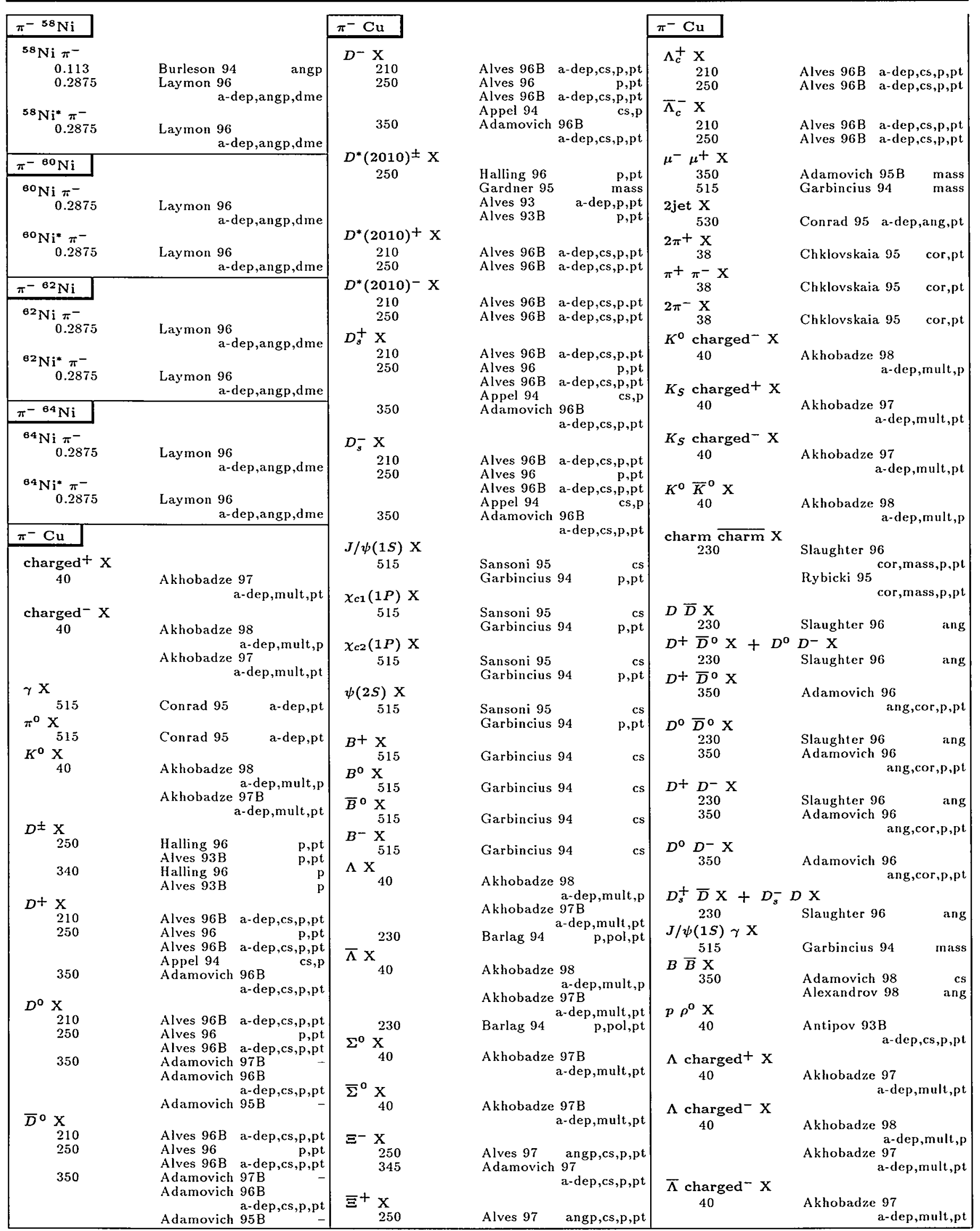




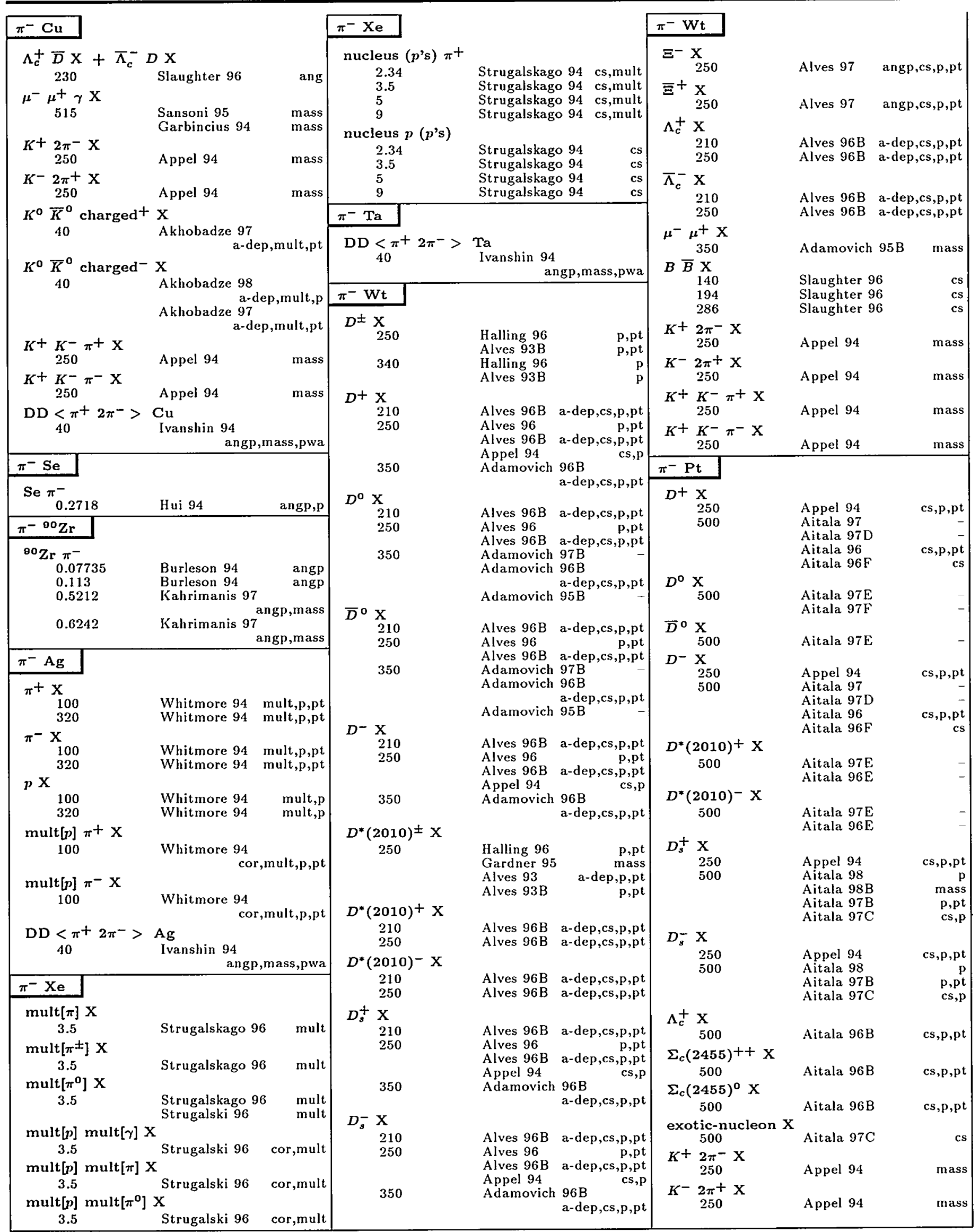

Entries are in order of beam name, then target name, then multiplicity of final state. Particle names are ordered as described in the legend on page 157 and as listed in the Particle Vocabulary. See also the Table of Contents of this Index beginning on the page 158 . A few chemical symbols for nuclei have been changed to avoid ambiguity with particle names (see the Particle Vocabulary). Beam momenta are $p_{l a b}$ in GeV/c, or in parentheses $E_{\mathrm{cm}}$ in $\mathrm{GeV}$. 


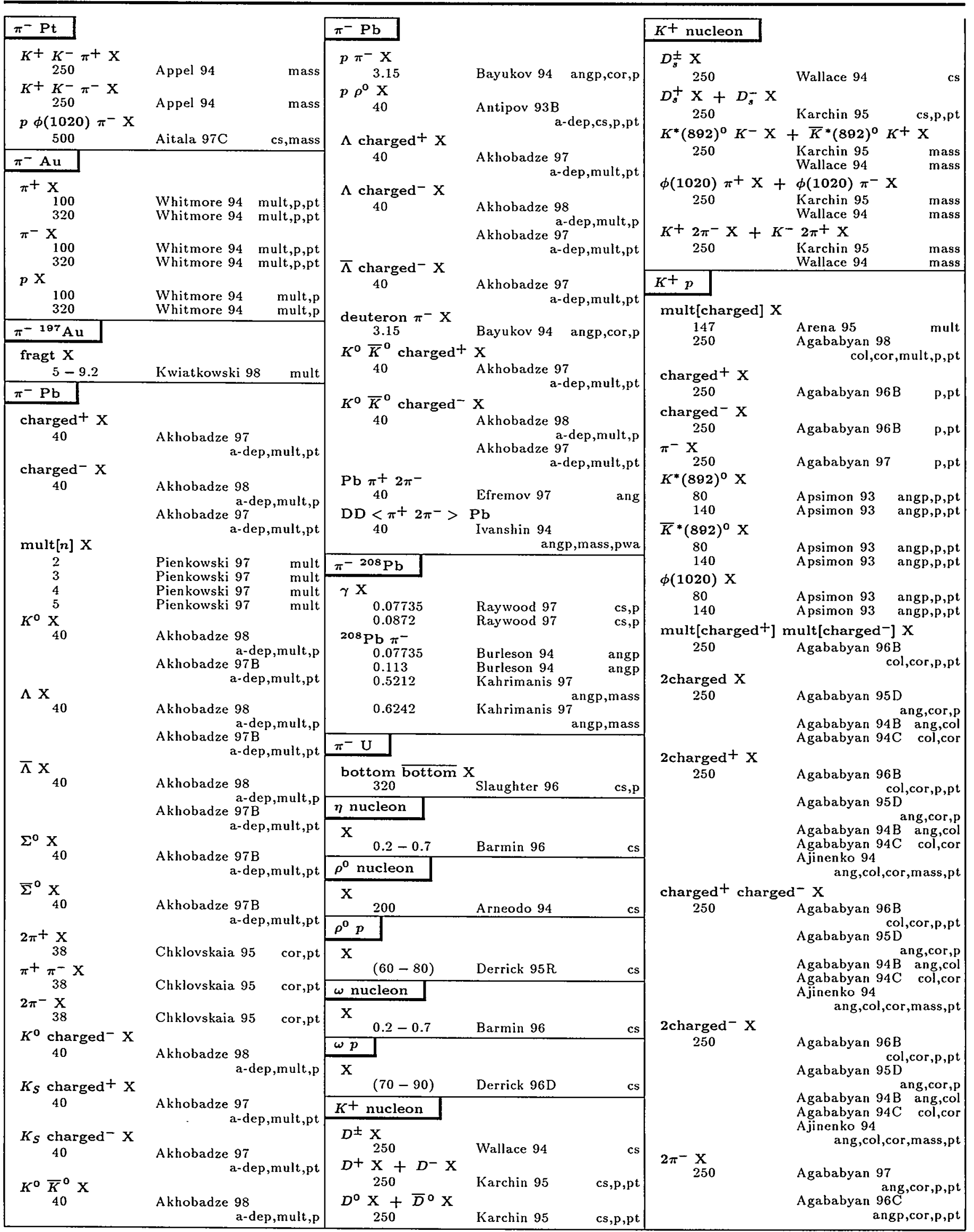




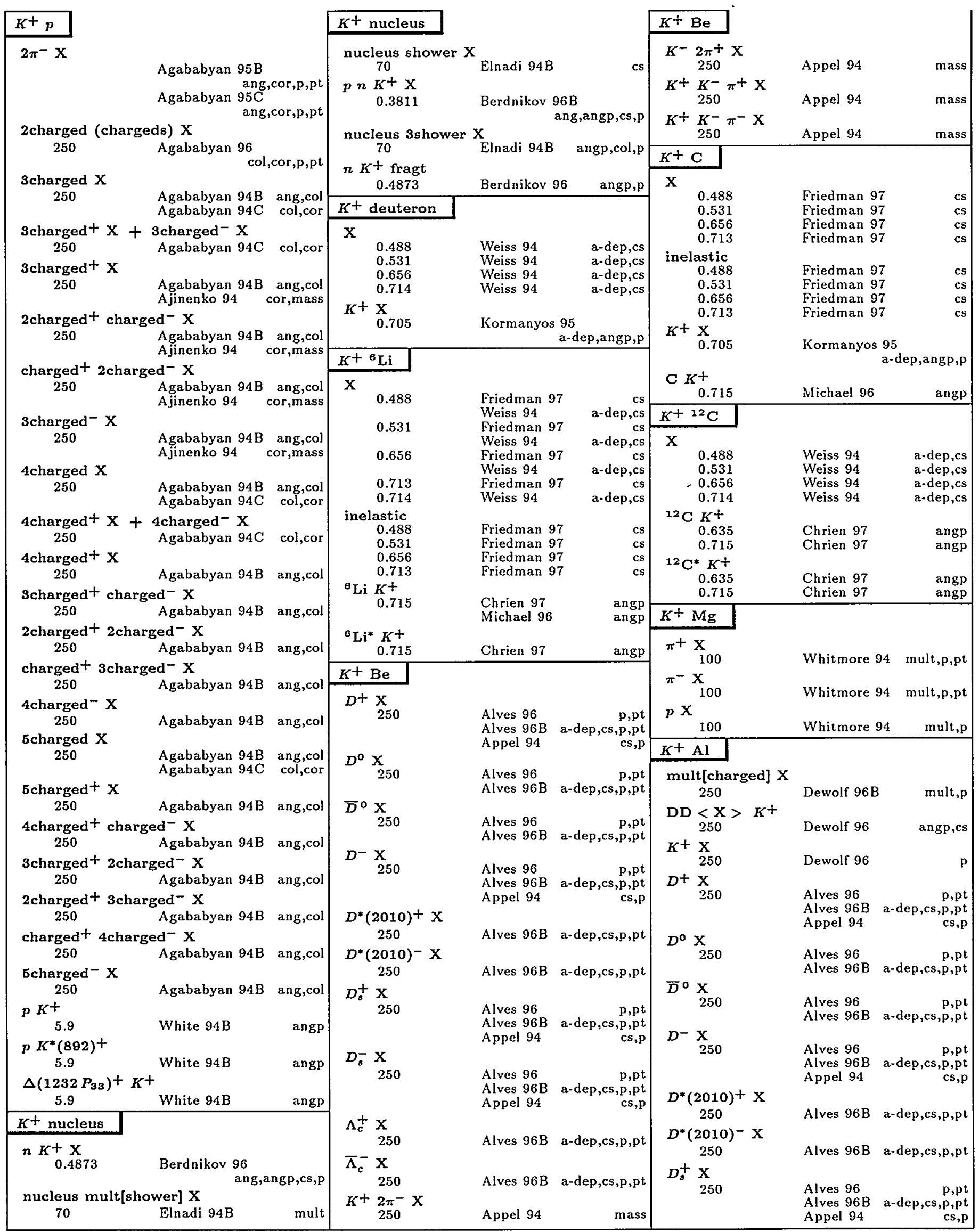

Entries are in order of beam name, then target name, then multiplicity of final state. Particle names are ordered as described in the legend on page 157 and as listed in the Particle Vocabulary. See also the Table of Contents of this Index beginning on the page 158. A few chemical symbols for nuclei have been changed to avoid ambiguity with particle names (see the Particle Vocabulary). Beam momenta are $p_{\text {lab }}$ in GeV/c, or in parentheses $E_{\mathrm{cm}}$ in $\mathrm{GeV}$. 


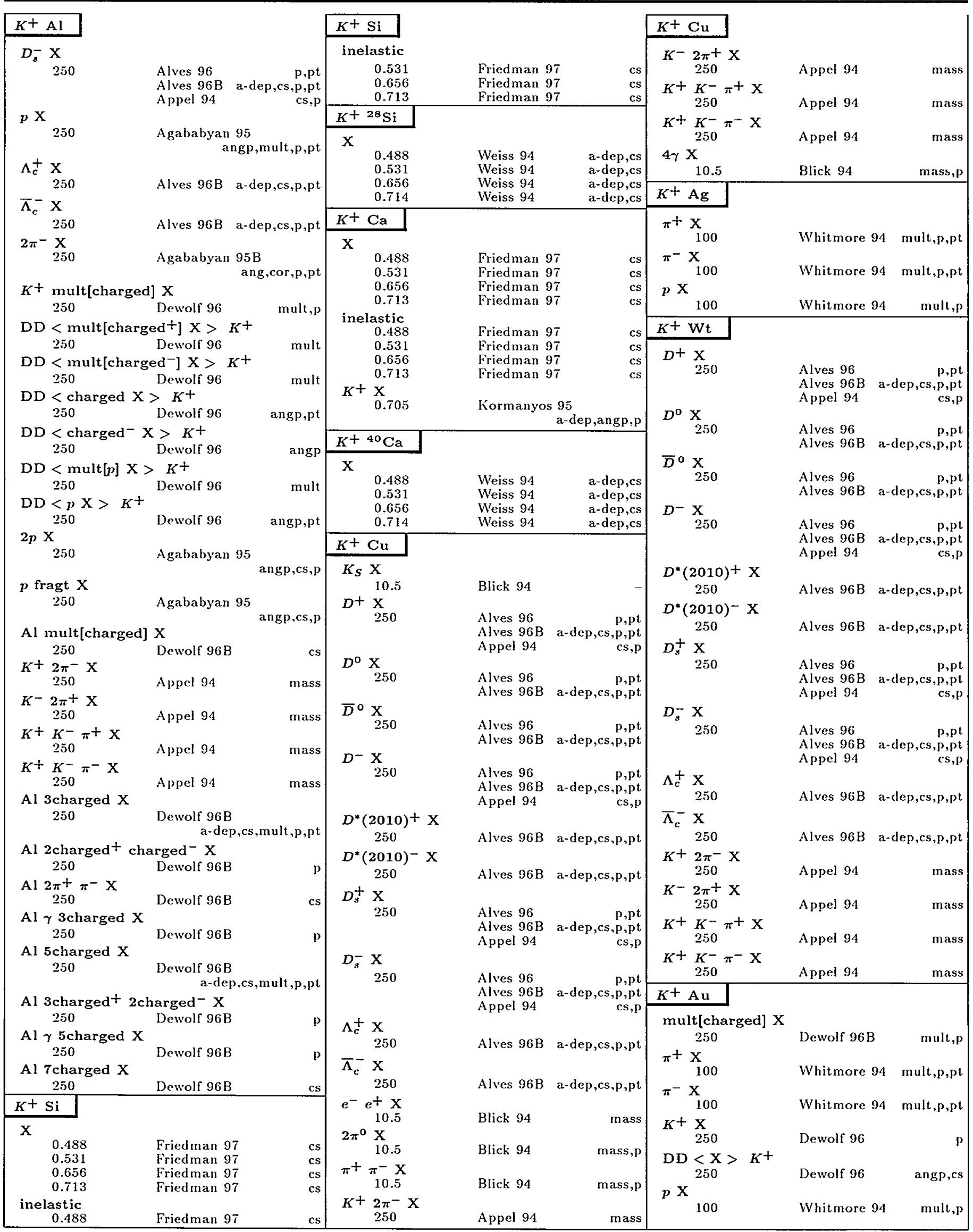




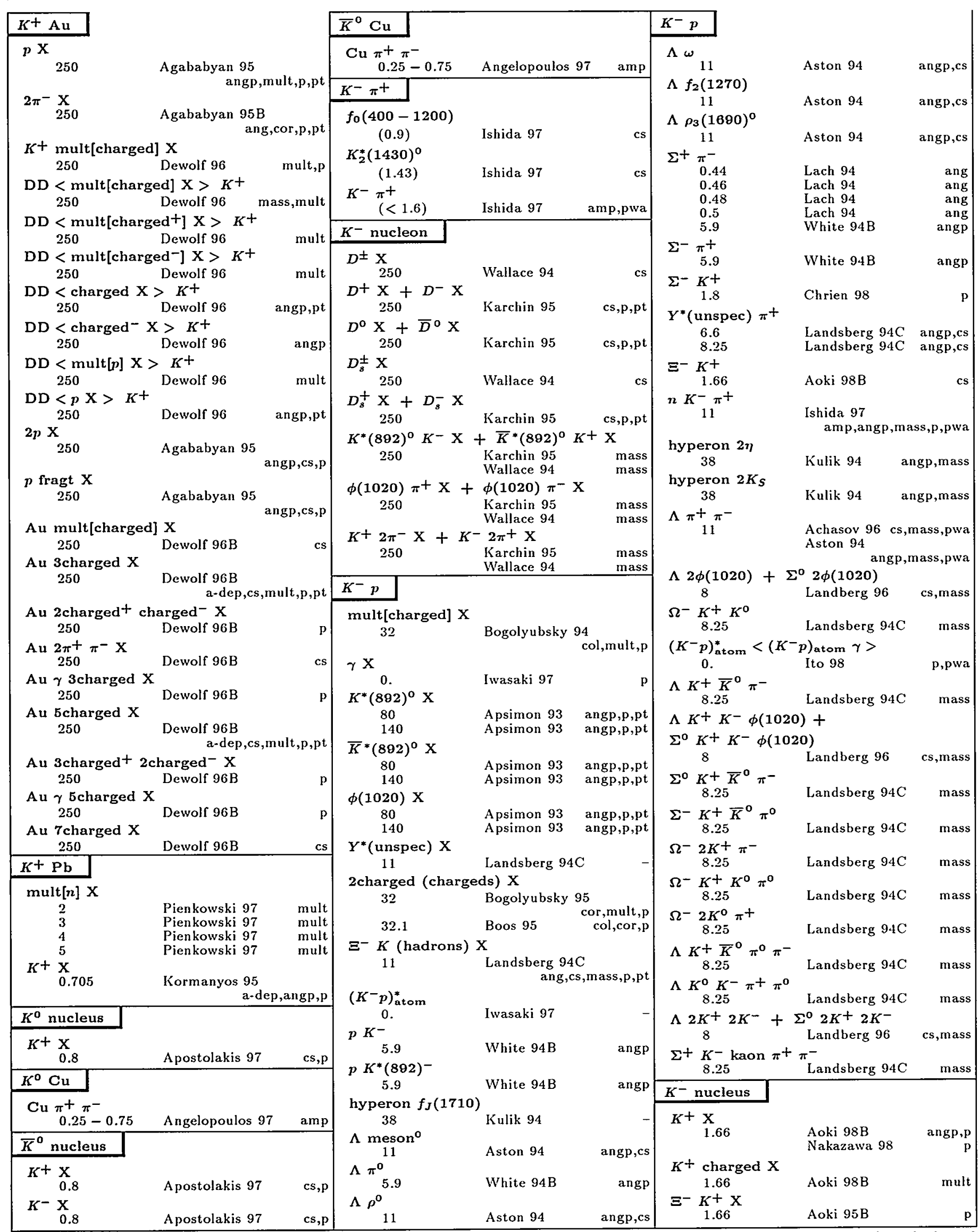

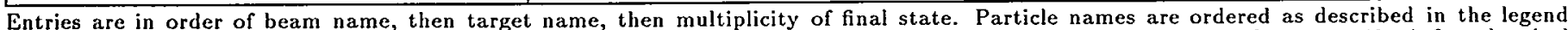

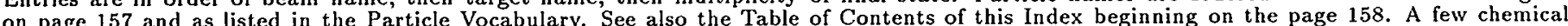

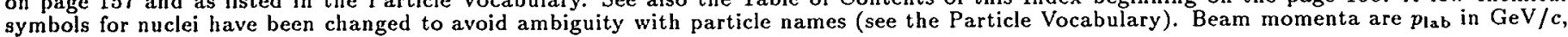
or in parentheses $E_{\mathrm{cm}}$ in $\mathrm{GeV}$. 
$K^{-}$nucleus $\rightarrow$ nucleus mult[shower] $\mathrm{X}$

$K^{-} \mathrm{Cu} \rightarrow D^{*}(2010)^{-} \mathrm{X}$

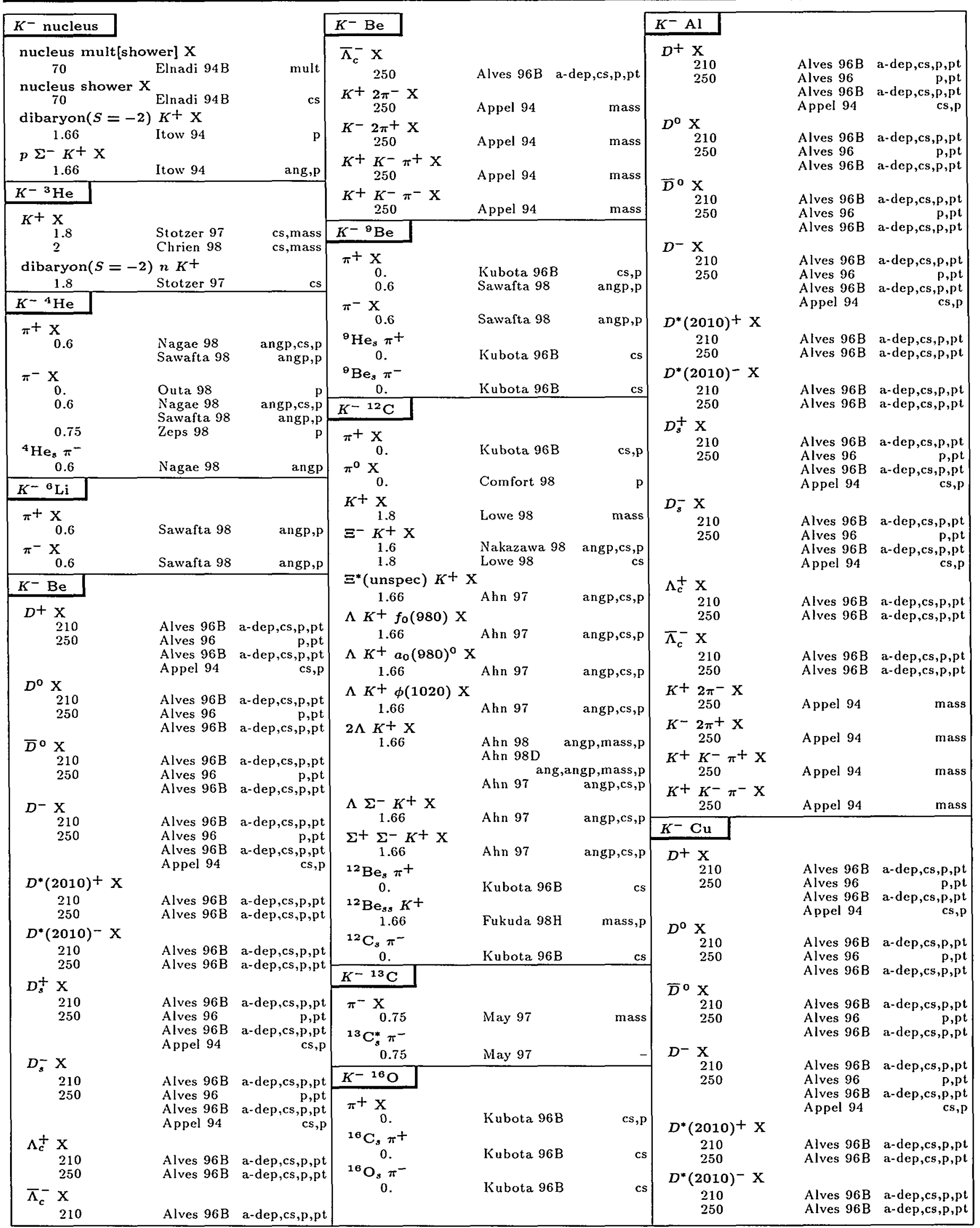




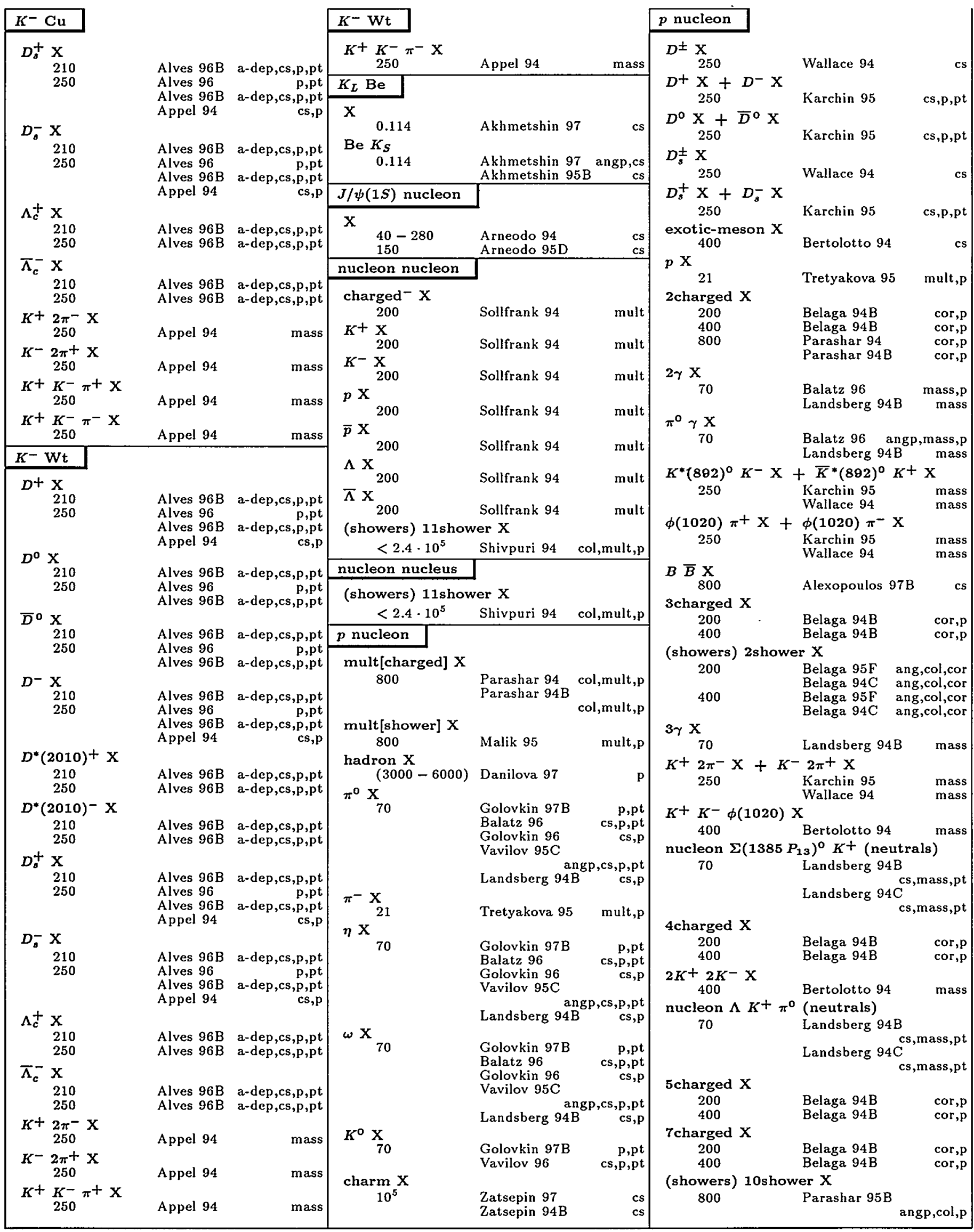

Entries are in order of beam name, then target name, then multiplicity of final state. Particle names are ordered as described in the legend on page 157 and as listed in the Particle Vocabulary. See also the Table of Contents of this Index beginning on the page 158. A few chemical symbols for nuclei have been changed to avoid ambiguity with particle names (see the Particle Vocabulary). Beam momenta are $p_{1 a b}$ in GeV/c, or in parentheses $E_{\mathrm{cm}}$ in $\mathrm{GeV}$. 


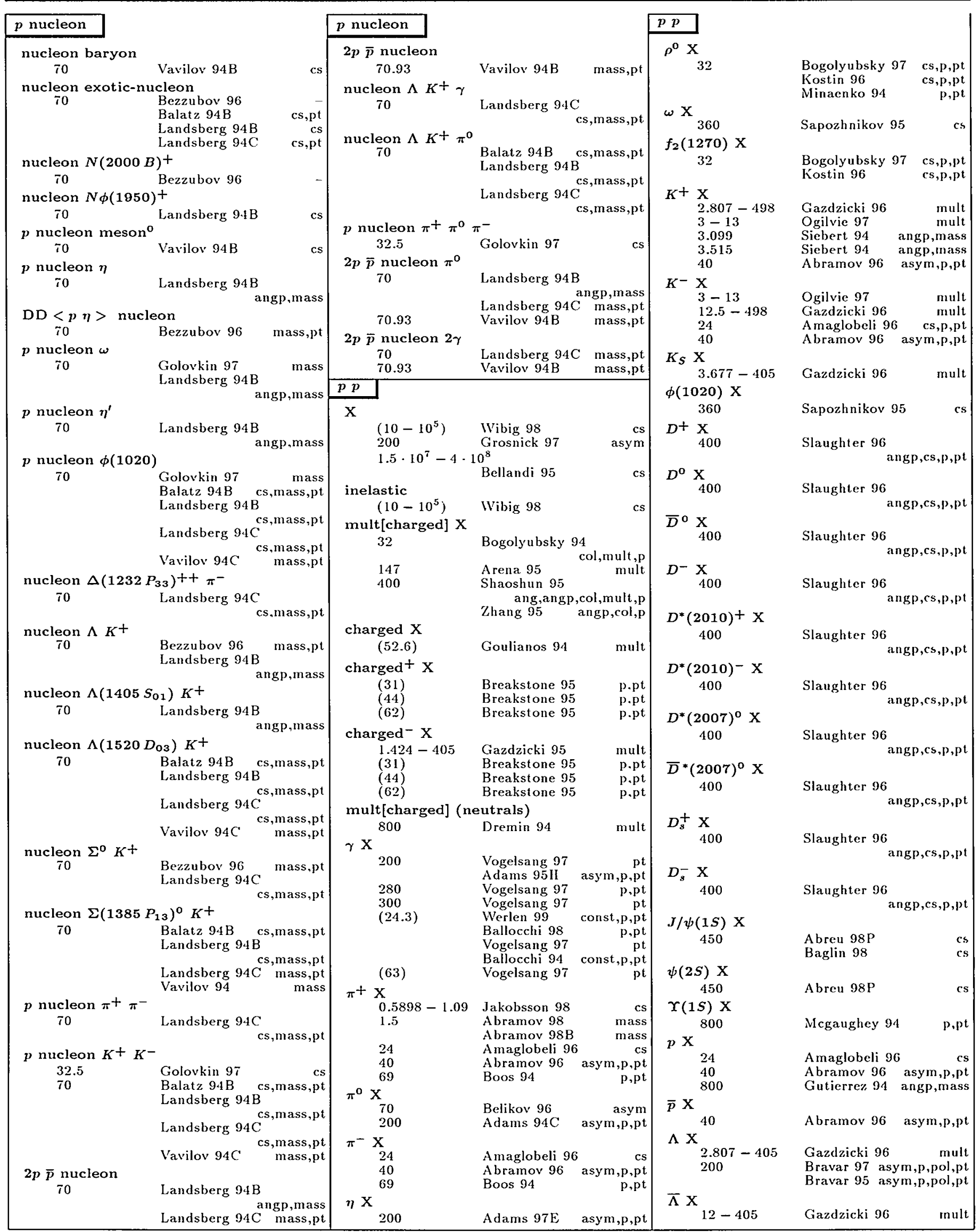




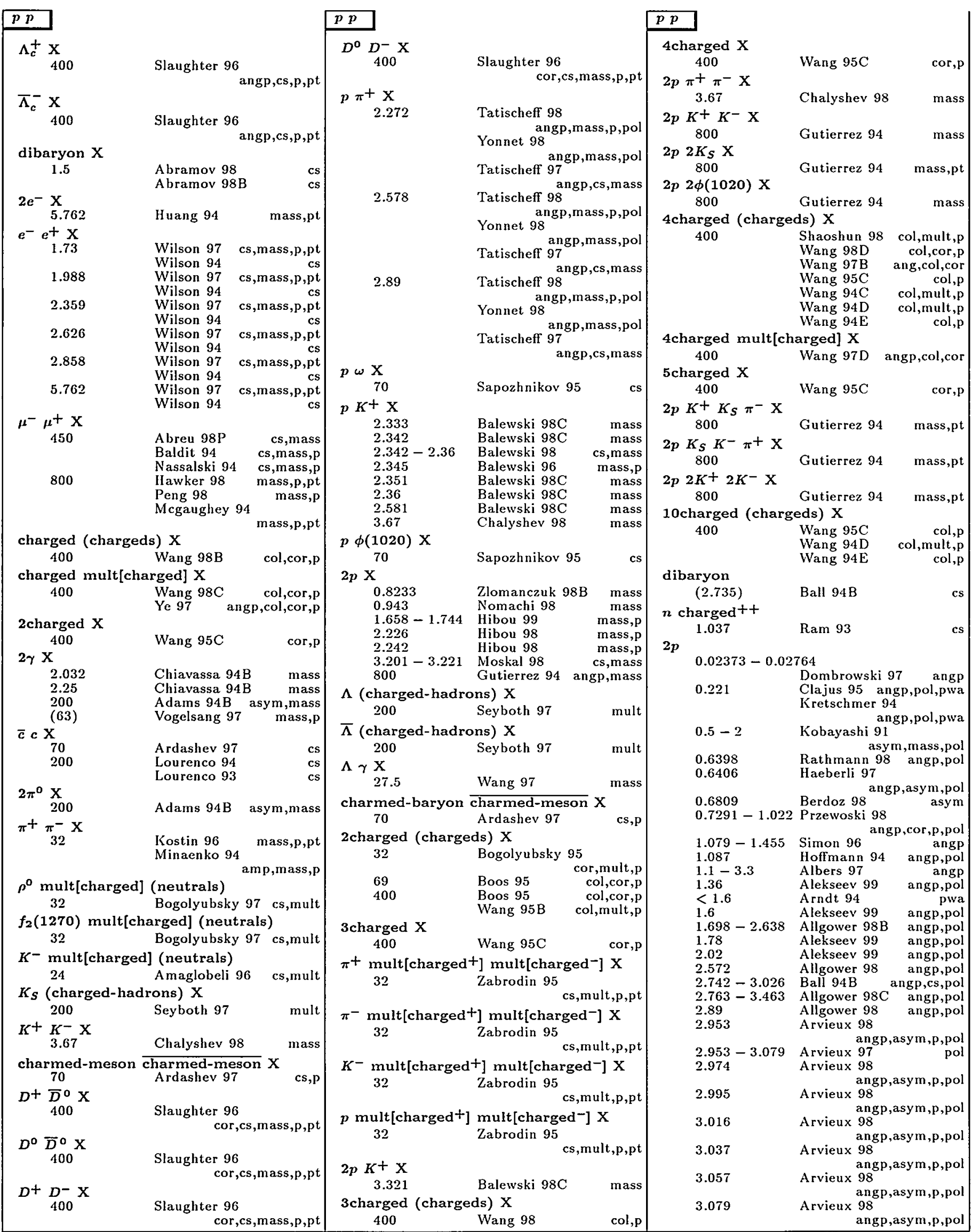

Entries are in order of beam name, then target name, then multiplicity of final state. Particle names are ordered as described in the legend on page 157 and as listed in the Particle Vocabulary. See also the Table of Contents of this Index beginning on the page 158. A few chemical symbols for nuclei have been changed to avoid ambiguity with particle names (see the Particle Vocabulary). Beam momenta are $p_{\text {lab }}$ in GeV/c, or in parentheses $E_{\mathrm{cm}}$ in $\mathrm{GeV}$. 


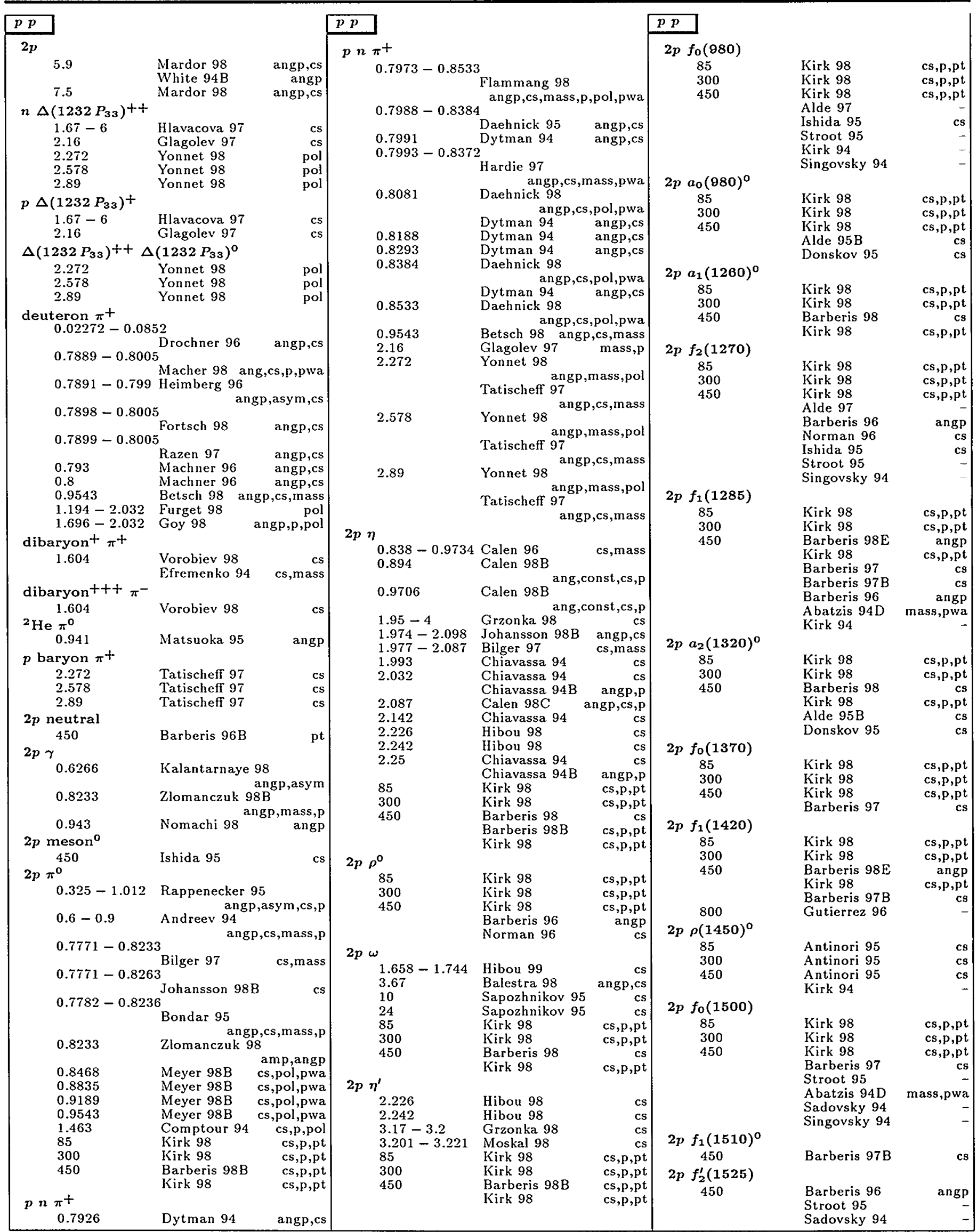


$p p$

$2 p f_{2}^{\prime}(1525)$

$2 p f_{2}(1565)$

85
300
450

$2 p \eta_{2}(1645)$

85

300

450

$2 p \pi_{2}(1670)^{\circ}$

85

300

450

$2 p f_{J}(1710)$

85

300

450

$2 p \eta_{2}(1870)$

85
300

450

$2 p f_{2}(1950)$

85

300

450

$2 p X(2000)$

450

$2 p f_{0}(2020)$

450

$2 p f_{2}(2150)$

450

$2 p f_{0}(2200)$

85

300

450

$2 p \phi(1020)$

$1.658-1.744$ Hibou 99

3.67

10

24
85

300

450

$2 p$ glueball

450

p $\wedge \mathrm{K}^{+}$

$2.32-2.36$

$2.342-2.36$

Balewski 98C

Brauksiepe 97

2.345

Balewski 96

(2.551 - 2.561) Sewerin 98B

$2.5 \quad$ Bilger 98

$2.5-3.7$
2.75

Grzonka 97

3.67

Grzonka 98

Bilger 98

Grzonka 97

Chalyshev 98

p $\boldsymbol{\Sigma}^{\circ} K^{+}$

$(2.625-2.638)$ Sewerin $98 \mathrm{~B}$ Grzonka 98

\section{$p p$}

\begin{tabular}{|c|c|}
\hline & $\begin{array}{c}\left.p \underset{70}{\Sigma(1385} P_{13}\right)^{\circ} K^{-} \\
\end{array}$ \\
\hline $\mathrm{cs}, \mathrm{p}, \mathrm{pt}$ & $\begin{array}{c}2 p(\pi \pi)_{L=0} \\
450\end{array}$ \\
\hline $\mathrm{cs}, \mathrm{p}, \mathrm{pt}$ & $2 p e^{-} e^{+}$ \\
\hline $\begin{array}{l}\mathrm{cs}, \mathrm{p}, \mathrm{pt} \\
\mathrm{cs}\end{array}$ & 0.6266 \\
\hline$c s, p, p t$ & $2 p 2 \gamma$ \\
\hline $\begin{array}{l}\mathrm{cs}, \mathrm{p}, \mathrm{pt} \\
\mathrm{cs}, \mathrm{p}, \mathrm{pt}\end{array}$ & $\begin{array}{c}450 \\
2 p 2 \pi^{\circ}\end{array}$ \\
\hline & 450 \\
\hline $\mathrm{cs}, \mathrm{p}, \mathrm{pt}$ & \\
\hline $\begin{array}{l}\mathrm{cs}, \mathrm{p}, \mathrm{pt} \\
\mathrm{cs} \\
\mathrm{cs}, \mathrm{p}, \mathrm{pt}\end{array}$ & $\begin{array}{r}2 p \pi^{+} \pi^{-} \\
1.403\end{array}$ \\
\hline$c s, p, p t$ & 1.604 \\
\hline $\begin{array}{r}c s, p, p t \\
c s, p, p t \\
\text { angp }\end{array}$ & $\begin{array}{l}85 \\
300 \\
(28) \\
450\end{array}$ \\
\hline
\end{tabular}

Balatz 93

Alde 97

Messchendorp 98

$2 p 2 \phi(1020)$

85

Messchendorp 98 mass

Abazov 96 cor,mass, $\mathrm{p}$

Barberis 98B mass

Alde 97 angp,mass, $p \quad 2 p \Lambda \bar{\Lambda}$

Donskov 95 mass $\quad 27.5$

Ishida 95 mass 450

Stroot 95 mass, $p$

\begin{tabular}{ll|l} 
Singovsky $94 \quad$ mass, $\mathrm{p}$ & $2 p \pi^{+} \pi^{\mathrm{o}} \pi^{-}$
\end{tabular}

450

Bilger 98B

Brodowski 96

Vorobiev 98

cs,mass

Kirk 98 mass

Kirk 98 mass

Norman 96 angp,mass

Kirk 98

Barberis 96 angp,mass

Barberis 96B mass,pt

Kirk 94

mass,pt

$2 p \eta \pi^{\circ}$

450

Alde 95B

Donskov 95 amp,mass

$2 p 2 \eta$

450

Stroot 95

Sadovsky 94

Singovsky 94

mass, $p$

cs

cs

$2 p 2 \rho^{\circ}$

300

450

$2 p \rho^{+} \rho^{-}$

300

$2 p \omega \rho^{\circ}$

300

$2 p 2 \omega$

300

Girone 94

ang,cs, mass,pwa
Abatzis $94 \mathrm{D}$ mass,pwa

Girone 94

ang,cs,mass,pwa

$2 p \eta \pi^{+} \pi^{-}$

450

$2 p \rho^{0} \pi^{+} \pi^{-}$

85

300

450

,

$2 p \omega \pi^{+} \pi^{-}$

24

$2 p f_{2}(1270) \pi^{+} \pi^{-}$

85

300

An

$$
450
$$

cs,mass,pwa

Antinori 95 cs, mass,pwa

$2 p f_{0}(1500) \pi^{+} \pi$

$$
\begin{aligned}
& 85 \\
& 300 \\
& 450
\end{aligned}
$$

$2 p K^{+} K_{S} \pi^{-}$

27.5

450

Girone 94

800

Antinori 95

Antinori 95

Antinori 95

Antinori 95 cs,mass,pwa

Antinori 95 cs,mass,pwa

Abatzis $94 \mathrm{D}$ mass,pwa

Sapozhnikov $95 \quad$ cs

Hartouni 94 mass,p Barberis 97B

mass,pt,pwa Gutierrez 96

Girone 94

\begin{tabular}{l|ll}
$\mathrm{cs}, \mathrm{p}, \mathrm{pt}$ \\
$\mathrm{cs}, \mathrm{p}, \mathrm{pt}$
\end{tabular}$\quad 2 p \quad a_{2}(1320)^{+} \pi^{-}$

cs,p,pt
cs,p,pt

$$
85
$$

300
450

Antinori $95 \mathrm{cs}$,mass, pwa

Antinori $95 \mathrm{cs}$ mass,pwa

Antinori 95 cs,mass,pwa

Abatzis 94D mass,pwa

$2 p K_{S} K^{-} \pi^{+}$

27.5

450

800

Antinori 95 cs,mass,pwa

angp,cs $2 p \quad a_{2}(1320)^{-} \pi^{+}$

85

300

Antinori $95 \mathrm{cs}$,mass, pwa

Antinori 95 cs,mass, pwa

Abatzis 94D mass,pwa

$2 p K^{+} \pi^{-}$

3.67

$2 p K^{+} K^{-}$

Chalyshev 98

mass

Grzonka 98

3.3

Balewski 98C

Brauksiepe 97

Kirk 98

$\begin{array}{lr}\text { Kirk } 98 & \text { mass } \\ \text { Norman } 96 & \text { angp,mass }\end{array}$

300

(28)

450

Kirk 98

mass

Barberis 96 angp,mass

Barberis 968 mass,pt

cs, mass, $p$

\begin{tabular}{r|r} 
cs,pol,pt & 800
\end{tabular}

Gutierrez 94

mass

Reyes 97 ang,mass,pwa

Gutierrez 94 mass,

800

$2 p 2 K_{S} \pi^{\circ}$

450

ang,mass,pt,pwa

Gutierrez 94 mass,pt

Hartouni 94 mass,p

Barberis 97B

Barberis $96 \mathrm{~B}^{\text {mass,pt,pwa }}$

Gutierrez 96

ang,mass,pt,pwa

Gutierrez 94 mass,pt

Barberis 97B

mass,pt,pwa

$2 p \phi(1020) \pi^{+} \pi^{-}$

$$
24 \text { Sapozhnikov } 95
$$

$2 p K^{+} K^{-} \phi(1020)$

${ }^{85} \pi^{\circ}$

450

p $\Lambda K^{+} \pi^{+} \pi^{-}$

27.5

$3 p \bar{\Lambda} K^{-}$

27.5

$2 p 4 \gamma$

450

$2 p \bar{K}^{*}(882) K^{*}(892)$

450

Barberis $98 \mathrm{D}$ cs,mass,pt

$$
2
$$

Girone 94

\section{)}

Barberis 98F

cs,mass,p,pt

Hartouni 94 mass,p

Hartouni 94 mass,p

Sadovsky 94

Singovsky 94

Barberis $98 \mathrm{~B}$
Bertolot to 94 cs,mass

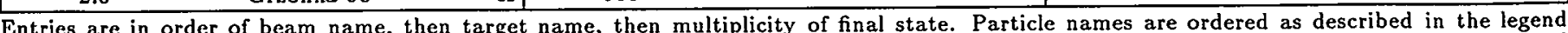

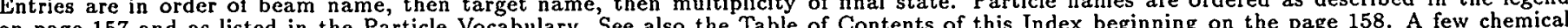

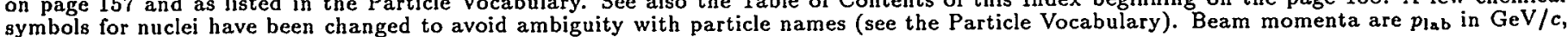
or in parentheses $E_{\mathrm{cm}}$ in $\mathrm{GeV}$. 


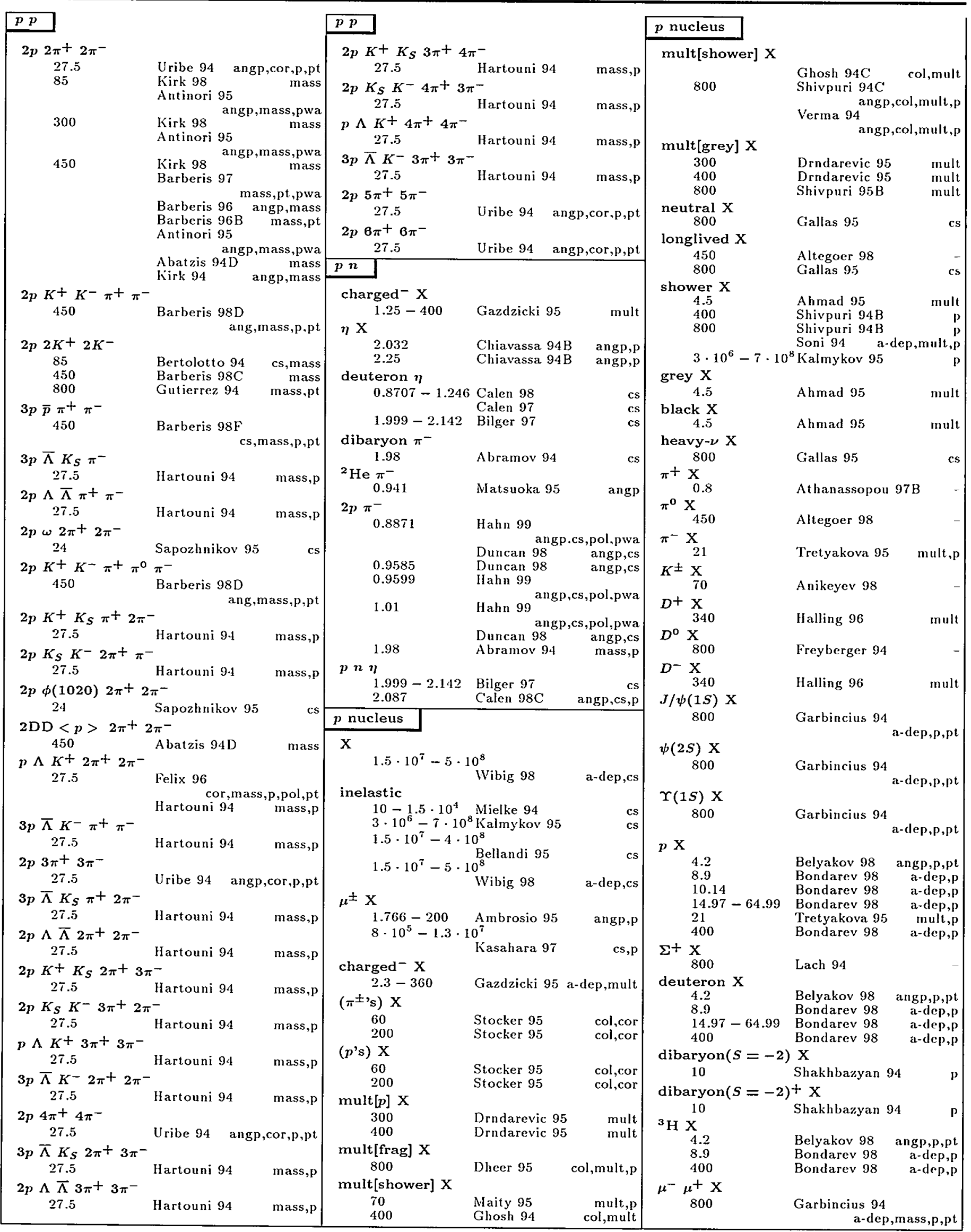




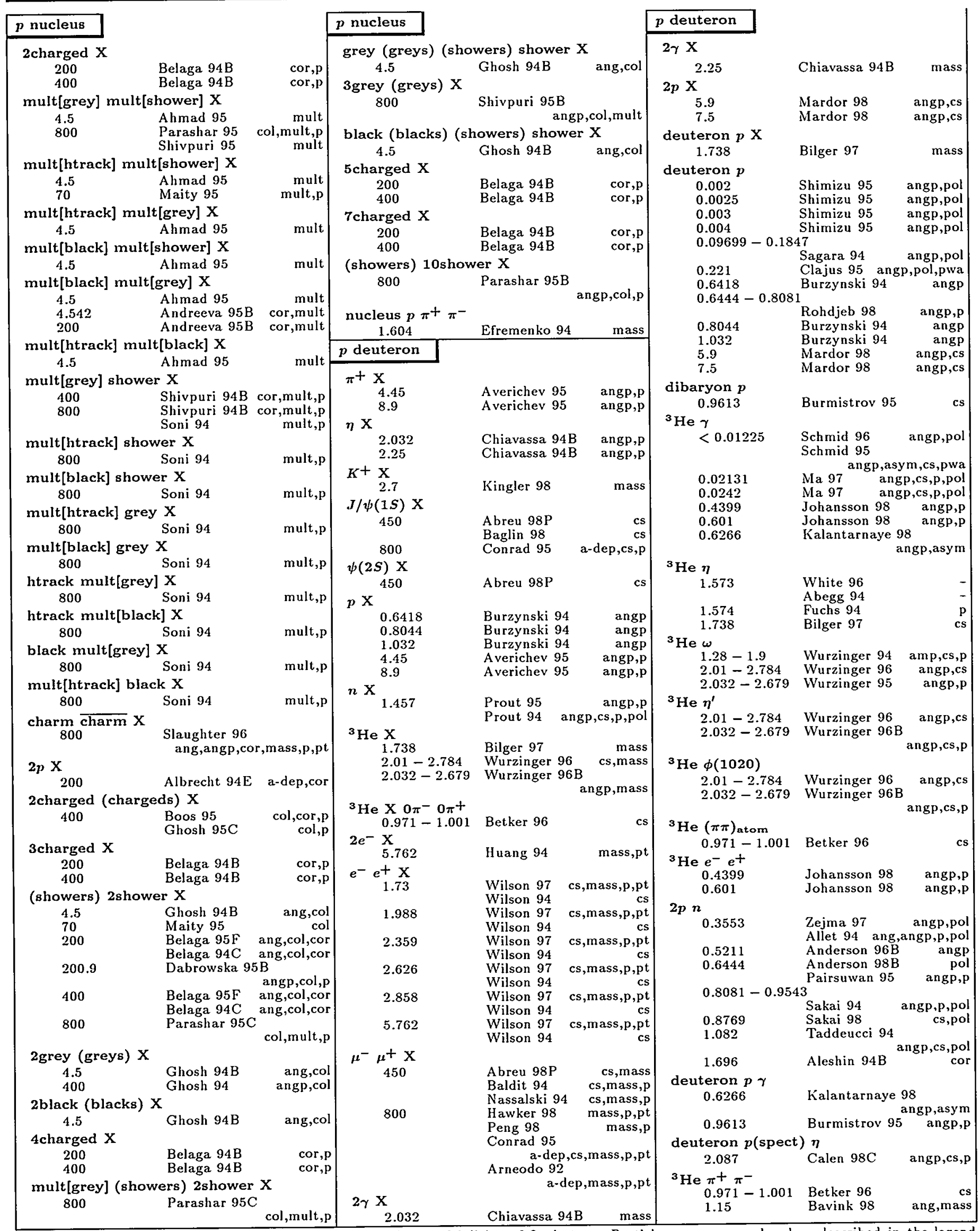

Entries are in order of beam name, then target name, then multiplicity of final state. Particle names are ordered as described in the legend on page 157 and as listed in the Particle Vocabulary. See also the Table of Contents of this Index beginning on the page 158. A few chemical symbols for nuclei have been changed to avoid ambiguity with particle names (see the Particle Vocabulary). Beam momenta are $p_{\text {lab }}$ in GeV/c, or in parentheses $E_{\mathrm{cm}}$ in $\mathrm{GeV}$. 


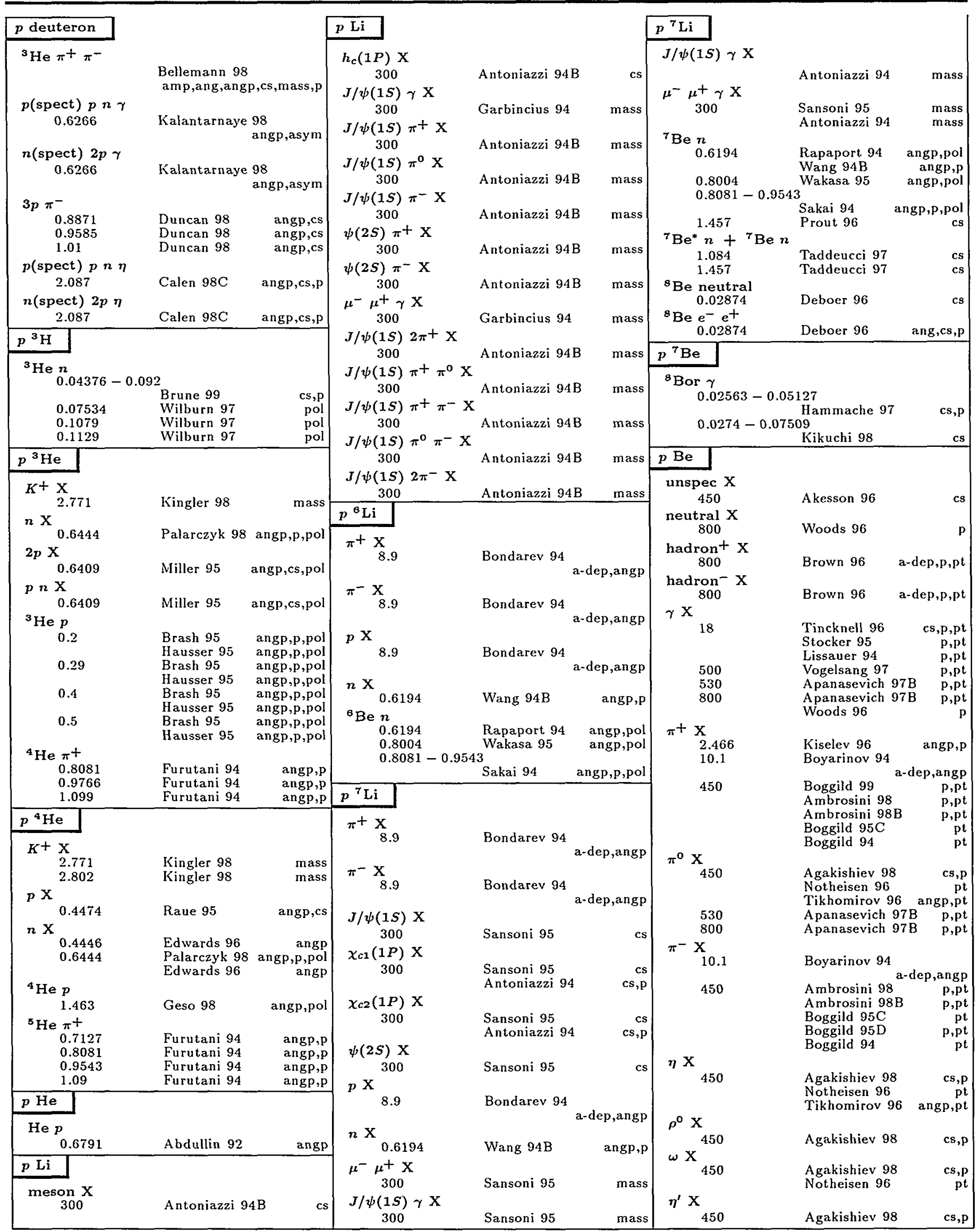




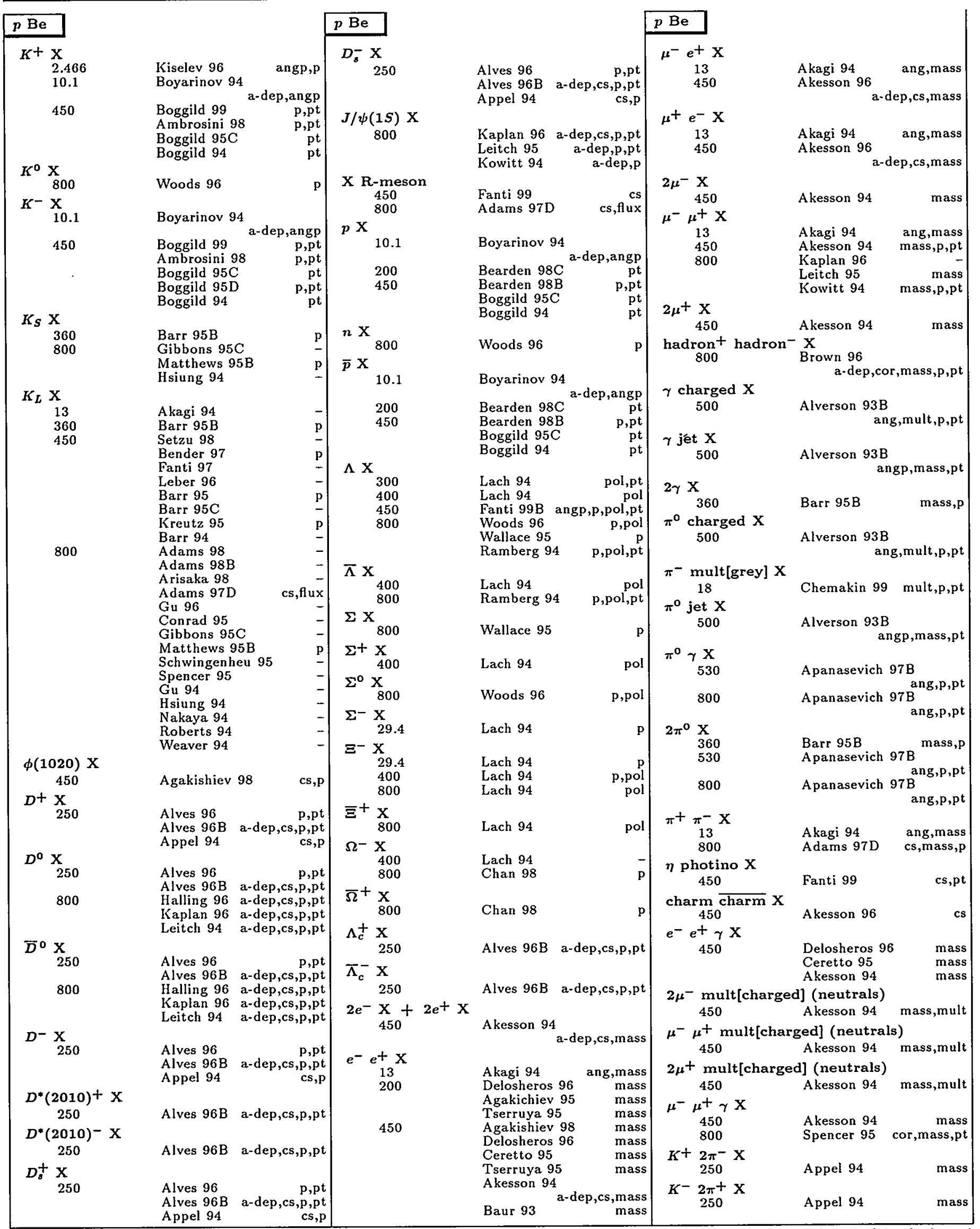

Entries are in order of beam name, then target name, then multiplicity of final state. Particle names are ordered as described in the legend on page 157 and as listed in the Particle Vocabulary. See also the Table of Contents of this Index beginning on the page 158. A few chemical symbols for nuclei have been changed to avoid ambiguity with particle names (see the Particle Vocabulary). Beam momenta are $p / s b$ in GeV/c, or in parentheses $E_{\mathrm{cm}}$ in $\mathrm{GeV}$. 


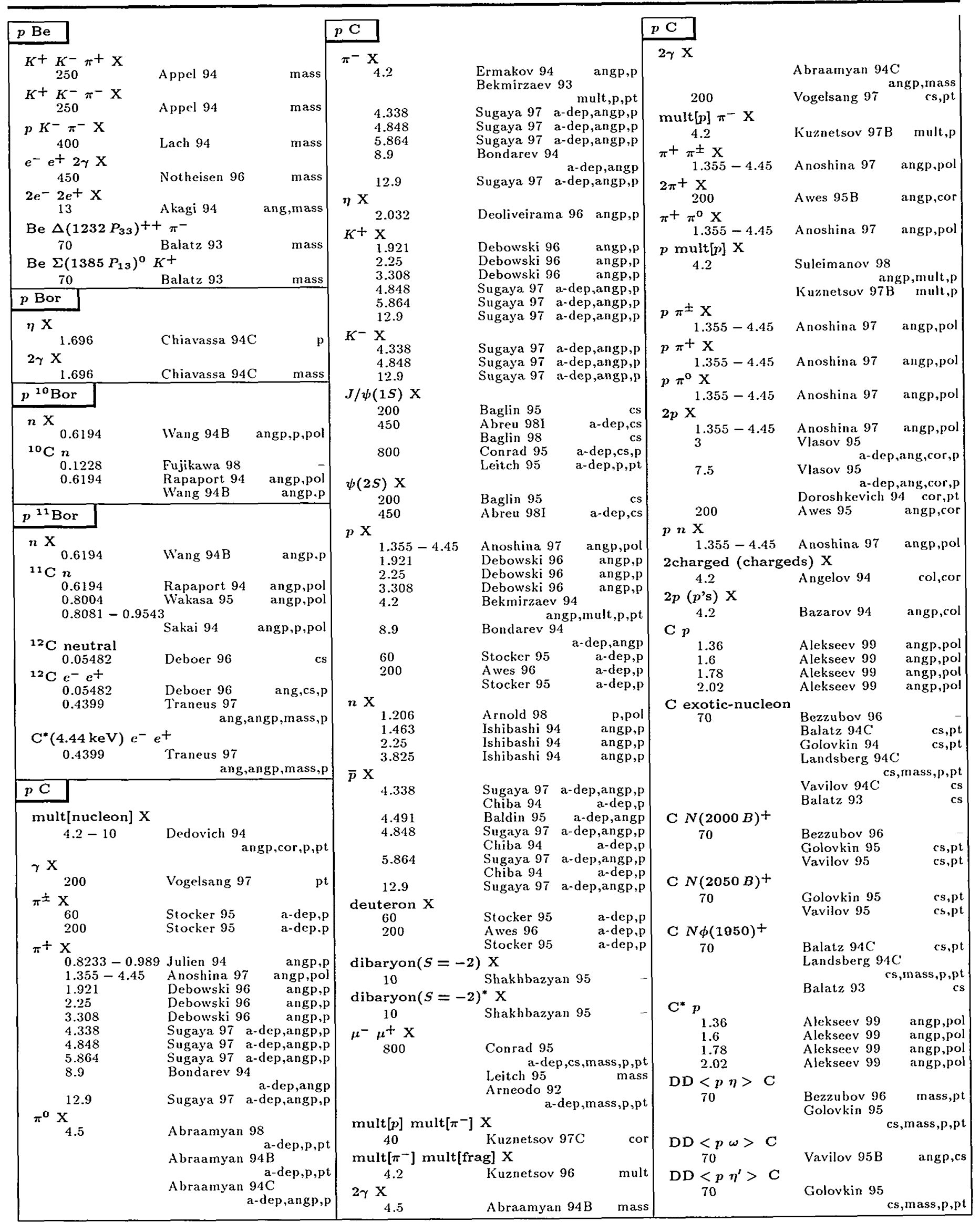




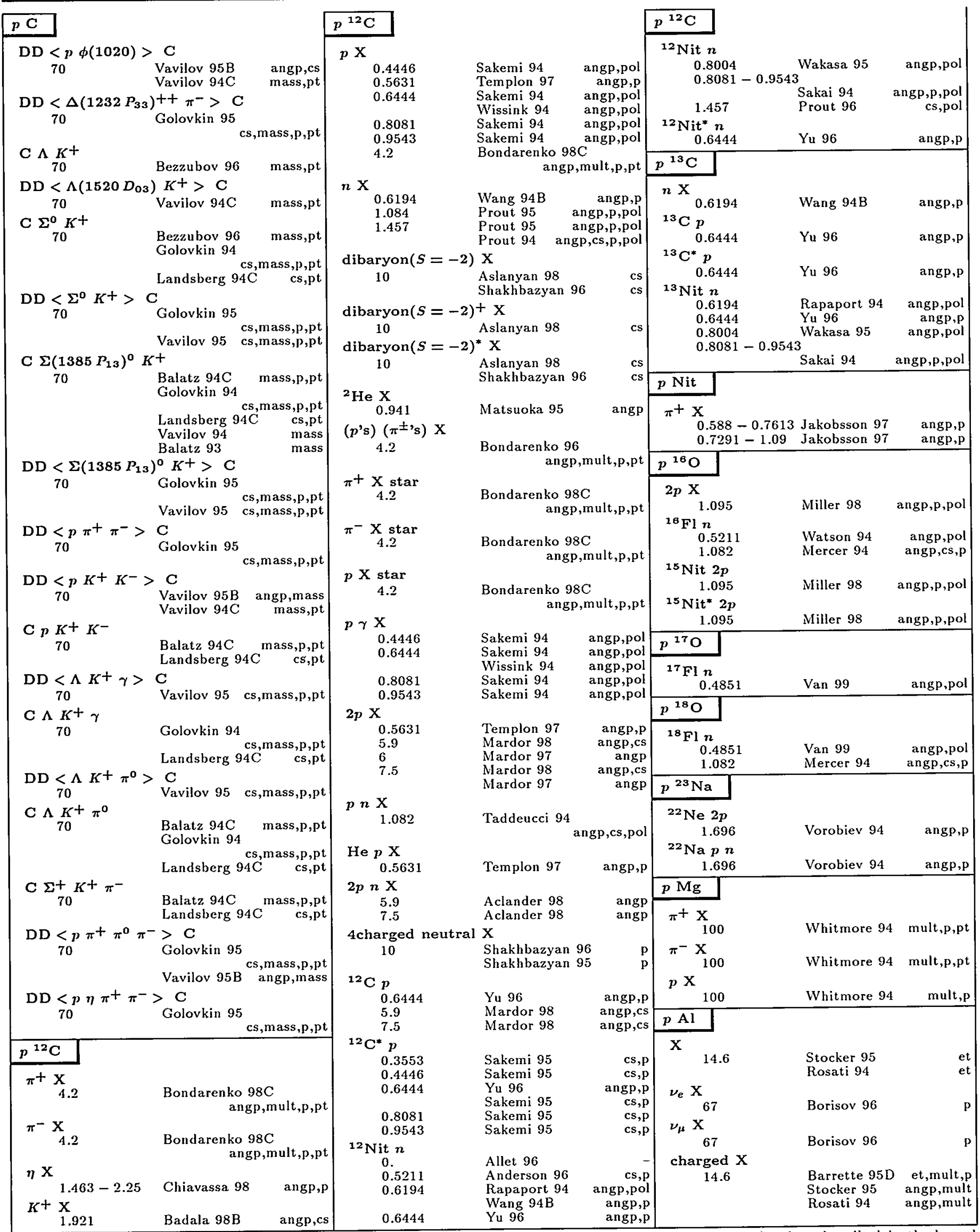

Entries are in order of beam name, then target name, then multiplicity of final state. Particle names are ordered as described in the legend Entries are in order of beam name, then target name, then muthe Table of Contents of this Index beginning on the page 158. A few chemical on page 157 and as listed in the Particle Vocabulary. See also the Table of Contents of this Index beginning on the page 158 . A few chem or in parentheses $E_{\mathrm{cm}}$ in GeV. 


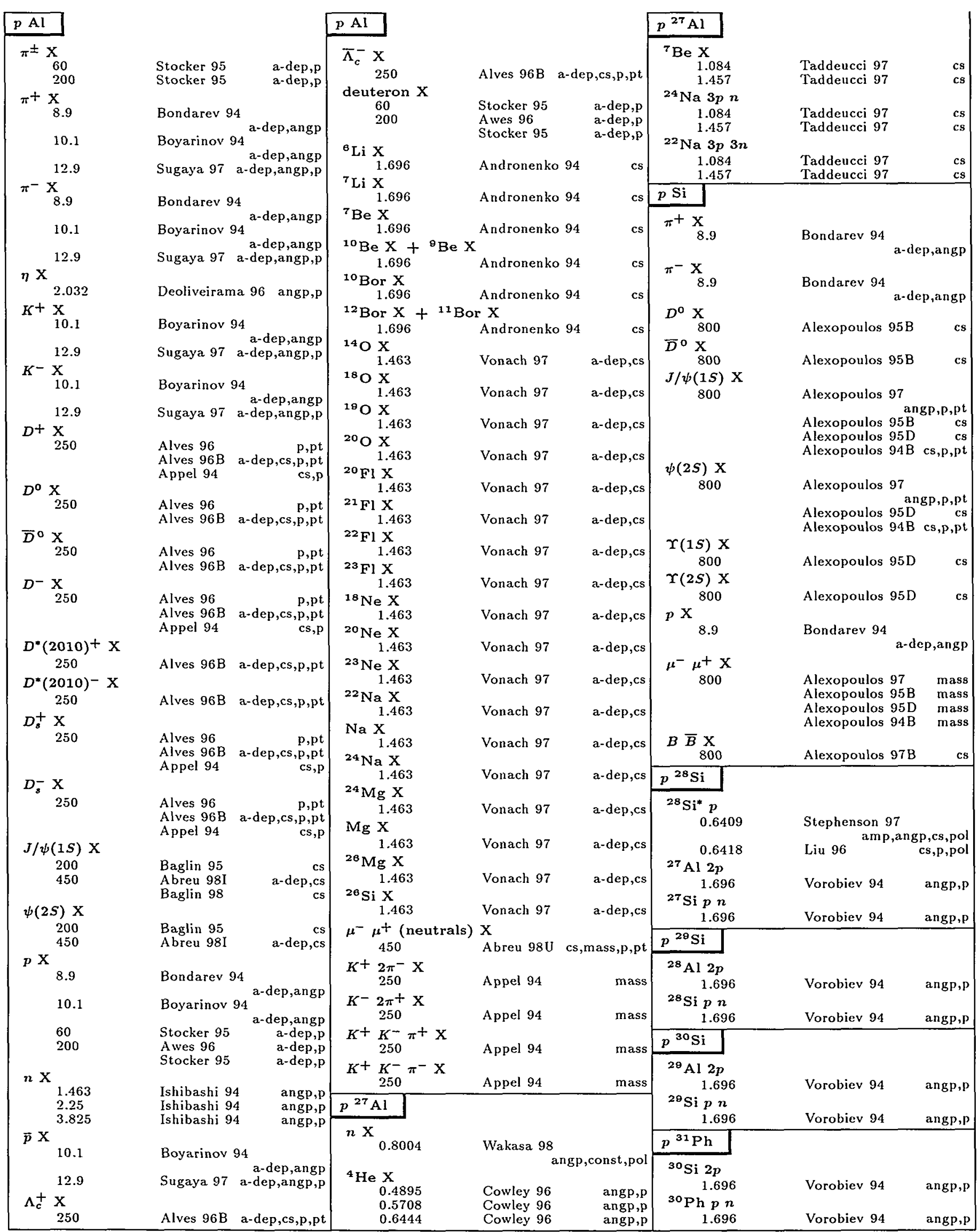




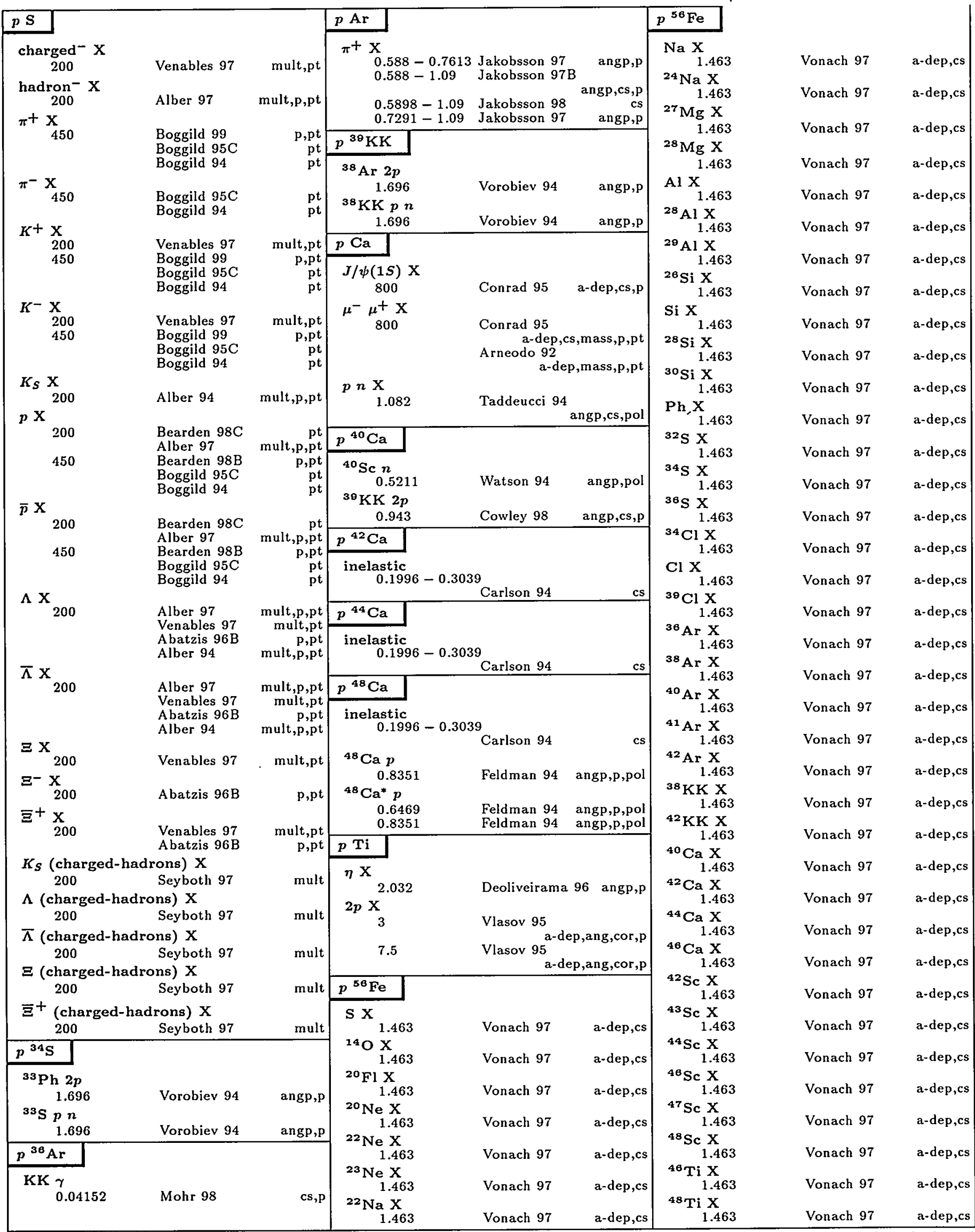

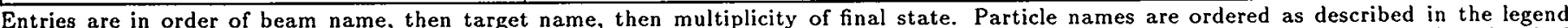

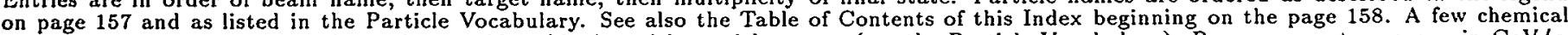

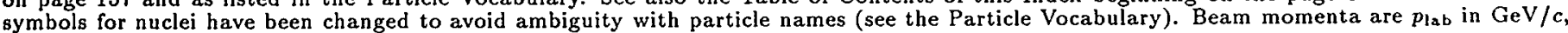
or in parentheses $E_{\mathrm{cm}}$ in $\mathrm{GeV}$. 


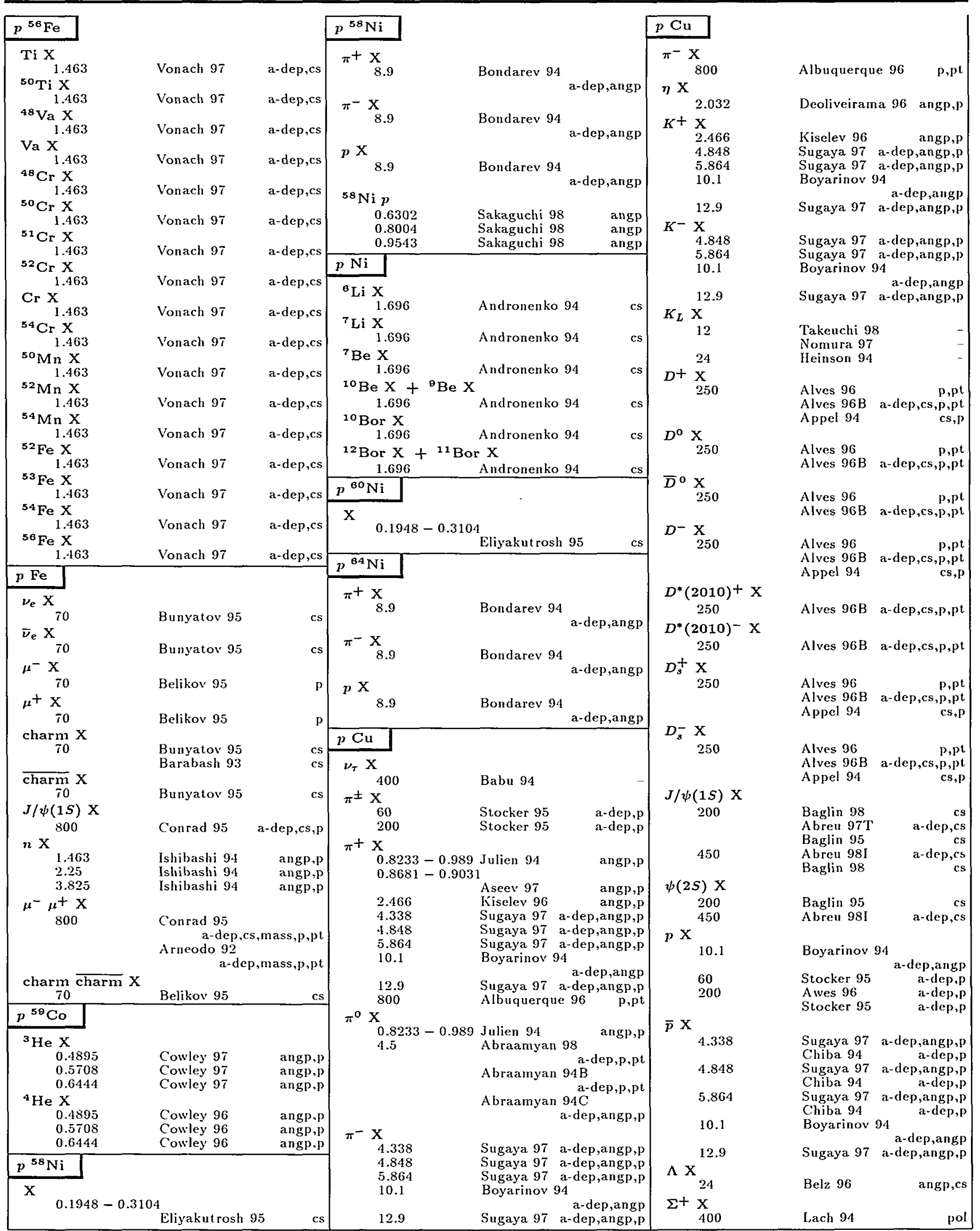




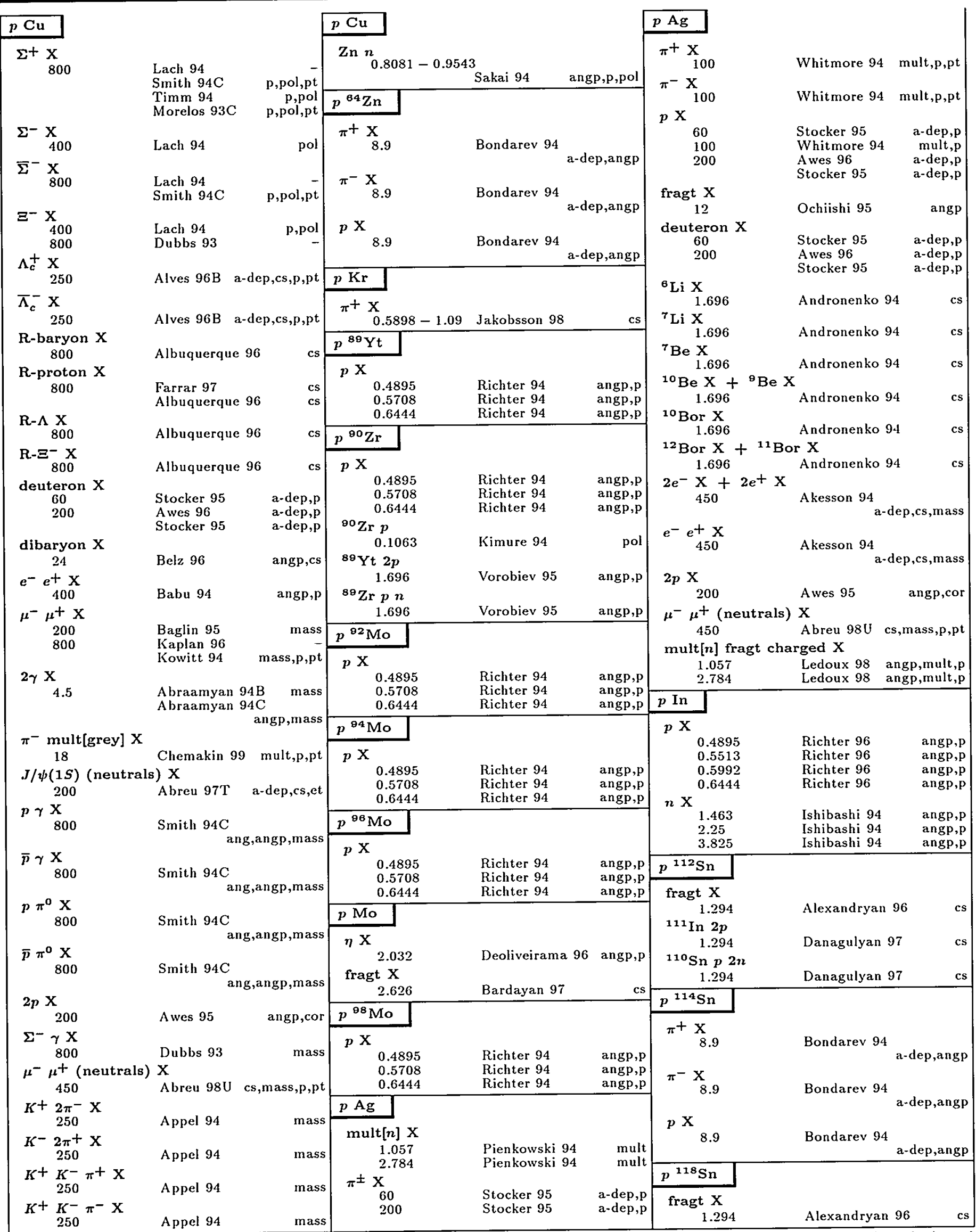

Entries are in order of beam name, then target name, then multiplicity of final state. Particle names are ordered as described in the legend on page 157 and as listed in the Particle Vocabulary. See also the Table of Contents of this Index beginning on the page 158. A few chemical symbols for nuclei have been changed to avoid ambiguity with particle names (see the Particle Vocabulary). Beam momenta are $p_{\text {lab }}$ in GeV/c, or in parentheses $E_{\mathrm{cm}}$ in $\mathrm{GeV}$. 


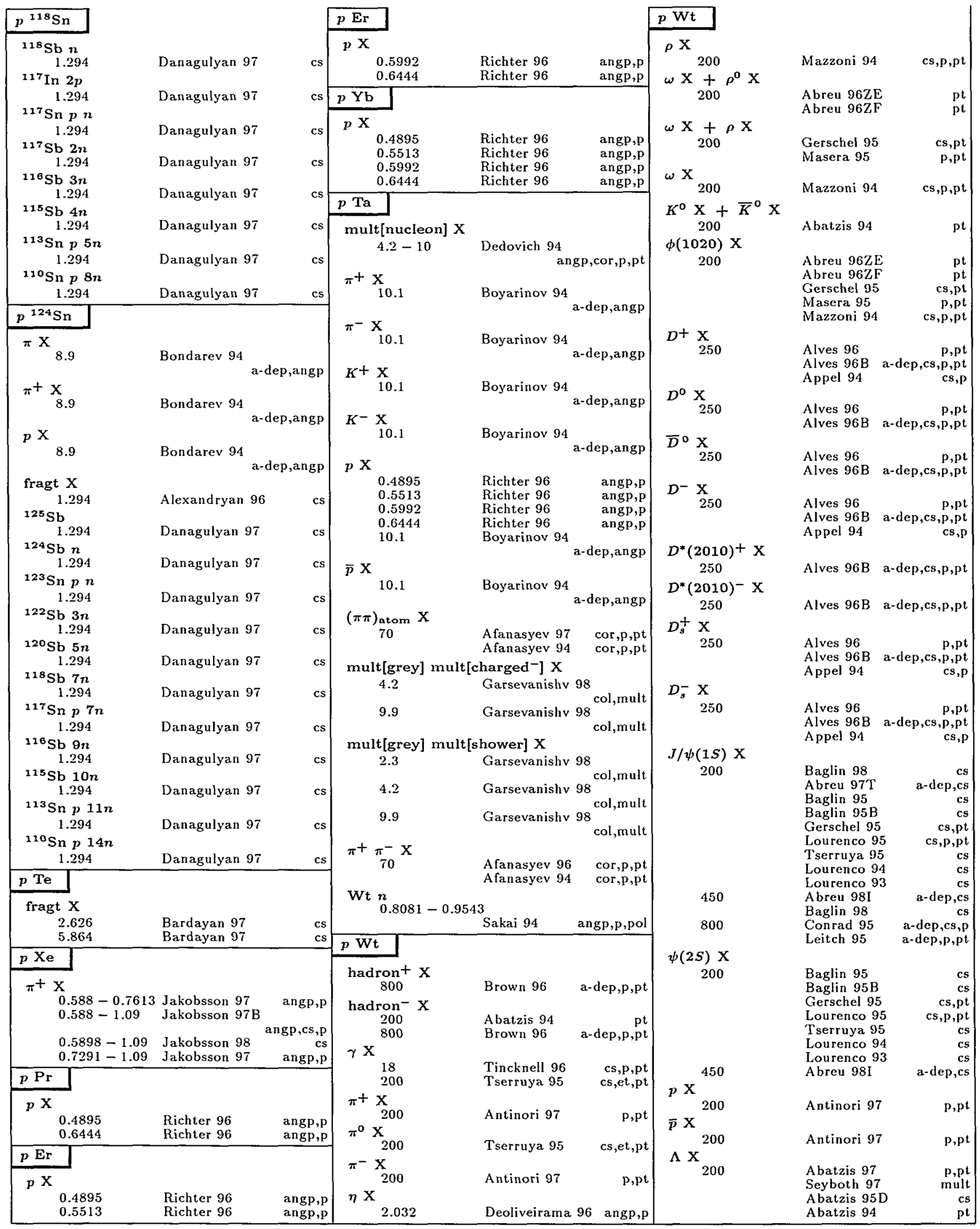




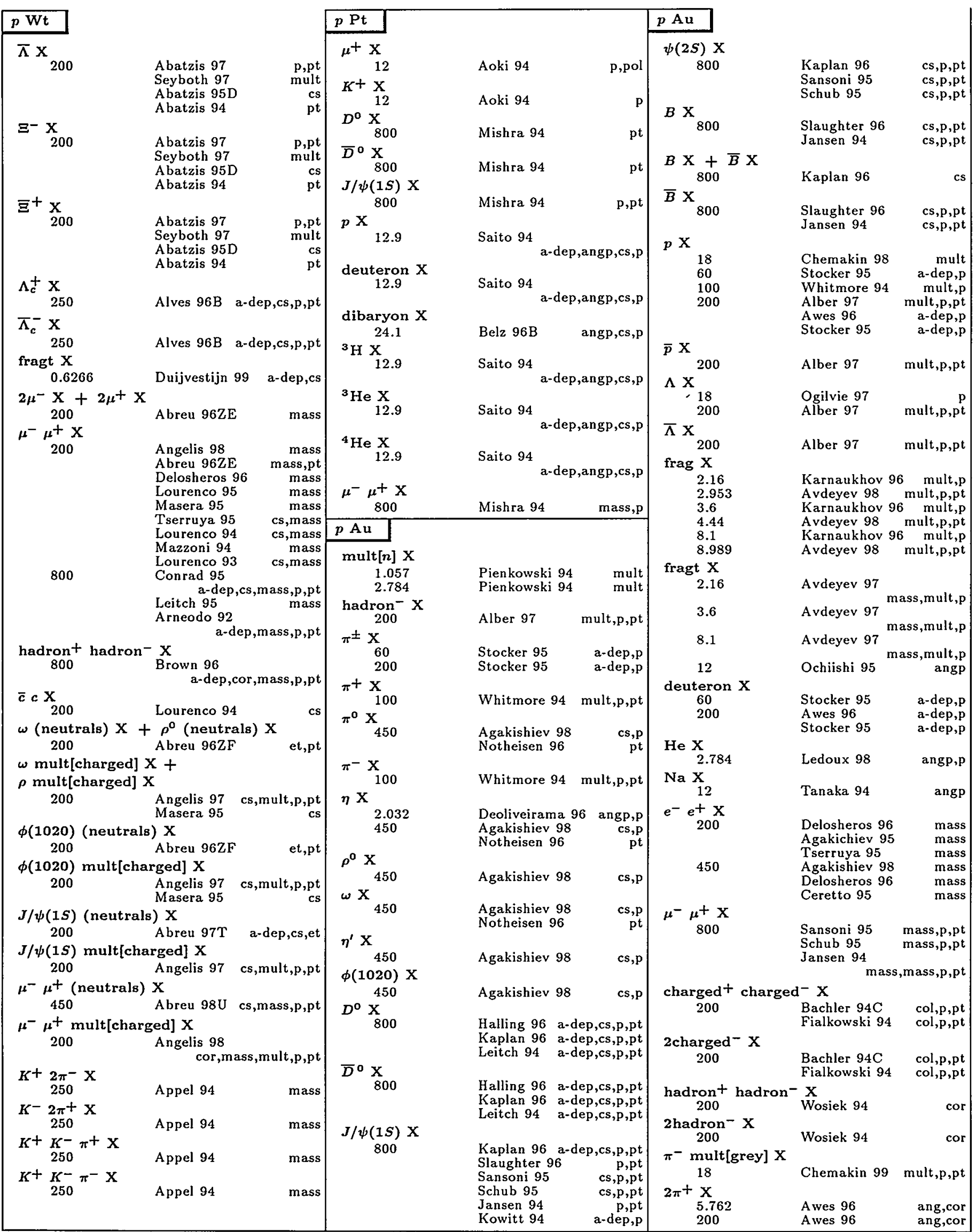

Entries are in order of beam name, then target name, then multiplicity of final state. Particle names are ordered as described in the legend on page 157 and as listed in the Particle Vocabulary. See also the Table of Contents of this Index beginning on the page 158 . A few chemical symbols for nuclei have been changed to avoid ambiguity with particle names (see the Particle Vocabulary). Beam momenta are $p_{l a b}$ in GeV/c, or in parentheses $E_{\mathrm{cm}}$ in $\mathrm{GeV}$. 


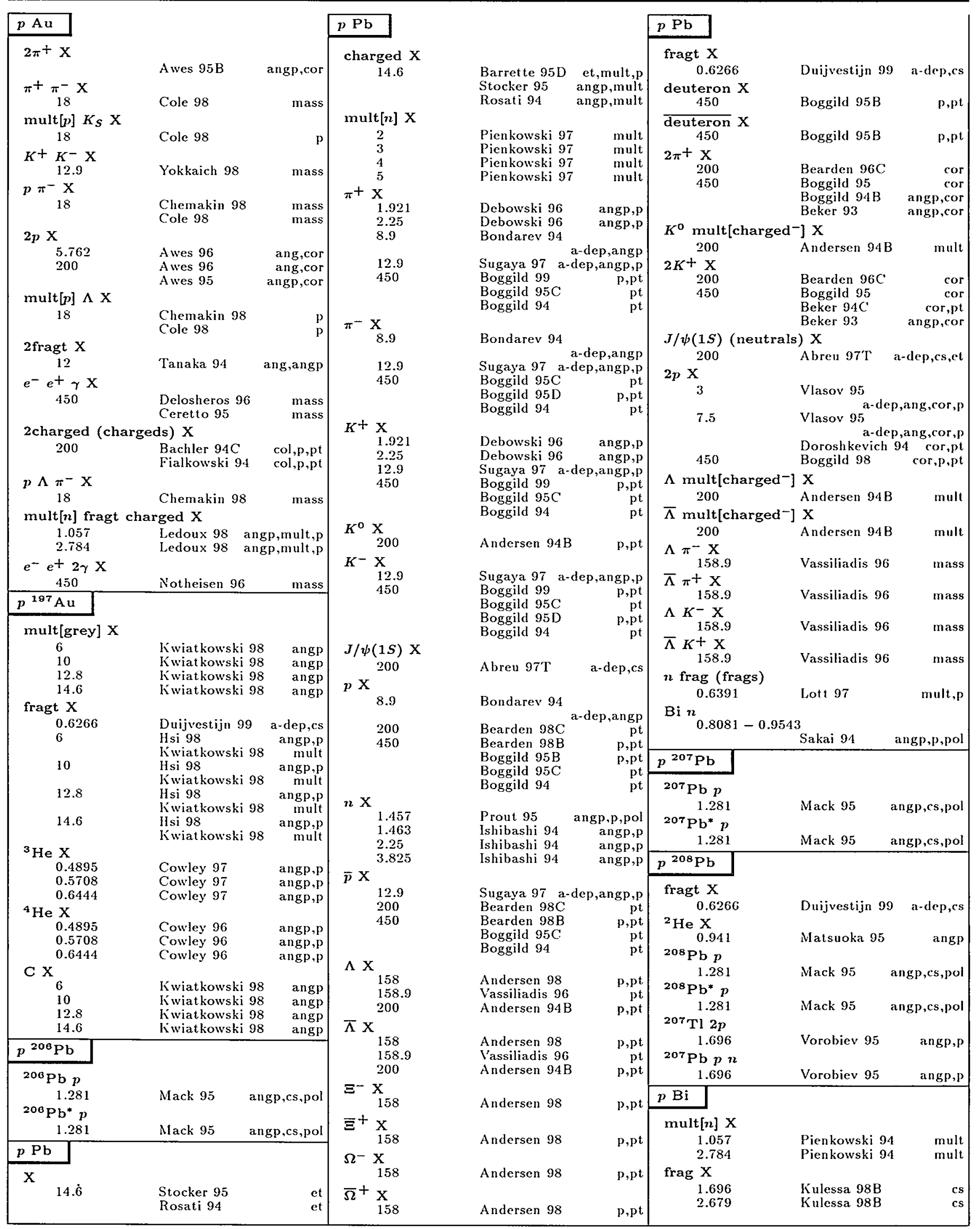




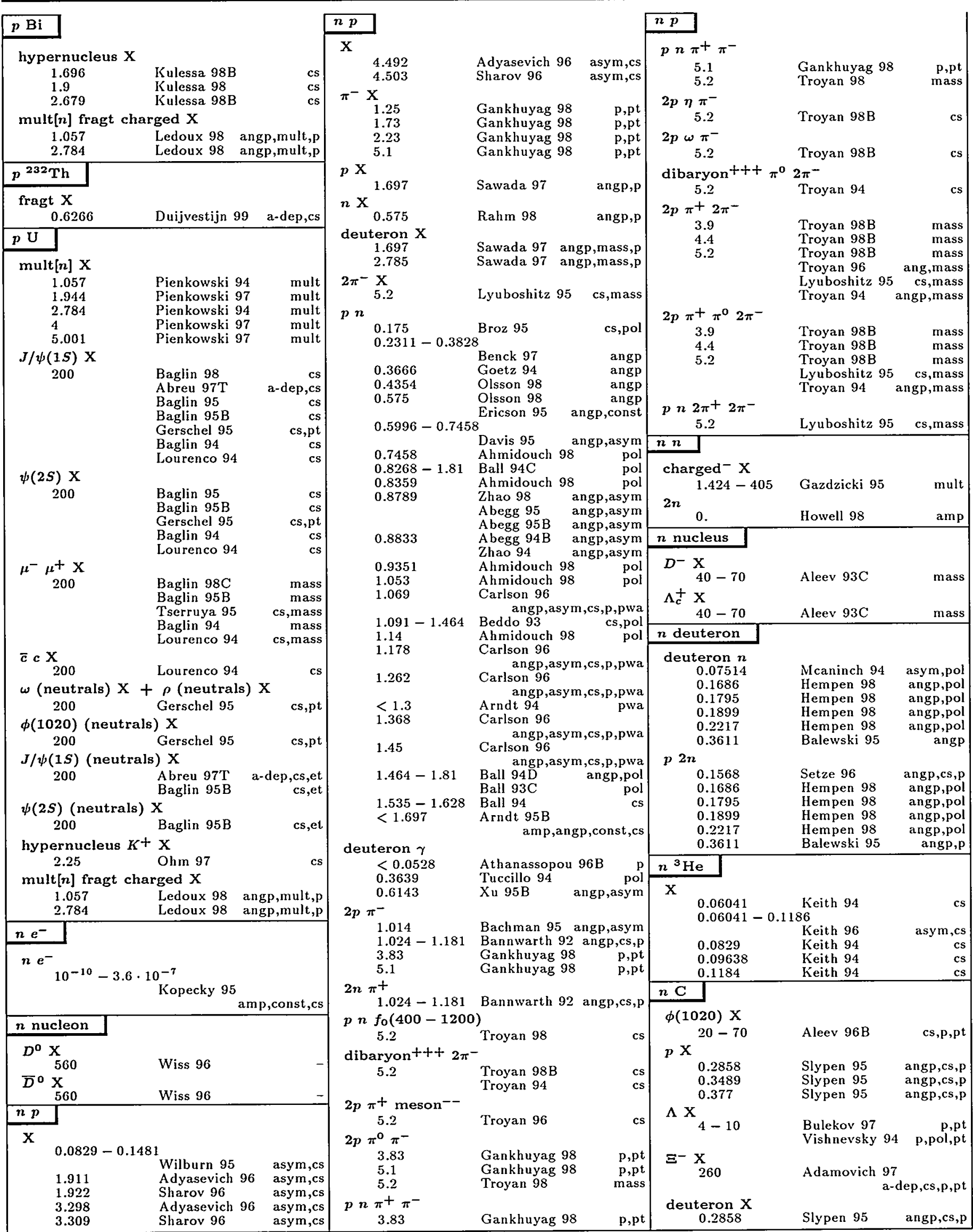

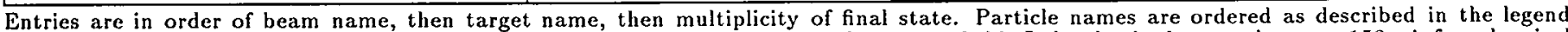

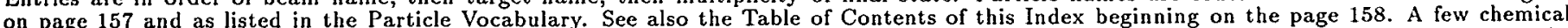

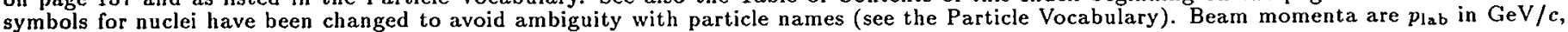
or in parentheses $E_{\mathrm{cm}}$ in $\mathrm{GeV}$. 
$n \mathrm{C} \rightarrow$ deuteron $\mathrm{X}$

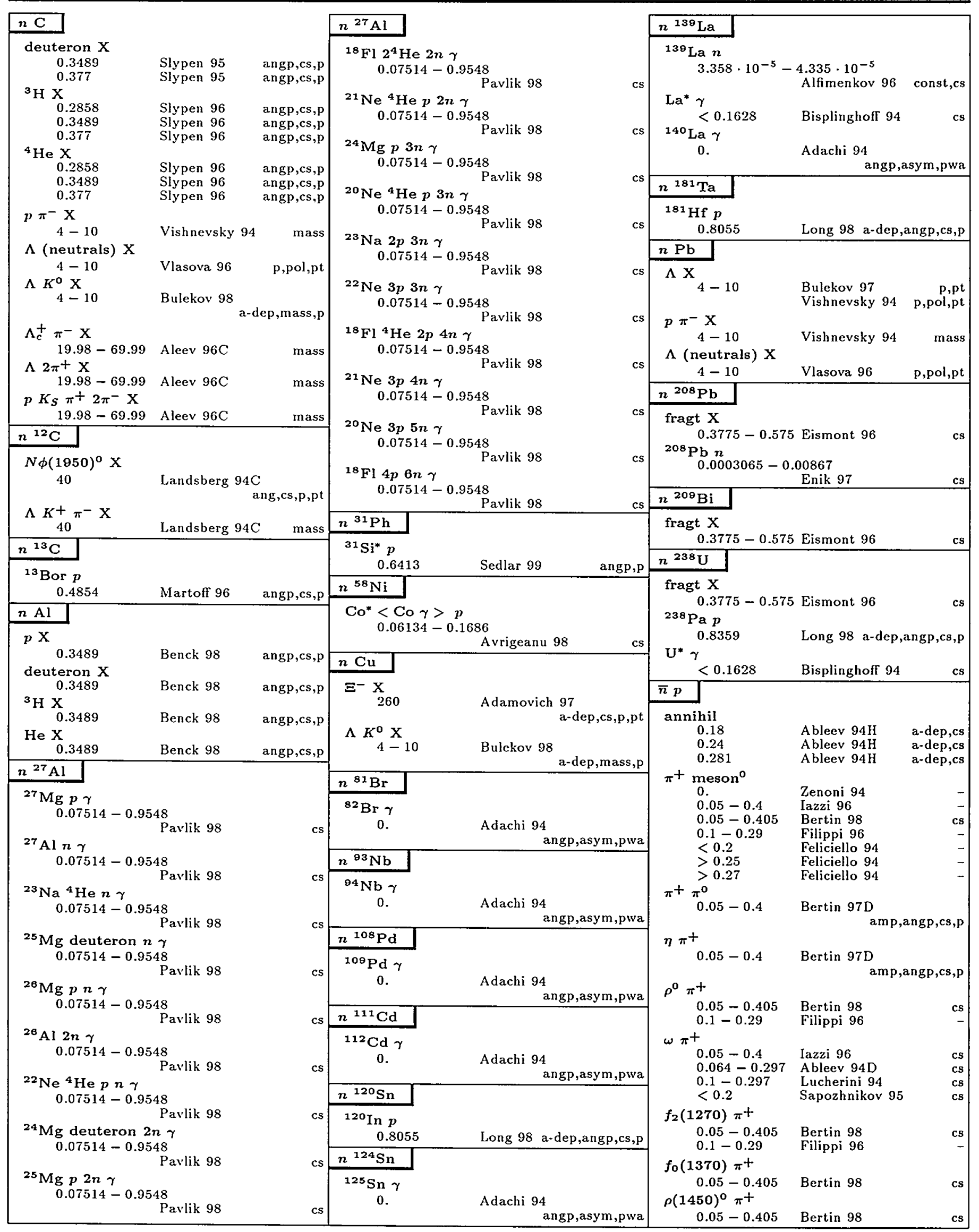




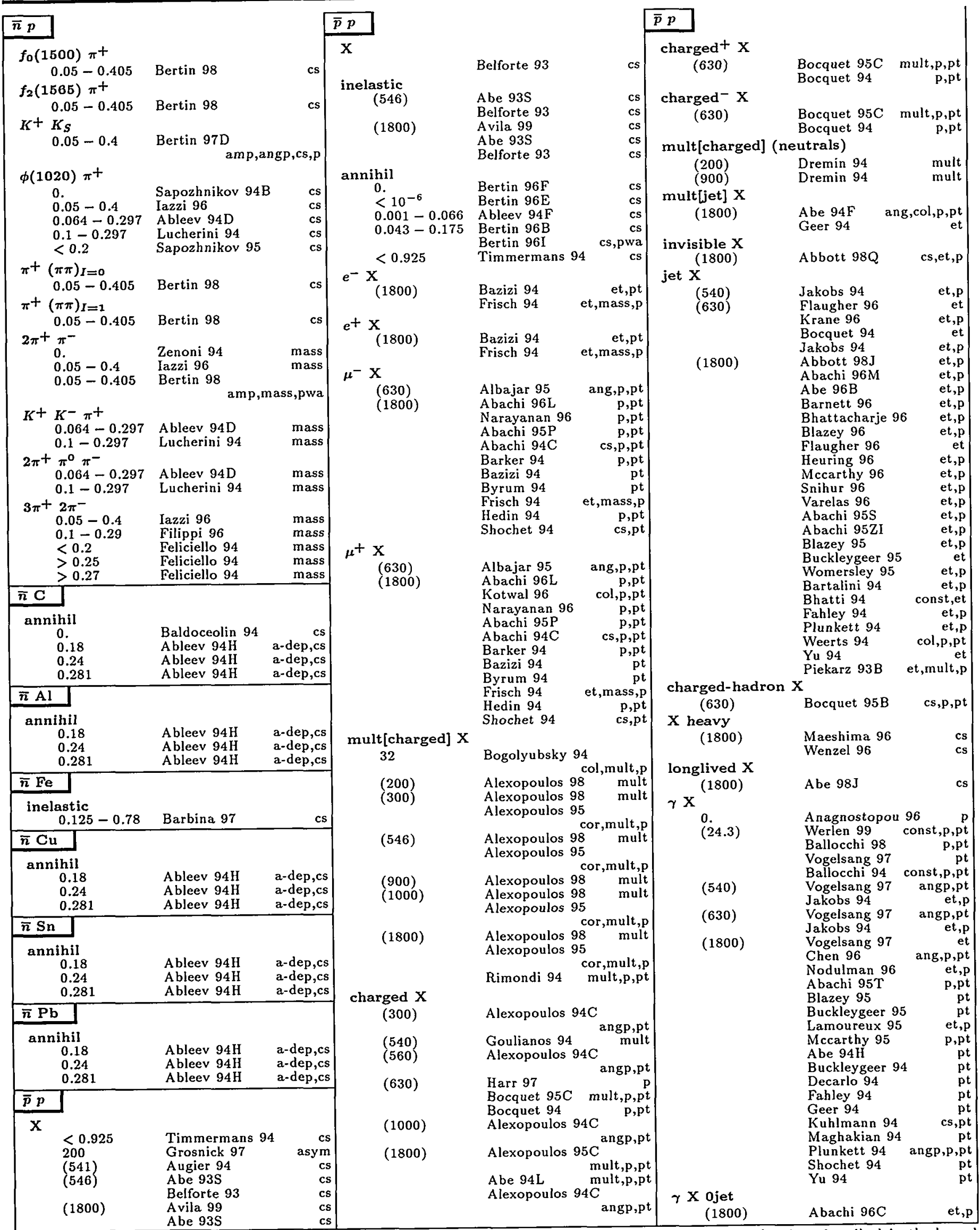

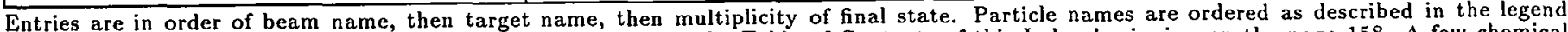

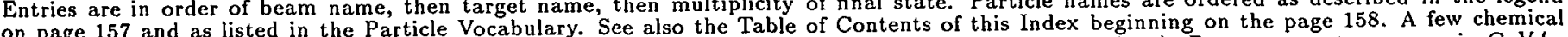

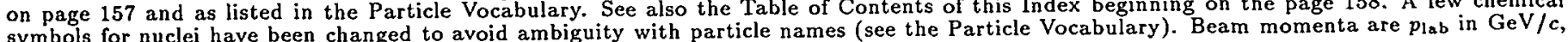
or in parentheses $E_{\mathrm{cm}}$ in $\mathrm{GeV}$. 


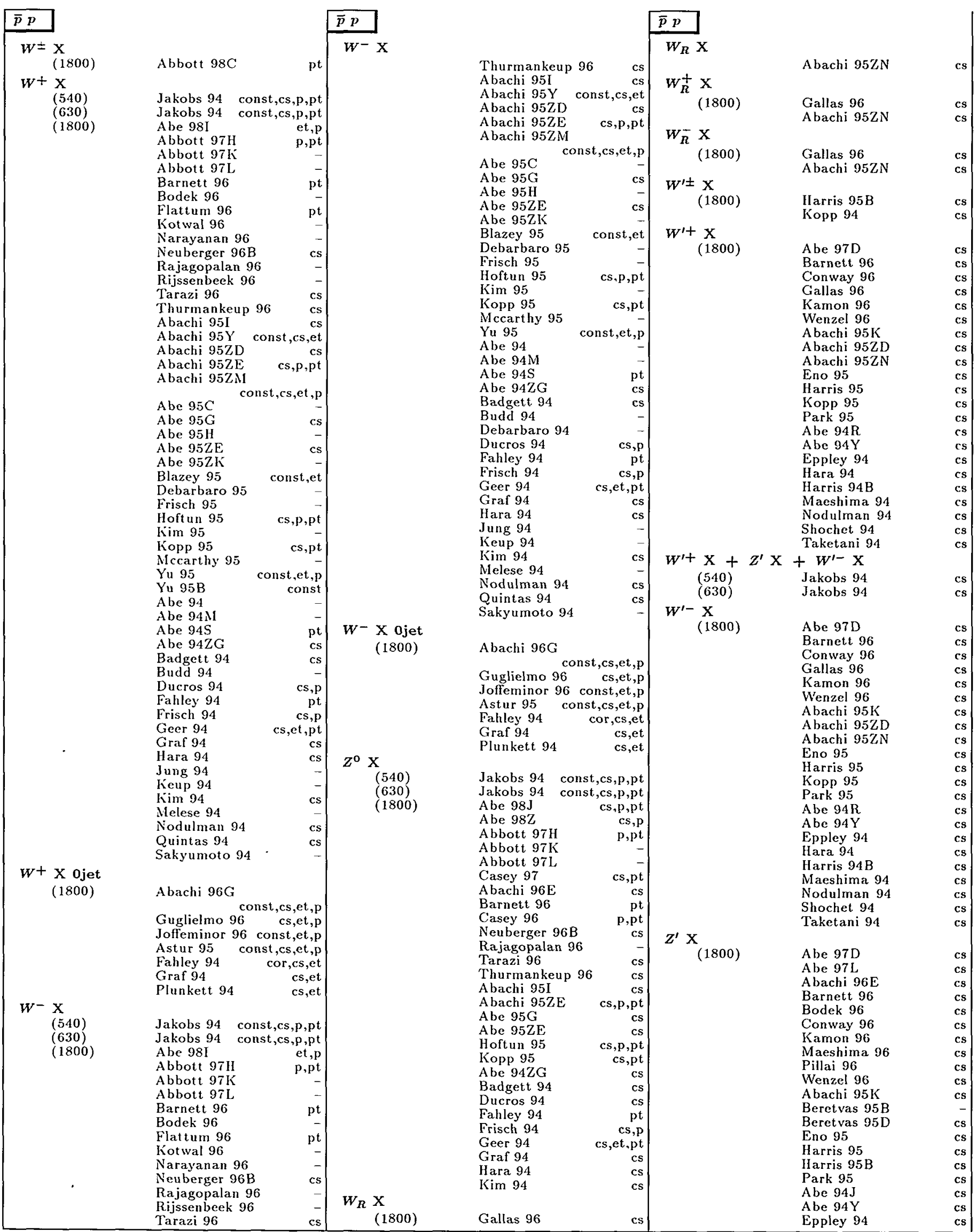




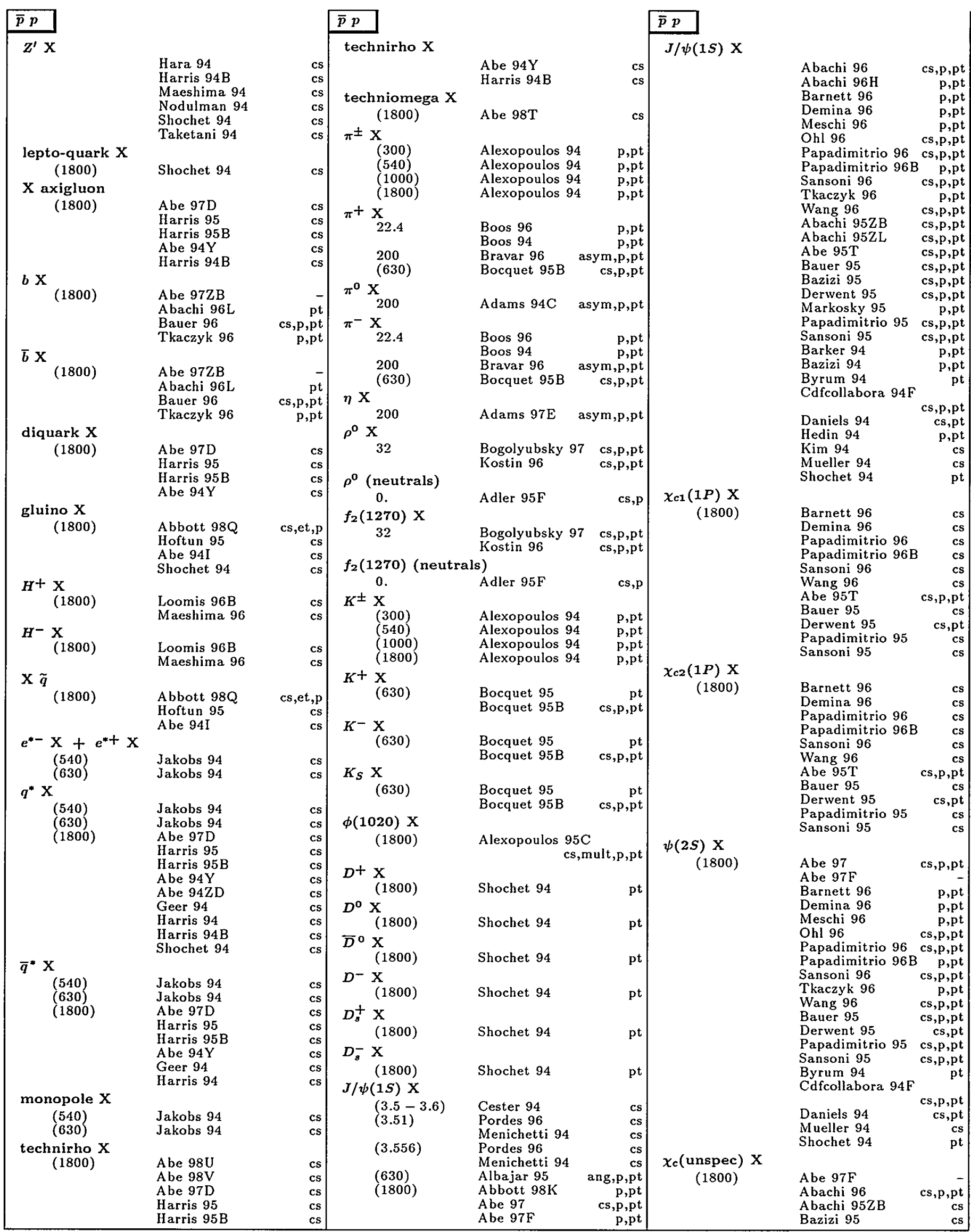

Entries are in order of beam name, then target name, then multiplicity of final state. Particle names are ordered as described in the legend on page 157 and as listed in the Particle Vocabulary. See also the Table of Contents of this Index beginning on the page 158 . A few chemical symbols for nuclei have been changed to avoid ambiguity with particle names (see the Particle Vocabulary). Beam momenta are $p_{l a b}$ in GeV/c, or in parentheses $E_{\mathrm{cm}}$ in $\mathrm{GeV}$. 
$\bar{p} p \rightarrow \chi_{c}$ (unspec) $\mathrm{X}$

$\bar{p} p \rightarrow B^{-} \mathrm{X}$

\begin{tabular}{|c|c|c|c|c|c|c|c|c|}
\hline $\bar{p} p$ & & & $\bar{p} p$ & & & $\overline{\bar{p} p}$ & & \\
\hline$\chi_{c}$ (unspec) $\mathrm{X}$ & & & $B+\mathrm{X}$ & & & $\bar{B}^{\circ} \mathrm{X}$ & & \\
\hline & Derwent 95 & cs & & $\begin{array}{l}\text { Hara } 94 \\
\text { Lewis } 94\end{array}$ & $\begin{array}{c}\text { cs } \\
\text { pt }\end{array}$ & & Abe $98 \mathrm{ZD}$ & cs \\
\hline & $\begin{array}{l}\text { Markosky } 95 \\
\text { Hedin } 94\end{array}$ & $\begin{array}{l}c s \\
c s\end{array}$ & & $\begin{array}{l}\text { Lewis } 94 \\
\text { Lewis } 94 \mathrm{~B}\end{array}$ & $\begin{array}{r}\mathrm{pt} \\
\mathrm{cs}, \mathrm{pt}\end{array}$ & & $\begin{array}{l}\text { Abe } 97 Z 1 \\
\text { Abe } 972 \mathrm{~K}\end{array}$ & - \\
\hline & $\begin{array}{l}\text { Hedin } 94 \\
\text { Mueller } 94\end{array}$ & $\begin{array}{c}\mathrm{cs} \\
\mathrm{cs}\end{array}$ & & Mueller 94 & cs,pt & & Abachi $96 \mathrm{I}$ & - \\
\hline bottom $\mathrm{X}$ & & & & Plunkett 94 & $\mathrm{cs}, \mathrm{pt}$ & & Abe 96D & - \\
\hline $\begin{array}{l}(630) \\
(1800)\end{array}$ & Albajar 93B & $\mathrm{cs}, \mathrm{pt}$ & $B^{\circ} \mathrm{X}$ & Shochet 94 & cs,pl & & $\begin{array}{l}\text { Abe } 96 \mathrm{E} \\
\text { A be } 96 \mathrm{~K}\end{array}$ & - \\
\hline$(1800)$ & $\begin{array}{l}\text { Abachi } 94 \mathrm{C} \\
\text { Bazizi } 94\end{array}$ & $\begin{array}{r}\mathrm{cs}, \mathrm{p}, \mathrm{pt} \\
\mathrm{cs}, \mathrm{pt}\end{array}$ & $(1800)$ & Abe $99 \mathrm{D}$ & cs & & Abe $96 \mathrm{P}$ & $c s, p, p t$ \\
\hline & Byrum 94 & $\mathrm{cs}, \mathrm{pt}$ & & $\begin{array}{l}\text { Abe } 98 \mathrm{E} \\
\text { Abe } 980\end{array}$ & - & & $\begin{array}{l}\text { Barnett } 96 \\
\text { Bauer } 96\end{array}$ & $\begin{array}{l}\text { cs,p,pt } \\
\text { cs,p,pt }\end{array}$ \\
\hline & Daniels 94 & cs,pt & & $\begin{array}{l}\text { Abe } 980 \\
\text { Abe } 98 Z D\end{array}$ & cs & & Huffman 96 & $\mathrm{cs}, \mathrm{p}, \mathrm{pt}$ \\
\hline & $\begin{array}{l}\text { Hedin } 94 \\
\text { Huehn } 94\end{array}$ & $\begin{array}{r}\mathrm{p}, \mathrm{pt} \\
\mathrm{cs}, \mathrm{pt}\end{array}$ & & A be $97 \mathrm{ZI}$ & - & & Laasanen 96 & $\mathrm{cs}, \mathrm{p}, \mathrm{pt}$ \\
\hline & Skarha 94 & & & $\begin{array}{l}\text { Abe } 972 \mathrm{~K} \\
\text { Abachi } 96 \mathrm{I}\end{array}$ & - & & $\begin{array}{l}\text { Lewis } 96 \\
\text { Lucchesi } 96\end{array}$ & $\mathrm{cs}, \mathrm{p}, \mathrm{pt}$ \\
\hline bottom $x$ & Abe $93 \mathrm{~N}$ & -1 & & $\begin{array}{l}\text { Abachi } 961 \\
\text { Abe } 96 \mathrm{D}\end{array}$ & - & & Meschi 96 & - \\
\hline $\begin{array}{r}\text { bottam } X \\
(1800)\end{array}$ & Abachi $94 \mathrm{C}$ & & & Abe $96 \mathrm{E}$ & - & & Miao 96 & - \\
\hline & $\begin{array}{l}\text { Abach1 94C } \\
\text { Bazizi } 94\end{array}$ & $\begin{array}{r}\mathrm{cs}, \mathrm{p}, \mathrm{pt} \\
\mathrm{cs}, \mathrm{pt}\end{array}$ & & $\begin{array}{l}\text { A be } 96 \mathrm{~K} \\
\text { A be } 96 \mathrm{P}\end{array}$ & - & & $\begin{array}{l}\text { Paulini } 96 \\
\text { Slaughter } 96\end{array}$ & $\mathrm{p}, \mathrm{pt}$ \\
\hline & Byrum 94 & $\mathrm{cs}, \mathrm{pt}$ & & $\begin{array}{l}\text { Abe } 96 \mathrm{P} \\
\text { Barnett } 96\end{array}$ & $\begin{array}{l}\mathrm{cs}, \mathrm{p}, \mathrm{pt} \\
\mathrm{cs}, \mathrm{p}, \mathrm{pt}\end{array}$ & & Speer 96 & $\mathrm{p}, \mathrm{pu}$ \\
\hline & Daniels 94 & $\mathrm{cs}, \mathrm{pt}$ & & Bauer 96 & $c s, p, p t$ & & Wang 96 & $\mathrm{cs}, \mathrm{pt}$ \\
\hline & $\begin{array}{l}\text { Hedin } 94 \\
\text { Huehn } 94\end{array}$ & $p, p t$ & & Huffman 96 & $\mathrm{cs}, \mathrm{p}, \mathrm{pt}$ & & Wenzel 96 & \\
\hline$B \mathrm{X}$ & Huehn 94 & $\mathrm{cs}, \mathrm{pt}$ & & $\begin{array}{l}\text { Laasanen } 96 \\
\text { Lewis } 96\end{array}$ & $\mathrm{cs}, \mathrm{p}, \mathrm{pt}$ & & $\begin{array}{l}\text { Yu } 96 \mathrm{~B} \\
\text { A be } 95 \mathrm{E}\end{array}$ & $\begin{array}{r}\mathrm{cs}, \mathrm{p}, \mathrm{pt} \\
\mathrm{p}, \mathrm{pt}\end{array}$ \\
\hline$(1800)$ & Abbott $98 \mathrm{~B}$ & -1 & & $\begin{array}{l}\text { Lewis } 96 \\
\text { Lucchesi } 96\end{array}$ & $\mathrm{cs}, \mathrm{p}, \mathrm{pl}$ & & A be $95 \mathrm{M}$ & \\
\hline & Abachi 96 & $\mathrm{cs}, \mathrm{p}, \mathrm{pt}$ & & & - & & Abe $95 \mathrm{~N}$ & - \\
\hline & $\begin{array}{l}\text { Abachi } 961 \\
\text { Demina } 96\end{array}$ & - & & $\begin{array}{l}\text { Miao } 96 \\
\text { Paulini } 96\end{array}$ & $\begin{array}{l}- \\
-\end{array}$ & & A be $95 \mathrm{R}$ & - \\
\hline & Papadimitrio 96B & & & Slaughter 96 & $\mathrm{p}, \mathrm{pt}$ & & A be $95 \mathrm{ZB}$ & - \\
\hline & $\begin{array}{l}\text { Wenzel 96B } \\
\text { Abachi 95P }\end{array}$ & $p, p t$ & & $\begin{array}{l}\text { Speer } 96 \\
\text { Wang } 96\end{array}$ & - & & $\begin{array}{l}\text { Abe } 95 \mathrm{ZC} \\
\text { Abe } 95 \mathrm{ZI}\end{array}$ & $\begin{array}{l}- \\
-\end{array}$ \\
\hline & Abachi $95 \mathrm{ZB}$ & $\mathrm{cs}, \mathrm{p}, \mathrm{pt}$ & & Wenzel 96 & cs,pl & & Anwaywiese 95 & 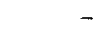 \\
\hline & Ваuer 95 & & & Yu 96B & $\mathrm{cs}, \mathrm{p}, \mathrm{pt}$ & & $\begin{array}{l}\text { Derwent 95 } \\
\text { Papadimitrio } 95\end{array}$ & $\operatorname{cs.pt}$ \\
\hline & Bazizi 95 & & & $\begin{array}{l}\text { Abe } 95 \mathrm{E} \\
\text { A be } 95 \mathrm{M}\end{array}$ & $\mathrm{p}, \mathrm{pt}$ & & & $\mathrm{cs}, \mathrm{pt}$ \\
\hline & $\begin{array}{l}\text { Derwent } 95 \\
\text { Markosky } 95\end{array}$ & $\begin{array}{r}\mathrm{p}, \mathrm{pt} \\
\mathrm{cs}, \mathrm{et}, \mathrm{p}\end{array}$ & & Abe $95 \mathrm{~N}$ & -1 & & Skarha 95 & - \\
\hline & Barker 94 & $\mathrm{cs}, \mathrm{p}, \mathrm{pt}$ & & A be $95 \mathrm{P}$ & - & & Ukegawa 95 & \\
\hline & Cdfcollabora $94 \mathrm{~F}$ & & & Abe $95 R$ & - & & Abe $942 F$ & $\begin{array}{r}c s, p, p t \\
c s, p t\end{array}$ \\
\hline & & $\mathrm{cs}, \mathrm{p}, \mathrm{pt}$ & & A be 95ZB & - & & $\begin{array}{l}\text { Byrum } 94 \\
\text { Cdfcollabora } 94 \mathrm{~B}\end{array}$ & pt \\
\hline & $\begin{array}{l}\text { Garbincius } 94 \\
\text { Luke } 93\end{array}$ & $\mathrm{p}, \mathrm{pt}$ & & $\begin{array}{l}\text { Abe } 95 \mathrm{ZC} \\
\text { A be } 95 \mathrm{ZI}\end{array}$ & - & & Dejongh 94 & \\
\hline$B \mathrm{X}+\bar{B} \mathrm{X}$ & & & & Anwaywiese 95 & - & & Garbincius 94 & $\mathrm{p}, \mathrm{pt}$ \\
\hline$(1800)$ & A be $97 \mathrm{~F}$ & - & & Derwent 95 & & & $\begin{array}{l}\text { Hara 94 } \\
\text { Karen } 94\end{array}$ & cs \\
\hline & Ohl 96 & $\mathrm{cs}, \mathrm{p}, \mathrm{pt}$ & & $\begin{array}{l}\text { Papadimitrio } 95 \\
\text { Ragan } 95\end{array}$ & $\mathrm{pt}$ & & Lewis 94 & $\mathrm{pt}$ \\
\hline$B+\mathrm{X}$ & & & & Skarha 95 & - & & Lewis $94 \mathrm{~B}$ & cs,pt \\
\hline$(1800)$ & A be 99D & cs & & Ukegawa 95 & - & & Plunkett 94 & cs,pt \\
\hline & $\begin{array}{l}\text { A be } 98 \mathrm{E} \\
\text { A be } 97 \mathrm{ZI}\end{array}$ & $z$ & & $\begin{array}{l}\text { Abe } 94 \mathrm{D} \\
\text { Abe } 94 Z \mathrm{~F}\end{array}$ & $c s, p, p t$ & $B^{-} \mathrm{X}$ & Shochet 94 & $\mathrm{cs}, \mathrm{pt}$ \\
\hline & A be $96 \mathrm{D}$ & - & & Bailey 94 & cs,pt & $(1800)$ & Abe $99 \mathrm{D}$ & cs \\
\hline & A be $96 \mathrm{E}$ & - & & Byrum 94 & cs,pt & $(1000)$ & Abe $98 \mathrm{E}$ & - \\
\hline & Abe $96 \bar{K}$ & - & & Cdfcollabora 94B & $\mathrm{pt}$ & & A be 980 & - \\
\hline & Abe $96 \mathrm{~N}$ & & & Cdfcollabora $94 \mathrm{E}$ & $\mathrm{cs}, \mathrm{pt}$ & & Abe $97 Z I$ & - \\
\hline & Barnett 96 & $\mathrm{cs}, \mathrm{p}, \mathrm{pt}$ & & Dejongh 94 & & & Abe $96 \mathrm{D}$ & - \\
\hline & Bauer 96 & $\mathrm{cs}, \mathrm{p}, \mathrm{pt}$ & & Garbincius 94 & $\mathrm{p}, \mathrm{pt}$ & & Abe $96 \mathrm{E}$ & - \\
\hline & Huffman 96 & $\mathrm{cs}, \mathrm{p}, \mathrm{pt}$ & & Hara 94 & $\mathrm{cs}$ & & A be $96 \mathrm{~K}$ & - \\
\hline & Laasanen 96 & $\mathrm{cs}, \mathrm{p}, \mathrm{pt}$ & & Karen 94 & $\mathrm{pt}$ & & Abe $96 \mathrm{~N}$ & - \\
\hline & Lewis 96 & $\mathrm{cs}, \mathrm{p}, \mathrm{pt}$ & & Lewis 94 & $\mathrm{pt}$ & & Barnett 96 & $\mathrm{cs}, \mathrm{p}, \mathrm{pt}$ \\
\hline & Lucchesi 96 & & & Lewis 94B & cs,pt & & Bauer 96 & $\mathrm{cs}, \mathrm{p}, \mathrm{pt}$ \\
\hline & Meschi 96 & & & Mueller 94 & $\mathrm{cs}, \mathrm{pt}$ & & Huffinan 96 & $\mathrm{cs}, \mathrm{p}, \mathrm{pt}$ \\
\hline & Miao 96 & - & & Plunkett 94 & $\mathrm{cs}, \mathrm{pt}$ & & Laasanen 96 & $\mathrm{cs}, \mathrm{p}, \mathrm{pt}$ \\
\hline & Paulini 96 & & & Shochet 94 & cs,pt & & Lewis 96 & $\mathrm{cs}, \mathrm{p}, \mathrm{pt}$ \\
\hline & Slaughter 96 & $p, p t$ & $\bar{B} \mathrm{X}$ & & & & Lucchesi 96 & \\
\hline & $\begin{array}{l}\text { Wang } 96 \\
Y_{u} 96 B\end{array}$ & $\begin{aligned} c s, p t \\
c s, p, p t\end{aligned}$ & $(1800)$ & Abbott $98 \mathrm{~B}$ & & & Meschi 96 & - \\
\hline & $\begin{array}{l}\text { Yu } 96 \mathrm{~B} \\
\text { Abe } 95 \mathrm{E}\end{array}$ & $\begin{array}{r}\mathrm{cs}, \mathrm{p}, \mathrm{pt} \\
\mathrm{p}, \mathrm{pt}\end{array}$ & & Abachi 96 & $\mathrm{cs}, \mathrm{p}, \mathrm{pt}$ & & $\begin{array}{l}\text { Miao } 96 \\
\text { Pauting }\end{array}$ & - \\
\hline & Abe $95 \mathrm{~N}$ & - & & Abachi 96I & & & Slaughter 96 & $\mathrm{p}, \mathrm{pt}$ \\
\hline & Abe 950 & - & & Demina 96 & & & Wang 96 & cs,pt \\
\hline & A be $95 \mathrm{P}$ & - & & $\begin{array}{l}\text { Papadimitrio 96B } \\
\text { Wenzel 96B }\end{array}$ & - & & Yu $96 \mathrm{~B}$ & $\mathrm{cs}, \mathrm{p}, \mathrm{pt}$ \\
\hline & $\begin{array}{l}\text { Abe } 95 X \\
\text { Abe } 95 Z I\end{array}$ & cs & & Abachi $95 \mathrm{P}$ & $\mathrm{p}, \mathrm{pt}$ & & Abe $95 \mathrm{E}$ & $\mathrm{p}, \mathrm{pt}$ \\
\hline & $\begin{array}{l}\text { Abe } 95 Z 1 \\
\text { Anwaywiese } 95\end{array}$ & - & & Abachi 95ZB & $\mathrm{cs}, \mathrm{p}, \mathrm{pt}$ & & $\begin{array}{l}\text { Abe } 95 \mathrm{~N} \\
\text { A be } 95 \mathrm{O}\end{array}$ & - \\
\hline & Derwent 95 & - & & $\begin{array}{l}\text { Bauer 95 } \\
\text { Bazizi 95 }\end{array}$ & $p, \overline{p t}$ & & Abe $95 \mathrm{P}$ & - \\
\hline & Papadimitrio 95 & $\mathrm{cs}, \mathrm{pt}$ & & $\begin{array}{l}\text { Baz1z1 } 95 \\
\text { Derwent } 95\end{array}$ & $\begin{array}{l}p, p t \\
\mathrm{p}, \mathrm{pt}\end{array}$ & & Abe $95 X$ & cs \\
\hline & $\begin{array}{l}\text { Ragan } 95 \\
\text { Skarha } 95\end{array}$ & - & & Markosky 95 & $\mathrm{cs}, \mathrm{et}, \mathrm{p}$ & & Abe 95ZI & - \\
\hline & Ukegawa 95 & - & & Barker 94 & $\mathrm{cs}, \mathrm{p}, \mathrm{pt}$ & & $\begin{array}{l}\text { Anwaywiese } 95 \\
\text { Derwent } 95\end{array}$ & 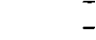 \\
\hline & A be $94 \mathrm{D}$ & - & & Cdfcollabora & $c s, p, p t \mid$ & & Papadimitrio 95 & $\mathrm{cs}, \mathrm{pt}$ \\
\hline & Anwaywiese 94 & $p, p t$ & & Garbincius 94 & $c s, p, p t$ & & Ragan 95 & 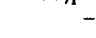 \\
\hline & Bailey 94 & cs,pt & $\bar{B} \mathbf{0} \times$ & Gatoliterus git & & & Skarha 95 & - \\
\hline & $\begin{array}{l}\text { Byrum 94 } \\
\text { Cdfcollabora } 94 \mathrm{E}\end{array}$ & $\begin{array}{l}c s, p t \\
c s, p t\end{array}$ & $B=A_{(1800)}$ & Abe $99 \mathrm{D}$ & cs & & Ukegawa 95 & - \\
\hline & Dejongh 94 & $\cos , p_{2}$ & & Abe $98 \mathrm{E}$ & -1 & & $\begin{array}{l}\text { Anwaywiese } 94 \\
\text { Byrum } 94\end{array}$ & $\begin{array}{r}\mathrm{p}, \mathrm{pt} \\
\mathrm{cs}, \mathrm{pt}\end{array}$ \\
\hline & Garbincius 94 & $\mathrm{p}, \mathrm{pt}$ & & A be 980 & - & & Dejongh 94 & - \\
\hline
\end{tabular}




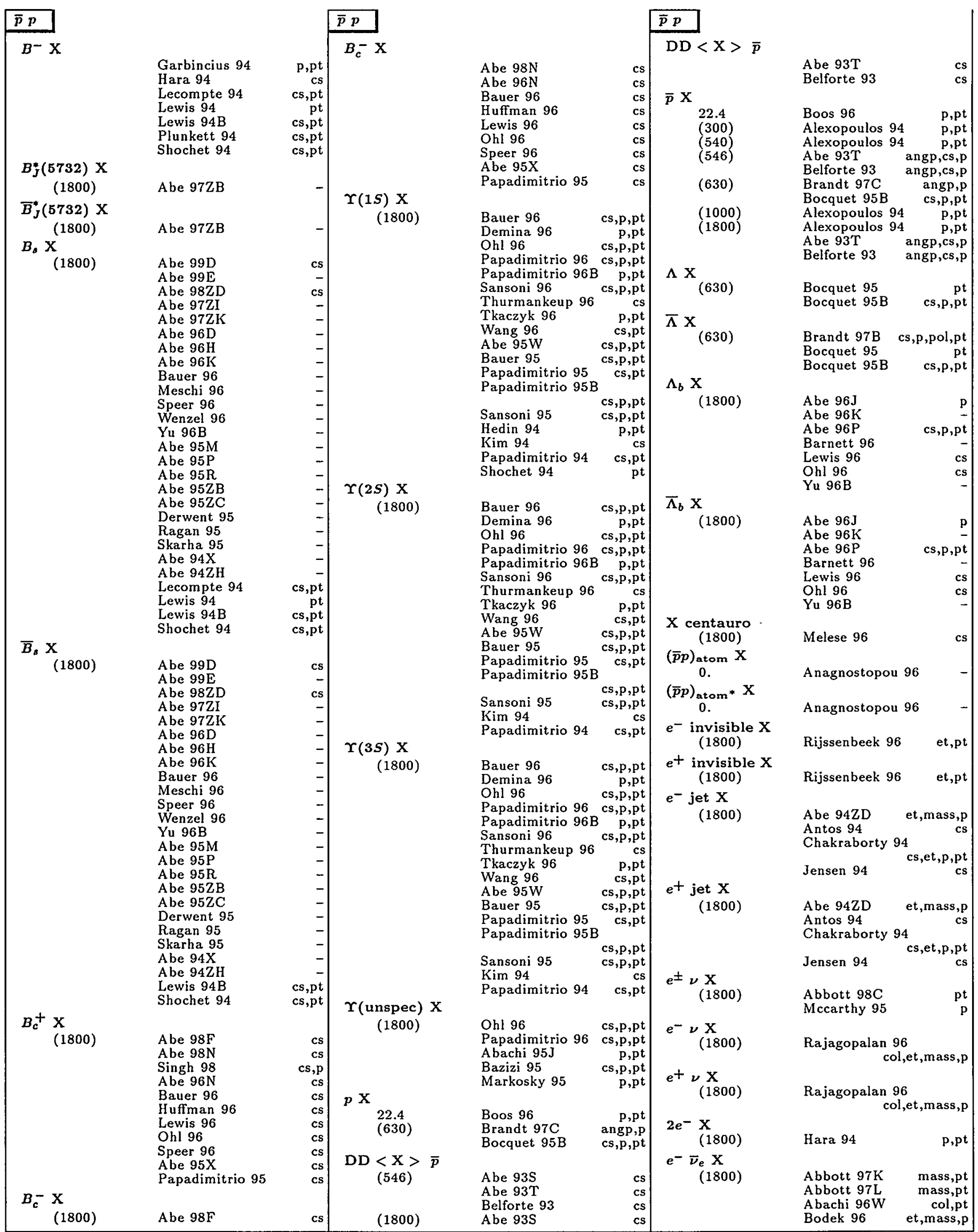

Entries are in order of beam name, then target name, then multiplicity of final state. Particle names are ordered as described in the legend on page 157 and as listed in the Particle Vocabulary. See also the Table of Contents of this Index beginning on the page 158. A few chemical symbols for nuclei have been changed to avoid ambiguity with particle names (see the Particle Vocabulary). Beam momenta are $p_{l a b}$ in GeV/c, or in parentheses $E_{c m}$ in $\mathrm{GeV}$. 


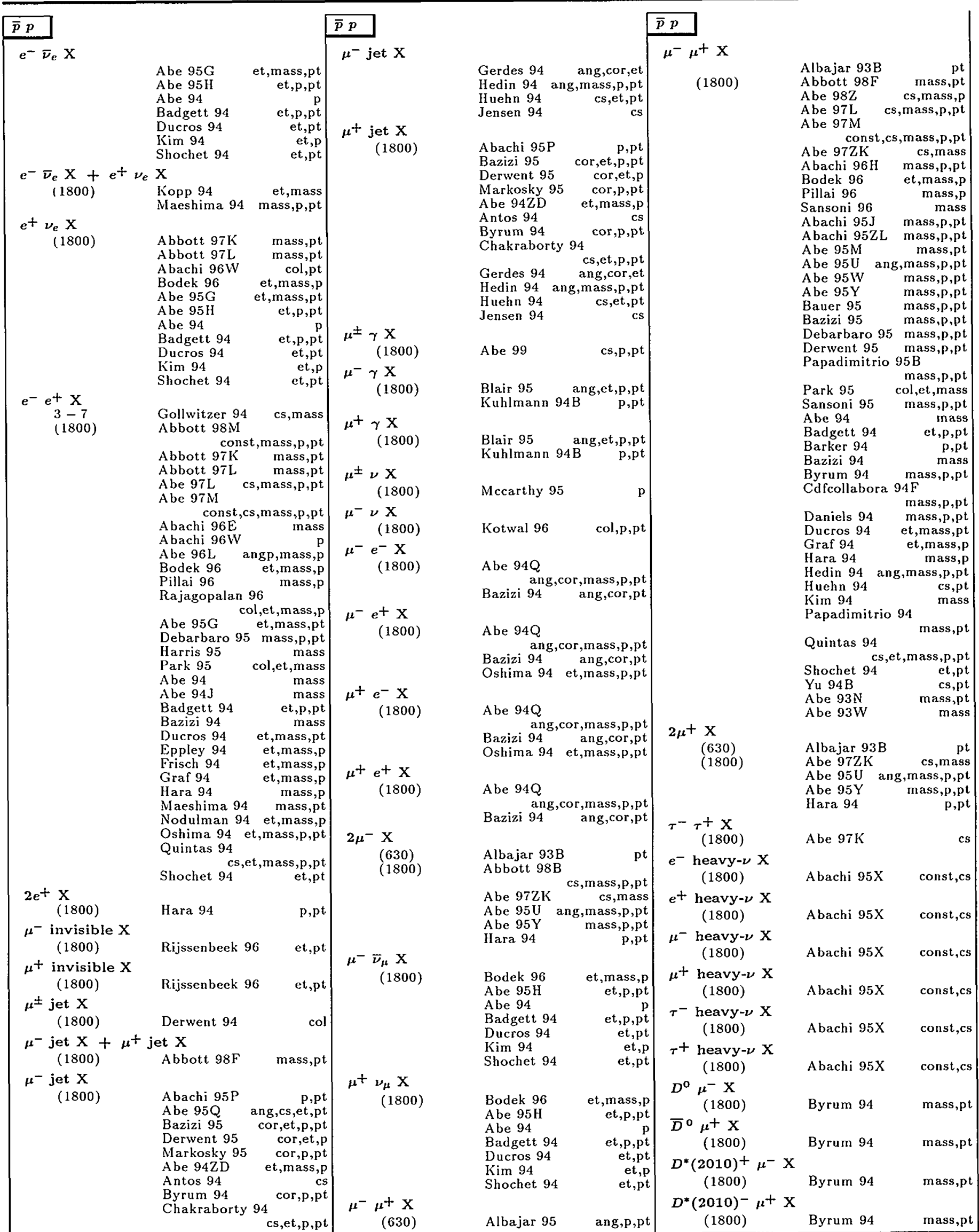




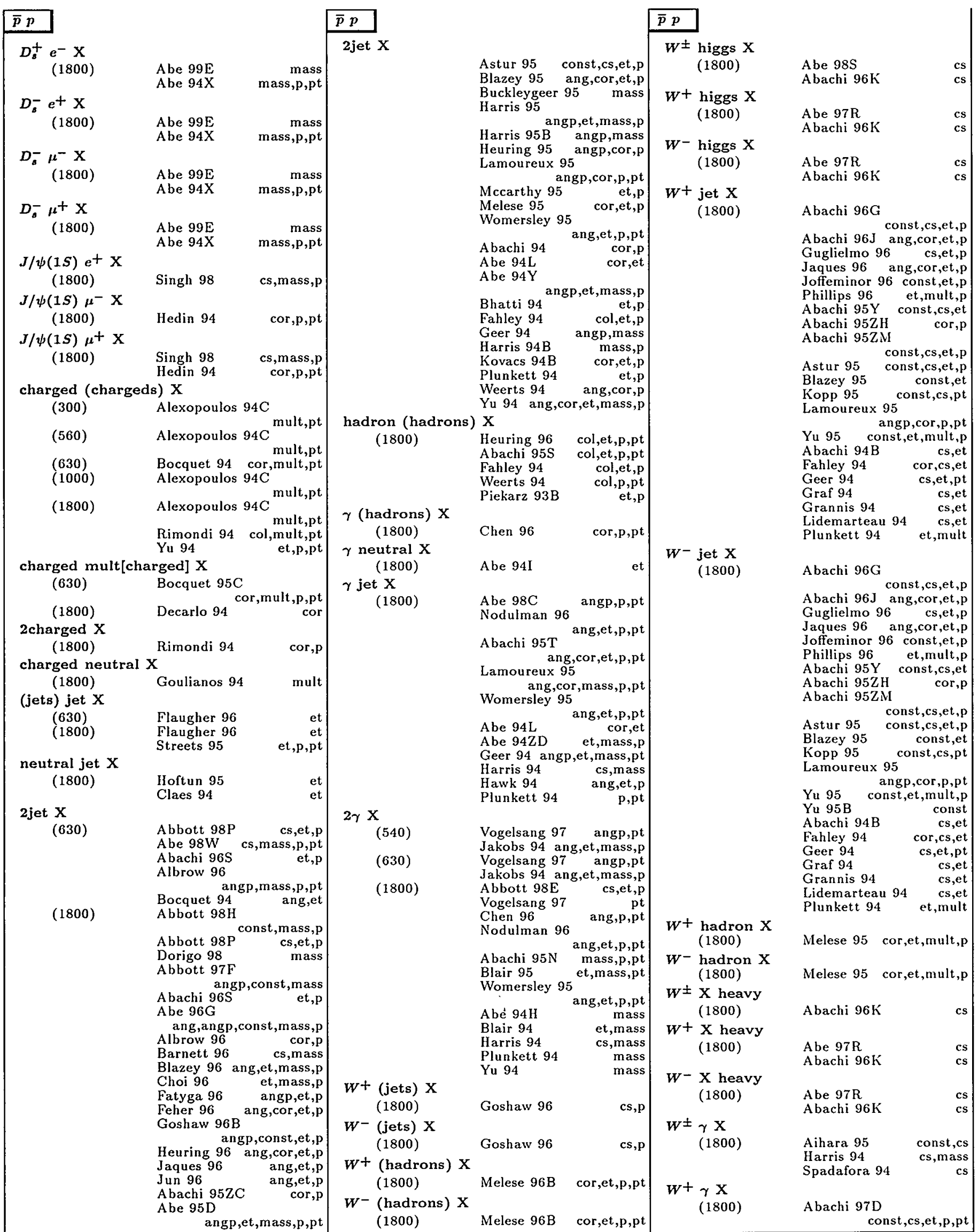

Entries are in order of beam name, then target name, then multiplicity of final state. Particle names are ordered as described in the legend on page 157 and as listed in the Particle Vocabulary. See also the Table of Contents of this Index beginning on the page 158. A few chemical symbols for nuclei have been changed to avoid ambiguity with particle names (see the Particle Vocabulary). Beam momenta are $p_{\mathrm{lab}}$ in GeV/c, or in parentheses $E_{\mathrm{cm}}$ in $\mathrm{GeV}$. 
$\bar{p} p$

$w+\gamma \mathrm{X}$

$W^{-} \gamma \mathrm{X}$

$(1800)$

$W^{-} \boldsymbol{\gamma} \mathrm{X}+\boldsymbol{W}^{+} \boldsymbol{\gamma}$

(1800)

$w-W+\mathrm{X}$

(1800)

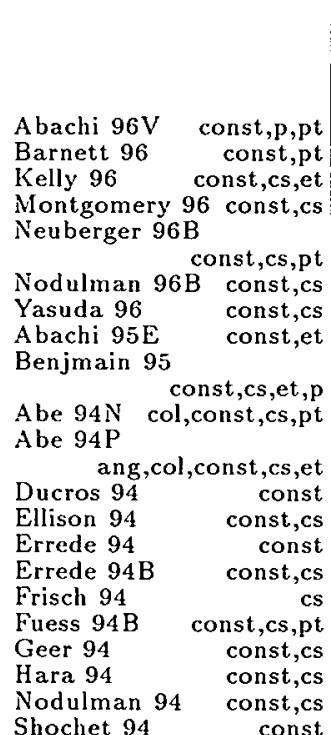

Shochet 94

const

Abachi $97 \mathrm{D}$

const,cs,et,p,pt

Abachi $96 \mathrm{~V}$ const,p,pt

Barnett 96 const,pt

Kelly 96 const,cs,et

Montgomery 96 const,cs

Neuberger $96 \mathrm{~B}$

Nodulman 96B const,cs

Yasuda 96 const,cs

Abachi $95 \mathrm{E}$ const,et

Benjmain 95

const,cs,et,p

Abe $94 \mathrm{~N}$ col,const,cs,pt

Abe $94 \mathrm{P}$

ang,col,const,cs,et

Ducros 94

Ellison 94

Errede 94

Errede 94B const,cs

Frisch 94

Fuess $94 \mathrm{~B}$

const, cs

Hara 94 const,cs

Nodulman 94 const,cs

$\begin{array}{lr}\text { Shochet } 94 & \text { const }\end{array}$

$\gamma \mathrm{X}$

Abbott $98 \mathrm{G}$

Abbott $98 \mathrm{D}$

Abbott $98 \mathrm{G}$

Abachi $97 \mathrm{D}$

Abbott const,cs,et,p,pt

Abbott 97C const,cs

Abachi $96 \mathrm{D}$

Abe $96 \mathrm{R}$ const,cs,p,pt

$\begin{array}{ll}\text { Abe } 96 R & \text { const,cs } \\ \text { Barnett } 96 & \text { const,pt }\end{array}$

Kelly 96 const,cs,et

Neuberger 96

Neuberger $96 \mathrm{~B}$

Nodulman 96B const,cs

Yasuda 96 const,cs

Abachi $95 \mathrm{C}$ const,cs

Abachi $95 U$ const,cs

Abachi 95ZF const,cs

Abe 95L const,cs,pt

Diehl 95

Fuess 95

Ducros 94

Errede 94

Frisch 94

Fuess 94B

Hara 94

Oshima 94

const,cs

const, cs,pt

const
const

$\bar{p} p$

$Z^{\circ}$ (jets) X

(1800)

$Z^{0}$ higgs $\mathrm{X}$

(1800)

$Z^{\circ}$ jet $\mathrm{X}$

(1800)

$Z^{\circ} \gamma \mathrm{X}$

(1800)

$Z^{\circ} w^{+} \mathrm{X}$

(1800)

$z^{\circ} w^{-X}$

(1800)

const, cs,pt const, cs

Shochet 94 $\mathrm{cs}$
const

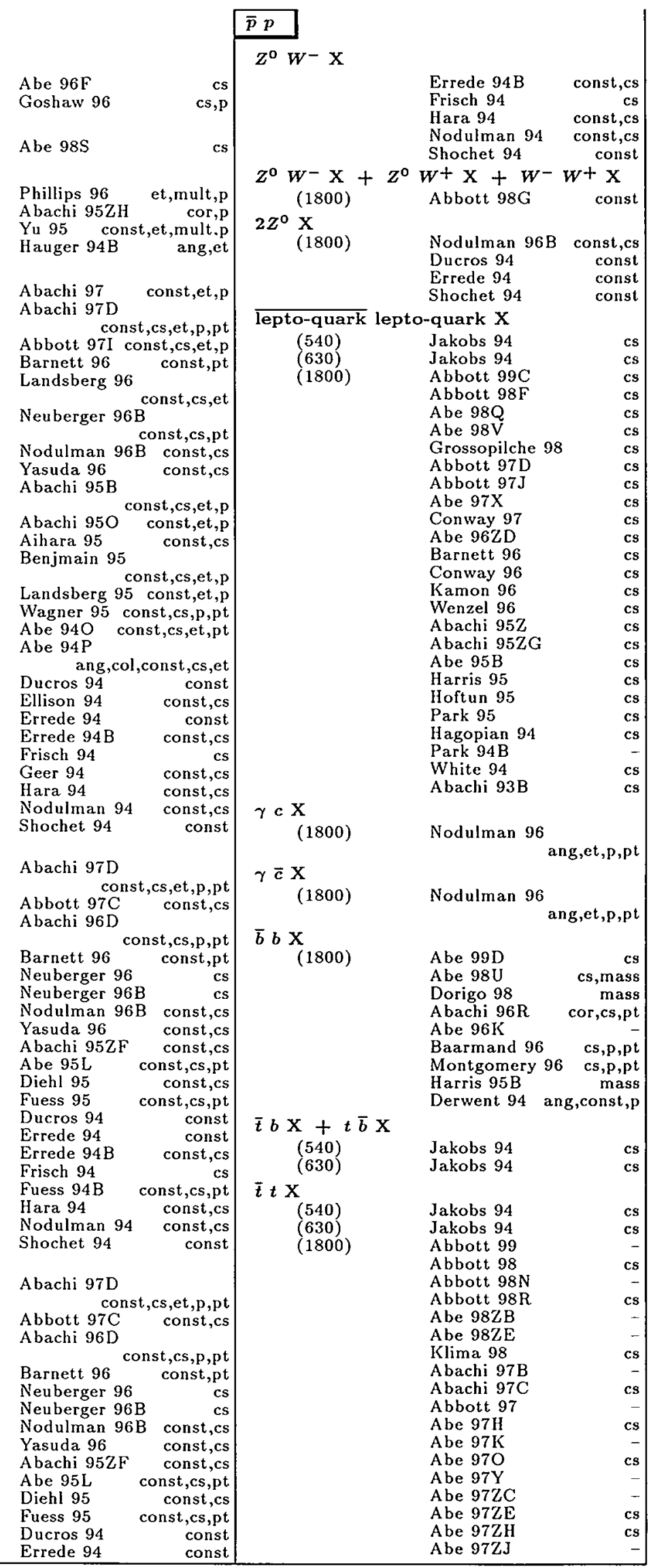




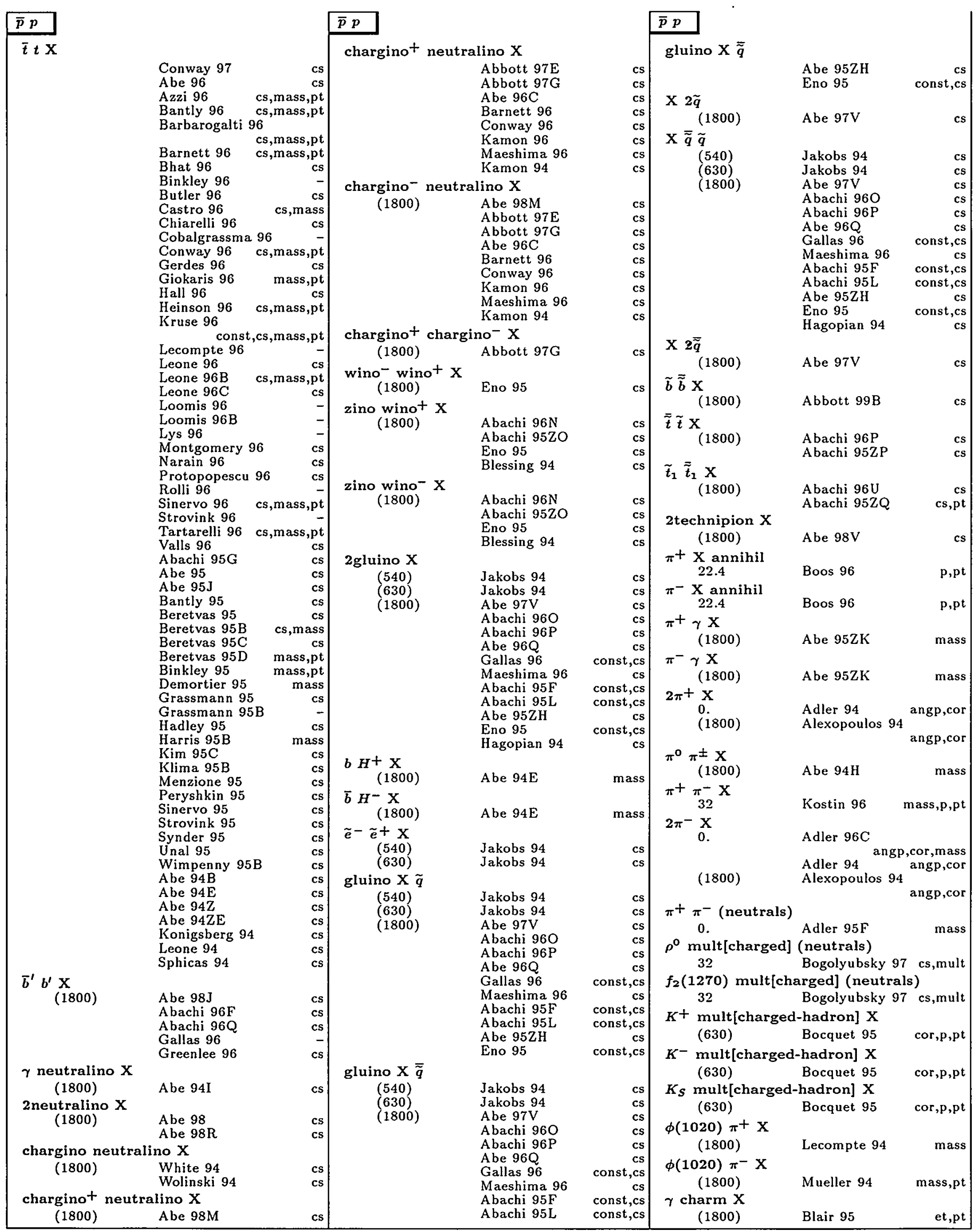

Entries are in order of beam name, then target name, then multiplicity of final state. Particle names are ordered as described in the legend on page 157 and as listed in the Particle Vocabulary. See also the Table of Contents of this Index beginning on the page 158 . A few chemical symbols for nuclei have been changed to avoid ambiguity with particle names (see the Particle Vocabulary). Beam momenta are $p_{\text {lab }}$ in GeV/c, or in parentheses $E_{\mathrm{cm}}$ in $\mathrm{GeV}$. 
$\bar{p} p \rightarrow \gamma$ charm X

$\bar{p} p \rightarrow e^{-}$hadron (hadrons) $\mathrm{X} 0 \mathrm{jet}$

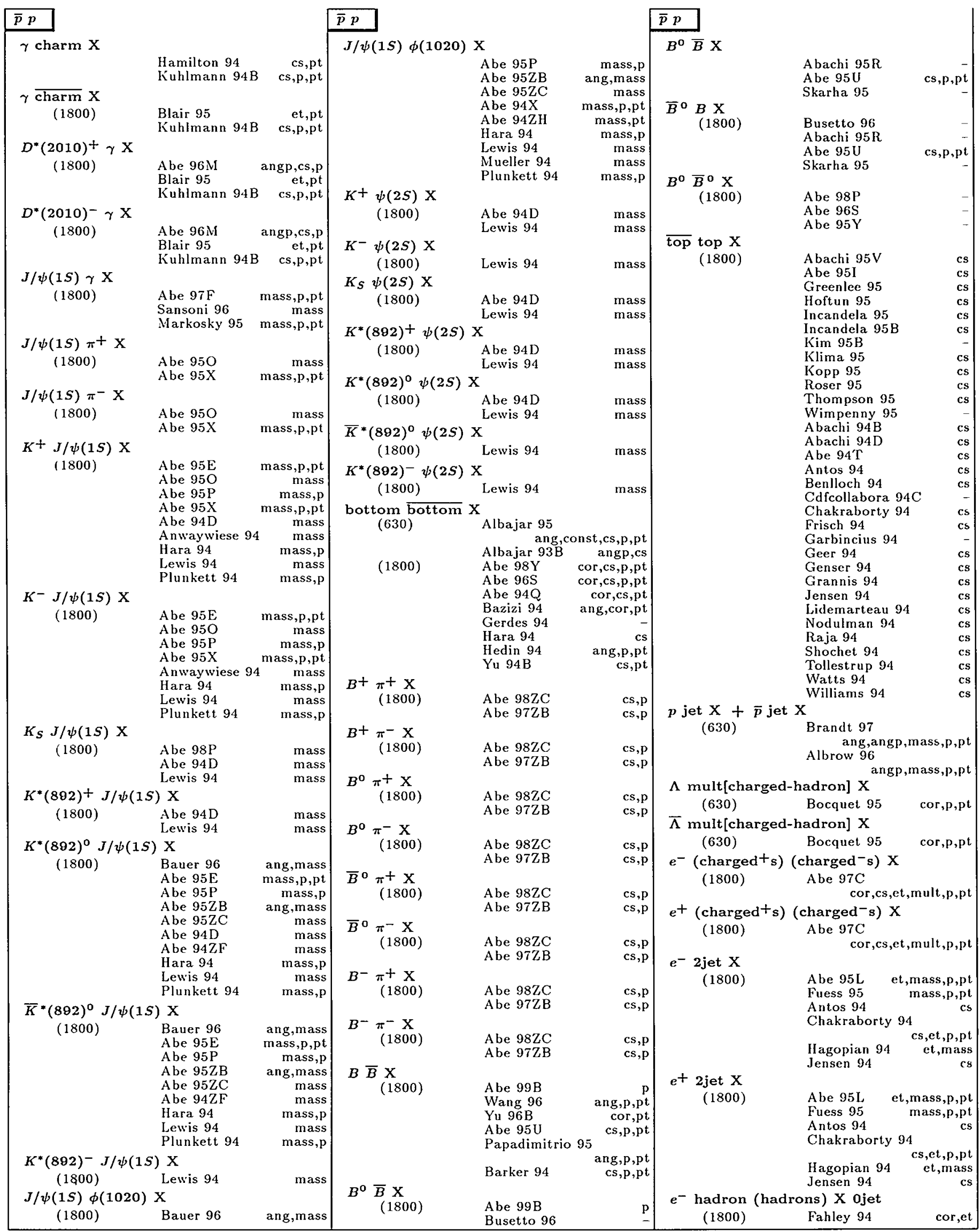




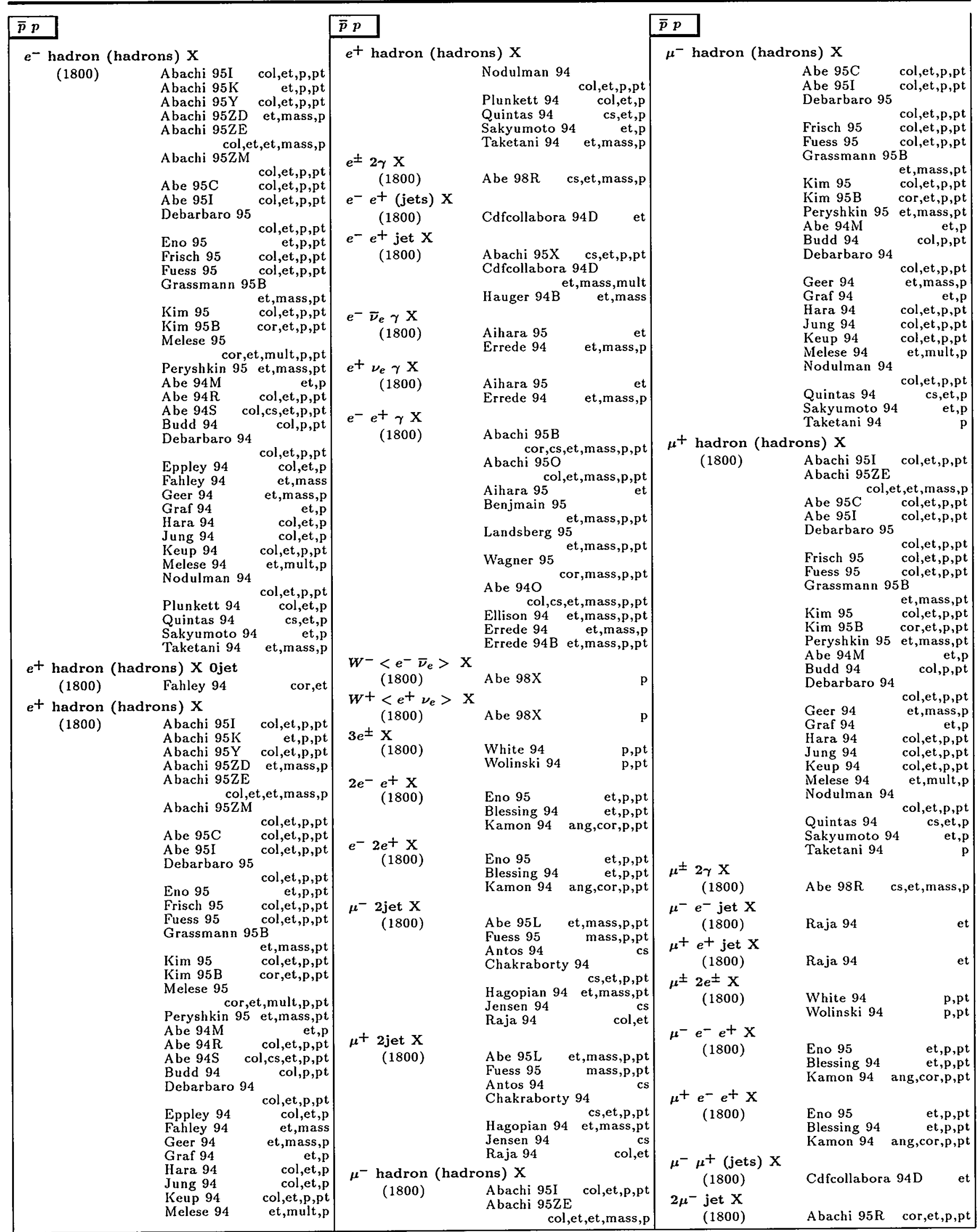

Entries are in order of beam name, then target name, then multiplicity of final state. Particle names are ordered as described in the legend on page 157 and as listed in the Particle Vocabulary. See also the Table of Contents of this Index beginning on the page 158. A few chemical symbols for nuclei have been changed to avoid ambiguity with particle names (see the Particle Vocabulary). Beam momenta are plab in GeV/c, or in parentheses $E_{\mathrm{cm}}$ in $\mathrm{GeV}$. 


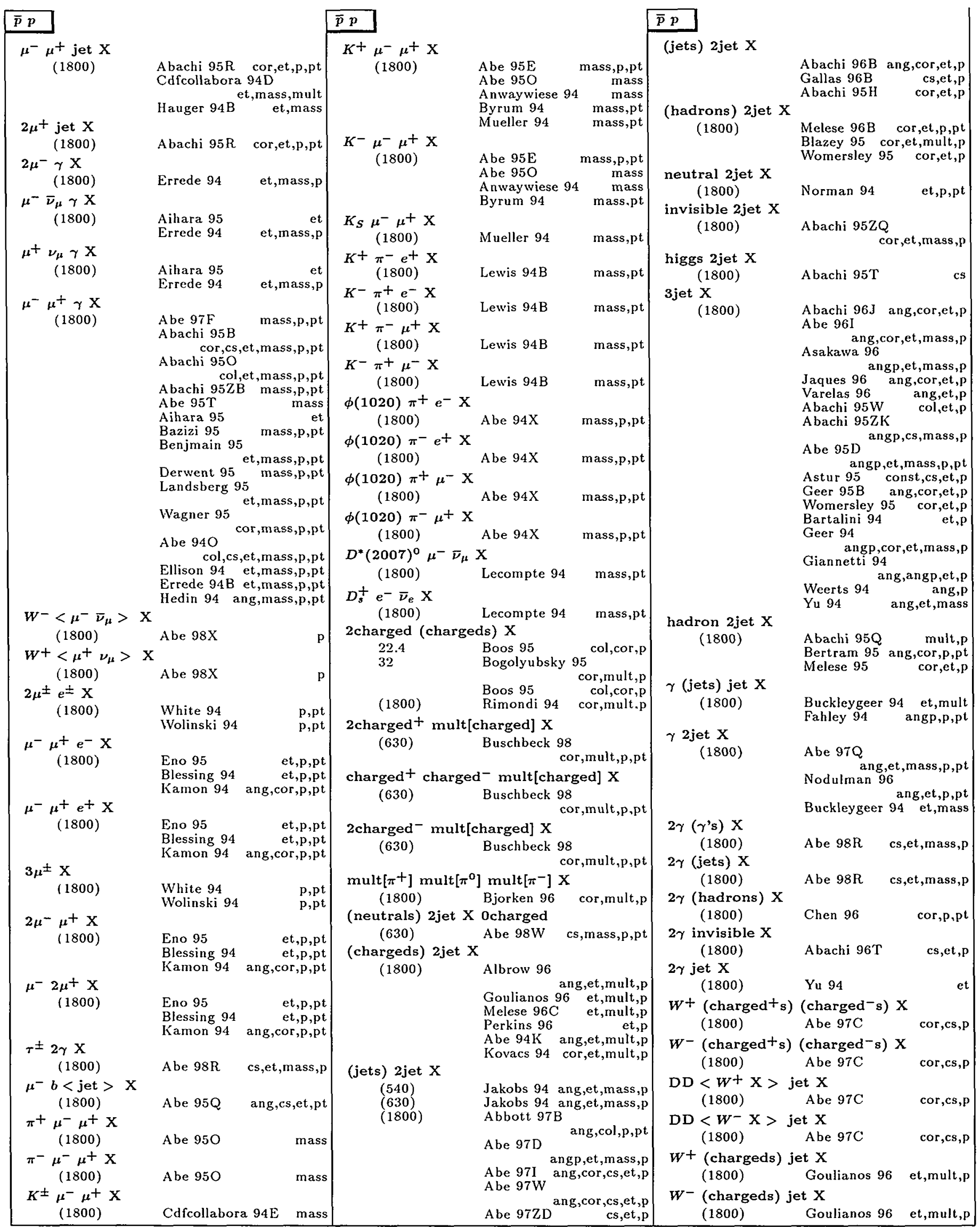




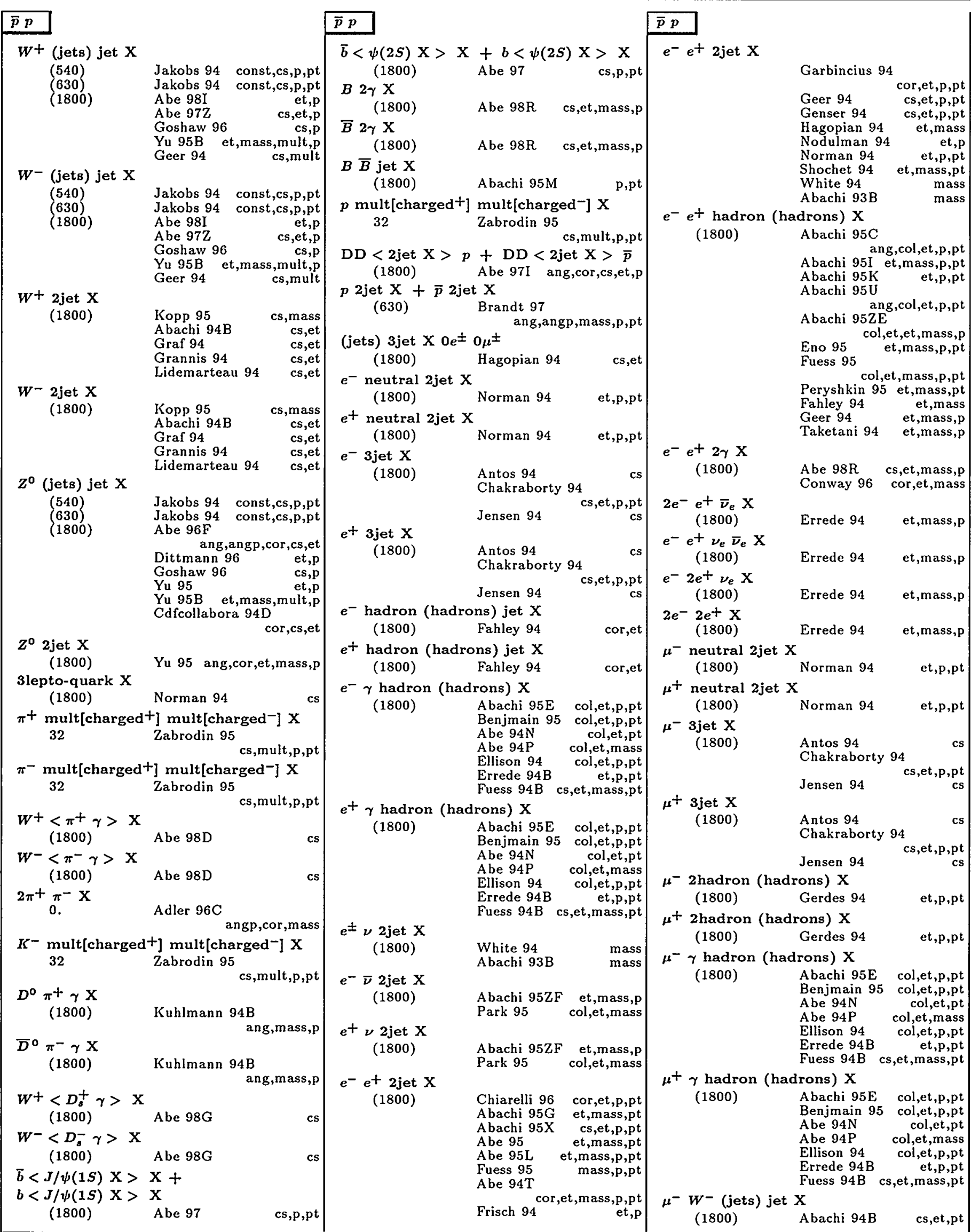

Entries are in order of beam name, then target name, then multiplicity of final state. Particle names are ordered as described in the legend on page 157 and as listed in the Particle Vocabulary. See also the Table of Contents of this Index beginning on the page 158. A few chemical symbols for nuclei have been changed to avoid ambiguity with particle names (see the Particle Vocabulary). Beam momenta are $p_{\text {lab }}$ in GeV/c, or in parentheses $E_{\mathrm{cm}}$ in $\mathrm{GeV}$. 


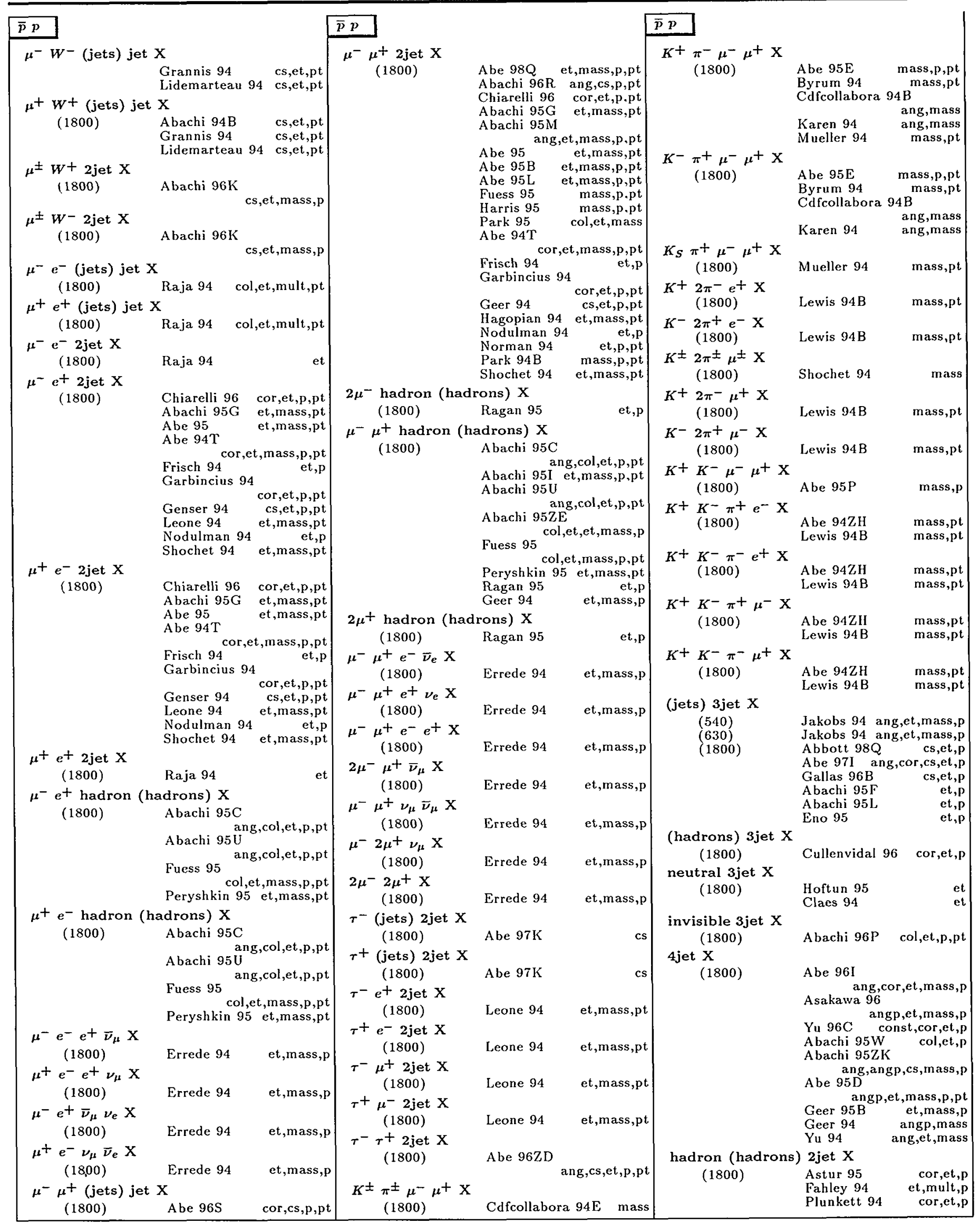




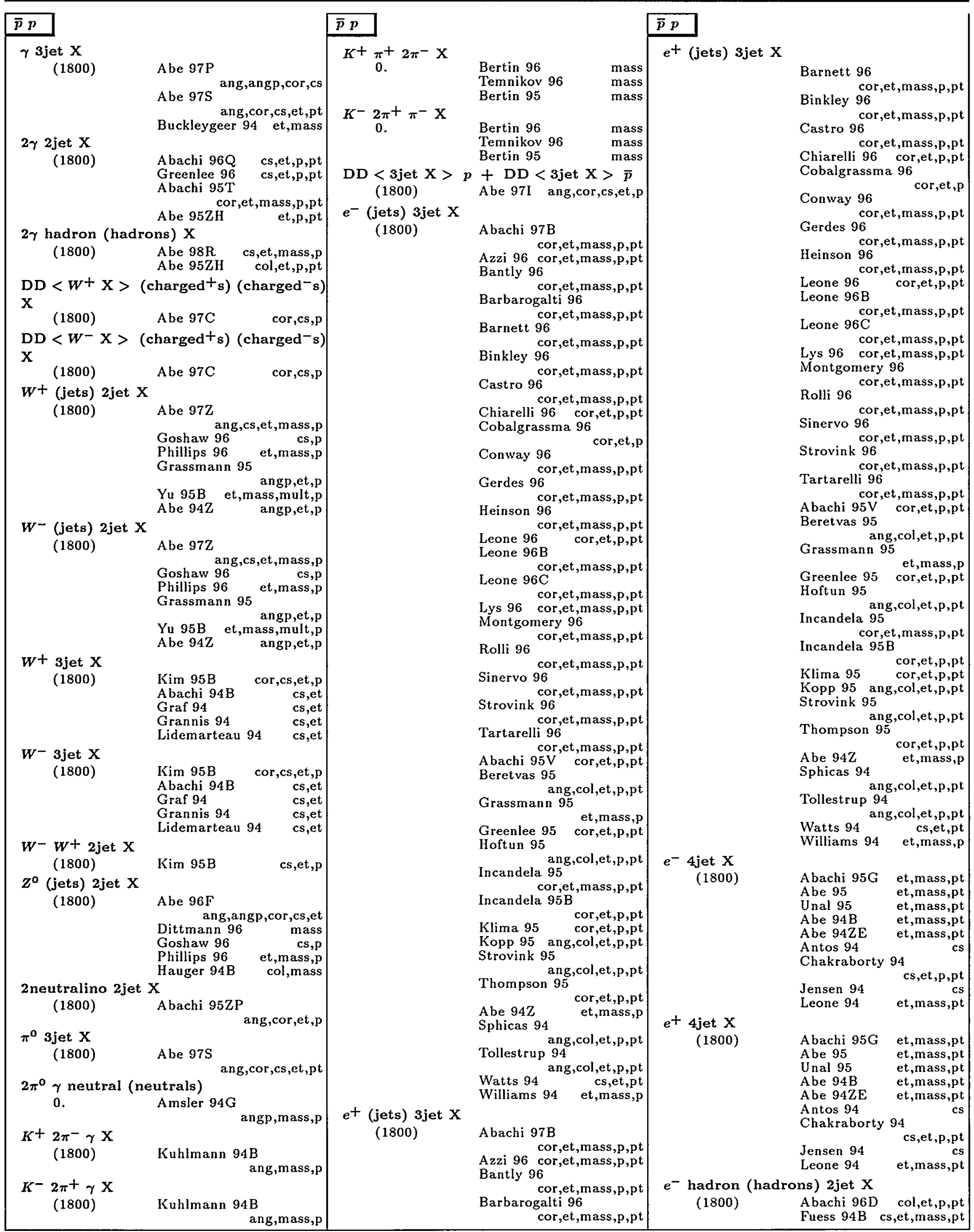

Entries are in order of beam name, then target name, then multiplicity of final state. Particle names are ordered as described in the legend on page 157 and as listed in the Particle Vocabulary. See also the Table of Contents of this Index beginning on the page 158. A few chemical symbols for nuclei have been changed to avoid ambiguity with particle names (see the Particle Vocabulary). Beam momenta are $p_{l a b}$ in GeV/c, or in parentheses $E_{\mathrm{cm}}$ in $\mathrm{GeV}$. 


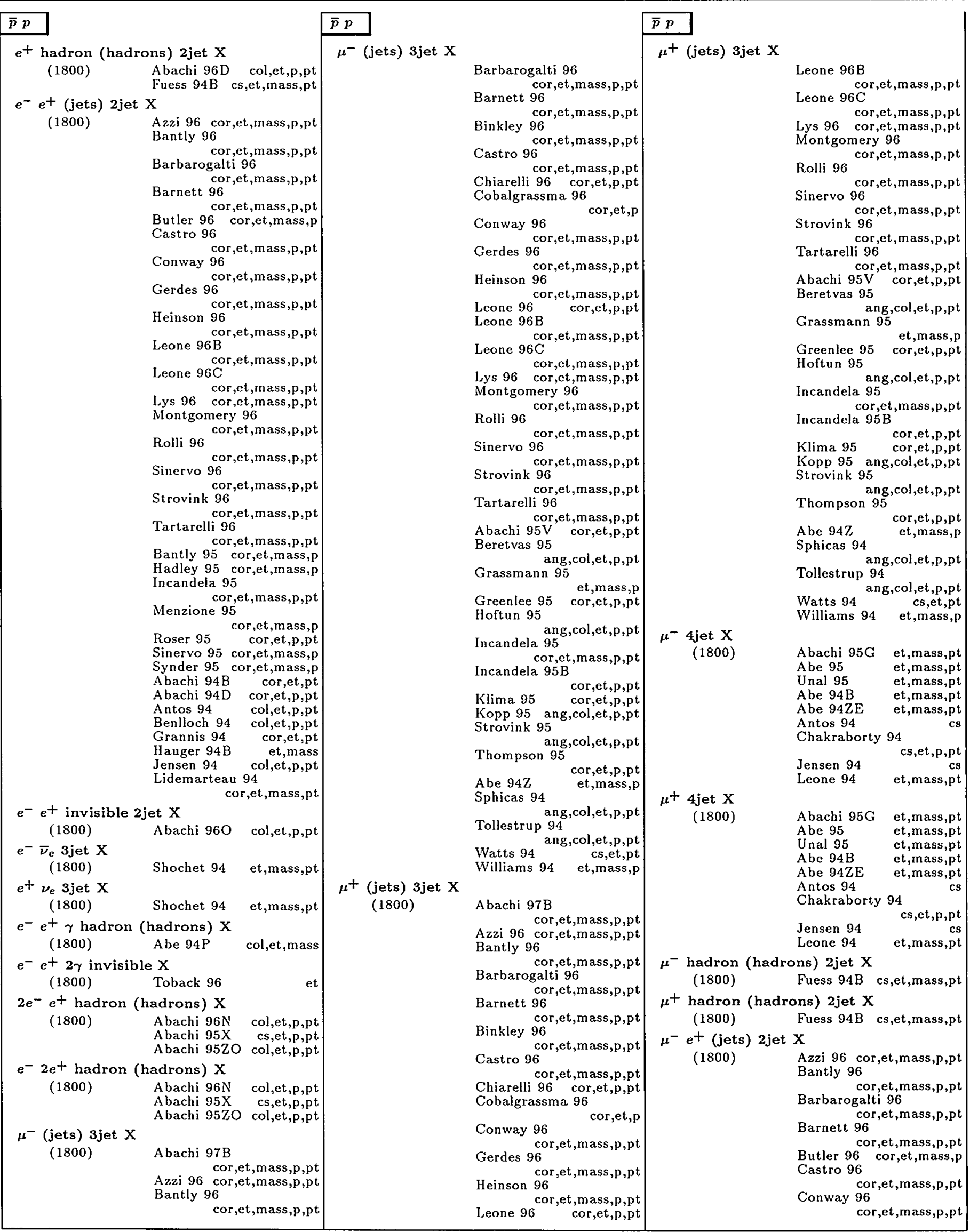




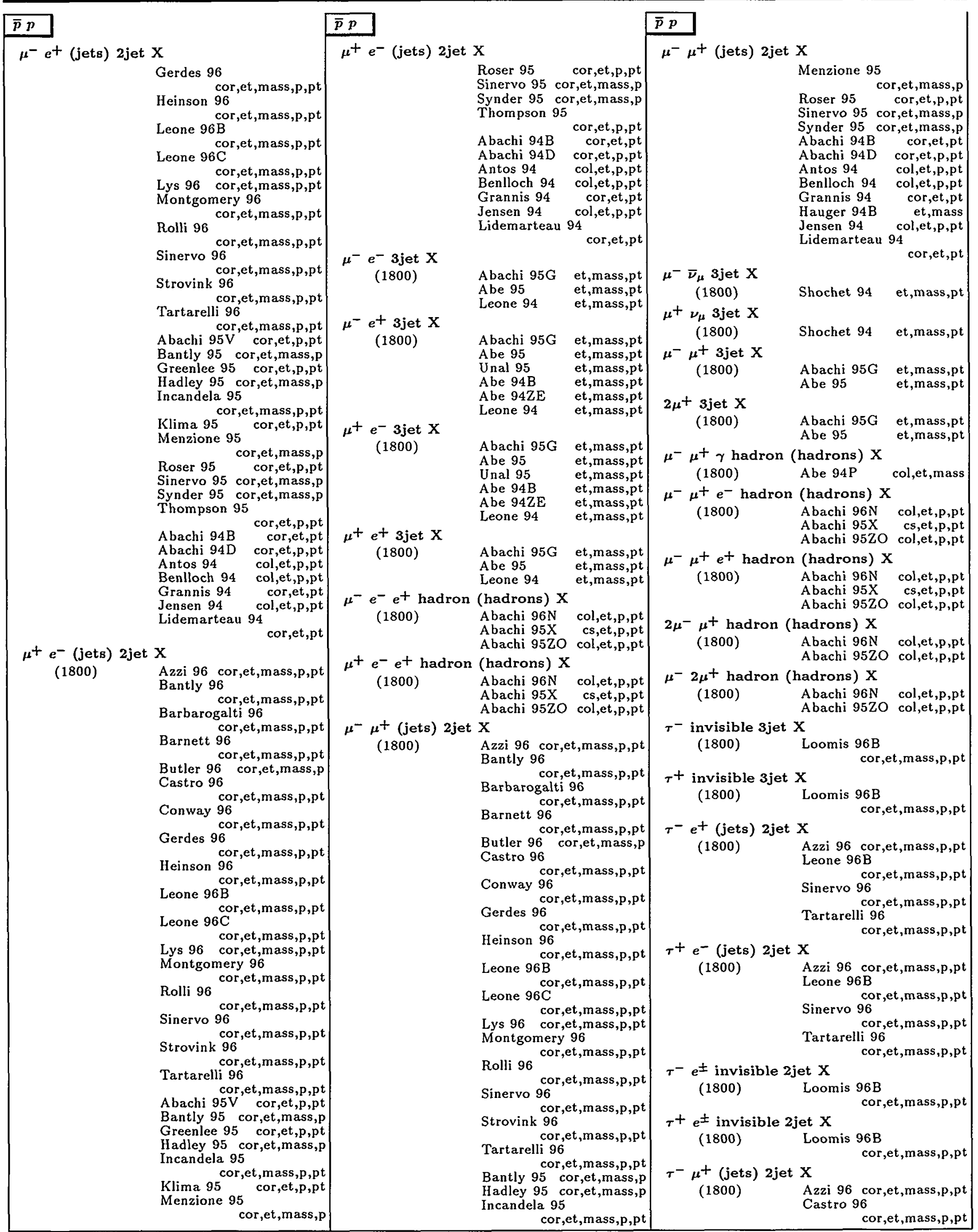

Entries are in order of beam name, then target name, then multiplicity of final state. Particle names are ordered as described in the legend on page 157 and as listed in the Particle Vocabulary. See also the Table of Contents of this Index beginning on the page 158. A few chemical symbols for nuclei have been changed to avoid ambiguity with particle names (see the Particle Vocabulary). Beam momenta are $p_{\text {lab }}$ in GeV/c, or in parentheses $E_{c m}$ in GeV. 


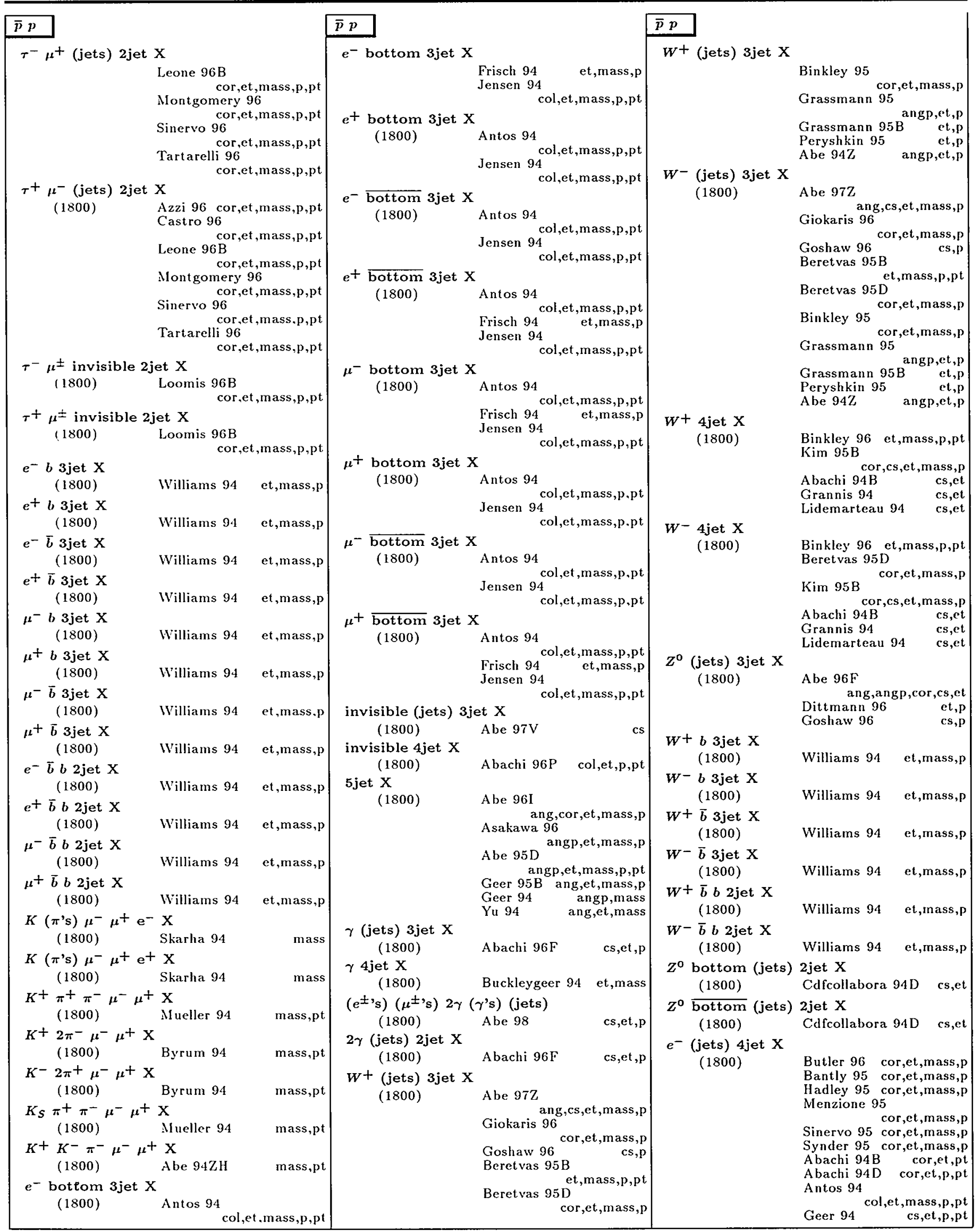




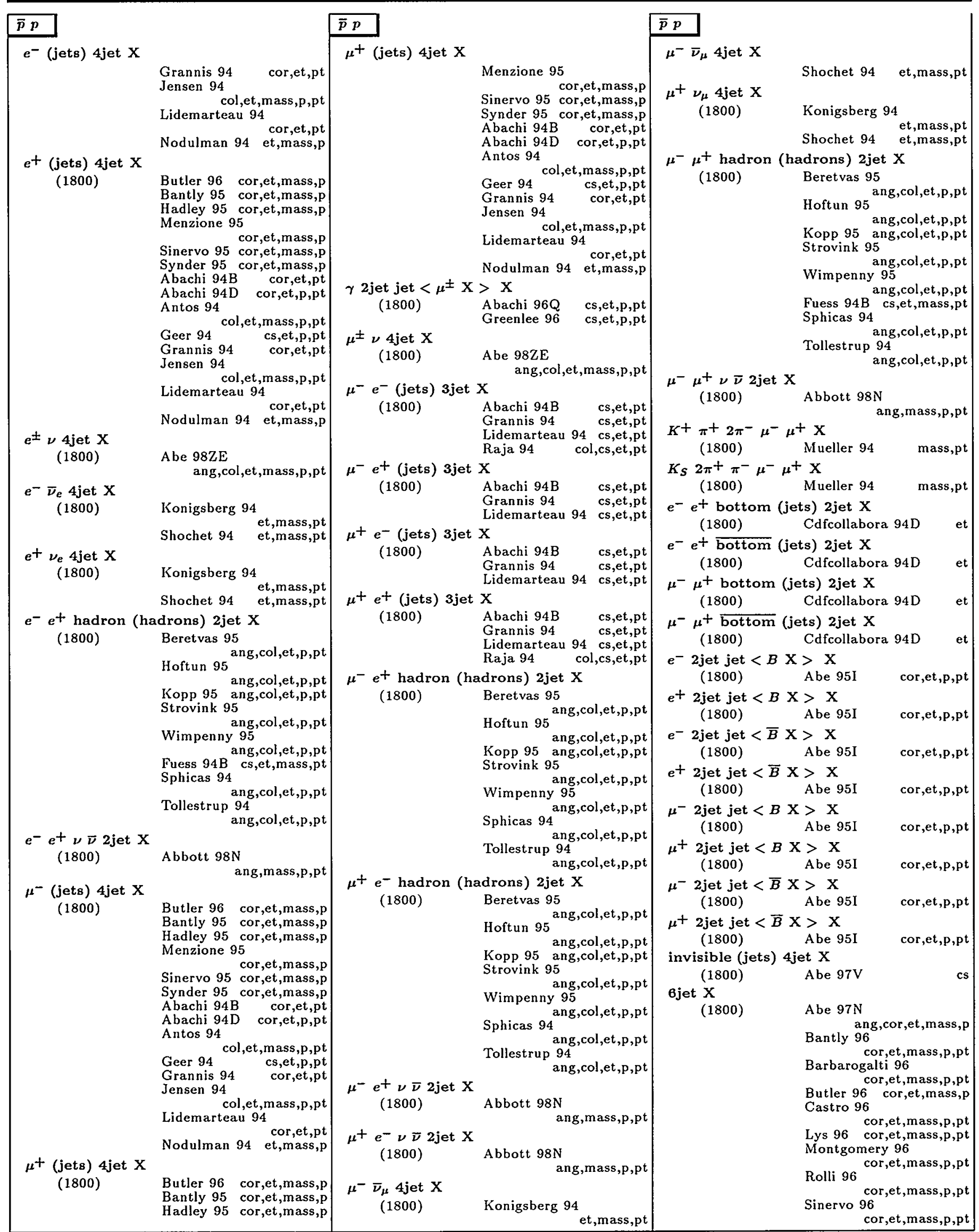

Entries are in order of beam name, then target name, then multiplicity of final state. Particle names are ordered as described in the legend on page 157 and as listed in the Particle Vocabulary. See also the Table of Contents of this Index beginning on the page 158. A few chemical symbols for nuclei have been changed to avoid ambiguity with particle names (see the Particle Vocabulary). Beam momenta are $p_{l a b}$ in GeV/c, or in parentheses $E_{\mathrm{cm}}$ in $\mathrm{GeV}$. 


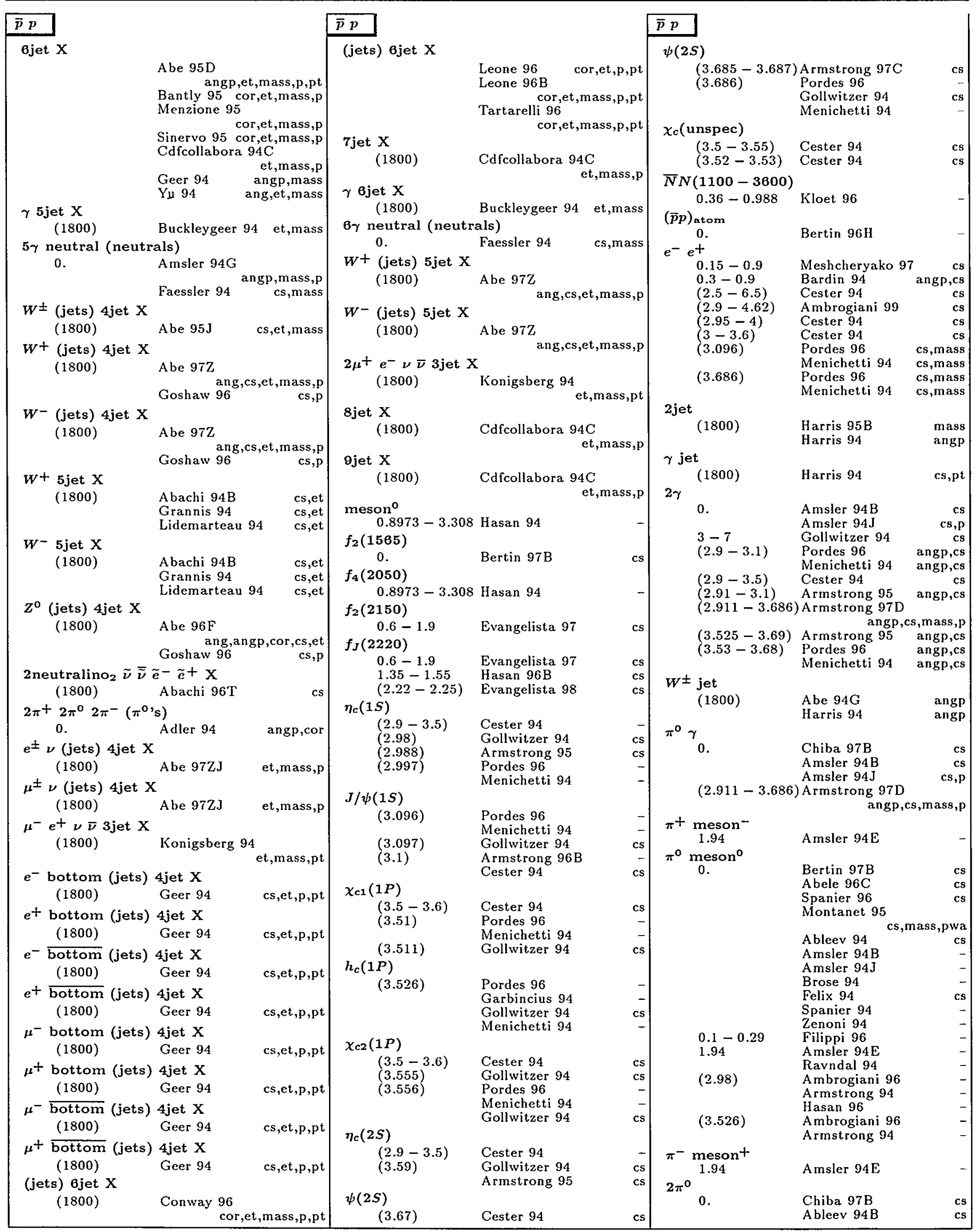




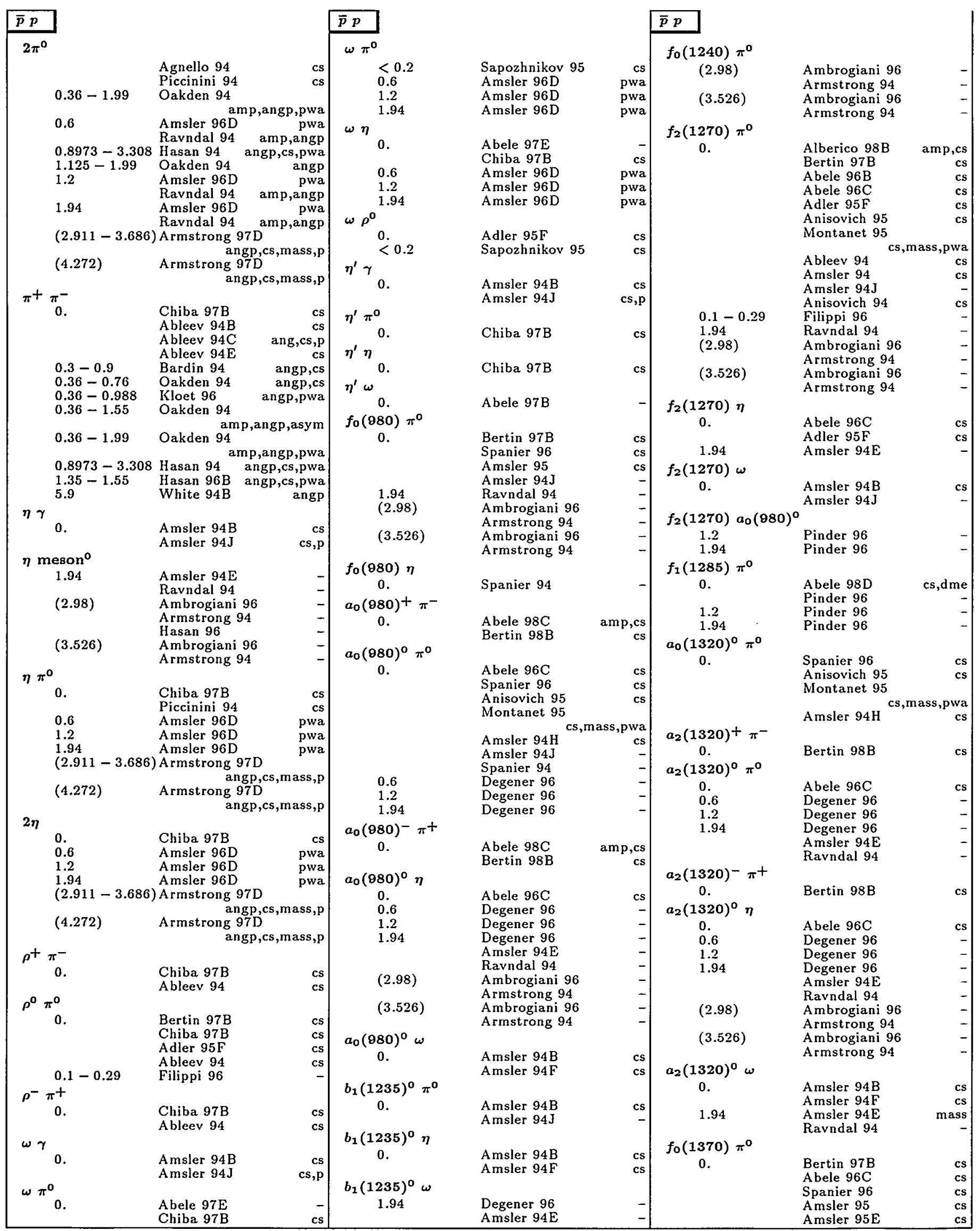

Entries are in order of beam name, then target name, then multiplicity of final state. Particle names are ordered as described in the legend on page 157 and as listed in the Particle Vocabulary. See also the Table of Contents of this Index beginning on the page 158. A few chemical symbols for nuclei have been changed to avoid ambiguity with particle names (see the Particle Vocabulary). Beam momenta are $p_{\text {lab }}$ in GeV/c, or in parentheses $E_{\mathrm{cm}}$ in $\mathrm{GeV}$. 


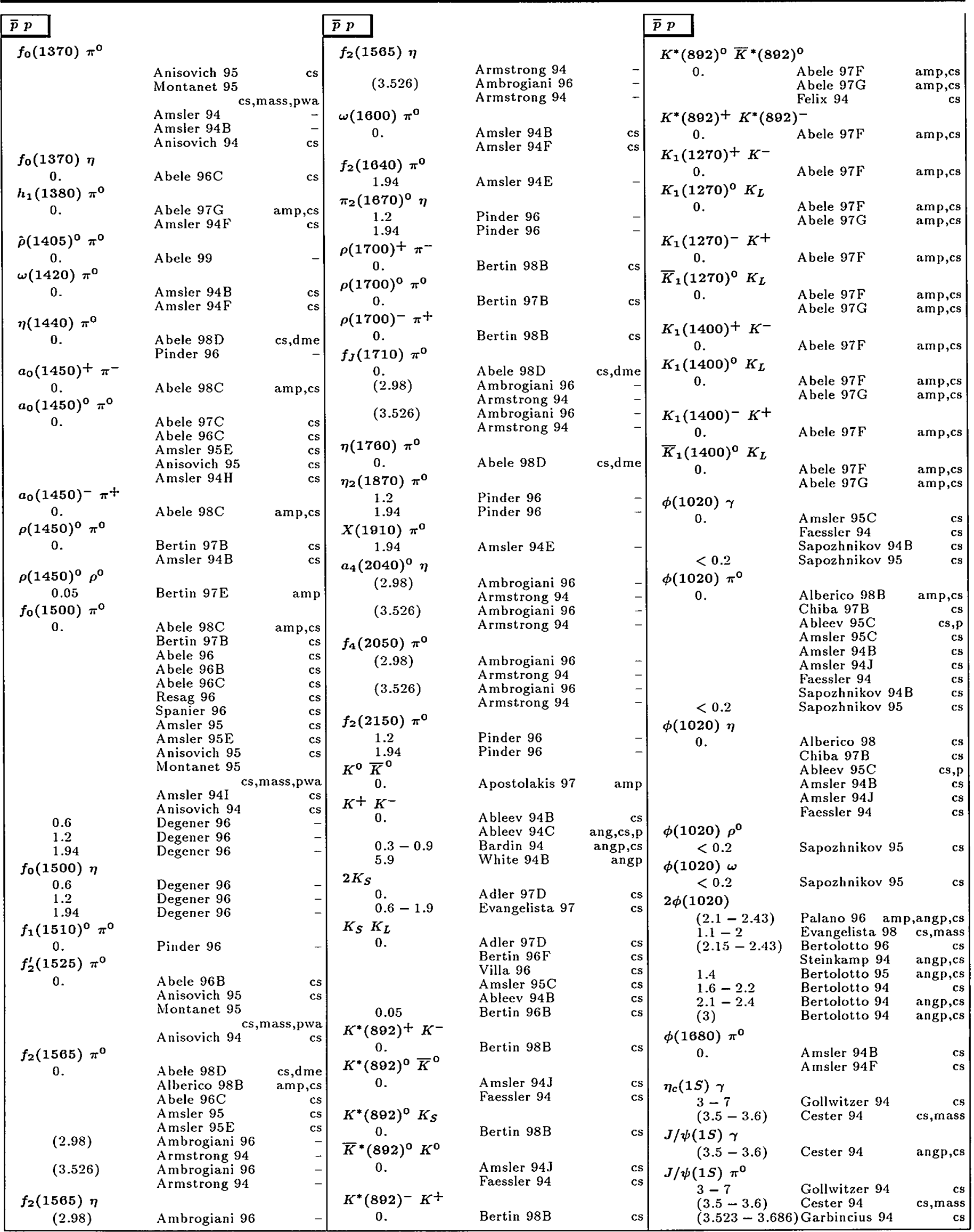




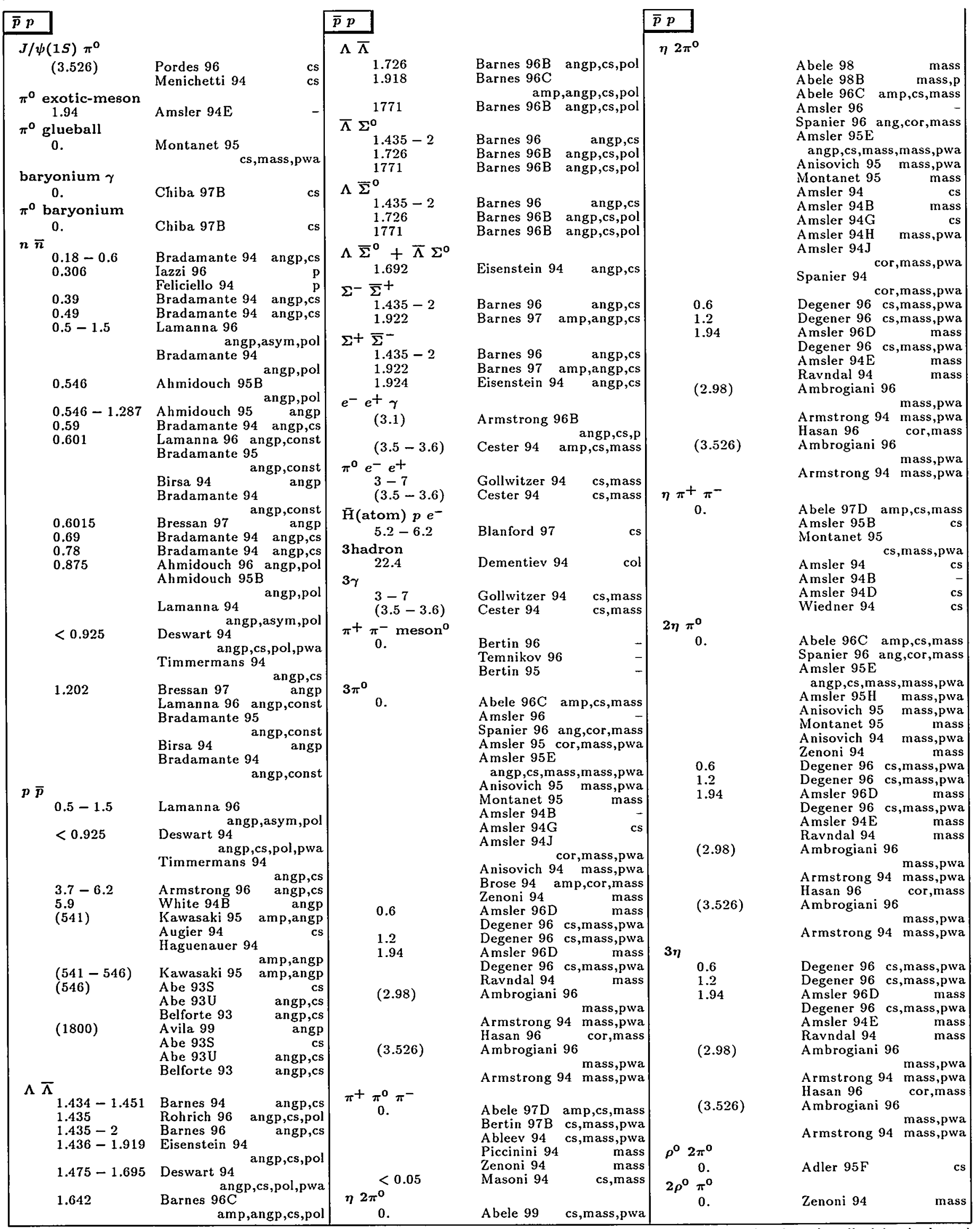

Entries are in order of beam name, then target name, then multiplicity of final state. Particle names are ordered as described in the legend on page 157 and as listed in the Particle Vocabulary. See also the Table of Contents of this Index beginning on the page 158. A few chemical symbols for nuclei have been changed to avoid ambiguity with particle names (see the Particle Vocabulary). Beam momenta are $p_{\text {lab }}$ in GeV/c, or in parentheses $E_{\mathrm{cm}}$ in $\mathrm{GeV}$. 


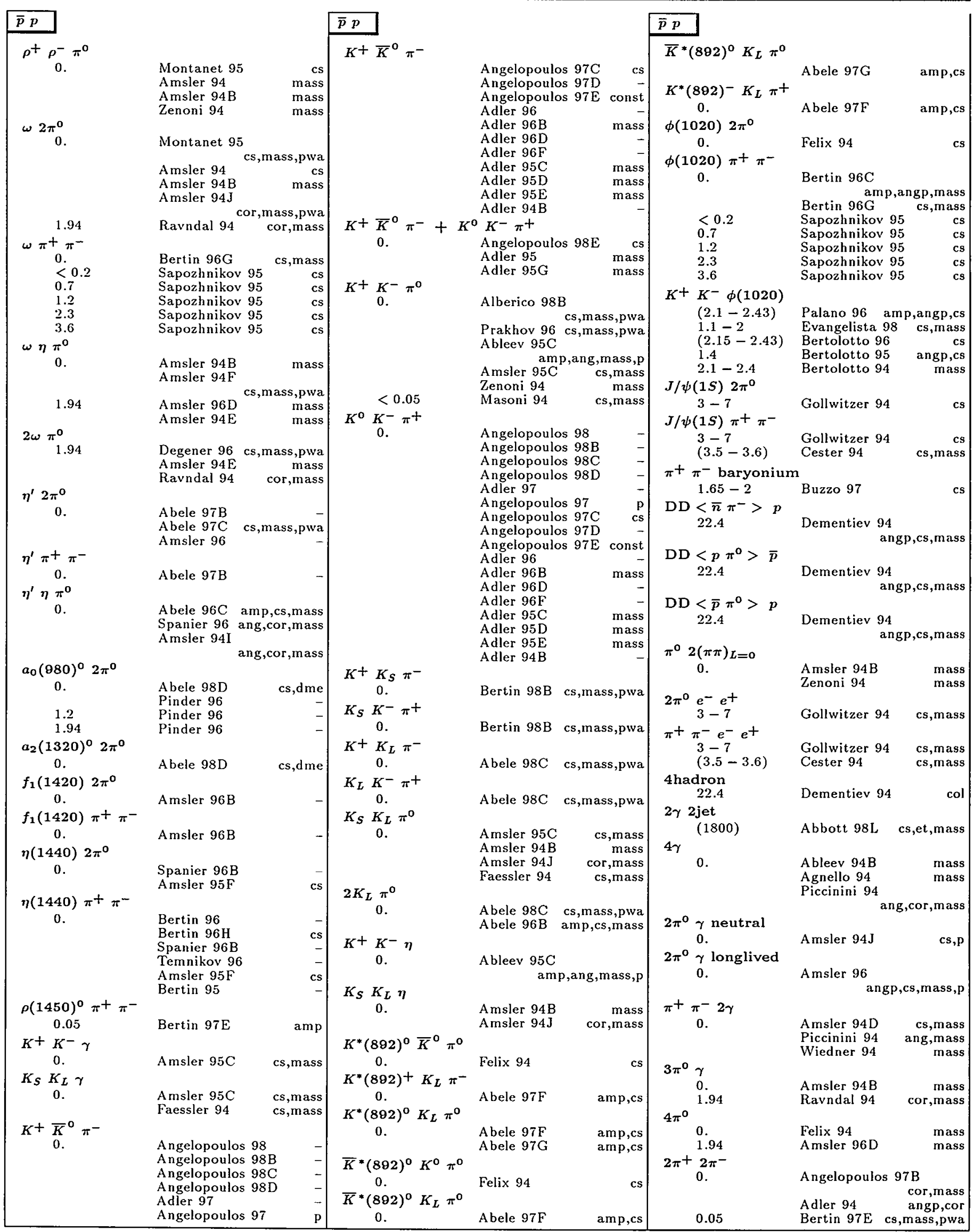




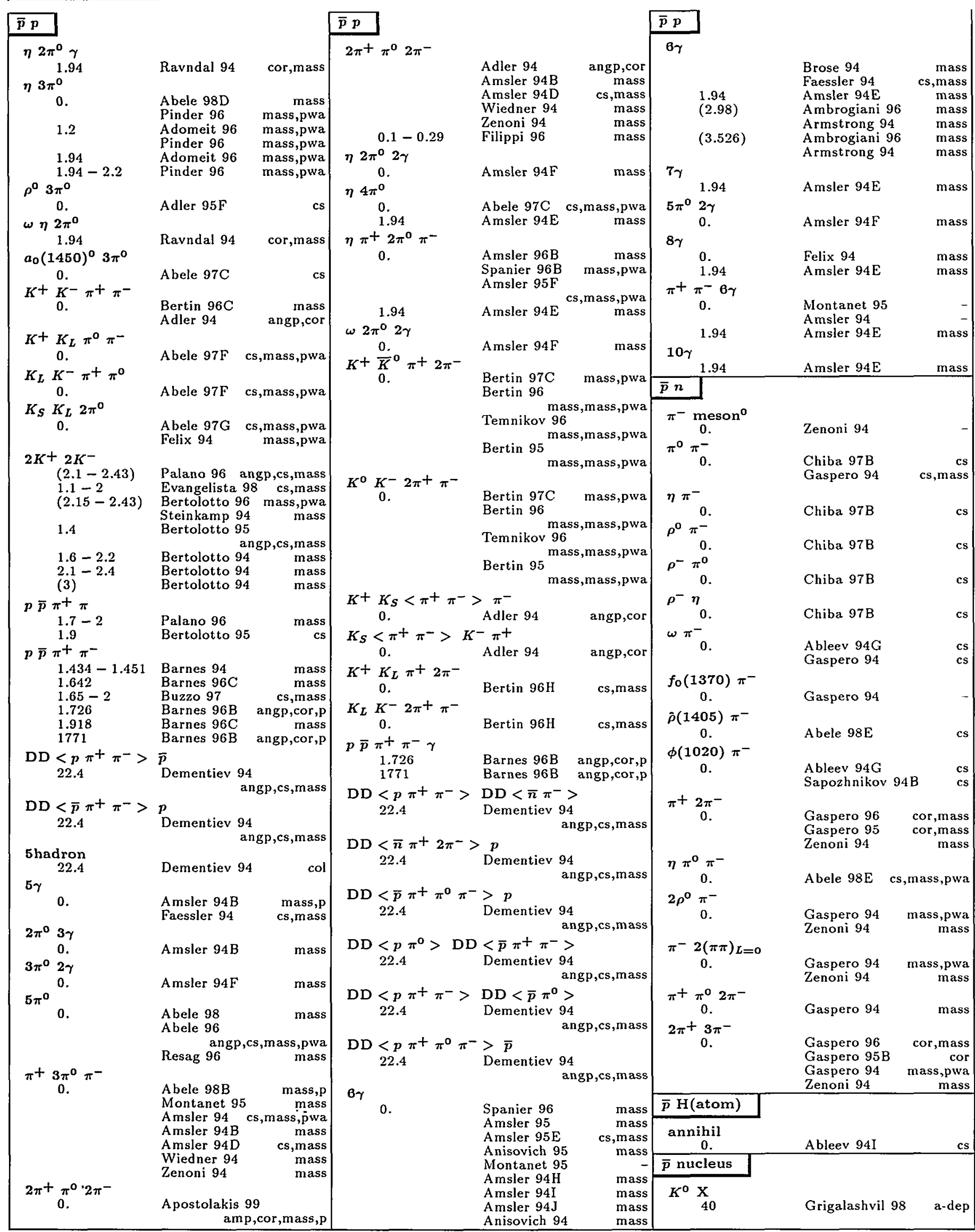

Entries are in order of beam name, then target name, then multiplicity of final state. Particle names are ordered as described in the legend on page 157 and as listed in the Particle Vocabulary. See also the Table of Contents of this Index beginning on the page 158. A few chemical symbols for nuclei have been changed to avoid ambiguity with particle names (see the Particle Vocabulary). Beam momenta are $p_{\text {lab }}$ in GeV/c, or in parentheses $E_{c m}$ in GeV. 
$\bar{p}$ nucleus $\rightarrow \Lambda \mathrm{X}$

$\bar{p}^{4} \mathrm{He} \rightarrow(\bar{p} \mathrm{He})_{\text {atom }}$

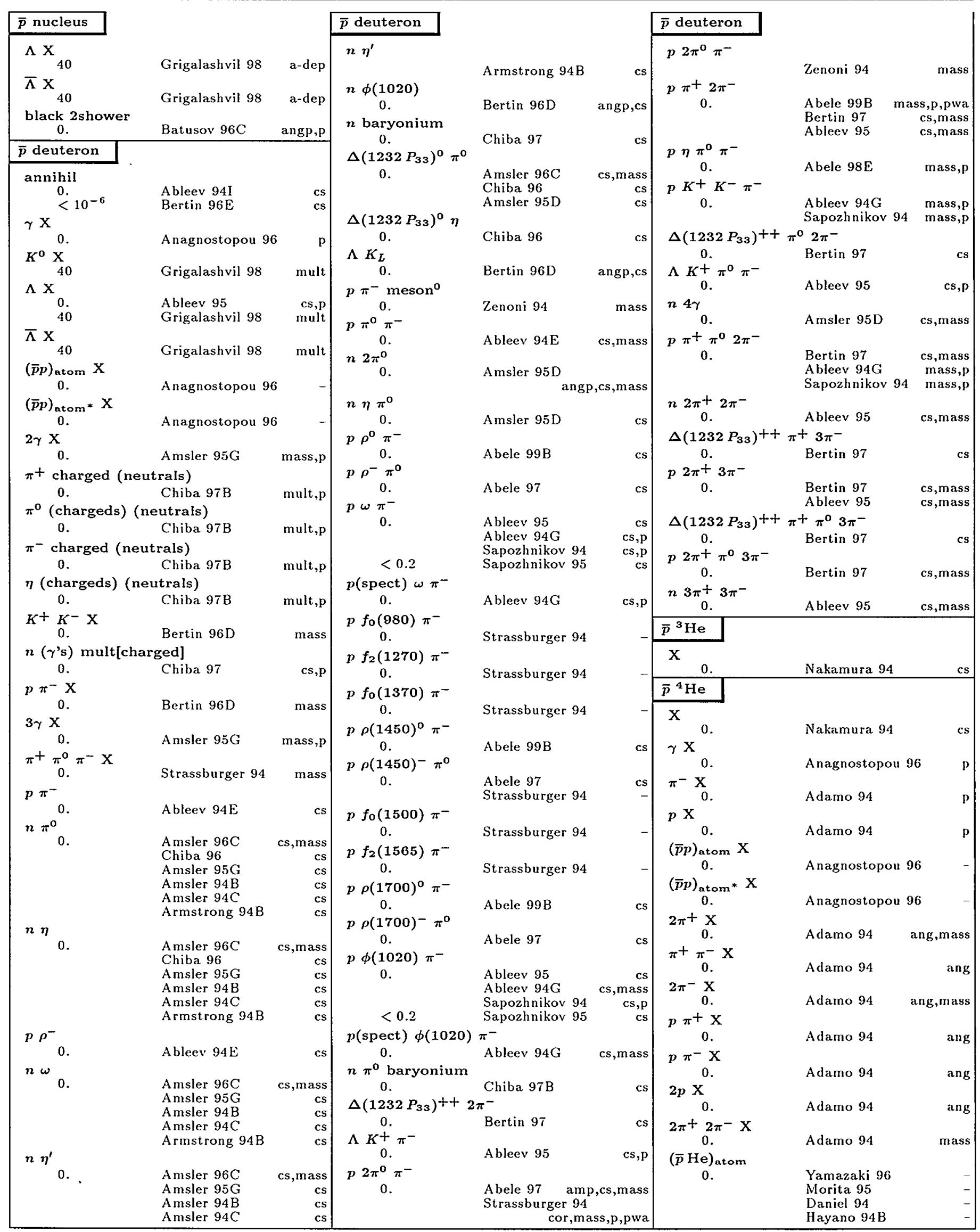




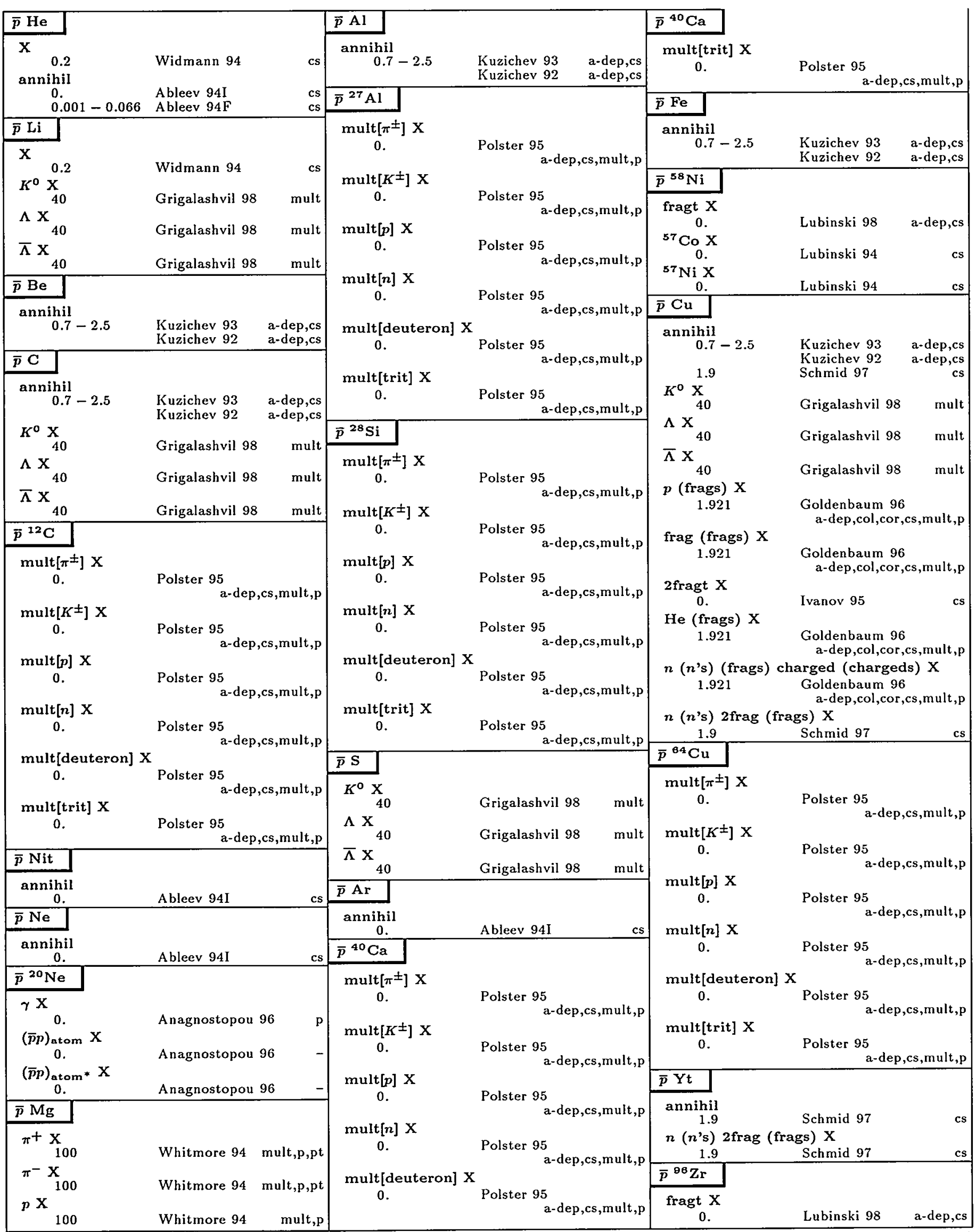

Entries are in order of beam name, then target name, then multiplicity of final state. Particle names are ordered as described in the legend on page 157 and as listed in the Particle Vocabulary. See also the Table of Contents of this Index beginning on the page 158 . A few chemical symbols for nuclei have been changed to avoid ambiguity with particle names (see the Particle Vocabulary). Beam momenta are $p_{\text {lab }}$ in GeV/c, or in parentheses $E_{\mathrm{cm}}$ in $\mathrm{GeV}$. 


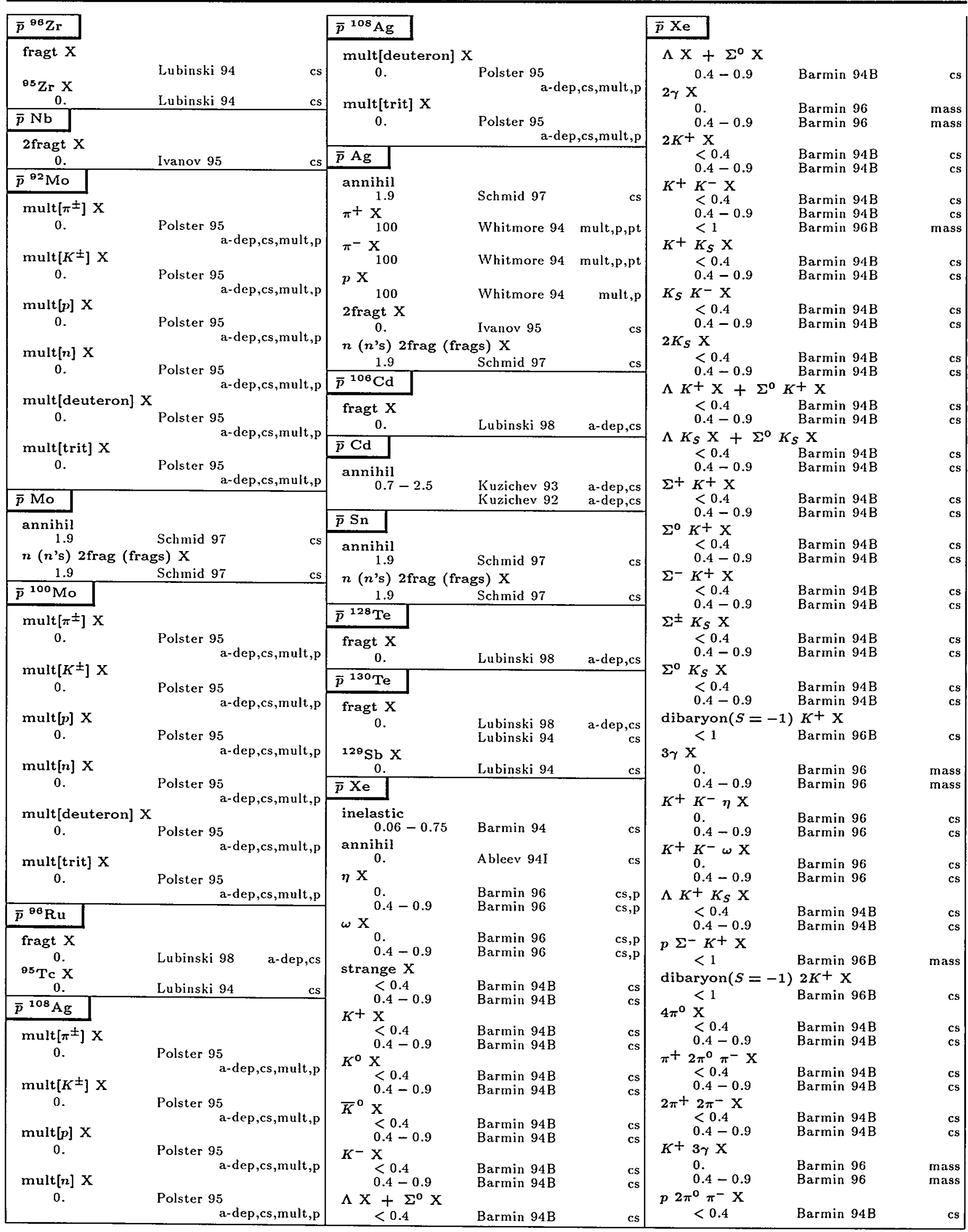




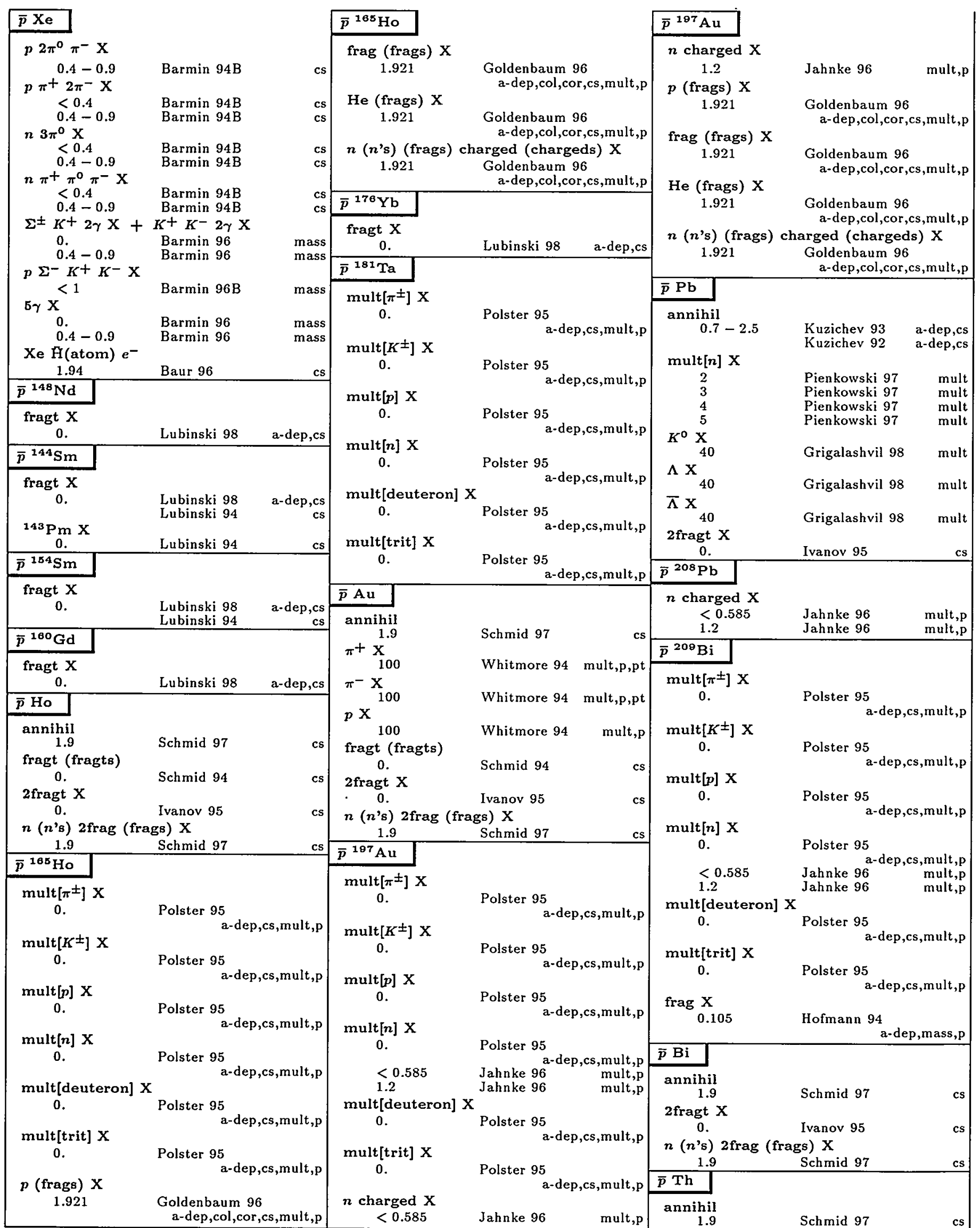

Entries are in order of beam name, then target name, then multiplicity of final state. Particle names are ordered as described in the legend on page 157 and as listed in the Particle Vocabulary. See also the Table of Contents of this Index beginning on the page 158. A few chemical symbols for nuclei have been changed to avoid ambiguity with particle names (see the Particle Vocabulary). Beam momenta are $p$ lab in GeV/c,
or in parentheses $E_{\mathrm{cm}}$ in $\mathrm{GeV}$. 


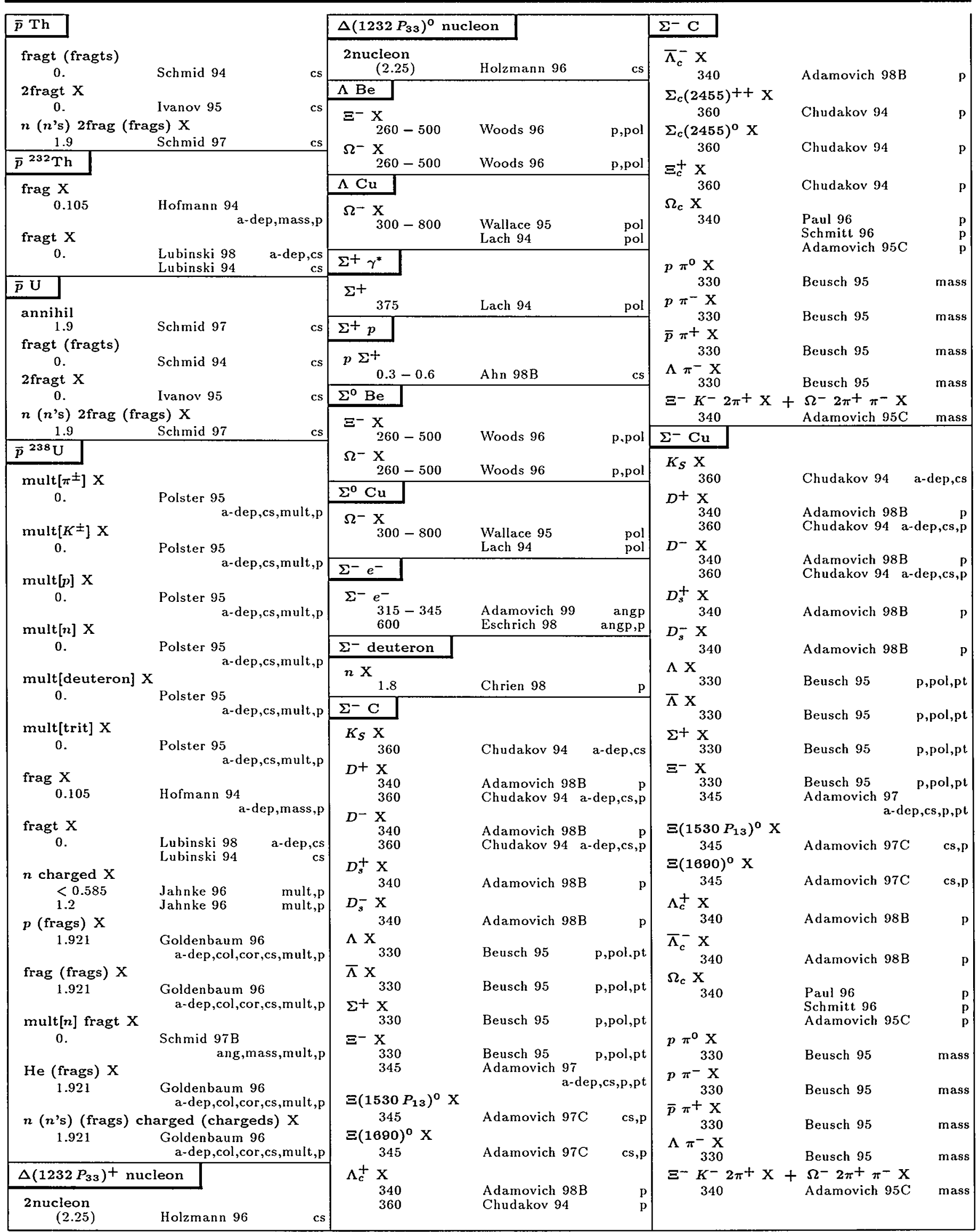




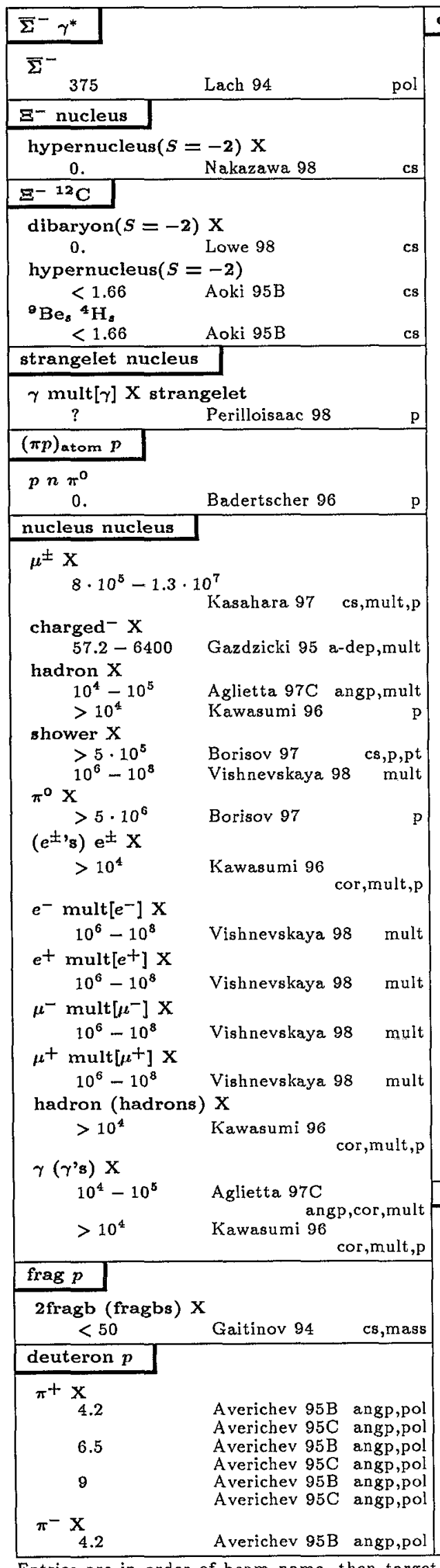

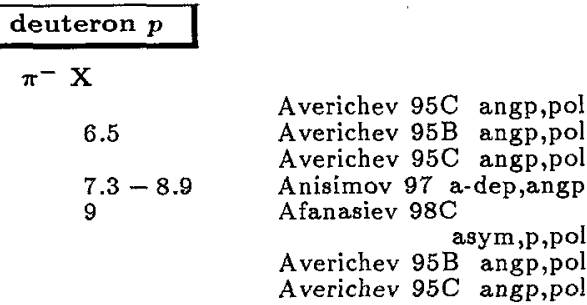

Averichev 95C angp,po Averichev 95B angp,pol Averichev 95C angp,pol Anisimov 97 a-dep,angp Afanasiev $98 \mathrm{C}$

asym, $p, p o$ Averichev 95B angp,po Averichev 95C angp,po p X $1.102-3.775$

Punjabi 94 Azhgirey 96 angp,p,pol deuteron $\mathrm{X}$ $4.24-6.5$ 4.495

4.5

5.5 5.532 8

Azhgirey 94

Azhgirey 97 Azhgirey 98

Azhgirey $95 \mathrm{~B}$

Azhgirey 95B

Azhgirey 98

Azhgirey 98

deuteron $p$

1.042

$1.102-3.773$

Sakamoto 96

$3.5-6$

$3.5-6.5$

Punjabi 95

Azhgirey 94B

\section{5}

5.5

dibaryont $p$

$$
3.3
$$

${ }^{8} \mathrm{He} \gamma$

\section{$<0.01732$ \\ 0.03519}

0.1587

$0.2186-0.2733$

0.4133

Azhgirey 97

Azhgirey 97B

Azhgirey 95

Azhgirey 95

ang $p, p, p o$.

angp,p,pol

angp, $p$

angp,p

angp, $p$

angp, $p$

angp,p

I

angp,pol

angp,pol

angp,p,pol

angp,

angp, $p, p o l$

Deloff 95

${ }^{3} \mathrm{He} \pi^{\circ}$

$1.284-1.34 \quad$ Nikulin 96

$2 p n$

0.4451

3.34

3.35

3.391

Schmid 96

an 7 angp,pol

angp,cs,p,pol

Browne 96 angp,pol

Schmid 96B

Anklin 98B

pol
angp,pol

angp,pol

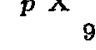

${ }^{8} \mathrm{He}{ }^{2} \mathrm{He}$

$c s, p o l \mid \begin{aligned} & { }^{\circ} \mathrm{He} \\ & { }_{0.6967}^{2} \mathrm{He}\end{aligned}$

deuteron ${ }^{7} \mathrm{Li}$

\section{Qin 95}

pol

Glagolev 98 angp,mass

Glagolev 95B angp,cs, $p$

Glagolev 96 angp,p

$\pi+\mathrm{x}$

Belostotsky 97 angp,

Belostotsky 97 angp,p,pol

Belostotsky 94

angp, $p, p o l$

Ero 94 angp

Azhgirey 97 angp,p,pol

Azhgirey 97B

$3 p \pi^{-}$

3.3

3.34

p 2 n $\pi^{+}$

3.34

deuteron nucleus

$\gamma \mathrm{X}$

$K^{\circ} \mathrm{X}^{2.5}$

$\mathrm{X}^{2.5}$

Troyan 94B

Deloff 95

Glagolev 98 angp,mass angp $, p, p o l$

$\Lambda \mathrm{X}$

$8.266-9.084$ Okonov 94

(showers) 2shower $\mathrm{X}$

angp, muit,p,pt

(showers) 2shower $\mathrm{X}$

2grey (greys) $\mathrm{X}$

Ghosh $94 \mathrm{~B}$

2black (blacks) X

Ghosh 94B

grey (greys) (showers) shower $\mathrm{X}$

9 Ghosh $94 \mathrm{~B}$ ang,col

${ }^{4} \mathrm{He} \mathrm{X}$

1.568
1.602

He $n$

He $\eta$

$\eta$

deuteron $p$

0.2125

$\pi+\mathrm{x}$

$\pi^{-\mathrm{X}}$

$\pi-\mathbf{X}$

$p \times$

deuteron $\mathrm{Be}$

$\pi^{-} \mathrm{X}$

inelastic

0.379

0.5

0.6122

deuteron $\mathrm{C}$

charged $\mathrm{X}$

8.4
$8.266-9.084$

\section{deuteron nucleus}

(showers) shower fragb (fragbs) $\mathrm{X}$

$$
9 \quad \text { Ghosh 94B ang,col }
$$

grey (greys) fragb (fragbs) $X$

black (blacks) fragb (fragbs) $\mathrm{X}$

Ghosh 94B ang,col

$2.337-2.36 \quad$ Willis $97 \quad \mathrm{cs}, \mathrm{p}, \mathrm{pol}$

$2.337 \quad$ Wurzinger 98

ngp, $p, p o l$

2.337 Frascaria 94 amp,cs

$2.338 \quad$ Frascaria 94 amp,cs

$2.34 \quad$ Frascaria 94 amp,cs

Frascaria 94

amp,cs

${ }^{4} \mathrm{He} 2 \pi^{\circ} \quad$ Felsher 97 angp,pol

Bondarev 94

Bondarep,angp

Bondarev 94

a-dep,angp

Bondarev 94

Afanasiev $98 \mathrm{C}$

asym, $\mathrm{p}, \mathrm{pol}$

deuteron ${ }^{9} \mathrm{Be}$

$0.8289-2.926$ Ladygin 97

mult[nucleon] $X$

Dedovich 94

angp,cor, $p, p t$

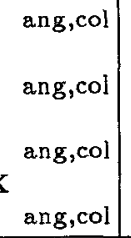

$\pi^{+} \mathrm{X}$

3.731

6.615

7.649
8.675

9

9.696
Debowski 97

Sugaya 97 a-dep,angp,p

Sugaya 97 a-dep,angp,p

Sugaya 97 a-dep,angp,p

Bondarev 94

Entries are in order of beam name, then target name, then multiplicity of final state. Particle names are ordered as described in the legend on page 157 and as listed in the Particle Vocabulary. See also the Table of Contents of this Index beginning on the page 158 . A few chemical symbols for nuclei have been changed to avoid ambiguity with particle names (see the Particle Vocabulary). Beam momenta are $p_{\text {lab }}$ in GeV/c, or in parentheses $E_{c m}$ in $\mathrm{GeV}$. 


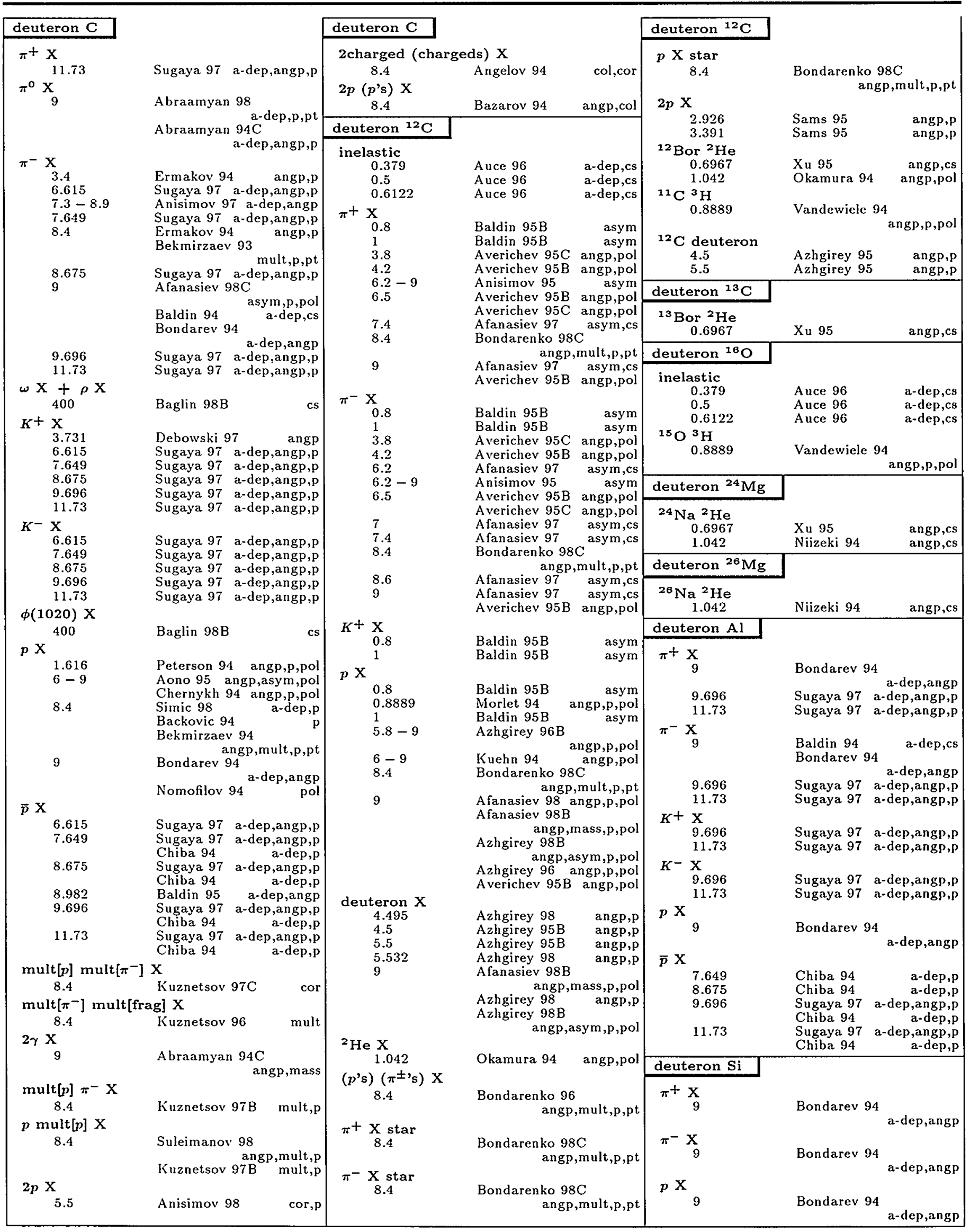




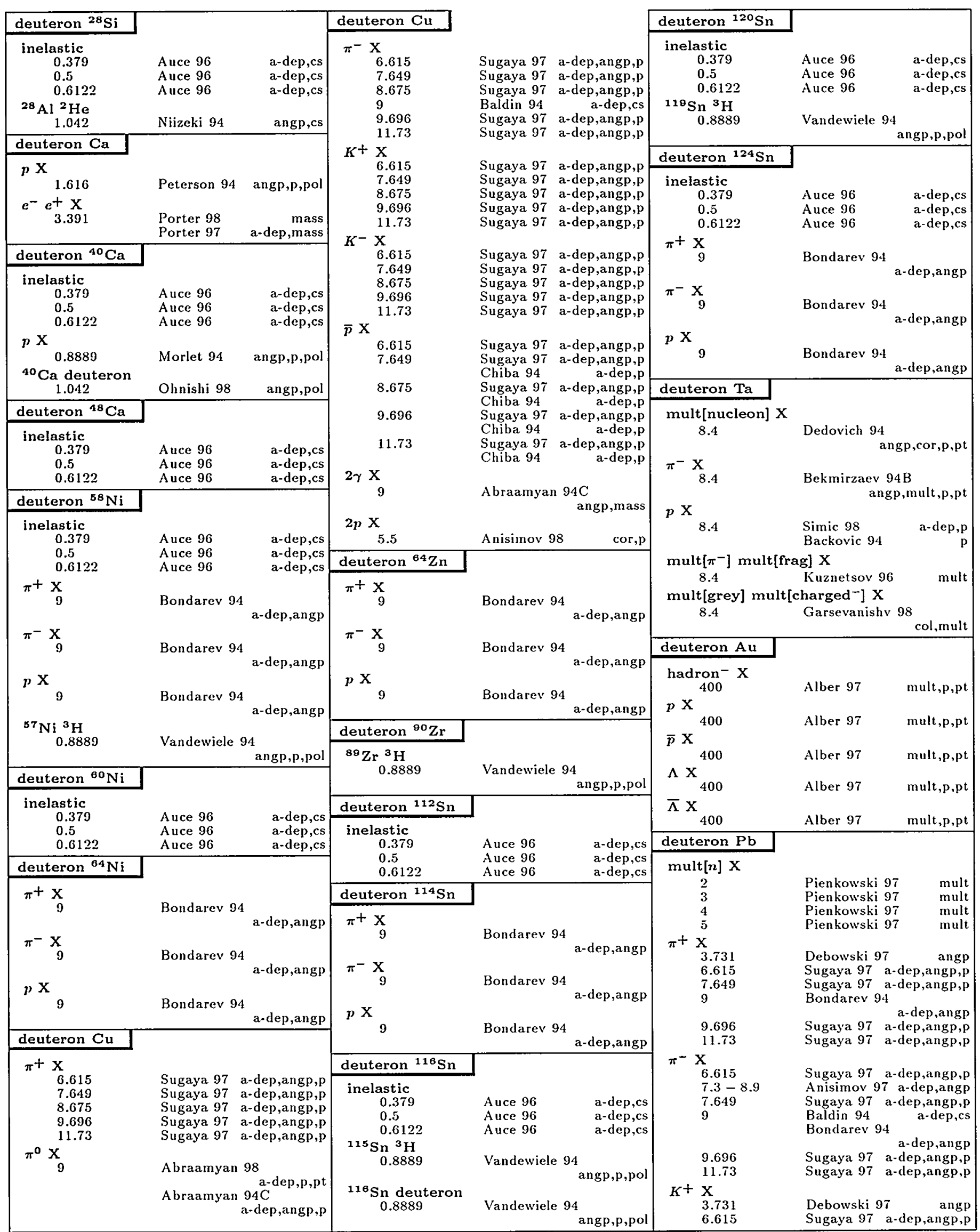

Entries are in order of beam name, then target name, then multiplicity of final state. Particle names are ordered as described in the legend on page 157 and as listed in the Particle Vocabulary. See also the Table of Contents of this Index beginning on the page 158 . A few chemical symbols for nuclei have been changed to avoid ambiguity with particle names (see the Particle Vocabulary). Beam momenta are $p_{\text {lab }}$ in GeV/c, or in parentheses $E_{\mathrm{cm}}$ in $\mathrm{GeV}$. 


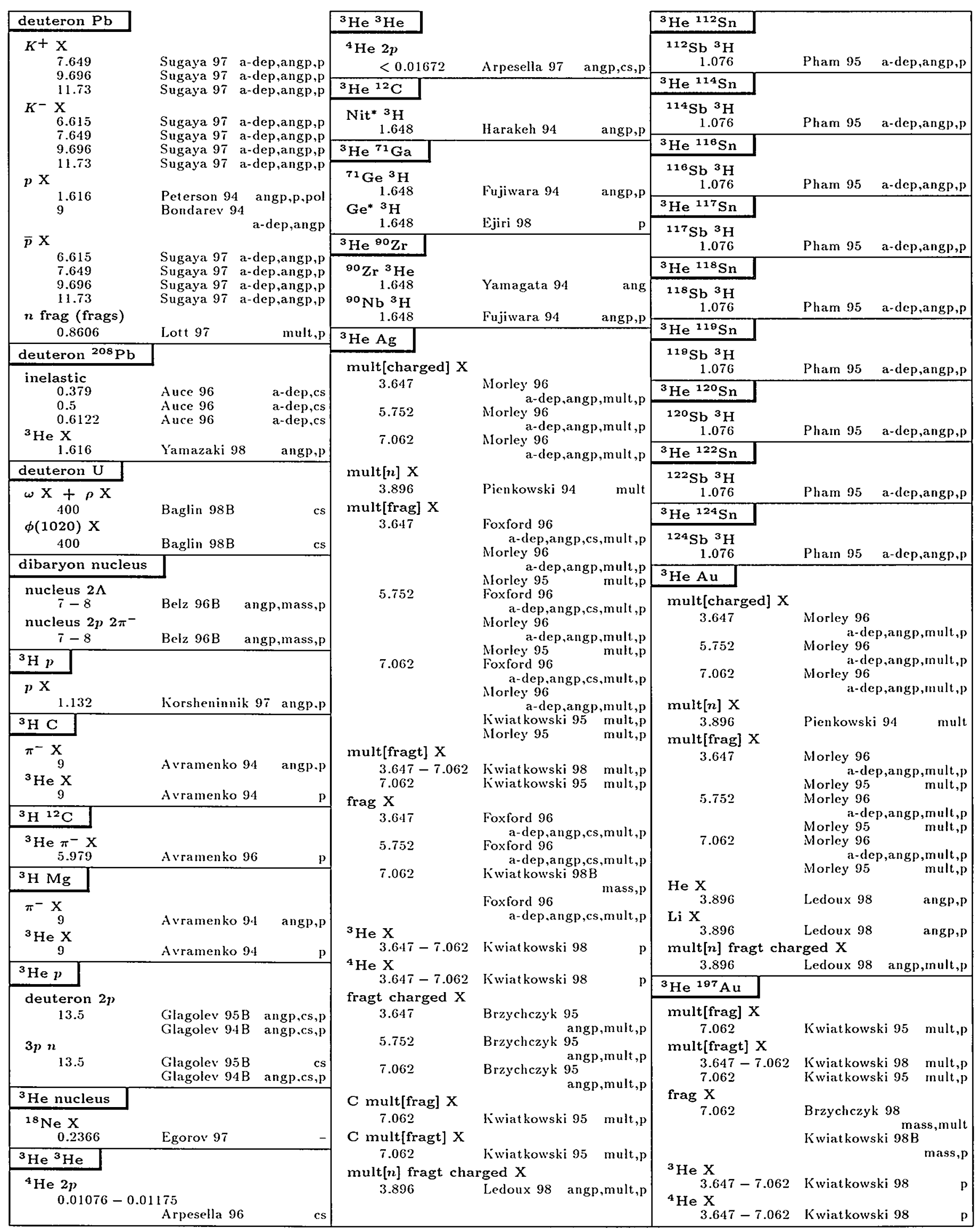




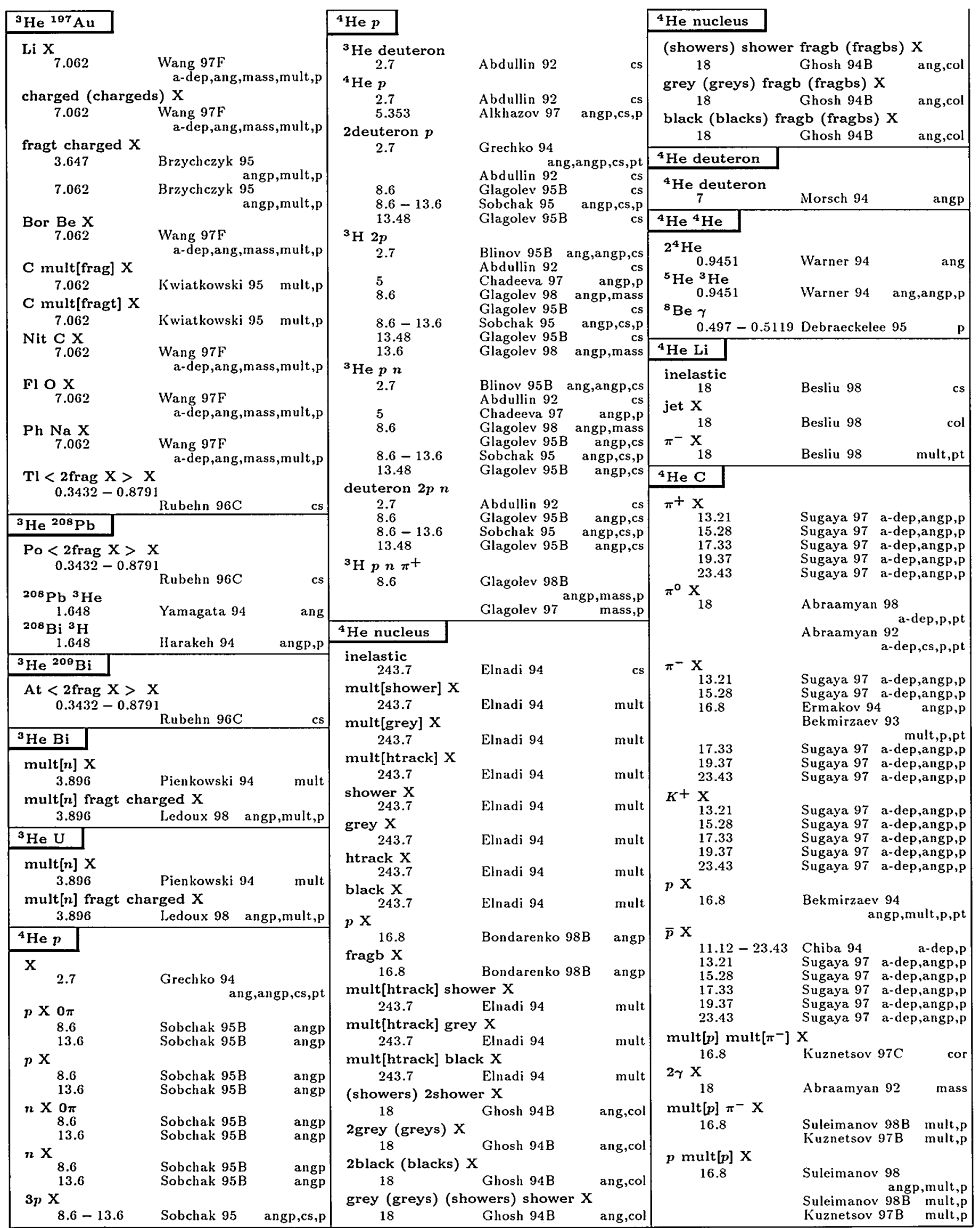

Entries are in order of beam name, then target name, then multiplicity of final state. Particle names are ordered as described in the legend on page 157 and as listed in the Particle Vocabulary. See also the Table of Contents of this Index beginning on the page 158. A few chemical symbols for nuclei have been changed to avoid ambiguity with particle names (see the Particle Vocabulary). Beam momenta are $p_{\text {lab }}$ in GeV/c, or in parentheses $E_{\mathrm{cm}}$ in $\mathrm{GeV}$. 


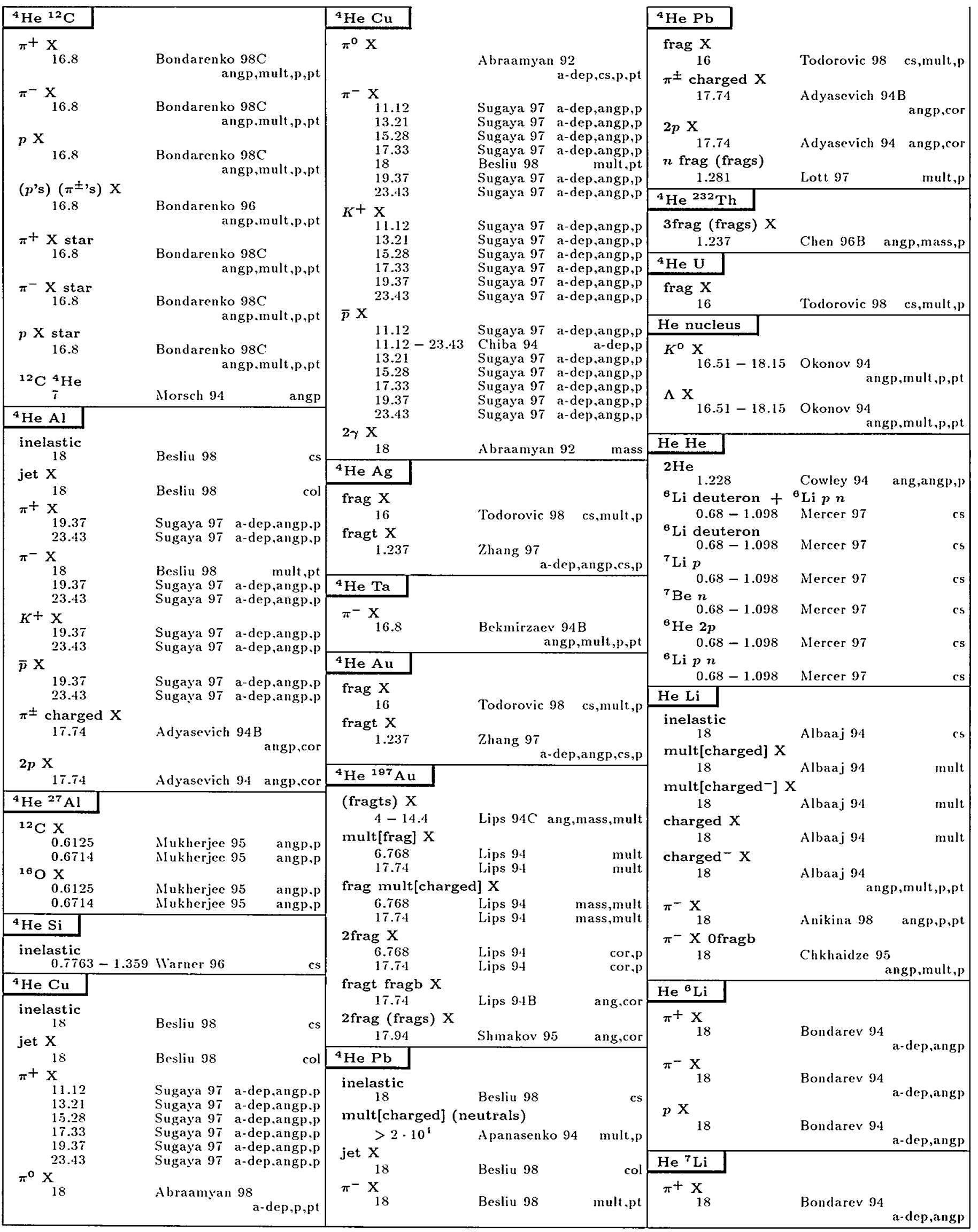




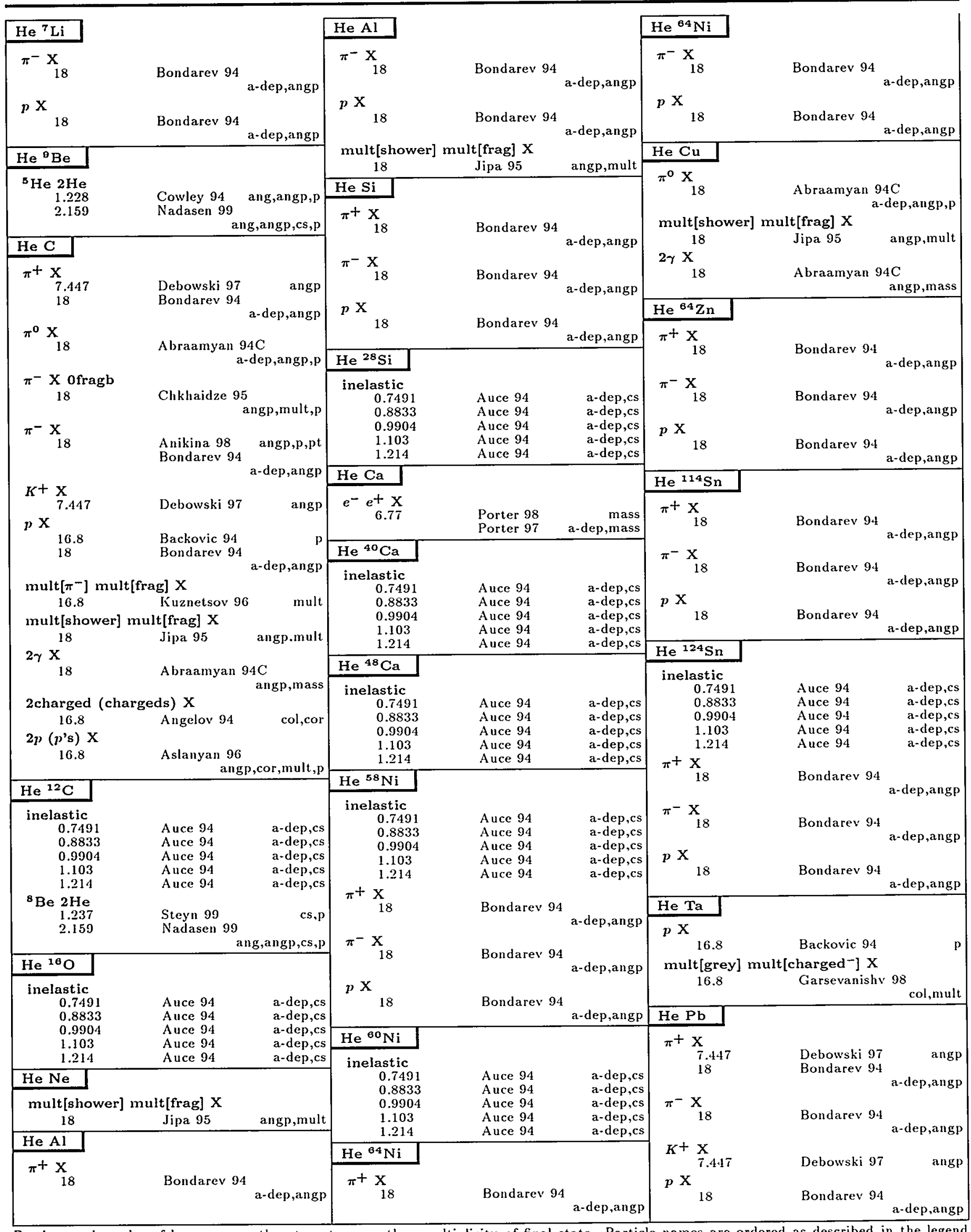

Entries are in order of beam name, then target name, then multiplicity of final state. Particle names are ordered as described in the legend on page 157 and as listed in the Particle Vocabulary. See also the Table of Contents of this Index beginning on the page 158 . A few chemical symbols for nuclei have been changed to avoid ambiguity with particle names (see the Particle Vocabulary). Beam momenta are $p_{\text {lab }}$ in GeV/c, or in parentheses $E_{\mathrm{cm}}$ in $\mathrm{GeV}$. 
$\mathrm{He} \mathrm{Pb} \rightarrow$ mult[shower] mult[frag] X

${ }^{10} \mathrm{Be} p \rightarrow{ }^{10} \mathrm{Be} p$

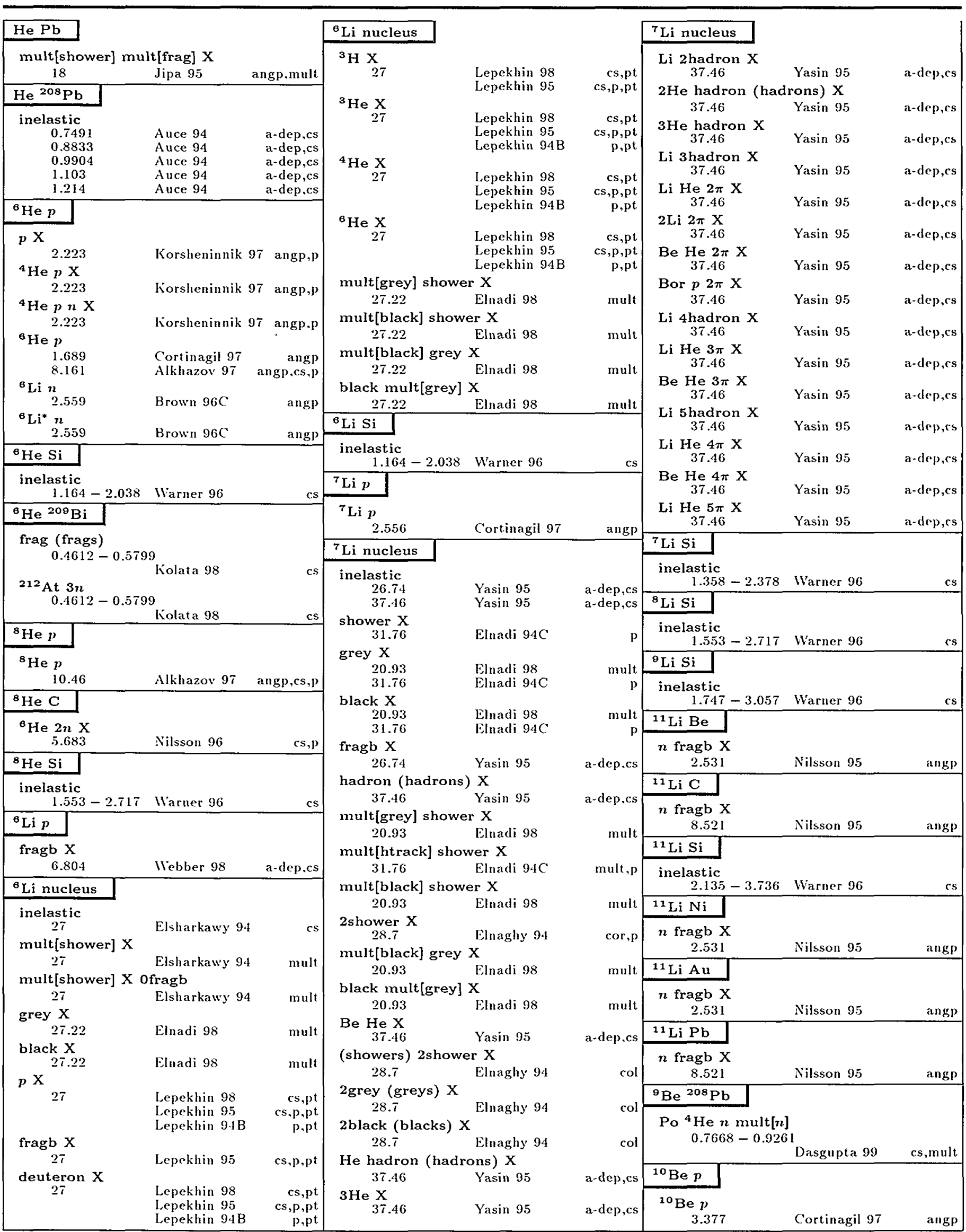




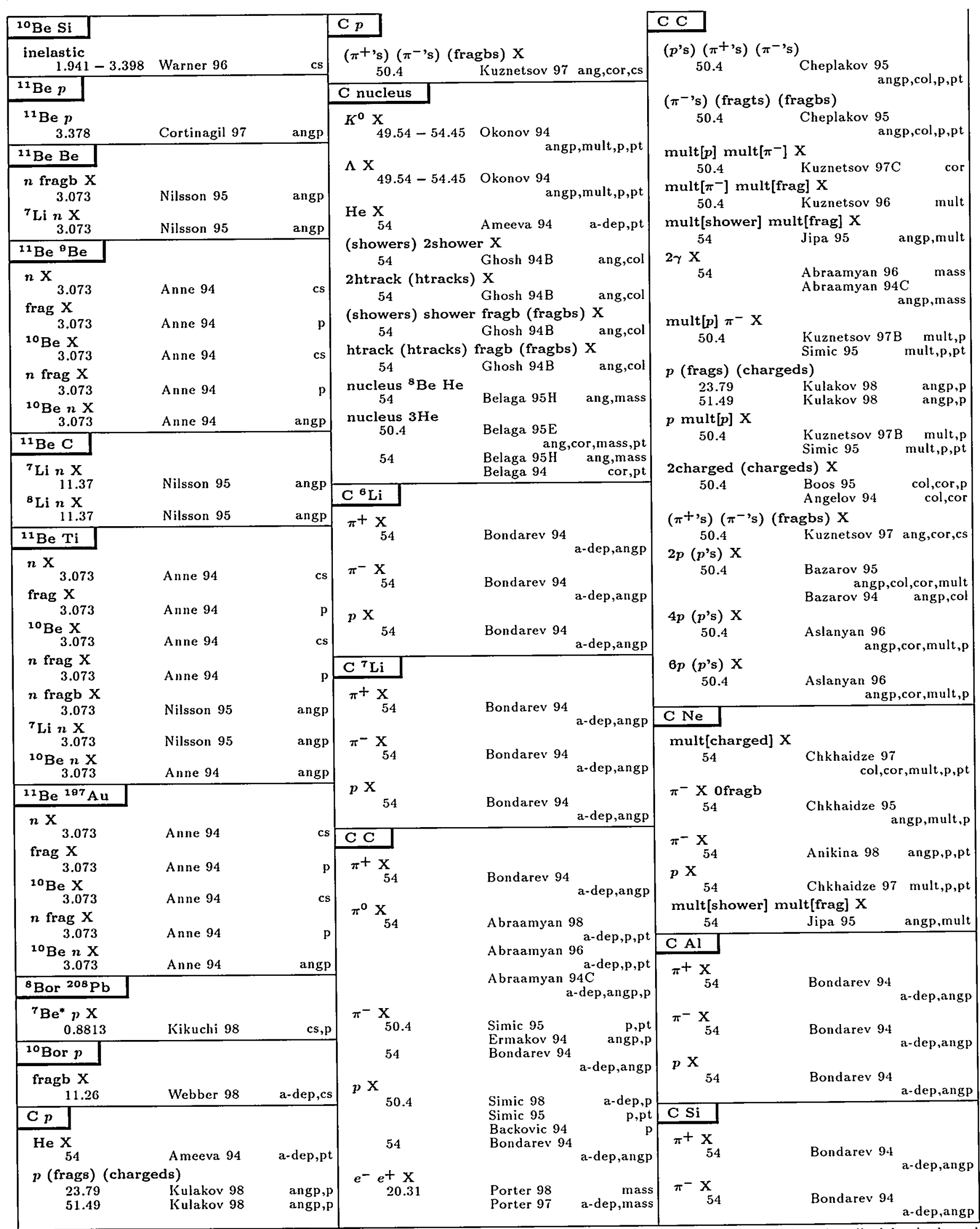

Entries are in order of beam name, then target name, then multiplicity of final state. Particle names are ordered as described in the legend En page 157 and as listed in the Particle Vocabulary. See also the Table of Contents of this Index beginning on the page 158. A few chemical symbols for nuclei have been changed to avoid ambiguity with particle names (see the Particle Vocabulary). Beam momenta are $p_{\text {lab }}$ in GeV/c, or in parentheses $E_{\mathrm{cm}}$ in $\mathrm{GeV}$. 


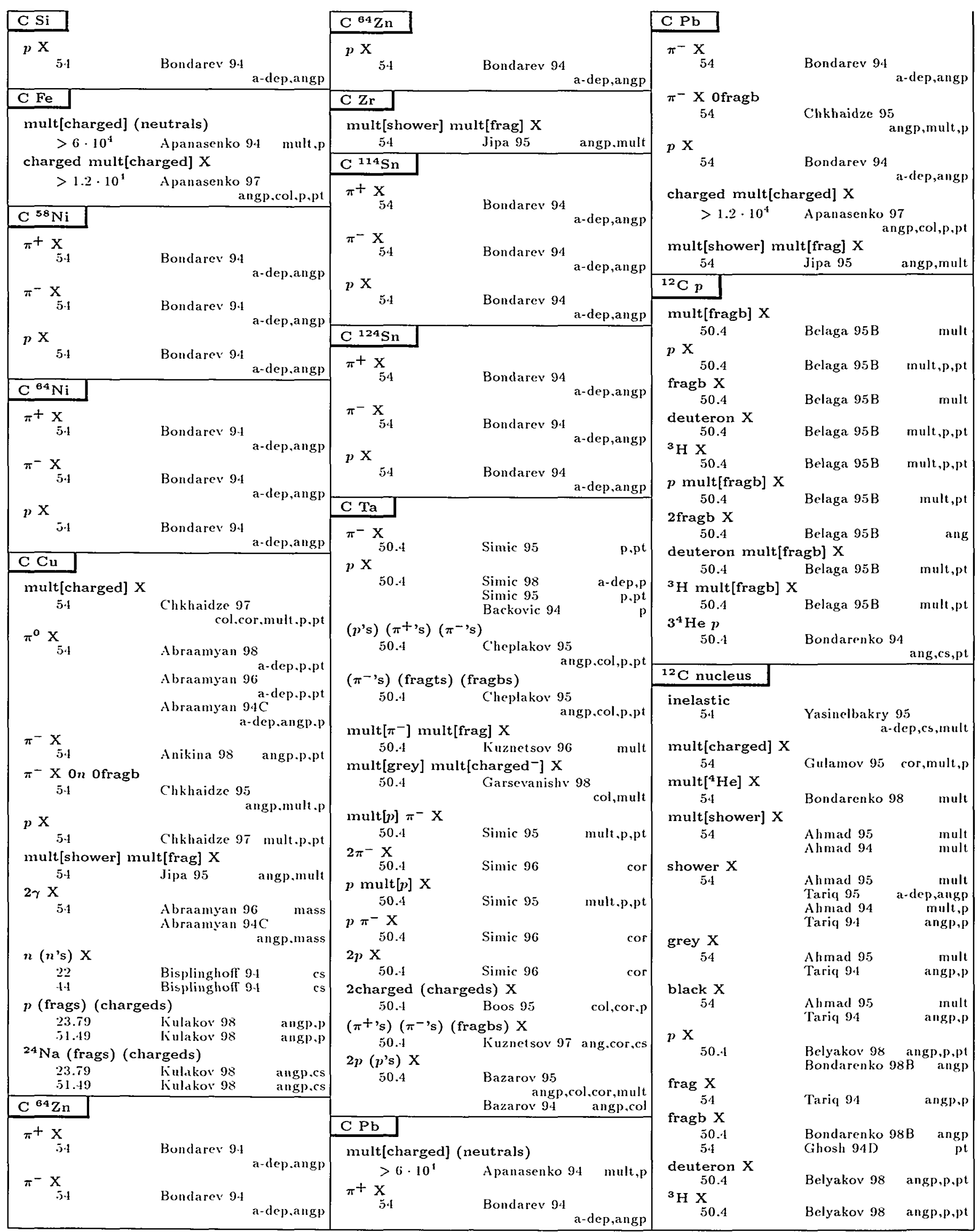




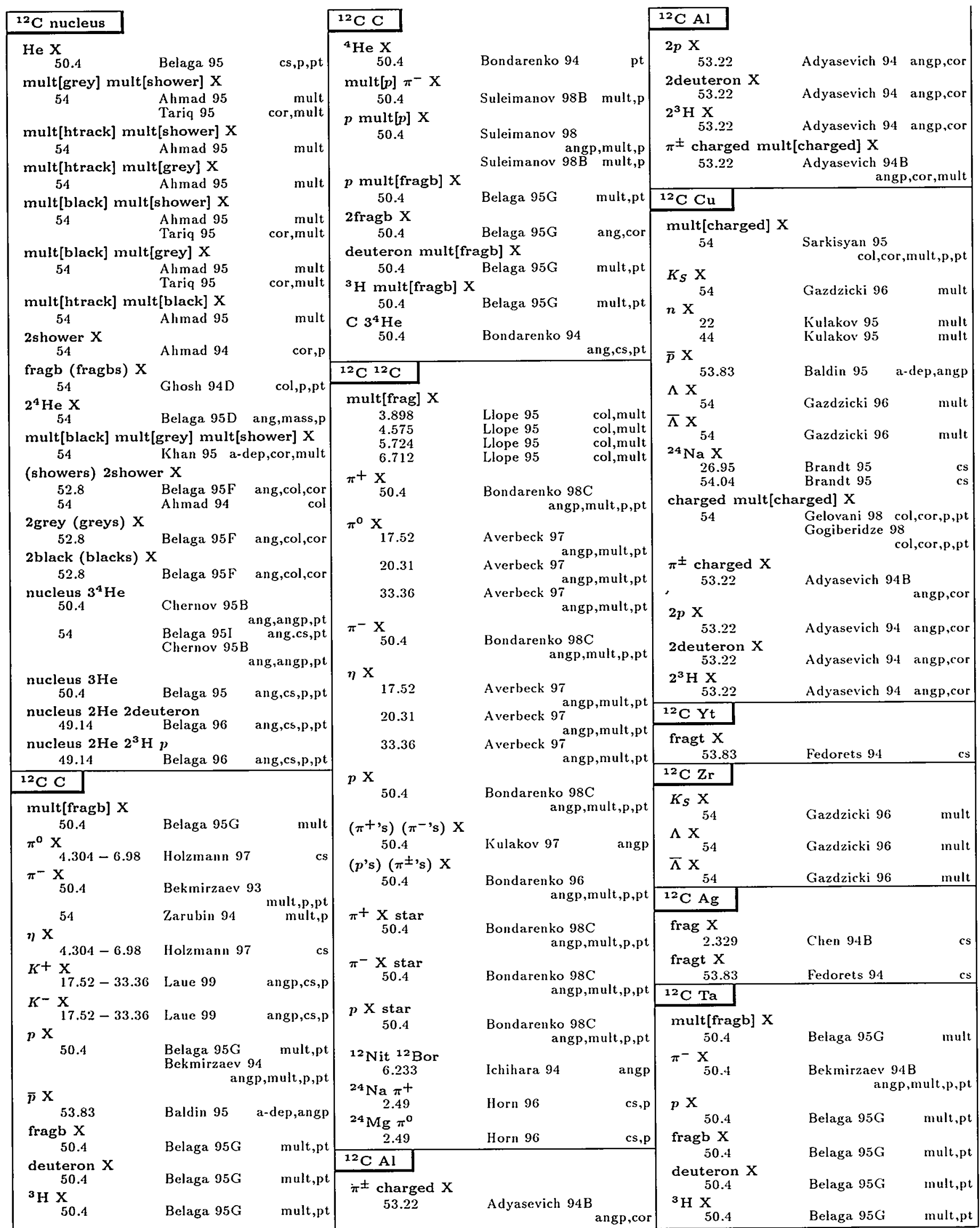

Entries are in order of beam name, then target name, then multiplicity of final state. Particle names are ordered as described in the legend on page 157 and as listed in the Particle Vocabulary. See also the Table of Contents of this Index beginning on the page 158 . A few chemical symbols for nuclei have been changed to avoid ambiguity with particle names (see the Particle Vocabulary). Beam momenta are $p_{l a b}$ in GeV/c, or in parentheses $E_{\mathrm{cm}}$ in $\mathrm{GeV}$. 
${ }^{12} \mathrm{C} \mathrm{Ta} \rightarrow{ }^{4} \mathrm{He} \mathrm{X}$

${ }^{16} \mathrm{O} p \rightarrow{ }^{3} \mathrm{He}$ mult[trit] X

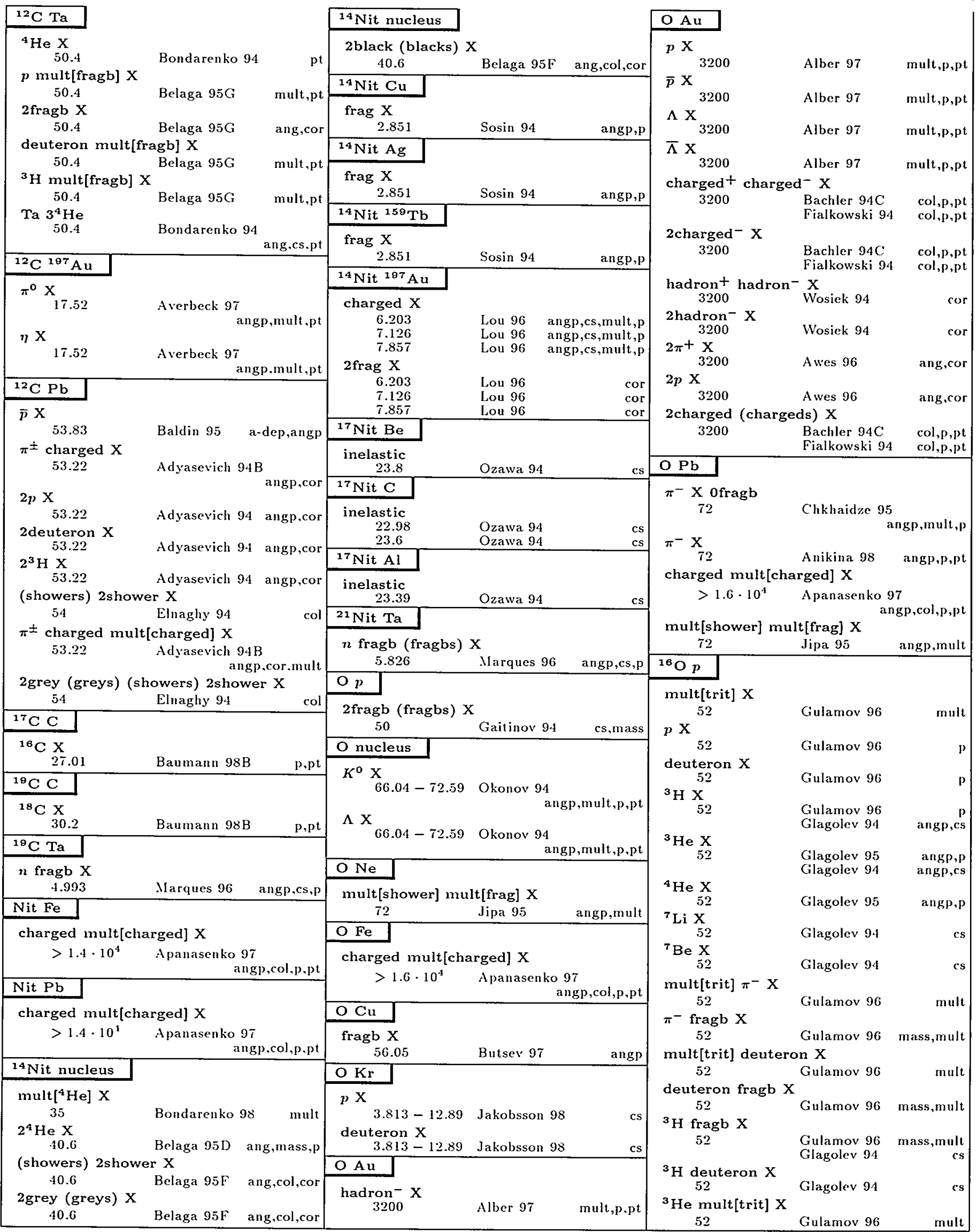




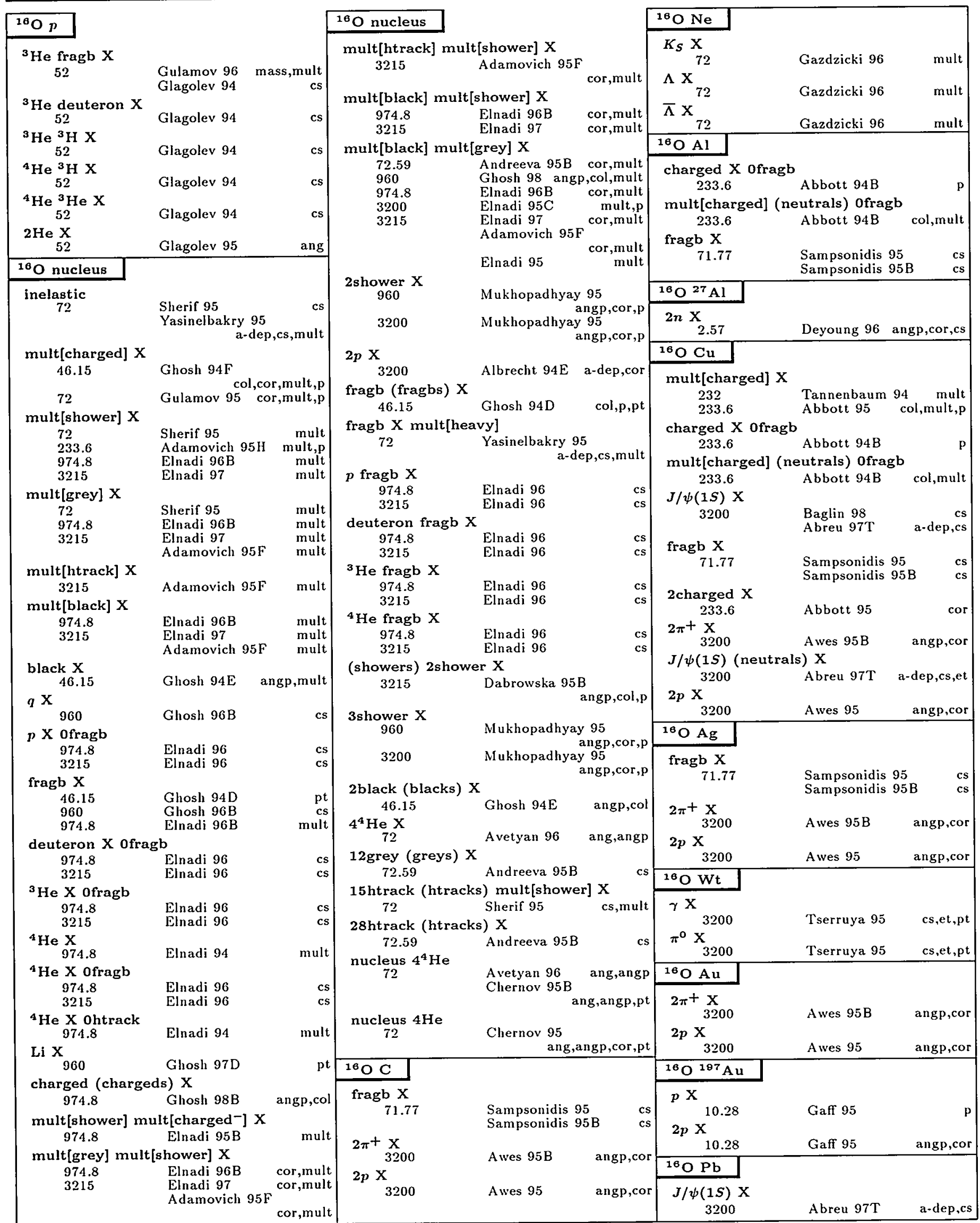

Entries are in order of beam name, then target name, then multiplicity of final state. Particle names are ordered as described in the legend on page 157 and as listed in the Particle Vocabulary. See also the Table of Contents of this Index beginning on the page 158. A few chemical symbols for nuclei have been changed to avoid ambiguity with particle names (see the Particle Vocabulary). Beam momenta are $p$ lab in GeV/c, or in parentheses $E_{\mathrm{cm}}$ in $\mathrm{GeV}$. 
${ }^{16} \mathrm{O} \mathrm{Pb} \rightarrow$ fragb $\mathrm{X}$

${ }^{22} \mathrm{Ne} p \rightarrow$ fragb $\mathrm{X}$

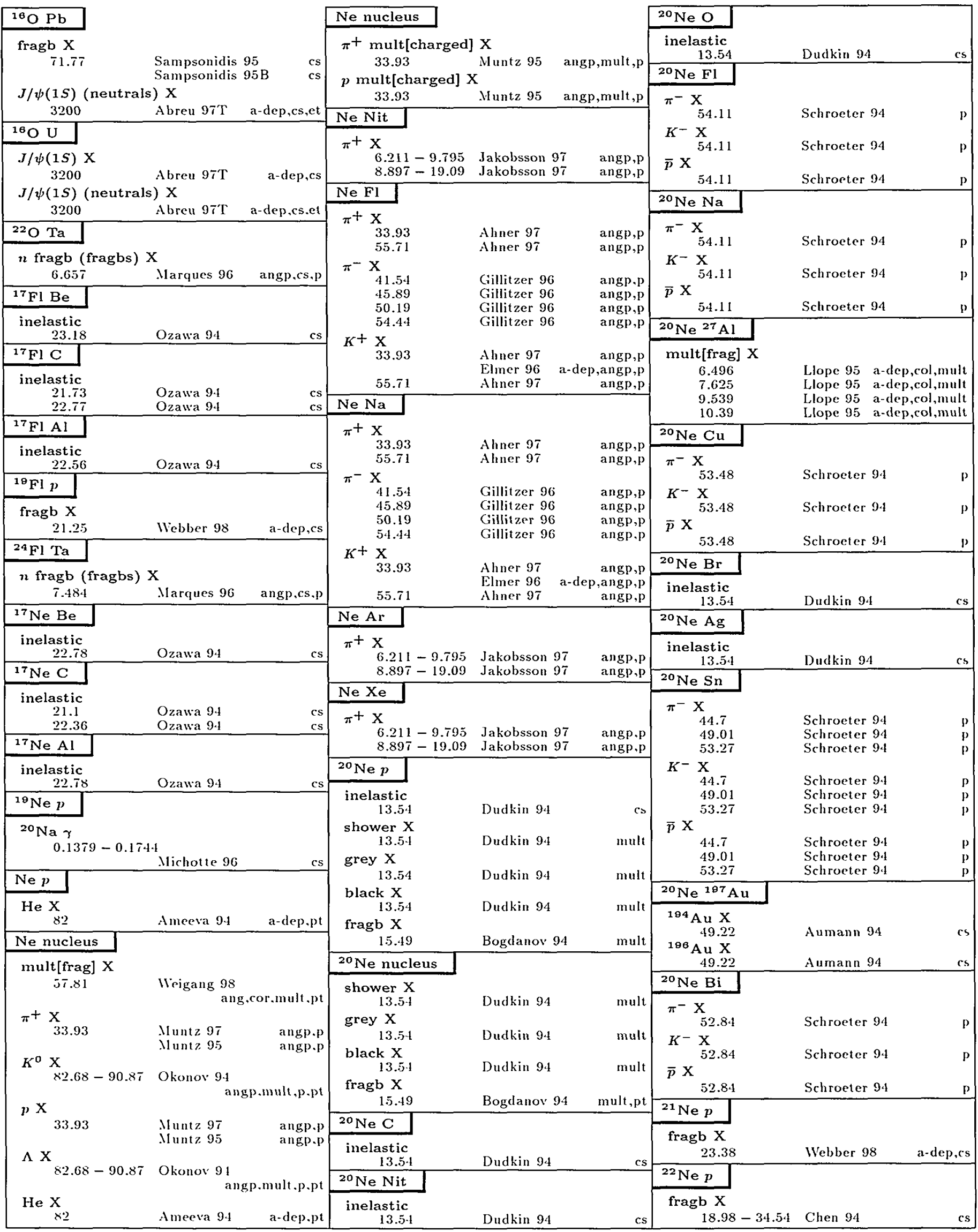




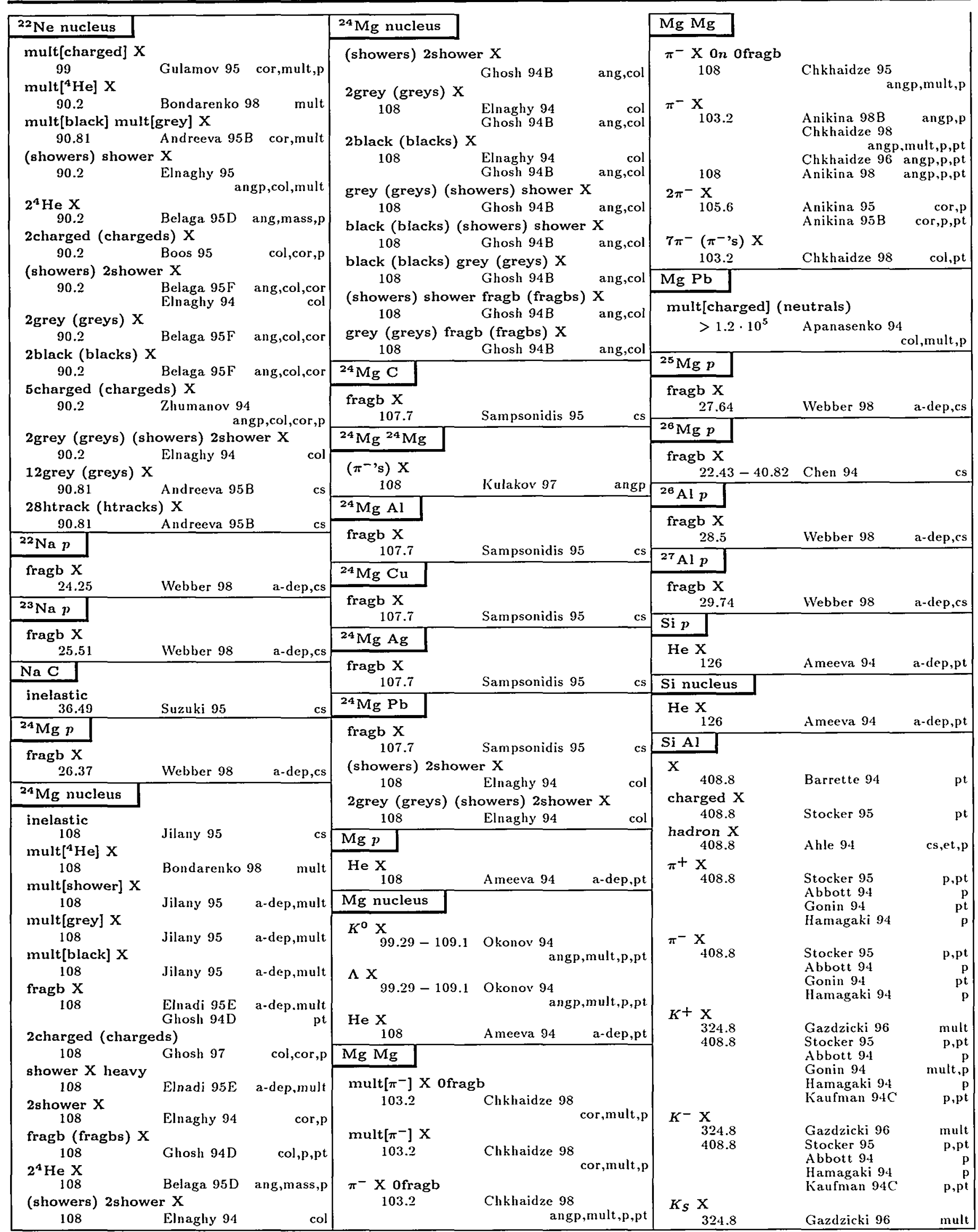

Entries are in order of beam name, then target name, then multiplicity of final state. Particle names are ordered as described in the legend on page 157 and as listed in the Particle Vocabulary. See also the Table of Contents of this Index beginning on the page 158. $A$ few chemical symbols for nuclei have been changed to avoid ambiguity with particle names (see the Particle Vocabulary). Beam momenta are plab in GeV/c, or in parentheses $E_{\mathrm{cm}}$ in $\mathrm{GeV}$. 


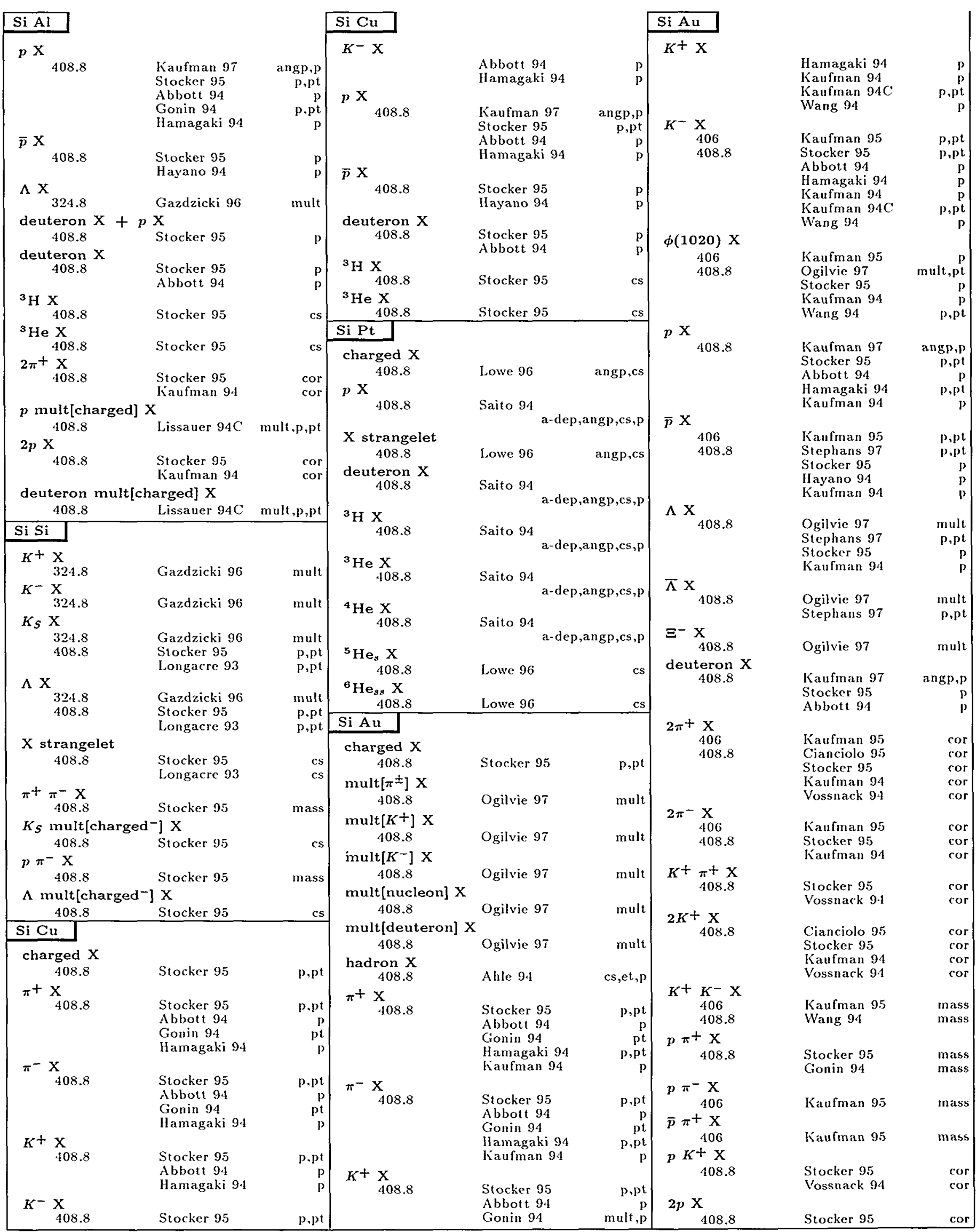




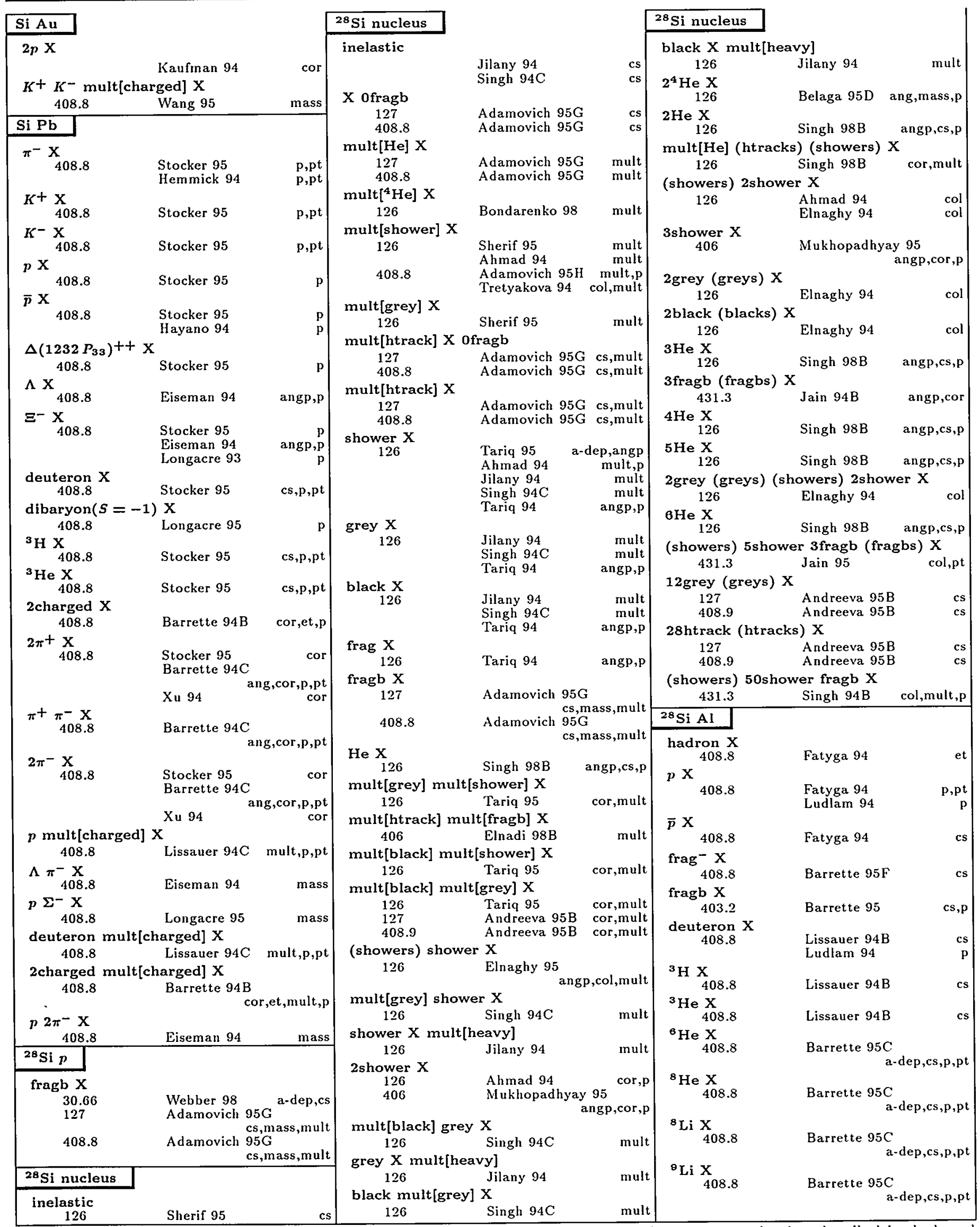

Entries are in order of beam name, then target name, then multiplicity of final state. Particle names are ordered as described in the legend on page 157 and as listed in the Particle Vocabulary. See also the Table of Contents of this Index beginning on the page 158 . A few chemical symbols for nuclei have been changed to avoid ambiguity with particle names (see the Particle Vocabulary). Beam momenta are $p_{\text {lab }}$ in GeV/c, or in parentheses $E_{\mathrm{cm}}$ in $\mathrm{GeV}$. 


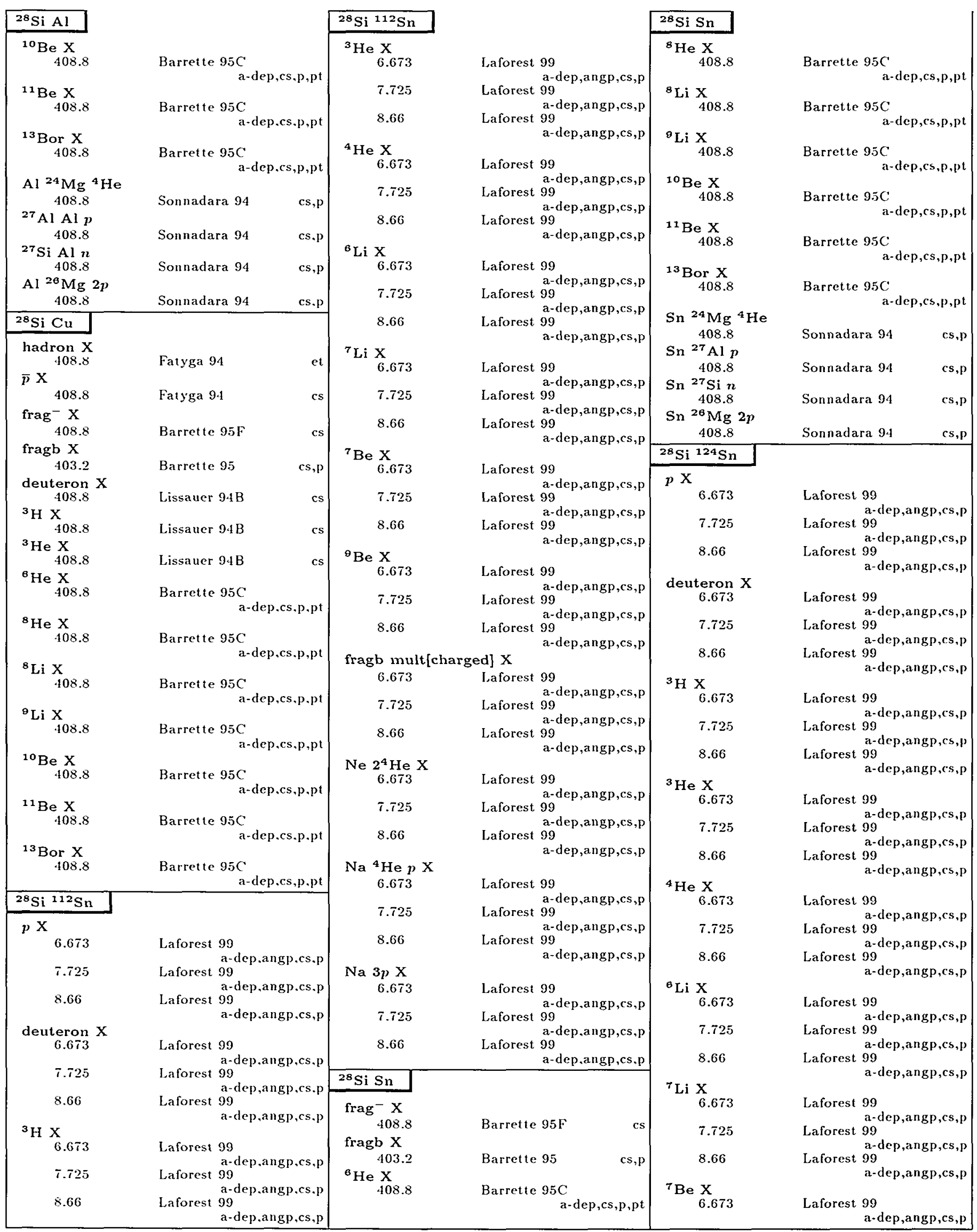




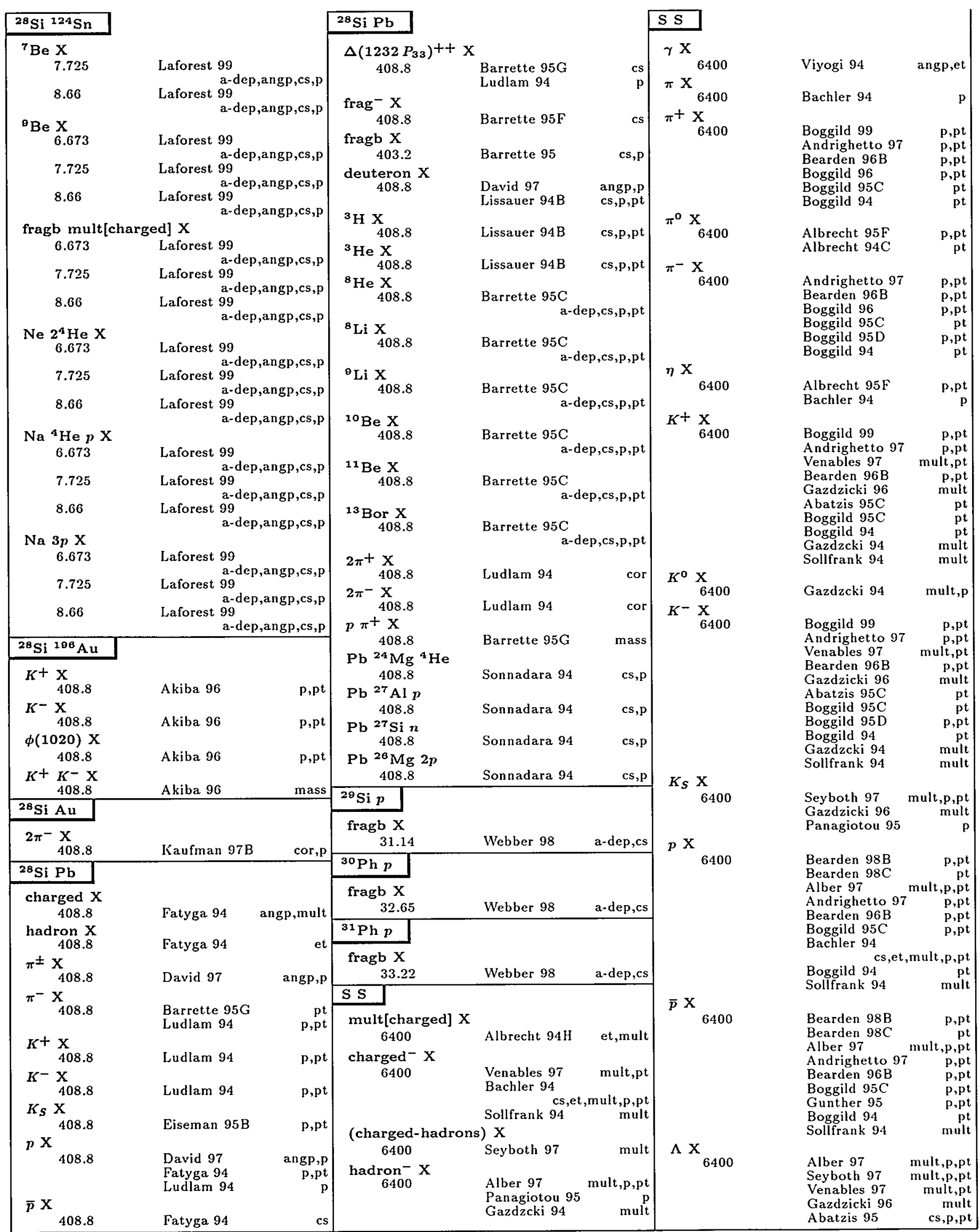

Entries are in order of beam name, then target name, then multiplicity of final state. Particle names are ordered as described in the legend on page 157 and as listed in the Particle Vocabulary. See also the Table of Contents of this Index beginning on the page 158. A few chemical symbols for nuclei have been changed to avoid ambiguity with particle names (see the Particle Vocabulary). Beam momenta are $p_{l a b}$ in GeV/c, or in parentheses $E_{\mathrm{cm}}$ in $\mathrm{GeV}$. 


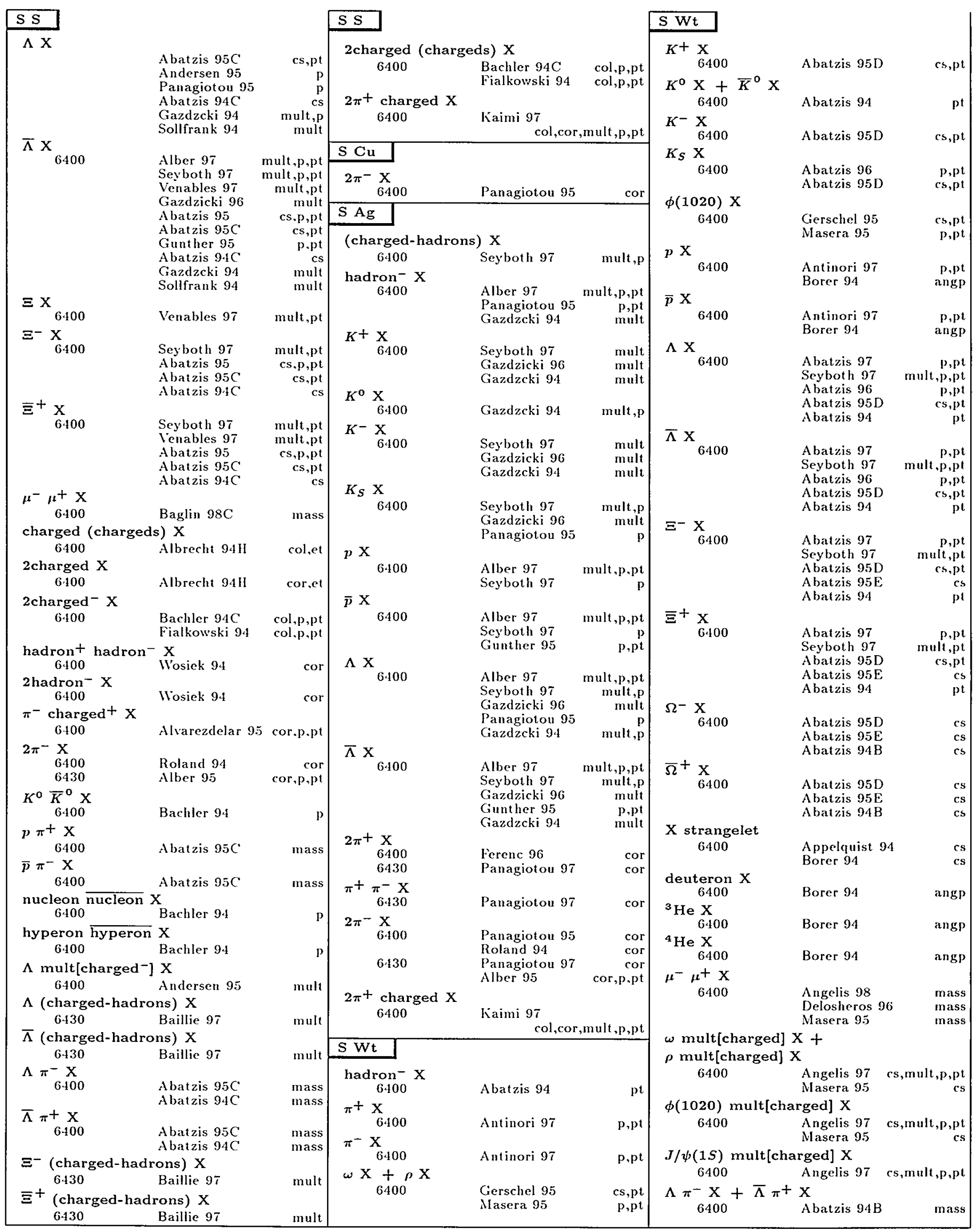




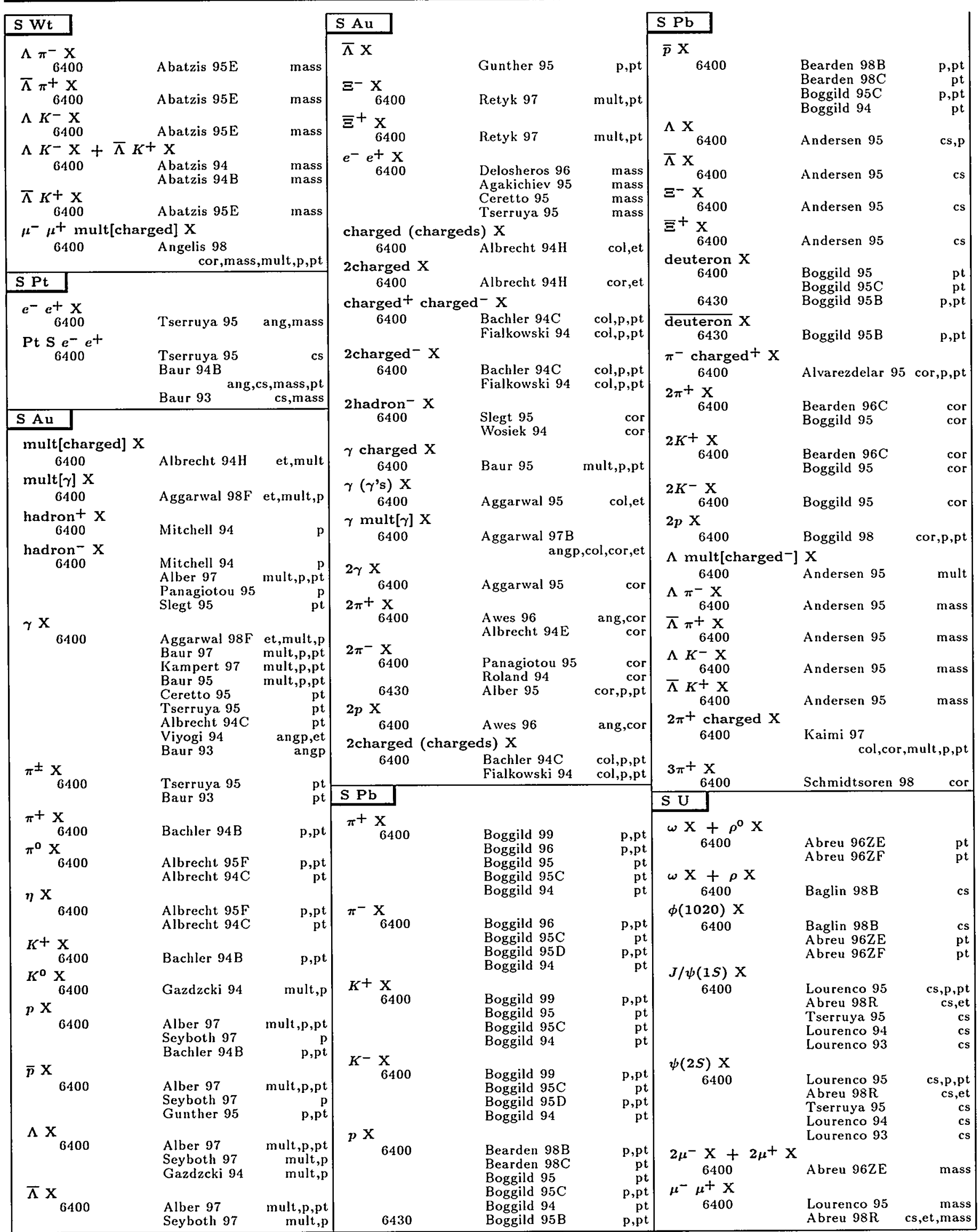

Entries are in order of beam name, then target name, then multiplicity of final state. Particle names are ordered as described in the legend on page 157 and as listed in the Particle Vocabulary. See also the Table of Contents of this Index beginning on the page 158. A few chemical symbols for nuclei have been changed to avoid ambiguity with particle names (see the Particle Vocabulary). Beam momenta are $p_{1 a b}$ in GeV/c, or in parentheses $E_{\mathrm{cm}}$ in $\mathrm{GeV}$. 


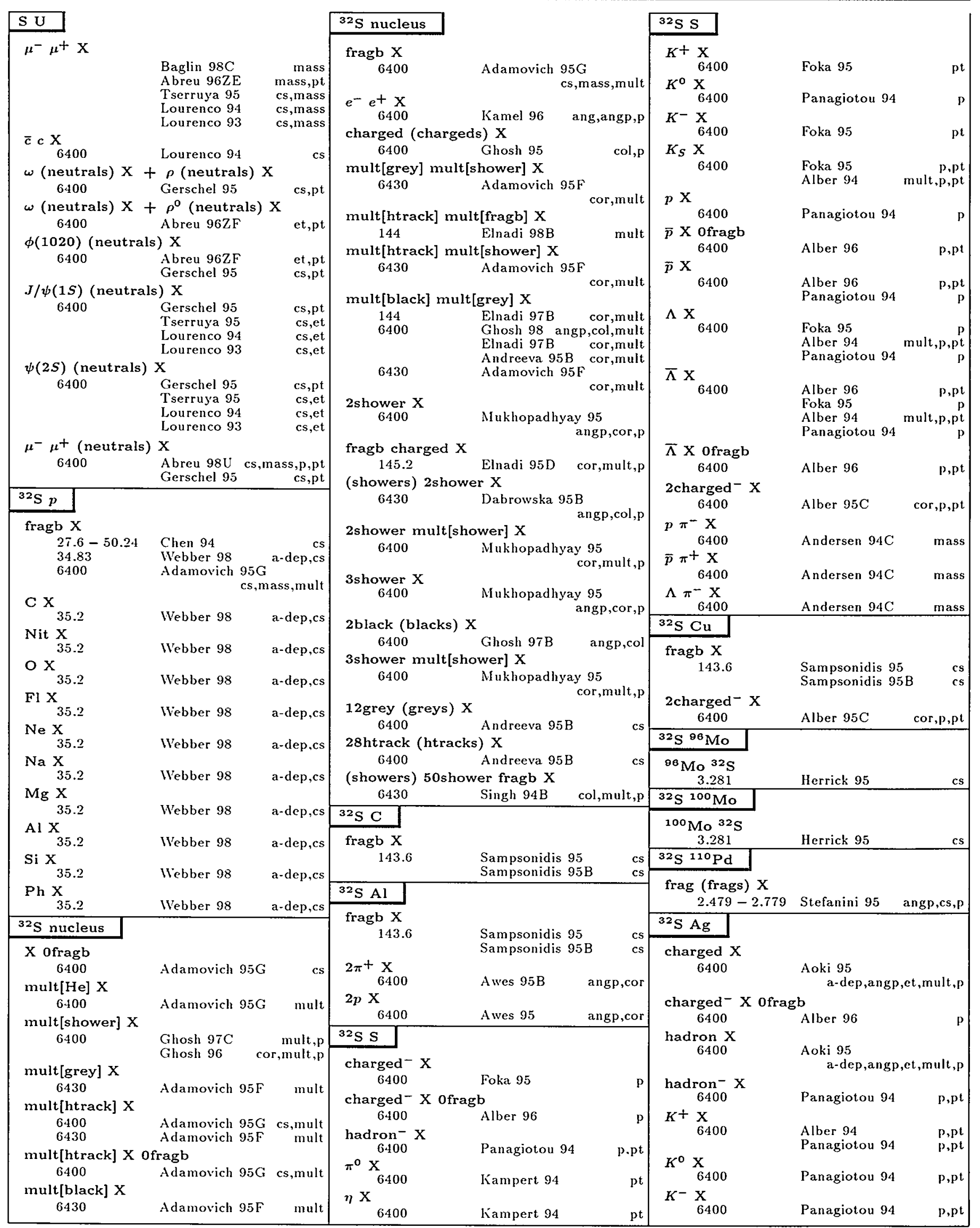




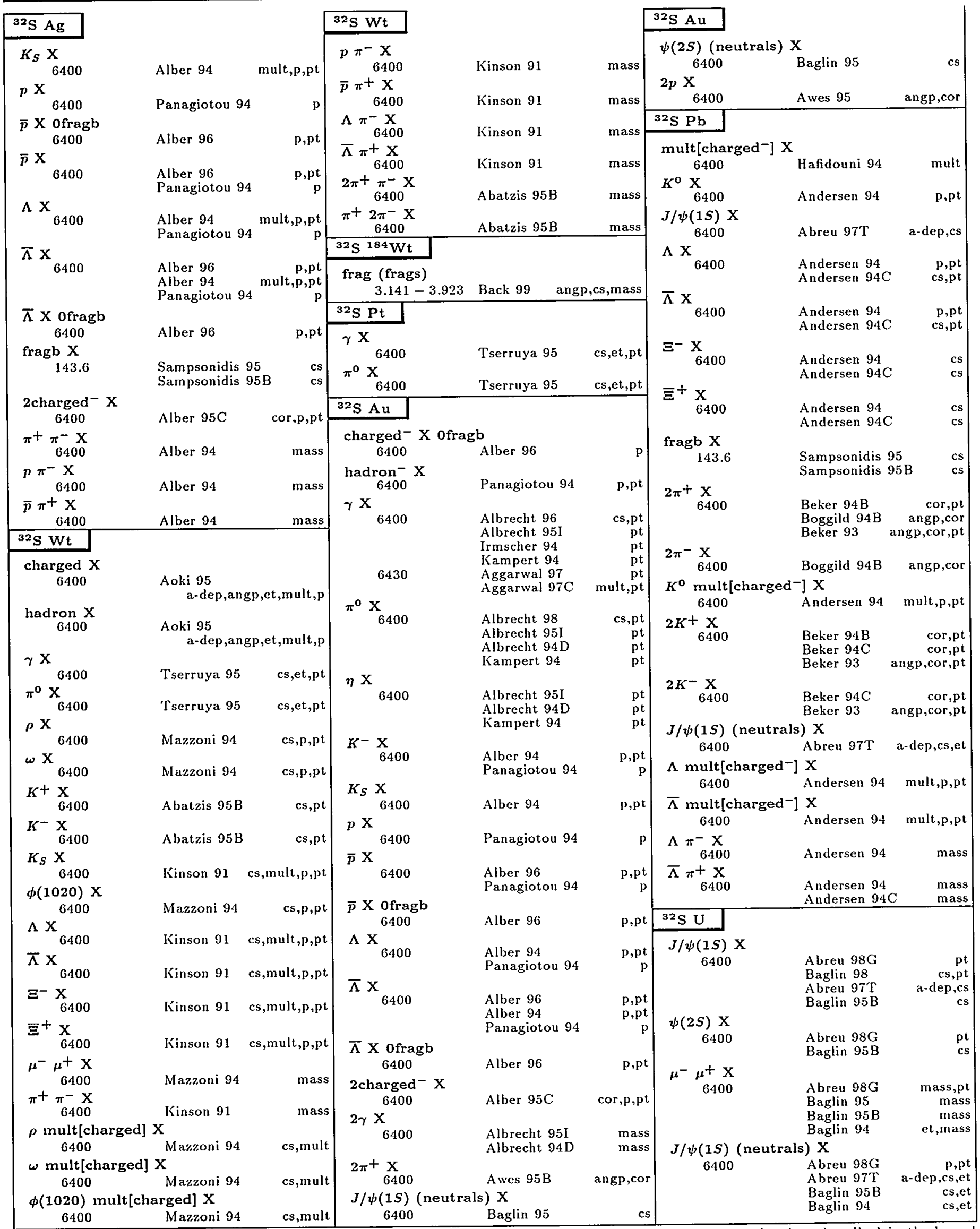

Entries are in order of beam name, then target name, then multiplicity of final state. Particle names are ordered as described in the legend

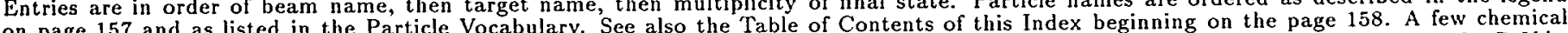
on page 157 and as listed in the Particle Vocabulary. See also the Table of Contents of this Index beginning on the page 158 . A ream momenta are $p_{\text {lab }}$ in GeV/c, or in parentheses $E_{\mathrm{cm}}$ in GeV. 


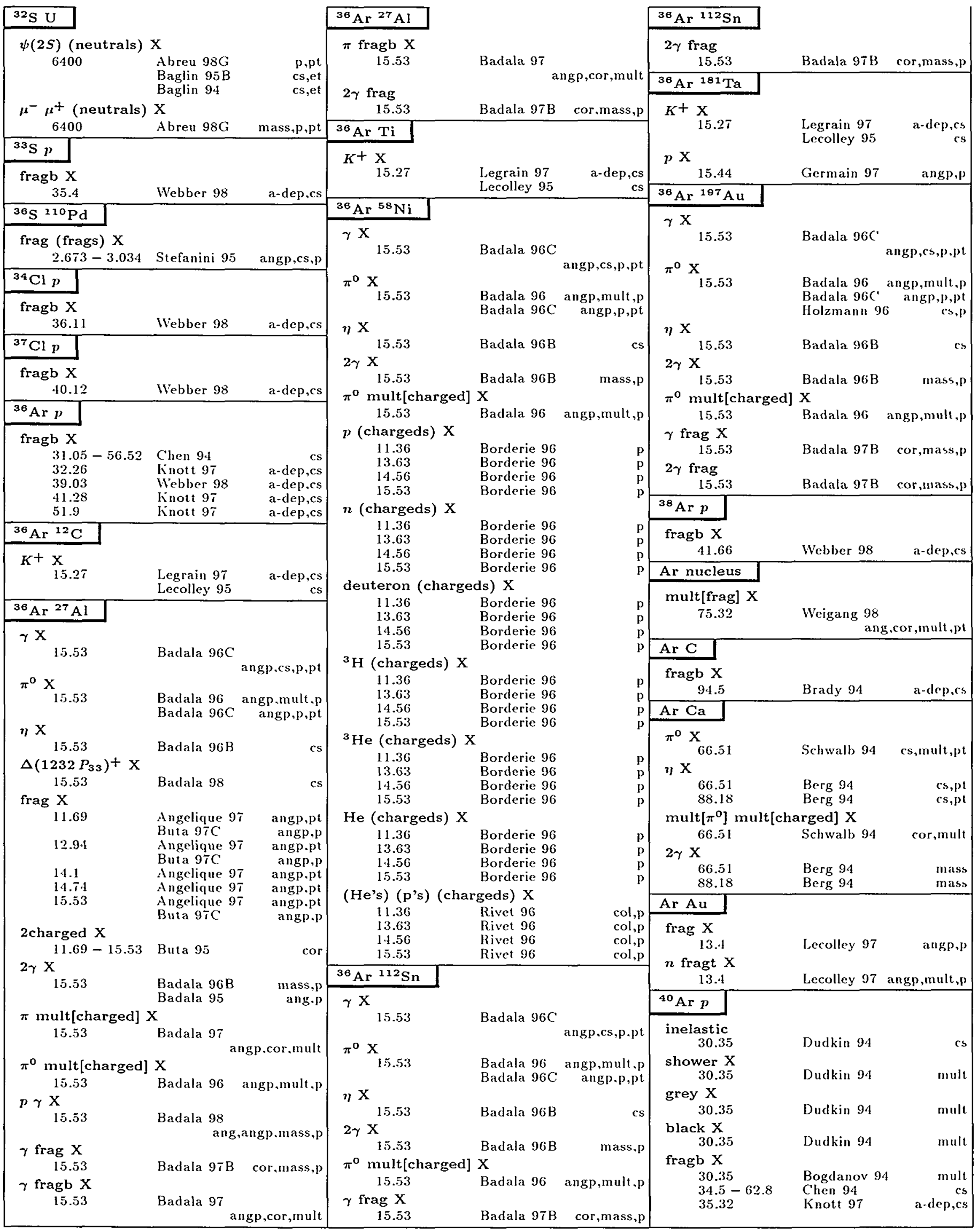




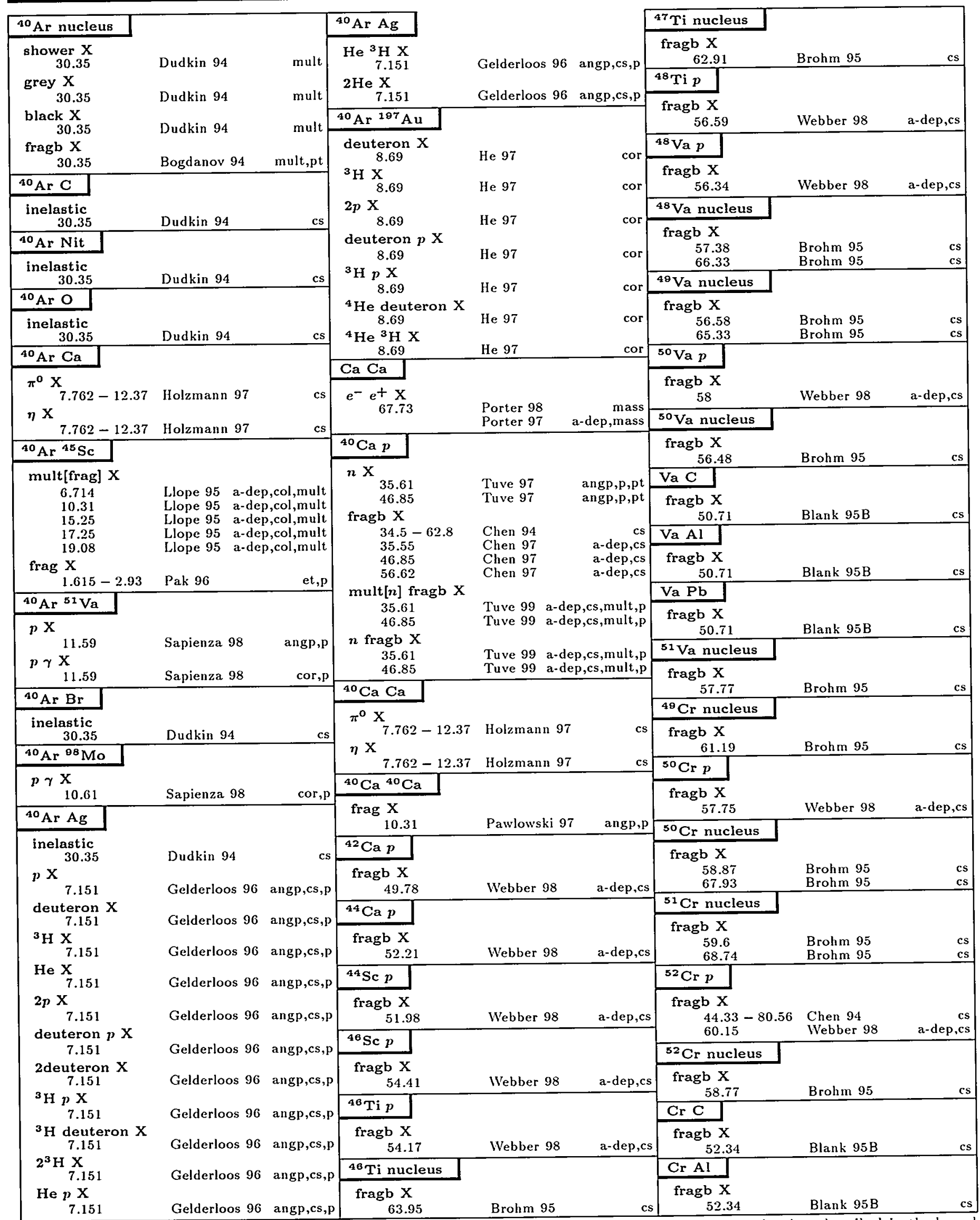

(n) Entries are in order of beam name, then target name, then multiplicity of final state. Particle nameginning on the page 158 . A few chemical on page 157 and as listed in the Particle Vocabulary. See wiso the Table of (see the Particle Vocabulary). Beam momenta are $p_{\text {lab }}$ in GeV/c, or in parentheses $E_{\mathrm{cm}}$ in $\mathrm{GeV}$. 


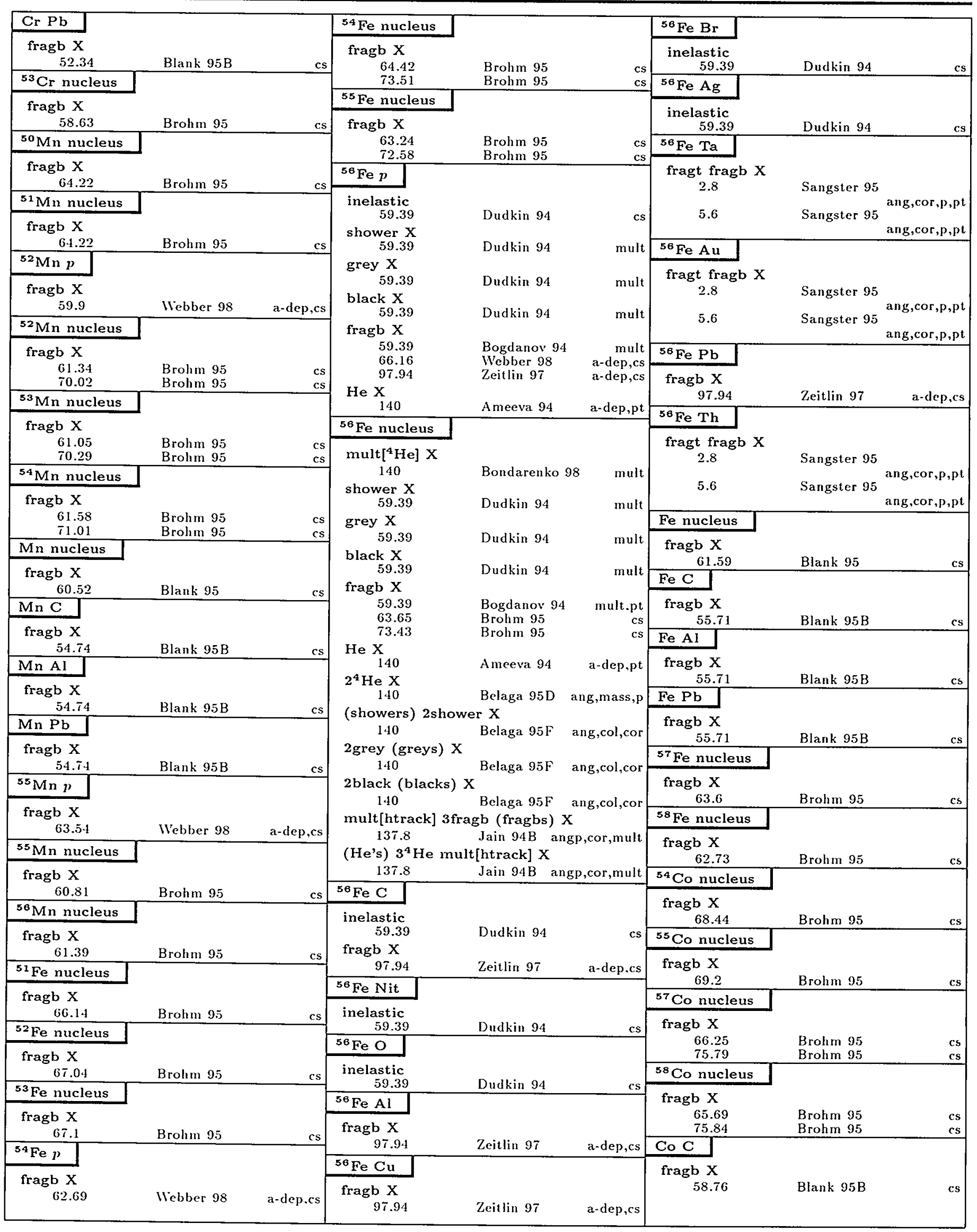




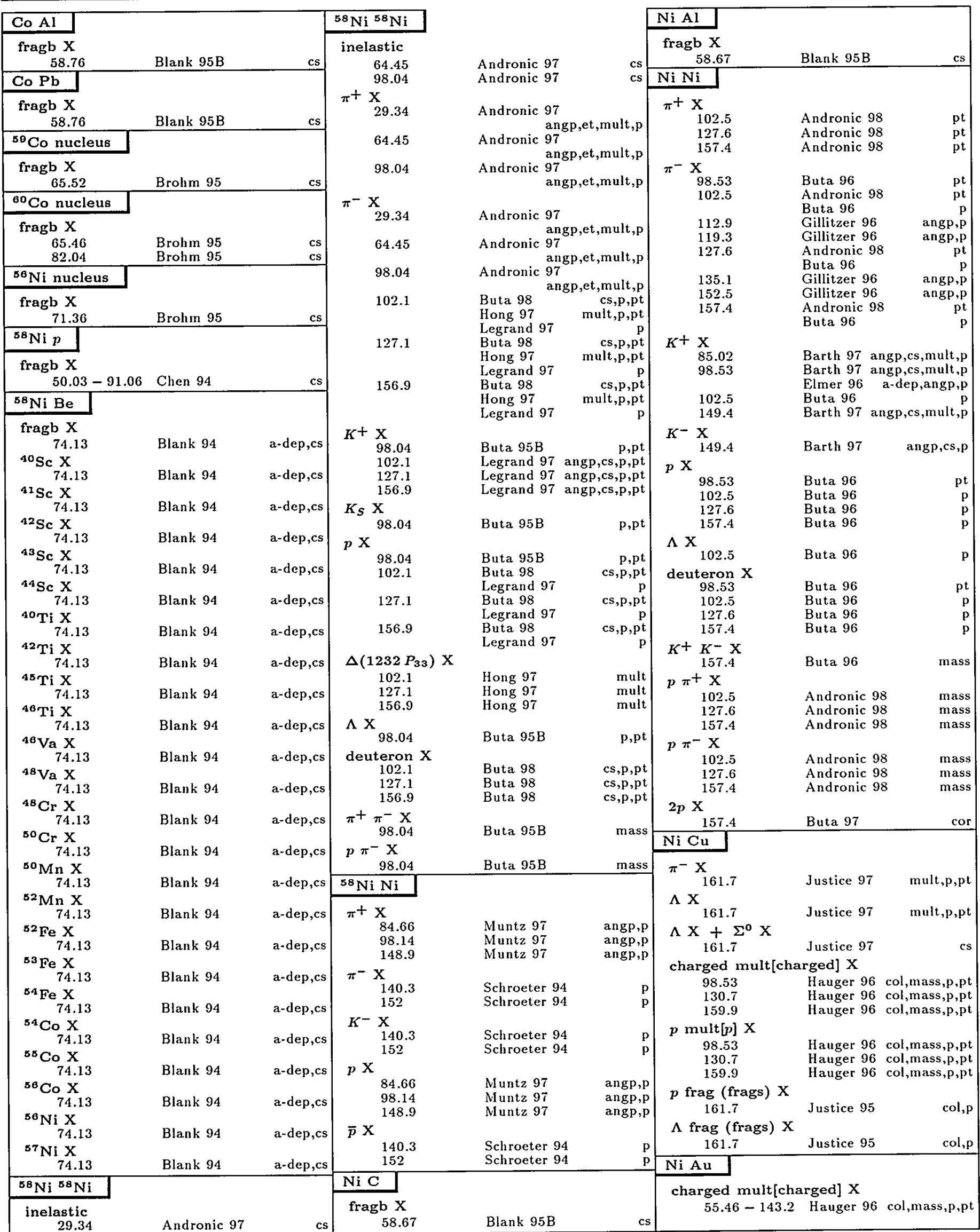

Entries are in order of beam name, then target name, then multiplicity of final state. Particle names are ordered as described in the legend on page 157 and as listed in the Particle Vocabulary. See also the Table of Contents of this Index beginning on the page 158. A few chemical symbols for nuclei have been changed to avoid ambiguity with particle names (see the Particle Vocabulary). Beam momenta are $p_{\text {lab }}$ in GeV/c, or in parentheses $E_{\mathrm{cm}}$ in $\mathrm{GeV}$. 


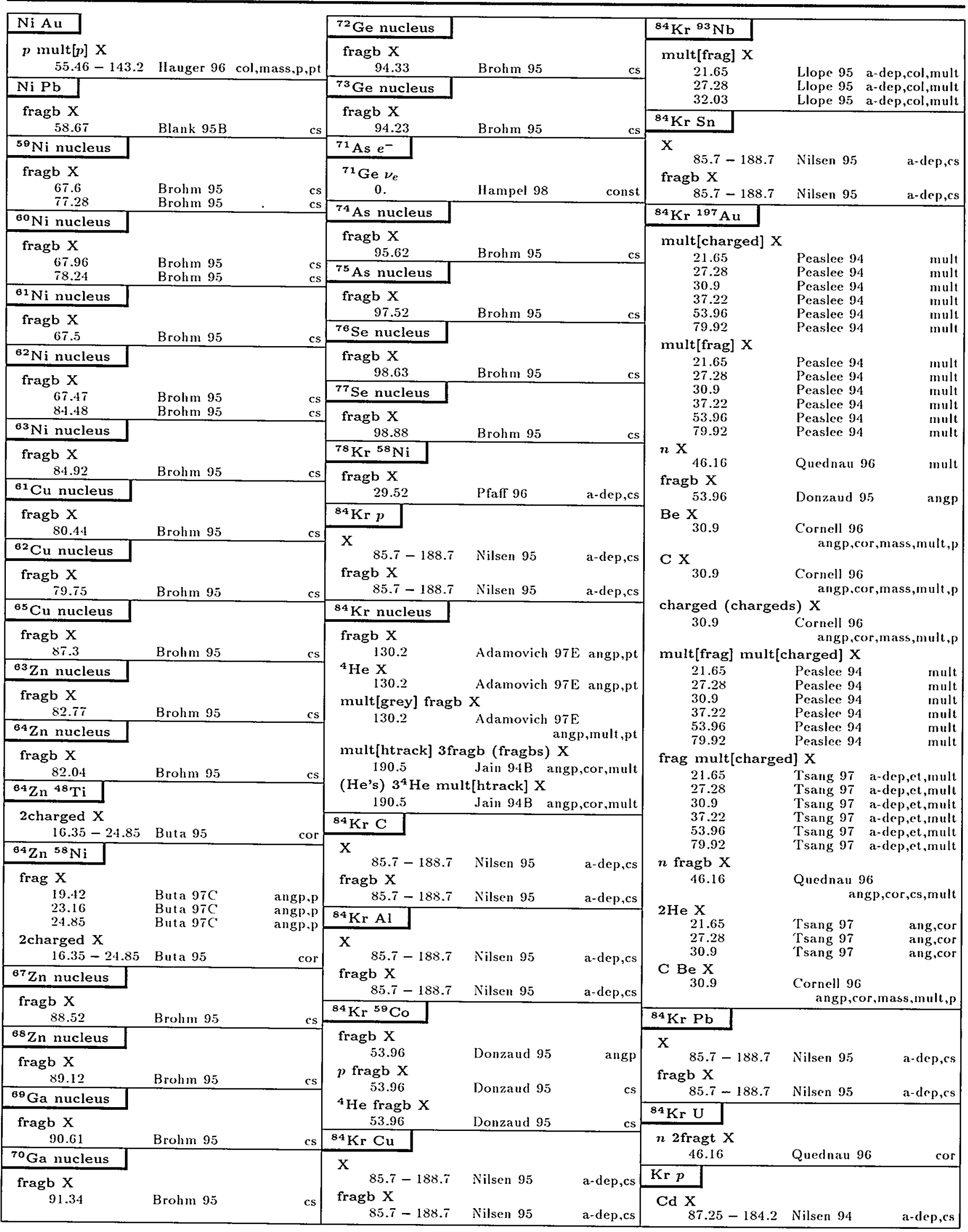




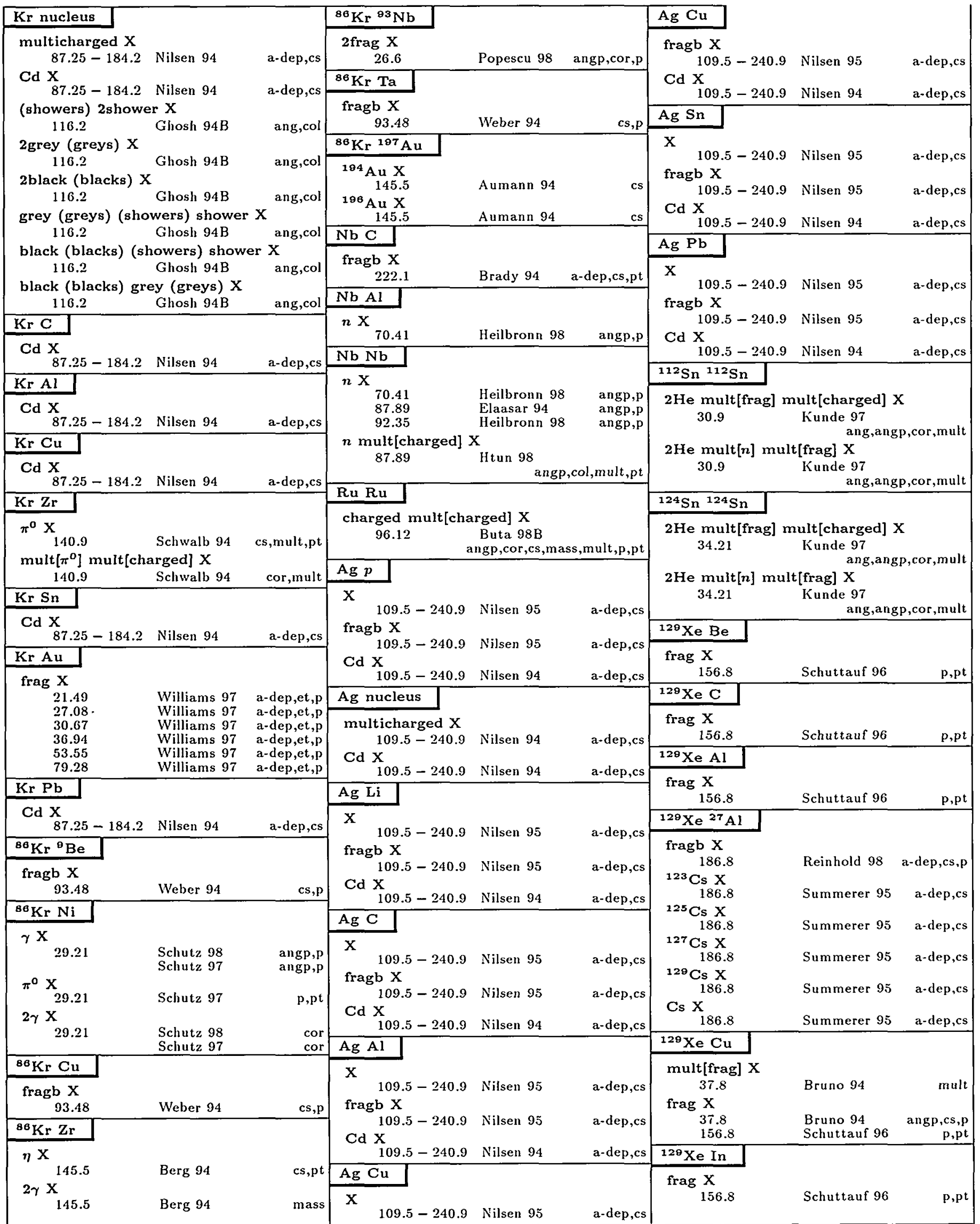

Entries are in order of beam name, then target name, then multiplicity of final state. Particle names are ordered as described in the legend on page 157 and as listed in the Particle Vocabulary. See also the Table of Contents of this Index beginning on the page 158 . A few chemical symbols for nuclei have been changed to avoid ambiguity with particle names (see the Particle Vocabulary). Beam momenta are $p$ lab in GeV/c, or in parentheses $E_{\mathrm{cm}}$ in $\mathrm{GeV}$. 


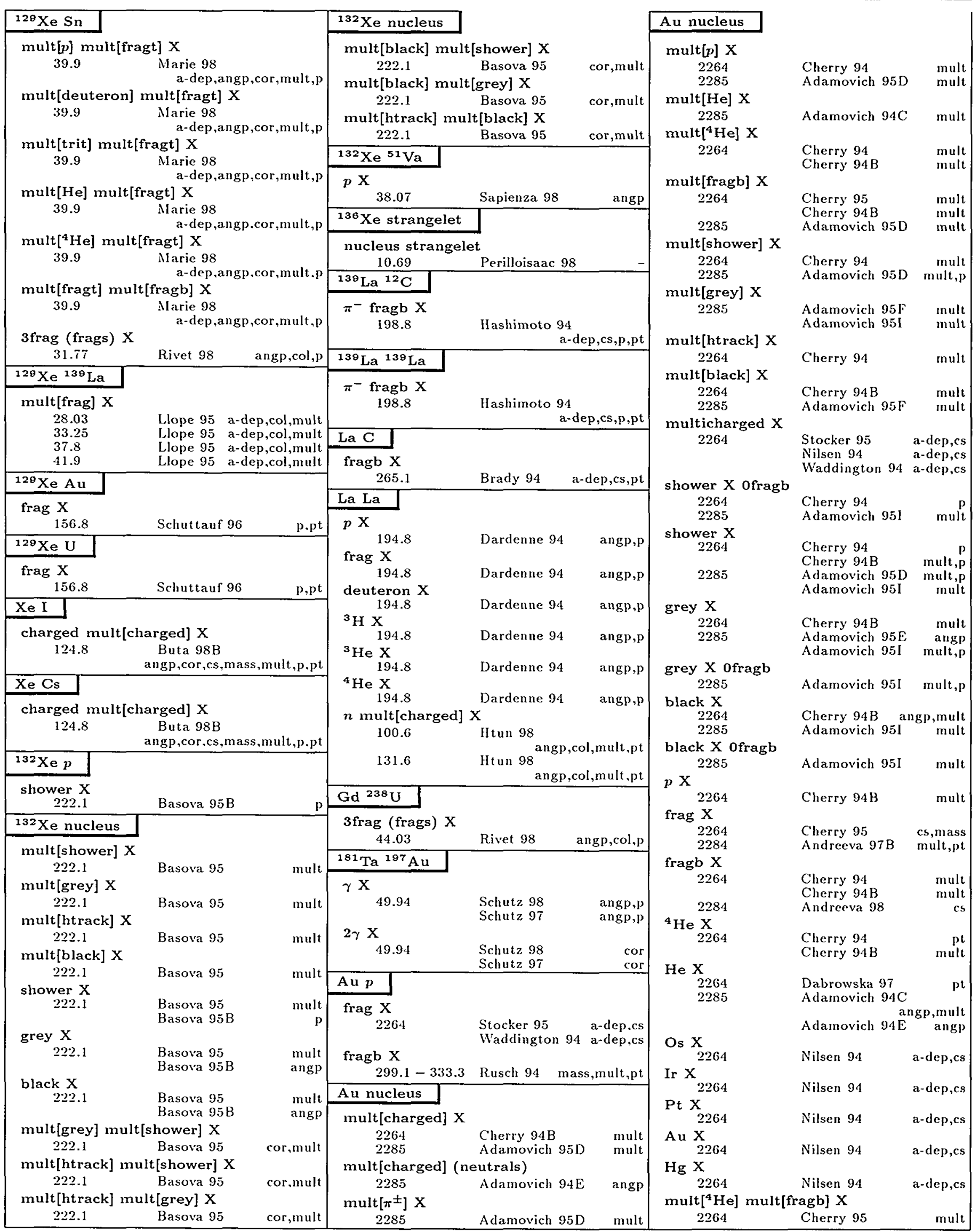




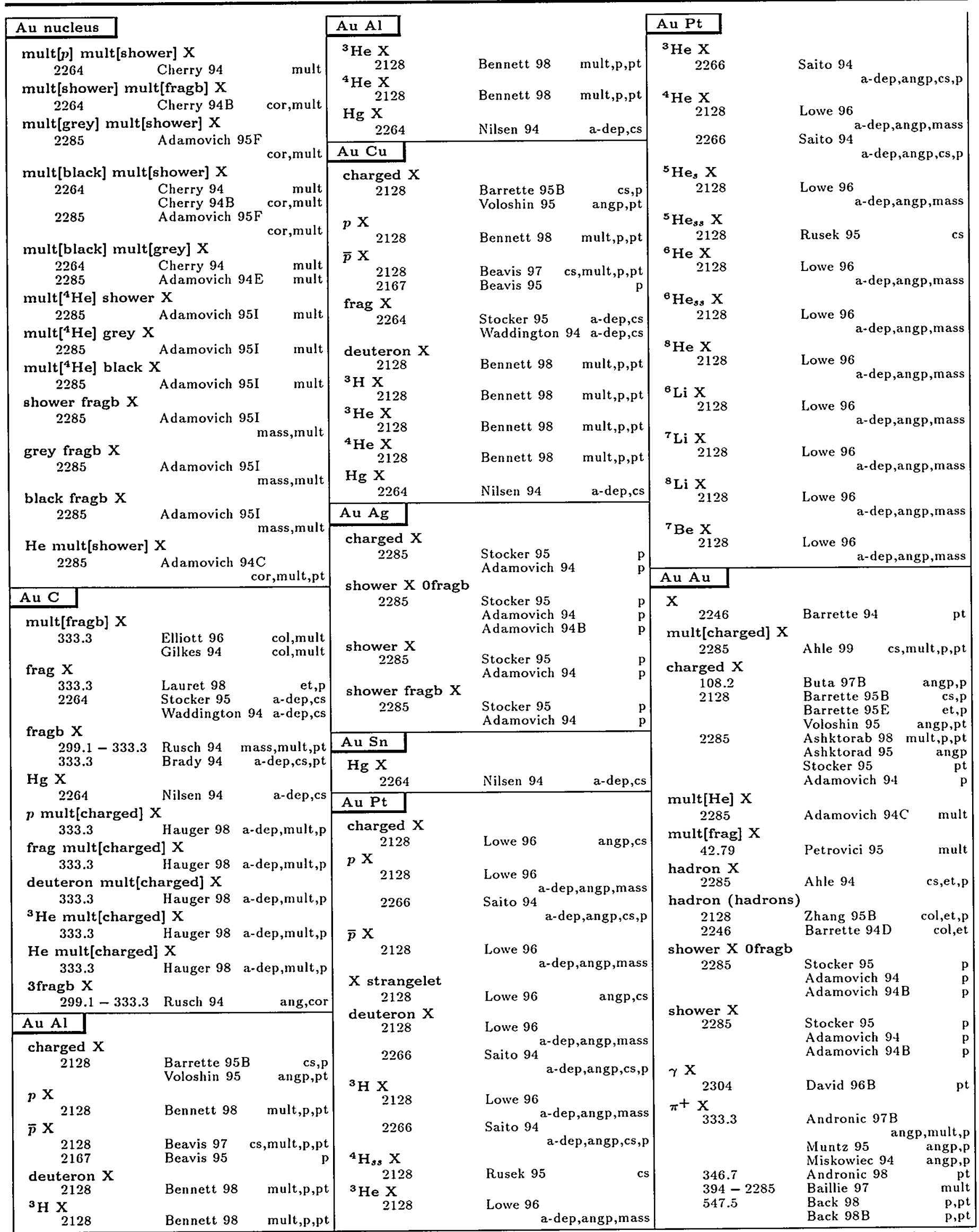

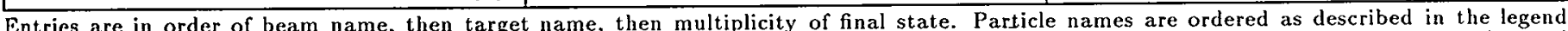

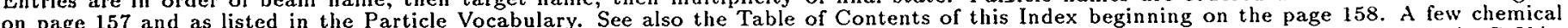

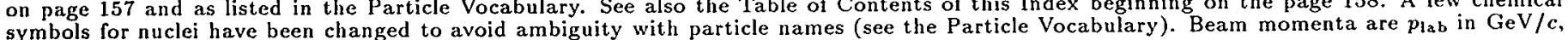
or in parentheses $E_{\mathrm{cm}}$ in $\mathrm{GeV}$. 
$\mathrm{Au} A \mathrm{u} \rightarrow \pi^{+} \mathrm{X}$

Au Au $\rightarrow \mathrm{He} \mathrm{X}$

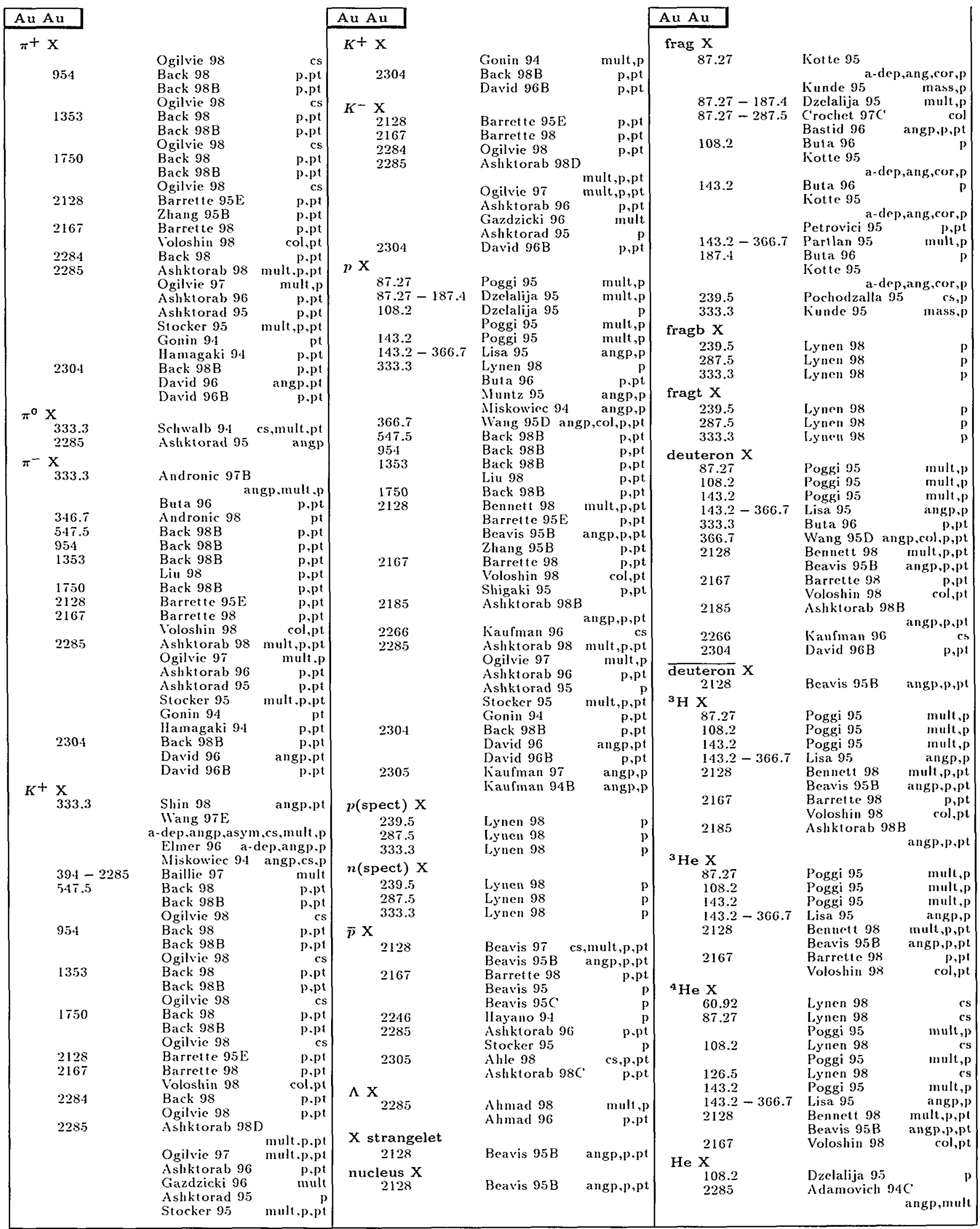




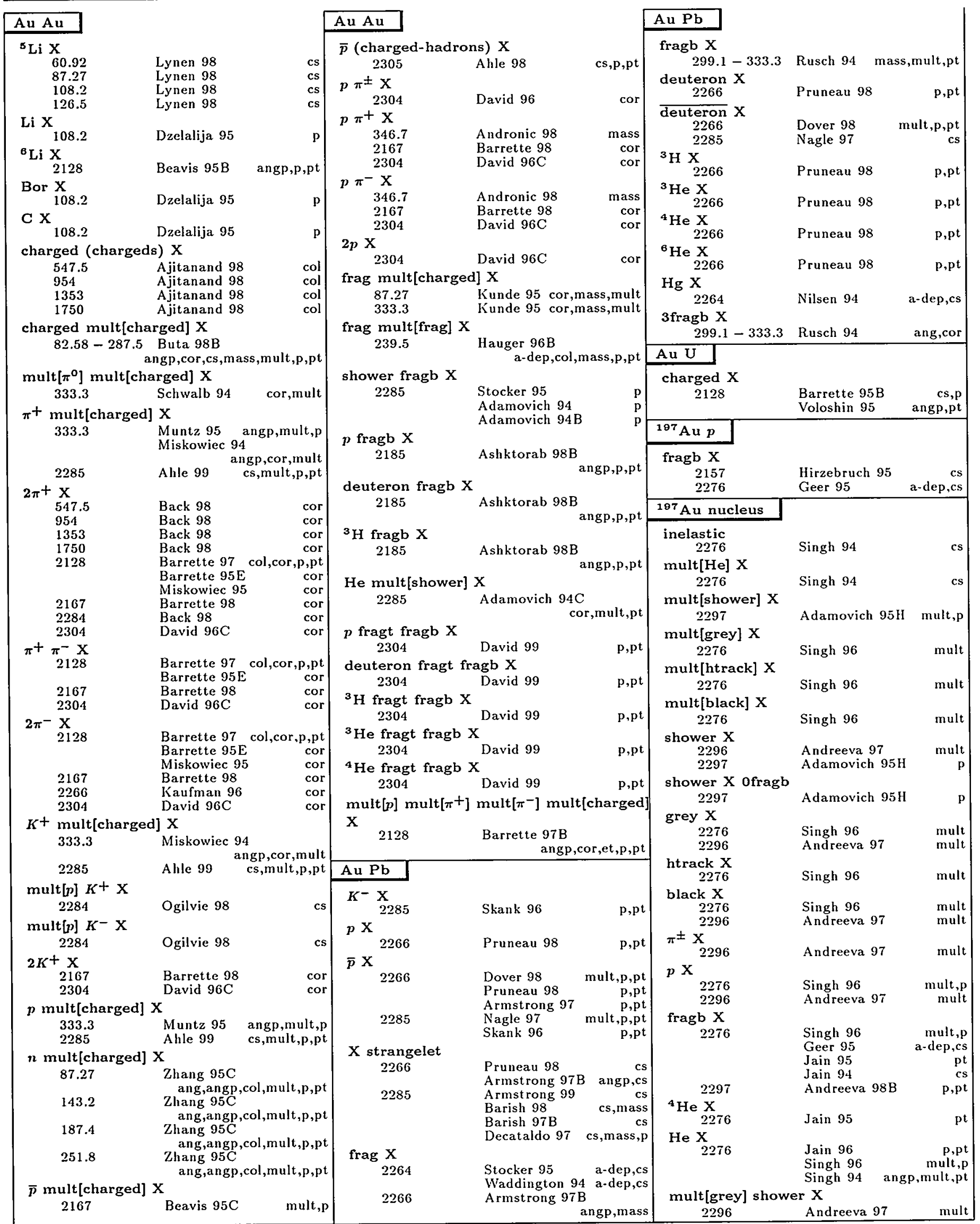

Entries are in order of beam name, then target name, then multiplicity of final state. Particle names are ordered as described in the legend on page 157 and as listed in the Particle Vocabulary. See also the Table of Contents of this Index beginning on the page 158. A few chemical symbols for nuclei have been clianged to avoid ambiguity with particle names (see the Particle Vocabulary). Beam momenta are $p_{1 a b}$ in GeV/c, or in parentheses $E_{\mathrm{cm}}$ in $\mathrm{GeV}$. 


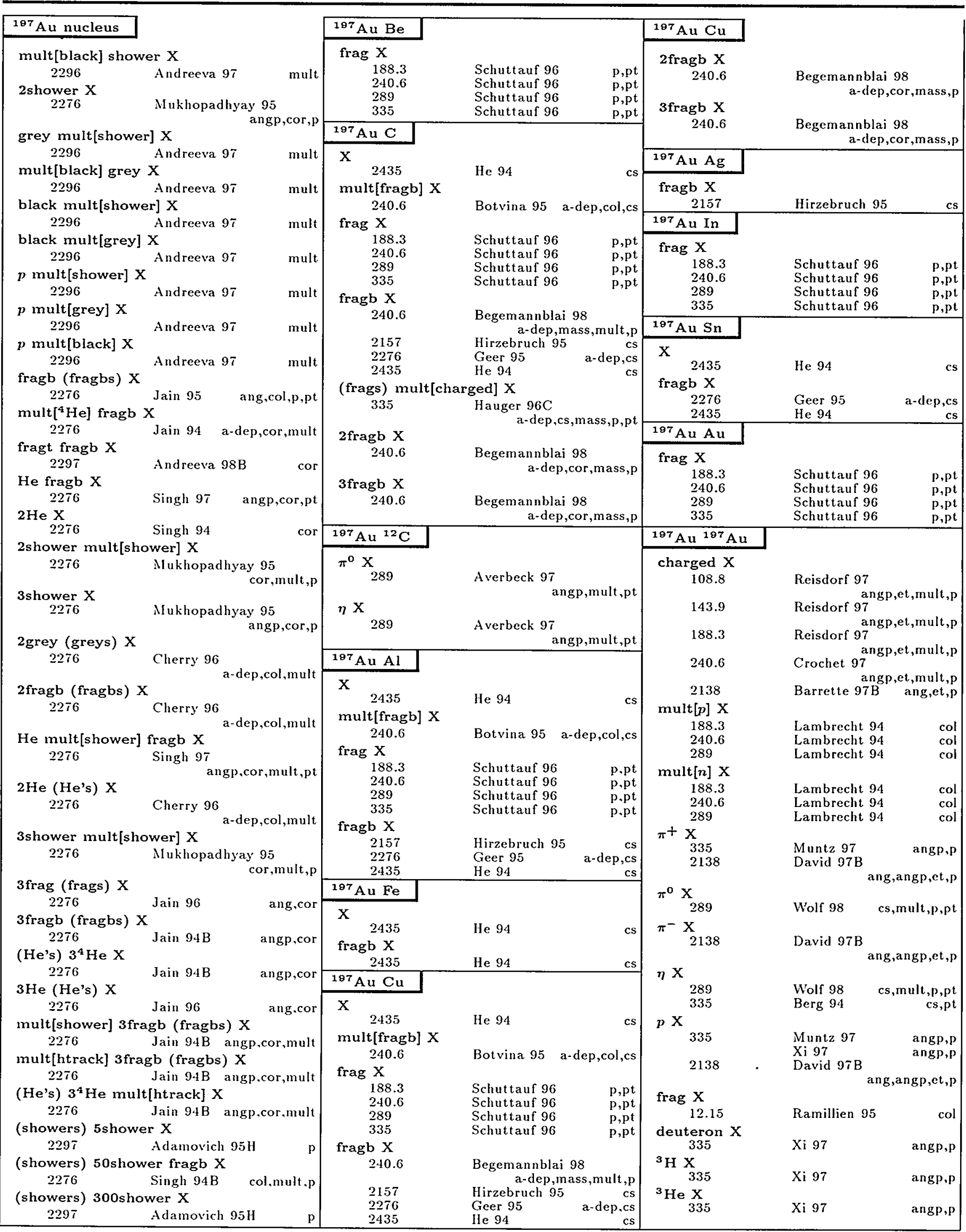




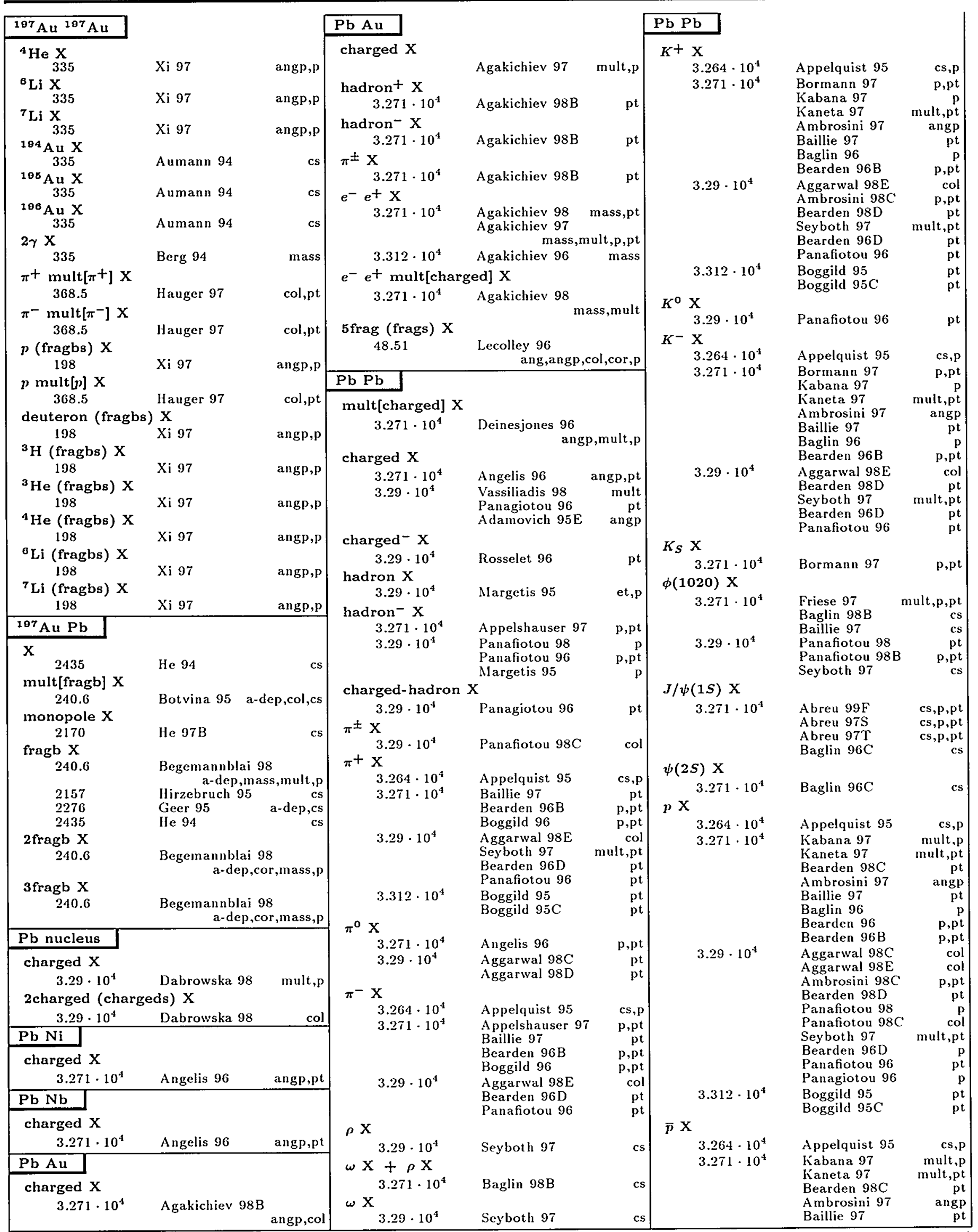

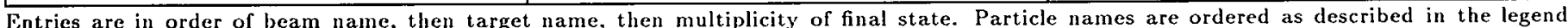

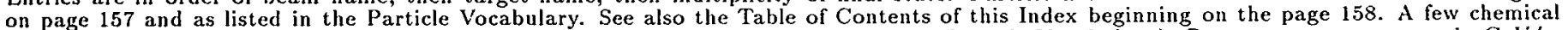

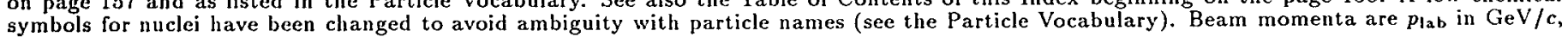
or in parentheses $E_{\mathrm{cm}}$ in $\mathrm{GeV}$. 


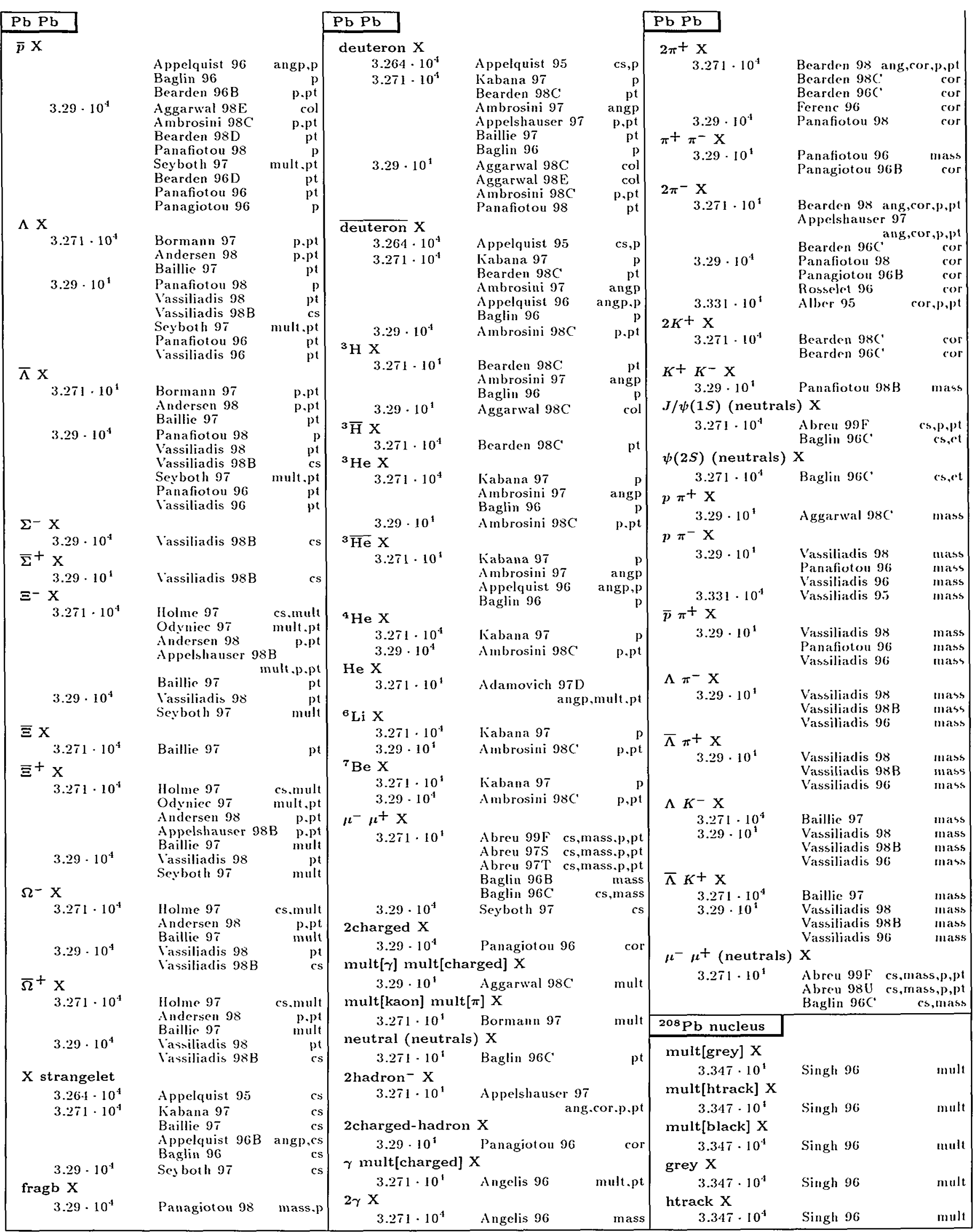




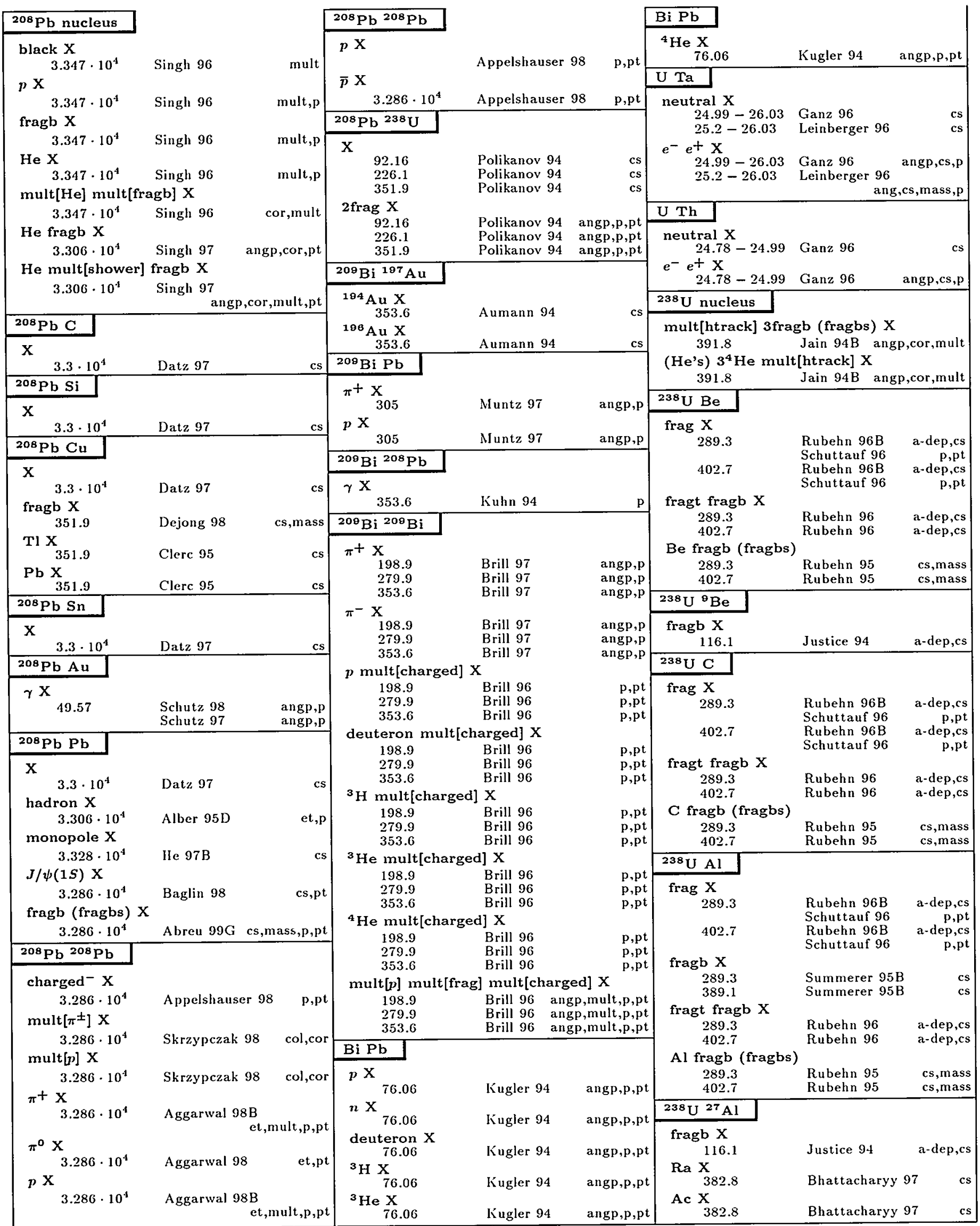

Entries are in order of beam name, then target name, then multiplicity of final state. Particle names are ordered as described in the legend on page 157 and as listed in the Particle Vocabulary. See also the Table of Contents of this Index beginning on the page 158. A few chemical symbols for nuclei have been changed to avoid ambiguity with particle names (see the Particle Vocabulary). Beam momenta are $p_{\text {Iab in }}$ GeV/c, or in parentheses $E_{\mathrm{cm}}$ in $\mathrm{GeV}$. 


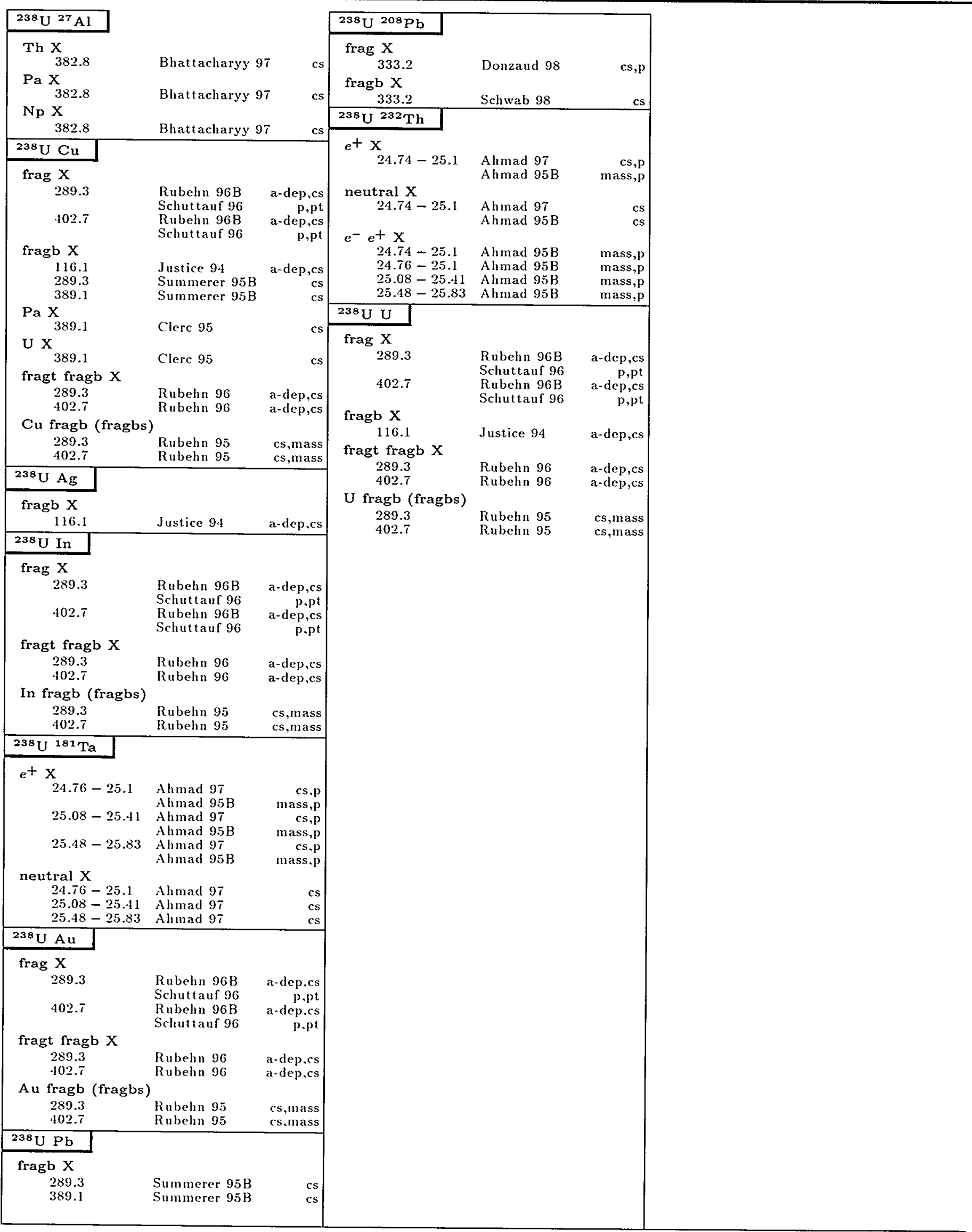


This index lists papers by names of particles studied or produced and their decays, ordered by particle name, then decay.

Particle names follow the ordering in the Particle Vocabulary of this compilation: Gauge bosons, leptons, mesons, baryons, atoms, and nuclei; and within each group the ordering is mainly by increasing mass. Within mesons and baryons, however, we further categorize these particles as having only light quarks, or as having as the heaviest quark, a strange, charmed, bottom, or top quark. To simplify searching in this Index, a short "Table of Contents" of the full Index comes first.

For a given particle, the decays are divided into two classes: inclusive and exclusive, with inclusive first. In each inclusive or exclusive sample, ordering is by the final-state multiplicity, with separation of semileptonic from nonleptonic final states; semileptonic appear first, then nonleptonic. The decays are further ordered by names of the final-state particles, taking into account the weak and strong isospin multiplet structures. When no decay mode is given, no mode was given in the original paper.

For a given decay, papers are ordered by year (most recent first), then first author name. For the full reference, see the ID/Reference/Title Index.

\section{Illustrative Key}

Particle: see the Particle Vocabulary for nomenclature.

Decay: decay mode of the particle.

\begin{tabular}{|l|l|}
\hline$a_{2}(\mathbf{1 3 2 0})^{+}$ & \\
& \\
& Ahmad 92 \\
& Baltrusaitis 91 \\
& Bisello 91B \\
& Kopke 89 \\
& Thorndike 88 \\
$\eta \pi^{+}$ & Atkinson 92C \\
& Landsberg 91 \\
$K^{+} K_{S}$ & Landsberg 91 \\
&
\end{tabular}

Document ID: see ID/Reference/Title Index for the full reference. 
This is a short "Table of Contents" of the full Particle/Decay Index

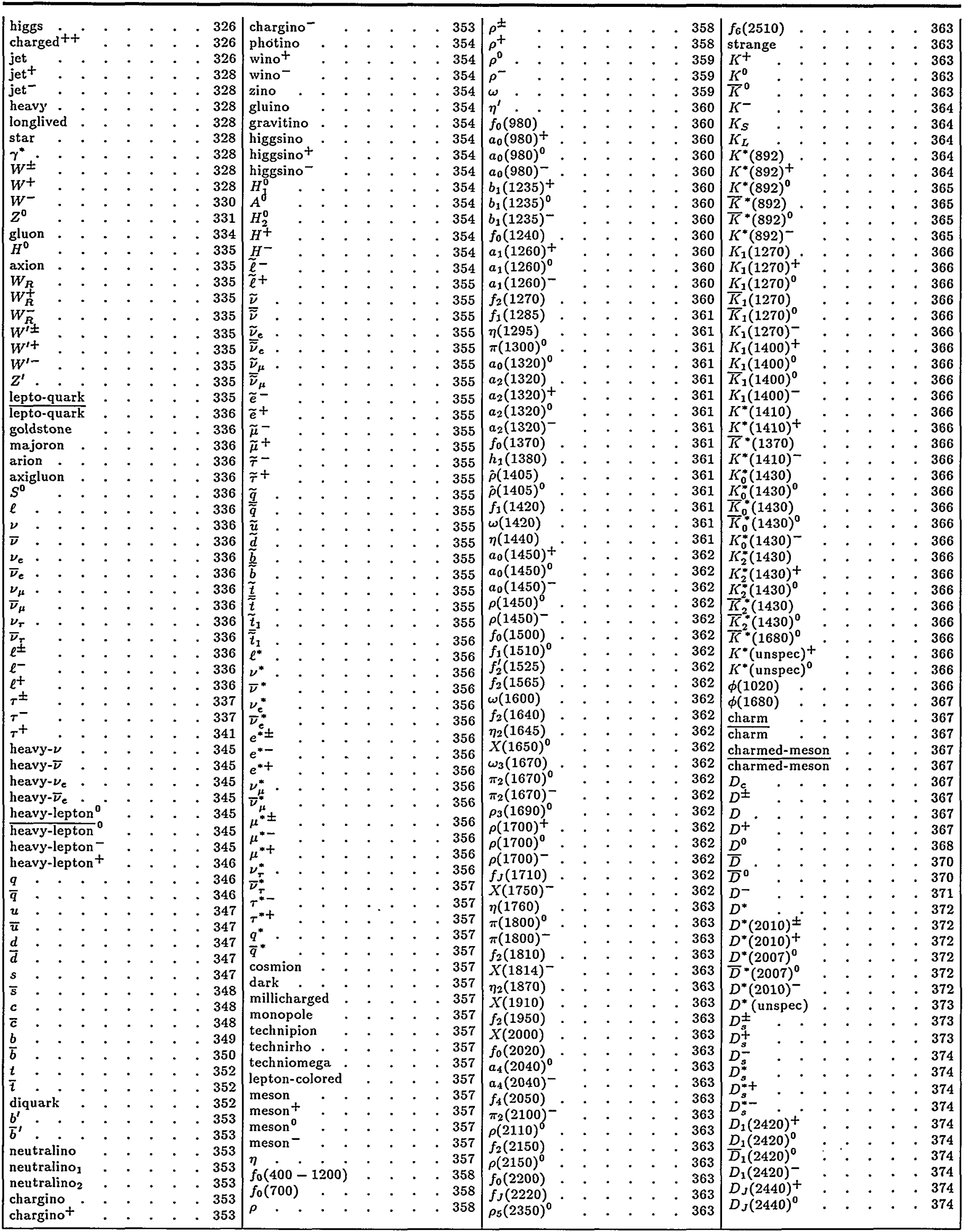


This is a short "Table of Contents" of the full Particle/Decay Index

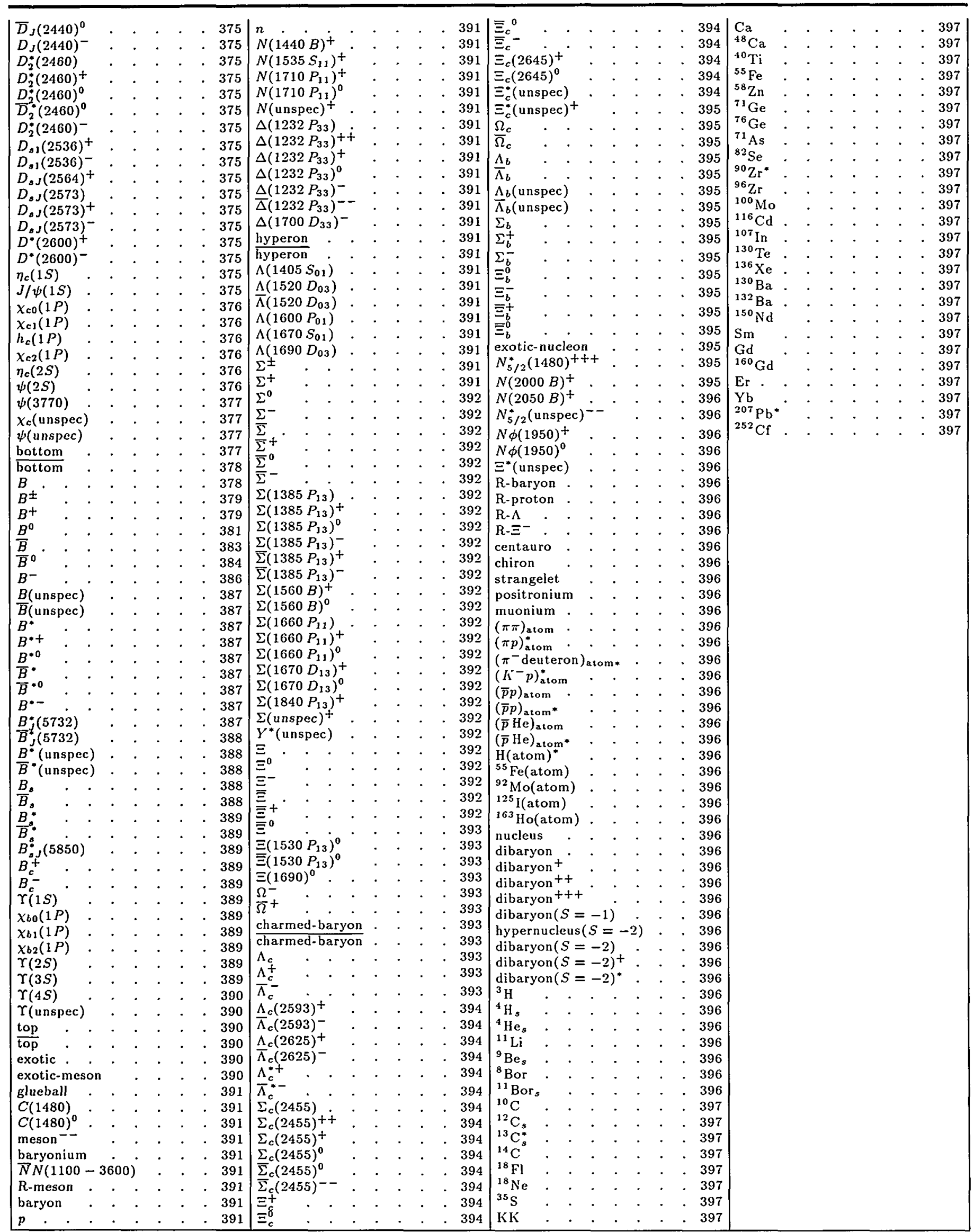




\begin{tabular}{|c|c|c|c|c|c|c|c|}
\hline higgs & & jet & & jet & & jet & \\
\hline $\begin{array}{l}\text { hadron (h } \\
\text { 2hadron ( } \\
\text { invisible } \\
e^{-} e^{+} \\
\mu^{-} \mu^{+} \\
\tau^{-} \tau^{+} \\
\text {2invisible } \\
\text { 2jet }\end{array}$ & $\begin{array}{l}\text { Barate } 99 \mathrm{~B} \\
\text { Bock } 98 \\
\text { Acciarri } 97 \mathrm{Q} \\
\text { Barate } 97 \mathrm{~L} \\
\text { Akers } 94 \mathrm{~F} \\
\text { Demin } 94 \\
\text { drons) } \\
\text { Richard } 94 \\
\text { adrons) } \\
\text { Abreu } 94 \mathrm{P} \\
\text { Acciarri } 96 \mathrm{~B} \\
\text { Acciarri } 96 \mathrm{~B} \\
\text { Acciarri } 96 \mathrm{~B} \\
\text { Abreu } 99 \mathrm{E} \\
\text { Abbiendi } 98 \mathrm{R} \\
\text { Acciarri } 98 \mathrm{~F} \\
\text { Acciarri } 98 \mathrm{~J} \\
\text { Barate } 98 \mathrm{R} \\
\text { Barate } 98 \mathrm{~S} \\
\text { Sopczak } 98 \\
\text { Abreu } 97 \mathrm{~L} \\
\text { Ackerstaff } 97 \mathrm{~V} \\
\text { Barate } 97 \mathrm{O} \\
\text { Abreu } 97 \mathrm{~L} \\
\text { Acciarri } 97 \mathrm{U} \\
\text { Abreu } 99 \mathrm{E} \\
\text { Buskulic } 96 \mathrm{~K} \\
\text { Alexander } 95 \mathrm{I} \\
\text { Kopp } 95 \\
\text { Abreu } 94 \mathrm{P} \\
\text { Janot } 94 \\
\text { Ackerstaff } 98 \mathrm{R} \\
\text { Ackerstaff } 97 \mathrm{R} \\
\text { Acciarri } 96 \mathrm{G} \\
\text { Abachi } 95 \mathrm{~T} \\
\text { Alexander } 95 \mathrm{I} \\
\text { Acciarri } 98 \mathrm{~F} \\
\text { Acciarri } 98 \mathrm{~J} \\
\text { Sopczak } 98 \\
\text { Acciarri } 97 \mathrm{U} \\
\text { Acciarri } 96 \mathrm{~B} \\
\text { Acciarri } 96 \mathrm{G} \\
\text { Alexander } 96 \mathrm{~W} \\
\text { Buskulic } 96 \mathrm{I} \\
\text { Abreu } 94 \mathrm{P} \\
\text { Abreu } 97 \mathrm{~L} \\
\text { Abreu } 99 \mathrm{E} \\
\text { Abbiendi } 98 \mathrm{R} \\
\text { Abe } 98 \mathrm{~S} \\
\text { Acciarri } 98 \mathrm{~J} \\
\text { Barate } 98 \mathrm{R} \\
\text { Barate } 98 \mathrm{~S} \\
\text { Abe } 97 \mathrm{R} \\
\text { Abreu } 97 \mathrm{~L} \\
\text { Ackerstaff } 97 \mathrm{~V} \\
\text { Barate } 97 \mathrm{O} \\
\text { Abachi } 96 \mathrm{~K} \\
\text { Stichelbaut } 96 \\
\text { Acciarri } 96 \mathrm{~B} \\
\text { Acciarri } 96 \mathrm{~B} \\
\end{array}$ & & 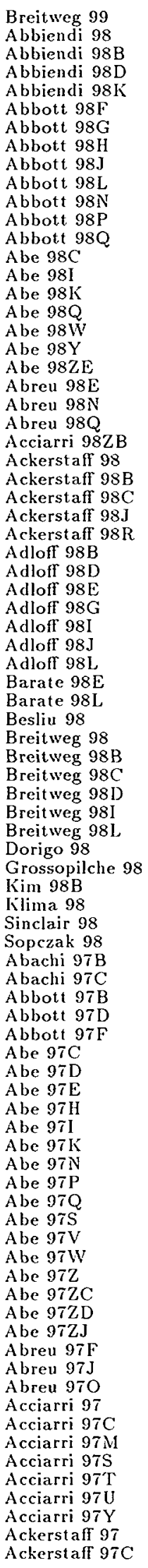 & & 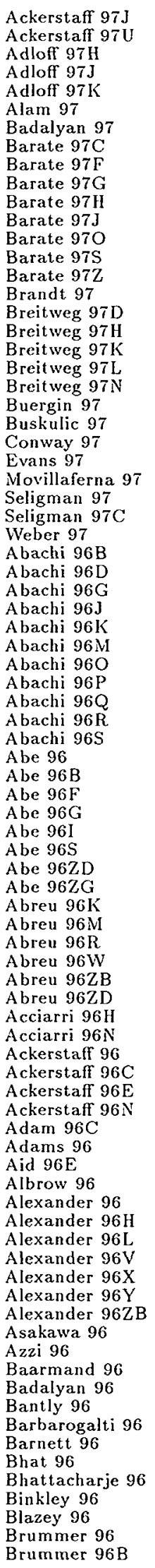 & & 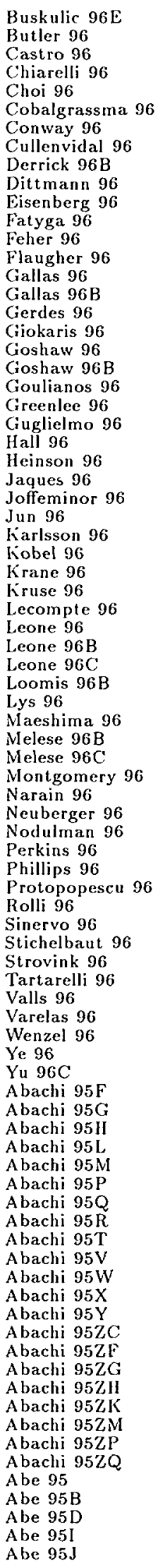 \\
\hline
\end{tabular}




\section{jet}

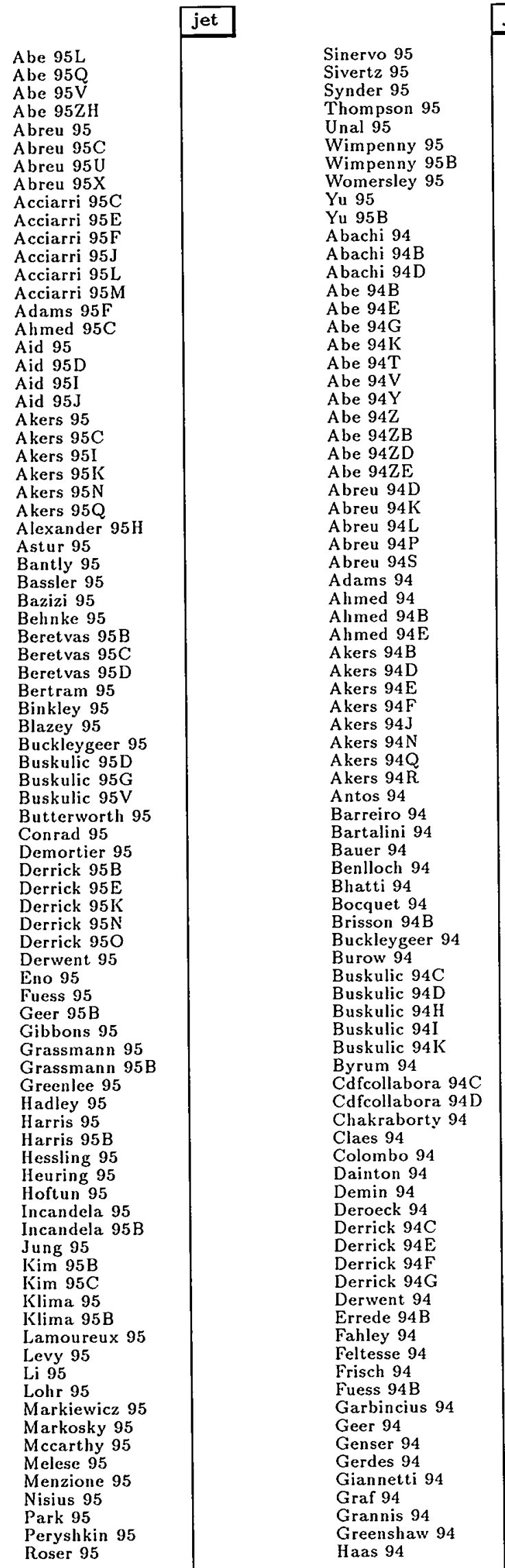

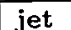

jet

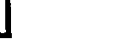

Thompson 95

Yu 95

Abachi 94D

Abe $94 \mathrm{~K}$

A be $94 \mathrm{~V}$

A be 94ZB

Abe 947E

Abreu 94D

Abreu 94

Abreu 94S

Alimed 94

Ahmed $94 \mathrm{E}$

A kers $94 \mathrm{~J}$

Bhatti 94

Bocquet 94

Buckleygeer 94

Burow 94

Buskulic $94 \mathrm{C}$

Buskulic 94I

Buskulic 94K

Cdfcollabora 94C

Chakraborty 94

Dainton 94

Deroeck 9

Derrick 94C

Derrick 94

Derrick $94 \mathrm{G}$

Derwent 94

Feltesse 94

Fuess 94B

Giannetti 94

Graf 94

Greenshaw 94

Haas 94

$\ell^{ \pm} \mathrm{X}$

$\mu^{ \pm} \mathrm{X}$

charged $\mathrm{X}$

hadron $\mathrm{X}$
Hagopian 94

Harnew 94

Harris 94

Harris 94B

Hauger 94B

Hawk 94

Hedin 94

Huehn 94

Jakobs 94

Janot 94

Jensen 94

Kiesling 94

Konigsberg 94

Kovacs 94

Kovacs 94B

Kramer 94

Kuhlen 94

Labarga 94

Leone 94

Lidemarteau 94

Martirena 94

Morgan 94

Nakano 94

Naples 94

Nodulman 94

Norman 94

Park 94B

Plunkett 94

Raja 94

Shirai 94

Shochet 94

Strohmer 94

Tauchi 94

Watts 94

Weerts 94

White 94

Williams 94

Abachi 93B

Abreu 93L

Adams 93C

A kers 93F

A kers $93 \mathrm{H}$

Alverson 93B

Derrick 93C

Krasny 93B

Liu 93

Piekarz 93B

Sakuda 93

Akers $94 \mathrm{G}$

Abachi $96 \mathrm{~F}$

Acciarri $97 \mathrm{G}$

Barate 97

Moutoussi 96

Snihur 96

Varelas 96

Alexander 95D

Buskulic 95Q

Buskulic 95R

charged $+x$

Abe $94 \mathrm{~L}$

charged $^{-} X$

Bordner 96

Bordner 96

charged (chargeds)

Baird 95

Abe $94 \mathrm{~W}$

Alton 96

Abachi 95ZI

Derrick 95J

Streets 95

hadron (hadrons)

Acciarri $97 \mathrm{H}$

Heuring 96

Mecarthy 96

Abachi 95S

Syed 95

charged-hadron $X$

Abreu 96B

Moutoussi 96

A kers $95 \mathrm{M}$ jet

\begin{tabular}{|c|c|}
\hline charged-ha & $\begin{array}{l}\text { A kers } 95 \mathrm{~V} \\
\text { Marti } 95 \\
\text { Ward 95 } \\
\text { dron (hadrons }\end{array}$ \\
\hline & Abreu $96 \mathrm{Q}$ \\
\hline$\pi^{+} \mathrm{X}$ & Abreu $97 \mathrm{C}$ \\
\hline$\pi^{-} \mathrm{X}$ & Abreu $97 \mathrm{C}$ \\
\hline $\boldsymbol{K}^{+} \mathbf{x}$ & Abreu $96 \mathrm{ZC}$ \\
\hline$K^{-} \mathbf{X}$ & Abreu $96 \mathrm{ZC}$ \\
\hline $\begin{array}{l}K_{S} \mathrm{X} \\
D^{*}(2010)^{ \pm}\end{array}$ & $\begin{array}{l}\text { Acciarri } 97 \mathrm{G} \\
\text { Abreu } 96 \mathrm{ZC} \\
\mathrm{X} \\
\text { Breitweg } 98 \mathrm{E}\end{array}$ \\
\hline bottom X & $\begin{array}{l}\text { Beretvas } 95 \\
\text { Kopp } 95 \\
\text { Strovink } 95 \\
\text { Sphicas } 94 \\
\text { Tollestrup } 94\end{array}$ \\
\hline
\end{tabular}

bottom $\mathrm{X}$

Beretvas 95

Kopp 95

Strovink 95

Sphicas 94

Tollestrup 94

$B \mathrm{X}$

Alexander 95I

$\bar{B} \times$

Alexander 95I

p X

Abreu 96ZC

$\bar{p} \mathrm{X}$

$\Lambda \mathrm{X}$

Abreu 96ZC

$\bar{\Lambda} \mathrm{x}$

Abreu 96ZC

Acciarri $97 \mathrm{G}$ Abreu 96ZC

charged (chargeds) $\mathrm{X}$ Yu 94

charged (chargeds)

(neutrals)

charged neutral (neutrals) Alexander 96G

hadron (hadrons) $\mathrm{X}$

Acciarri $95 \mathrm{~N}$

2hadron (hadrons)

Hasegawa 95B

charged-hadron

(charged-hadrons) (neutrals) Buskulic $96 \mathrm{H}$

2charged-hadron $X$

Abreu 96B

Moutoussi 96

Marti 95

2charged-hadron (hadrons)

$2 \pi+X$ Abe 95ZP

Abreu $97 \mathrm{C}$

Abreu 97C

A Abreu 97C

$-\pi^{+} \mathrm{X}$

$D_{s}^{-} \pi^{+} \mathrm{X}$

$K^{+} 2 \pi^{-} \mathrm{X}$

Akers $94 \mathrm{H}$

$K^{-} 2 \pi+\mathrm{X}$ Akers 94G

Akers $94 \mathrm{G}$

$\bar{D}^{0} \pi^{+} \pi^{-} \mathrm{X}$

$K^{+} K^{-} J / \psi(1 S) \times$

$+\quad$ Akers 94H

$K^{+} K^{-} e^{-} e^{+} \mathrm{X}$

Akers 94

Entries in order of particle name, then decay. A few chemical symbols for nuclei have been changed to avoid ambiguity with particle names (see the Particle Vocabulary). See the legend on page 323. 


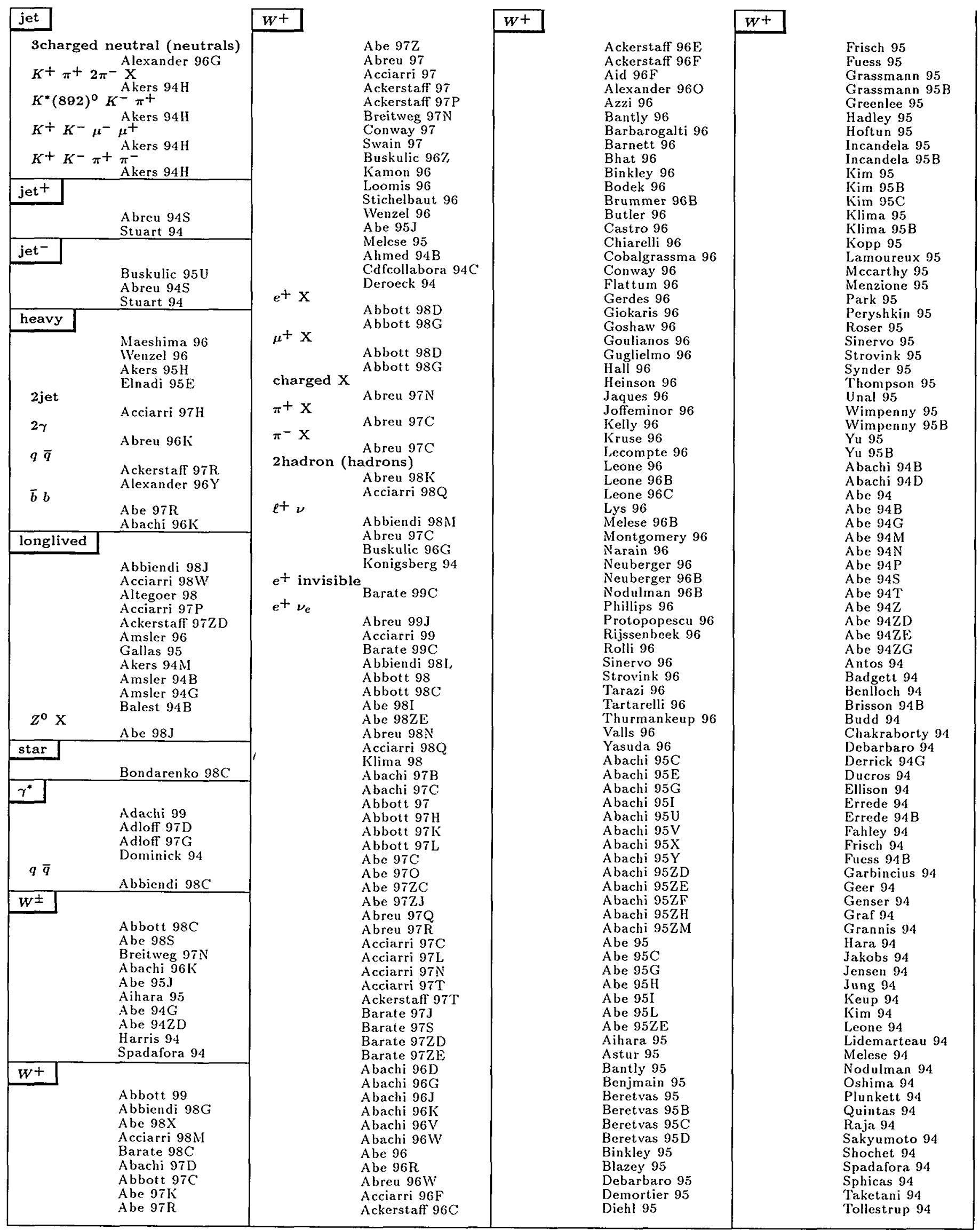




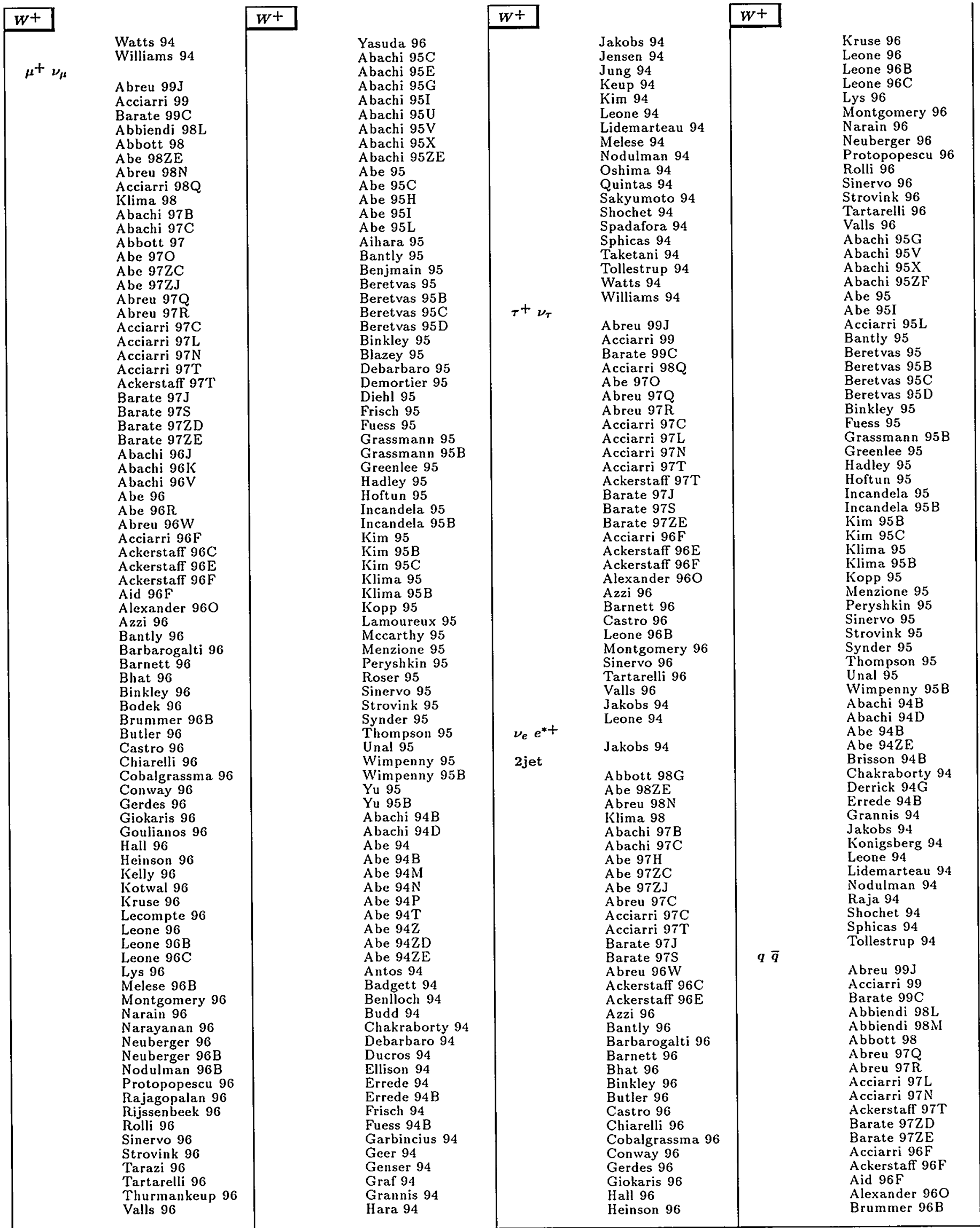

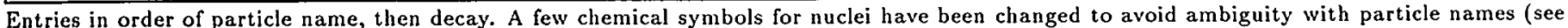
the Particle Vocabulary). See the legend on page 323. 


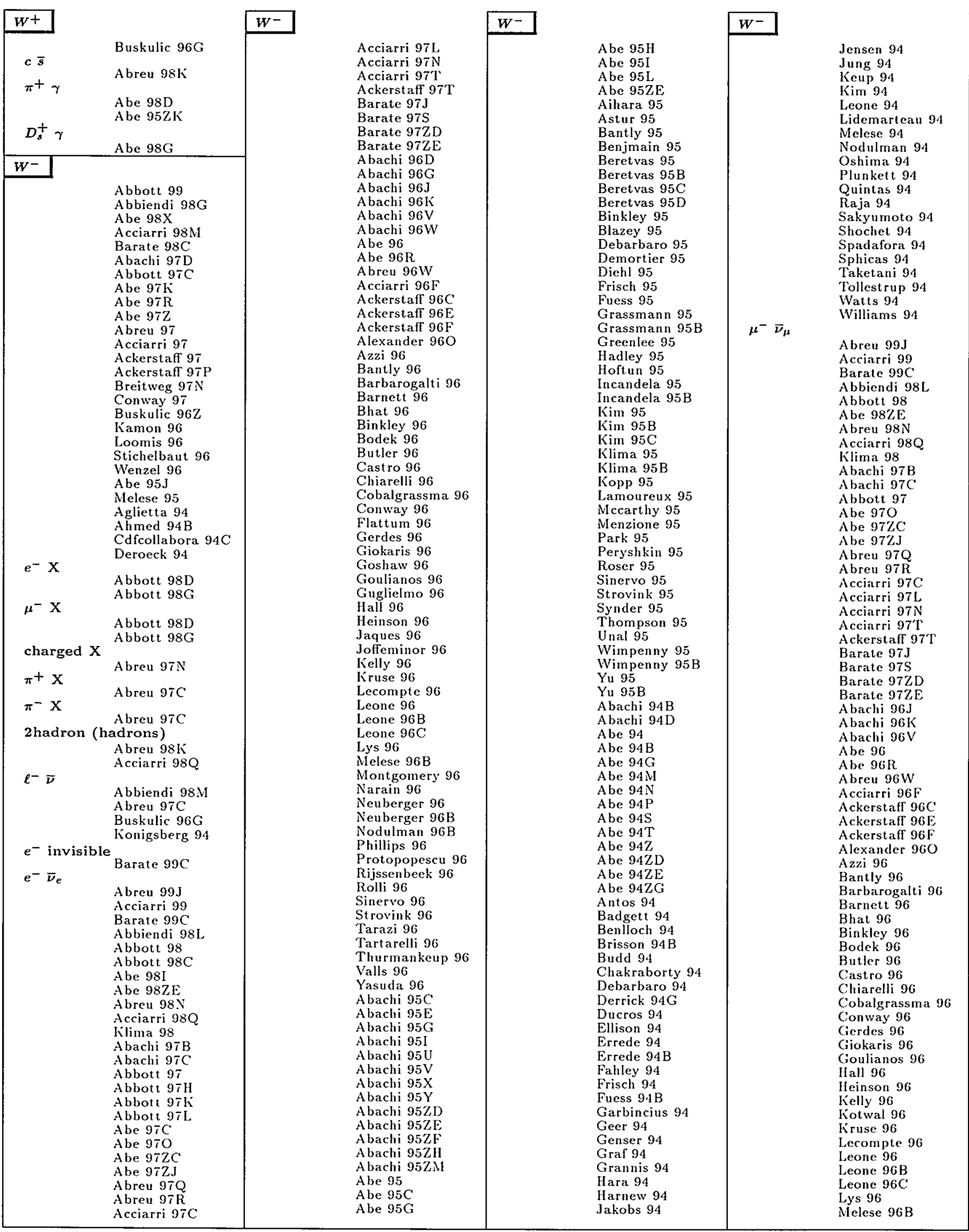




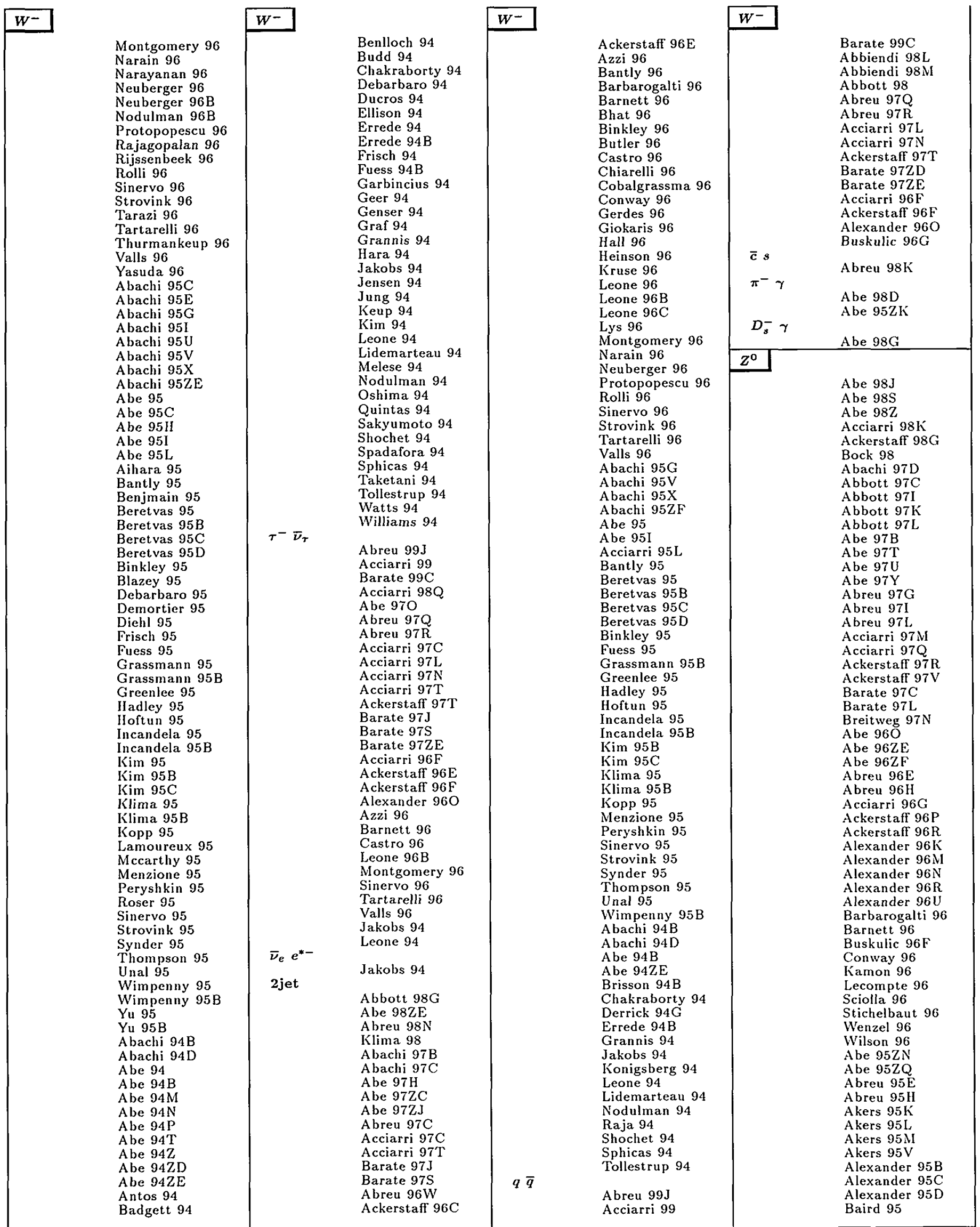




\begin{tabular}{|c|c|}
\hline \multirow[t]{2}{*}{$Z^{\mathbf{0}}$} & \\
\hline & $\begin{array}{l}\text { Hoftun } 95 \\
\text { Musolino } 95 \\
\text { Abe } 94 \mathrm{U} \\
\text { Abe } 94 \mathrm{~V} \\
\text { Abe } 94 \mathrm{WV} \\
\text { Acciarri } 94 \mathrm{E} \\
\text { Ahmed } 94 \mathrm{~B} \\
\text { Deroeck } 94 \\
\text { Fero } 94 \\
\text { Nodulman } 94 \\
\text { Shochet } 94\end{array}$ \\
\hline$e^{x} \times$ & Behnke 95 \\
\hline$e^{-x}$ & Carr 94 \\
\hline$e^{+} \mathrm{X}$ & Carr 94 \\
\hline$\mu^{ \pm} \mathrm{X}$ & \\
\hline$\mu^{-} \mathrm{X}$ & Behnke 95 \\
\hline$\mu^{+} \mathrm{X}$ & Carr 94 \\
\hline$b \times$ & Carr 94 \\
\hline $\bar{b} \mathbf{X}$ & $\begin{array}{l}\text { Goy } 96 \\
\text { Buskulic } 95 \mathrm{~V}\end{array}$ \\
\hline$\pi^{ \pm} x$ & $\begin{array}{l}\text { Goy } 96 \\
\text { Buskulic } 95 \mathrm{~V}\end{array}$ \\
\hline $\begin{array}{ll}\pi^{2} & \mathrm{X} \\
\pi^{0} \mathrm{X}\end{array}$ & Barate $97 \mathrm{ZC}$ \\
\hline$\eta \mathrm{X}$ & $\begin{array}{l}\text { Acciarri } 96 \mathrm{~N} \\
\text { Malinin } 96\end{array}$ \\
\hline$\rho^{0} \mathrm{X}$ & $\begin{array}{l}\text { Acciarri } 96 \mathrm{~N} \\
\text { Malinin } 96\end{array}$ \\
\hline & $\begin{array}{l}\text { Buskulic } 96 \mathrm{X} \\
\text { Chapkin } 96\end{array}$ \\
\hline $\mathrm{Y}$ & $\begin{array}{l}\text { Buskulic } 96 \mathrm{X} \\
\text { Malinin } 96\end{array}$ \\
\hline$\eta^{\prime} \times$ & Malinin 96 \\
\hline$f_{0}(980) \times$ & Chapkin 96 \\
\hline $\begin{array}{l}f_{2}(1270) X \\
K^{ \pm} \mathrm{X}\end{array}$ & Chapkin 96 \\
\hline$K_{S} \mathrm{X}$ & $\begin{array}{l}\text { Barate } 97 \mathrm{ZC} \\
\text { Abreu } 95 \mathrm{D}\end{array}$ \\
\hline & $\begin{array}{l}\text { Malinin } 96 \\
\text { Baird } 94\end{array}$ \\
\hline $\begin{array}{l}K^{*}(892)^{+} X \\
K^{*}(892)^{\circ} \mathrm{X}\end{array}$ & Chapkin 96 \\
\hline & $\begin{array}{l}\text { Abreu } 96 \mathrm{~J} \\
\text { Buskulic } 96 \mathrm{X} \\
\text { Akers } 95 \mathrm{G}\end{array}$ \\
\hline $\bar{K}^{*}(892)^{0} X$ & $\begin{array}{l}\text { Abreu 96J } \\
\text { Buskulic } 96 \mathrm{X} \\
\text { Akers } 956 \mathrm{i}\end{array}$ \\
\hline$K^{*}(892)^{-} \mathrm{X}$ & Chapkin 96 \\
\hline$K_{2}^{*}(1430)^{0}$ & $x^{\text {Chapkin } 96}$ \\
\hline $\bar{K}_{2}^{*}(1430)^{0}$ & $\begin{array}{l}\text { Abreu } 96 \mathrm{~J} \\
\text { A kers } 95 \mathrm{G} \\
\mathrm{X}\end{array}$ \\
\hline$\phi(1020) \mathrm{X}$ & $\begin{array}{l}\text { Abreu } 96 \mathrm{~J} \\
\text { Akers } 95(;\end{array}$ \\
\hline & $\begin{array}{l}\text { Abreu } 96 \mathrm{~J} \\
\text { Buskulic } 96 \mathrm{X} \\
\text { Akers } 95 \mathrm{G}\end{array}$ \\
\hline & $\begin{array}{l}\text { Behnke } 95 \\
\text { Buskulic 93P }\end{array}$ \\
\hline & $\begin{array}{l}\text { Alexander } 96 \mathrm{Q} \\
\text { Behnke } 95 \\
\text { Colas } 94\end{array}$ \\
\hline
\end{tabular}

$Z^{0}$

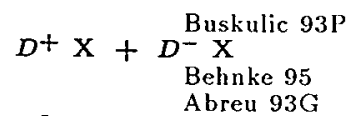

$D^{\circ} \mathrm{X}$

Alexander 96Q

Behnke 95

Colas 94

$D^{0} \mathrm{X}+\bar{D}^{\mathrm{O}}$ Buskulic 93P

$\mathrm{X}$

Behnke 95

$\bar{D}^{\circ} \mathrm{X}$

Abreu 93G

Alexander $96 \mathrm{Q}$

Behnke 95

Buskulic 93P

$D^{-} \mathrm{X}$

Alexander $96 \mathrm{Q}$

Behnke 95

$D^{*}(2010)^{ \pm} \mathrm{X}^{\text {Buskulic 93P }}$

Behnke 95

A kers $94 \mathrm{~S}$

$D^{*}(2010)+\mathrm{X}$

Buskulic 93P

Kobel 96

Behnke 95

Buskulic 95J

Colas 94

Buskulic 93P

$D^{*}(2010)+\mathrm{X}+$

$D^{*}(2010)^{-} \mathrm{X}$

Behnke 95

$D^{*}(2010)-\mathrm{X}$

Abreu $93 \mathrm{G}$

Kobel 96

Behnke 95

Buskulic 95J

$D^{*}(\mathbf{2 0 0 7})^{\circ} \mathrm{X}$

Buskulic 93P

Behnke 95

$\bar{D}^{*}(2007)^{\circ} \mathrm{X}^{\text {Buskulic 93P }}$

Behnke 95
Buskulic 93P

$D_{s}^{+} \mathrm{X}$

Alexander $96 \mathrm{Q}$

$D_{s}^{-} \mathrm{X}$

$D_{s}^{*+} \mathrm{X}$

Alexander $96 \mathrm{Q}$

$D_{3}^{*-\mathrm{X}}$

Acciarri $96 \mathrm{~L}$

$D_{1}(2420)^{\circ} \mathrm{X}^{A}$ Acciarri $96 \mathrm{~L}$

Furtjes 96

$\bar{D}_{1}(2420)^{\circ} \mathrm{X}$

Colas 94

$D_{2}^{*}(2460)^{0} \mathrm{X}^{\text {Furtjes } 96}$

Furtjes 96

$\bar{D}_{2}^{*}(2460)^{0}$ Colas 94

$J / \psi(1 S) \times$ Furtjes 96

Acciarri $982 \mathrm{~B}$

Acciarri $97 \mathrm{~F}$

Alexander $96 \mathrm{E}$

Keranen 96

Abreu 950

Alexander $95 \mathrm{E}$

Behnke 95

Abreu $94 \mathrm{M}$

$\chi_{c 1}(1 P) \mathrm{X}$

$\chi_{c 2}(1 P) \mathrm{X}+\chi_{c 1}(1 P) \mathrm{x}$

Abreu $94 \mathrm{M}$

$\chi_{c 2}(1 P) \mathrm{X}$

$\psi(2 S) \mathrm{X}$

Acciarri $97 \mathrm{~F}$

Kieranen 96 Abreu 950
$Z^{0}$

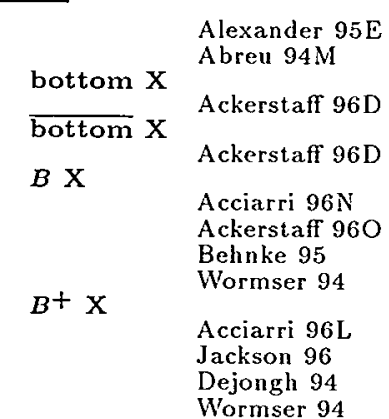

$B^{\circ} \mathrm{X}+B_{s} \mathrm{X}$

$B^{\mathrm{o}} \mathrm{X}$

A breu 94J

$B^{\circ} \mathrm{X}$ Abreu $96 \mathrm{~V}$

Acciarri 961

Acciarri $96 \mathrm{~J}$

Ackerstaff $96 \mathrm{~J}$

Jackson 96

Acciarri 95P

Dejongh 94

Wormser 94

$\bar{B} \times$

Acciarri $96 \mathrm{~N}$

Ackerstaff 960

Behnke 95

$\bar{B}^{\circ} \mathrm{X}+\bar{B}_{s} \mathrm{X}$

Vormser 94

$\bar{B} \circ \mathrm{X}$ Abreu $94 \mathrm{~J}$

Abreu $96 \mathrm{~V}$

Acciarri 96I

Acciarri 96J

Ackerstaff $96 \mathrm{~J}$

Jackson 96

Dejongh 94

Wormser 94

$B^{-} \mathrm{X}$

Acciarri 96L

Jackson 96

Dejongh 94

Wormser 94

$B^{*} \mathrm{X}$

Furtjes 96

$B^{*+} \mathrm{X}$

Furtjes 96

$B^{* 0} \mathrm{X}$

Furtjes 96

$\bar{B}^{*} \mathrm{X}$

$\bar{B}^{* 0} \mathrm{X}$

Furtjes 96

$B^{*-} \mathrm{X}$

Furtjes 96

Furtjes 96

$B^{*}$ (unspec) X

Podobrin 95

$\bar{B}^{*}$ (unspec) X

$B_{3} \mathrm{X}$

Pod

Podobrin 95

Acciarri 96I

Acciarri $96 \mathrm{~J}$

Acciarri 95P

Podobrin 95

Abreu 94I

IVormser 94

$\bar{B}_{s} \mathrm{X}$

Acciarri 96I

Acciarri 96.

Podobrin 95

Abreu 941

Wormser 94

$B_{c}^{+} \mathrm{X}$

Ackerstaff $97 \mathrm{ZB}$

$B_{c}^{-} \mathrm{X}$

Alexander $95 \mathrm{E}$

Ackerstaff 97ZB

$\Upsilon(1 S) \mathrm{X}$
$Z^{\mathrm{o}}$

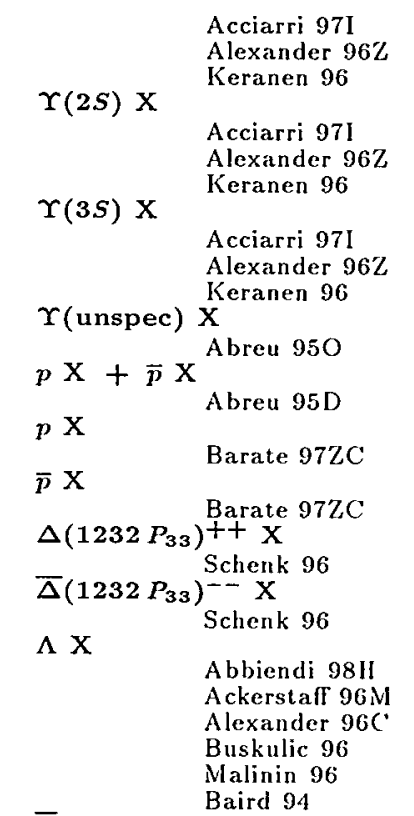

$\bar{\Lambda} \mathrm{X}$

Abbiendi $98 \mathrm{H}$ Ackerstaff $96 \mathrm{M}$ Alexander 96C Buskulic 96 Malinin 96

$\Lambda\left(1520 D_{03}\right) \mathrm{X}$

Baird 94

$\bar{\Lambda}\left(1520 D_{03}\right) \stackrel{\text { X }}{A}$

Alexander $96 \mathrm{C}$

$\Sigma+\mathrm{x}$

Alexander $96 \mathrm{C}^{\circ}$

$\Sigma^{0} \mathrm{X}$

Alexander 96D

$\Sigma^{-} \mathrm{X}$

Alexander 96D

$\bar{\Sigma}^{+} \mathrm{X}$

$\bar{\Sigma}^{0} \mathrm{X}$

Alexander 96D

$\bar{\Sigma}^{-} \mathrm{X}$

Alexander 96D

$\Sigma\left(1385 P_{13}\right)^{+} \mathrm{Ale}$

Alexander 96D

Alexander $96 \mathrm{C}$

Allport 96

Schenk 96

$\Sigma\left(1385 P_{13}\right)^{-} \mathrm{X}$

Alexander $96 \mathrm{C}$

Allport 96

Schenk 96

$\bar{\Sigma}\left(1385 P_{13}\right)^{+} \mathrm{X}$

Alexander $96 \mathrm{C}$

Allport 96

Schenk 96

$\bar{\Sigma}\left(1385 P_{13}\right)^{-X}$

Alexander $96 \mathrm{C}$

Allport 96

Schenk 96

$\Xi^{-} \mathrm{x}$

Alexander $96 \mathrm{C}$ Allport 96

$\Xi^{+} \mathrm{x}$

Alexander $96 \mathrm{C}^{\circ}$ Allport 96

$\Xi\left(1530 P_{13}\right)^{\mathrm{o}} \mathrm{X}$

Alexander $96 \mathrm{C}$

Allport 96

$\Xi\left(1530 P_{13}\right)^{\circ} \mathrm{X}$ chenk 96

Alexander $96 \mathrm{C}$

Allport 96

Schenk 96 


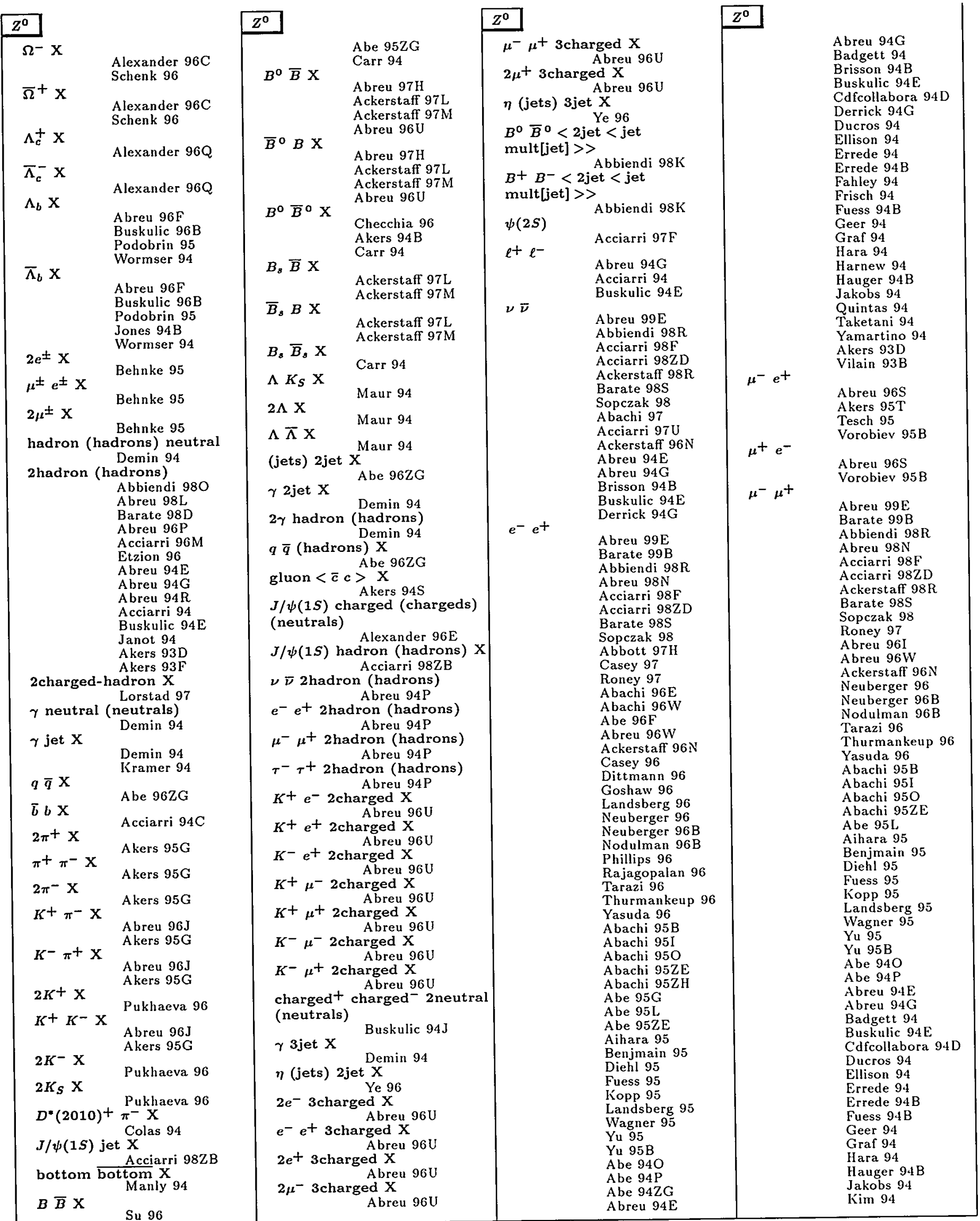




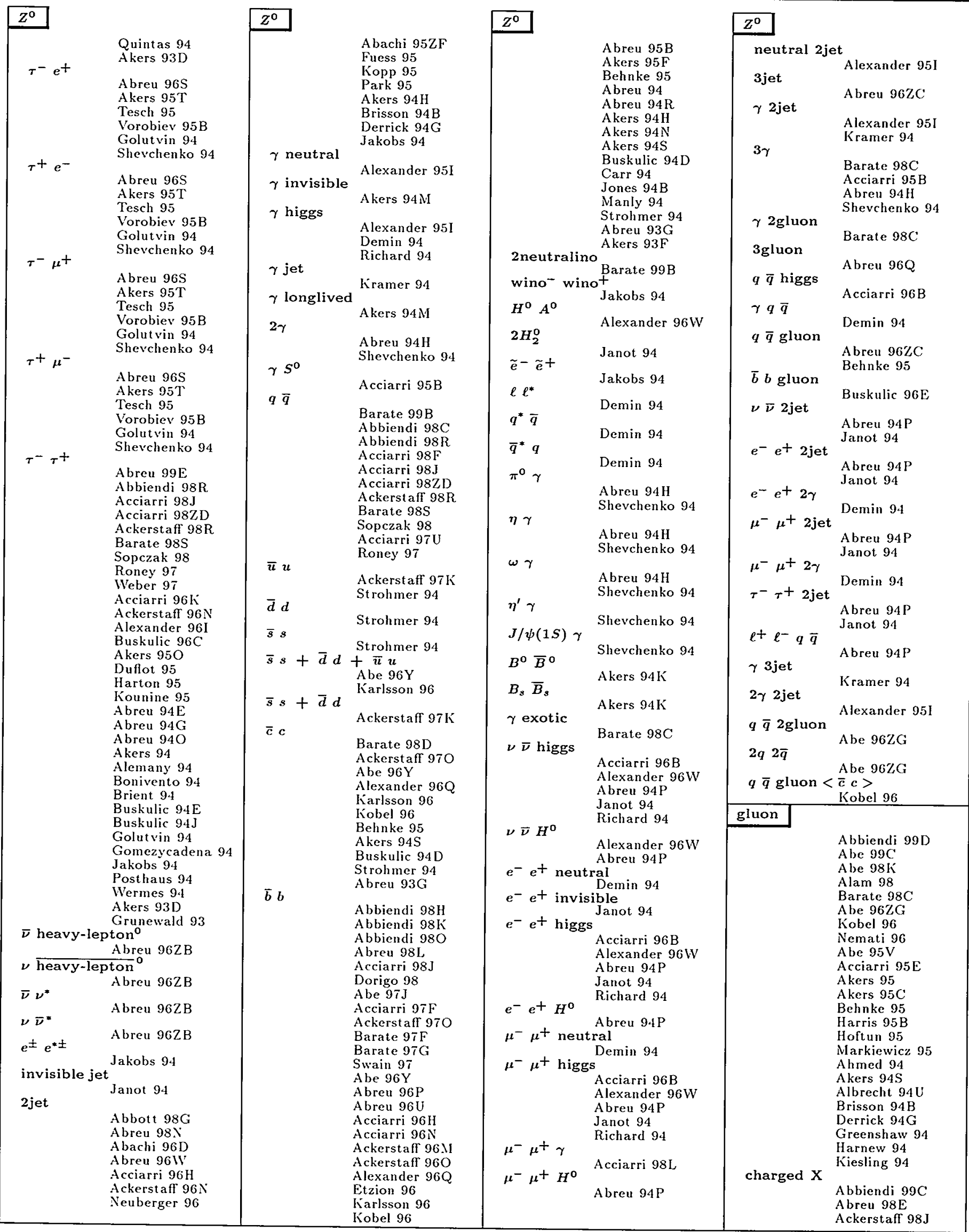




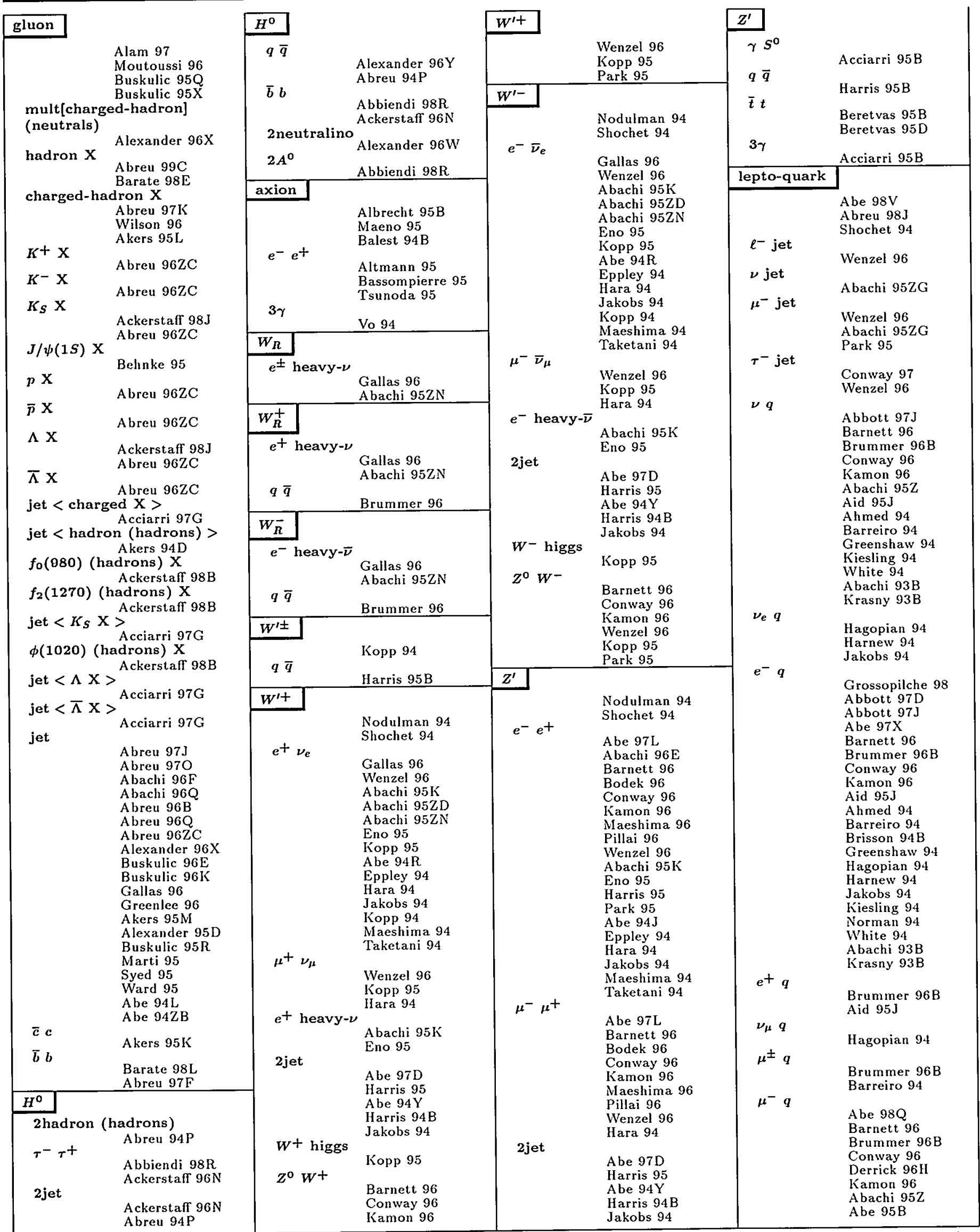

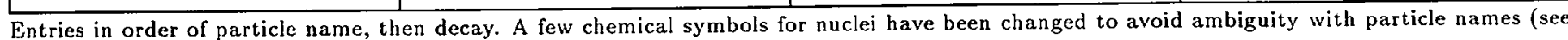
the Particle Vocabulary). See the legend on page 323. 


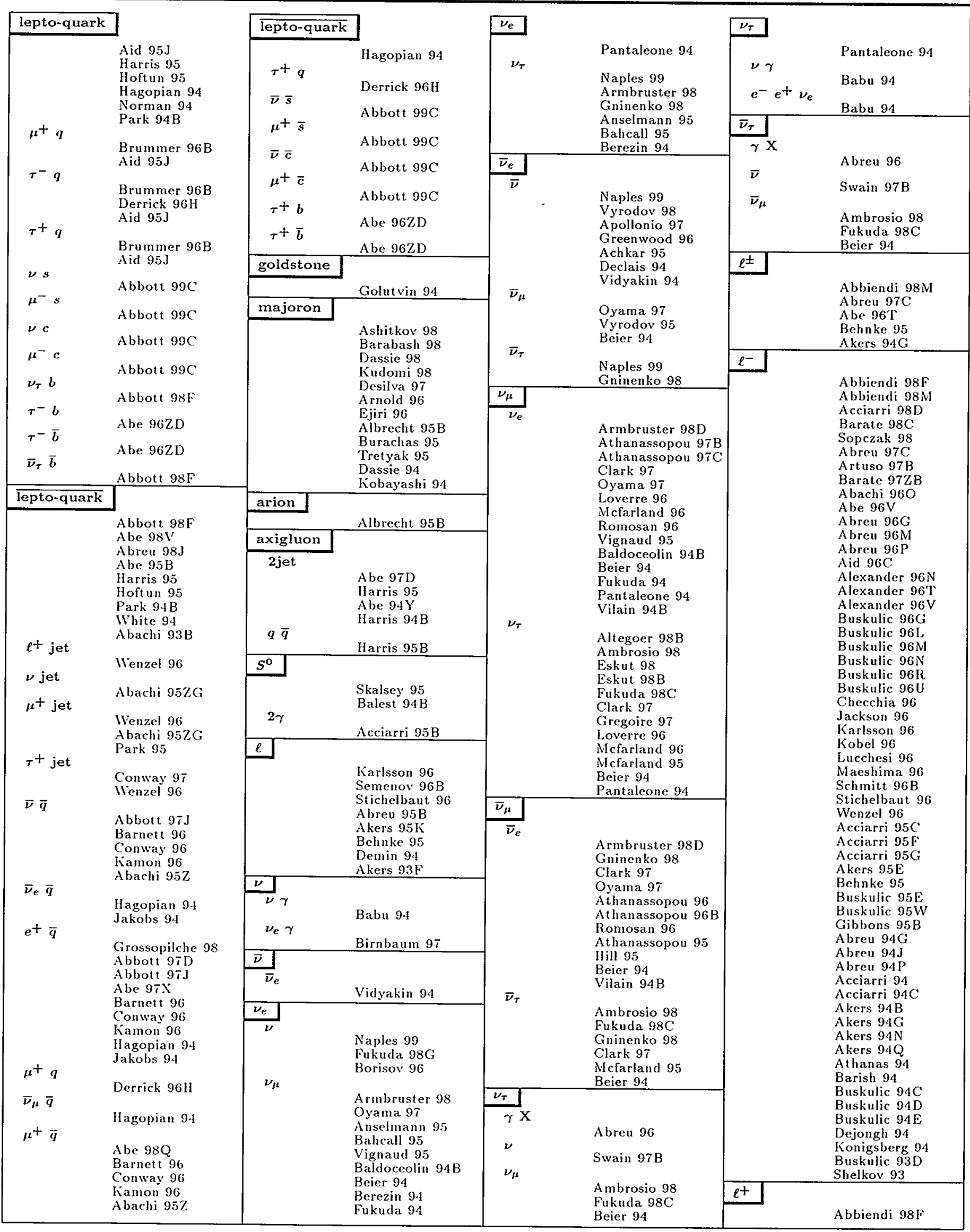




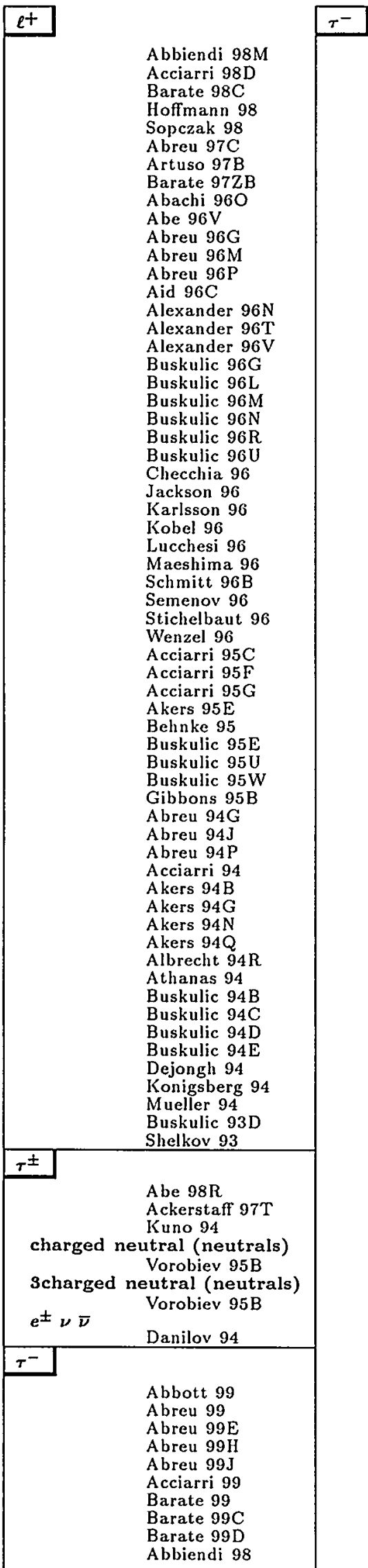

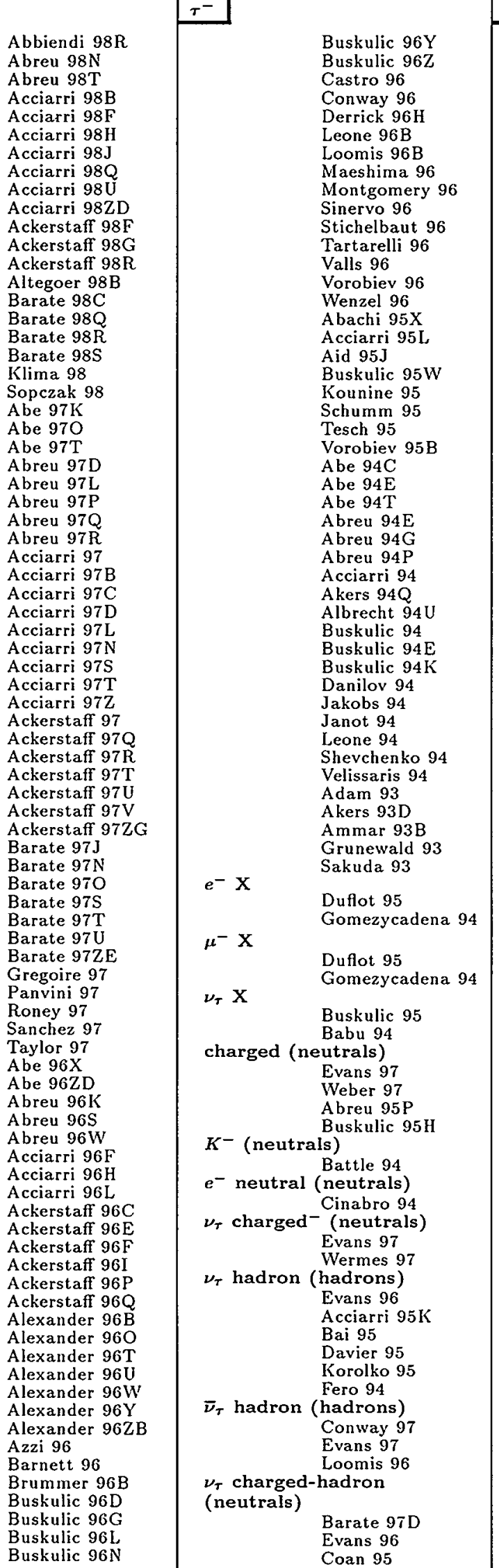

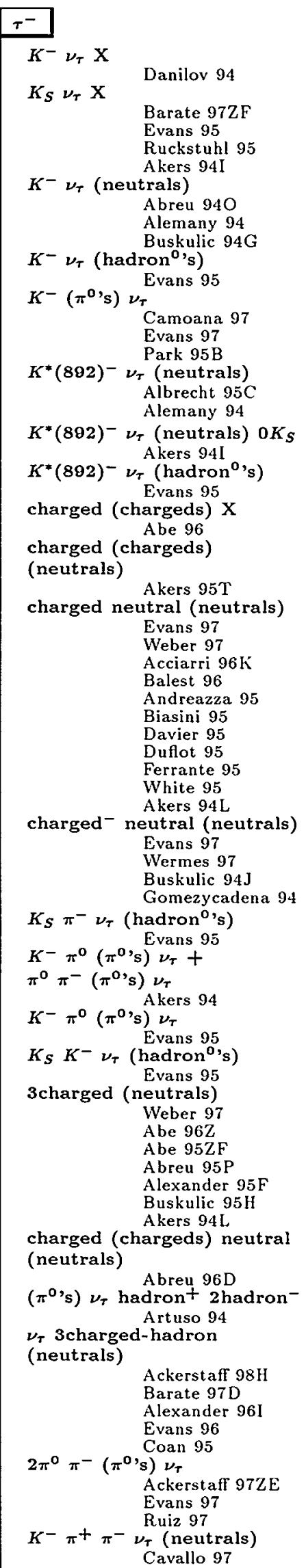

Entries in order of particle name, then decay. A few chemical symbols for nuclei have been changed to avoid ambiguity with particle names (see the Particle Vocabulary). See the legend on page 323. 


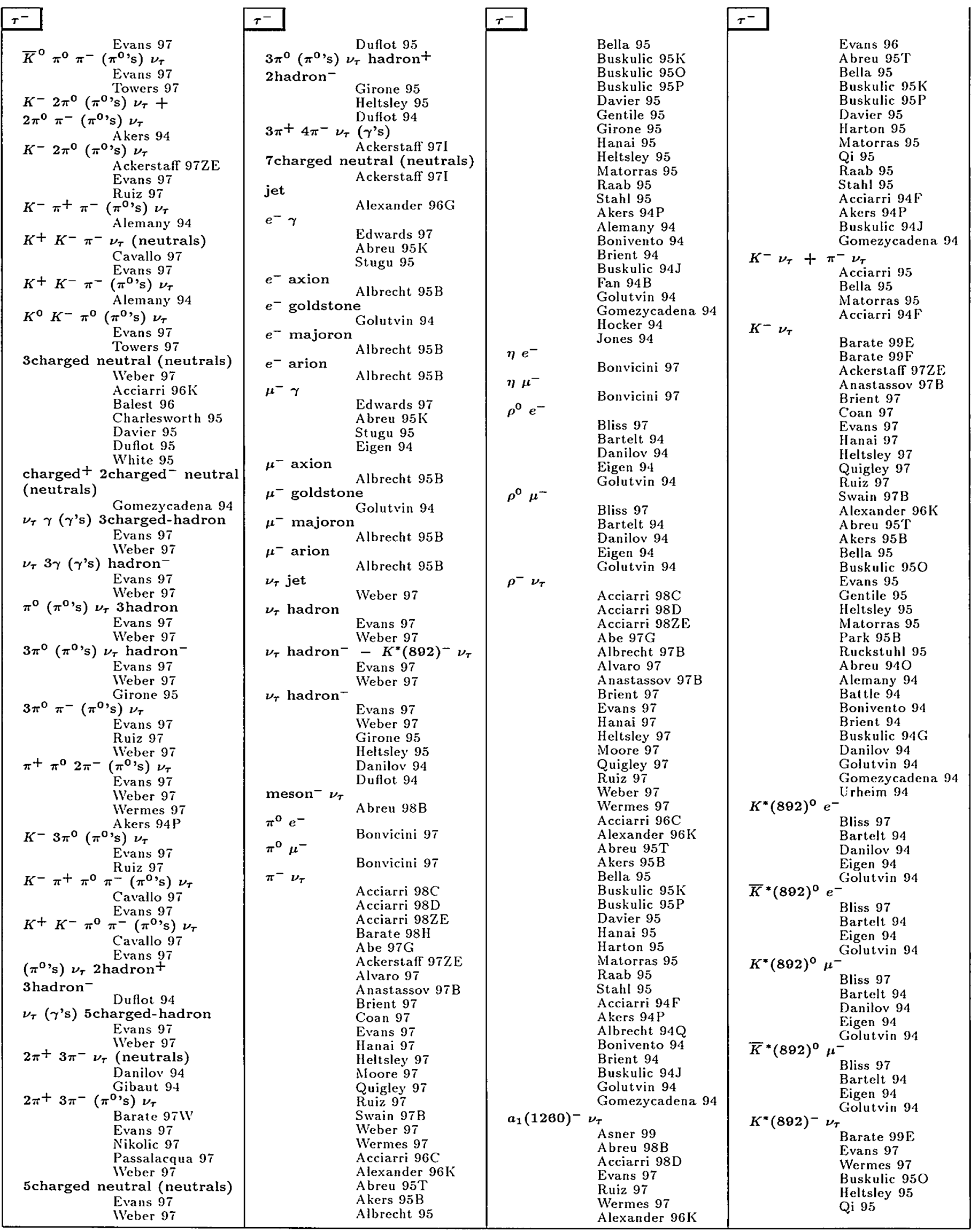


$K_{1}(1270)^{-} \nu_{r}$

$K_{1}(1400)^{-} \nu_{\tau}$

Barate $99 \mathrm{E}$

auer 93B

$K^{*}(1410)^{-}{ }_{\nu_{r}}$

$\phi(1020)$ Barate $99 \mathrm{E}$

$\phi(\mathbf{1 0 2 0}) \mu^{-} \begin{aligned} & \text { Bliss } 97 \\ & \text { Bliss } 97\end{aligned}$

\section{$\bar{p} \gamma$ \\ Bartelt 98B}

$\bar{p} \pi^{\circ}$

Bartelt 98B

$\bar{p} \eta$

$\ell^{-} \nu_{\tau} \bar{\nu}$

$2 e^{-} e^{+}$

Bartelt 98B

Acciarri $94 \mathrm{C}$

Bliss 97

Bartelt 94

Danilov 94

Eigen 94

Golutvin 94

$e^{-} \nu_{\tau} \bar{\nu}_{e}$

Abbiendi 98I

Acciarri 98D

Acciarri 98ZE

Ackerstaff $98 \mathrm{~S}$

A be $97 \mathrm{G}$

Albrecht 97B

Alexander 97

Anastassov $97 \mathrm{~B}$

Brient 97

Chadha 97B

Edsall 97

Evans 97

Hanai 97

Heltsley 97

Kounine 97

Lohmann 97

Moore 97

Quigley 97

Sobie 97

Swain 97B

Weber 97

Wermes 97

Acciarri 96C

Ackerstaff $96 \mathrm{~N}$

Alexander $96 \mathrm{~K}$

Evans 96

Waldi 96

Abreu 95F

Abreu 95T

Akers 95B

A kers 95D

Albrecht 95

Alcaraz 95

Alexander 95G

Artuso 95

Bai 95

Bella 95

Buskulic 95K

Buskulic 95N

Buskulic 95P

Coan 95

Dam 95

Davier 95

Duflot 95

Hanai 95

Heltsley 95

Matorras 95

Raab 95

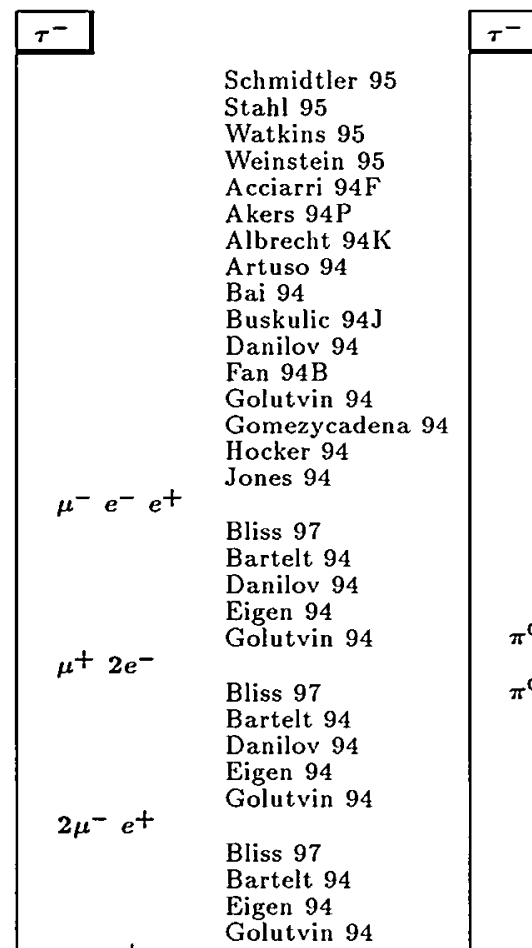

$\mu+2 e^{-}$

Bliss 97

Bartelt 94

Danilov 94

Eigen 94

Golutvin 94

$2 \mu^{-} e^{+}$

Bliss 97

Bartelt 94

Eigen 94

Golutvin 94

$\mu^{-} \mu^{+} e^{-}$

Bliss 97

Bartelt 94

Danilov 94

Eigen 94

Golutvin 94

$2 \mu^{-} \mu^{+}$

Bliss 97

Bartelt 94

Danilov 94

Eigen 94

Golutvin 94

$\mu^{-} \nu_{\tau} \bar{\nu}_{\mu}$

Acciarri 98D

Acciarri 98ZE

Ackerstaff $98 \mathrm{~S}$

Abe $97 \mathrm{G}$

Alexander 97

Anastassov 97B

Brient 97

Chadha 97B

Edsall 97

Evans 97

Hanai 97

Heltsley 97

Kounine 97

Lohmann 97

Moore 97

Quigley 97

Sobie 97

Swain 97B

Weber 97

Wermes 97

Acciarri 96C

Ackerstaff $96 \mathrm{~N}$

Alexander $96 \mathrm{~K}$

Waldi 96

Abreu 95F

Abreu 95T

A kers 95B

A kers 95D

Albrecht 95

Alcaraz 95

Artuso 95

Bai 95

Bella 95

Buskulic 95K

Buskulic 95N

Buskulic 95P

Dam 95

Davier 95 $\pi^{0} \nu_{\tau}$ charged

Duflot 95

Hanai 95

Heltsley 95

Korolko 95

Matorras 95

Raab 95

Schmidtler 95

Stahl 95

Watkins 95

Weinstein 95

Acciarri 94F

Akers $94 \mathrm{P}$

Albrecht $94 \mathrm{~K}$

Artuso 94

Bai 94

Buskulic 94J

Danilov 94

Fan 94B

Golutvin 94

Gomezycadena 94 Jones 94

$\pi^{0} \nu_{\tau}$ hadron

Urheim 94

Evans 97

Weber 97

Girone 95

Heltsley 95

Weinstein 95

Artuso 94

Danilov 94

Duflot 94

Golutvin 94

Gomezycadena 94

$2 \pi^{0} e^{-}$

$\pi^{+} \pi^{-} e^{-}$

Bonvicini 97

Bliss 97

Bartelt 94

Danilor 94

Eigen 94

Golutvin 94

$2 \pi^{-} e^{+}$

Bliss 97

Bartelt 94

Danilov 94

Eigen 94

Golutvin 94

$2 \pi^{0} \mu^{-}$

Bonvicini 97

$\pi^{+} \pi^{-} \mu^{-}$

Bliss 97

Bartelt 94

Danilov 94

Eigen 94

Golutvin 94

$2 \pi^{-} \mu^{+}$

Bliss 97

Bartelt 94

Danilov 94

Eigen 94

Golutvin 94

$\pi^{0} \pi^{-} \nu_{\tau}$

Ackerstaff $98 \mathrm{~T}$

Barate $98 \mathrm{H}$

Ackerstaff $97 \mathrm{ZE}$

Alemany 97

Alexander 97

Barate $97 \mathrm{E}$

Chadha 97B

Evans 97

Ruiz 97

Urheim 97

Buskulic 950

Gentile 95

Girone 95

Heltsley 95

Weinstein 95

Albrecht 94Q

Danilov 94

Hocker 94

Urheim 94

$\tau^{-}$

$$
\begin{aligned}
& \pi^{0} \pi^{-} \nu_{\tau} \\
& K^{-}<\pi^{0} \pi^{-}>\nu_{\tau} \\
& \text { Evans } 97 \\
& \eta \pi^{0} \mathrm{e}^{-} \\
& \text {Weber } 97 \\
& \eta \pi^{0} \mu^{-} \\
& \eta \boldsymbol{\pi}^{-} \boldsymbol{\nu}_{\tau}
\end{aligned}
$$

$2 \eta e^{-}$

Bonvicini 97

$2 \eta \mu^{-}$

Bonvicini 97

$\omega \nu_{\tau}$ hadron

Heltsley 95

Danilov 94

$\omega \pi^{-} \nu_{\tau}$

Barate $98 \mathrm{H}$

Evans 97

Rouge 97

Buskulic 96C

Balest 95

Bourdon 95

$\eta^{\prime} \pi^{-} \nu_{\tau}$

$f_{1}(1285) \pi-\nu_{\tau}$

Bergfeld 97

Evans 97

Shelkov 97

$K^{0} \nu_{\tau}$ charged

$\bar{K}^{\circ} \nu_{\tau}$ had Urheim 94

$\pi^{0} \nu_{\tau}$ hadron ${ }^{-}$

$K_{S} \nu_{\tau}$ hadron-

Cavallo 97

Evans 97

Heltsley 95

Smith 94

$K^{+} \pi^{-} e^{-}$

Bliss 97

Bartelt 94

Danilov 94

Eigen 94

Golutvin 94

$K^{-} \pi^{+} e^{-}$

Bliss 97

Bartelt 94

Danilov 94

Eigen 94

Golutvin 94

$K^{-} \pi^{-} e^{+}$

Bliss 97

Bartelt 94

Danilov 94

Eigen 94

Golutvin 94

$K^{+} \pi^{-} \mu^{-}$

Bliss 97

Bartelt 94

Danilov 94

Eigen 94

Golutvin 94

$K^{-} \pi^{+} \mu^{-}$

Bliss 97

Bartelt 94 


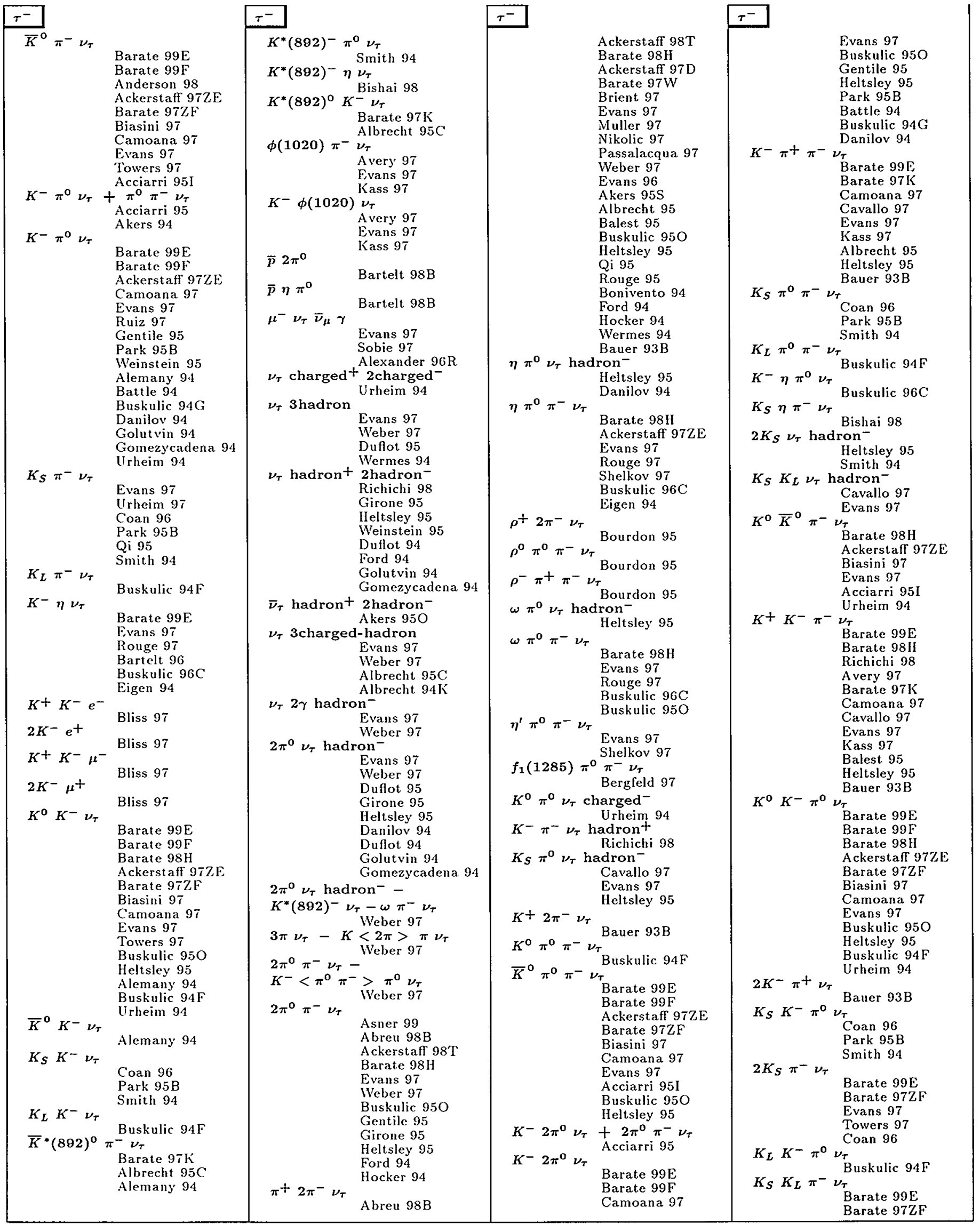




\begin{tabular}{|c|c|c|c|c|}
\hline$\tau^{-}$ & $\tau^{-}$ & $\tau^{-}$ & $\tau^{+}$ & \\
\hline 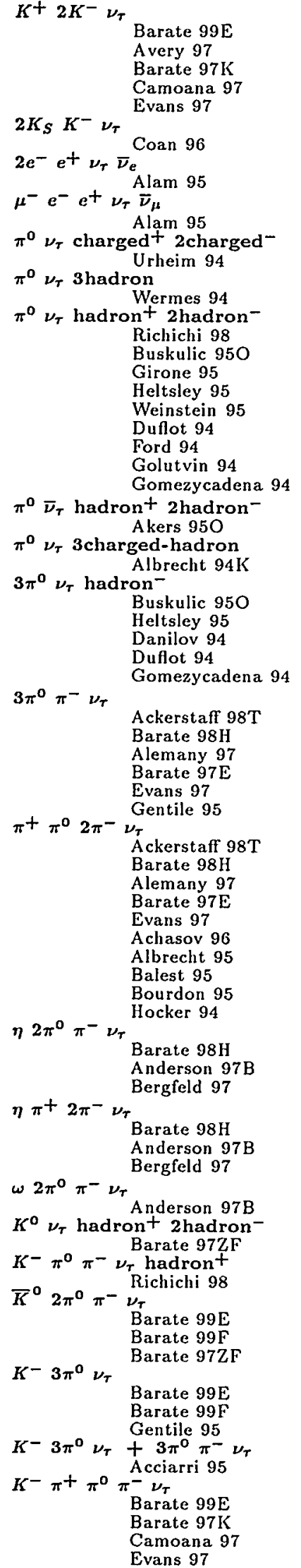 & 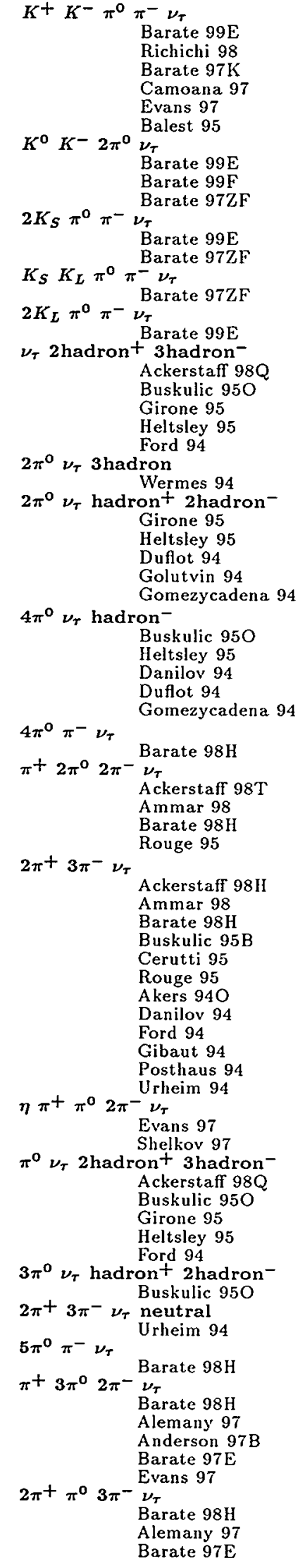 & 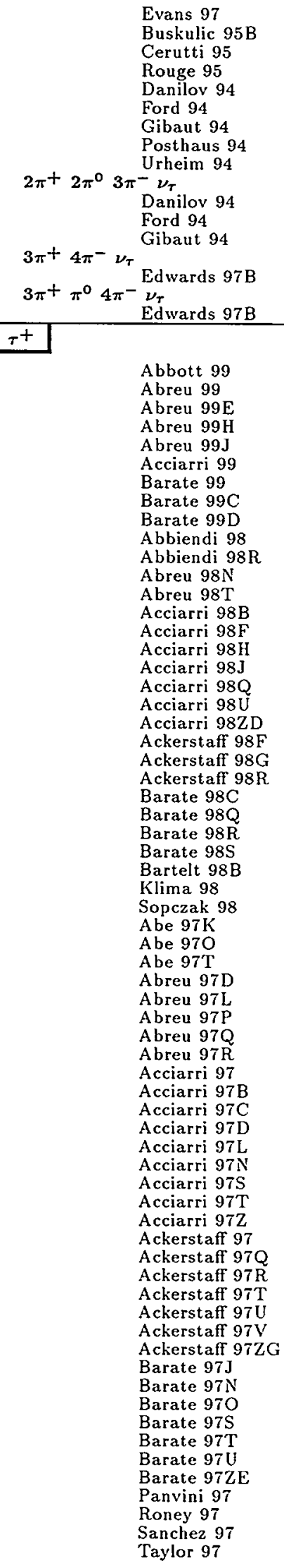 & & 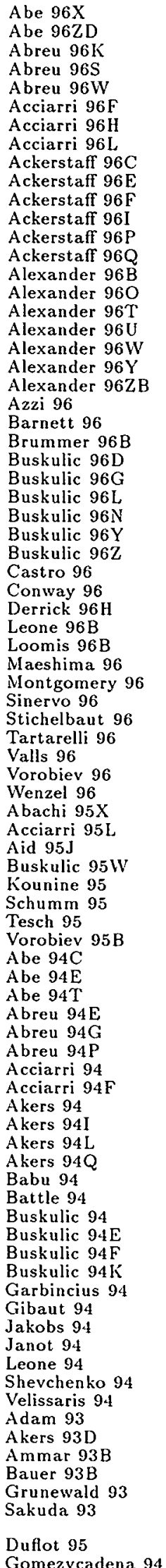 \\
\hline
\end{tabular}

Entries in order of particle name, then decay. A few chemical symbols for nuclei have been changed to avoid ambiguity with particle names (see the Particle Vocabulary). See the legend on page 323. 


\begin{tabular}{|c|c|c|c|c|}
\hline$\tau^{+}$ & $\tau^{+}$ & & $\tau^{+}$ & \\
\hline 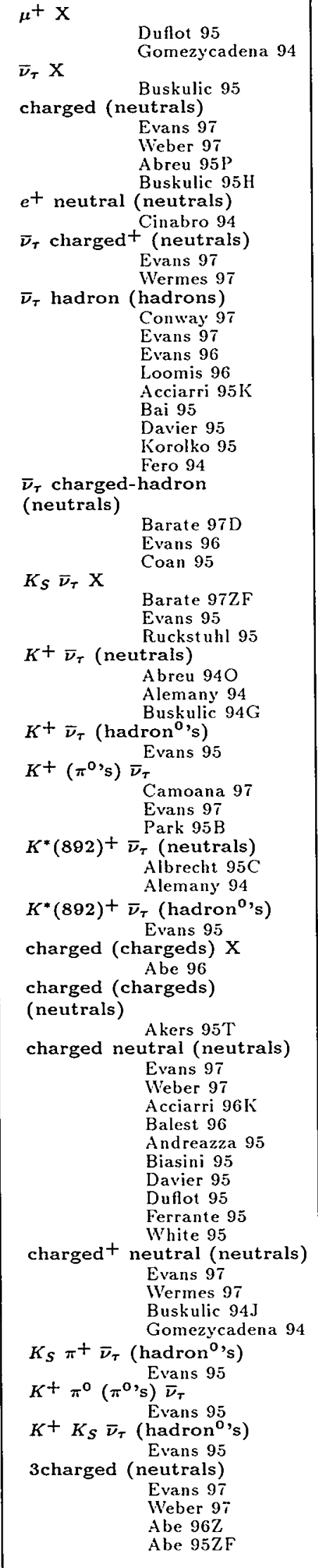 & 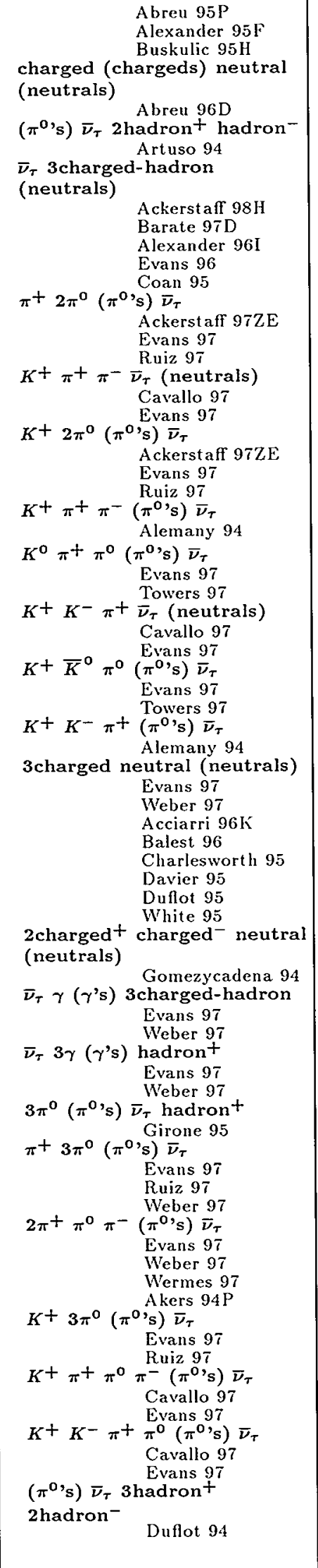 & 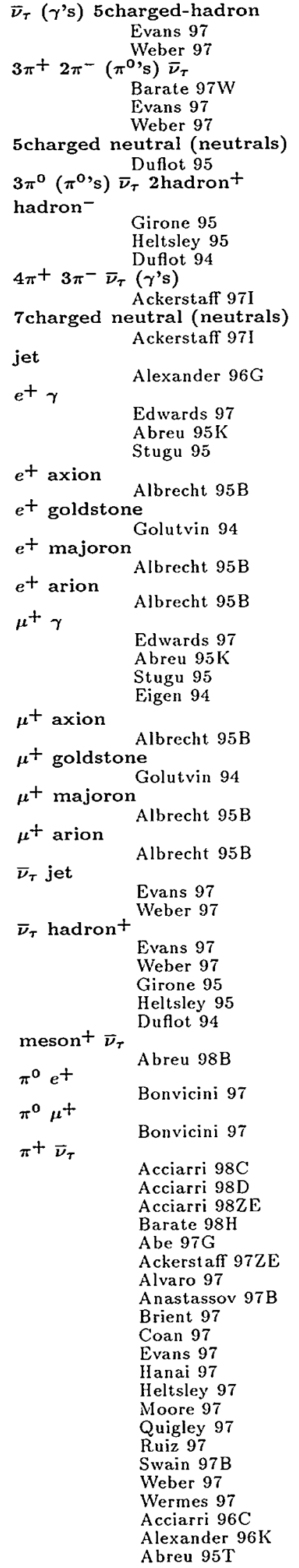 & $\begin{array}{l}\eta e^{+} \\
\eta \mu^{+} \\
\rho^{0} e^{+}\end{array}$ & 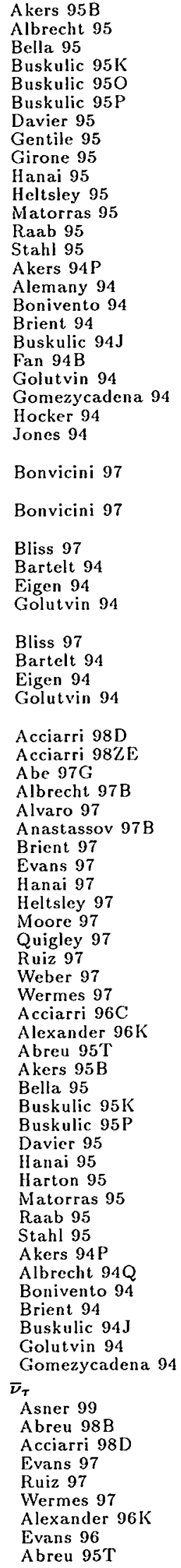 \\
\hline
\end{tabular}




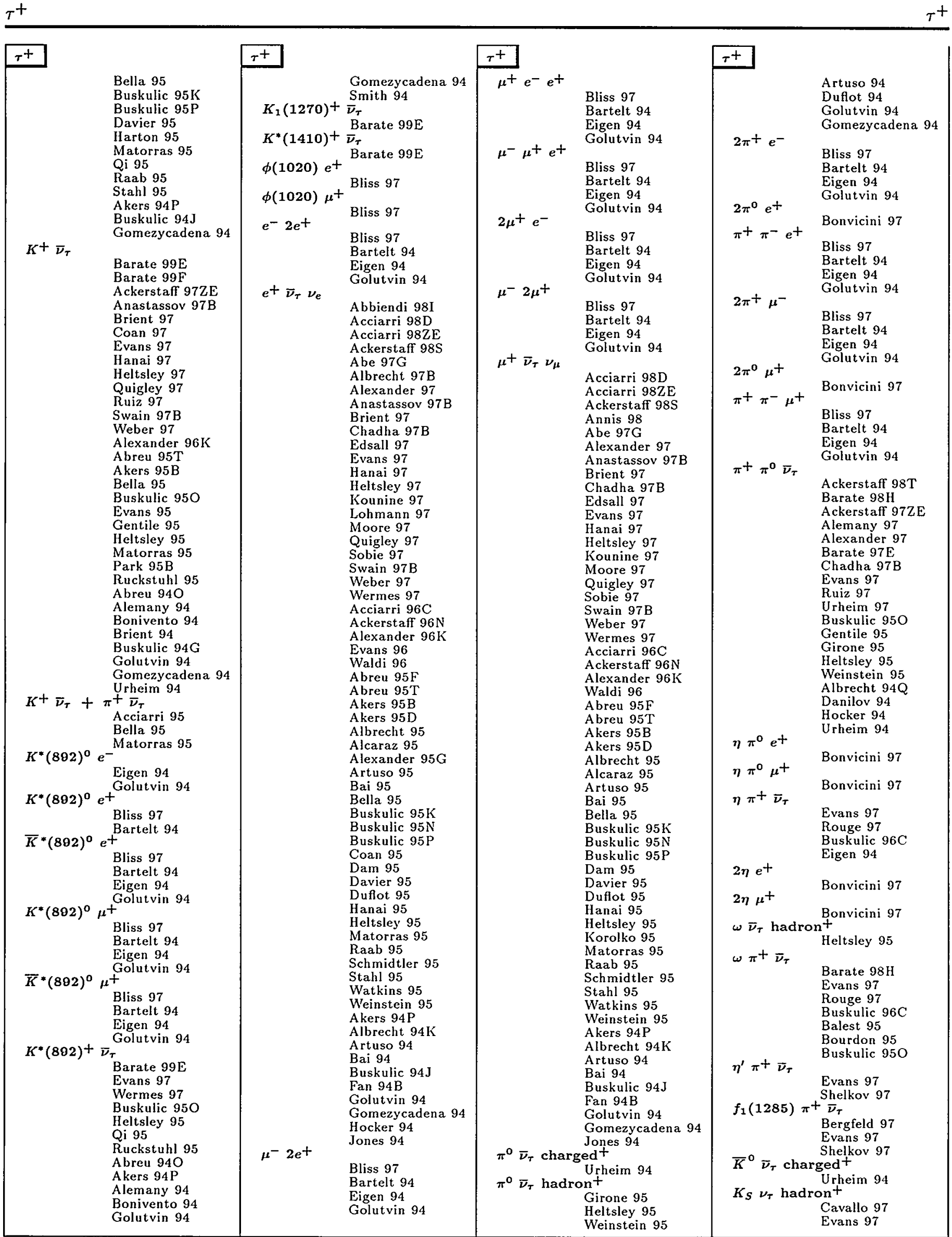




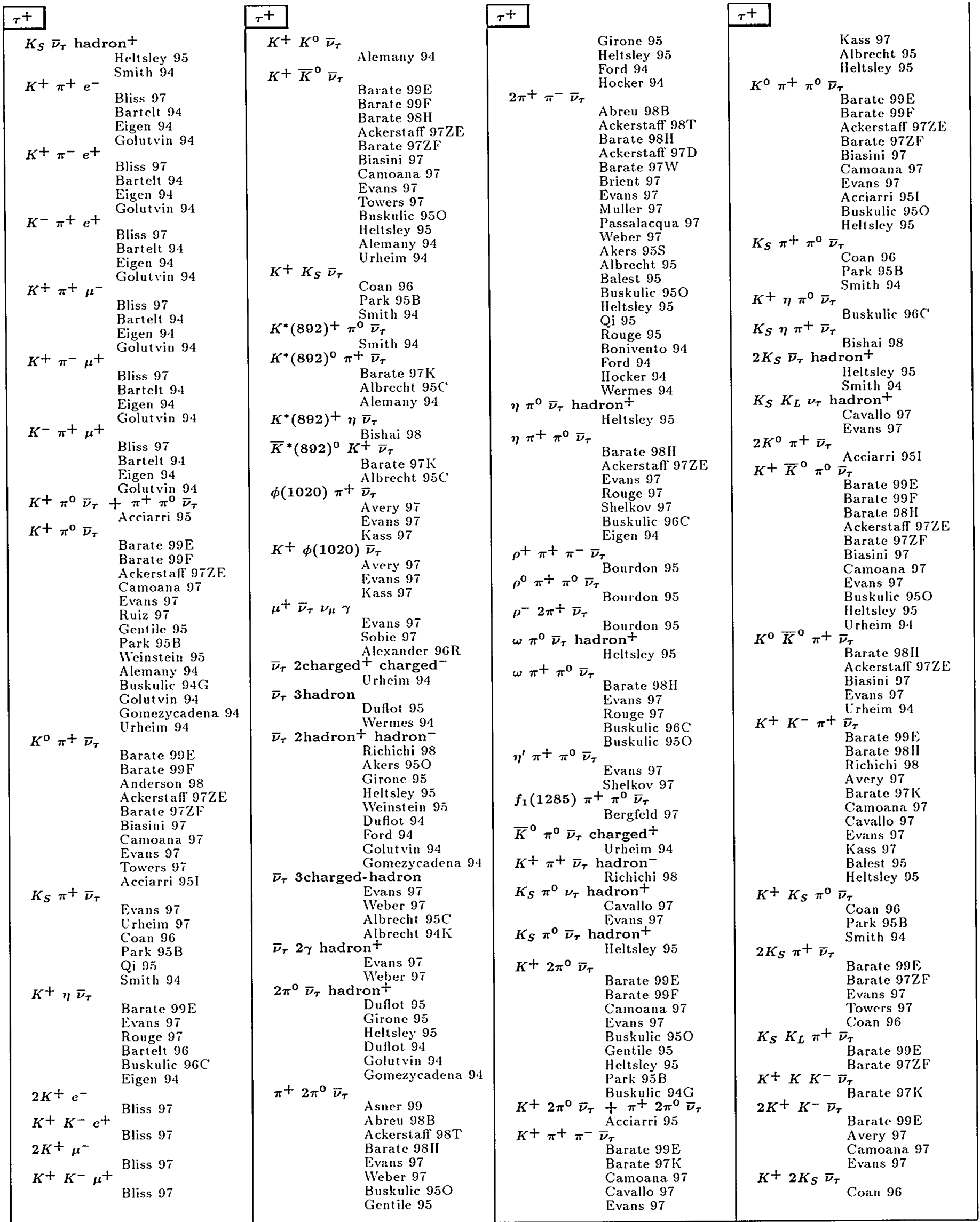




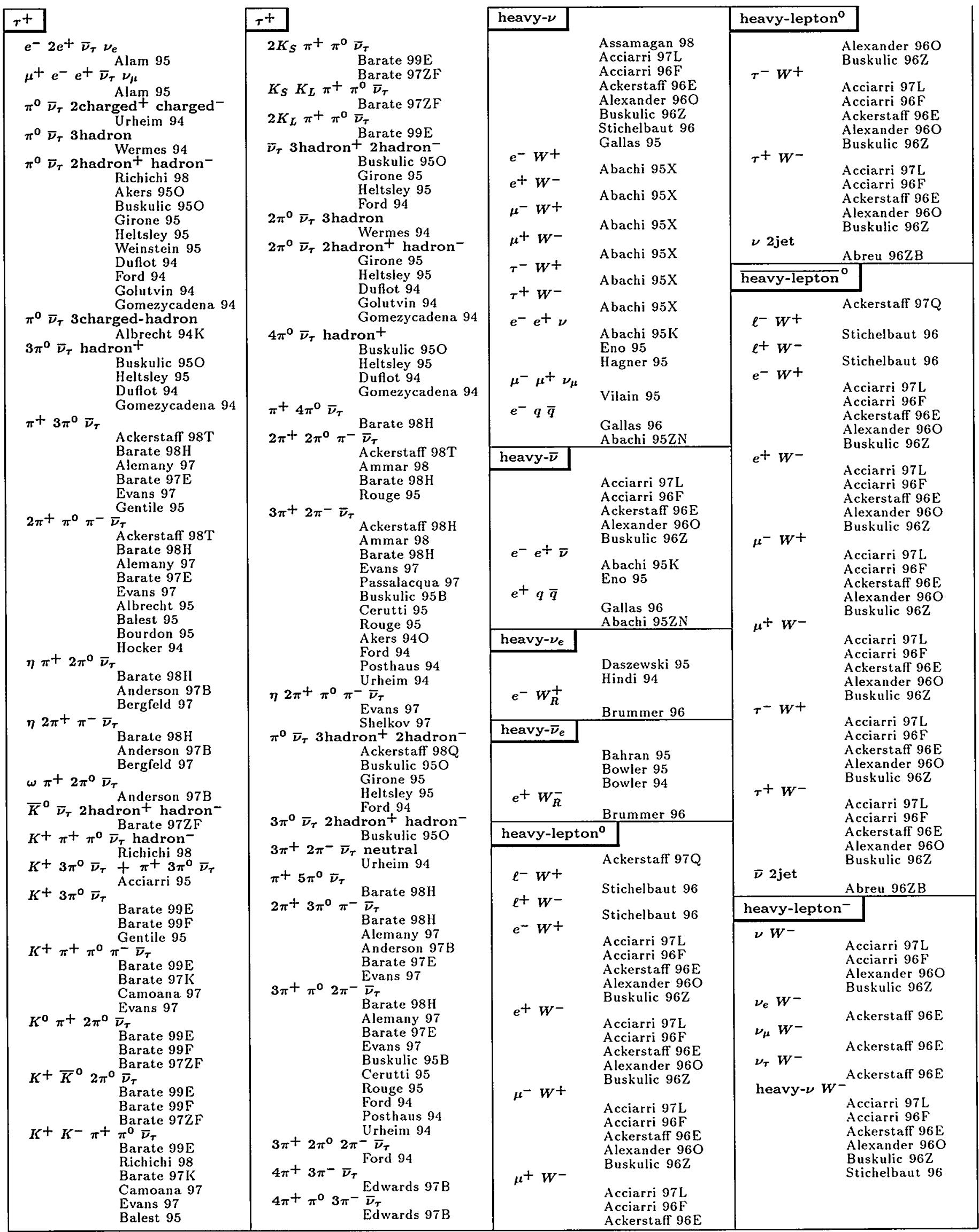

Entries in order of particle name, then decay. A few chemical symbols for nuclei have been changed to avoid ambiguity with particle names (see the Particle Vocabulary). See the legend on page 323. 


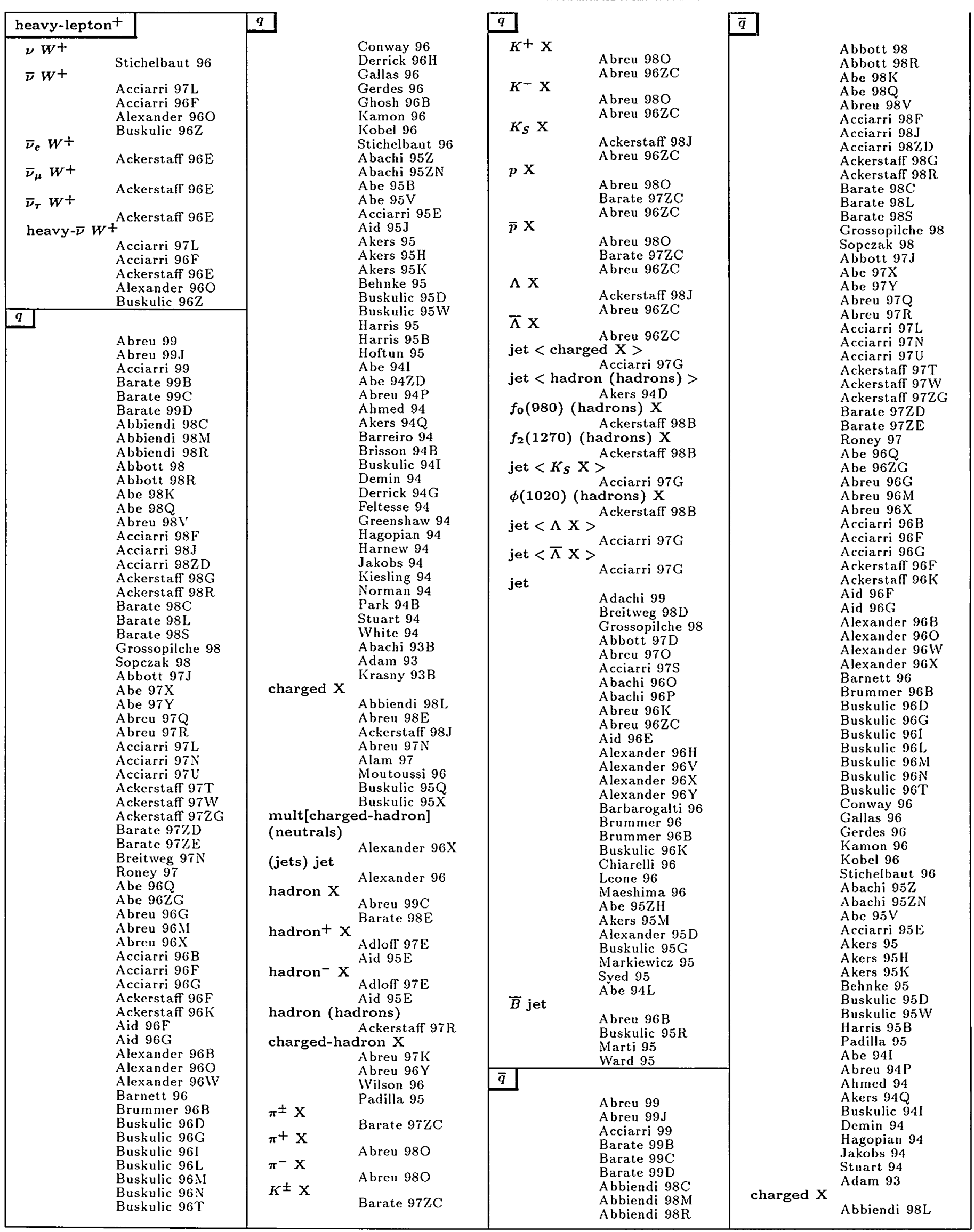




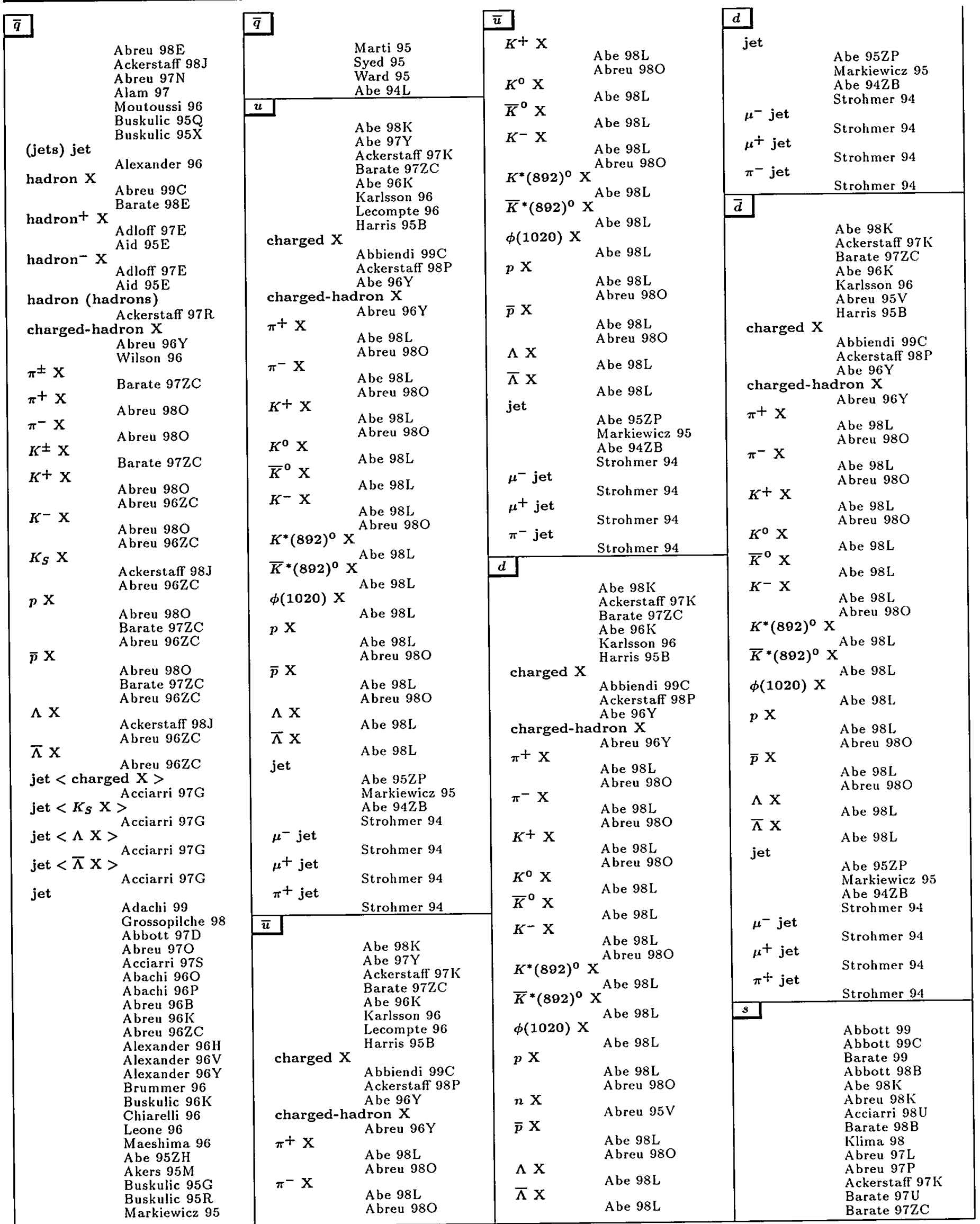

Entries in order of particle name, then decay. A few chemical symbols for nuclei have been changed to avoid ambiguity with particle names (see the Particle Vocabulary). See the legend on page 323. 


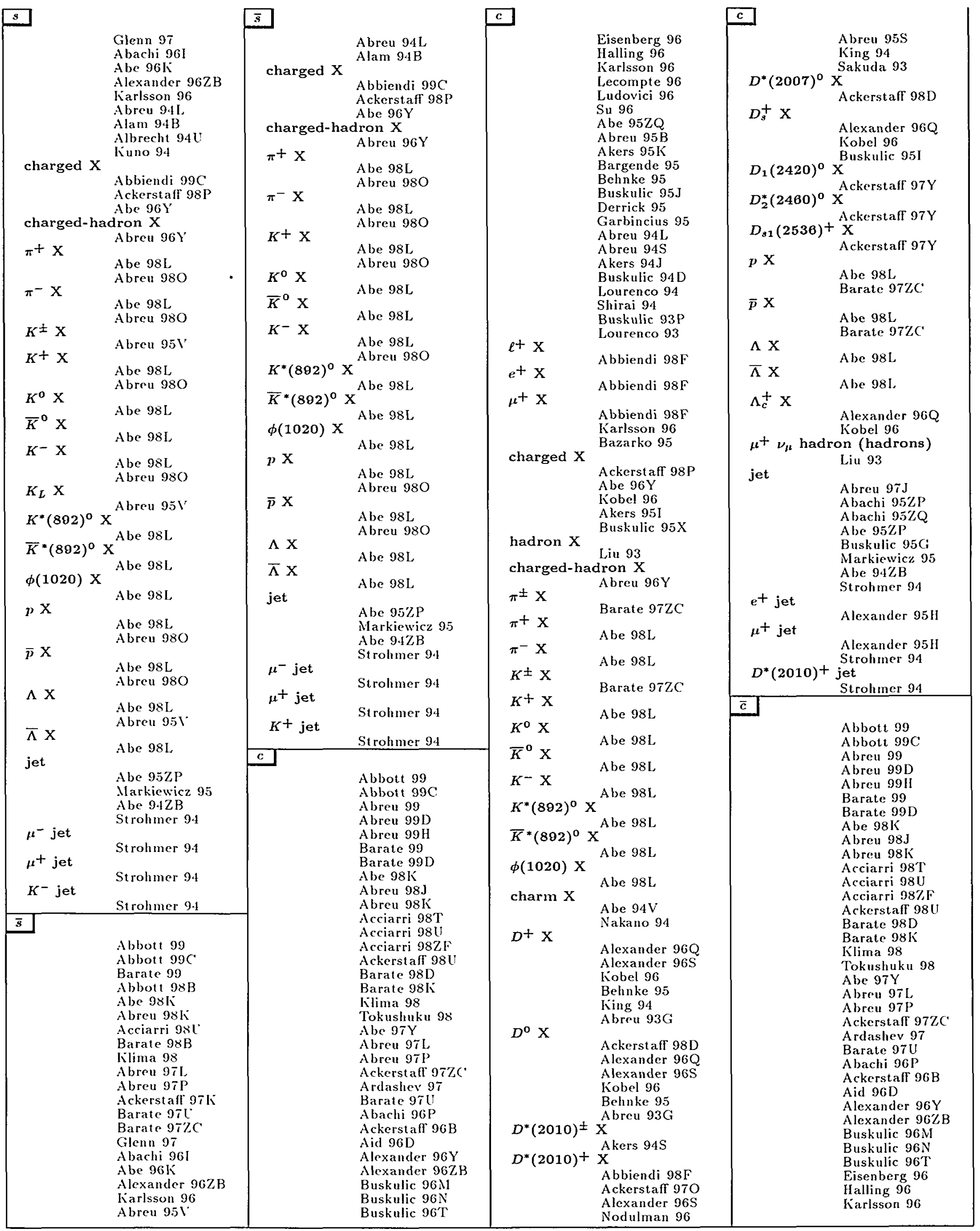


$\bar{c}$

Lecompte 96

Ludovici 96

Su 96

Abe $95 \mathrm{ZQ}$

A kers $95 \mathrm{~K}$

Alexander $95 \mathrm{H}$

Bargende 95

Behnke 95

Buskulic 95J

Derrick 95

Garbincius 95

Abreu $94 \mathrm{~L}$

Abreu 94S

Akers $94 \mathrm{~S}$

Buskulic $94 \mathrm{D}$

Lourenco 94

Abreu 93G

Buskulic 93P

Lourenco 93

$\ell^{-} \mathrm{x}$

Abbiendi $98 \mathrm{~F}$

$e^{-X}$

Abbiendi $98 \mathrm{~F}$

$\mu^{-} \mathrm{X}$

Abbiendi $98 \mathrm{~F}$

charged $\mathrm{X}$

Karisson 96

Ackerstaff $98 \mathrm{P}$

A be $96 \mathrm{Y}$

Kobel 96

Akers 95I

Buskulic 95X

hadron $\mathrm{X}$

Liu 93

charged-hadron $X$

$\pi^{ \pm} \mathrm{X}$

Abreu $96 \mathrm{Y}$

$\pi+\mathrm{x}$

Barate 97ZC

$\pi^{-} \mathrm{X}$

Abe $98 \mathrm{~L}$

$K^{ \pm} \mathrm{X} \quad$ Abe $98 \mathrm{~L}$

$K^{+} \mathrm{X}$

Barate 97ZC

$K^{\circ} \mathrm{X}$

A be $98 \mathrm{~L}$

$\bar{K}^{\circ} \mathrm{X}$

Abe $98 \mathrm{~L}$

$K^{-} \mathrm{X}$

Abe $98 \mathrm{~L}$

$K^{*}(892)^{\circ} \mathrm{A}$ be $98 \mathrm{~L}$

$\bar{K}^{*}(892)^{\circ} X^{\text {Abe } 98 \mathrm{~L}}$

$\phi(1020) \mathrm{X}$ Abe $98 \mathrm{~L}$

charm $\times$ Abe 98L

charm X Abe $94 \mathrm{~V}$

$\bar{D} \circ \mathrm{X} \quad$ Nakano 94

Ackerstaff $98 \mathrm{D}$

Alexander $96 \mathrm{Q}$

Alexander 96S

$D^{-} \mathrm{X}$

Alexander $96 \mathrm{Q}$

Alexander $96 \mathrm{~S}$

Kobel 96

King 94

$D^{*}(2010)^{-} \mathrm{X}$

Abbiendi $98 \mathrm{~F}$

A ckerstaff 970

Alexander $96 \mathrm{~S}$

Nodulman 96

Abreu 95S

King 94

Sakuda 93

$\bar{D}^{*}(\mathbf{2 0 0 7})^{\circ} \mathrm{X}$

$\mathrm{X}$

$D_{s}^{-} \mathrm{X}$

Alexander $96 \mathrm{Q}$

Kobel 96

Buskulic 95I
$\bar{D}_{1}(2420)^{\circ} \mathrm{X}$

$\bar{D}_{2}^{*}(2460)^{\circ} \mathrm{X}$

Ackerstaff $97 Y$

$D_{s 1}(2536)^{-} \mathrm{X}$

$p$ X

Ackerstaff $97 \mathrm{Y}$

A be $98 \mathrm{~L}$

$\bar{p} \mathrm{X}$

Abe $98 \mathrm{~L}$

$\Lambda \mathrm{X}$

Barate 97ZC

$\bar{\Lambda} \mathrm{X}$

Abe $98 \mathrm{~L}$

$\bar{\Lambda}_{c}^{-} \mathrm{X}$

Abe $98 \mathrm{~L}$

Alexander $96 \mathrm{Q}$

Kobel 96

$\mu-\bar{\nu}_{\mu}$ hadron (hadrons)

jet

Liu 93

Abreu $97 \mathrm{~J}$

Abachi 95ZP

Abachi 95ZQ

A be 95ZP

Buskulic 95G

Markiewicz 95

A be $94 \mathrm{ZB}$

Strohmer 94

$\mu^{-}$jet

Strohmer 94

$D^{*}(2010)^{-}$jet

Strohmer 94

\section{b}

Abbiendi 99D

Abbott 99

Abbott 99B

A be $99 \mathrm{C}$

Abreu 99

Abreu 99D

Abreu $99 \mathrm{E}$

Abreu $99 \mathrm{H}$

Barate 99D

Abbiendi 98

Abbiendi $98 \mathrm{~K}$

Abbiendi 98R

Abbott 98

Abbott $98 \mathrm{~F}$

Abbott $98 \mathrm{~N}$

Abbott $98 \mathrm{R}$

Abe $98 \mathrm{H}$

A be $98 \mathrm{~J}$

$A$ be $98 \mathrm{~K}$

Abe $98 \mathrm{~S}$

A be $98 \mathrm{~T}$

A be $98 \mathrm{U}$

Abe $98 \mathrm{ZB}$

Abreu $98 \mathrm{~L}$

Abreu 98M

Acciarri 98J

Acciarri $98 \mathrm{Y}$

Acciarri 98ZF

Ackerstaff $98 \mathrm{G}$

Ackerstaff $98 \mathrm{U}$

Barate $98 \mathrm{~F}$

Barate $98 \mathrm{~L}$

Barate $98 \mathrm{R}$

Barate $98 \mathrm{~S}$

Dorigo 98

Hoffmann 98

Abbott $9 \bar{T}$

A be 97

Abe $97 \mathrm{~K}$

A be 970

A be $97 \mathrm{R}$

A be $97 \mathrm{ZB}$

Abe $972 \mathrm{~J}$

Abreu $97 \mathrm{~F}$

A breu $97 \mathrm{~L}$

Ackerstaff $97 \mathrm{U}$
Barate $97 \mathrm{ZC}$

Ackerstaff $97 \mathrm{~V}$

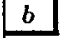

\section{b}

Ackerstaff $97 \mathrm{ZC}$

Conway 97

Swain 97

Abachi $96 \mathrm{U}$

Abe 96ZD

Abreu 96U

Ackerstaff $96 \mathrm{~B}$

Buskulic 96T

Etzion 96

Gallas 96

Loomis 96

Loomis $96 \mathrm{~B}$

Ludovici 96

Protopopescu 96

Slaughter 96

Stichelbaut 96

Su 96

Abe $95 \mathrm{~J}$

Abe $95 \mathrm{Q}$

Abreu 95X

Akers 95C

Buskulic 95J

Buskulic 95V

Harris 95B

Abe $94 \mathrm{ZC}$

Abreu 94R

Abreu 94S

Acciarri 94D

A kers $94 \mathrm{H}$

A kers 94Q

Carr 94

Jakobs 94

Jesik 94

A kers 93F

Buskulic 93P

$\ell^{-} \mathrm{X}$

Karlsson 96

$\bar{\nu} \mathrm{X}$

$e^{-X}$

Acciarri $96 \mathrm{H}$

Bauer 96

Behnke 95

Grassmann 95

A be $94 Z$

$\mu^{ \pm} \mathrm{X}$

Karlsson 96

$\mu^{-} \mathrm{X}$

Abachi $96 \mathrm{~L}$

Baarmand 96

Ueno 96

A bachi 95G

Abe 95

Behnke 95

Grassmann 95

A be $94 Z$

Leone 94

charged $\mathrm{X}$

Ackerstaff $98 \mathrm{P}$

Abe 96Y

Kobel 96

Akers 95I

Behnke 95

Buskulic 95X

A kers $93 \mathrm{H}$

jet $\mathrm{X}$

Derwent 94

hadron $\mathrm{X}$

Behnke 95

Liu 93

hadron (hadrons)

Abbiendi 980

Abe $97 \mathrm{~J}$

Beretvas 95C

charged-hadron $\mathrm{X}$

Abreu 96Y

$\ell \mathrm{X}$

Padilla 95

Karlsson 96

Abreu 95B

u X

Behnke 95

$d \mathrm{X}$

Abe $96 \mathrm{~K}$

Abe $96 \mathrm{~K}$

$b$

\begin{tabular}{|c|c|}
\hline $\mathrm{X}$ & A be $96 \mathrm{~K}$ \\
\hline $\mathbf{x}$ & Behnke 95 \\
\hline $\mathrm{x}$ & Denne 90 \\
\hline$+x$ & $\begin{array}{l}\text { Barate } 972 \mathrm{C} \\
\text { Abe } 98 \mathrm{~L} \\
\text { Abreu } 98 \mathrm{O}\end{array}$ \\
\hline$\pi^{-} \mathrm{X}$ & $\begin{array}{l}\text { Abe } 98 \mathrm{~L} \\
\text { Abreu } 980\end{array}$ \\
\hline$K^{ \pm} \mathrm{X}$ & Barate $97 Z C$ \\
\hline$+x$ & $\begin{array}{l}\text { Abe } 98 \mathrm{~L} \\
\text { Abreu } 980\end{array}$ \\
\hline $\begin{array}{l}K^{0} \mathrm{X} \\
\bar{K}^{0} \mathrm{X}\end{array}$ & Abe $98 \mathrm{~L}$ \\
\hline$K^{-} \mathrm{X}$ & $\begin{array}{l}\text { Abe } 98 \mathrm{~L} \\
\text { Abe } 98 \mathrm{~L} \\
\text { Abreu } 980\end{array}$ \\
\hline $\begin{array}{l}K^{*}(892)^{0} \mathrm{X} \\
\bar{K}^{*}(892)^{0}\end{array}$ & Abe $98 \mathrm{~L}$ \\
\hline$\phi(1020) \mathrm{X}$ & $\begin{array}{l}\text { A be } 98 \mathrm{~L} \\
\text { A be } 98 \mathrm{~L}\end{array}$ \\
\hline
\end{tabular}

$D^{+} \mathrm{X}$

Alexander $96 \mathrm{Q}$

Buskulic 960

Kobel 96

Behnke 95

$D^{\circ} \mathrm{X}$

Abreu 93G

Alexander $96 \mathrm{Q}$

Buskulic 960

Kóbel 96

Behnke 95

$D^{*}(2010)^{ \pm} \mathrm{X}$

$D^{*}(2010)+\mathrm{X}$

$D_{s}^{+} \mathrm{X}$

Ackerstaff 970

Alexander $96 \mathrm{Q}$

Buskulic 96O

Kobel 96

$D_{s}^{-} \mathrm{X}$

$D_{1}(2420)^{\circ} \mathrm{X}^{\mathrm{A}}$

$D_{2}^{*}(2480)^{\circ} \mathrm{X}$

$J / \psi(1 S) \mathrm{X}$

Abe $99 \mathrm{D}$

Ackerstaff $97 \mathrm{Y}$

Ackerstaff $97 Y$

Acciarri 97F

Baarmand 96

Tkaczyk 96

Behnke 95

Abreu 94M

$\chi_{c 1}(1 P) \mathrm{X}$

Acciarri $97 \mathrm{~F}$

$\chi_{c 2}(1 P) \mathrm{X}+\chi_{c 1}(1 P) \mathrm{X}$

Abreu 94AI

$\psi(2 S) \mathrm{X}$

Acciarri $97 \mathrm{~F}$

Tkaczyk 96 


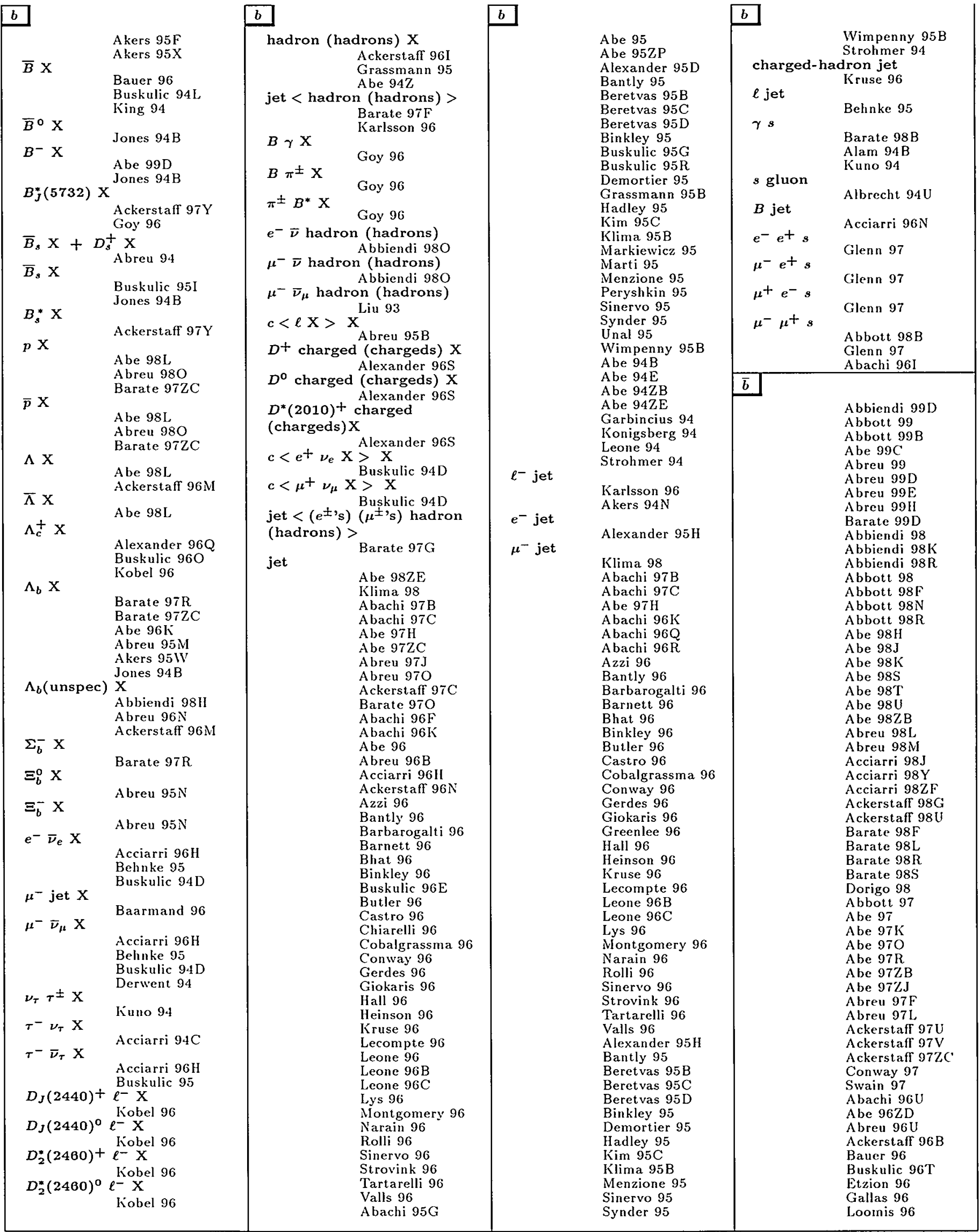


$\bar{b}$

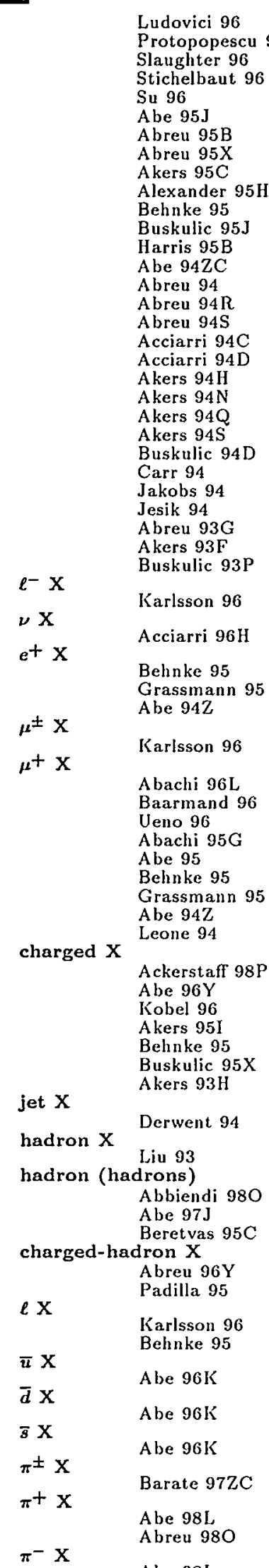

$\bar{b}$

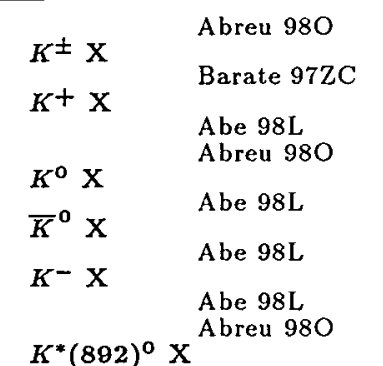

$\bar{K}^{*}(882)^{\circ} \mathrm{x}^{\text {Abe } 98 \mathrm{~L}}$

Abe $98 \mathrm{~L}$ $\phi(1020) \mathrm{X}$

$\bar{D}^{\circ} \mathrm{X}$

Abe $98 \mathrm{~L}$

Alexander 96Q Buskulic 960 Kobel 96

$D^{-} \mathrm{X}$

Alexander 96Q Buskulic 960

$D^{*}(2010)-\mathrm{X}$ Kobel 96

$D_{s}^{+} \mathrm{X}$

$D_{s}^{-} \mathrm{X}$

Ackerstaff 970

Abe $99 \mathrm{D}$

$\bar{D}_{1}(2420)^{\circ} \mathrm{X}$

Alexander $96 \mathrm{Q}$ Buskulic 960 Kobel 96

$\bar{D}_{\mathbf{2}}^{*}(\mathbf{2 4 6 0})^{\circ} \mathrm{X}^{\text {Ackerstaff } 97 \mathrm{Y}}$

$J / \psi(1 S) \times$ Ackerstaff $97 Y$

Acciarri $97 \mathrm{~F}$

Baarmand 96

Tkaczyk 96

$\chi_{c 1}(1 P) \times$

Abreu $94 \mathrm{M}$

Acciarri $97 \mathrm{~F}$

$\chi_{c 2}(1 P) \times+\chi_{c 1}(I P) \times$

$\psi(2 S) \mathrm{X}$

Abreu 94M

\section{cciarri 97F \\ Tkaczyk 96 \\ Abreu $94 \mathrm{M}$ \\ bottom $\mathrm{X}$}

Abreu 96P

Buskulic $96 \mathrm{Q}$

A be $95 \mathrm{ZR}$

A be $94 \mathrm{U}$

A be $94 \mathrm{~V}$

Manly 94

Nakano 94

Williams 94

$B \times$

Buskulic 94L

King 94

$B+\mathbf{X}$

Abe $99 \mathrm{D}$

A kers $94 \mathrm{R}$

Jones $94 \mathrm{~B}$

$B^{\circ} \mathbf{X}$

Jones $94 \mathrm{~B}$

$\bar{B} \times$

Barate $97 \mathrm{ZC}$

Ackerstaff $96 \mathrm{M}$

Ackerstaff 960

Abreu $95 \mathrm{~S}$

Akers 95F

A kers 95X

$\bar{B}_{J}^{*}(5732) \mathrm{X}$

Ackerstaff $97 \mathrm{Y}$

$B_{s} \times$ $\bar{b}$

$\bar{B}_{s}^{*} \mathrm{X}$ $B^{*}(5850) \mathrm{X}^{\text {Ackerstaff } 97 \mathrm{Y}}$

$\begin{array}{ll}p \times & \text { Akers } 94 \mathrm{R} \\ & \text { Abe } 98 \mathrm{~L}\end{array}$

Abreu 980

$\bar{p} \times$

Barate $97 \mathrm{ZC}$

Abe $98 \mathrm{~L}$

Abreu 980

$\Lambda \mathrm{X} \quad$ Barate $97 Z \mathrm{C}$

$\bar{\Lambda} \mathrm{X}$

Abe $98 \mathrm{~L}$

Abe $98 \mathrm{~L}$

$\bar{\Lambda}_{c}^{-} \mathbf{X}$

Ackerstaff $96 \mathrm{MI}$

Alexander $96 \mathrm{Q}$

Buskulic 960

$\bar{\Lambda}_{b} \mathrm{X}$

Kobel 96

Barate $97 \mathrm{R}$

Barate $97 Z \mathrm{C}$

Abe $96 \mathrm{~K}$

Abreu $95 \mathrm{M}$

Akers $95 \mathrm{~W}$

$\bar{\Lambda}_{b}$ (unspec) $\mathrm{X}$

Jones $94 \mathrm{~B}$

Abbiendi $98 \mathrm{H}$

Abreu $96 \mathrm{~N}$

$\boldsymbol{\Sigma}_{b}^{+} \mathbf{X}$

Ackerstaff $96 \mathrm{M}$

$\Xi_{b}^{+} \mathrm{X}$

Barate $97 \mathrm{R}$

$\bar{\Xi}_{b}^{0} \mathrm{X}$

$e^{+} \nu_{e} \mathrm{X}$

Abreu $95 \mathrm{~N}$

$\mu^{+}$jet $X$

Abreu $95 \mathrm{~N}$

$\mu^{+} \nu_{\mu} \mathrm{X}$

Acciarri $96 \mathrm{H}$

Baarmand 96

$\tau^{+} \nu_{\tau} \mathrm{X}$

Acciarri $96 \mathrm{H}$

Derwent 94

$\nu_{\tau} \mathrm{X}$ Acciarri $96 \mathrm{H}$

$\bar{D}_{J}(2440)^{\circ} \ell^{+}$X X

Kobel 96

$D_{J}(2440)^{-} \ell^{\dagger} \mathrm{X}$

$\bar{D}_{2}^{*}(2460)^{\circ} \ell^{+} \mathrm{X}$

$D_{2}^{*}(2460)-e^{+} \mathrm{X}$

$e^{+} \mathrm{X}$

hadron (hadrons) $\mathrm{X}$

Ackerstaff $96 \mathrm{I}$

Grassmann 95 Abe 942

jet $<$ hadron (hadrons) $>$ Barate $97 \mathrm{~F}$

Barate $97 \mathrm{G}$

Karlsson 96

$B \boldsymbol{\gamma} \mathrm{X}$

Buskulic $95 \mathrm{~V}$

$\bar{B} \boldsymbol{\gamma} \mathbf{X}$

$B \pi^{ \pm} \mathrm{X}$

Goy 96

$\bar{B} \pi^{ \pm} \mathrm{X}$

Buskulic $95 \mathrm{~V}$

$\pi^{ \pm} B^{*} \mathrm{X}$

Goy 96

$\pi \pm \bar{B}^{*} \mathrm{X}$

Buskulic $95 \mathrm{~V}$

$e^{+} \nu$ hadron (hadrons)

Abbiendi 980

Abbiendi 980

Buskulic 95I

Jones $94 \mathrm{~B}$ $\bar{b}$

$\mu^{+} \nu_{\mu}$ hadron (hadrons) Liu 93

$\bar{D}^{\circ}$ charged (chargeds) $\mathrm{X}$ Alexander $96 \mathrm{~S}$

$D^{-}$charged (chargeds) $\mathrm{X}$ Alexander $96 \mathrm{~S}$

$D^{*}(2010)^{-}$charged (chargeds) $\mathrm{X}$

Alexander 965 jet $<\left(e^{ \pm \prime} s\right)$ ( $\left.\mu^{ \pm \prime s}\right)$ hadron (hadrons) $>$

jet

Barate $97 \mathrm{G}$

A be $98 \mathrm{ZE}$

Klima 98

Abachi 97B

Abachi $97 \mathrm{C}$

Abe $97 \mathrm{H}$

A be $97 \mathrm{ZC}$

Abreu 97J

Abreu 970

Ackerstaff $97 \mathrm{C}$

Barate 970

Abachi $96 \mathrm{~F}$

Abachi $96 \mathrm{~K}$

Abe 96

Acciarri $96 \mathrm{H}$

Ackerstaff $96 \mathrm{~N}$

Azzi 96

Bantly 96

Barbarogalti 96

Barnett 96

Bhat 96

Binkley 96

Buskulic 96E

Butler 96

Castro 96

Chiarelli 96

Cobalgrassma 96

Conway 96

Gerdes 96

Giokaris 96

Hall 96

Heinson 96

Kruse 96

Lecompte 96

Leone 96

Leone 96B

Leone $96 \mathrm{C}$

Lys 96

Montgomery 96

Narain 96

Rolli 96

Sinervo 96

Strovink 96

Tartarelli 96

Valls 96

Abachi 95G

Abe 95

Abe 95ZP

Alexander 95D

Bantly 95

Beretvas 95B

Beretvas 95C

Beretvas 95D

Binkley 95

Buskulic 95G

Demortier 95

Grassmann 95B

Hadley 95

Kim 95C

Klima $95 \mathrm{~B}$

Markiewicz 95

Menzione 95

Peryshkin 95

Sinervo 95

Synder 95

Unal 95

Wimpenny 95B

Abe $94 \mathrm{~B}$

Abe $94 \mathrm{E}$

Abe $942 B$

Abe $942 E$ 


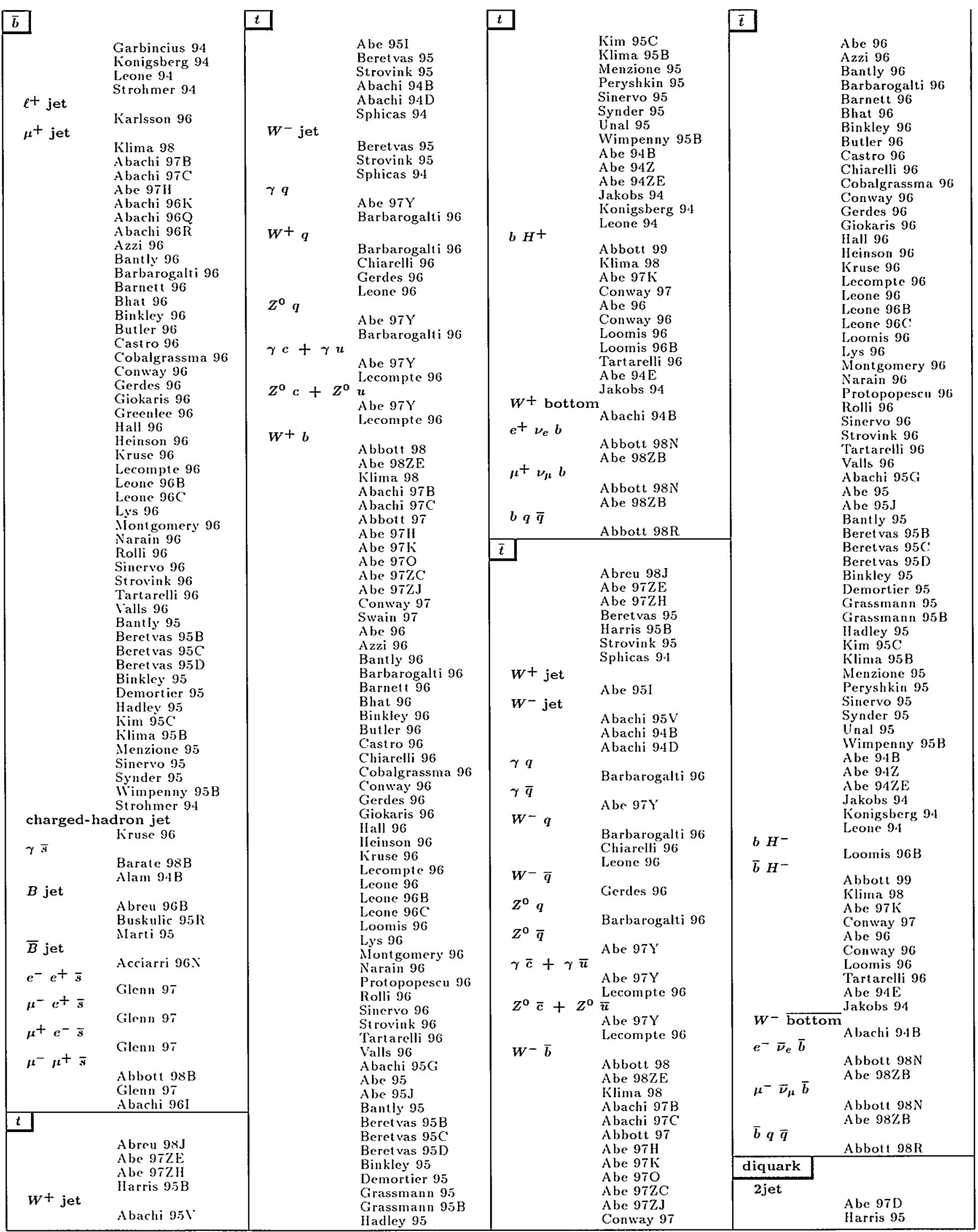




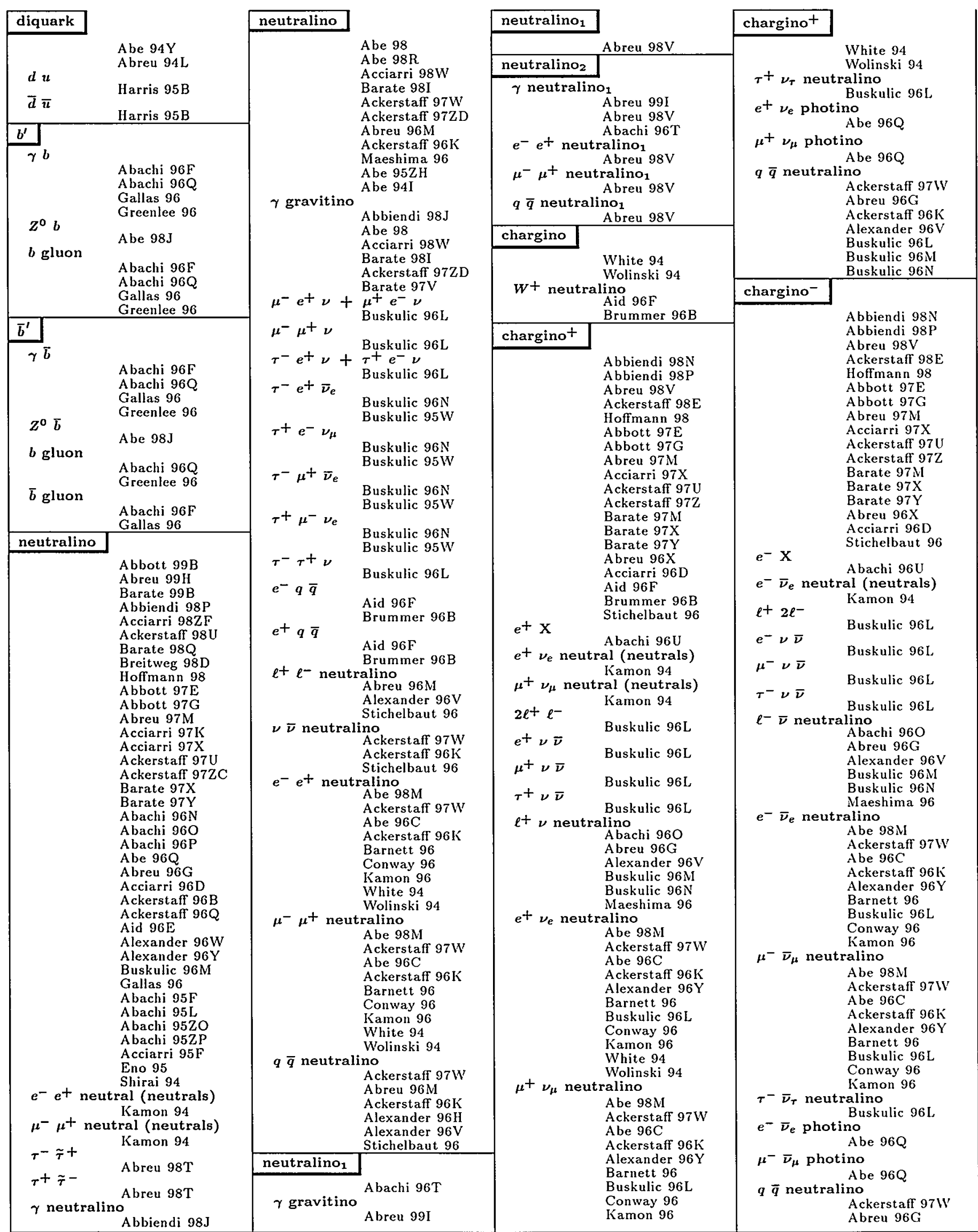

Entries in order of particle name, then decay. A few chemical symbols for nuclei have been changed to avoid ambiguity with particle names (see the Particle Vocabulary). See the legend on page 323. 


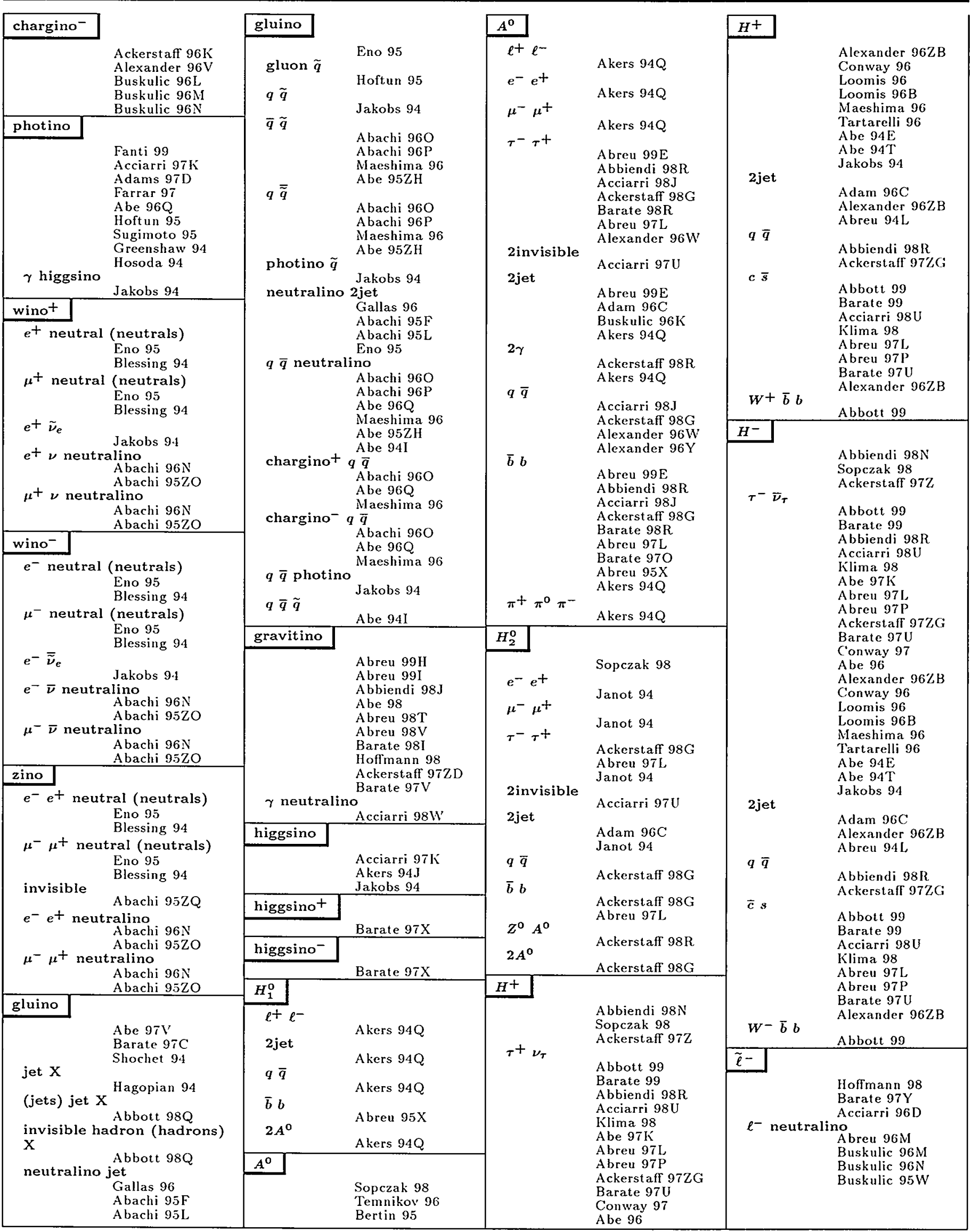




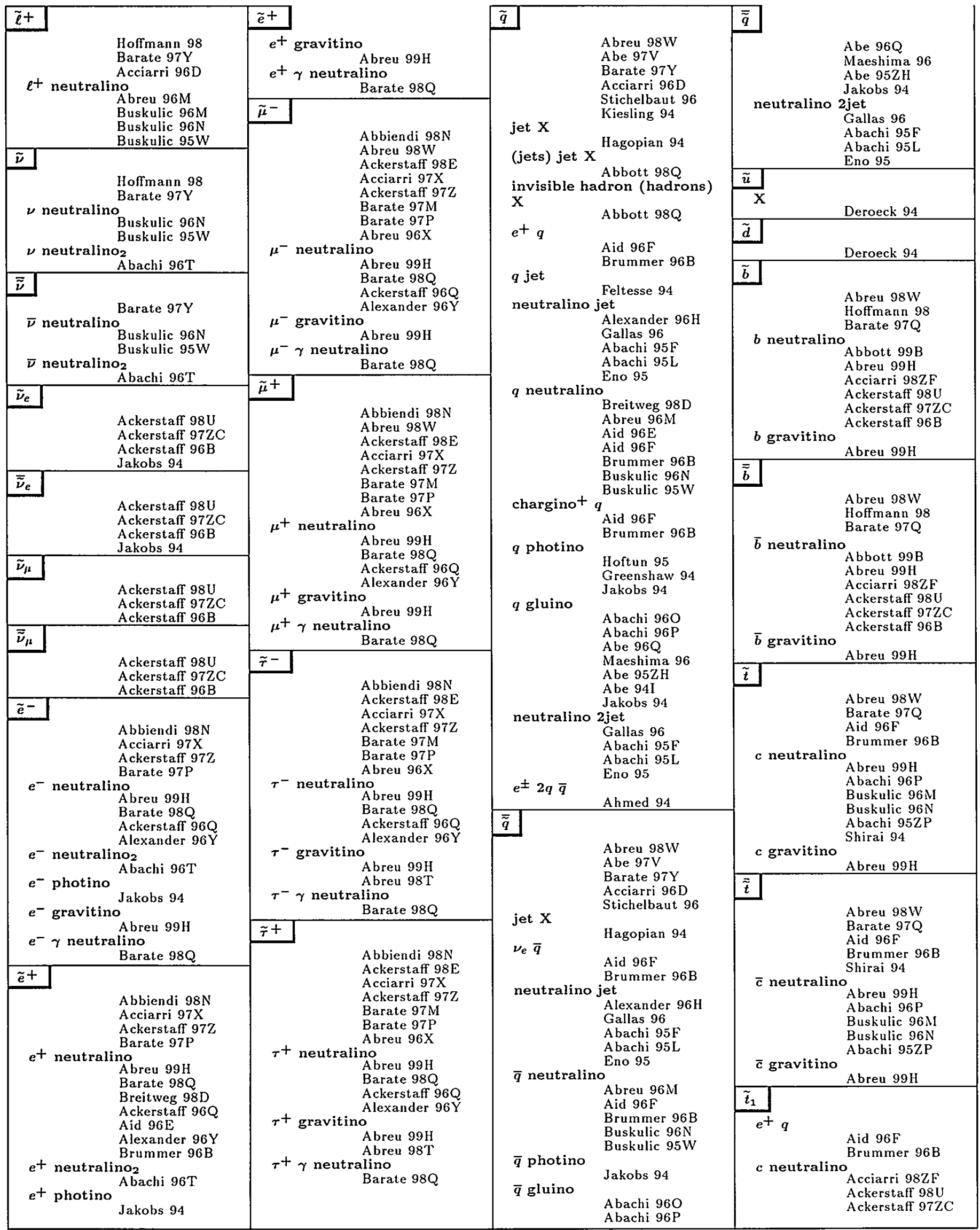

Entries in order of particle name, then decay. A few chemical symbols for nuclei have been changed to avoid ambiguity with particle names (see the Particle Vocabulary). See the legend on page 323. 


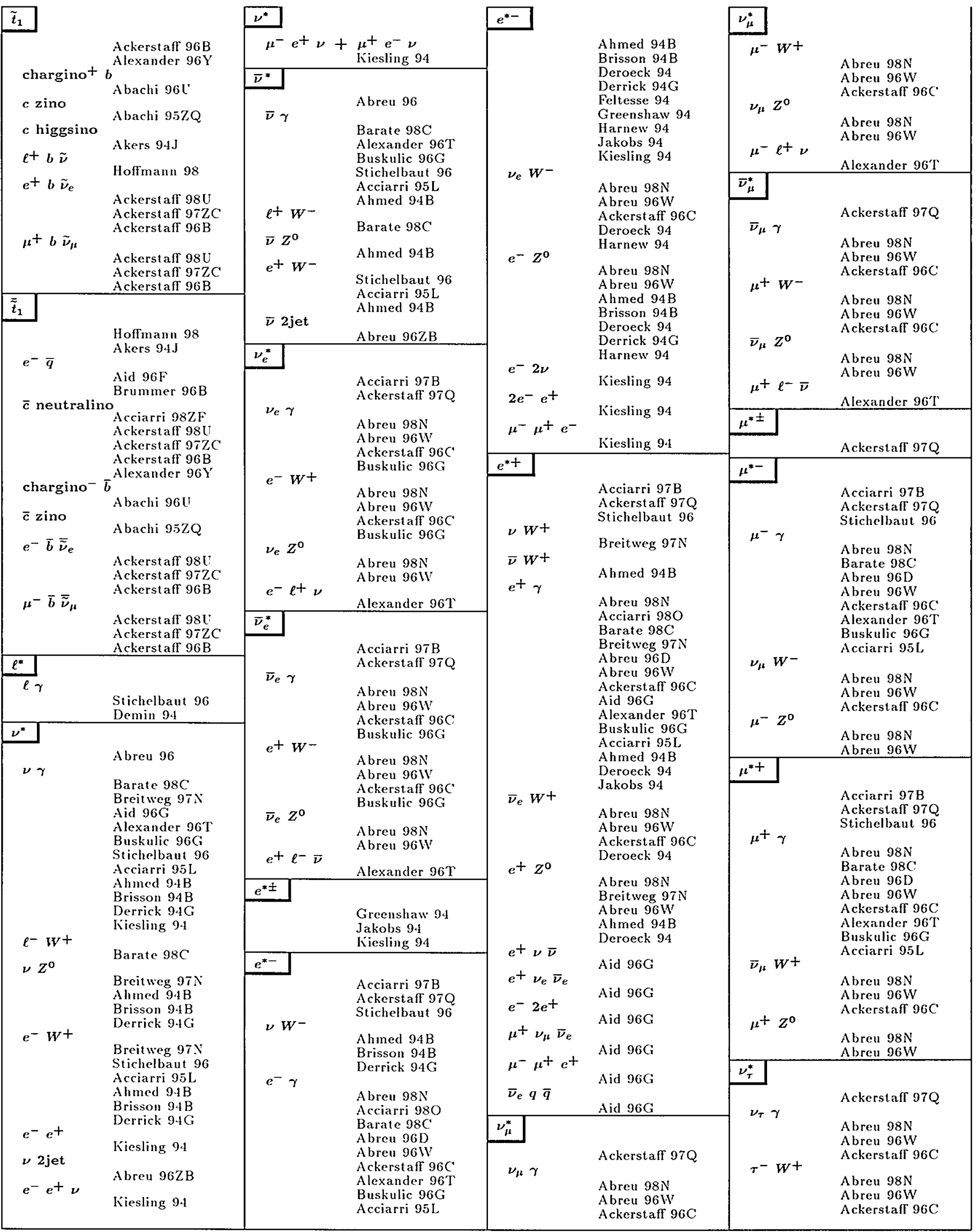




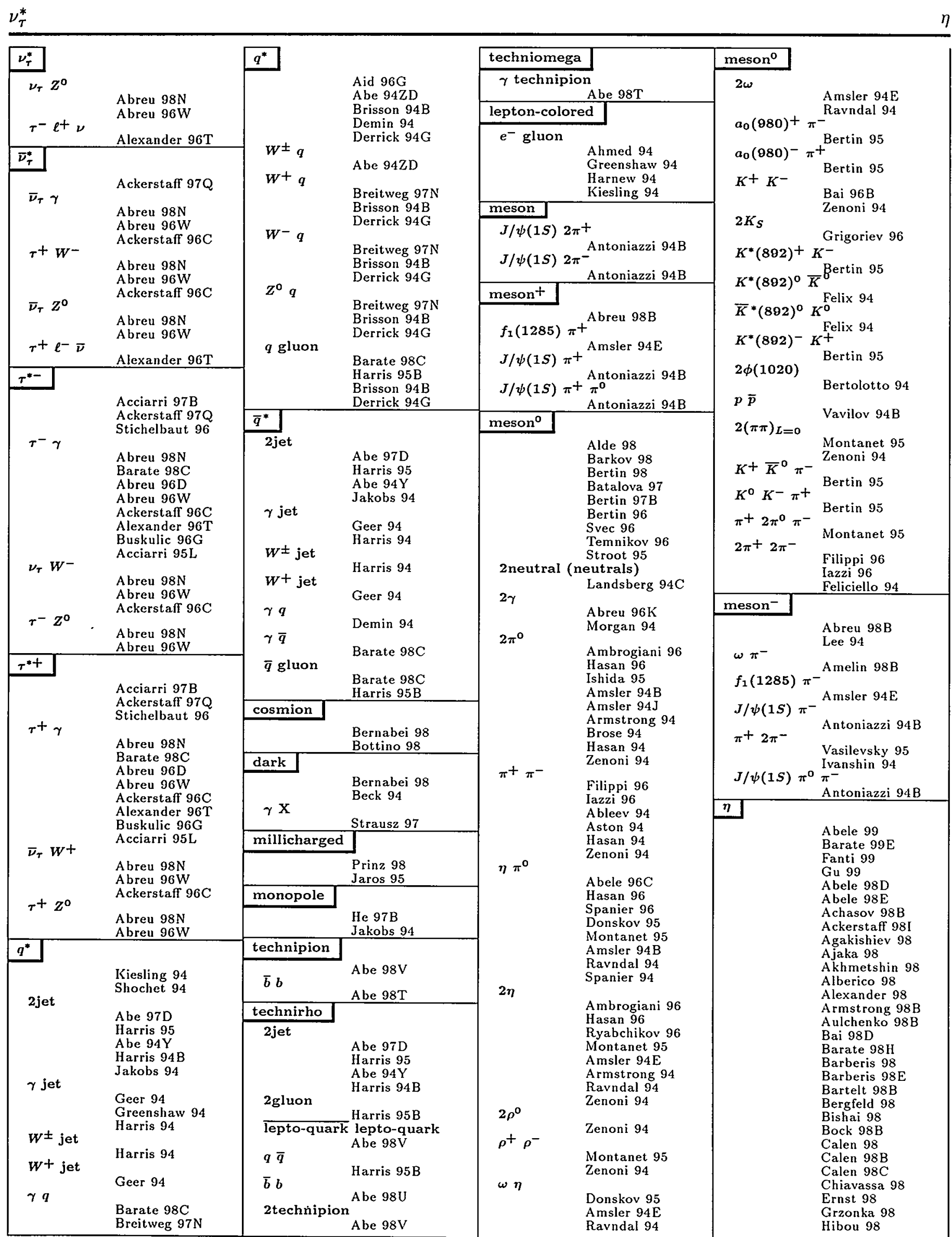

Entries in order of particle name, then decay. A few chemical symbols for nuclei have been changed to avoid ambiguity with particle names (see the Particle Vocabulary). See the legend on page 323. 


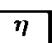

H и 98

Johansson $98 \mathrm{~B}$

Kirk 98

Smith 98

Troyan 98B

Wolf 98

Wurzinger 98

A be $97 \mathrm{~F}$

Abele $97 \mathrm{C}$

A bele 97 D

Abele $97 \mathrm{E}$

Ackerstaff $97 \mathrm{ZE}$

Adams 97E

Akhmetshin 97C

Anderson $97 \mathrm{~B}$

Armstrong $97 \mathrm{C}$

Armstrong $97 \mathrm{D}$

Averbeck 97

Balest 97

Bartelt 97

Behrens 97

Bergfeld 97

Bertin $97 \mathrm{D}$

Bilger 97

Bonvicini 97

Calen 97

Chiba 97B

Evans 97

Golovkin 978

Hoffmannroth 97

Holzmann 97

Jessop 97B

Krusche 97

Nemati 97

Rouge 97

Shelkov 97

Thompson 97

Willis 97

Abele $96 \mathrm{C}$

Acciarri $96 \mathrm{~N}$

Achasov 96

Adomeit 96

A mbrogiani 96

Amsler 96B

Amsler 96D

Badala $96 \mathrm{~B}$

Barberis 96B

Barmin 96

Baru 96

Bezzubov 96

Calen 96

Cason 96

Degener 96

Deoliveirama 96

Hasan 96

Pinder 96

Prokoshkin 96E

Ryabchikov 96

Semenov 96

Spanier 96B

Tikhomirov 96

Zachariadou 96

Acciarri $95 \mathrm{MI}$

Adler $95 \mathrm{~F}$

Albrecht 95I

Amsler $95 \mathrm{E}$

Breuer 95

Donskov 95

Helisley 95

Price 95

Ross 95

Savinov 95

Abreu $94 \mathrm{H}$

Acciarri 94

Alam $94 \mathrm{C}$

Albrecht 94C

Albrecht 94D

Amelin 94

Armstrong 94

Armstrong 94B

Bachler 94

Chiavassa 94

Chiavassa $94 \mathrm{~B}$

Danilov 94

Eigen 94

$\eta$
Fuchs 94
Futrals)

longlived

$e^{-} e^{+}$

Amsler $94 \mathrm{G}$

Achasov 97

Browder 97

White 96

$\mu^{-} e^{+}$

White 96

$\mu+e^{-}$

White 96

$\mu^{-} \mu^{+}$

Browder 97

$\gamma$ longlived

$2 \gamma$

Amsler 96

Barberis $98 \mathrm{~B}$

Browder 97

Gronberg 97

Acciarri 96J

Acciarri $96 \mathrm{M}$

Alde 96

Amsler $96 \mathrm{C}$

Balatz 96

Bartelt 96

Benayoun 96

Buskulic 96C:

Chiba 96

Dowd 96

Freyberger 96

Golovkin 96

Jin 96

Kubota 96

Malinin 96

Notheisen 96

Prokoshkin 96

Prokoshkin 96B

Prokoshkin 96C

Prokoshkin 96D

Spanier 96

Ye 96

Zaitsev 96

Acciarri 95 $\mathrm{P}$

Albrecht $95 \mathrm{~F}$

Alde 95

Alde 95B

Amelin $95 \mathrm{C}$

Ammar 95

Amsler 95B

Amsler 95D

Amsler 95F

Amsler 95G

Amsler 95H

Anisovich 95

Becattini 95

Brandenburg 95

Donskov 95

Dytman 95

Gibaut 95

Golovkin 95

Krusche 95C

Montanet 95

Stroot 95

Vavilov $95 \mathrm{C}$

Achasov 94

Albrecht $94 \mathrm{~J}$

Alde 94

Amsler $94 \mathrm{C}$

Amsler 94D

Amsler 94E

Amsler $94 \mathrm{~F}$

Amsler $94 \mathrm{G}$

Amsler 94

Amsler 941

$\eta$

Amsler 94J

Anisovich 94

Aoyagi 94

Berg 94

Castro 94

Chiavassa $94 \mathrm{C}$

Donskov 94

Faessler 94

Fan 94

Fan 94B

Fuchs 94

Kampert 94

Kulik 94

Landsberg 94B

Piccinini 94

Prokoshkin 94

Sadovsky 94

Singovsky 94

Wiedner 94

Zaitsev 94

$2 \pi^{0}$

Achasov 98

Achasov 97

$\pi^{+} \pi^{-}$

$e^{-} e^{+} \gamma$

$\pi^{+} \pi^{-} \gamma$

Akhmetshin 97B

Notheisen 96

Amelin 98

Gibaut 94B

$3 \pi^{0}$

Abele 98

Achasov 98C

Gronberg 97

Golovkin 96

Amsler 95B

Krusche 95

Krusche 95B

Krusche $95 \mathrm{C}$

Montanet 95

Stroot 95

Amsler 94

Amsler 94B

Amsler 94D

Amsler 94F

Wiedner 94

$\pi^{+} \pi^{0} \pi^{-}$

Abele $98 \mathrm{~B}$

Gronberg 97

Buskulic $96 \mathrm{C}$

Zaitsev 96

Ableev $95 \mathrm{C}$

Akhmetshin 95

Akhmetshin $95 \mathrm{~B}$

Amelin 95C

Amsler 95B

Golovkin 95

Montanet 95

Achasov 94

Amsler 94

Amsler 94B

Amsler $94 \mathrm{D}$

Gibaut $94 \mathrm{~B}$

Landsberg $94 \mathrm{~B}$

Shevchenko 94

Wiedner 94

Zaitsev 94

$f_{0}(400-1200)$

\begin{tabular}{|c|c|}
\hline $\begin{array}{l}\pi^{+} \pi^{-} \\
K^{-} \pi^{+}\end{array}$ & $\begin{array}{l}\text { Svec } 96 \\
\text { Troyan } 98 \\
\text { Ishida } 97\end{array}$ \\
\hline$f_{0}(700)$ & Alekseev 97 \\
\hline & $\begin{array}{l}\text { Baglin } 98 \mathrm{~B} \\
\text { Bai } 98 \mathrm{~L} \\
\text { Geiger } 98 \\
\text { Thompson } 98 \\
\text { Angelis } 97 \\
\text { Gronberg } 97\end{array}$ \\
\hline
\end{tabular}

$\rho$

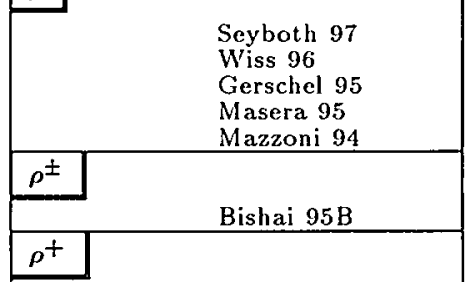

Acciarri $98 \mathrm{C}$

Acciarri 98D

Acciarri 98ZE

Ackerstaff 98I

Barberis 98

Bergfeld 98

Bulekov 98

Smith 98

A be $97 \mathrm{G}$

Albrecht $97 \mathrm{~B}$

Alvaro 97

Anastassov $97 \mathrm{~B}$

Behrens 97

Brient 97

Chiba 97B

Evans 97

Hanai 97

Heltsley 97

Jessop $97 \mathrm{~B}$

Moore 97

Quigley 97

Ruiz 97

Weber 97

Wermes 97

Achasov 96

Adams $96 \mathrm{~B}$

Albrecht $96 \mathrm{~B}$

Artuso 96

Wiss 96

Akers 95B

Asner 95

Bella 95

Buskulic 95K

Buskulic 95P

Davier 95

Gibbons 95B

Hanai 95

Harton 95

Kodama 95

Matorras 95

Raab 95

Ross 95

A bleev 94

Akers 94P

Amsler 94B

Buskulic 94

Buskulic 94J

Golutvin 94

Payne 94

White 94B

Zenoni 94

Muheim 93B

Landsberg 89

$\pi^{+} \pi^{0}$

Acciarri $96 \mathrm{C}$ 


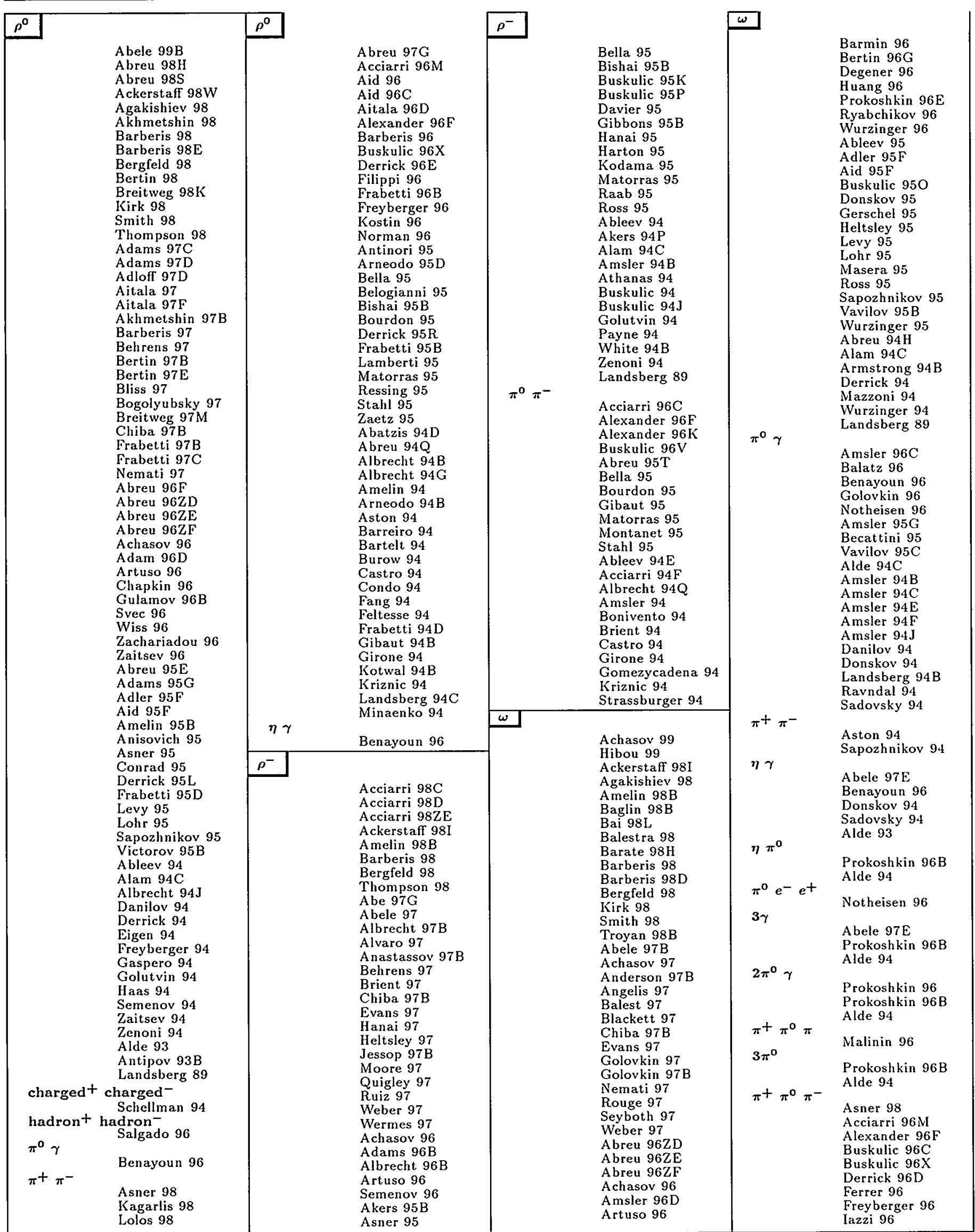

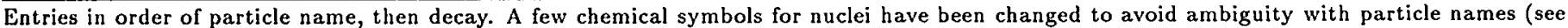
the Particle Vocabulary). See the legend on page 323. 


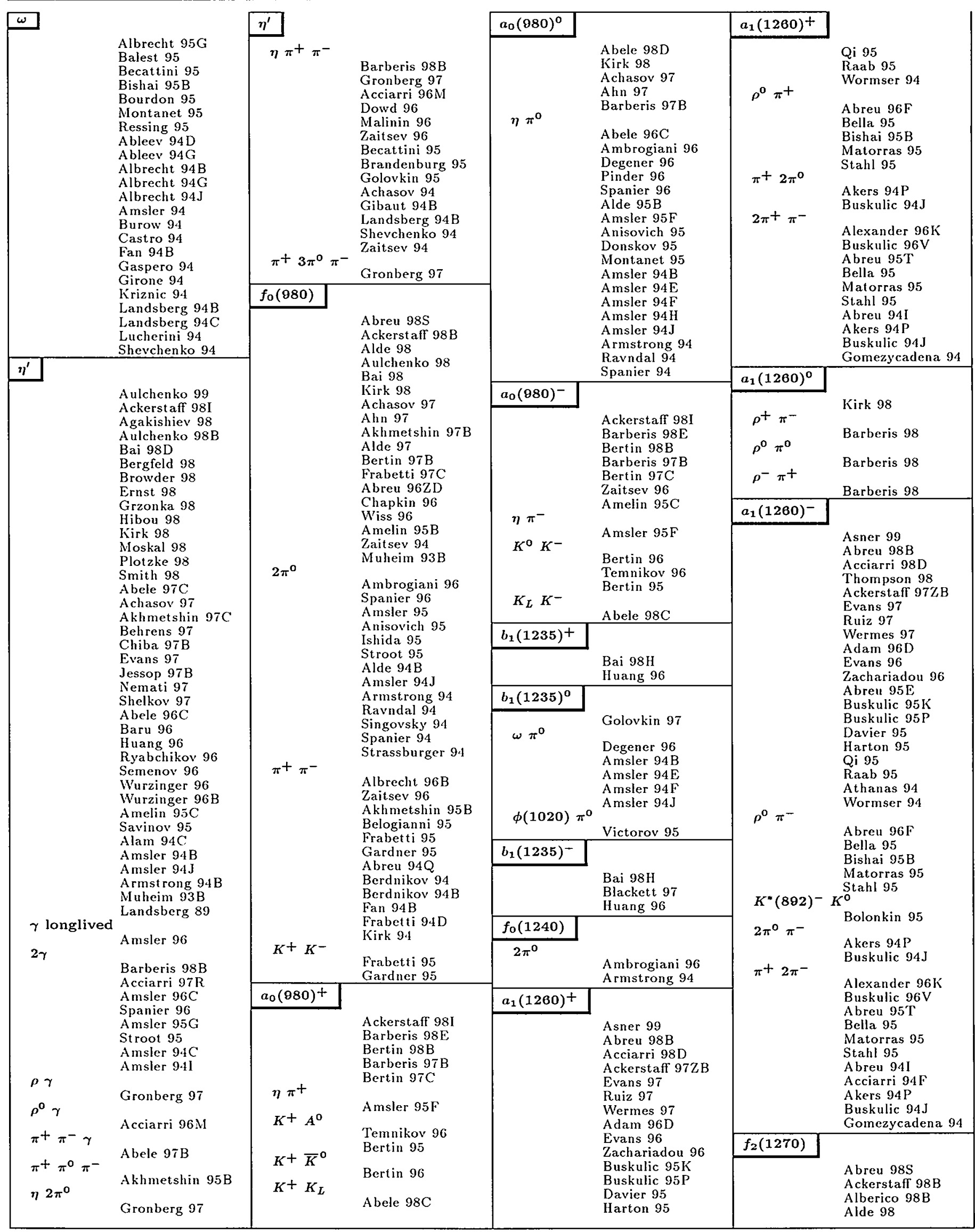




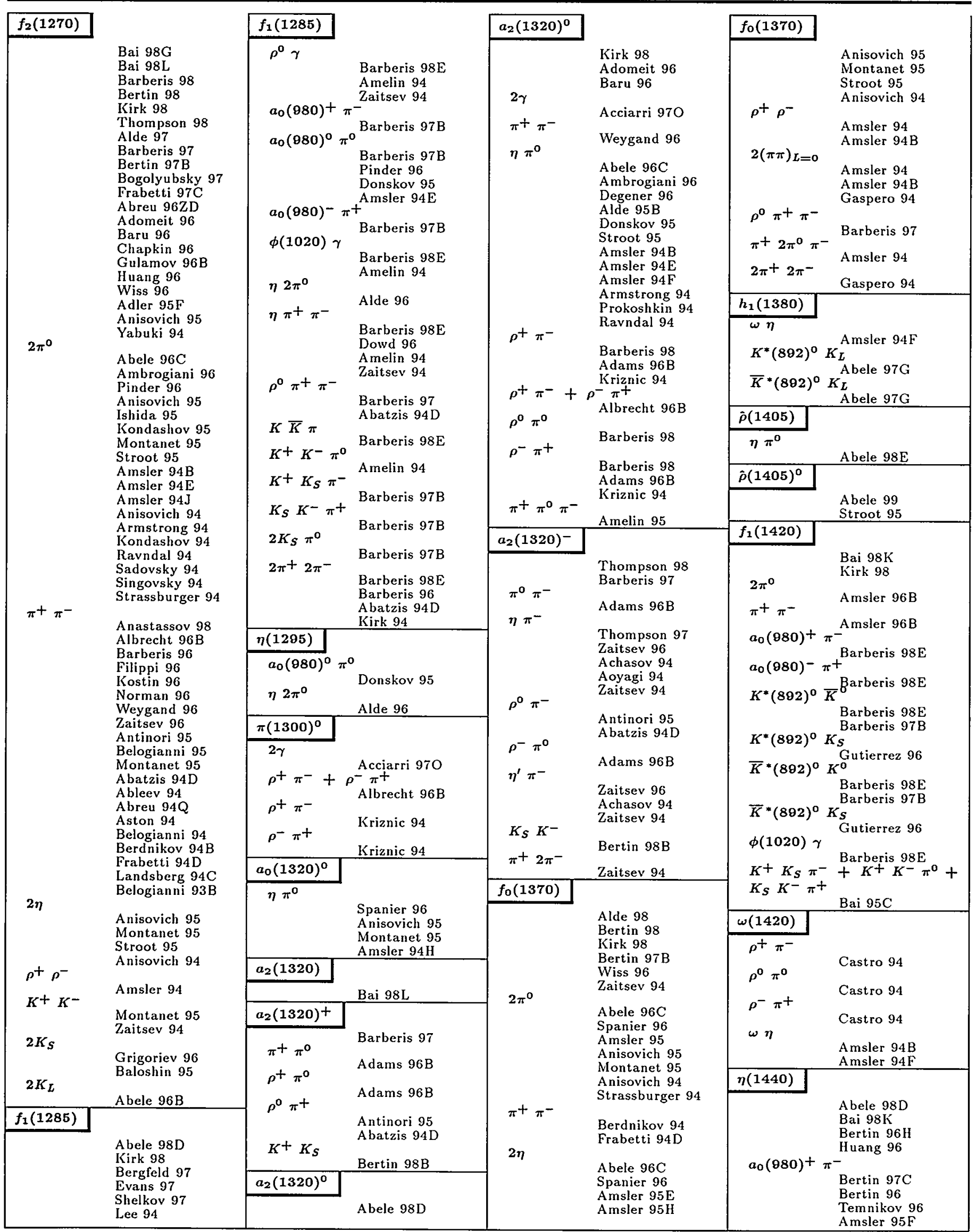

Entries in order of particle name, then decay. A few chemical symbols for nuclei have been changed to avoid ambiguity with particle names (see the Particle Vocabulary). See the legend on page 323. 


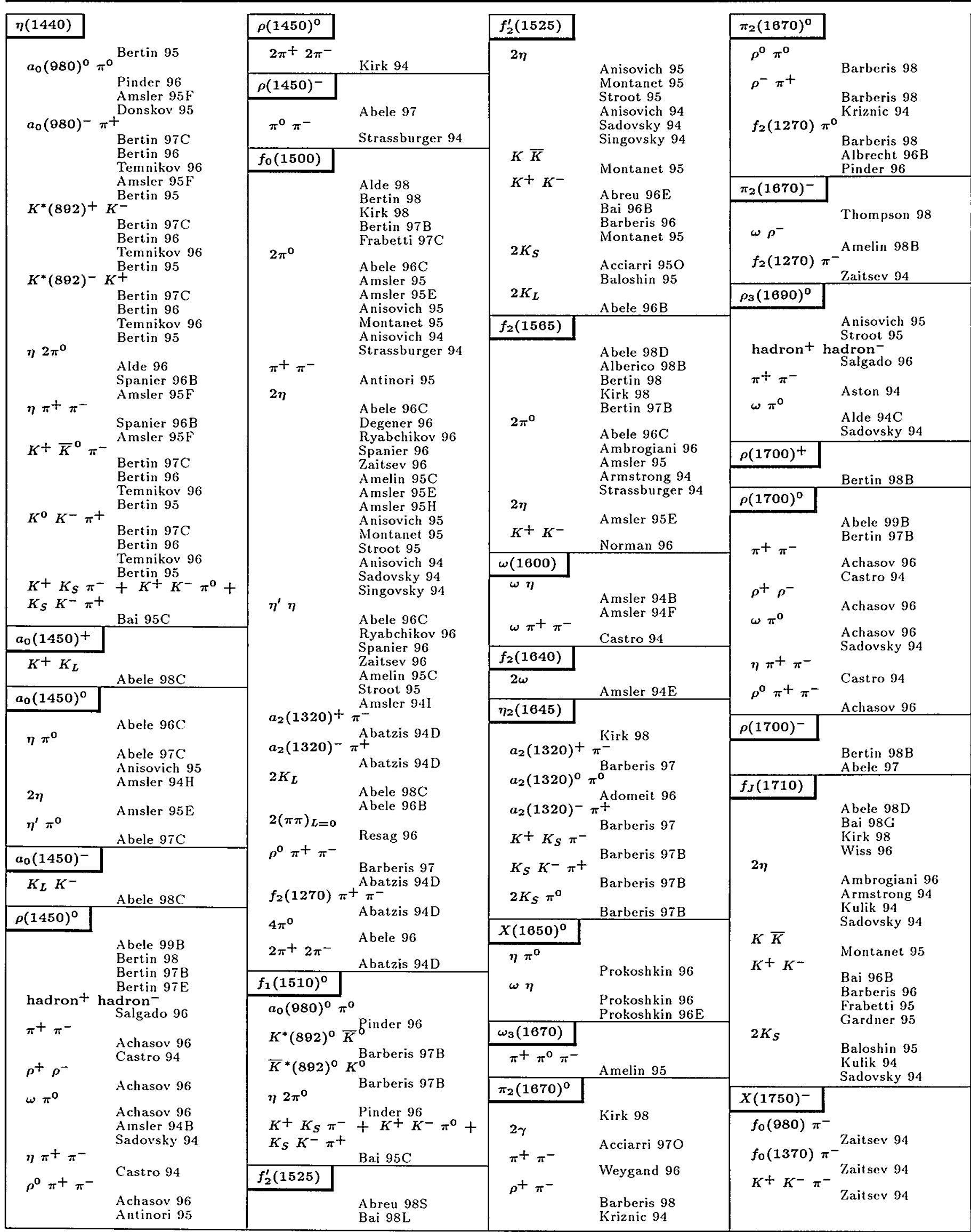




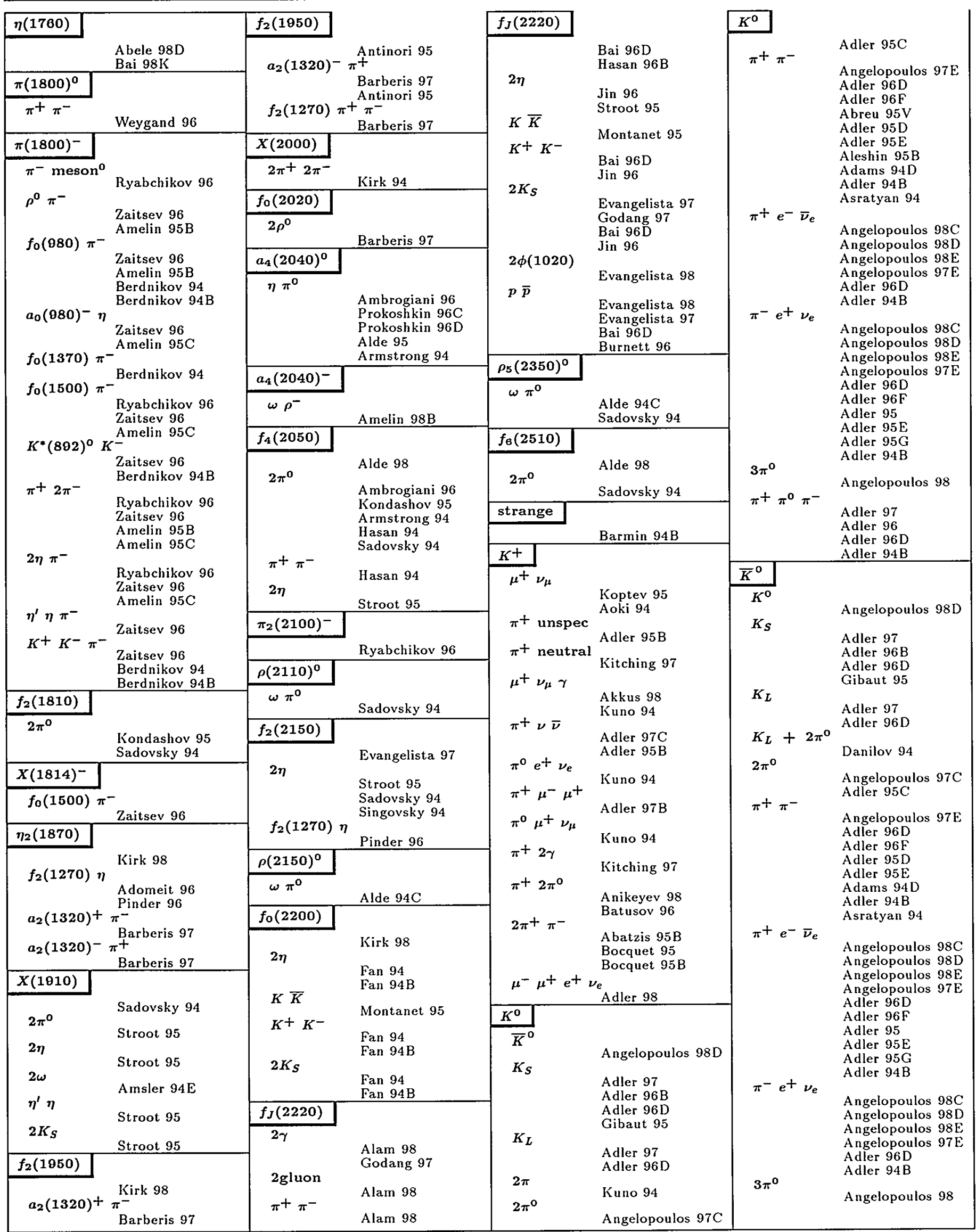

Entries in order of particle name, then decay. A few chemical symbols for nuclei have been changed to avoid ambiguity with particle names (see the Particle Vocabulary). See the legend on page 323. 


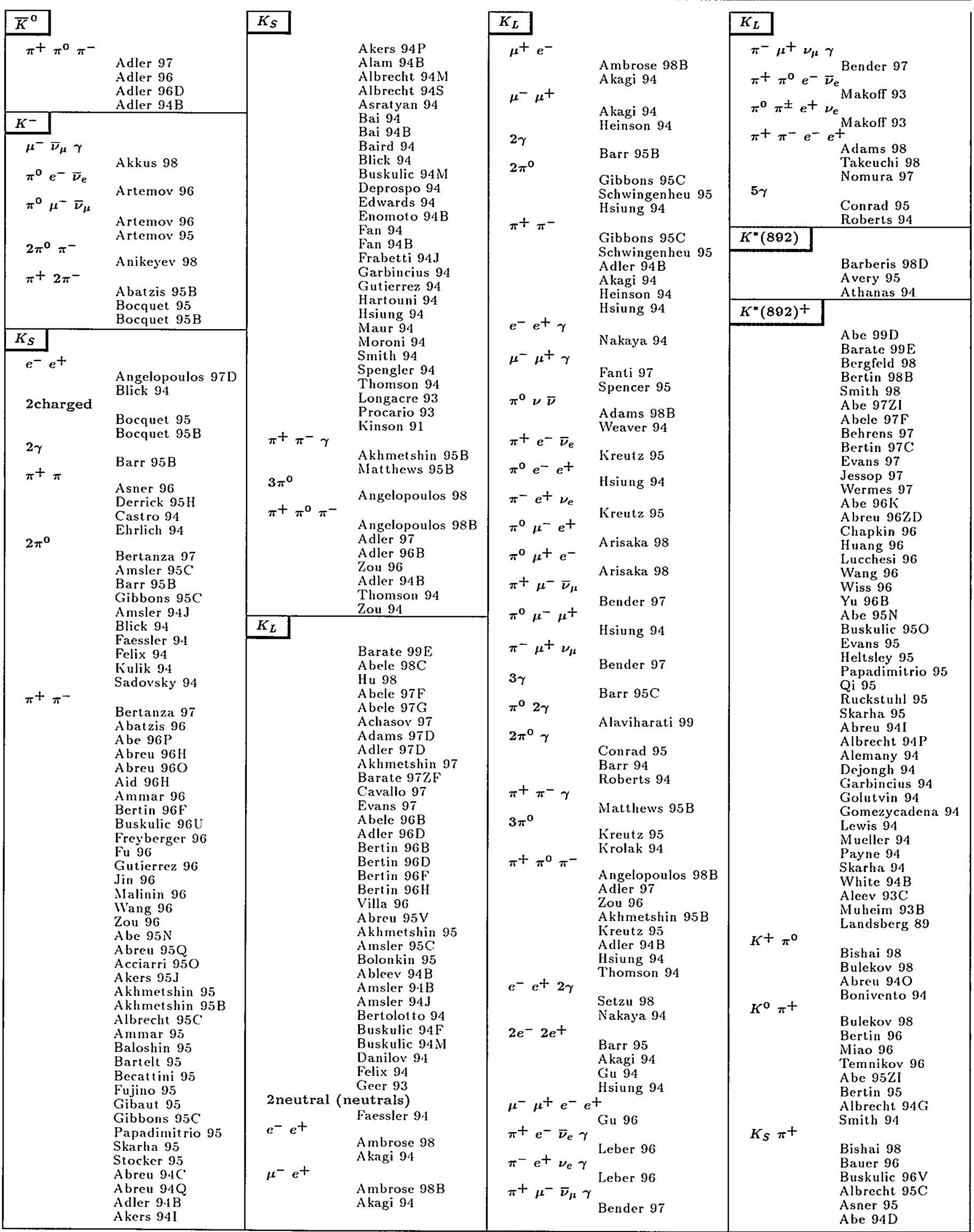




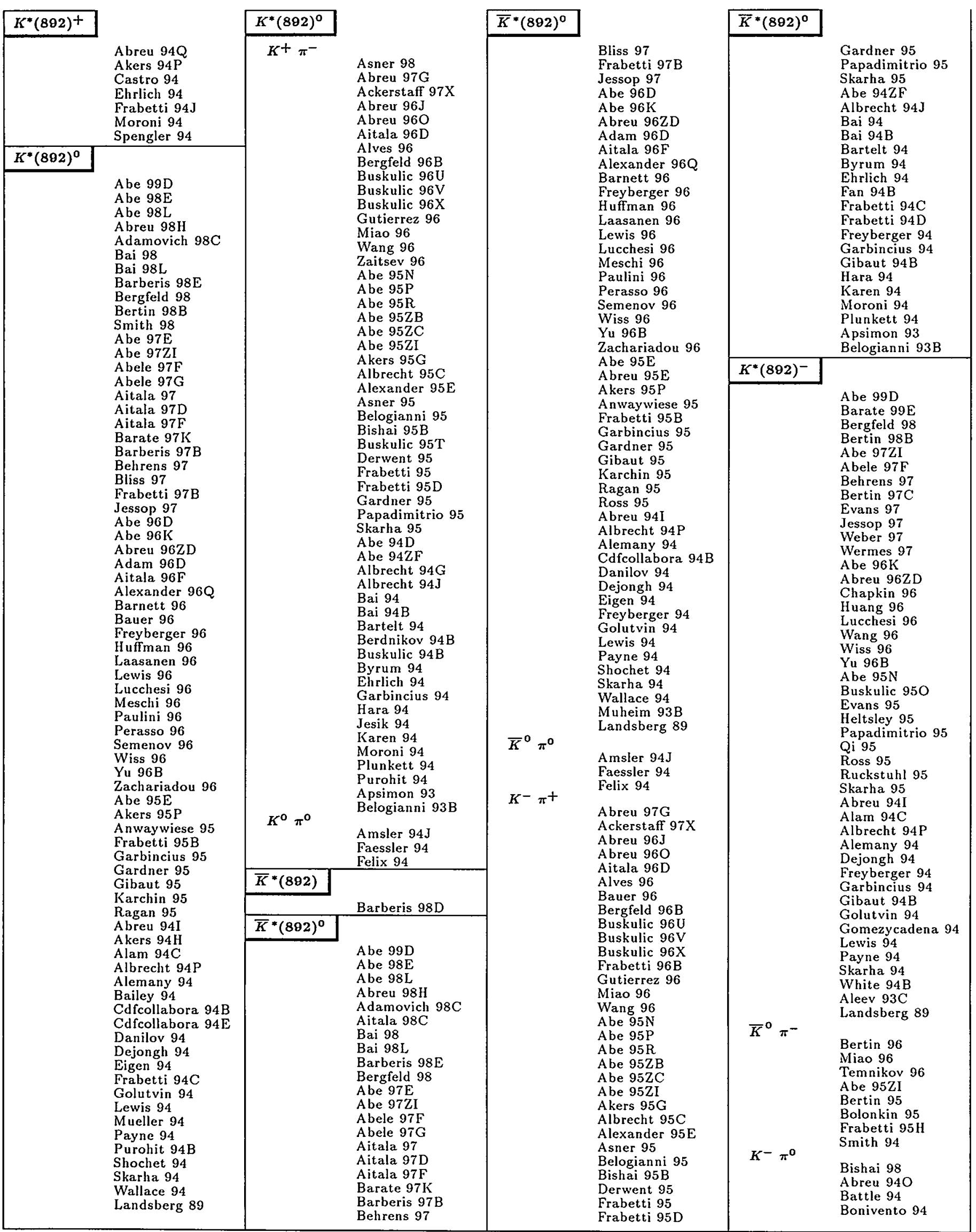

Entries in order of particle name, then decay. A few chemical symbols for nuclei have been changed to avoid ambiguity with particle names (see the Particle Vocabulary). See the legend on page 323. 


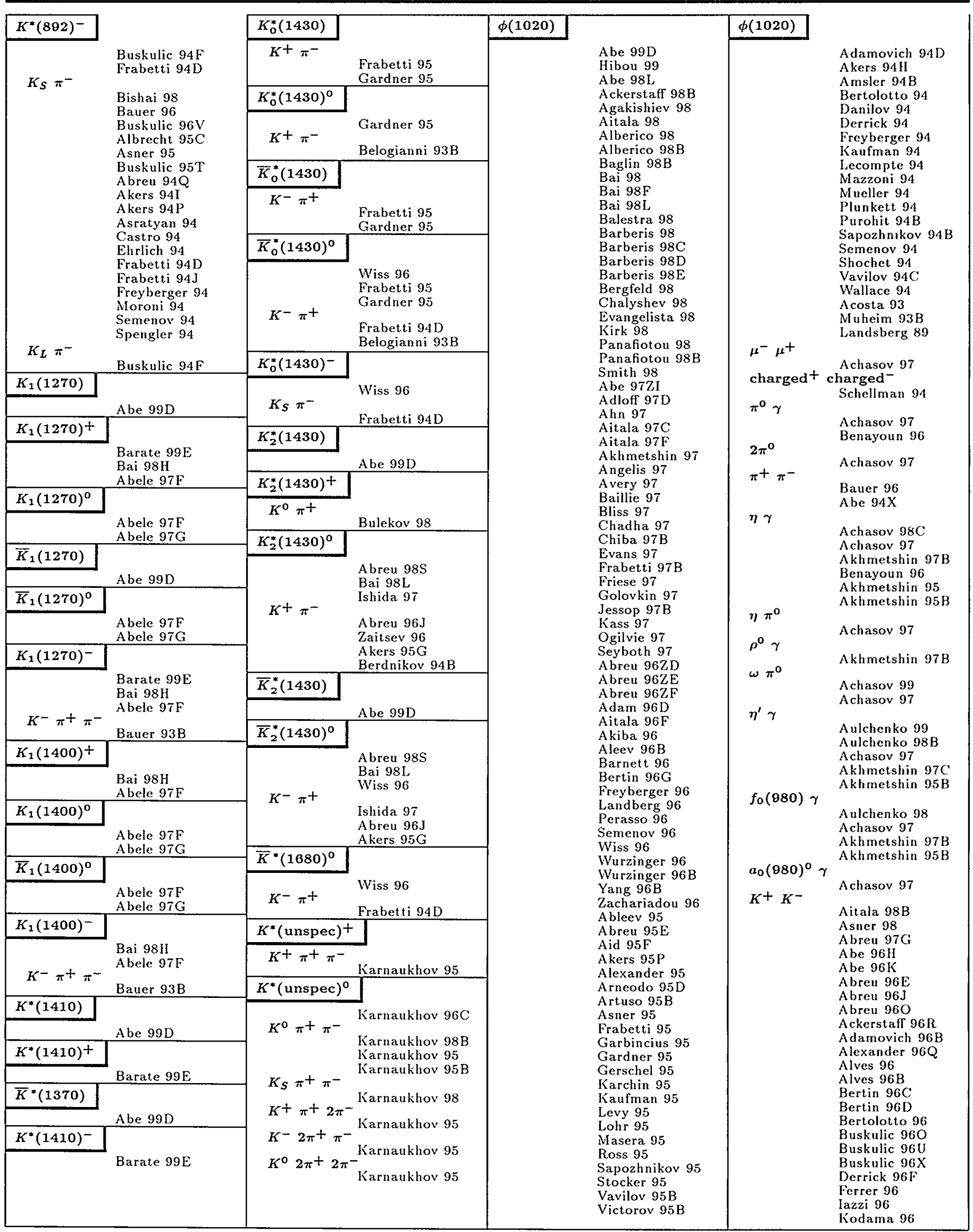




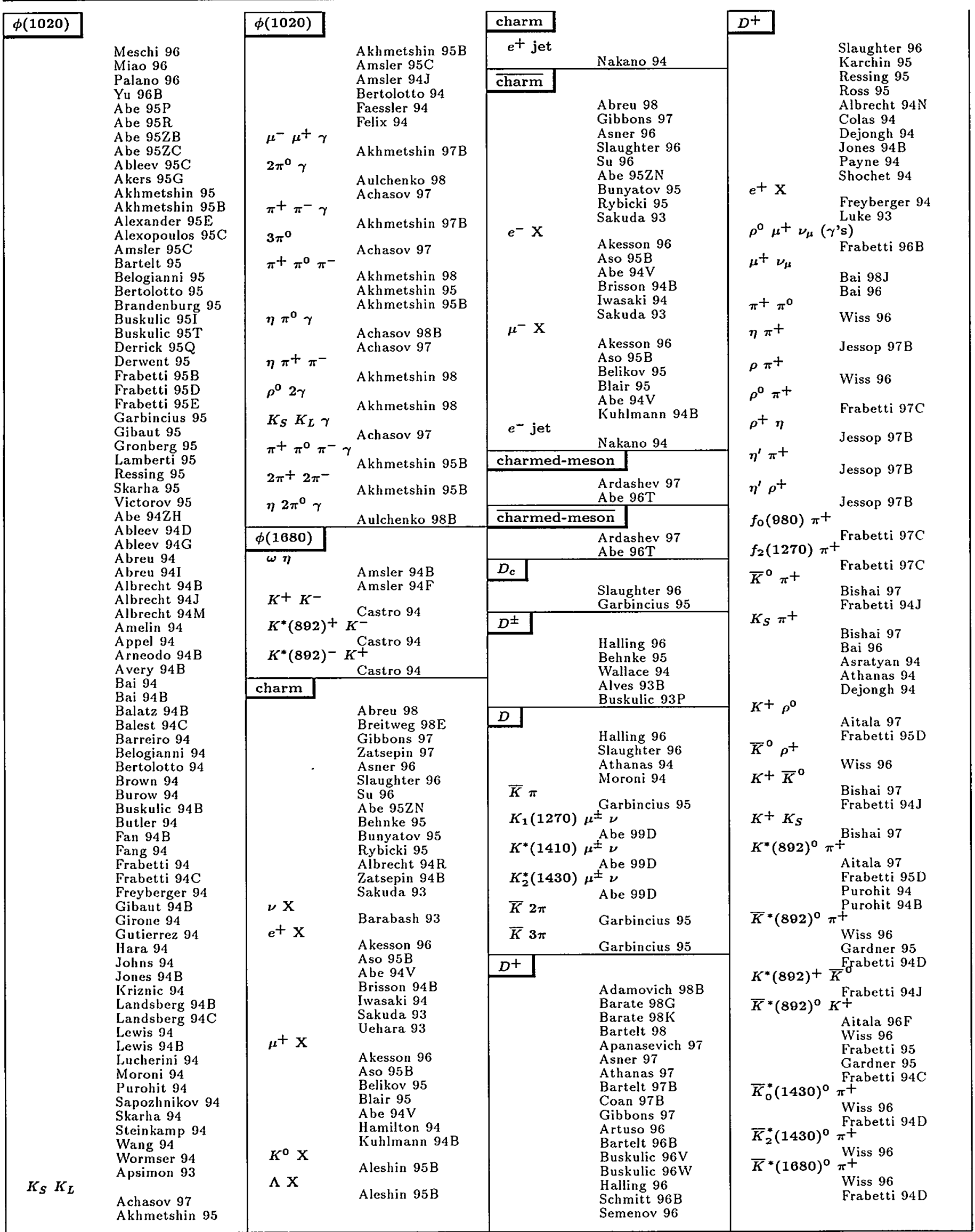

Entries in order of particle name, then decay. A few chemical symbols for nuclei have been changed to avoid ambiguity with particle names (see the Particle Vocabulary). See the legend on page 323. 


\begin{tabular}{|c|c|c|c|}
\hline$D^{+}$ & & $D^{\circ}$ & \\
\hline
\end{tabular}




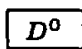

Buskulic $96 \mathrm{Q}$

Buskulic 96R

Buskulic 96T

Buskulic 96V

Buskulic 96W

Kaplan 96

Kobel 96

Lucchesi 96

Nodulman 96

Semenov 96

Semenov 96B

Spaan 96

Wang 96

Wang $96 \mathrm{~B}$

Wiss 96

A be $952 Q$

Abreu 95L

Abreu 95S

Adamovich 95

A kers $95 \mathrm{~F}$

Akers 95R

Albrecht 95D

Albrecht $95 \mathrm{H}$

Alexander 95C

Artuso 95B

Asratyan 95

Bargende 95

Bartelt 95

Behnke 95

Buskulic 95C

Buskulic 95J

Buskulic 95L

Buskulic 95S

Derrick 95

Dubosca 95

Frabetti 95H

Frabetti 95I

Garbincius 95

Gardner 95

Gibaut 95

Kubota 95

Papadimitrio 95

Ressing 95

Ross 95

Skarha 95

Adamovich 94D

Akers 94B

Alam $94 \mathrm{C}$

Albrecht $94 \mathrm{~L}$

Asratyan 94

Athanas 94

Balest 94

Barish 94

Barreiro 94

Bergfeld 94B

Brisson 94B

Brown 94

Buskulic 94L

Colas 94

Danilov 94

Dejongh 94

Frabetti 94C

Frabetti 94E

Frabetti $94 \mathrm{~F}$

Frabetti 94G

Frabetti 94I

Johns 94

Kubota 94

Kuhlmann 94B

Leitch 94

Lewis 94B

Litvintsev 94

Moroni 94

Purohit 94

Skarha 94

Wormser 94

Abreu 93G

Albrecht 93D

Alves 93

Alves 93B

Buskulic 93D

Buskulic 93P

Cinabro 93B

Sakuda 93

\section{$D^{\mathbf{0}}$}

$K_{S} \pi^{\circ}$

$\bar{K}^{\circ} \rho^{0}$

Athanas 94

Dejongh 94

Wiss 96

$K^{-} \rho^{+}$

Frabetti 94D

Wiss 96

$K_{s} \rho^{\circ}$

Frabetti 94D

$\bar{K}^{\circ} \omega$

Wiss 96

$\bar{K}^{0} f_{0}(880)$

Ross 95

Wiss 96

$\bar{K}^{\mathrm{O}} f_{2}(1270)$

Frabetti $94 \mathrm{D}$

Wiss 96

$\bar{T}^{\circ}$ Frabetti 94D

Wiss 96

$K^{\circ} \bar{K}^{\circ} \quad$ Frabetti $94 \mathrm{D}$

Asner 96

Wiss 96

Frabetti 941

$K^{+} K^{-}$

Aitala $97 \mathrm{E}$

Asner 96

Bartelt 95

Ross 95

Adamovich 94D

Frabetti 94C

Frabetti 94F

$K^{*}(892)^{\circ} \gamma$

$\bar{K}^{*}(\mathbf{8 8 2})^{0} \pi^{\text {Asner } 98}$ Wiss 96

$K^{*}(882)^{-} \pi^{+}$

Frabetti 94D

Wiss 96

Frabetti $95 \mathrm{H}$

Asratyan 94

Frabetti 94D

$\bar{K}^{*}(892)^{\circ} \eta$

Semenov 94

$K^{*}(\mathbf{8 \theta 2})+K^{R}$

$K^{*}(\mathbf{8 9 2})^{0} \bar{K}^{\text {Wiss } 96}$

$\bar{K}^{*}(\mathbf{8 9 2})^{\circ} K^{\text {Wiss } 96}$

$K^{*}(892)-$ Wiss 96

Wiss 96

$K^{*}(892)^{\circ} \bar{K}^{*}(892)^{\circ}$

Aitala $97 \mathrm{~F}$

Wiss 96

Frabetti 95B

$K_{0}^{*}(1430)^{-} \pi^{+}$

Wiss 96

$\phi(1020)$

$\phi(1020) \pi^{0}$ Asner 98

$\phi(1020) \eta$

Albrecht 94J

$\phi(1020) \rho^{0}$

Albrecht $94 \mathrm{~J}$

Aitala $97 \mathrm{~F}$

Wiss 96

Frabetti 95B

Albrecht 94

Danilov 94

Semenoy 94

$\phi(1020) \omega$

$\bar{K}^{\circ} \phi(1020){ }_{\text {Wiss } 96}^{\text {Albrecht } 94 \mathrm{~J}}$
$D^{\mathbf{0}}$

$K_{S} \phi(1020)$

$\pi^{0} e^{-} e^{+}$

Bartelt 95

$\pi^{-} e^{+} \nu_{e}$

Freyberger 96

Frabetti 96

Semenov 96

Spaan 96

Butler 95

Freyberger 94

$\pi^{0} \mu^{-} e^{+}$

Freyberger 96

$\pi^{0} \mu^{+} e^{-}$

Freyberger 96

$\pi^{0} \mu^{-} \mu^{+}$

Freyberger 96

Kodama 95

$\pi^{-} \mu^{+} \nu_{\mu}$

$\eta e^{-} e^{+}$

Frabetti 96

$\eta \mu^{-} e^{+}$

Freyberger 96

Freyberger 96

$\eta \mu^{+} e^{-}$

Freyberger 96

$\eta \mu^{-} \mu^{+}$

$\rho^{\mathbf{o}} e^{-} e^{+}$

$\rho^{0} \mu^{-} e^{+}$

$\rho^{\mathbf{o}} \mu^{+} e^{-}$

$\rho^{0} \mu^{-} \mu^{+}$

$\omega e^{-} e^{+}$

$\omega \mu^{-} e^{+}$

$\omega \mu^{+} e^{-}$

$\omega \mu^{-} \mu^{+}$

$K^{-} e^{+} \nu$

$\bar{K}^{0} e^{-} e^{+}$

$K^{-} e^{+} \nu_{e}$

Aitala $96 \mathrm{E}$

Alexander $96 \mathrm{~S}$

Frabetti 96

Perasso 96

Semenov 96

Spaan 96

Butler 95

Freyberger 94

$\bar{K}^{\circ} \mu^{-} e^{+}$

Freyberger 96

$\bar{K}^{0} \mu^{+} e^{-}$

$\bar{K}^{\circ} \mu^{-} \mu^{+}$

$K^{-} \mu^{+} \nu_{\mu}$

Freyberger 96

Freyberger 96

Aitala $96 \mathrm{E}$

Alexander $96 \mathrm{~S}$

Frabetti 96

Perasso 96

Frabetti 95H

Freyberger 94

Gibaut 94B

Johns 94

Kodama 94

$\bar{K}^{*}(892)^{\circ} e^{-} e^{\dagger}$

Freyberger 96

$K^{*}(892)^{-} e^{+} \nu_{e}$

Freyberger 94

Freyberger 94

$D^{\mathbf{o}}$

$$
\begin{gathered}
\bar{K}^{*}(\mathbf{8 9 2})^{\circ} \mu^{-} e^{+} \\
\text {Freyberger } 96 \\
\bar{K}^{*}(\mathbf{8 9 2})^{\circ} \mu^{+} e^{-} \\
\text {Freyberger } 96 \\
\bar{K}^{*}(\mathbf{8 9 2})^{\circ} \mu^{-} \mu^{+} \\
\text {Freyberger } 96 \\
K^{*}(\mathbf{8 9 2})^{-} \mu^{+} \nu_{\mu} \\
\phi(\mathbf{1 0 2 0}) e^{-} e^{+} \\
\text {Freyberger } 96 \\
\phi(\mathbf{1 0 2 0}) \mu^{-} e^{+} \\
\text {Freyberger } 96 \\
\phi(\mathbf{1 0 2 0}) \mu^{+} e^{-} \\
\text {Freyberger } 96 \\
\phi(\mathbf{1 0 2 0}) \mu^{-} \mu^{+}
\end{gathered}
$$

$\bar{K}^{0} \pi^{+} \pi^{-}$

Freyberger 96

$$
K^{-} \pi^{+\pi^{\circ}} \quad \begin{aligned}
& \text { Ross } 95 \\
& \text { Abe } 96 \mathrm{E}
\end{aligned}
$$

Abreu 96C

Alexander 965

Barish 96

Bauer 96

Buskulic 96Q

Buskulic $96 \mathrm{R}$

Buskulic $96 \mathrm{~V}$

Lucchesi 96

Spaan 96

Wiss 96

Abe $952 Q$

Abreu $95 \mathrm{~S}$

Artuso 95B

Behnke 95

Buskulic 95C

Buskulic 95J

Duboseq 95

Gardner 95

Gibaut 95

Ross 95

Akers 94B

Alam 94C

Albrecht $94 U$

Athanas 94

Colas 94

Dejongh 94

Frabetti 94D

King 94

Kubota 94

Skarha 94

Alves 93

Alves 93B

Buskulic 93P

Sakuda 93

nonres $\left\langle K^{-} \pi^{+} \pi^{0}>\right.$

Wiss 96

$K_{S} \pi^{+}$

Frabetti 94D

Bai 96

Buskulic $96 \mathrm{Q}$

Buskulic $96 \mathrm{~W}$

Wiss 96

Asratyan 95

Buskulic 95S 


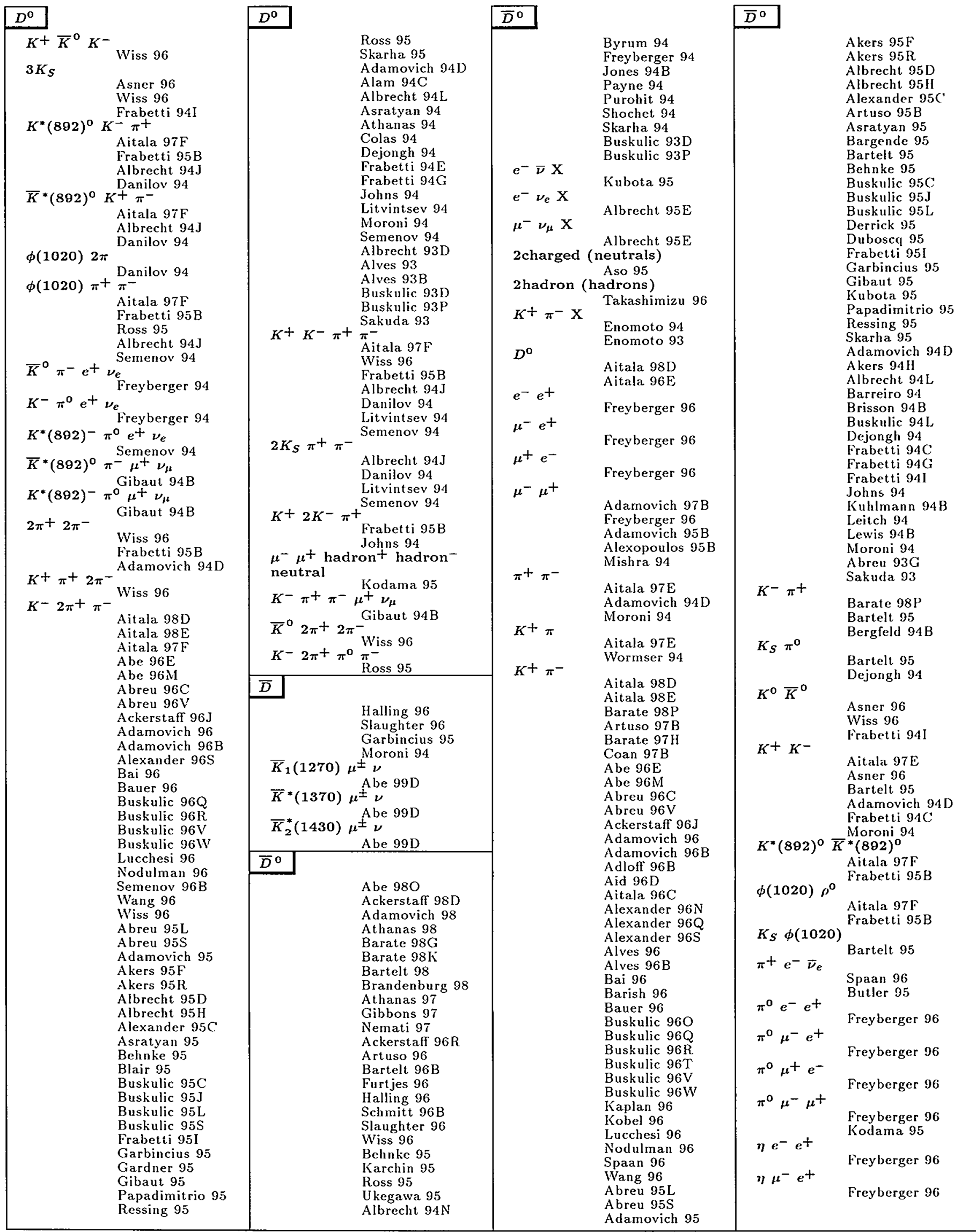


$\eta \mu+e^{-}$

$\eta \mu^{-} \mu^{+}$

$\rho^{0} e^{-} e^{+}$

$\rho^{0} \mu^{-} e^{+}$

$\rho^{0} \mu+e^{-}$

$\rho^{0} \mu^{-} \mu^{+}$

$\omega e^{-} e^{+}$

$\omega \mu^{-} e^{+}$

$\omega \mu+e^{-}$

$\omega \mu^{-} \mu^{+}$

$K^{+} e^{-} \bar{\nu}$

$K^{+} e^{-} \bar{\nu}_{e}$

Kubota 95

Aitala $96 \mathrm{E}$

Alexander $96 \mathrm{~S}$

Perasso 96

Spaan 96

$K^{0} e^{-} e^{+}$

Butler 95

$K^{0} \mu^{-} e^{+}$

Freyberger 96

$K^{0} \mu^{+} e^{-}$

Freyberger 96

$K^{+} \mu^{-} \bar{\nu}_{\mu}$

Freyberger 96

Aitala $96 \mathrm{E}$

Alexander $96 \mathrm{~S}$

Perasso 96

Johns 94

$K^{0} \boldsymbol{\mu}^{-} \mu^{+}$

Freyberger 96

$K^{*}(892)^{0} e^{-} e^{t}$

Freyberger 96

$K^{*}(802)^{0} \mu^{-} e^{+}$

Freyberger 96

$K^{*}(892)^{0} \mu^{+} e^{-}$

Freyberger 96

$K^{*}(802)+\mu^{-} \bar{\nu}_{\mu}$

$K^{*}(892)^{\circ} \mu^{-} \mu^{+}$

Freyberger 96

$\phi(1020) e^{-} e^{+}$

Freyberger 96

$\phi(1020) \mu^{-} e^{t}$

$\phi(1020) \mu^{+} e^{-}$

Freyberger 96

$\phi(1020) \mu^{-} \mu^{+}$

Freyberger 96

Freyberger 96

$K^{+} \pi^{0} \pi^{-}$

Abe $96 \mathrm{E}$

Abreu $96 \mathrm{C}$

Alexander 96S

Barish 96

Bauer 96

Buskulic 96Q

Buskulic 96R

Buskulic 96V

Lucchesi 96

Spaan 96

Abreu $95 \mathrm{~S}$

Artuso 95B

Buskulic 95C

Buskulic 95 J

Duboscq 95

Gibaut 95

Dejongh 94

\section{$\bar{D}^{\mathbf{0}}$}

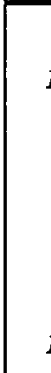

$\begin{array}{ll}K^{+} K^{-} \pi^{0} & \text { Asner } 96 \\ \mathbf{2} K_{S} \pi^{\mathbf{0}} & \text { Asner } 96 \\ \mathbf{3} K_{S} & \text { Asner } 96 \\ & \text { Frabetti } 94\end{array}$

$K^{*}(892)^{\circ} K^{-} \pi^{+}$

$\bar{K}^{*}(892)^{\circ} K^{+} \pi^{-}$

$\begin{array}{ll} & \text { Aitala } 97 \mathrm{~F} \\ & \text { Frabetti } 95 \mathrm{~B}\end{array}$

$\phi(1020) \pi^{+} \pi^{-}$

$\pi^{-}$Aitala $97 \mathrm{~F}$

$2 \pi^{+} 2 \pi^{-} \quad$ Frabetti 95B

$K^{+}+2 \pi^{-}$Adamovich 94D

Aitala $98 \mathrm{D}$

Aitala $98 \mathrm{E}$

Aitala $97 \mathrm{~F}$

A be $96 \mathrm{E}$

Abe $96 \mathrm{M}$

Abreu 96C

Abreu $96 \mathrm{~V}$

Ackerstaff $96 \mathrm{~J}$

Adamovich 96

Adamovich $96 \mathrm{~B}$

Alexander $96 \mathrm{~S}$

Bai 96

Bauer 96

Buskulic 96Q

Buskulic 96R

Buskulic 96V

Buskulic 96W

Lucchesi 96

Nodulman 96

Wang 96

Abreu 95L

Abreu 95S

Adamovich 95

A kers 95F

A kers 95R

Albrecht 95D

Albrecht $95 \mathrm{H}$

Alexander 95C

Asratyan 95

Blair 95

Buskulic 95C

Buskulic 95J

Buskulic 95L

Frabetti 95I

Garbincius 95

Gibaut 95

Papadimitrio 95

Ressing 95

Skarha 95

Adamovich 94D

Albrecht 94L

Albrecht $94 \mathrm{U}$

Dejongh 94

Frabetti 94G

Moroni 94

Sakuda 93

$K^{+} K^{-} \pi^{+} \pi^{-}$

Aitala 97F

Frabetti 95B

$2 K^{+} K^{-} \pi^{-}$

Frabetti 95B

$\mu^{-} \mu^{+}$hadron+ hadronneutral

Kodama 95

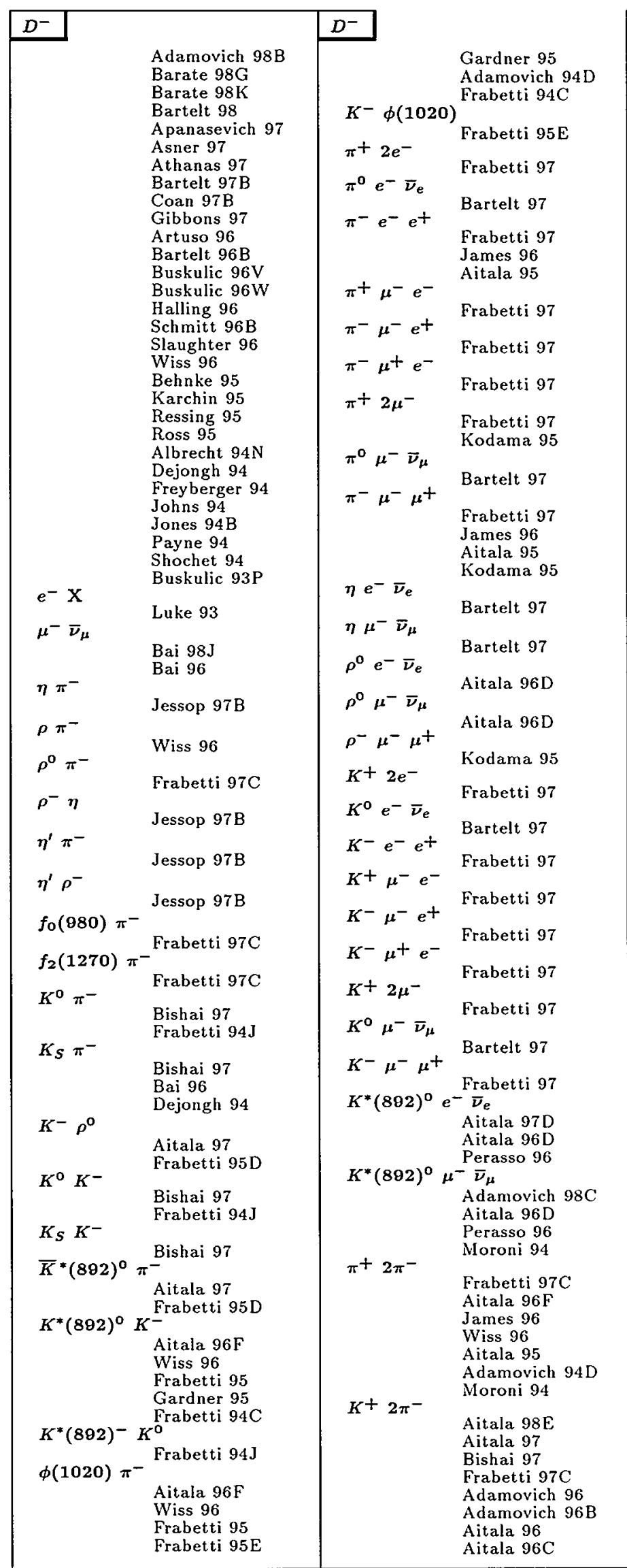

Entries in order of particle name, then decay. A few chemical symbols for nuclei have been changed to avoid ambiguity with particle names (see the Particle Vocabulary). See the legend on page 323. 


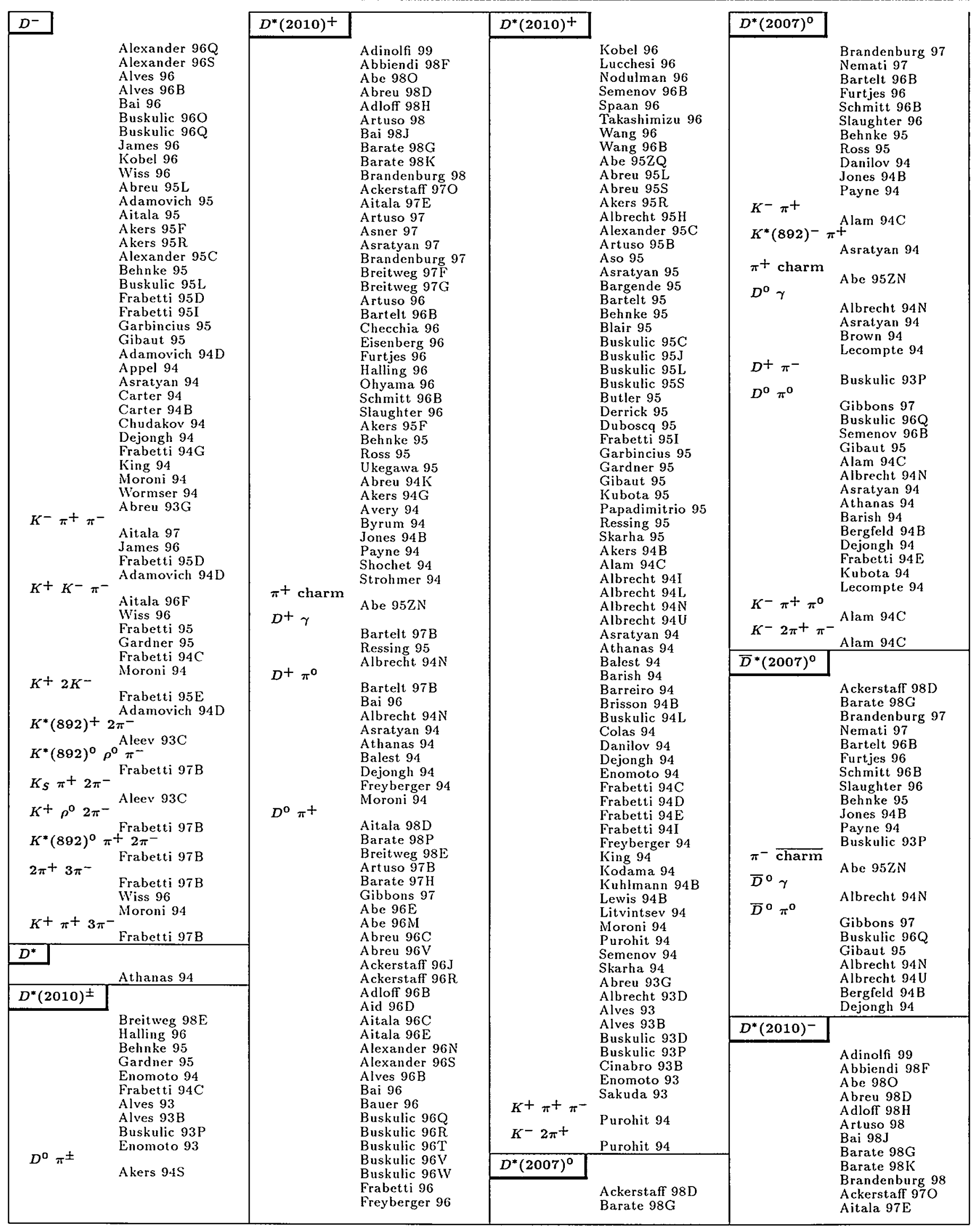




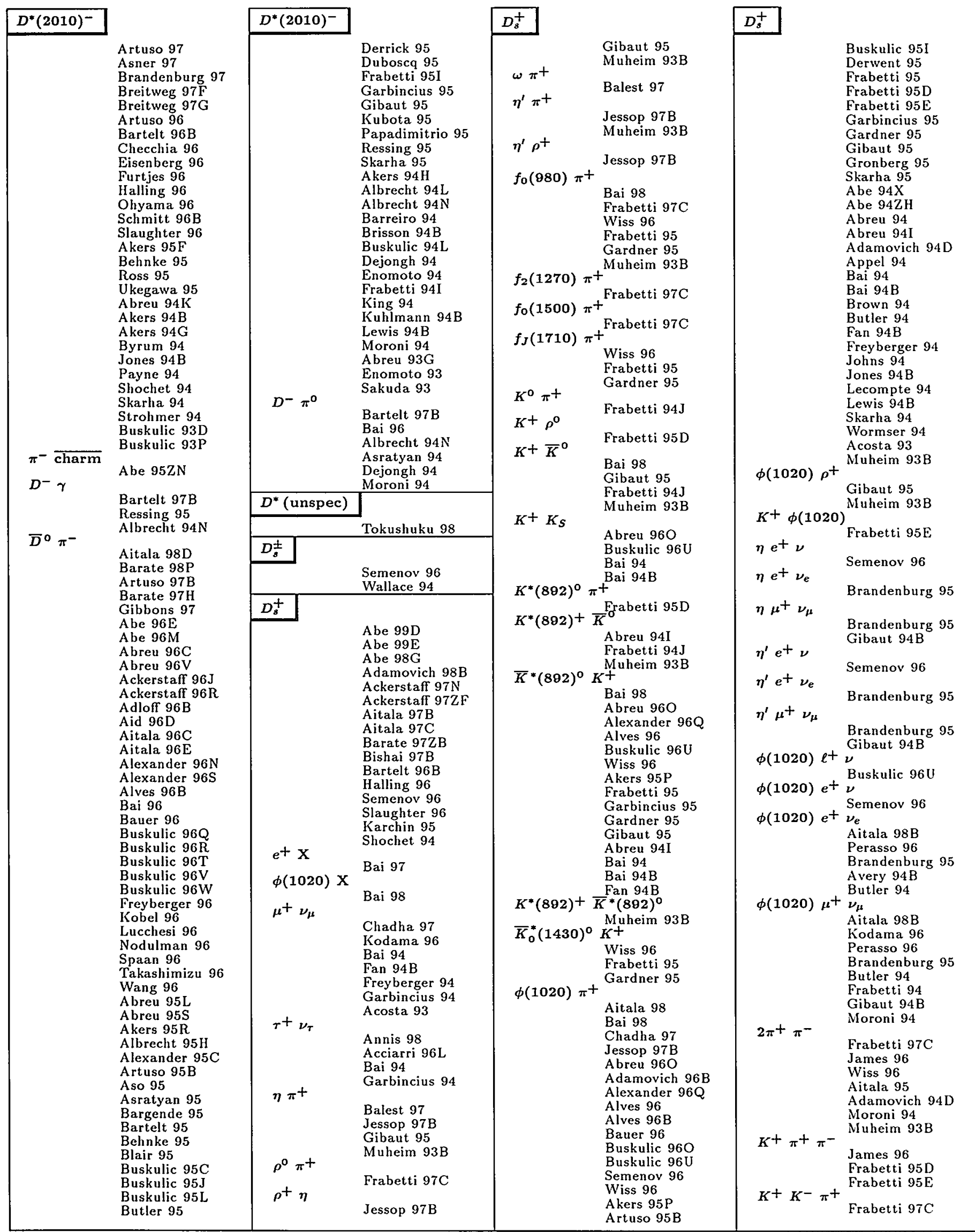

Entries in order of particle name, then decay. A few chemical symbols for nuclei have been changed to avoid ambiguity with particle names (see the Particle Vocabulary). See the legend on page 323. 


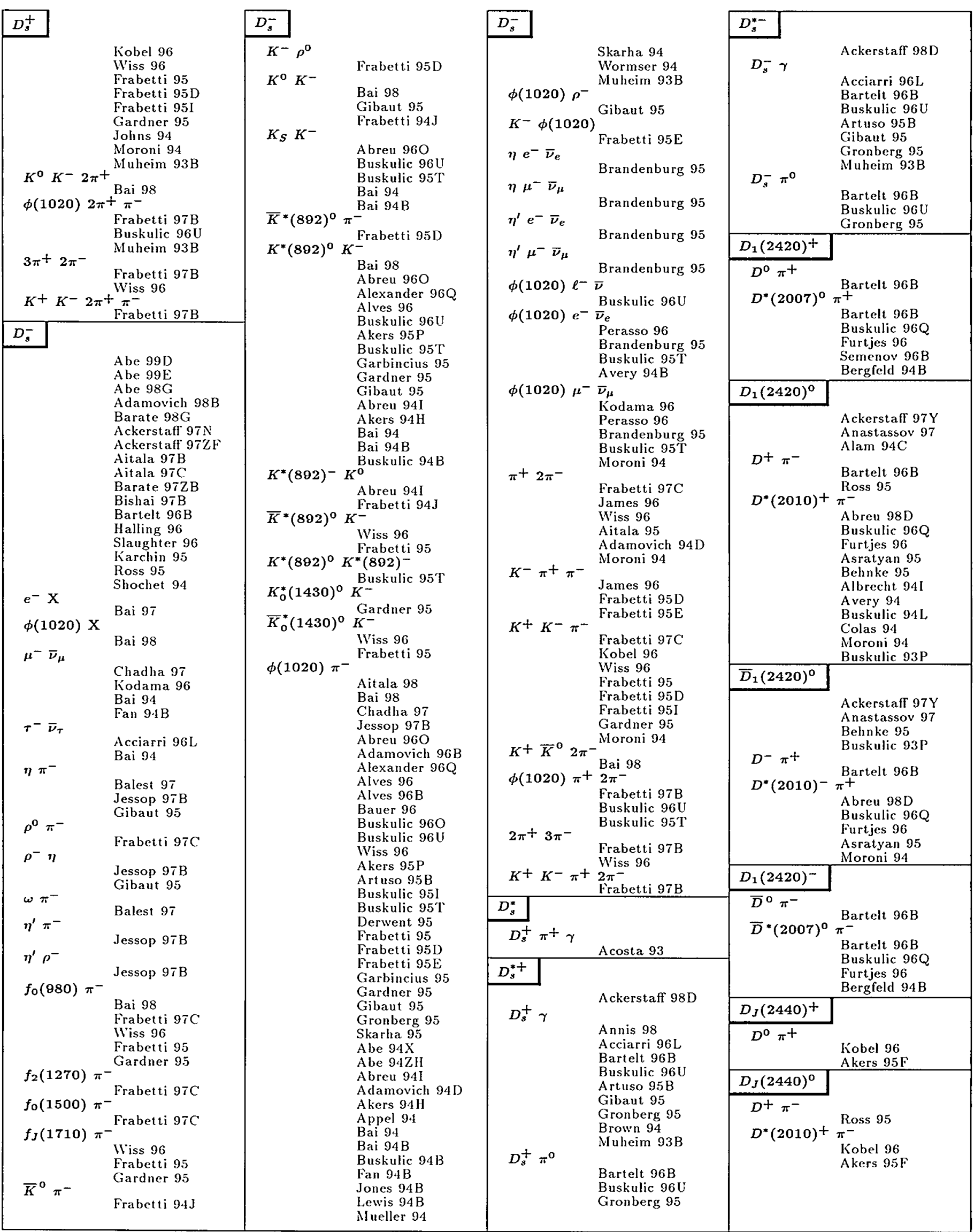




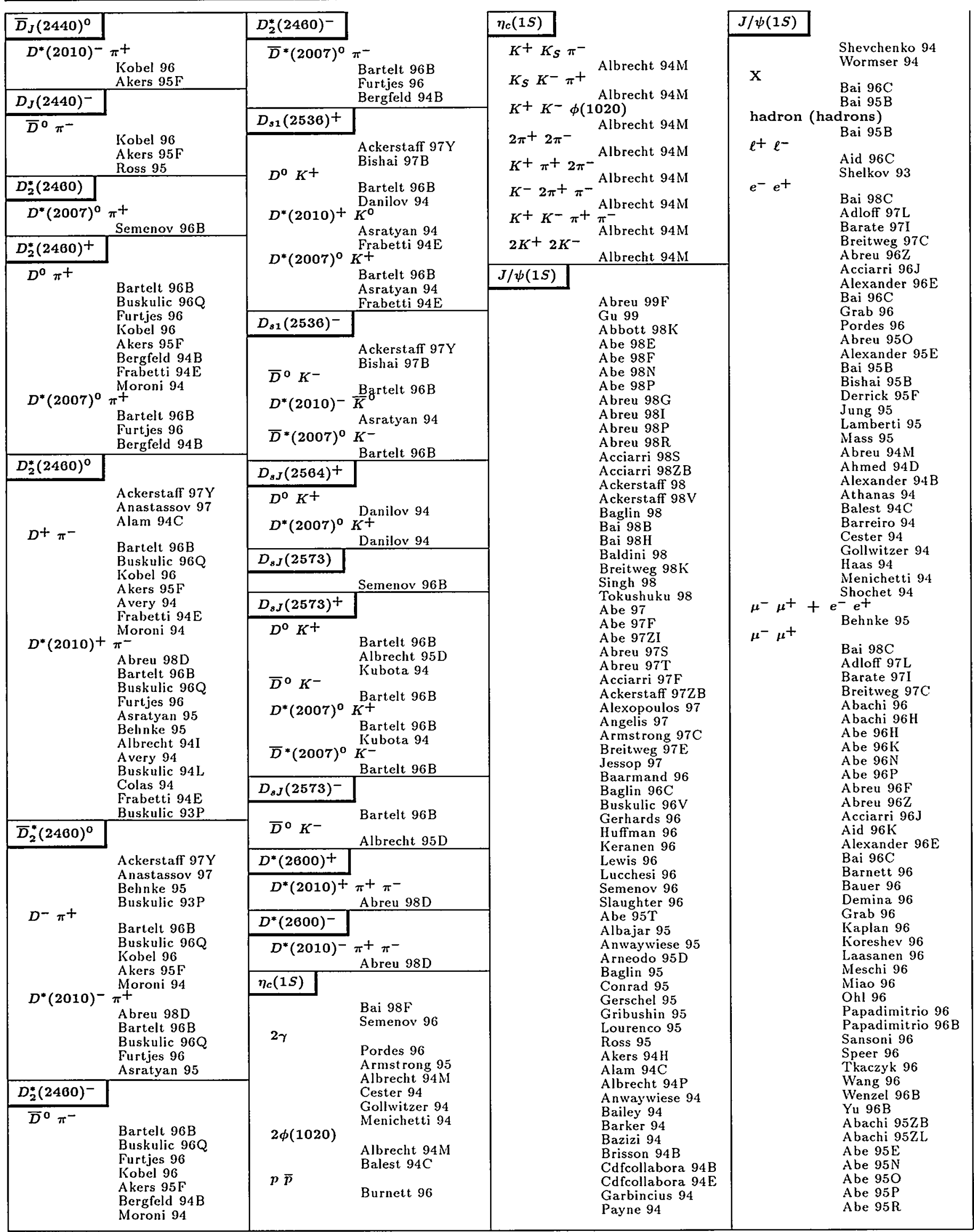

Entries in order of particle name, then decay. A few chemical symbols for nuclei have been changed to avoid ambiguity with particle names (see the Particle Vocabulary). See the legend on page 323. 


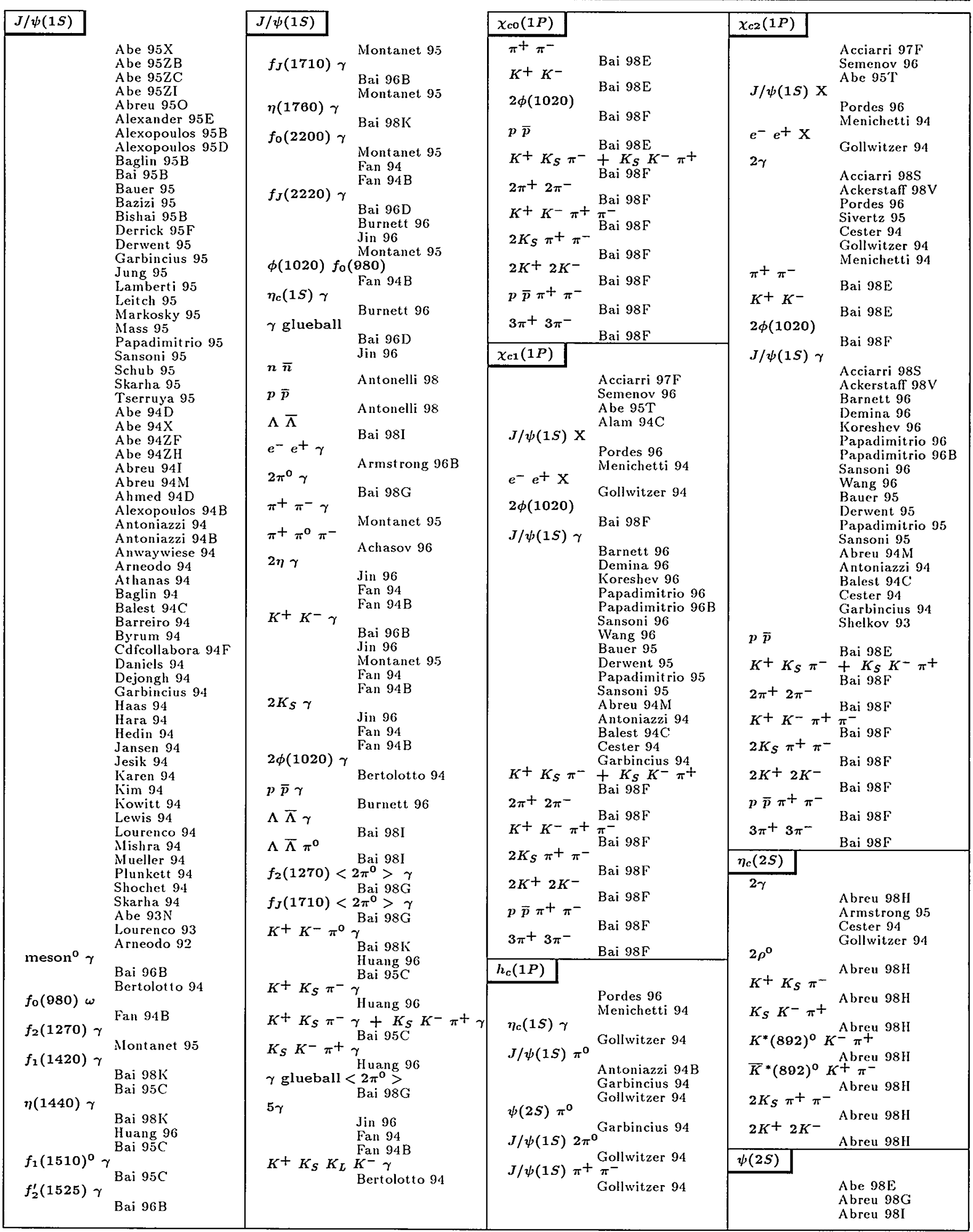




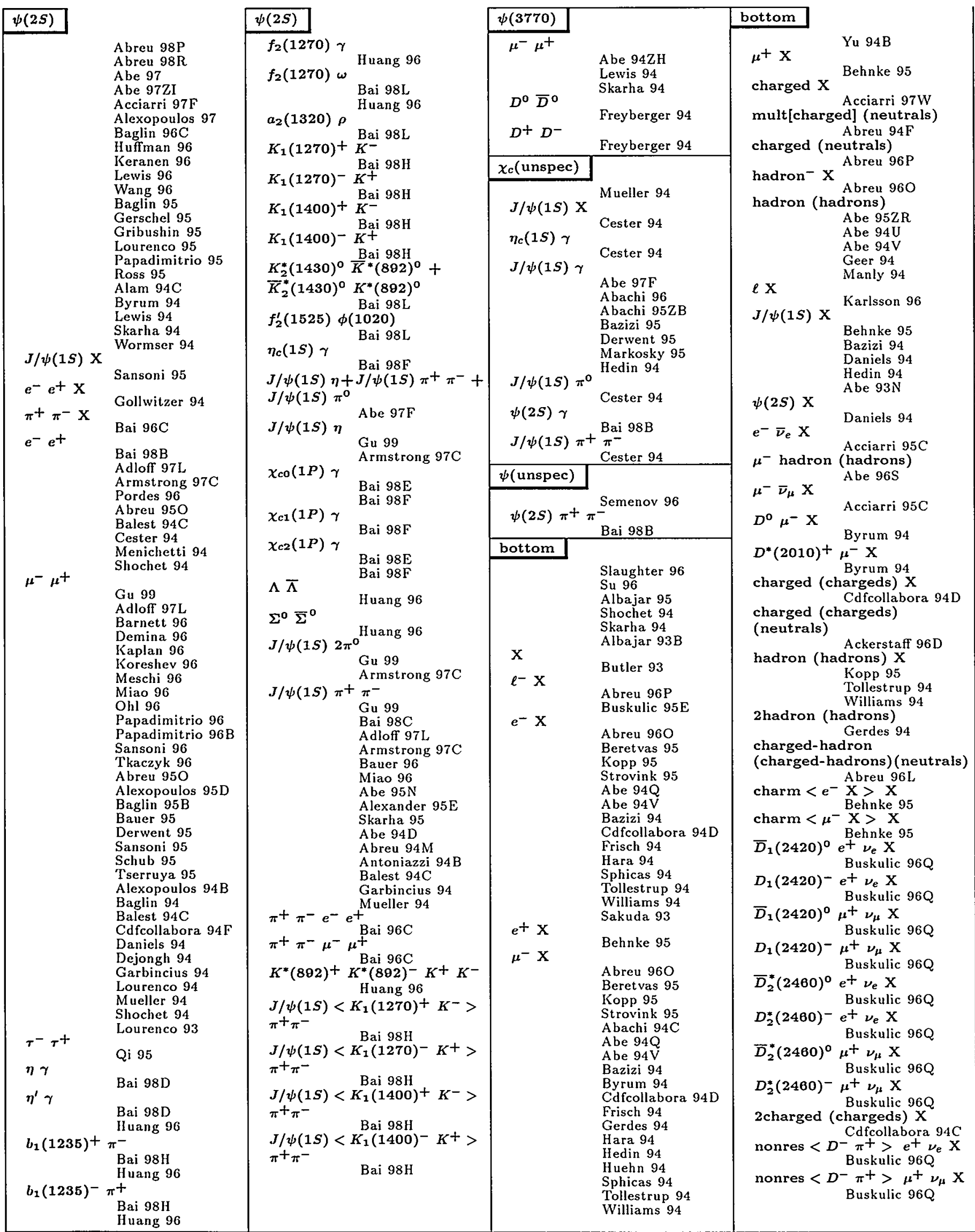

Entries in order of particle name, then decay. A few chemical symbols for nuclei have been changed to avoid ambiguity with particle names (see the Particle Vocabulary). See the legend on page 323. 


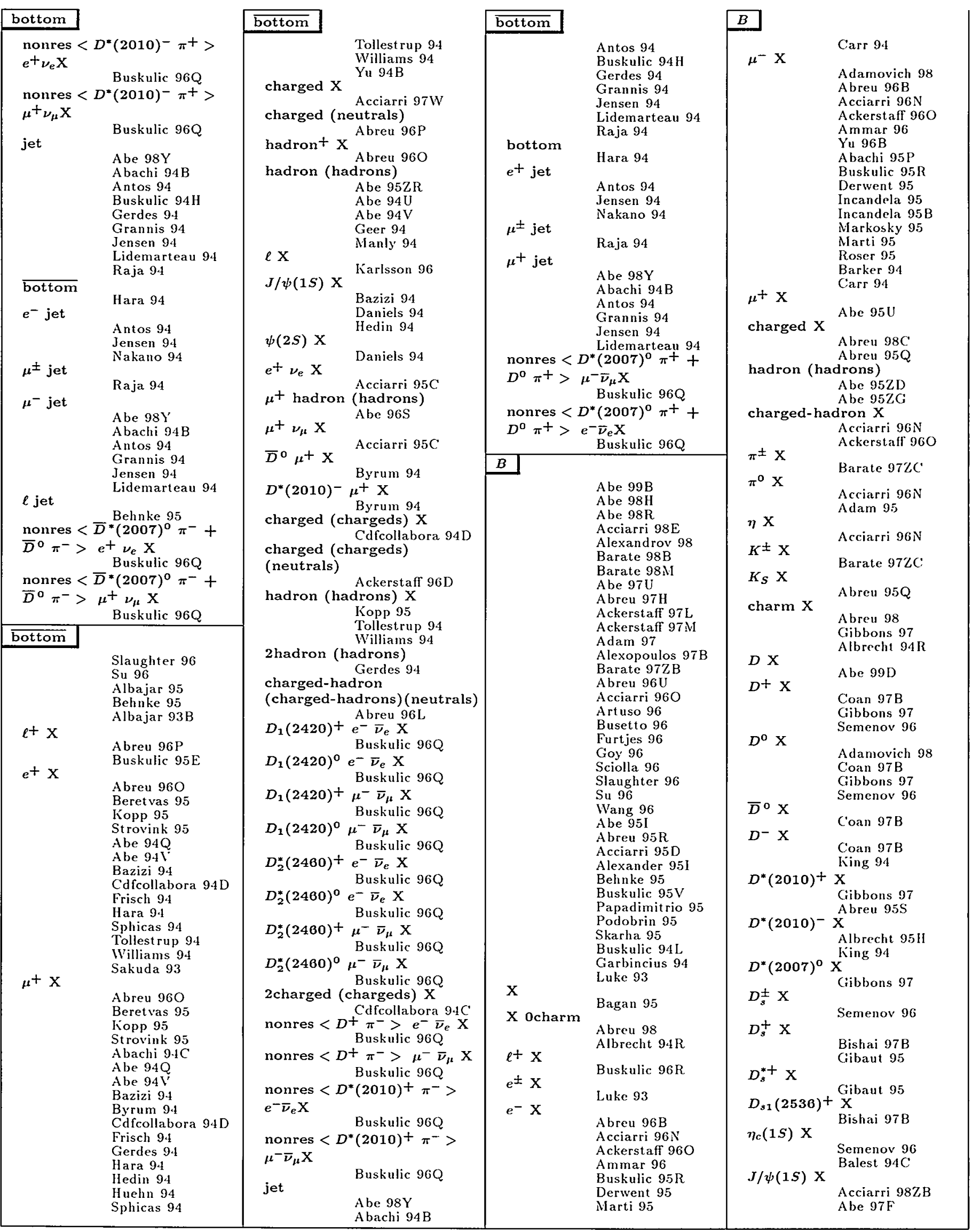


$\chi_{c 1}(1 P) \times$

Demina 96

Papadimitrio 96B

Semenov 96

Bauer 95

Balest $94 \mathrm{C}$

$\chi_{c 2}(1 P) \mathrm{X}$

Demina 96

Papadimitrio 96B

Semenov 96

Bauer 95

Balest 94C

$\psi(2 S) \mathrm{X}$

Oh! 96

Alexander $95 \mathrm{E}$

Bauer 95

Derwent 95

$\psi$ (unspec) $\mathrm{X}$

Balest 94C

$\mathrm{X} \quad$ Semenov 96

$\bar{p} \mathrm{X} \quad$ Barate $97 Z \mathrm{C}$

$\Lambda \mathrm{X} \quad$ Barate $97 Z \mathrm{C}$ Abreu 95Q

$\Lambda_{c} \mathrm{X}$

Semenov 96

$\Lambda_{c}^{+} \mathrm{X}$

Ammar 96

Fujino 95

$\bar{\Lambda}_{c}^{-} \mathrm{X}$

Bonvicini 97B

Ammar 96

$\Sigma_{c}(2455)++X$

$\Sigma_{c}(2455)+\mathrm{X}$

Procario 93

Procario 93

Procario 93

$\Xi_{c}^{+} \mathrm{X}$

Semenov 96

$\Xi_{c}^{0} \mathbf{X}$

Fujino 95

Semenov 96

$\Xi_{c}^{0} \mathrm{X}$

Fujino 95

$\Xi_{c}^{-} \mathrm{X}$

Barish 97

$e^{-\bar{\nu} \mathrm{X}}$

Barish 97

$e^{+} \nu_{e} \mathrm{X}$

Barislı 95

Wang 96B

Bagan 95

$e^{-} e^{+x}$

$\mu^{-}$jet $X$

Balest 94C

$\mu^{+} \nu_{\mu} \mathrm{X}$

Markosky 95

$\mu^{-} \mu^{+} \mathrm{X}$

Wang 96B

Abbott 98B

Abachi 96I

Balest 94C
$B$

$D^{+} e^{-\mathrm{X}}$

$D^{0} e^{-\mathrm{X}}$

A kers $95 \mathrm{~F}$

$D^{+} \mu^{-\mathrm{X}}$

Akers $95 \mathrm{~F}$

$D^{0} \mu^{-} \mathrm{X}$

Akers $95 \mathrm{~F}$

$D^{*}(\mathbf{2 0 1 0})+$ Akers $95 \mathrm{~F}$

Akers 95F

$D^{*}(2010)^{+} \mu^{-} \mathrm{X}$

Akers 95F

$D_{J}(2440)+e^{-X}$

$D_{J}(\mathbf{2 4 4 0})^{\circ} e^{-\mathrm{X}}$

$D_{J}(2440)+{ }^{-}{ }^{-} \mathrm{X}$ (kers $95 \mathrm{~F}$

$D_{J}(\mathbf{2 4 4 0})^{\circ}$ A kers $95 \mathrm{~F}$

Akers 95F

$D_{2}^{*}(2480)+e^{-} \mathrm{X}$

$D^{*}(2460)^{\circ}$ Akers $95 \mathrm{~F}$

Akers 95F

$D_{2}^{*}(2460)+\mu^{-} \mathrm{X}$

Akers $95 \mathrm{~F}$

$D_{2}^{*}(2480)^{\circ} \mu^{-} \mathrm{X}$

$\bar{\Lambda}_{c}^{-} e^{+} \mathrm{X}$

Akers $95 \mathrm{~F}$

Bonvicini $97 \mathrm{~B}$

charged (chargeds) $X$ Buskulic 95R

Wormser 94

charged (chargeds)

(neutrals)

Buskulic 96R Akers $95 \mathrm{X}$

hadron (hadrons) $\mathrm{X}$

$2 \phi(1020) X$ Abreu 95Q

Balest $94 \mathrm{C}$

charm charm $X$

$D^{\mathrm{o}} \bar{D}^{\mathrm{o}} \mathrm{X}$

$D^{+} D^{-} \mathrm{X}$ Barate 98G

Barate $98 \mathrm{G}$

$D^{\circ} D^{-} \mathrm{X} \quad$ Barate $98 \mathrm{G}$

$D^{*}(2010)+D^{-} \mathrm{X}$

Barate 98G

$D^{*}(2010)^{-} D^{+} \mathrm{X}$

$D^{*}(2010)-D^{0}$ Xate $98 \mathrm{G}$

Barate $98 \mathrm{G}$

$D^{*}(2010)+D^{*}(2010)^{-} \mathrm{X}$

$D_{s}^{-} D^{+} \mathrm{X}$

Barate $98 \mathrm{G}$

$D_{s}^{-} D^{\mathrm{o}} \mathrm{X}$

Barate 98G

Barate $98 \mathrm{G}$

$D_{s}^{-} D^{*}(2010)^{+} \mathrm{X}$

$J / \psi(1 S) \gamma \mathrm{X}$

Barate $98 \mathrm{G}$

$\bar{\Lambda}_{c}^{-} p \mathrm{X}$

Balest 94C

Bonvicini 97B

$e^{-} \bar{\nu}$ hadron (hadrons)

Ocharm

Acciarri 98P Barate 98J

$\mu^{-} \bar{\nu}$ hadron (hadrons)

Ocharm

Acciarri 98P

Barate $98 \mathrm{~J}$

kaon $\left(\pi^{\prime} s\right) e^{-} e^{+}$

Glenn 97

kaon $\left(\pi^{\prime} s\right) \mu^{-} e^{+}$ Glenn 97

kaon $\left(\pi\right.$ 's) $\mu^{+} \mathrm{e}^{-}$ Glenn 97
$B$

kaon ( $\left.\pi^{\prime} s\right) \mu^{-} \mu^{+}$

$\bar{D}^{\circ} e^{+} \nu_{e} \mathrm{x}^{\text {Glenn } 97}$

$D^{-} e^{+} \nu_{e} \mathrm{X}^{\text {Coan 97B }}$

$\bar{D}^{\circ} \mu^{+} \nu_{\mu} \mathrm{X}^{\text {Coan 97B }}$

$D^{-}+\nu_{\mu} \mathrm{x}^{\text {Coan 97B }}$

D+ ${ }^{-} e^{-} \mathrm{x}^{\text {Coan 97B }}$

$D^{+} \pi^{-} e^{-} \mathrm{X}$ A kers 95F

$D^{\circ} \pi^{+} e^{-\mathrm{X}}$ Akers 95F

$D^{+} \pi^{-} \mu^{-} \mathrm{X}$

$D^{\circ} \pi^{+} \mu^{-} \mathrm{x}^{\text {Akers } 95 \mathrm{~F}}$

$D^{*}(2010)-\ell^{+}$Akers 95F

Artuso $97 \mathrm{~B}$

$D^{*}(\mathbf{2 0 1 0})+e^{-\bar{\nu}_{e} X}$

Abreu 96C

$D^{*}(2010)+\mu^{-} \bar{\nu}_{\mu} \mathrm{X}$

$D^{*}(2010)+\pi^{-} e^{-} \mathrm{X}$

Akers 95F

$D^{*}(\mathbf{2 0 1 0})+\pi^{-} \mu^{-} \mathrm{X}$

3charged-hadron $X$

$J / \psi(15) \pi+\frac{\text { Adamovich } 98}{\pi-X}$

Balest $94 \mathrm{C}$

$\pi^{+} \pi^{-} e^{-} e^{+} \mathrm{X}$

Balest $94 \mathrm{C}$

$\pi^{+} \pi^{-} \mu^{-} \mu^{+}$X

$2 K^{+} K^{-} \mathrm{x}$ Balest 94C

$2 K^{-} \mathrm{X}$ Balest $94 \mathrm{C}$

jet

Incandela 95 Incandela $95 \mathrm{~B}$

Roser 95

$\mu^{-}$jet

Abachi $95 \mathrm{M}$

Abachi 95P

Bazizi 95

Derwent 95

$\mu^{+}$jet

Abachi 95R

$\Sigma_{c}(\mathbf{2 4 5 5})^{\circ} \frac{\mathrm{Abachi}}{\text { nucleon }}$

Procario 93

kaon $\pi \gamma$

Alam 94B

$K_{S} \pi \gamma$

Alam 94B

kaon $2 \pi \gamma$

Alam $94 \mathrm{~B}$

kaon $\pi^{0} \pi \gamma$

Alam $94 \mathrm{~B}$

$K_{S} 2 \pi \gamma$

$K_{S} \pi^{\circ} \pi$ Alam 94B

Alam $94 \mathrm{~B}$

kaon $3 \pi \gamma$ Alam $94 \mathrm{~B}$

kaon $\pi^{\circ} 2 \pi \gamma$

Alam $94 \mathrm{~B}$

$K_{S} 3 \pi \gamma$

Alam 94B

$K_{S} \pi^{\circ} 2 \pi \gamma$ Alam 94B

kaon $4 \pi \gamma$ Alam $94 \mathrm{~B}$

kaon $\pi^{\circ} 3 \pi \gamma$

Alam $94 \mathrm{~B}$

$K_{S} 4 \pi \gamma$

Alam $94 \mathrm{~B}$

$K_{S} \pi^{0} 3 \pi \gamma$

$B^{ \pm}$ Alam 94B

Akers $94 \mathrm{R}$
$B^{ \pm} J / \psi(1 S)$

\begin{tabular}{|l|}
\multicolumn{2}{|c}{ Jesik 9 } \\
\hline$B^{+}$
\end{tabular}

Abe $98 \mathrm{ZC}$

Acciarri $98 \mathrm{X}$

Barate 98

Abe 972B

Ackerstaff $97 \mathrm{ZH}$

Coan 97B

Ackerstaff $96 \mathrm{R}$

Furtjes 96

Slaughter 96

A breu $95 \mathrm{H}$

Albrecht 95H

Ross 95

Akers 94R

Albrecht 94I

Barish 94

Garbincius 94

Buskulic 93D

$\ell^{+} \mathrm{X}$

Jackson 96

$e^{+} \mathrm{X}$

Abe 97B

Artuso 97

$\mu+\mathrm{x}$

Abe $97 \mathrm{~B}$

Artuso 97

hadron (hadrons)

Jackson 96

Dejongh 94

$\boldsymbol{\eta}^{\prime} \mathrm{X}$

$K+\mathrm{x}$

Browder 98

$K^{-} \mathrm{X}$

Albrecht $94 \mathrm{R}$

$K_{S} \mathrm{X}$

Albrecht 94R

$J / \psi(1 S) \times$ Albrecht 94R

Garbincius 94

$\ell^{+} \nu \mathrm{X}$

Schmitt 96B

charmed-meson $\ell^{ \pm} \mathrm{X}$

$\bar{D}^{\circ} \ell^{+} \mathrm{X}$

$D^{0} e^{+} \mathrm{X}$

$\bar{D}^{0} e^{+} \mathrm{X}$

Behnke 95

Lewis $94 \mathrm{~B}$

Shochet 94

$\bar{D}^{\circ} \mu^{-} \mathrm{X}$ Wormser 9

$\bar{D}^{\circ}{ }_{\mu}+\mathrm{X}$

Lewis $94 \mathrm{~B}$

Dejongh 94

Wormser 94

$D^{*}(2010)^{-} e^{+} \mathrm{X}$

Buskulic 96V Lewis $94 \mathrm{~B}$

$D^{*}(2010)^{-} \mu^{+} \mathrm{X}$

Buskulic 96V

Lewis $94 \mathrm{~B}$

$D^{*}(2007)^{\circ} e^{+} \mathrm{X}$

Jones 94B

charged (chargeds)

(neutrals)

Abe $96 \mathrm{U}$

Abe 96ZE

Abe $96 \mathrm{ZF}$

$K^{+} e^{+} \nu \mathrm{X}$

dam 95B

Albrecht 94 $\mathrm{R}$

$K^{-} \ell^{+} \nu \mathrm{X}$ Albrecht $94 \mathrm{R}$

$K_{S} \ell^{+} \nu \mathrm{X}$

Entries in order of particle name, then decay. A few chemical symbols for nuclei have been changed to avoid ambiguity with particle names (see the Particle Vocabulary). See the legend on page 323. 


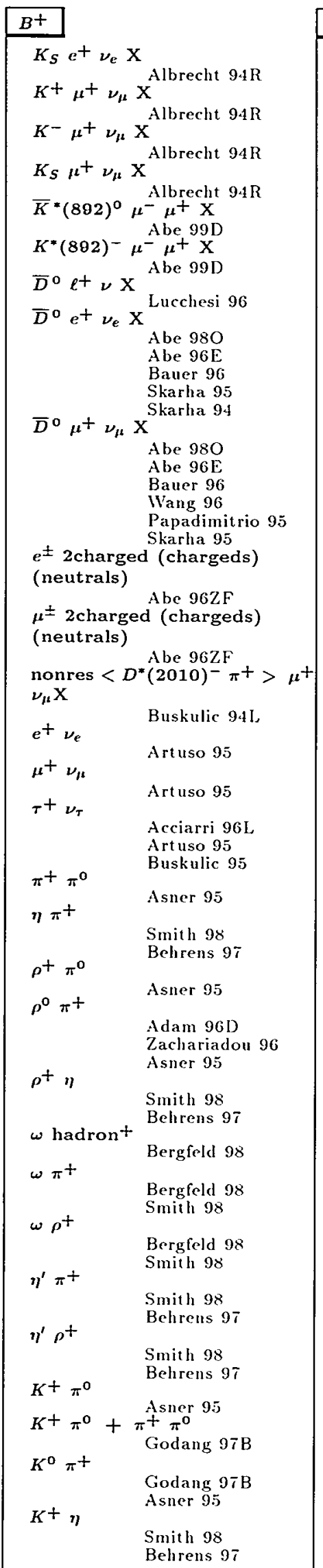

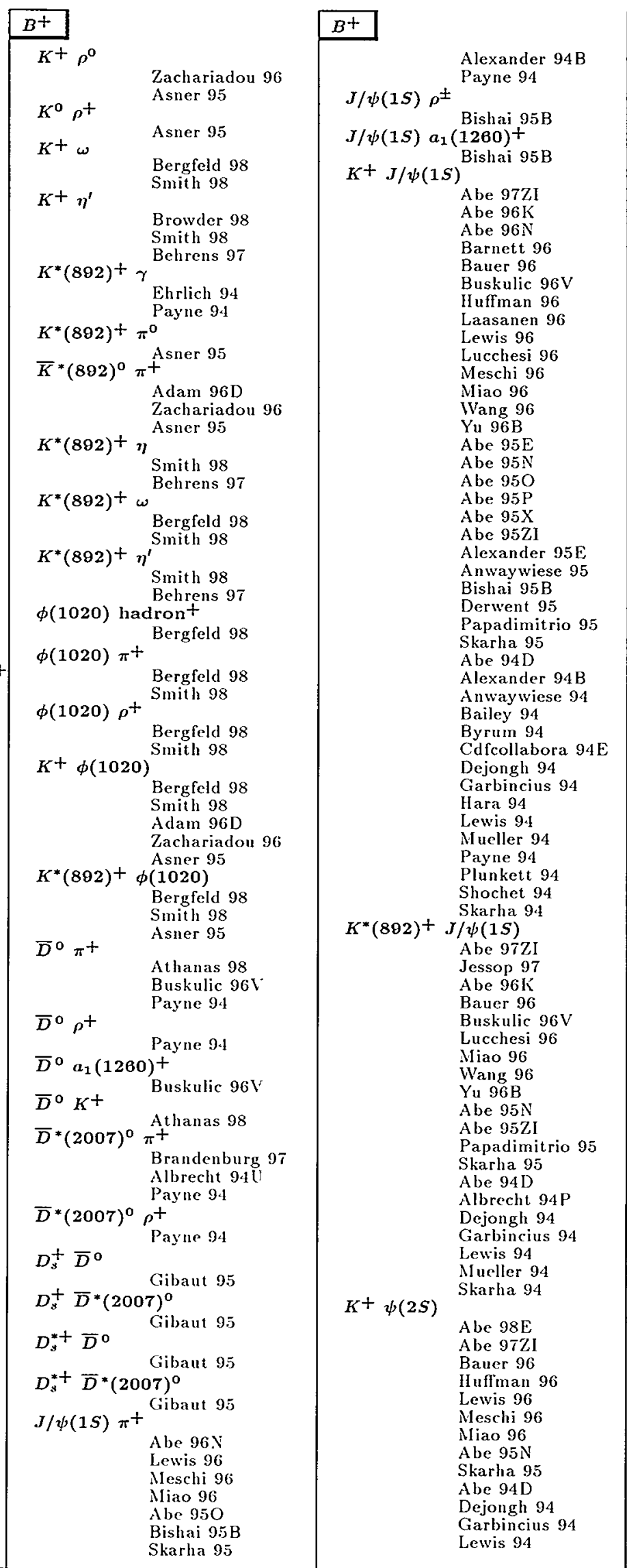

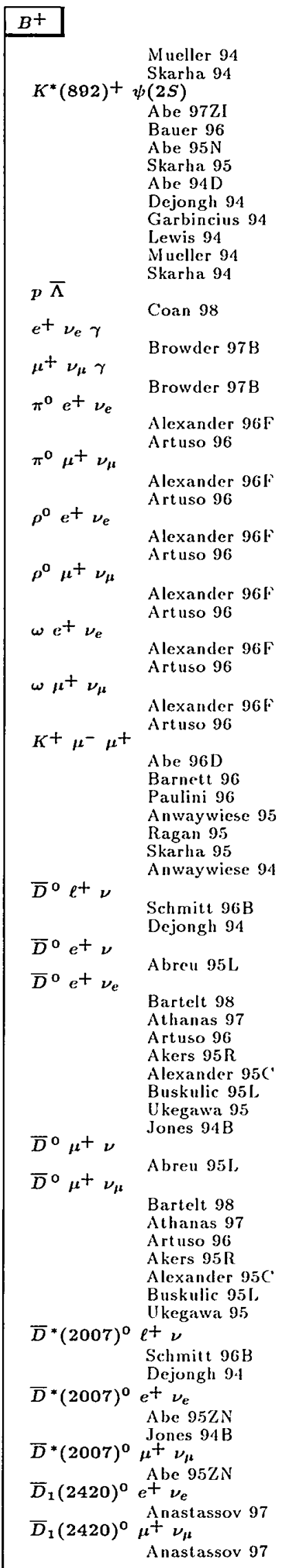




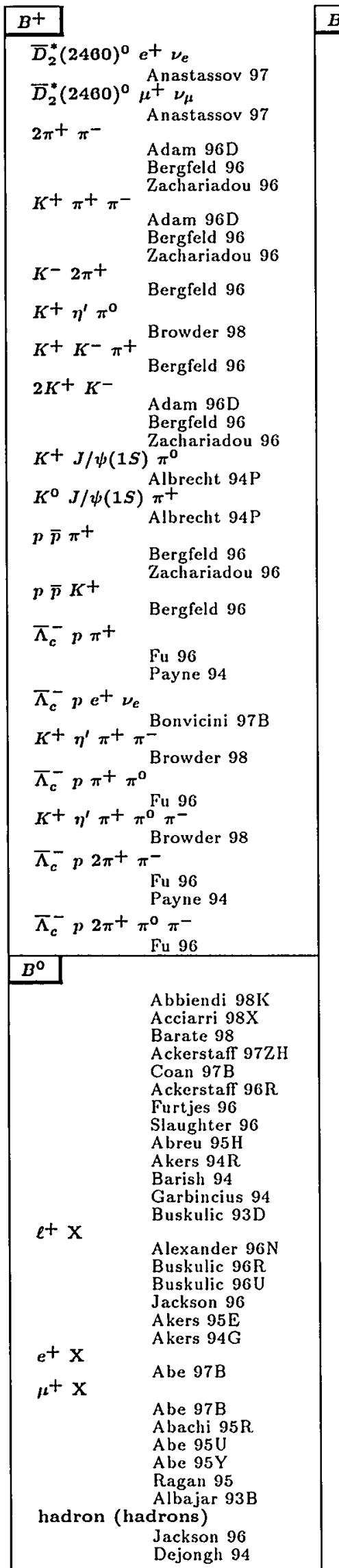

$B^{\mathbf{0}}$

$\eta^{\prime} \mathrm{X}$

$K^{+} \mathrm{X}$

Browder 98

$K^{-} \mathrm{X}$

Albrecht 94R

$K_{S} \mathrm{X}$

Albrecht 94R

$D^{*}(2010)-\mathrm{X}$

Albrecht 94R

Buskulic 96R

Albrecht $95 \mathrm{H}$

A breu $94 \mathrm{~K}$

$J / \psi(1 S) \mathrm{X}$

A kers 94G

Q+ $\nu \mathrm{X}$ Garbincius 94

$e^{+}$charged $X$

Abreu $96 \mathrm{U}$

$e^{+} \nu_{e} \mathrm{X}$

$\mu^{+}$charged $X$

Wang $96 \mathrm{~B}$

$\mu^{+} \nu_{\mu} \mathrm{X}$

Abreu $96 \mathrm{U}$

Wang $96 \mathrm{~B}$

charmed-meson $\ell^{ \pm} \mathrm{X}$

$\bar{D}^{\mathrm{a}} e^{-\mathrm{X}}$

A be $96 \mathrm{~T}$

$\bar{D}^{\circ} e^{+\mathrm{X}}$

Lewis $94 \mathrm{~B}$

$D^{-} e^{+} \mathrm{X}$

Lewis $94 \mathrm{~B}$

$\bar{D}^{0} \mu^{+} \mathrm{X}$

Jones 94B

Wormser 94

$D^{-} \mu^{+} \mathrm{X}$

Lewis $94 \mathrm{~B}$

$\mu+x$

Dejongh 94

$D^{*}(2010)^{-} e^{+} \mathrm{X}$

Abreu $96 \mathrm{~V}$

Buskulic 96V

Jones 94B

Lewis $94 \mathrm{~B}$

Shochet 94

$D^{*}(2010)^{-} \mu^{+} \mathrm{X}$

Abreu 96V

Buskulic 96V

Dejongh 94

Lewis $94 \mathrm{~B}$

charged (chargeds)

(neutrals)

Abe $96 \mathrm{ZE}$

charged-hadron

(charged-hadrons) $\mathrm{X}$

$K^{+}$charged $X$

Buskulic $96 \mathrm{U}$

Abreu 96U

$\ell^{-}$charged (chargeds)

(neutrals)

$e^{ \pm}$charged (chargeds)

(neutrals)

Abe 962F

(neutrals)

Buskulic 96F

(neutrals)

Abe 96ZF

$\mu^{+}$hadron (hadrons) $\mathrm{X}$

Ragan 95

$\mu^{-}$hadron (hadrons)

(neutrals)

Buskulic 96F

$K^{-} \ell^{+} \nu \mathrm{X}$ Albrecht 94R
$B^{\mathbf{0}}$

$K_{S} \ell^{+} \nu \mathrm{X}$

$K^{+} e^{+} \nu_{e} \mathrm{X}^{\text {Albrecht } 94 \mathrm{R}}$

$K^{-} e^{+} \nu_{e} \mathrm{X}^{\text {Albrecht } 94 \mathrm{R}}$

$K_{S} e^{+} \nu_{e} \mathrm{X}$ Albrecht 94R

$K_{S} e^{+} \nu_{e} \mathrm{X}$ Albrecht $94 \mathrm{R}$

$K^{+} \mu^{+} \nu_{\mu} \mathrm{X}$ Albrecht $94 \mathrm{R}$

$K^{-} \mu^{+} \nu_{\mu} \mathrm{X}^{\text {Albrecht } 94 \mathrm{R}}$

$K_{S} \mu^{+} \nu_{\mu} \mathrm{X}$ Albrecht 94R

$K^{*}(892)+\mu-\mu+\mathrm{X}$

$K^{*}(892)^{0} \mu^{-} \mu^{+}{ }^{-} \mathrm{X}$

Abe $99 \mathrm{D}$

jet $\left\langle D^{*}(2010)^{-} \ell^{+} \mathrm{X}\right\rangle$

$D^{*}(2010)^{-} \ell^{+} \nu \mathrm{X}$

Alexander $96 \mathrm{~N}$ Lucchesi 96

$D^{*}(2010)+e^{-} \bar{\nu}_{e} X$

Skarha 95

$e^{+} \nu_{e} \mathrm{X}$

A be 980

Abe $96 \mathrm{E}$

Abreu $96 \mathrm{C}$

Bauer 96

Skarha 94

$D^{*}(2010)+\mu^{-} \bar{\nu}_{\mu} \mathrm{X}$

Wang 96

Papadimitrio 95

Skarha 95

$D^{*}(2010)^{-} \mu^{+} \nu_{\mu} \mathrm{X}$

Abe 980

Abe $96 \mathrm{E}$

Abreu 96C

2charged (chargeds)

(neutrals)

A be $96 \mathrm{U}$

Adam 95B

$\mathrm{K}^{-}$charged (chargeds)

(neutrals)

$\bar{B}^{\circ}$

Abe $96 \mathrm{~W}$

Abe 99B

Abe $98 \mathrm{P}$

A be $98 \mathrm{ZC}$

Acciarri 98E

Acciarri 98V

Ackerstaff 98

A be $97 \mathrm{ZB}$

Abreu $97 \mathrm{H}$

Ackerstaff $97 \mathrm{~L}$

Ackerstaff $97 \mathrm{M}$

Abe $96 \mathrm{~S}$

Abe $96 \mathrm{~V}$

Abe $96 \mathrm{~W}$

Abreu $96 \mathrm{U}$

Acciarri 960

Alexander $96 \mathrm{~N}$

Barnett 96

Buset to 96

Buskulic 96F

Buskulic $96 \mathrm{R}$

Checchia 96

Lucchesi 96

Abachi 95R

A be $95 \mathrm{U}$

Abe $95 \mathrm{Y}$

A kers $95 \mathrm{E}$

Albrecht $95 \mathrm{H}$

Alexander $95 \mathrm{H}$

Behnke 95

Derwent 95

Ragan 95

Skarha 95

Abreu 94J

Abreu $94 \mathrm{~K}$
$B^{\circ}$

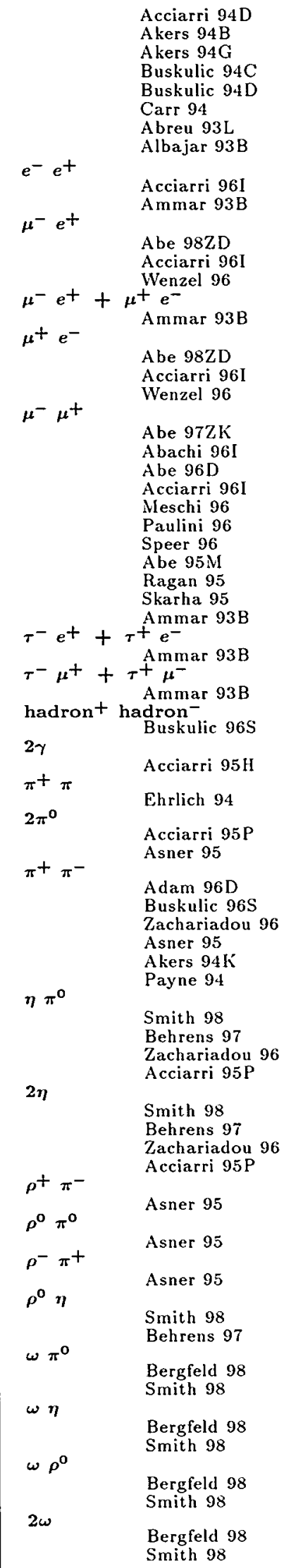

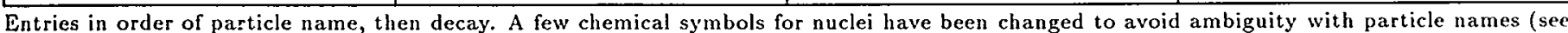
the Particle Vocabulary). See the legend on page 323. 


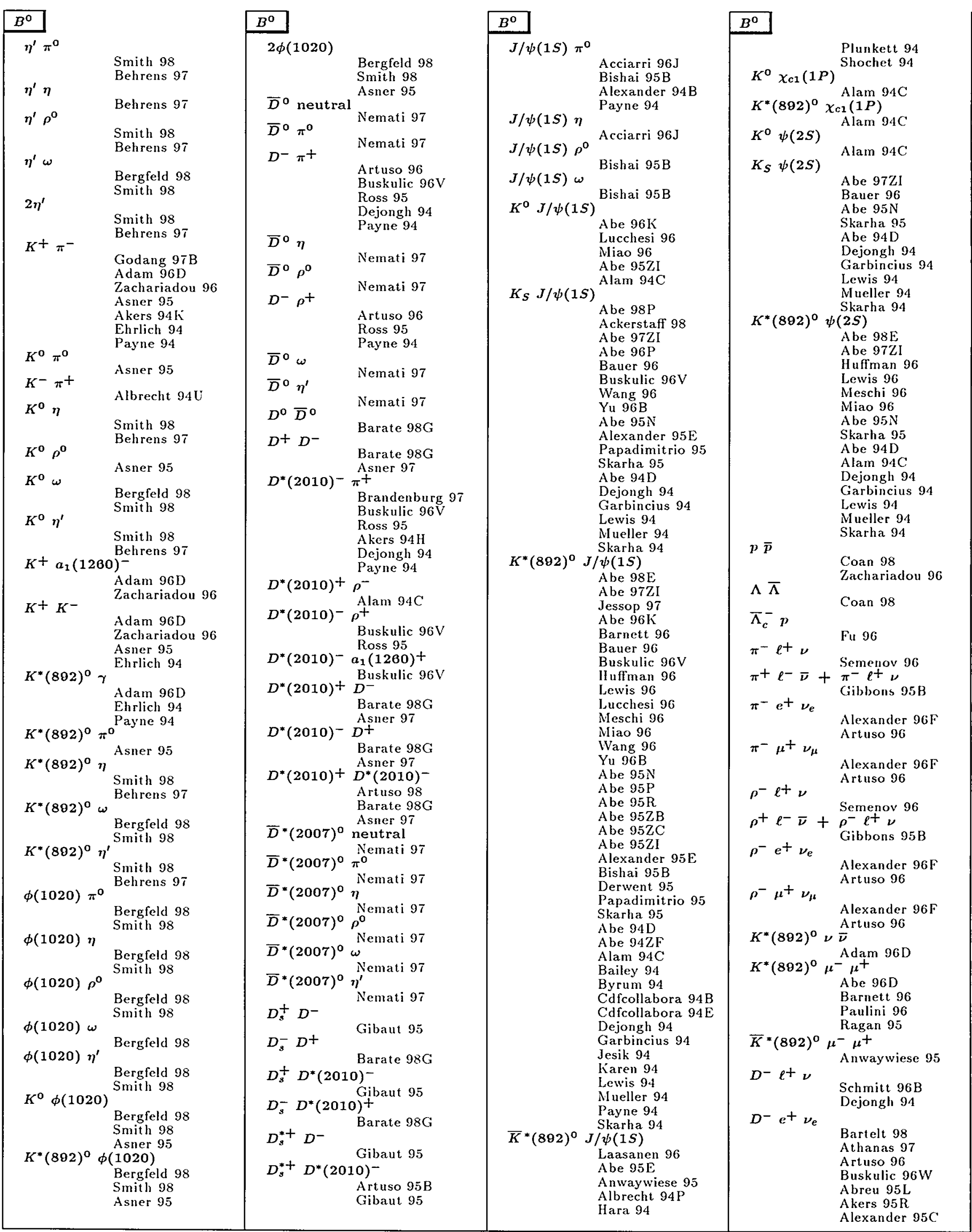




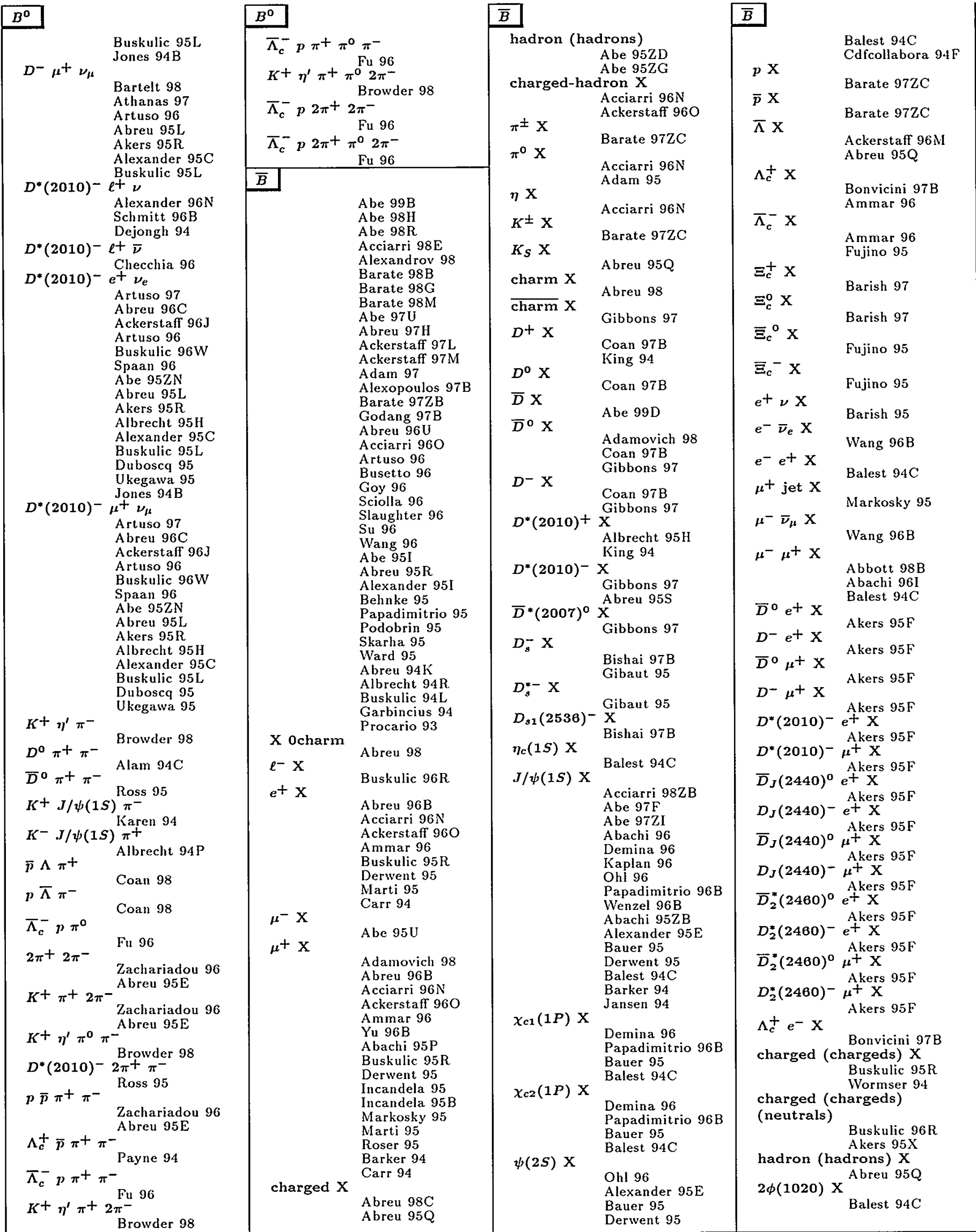

Entries in order of particle name, then decay. A few chemical symbols for nuclei have been changed to avoid ambiguity with particle names (see the Particle Vocabulary). See the legend on page 323. 
$\bar{B}$

charm charm $X$

$J / \psi(1 S) \gamma$ Abreu 98

Balest $94 \mathrm{C}$

$\Lambda_{c}^{+} \bar{p} \mathrm{X} \quad$ Bonvicini $97 \mathrm{~B}$

$e^{+} \nu$ hadron (hadrons)

Ocharm

Acciarri 98P

Barate 98J

${ }^{+}+\nu$ hadron (hadrons)

Ocharm

Acciarri $98 \mathrm{P}$

Barate $98 \mathrm{~J}$

kaon ( $\left.\pi^{\prime} s\right) \mathrm{e}^{-} \mathrm{e}^{+}$

Glenn 97

kaon ( $\left.\pi^{\prime} s\right) \mu^{-} \mathrm{e}^{+}$

Glenn 97

kaon $\left(\pi^{\prime} s\right) \mu^{+} e^{-}$

Glenn 97

kaon $\left(\pi\right.$ 's) $\mu^{-} \mu^{+}$

$D^{+} e^{-} \bar{\nu}_{e} \mathrm{X}^{\text {Glenn } 9 \bar{\tau}}$

$D^{\mathrm{o}} e^{-\bar{\nu}_{e}} \mathrm{X}^{\text {Coan } 9 \tau \mathrm{B}}$

Coan $97 \mathrm{~B}$

$D^{+} \mu^{-} \bar{\nu}_{\mu k} \mathrm{X}$

Bauer 96

$D^{0} \mu^{-} \bar{\nu}_{\mu} \mathrm{X}^{\operatorname{Coan} 97 \mathrm{~B}}$

$\bar{D}^{\circ} \pi^{-} e^{+} \mathrm{X}$

$\pi$ X $e^{+}$kers $95 \mathrm{~F}$

$D^{-} \pi^{+} e^{+} \mathrm{X}$

$\bar{D}^{0}-\mathrm{A}^{-}+\mathrm{X}^{\text {Akers } 95 \mathrm{~F}}$

$D^{-}+\mathrm{X}^{\text {Akers } 95 \mathrm{~F}}$

Akers $95 \mathrm{~F}$

$D^{*}(2010)+\ell^{-} \bar{\nu} \mathrm{X}$

D*(2010)- Artuso 97B

Abreu $96 \mathrm{C}$

$D^{*}(2010)^{-} \mu^{+} \nu_{u} \mathrm{X}$

Abreu 960

$D^{*}(2010)^{-} \pi^{+} e^{+} \mathrm{X}$

$D^{*}(\mathbf{2 0 1 0})^{-} \pi^{+} \mu^{+}{ }^{+} X$

Akers $95 \mathrm{~F}$

3charged-hadron $X$

Adamovich 98

$+{ }^{-}$Balest 94C

$\pi^{+} \pi^{-} e^{-} e^{+} \mathrm{X}$

$\pi^{+} \pi^{-} \mu^{-} \mu^{+} \mathrm{X}$

Balest $94 \mathrm{C}^{\circ}$

$2 K^{+} 2 K^{-} \mathrm{X}_{\text {Balest } 94 \mathrm{C}}$

jet

Incandela 95

Incandela $95 \mathrm{~B}$

Roser 95

$\mu^{-}$jet

Abachi $95 R$

$\mu^{+}$jet

Abachi 95.11

Abachi 95P

Bazizi 95

Derwent 95

kaon $\pi \gamma$

Alam 94B

$K_{S} \pi \gamma$

Alam 9.4B

kaon $2 \pi \gamma$

kaon $\pi^{0} \pi \gamma$

Alam 94B

$K_{S} 2 \pi \gamma \quad$ Alam $94 \mathrm{~B}$

$K_{S} \pi^{\circ} \pi \gamma$ Alam 94B

Alam 94B
$\bar{B}$

kaon $3 \pi \gamma$

Alam 94B

$\gamma$

Alam 94B

$K_{S} 3 \pi \gamma \quad$ Alam $94 \mathrm{~B}$

$K_{S} \pi^{\mathrm{o}} 2 \pi \gamma \quad \begin{aligned} & \text { Alam 94B } \\ & \text { Alam 94B }\end{aligned}$

kaon $4 \pi \gamma$ Alam 94B

kaon $\pi^{0} 3 \pi \gamma$

$K_{S} 4 \pi \quad$ Alam 94B

$K_{S} \pi^{0} 3 \pi \gamma$ Alam 94B

Alam 94B

\begin{tabular}{|l|l} 
& (neutrals) \\
& Abe $96 \mathrm{ZE}$ \\
A be $96 \mathrm{~F}$
\end{tabular}

Abbiendi $98 \mathrm{~K}$

Barate 98

Smith 98

Ackerstaff $97 \mathrm{ZII}$

Coan 97B

Ackerstaff $96 \mathrm{R}$

Furtjes 96

Slaughter 96

Abreu $95 \mathrm{H}$

Acciarri $95 \mathrm{H}$

Behnke 95

Acciarri 94D

$A$ kers $94 \mathrm{R}$

Buskulic 94C

Buskulic 94 D

Garbincius 94

Lewis $94 \mathrm{~B}$

$\ell^{-} \mathrm{X}$

Abreu 93L

Alexander $96 \mathrm{~N}$

Buskulic 96R

Buskulic 96U

Jackson 96

Akers $95 \mathrm{E}$

Akers $94 \mathrm{G}$

$e^{-x}$

$\mu^{-\mathrm{X}}$

Abe 97B

Abe $97 \mathrm{~B}$

Abachi 95R

Abe $95 \mathrm{U}$

Abe $95 \mathrm{Y}$

Ragan 95

Albajar 93B

hadron (hadrons)

Jackson 96

Dejongh 94

$\eta^{\prime} \mathrm{X}$

$K^{-} \mathrm{x}$

$D^{*}(2010)+$

Albrecht $95 \mathrm{H}$

Buskulic 96R

Albrecht $95 \mathrm{H}$

Abreu $94 \mathrm{~K}$

$J / \psi(1 S) \times$

Akers $94 \mathrm{G}$

Garbincius 9

$e^{-}$charged $\mathrm{X}$

$-\bar{\nu}_{0} \mathrm{X}$ Abreu $96 \mathrm{U}$

Ross 95

$\mu^{-}$charged $\mathrm{X}$

$\mu^{-} \bar{\nu}_{\mu} \mathrm{X}$ Abreu $96 \mathrm{U}$

Ross 95

charmed-meson $\ell^{ \pm} \mathrm{X}$

$D^{+} \ell^{-} \mathrm{X}$

$D^{+} e^{-} \mathrm{X}$

$\bar{B}^{\circ}$

$D^{+} \mu^{-} \mathrm{X}$

$D^{*}(2010)+\ell^{-} \mathrm{X}$

Dejongh 94

Behnke 95

$D^{*}(2010)+e^{-X}$

Abreu $96 \mathrm{~V}$

Buskulic 96V

Jones $94 \mathrm{~B}$

$D^{*}(2010)^{+} \mu^{-} \mathrm{X}$

$\mu^{-} \mathrm{X}$

Buskulic 96V

Dejongh 94

charged-hadron

(charged-hadrons) $\mathrm{X}$

$K^{-}$charged $\mathrm{X}$ Buskulic $96 \mathrm{U}$

Abreu 96U

$\ell^{+}$charged (chargeds)

(neutrals)

$e^{ \pm}$charged (chargeds)

(neutrals)

$e^{+}$hadron (hadrons)

(neutrals)

$\mu^{ \pm}$charged (chargeds)

(neutrals)

Abe 96ZF

$\mu$ hadron (hadrons)

$\mu^{+}$hadron (hadrons) (neutrals)

Buskulic $96 \mathrm{~F}$

$K^{*}(892)^{0} \mu^{-} \mu^{+} \mathrm{X}$

$K^{*}(892)-A$ be $99 \mathrm{D}$

$A$ be $99 \mathrm{D}$

jet $\left\langle D^{*}(2010)^{+} \ell^{-} \mathrm{X}\right\rangle$

$D^{*}(2010)+\ell^{-} \bar{\nu}$ crs 94

Alexander $96 \mathrm{~N}$

Lucchesi 96

$D^{*}(2010)+e^{-\bar{\nu}_{e} \mathrm{X}}$

A be 980

Abe $96 \mathrm{E}$

Abreu 96C

Baver 96

Skarha 91

$D^{*}(2010)^{-} e^{+} \nu_{e} \mathrm{X}$

$D^{*}(\mathbf{2 0 1 0})+\mu^{-} \bar{\nu}_{\mu} \mathrm{X}$

A be 980

Abe $96 \mathrm{E}$

Abreu 96C

Bauer 96

$D^{*}(2010)-\mu^{+} \nu_{u k} \mathrm{X}$

Wang 96

Papadimitrio 95

Skarha 95

2charged (chargeds)

(neutrals)

Abe $96 \mathrm{U}$

Adam 95B

$K^{+}$charged (chargeds)

(neutrals)

$B$

A be $96 W^{r}$

\begin{tabular}{l|l} 
Behnke 95 & $B^{0}$
\end{tabular}

Jones $94 \mathrm{~B}$

Wormser 94

$\bar{B}^{0}$

Acciarri $98 \mathrm{~V}$

Ackerstaff 98

A be $97 Z B$

Abreu $97 \mathrm{H}$

Ackerstaff 97L

Ackerstaff $97 \mathrm{M}$

A be $96 \mathrm{~S}$

A be $96 \mathrm{~V}$

A be $96 \mathrm{~W}$

Abreu $96 \mathrm{U}$

Alexander $96 \mathrm{~N}$

Barnett 96

Buset to 96

Buskulic 96F

Buskulic 96R

Checchia 96

Lucchesi 96

Abachi 95R

Abe $95 \mathrm{U}$

Abe $95 \mathrm{Y}$

A kers 95E

Albrecht 95I

Alexander $95 \mathrm{H}$

Behnke 95

Derwent 95

Ragan 95

Skarha 95

Abreu 94J

Akers 94B

Akers $94 \mathrm{G}$

Carr 94

$\overleftarrow{B}$

Abreu $94 \mathrm{~K}$

$e^{-} e^{+}$

Acciarri 96I

$\mu^{-} e^{+}$

A be $98 Z \mathrm{D}$

Acciarri 96I

Wenzel 96

$\mu^{+} e^{-}$

Abe $98 \% \mathrm{D}$

Acciarri 96I

Wenzel 96

$\mu^{-} \mu^{+}$

Abe $972 \mathrm{~K}$

Abachi 961

Abe 96D

Acciarri 96

Meschi 96

Paulini 96

Speer 96

Abe $95 \mathrm{M}$

Ragan 95

Skarha 95

hadron ${ }^{+}$hadron-

$\pi^{+} \pi$

$2 \pi^{\circ}$

$\pi^{+} \pi^{-}$

Buskulic $96 \mathrm{~S}$

Ehrlich 94

Asner 95

Adam 96D

Buskulic 96S

Zachariadon 96

Asner 95

A kers $94 \mathrm{~K}$

Payne 94

$\eta \pi^{\mathrm{o}}$

Bchrens 97

Zachariadou 96

$2 \eta$

Behrens 97

Zachariadou 96

$\rho^{+} \pi^{-}$

Asner 95

$\rho^{0} \pi^{0}$

Asner 95

$\rho^{-} \pi^{+}$

Asner 95

$\rho^{\circ} \eta$

Behrens 97

$\omega \pi^{\mathrm{o}}$

Bergfeld 98 


\begin{tabular}{|c|c|c|c|c|c|c|c|}
\hline $\bar{B}^{0}$ & & $\bar{B}^{\circ}$ & & $\bar{B}^{\circ}$ & & $\bar{B}^{\circ}$ & \\
\hline 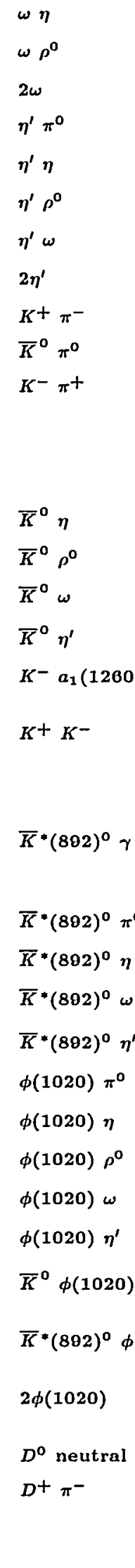 & 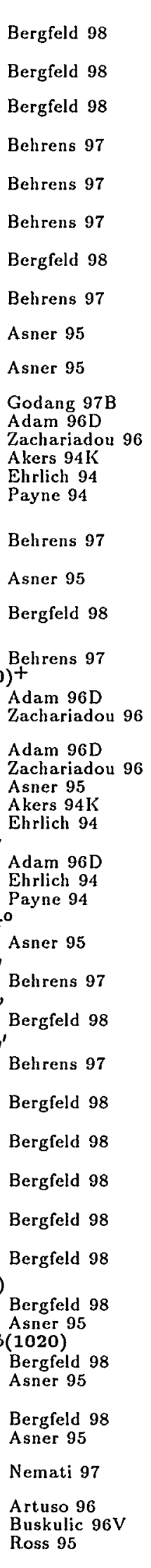 & $\begin{array}{l}D^{0} \pi^{0} \\
D^{0} \eta \\
D^{+} \rho^{-} \\
D^{0} \rho^{0} \\
D^{0} \omega \\
D^{0} \eta^{\prime} \\
D^{+} D^{-} \\
D^{*}(2010)^{+} \\
J^{+} \psi(1 S) \pi^{0}\end{array}$ & 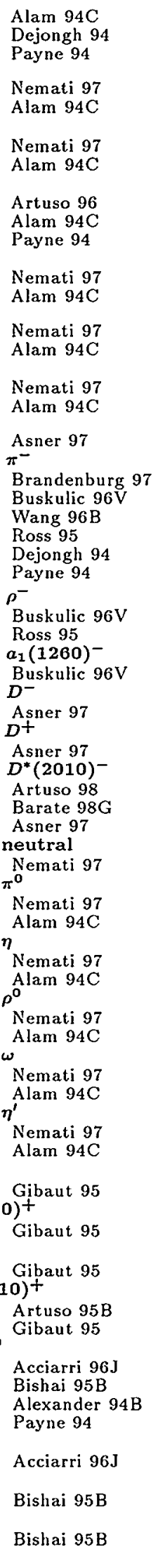 & & 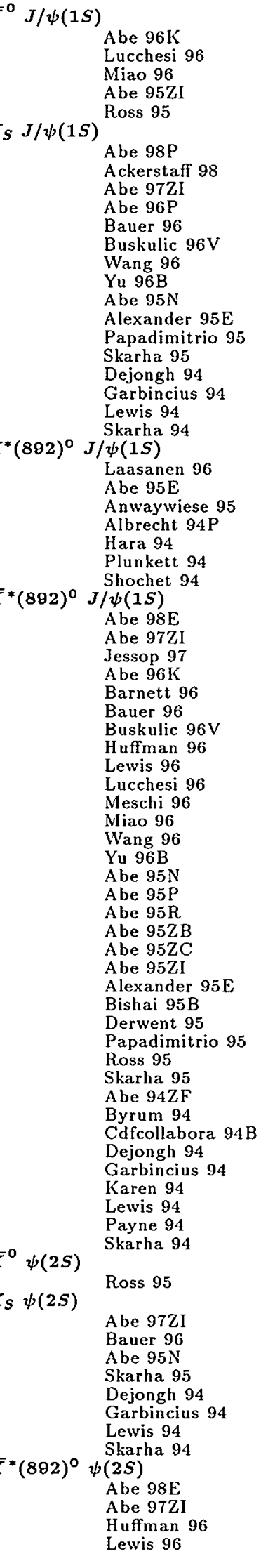 & $\begin{array}{l}\rho \bar{p} \\
\Lambda \bar{\Lambda} \\
\Lambda_{c}^{+} \bar{p} \\
\Sigma_{c}(2455)^{+} \\
\pi^{+} \ell^{-} \bar{\nu}+ \\
\pi^{+} e^{-} \bar{\nu}_{e} \\
\pi^{+} \mu^{-} \bar{\nu}_{\mu} \\
\rho^{+} \ell^{-} \bar{\nu}+ \\
\rho^{+} e^{-} \bar{\nu}_{e} \\
\rho^{+} \mu^{-} \bar{\nu}_{\mu} \\
\bar{K}^{*}(892)^{\circ}{ }_{\nu} \\
K^{*}(892)^{\circ} \mu \\
\bar{K}^{*}(892)^{\circ}\end{array}$ & 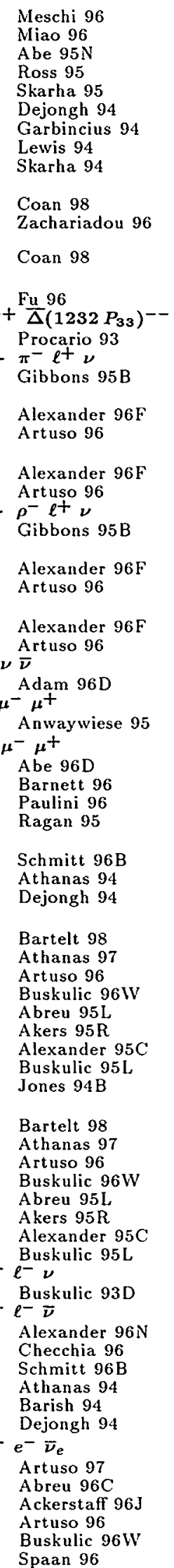 \\
\hline
\end{tabular}

Entries in order of particle name, then decay. A few chemical symbols for nuclei have been changed to avoid ambiguity with particle names (see the Particle Vocabulary). See the legend on page 323. 


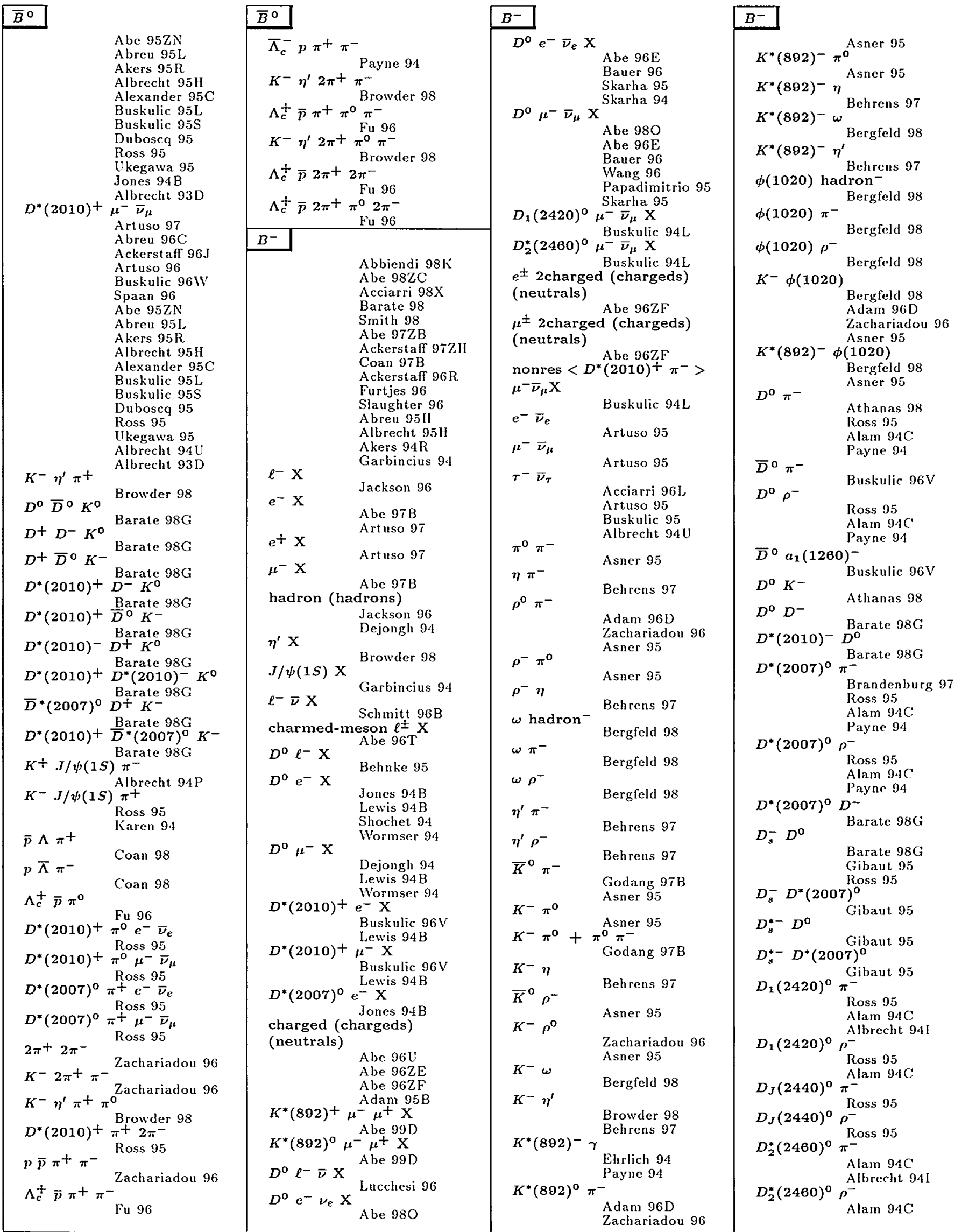




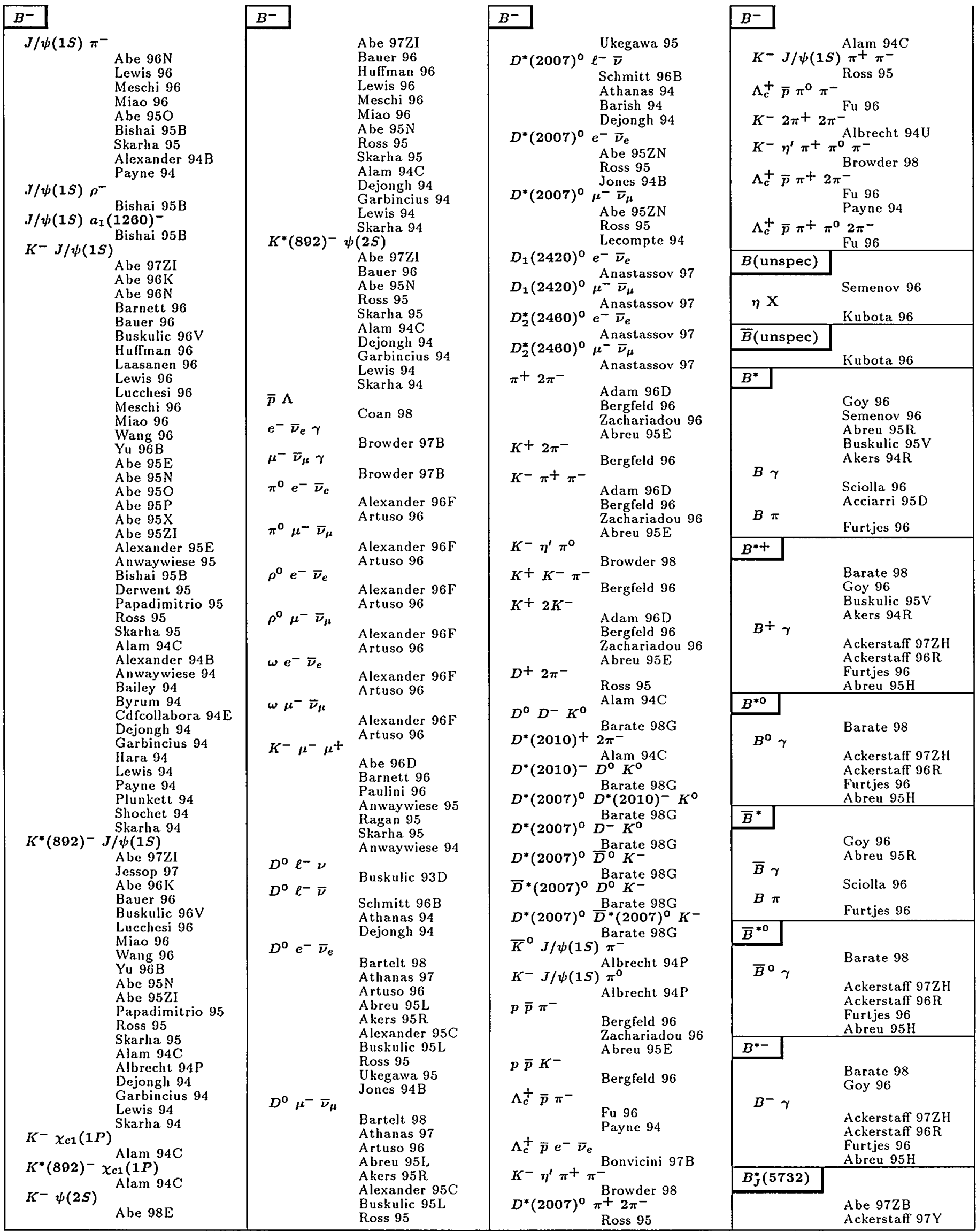

Entries in order of particle name, then decay. A few chemical symbols for nuclei have been changed to avoid ambiguity with particle names (see the Particle Vocabulary). See the legend on page 323. 


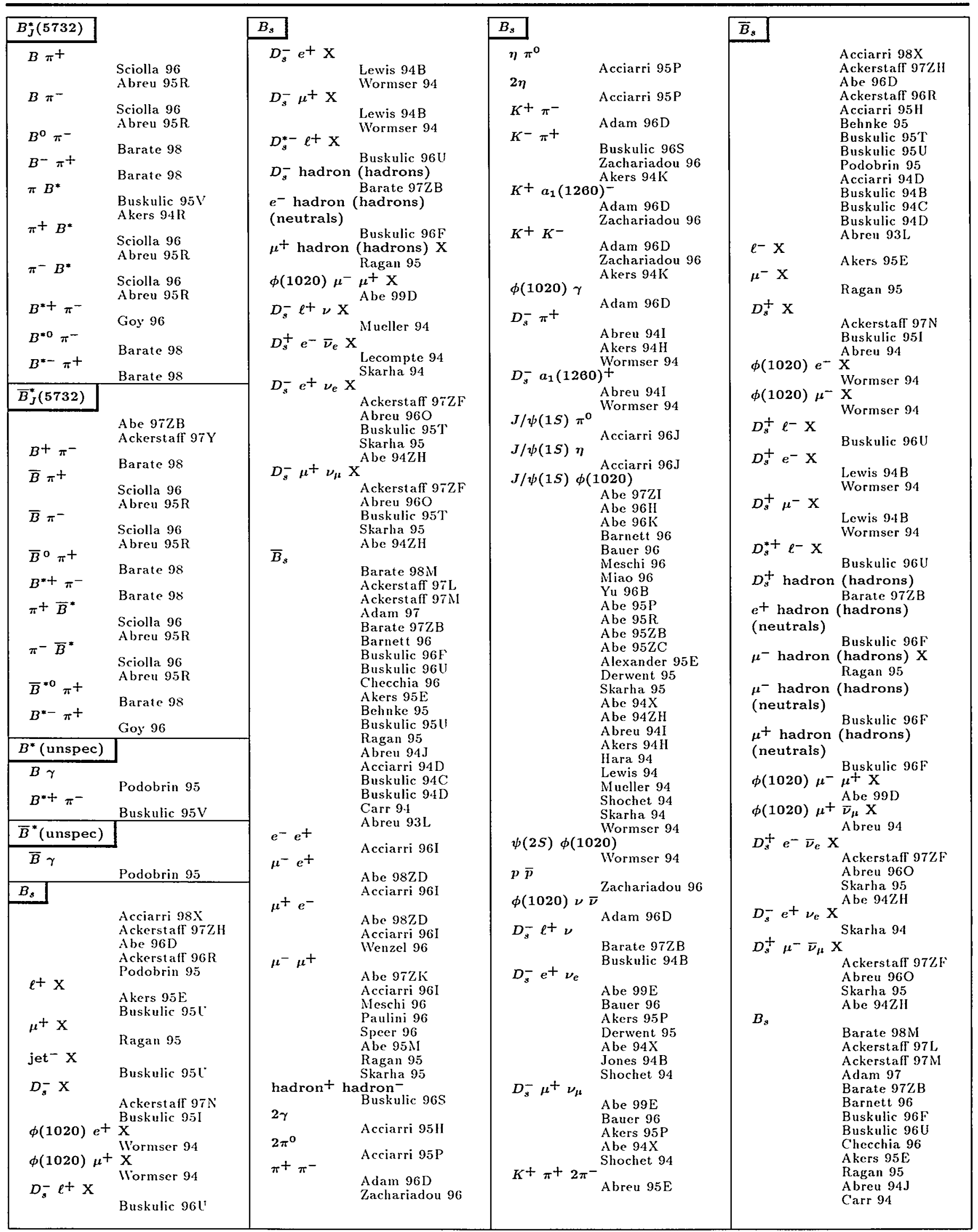




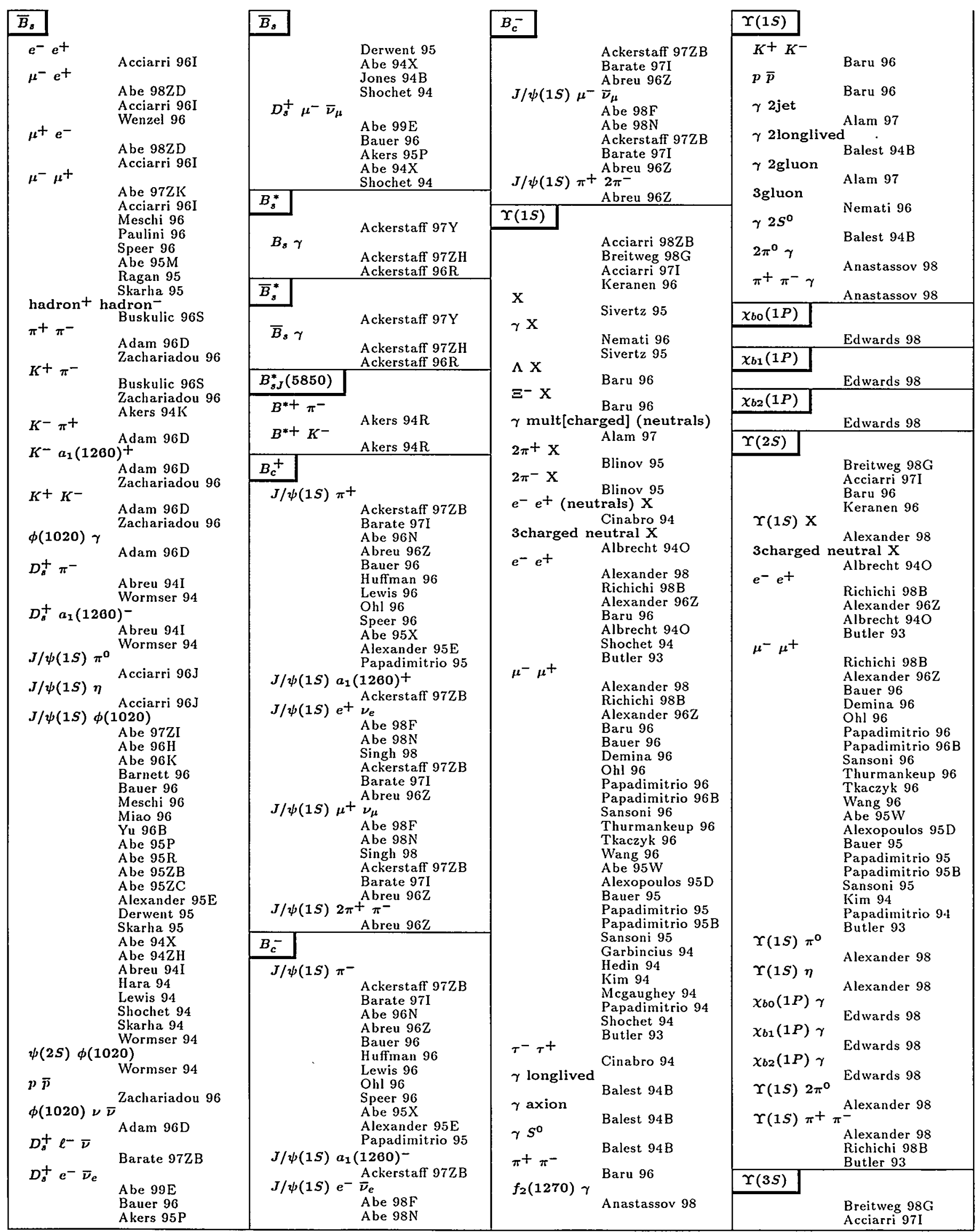

Entries in order of particle name, then decay. A few chemical symbols for nuclei have been changed to avoid ambiguity with particle names (see the Particle Vocabulary). See the legend on page 323 . 


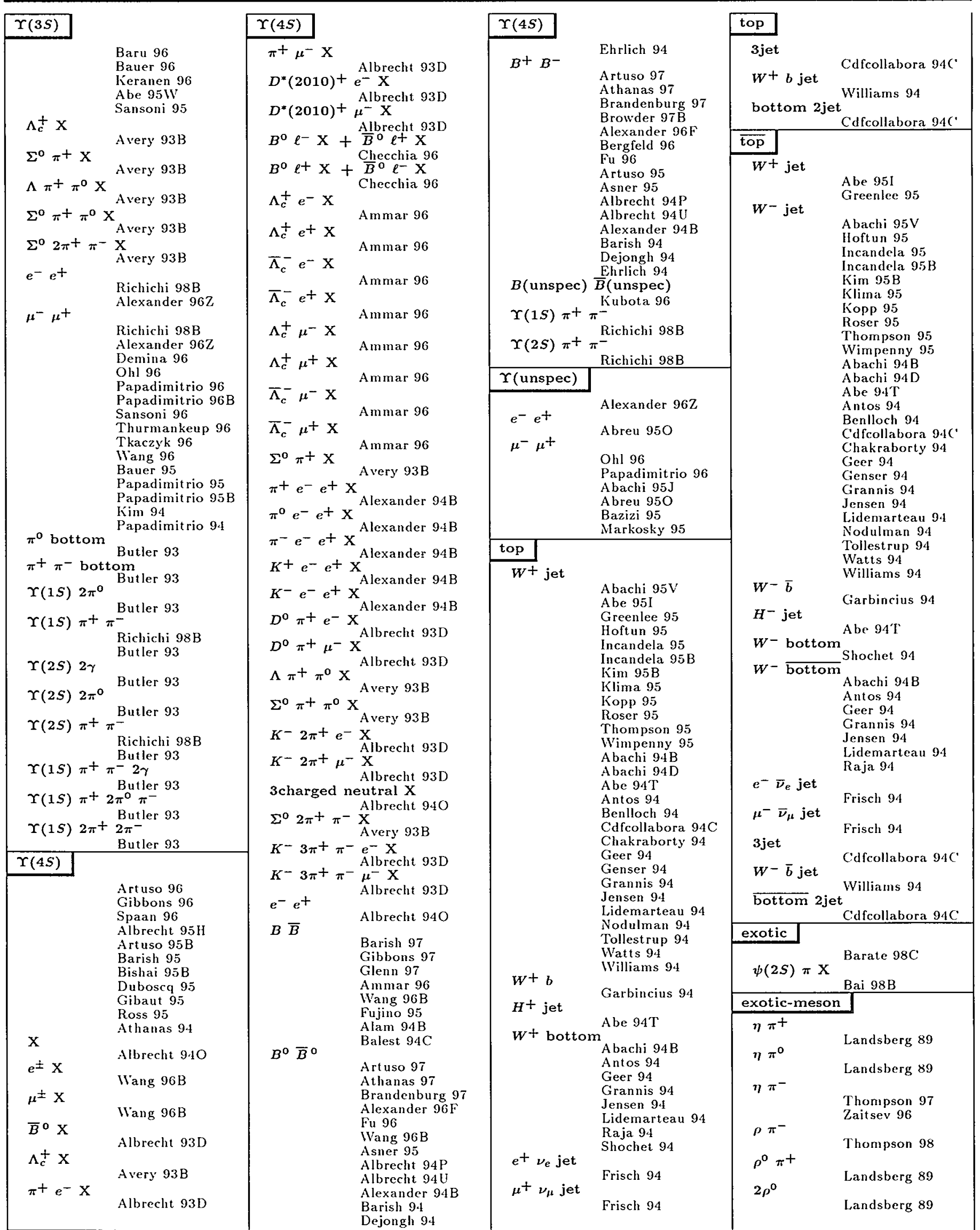




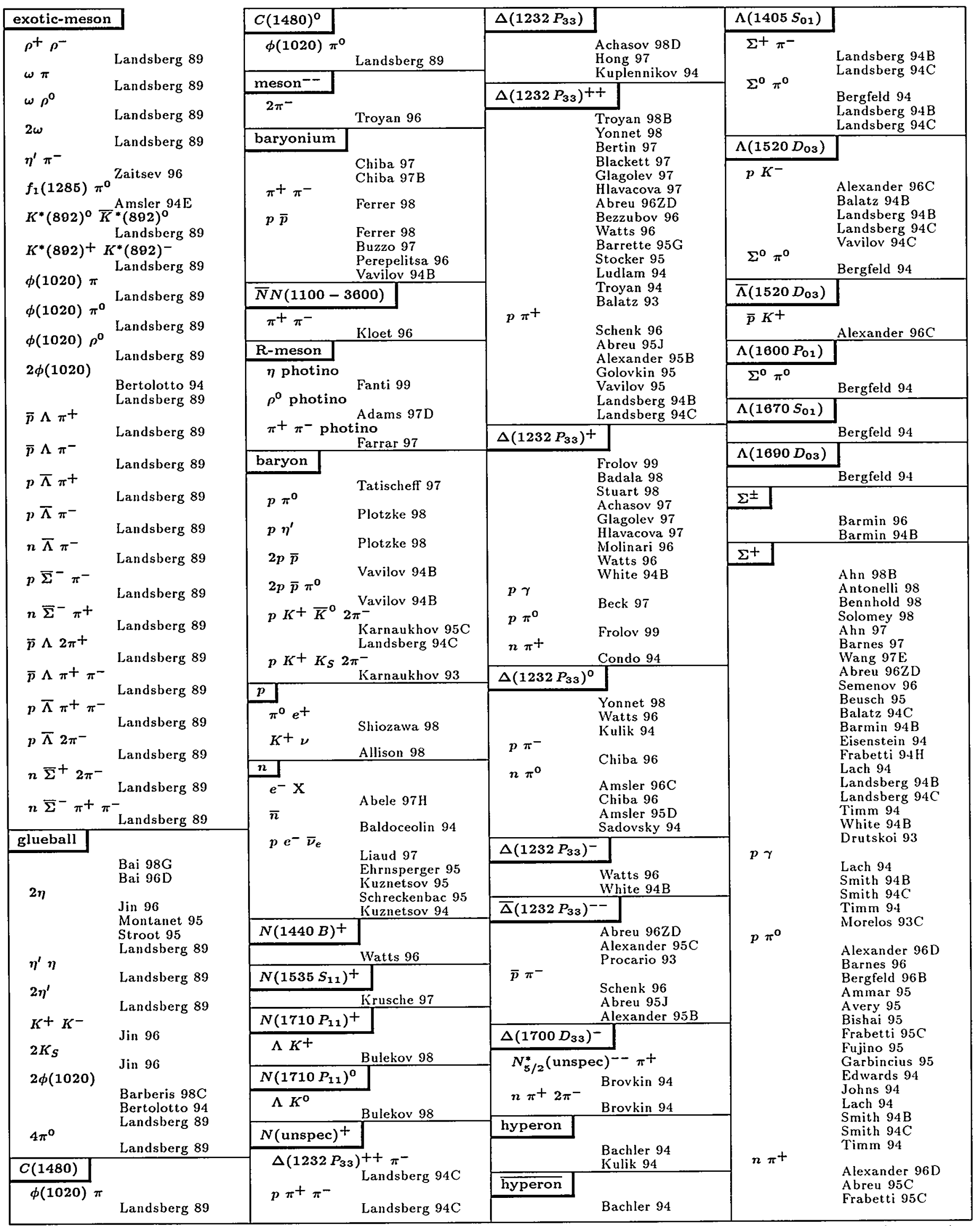

Entries in order of particle name, then decay. A few chemical symbols for nuclei have been changed to avoid ambiguity with particle names (see the Particle Vocabulary). See the legend on page 323. 


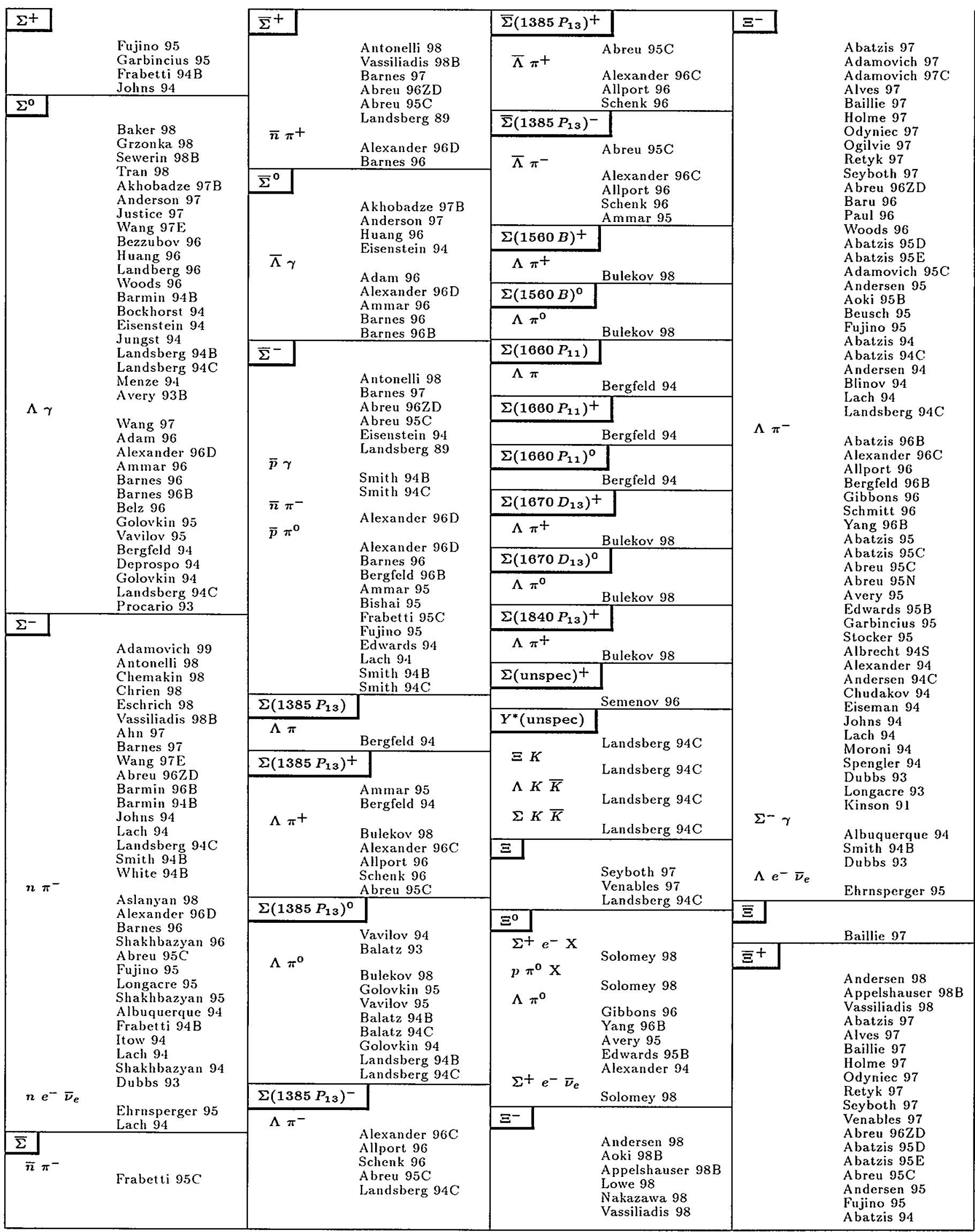




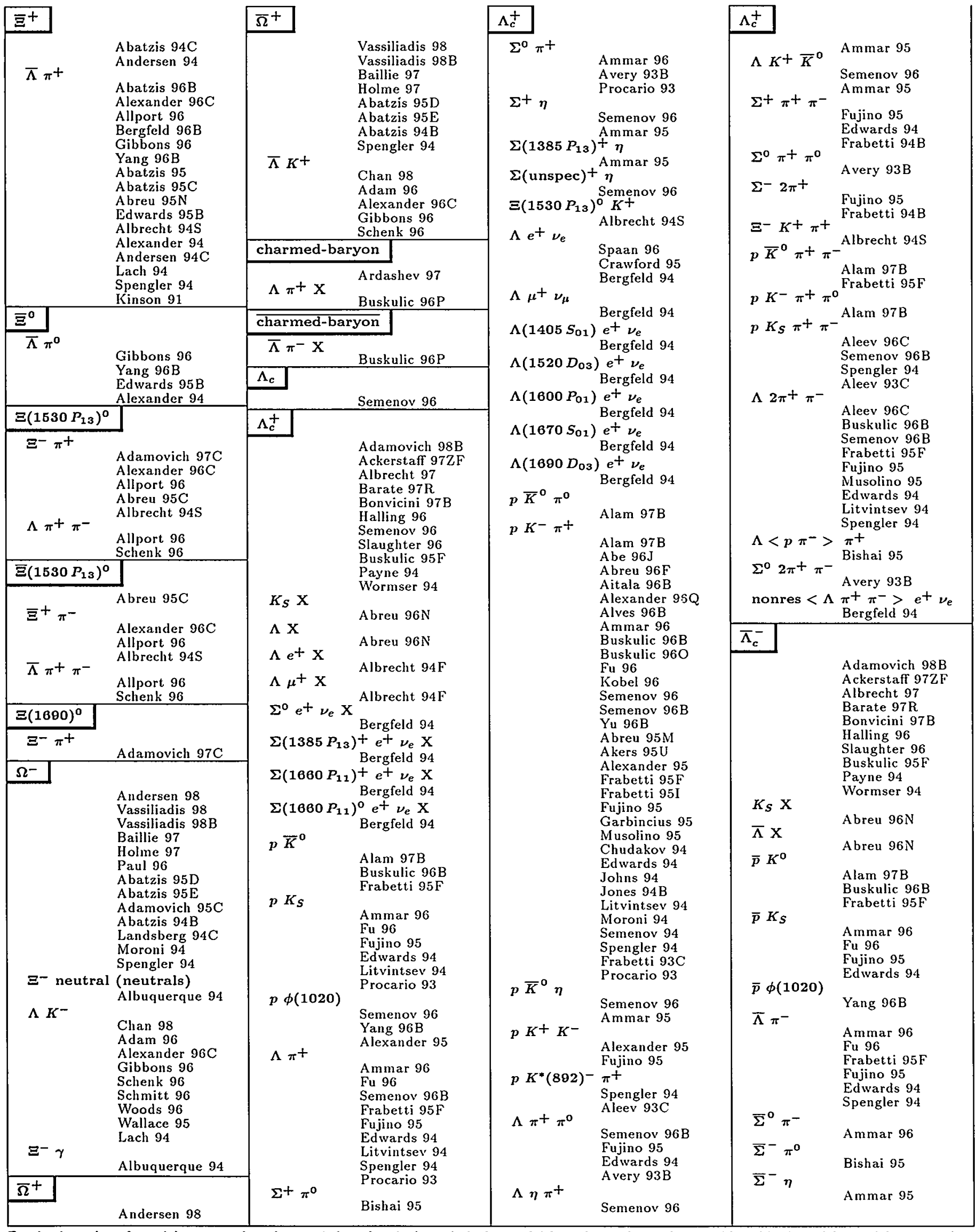

Entries in order of particle name, then decay. A few chemical symbols for nuclei have been changed to avoid ambiguity with particle names (see the Particle Vocabulary). See the legend on page 323. 


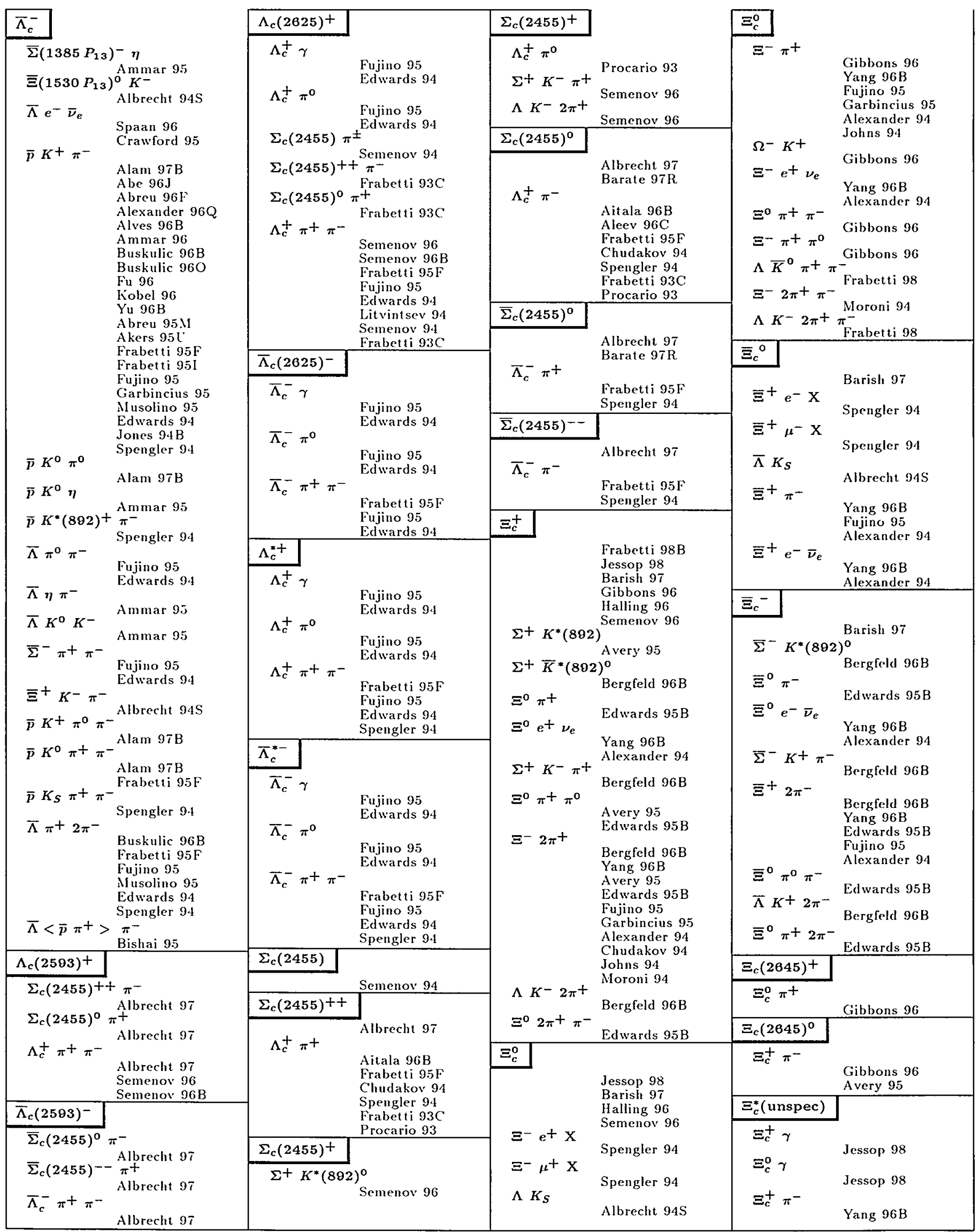




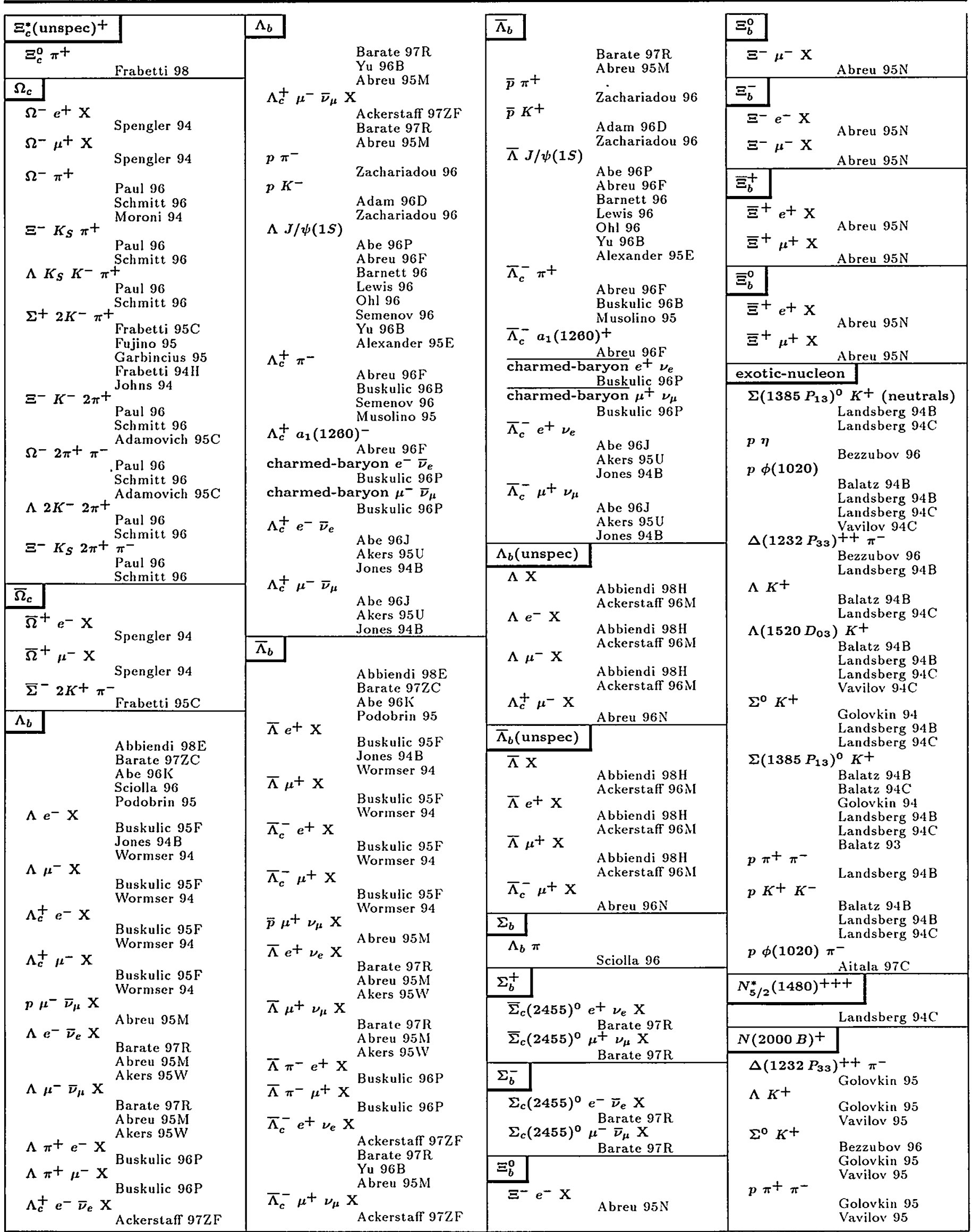

Entries in order of particle name, then decay. A few chemical symbols for nuclei have been changed to avoid ambiguity with particle names (see the Particle Vocabulary). See the legend on page 323. 


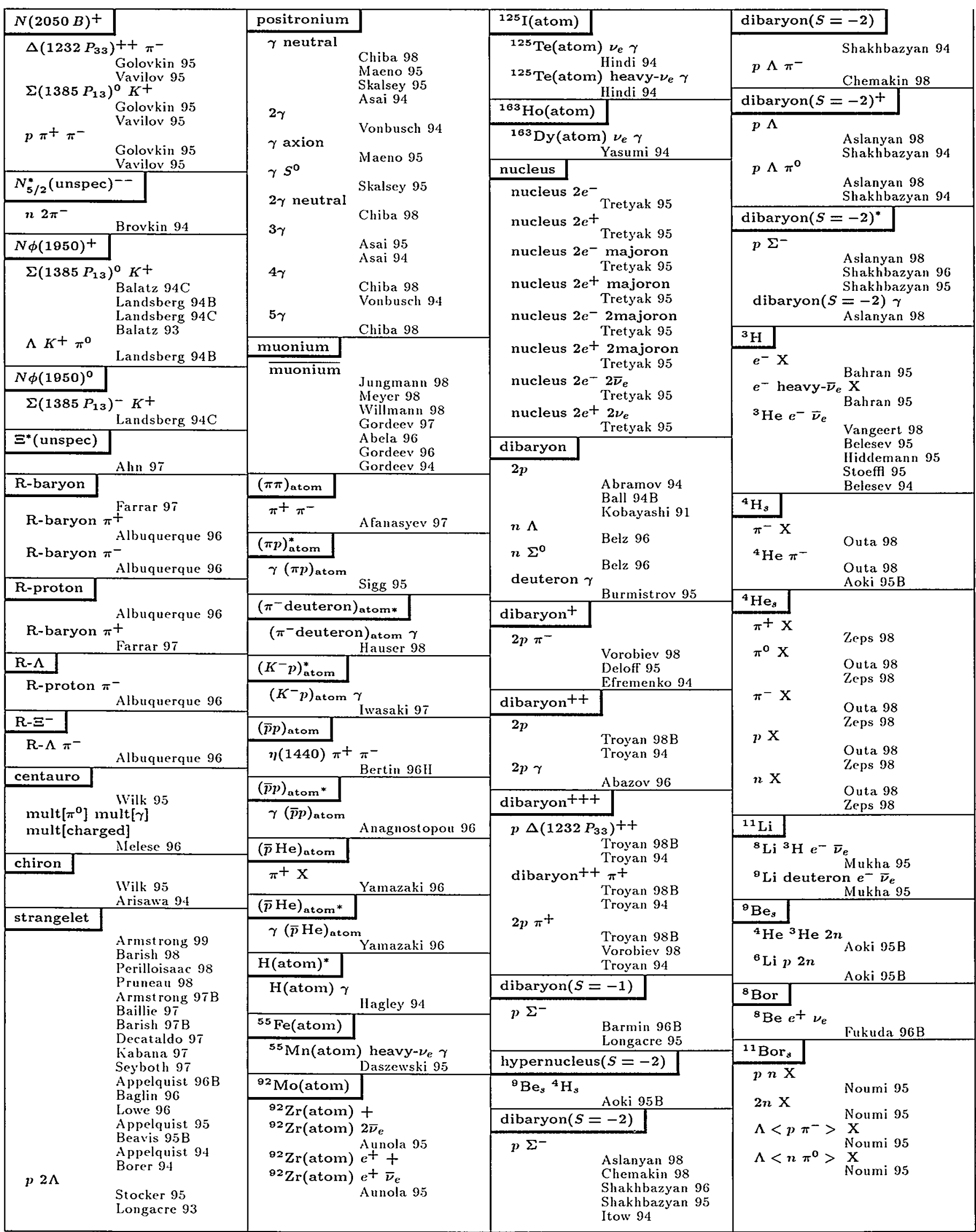




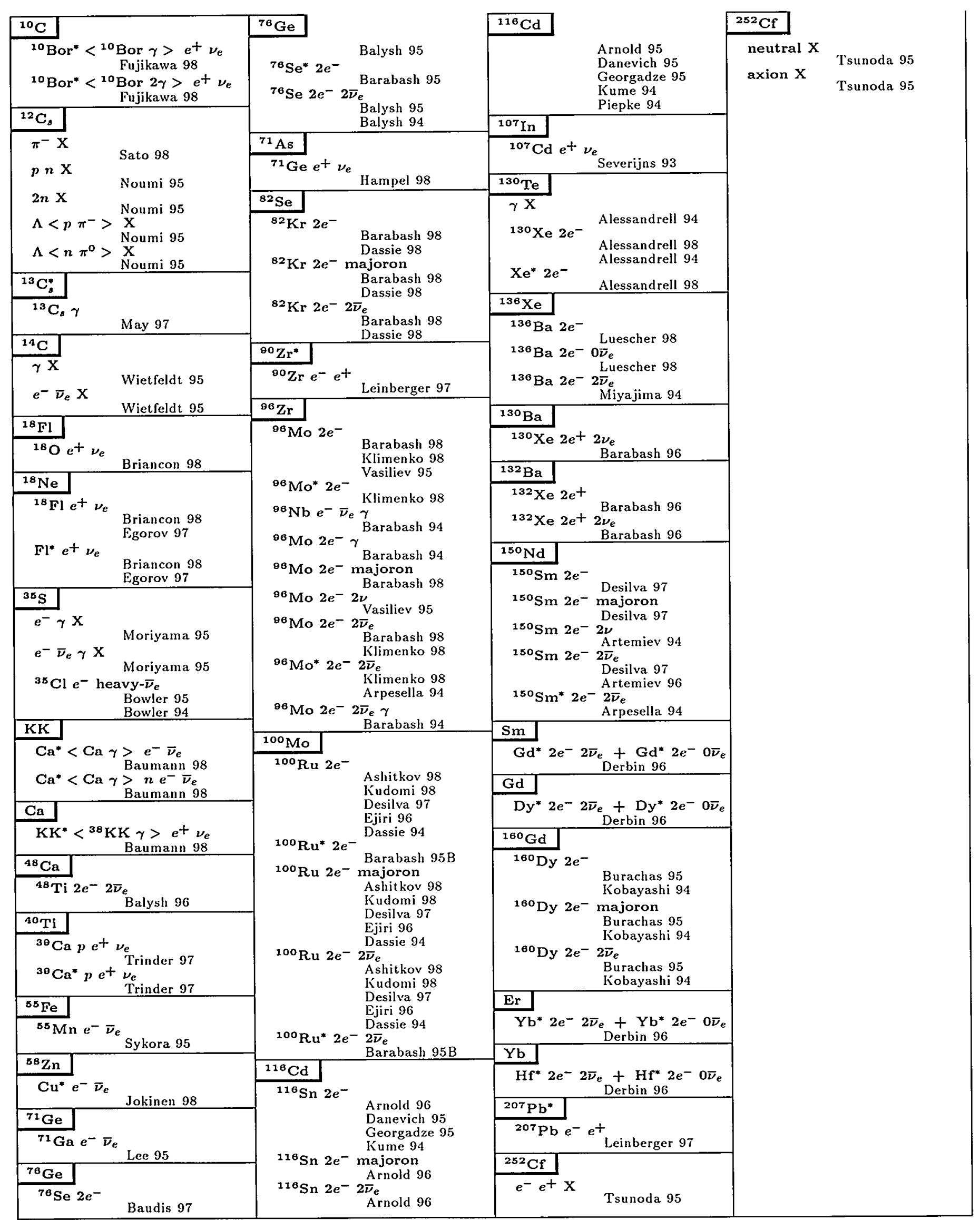

Entries in order of particle name, then decay. A few chemical symbols for nuclei have been changed to avoid ambiguity with particle names (see the Particle Vocabulary). See the legend on page 323. 
This index lists papers by the accelerator, the experiment number, and the detector used, ordered alphabetically. The Accelerator Vocabulary and the Detector Vocabulary list all the facilities used by papers indexed in this book.

A question mark indicates that the information is missing, usually because it was not given in the paper. A dash mark indicates that no experiment number or name is available.

\section{Illustrative Key}

Accelerator: see the Accelerator Vocabulary for definitions.

Detector: see the Detector Vocabulary for definitions.

\begin{tabular}{|cl|}
\hline ANL & \\
\hline ANL-E-412 & \\
DBC-12FT & Mann 86 \\
ANL-E-435 & \\
CNTR & Auer 86 \\
SPEC & Auer 86B \\
WIRE & Auer 86 \\
ANL-E-441 & . \\
EMS & Finley 85 \\
ANL-E-447 & \\
SPEC & Auer 88 \\
ANL-E-451 & \\
EMS & Wicklund 85 \\
& Wicklund 87 \\
\hline BNL & \\
BNL-673-593 & \\
MPS & Bensinger 85 \\
BNL-701 & \\
COMB & Franklin 87 \\
BNL-702 & \\
COMB & Snow 85 \\
BNL-723 & \\
SPEC & Gall 88 \\
BNL-726 & Hertzog 88 \\
WIRE & Christenson 85 \\
BNL-732 & Chiang 86 \\
CALO & Chan
\end{tabular}

Document ID: see the ID/Reference/Title Index for the full reference.

Experiment: the experiment's number, where known. 


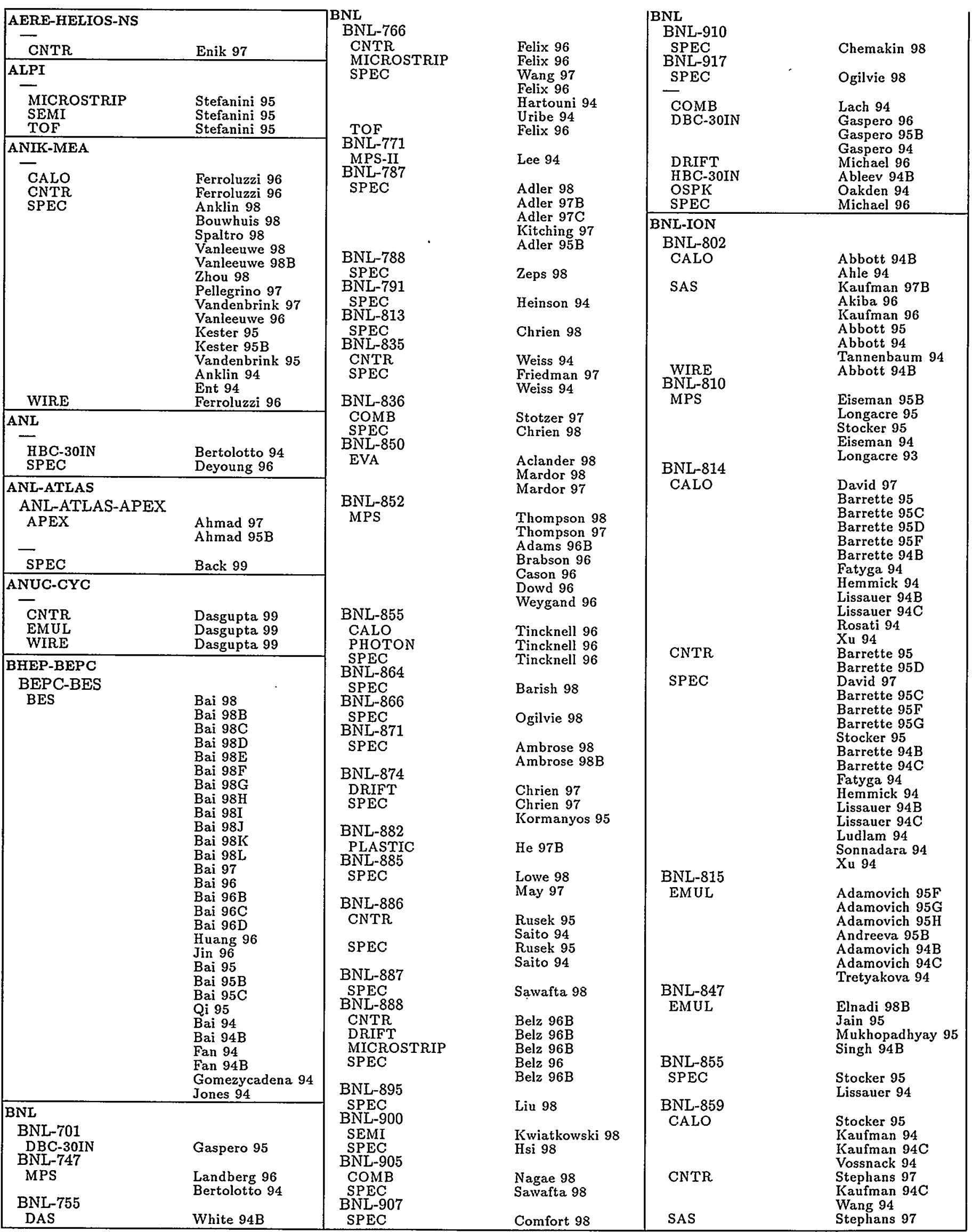




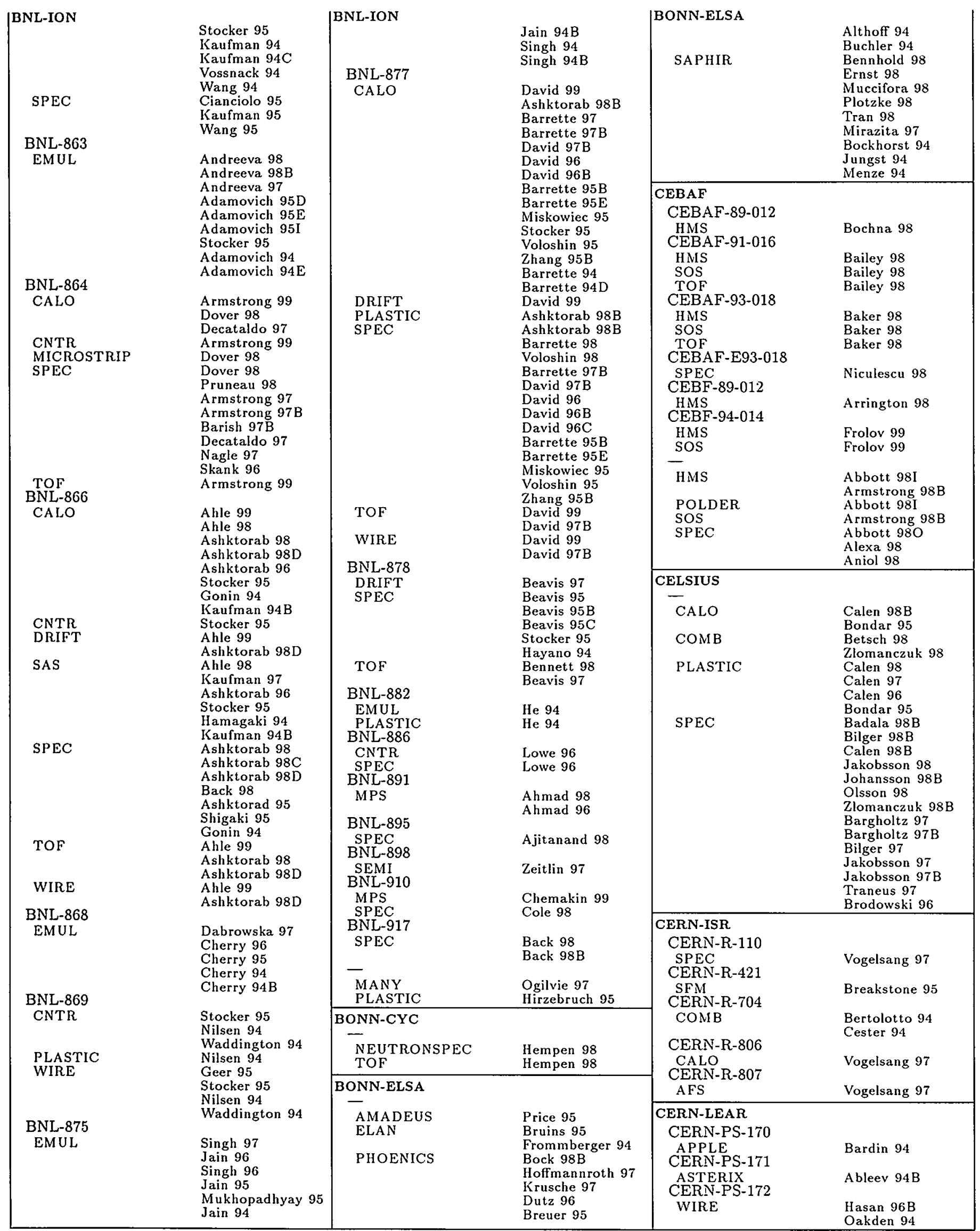

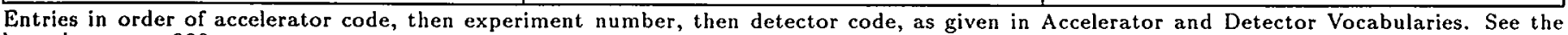
legend on page 399. 


\section{CERN-LEAR \\ CERN-PS-173 \\ CONB \\ CERN-PS-179 \\ EMUL \\ CERN-PS-182 \\ SPEC \\ CERN-PS-185}

WIRE

CERN-PS-186

DAS

CERN-PS-195

CPLEAR

CERI-PS-196

OTHER

CERN-PS-197

CRYS-BARREL

\section{CERN-LEAR}

Bradamante 94

Batusov 96C

Ableev 94B

Barnes 97

Barnes 96

Barnes 96B

Barnes 96C

Rohrich 96

Barnes 94

Eisenstein 94

Hofmann 94

A postolakis 99

Angelopoulos 98

Angelopoulos 98B

Angelopoulos $98 \mathrm{C}$

Angelopoulos $98 \mathrm{D}$

Angelopoulos $98 \mathrm{E}$

Adler 97

Adler $97 \mathrm{D}$

Angelopoulos 97

Angelopoulos $97 \mathrm{~B}$

Angelopoulos $97 \mathrm{C}$

Angelopoulos 97D

Angelopoulos 97 E

A postolakis 97

Adler 96

Adler $96 \mathrm{~B}$

Adler 96C

Adler 96D

Adler $96 \mathrm{~F}$

Adler 95

Adler $95 \mathrm{C}$

Adler $95 \mathrm{D}$

Adler $95 \mathrm{E}$

Adler $95 \mathrm{~F}$

Adler $95 \mathrm{G}$

Ableev 94B

Adler 94

Adler $94 \mathrm{~B}$

Gabriclse 95

Abele 99

A bele 99B

A bele 98

A bele $98 \mathrm{~B}$

A bele $98 \mathrm{C}$

A bele 98D

A bele $98 \mathrm{E}$

A bele 97

A bele $97 \mathrm{~B}$

A bele $97 \mathrm{C}$

A bele $97 \mathrm{D}$

Abele $97 \mathrm{E}$

Abele $9 \pi \mathrm{F}$

A bele $97 \mathrm{G}$

Abele 96

Abele $96 \mathrm{~B}$

$A$ bele $96 \mathrm{C}$

Adomeit 96

Amsler 96

Amsler $96 \mathrm{~B}$

Amsler $96 \mathrm{C}$

Amsler 96D

Degener 96

Pinder 96

Resag 96

Spanier 96

Spanier 96B

Amsler 95

Amsler 95B

Amsler 95C

Amsler 95D

Amsler 95E

Amsler $95 \mathrm{~F}$

Amsler $95 \mathrm{G}$

Amsler $95 \mathrm{H}$

Anisovich 95

Montanet 95

CNTR

OBELIX

SPEC

JETSET

PIIOTON

SEMI

TOF
WIRE
Ableev $94 \mathrm{~B}$

Amsler 94

Amsler 94B

Amsler 94C

Amsler 94D

Amsler 94E

Amsler 94F

Amsler $94 \mathrm{G}$

Amsler $94 \mathrm{H}$

Amsler 94I

Amsler 94. J

Anisovich 94

Armstrong 94 B

Brose 94

Faessler 94

Felix 94

Ravndal 94

Spanier 94

Strassburger 94

Wiedner 94

CERN-PS-199

CERN-PS-201

Barbina 97

Ahmidouch 96

Lamanna 96

Ahmidouch 95

Ahmidouch 95B

Lamanna 94

Alberico 98

Alberico $98 \mathrm{~B}$

Bertin 98

Bertin 98B

Bertin 97

Bertin 97B

Bertin 97D

Bertin 97E

Bertin 96I

Filippi 96

Iazzi 96

Prakhov 96

Ableev 95C

Ableev 94

Ableev 94B

Ableev 94C

A bleev 94D

A bleev 94G

Ableev $94 \mathrm{H}$

Ableev 94I

Adamo 94

Agnello 94

Feliciello 94

Lucherini 94

Masoni 94

Piccinini 94

Sapozhrikov 94

Sapozhnikov 94B

Bertin $97 \mathrm{C}$

Bertin 96

Bertin 96B

Bertin 96C

Bertin 96D

Bertin $96 \mathrm{E}$

Bertin $96 \mathrm{~F}$

Bertin 96G

Bertin $96 \mathrm{H}$

Temnikov 96

Villa 96

Ableev 95

Bertin 95

Ableev $94 \mathrm{E}$

CERN-PS-202

CERN-PS-203

Ableev $94 \mathrm{~F}$

Evangelista 98

Buzzo 97

Evangelista 97

Bertolot to 96

Palano 96

Bertolotto 95

Bertolot to 94

Steinkamp 94

Lubinski 94

Polster 95

Schmid 94

Ivanov 95
CERN-LEAR

CERN-PS-205

CNTR

PLASTIC

CERN-PS-206

CNTR

COMB

TOF

CERN-PS-207

SPEC

CERN-PS-208

CNTR

COMB

NEUTRONSPEC

Yamazaki 96

Morita 95

Hayano 94B

Nakamura 94

Widmann 94

Daniel 94

Bressan 97

Lamanna 96

Bradamante 95

Birsa 94

Bradamante 94

Bradamante 94

Bressan 97

Lamanna 96

Bradamante 95

Birsa 94

Anagnostopou 96

Lubinski 98 Goldenbaum 96

Jahnke 96

Schmid 97

Schmid 97B

Goldenbaum 96

SPEC

TOF

Schmid 97B

Schmid 97B

Goldenbaum 96

CERN-PS-210

CNTR

Baur 96

WIRE

Kloet 96

CERN-LEP

CERN-LEP-ALEPH

ALEPH

Barate 99

Barate 99B

Barate 99C

Barate 99D

Barate $99 \mathrm{E}$

Barate $99 \mathrm{~F}$

Barate 98

Barate 98B

Barate 98C

Barate 98D

Barate 98E

Barate $98 \mathrm{~F}$

Barate 98G:

Barate 98H

Barate 98I

Barate 98J

Barate $98 \mathrm{~K}$

Barate 98L

Barate $98 \mathrm{M}$

Barate $98 \mathrm{~N}$

Barate 980

Barate 98P

Barate $98 \mathrm{Q}$

Barate 98R

Barate $98 \mathrm{~S}$

Bock 98

Alemany 97

Barate 97

Barate $97 \mathrm{~B}$

Barate $97 \mathrm{C}$

Barate 97D

Barate $97 \mathrm{E}$

Barate 97F

Barate $97 \mathrm{G}$

Barate $97 \mathrm{H}$

Barate 97I

Barate 97J

Barate $97 \mathrm{~K}$

Barate 97L

Barate $97 \mathrm{M}$

Barate $97 \mathrm{~N}$

Barate 970

Barate $97 \mathrm{P}$

Barate $97 \mathrm{Q}$

Barate $97 \mathrm{R}$

Barate 97S 
CERN-LEP

Barate 97

Barate $97 \mathrm{~W}$

Barate $97 \mathrm{X}$

Barate $97 Y$

Barate $97 \mathrm{Z}$

Barate 97ZB

Barate 97ZC

Barate 97ZD

Barate 97ZE

Barate 972F

Buskulic 97

Camoana 97

Nikolic 97

Rouge 97

Sanchez 97

Weber 97

Wermes 97

Abreu 96Y

Barate 96

Buskulic 96

Buskulic 96B

Buskulic 96C

Buskulic 96D

Buskulic 96E

Buskulic 96F

Buskulic 96G

Buskulic 96H

Buskulic 96I

Buskulic 96J

Buskulic 96K

Buskulic 96L

Buskulic 96M

Buskulic 96N

Buskulic 960

Buskulic 96P

Buskulic $96 \mathrm{Q}$

Buskulic 96R

Buskulic 96S

Buskulic 96T

Buskulic 96U

Buskulic 96V

Buskulic 96W

Buskulic 96X

Buskulic 96Y

Buskulic 96Z

Checchia 96

Evans 96

Goy 96

Karlsson 96

Moutoussi 96

Semenov 96

Andreazza 95

Behnke 95

Bella 95

Bourdon 95

Buskulic 95

Buskulic 95B

Buskulic 95C

Bushulic 95D

Buskulic 95E

Buskulic 95F

Buskulic 95G

Buskulic $95 \mathrm{H}$

Buskulic 95I

Buskulic 95J

Buskulic 95K

Buskulic 95I

Buskulic 95M

Buskulic 95N

Buskulic 95O

Buskulic 95P

Buskulic 95Q

Buskulic 95R

Buskulic 95S

Buskulic 95T

Buskulic 95U

Buskulic 95V

Buskulic 95 $\mathrm{W}^{\prime}$

Buskulic 95X

Cerutti 95

Charlesworth 95

Davier 95

Duflot 95

Ferrante 95

Girone 95
CERN-LEP

Harton 95

Kounine 95

Matorras 95

Musolino 95

Padilla 95

Park 95B

Raab 95

Rouge 95

Stahl 95

Vorobiev 95B

Alemany 94

Brient 94

Buskulic 94

Buskulic 94B

Buskulic 94C

Buskulic 94D

Buskulic 94E

Buskulic 94F

Buskulic 94G

Buskulic 94

Buskulic 94I

Buskulic 94J

Buskulic $94 \mathrm{~K}$

Buskulic 94L

Buskulic 94M

Buskulic 94N

Carr 94

Colas 94

Danilov 94

Dejongh 94

Dremin 94

Duflot 94

Golutvin 94

Gomezycadena 94

Janot 94

Kramer 94

Richard 94

Wormser 94

Buskulic 93D

CERN-LEP-DELPHI

\section{DELPHI}

Abreu 99

Abreu 99B

Abreu 99C

Abreu $99 \mathrm{D}$

Abreu 99E

Abreu $99 \mathrm{H}$

Abreu 99I

Abreu 99J

Abreu 99K

Abreu 98

Abreu $98 \mathrm{~B}$

Abreu $98 \mathrm{C}$

Abreu 98D

A breu $98 \mathrm{E}$

Abreu $98 \mathrm{~F}$

Abreu $98 \mathrm{H}$

Abreu 98J

Abreu $98 \mathrm{~K}$

Abreu 98L

Abreu $98 \mathrm{MI}$

Abreu $98 \mathrm{~N}$

A breu 980

A breu $98 \mathrm{Q}$

Abreu $98 \mathrm{~S}$

Abreu 98T

Abreu 98V

Abreu 98W

Bock 98

Abreu 97

Abreu 97B

Abreu 97C

Abreu $97 \mathrm{D}$

Abreu $97 \mathrm{E}$

Abreu $97 \mathrm{~F}$

Abreu 97G

Abreu 97

Abreu 97I

Abreu 97J

Abreu $97 \mathrm{~K}$

Abreu 97L

Abreu $97 \mathrm{M}$

Abreu $97 \mathrm{~N}$

Abreu 970

Abreu 97P
CERN-LEP

Abreu $97 \mathrm{Q}$

Abreu 97R

Adam 97

Batyunya 97

Cavallo 97

Edsall 97

Lorstad 97

Ruiz 97

Weber 97

Abreu 96

Abreu $96 \mathrm{~B}$

Abreu 96C

Abreu $96 \mathrm{D}$

Abreu $96 \mathrm{E}$

Abreu $96 \mathrm{~F}$

Abreu $96 \mathrm{G}$

Abreu $96 \mathrm{H}$

Abreu 96I

Abreu $96 \mathrm{~J}$

Abreu 96K

Abreu 96L

Abreu $96 \mathrm{M}$

Abreu $96 \mathrm{~N}$

Abreu $96 \mathrm{O}$

Abreu $96 \mathrm{P}$

Abreu 96Q

Abreu 96R

Abreu 96S

Abreu $96 \mathrm{~T}$

Abreu $96 \mathrm{U}$

Abreu $96 \mathrm{~V}$

Abreu $96 \mathrm{~W}$

Abreu 96X

Abreu $96 \mathrm{Y}$

Abreu $96 \mathrm{Z}$

Abreu 962B

Abreu 96ZC

Abreu 962D

Adam 96

Adam 96B

Adam 96D

Allport 96

Chapkin 96

Checchia 96

Karlsson 96

Keranen 96

Moutoussi 96

Pukhaeva 96

Sciolla 96

Semenov 96

Abreu 95

Abreu 95B

Abreu $95 \mathrm{C}$

Abreu $95 \mathrm{D}$

Abreu 95E

Abreu $95 \mathrm{~F}$

Abreu 95G

Abreu $95 \mathrm{H}$

Abreu 95I

Abreu 95J

Abreu $95 \mathrm{~K}$

Abreu 95L

Abreu 95

Abreu $95 \mathrm{~N}$

Abreu 95O

Abreu 95P

Abreu $95 \mathrm{Q}$

Abreu 95R

Abreu $95 \mathrm{~S}$

Abreu 95T

Abreu $95 \mathrm{U}$

Abreu 95V

Abreu 95X

Adam 95

Adam 95B

Andreazza 95

Behnke 95

Bella 95

Charlesworth 95

Dam 95

Davier 95

Ferrante 95

Harton 95

Kounine 95

Marti 95

Entries in order of accelerator code, then experiment number, then detector code, as given in Accelerator and Detector Vocabularies. See the legend on page 399. 


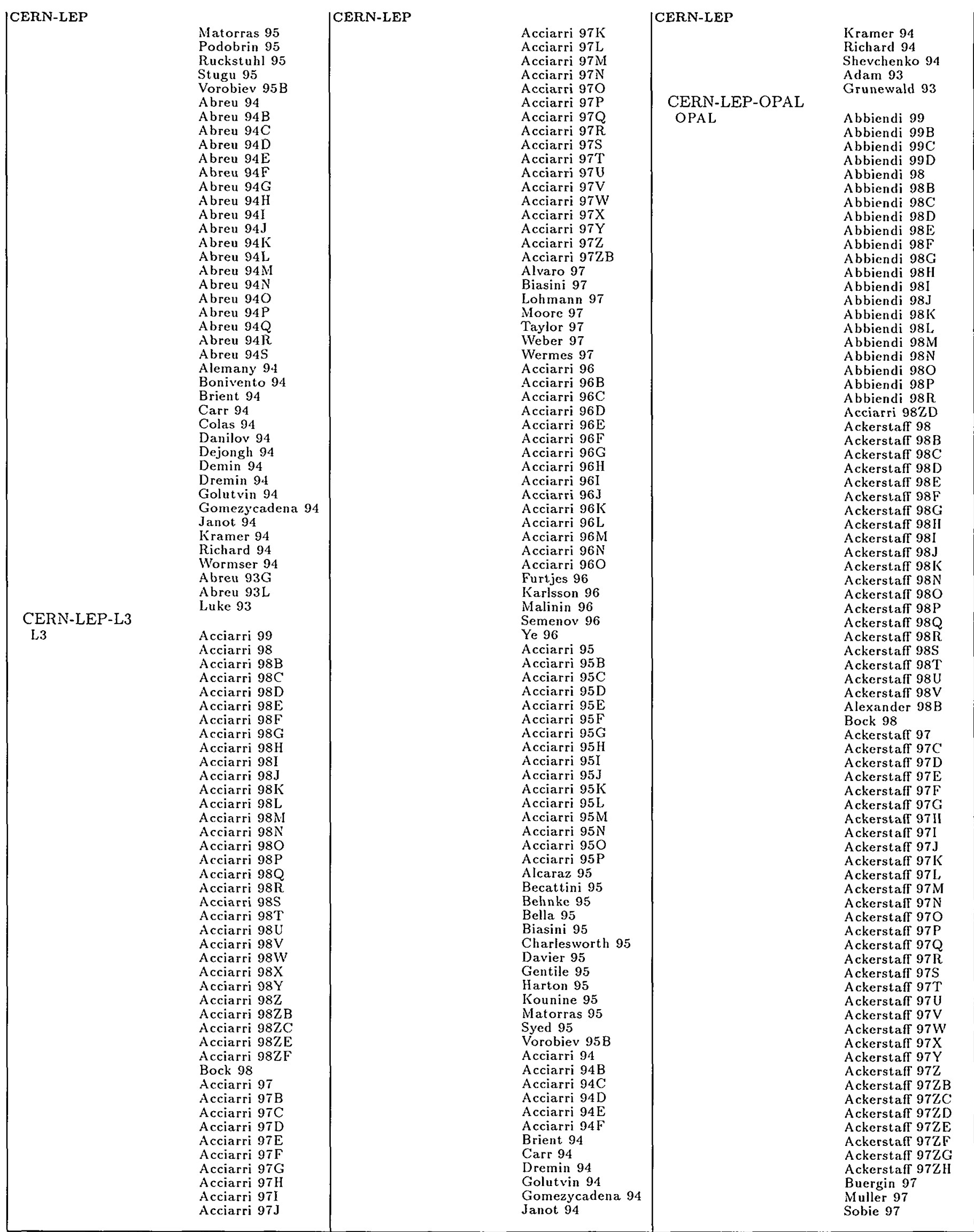


CERN-LEP

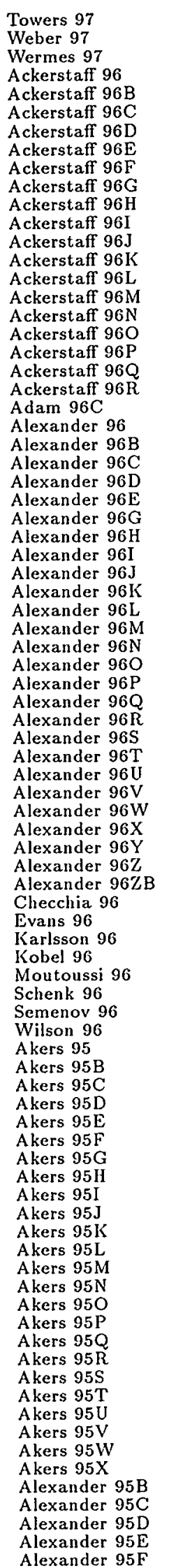

CERN-LEP

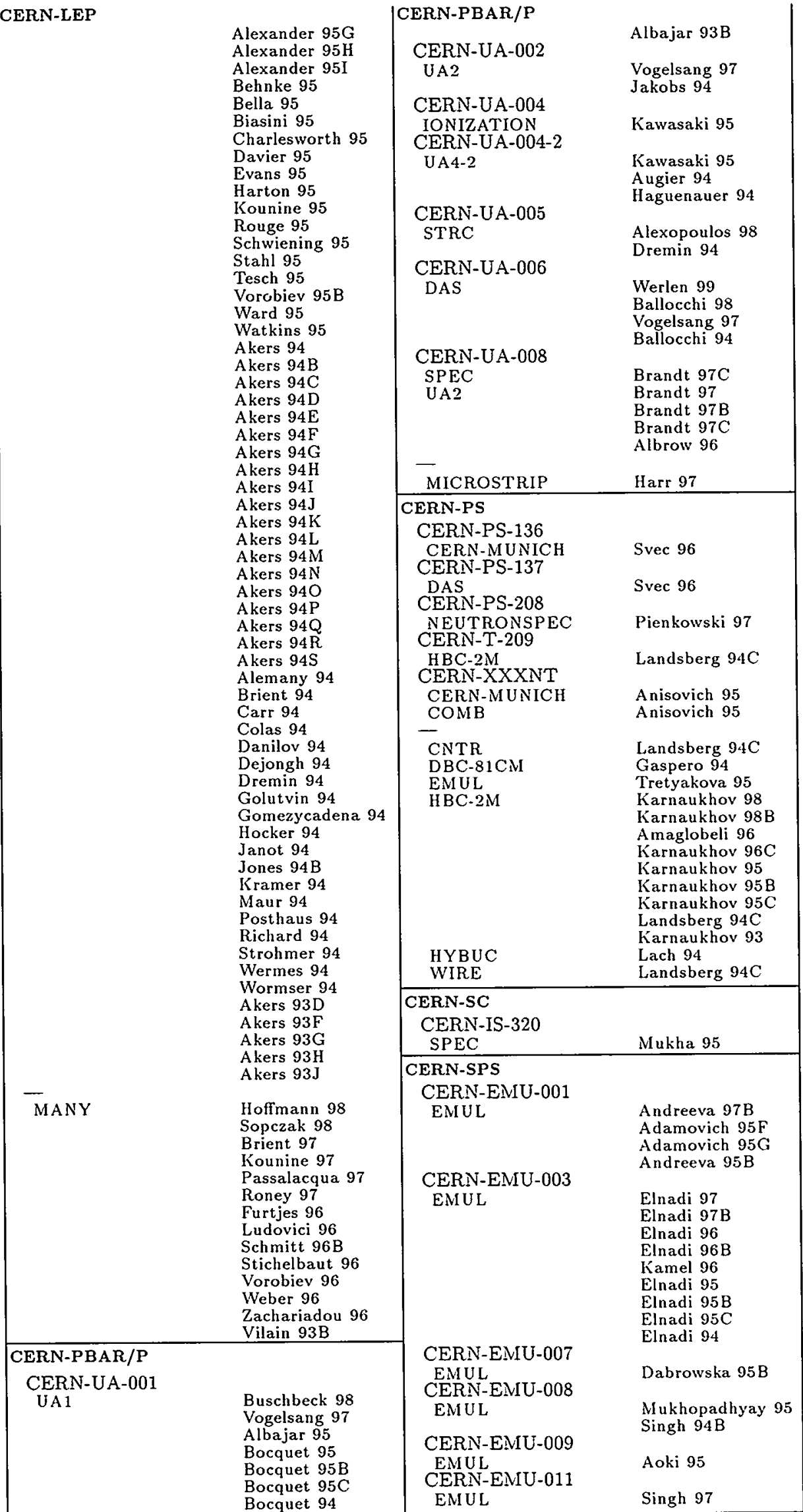




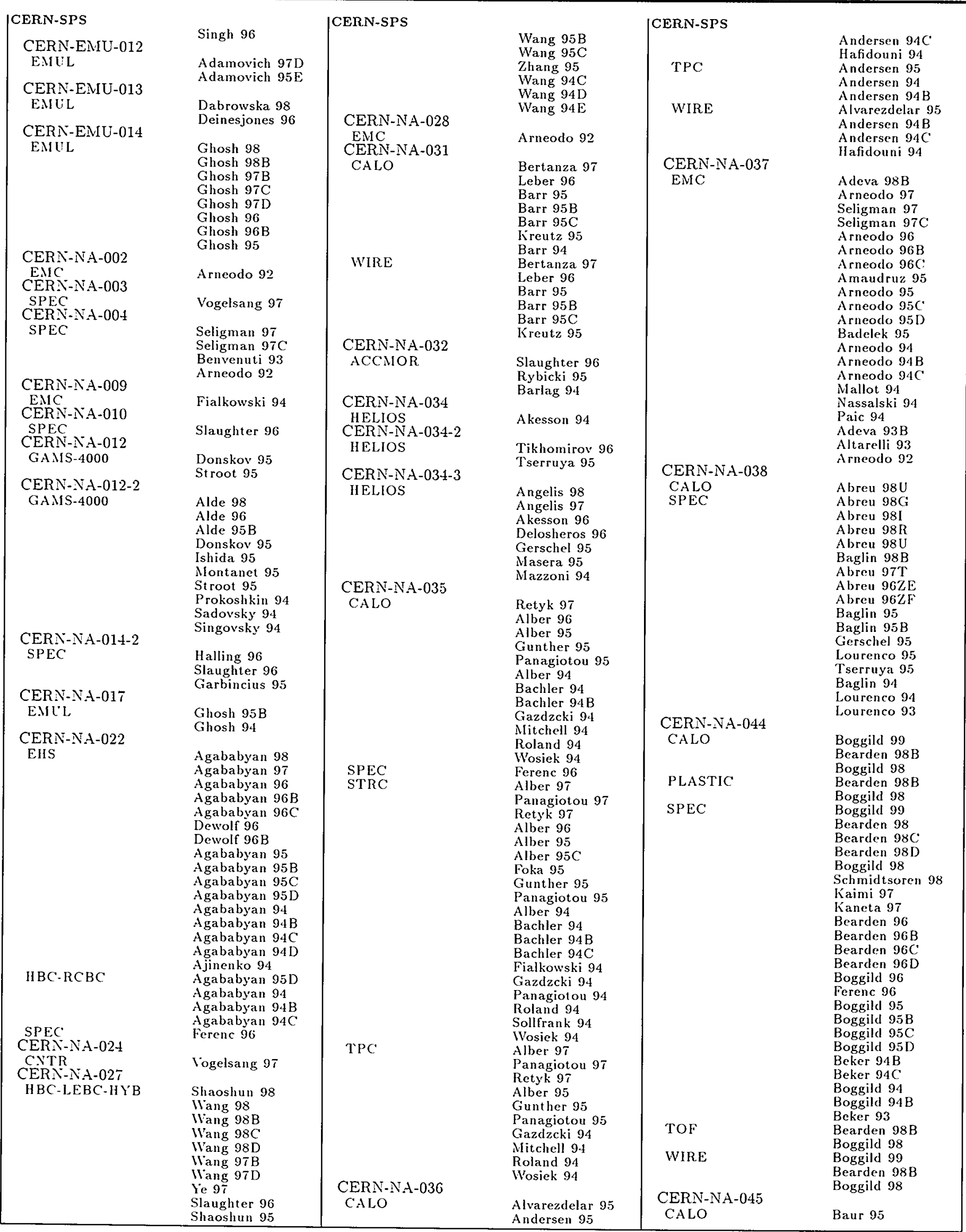




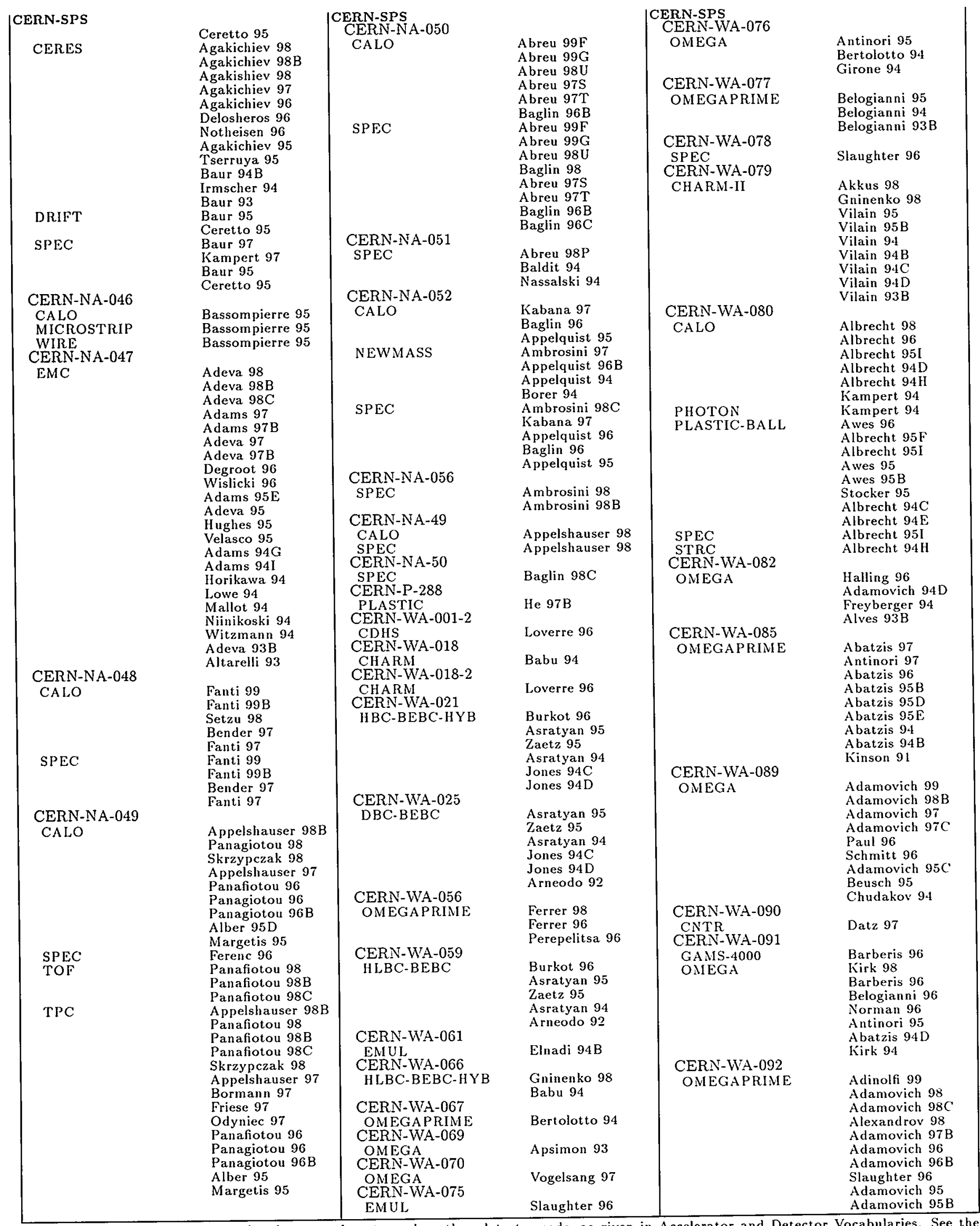

Entries in order of accelerator code, then experiment number, then detector code, as given in Accelerator and Detector Vocabularies. See the legend on page 399 . 


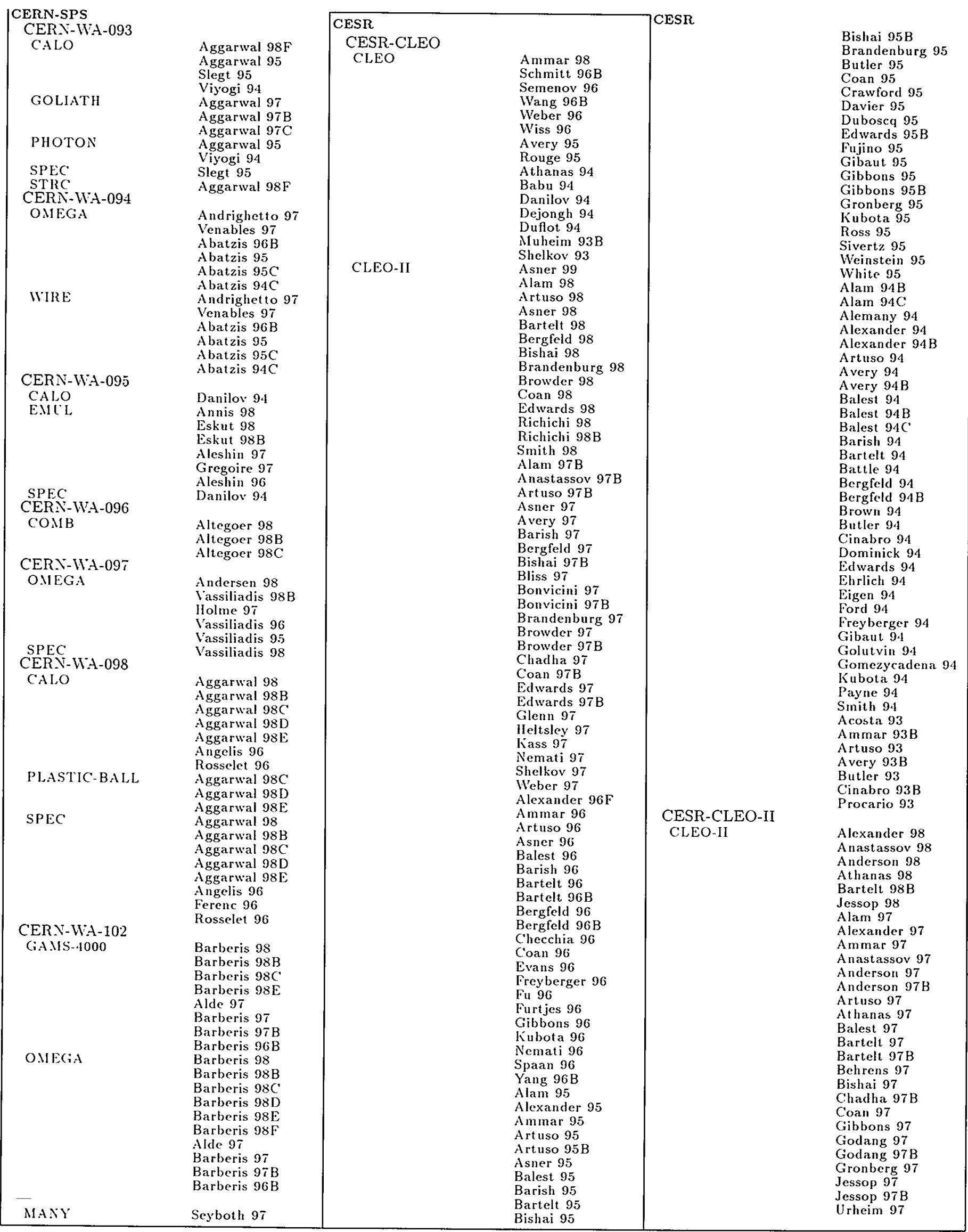




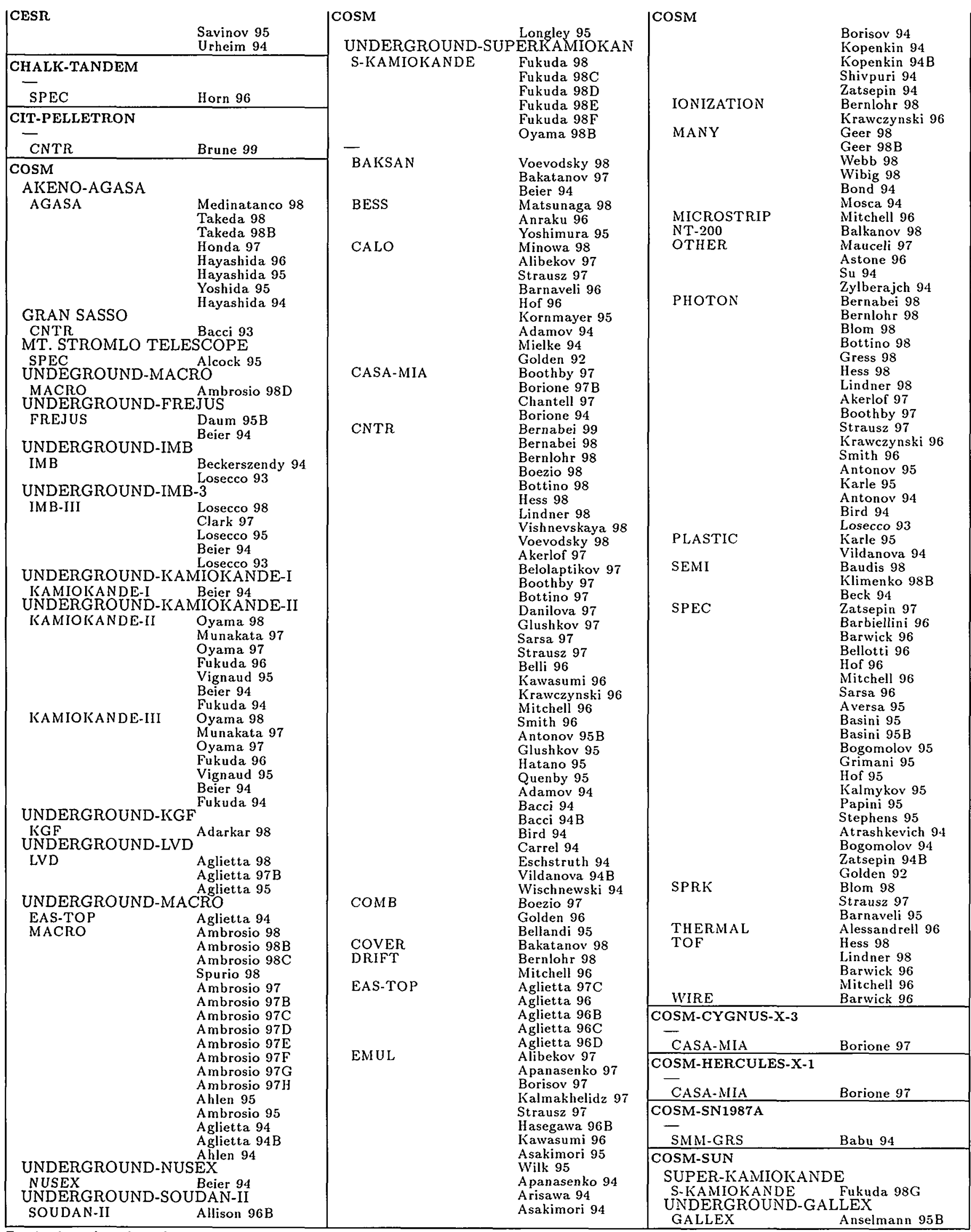




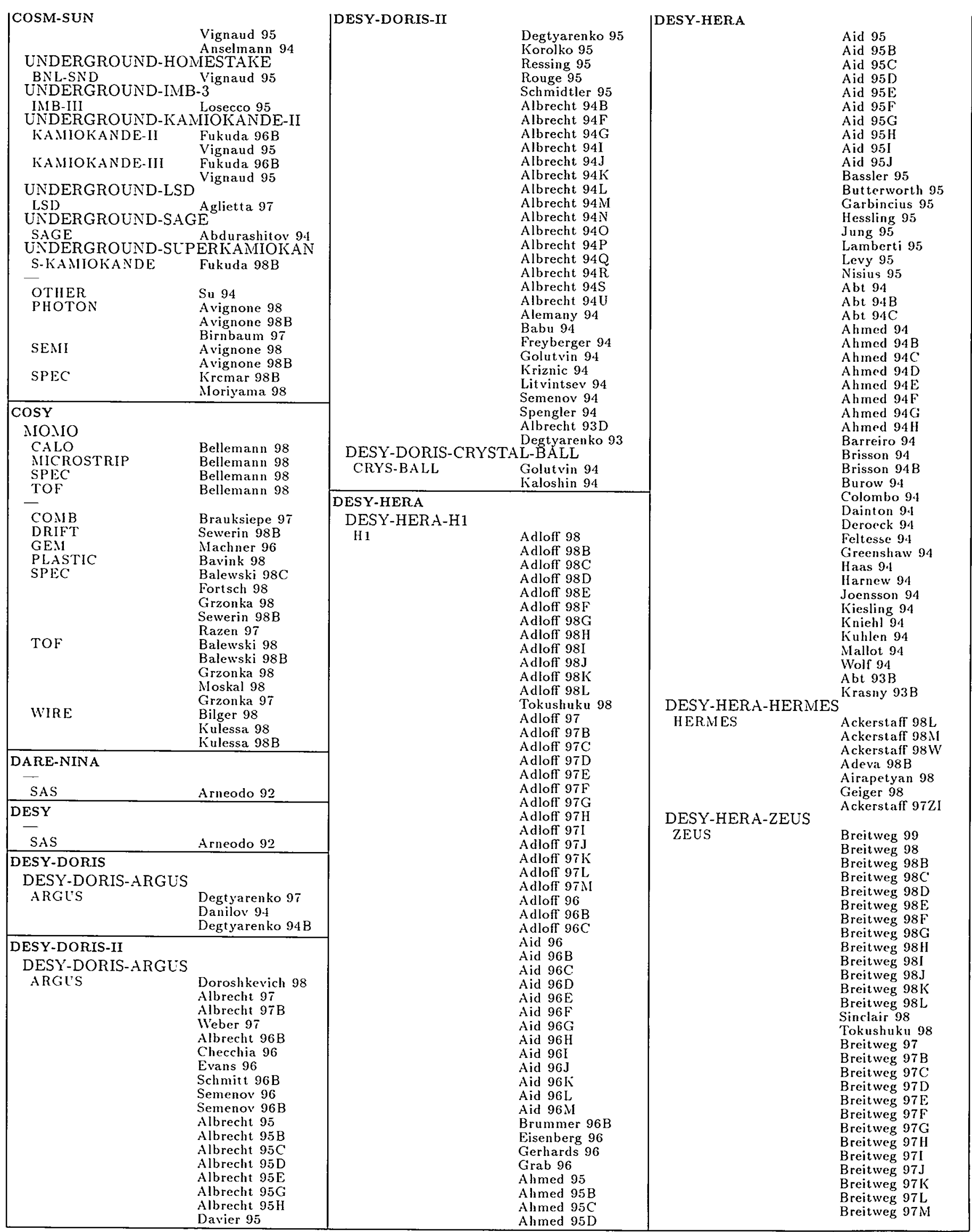




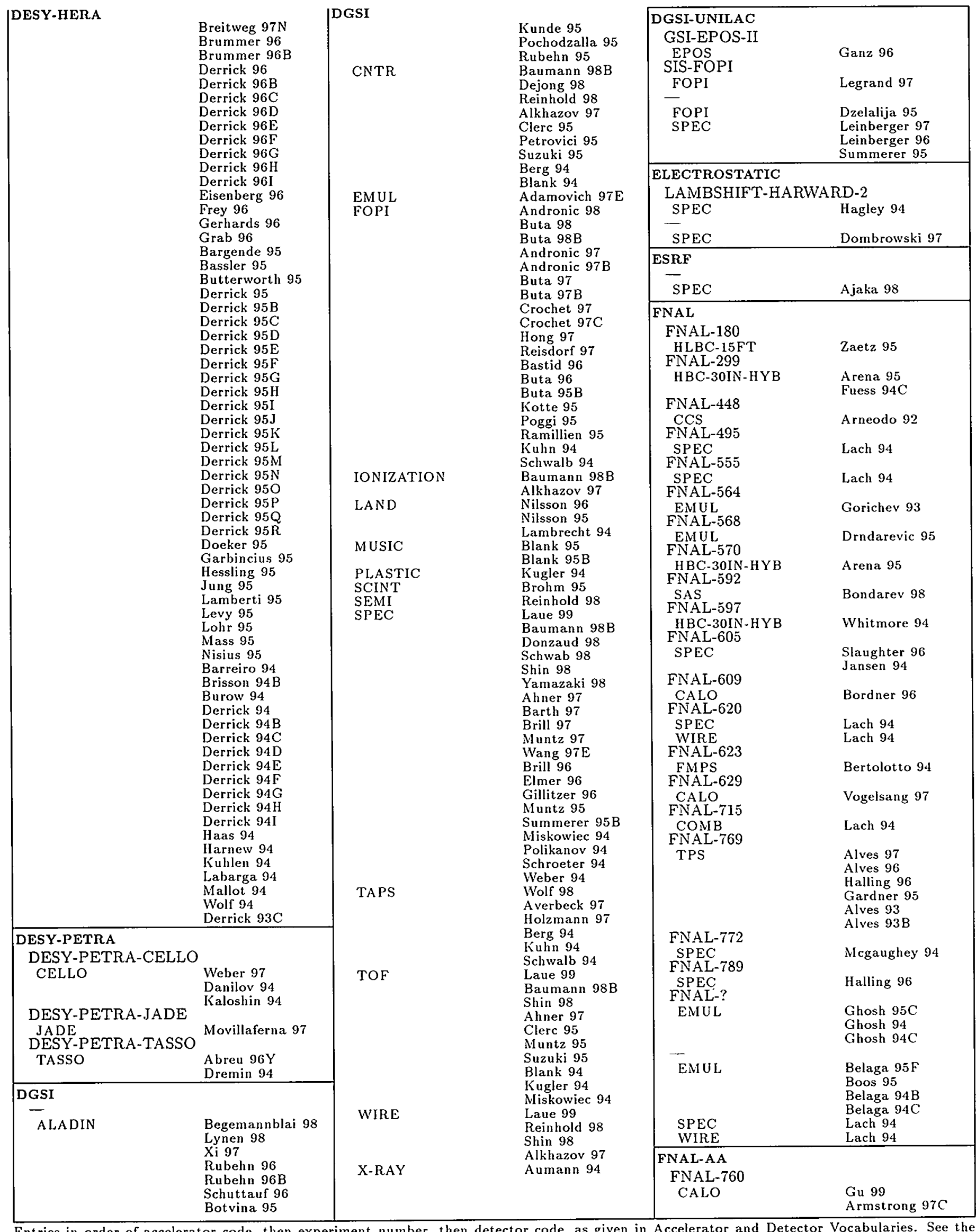




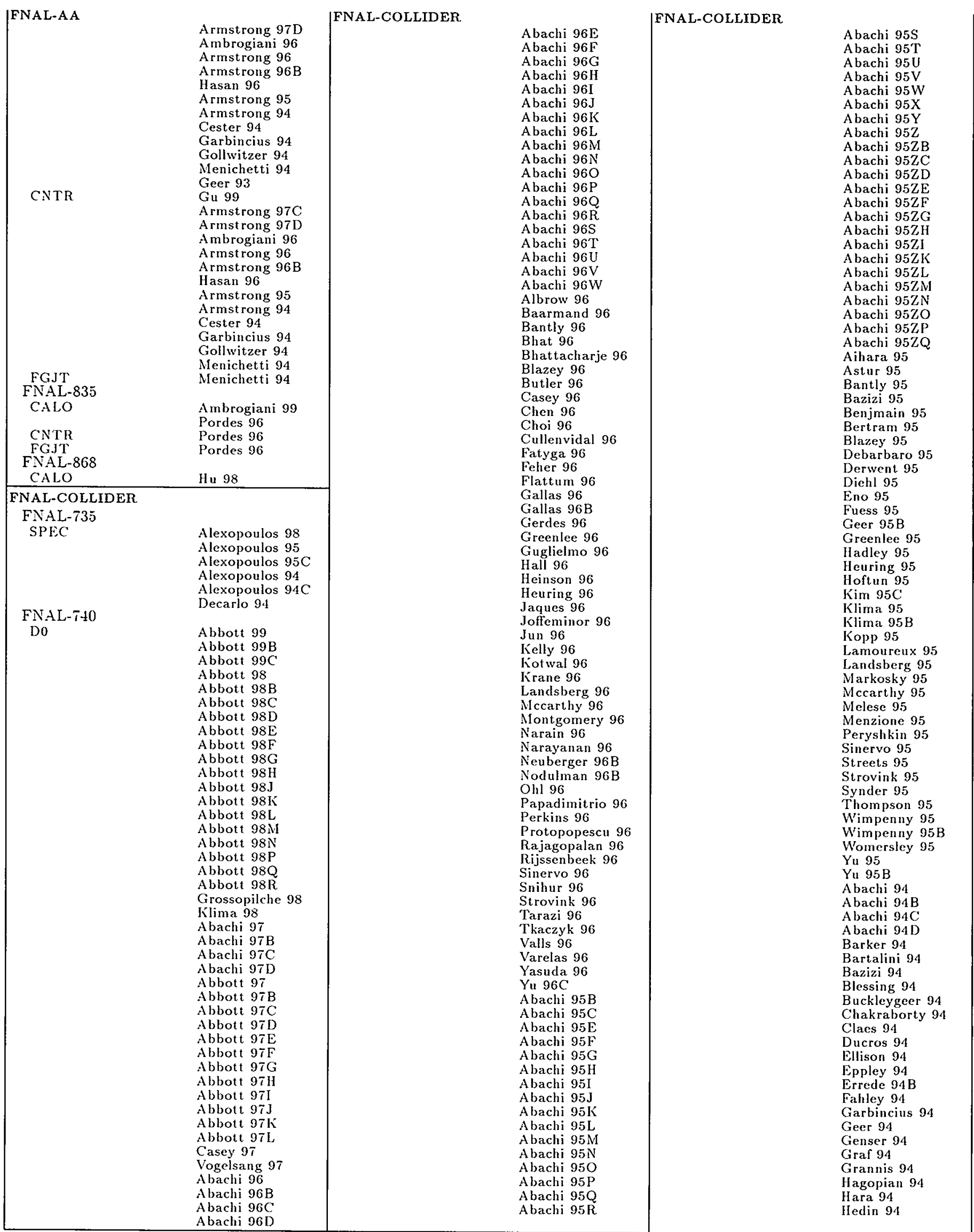


FNAL-COLLIDER

FNAL-741

CDF

Huehn 94
Jung 94
Lidemarteau 94
Nodulman 94
Norman 94
Oshima 94
Plunkett 94
Quintas 94
Raja 94
Spadafora 94
Taketani 94
Tollestrup 94
Weerts 94
White 94
Wolinski 94
Yu 94
Abachi $93 \mathrm{~B}$
Piekarz 93B

Abe 99

A be $99 \mathrm{~B}$

Abe 99D

Abe $99 \mathrm{E}$

Abe 98

A be $98 \mathrm{C}$

Abe $98 \mathrm{D}$

Abe $98 \mathrm{E}$

A be $98 \mathrm{~F}$

Abe 98G

A be $98 \mathrm{I}$

A be $98 \mathrm{~J}$

A be $98 \mathrm{M}$

A be $98 \mathrm{~N}$

Abe 980

A be $98 \mathrm{P}$

Abe $98 Q$

Abe $98 \mathrm{~S}$

Abe $98 \mathrm{~T}$

A be $98 \mathrm{U}$

A be $98 \mathrm{~V}$

Abe $98 \mathrm{~W}$

$A$ be $98 \mathrm{X}$

A be $98 \mathrm{Y}$

A be $98 \mathrm{Z}$

Abe 98ZB

Abe $98 \mathrm{ZC}$

Abe 98ZD

Abe 98ZE

Dorigo 98

Grossopilche 98

Singh 98

A be $97 \mathrm{D}$

A be $97 \mathrm{~F}$

$A$ be $97 \mathrm{H}$

Abe $97 \mathrm{~K}$

A be $97 \mathrm{~L}$

A be $97 \mathrm{M}$

Abe $97 \mathrm{~N}$

Abe $97 \mathrm{O}$

Abe 97P

A be $97 \mathrm{Q}$

Abe $97 R$

Abe 97S

Abe $97 \mathrm{~V}$

Abe $97 \mathrm{~W}$

Abe $97 \mathrm{X}$

Abe $97 \mathrm{Y}$

A be $97 \mathrm{Z}$

Abe $972 \mathrm{~B}$

Abe $97 \mathrm{ZC}$

A be 97ZD

A be $97 \mathrm{ZE}$

Abe $97 \mathrm{ZH}$

Abe $97 \mathrm{ZI}$

Abe 97ZJ

Abe $97 \mathrm{ZK}$

Conway 97

Vogelsang 97

Abe 96

A be $96 \mathrm{~B}$

Abe $96 \mathrm{C}$

A be 96D

A be $96 \mathrm{E}$

Abe $96 \mathrm{~F}$
FNAL-COLLIDER

A be $96 \mathrm{C}$

Abe $96 \mathrm{H}$

Abe $96 \mathrm{I}$

Abe $96 \mathrm{~J}$

Abe $96 \mathrm{~K}$

Abe $96 \mathrm{~L}$

Abe $96 \mathrm{M}$

Abe $96 \mathrm{~N}$

Abe $96 \mathrm{Q}$

Abe 96R

A be $96 \mathrm{~S}$

Abe $96 \mathrm{ZD}$

Albrow 96

Asakawa 96

Azzi 96

Barbarogalti 96

Barnett 96

Bauer 96

Binkley 96

Bodek 96

Busetto 96

Castro 96

Chiarelli 96

Cobalgrassma 96

Conway 96

Demina 96

Dittmann 96

Flaugher 96

Gerdes 96

Giokaris 96

Goshaw 96

Goshaw 96B

Goulianos 96

Huffman 96

Kamon 96

Kruse 96

Laasanen 96

Lecompte 96

Leone 96

Leone 96B

Leone 96

Lewis 96

Loomis 96

Loomis $96 \mathrm{~B}$

Lucchesi 96

Lys 96

Maeshima 96

Melese 96

Melese 96B

Melese 96C

Meschi 96

Miao 96

Neuberger 96

Neuberger 96B

Nodulman 96

Nodulman 96B

Ohl 96

Papadimitrio 96

Papadimitrio 96B

Paulini 96

Phillips 96

Pillai 96

Rijssenbeek 96

Rolli 96

Sansoni 96

Sinervo 96

Slaughter 96

Speer 96

Tartarelli 96

Thurmankeup 96

Tkaczyk 96

Toback 96

Valls 96

Varelas 96

Wang 96

Wenzel 96

Wenzel 96B

Yu 96B

Abe 95

Abe $95 \mathrm{~B}$

Abe 95C

A be 95D

Abe $95 \mathrm{E}$

Abe 95G

Abe $95 \mathrm{H}$
FNAL-COLLIDER

Abe 95I

Abe 95J

Abe $95 \mathrm{~L}$

Abe $95 \mathrm{M}$

Abe $95 \mathrm{~N}$

Abe 950

Abe 95P

Abe $95 \mathrm{Q}$

Abe $95 \mathrm{R}$

Abe $95 \mathrm{~T}$

Abe $95 \mathrm{U}$

A be $95 \mathrm{~W}$

Abe 95X

Abe $95 \mathrm{Y}$

Abe $95 \mathrm{ZB}$

Abe $95 \mathrm{ZC}$

A be $95 \mathrm{ZE}$

Abe $95 \mathrm{ZH}$

Abe 95ZI

Abe 95ZK

Aihara 95

Anwaywiese 95

Bauer 95

Benjmain 95

Beretvas 95

Beretvas 95B

Beretvas 95C

Beretvas 95D

Bertram 95

Binkley 95

Blair 95

Blazey 95

Buckleygeer 95

Debarbaro 95

Demortier 95

Derwent 95

Diehl 95

Frisch 95

Fuess 95

Geer 95B

Grassmann 95

Grassmann 95B

Harris 95

Harris 95 B

Incandela 95

Incandela 95B

Kim 95

Kim 95B

Kim 95C

Kopp 95

Lamoureux 95

Mccarthy 95

Melese 95

Menzione 95

Papadimitrio 95

Papadimitrio 95B

Park 95

Ragan 95

Roser 95

Sansoni 95

Sinervo 95

Skarha 95

Streets 95

Ukegawa 95

Unal 95

Wagner 95

Womersley 95

Yu 95

Yu 95B

Abe 94

A be $94 \mathrm{~B}$

Abe $94 \mathrm{D}$

Abe $94 \mathrm{E}$

Abe $94 \mathrm{~F}$

A be $94 \mathrm{G}$

A be $94 \mathrm{H}$

Abe $94 \mathrm{I}$

Abe $94 \mathrm{~J}$

Abe $94 \mathrm{~K}$

Abe $94 \mathrm{~L}$

Abe $94 \mathrm{M}$

Abe $94 \mathrm{~N}$

Abe 940

Abe 94P

A be $94 Q$ 
|FNAL-COLLIDER
Abe $94 \mathrm{R}$

A be $94 \mathrm{~S}$

A be $94 \mathrm{~T}$

Abe $94 X$

Abe $94 \mathrm{Y}$

A be $94 Z$

A be $94 Z \mathrm{D}$

A be $942 \mathrm{E}$

A be $94 Z F$

Abe $94 Z G$

Abe 94ZH

Antos 94

Anwaywiese 94

Badgett 94

Bailey 94

Bartalini 94

Bazizi 94

Benlloch 94

Bhatti 94

Blair 94

Buckleygeer 94

Budd 94

Byrum 94

Cdfcollabora 94B

Cdfcollabora 94C

Cdfcollabora 94D

Cdfcollabora $94 \mathrm{E}$

Cdfcollabora $94 \mathrm{~F}$

Daniels 94

Debarbaro 94

Dejongh 94

Derwent 94

Ducros 94

Errede 94

Errede 94B

Frisch 94

Fuess 94B

Garbincius 94

Geer 94

Gerdes 94

Giannetti 94

Hagopian 94

Hamilton 94

Hara 94

Harris $94 \mathrm{~B}$

Hauger $94 \mathrm{~B}$

Hawk 94

Jensen 94

Jung 94

Kamon 94

Karen 94

Keup 94

fim 94

Konigsberg 94

Kopp 94

Kovacs 94

Kiovacs $94 \mathrm{~B}$

Kuhlmann 94

luhlmann 94B

Lecompte 94

Leone 94

Lewis 94

Lewis $94 \mathrm{~B}$

Maeshima 94

Maghakian 94

Melese 94

Mueller 94

Nodulman 94

Papadimitrio 9

Park 94B

Plunket t 94

Rimondi 94

Sakyumoto 94

Shochet 94

Skarha 94

Sphicas 94

Tollestrup 94

Watts 94

Williams $9-1$

Yu 94

Yu 94B

A be $93 \mathrm{~N}$

$A$ be $93 \mathrm{~S}$

A be $93 \mathrm{~T}$

Abe 93U

A be $931 \mathrm{~W}$

\section{FNAL-COLLIDER}

FNAL-775

$\mathrm{CDF}$

FNAL-811

SCINT

FNAL-864

MINIMAX

MANY

FNAL-TEV

FNAL-400

SPEC

FNAL-581

SPEC

FNAL-581-704

SPEC

FNAL-621

WIRE

FNAL-632

HLBC-15FT-HY'B

FNAL-653

EMUL

SPEC

\section{FNAL-665}

CCM

FNAL-667

EMUL

FNAL-672

FMPS

FNAL-683

CALO

SPEC

\begin{tabular}{l|l} 
Belforte 93 & FNAL-TEV \\
Luke 93 & FNAL-687 \\
CALO
\end{tabular}

SPEC

A be $97 \mathrm{C}$

A be $97 \mathrm{I}$

Abe $96 \mathrm{P}$

Avila 99

Bjorken 96

Harris 94

iss 96

Grosnick 97

Adams $94 \mathrm{~B}$

Zou 96

Thomson 94

Zou 94

Asratyan 97

Deprospo 94

Kodama 96

Slaughter 96

Kodama 95

Freyberger 94

Garbincius 94

Gibaut $94 \mathrm{~B}$

Kodama 94

Kodama 96

Slaughter 96

Kodama 95

Freyberger 94

Garbincius 94

Gibaut 94B

Kódama 94

Adams $97 \mathrm{C}$

Adams $97 \mathrm{~F}$

Seligman 97

Seligman $97 \mathrm{C}$

Adams 96

Salgado 96

Adams 95

Adams $95 \mathrm{C}$

Adams 95D

Adams $9.5 \mathrm{~F}$

Adams $95 \mathrm{G}$

Badelek 95

Conrad 95

Kotwa! 95

Adams $94 \mathrm{D}$

Adams 94E

Adams 94F

Adams $94 \mathrm{H}$

Fang 94

Kotwal 94

Ḱotwal 94B

Mallot 94

Schellman 94

Spentzouris 94

Wolbers 94

Adams $93 \mathrm{C}$

Adams 93D

Adams $93 \mathrm{~F}$

Arneodo 92

Cherry 94C

Garbincius 94

Alton 96

Conrad 95

Adams 94

Naples 94

Alton 96

Conrad 95

Adams 94
FNAL-690

SPEC

FNAL-691

TPS

FNAL-704

SPEC

FNAL-705

SPEC

FNAL-706

FMIPS

FNAL- 731

SPEC

FNAL-733

FMM

FNAL-743

FNIPS

HBC-LEBC-HYB

FNAL-744

LAB-E
Naples 94

Halling 96

Garbincius 95

Frabetti $94 \mathrm{G}$

Johns 94

Frabetti 98

Frabetti 98B

Frabetti 97

Frabetti 97B

Frabetti 97C

Frabetti 96

Frabetti 96B

Halling 96

Semenov 96

Slaughter 96

Wiss 96

Frabetti 95

Frabetti 95B

Frabetti 95C

Frabetti 95D

Frabetti 95E

Frabetti $95 \mathrm{~F}$

Frabett $95 \mathrm{H}$

Frabetti 95

Fujino 95

Garbincius 95

Gardner 95

Frabetti 94

Frabetti 94B

Frabetti 94C

Frabetti 94D

Frabetti $94 \mathrm{E}$

Frabetti $94 \mathrm{~F}$

Frabetti 94G

Frabetti $94 \mathrm{H}$

Frabetti 941

Frabetti 04J

Johns 94

Moroni 94

Frabetti 93C'

Reyes 97

Gutierrez 96

Guticrrez 94

Wiss 96

Garbincius 95

Freyberger 94

Adams $97 \mathrm{E}$

Bravar 97

Vogelsang 97

Bravar 96

Adams 9.5H

Bravar 95

Adams $94 \mathrm{C}^{\circ}$

Sansoni 95

Antoniazzi 94

Antoniazzi $94 \mathrm{~B}$

Garbincius 94

Gu 99

A panasevich 97

A panasevich $97 \mathrm{~B}$

Vogelsang 97

Koreshev 96

Slaughter 96

Conrad 95

Gribushin 95

Sansoni 95

Jesik 94

Alverson 93B

Gibbons $95 \mathrm{C}^{\circ}$

Hsiung 94

Makoff 93

Gallas 95

Dremin 94

Dremin 94

Mcfarland 9 


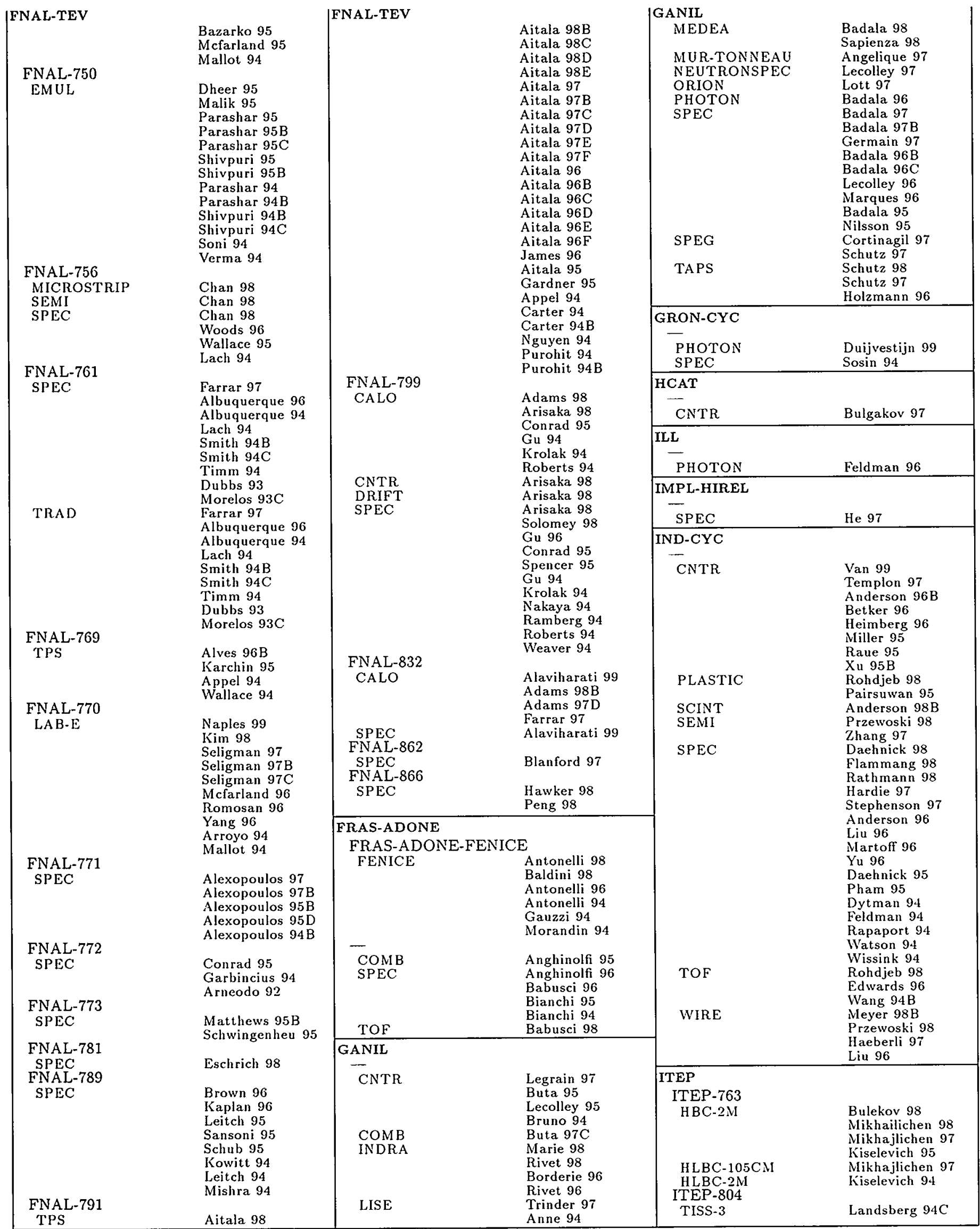

Entries in order of accelerator code, then experiment number, then detector code, as given in Accelerator and Detector Vocabularies. See the legend on page 399 . 


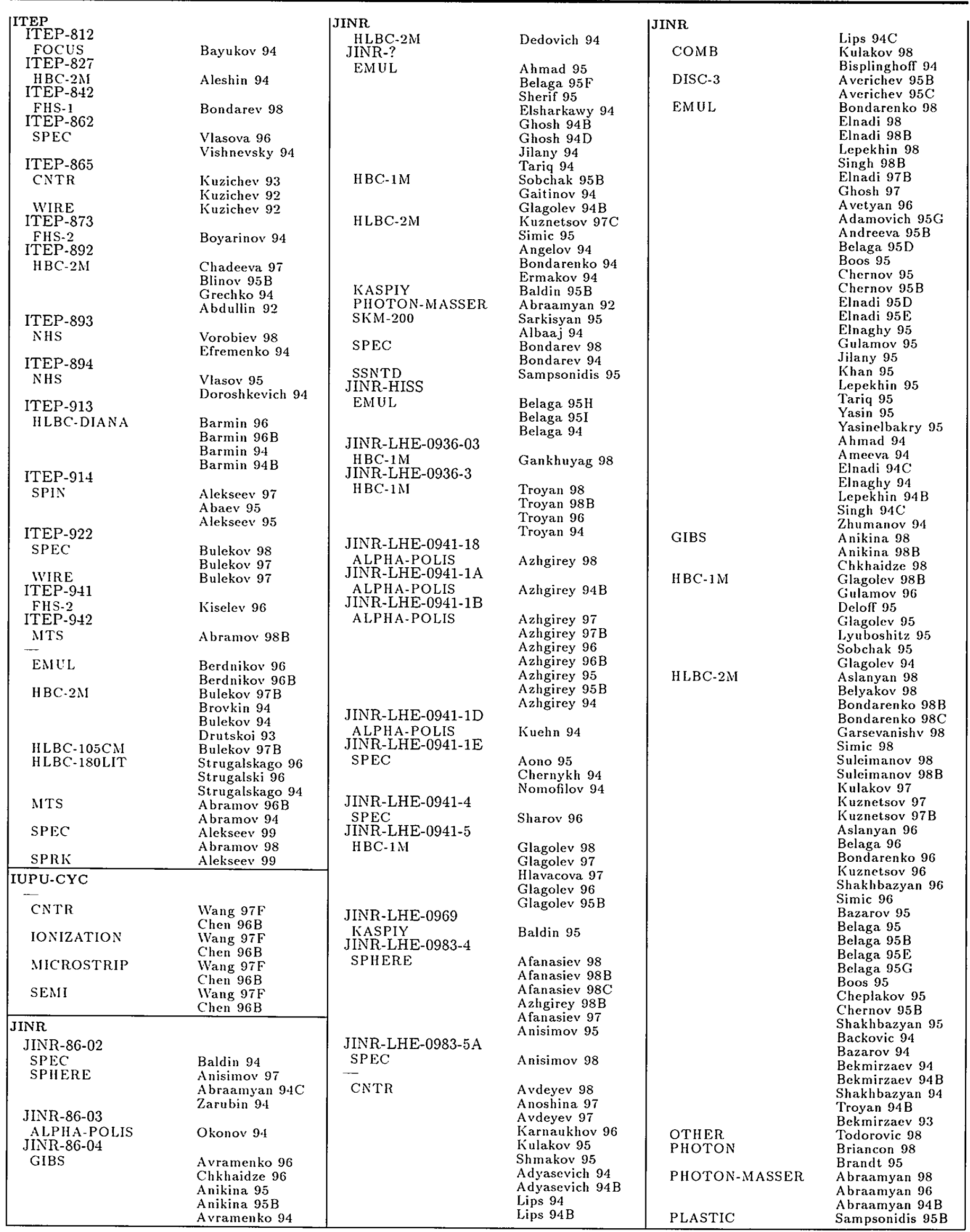




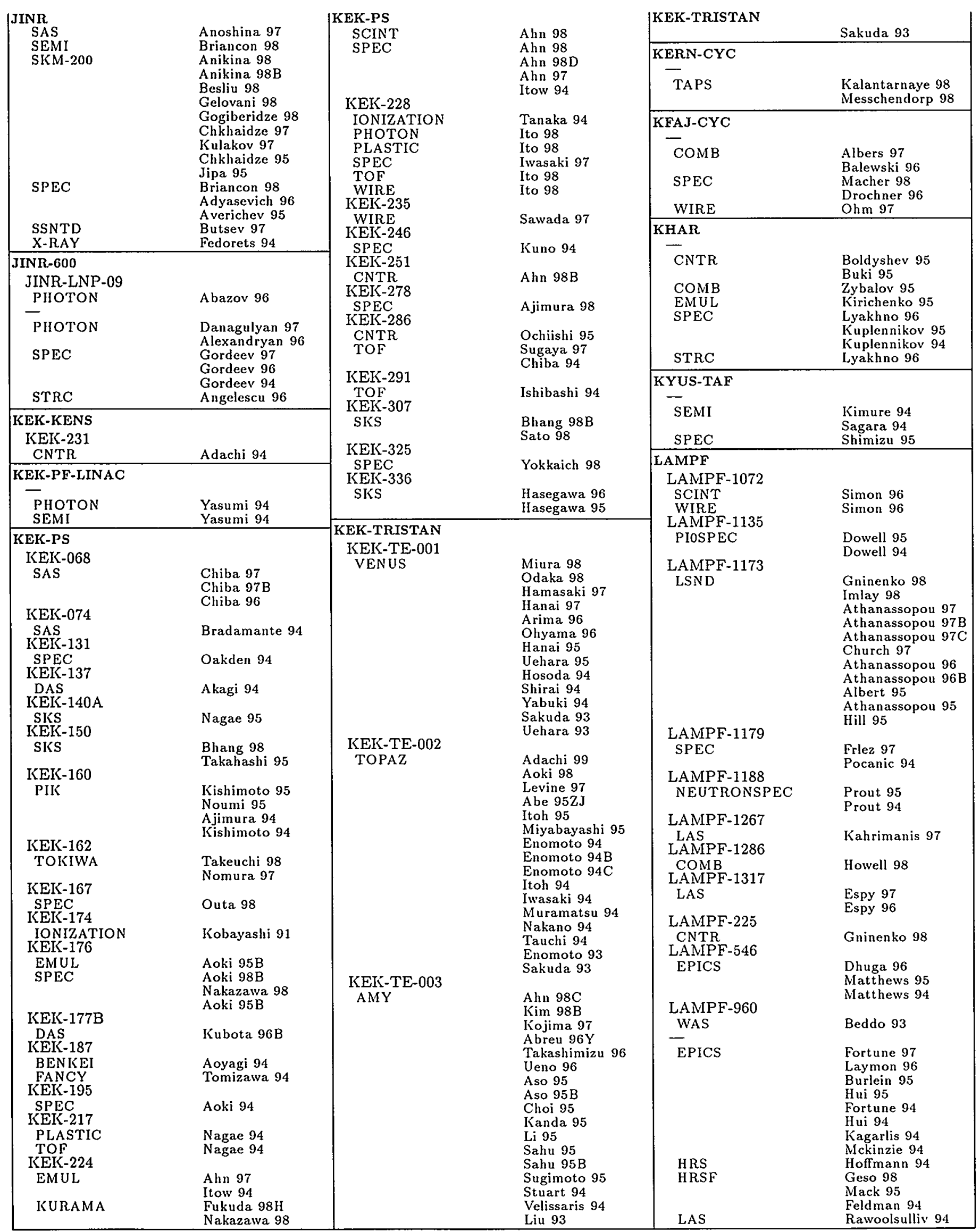

Entries in order of accelerator code, then experiment number, then detector code, as given in Accelerator and Detector Vocabularies. See the legend on page 399 . 


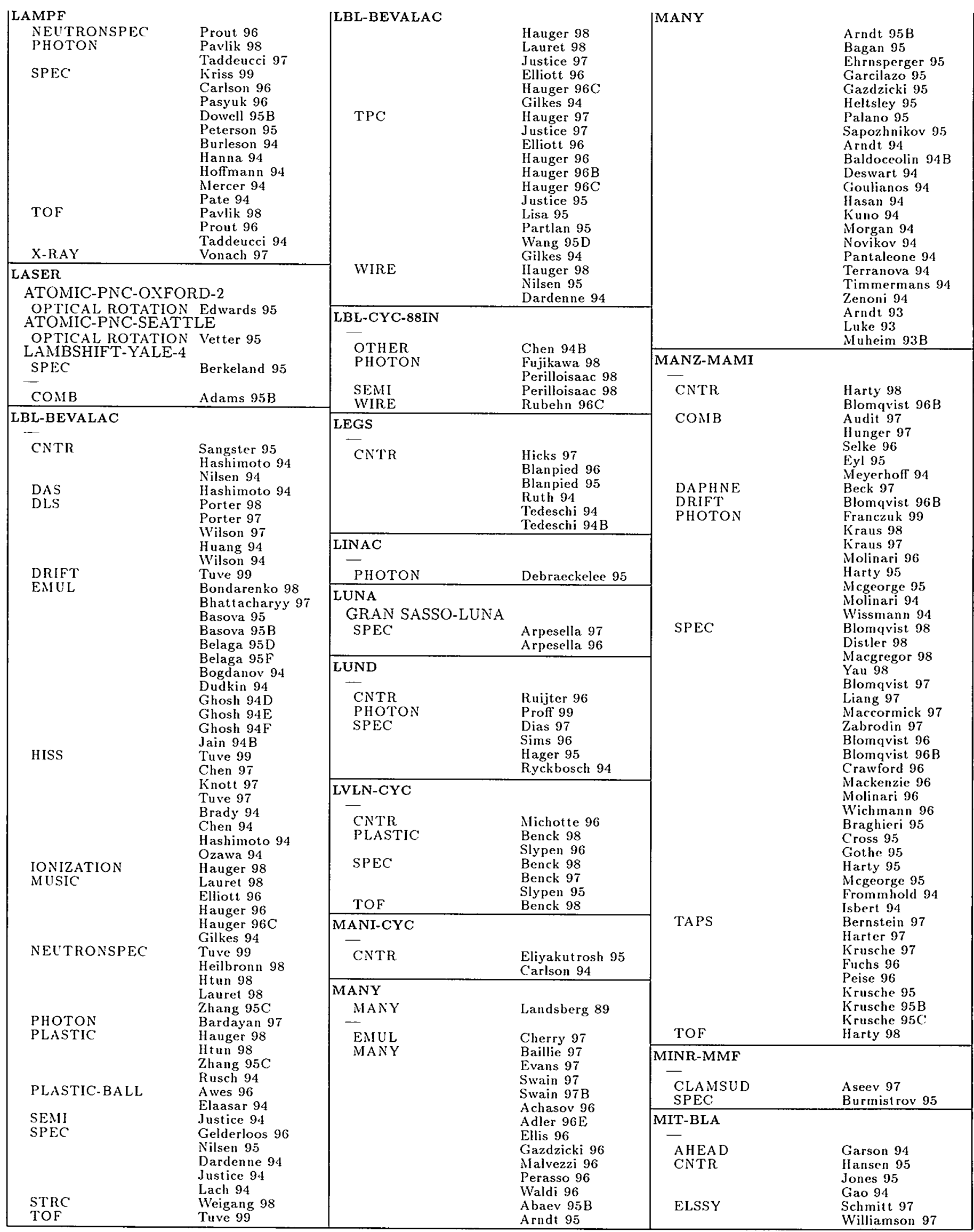




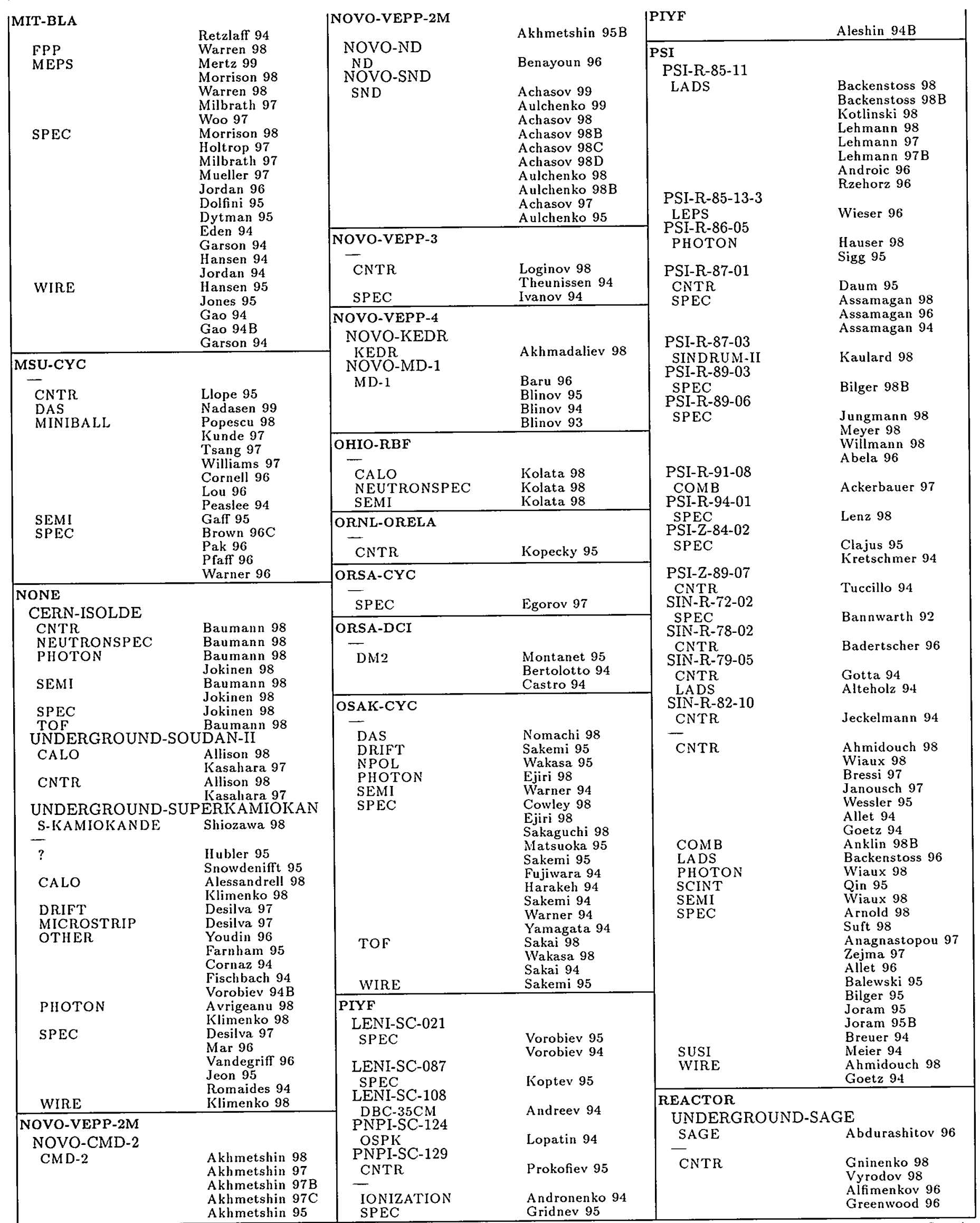




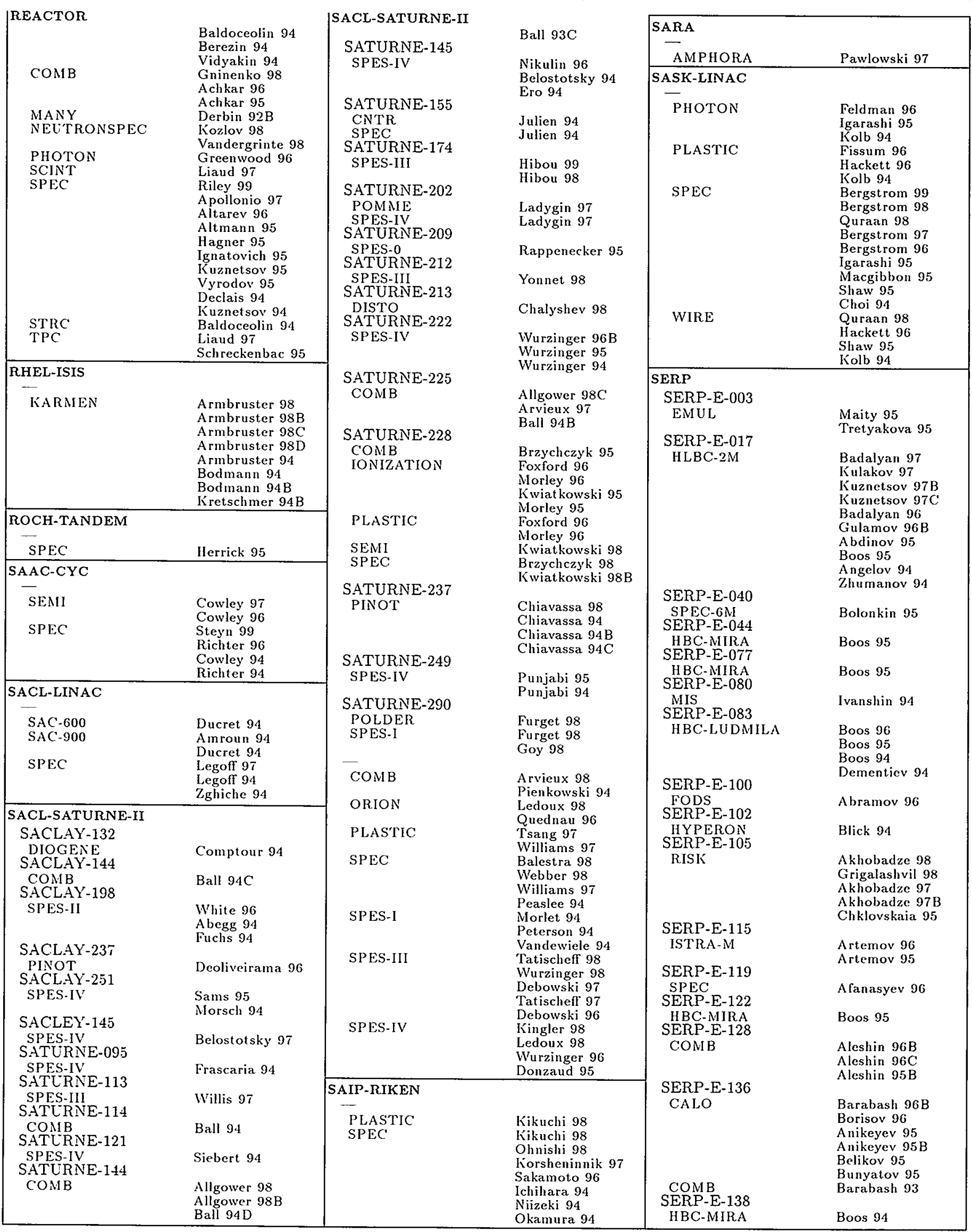




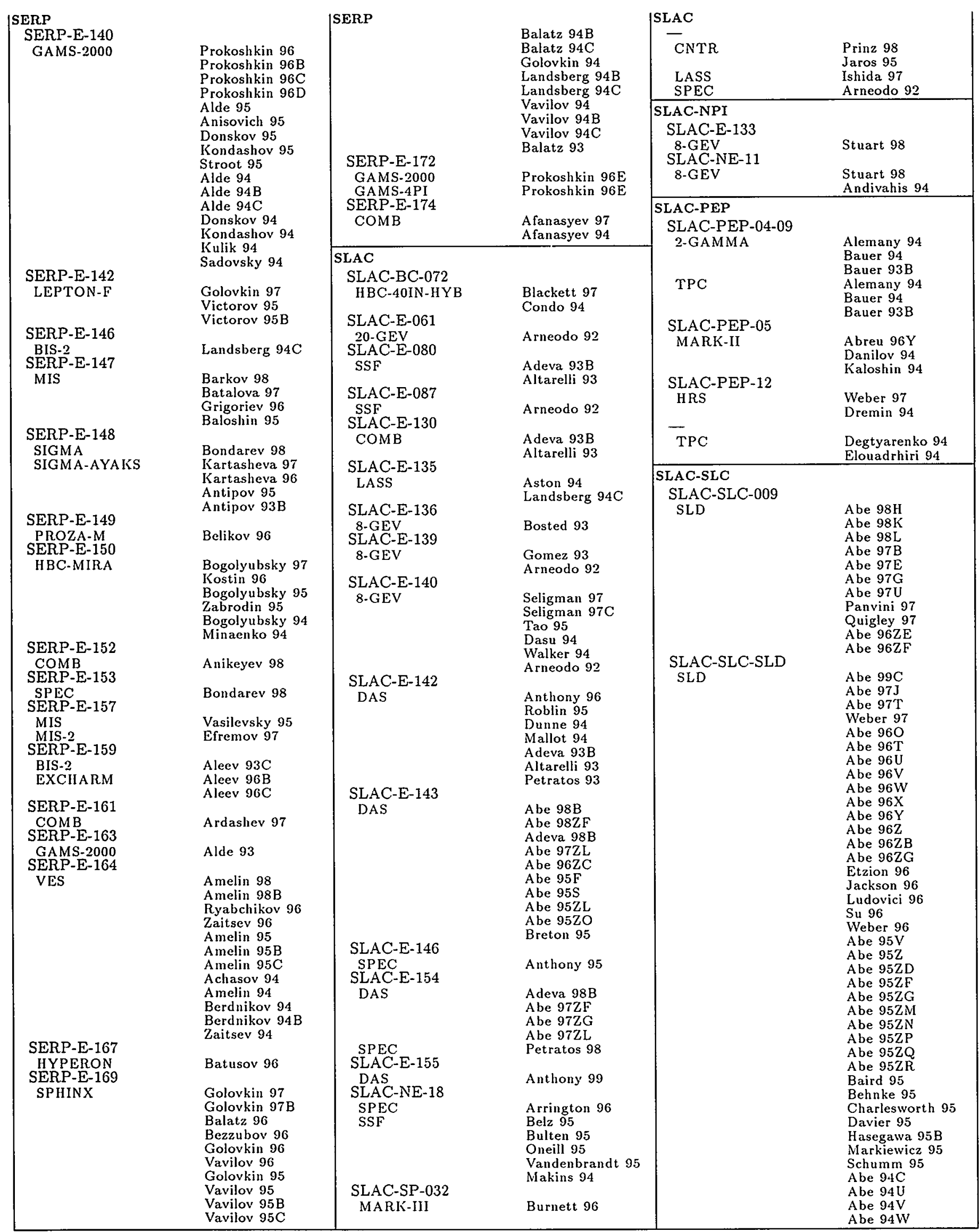


SLAC-SLC

UUPP-CYC

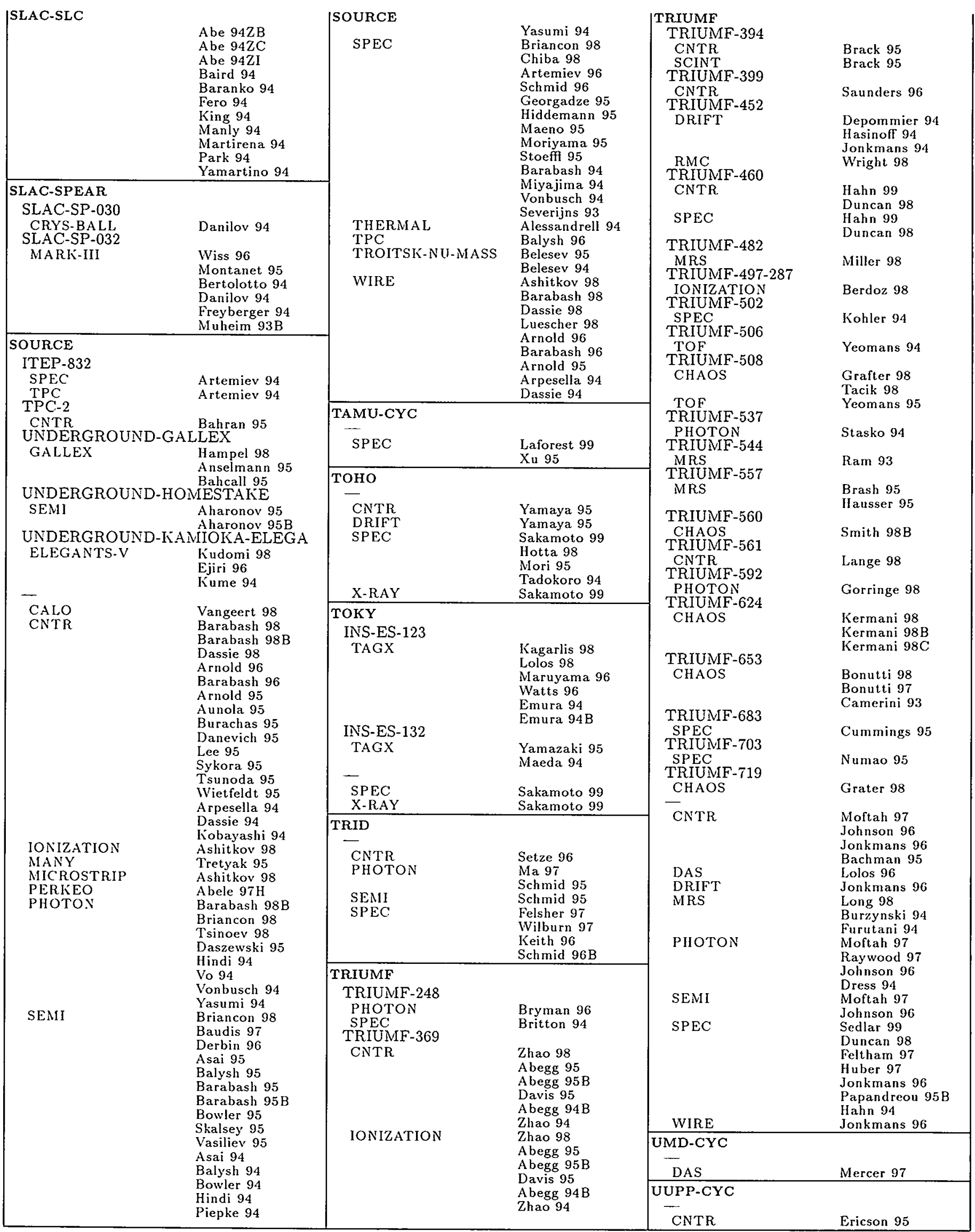




\begin{tabular}{|c|c|}
\hline $\begin{array}{l}\text { UUPP-CYC } \\
\text { NEUTRONSPEC } \\
\text { PHOTON } \\
\text { PLASTIC } \\
\text { SPEC } \\
\end{array}$ & $\begin{array}{l}\text { Auce } 94 \\
\text { Rahm } 98 \\
\text { Eismont } 96 \\
\text { Calen } 98 \mathrm{C} \\
\text { Auce } 96 \\
\text { Johansson } 98 \\
\end{array}$ \\
\hline $\begin{array}{l}\text { VAN-DE-GRAAFF } \\
\text { CNTR } \\
\text { PHOTON } \\
\text { SEMI } \\
\text { SPEC }\end{array}$ & $\begin{array}{l}\text { Wilburn } 95 \\
\text { Mohr } 98 \\
\text { Mohr 98 } \\
\text { Hammache } 97 \\
\text { Deboer 96 } \\
\text { Keith 94 } \\
\end{array}$ \\
\hline $\begin{array}{l}\text { VECC } \\
\overline{\text { SSNTD }}\end{array}$ & Mukherjee 95 \\
\hline$\frac{\text { WISC }}{\text { SEMI }}$ & $\begin{array}{l}\text { Browne } 96 \\
\text { Mcaninch } 94 \\
\end{array}$ \\
\hline $\begin{array}{l}\text { YERE-ARUS } \\
\text { - COMB } \\
\text { DEUTRON-2 } \\
\text { OSPK } \\
\text { PHOTON } \\
\text { SPEC }\end{array}$ & $\begin{array}{l}\text { Arakelyan } 95 \\
\text { Alanakyan } 98 \\
\text { Alanakyan } 97 \\
\text { Markaryan } 95 \\
\text { Danagulyan } 97 \mathrm{~B} \\
\text { Alexandryan } 94 \\
\text { Vartapetyan } 95\end{array}$ \\
\hline
\end{tabular}


We list here all particle names used to describe decays and reactions. Most of the names are obvious. The first column gives the usual particle symbol, the second gives the English spelling, and the third gives a brief definition. The English spelling is the "computer name," the version to be typed in searching our computer databases.

Particle names follow the ordering in the Particle Vocabulary of this compilation: Gauge bosons, leptons, mesons, baryons, atoms, and nuclei; and within each group the ordering is mainly by increasing mass. Within mesons and baryons, however, we further categorize these particles as having only light quarks, or as having as the heaviest quark, a strange, charmed, bottom, or top quark.

In the indices, the order of the charge states is $++,+, 0,-,-$. For antiparticles, we use the actual charge of the antiparticle, as in $\bar{\Delta}^{--}$. Unless an antiparticle has a common name of its own, as in $K^{+}$, all antiparticles are spelled with the suffix "BAR" appended to the English portion of the names, as PBAR for $\bar{p}$, DELTABAR(1900S31)0 for $\bar{\Delta}\left(1900 S_{31}\right)^{0}$, and charmBar for charm.

We use the chemical symbols for nuclei except in a few cases where an ambiguity with a particle name exists. For example, we use kK for potassium to avoid confusion with the $K$ meson, and sit for nitrogen to avoid confusion with the neutron, nucleon or nickel.

The names we use in writing any reaction are based on those used by the authors of the paper. For example, one paper might refer to $\pi^{+}, \pi^{-}$, and $\pi^{0}$, while another just uses $\pi$ to mean all three.

Some of the "particle names" represent a group of particles whose exact number is unknown or is varied in some limited range; for example MULT [PI+] means a limited number of $\pi^{+}$particles or a multiplicity distribution on the number of $\pi^{+}$particles. Names like these are treated as a single particle in the Reaction/Momentum Index, where the reaction final states are ordered by increasing multiplicity. We do give the exact number of particles when known, e.g., " $3 \pi^{+}$" or " 2 charm," and these forms are treated as the stated number of particles. 


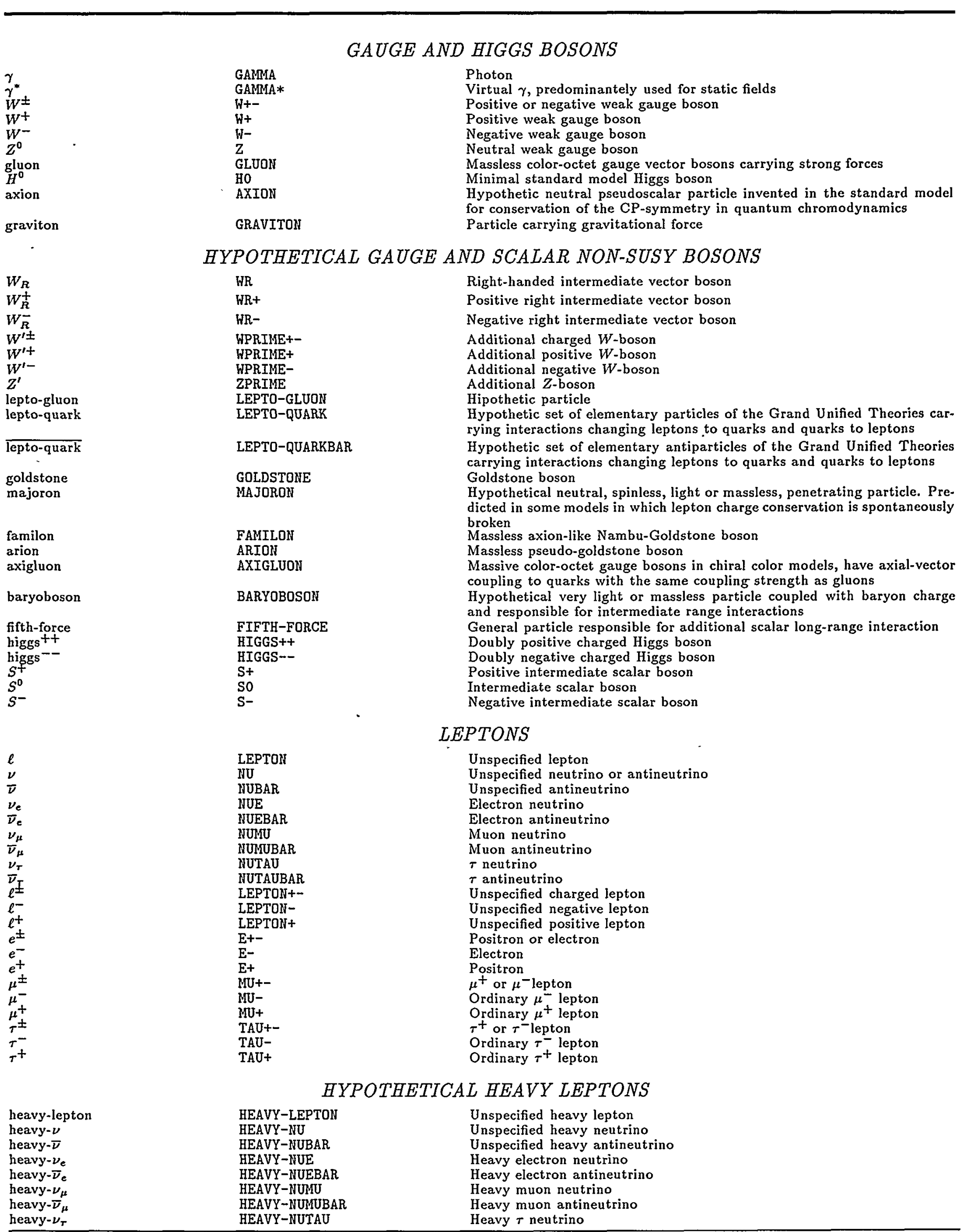




Particle Name Computer Name Explanation

heavy $-\bar{\nu}_{r}$ heavy-lepton ${ }^{0}$ heavy-lepton 0 heavy-lepton ${ }^{ \pm}$ heavy-lepton heavy-lepton ${ }^{+}$ heavy-e heavy- $e^{0}$ heavy- $\mu$ heavy $-\mu^{0}$ heavy- $\tau$

neutralino 2

chargino

chargino ${ }^{+}$

chargino-

photino

photino

wino

winot

wino $^{-}$

zino

gluino

axino

gravitino

gravitino

higgsino

higgsino $(C P=-1)^{0}$

higgsino $^{+}$

higgsino $^{0}$

higgsino-

goldstino

goldstino

$H_{1}^{0}$
$A^{0}$
$H_{2}^{0}$
$H^{ \pm}$
$H^{+}$
$H^{-}$
$\tilde{\ell}$
$\tilde{\ell}^{-}$
$\tilde{\ell}^{+}$

HEAVY-NUTAUBAR
HEAVY-LEPTONO
HEAVY-LEPTONBARO
HEAVY-LEPTON+-
HEAVY-LEPTON-
HEAVY-LEPTON+
HEAVY-E
HEAVY-EO
HEAVY-MU
HEAVY-MUO
HEAVY-TAU

Heavy $\tau$ antineutrino

Unspecified neutral heavy lepton

Unspecified neutral heavy antilepton

Unspecified charged heavy lepton

Unspecified negative heavy lepton

Unspecified positive heavy lepton

Unspecified heavy electron

Neutral heavy electron

Unspecified heavy muon

Neutral heavy muon

Unspecified heavy $\tau$ lepton

\section{QUARKS AND DIQUARKS}

Quark of unspecified charge

Antiquark of unsprcified charge

Up quark

Up antiquark

Down quark

Down antiquark

Strange quark

Strange antiquark

Charmed quark

Charmed antiquark

Bottom quark

Bottom antiquark

Top quark

Top antiquark

Diquark of unspecified charge and flavor

\section{HYPOTHETICAL QUARKS}

BQPRIME

BQPRIMEBAR

Bottom quark of fourth generation

Bottom antiquark of fourth generation

\section{SUSY PARTICLES: FERMIONS}

SPARTICLE

GAUGINO

IEUTRALINO

NEUTRALINO-1

NEUTRALINO-2

CHARGINO

CHARGINO+

CHARGINO-

PHOTINO

PHOTINOBAR

WINO

WINO+

WINO-

ZIHO

GLUIMO

AXINO

GRAVITIND

GRAVITINOBAR

HIGGSINO

HIGGSINO $(\mathrm{CP}=-1) 0$

HIGGSINO+

HIGGSINOO

HIGGSINO-

GOLDSTINO

GOLDSTINOBAR

Supersymmetric partner of any ordinary particle

Spin-1/2 supersymmetric partner of any gauge boson

Any supersymmetric partner of an ordinary neutral particle

The lightest supersymmetric partner of the ordinary neutral particles which appeared in the supersymmetric extention of the SM

The second supersymmetric partner of the ordinary neutral particles which appeared in the supersymmetric extention of the SMI

Mixture of wino and charged higgsino

Mixture of wino and charged higgsino

Mixture of wino and charged higgsino

Spin-1/2 supersymmetric partner of the photon

Spin-0 supersymmetric partner of the antiphoton

Spin-1/2 supersymmetric partner of the $W^{ \pm}$

Spin-1/2 supersymmetric partner of the $W^{+}$

Spin-1/2 supersymmetric partner of the $W^{-}$

Spin-1/2 supersymmetric partner of the $Z^{0}$

Spin-1/2 supersymmetric partner of the gluon

Spin-1/2 supersymmetric partner of the axion

Spin-3/2 supersymmetric partner of the graviton

Antigravitino

Spin-1/2 supersymmetric partner of any Higgs boson

Spin-1/2 supersymmetric partner of neutral CP-odd Higgs boson

Spin-1/2 supersymmetric partner of the positive Higgs boson

Spin-1/2 supersymmetric partner of any neutral CP-even Higgs boson

Spin-1/2 supersymmetric partner of the negative Higgs boson

Supersymmetric partner of the Goldstone boson

Spin-0 supersymmetric partner of the antigoldstino

\section{SUSY PARTICLES: BOSONS}

$\mathrm{H}(1) \mathrm{O}$
$\mathrm{A} 0$
$\mathrm{H}(2) \mathrm{O}$
$\mathrm{H}+-$
$\mathrm{H}+$
$\mathrm{H}-$
SLEPTON
SLEPTON-
SLEPTON+

Minimal supersymmetric model CP-even Higgs boson (lightest)

Minimal supersymmetric model $\mathrm{CP}$-odd Higgs boson

Minimal supersymmetric model $\mathrm{CP}$-even Higgs boson (heaviest)

Minimal supersymmetric charged Higgs boson

Minimal supersymmetric positive Higgs boson

Minimal supersymmetric model negative Higgs boson

Spin-0 supersymmetric lepton partner

Negative spin-0 supersymmetric lepton partner

Positive spin-0 supersymmetric lepton partner

See the legend on page 425 . 


\section{Particle Name Computer Name Explanation}

SLEPTONO

SNU

SNUBAR

SNUE

SNUEBAR

SNUMU

SNUMUBAR

SNUTAU

SNUTAUBAR

SELECTRON

SELECTRON-

SELECTRON+

SMUON

SMUON-

SMUON+

STAU

STAU-

STAU+

SQUARK

SQUARKBAR

S-UQ

$S-D Q$

S-SQ

$S-C Q$

$S-B Q$

S-BQBAR

S-TQ

S-TQBAR

S-TQ1

S-TQ1BAR

S-TQ2

S-TQ2BAR

GLUINIUM
Neutral spin-0 supersymmetric lepton partner

Spin-0 supersymmetric partner of the neutrino

Spin-0 supersymmetric partner of the antineutrino

Spin-0 supersymmetric partner of the $\nu_{e}$

Spin-0 supersymmetric partner of the $\bar{\nu}_{e}$

Spin-0 supersymmetric partner of the $\nu_{\mu}$

Spin-0 supersymmetric partner of the $\bar{\nu}_{\mu}$

Spin-0 supersymmetric partner of the $\nu_{r}$

Spin-0 supersymmetric partner of the $\bar{\nu}_{T}$

Spin-0 supersymmetric partner of the positron or electron

Spin-0 supersymmetric partner of the electron

Spin-0 supersymmetric partner of the positron

Spin-0 supersymmetric partner of muon

Spin-0 supersymmetric partner of $\mu^{-}$

Spin-0 supersymmetric partner of $\mu^{+}$

Spin-0 supersymmetric partner of $\tau$ lepton

Spin-0 supersymmetric partner of $\tau^{-}$lepton

Spin-0 supersymmetric partner of $\tau^{+}$lepton

Spin-0 supersymmetric quark partner

Spin-0 supersymmetric antiquark partner

Spin-0 supersymmetric partner of the up quark

Spin-0 supersymmetric partner of the down quark

Spin-0 supersymmetric partner of the strange quark

Spin-0 supersymmetric partner of the charm quark

Spin-0 supersymmetric partner of the bot tom quark

Spin-0 supersymmetric partner of the bottom antiquark

Spin-0 supersymmetric partner of the top quark

Spin-0 supersymmetric partner of the top antiquark

Lightest mass eigenstate of the mixed spin-0 supersymmetric partners of the left and right top quarks

Lightest mass eigenstate of the mixed spin-0 supersymmetric partners of the left and right top quarks

Heaviest mass eigenstate of the mixed spin-0 supersymmetric partners of the left and right top quarks

Heaviest mass eigenstate of the mixed spin-0 supersymmetric partners of the left and right top quarks

Bound state of gluinos

\section{COMPOSITE LEPTONS AND QUARKS}

$C^{*}$
$\nu^{*}$
$\nu^{*}$
$\nu_{c^{*}}^{*}$
$\bar{\nu}_{e}^{*}$
$e^{*} \pm$
$e^{*}-$
$\epsilon^{*+}$
$\nu_{\mu}^{*}$
$\bar{\nu}_{\mu^{*}}^{*}$
$\mu^{*}$
$\mu^{*}$
$\mu^{*+}$
$\nu_{\bar{*}}^{*}$
$\bar{\nu}_{T}^{*}$
$\tau^{*}$
$\tau^{*}$
$\tau^{*}+$
$q^{*}$
$\bar{q}^{*}$

cosmion

dark

millicharged monopole technipion technipion ${ }^{+}$ technipion technirho

techniomega
Unspecified exited lepton

Excited generic neut rino

Excited generic anti-neutrino

Composite electron neutrino

Composite electron anti-neutrino

Excited positron or electron

Excited electron

Excited positron

Composite muon neutrino

Composite muon anti-neutrino

Excited charged muon

Excited $\mu^{-}$

Excited $\mu^{+}$

Composite tau neutrino

Composite tau anti-neutrino

Excited $\tau$ of unspecified charge

Excited $\tau^{-}$

Excited $\tau^{+}$

Excited quark

Excited antiquark

\section{MISCELLANEOUS HYPOTHETICAL PARTICLES}

COSMION

DARK

MILLICHARGED

MONOPOLE

TECHNIPION

TECHNIPION+

TECHNIPION-

TECHNIRHO

TECHNIOMEGA
Weakly interacting massive particle (WIMP), have been proposed to solve simultaneously the problem of dark matter, and deflict of B8 neutrinos from the Sun

A generic name for the dark matter in the galactic halo. May be a weakly interacting particle (neutrino, axion, stable particle, etc.) or strongly interacting particles (quark nuggets)

Hypothetic particle with electric charge less than $10^{-3}$ e

Magnetic monopole

Technicolor pion

Positive technicolor pion

Negative technicolor pion

Technicolor $\rho$

Technicolor omega 
color

lepton-colored

lepton-colored

e-color \pm

$e^{++}$

wquark

wquark

tacliyon

tachyon ${ }^{+}$

tachyon ${ }^{-}$

$e$-nonpauli

$p$-nonpaul

unspec

neutral

invisible

higgs

clarged

charged ${ }^{+t}$

charged +

charged -

charged ${ }^{--}$

multicharged

even-charged

odd-charged

parton

jet

jet ${ }^{+}$

jet $^{-}$

hadron

hadron 0

charged-hadron

hadron ${ }^{t}$

hadron-

charged-meson

vmeson

heavy

heavy-flavor

longlived

longlived ${ }^{+}$

longlived $^{0}$

longlived -

narrow

vee

kink+

kink-

star

shower

shower ${ }^{+}$

shower-

grey

htrack

black

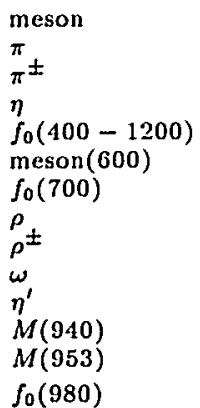

COLOR

LEPTON-COLOR

LEPTON-COLORBAR

E-COLOR+-

LEPTON++

WQUARK

HQUARKBAR

TACHYON

TACHYON+

TACHYON-

NONPAULI (E-)

NONPAULI (P)
Unspecified particle carrying color

Unspecified lepton carrying color

Unspecified lepton carrying color

Colored electron of unspecified charge

Doubly charged lepton

Hypothetic fractionally charged particle interacting only weakly

Weak antiquark

Hypothetic faster-than-light particle of unspecified charge

Hypothetic faster-than-light positively charged particle

Hypothetic faster-than-light negatively charged particle

Electron-like particle but not fermionic, used to encode experiments on search for Pauli principle violation in exotic atoms

Proton-like particle but not fermionic, used to encode experiments on search for Pauli principle violation in exotic nuclei

\section{GENERIC PARTICLE NAMES}

UNSPEC

NEUTRAL

INVISIBLE

HIGGS

CHARGED

CHARGED++

CHARGED+

CHARGED-

CHARGED--

MULTICHARGED

EVEN-CHARGED

ODD-CHARGED

PARTON

JET

$\mathrm{JET}+$

JET-

HADRON

HADRONO

CHARGED-HADRON

HADRON+

HADRON-

CHARGED-MESON

VMESON

HEAVY

HEAVY -FLAVOR

LONGLIVED

LONGLIVED+

LONGLIVEDO

LONGLIVED-

NARROW

VEE

KINK+

KINK-

STAR

SHOWER

SHOWER+

SHOWER-

GREY

HTRACK

BIACK
Particle of unspecified type

Unspecified neutral particle

One or more neutral weakly interacting particles not visible in the current detecting system

Generic name for Higgs bosons

Unspecified charged particle

Unspecified doubly charged positive particle

Positive particle of unspecified type

Negative particle of unspecified type

Unspecified doubly charged negative particle

Stable particle with electric charge greater than 1

An even number of charged particles

An odd number of charged particles

Hypothetic particle

Jet detected as a whole

Jet with positive net charge

Jet with negative net charge

Unspecified hadron

Unspecified neutral hadron

Unspecified charged hadron

Unspecified positive hadron

Unspecified negative hadron

Unspecified charged meson

Neutral vector meson of unspecified mass

Unspecified stable particle with mass greater than proton's mass

Any unspecified particle carrying a flavor heavier than strange

Unspecified particle stable under strong and electromagnetic decay

Unspecified positive-charge particle stable under strong and electromagnetic decay

Unspecified neutral-charge particle stable under strong and electromagnetic decay

Unspecified negative-charge particle stable under strong and electromagnetic decay

Unspecified narrow resonance

Unspecified neutral strange particle decay

Negative kinking track observed in track detector

High charged multiplicity final state

Shower track

Positive shower track

Negative shower track

Emulsion track reported as grey

(mostly protons in the range $30-400 \mathrm{MeV} / \mathrm{c}$ )

Heavy tracks (black or grey) in emulsion

Heavily ionizing track in emulsions
Positive kinking track observed in track detector

\section{HADRONS: LIGHT QUARK MESONS}

MESON
PI
PI+-
ETA
FO $(400-1200)$
MESON $(600)$
FO $(700)$
RHO $(770)$
RHO $(770)+-$
OMEGA $(783)$
ETAPRIME(958)
M(940)
M(953)
FO(980)

Unspecified meson

Pion of unspecified charge

$\pi^{+}$or $\pi^{-}$meson

$\eta(549)$ meson

Was $\sigma$. Broad $\pi \pi S$-wave enhansement needed in PWA

Neutral meson

Was $\epsilon(700) . \pi \pi S$-wave (near $700 \mathrm{MeV}$ )

$\eta \eta^{\prime}(958)$ meson

Nonstrange, $I=0$ meson resonance

Nonstrange, $I=0$ meson resonance

$I=1, S$-wave $K \bar{K}$ enhancement

See the legend on page 425 . 
$a_{0}(980)$

$h(990)$

M(1033)

$\eta(1080)$

$M(1150)$

$\omega \pi(1160)$

$h_{1}(1170)$

$b_{1}(1235)$

$f_{0}(1240)$

$A_{1.5}(1170)^{+}$

$a_{1}(1260)$

$a_{1}(1260)^{ \pm}$

$f_{2}(1270)$

$f_{1}(1285)$

$\eta(1295)$

$\pi(1300)$

$a_{0}(1320)$

$a_{2}(1320)$

$a_{2}(1320)^{\ddagger}$

$f_{0}(1370)$

$h_{1}(1380)$

$\hat{\rho}(1405)$

$f_{1}(1420)$

$\omega(1420)$

$f_{2}(1430)$

$7(1440)$

$X_{1}(1440)^{+}$

$a_{0}(1450)$

$\rho(1450)$

$\eta(1490)$

$f_{0}(1500)$

$f_{2}^{\prime}(1525)$

$f_{2}(1565)$

$\omega(1600)$

$f_{2}(1640)$

$\eta_{2}(1645)$

$X(1650)$

$\omega_{3}(1670)$

$\pi_{2}(1670)$

$\rho_{3}(1690)$

$\mathrm{X}(1690)^{0}$

$\rho(1700)$

$f_{J}(1710)$

$\therefore(1750)^{-}$

$f_{0}(1750)$

$\eta(1760)$

$X(1775)$

$a_{5}(1790)^{+}$

$\pi(1800)$

$f_{2}(1810)$

I (181.1)

$\eta_{2}(1870)$

I $(1910)$

$f_{2}(1920)$

f(1935)

$f_{2}(1950)$

$f_{2}(2010)$

$f_{0}(2020)$

$a_{4}(2040)$

$f_{4}(2050)$

$f_{0}(2060)$

$\eta(2100)$

$\pi_{2}(2100)$

$\rho(2110)$

$f_{2}(2150)$

$\rho(2150)$

$f_{2}(2175)$

$f_{0}(2200)$

$f_{J}(2220)$

$\eta(2225)$

$\rho_{3}(2250)$

$f_{2}(2300)$

$f_{4}(2300)$

$f_{2}(2340)$
AO (980)

$\mathrm{H}(990)$

$M(1033)$

ETA (1080)

$M(1150)$

OMEGAPI (1160)

$\mathrm{H} 1$ (1170)

$\mathrm{B} 1$ (1235)

Fo (1240)

A $1.5(1170)$ +

A1 (1260)

A1 (1260) +-

F2 (1270)

F1 (1285)

ETA (1295)

PI (1300)

$\mathrm{AO}(1320)$

A2 (1320)

A2 (1320)+-

Fo (1370)

H1 (1380)

RHOHAT (1405)

F1 (1420)

OMEGA (1420)

F2(1430)

ETA (1440)

$\mathrm{X} 1(1440)+$

$\mathrm{AO}(1450)$

RHO (1450)

$\operatorname{ETA}(1490)$

F0 (1500)

F2PRIME (1525)

F2 (1565)

OMEGA (1600)

F2 (1640)

ETA2 (1645)

$X(1650)$

OMEGA3 (1670)

PI2(1670)

RHO3 (1690)

$X(1690) 0$

RHO (1700)

$\mathrm{F} / \mathrm{J}(1710)$

$X(1750)$ -

FO (1750)

ETA (1760)

$\mathrm{X}(1775)$

A5 (1790)+

PI (1800)

F2 (1810)

$\mathrm{X}(1814)$

ETA2(1870)

$\mathrm{X}(1910)$

F2 (1920)

$X(1935)$

F2 (1950)

F2 (2010)

Fo $(2020)$

$A 4(2040)$

F4 (2050)

F0 (2060)

ETA $(2100)$

PI2(2100)

RHO (2110)

F2 (2150)

RHO (2150)

F2 (2175)

FO (2200)

$\mathrm{F} / \mathrm{J}(2220)$

ETA (2225)

$\mathrm{RHO3}(2250)$

F2 (2300)

F4 (2300)

F2 (2340)
Nonstrange, $I=0$ meson resonance

Nonst range, $I=0$ meson resonance

Reported meson state decaying to $\omega \pi$

"Buddha" meson

Was $g_{s}(1240)$

Was $D(1285)$

Intensify peaking in mass of $\eta \pi$ system at $a_{2}$ meson mass and with com. parable width

Was $\epsilon(1300) . \pi \pi S$-wave (near $1300 \mathrm{MeV}$ )

Seen in partial-wave analysis of the $K \bar{K} \pi$ system. Needs confirmation

Seen in the P-wave intensity of the $\eta \pi^{0}$ system

Was $E(1420)$

Meson resonance

Was $\iota(1440)$ - glueball candidate

Meson resonance

Seen in the $\eta^{\prime} \pi^{0}$ system

Was $A(1680)$

Was $\theta(1690)$ - glueball candidate

Meson decaying into $3 \pi$

Was $S(1730)$

Seen by CONDO 91 in 3 pion system produced in charge exchange photoproduction

Triplet of hadronic resonance with quantum numbers $I^{G}\left(J^{P}\right)=1^{-}\left(0^{-}\right)$

Seen in coherent production of $\pi^{-} \eta \eta$ system on a carbon nucleus

Was $S(1935)$

Glueball candidate

Was $\delta(2040)$

Was $h(2030) . I=0, J^{P}=4^{+}$meson resonance

Seen in the $\left(\omega \pi^{0}\right)$ and $(\omega 3$ pion) systems

Was $\epsilon(2150)$

Was $\xi(2220)$. Meson seen in $J / \psi(1 S)$ decays

Seen in $\bar{p} p$ formation experiments

Was $g_{T}(2320)$

Was $\epsilon(2300)$ 
$\rho_{5}(2350)$

$a_{6}(2450)$

$f_{6}(2510)$

meson(2950)

$X(3250)$

$\left(e^{+} e^{-(1100-2200))}\right.$

$X(1900-3600)$

strange

strange ${ }^{o}$

kaon

IK

$K \pm$

$K_{S}$

$K_{L}$

$K^{*}(892)$

$K^{*}(892)^{ \pm}$

$K_{1}(1270)$

$K_{1}(1400)$

$K^{*}(1410)$

$K_{0}^{*}(1430)$

$K_{2}^{\prime}(1430)$

$K(1460)$

$K_{2}(1580)$

$K_{1}(1650)$

$K^{*}(1680)$

$K_{2}(1770)$

$K_{3}^{*}(1780)$

$K_{2}(1820)$

$\kappa(1830)$

$K_{0}^{*}(1950)$

$\kappa_{2}^{*}(1980)$

$K_{4}^{*}(2045)$

$\kappa_{2}(2250)$

$K_{3}(2320)$

$K_{s}^{*}(2380)$

$K_{1}(2500)$

$I(2600)$

K(3100)

$K^{*}$ (unspec)

strangeonium

$\phi(1020)$

$\phi(1680)$

$\phi_{3}(1850)$

K $\bar{K}(2200)^{0}$

$\phi_{5}(2400)$

\section{charm}

charm ${ }^{ \pm}$

charmed-meson

$D_{c}$

$D^{ \pm}$

$D$ (unspec)

$D^{*}$

$D^{*}$ (unspec)

$D^{ \pm}$

$D_{1}(2420)$

$D_{J}(2440)$

$D_{2}^{*}(2460)$

$D_{j}^{*}(2470)$

$D_{a 1}(2536)^{ \pm}$

$D^{*}(2547)^{+}$

$D_{a} J(2564)^{+}$

$D_{4 J}(2573)$

$D^{*}(2600)^{+}$

$D_{*}^{*}(2790)$
RHO5 (2350)

A6 (2450)

Was $\delta(2450)$

$\operatorname{MESON}(2950) \quad$ Bump seen in $p \bar{p} \pi$

$\mathrm{X}(3250)$

\section{HADRONS: OTHER UNFLAVOURED MESONS}

$E+E-(1100-2200)$

$\mathrm{X}(1900-3600)$

Vector meson

Any meson bump seen in production experiments in that mass region

\section{HADRONS: MESONS WITH HEAVIEST QUARK STRANGE}

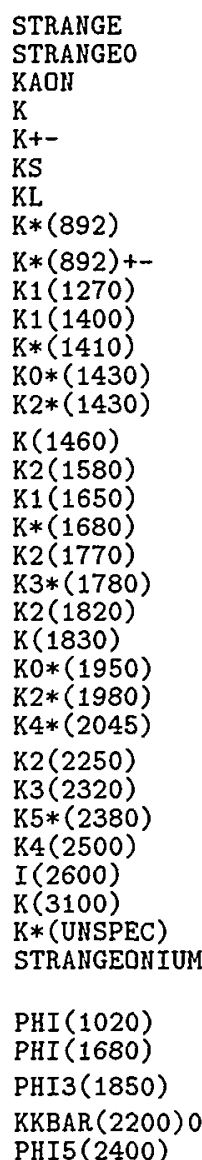

Unspecified strange particle

Represents a $\Lambda$ or $\Sigma^{b}$ or low-mass neutral $Y^{*}$

Kaon or antikaon of unspecified charge

$\Lambda$ meson

Ordinary $\mathrm{K}^{+}$or $\mathrm{K}^{-}$meson

$K_{\text {short }}$, neutral $K$ meson

$K_{\text {long }}$, neutral $K$ meson

Was $Q(1280)$

Was $Q(1400)$

Was $r(1350)$

$J^{P}=0^{-}$state seen in PWA decaying predominantly into $\epsilon K^{-}$

Seen in PWA of the $K 2 \pi$ system. Was $L(1580)$

Various peaks in $K \phi$ and $K^{\prime} 2 \pi$ reported in PWA

Seen in PWA of $K \phi$ system

Seen in PWA of $K 2 \pi$ system

$\Lambda \bar{p}$ or $\bar{\Lambda} p$ state with quantum numbers of kaon

Strange meson

Exotic meson possibly seen in $\Lambda \bar{p}$ plus pions

Unspecified $K^{*}$

Unspecified meson whose quark content is dominantly $s \bar{s}$, such as the $\phi(1020)$

Bump in $K^{+} K^{-}$mass

Reported spin 5 boson resonance

\section{HADRONS: MESONS WITH HEAVIEST QUARK CHARMED}
CHARM
CHARM+-
CHARMED-MESON
DC
$\mathrm{D+-}$
$\mathrm{D}$
D(UISPEC)
D*
D*(UNSPEC)
$\mathrm{D} / \mathrm{S}+-$
$\mathrm{D} / \mathrm{S} *$
D1 $(2420)$
$\mathrm{D} / \mathrm{J}(2440)$
$\mathrm{D} 2 *(2460)$
$\mathrm{D} / \mathrm{J} *(2470)$
$\mathrm{D} / \mathrm{S} 1(2536)+-$
$\mathrm{D} / \mathrm{S} *(2547)+$
$\mathrm{D} / \mathrm{S} / \mathrm{J}(2564)+$
$\mathrm{D} / \mathrm{S} / \mathrm{J}(2573)$
$\mathrm{D} *(2600)+$
$\mathrm{D} / \mathrm{S} *(2790)$

See the legend on page 425 .

nspecified charmed particle

Unspecified charged charmed particle

Unspecified charmed meson

$D$ or $\bar{D}$ charmed meson

$D(1869)^{+}$or $D(1869)^{-}$charmed nonstrange meson

$D^{+}$or $D^{0}$ charmed meson

Unspecified charmed nonstrange meson

Excited charmed nonstrange meson

Was $F . D_{s}^{+}$or $D_{s}^{-}$charmed strange meson

Was $F^{*}(2140)$. Excited charmed strange meson

Excited charmed nonstrange meson

Excited charmed nonstrange meson seen in $D^{0} \pi^{+}$

Meson resonance. $C=S= \pm 1$. Seen in $D^{*}(2010)^{+} K^{* 0}$.

Charmed, strange meson of unspecified charge 
$D_{s}$ (unspec)

charmonium

I $(2830)$

$\backslash(2850)$

$\eta_{\mathrm{c}}(1 S)$

$\psi(3040)$

$J / \psi(1 S)$

$x(3180)$

$x(3300-3500)$

$\chi_{c 0}(1 P)$

$x_{c}(3455)$

$\lambda_{c 1}(1 P)$

$h_{c}(1 P)$

$\chi_{c 2}(1 P)$

$\eta_{\mathrm{c}}(2 S)$

$\psi(2 S)$

$\psi(3770)$

$\psi(4040)$

$\psi(4160)$

$\psi(4415)$

Ic(unspec)

$\psi$ (unspec)

bottom

bottom \pm

$B$

$B$ (unspec)

$B^{*}$

$B_{J}^{*}(5732)$

$B^{*}$ (unspec)

$B_{s}$.

$B_{s j}^{*}(5850)$

$B_{c}^{+}$

$B_{c}^{-}$

bottomonium

$\zeta(8300)$

$\eta_{b}$

$\Upsilon(1 S)$

$h_{b}(1 P)$

$\checkmark 60(1 P)$

$\backslash b_{1}(1 P)$

$\backslash b_{2}(1 P)$

$\Upsilon(1 D)$

$\Upsilon(2 S)$

$\backslash b_{0}(2 P)$

$\backslash \iota_{1}(2 P)$

$\backslash 62(2 P)$

$\Upsilon(3 S)$

$\Upsilon(4 S)$

$\Upsilon(10860)$

$\Upsilon(11020)$

xb(unspec)

$\Upsilon$ (unspec)

top

toponium

D/S (UNSPEC)
CHARMONIUM
X(2830)
CHI (2850)
ETA/C(1S)
PSI (3040)
J/PSI (1S)
CHI $(3180)$
CHI (3300-3500)
CHI/CO(1P)
CHI/C(3455)
CHI/C1(1P)
H/C (1P)
CHI/C2(1P)
ETA/C(2S)
PSI (2S)
PSI (3770)
PSI (4040)
PSI (4160)
PSI (4415)
CHI/C(UNSPEC)
PSI (UNSPEC)

Unspecified charmed strange meson

Unspecified charm-anticharm state

$J^{P}=0^{-}$charmonium state

Charmonium meson

Generic name for charmonium $\lambda$ states in the $3300-3500 \mathrm{MeV}$ mass region Particle observed in $e^{+} e^{-}-\mu^{+} \mu^{-} 2 \gamma$

Radiative decay product of $\psi(2 S)$

Observed in $e^{+} e^{-2} \gamma$ final state

${ }^{1} P_{1}(c \bar{c})$ bound state

Charmonium meson

Charmonium meson

Unspecified $\psi$ meson

\section{HADRONS: MESONS WITH HEAVIEST QUARK BEAUTY}

BOTTOM

BOTTOM+-

B

$\mathrm{B}+-$

$B$ (UNSPEC)

B*

$\mathrm{B} / \mathrm{J} *(5732)$

$\mathrm{B} *$ (UNSPEC)

$\mathrm{B} / \mathrm{S}$

$B / S *$

$\mathrm{B} / \mathrm{S} / \mathrm{J} *(5850)$

$\mathrm{B} / \mathrm{C}+$

$\mathrm{B} / \mathrm{C}-$

BOTTOMONIUM

ZETA (8300)

ETA/B(1S)

UPSI(1S)

$\mathrm{H} / \mathrm{B}$ (1P)

CHI/BO (1P)

$\mathrm{CHI} / \mathrm{B} 1$ (1P)

$\mathrm{CHI} / \mathrm{B} 2$ (1P)

UPSI (1D)

UPSI (2S)

$\mathrm{CHI} / \mathrm{BO}$ (2P)

CHI/B1 (2P)

$\mathrm{CHI} / \mathrm{B} 2$ (2P)

UPSI (3S)

UPSI (4S)

UPSI (10860)

UPSI (11020)

CHI/B (UNSPEC)

UPSI (UNSPEC)
Unspecified particle with naked bottom

Generic name for charged particle with naked beauty

$B^{+}$or $B^{0}$ bottom meson

Charged open beauty meson

Meson of unspecified mass with antibeauty quark

Excited bottom meson

Orbitally excited positive or neutral (isodublet) bottom meson

Vector beauty meson

Beauty-antistrange meson

Beauty-antistrange excited meson

Antibeauty-charmed meson

Beauty-charmed meson

Unspecified bottom-antibot tom state

Lowest mass $J^{P}=0^{-}-6 \bar{b}$ state

Lowest mass $J^{P}=1^{+} b \bar{b}$ state

Bottomonium meson

Bottomonium meson

Bottomonium meson

$J^{P}=1^{-} b \bar{b}$ state

Bottomonium meson

Bottomonium meson

Bottomonium meson

Bot tomonium meson

Unspecified $\Upsilon$ particle

\section{HADRONS: MESONS WITH HEAVIEST QUARK TOP}

TOP

TOPONIUM
Unspecified particle with naked top

Unspecified top-antitop state

\section{HADRONS: EXOTIC MESONS AND MESON-LIKE OBJECTS}

exotic

exotic-meson

glueball

pomeron

$C(1480)$

$\mathrm{N}(1600)$

meson --

baryonium

baryonium $(S=-1)$
EXOTIC

EXOTIC-MESON

GLUEBALL

POMERON

$C(1480)$

$X(1600)$

MESON--

BARYONIUM

BARYONIUM $(S=-1)$
Unspecified particle which cannot be fit into $q \bar{q}$ or $q q q$ model Manifestly exotic meson that cannot be formed of quark-antiquark Unspecified glueball

Meson decaying into $\phi \pi$

Exotic $I^{G}\left(J^{P C}\right)=2^{+}\left(2^{++}\right)$meson resonance

Charged -2 meson of unspecified mass

Unspecified nucleon-antinucleon particle

Strange mesons that couple predominately to baryon-antibaryon 
baryonium $(S=+1)$

R-meson

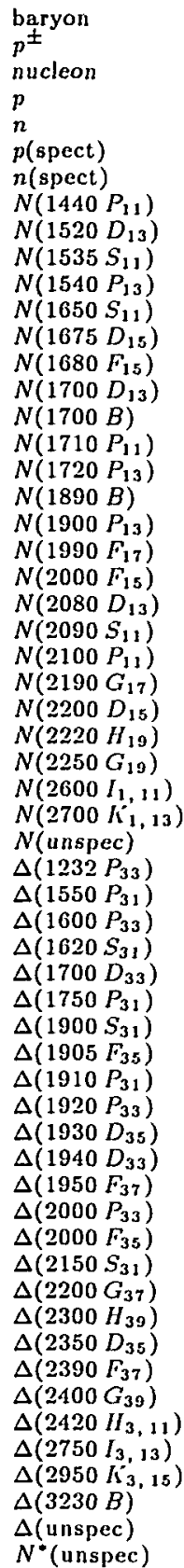

hyperon

$\Lambda$

$\Lambda(1330 B)$

$\Lambda\left(1405 S_{01}\right)$

$\Lambda\left(1520 D_{03}\right)$

$\Lambda\left(1600 P_{01}\right)$

$\Lambda\left(1670 S_{01}\right)$

$\Lambda\left(1690 D_{03}\right)$

$\Lambda(1690 B)$

$\Lambda\left(1800 S_{01}\right)$

$\Lambda\left(1800 G_{09}\right)$

$\Lambda(1800 B)$

$\Lambda\left(1810 P_{01}\right)$

\author{
BARYONIUM $(S=+1)$ \\ R-MESON \\ Strange mesons that couple predominately to baryon-antibaryon \\ Generic exotic meson containing gluino or squarks
}

\section{HADRONS: LIGHT QUARK BARYONS}

$\begin{array}{ll}\text { BARYON } & \text { Unspecified baryon } \\ \text { P+- } & \text { Proton or antiproton } \\ \text { NUCLEON } & \text { Unspecified nucleon } \\ \text { P } & \text { Proton } \\ N & \text { Neutron } \\ \text { P(SPECT) } & \text { Spectator proton } \\ \text { N(SPECT) } & \text { Spectator neutron }\end{array}$

Bump in production experiment

Enhancement seen in $\pi p \rightarrow \eta^{\prime} n$ cross section

Unspecified $I=1 / 2, S=0$ baryon

Unspecified $I=3 / 2, S=0$ baryon

$S=0$ baryon with unspecified mass and isospin

\section{HADRONS: BARYONS WITH HEAVIEST QUARK STRANGE}
HYPERON
LAMBDA
$\operatorname{LAMBDA}(1330 \mathrm{~B})$
LAMBDA (1405S01)
LAMBDA (1520D03)
LAMBDA (1600P01)
LAMBDA (1670S01)
LAMBDA (1690D03)
LAMBDA (1690B)
LAMBDA (1800S01)
LAMBDA (1800G09)
LAMBDA (1800B)
LAMBDA (1810P01)

Unspecified hyperon

Ordinary $\Lambda$ hyperon

Bump in production experiment

See the legend on page 425 . 
EXPLANATION

$\Lambda\left(1820 F_{05}\right)$

$\Lambda\left(1830 D_{05}\right)$

$\Lambda\left(1890 P_{03}\right)$

$\Lambda(2000)$

$\Lambda\left(2020 F_{0 \tau}\right)$

$A\left(2100 G_{07}\right)$

$A(2100 B)$

$.1\left(2110 F_{05}\right)$

$\Lambda\left(2325 D_{03}\right)$

$\Lambda\left(2350 H_{09}\right)$

$.1(2350 B)$

$A(2585 B)$

(unspec)

$\Sigma$

$\Sigma\left(1385 P_{13}\right)$

$\Sigma\left(1385 P_{13}\right)^{ \pm}$

$\Sigma(1480 B)$

$\Sigma(1560 B)$

$\Sigma\left(1580 D_{13}\right)$

$\Sigma\left(1620 s_{11}\right)$

$\Sigma(1620 B)$

$\Sigma\left(1650 P_{11}\right)$

$\Sigma\left(1660 P_{11}\right)$

$\Sigma\left(1670 D_{13}\right)$

$\Sigma(1690 \mathrm{~B})$

$\Sigma\left(1750 s_{11}\right)$

$\Sigma(170 B)$

$\Sigma\left(1770 P_{11}\right)$

$\Sigma\left(1775 D_{15}\right)$

$\Sigma\left(1840 P_{13}\right)$

$\Sigma\left(1880 P_{11}\right)$

$\Sigma\left(1900 S_{11}\right)$

$\Sigma\left(1915 F_{15}\right)$

$\Sigma(1915 B)$

$\Sigma\left(1920 P_{13}\right)$

$\Sigma\left(1940 D_{13}\right)$

$\Sigma\left(2000 S_{11}\right)$

$\Sigma(2030 \mathrm{~F})$

$\Sigma(2030 \mathrm{~B})$

$\Sigma\left(2070 F_{15}\right)$

$\Sigma\left(2080 P_{13}\right)$

$\Sigma\left(2100 G_{17}\right)$

$\Sigma\left(2140 P_{13}\right)$

$\Sigma(2250 \mathrm{~B})$

$\Sigma(24.55 B)$

$\Sigma(26 \div 0 B)$

$\Sigma(3000 B)$

$\Sigma(3170 B)$

E(unspec)

$Y^{*}$ (unspec)

$\equiv$

$\Xi\left(1530 P_{13}\right)$

$\Xi(1620)$

$\Xi(1690)$

$\Xi(1760)$

$\Xi\left(1820 \mathrm{D}_{13}\right)$

$\Xi(1950)$

$\Xi(2030)$

$\Xi(2120)$

$\Xi(2250)$

$\Xi(2370)$

$\Xi(2500)$

$\Xi(2600)$

$\Xi$ (unspec)

$\Omega^{ \pm}$

$\Omega^{-}$

$\Omega(2250)=$

$\Omega(2250)^{-}$

$\Omega(2380)=$

$\Omega^{*}\left(\right.$ unspec $^{-}$

$\lambda_{c}$

$\Lambda_{c}(2593)^{ \pm}$

$\Lambda_{c}(2593)^{+}$

$\Lambda_{c}(2625)$

\author{
LAMBDA (1820F05) \\ LAMBDA (1830D05) \\ LAMBDA (1890P03) \\ IAMBDA (2000) \\ LAMBDA (2020F07) \\ LAMBDA (2100G07) \\ IAMBDA (2100B) \\ IAMBDA (2110F05) \\ LAMBDA (2325D03) \\ LAMBDA (2350H09) \\ LAMBDA (2350B) \\ LAMBDA (2585B) \\ LAMBDA (UNSPEC) \\ SIGMA \\ Bump in production experiment \\ Bump in production experiment \\ Bump in production experiment \\ Unspecified $I=0, S=-1$ baryon \\ Ordinary $\Sigma$ hyperon
}

SIGMA+-

SIGMA (1385P13)

SIGMA (1385P13) +-

SIGMA (1480B)

SIGMA (1560B)

SIGMA (1580D13)

SIGMA (1620S11)

$\operatorname{SIGMA}(1620 B)$

SIGMA (1650P11)

SIGMA (1660P11)

SIGMA (1670D13)

SIGMA (1690B)

SIGMA (1750S11)

SIGMA (1770B)

SIGMA (1770P11)

SIGMA (1775D15)

SIGMA (1840P13)

SIGMA (1880P11)

SIGMA (1900S11)

SIGMA (1915F15)

SIGMA (1915B)

SIGMA (1920P13)

SIGMA (1940D13)

SIGMA (2000S11)

SIGMA (2030F 17)

SIGMA (2030B)

SIGMA (2070F15)

SIGMA (2080P13)

SIGMA (2100G17)

SIGMA (2140P13)

SIGMA (2250B)

SIGMA (2455B)

SIGMA (2620B)

SIGMA (3000B)

SIGMA (3170B)

SIGMA (UNSPEC)

Y* (UNSPEC)

$X I$

XI (1530P13)

$X I(1620)$

XI (1690)

XI (1760)

XI (1820D13)

XI (1950)

XI (2030)

XI (2120)

$X I(2250)$

$X I(2370)$

XI (2500)

XI (2600)

XI (UNSPEC)

OMEGA+-

OMEGA-

OMEGA (2250)+-

OMEGA (2250)-

OMEGA $(2380)+-$

OMEGA* (UNSPEC) -

Bump in production experiment

Bump in production experiment

Seen in DPWA

Suggested by some but not all PWA

Seen in DPWA

Bump in production experiment

Seen in production experiment

Unspecified $I=1, S=-1$ baryon

$S=-1$ baryon with unspecified mass and isospin

Ordinary $\Xi$ hyperon

Seen in $\equiv \pi$ channel

Seen in production experiment

Seen in production experiment

Seen in production experiment

Not confirmed

Unspecified $I=1 / 2, S=-2$ baryon

Ordinary $\Omega^{-}$hyperon

$S=-3$ baryon with unspecified mass and isospin

\title{
HADRONS: BARYONS WITH HEAVIEST QUARK CHARMED
}

\author{
LAMBDA/C \\ LAMBDA/C (2593)+- \\ LAMBDA /C (2593)+ \\ LAMBDA /C (2625)
}

$\Lambda_{c}(2281)^{+}$or $\bar{\Lambda}_{c}(2281)^{-}$

$\Lambda_{c}(2593)^{+}$charmed baryon or $\bar{\Lambda}_{c}(2593)^{-}$charmed antibaryon, $I=0$

$\Lambda_{c}(2593)^{+} I=0$ charmed baryon 


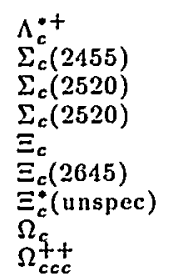

$\Lambda_{\iota}$ $\Lambda_{b}$ (unspec)

$\Sigma_{b}$

$\Omega_{\bar{b}}^{-}$

$\Omega_{c b}^{0}$

$\Omega_{\bar{L}}$

exotic-nucleon $N_{3 / 2}^{*}(1380)^{t++}$

$N_{5 / 2}^{*}(1390)^{+++}$

$N_{5 / 2}^{*}(1480)^{+++}$

$N_{5 / 2}^{*}(1650)$

$N_{5 / 2}^{*}(1760)^{+++}$

$N_{5 / 2}^{*}(1760)^{+}$

$N(2000 B)^{+}$

$N(2050 B)^{+}$

$N_{5 / 2}^{*}(2070)^{+++}$

$N_{5 / 2}^{*}$ (unspec)

$N(2170 B)^{+}$

$N(3520 B)^{+}$

$N \phi(1950)^{+}$

$N \phi(1950)^{0}$

$Z_{0}\left(1780 P_{01}\right)$

$Z_{0}\left(1865 D_{03}\right)$

$Z_{0}$ (unspec)

$Z_{1}\left(1900 P_{13}\right)$

$Z_{1}(2150)$

$Z_{1}(2500)$

$Z_{1}$ (unspec)

$Z$ (unspec)

$Y_{2}^{*}$ (unspec)

$\Xi_{3 / 2}^{*}$ (unspec)

R-baryon

R-proton

R- $\Delta^{++}$

$\mathrm{R}-\Lambda$

R- $\Xi^{-}$

LAMBDA/C*+
SIGMA/C (2455)
SIGMA/C (2520)
SIGMA/CBAR(2520)
XI/C
XI/C (2645)
XI/C* (UNSPEC)
OMEGA/C
OMEGA/CCC++

LAMBDA/C*+

SIGMA/C (2455)

SIGMA/CBAR (2520)

$\mathrm{XI} / \mathrm{C}$

OMEGA/C
Exited $\Lambda_{c}^{+}$charmed baryon

$I=1$ charmed baryon triplet

Charmed baryon with $\mathrm{I}=1$

Charmed baryon with $\mathrm{I}=1$

Baryon with quark content usc

Unspecified excited charmed strange baryon ( $q s c$ )

$I=0$ charmed doubly strange baryon

Baryon with quark content ccc

\section{HADRONS: BARYONS WITH HEAVIEST QUARK BEAUTY}

LAMBDA/B
LAMBDA/B(UNSPEC)
SIGMA/B
XI/B
OMEGA/B-
OMEGA/CBO
OMEGA/BB-

$\Lambda_{b}(5500)^{0} I=0$ bottom baryon

Unspecified $I=0$ bottom baryon

Ground state $I=1$ bottom baryon ( $q q b)$

Ground state $I=1 / 2$ strange bottom baryon ( $q s b)$

\section{HADRONS: EXOTIC BARYONS}

$\begin{array}{ll}\text { EXOTIC-NUCLEON } & \text { Cannot be formed of } q q q \\ \mathbb{N} * 5 / 2(1380)+++ & \text { Exotic baryon }\end{array}$

E*5/2(1380) ++t

$N * 5 / 2(1390)+++$

H $* 5 / 2(1480)+++$

$\mathrm{l} * 5 / 2(1650)$

$\mathrm{N} * 5 / 2(1760)+++$

$11 * 5 / 2(1760)+$

EXOTIC-N (2000B)+

EXOTIC-N (2050B)+

$\mathrm{N} * 5 / 2(2070)+++$

$\mathrm{N} * 5 / 2$ (UNSPEC)

EXOTIC-N (2170B)+

EXOTIC-N (3520B)+

NPHI (1950)+

NPHI (1950) O

ZO(1780P01)

$\mathrm{ZO}(1865 \mathrm{DO3})$

Z0 (UNSPEC)

Z1(1900P13)

$\mathrm{Z1}(2150)$

$\mathrm{Z1}(2500)$

Z1 (UNSPEC)

$2 *$ (UNSPEC)

$\mathrm{Y} * 2$ (UNSPEC)

$X I *$ (UNSPEC)

$X I * 3 / 2$ (UNSPEC)

R-BARYOH

R-PROTON

R-DEL++

R-LAMBDA

$R-X I-$
Exotic $I=5 / 2$ nonstrange baryon

Exotic $I=5 / 2$ nonstrange baryon

$I=5 / 2, Y=1$ baryon of unspecified charge

Exotic baryon

Exotic baryon

Bump in production experiment, candidate for cryptoexotic pentaquark baryons with hidden strangeness

Bump in production experiment, candidate for cryptoexotic pentaquark baryons with hidden strangeness

Exotic baryon

Unspecified $I=5 / 2, S=0$ baryon

Bump in production experiment, candidate for cryptoexotic pentaquark baryons with hidden strangeness

Bump in production experiment, candidate for cryptoexotic pentaquark baryons with hidden strangeness

Reported baryon with $s \bar{s}$ and 3 other quarks

Reported baryon with $s \bar{s}$ and 3 other quarks

Exotic $I=0, S=+1$ baryon of unspecified mass

Exotic $I=1, S=+1$ baryon of unspecified mass

Exotic $I=$ unspecified, $S=+1$ baryon of unspecified mass

Unspecified $I=2, S=-1$ baryon

$I=$ unspecified, $S=-2$ baryon of unspecified mass

Unspecified $I=3 / 2, S=-2$ baryon

Generic exotic baryon containing one gluino in addition to the usual quarks (3q gluino)

Exotic hadron containing one gluino in addition to the usual quarks ( $u u$ d gluino)

Exotic hadron containing one gluino in addition to the usual quarks ( $u u$ u gluino)

Exotic hadron containing one gluino in addition to the usual quarks ( $u u$ $s$ gluino)

Exotic hadron containing one gluino in addition to the usual quarks ( $s s$ d gluino)

\begin{tabular}{ll} 
anomalon & ANOMALON \\
fireball & FIREBALI \\
centauro & CENTAURO \\
mini-centauro & MINI-CENTAURO \\
chiron & CHIRON \\
geminion & GEMINION \\
\hline
\end{tabular}

\section{EXOTIC MATTER}

Anomalous nuclear fragment

Final state with 50 or more charged particles and no $\pi^{0}$ 's New type of final state with 20 or less charged particles, no $\pi^{0}$ 's

Hypothetical particle

See the legend on page 425 . 
strangelet

posit ronium

muonium

$(\pi \mu)_{\text {atom }}$

mupatom

$\left(\mu^{-3} \mathrm{He}\right)$ atom

$(\pi \pi)_{\text {atom }}$

taonium

$(\pi p)_{\text {atom }}$

( $\pi^{-}$deuteron $)_{\text {aiom }}$

$\left(K^{-} p\right)_{\text {atom }}$

$(\bar{p} p)_{\text {azom }}$

( $\bar{p}$ deuteron ) atom

( $\bar{p}$ He)atom

atom

$\mathrm{H}$ (atom)

${ }^{2} \mathrm{H}$ (atom)

$\mathrm{C}$ (atom)

${ }^{19} \mathrm{Fl}$ (atom)

Nit(atom)

$\mathrm{Ne}(\mathrm{atom})$

Al(atom)

Ar(atom)

Ca(atom)

${ }^{50} \mathrm{Ti}($ atom $)$

${ }^{50} \mathrm{Cr}$ (atom)

${ }^{55} \mathrm{Mn}$ (atom)

$\mathrm{Fe}($ atom)

${ }^{58} \mathrm{Ni}($ atom $)$

Cu(atom)

${ }^{64} \mathrm{Zn}$ (atom)

${ }^{67} \mathrm{Ga}(\mathrm{atom})$

${ }^{i 1} \mathrm{Ge}(\mathrm{atom})$

${ }^{92} \mathrm{Zr}$ (atom)

${ }^{92}$. Io(atom)

${ }^{106} \mathrm{Pd}$ (atom)

Ag(atom)

${ }^{106} \mathrm{Cd}$ (atom)

$\mathrm{Sn}(\mathrm{atom})$

124 Te(atom)

${ }^{124} \mathrm{Xe}(\mathrm{atom})$

${ }^{125} \mathrm{I}$ (atom)

Cs(atom)

${ }^{163}$ Dy (atom)

${ }^{163} \mathrm{Ho}$ (atom)

${ }^{180} \mathrm{Wit}$ (atom)

$\mathrm{Pt}$ (atom)

Au(atom)

$\mathrm{Hg}$ (atom)

Tl(atom)

$\mathrm{Pb}$ (atom)

Bi(atom)

ion

${ }^{76} \mathrm{Ge}\left(\right.$ ion $\left.{ }^{+}\right)$

${ }^{163} \mathrm{Ho}\left(\right.$ ion $\left.66^{+}\right)$

${ }^{238} \mathrm{~L}^{-}\left(\right.$ion $\left.90^{+}\right)$

nucleus

frag

fragb

fragt

hypernucleus

charmed-nucleus

deuteron

deut ${ }^{*}(2170)^{\circ}$

deut ${ }^{*}(2500)^{+}$

demon
STRANGELET

Proposed new form of strange hadronic matter. Quark nuggets.

\section{ELECTROMAGNETIC COMPOSITES}

POSITRONIUM

MUEATOM

PIMUATOM

MUPATOM

MUHE3ATOM

PIPIATOM

TAONIUM

PIPATOM

PIDEUTATOM

KAONPATOM

PBARPATOM

PBARDEUTATOM

PBARHEATOM

ATOM

$\operatorname{ATOM}(P)$

ATOM (DEUT)

$\operatorname{ATOM}(\mathrm{C})$

ATOM(FL19)

ATOM (NIT)

ATOM (NE)

$\operatorname{ATOM}(\mathrm{AL})$

ATOM (AR)

$\operatorname{ATOM}(\mathrm{CA})$

ATOM(TI50)

ATOM (CR50)

ATOM(MN55)

$\operatorname{ATOM}(\mathrm{FE})$

ATOM (NI58)

ATOM (CU)

ATOM(ZN64)

ATOM(GA67)

ATOM(GE71)

ATOM (ZR92)

ATOM(M092)

ATOM (PD106)

ATOM(AG)

ATOM (CD106)

ATOM(SN)

ATOM(TE124)

ATOM (XE124)

ATOM(I125)

ATOM(CS)

ATOM(DY163)

$\operatorname{ATOM}(\mathrm{H0163})$

ATOM (WT180)

ATOM(PT)

$\operatorname{ATOM}(\mathrm{AU})$

ATOM (HG)

ATOM(TL)

$\operatorname{ATOM}(\mathrm{PB})$

ATOM(BI)

IDN

ION (GE76) +

ION (H0163) 66*

ION (U238) $90+$ $\mu^{+} e^{-}$atom

$\pi \mu$ coulomb bound state

$\mu^{-} p$ atom
$\left(\mu^{-3} \mathrm{He}\right)$ coulomb bound state

$\pi \pi$ coulomb bound state

$\tau^{+} \tau^{-}$coulomb bound state

$\left(\pi^{-} p\right)$ coulomb bound state

Pion-deuterium coulombic atom

$\left(K^{-} p\right)$ coulomb bound state

Antiproton proton coulombic atom

Antiproton deuterium coulombic atom

Antiproton helium coulombic atom

Unspecified conventional atom

Hydrogen atom

Deuteron atom

Carbon atom

Fluorine-19 atom - note name is not same as chemical symbol

Nitrogen atom

Neon atom

Aluminum atom

Argon atom

Manganese- 55 atom

Iron atom

Copper atom

Gallium-67 atom

Silver atom

Tin atom

Tellur-124 atom

Atom of xenon-124 radioactive isotope

Iodine- 125 atom

Cesium atom

Platinum atom

Gold atom

Mercury atom

Bismuth atom

Unspecified ion of an atom

Ion of germanium -76 atom

66-charged holmium-163 ion

90-charged uranium-238 ion

NUCLEI. MULTIBARYONS, HYPER-AND SUPERNUCLEI

NUCLEUS
FRAG
FRAGB
FRAGT
HYPERNUCLEUS
CHARMED-NUCLEUS
DEUT
DEUT* $(2170) 0$
DEUT** $(2500)+$
DEMON

Unspecified nucleus

Nuclear fragment

Fragment of beam

Fragment of target

Unspecified hypernucleus, generally containing more than two baryons

Unspecified charmed nucleus

Deuteron

Low-mass $d \pi \pi$ bump

Exotic 6-quark deuteron-like state 


\section{Particle Name}

Computer Name

EXPLANATION

\section{dibaryon}

dineutron

$N N\left(2170^{1} D_{2}\right)$

$N N\left(2250^{3} F_{3}\right)$

$N N\left(2900^{1} H_{6}\right)$

dibaryon $(S=-1)$

$\Lambda N\left(2130^{3} S_{1}\right)$

$\Lambda N\left(2130^{3} S_{1}\right)^{+}$

hypernucleus $(S=-2)$

dibaryon $(S=-2)$
dibaryon $(S=-2)$

$(\Xi N)^{+}$

${ }^{3} \mathrm{II}$

trineutron

tribaryon

${ }^{i} \mathrm{H}$

tetraneutron

${ }^{4} \mathrm{H}_{3}$

${ }^{5} \mathrm{H}$

${ }^{6} \mathrm{H}$

${ }^{\mathrm{He}} \mathrm{He}_{\mathrm{s}}$

${ }_{5}^{5} \mathrm{He}$

${ }^{5} \mathrm{He}$.

${ }^{5} \mathrm{He}_{s \mathrm{~s}}$

${ }^{6} \mathrm{Hess}^{9} \mathrm{Hes}^{5}$

$\mathrm{Li}$

${ }_{8}^{7} \mathrm{Li}$.

${ }^{8} \mathrm{Li}$ 。

${ }^{9} \mathrm{Li}_{s}$

${ }^{9} \mathrm{Be}_{a}$

${ }^{10} \mathrm{Be}_{s}$

${ }^{11} \mathrm{Be}_{s s}$

${ }_{12} \mathrm{Be}_{s}$

${ }^{12} \mathrm{Bess}_{s s}$

Bor

$\mathrm{Bor}_{s}$

${ }^{30}$ Bor,

"Bors

12 Bor $_{s}$

${ }^{13} \mathrm{Bor}_{s}$ s

${ }_{12}^{C} \mathrm{C}_{s}$

${ }^{12} \mathrm{C}$.

$\mathrm{C}^{*}(4.44 \mathrm{keV})$

${ }^{13} \mathrm{C}_{8}$

${ }^{16} \mathrm{C}$.

$\mathrm{Nit}$

$\mathrm{O}$
$\mathrm{O}_{s}$

${ }_{16} \mathrm{O}_{4}$

Fl

$\mathrm{Ne}$

$\mathrm{Na}$

$\mathrm{Mg}$

$\mathrm{Al}$
$\mathrm{Si}$

${ }^{28} \mathrm{Si}_{\text {。 }}$

$\mathrm{Ph}$

S

CI

Ar

KK

$\mathrm{Ca}_{s}$

${ }^{10} \mathrm{Ca}_{s}$

$\mathrm{Sc}$

$\mathrm{Ti}$

\section{DIBARYON}

DINEUTRON

NN (2170/1D2)

$\operatorname{NN}(2250 / 3 \mathrm{~F} 3)$

NN (2900/1H6)

DIBARYON $(S=-1)$

LAMBDAN (2130/3S1)

$\operatorname{LAMBDAN}(2130 / 3 \mathrm{~S} 1)+$

HYPERNUCLEUS $(S=-2)$

DIBARYON $(\mathrm{S}=-2)$

DIBARYON $*(\mathrm{~S}=-2)$

XIN+

TRITIUIM

H3/S

TRINEUTRON

TRIBARYON

H4

TETRANEUTRON

$\mathrm{H} 4 / \mathrm{S}$

$\mathrm{H} 4 / \mathrm{SS}$

H5

H6

$\mathrm{H} 7$

HE

HE $4 / S$

HE5

HE5/S

HE5/SS

HE6/SS

HE9/S

LI

$\mathrm{LI} 7 / \mathrm{S}$

$\mathrm{LI8/S}$

LIg/S

$\mathrm{BE7} / \mathrm{S}$

$\mathrm{BE}$

BE $9 / S$

BE10/SS

$\mathrm{BE} 11 / \mathrm{SS}$

BE12/S

BE12/SS

BOR

$\mathrm{BOR} / \mathrm{S}$

BOR10/S

BOR11/S

BOR12/S

BOR13/SS

$\mathrm{C}$

C12/S

$\mathrm{C} / \mathrm{S}$

C12/S*

C* (4.44)

C13/S

C13/S*

C16/S

NIT

0

$0 / \mathrm{S}$

$016 / \mathrm{S}$

FL

NE

NA

MG

AL

SI

SI28/S

$\mathrm{PH}$

$\mathrm{S} / \mathrm{S}$

AR

AR

$\mathrm{CA}$

$\mathrm{CA} / \mathrm{S}$

$\mathrm{CA} 40 / \mathrm{S}$

$\mathrm{SC}$

TI
Dibaryon resonance

Dineutron resonance

Dibaryon resonance

Unspecified $S=-1$ dibaryon resonance

$S=-1$ dibaryon resonance

Unspecified hypernucleus with strangeness $=-2$

Unspecified $S=-2$ dibaryon resonance

Unspecified $S=-2$ excited dibaryon resonance

Dibaryon with $S=-2$

Tritium nucleus

Hypernucleus with $\Lambda$ instead of neutron

Reported 3-neutron bound state

Reported 3-baryon state

Hydrogen-4 nucleus

Hypothetic 4-neutron bound state

Hypernucleus with $\Lambda$ instead of neutron

Hydrogen-4 hypernucleus with strangeness $=-2$

Hydrogen-5 nucleus

Hydrogen-6 nucleus

Hydrogen- 7 nucleus

Helium nucleus

Helium hypernucleus with strangeness $=-1$

Helium-5 nucleus

Helium-5 hypernucleus with strangeness $=-1$

Helium-5 hypernucleus with strangeness $=-2$

Helium- 6 hypernucleus with strangeness $=-2$

Helium neutron-rich hypernucleus with strangeness $S=-1$

Lithium nucleus

Hypernucleus with $\Lambda$ instead of neutron

Hypernucleus with $\Lambda$ instead of neutron

Beryllium-7 strange nucleus

Beryllium nucleus

Beryllium-9 hypernucleus with strangeness $=-1$

Beryllium-10 hypernucleus with strangeness $=-2$

Beryllium-11 hypernucleus with strangeness $=-2$

Beryllium-9 neutron-rich hypernucleus with strangeness $S=-1$

Beryllium-12 hypernucleus with strangeness $=-2$

Boron nucleus - note name is not same as chemical symbol

Boron hypernucleus

Boron-10 hypernucleus with strangeness $=-1$

Boron-11 hypernucleus

Boron-12 hypernucleus with strangeness $=-1$

Boron-13 hypernucleus with strangeness $=-2$

Carbon nucleus

Carbon-12 hypernucleus

Carbon hypernucleus with strangeness $=-1$

Excited hypernucleus C12(lambda)

$4.44 \mathrm{keV}$ excited carbon nucleus

Carbon-13 hypernucleus

Excited Carbon- 13 hypernucleus

Neutron-rich hypernucleus $\mathrm{C} 16(\Lambda)$

Nitrogen nucleus - note name is not same as chemical symbol

Oxygen nucleus

Oxygen hypernucleus

Oxygen-16 hypernucleus

Fluorine nucleus - note name is not same as chemical symbol

Neon nucleus

Sodium nucleus

Magnesium nucleus

Aluminum nucleus

Silicon nucleus

Silicon-28 hypernucleus

Phosphorus nucleus - note name is not same as chemical symbol

Sulfur nucleus

Sulfur hypernucleus

Chlorine nucleus

Argon nucleus

Potassium nucleus - note name is not same as chemical symbol

Calcium nucleus

Calcium hypernucleus

Calcium- 40 hypernucleus

Scandium nucleus

Titanium nucleus

Vanadium nucleus - note name is not same as chemical symbol

See the legend on page 425 . 


Particle Name Computer name Explanation

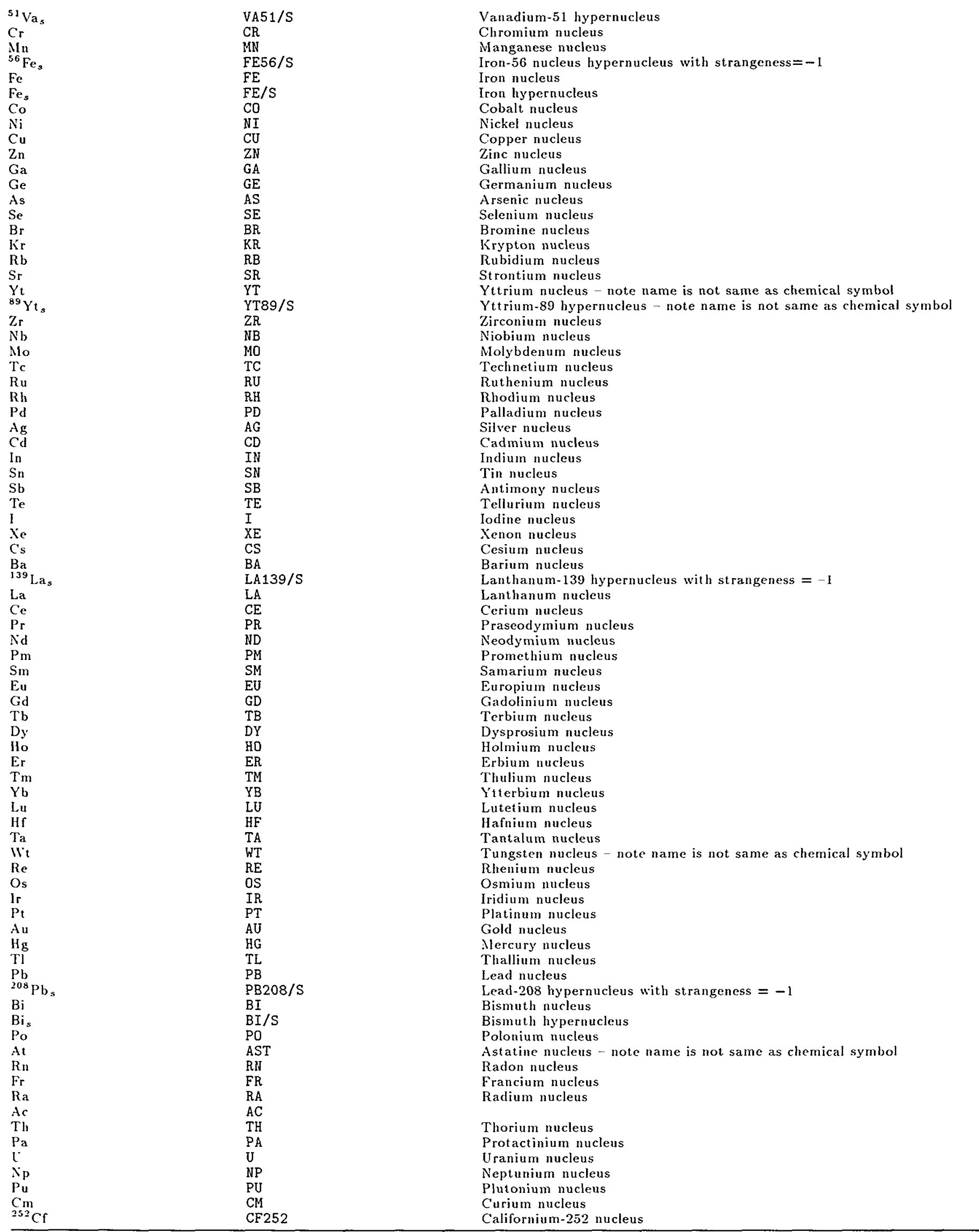




\section{Particle Name}

Computer NAME

SUPERNUCLEUS

Super heavy nucleus

\section{GLOBAL ATTRIBUTES OF INCLUSIVE REACTIONS}

$\mathrm{x}$

inelastic

annihil

(neutrals)

(chargeds)

(charged $^{+} s$ )

(charged $^{-} \mathrm{s}$ )

(vees)

( $\gamma$ 's)

(gluons)

(leptons)

( $\nu$ 's)

( $e^{ \pm \prime s}$ )

( $\left.e^{-\prime s}\right)$

$\left(e^{+, s}\right)$

( $\mu^{ \pm \prime s)}$

(jets)

(hadrons)

(hadrono's)

(charged-hadrons)

(mesons)

(charged-mesons)

( $\pi$ 's)

( $\left.\pi^{ \pm} \mathrm{s}\right)$

$\left(\pi^{+} \cdot s\right)$

( $\pi^{0}$ s)

$\left(\pi^{-} \mathrm{s}\right)$

$(\eta$ 's $)$

( $\rho^{+' s)}$

( $\left.\rho^{0} \mathrm{~s}\right)$

( $\rho^{-' s)}$

(stranges)

(kaons)

( $\mathrm{K}^{ \pm}$s)

(Ks)

$\left(K_{L}\right)$

(K's's)

(D(unspec)'s)

(nucleons)

(p's)

(n's)

( $\Lambda$ 's)

(He's)

(frags)

(fragbs)

(fragts)

(showers)

(greys)

(htracks)

(blacks)
$\mathbf{X}$

INELASTIC AHHIHIL
EXPLANATION

For use in inclusive reactions. Also for total cross-section data, as in $\mathrm{K}^{-} \mathrm{p} \rightarrow \mathrm{X}$

Same as X, (ANYTHING), except elastic excluded

Pure annihilation final state in nucleon-antinucleon scattering

\section{ATTRIBUTES OF SEMIINCLUSIVE REACTIONS}

$\begin{array}{ll}\text { (NEUTRALS) } & \text { Zero or more neutral particles } \\ \text { (CHARGEDS) } & \text { Zero or more charged particles plus possible neutrals } \\ \text { (CHARGED+S) } & \text { Zero or more positive charged particles plus possible neutrals } \\ \text { (CHARGED-S) } & \text { Zero or more negative charged particles plus possible neutrals } \\ \text { (VEES) } & \text { Zero or more unspecified neutral strange particle decays } \\ \text { (GAMMAS) } & \text { Zero or more } \gamma \text { 's }\end{array}$

(GAMMAS)

(GLUONS)

(LEPTONS)

(NUS)

$(\mathrm{E}+-\mathrm{S})$

$(\mathrm{E}-\mathrm{S})$

$(\mathrm{E}+\mathrm{S})$

$(M U+-S)$

(JETS)

(HADRONS)

(HADRONOS)

(CHARGED-HADRONS)

(MESONS)

(CHARGED-MESOHS)

(PIONS)

$(\mathrm{PI}+-\mathrm{S})$

(PI+S)

(PIOS)

(PI-S)

(ETAS)

(RHO+S)

(RHOOS)

(RHO-S)

(STRANGES)

(KAONS)

$(\mathrm{K}+-\mathrm{S})$

(KS)

(KL)

(KSS)

(D (UMSPEC)S)

(NUCLEONS)

(PROTONS)

(NS)

(LAMBDAS)

(HES)

(FRAGS)

(FRAGBS)

(FRAGTS)

(SHOWERS)

(GREYS)

(HTRACKS)

(BLACKS)
Zero or more unspecified leptons

Zero or more unspecified neutrinos

Zero or more electrons or positrons

Zero or more electrons

Zero or more positrons

Zero or more muons

Zero or more jets

Zero or more hadrons

Zero or more hadrons

Zero or more charged hadrons

Zero or more mesons

Zero or more charged mesons

Zero or more pions

Zero or more $\pi^{ \pm \prime}$ 's

Zero or more $\pi^{+}$'s

Zero or more $\pi^{0}$ 's

Zero or more $\pi^{-}$'s

Zero or more $\rho^{+ \text {'s }}$

Zero or more $\rho^{0}$ 's

Zero or more $\rho^{-}$'s

Zero or more unspecified strange particles

Zero or more unspecified kaons

Zero or more $K^{ \pm}$'s

Zero or more $K_{\text {short }}$

Zero or more $K_{\text {long }}$

Zero or more $k s$ 's

Zero or more unspecified nucleons

Zero or more protons

Zero or more neutrons

Zero or more $\Lambda$ 's

Zero or more He nuclei

Zero or more beam fragments

Zero or more target fragments

Zero or more grey tracks, usually in emulsions

Zero or more heavy tracks, usually in emulsions

Zero or more black tracks, usually in emulsions
Zero or more unspecified charmed nonstrange mesons

Zero or more nuclear fragments

\section{mult [charged] mult [charged ${ }^{++}$] mult [charged ${ }^{+}$] mult[charged $\left.{ }^{-}\right]$ mult[neutral] mult[vee] mult $[\gamma]$ mult[lepton] mult $[\nu]$ mult $\left[e^{ \pm}\right]$ mult $\left[e^{-}\right]$ mult $\left[e^{+}\right]$ mult $\left[\mu^{-}\right]$}

\section{ATTRIBUTES OF TOPOLOGICAL REACTIONS}

MULT (CHARGED)

MULT (CHARGED++)

MULT (CHARGED+)

MULT (CHARGED-)

MULT (NEUTRAL)

MULT (VEE)

MULT (GAMMA)

MULT (LEPTON)

MULT (NU)

$\operatorname{MULT}\left(E^{+-}\right)$

MULT(E-)

$\operatorname{MULT}(E+)$

$\operatorname{MULT}(M U-)$
Multiplicity distribution for unspecified charged particle Multiplicity distribution for unspecified double charged positive particle Multiplicity distribution for unspecified positive particle

Multiplicity distribution for unspecified negative particle Multiplicity distribution for unspecified neutral particle Multiplicity distribution for strange visible neutral vee (not $\gamma$ conversions) Multiplicity distribution for $\gamma$

Multiplicity distribution for unspecified lepton

Multiplicity distribution for unspecified $\nu$

Multiplicity distribution for positron or electron

Multiplicity distribution for electron

Multiplicity distribution for positron

Multiplicity distribution for $\mu^{-}$ 


\section{Particle NaMe} Computer Name

EXPLANATION

mult $\left[\mu^{+}\right]$

mult $[\mu]$

muli [jet]

mult [hadron]

mult[charged-hadron]

mult[hadron ${ }^{+}$]

mult[hadron $\left.{ }^{\circ}\right]$

mult [hadron ${ }^{-}$]

mult [meson]

muli[charged-meson]

mult [a]

mult $\left[\pi^{ \pm}\right]$

mult $\left[\pi^{+}\right]$

mult $\left[\pi^{0}\right]$

mult $\left[\pi^{-}\right]$

mult $[\eta]$

mult $[\rho]$

mult $\left[\rho^{\top}\right]$

$\operatorname{mult}\left[\rho^{\circ}\right]$

mult $\left[\rho^{-}\right]$

mult [w]

mult $[\omega(783)]$

mult $\left[f_{2}(1270)\right]$

$\operatorname{mult}\left[\rho_{3}(1690)^{0}\right]$

mult [st range]

mult [kaon]

mult $\left[K^{ \pm}\right]$

mult $\left[K^{++}\right]$

mult $\left[K^{-0}\right]$

mult $\left[K_{L}\right]$

mult $\left[K_{s}\right]$

mult $\left[\bar{K}^{0}\right]$

mult $\left[K^{-}\right]$

mult $\left[K^{*}(892)^{\dagger}\right]$

mult $\left[K^{*}(892)^{0}\right]$

mult $\left[\bar{K}^{*}(892)^{0}\right]$

mult $\left[K^{*}(892)^{-}\right]$

mult $\left[K_{2}^{*}(1430)^{+}\right]$

mult $\left[K_{2}(1430)^{0}\right]$

mult $\left[\bar{K}_{2}^{*}(1430)^{\circ}\right]$

mult $\left[K_{2}^{*}(1430)^{-}\right]$

$\operatorname{mult}[0]$

mult $\left[D^{ \pm}\right]$

mult [D(unspec)]

mult $\left[p^{ \pm}\right]$

muli[nucleon]

mult $[p]$

mult $[n]$

mult [in]

mult [p]

mult $\left[\Delta^{++}\right]$

mult $\left[\Delta^{+}\right]$

mult $\left[\Delta^{0}\right]$

mult $\left[\Delta^{-}\right]$

mult $\left[\bar{\Delta}^{+}\right]$

mult $\left[\bar{\Delta}^{0}\right]$

mult $\left[\bar{\Delta}^{-}\right]$

\section{mult $\left[\overline{3}^{--}\right]$}

mult $[1]$

mult $[\bar{\pi}]$

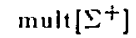

mult $\left[5^{0}\right]$

mult $[\Sigma-]$

mult $[\bar{E}+]$

mult [50]

mult $\left[\bar{\Sigma}^{-}\right]$

$\operatorname{mult}\left[\Sigma(1385)^{+}\right]$

mult $\left[\Sigma(1385)^{-}\right]$

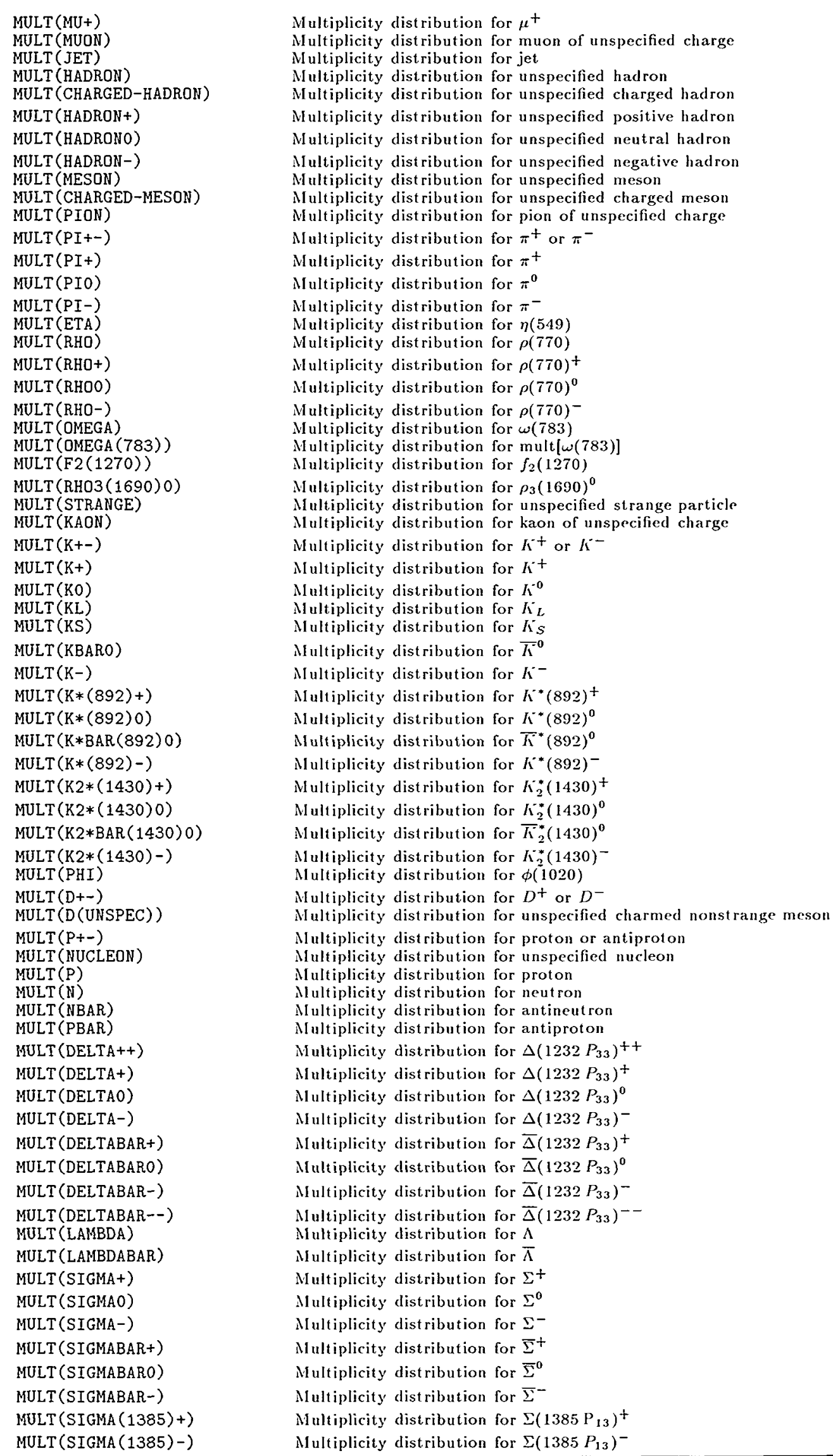

MULT (MU+)

MULT (MUON)

MULT (JET)

MULT (HADRON)

MULT (CHARGED-HADRON)

MULT (HADRON+)

MULT (HADRONO)

MULT (HADRON-)

MULT (MESON)

MULT (CHARGED-MESON)

MULT (PION)

$\operatorname{MULT}(P I \div-)$

$\operatorname{MULT}(P I+)$

$\operatorname{MULT}($ PIO)

MULT(PI-)

MULT(ETA)

MULT (RHO)

$\operatorname{MULT}(\mathrm{RHO}+)$

MULT (RHOO)

MULT (RHO-)

MULT (OMEGA)

MULT (OMEGA (783))

MULT(F2(1270))

MULT (RHO3 (1690) 0)

MULT (STRANGE)

MULT (KAON)

$\operatorname{MULT}(\mathrm{K}+-)$

MULT (K+)

MULT(KO)

$\operatorname{MULT}(\mathrm{KL})$

MULT(KS)

MULT (KBARO)

MULT(K-)

$\operatorname{MULT}(K *(892)+)$

$\operatorname{MULT}(\mathrm{K} *(892) 0)$

$\operatorname{MULT}(\mathrm{K} * \operatorname{BAR}(892) 0)$

$\operatorname{MULT}(K *(892)-)$

$\operatorname{MULT}(K 2 *(1430)+)$

$\operatorname{MULT}(K 2 *(1430) 0)$

$\operatorname{MULT}(K 2 * \operatorname{BAR}(1430) 0)$

MULT (K2*(1430)-)

$\operatorname{MULT}(\mathrm{PHI})$

$\operatorname{MULT}\left(D_{+-}\right)$

MULT(D (UNSPEC))

$\operatorname{MULT}\left(P_{+-}\right)$

MULT (NUCLEON)

$\operatorname{MULT}(P)$

$\operatorname{MULT}(N)$

MULT (NBAR)

MULT (PBAR)

MULT (DELTA++)

MULT(DELTA+)

MULT (DELTAO)

MULT (DELTA-)

MULT(DELTABAR+)

MULT (DELTABARO)

MULT (DELTABAR-)

MULT(DELTABAR--)

MULT (LAMBDA)

MULT (LAMBDABAR)

MULT(SIGMA+)

MULT (SIGMAO)

MULT(SIGMA-)

MULT(SIGMABAR+)

MULT (SIGMABARO)

MULT (SIGMABAR-)

$\operatorname{MULT}(\operatorname{SIGMA}(1385)+$ )

MULT (SIGMA (1385)-)

Multiplicity distribution for $\mu^{+}$

Multiplicity distribution for muon of unspecified charge

Multiplicity distribution for jet

Multiplicity distribution for unspecified hadron

Multiplicity distribution for unspecified charged hadron

Multiplicity distribution for unspecified positive hadron

Multiplicity distribution for unspecified neutral hadron

Multiplicity distribution for unspecified negative hadron

Multiplicity distribution for unspecified meson

Multiplicity distribution for unspecified charged meson

Multiplicity distribution for pion of unspecified charge

Multiplicity distribution for $\pi^{+}$or $\pi^{-}$

Multiplicity distribution for $\pi^{+}$

Multiplicity distribution for $\pi^{0}$

Multiplicity distribution for $\pi^{-}$

Multiplicity distribution for $\eta(549)$

Multiplicity distribution for $\rho(770)$

Multiplicity distribution for $\rho(770)^{+}$

Multiplicity distribution for $\rho(770)^{0}$

Multiplicity distribution for $\rho(770)^{-}$

Multiplicity distribution for $\omega(783)$

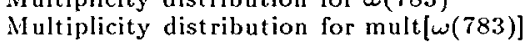

Multiplicity distribution for $f_{2}(1270)$

Multiplicity distribution for $\rho_{3}(1690)^{0}$

Multiplicity distribution for unspecified strange particle

Multiplicity distribution for kaon of unspecified charge

Multiplicity distribution for $\mathrm{K}^{-+}$or $\mathrm{K}^{-}$

Multiplicity distribution for $K^{+}$

Multiplicity distribution for $K^{-0}$

Multiplicity distribution for $K_{L}$

Multiplicity distribution for $\kappa_{S}$

Multiplicity distribution for $\bar{\kappa}^{-0}$

Multiplicity distribution for $K^{-}$

Multiplicity distribution for $K^{-*}(892)^{*}$

Multiplicity distribution for $K^{-*}(892)^{0}$

Multiplicity distribution for $\bar{K}^{*}(892)^{0}$

Multiplicity distribution for $K^{*}(892)^{-}$

Multiplicity distribution for $K_{2}^{*}(1430)^{4}$

Multiplicity distribution for $\kappa_{2}^{*}(1430)^{0}$

Multiplicity distribution for $\bar{K}_{2}^{*}(1430)^{0}$

Multiplicity distribution for $K_{2}^{*}(1430)^{-}$

Multiplicity distribution for $\phi(1020)$

Multiplicity distribution for $D^{+}$or $D^{-}$

Multiplicity distribution for unspecified charmed nonstrange meson

Multiplicity distribution for proton or antiproton

Multiplicity distribution for unspecified nucleon

Multiplicity distribution for proton

Multiplicity distribution for neutron

Multiplicity distribution for antineut

Multiplicity distribution for antiproton

Multiplicity distribution for $\Delta\left(1232 P_{33}\right)^{++}$

Multiplicity distribution for $\Delta\left(1232 P_{33}\right)^{+}$

Multiplicity distribution for $\Delta\left(1232 P_{33}\right)^{0}$

Multiplicity distribution for $\Delta\left(1232 P_{33}\right)^{-}$

Multiplicity distribution for $\bar{\Delta}\left(1232 P_{33}\right)^{+}$

Multiplicity distribution for $\bar{\Delta}\left(1232 P_{33}\right)^{0}$

Multiplicity distribution for $\bar{\Delta}\left(1232 P_{33}\right)^{-}$

Multiplicity distribution for $\bar{\Delta}\left(1232 P_{33}\right)^{--}$

Multiplicity distribution for $\Lambda$

Multiplicity distribution for $\bar{\Lambda}$

Multiplicity distribution for $\Sigma^{+}$

Multiplicity distribution for $\Sigma^{0}$

Multiplicity distribution for $\Sigma^{-}$

Multiplicity distribution for $\Sigma^{+}$

Multiplicity distribution for $\bar{\Sigma}^{0}$

Multiplicity distribution for $\bar{\Sigma}-$

Multiplicity distribution for $\Sigma\left(1385 \mathrm{P}_{13}\right)^{+}$

Multiplicity distribution for $\Sigma\left(1385 P_{13}\right)^{-}$ 


\section{PARTICLE NAME}
COMPUTER NAME
EXPLANATION

\begin{tabular}{|c|c|}
\hline mult $\left[\Xi^{-}\right]$ & $\operatorname{MULT}(\mathrm{XI}-)$ \\
\hline $\begin{array}{l}\text { mult }\left[\bar{\Xi}^{+}\right] \\
\text {mult[deuteron] } \\
\text { mult[trit] } \\
\text { mult[He] }\end{array}$ & $\begin{array}{l}\text { MULT (XIBAR+) } \\
\text { MULT (DEUTERON) } \\
\text { MULT (TRIT) } \\
\text { MULT (HE) }\end{array}$ \\
\hline mult $\left[{ }^{4} \mathrm{He}\right]$ & MULT (HE4) \\
\hline mult[frag] & MULT (FRAG) \\
\hline mult[fragb] & MULT (FRAGB) \\
\hline mult[fragt] & MULT (FRAGT) \\
\hline mult[star] & MULT(STAR) \\
\hline mult[shower] & MULT (SHOWER) \\
\hline mult [grey] & MULT (GREY) \\
\hline mult[htrack] & MULT (HTRACK) \\
\hline mult[black] & MULT (BLACK) \\
\hline mult [heavy] & MULT (HEAVY) \\
\hline
\end{tabular}

Multiplicity distribution for $\Xi^{-}$

Multiplicity distribution for $\bar{\Xi}^{+}$
Multiplicity distribution for deuteron

Multiplicity distribution for triton

Multiplicity distribution for helium nucleus

Multiplicity distribution for ${ }^{4} \mathrm{He}$ nucleus

Multiplicity distribution for nuclear fragment

Multiplicity distribution for beam fragment

Multiplicity distribution for target fragment

Multiplicity distribution for star

Multiplicity distribution for shower track

Multiplicity distribution for grey track

Multiplicity distribution for heavy tracks (black or grey) in emulsion

Multiplicity distribution for black track

\section{QUALIFIERS DESCRIBING ABSENCE OF PARTICLE IN THE FINAL STATE}

\begin{tabular}{|c|c|c|}
\hline $\begin{array}{l}0 \text { charged } \\
0 \text { charged } \\
0 \text { charged } \\
0 \text { charged-hadron } \\
0 \text { jet } \\
0 \text { vee } \\
0 \gamma \\
0 \text { lepton } \\
0 \nu_{\xi} \\
0 e^{ \pm} \\
0 e^{-} \\
0 e^{+} \\
0 \bar{\nu}_{c} \\
0 \nu_{\mu}\end{array}$ & $\begin{array}{l}\text { OCHARGED } \\
\text { OCHARGED+ } \\
\text { OCHARGED- } \\
\text { OCHARGED-HADRON } \\
\text { OJET } \\
\text { OVEE } \\
\text { OGAMMA } \\
\text { OLEPTON } \\
\text { OHUE } \\
\text { OE+- } \\
\text { OE- } \\
\text { OE+ } \\
\text { ONUEBAR } \\
\text { ONUMU }\end{array}$ & $\begin{array}{l}\text { Exactly zero charged particles } \\
\text { Exactly zero positive particles } \\
\text { Exactly zero negative particles } \\
\text { Exactly zero charged hadrons } \\
\text { Exactly zero jets } \\
\text { Exactly zero neutral strange particle decays } \\
\text { Exactly zero } \gamma \text { 's } \\
\text { Exactly zero leptons } \\
\text { Exactly zero } \nu_{e}^{\prime} \text { 's } \\
\text { Exactly zero electrons and positrons } \\
\text { Exactly zero electrons } \\
\text { Exactly zero positrons } \\
\text { Exactly zero } \nu_{e}^{\prime} s \\
\text { Exactly zero } \nu_{\mu} \text { 's }\end{array}$ \\
\hline $\begin{array}{l}0 \mu^{ \pm} \\
0 \mu^{-} \\
0 \mu^{+} \\
0 \bar{\nu}_{\mu} \\
0 \pi^{\prime} \\
0 \pi^{ \pm} \\
0 \pi^{+} \\
0 \pi^{0} \\
0 \pi^{-} \\
0 \text { strange } \\
0 K_{S} \\
0 K_{0}^{\prime} \\
0 p \\
0 n \\
0 \Lambda \\
0 \Lambda \\
0 \text { charm } \\
0 \text { bottom } \\
0 \text { fragb } \\
0 \text { shower } \\
0 \text { grey } \\
0 \text { htrack } \\
0 \text { He }\end{array}$ & $\begin{array}{l}\text { OMU+- } \\
\text { OMU- } \\
\text { OMU+ } \\
\text { ONUMUBAR } \\
\text { OPI } \\
\text { OPI+- } \\
\text { OPI+ } \\
\text { OPI0 } \\
\text { OPI- } \\
\text { OSTRANGE } \\
\text { OKS } \\
\text { OKO } \\
\text { OP } \\
\text { ON } \\
\text { OLAMBDA } \\
\text { OLAMBDABAR } \\
\text { OCHARM } \\
\text { OBOTTOM } \\
\text { OFRAGB } \\
\text { OSHOHER } \\
\text { OGREY } \\
\text { OHTRACK } \\
\text { OHE }\end{array}$ & 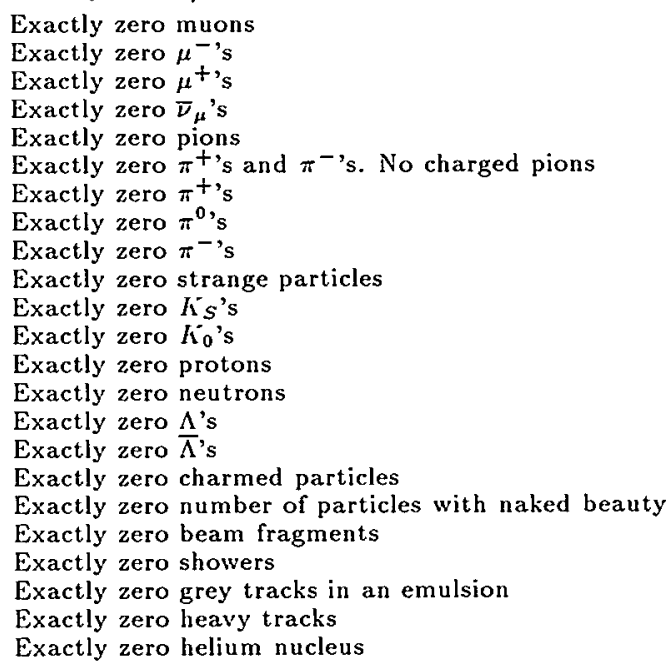 \\
\hline \multicolumn{3}{|c|}{ QUALIFIERS OF PARTICLE GROUP IN A FINAL STATE } \\
\hline $\begin{array}{l}\text { nonres } \\
\text { cc } \\
\text { DD }\end{array}$ & $\begin{array}{l}\text { NONRES } \\
\text { CC } \\
\text { DD }\end{array}$ & $\begin{array}{l}\text { Unspecified non resonant state } \\
\text { Charge conjugate final state } \\
\text { Unspecified diffraction dissociation final state }\end{array}$ \\
\hline
\end{tabular}

\section{MISCELLANEOUS TERMS}

$\begin{array}{ll}\text { unlinked } & \text { UNLINKED } \\ \text { crystal } & \text { CRYSTAL } \\ (\pi \pi)_{L=0} & \text { PIPI }(L=0) \\ \bar{N} N(I=0) & \text { NBARN }(I=0) \\ \bar{N} N(I=1) & \text { NBARI }(I=1) \\ (\pi \pi)_{I=0} & \operatorname{PIPI}(I=0) \\ (\pi \pi)_{I=1} & \operatorname{PIPI}(I=1) \\ (\pi \pi)_{I=2} & \operatorname{PIPI}(I=2) \\ (\pi \pi)_{L=0}^{0} & \operatorname{PIPI}(\mathrm{I}=0) 0 \\ (K)_{L=0} & \mathrm{KK}(\mathrm{L}=0) \\ \left(K^{*} \pi\right)_{l=1 / 2} & \mathrm{~K} * \mathrm{PI}(I=1 / 2) \\ \left(K^{*} \pi\right)_{I=3 / 2} & \mathrm{~K} * \mathrm{PI}(I=3 / 2) \\ (K \pi)_{l=1 / 2} & \mathrm{KPI}(\mathrm{I}=1 / 2)\end{array}$

Pseudo-particle used as a complete reaction by itself General target for channeling expts - target is not individual particles $\pi \pi S$-wave state

$\bar{N} N I=0$ initial state (and elastic final state)

$\bar{N} N I=1$ initial state (and elastic final state)

$\pi \pi I=0$ state

$\pi \pi I=1$ state

$\pi \pi I=2$ state

$\pi \pi L=0$ state

$\Lambda^{*} \pi I=1 / 2$ state

$K^{*} \pi I=3 / 2$ state

$\kappa \pi I=1 / 2$ state

See the legend on page 425 . 


\section{Particle Name}

$(K \pi)_{I=3 / 2}$

$(K N)_{i=0}$

$(K \cdot)_{l=1}$

$(\bar{k} \times)_{l=0}$

$(\bar{K} N)_{l=1}$

$(\pi \cdot)_{l=1}$

$(\pi \Lambda)_{I=1}^{+}$

$(\pi .1)_{I=1}^{0}$

$(\pi \Lambda)_{I=1}^{-}$

$(\pi \Sigma)_{t=0}$

$(\pi \Sigma)_{I=1}$

$(\pi \Sigma)_{i=1}^{+}$

$(\pi \Sigma)_{I=1}^{0}$

$(\pi \Sigma)_{t=1}^{-}$

$(\pi N)_{I=1 / 2}$

$\left(\pi V^{\prime}\right)_{I=3 / 2}$

$\wedge \rho(I=1 / 2)$

$N \rho(I=3 / 2)$

$(N \pi \pi)_{l=1 / 2}$

$(\pi \Delta)_{I=1 / 2}$

$(\pi \Delta)_{I=3 / 2}$

$(K \Sigma)_{I=1 / 2}$

$(K \Sigma)_{l=3 / 2}$

$N N(I=0)$

$N N^{\prime}(I=1)$

$f_{0}(995)$

$\Upsilon(5970)$

$\vec{K} N(1700)^{0}$

\section{Computer Name}

$\operatorname{KPI}(\mathrm{I}=3 / 2)$

$\mathrm{KN}(\mathrm{I}=0)$

$\operatorname{KN}(I=1)$

$\operatorname{KBARN}(I=0)$

$\operatorname{KBARN}(I=1)$

$\operatorname{PILAMBDA}(I=1)$

$\operatorname{PILAMBDA}(I=1)+$

$\operatorname{PILAMBDA}(I=1) 0$

$\operatorname{PILAMBDA}(I=1)-$

$\operatorname{PISIGMA}(I=0)$

$\operatorname{PISIGMA}(I=1)$

$\operatorname{PISIGMA}(I=1)+$

$\operatorname{PISIGMA}(I=1) 0$

$\operatorname{PISIGMA}(I=1)$ -

$\operatorname{PIN}(I=1 / 2)$

$\operatorname{PIN}(I=3 / 2)$

$\operatorname{NRHO}(I=1 / 2)$

$\operatorname{NRHO}(I=3 / 2)$

$\operatorname{NEPSILON~}(I=1 / 2)$

$\operatorname{PIDELTA}(I=1 / 2)$

$\operatorname{PIDELTA}(I=3 / 2)$

$\operatorname{KSIGMA}(I=1 / 2)$

$\operatorname{KSIGMA}(I=3 / 2)$

NN $(I=0)$

$\operatorname{NN}(I=1)$

\section{MISCLASSIFIED OR DEAD PARTICLES}

F0 (995)

UPSI (5970)

$\operatorname{KNBAR}(1700) 0$
$K \pi I=3 / 2$ state

$K N I=0$ initial state (and elastic final state)

Kn $I=1$ initial state (and elastic final state)

$\bar{K} N I=0$ initial state (and elastic final state)

$\bar{K} N I=1$ initial state (and elastic final state)

$\pi \Lambda I=1$ system of unspecified mass, for PWA

$\pi \Lambda I=1$ system of unspecified mass, for PWA

$\pi \Lambda I=1$ system of unspecified mass, for PWA

$\pi \Lambda I=1$ system of unspecified mass, for PWA

$\pi \Sigma I=0$ system of unspecified mass, for PWA

$\pi \Sigma I=1$ system of unspecified mass, for PWA

$\pi \Sigma I=1$ system of unspecified mass, for PWA

$\pi \Sigma I=1$ system of unspecified mass, for PWA

$\pi \Sigma I=1$ system of unspecified mass, for PWA

$\pi N I=1 / 2$ initial state (and elastic final state)

$\pi N I=3 / 2$ initial state (and elastic final state)

$N \rho I=1 / 2$ final state

$N \rho I=3 / 2$ final state

Nucleon and $S$-wave dipion $\mathrm{I}=1 / 2$ final state

$\pi \Delta I=1 / 2$ final state

$\pi \Delta I=3 / 2$ final state

$K \Sigma I=3 / 2$ state

$K \Sigma I=3 / 2$ state

$N N I=0$ initial state (and elastic final state)

NNI = 1 initial state (and elastic final state)

$S$-wave $\pi^{0} \pi^{0}$ enhancement

Early reported $\Upsilon$ to $e^{+} e^{-}$

Possible Ki state 
Our names for accelerators are collected below. Usually the name is simply an abbreviation of the name of the institution at which the accelerator is located. Where there is more than one accelerator at an institution, an appropriate modifier is appended, as in CERN-SPS.

For a cosmic ray experiment, we use COSM as the accelerator name, sometimes combined with a specification of the source of the rays.

We use NONE for certain cases, such as proton decay experiments, in which no particle beam is used. We use MANY for certain rapporteur talks or other papers surveying a number of experiments at several (often unspecified) accelerators.

Energies listed are the approximate maximum energies of the circulating beams. 
AERE-HELIOS-NS

ALPI

ANIK-MEA

ANL

ANL-ATLAS

ANUC-CYC

BHEP-BEPC

BNL

BNL-ION

BONN-CYC

BONN-ELSA

CEBAF

CELSIUS

GERN-ISR

CERN-LEAR

CERN-LEP

CERN-PBAR/P

CERN-PS

CERN-SC

GERN-SPS

CESR

CHALK-TANDEM

CIT-PELLETRON

COSM

COSM-CYGNUS-X-3

COSM-HERCULES-X-1

COSM-SN1987A

COSM-SUN

COSY

DARE-NINA

DESY

DESY-DORIS

DESY-DORIS-II

DESY-HERA

DESY-PETRA

DGSI

DGSI-UNILAC

ELECTROSTATIC

ESRF

FNAL

FNAL-AA

FNAL-COLLIDER

FNAL-TEV

FRAS-ADONE

GANIL

GRON-CYC

HCAT

ILL

IMPL-HIREL

IND-CYC

ITEP

IUPU-CYC

JINR

JINR-600

KEK-KENS

KEK-PF-LINAC

KEK-PS

KEK-TRISTAN

KERN-CYC

KFAJ-CYC

KHAR

KYUS-TAF

LAMPF

LASER

LBL-BEVALAC

LBL-CYC-88IN

LEGS

LINAC

LUNA

LUND

LVLN-CYC

MANI-CYC

MANY

MANZ-MAMI

MINR-MMF

MIT-BLA

MSU-CYC

NONE

NOVO-VEPP-2M
HELIOS neutron source at Harwell

Superconducting linac to accelerate ions (Legnaro, Italy)

MEA $e^{-}$linac at NIKHEF

Argonne (ZGS) proton synchrotron $\left(12.7 \mathrm{GeV} / c p_{\mathrm{lab}}\right)$

Superconducting linear accelerator at the Argonne National Laboratory

Canberra cyclotron

Beijing electron-positron colliding ring $\left(5.6 \mathrm{GeV} E_{c m}\right.$ )

Brookhaven (AGS) proton synchrotron $\left(33 \mathrm{GeV} / c p_{\mathbf{l a b}}\right)$

Heavy ion accelerator at BNL

Bonn CYClotron

Electron-Stretcher-Accelerator (2-3.5 GeV Plab)

Continuous Electron Beam Accelerator Facility ( $\mathrm{E}=0.4-4 . \mathrm{GeV}, 0.2 \mathrm{~mA}$ current)

Storage ring at the Svedberg Laboratory in Uppsala

CERN proton-proton collider $\left(62 \mathrm{GeV} E_{\mathrm{cm}}\right)$

CERN low-energy antiproton ring

CERN large electron-positron collider $\left(120 \mathrm{GeV} E_{\mathrm{cm}}\right)$

CERN $\bar{p} p$ collider $\left(540 \mathrm{GeV} E_{\mathrm{cm}}\right)$

CERN proton synchrotron $\left(28 \mathrm{GeV} / c p_{\text {lab }}\right)$

CERN synchrocyclotron (600 $\mathrm{MeV} T_{\mathrm{ab}}$ )

CERN super proton synchrotron $\left(450 \mathrm{GeV} / c p_{\mathrm{lab}}\right)$

Cornell electron-positron storage ring $\left(12 \mathrm{GeV} E_{\mathrm{cm}}\right)$

Chalk River MP tandem

California Institute of Technology 3-MeV Pelletron accelerator

Cosmic rays

Local source of cosmic ray particles from direction of Cygnus X-3

Local source of cosmic ray particles from direction of Hercules X-1

Local source of cosmic ray particles from direction of SN $1987 \mathrm{~A}$

Local source of cosmic ray particles from direction of the Sun

COoler SYnchrotron - JULIC cyclotron as injector and a synchrotron with the possibilities to coll the beam

Daresbury electron synchrotron $\left(5.2 \mathrm{GeV} / c p_{\mathrm{lab}}\right)$

Hamburg Deutches electron synchrotron $\left(7.5 \mathrm{GeV} / c p_{\mathrm{lab}}\right)$

DESY DOppel RIng Speicher $\left(2-6 \mathrm{Gev} E_{\mathrm{cm}}\right)$

DESY DORIS upgraded in $1977\left(11.2 \mathrm{GeV} E_{\mathrm{cm}}\right)$

$e-p$ machine at DESY

DESY electron-positron collider $\left(40 \mathrm{GeV} E_{\mathrm{cm}}\right)$

Darmstadt heavy ion facility (SIS/ESR)

Heavy ion accelerator at GSI

General electrostatic accelerator

European Synchrotron Radiation Facility, GRENOBLE

FNAL proton synchrotron $\left(500 \mathrm{GeV} / c p_{\text {lab }}\right)$

Antiproton accumulator for collider

FNAL $\vec{p} p$ collider $\left(2000 \mathrm{GeV} E_{\mathrm{cm}}\right)$

FNAL fixed target machine $(1000 \mathrm{GeV})$

Frascati electron-positron ring $\left(3 \mathrm{GeV} E_{\mathrm{cm}}\right)$

Two coupled isochronous cyclotrons for heavy ions

KVI Cyclotron at Groningen

High-current accelerator of the Institute of High-Current Electronics Electronics (TOMSK, RUSSIA)

Univ. of Ill. electron synchrotron $\left(300 \mathrm{MeV} / c p_{l_{\mathrm{ab}}}\right)$

Heavy Ion REsearch faciLity

Indiana University cyclotron facility

ITEP Moscow proton synchrotron ( $\left.7 \mathrm{GeV} / c p_{\mathrm{lab}}\right)$

Indiana University cyclotron

JINR Dubna proton synchrotron $\left(10 \mathrm{GeV} / c p_{\mathrm{lab}}\right)$

JINR Dubna synchrocyclotron $\left(600 \mathrm{MeV} T_{\mathrm{ab}}\right)$

KEK Spallation Neutron Source

KEK Photon Factory, storage ring using $2.5 \mathrm{GeV}$ electron beam from the injector LINAC

KEK proton synchrotron ( $12 \mathrm{GeV} p_{\text {lab }}$ )

KEK electron-positron collider $\left(64 \mathrm{GeV} E_{\mathrm{cm}}\right)$

Kernfysisch Versneller Inst. superconducting cyclotron

Institute of nuclear physics of KFA. (JULICH, GERMANY)

Electron linear accelerator ( $2 \mathrm{GeV} p_{\mathrm{lab}}$ ) at Kharkov Physico-Technical Inst., Ukr. Acad. Sci.

Tandem accelerator facility of the Kyushu University

Los Alamos meson/proton factory

Laser as a source of $\gamma$ 's

Tandem combination of LBL-HILAC and Bevatron. Accelerates ions up to Fe $(2.1 \mathrm{GeV} /$ nucleon for charge/mass $=$ $0.5,4.9$ for protons)

The LBL 88 inch cyclotron (160 MeV for alphas)

Laser Electron Gamma Source at BNL.

unspecified linac

The $50 \mathrm{kV}$ accelerator facility consisted of a duoplasmatron ion source (Laboratori Nazionali del Gran Sasso (LNGS))

Electron synchrotron at Lund $\left(1.1 \mathrm{GeV} / c p_{\mathrm{lab}}\right)$

Isochronous cyclotron at University of Louvain

University of Manitoba cyclotron

Used (rarely) for reviews and compilations

Mainz electron Microtron on the energy up to $855 \mathrm{MeV}$ and 100 microamps current

Moscow Meson Factory, proton and $H^{-}$ion accelerator with the energy of $600 \mathrm{MeV}$

MIT electron LINAC ( $780 \mathrm{MeV} E_{\mathrm{lab}}$ )

Michigan State Univ. superconducting cyclotron to $40 \mathrm{MeV}$

No accelerator used

Electron-positron storage ring at Novosibirsk $\left(1.4 \mathrm{GeV} E_{\mathrm{cm}}\right)$ 


\section{NOVO-VEPP-3}

\section{NOVO-VEPP-4}

OHIO-RBF

ORNL-ORELA

ORSA-CYC

ORSA-DCI

OSAK-CYC

PIYF

PSI

REACTOR

RHEL-ISIS

ROCH-TANDEM

SAAC-CYC

SACL-LINAC

SACL-SATURNE-II

SAIP-RIKEN

SARA

SASK-LINAC

SERP

SLAC

SLAC-NPI

SLAC-PEP

SLAC-SLC

SLAC-SPEAR

SOURCE

TAMU-CYC

TOHO

TOKY

TRID

TRIUMF

UMD-CYC

UUPP-CYC

VAN-DE-GRAAFF

VECC

WISC

YERE-ARUS
Novosibirsk (VEPP-3) electron-positron storage ring, used primarily as synchrotron radiation source, will be used as intermediate storage for VEPP-4 $\left(2.2 \mathrm{GeV} E_{\mathrm{cm}}\right)$

Electron-positron ring at Novosibirsk, also a synchrotron radiation source $\left(7-10.4 \mathrm{GeV} E_{\mathrm{cm}}\right)$

Notre Dame-Michigan-Ohio state radiative beam fasility

Oak Ridge Electron Linear Acelerator pulsed neutron source

Orsay synchrocyclotron $\left(150 \mathrm{MeV} / c p_{\text {lab }}\right)$

Orsay electron-positron storage ring $\left(3.4 \mathrm{GeV} E_{\mathrm{cm}}\right)$

$230 \mathrm{~cm}$ AVF cyclotron

Peterburg Inst. of Nucl. Phys. synchrocyclotron ( $\left.1 \mathrm{GeV} T_{\mathrm{lab}_{\mathrm{a}}}\right)$

Isochronous cyclotron ( $590 \mathrm{MeV}$ Tlab) at PSI

General nuclear reactor

Pulsed neutron source at RAL (1 - $1000 \mathrm{MeV}$ energy)

Tandem Electrostatic accelerator at Univ. of Rochester's Nuclear Structure Research Laboratory

National Accelerator Center Cyclotron, South Africa

Saclay electron LINAC

Saclay proton, deuteron, nucleus accelerator $\left(2.95 \mathrm{GeV} T_{\text {lab }}\right.$ for protons $)$

Ring cyclotron at Saitama Inst. of Phys. and Chem. Reserches

Coupled cyclotron accelerator system in CEN, Grenoble

LINAC at University of Saskatewan

IHEP Serpukhov proton synchrotron $\left(76 \mathrm{GeV} / c p_{\text {lab }}\right)$

Stanford electron linear accelerator $\left(40 \mathrm{GeV} / c p_{\text {lab }}\right)$

Stanford nuclear physics injector

Stanford electron-positron ring ( $\left.30 \mathrm{GeV} E_{\mathrm{cm}}\right)$

Stanford linear electron-positron collider $\left(100 \mathrm{GeV} E_{\mathrm{cm}}\right)$

Stanford electron-positron ring $\left(8.4 \mathrm{GeV} E_{\mathrm{cm}}\right)$

Generic name for radioactive source used for particle physics experiments

Texas A\&M university cyclotron

Low energy electron linear accelerator at Tohoku University

INS Tokyo electron synchrotron $\left(1.3 \mathrm{GeV} / c p_{\mathrm{lab}}\right)$

Triangle Universities Nuclear Laboratory accelerator

Canadian TRIangle University Meson Facility (520 MeV $T_{\text {lab }}$ )

University of Maryland cyclotron lab

Uppsala cyclotron

General Van-de-Graaff accelerator

The zero degree port of the Variable Energy Cyclotron Centre, Calcutta

$\mathrm{U}$. Wisconsin electrostatic $\left(2.5 \mathrm{MeV} / \mathrm{c} p_{\mathrm{lab}}\right)$

Yerevan (ARUS) electron synchrotron $\left(6.1 \mathrm{GeV} / c p_{\mathrm{lab}}\right)$ 
We list here detectors and the laboratories at which they are used.

Bubble chamber detector names give the fill - hydrogen (HBC), deuterium (DBC), helium (HEBC) or heavy liquid (HLBC) - then the chamber name (which is usually simply its size), and finally any qualifiers. The qualifiers are HYB for a predominantly hybrid mode of operation, RAP for a rapid-cycling chamber, and TST for a chamber containing a track-sensitive target. When more than one qualifier is appropriate, we use the one most important to the data at hand. 


\section{DETECTOR ACCELERATOR EXPLANATION}

2-GAMMA

20-GEV

8-GEV

?

ACCMOR

AFS

AGASA

AHEAD

ALADIN

ALEPH

ALPHA-POLIS

AMADEUS

AMPHORA

AMY

APEX

APPLE

ARGUS

ASTERIX

BAKSAN

BENKEI

BES

BESS

BIS-2

BNL-SND

CALO

CASA-MIA

CCM

CCS

CDF

CDHS

CELLO

CERES

CERN-MUNICH

CHAOS

CHARM

CHARM-II

CLAMSUD

CLEO

CLEO-II

CMD-2

CNTR

COMB

\section{COVER}

CPLEAR

CRYS-BALL

CRYS-BARREL

DO

DAPHNE

DAS

DBC-30IN

DBC-30IN

DBC-30IN

DBC-30IN

DBC-35CM

DBC-81CM

DBC-BEBC

DELPHI

DEUTRON-2

DIOGENE

DISC-3

DISTO

DLS

DM2

DRIFT

EAS-TOP

EHS

ELAN

ELEGANTS-Y

ELSSY

EMC

EMUL

EPICS

EPOS

EVA

EXCHARM

FANCY
SLAC-PEP

SLAC

SLAC

CERN-SPS

CERN-ISR

COSM

MIT-BLA

DGSI

CERN-LEP

JINR

BONN-ELSA

SARA

KEK-TRISTAN

ANL-ATLAS

CERN-LEAR

DESY-DORIS

CERN-LEAR

UNDERGROUND

KEK-PS

BHEP-BEPC

COSM

SERP

UNDERGROUND

COSM

FNAL-TEV

FNAL

FNAL-COLLIDER

CERN-SPS

DESY-PETRA

CERN-SPS

CERN-PS

TRIUMF

CERN-SPS

CERN-SPS

MINR-MMF

CESR

CESR

NOVO-VEPP-2M

COSM

CERN-LEAR

SLAC-SPEAR

CERN-LEAR

FNAL-COLLIDER

MANZ-MAMI

ANL

BNL

LBL

FNAL

SACL

SACL

CERN-PS

CERN-LEP

YERE-ARUS

SACL-SATURNE-II

JINR

SACL-SATURNE-II

LBL-BEVALAC

ORSA-DCI

CosM

CERN-SPS

BONN-ELSA

UNDERGROUND

MIT-BLA

CERN-SPS

LAMPF

DGSI-UNILAC

CESR

SERP

KEK-PS
System of forward detectors for studying mainly the 2- $\gamma$ process

$8-\mathrm{GeV}$ spectrometer

Unknown detector

Large aperture forward magnetic spectrometer

Axial field spectrometer

Akeno Giant Air Shower Array

Alberta high efficiency analyzer for deuterons

Forward spectrometer

LEP detector

Scintillation detector in the PHOENICS area at BONN-ELSA

High resolution lepton detector

ATLAS positron experiment

Apparatus for precision measurements of the proton electromagnetic form factors in the time-

like region and vector meson spectroscopy.

ARGUS detector at DORIS

Antiproton stop experiment with trigger on initial $x$ rays

Baksan underground scintillation telescope

BENKEI spectrometer system at KEK proton synchrotron

BEijing Spectrometer

Balloon Borne Experiment with a Superconducting Spectrometer

Upgrade of BIS

Homestake gold mine Brookhaven solar neutrino detector at Lead, South Dakota

Calorimeter

Chicago Air Shower Array with Michigan Muon Array

Chicago cyclotron magnet spectrometer

Chicago cyclotron spectrometer at Fermilab

Collider detector at Fermilab

CERN-Dortmund-Heidelberg-Saclay-Bologna neutrino detector at SPS (135 tons)

CELLO detector at DESY

CErenkov Ring Electron Spectrometer

CERN-Max Planck I (Munich) spectrometer

CERN-Hamburg-Amsterdam-Rome-Moscow neutrino detector

Upgraded charm detector

MINR-MMF magnetic spectrometer.

Solenoidal magnetic spectrometer

CLEO II detector at CESR

Cryogenic magnetic detector, $1.5 \mathrm{~T}$ solenoid, CsI and BGO calorimeters

Counters (no chambers)

Combinations of different types of detectors. Can include a hybrid system involving a bubble

chamber, if the bubble chamber is a minor part of the system

Shower detector at Baksan

Apparatus for precision measurements of CP violation and CPT tests with $K^{0} \bar{K}^{0}$.

Crystal ball

Crystal Barrel large-solid-angle detector

FNAL collider detector

Cilindrically symmetric $4 \pi$ detector

Double arm spectrometer

Deuterium bubble chamber

Deuterium bubble chamber

Deuterium bubble chamber

Deuterium bubble chamber

Deuterium bubble chamber

Deuterium bubble chamber

Deuterium filled Big European Bubble Chamber

LEP detector

Modification of DEUTRON detector

Pictorial drift chamber

Double-arm magnetic spectrometer

Dubna, Indiana, Saclay TOrino spectrometer with scintillating fibers

Double arm spectrometer

Detecteur magnetique no. 2

Generic drift chamber detector

Extended air shower detector on top of the Gran Sasso Mt., at the Gran Sasso National Labo-

ratory, L'Aquila, Italy

European hybrid spectrometer

ELEectron GAmma-ray NeuTrino Spectrometer $V$ at Kamioka underground laboratory

Electron spectrometer system

European muon collaboration

Emulsion. Also used for detectors like PLASTIC where tracks are "frozen" in a solid medium

Energetic pion spectrometer and detection system

Electron-positron solenoid spectrometer

Exclusive variable apparatus, built around CLEO I solenoid

Further modification of BIS

Forward and cylindrical detector system, large acceptance spectrometer covering both projectile and target regions 


DETECTOR ACCELERATOR EXPLANATION

\begin{tabular}{|c|c|}
\hline $\begin{array}{l}\text { FENICE } \\
\text { FGJT } \\
\text { FHS-1 } \\
\text { FHS-2 } \\
\text { FMM } \\
\text { FMPS } \\
\text { FOCUS } \\
\text { FODS } \\
\text { FOPI }\end{array}$ & $\begin{array}{l}\text { FRAS-ADONE } \\
\text { FNAL } \\
\text { ITEP } \\
\text { ITEP } \\
\text { FNAL } \\
\text { FNAL } \\
\text { ITEP } \\
\text { SERP } \\
\text { DGSI }\end{array}$ \\
\hline $\begin{array}{l}\text { FPP } \\
\text { FREJUS } \\
\text { GALLEX }\end{array}$ & $\begin{array}{l}\text { GANIL } \\
\text { UNDERGROUND } \\
\text { UNDERGROUND }\end{array}$ \\
\hline $\begin{array}{l}\text { GAMS-2000 } \\
\text { GAMS-4000 } \\
\text { GAMS-4PI } \\
\text { GEM } \\
\text { GIBS } \\
\text { GOLIATH } \\
\text { H1 } \\
\text { HBC-1M } \\
\text { HBC-1M } \\
\text { HBC-2M } \\
\text { HBC-2M } \\
\text { HBC-30IN } \\
\text { HBC-30IN } \\
\text { HBC-30IN } \\
\text { HBC-30IN } \\
\text { HBC-30IN-HYB } \\
\text { HBC-40IN-IIYB } \\
\text { HBC-40IN-IIYB } \\
\text { HBC-BEBC-HYB } \\
\text { HBC-LEBC-HYB } \\
\text { HBC-LUDMILA } \\
\text { HBC-MIRA } \\
\text { HBC-RCBC } \\
\text { HELIOS } \\
\text { HERMES } \\
\text { HISS } \\
\text { HLBC-105CM } \\
\text { HLBC-15FT } \\
\text { HLBC-15FT-HYB } \\
\text { HLBC-180LIT } \\
\text { HLBC-2M } \\
\text { HLBC-2M } \\
\text { HLBC-BEBC } \\
\text { HLBC-BEBC-HYB } \\
\text { HLBC-DIANA } \\
\text { HMS } \\
\text { HRS } \\
\text { HRSF } \\
\text { HYBUC } \\
\text { HYPERON }\end{array}$ & $\begin{array}{l}\text { SERP } \\
\text { CERN-SPS } \\
\text { SERP } \\
\text { COSY } \\
\text { JINR } \\
\text { CERN-SPS } \\
\text { DESY-HERA } \\
\text { KEK-PS } \\
\text { JINR } \\
\text { ITEP } \\
\text { CERN-PS } \\
\text { ANL } \\
\text { LBL } \\
\text { BNL } \\
\text { FNAL } \\
\text { FNAL } \\
\text { RHEL } \\
\text { SLAC } \\
\text { CERN-PS } \\
\text { CERN-SPS } \\
\text { SERP } \\
\text { SERP } \\
\text { CERN-SPS } \\
\text { CERN-SPS } \\
\text { HERA } \\
\text { LBL-BEVALAC } \\
\text { ITEP } \\
\text { FNAL } \\
\text { FNAL } \\
\text { ITEP } \\
\text { SERP } \\
\text { JINR } \\
\text { CERN-PS } \\
\text { CERN-PS } \\
\text { ITEP } \\
\text { CEBAF } \\
\text { SLAC-PEP } \\
\text { LAMPF } \\
\text { CERN-SPS } \\
\text { SERP }\end{array}$ \\
\hline $\begin{array}{l}\text { IMB } \\
\text { IMB-III } \\
\text { INDRA } \\
\text { IONIZATION } \\
\text { ISTRA-M } \\
\text { JADE } \\
\text { JETSET } \\
\text { KAMIOIKANDE-I } \\
\text { KAMIOKANDE-II } \\
\text { KAMIOKANDE-III } \\
\text { KARMEN } \\
\text { KASPIY } \\
\text { KEDR } \\
\text { KGF } \\
\text { KURAMA } \\
\text { L3 } \\
\text { LAB-E } \\
\text { LADS } \\
\text { LAND } \\
\text { LAS } \\
\text { LASS } \\
\text { LEPS } \\
\text { LEPTON-F } \\
\text { LISE } \\
\text { LSD }\end{array}$ & $\begin{array}{l}\text { UNDERGROUND } \\
\text { UNDERGROUND } \\
\text { GANIL } \\
\text { SERP } \\
\text { DESY-PETRA } \\
\text { CERN-LEAR } \\
\text { UNDERGROUND } \\
\text { UNDERGROUND } \\
\text { UNDERGROUND } \\
\text { RHEL-ISIS } \\
\text { JINR } \\
\text { NOVO-VEPP-4M } \\
\text { UNDERGROUND } \\
\text { KEK-PS } \\
\text { CERN-LEP } \\
\text { FNAL } \\
\text { PSI } \\
\text { DGSI } \\
\text { LAMPF } \\
\text { SLAC } \\
\text { PSI } \\
\text { SERP } \\
\text { GANIL } \\
\text { UNDERGROUND }\end{array}$ \\
\hline
\end{tabular}

Non-magnetic detector

FERMILAB Gas Fet Target

Focusing Hadron Spectrometer

Second arm of FHS-1

Neutrino calorimeter at FNAL

Multiparticle spectrometer at Fermilab

FOrward particle \& CUmulative particle Spectrometer

Double-arm spectrometer

Detector for an identification of charged particles $(Z \leq 15)$. High granularity time-of-flight wall (764 scintillators) and an inner shell of thin energy-loss detectors (188 elements)

Focal-Plane Polarimeter

Proton decay experiment, tracking calorimeter in the Alps

Solar neutrino detector in hall A of the Gran Sasso National Laboratory, L'Aquila, Italy. Uses

30 tons of Gallium in the form of $\mathrm{GaCl} 3$

Hodoscope gamma-spectrometer

Hodoscope gamma-spectrometer

GAMS-2000 modification

High resolution hybrid detector

Combination of scintillator counters and streamer chamber

CERN Goliath spectrometer

$\mathrm{H} 1$ detector at DESY/HERA

Hydrogen bubble chamber

Hydrogen bubble chamber

Hydrogen bubble chamber

Hydrogen bubble chamber

Hydrogen bubble chamber

Hydrogen bubble chamber

Hydrogen bubble chamber

Hydrogen bubble chamber

Hydrogen bubble chamber

Hydrogen bubble chamber

SLAC hybrid facility rapid cycling $\mathrm{HBC}$ (retired)

Big European bubble chamber with hybrid system

Little European bubble chamber with hybrid system

Also known as DUBNA chamber, but at Serpukhov

$4.5 \mathrm{~m} \times 1.6 \mathrm{~m} \times 1.1 \mathrm{~m}$ cold chamber

Spectrometer at DESY/HERA

Heavy Ion Spectrometer System

Heavy-liquid bubble chamber

Heavy-liquid bubble chamber

Heavy-liquid bubble chamber

Heavy-liquid bubble chamber

180-liter propane chamber

180-liter propane chamber built by Dubna

Heavy-liquid bubble chamber

Heavy-liquid bubble chamber

Heavy-liquid bubble chamber

High Momentum Spectrometer

High resolution spectrometer

High resolution spectrometer facility

Hydrogen bubble chamber at CERN SPS

Single arm magnetic spectrometer with big spark and proportional chambers and gas hodoscope counter

Irvine-Michigan-Brookhaven underground Cherenkov detector

Irvine-Michigan-Brookhaven underground Cherenkov detector

Generic detector looking for ionization

JADE detector at DESY

Compact general purpose detector

Kamioka nucleon decay detector

Kamioka nucleon decay detector, stage-2

Kamioka nucleon decay detector, stage-3, started operation on November 1990

$56 \mathrm{t}$ liquid scintillator calorimeter

Channel and $\pi$-meson spectrometer with final particle energy up to $1 \mathrm{GeV}$

Kolar Gold Field underground detector

LEP detector

1100-ton target-calorimeter muon-spectrometer detector for neutrino physics

Large Acceptance Detector System

Large area neutron detector

Large aperture solenoid spectrometer

Low energy magnetic spectrometer

Magnetic recoil spectrometer 


\section{DETECTOR ACCELERATOR EXPLANATION}

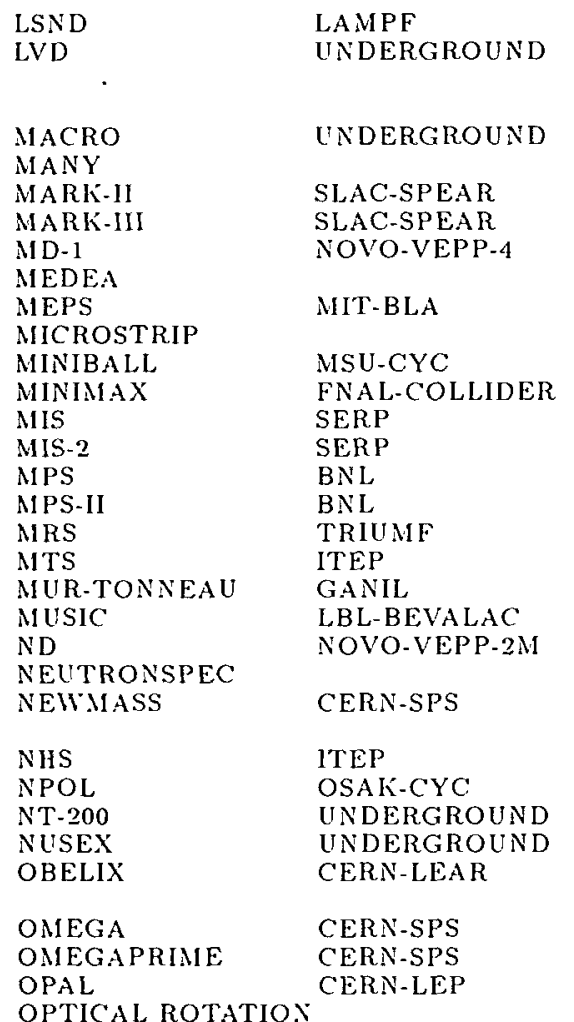

OPTICAL ROTATION

ORION

OSPK

OTHER

PERKEO

PHOENICS

PHOTON

PHOTON-MASSER

PIOSPEC

PIK

PINOT

PLASTIC

PLASTIC-BALL

POLDER

POMME

PROZA-M

RISK

RMC

S-KAMIOKANDE

SAC -600
$S A C-900$

SAGE

SAPHIR

SAS

SCINT

SEMII

SFM

SIGMA

SIGMA-AYAKS

SINDRLM-II

SKM-200

SISS

SLD

SMM-GRS

SND

SOS

SOUDAN-II

SPEC

SPEC-6N

SPEG

SPES-0

SPES-I

SPES-II
SACL-SATURNE-II

BONN-ELSA

JINR

LA.MPF

KEK-PS

SACL-SATURNE-II

LBL-BEVALAC

SACL-SATURNE-II

SACL-SATURNE-II

SERP

SERP

TRIUMF

UNDERGROUND

SACL-LINAC

SACL-LINAC

UNDERGROUND

BONN-ELSA

GANIL

CERN-PS

SERP

SERP

PSI

JINR

KEK-PS

SLAC-SLC

COS.II

NOVO-VEPP-2M

CEBAF

UNDERGROUND

SERP

GANIL

SACL-SATURNE-II

SACL-SATURNE-II

CERN-LEAR
Liquid Scintillator Neut rino detector

Large volume detector, installed in hall $A$ of the Gran Sasso National Laboratory, LAquila, Italy. Operated by an Italian-Soviet collaboration. Using 1600 tons of liquid scintillator and limited streamer tubes. Experiment aiming at the detection of $n$

Large area detector

Many different detectors

SLAC Mark-II detector

SLAC-SPEAR spectrometer system (not related to MARK-II)

Magnetic detector

MEDEA is multidetector at GANIL

Medium energy pion spect rometer

Generic microstrip detector

The apparatus is used in FNAL-864 experiment. Its main part is a tracking telescope Multiparticle spectrometer

Multiparticle spect rometer

Updated BNL MPS

Medium resolution spectrometer

3-m magnetic spectrometer with spark chambers

Axially symmetric multi-detector system at GANIL

MUltiple-Sampling Ionization Chamber, component of IIISS

Neutral detector at VEPP.2M

Neutron spectrometer

Detector to look for charged massive object at $0^{\circ}$ production angle, using $\mathrm{H} 6$-beamline in the North Experimental Area as a charged particle spectrometer

Spectrometer without magnetic field

Neutron Polarimeter

Neutrino Telescope with 200 phototubes on Baikal lake

NUSEX nucleon decay detector, Mont Blanc tunnel

A large acceptance and high resolution detector based on the Open Axial Field Spectrometer to study of PBAR and NBAR annihilation.

CERN OMEGA spectrometer

LEP detector

Generic detector system to measure the rotation angle of the polarization plane of the laser light.

neutron calorimeter, moved from GANIL

Optical spark chamber

Rare nonelectronic detectors (c.g. moon, ocean floor)

Solenoidal electron spect rometer

Photon spectrometer

Lead Glass Photon Spectrometer

Los Alamos $\pi^{0}$ spectrometer

Medium resolution spect rometer for (PI,K) nuclear spectroscopy

Saclay high resolution pio and eta detector

Lexan or other such material in which tracks are frozen (except emulsion)

Plastic ball detector

Tensor deuteron polarimeter

\section{Modified PROZA}

$4.7 \times 0.9 \times 0.8 \mathrm{~m}^{3}$ streamer chamber in magnetic field

Radiative Muon Capture spectrometer

Very large water Cherenkov detector with a total mass of 50000 tons, 11200 20-inch PMT, trigger threshold $5 \mathrm{MeV} / \mathrm{c}$ for electron

High resolution electron scattering detector

High resolution electron scattering detector

Soviet-American Gallium Experiment in the Baksan Neutrino Observatory

Single arm spectrometer

Semiconductor detector

Split field magnet

CERN-IHEP magnetic spectrometer at Serpukhov

modified SIGMA spectrometer with cumulative particles arm

Upgraded large-angle solenoid detector

2-m neon filled streamer chamber

high resolution large acceptance superconducting spectrometer for nuclear spectroscopy

SLac Detector

Gamma ray spectrometer of the solar maximum mission satellite facility

Spherical Neutral Detector, 1680 NaI(TI) counters

Short Orbit Spectrometer

Detector with drift calorimeter modules, 1030 tons, at Minnesota

General spectrometer system not filling one of the others or where specific type not given

$6-m$ spect rometer

The energy loss magnetic spectrometer

Modular lead-glass Cerenkov detector

High resolution spectrometer

High resolution spectrometer 


\begin{tabular}{|c|c|c|}
\hline DETECTOR & ACCELERATOR & EXPLANATION \\
\hline SPES-III & SACL-SATURNE-II & Saclay Saturne spectrometer \\
\hline SPES-IV & SACL-SATURNE-II & High resolution spectrometer \\
\hline SPHERE & JINR & $4-\pi$ detector \\
\hline SPHINX & SERP & \\
\hline SPIN & ITEP & Magnetic superconducting spectrometer with horizontal magnetic field \\
\hline SPRK & & Spark chamber of unspecified type (use WIRE or OSPK, if possible) \\
\hline SSF & SLAC & SLAC spectrometer facility - $1.6,8$, and/or $20 \mathrm{GeV}$ spectrometers \\
\hline SSNTD & JINR & Solid State Nuclear Track Detector \\
\hline STRC & & Streamer chamber \\
\hline SUSI & PSI & Pion spectrometer \\
\hline TAGX & TOKY & Large-aperture spectrometer system \\
\hline TAPS & DGSI & Two arms photon spectrometer \\
\hline TASSO & DESY-PETRA & DESY TASSO detector \\
\hline THERMAL & & General detector based on measuring of temerature increase after particle passing \\
\hline TISS-3 & ITEP & Spark Chamber of $1.5 \mathrm{~m}$ lenght \\
\hline TOF & & Time Of Flight detecting system \\
\hline TOKIWA & KEK-PS & KEK spectrometer \\
\hline TOPAZ & KEK-TRISTAN & Solenoidal spectrometer with time projection chamber \\
\hline TPC & SLAC-PEP & Time projection chamber \\
\hline TPS & FNAL & Tagged photon spectrometer \\
\hline TRAD & & A general transition radiation detector \\
\hline TROITSK-NU-MASS & & $\begin{array}{l}\text { Setup to measure electron neutrino mass. An integral electrostatic spectrometer with adiabatic } \\
\text { magnetic collimation and gaseous tritium source }\end{array}$ \\
\hline UA 1 & CERN-PBAR/P & UA1 detector at CERN \\
\hline UA2 & CERN-PBAR/P & UA2 detector at CERN \\
\hline UA4-2 & CERN-PBAR/P & $\begin{array}{l}\text { Two pairs of "Roman Pots" placed symmetrically at } 45 \mathrm{~m} \text { from the crossing point. Main detec- } \\
\text { tor components: drift chambers, hodoscopes based on scintillating fibres, scintillation counters } \\
\text { for trigger }\end{array}$ \\
\hline VENUS & KEK-TRISTAN & Versatile economical and novel universal spectrometer \\
\hline VES & SERP & Cerenkov counter combined with gamma spectrometer \\
\hline WAS & & Wide angle spectrometer \\
\hline WIRE & & $\begin{array}{l}\text { Wire chambers (proportional wire chambers, drift chambers). Includes all nonoptical spark } \\
\text { chambers }\end{array}$ \\
\hline $\begin{array}{l}\text { X-RAY } \\
\text { ZEUS }\end{array}$ & DESY-HERA & $\begin{array}{l}\text { X-RAY spectrometer } \\
\text { ZEUS detector at DESY/HERA }\end{array}$ \\
\hline
\end{tabular}


We give here the symbols used in the Reaction/Momentum/Data Descriptor Index to indicate what quantities are measured in an experiment. 
A-DEP

AMP

ANG

ANGP

\section{ANGP*MASS \\ ANGP*P \\ ANGP*PT \\ ASYM}

COL

CONST

COR

CS

DME

ET

FLUX

FV

MANY

MASS

MASS*MASS

MULT

$\mathrm{P}$

$\mathrm{P} * \mathrm{P}$

POL

PT

PT*MASS

$\mathrm{PT}^{*} \mathrm{P}$

PWA

QNC
Atomic number dependence

Amplitudes not decomposed into states of definite angular momentum: Re/Im ratio, helicity amplitude, etc. Angular distributions between particles in the final state. Includes also angular distributions involving decay products of particles listed in the reaction, even though those decay products are not themselves explicitly listed. Includes angles used to study the decay of a system in the final state, even though the coordinate system axes may be def ined with respect to the incident particles (e.g., Jackson angles, etc.). Also the equivalent, expressed as moments, etc.

Production angular distributions of one or more of the outgoing particles relative to one of the incident particles. Also the equivalent, expressed as moments or polynomial expansion coefficients. Also invariant cross sections as a function of production angle or $t$. By convention, does not include rapidity or its approximation (see $P$ )

Biplot, note that order of description is essential

Biplot, note that order of description is essential

Biplot, note that order of description is essential

Asymmetry in scattering off a polarized target and/or with a polarized beam (with exception of special case noted under POL)

Collective variables (sphericity, thrust, etc.)

Physical constant (Fermi constant, Weinberg angle, etc.). Used toexpress that model parameters are extracted from data

General correlator (on momentum, rapidity, etc.)

Cross section, cross section ratio, or cross section upper limit.Can also be listed for very rare reactions whose existence is being established, even though the number of events has not been converted to a cross section. Does not include parametrizations of the cross section, e.g. as a function of energy

Density matrix elements, including joint density matrix elements

Transverse energy

Cosmic-ray particle flux

For proposals only. Experiment proposes to measure complete four-vector, without specifying exactly what analysis of them will be done

For rare cases when there are many types of data measured

Mass spectrum, or invariant cross section as a function of mass

Multiplicity distribution, its average, ratio or moments

Any function of outgoing momentum or energy not included in any others. Includes rapidity and Feynman scaling variables

Final state spin polarization measurement, including Wolfenstein spin rotation parameters, and measurements of the asymmetry off a polarized target when it is equal to the final state polarization

Transverse momentum spectrum, or invariant cross section as a function of above. Does not include a momentum transfer spectrum (see ANGP). Includes transverse mass, unless the particle mass is also variable

Partial wave amplitudes, including formation partial waves and production partial waves. Any attempt to measure amplitudes of definite angular momentum. Includes scattering length and effective range

Test of quantum-number conservation 